$(200)$

B no. 44 cp. 2

Professional Paper No. 44

$$
\begin{aligned}
& \text { U.J. 3 ing } \\
& 28 \times 1 \text { pent }
\end{aligned}
$$

Series $\left\{\begin{array}{l}\text { B, Descriptive Geology, } 71 \\ 0, \text { Underground Waters, } 39\end{array}\right.$

DEPARTMENT OF THE INTERIOR

UNITED S'TATES GEOLOGICAL SURVEY

ChaRLES D. WALCOT', DHector

\title{
UNDERGROUND WATER RESOURCES OF LONG ISLANI, NEW YORK
}

$\mathrm{BY}$

A. C. VEATCH, C. S. SLICHTER, ISAIAH BOWMAN, W. O. CROSBY, AND R. E. HORTON

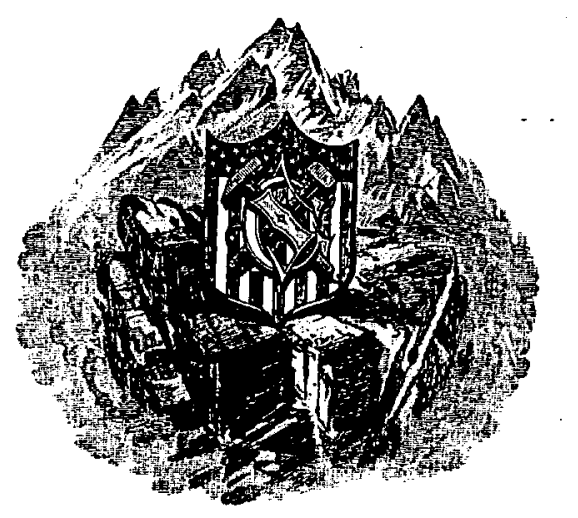

\section{8}

WASHINGTON

GOVERNMENT PRINTING OFFICE
1906





\section{CONTENTS.}

Page.

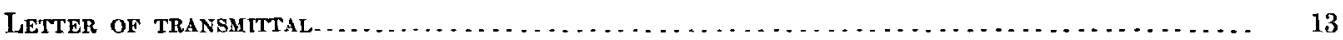

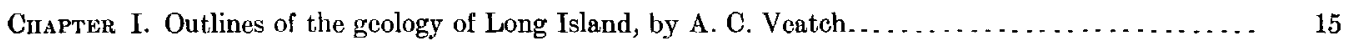

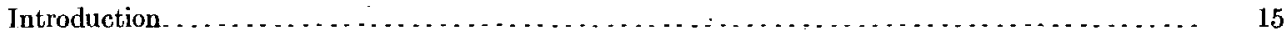

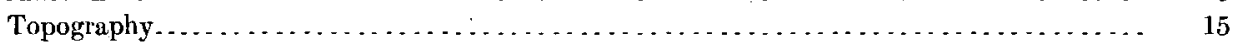

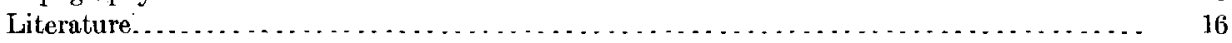

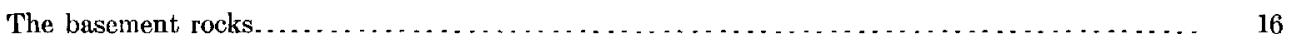

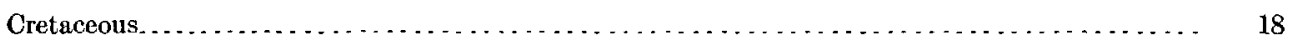

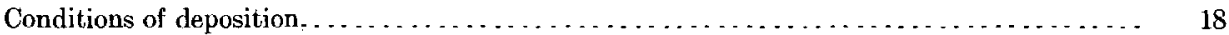

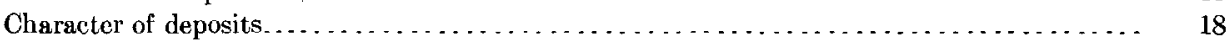

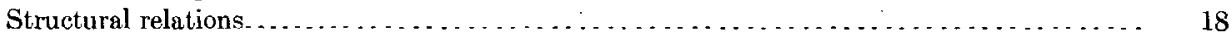

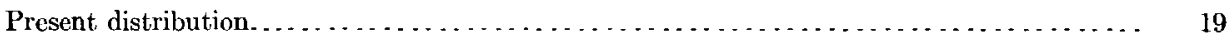

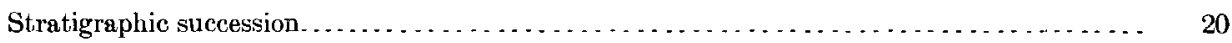

Relation to adjacent areas. . . . . . . . . . . . . . . . . .

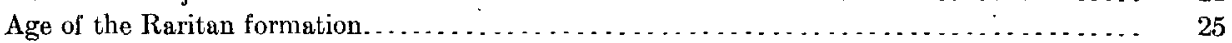

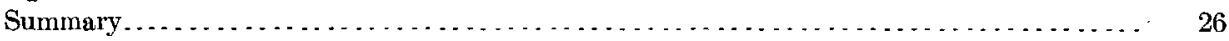

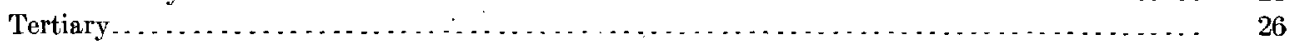

General conditions. . . . . . . . . . . . . . . . . .

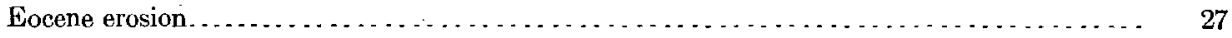

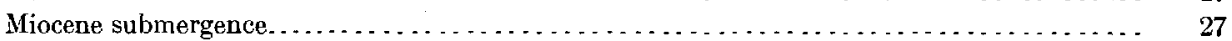

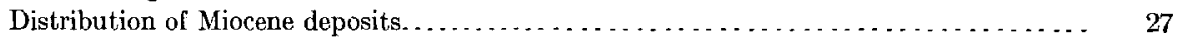

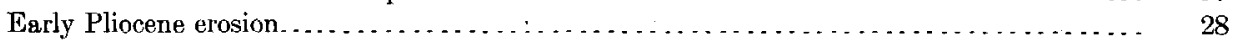

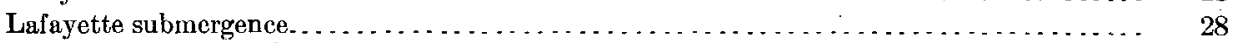

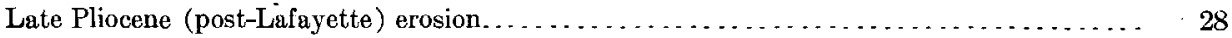

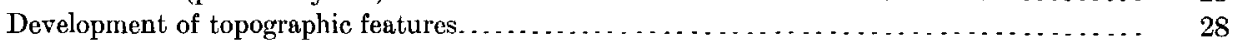

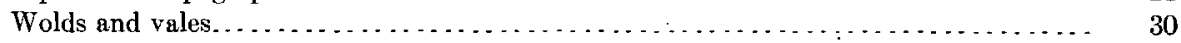

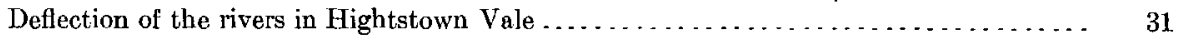

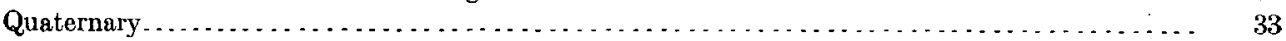

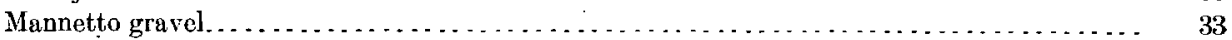

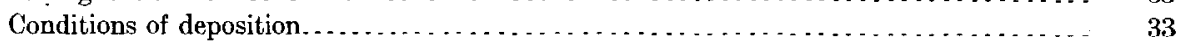

Character of deposits........

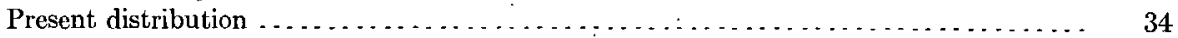

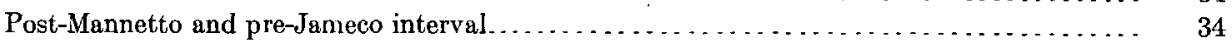

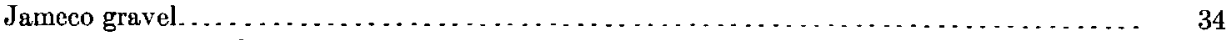

Conditions of deposition. . . . . . .

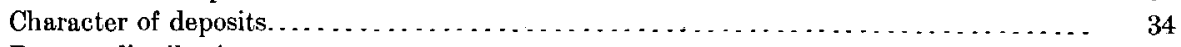

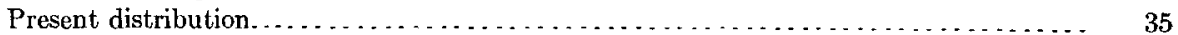

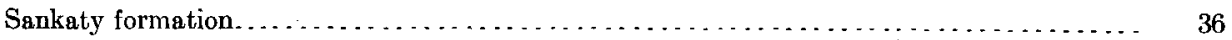

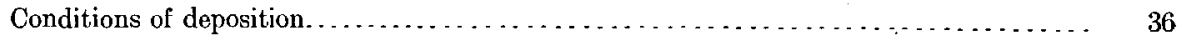

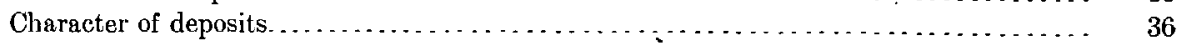

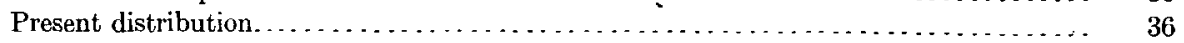

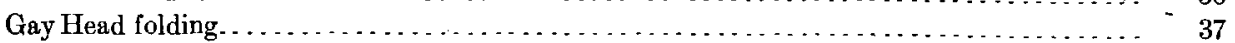

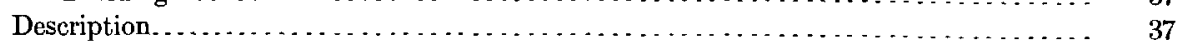

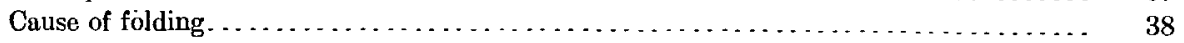

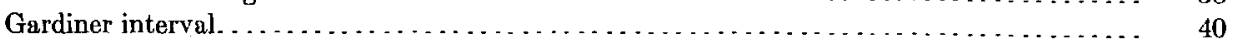

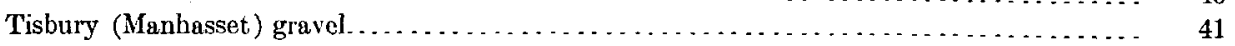

Conditions of deposition. . . . . . . . . . . . . . .

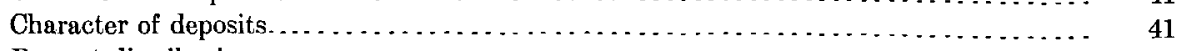

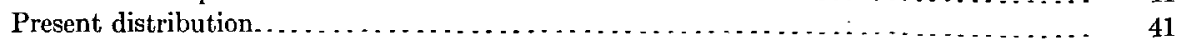


Chapter 1. Outlines of the geology of Long Island, by A. C. Veatch-Continued. Page. Quaternary-Continued.

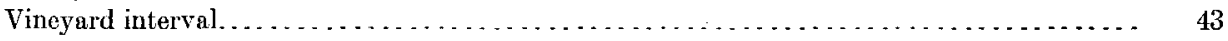

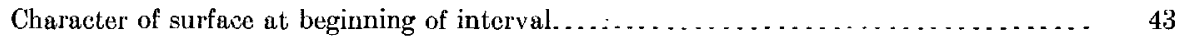

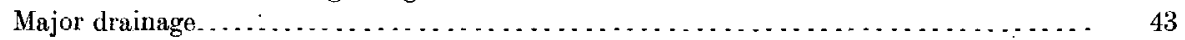

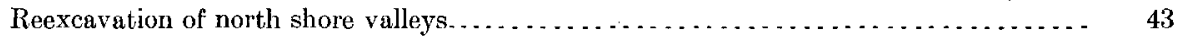

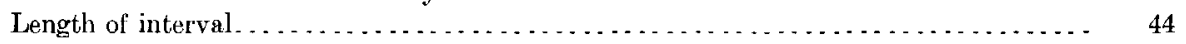

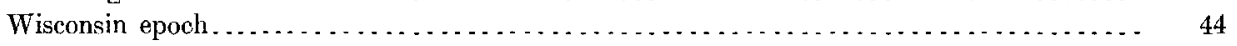

General conditions of deposition. . . . . . . . . . . . .

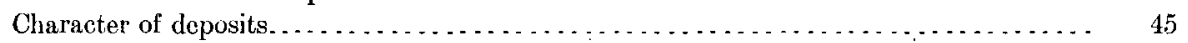

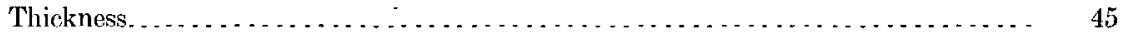

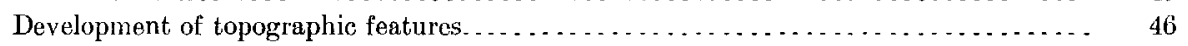

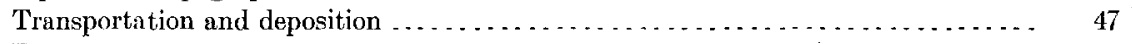

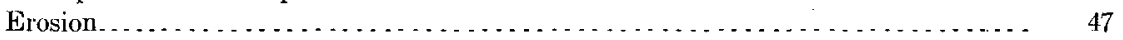

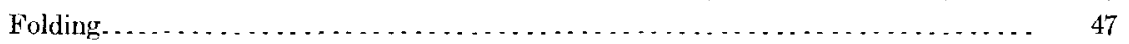

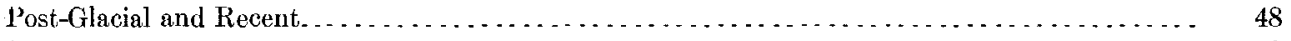

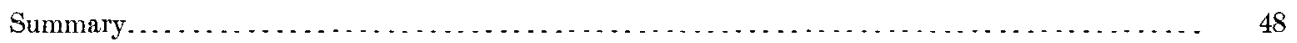

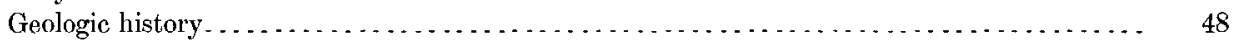

Topographic history . . . . . . . . . . . .

Chapter II. Underground water conditions of Long Island, by A: C. Veatch . . . . . . . . . 53

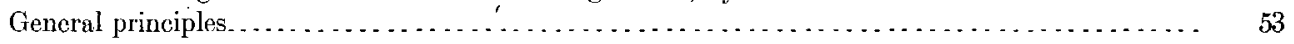

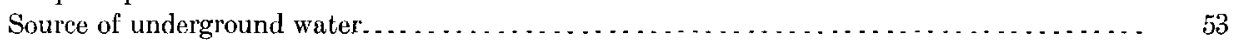

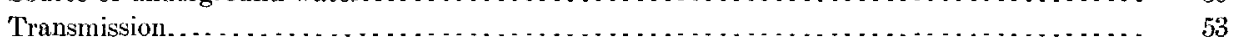

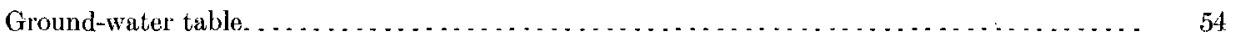

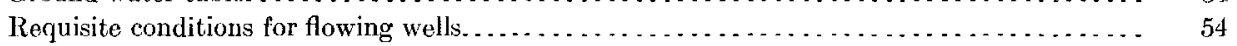

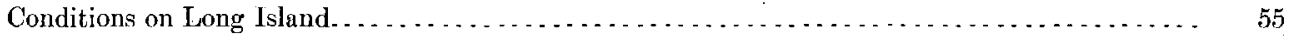

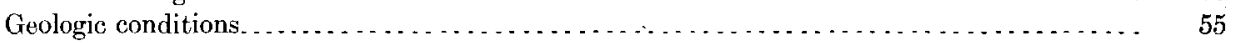

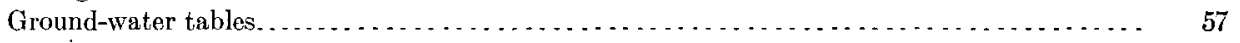

Perched water tables. . . . . . . . . . . . . . . . . . . . . . . . . . . . . . . . . . . . 57

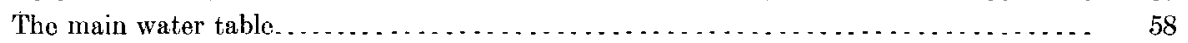

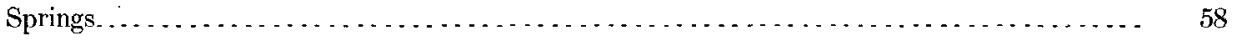

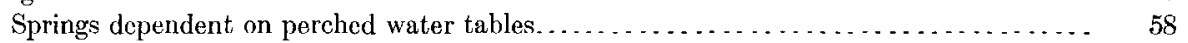

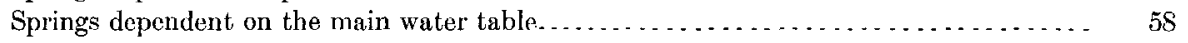

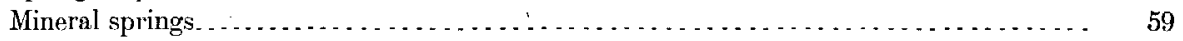

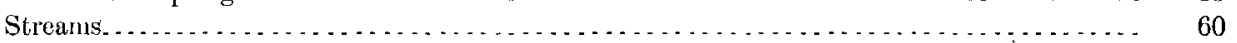

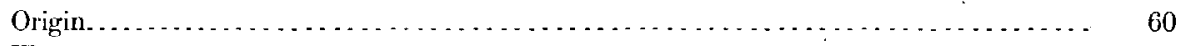

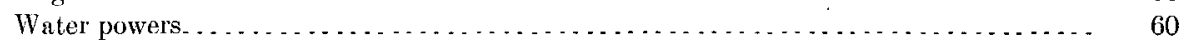

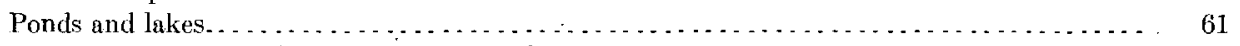

Ponds and lakes dependent on perched water tables . . . . . . . . . . . . . . . . . 61

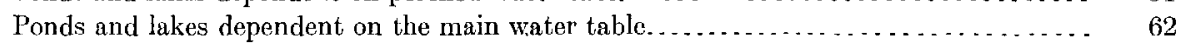

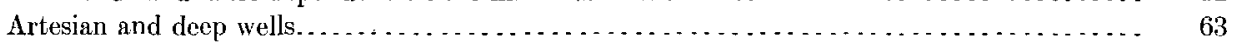

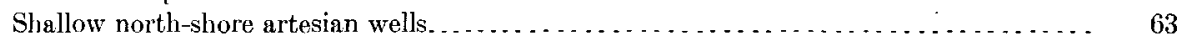

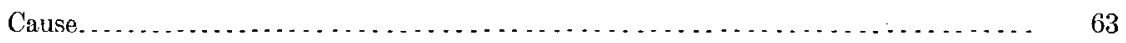

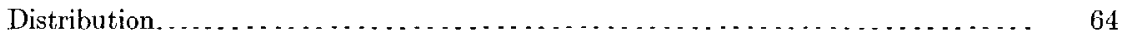

Predictions................

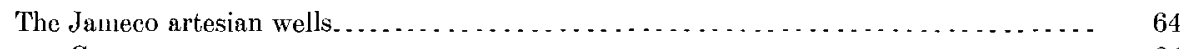

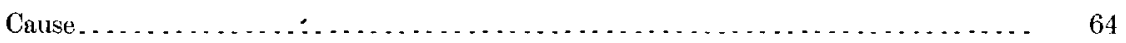

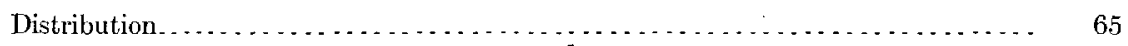

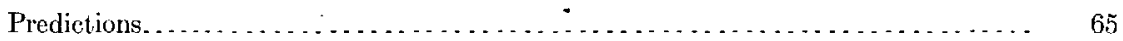

The Cretaceous artesian wells.

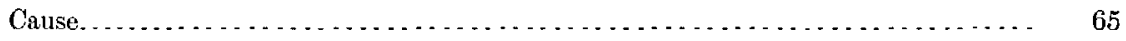

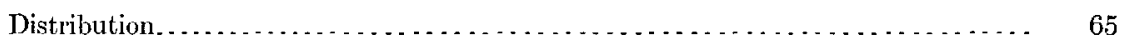

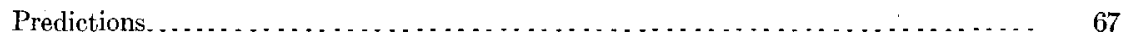

Requisite conditions for successful wells on Long Island . . . . . . . . . . . . . . . . 67

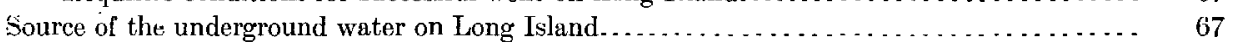


Chapter II Underground water conditions of Long Island, by A C Veatch-Contınued Page

Conditions on Long Island-Continued

Causes producing fluctuations of the ground-water table. . . . . . . . . . . . . . . 69

Natural causes........................ . 69

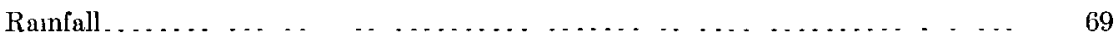

Tides. .............................................. 71

Thermometric and barometric changes............................. 72

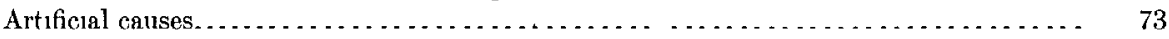

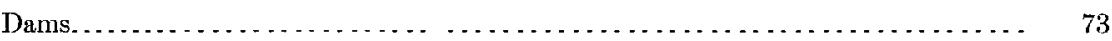

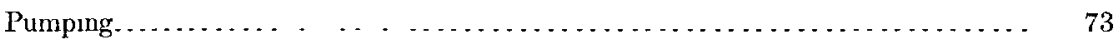

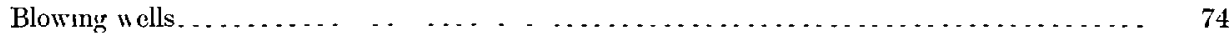

Waterworks. . . . . . . . . . . . . . . . . . . . . . . . . . . . 74

Chapter. III Measurements of velocity of underflow on Long Island, by Charles S Slıchter........ 86

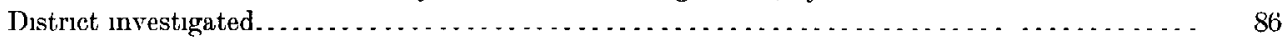

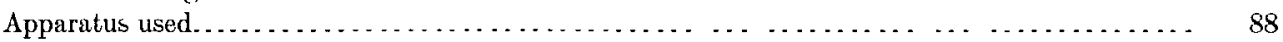

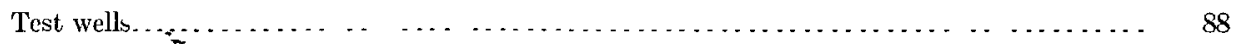

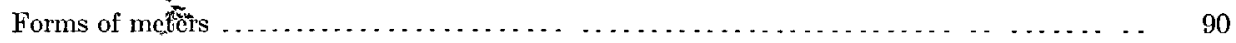

Direct-reãdıng meters. . . . . . . . . . . . . . . . .

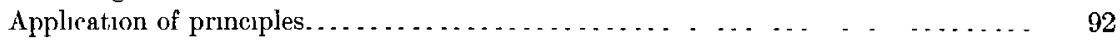

Self-recording meter........................................... 97

Principles involved. . . . . . . . . . . . . . . . . . . . . . . . . . . . . . . . . . 99

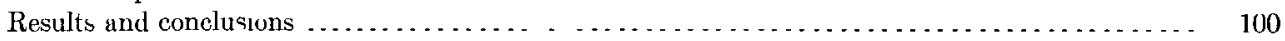

Existence of underflow. ... . . . . . . . . . . . . . . . . . . . . . . . . . 100

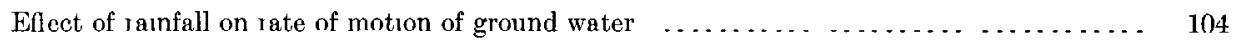

Effect of seepage water from ponds and reservors on rate of motion $\ldots \ldots \ldots \ldots \ldots$

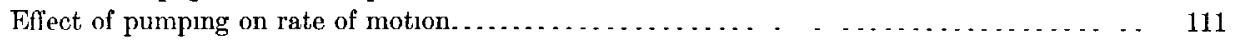

Specific capacity .......................................... 114

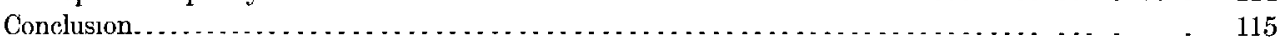

Chapter IV Well records, compled by A $\mathrm{C}$ Veateh and Isalah Bowman.................. 116

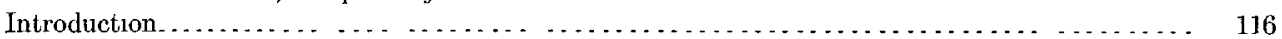

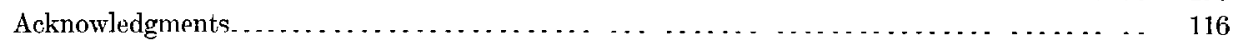

Representative wells. . . . . . . . . . . . . . . . . . . . . . . . . . . . . . . 118

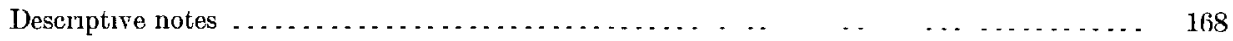

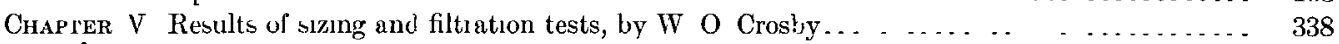

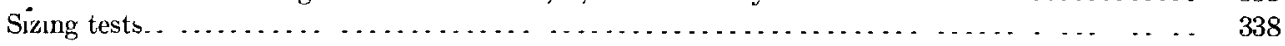

Filtration tests .. . . . . . . . . . . . . . . . . . . . . . . . . . . . . 354

Chapter VI The surface streams of Long Island, by $\mathrm{R}$ F Horton $\ldots \ldots \ldots \ldots \ldots$

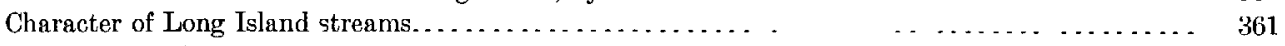

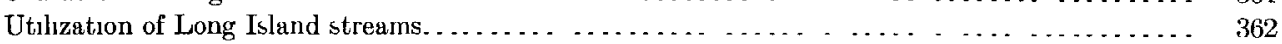

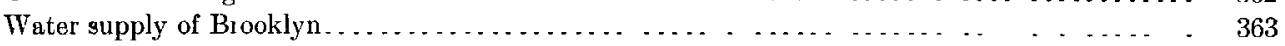

Gagings of Long Island streams. . . . . . . . . . . . . . . . . . . . . . . . . . . . $\quad \ldots 65$

East Mcadow Brook near Freeport. . . . . . . . . . . . . . . . . . . . . . . . . . . 368

Newbridge streams near Merrick . . . . . . . . . . . . . . . . . . . . . . . . . . . . . . . 370

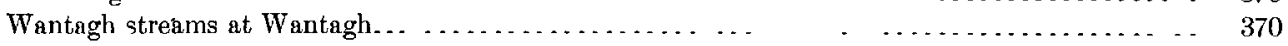

Massapequa Creek at Farmingdale and Freeport. . . . . . . . . . . . . . . . . . . . . 371

Carlls River at Babylon. . . . . . . . . . . . . . . . . . . . . . . . . . . . . . . . . . . 373

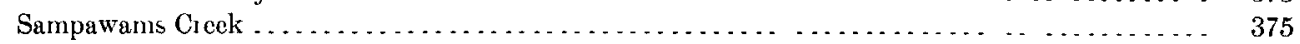

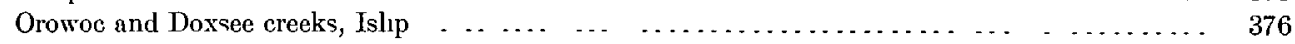

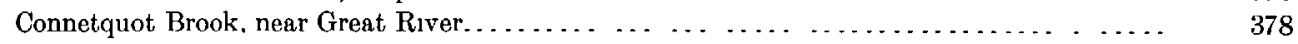

Lake Ronkonkoma and adjacent streams........... . . . . . . . . . . . . . 379

Carmans River (or Connecticut River of Long Island) ...... . . . . . . . . . . . . . 380

Pecontc River......................................................... 381

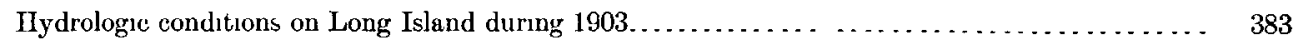





\section{T A B LES.}

T. Chete. Page.

I. Cretaccous and Tertiary formations of New Jersey........................... 21

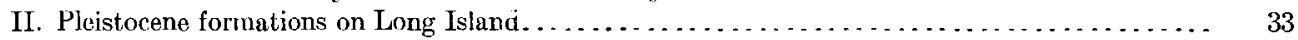

III. Thirkness of late Pleistocene deposits in wells on the north shore of Long Island . . . . . . . . . . . 42

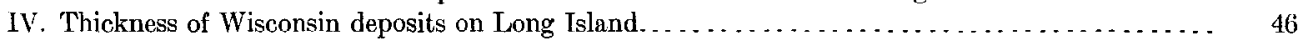

V. Wells in the Lloyd gravel. ... . . . . . . . . . . . . . . . . . . . . . . . . 65

VI. Analyses showing difference between water from the Lloyd sand and from the rock wells of Connecticut.

VII. The effect of ground-water pumping in diminishing stream flow from 1873 to 1889 in the old watershed of the Brooklyn waterworks, comparing five-year periods . . . . . . . . . . . . .

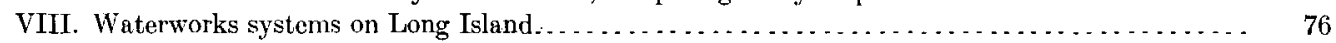

1X. Station No. 1, Massapequa, Long Island, June 21, 1903: Field record of electric current reading

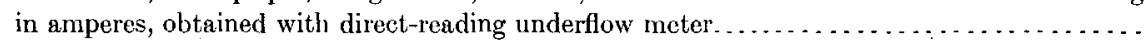

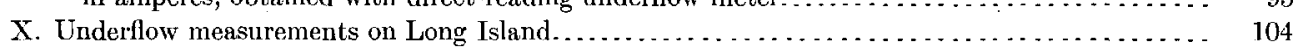

XI. Representative wells on Long Island. . . . . . . . . . . . . . . . . . . . . . . . . . . . 118

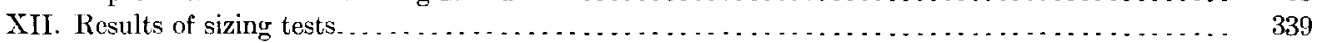

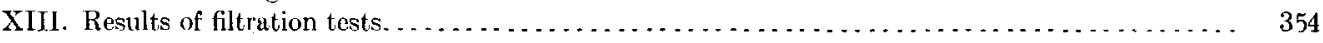





\section{ILLUSTRATIONS.}

Plate I. Map showing data bearing on the position of bed rock in western Long Island, and vicinity

II. Map showing structure of the basal Cretaceous beds on Long Island, and their relation to

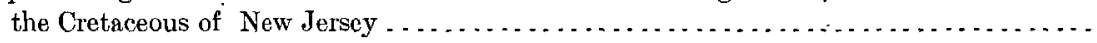

III. Map showing the distribution of the Cretaceous on western Long Island. . . . . . . . . . . .

IV. $A$, Mannetto gravel near top of Melville section; $B$, Cretaceous sand near the base of the MeI-

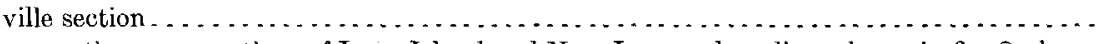

V. Comparative cross sections of Long Island and New Jersey, along lines shown in fig. 8, show-

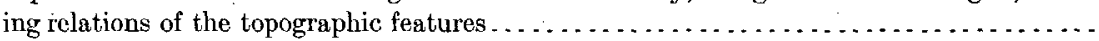

VI. Development of the major drainage of the North Atlantic coastal plain . . . . . . . . . . . .

VII. Broken Grounds, near Fresh Pond, Long Island . . . . . . . . . . . . . . . . . . . . . .

VIH. $A$ and $B$, King's sand pit, Hempstead Harbor, showing the Manhasset bowlder bed. . ......

IX. $A$, A portion of the Harbor Hill outwash plain over the Tisbury terrace, south of Huntington, N. Y.; $B$, A bowldery portion of the Harbor Hill moraine near Creedmoor, N. Y. . . . .

$\mathrm{X}$. Hooked sand spit at entrance to Smithtown Harbor, Long Island . . . . . . . . . . . . . . .

$\mathrm{XI}$. Cross sections of Long Island, along lines given on Pl, XII . . . . . . . . . . . . . . .

XII. Map showing position of the main ground-water table on Long Island, on July 1, 1903.. In' pocket.

XIII. Views showing head developed in the north shore artesian wells: $A$, At Oyster Bay, Burgess well; $B$, At Cold Spring Harbor, Jones well . . . . . . . . . . . . . . . . . . . . . . . .

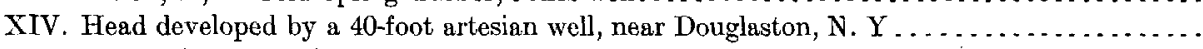

XV. Map of Long Island, showing north shore and Jameco artesian well areas . . . . . . . . . . . .

XVI. Map of Long Island, showing probable Cretaceous artesian well area; and depth of Lloyd gravel below sea level. . . . . . . . . . . . . . . . . . . . . . . . . . . . . . . . . .

XVII. Fluctuations of the main ground-water table on Long Island . . . . . . . . . . . . . . . 70

XVIII. Examples of fluctuations due to thermometric and barometric changes.............. 72

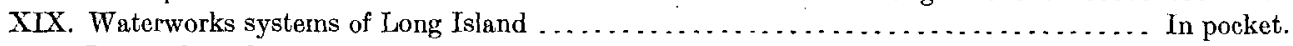

$\mathrm{XX}$. Electrode and perforated brass buckets used in charging wells . . . . . . . . . . . . . 90

XXI. $A$, Underflow meter showing connections when used as direct reading apparatus; $B$, Commu-

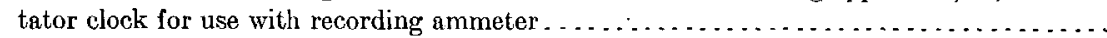

XXII. $A$, Commutator clock for use with recording ammeter; $B$, View of recording ammeter, commu-

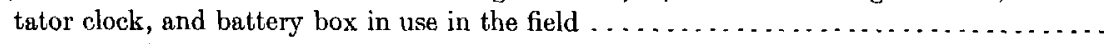

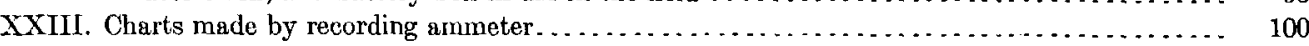

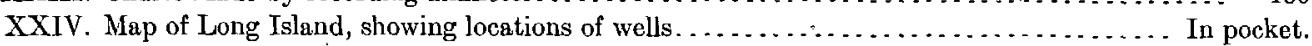

XXV. Plan and longitudinal section of strata encountered in the South Brooklyn sewer tunnel.... 168

XXVI. 'Test borings of Rapid Transit Railroad Commission across East River. . . . . . . . . . . . . . 170

XXVII. Test borings of Rapid Transit Railroad Commission from East River to De Kalb avenue,

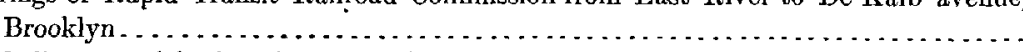

XXVIII. Map and diagram of borings for Pennsylvania, New York and Long Island Railroad tun-

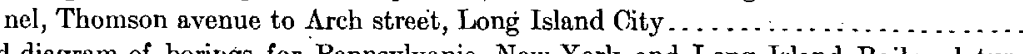

XXIX, Map and diagram of borings for Pennsylvania, New York and Long Island Railroad tunnel, Arch street to Vernon avenue, Long Island City . . . . . . . . . . . . . . . . .

16

18

20

22

30 .

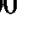
s8

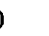


Plate XXX. Map and diagram of borings for Pennsylvania, Now York and Long Island Railroad tunnel, Vernon avenue to East River, Long Island City . . . . . . . . . . . . . . . . . .

XXXI. Map and diagram of borings for Pennsylvania, New York and Long Island Railroad

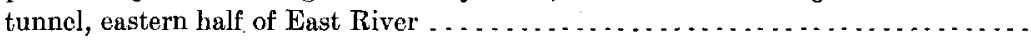

XXXII. Map and diagram of borings for Pennsylvania, New York and Long Island Railroad tunnel, western half of East River . . . . . . . . . . . . . . . . . . . . . . . . . . . .

XXXIII. Map and diagram of borings for Pennsylvania, New York and Long Island Railroad tunnel, East River to First avenue, New York City . . . . . . . . . . . . . . . .

XXXIV. Record of test borings made at Long Island City pumping station No. 3 (No, 99) ....

FIG. 1. Sections from Hudson River to Long Island, showing the general folded and eroded character of the bed rock underlying Long Island . . . . . . . . . . . . . . . . . . . . . . . . . .

2. Map showing dip of Cretaceous beds near Setauket, N. Y . . . . . . . . . . . . . . . .

3. Section from Delaware River to Pipers Corner, N. J . . . . . . . . . . . . . . . . . . . . .

4. Sketch map showing known distribution of the Mioceno near Long Island . . . . . . . . . . . .

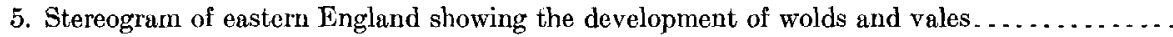

6. Diagram showing the three uses of "escarpment" as applied to wold $\ldots \ldots \ldots \ldots \ldots \ldots \ldots$.

7. Diagram showing relations of wold, vale, cuesta, and bajada . . . . . . . . . . . . . . .

8. Sketch map showing locations of sections shown on Pl. V . . . . . . . . . . . . . . . . .

9. Comparative maps, showing deflection of streams in the Hightstown Vale, and the deflection which would be produced by the large Texas bars if the land were elevated ...............

10. Section from near Ridgewood, Brooklyn, to Valley Stream, showing position of the Wisconsin, Tisbury, Sankaty, Jameco, and Cretaceous beds, and the east side of the Sound River Valley

11. Section near middle of northeast shore of Gardiners Island, New York . . . . . . . . . . . . . .

12. Section on west side of the hollow which afforded the section in fig. 11, about 200 feet farther

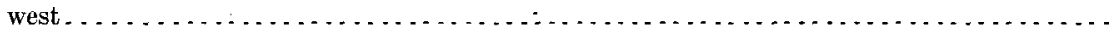

13. Section from Wards Island to Barnums Island, showing fold at Rockaway Ridge (Hewlett), and relations of the (1) Sankaty, (2) Jameco, (3) Cretaceous, and (4) "bed rock" .....

14. Section at Tobacco Point, east side of Gardiners Island, New York . . . . . . . . . . . . . . .

15. Section near Cherry Hill Point, Gardiners Island, showing location of fossil-bearing stratum..

16. Cross section through Oyster Bay and Center Island, showing relations of clay and waterbearing horizons encountered in the Oyster Bay wells to the Cretaccous clays and Lloyd

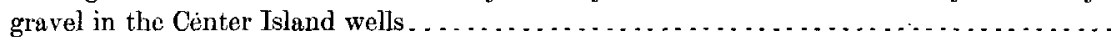

17. Cross section at Gay Head, Marthas Vineyard . . . . . . . . . . . . . . . . . . . . .

18. Sections exposed at Browns Point, after storm of October 11 and $12,1836 \ldots \ldots \ldots \ldots \ldots$

19. Diagram illustrating factors giving spring phenomena great power in reexcavating the north shore valleys . . . . . . . . . . . . . . . . . . . . . . . . . . . . . . . . . . . . . . .

20. Sketch map showing relative positions of the ice during the Ronkonkoma and Harbor Hill

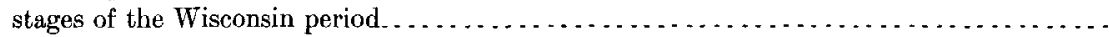

21. Diagram showing ground-water table unaffected by surface features. $\ldots \ldots \ldots \ldots \ldots \ldots$.

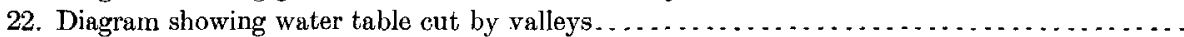

23. Diagram showing common arrangement of factors producing artesian wells . . . . . . . . . .

24. Diagrammatic cross section of Long Island, showing general water conditions and cause of flow-

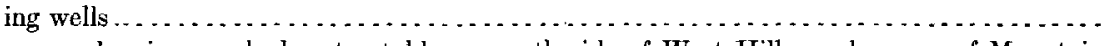

25. Diagram showing perched water table on north side of West Hills, and source of Mountain Mist Springs ....

26. Diagrams showing analogy between a well and a channel that cuts the ground-water table...

27. Sketch map showing increase in spring flow along Hempstead Brook. From data collected by the Brooklyn waterworks.

28. Sketch map of Long Island, showing distribution of water power developments, 1800-1900.

29. Lake Success; an example of a kettle-hole lake depending on local impervious strata........

30. Diagram showing effect of a pond on the ground-water table, and the consequent decrease in

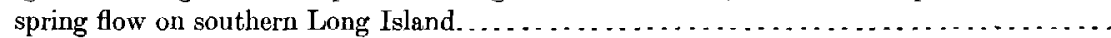

31. Diagram showing loss of water by leakage from pond whose surface is above the adjacent ground-water table.

Page. 
FIG. 32. Lake Ronkonkoma; an example of a kettle-hole lake depending on the main ground-water

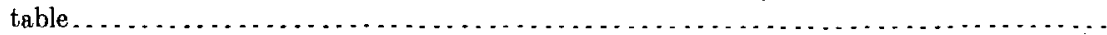
33. Artesian well or spring (No. 335) at Manhasset, from a drawing by J. H. L'Hommedieu..... 34. Autograph record of water level in a 386-foot well at Long Beach, N. Y., showing fluctuations due to tides.

Page.

35. Record of water level in a 40-foot well of the Citizens' Water Supply Company at Douglaston,

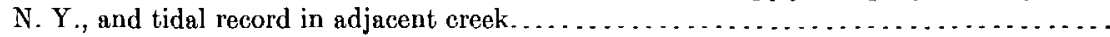

36. Diagram showing cone of depression produced by a pumping station, and its effect on a nearby pond and well.

37 Map of southern Long Island showing location of underflow stations at which determinations of

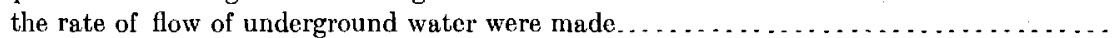

38. Plan of arrangement of test wells used in determining the velocity and direction of motion of

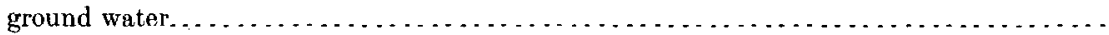

39. Diagram showing clectric method of determining the velocity of underground water .......

40. Curve of velocity and underflow measurements, San Gabriel River, California . . . . . . . . . . .

41. Curves showing the possibility of using direct-reading apparatus when well points are not used .

42. Diagram showing manner in which the electrolyte spreads in passing downstream . . . . . . . .

43. Diagram showing spread of electrolyte from a well in which the water is moving about twice as fast as in fig. 42 ....

44. Diagram showing velocity and direction of flow of underoround water at Wantagh pumping station.

45. Diagram showing velocity and direction of flow of underground water at Agawam pumping station (station 5) . . . . . . . . . . . . . . . . . . . . . . . . . . . . . . . . . .

46. Diagram showing velocity and direction of flow of underground water at Agawam pumping

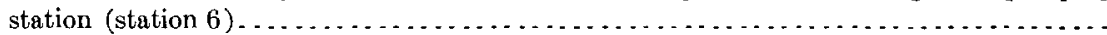

47. Diagram showing velocity and direction of flow of underground water at East Meadow Brook and Babylon road (station 7 ) . . . . . . . . . . . . . . . . . . . . . . . . . . . . .

48. Diagram showing velocity and direction of flow of underground water near Merrick pumping

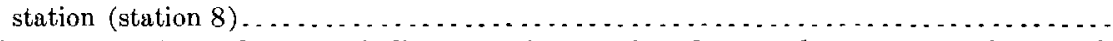

49. Diagram showing velocity and direction of flow of underground water at Cedar Brook (station 10) . . . . . . . . . . . . . . . . . . . . . . . . . . . . . . . . . . . .

50. Diagram showing velocity and direction of flow of underground water at Grand avenue and Newbridge Brook (station 12) . . . . . . . . . . . . . . . . . . . . . . . . . . . . .

51. Diagram showing velocity and direction of flow of underground water at Bellevue road

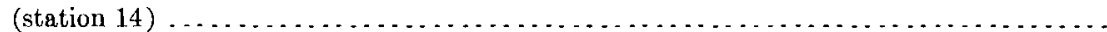

52. Diagram showing velocity and direction of flow of underground water at Bellevue road

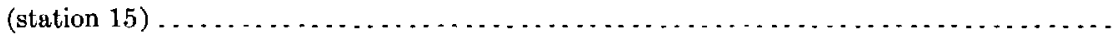

53. Diagram showing velocity and direction of flow of underground water at Bellevue road

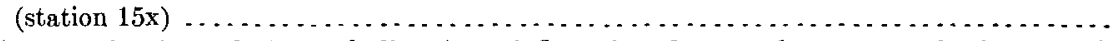

54. Diagram showing velocity and direction of flow of underground water south of Wantagh

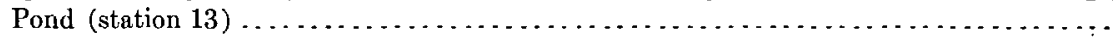

55. Diagram showing velocity and direction of flow of underground water at Wantagh Pond (station $16 \mathrm{x}$ )

56. Diagram showing velocity and direction of flow of underground water at Wantagh Pond (station 17).

57. Diagram showing velocity and direction of flow of underground water above Wantagh Pond

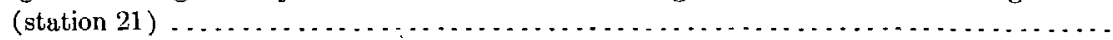

58. Map showing locations of stations 5 and 6 with reference to Agawam pumping station and East Meadow Pond .

59. Vertical section throul stations 5 and 7 aid test wells in Agawam Pond shown in fi....

60. Map showing locations of stations $2,13,16$, and 17, near Wantagh pumping station and Wantagh Pond

61. Sketch map showing location of deep wells of the Fleischmann Manufacturing Company at

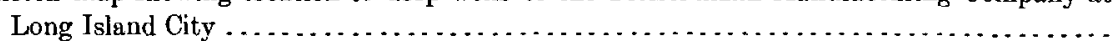

63

64

70

71

72

87

88

89

91

92

93

94

98

99

100

101

102

103

105

106

107

108

109

110

111

112

113

112

114

180 
Fis. 62 Index map showing location of PIs. XXVIII-XXXIII

63. Type of well used at the Montauk waterworks plant, at Dunton, N. Y $\ldots \ldots \ldots \ldots \ldots \ldots$

64. Sketch map showing location of test borings at Bayside pumping station . . . . . . . . . . . . .

65. Sketch map giving locations of wells of the Queens County Water Company, shown in fig. 66. .

66. Sections of wells of the Queens County Water Company, by Charles R. Bettes, chief engincer.

67. Sketch map showing location of wells described at Oyster Bay $\ldots \ldots \ldots \ldots \ldots \ldots \ldots \ldots$.

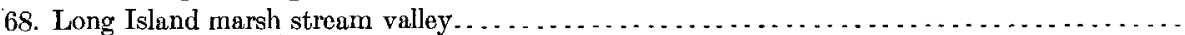

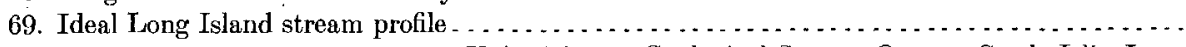

70. Temporary gaging station of the United States Geological Survey, Orowoc Creek, Islip, Long Island, June 7, 1903. Plan shows bridge floor removed . . . . . . . . . . . . . . . . .

71. Weir on private pond, Cutting Creek, near Great River, Long Island . . . . . $\ldots \ldots \ldots \ldots$ 


\title{
LETTER OF TRANSMITTAL.
}

\author{
Department of the Interior, \\ United States Geological Survey, \\ Hydrograpuic Branch, \\ Washington, D. C., July 7, 1904 .
}

SIR: I transmit herewith the manuscript of a paper entitled "The Underground Water Resources of Long Island, New York," by Messrs. A. C. Veatch, Charles S. Slichter, Isaiah Bowman, W. O. Crosby, and R. E. IIorton. The field work upon which the report is based formed a part of a detailed investigation of the geology and water resources of the island conducted by Mr. M. L. Fuller, chief of the eastern scetion of the division of hydrology, assisted by Mr. Veatch, to whom was given the immediate supervision of problems relating to underground waters.

The paper deals with an area in which the problems relating to underground waters are of great importance, especially as they affect city and town supplies. Great interest is manifested in such waters throughout the area, and it is thought that the report, which is the result of unusually detailed work, will prove of great value to engineers and others who may be interested in public or private supplies from underground sources.

A separate report, treating the geology of the island in more detail, has been prepared by Mr. Fuller and will soon be transmitted for publication.

Very respectfully,

Hon. Charles D. WalcotT,

$$
\begin{aligned}
& \text { F. H. NeweLL, } \\
& \text { Chief Engineer. }
\end{aligned}
$$

Director United States Geological Survey. 



\title{
UNDERGROUND WATER RESOURCES OF LONG ISLAND, NEW YORK.
}

\author{
By A. C. Veatch, Charles S. Slichter, Isaiah Bowman, W. O. Crosby, \\ and R. E. Horton. \\ C H A P T E R I. \\ OUTLINES OF THE GEOLOGY OF LONG ISLAND.
}

Bу А. С. Уеaтсн. ${ }^{a}$

INTRODUCTION.

As Long Island is the largest island on the eastern coast of the United States, and is of such size, 120 miles long and 23 miles wide, that it is a more or less noticeable feature on even very small-scale maps, little need be said of its general geographic position.

TOPOGRAPHY.

In shape Long Island resembles a huge fish, with the head toward New York. This rude resemblance caused the early whalers to apply the names North Fluke and South Fluke to the two projections which form the tail.

A range of hills having a relief of from 100 to 200 feet gives topographic expression to each of the flukes; and continuing westward, these ranges coalesce north of the center of the island near the Suffolk-Nassau county line, where they reach their maximum elevation of 420 feet at High Hill. Westward a group of rolling hills, occasionally reaching a height of over 300 feet, and not separable into distinct lines, continues to the Narrows at Brooklyn. South of these hill ranges the land is comparatively level and slopes off gently to the sea or forms more or less elevated table-lands between the two lines of hills. The northern shore, skirted by the hills, is rugged and precipitous, with long, narrow bays, while the southern shore passes gradually from a gently sloping plain into a salt marsh inclosing broad, shallow bays, beyond which is a barrier beach.

" $A$ more detailed report on the geology of Long Island is now in preparation, and the discussion of local data, as well as questions of correlation, has therefore been omitted in this outline, which has been condensed from the writer's complete report on the geology. 
The hills are very irregular and even the plains between the two ranges of hills are by no means level, but are pitted by somewhat circular depressions found in glaciated regions and commonly called "kettle holes." On Long Island many of those contain water, forming charming little lakes and ponds, which add much to the picturesqueness of the region.

In general the topography has a glacial aspect, and the glacial forms are so prominent that the fact that the major topographic features are of pre-Glacial origin is commonly overlooked.

\section{LITERATURE.}

The literature dealing with the geology and water resources of Long Island is very extensive, and will be presented in detail in a paper on the Geology of Long Island, now in preparation. Only a few of the more important titles are presented in the accompanying list:

Mather, W. W. Geology of the first geological district. Geol. New York, pt. 1, 1843.

Lewis, E. Ups and downs of Long Island. Pop. Sci. Monthly, vol. 10, 1877, pp. 434-446.

Upham, Warren. Terminal moraines of the North American ice sheet. Am. Jour. Soi., 3d ser., vol. 18, 1879, pp. 81-92, 197-209.

DANA, J. D. Long Island Sound in the Quaternary age, with observations on the submarine Hudson River channel. Am. Jour. Sci., 3d ser., vol. 40, 1890, pp. 425-437.

Merrili, F. J. IT. Geology of Long Island. Annals New York Acad. Sci., vol. 3, 1886, pp. 341-364.

Hollick, Arthur. Preliminary contributions to our knowledge of the Cretaceous formation of Long Island and eastward. Trans. New York Acad. Sci., vol. 12, 1893, pp. 222-237.

Some further notes on the geology of the north shore of Long Island. Trans. New York Acad. Sci., vol. 13, 1894, pp. 122-130.

- Dislocations in certain portions of the Atlantic constal plain strata and their probable causes. Trans. . New York Acad. Sci., vol. 14, 1894, pp. 8-20.

De Varona, I. M. History and description of the water supply of the eity of Brooklyn. 1896, $306 \mathrm{pp}$., 8 tables, 45 pls. (Gives bibliography of the Brooklyn waterworks on pp. 301-306.)

Freeman, John R. Report upon New York's water supply. New York, 1900, 587 pp., 113 figs.

The Merohants' Assoctation. The water supply of the city of New York. 1900, 62 pp., 25 pls.

Ries, Heinrich: Clays of New York. Bull. New York State Mus., No. 35, 1900, pp. 495, 5572, 573, 595-607, $692,817-822$.

Woodworth, JAY Backus. Pleistocene geology of portions of Nassau County and Borough of Queens. Bull. New York State Mus., No. 48, 1901.

Salisbury, R. D. Description of New York City. Geologic Atlas U. S., folio 83, U. S. Geol. Survey, 1902.

Spear, WaLter E. Long Island sources. Rept. Commission on Additional Water Supply for the City of New York, Nov. 30, 1903, New York, 1904, appendix 7, pp. 617-806.

THE BASEMENT ROCKS.

Although bed rock underlies all Long Island at a greater or less depth, it outcrops only along East River, at Long Island City and Astoria, where Merrill has recognized two divisions-the Fordham gray gneiss and the Stockbridge dolomite, the former of probable pre-Cambrian and the latter of Silurian or CambroSilurian age." In the Fordham gray gneiss are occasional dikes and bosses of granite and intrusions of diorite.

These rocks are the remnants of strata which were profoundly altered by pressure and heat, by folding and faulting, and then reduced by erosion (fig. 1), dur-

a Merrill, F. J. H., Description New York City, Geologic Atlas U. S., folio 83, U. S. Geol. Survey, 1902, pp. 3-5. 
ing the ages that elapsed between the Silurian and the Cretaceous periods. The many changes of this old land surface and its topographic aspect at different stages before the Cretaceous can only be partly outlined, but the history since the beginning of the Cretaceous can be inferred more or less completely.

The surface of these older beds, so far as it has been revealed on Long Island by borings that penetrate the mantle which has protected it from erosion since the early Cretaceous, has a few minor irregularities, but, on the whole, slopes gently to the south and east at a rate of about 100 feet per mile. The unevenness of the present surface is very slight when compared with the great irregularity (fig. 1) indicated by the structure On Pl. I is shown the depth to bed rock in the western portion of the island; in the eastern part of the island the depths at which bed rock was encountered, at 655 feet at Greenport $\left(892^{\prime \prime}\right)$ and at 150 feet at Fishers Island $\left(919^{a}\right)$, show a similar slope.
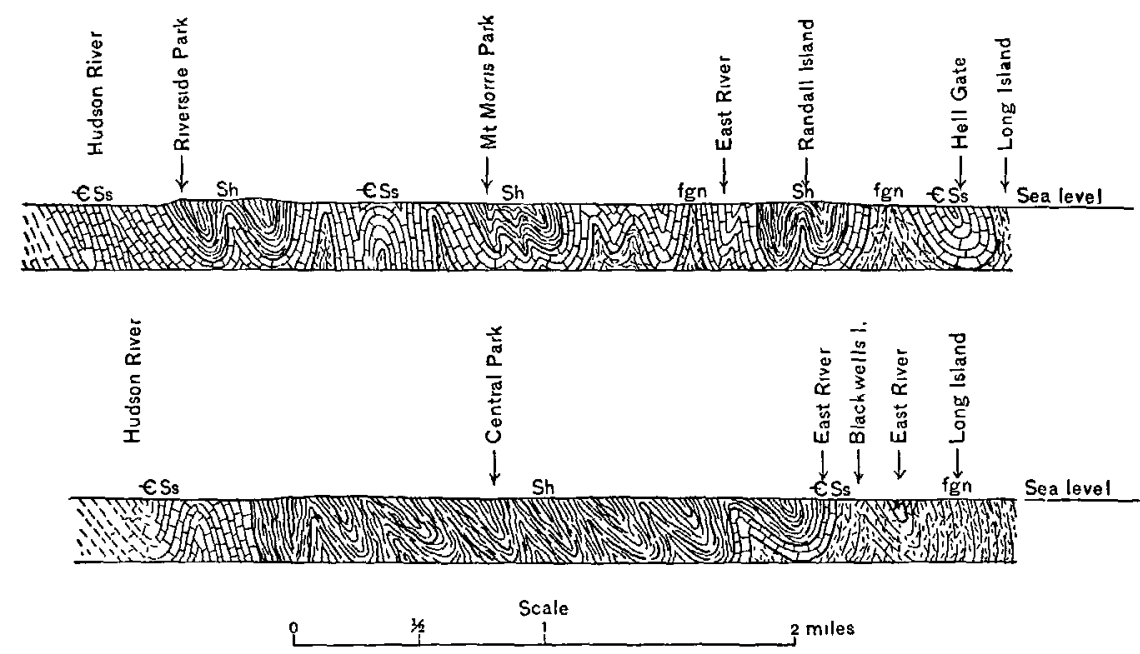

Fig 1 -Sections from Hudson River to Long Island, showing in a general way the folded and eroded character of bed rock underlyung Long Island (Merrill 1902), fgn Fordham guess (pre-Cambrian), ess, Stockbridge dolomite (CambroSilurian), Sh, JIudson schist (Silurıa)

This sloping surface, with its minor irregularities, was probably at one time nearly horizontal and formed a part of the great, almost level, plain known as the Schooley peneplain, which extended over a large part of the eastern United States and which resulted from long-continued erosion under very uniform conditions. It owes its present slope or dip to the very broad folding which began near the beginning of the Cretaceous and which, after several minor halts and fluctuations, elevated the Schooley Mountain in New Jersey 1,500 feet and depressed the old surface in the Long Island region.

a The numbers given in parentheses throughout this paper correspond with those used on Pl XXIV and in Chapter IV, where detaled records are given.

bDavis, W M., and Wood, J. W, Geographic development of northern New Jersey. Proc Boston Soc. Nat. Hist, vol. 24, 1890, pp 365-423 Willis, Bailey, The northern Appalachians. Mon Nat. Geog. Soc., vol 1, No.6, pp. 169-202, 1895, Salisbury, R D. Phys. Geog New Jersey Final report State geologist New Jersey, vol. 4, 1898, pp. 83-85. 
CRETACEOUS.

CONDITIONS OF DEPOSITION.

By this change of level at the beginning of the Cretaceous period the sea again covered this area, and the rejuvenated streams carried into it the deeply weathered material from the surface of the old Schooley peneplain. The strata for 300 or 400 feet above the bed rock are therefore composed almost entirely of the products of long-continued weathering and present a peculiar mingling of sand and plastic clays, often brightly colored, which are more or less distinct from the beds that follow.

CHARACTER OF DEPOSITS.

These irregular-bedded varicolored clays with light-colored quartz sands and gravels, which characterize the base of the Cretaceous system in this region, show an increasing percentage of sand in their upper portions, and pass more or less gradually, on the north shore, into the light-colored quartz sands with occasional irregular clay beds which form the upper strata of the pre-Pleistocene series, and, on the south shore, into the fine gray lignite-bearing sands and clays of the same age. The thick greensand marls of the New Jersey section are almost wholly absent, their presence being merely suggested in the West Hills, at Quogue and Bridgehampton. The absence of greensand marls, the extreme scarcity of marine fossils, and the presence of plant remains, indicate shoal water, or near-shore conditions during the several epochs in which these rocks were deposited.

As a result of the long-continued weathering to which all the material compos- ing these beds has been subjected, the sand beds lack the readily broken-down minerals so common in glacial deposits, and the gravel beds do not contain compound crystalline or transported clastic pebbles. The gravels from the lowest to the highest (with but one doubtful exception) are composed of quartz or locally derived quartz-conglomerate, with occasional very much decomposed milk-white chert fragments. This difference in composition is the most serviceable criterion for separating the pre-Pleistocene from the Pleistocene beds in this region.

\section{STRUCTURAL RELATIONS.}

These Cretaceous beds are now not only almost entirely hidden by Pleistocene deposits, but are so disturbed in the few limited outcrops on the north shore (PI. III) that neither the original slope of the strata, the amount of deformation, either horizontal or vertical, nor the relation of one outcrop to another can be satisfactorily determined. The apparently undisturbed outcrop in the West Hills furnishes no extended exposure, and even here the structure is concealed by hill creep and landslides.

Any knowledge of the structure is, therefore, dependent upon well records, and these have fortunately revealed a key bed that is not only satisfactorily persistent on the island, but continues in New Jersey, and furnishes a new basis for a comparison of the stratigraphy. A critical study showed that the top of a waterbearing sand situated 150 to 200 feet above bed rock in 14 north shore wells (see 


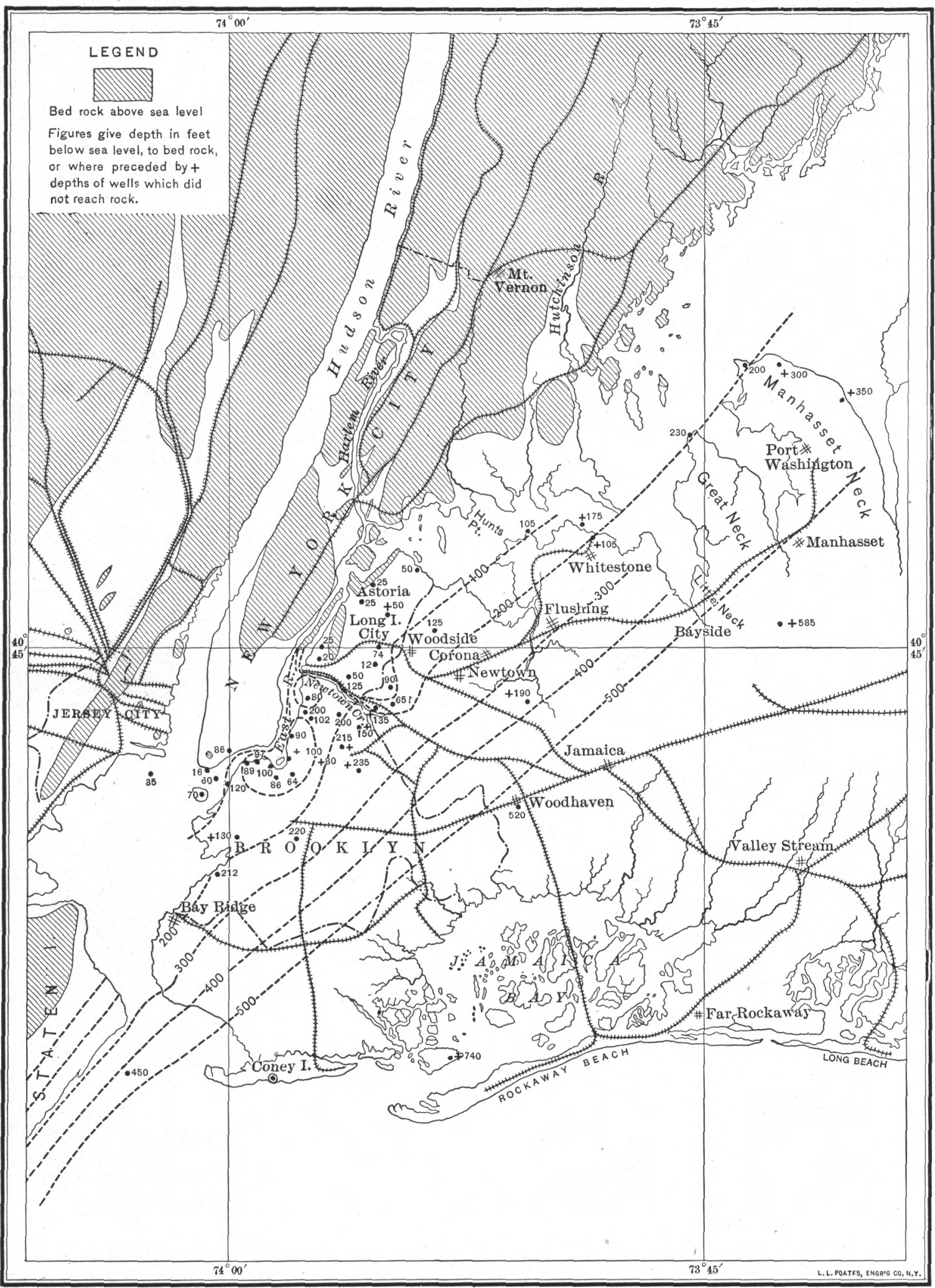

MAP SHOWING DATA BEARING ON THE POSITION OF BED ROCK IN WESTERN LONG ISLAND, NEW YORK, AND VICINITY.

By A. C. Veatch. 


p. 65) has a very regular southeastward dip (Pl. II) and a continuation of the lines of equal depth parallel to the line of strike showed that not only the nonwaterbearing gravels of the Woodhaven well (143) and the good water carriers of the Barren Island wells (129-132) belonged to the same horizon, but also the waterbearing beds in certain wells in New Jersey, which encounter a gravel horizon at a somewhat similar height above bed rock. The position of the top of this gravel and sand, which it will be convenient to call the Lloyd sand from its fossiliferous development in the well (633) on Lloyd Neck, is shown in Pl. II.

As indicated on Pl. II, in northern Long Island on a 6-mile line, the dip is as much as 80 feet per mile, while in New Jersey on a 22-mile line it is only half so much. It is quite probable, therefore, that the dip on Long Island becomes somewhat less to the south, and that on the south shore, about Amityville and Babylon; wells will strike this sand at even a less depth than indicated on Pls. II and XVI.

Some additional evidence bearing on the general structure of this region is furnished by the dip indicated by a few wèlls near Setauket (fig. 2), which

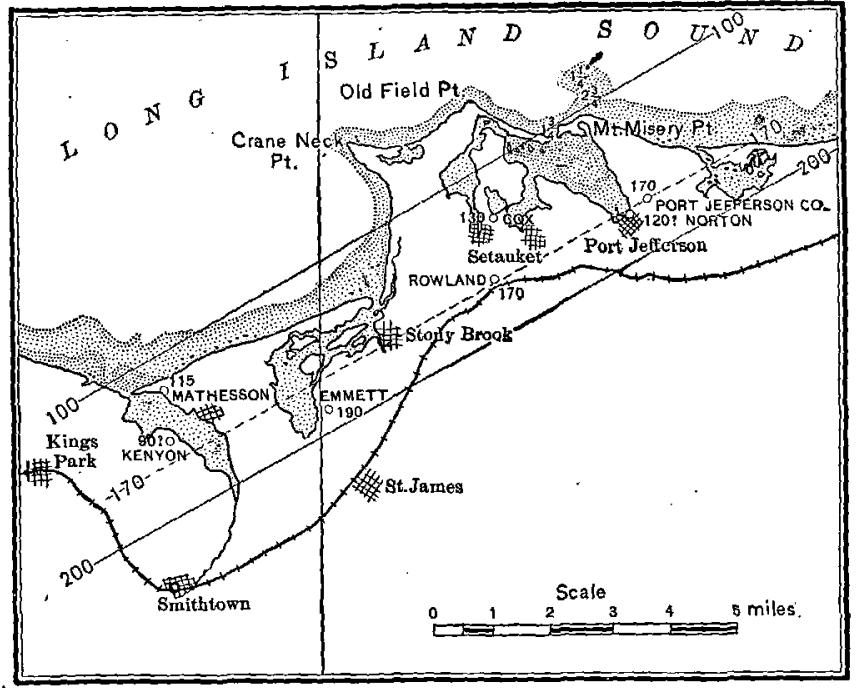
FIG. 2.-Map showing dip of Cretaceous beds near Setauket, N. Y. Figures at wells give depth of water-bearing stratum below sea level. reach a coarse sand and gravel about 600 or 700 feet above the Lloyd sand. The original calculation of the dip, based on the similarity suggested by the Cox (763), Rowland (760), and Emmett (752) records, has been confirmed by the record and samples from the Port Jefferson Company well (811). A comparison of the strike of this bed (fig. 2) with that of the Lloyd sand (Pl. II) shows it to be very nearly parallel, although the dip is much nearer that usually found in New Jersey-about 40 feet per mile.

\section{PRESENT DISTRIBUTION.}

A detailed knowledge of the distribution of the Cretaceous on Long Island is, like the determination of the structure, almost wholly dependent on well records. The available data are shown on Pl. III. This map emphasizes two points: Although (1) the Cretaceous beds have determined the major topographic relief of the island (see also Pl. V, $A-A$ ), (2) near the western end they have been deeply trenched by a broad north-south valley, representing the outlet of the Sound River (Pl. VI).

The most important outcrop of Cretaceous rocks is in the West Hills, on the road leading from Melville to Hicksville (PI. III). The following section was observed 
at this point early in the spring of 1903 , just after the landslips of the previous winter had been removed by road graders and the section further cleaned up with a spade:

Section just west of Melville, N.Y.

Pleistocene:

[Top of section about 300 fect above tide.]

1. Horizontally bedded yellow sand and quartz gravel, with a few very much weathered compound pebbles. Near the upper part of the section the gravel is a very bright orange. (PI.IV,A) . 35 Miocene (?): Fluffy (Beacon IIill) sand:

2. Orange clayey sands, fine, micaceous, containing iron scales and small gravel; closely resembles sand at Kirkwood, N. J............................................ . 3 Cretaceous:

3. Dark-colored, lavender, green, and black sandy clay, weathering yellow . . . . . . . . . 3

4. Horizontally bedded, finely laminated red clayey sand, with a few rounded quartz pebbles

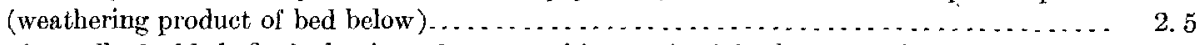

5. Horizontally bedded, fincly laminated green, white, and pink clayey sand, containing some greensand grains and rounded quartz pebbles.............................. 3

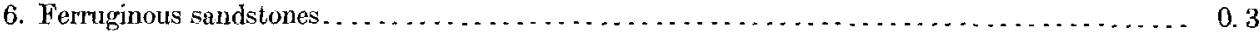

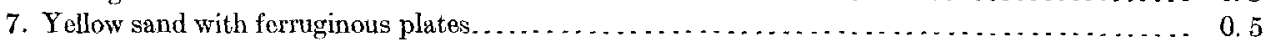

8. Irregularly bedded gray clayey sand, blotched with red and yellow, becoming more sandy above,

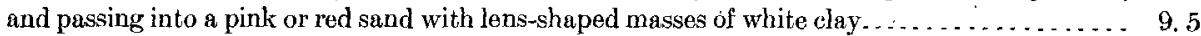

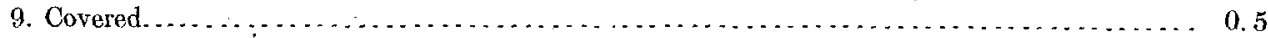

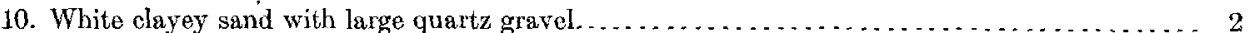

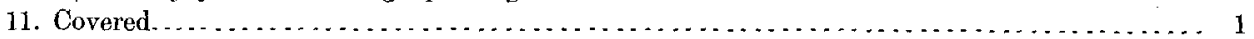

12. Stratified orange-colored sandy clay, with ferruginous plates. $\ldots \ldots \ldots \ldots \ldots \ldots \ldots \ldots \ldots$

13. Very black sand and gravel, stained, probably with manganese dioxide $\ldots \ldots \ldots \ldots \ldots \ldots \ldots . .2$

14. Coarse white sand and yellow clayey sand, horizontally, though rather irregularly, bedded, the bedding lines being darker and rather more clayey than the rest. (IPI IV,$B$ ) $\ldots \ldots \ldots \ldots$ 19

\section{STRATIGRAPHIC SUCCESSION.}

A study of the local data indicates that from a stratigraphic standpoint the greensand beds in the Quogue (858-859) and Bridgehampton (897) wells, and the impure greensand marls in the Melville section (p. 20) are to be regarded as the highest beds of the pre-Pleistocene series which have thus far been recognized. Wells which might show younger beds may be looked for east of Babylon, but the wells in this region, except those at Quogue and Bridgehampton, are so shallow and the data so meager that the lignitiferous sands of the Pleistocene can not with certainty be separated from the older, and while a portion of these sands are doubtless pre-Pleistocene, their thickness can not be very great and a generalized section of the pre-Pleistocene beds may be commenced with the greensand layer above mentioned.

$$
\text { Ge zeralized section of pre-Pleistocene deposits on Long Island. }
$$

1. Impure greensand marl developed in about the same stratigraphic position in the Melville section

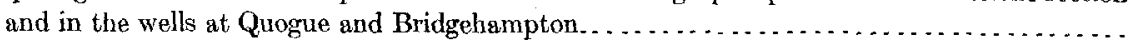

2. Sands with irregular clay beds. The beds, though showing considerable lignitic material, are commonly lighter on the north shore and in the hill lands than on the south shore. They are shown in detail in the many shallow wells in northern Oyster Bay Township, in the Lake Success well (317), the Hollis well (220), the Wheatley Hill wells (particularly 431), in the Molville section, in the Barren Island wells, and in many of the test wells of the Brooklyn waterworks east of Jameco, as well as in the Long Beach, Barnum Island, Quogue, and Riverhead wells.

Feet.

$50 \pm$ 


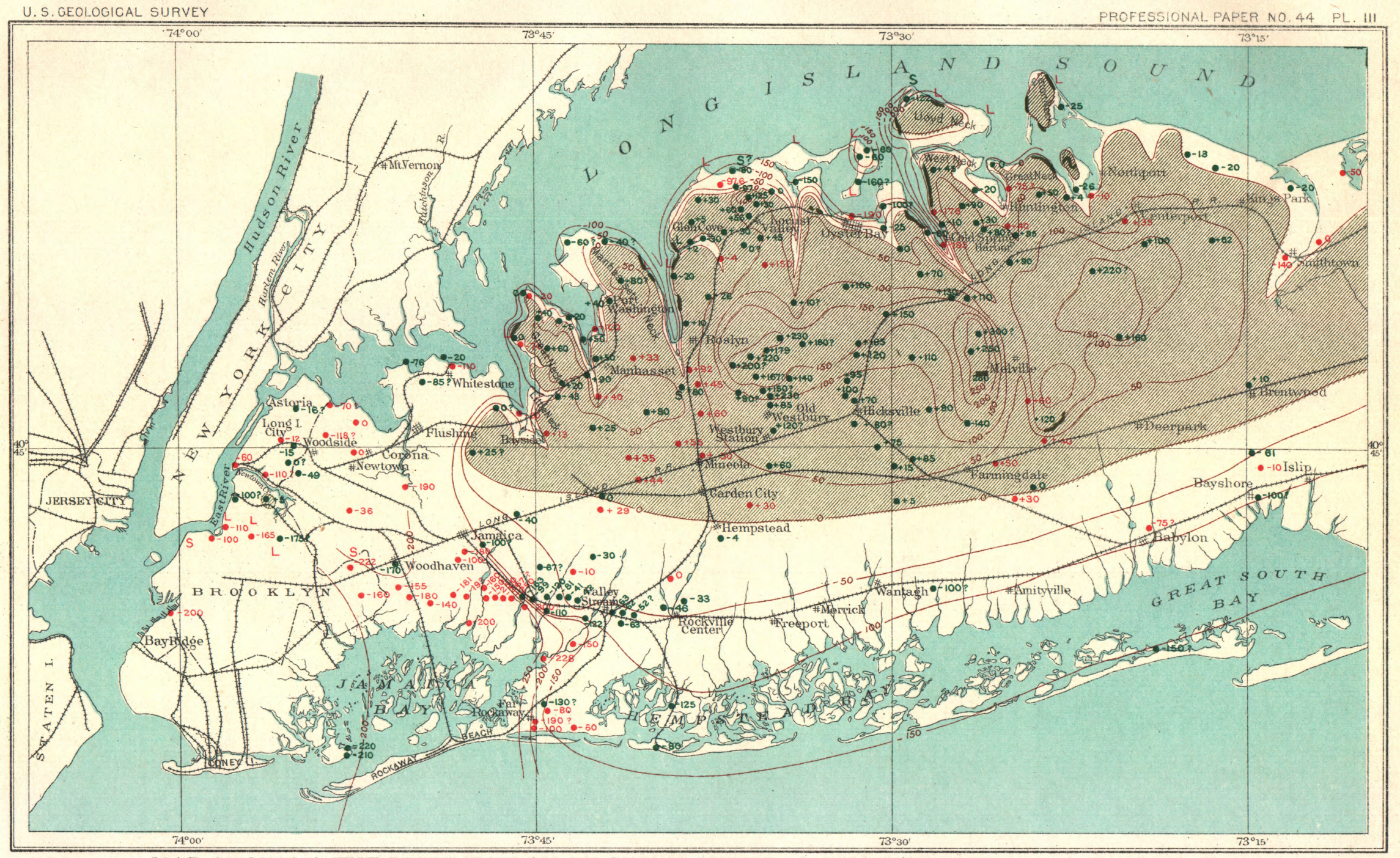

MAP SHOWING THE DISTRIBUTION OF CRETACEOUS ON WESTERN LONG ISLAND, NEW YORK A.C. Veatch 

3 Varicolored clay, often bright red, in wells on the north shore, may be entirely absent or very thin, as in the Cox well (564) in Hempstead Harbor, and the Bevin well (670) on Eaton Neck, with the clay layers in the sueceeding beds it sometimes reaches a thickness of between 400 and 500 feet, as in the Ward well (628) near Huntington, but this thickness, as shown by near-by wells (620), is a.bnormal, the average thickness being about 100 to 150 feet.

4 Lloyd sand Yellow to white quartz sand and gravel, with occasional clay layers, as at Woodhaven, separated from bed rock by clay beds, but at Greenport apparently resting directly upon it, contains much decayed white chert, and in one case (633) marme fossils Maximum thickness shown at Peacock Point and Lake Success (317) Lithologically this giavel is identical with the older portions of the yellow gravel of New Jersey, and suggests that a part of this complex may represent undisturbed Cretaceous outerops. .................

5 Probable thickness of beds between the Lloyd gravel and bed rock ...................

\section{RELATION TO ADJACENT AREAS}

Fortunately for the purposes of this study the pre-Pleistocene beds in New Jersey, partıcularly those belonging to the Cretaceous, are not only well developed but well known, and furnish a ready near-by standard with which to compare the Long Island section. Before undertaking this comparison in detail, it will be necessary to review briefly the geologic succession in that region, and to give the thickness and general character of the mam lithologic units In various reports of the New Jersey geological survey these details are given at length, and it is from this source that the following abstract has been prepared

TARLE I-Cretaceous and Tertzary formations of New Jersey

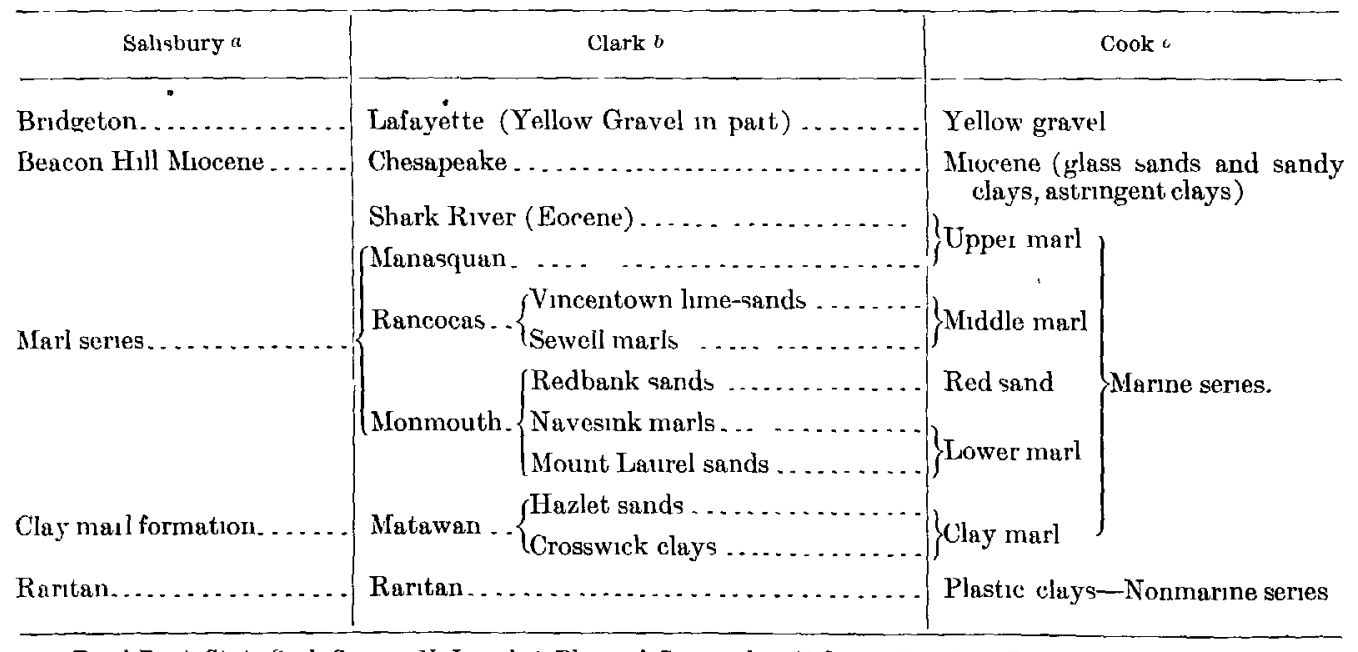

"Funal Rept State Geol Survey N J , vol 4, Physical Geography, 189R, p 117 Ann Rept N J Geol Survey, pp 13-15, 1898 Rept N J Geol Survey p 334, 1894, Bull Geol Soc Am, vol 8, pp 315-358, 1897, Ann Rept N J Geol Survey, p 174, 1898 . $a$ Geology of New Jersey, 1868 , and subsequent publication
that it has not the stratigraphic position indicated by Cook

The Miocene strata which unconformably overle the Cretaceous and Eocene beds are as a rule coarse and lighter colored at the outcrop than in the embed At the outcrop these beds are commonly yellow or brown, whle in the embed they are darker and the percentage of clay material is greater. They cap many of the 
high hills of the coastal plain as outliers in the Cretaceous area, and underlie all of the plain south of the Cretaceous outcrop.

The Cretaceous, including the lithologically similar Eocene Shark River beds, may be divided on lithologic grounds into (1) the marl series or greensand beds, (2) the clay marls (or Matawan), and (3) the plastic clays (or Raritan). The general character and relation of these beds are well shown graphically in fig. 3 and Pl. II, and may be briefly stated as follows:

1. Marl series. Greensand marl, sometimes with some claycy material which produces gray or chocolate-colored marls, generally quite fossiliferous, and at times calcareous. 'Toward the base the amount of sandy material increases and the beds take on a ferruginous aspect with a decreasing percentage of glauconite . . . . . . . . . . . . . . . . . . . . . . . . . . . 262-a 430

2. Clay marls or Matawan. Highly ferruginous brown sands, at times coarse and white, passing into slate and drab-colored clays interstratified with white sand, and finally into dark-colored or black clays. Marine fossils are by no means as abundant as in the overlying layers, and are as

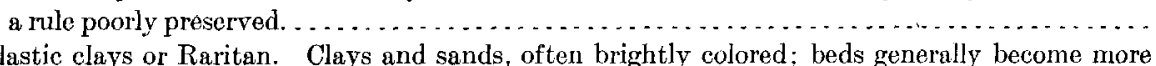
Feet. Plastic clays or Raritan. Clays and sands, often brightly colored; beds generally become more
sandy in the upper portion, though they sometimes contain dark-colored clay, and are then not separable from the overlying Matawan or clay marl. The differentiation of this horizon has, rested on the plastic clays which it contains, and its general nonmarine character . . . . .

A comparison of this section with the general section found on Long Island shows little similarity. In part this difference is due to the relatively small amoun.

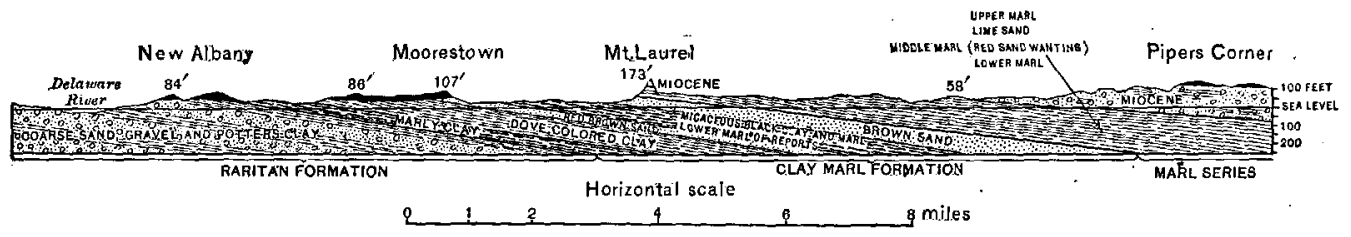

FIg. 3.- Section from Delaware River to Pipers Corner, N. J., showing character and relation of Cretaceous horizons. (Salisbury, 1896.b) Black represents surficial deposits. Length, 20 miles; height, 473 feet.

of information available regarding the older beds on Long Island, and will probably disappear as the data increase. In part, however, it is real; for although the Pleistocene deposits effectually mantle almost the entire island and prevent a careful study of the older beds, the well data are now complete enough to positively indicate the absence of any great fossiliferous greensand marl bed 250 to 450 feet thick, such as occurs in New Jersey. Only in the lower beds is there any similarity, and these have thus far furnished the only bases for the correlation of the two sections. The manifest lithologic resemblance of the few outcrops on the north shore to the Raritan beds of New Jersey caused Mather at a very early date to correlate them. Later the work of Newberry, Hollick, and White on the fossil plants of Long Island and the New. England islands confirmed this tentative correlation. To these data it is now possible to add direct stratigraphic evidence, which confirms the conchusion reached by Ward from a study of the flora: That the beds furnishing the fossil leaves on Long Island (the Island series) are somewhat younger, and therefore stratigraphically higher than the Amboy clays. ${ }^{c}$

a For thickness shown in the Asbury Park well, see Rept. N. J. Geol. Survey, 1896, p. 73.

b Ann. Rept. New Jersey Geol. Survey tor 1895, 1896, PI. II.

c Ward, Lester F., The Potomac formation: Fifteenth Ann. Rept. U. S. Geol. Survey, 1895, p. 335; Age of the Islund series: Science, new ser., vol. 4, 1896, pp. 757-760. 


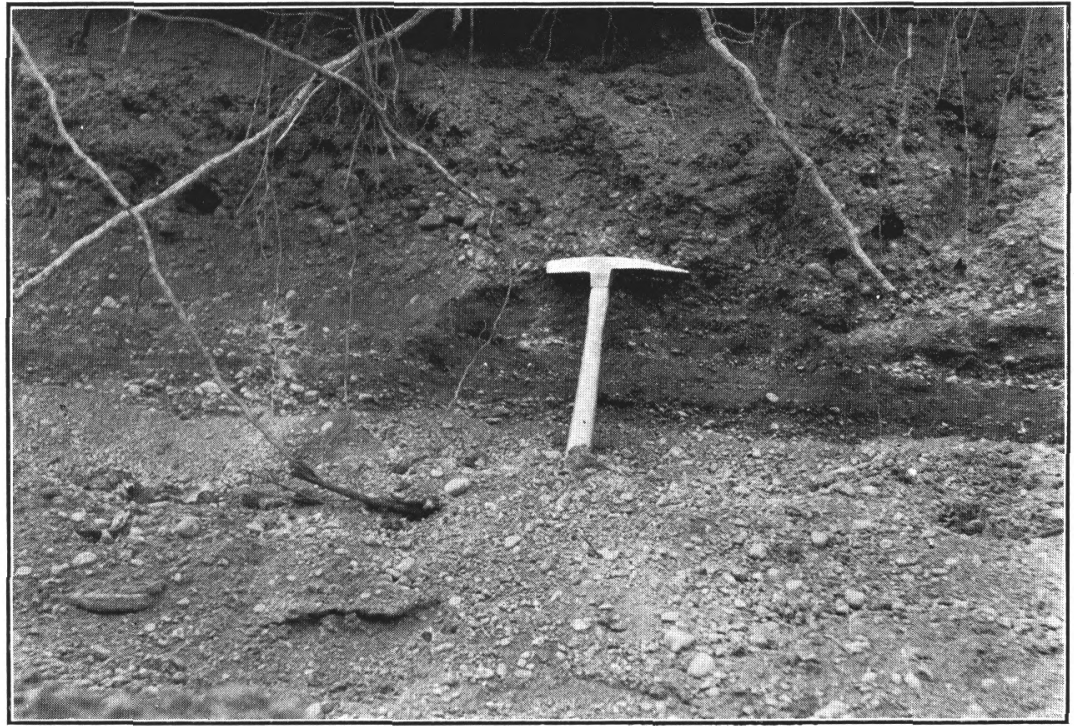

A. MANNETTO GRAVEL NEAR TOP OF MELVILLE SECTION.

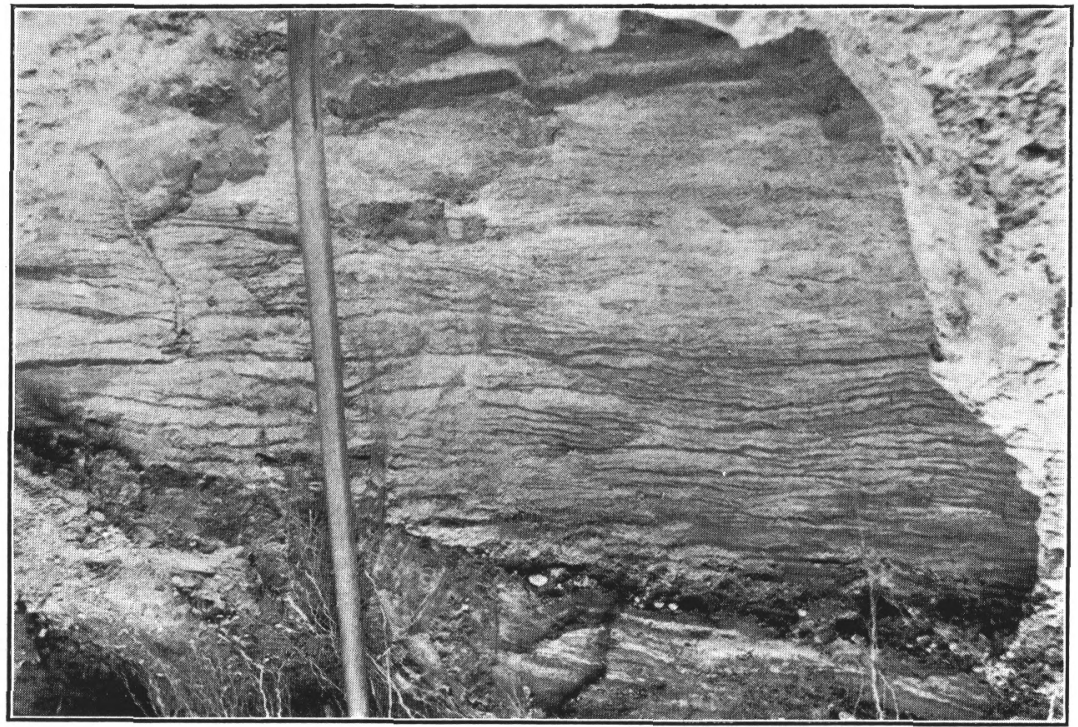

B. CRETACEOUS SAND NEAR BASE OF MELVILLE SECTION. 

In this work the top of the Lloyd sand has proved a convenient plane of reference, and a study of the New Jersey records shows that it contmues into New Jersey and can therefore be made a basis of correlation between the two sections This extension is graphically shown in Pl II The considerations on which the prolongation of these lines from Long Island were based are (1) The general line of strike of the Cretaceous beds; (2) a water horizon in the wells at Runyon" (white sand beneath 100 feet of white, red, and blue clay), Yardville," Hightstown, "Jamesburg," Asbury Park, ${ }^{\circ}$ and Ocean Grove, ${ }^{e}$ which is 150 to 200 feet below the base of the Matawan

Woolman ' has suggested that the Woodhaven and Barren Island horizons are a continuation of the horizons developed at Keyport, ${ }^{g}$ Matawan, ${ }^{h}$ Atlantic Highlands, ${ }^{2}$

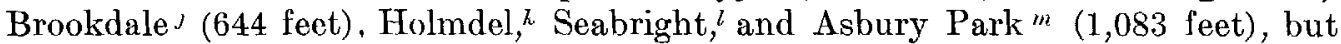
an attempt to include the horizon developed in these New Jersey wells causes the lines of equal depth to diverge from the general line of strike, and does not account for the depth reached in the Asbury Park and Ocean Grove wells Woolman explains this greater depth by an assumed thickenung of the Matawan, but Clark in reviewing the evidence is inclined to give to the Matawan in these wells' a thockness of only about 400 feet $m$

According to this hypothesis the lowest water-beanng layer would have about the position of the beds which were struck by the Runyon, Jamesburg, Hightstown, and Yardville wells, and which are 200 feet below the beds of the Matawan Moreover, a water-bearing sand occurs in the Asbury Park well at a depth of 954 feet which seems the true continuation of this upper horizon. If the 200-foot line on $\mathrm{Pl}$ II is called 0 and the other lines renumbered accordingly, the position of this upper hortzon will be approximately indicated in all of the wells. Thus, near the 200-foot line will be found the Matawan and Keyport wells (215-220), near the 400 -foot line, the Holmdel well (450), ${ }^{n}$ near the 500-foot line the 465-foot horizon in the Atlantic Highlands well; near the 600, the 606 horszon of the Brookdale well; near the 700 , the 670 of the Seabright well, and near the 900, the 954 Asbury Park horizon. The Lloyd sand is therefore equivalent to the lower horizon in the Asbury Park and Ocean Grove wells and is about 200 feet below the horizon in the other wells to which Woolman referred it. This upper horizon is regarded as either basal Matawan or uppermost Raritan, and the Lloyd sand is therefore a horizon in the Raritan about 200 feet below the base of the Matawan. In a general way, then, the 200 or 300-foot line marks the line of parting between the so-called marine and nonmarine

a Ann Rept New Jerscy Geol Survey for 1897-98, p 246

$b$ lbid, p 281

c Ann Rept New Jersey Geol Survey, 1895, pp 200, 201, Bull U S Geol Survey No 138, 1896, pp 66-67

I Ann Rept New Jersey Geol Survey, 1880, pp 166-168, Bull U S Geol Survey No 138, 1896, pp 67, 68

- Ann Rept New Jersey Geol Survey 1896, pp 72-75

$f$ Woolman, Lewis, Ann Rept New Jersey Geol Survey, 1900, p 77

$y$ Ann Rept Now Jersey Geol Survey, 1898, pp 245-246

$h$ Ibid, $\mathrm{p} 24 \mathrm{~h}$

I Ibid, p 244

3 Ibld, $\mathrm{p} 228$

4 Ibld , 1897, pp 147-148.

$l$ Ib1d 1900, pp 76- 77

$m$ Ibld, 1898, pp 176-177

$n$ The difference in this case 19 clearly due to the generalized character of the record, quicksand is reported for some distance above the water-bearng layer, and doubtless in pait represents the upward extension of the sand bed 
Cretaceous, and Cretaceous fossils would be expected south of this line on Long Island.

From these data the outcrops at Glen Cove and Sea Cliff are to be regarded as uppermost Raritan which has been, perhaps, slightly disturbed by folding, while the gray sands and clays at Greenwood are clearly Matawan, and the Terebratula found in the Roslyn well ${ }^{a}$ naturally falls near the base of the Matawan. In the same way the Lloyd Beach clays are to be regarded as Matawan, unless they have been more profoundly disturbed by ice pressure than now appears; and the Little Neck and Fresh Pond areas fall far south in the Matawan. On account of the leaf remains found at Little Neck this locality has been referred to the Raritan, but the recent collections of Berry ${ }^{b}$ in the Matawan show essentially the same fauna, and there is therefore no conflict between the paleontologic and stratigraphic evidence.

In all cases there is the ever present question of how much the beds may have been disturbed, and as the folding amounts to as much as 100 or 200 feet in the islands to the east, this is not always a negligible factor. In the wells on the south shore, as was early noticed by Woolman, the somber-colored lignite-bearing sands and clays are fair lithologic representatives of the Matawan, but in this region greensand must be almost entirely absent in the Matawan, for it is not represented in any of the samples from the wells of the Brooklyn waterworks or from any of the neighboring wells, the only suggestion of it being in the Pleistocene deposits in the Queens County well at Valley Stream (273) and at Long Beach (373), in both of which it occurs in coarse sand, evidently redeposited. This occurrence is so suggestive that it is confidently expected that fossiliferous greensand will be found in wells north and east of these localities.

The Cretaceous fossils found at different points in the drift at Brooklyn are also suggestive, though in all cases they are so separated from the Cretaceous beds that their real source can be only conjectured. They are perhaps Matawan, and may even be in part representatives of the occasional forms which are known to occur in the upper part of the Raritan.

On the north shore the beds in the same position as a rule more strongly resemble the underlying Raritan, though in the dark clays at Greenwood, Little Neck, West Neck (in the Ward well), and possibly at Elm Point, the darker beds are suggested. The sandy layers in part correspond to the Hazlet sands, but above the Matawan there is absolutely no similarity in the two sections. In place of from 250 to 450 feet of greensand marls there are sands and clays in no way different from the underlying beds which are known to be Matawan because of their lithologic character and position with relation to the Lloyd sand. Greensand beds have been reported only in the West Hills and in the Quogue and Bridgehampton wells, and in the latter cases there are some reasons for believing them similar to the Miocene greensands of Marthas Vineyard. ${ }^{c}$

So radical a change in the character of the deposits naturally raises the question of the cause. In some respects these light sands in the hills and the dark clays

a See well No. 437, p. 281

b Berry, E. W., Am. Nat, vol. 37, 1903, pp. 677-684; Bull. New Y.rk Bot. Gar., vol. 3, No. 9, 1903, pp. 45-103, pls. 43-57; Bull. Torrey Bot. Club, vol. 31, 1904, pp. 67-82, pls. 1-5.

c Bull Geol. Soc. Am., vol. 8, 1897, pp. 202, 203. 
of the south shore above the beds regarded as Matawan suggest Mrocene, but a comparison with the known position of the Miocene in adjacent areas renders this correlation very doubtful It will be seen from fig 4 and $\mathrm{Pl} V$ that, so far as present knowledge goes, Long Island lies north of the main Miocene deposits, and that if the Mrocene occurs at all it is to be expected as mere erosion outliers occupying the highest hills Moreover, Mr G. N Knapp, who, by reason of his long and extensive field work in New Jersey, is well fitted to judge, has examined the beds in the Melville section ( $\mathrm{p} 20$ ) and regards them as Cretaceous, with the possible exception of a thin layer between the upper gravel and the impure mall, which resembles Miocene In order that any other portion of these beds may be Miocene, it is necessary to assume a much greater discordance of structure than is known to exist anywhere in this region between the Miocene and Cretaceous. These facts, with the agreement of the thickness of the beds below the Miocene (?) of the West Hill section with the thickness of the Cretaceous deposits of northern New Jersey, and the fact that Long Island is to be regarded as the normal continuation of New Jersey, both geologically and topographically, with the addition of a mantle of glacial deposits, throw the burden of proof on the person arguing for the Mrocene age of these beds. The total absence of large greensand beds indicates a change in the local conditions Perhaps the ancestral Hudson and Connecticut rivers may have had something to do with it, perhaps the ocean currents are responsible, for it is well known that both these factors tend to interfere with the formation of grcensand, and glauconitic deposits are therefore seldom continuous over great areas ${ }^{a}$

This sandy phase reappears on Marthas Vineyard above the basal plantbearing beds, though at this point it contains fossils," and while the data are not conclusive, they furnish further evidence of the change from the New Jersey conditions which is indicated on Long Island

\section{AGE OF THE RARITAN FORMATION}

After the early correlations, which were based on very meager data, the Raritan was referred to the Upper Cretaceous, and it was not until the work of Ward in connection with the much disputed Potomac group that it was referred to the Lower Cretaceous ${ }^{c}$ It was shown by Newberry ${ }^{d}$ and Hollick ${ }^{e}$ to be rather closely related to the Dakota and the Patoot and Atane beds of Greenland, all of which are regarded as Upper Cretaceous.

The work of Berry has now shown that there is no essential break between this fauna and that of the Cliffwood section, which is clearly Upper Cretaceous $f^{f}$

$a$ Clark, W. B , New Jersey Geol Survey, 1893, p 225

$b$ Woodworth, J B Bull Geol Soc America, vol 8, 1897, pp 199-200

- Ward, L. F, The Potomac Formation Fifteenth Ann Rept U S Geol Survev, 189;, pp 345-346, Age of the Island senes, Sol, new series, vol 4, 1896, pp 757-760, Professor Fontane and Professor Newber ry on the age of the Potomac formations, $\mathrm{Scl}$, new series, vol $5,1897, \mathrm{p} 420$

d Newberry, J S, The flota of the Amboy clay, u posthumous work edited by Artnil Hollick Monograph U S Geol Sur vey, vol 26,1895 , pp 23,33

e Holluck, Arthur Proc Am Assoc Adv Science, vol 47, 1898, pp 292-293, Science, new series, vol 7, 1898, pp 467-468, Am Gieol, vol 22, 1898, pp 25̄̄-256

$f$ Berry, Edward W, Plants from the Matawan Am Nat, vol 37, pp 677-684, 1903, Flora of the Matawan formation (Crosswick's clays) Bull New York Bot Gar, vol 3, No 9, 1903, pp 45-103, pls 43-57, Additions to the lora of the Matawan formation Bull Torr Bot Club, vol 31, 1904, pp 67-82, pls 1-5 
Mr. David White informs me that he regards the Marthas Vineyard flora, on which Ward based his Island series, as essentially the same as the Cliffwood. The Long Island plant remains described by Hollick represent a horizon 100 or 200 feet above the Lloyd sand, and are therefore stratigraphically between the Amboy clays (Woodbridge, South Amboy, and Sayreville horizons) and the Cliffwood or basal Matawan. The stratigraphic sequence is, then, as follows: (1) Amboy clays; (2) Long Island red leaf-bearing concretions; (3) Cliffwood, Marthas Vineyard, East Neck."

The few fragmentary marine remains obtained from the Lloyd sand at Lloyd Point are regarded by Stanton as Upper Cretaceous, and therefore confirm the gencral drift of the plant evidence, as do the molluscan remains (including Exogyra) reported by Woolman from a similar horizon in the Asbury Park well. ${ }^{b}$ On the one hand marine fossils indicate the flora in the upper beds as clearly Upper Cretaceous; on the other, the flora shows that there is no. essential break between the upper and lower beds of the Raritan. There is, however, a sharp floral break at the base of the Raritan ${ }^{c}$ and it seems, therefore, necessary to return to the view of Newberry and regard the Raritan as basal Upper Cretaceous, and essentially equivalent to the Dakota and the Woodbine. ${ }^{d}$

\section{SUMMARY OF THE CRETACEOUS.}

The more important points relative to the pre-Pleistocene on Long Island may be briefly summarized as follows:

1. The bulk of the pre-Pleistocene deposits on Long Island are Cretaceous.

2. The basal beds are the stratigraphic equivalents of the Raritan, and are Upper Cretaceous.

3. The Matawan beds are apparently well represented, but their lithological character changes in going eastward.

- 4. No greensand beds comparable to the great greensand marl beds of New Jersey have been found, their stratigraphic position being occupied by fine lignitiferous sand with occasional clay beds.

TERTIARY.

\section{GENERAL CONDITIONS.}

Although there are no indications on Long Island of any break in the sedimentation during the Cretaceous, Doctor Clark has found in New Jersey evidence of perhaps two unconformities which indicate land periods of comparatively short duration.e It was, however, not until rather late Tertiary time that this region commenced to undergo the profound erosion which has given rise to the present land forms. These stages are imperfectly shown on Long Island, but in adjoining portions of the coastal plain the following major stages have been found: Late Pliocene (post-Lafayette) erosion, Lafayette submergence, early Pliocene erosion, Miocene submergence, Eocene erosion.

$a$ The East Neck locality is perhaps a little higher stratigraphically than the other two.

$b$ Ann. Rept. N. J. Geol. Survey, 1895, pp. 72-75, 1896.

c Science, new series, vol. 4, 1896, p. 759 .

$d$ Twenty-first $\Lambda$ nn. Rept. U. S. Geol. Survey, pt. 7, 1901, pp. 318-322.

e Bull. Geol. Soc. America, vol. 8, 1897, pp. 328, 337-338. 
EOCENE EROSION.

The absence of the greater portion of the Eocene in New Jersey indicates a period of elevation, but the absence of any great unconformity between the Cretaceous or Eocene and the Miocene strata indicates that either this elevation was slight or that the period was of such a duration that the land was essentially. base-leveled.

\section{MIOCENE SUBMERGENCE.}

While the deposits of the Miocene were clearly very thick toward the sea and thin toward the land, the exact position of their landward edge is uncertain.

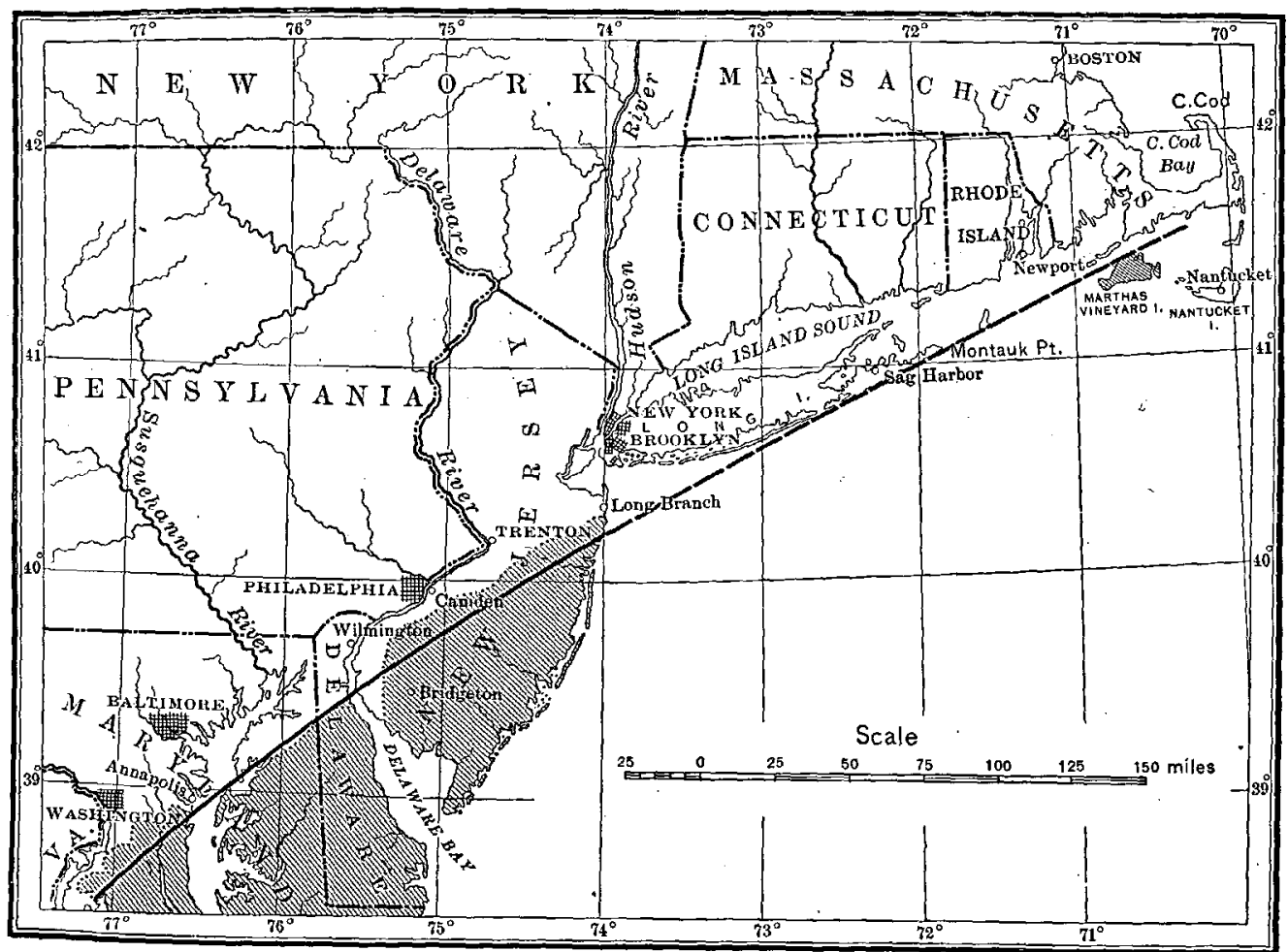

Fig. 4.-Sketch map showing known distribution of the Miocene near Long Island. Shaded area is underlain by Miocerie. Heavy black line gives general direction of strike and shows approximate point at which base of Miocene reaches sea level.

It may, however, be regarded as reasonably certain that over most of the Atlantic coastal plain they were of sufficient extent and thickness to obliterate the low features developed in the underlying Cretaceous and Eocene beds during the preceding erosion period.

Distribution of Miocene deposits.--In the Long Island region and in the New Jersey region the Miocene sediments were deposited under similar conditions, and as these two areas have been subjected to the same forces, except glacial action, their distribution in both should be similar. The only bed thus far seen on Long 
Island which is regarded as possibly Miocene is a thin bed of "fluffy sand" which Mr. G. N. Knapp recognized in the upper part of the Melville section (p. 20), and which is the counterpart of certain sands occurring in the Miocene of New Jersey. A comparison of the sections shown in $\mathrm{Pl} . \mathrm{V}$ indicates that if the structure is normal, and there is every reason to believe it is, a Miocene outlier should be expected at this point. The same evidence shows the absence of the Miocene above sea level (fig. 4 and Pl. V) on southern Long Island, except possibly along a portion of the South Fluke. This line of argument is important, for it shows that the Tertiary deposits can not be expected on the north shore any more than in the Hightstown Vale (p. 30) in New Jersey, and that the occurrences on Long Island are probably limited to erosion outliers, with the embed beneath the Atlantic.

\section{EARLY PLIOCENE EROSION.}

In the succeeding erosion period the first forerunners of the present topography were developed. Erosion was active, the mantle of Miocene beds was partly removed and the underlying Cretaceous exposed near the old shore line.

\section{LAFAY ETTE SUBMERGENCE.}

During Lafayette time the rather low topography developed in this region was buried by a mantle of littoral deposits. The smaller depressions were obliterated but the broader features persisted.

\section{LATE PLIOCENE (POST-LAFAYETTE) EROSION.}

After the Lafayette submergence there was a long period of erosion in which the land stood relatively high and the essential features of the present topography were developed.

\section{DEVELOPMENT OF TOPOGRAPHIO FEATURES.}

The most pronounced topographic feature resulting from or accentuated by the early and late Pliocene erosion epochs is a more or less persistent line of hills

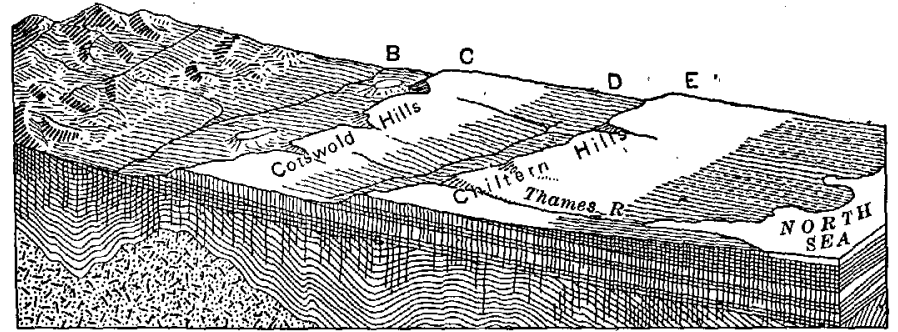

Fia. 5.-Shereogram of eastern England (after Davis), showing the development of wolds and vales. B, D, vales; C, E; wolds. overlooking a landward depression which extends from the Mannetto (West) and Wheatley hills on Long Island through the highlands in the coastal plain of New Jersey and Maryland to the Potomac River near Washington. Such a degradational feature is common in all

regions of gently inclined rocks of unequal hardness. By weathering and erosion the softer beds are removed and the more resistant ones stand out as chains of hills. Marked topographic forms depending on these factors extend over wide areas and it seems desirable to have distinctive topographic terms for them. 
Fortunately names are readily obtainable by analogy with eastern England where, in the gently sloping rocks of the post-Paleozoic series, similar features are well developed (fig. 5). There the ranges of hills are in many places called wolds-as the Cotswold Hills and the Lincolnshire and Yorkshire wolds-and the accompanying longitudinal depressions have been termed vales-as the vales of Pickering, Blackmore, White Horse; Red Horse, Pewsey, and Wardour. These terms are, therefore, appropriate for lines of hills and parallel valleys of a similar

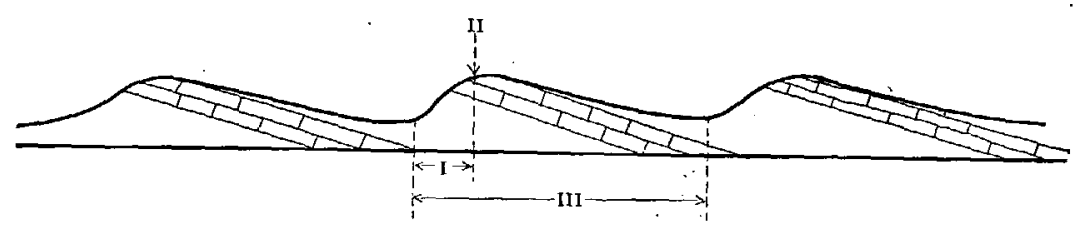

FIG. 6.-Diagram showing the three uses of "escarpment" as applied to topographic features.

type and origin. As a definite physiographic term wold may then be defined as a range of hills produced by differential erosion from inclined sedimentary rocks, and vale as the accompanying depression or strike valley (fig. 7).

Wold has, so far as the writer is aware, never before been used as a distinct term for a definite topographic form, but vale has been extensively employed by Woodward in describing the longitudinal valleys in eastern England."

As a geographic term, vale, although generally applied to these strike valleys, has cccasionally been used for valleys of other origin-as the Vale of Eden, in Westmoreland and Cumberland, in which a portion of the depression has been

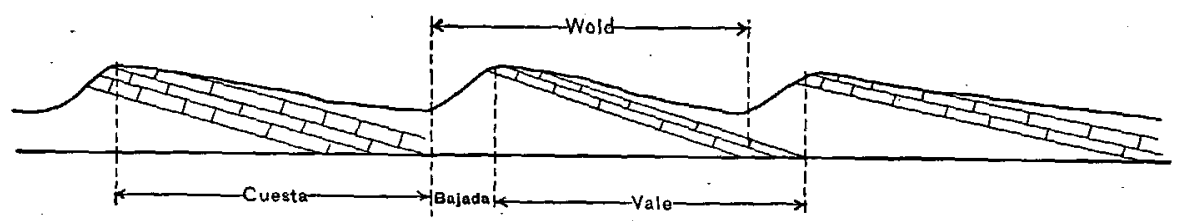

FIG. 7.-Diagram showing relations of wold, vale, cuesta, and bajada.

produced by faulting ${ }^{b--b u t}$ these may be regarded as exceptional cases, and the word used in a physiographic sense as the direct antithesis of wold, or wolds, without confusion.

To the feature here defined as a wold, the term escarpment has often been applied, but, as already pointed out by Davis," this usage is objectionable, for when escarpment is used for the whole hill feature it is given a meaning quite different from that usually associated with it. It is commonly used for a very steep declivity or cliff,,$l$ but has been extended to mean: (1) The steeper slope of a

a Woodward, Horace B., The Jurassic rocks of Britain: Memoirs Geol, Survey Gt. Brit., vol. 3, 1893, pp. 309-313; vol. 4, 1894, p. 459; rol. 5, 1895, p. 297. The geology of England and Wales, 1887, p. 599.

b Marr, John E., The scientific study of scenery, London, 1900, p. 113. Ramsey, A. C., Physical geology and geography of Great Britain, 6th ed., 1899, pp. 362-363, fig. 129.

c.Proc. Geol. Assoc. Lond., vol. 16, 1899, p. 77.

$d$ Geikie. Archibald, Text-book of geology, vol. 2, 1903;p. 13. Example cited: The face of a mesa. 
wold; ${ }^{a}$ (2) the top or crest line of a wold $;^{b}$ (3) the whole hill feature-exactly synonymous with wold (fig. 6). $c$

The.word cuesta is used in the southwestern United States for a sloping plain which is terminated on one side by a steep slope. ${ }^{d}$ It seems to have no relation to structure, but only to topographic form, and while the long slope of a wold, or dip slope, is a cuesta, a cuesta is not always a dip slope. The word has been applied by Davis to many of the dip slopes of wolds in the United States, and has been extended by him to include the whole topographic form, with the remark that while there may be objection to this use of the word it will, until a better name is suggested, serve

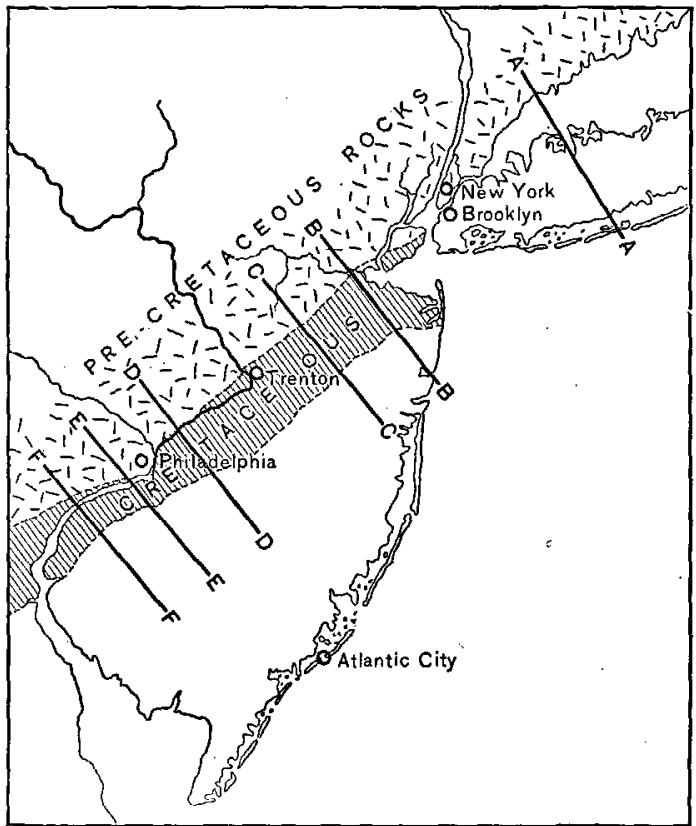

Fia. 8.-Skotch map showing locations of sections shown on P1. V. a useful purpose. ${ }^{e}$ Cuesta should doubtless be restricted to its original usage, and apply only to the gently sloping plain. A name for the shorter slope or inface can likewise be obtained in the same region in the companion terms to cuesta of $c e j a$ and $b a-$ jada, the first referring to an escarpment and the second to "a gradually descending slope as distinguished from a more vertical escarpment." $f$ Bajada would then be applied where there is no escarpment or where the escarpment feature was an insignificant portion of the whole slope; while ceja would be applied where the scarp forms the major part of the boundary between two successive cuestas (fig. 7).

WOLDS AND VALES.

In the coastal plain of New Jersey there is a well-marked vale and wold (Pl. V) and a less perfectly developed pair. The innermost vale may be traced more or less continuously from the Potomac River near Washington to northern Long Island, and perhaps to southeastern Massachusetts; in it are found Long Island Sound and the northeast and southwest portions of the Delaware, Susquehanna, and Potomac rivers. Through New Jersey it is particularly well marked, and may be named the Hightstown Vale, from Hightstown, in Mercer County, where it is typically developed (P's. II and V,C).

Coastward of the Hightstown Vale and overlooking it is a range of rolling hills, highest to the northeast at Beacon Hill and Telegraph Hill, N. J., and Mannetto Hills,

a Harrison, W. Jerome, Geology of the counties of England, 1882, p. 344. Geikie, James, Earth sculpture, 1898, p. 58 , fig. 15. $b$ Marr, John E., The scientific study of scenery, 1900, p. 117.

c Geikie, James, Earth sculpture, 1898, pp. 65, 70, fig. 23. Woodward, Horace B., The geology of England and Wales, 1887, p: 599; The Jurassic rocks of Britain: Memoirs Geol. Survey of United Kingdom, vol. 4, 1894, p. 459; ibid., vol. 5, 1895, p. 297.

$d$ Hill, R. T., Description of topographic terms of Spanish America: Nat. Geog. Mag., vol. 7, 1896, p. 295.

e Davis, W. M., The drainage of cuestas: Proc. Geol. Assoc. London, vol. 16, 1899, pp. 76, 77.

$f$ Nat. Geog. Mag., vol. 7, 1896, p. 297. 


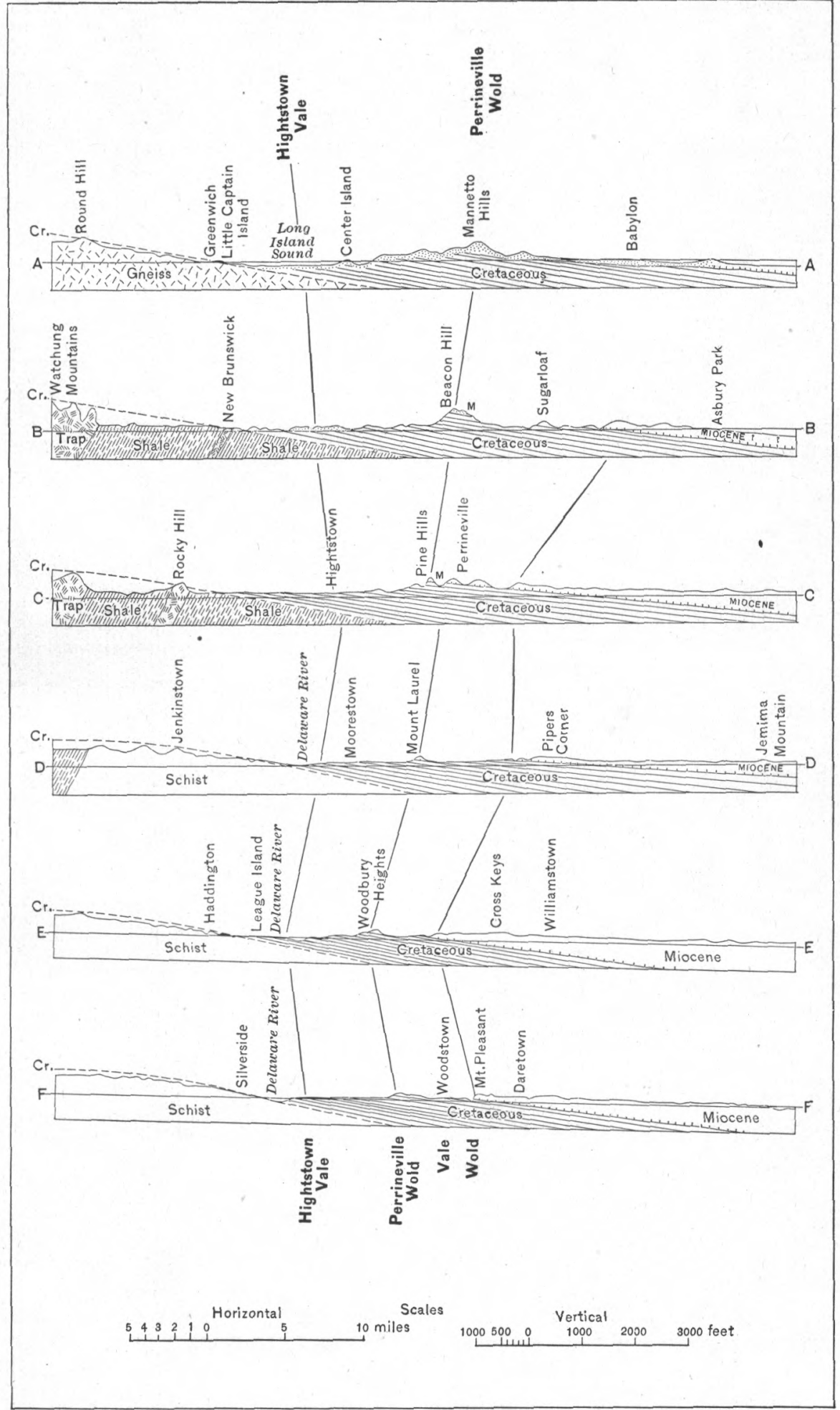

COMPARATIVE CROSS SECTIONS OF LONG ISLAND AND NEW JERSEY ALONG LINES SHOWN IN FIGURE 8, SHOWING RELATIONS OF THE TOPOGRAPHIC FEATURES.

By A. C. Veatch, 1904.

Dotted portion of sections A-A and B-B represents Pleistocene deposits. Broken line marked Cr. shows pre-Cretaceous peneplain. 

Long Island, but gradually becoming lower and of less importance topographically to the south. This range of hills is typically developed at Perrineville, in Monmouth County, N. J., 5 or 6 miles east of Hightstown, and is, therefore, named the Perrineville Wold. Both the Hightstown Vale and Perrineville Wold have been produced by the differential erosion of Cretaceous strata. Of the minor and but partly developed vale and wold to the east of the Perrineville Wold little need be said at this time, except to point out their general resemblance to the major topographic features of this type.

DEFLECTION OF RIVERS IN HIGHTSTOWN VALE.

In studying the abnormal deflection of the rivers in the Hightstown Vale it is necessary to commence with the uplift which marked the beginning of the post-Miocene erosion cycle. At that time the streams issuing from the valleys of the older land followed the retreating sea directly across the emerging coastal plain and adapted themselves to its minor irregularities and gentle slope (Pl. VI, $A$ ). During this period, in the region north of Virginia, the streams near the landward edge of the Miocene rocks cut through the Miocene and reached the Cretaceous. The soft basal Cretaceous rocks were more easily eroded than the overlying ones, and a shallow vale, overlooked by a low, northwest-facing wold broken by the transverse or dip valleys of the main streams, was developed parallel to the old shore line. This ancestral Hightstown Vale and Perrineville Wold was farther inland than to-day and, though not prominent, was doubtless well marked.

In the succeeding Lafayette submergence a mantle of littoral sediments was spread over the coastal plain. The narrow transverse valleys through the wold (fig. 5) were more nearly obliterated by this mantle than the broad vale, and when the land was again elevated the ancestral Connecticut, Delaware, Susquehanna, and Potomac rivers discharged into a slightly depressed trough. Had there been no tilting in either direction in this uplift these rivers would have overflowed the barrier afforded by the wold and the more or less completely filled, narrow, transverse valleys and cut new channels directly to the sea; but if there was tilting in either direction the rivers would have flowed down the vale in direction of the tilting and finally escaped seaward through the partly filled depressions of lower transverse stream valleys. As these streams were favored by softer strata and by greater volumes, they maintained their ascendancy over the smaller streams which developed east of the crest of the Perrineville Wold, and so persisted in their deflected courses (Pl. VI, $B$ ).

In much of Virginia and North Carolina where the more recent deposits overlying these Cretaceous beds have not been removed, no such deflection of the rivers occurs; but in Alabama where this mantle is no longer present the Coosa is deflected into an east-west course at the point where it leaves the older land; farther north the Tennessee is deflected under conditions very similar to those on the North Atlantic coast.

Two other explanations have been offered for this deflection, the first by McGee, ${ }^{a}$ and the second by Darton. ${ }^{b}$ In the first the deflection is attributed to faulting and in the second to the action of coastal bars. In the first case it must be regarded as a

a McGee, W J, The geology of the head of Chesapeake Bay: Seventh Ann. Rept. U. S. Geol. Survey, 1888, pp. 616-634.

${ }^{b}$ Darton, N. H., Jour. geol., vol. 2, 1894, p. 581; also Newsom, J. F., The effect of sea barriers upon ultimate ärainage: Jour. Geol., vol. 7, 1899, pp. 445-451. 
very strange coincidence that a fault should follow the curved line of strike of the Cretaceous when this is not parallel to the mountain chains and produce a valley just where a vale should be produced by differential erosion. Moreover, the rock surface beneath the plain and the remnants of the old surface preserved in the crests or flat tops of the hills through New Jersey show no break such as would have been produced by a fault.

Darton's explanation was proposed when further field work had proved the nonexistence of this hypothetical fault, and was based on the prevailing southward drift of the sands of the Atlantic coast. This is assumed to have prevailed since early Cretaceous time, and to have produced the ultimate deflection of the rivers by building spits or bars along the shores. There are two objections to this hypothesis: (1) it does not explain why the deflection is confined to the outcrop of the soft layers of the Cretaceous - why it does not extend continuously southward through the coastal plain, but reappears when the Cretaceous is again exposed; (2) all the coastal bars now
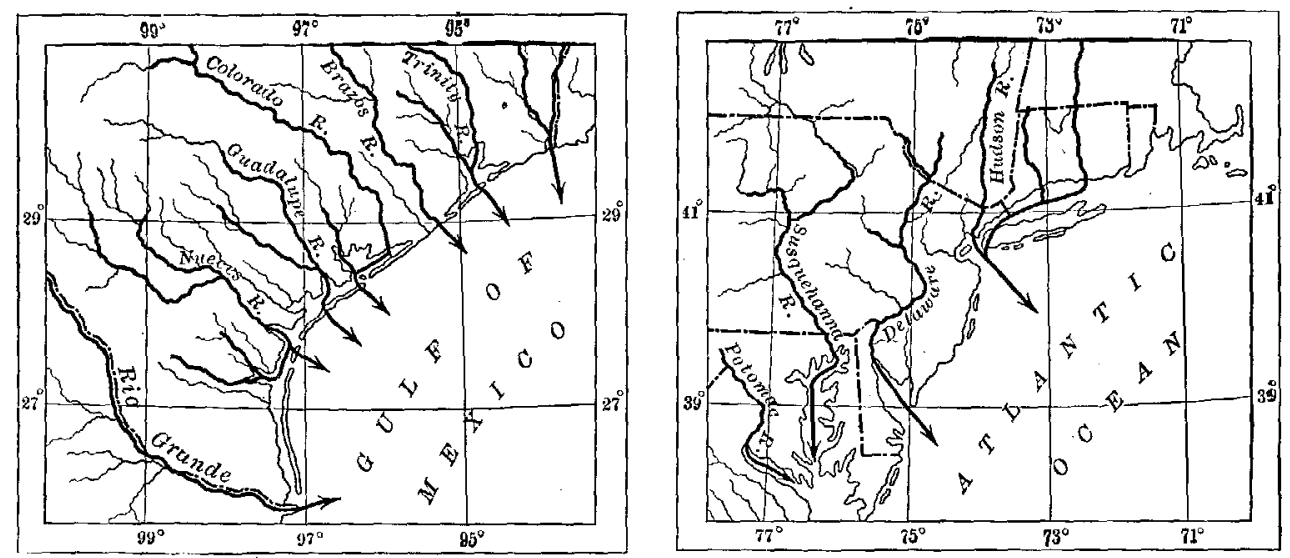

FIG. 9.-Comparative maps showing deflection of streams in the Hightstown Vale and the deflection which would be produced by the large Texas bars if the land were elevated.

forming are cut by important breaks, or tidal guts, and while these might, if the land were elevated, produce minor deflections under certain favorable conditions, they could not cause deflections of this magnitude, and the deflections would not have the same uniformity in direction. The long Texas bars offer, perhaps, the closest analogy to hypothetical bars necessary for the diversion of these northern rivers, both in the length of the bars and the size of the rivers discharging into the coastal lagoon behind them. However, careful study of the Coast Survey charts shows that where the rivers are carrying a moderate amount of sediment, as the Brazos and the Rio Grande, they have extended their mouths to the coastal barrier, and that where they are not so laden there is always a deep channel or tidal gut in the bar so situated that the deflection on elevation would be comparatively small. The comparatively insignificant effect that these bars would have in case the land were elevated is shown in fig. 9. Rivers may be deflected, as in the case of the Colorado, but it is regarded as extremely improbable that they could be deflected to the extent and with the regularity of the rivers in the Hightstown Vale. 


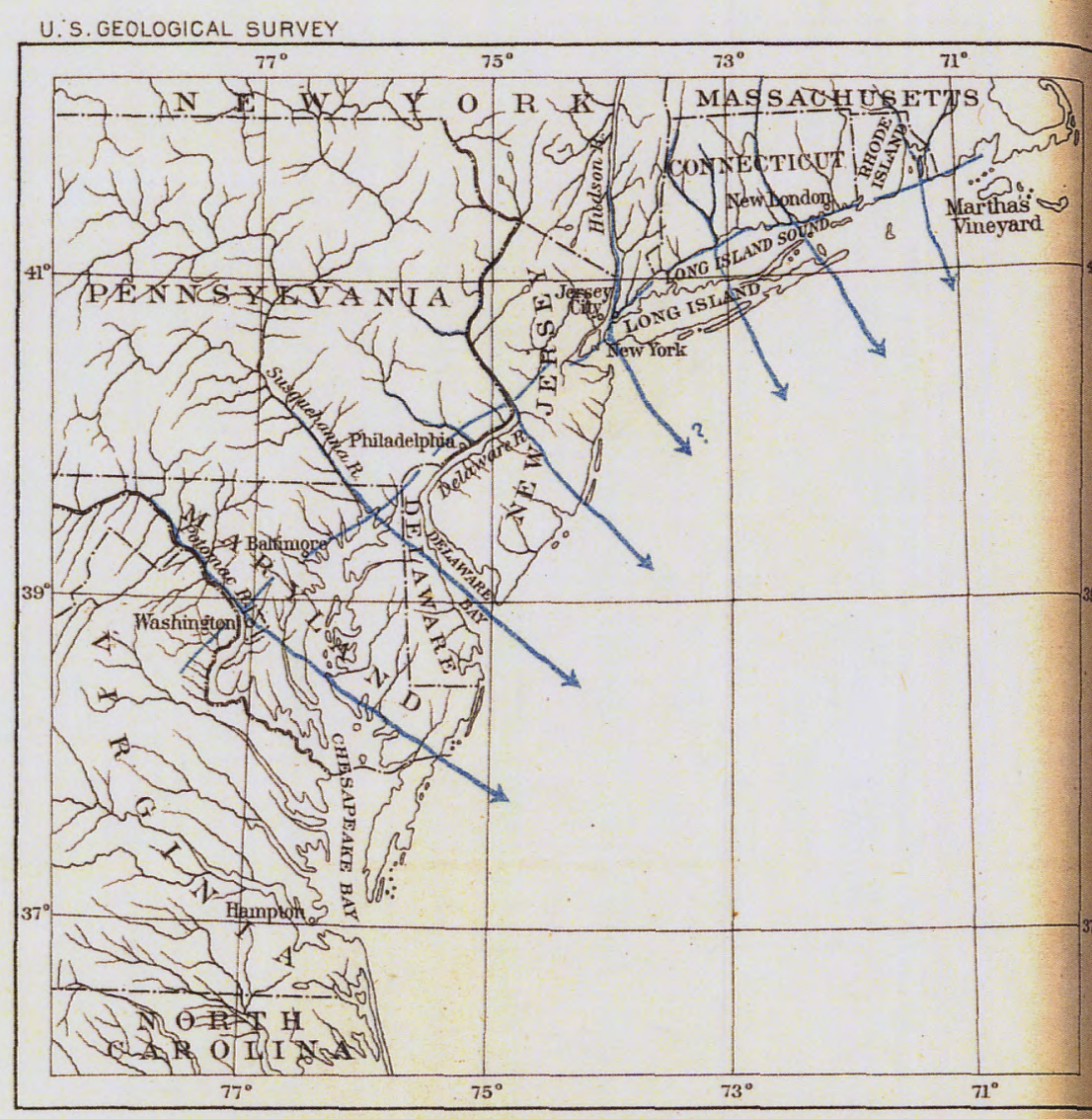

(A) POST-MIOCENE EROSION INTERVAL

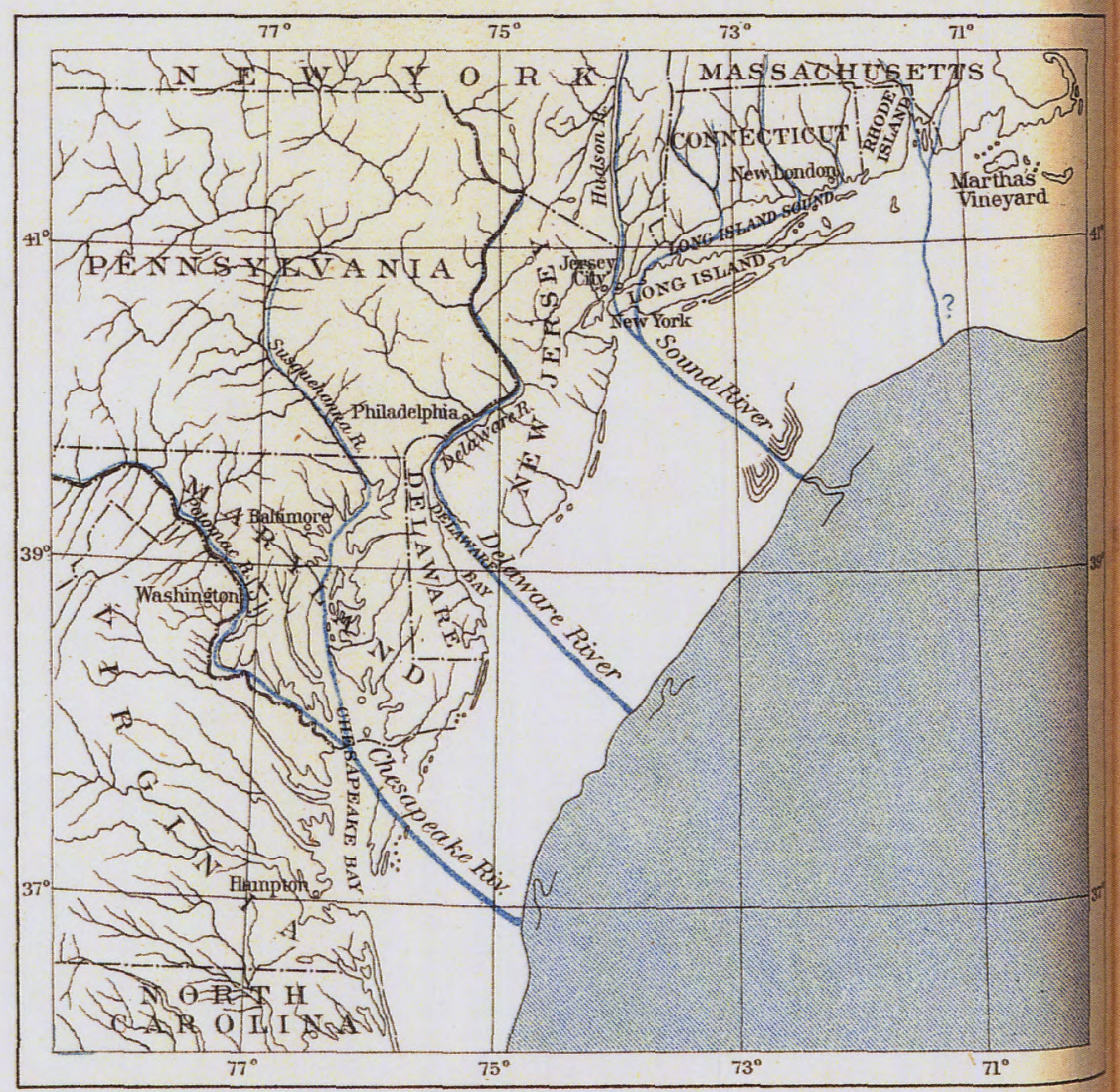

C) POST - MANNETO EROSION INTERVAL

DEVELOPMENT OF MAJOR DRATNA
PROFESSIONAL PAPER NO.44 PL.VI

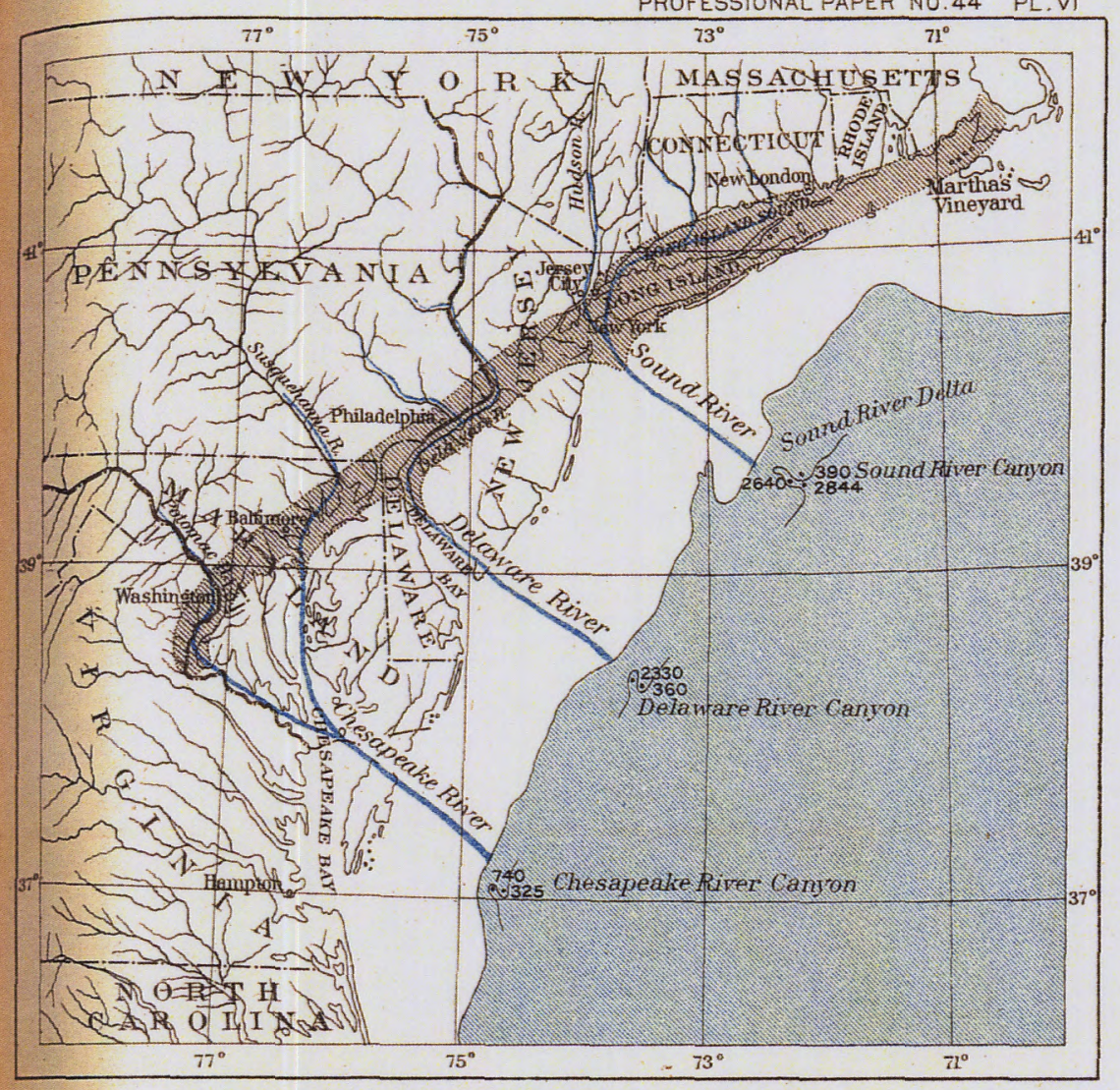

LEGEND

arich

Present drainage

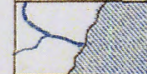

Former drainage
and shore line

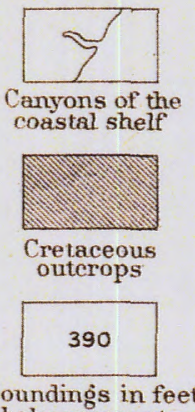

Soundings in feet
belowpresent sealevel
sent

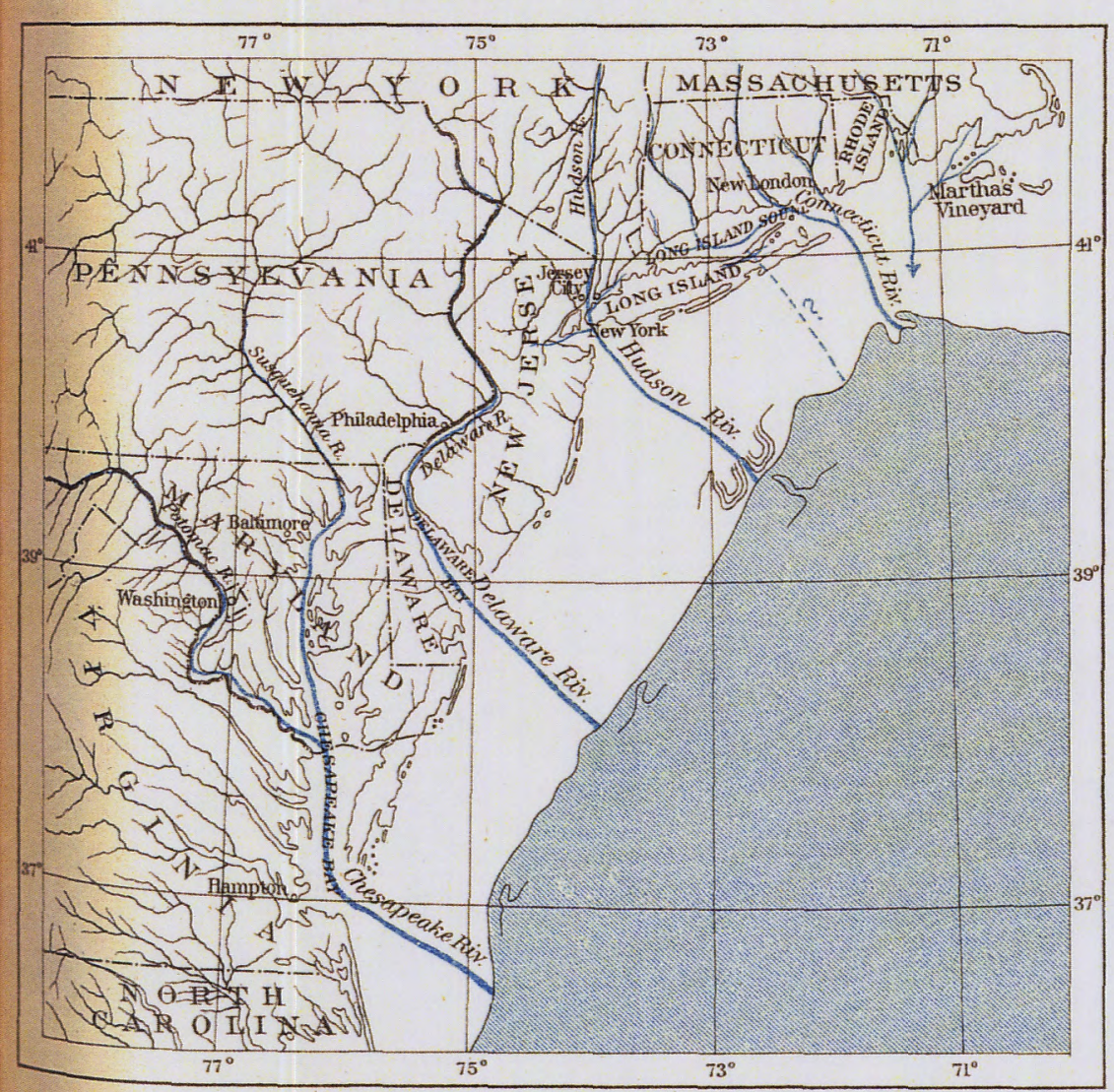

(D) VINEYARD EROSION INTERVAL

F NORTH ATLANTIC COASTAL PLAIN 



\section{QUATERNARY.}

While during the Cretaceous and Tertiary the portion of the Atlantic coastal plain between Cape Hatteras and Nantucket was subjected to very nearly the same conditions and the development was therefore the same in both periods, in the Quaternary new factors arose which affected only the region from Long Island eastward, and gave to it a surficial aspect differing decidedly. from that of the other portions of the coastal plain to the south. Although the several ice advances directly affected Long Island and the region eastward, none of them reached the coastal plain of New Jersey and Maryland, and here the only records of Pleistocene time are therefore the terraces formed in such positions that they were not destroyed by subsequent submergences.

In the region affected by the glaciers the following divisions of the Pleistocene have been recognized:

TABLE II.-Pleistocene formations on Long'Island.

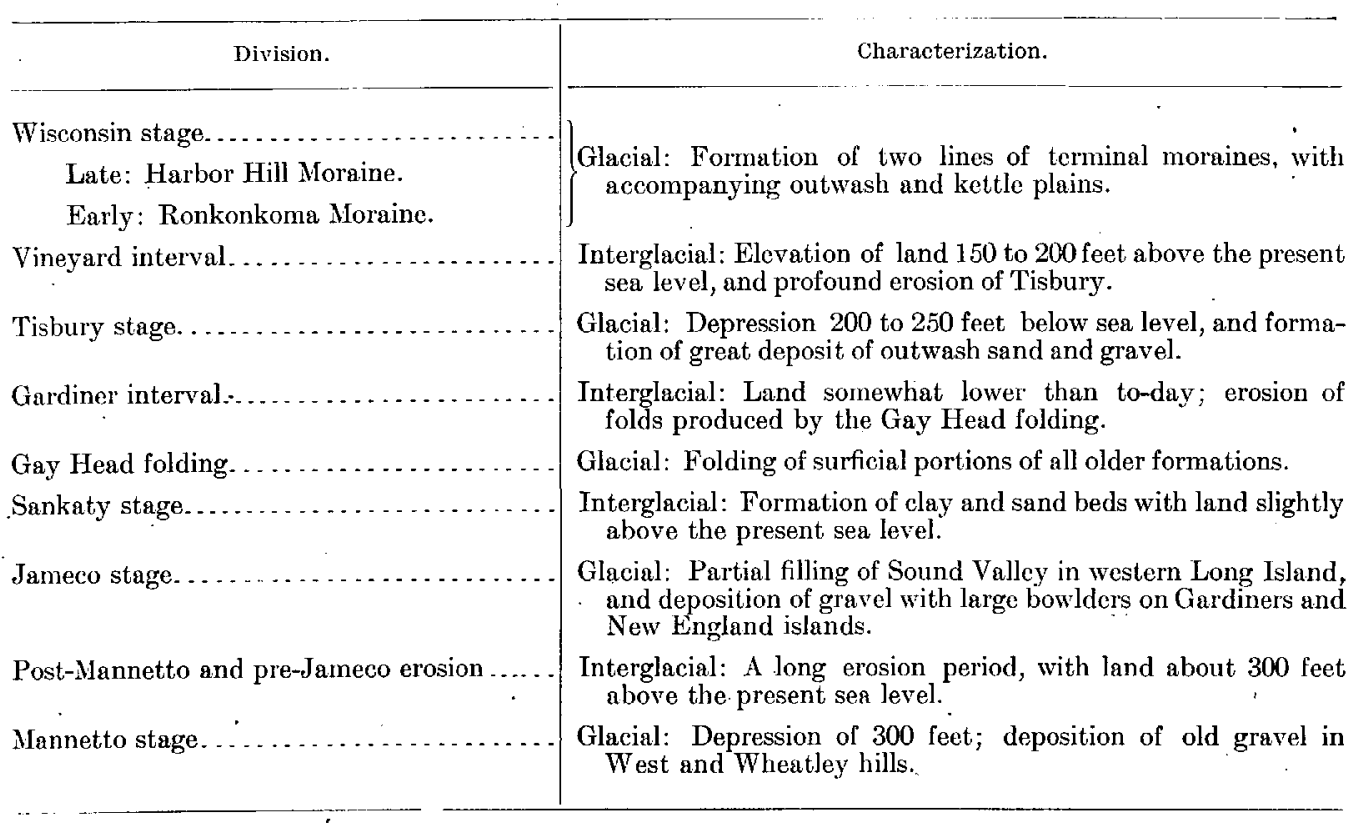

\section{MANNE'TO GRAVEL.}

CONDITIONS OF DEPOSITION

Following the long post-Lafayette erosion epoch, when the drainage was approximately as shown in Pl. VI, $B$, the land was submerged to a depth of about 300 feet at Long Island, and a mantle of gravel and loam spread over the irregular surface developed during the Tertiary. 
34 ' 'UNDERGROUND WATER RESOUROFS OF LONG ISLAND, NEW YORK.

CIIAR.AOTER OF DEPOSITS

In this region these deposits are for the most part composed of quartz gravel, but contain also some very much decayed pebbles and bowlders of probable glacial origin, in which respect they agree with the earliest Pleistocene deposits which Salisbury has recognized in New Jersey.

PRESENT DISTRIBUTION

Because of the destructive and reconstructive effects of the succeeding periods the deposits of this age are now recognizable, as a rule, only in the higher levels, and the typical examples on Long Island are, therefore, on the highest hills of the prePleistocene, as in the Mannetto (West) and Wheatley hills, from the first of which the formation has been named.

\section{POST-MANNETTO AND PRE-JAMECO INTERVAL}

Following the deposition of the Mannetto gravel the land was again lifted, this time to a height of something of over 250 feet, and the work of the preceding erosion epochs was continued The Mannetto deposits were to a large extent removed and the valleys somewhat deepened.

JAMECO GRAVEL

GONDITIONS OF DEPOSITION

The ice sheet, again advancing, appears to have about reached the present north shore of Long Island, and to have extended well down toward Block Island

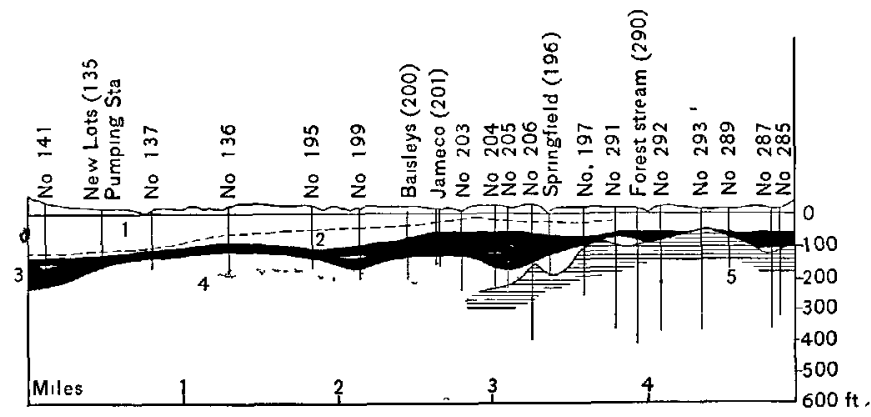

FIG 10 -Section from niear Ridgeway, Brooklyn, to Valley Stream, showng position of (1) Wisconsin, (2) Tisbury, (3) Sankaty, (4) Jameco. (5) Cretaceous beds, and the east side of the Sound River Valley Figures correspond with

those used in Pl XXIV and in Chapter IV $\quad$ was deposited along the upland. The deposits are then thickest in and near the old valley; they are poorly developed on the south shore east of this valley, but reappear in force on eastern Long Island and the islands to the east, where they have been brought up by folding.

CHARACTER OF DEPOSITS

In western Long Island the Jameco gravels consist of dark-colored sands and gravels that vary, considerably in coarseness and are distinguished by the small per- 
centage of quartz which they contain. Even the surface gravel, which represents the outwash when the ice was but a few miles to the north, contains a very much higher percentage of quartz; the only gravel beds on the island resembling these occur in the Wisconsin deposits in and north of the moraine. So pronounced was the glacial character of these old gravels that when they were first examined it

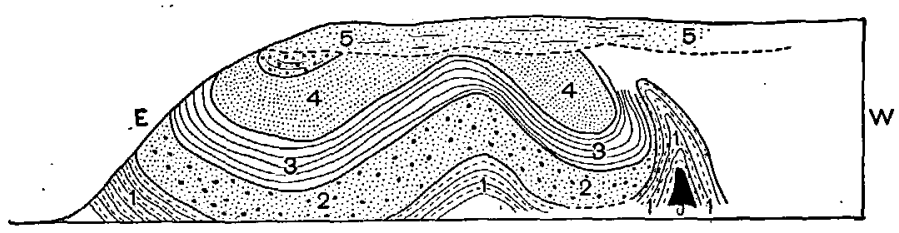

Frg. 11.- Section near middle of northeast shore of Gardiners Island, N. Y.; (0) black Cretaceous clay; (1) fine gray micaceous sand (Cretaceous); (2) Jameco gravel; (3) red clay (Sankaty); (4) silty sand (Sankaty); (5) Wisconsin till and outwash gravel. Height of section, 60 feet.

was thought they surely represented surface Wisconsin deposits, and that some. careless clerk had inverted the tube and labeled it upside down; " but this theory became untenable as well record after well record was examined, and all, in certain regions, showed the following succession:

Geologic succession in wells in western Long Island.

1. Quartz sand and gravel with a noticeable percentage of erratic material (Wisconsin).

2. Quartz sand, gray or yellow, with little if any material of recognizable glacial origin (Tisbury):

3. Blue clay with wood (Sankaty).

4. Dark, multicolored, highly erratic gravel (Jameco).

East of this valley and the delta-like extension at its opening the only repre-

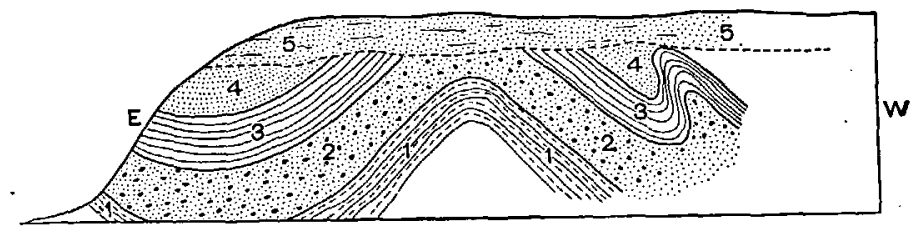

F19. 12.-Section on west side of hollow which afforded the section in fig. 11, about 200 feet farther west. The numerals indicate same beds as in fig. 11 . sentatives of this period are the normal coastal sands and gravels similar to the beds above and below, and seldom separable from them.

At Gardiners Island and on Marthas Vineyard the percentage of fine, yellow, gravel is much greater, and the beds contain very large bowlders of compound quartz crystalline rocks, indicating the nearness of the ice. These beds, which are here brought up by folding, are likewise separated from the younger gravels by the clay deposits of the Sankaty (figs. 11, 12).

PRESENT DISTRIBUTION.

On the north shore the Jameco beds have been considerably eroded and disturbed and are not always readily separable from the succeeding deposits. Occasional remnants of considerable local importance as sources of water supply, however, have been encountered, as indicated in the well records.

On the south shore where the Jameco beds have not been eroded and are typically developed in the region of the old valley (fig. 10) they form one of the most important water horizons of the island.

a The samples of the borings of the Brooklyn waterworks are preserved in the Municipal Building, Brooklyn, N. Y., in glass tubes representing miniature reproductions of the borings. 


\section{SANKATY FORMATION.}

CONDITIONS OF DEPOSITION.

The effect of the Jameco epoch was to partly fill the Sound Valley on western Long Island and to spread a relatively thin cover of gravel and sand over the areas not in the lee of the old land masses. With the continuance of the progressive subsidence, which appears to have begun near the close of the post-Mannetto erosion interval, the coarser beds of the Jameco were succeeded by finer sediments, and as the ice retreated, temperate water forms similar to those living to-day occupied the waters. The land stood about 50 feet higher than to-day, and there existed an ancestral Long Island rudely resembling the present island. The beds forming near its shore were predominantly swampy and in many ways similar to those accumulating on a minor scale at present. These swamp conditions gave place in deeper water to more truly marine ones, where marine forms were included, in greater or less numbers, in the sediments deposited.

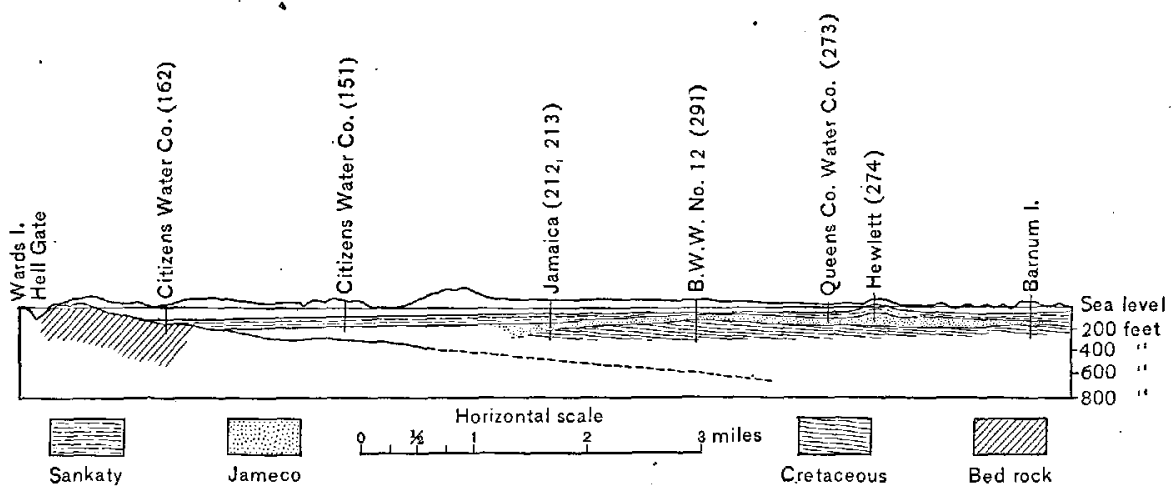

Fig. 13.-Section from Wards Island to Barnum Island, showing fold at Rockaway Ridge (Hewlett), and the relations of the Sankaty, Jameco, cretaceous, and "bed rock." Figures correspond to those used on Pl. XXIV and in Chapter IV

CHARACTER OF DEPOSI'T.

These sediments therefore vary from truly swampy deposits on the one hand to relatively fine sands and clays, which show no trace of swamp origin, and which contain shallow-water mollusks on the other; thus on western Long Island, where the partly filled channel of the Sound Valley favors the formation of swamp deposits, there are irregular beds of dark-colored clay (figs. 10,13), containing considerable lignite and lignitized wood, occasional lenticular beds of silty sand and gravel from 5 to 10 feet thick, and, toward the coast, a few marine shells. On the other hand, the beds of this age on Gardiners Island (figs. 14, 15), which have been brought up by folding, were formed farther from the shore, contain no lignitic material, and carry a good molluscan fauna. In general this formation is about 50 feet thick, although some of the Brooklyn waterworks test borings show a thickness of 150 feet near the axis of the old valley.

PRESENT DISTRIBUTION.

The Sankaty deposits, like the Jameco, occur'on the north shore merely as erosion remnants, more or less disturbed by folding, and associated with some- 
what similar Cretaceous deposits. They afford some of the local clay layers which are the retaining layers in some of the shallow north shore artesian wells (fig. 16).

On the south shore these beds are most typically developed in the region of the old valley, where they form the retaining layer for the water in the Jameco gravels (fig. 13). East of Springfield they are less typical, although well developed at the Queens County Water Company's plant and under Rockaway Ridge. Their presence is suggested by the silty clays overlying the artesian horizons at a number of the Brooklyn plants east of Millburn.

\section{GAY HEAD FOLDING}

DESCRIPTION.

The exact conditions immediately following the deposition of the Sankaty are not known, but there is no evidence indicating that the relative positions of

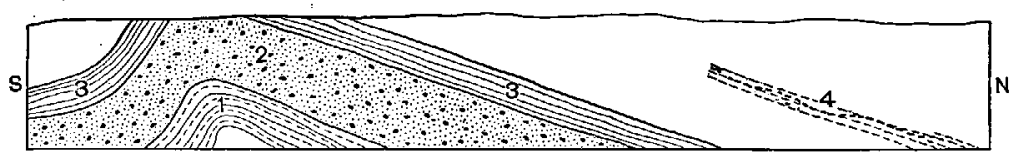

FIG. 14.--Section at Tobacco Point, east side of Gardiners Island, N. Y. 1, Cretaceous; 2, Jameco gravel; 3, red clay (Sankaty); 4, fossil bed with bowlders (Sankaty). Height, 20 feet; length, 1,200 1 feet. Surface beds omitted.

the land and sea were materially changed. The increasing sandiness of the upper part of the Sankaty on Gardiners Island suggests a slight change from the progressive subsidence which began in the post-Mannetto interval, but the change was not of a very great order. The important and distinctive feature of this period is the wonderful folding and disturbance of the beds along the north shore of Long Island and the islands eastward. These folded and faulted strata can now best be seen at Gay Head on Marthas Vineyard (fig. 17) and on Gardiners Island (figs. 11, 12, 14, 15). At Gay Head Woodworth has very carefully worked out a section showing a deformation of more than 200 feet and a wonderful series of closely compacted folds and faults. On Gardiners Island

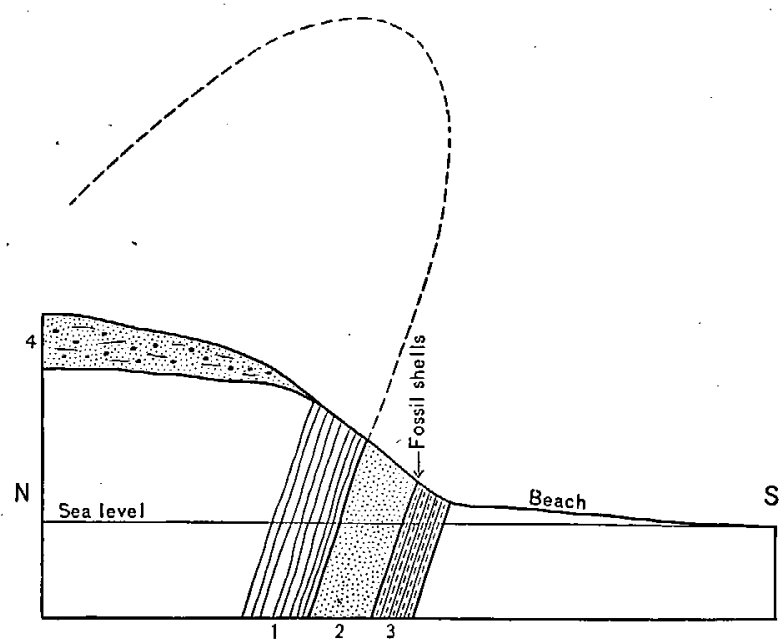

FIG. 15.-Section near Cherry Hill Point, Gardiners Island, showing location of fossil-bearing stratum. 1, Laminated red clay and sand; 2, mottled-gray, brown, and yellow sand; 3 , dark, yellowish-brown, silty clay; 4, till. 1-3 are Sankaty; 4 is Wisconsin. the folds are as complicated as on Marthas Vineyard, and the opportunities for study even better. It is regarded as particularly unfortunate that time was not available in which to work out the detailed maps and sections, which are urgently needed at this locality. At present 
it can only be stated that while the axes of a series of adjacent folds are generally parallel, they are not parallel to the axes of a series at no very great distance. Thus, near the center of the northeast shore a series of four folds was seen whose axes are $\mathrm{N} .20^{\circ} \mathrm{W}$., while a little farther east, near Eastern Plain Point, the axis of three or four sharp overturned folds is almost due east and west.

Ries observed a similar folded structure on Fishers Island, where excavation has shown that the folding does not extend downward over 20 or 30 feet." On Long Island, near Orient, Mather observed the same phenomena (fig. 18) and noted their superficial character. ${ }^{b}$ Some folding and disturbance of strata can be observed in nearly all of the outcrops of the older beds on the north shore, among which should be noted particularly those near Lloyd Beach in Cold Spring Harbor, at the southern end of Center Island, and at Glen Cove and Sea Cliff. In these regions well borings have clearly shown that the folding is entirely superficial (Pl. II, fig. 16).

OAUSE OF FOLDING.

In studying the cause of this folding four principal points need to be considered: (1) As the folding involves glacial deposits, it is clearly Pleistocene; (2) it is essen-

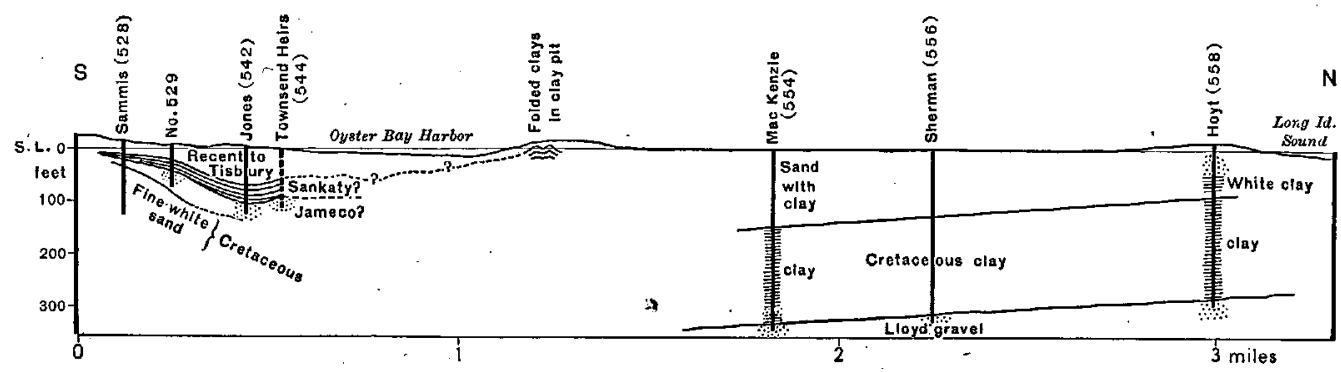

FIG. 16.-Cross section through Oyster Bay and Center Island, showing relations of clay and water-bearing horizons eneountered in the Oyster Bay wells to the Cretaceous clays and Lloyd gravel in the Center Island wells.

tially superficial and, therefore, can not be of orogenic origin; (3) it occurs wholly in a glaciated region, other portions of the coastal plain showing no analogous phenomena; (4) the general direction, as well as the local irregularities of the folds, are such as would be expected from ice thrusts. To account for these folds three theories have been advanced: (1) That they are due to landslips; ${ }^{c}$ (2) that they were formed by mountain-building forces; ${ }^{d}$ (3) that they were produced by the lateral shove of a continental ice sheet. ${ }^{e}$

It is well known that landslip or hill creep can produce local disturbances of considerable importance, and these phenomena may be observed to-day in all bluff sections or steep slopes in this region, particularly at the Broken Grounds or Ragged Ground near Fresh Pond, north of Northport (Pl. VII). At these places, however,

$a$ Bull. New York State Mus. No. 35, 1900, p. 603.

$b$ Geology of the First District, 1843 , pp. 249, 259.

c Mather, W. W., Report of the first district, 1843, p. 249. Dana, Manual of Geology, 1895, p. 1021.

d Shaler, N. S., Seventh Ann. Rept. U. S. Geol. Survey, 1888, pp. 343-347; Bull. Geol. Soc. Am., vol. 5, 1894, pp. 199-202; Bull. Geol. Soc. Am., vol. 6, I895, p. 7. Dana, Manual of Geology, 1895, p. 934 .

e Merrill, F. J. H., Proc. Am. Assoc. Adv. Sci., vol. 35, 1886, pp. 228-229. Hollick, Arthur, Trans. New York Acad. Sci., vol. 13, 1894, p. 123; Bull. Geol. Soc. Am., yol. 6, 1895, pp. 5-7, Ries, Heinrich, Bull. New York State Museum, No. 35, 1900, p. 603. 


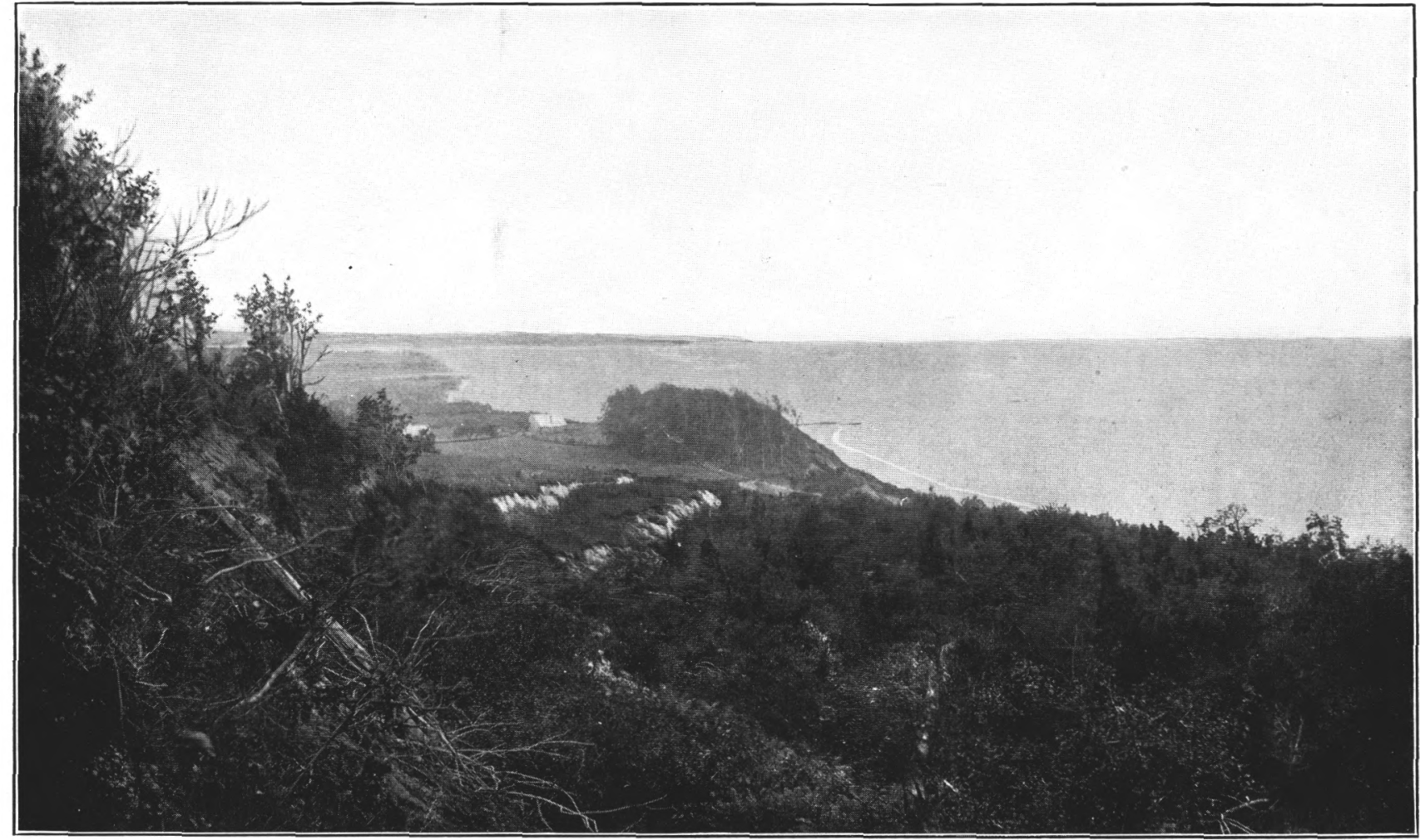

LANDSLIPS DUE TO PARTIAL UNDERMINING OF CLIFF FACE BY OCEAN AT THE BROKEN GROUNDS, OR RAGGED GROUND, NEAR FRESH POND, NORTHEAST OF HUNTINGTON, NORTH SHORE OF LONG ISLAND.

Photograph by Edward P. Buffet. 

the folding is essentially local, and presents neither the characteristics nor the magnitude of the occurrences at Gardiners Island, Block Island, and Marthas Vineyard, where the materials have been forced up-not let down. Moreover, no analogous foldings occur in the southward extension of the coastal plain beyond the limits of ice action.

The theory of orogenic origin is not only ruled out by the superficial character of the folding, but has other insurmountable objections.

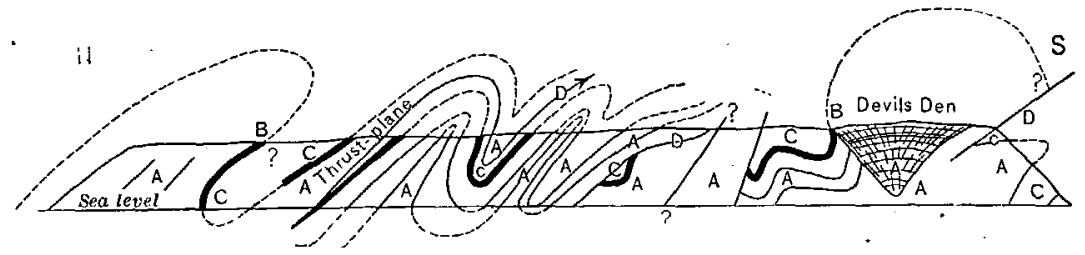

Fig. 17.-Cross section at Gay Head, Marthas Vineyard. After Woodworth. A, Cretaceous; B, Miocene, with prohably Pliocene; C, Jameco and Sankaty; D, thrust planes and faults. Surface morainal deposits omitted. Height, 120 feet; length, 1 mile

The only hypothesis which explains all of the phenomena observed is that the folding was produced by the thrust or drag of a continental ice sheet. As the major portion of the folding occurred at one time, or in the same epoch, and

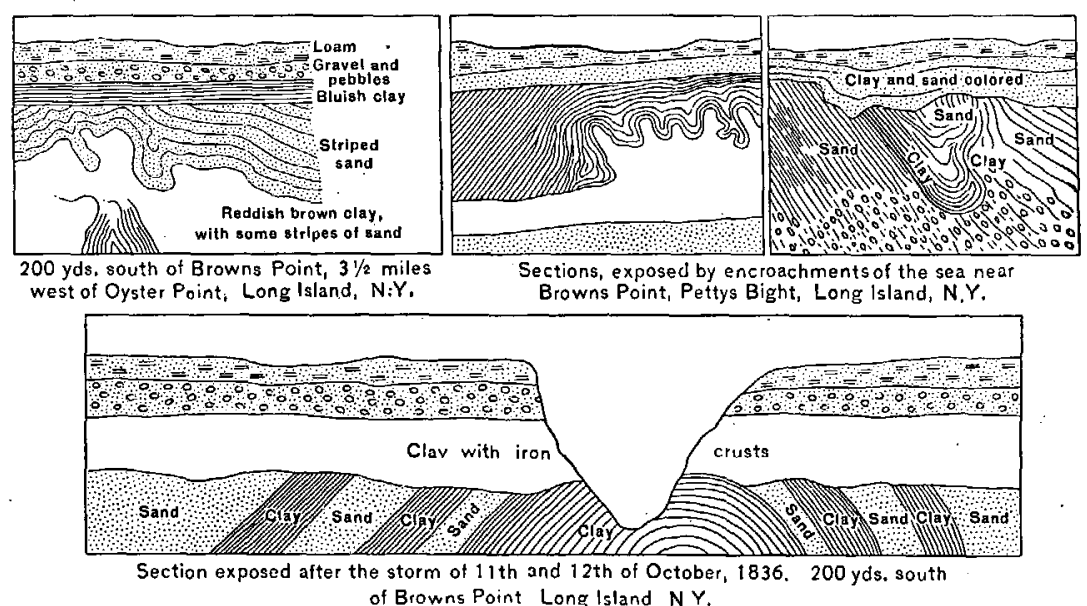

Fia. 18.-Sections exposed at Browns Point after storm of October 11 and 12, 1836. After Mather, 1843.

as later deposits show only minor disturbances, it is necessary to suppose that the conditions were more favorable during the Gay Head stage than during the Wisconsin stage, which is the only other advance which approached this one in extent. Among the conditions which may have been effective in producing this difference in results, the following may be enumerated: (1) The ice producing this folding extended farther south than any previous advance, and therefore was resisted by more of the original irregularities of the surface; (2) the clayey character of the strata against which it pressed was particularly favorable for the production and preservation of the folds, while before the Wisconsin all the older beds had been covered with a heavy mantle of Tisbury gravels which did not lend themselves so readily either to deformation, or the preservation of records of deformation. 
In the two most noted examples of disturbance by glacial action in Europethe Norfolk Cliffs in England, and the cliffs of the islands of Möen and Rügen ${ }^{a}$ in Denmark and Germany - the conditions were very similar to these in this region. The ice, coming from the harder, pre-Cretaceous rocks, passed across a depression, which 'may have been filled with water, and, impinging against the higher upper Cretaceous beds covered with glacial sands and clays of a former advance, produced very remarkable dislocations and contortions. The Cretaceous chalk, being more brittle than the Cretaceous clays of the coast of the United States, was more often broken, and great masses were pushed up bodily and commingled with the glacial beds.

The same suggestions of origin have been proposed for these European icemade folds and faults, with the addition, in England, of an iceberg hypothesis. This theory, first proposed by Lyell, ${ }^{b}$ was widely adopted in England, and it was not until the extended, careful work of Reid that it was shown to be untenable.

\section{GARDINER INTERVAL.}

After the Gay Head folding the tops of the folds were truncated. While this truncation might be produced by the overriding of the ice, the exposures on Gardiners Island show no evidence that it was accomplished in this manner. The truncation is clean, not dragged as it would be if it had been produced by ice, and bears all the aspects of having been produced by water erosion. Woodworth has arrived at a similar conclusion from a study of the exposures on the New England islands, and feels that a considerable erosion period is indicated. The truncation, as was first observed by Mr. Isaiah Bowman, is more nearly that which would be produced on a slowly subsiding coast by wave action than that caused by stream erosion at a high level. Of course, very long-continued erosion would eventually produce a base-level condition, but the decapitation of the folds on such limited areas as Gardiner, Block, and Nantucket islands, and Marthas Vineyard, under such favorable conditions as must have existed there, would be much more quickly and normally accomplished by wave action than by run-off. ${ }^{d}$ It is therefore felt that the land during this erosion interval, instead of standing higher than to-day, was 50 to 100 feet lower. As the name Gay Head belongs more properly to the folding $f$ than to the erosion interval which followed, the name Gardiner interval is suggested for the latter, from Gardiners Island, where the truncated folds can be well observed.

a Johnstrup, F., Über die Lagerungsverhälttnisse und dỉ Hebungsphünomene in die Kreidefelsen auf Möen und Rügen: Zeitschrift Deutschen Geol. Gesell., Band 26, 1874, pp. 533-585. Reid, Clement, The glacial deposits of Cromer: Geol. Mag., ncw series, vol. 7, 1880, pp. 55-66, 238-239; The geology of the country around Cromer: Memoirs Geol. Survey England and Wales, 1882. Geikie, James, The Great Ice $A$ ge, 1894, pp. 339-341, 426-430.

$b$ Lyell, Charles, on the bowlder formation or drift and the associated fresh-water deposits composing the mud cliffs of eastern Norfolk, London, and Ndinburg: Phil. Mag., vol. 16, 3d ser., 1840, p. 379.

c Bull. Geol. Soc. Am., vol. 8, 1897, pp. 207-211.

a See roferences to destruction of European coast by wave action in Lyell, Principles of geology, vol. 1, 1872, pp. 507-564; Geikie, Text-book of geology, vol. 1, 1903, pp. 571-593; also Chamberluin and Salisbury, Geology, 1904, pp. 326-331; Shaler, Sea and land, pp. 1-30; Tarr, Physical geography, pp. 332-333.

e Seventcenth Ann. Rept. U. S. Geol. Survey, pt. 1, 1896, table facing p. 988.

$f$ Sec usage of "Gay Head diastrophe," by Woodworth, Bull. Geol. Soe. Am., vol. 8, 1897, pp. 207-210. Professor Woodworth writes, December 5, 1904: "In regard to the use of the phrase "Gay Head folding " or its synonym, "Gay Head diastrophe" I agree with you that it is desirable to restrict it to the merc fact of the episode of the dislocation and to frec it from the idea of erosion which followed the time of folding. It was an oversight on my part in not specifically abandoning the earlier term of the "Gay Head interval," which covered the whole question of the unconformity." 
U. S. GEOLOGICAL SURVEY

PROFESSIONAL PAPER NO. 44 PL. VIII

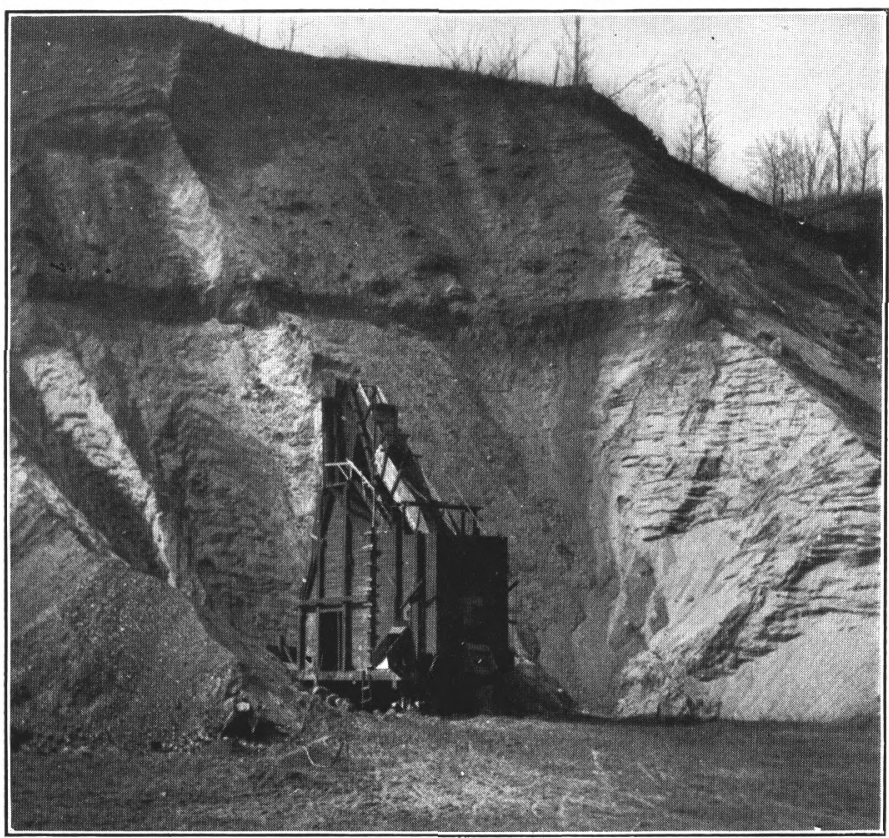

$A$

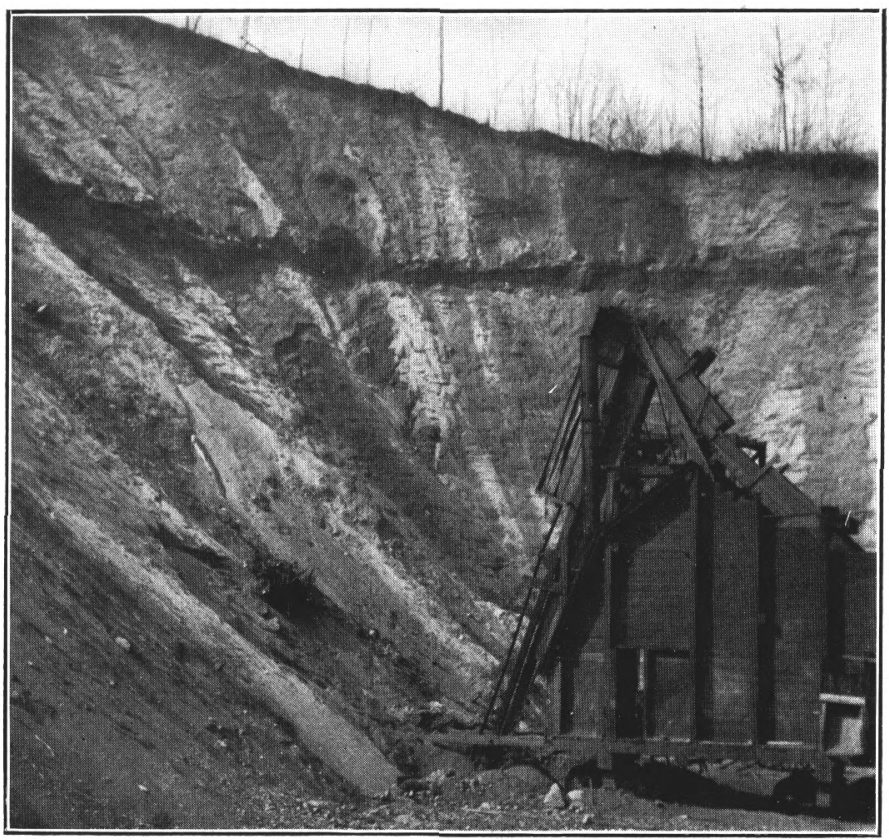

$B$

HORIZONTALLY STRATIFIED TISBURY (MANHASSET) SAND AND GRAVEL BEDS, WITH INCLUDED LAYER OF BOWLDER CLAY (DARK-COLORED BAND), MANHASSET BOWLDER BED, KING'S SANDPIT, HEMPSTEAD HARBOR, N. Y. 

TISBURY (MANHASSET) GRAVEL

CONDITTONS OF DEPOSITION

The subsidence that began near the close of the post-Mannetto erosion interval, and gradually mcreased during the Jameco, the Sankaty, the Gay Head, and the Gardiner culminated in the Tisbury epoch, with a total depression of 200 to 250 feet below the present sea level The ice again advancing passed beyond the continental border and deposited gieat beds of outwash gravel in the border of the sea and around the higher parts of Long Island, then a group of rather small sslands As these deposits were perhaps laid down to a great extent by a retreating ice sheet, it is possible that the ice extended south of the Sound and that the giavel capping the Half Hollow Hills south of the Wisconsin deposits, and lapping around the southern edge of the West Hills was deposited in this earlier greater advance The greater portion, however, was formed when the ice was but a short distance north of the present shore, the northern edge of these deposits terminating along this line in the rather abrupt, scarp of a sand plam The deposition took place very near sea level, and at times the submergence was sufficient to allow floating ice Such conditions are thought to have controlled the formation of the bowlder bed in the midst of the stratified gravels in the region about Hempstead Harbor and caused the irregular distribution of bowlders through beds of the same age on Marthas Vineyard (See Pl VIII.)

CHAKLCTER OF DEPOSIL .

The deposits of this epoch consist of quartz sand and gravel, containing a relatively small proportion of slightly weathered compound crystalline pebbles. They lie horizontally on the truncated folds produced by the Gay Head folding and Gardiner erosion and are separated from the Wisconsin deposits by a marked unconformity On Long Island they differ from the Jameco in the small amount of erratic material which they contam and from the still older Mannetto in the very slight weathering of the compound pebbles These lithological distinctions can not, however, be regarded as absolute, and confirmatory stratigraphic evidence must be sought in all cases

PRESENT DISTRIBUTION

Woodworth has shown that on the north shore of Long Island the Tisbury sunds were deposited as a comparatively level, plateau-like plain, reaching a height of over 200 feet In this region the beds are most characteristically developed and attam a maximum thickness of 150 to 250 feet Deposits are commonly thinner near the axes of the penunsulas and thicken toward the valleys, as would be expected from theu deposition over an antecedent topography

The surface exposures show that the Wisconsin is relatively thin, and while there is always a chance of correlating some Mannetto or Cretaceous with this gravel, or of including stratified sand and gravel of Wisconsin age, the following table may be regarded as giving a falr approximation of the thickness of these beds in this region. 
UNDERGROUND WATER RESOURCES OF LONG ISLAND, NEW YORK.

TABLE III.-Thickness of late Pleistocene deposits in wells on the north shore of Long Island.

\begin{tabular}{|c|c|c|c|c|}
\hline Noa & Location. & $\begin{array}{l}\text { Total } \\
\text { depth. }\end{array}$ & $\begin{array}{l}\text { Recent to } \\
\text { Wisconsin: }\end{array}$ & Tisbury. \\
\hline 151 & Corona $\ldots \ldots \ldots \ldots \ldots \ldots \ldots$ & . 190 & $?$ & $90 \pm$ \\
\hline 239 & 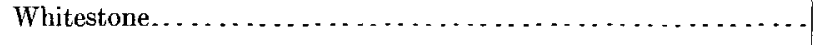 & 95 & $25 \pm$ & $?$ \\
\hline 241 & 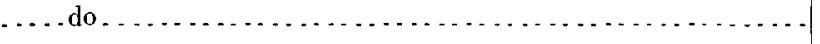 & 120 & 15 & 45 \\
\hline 246 & 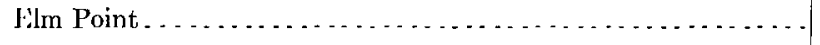 & 104 & 12 & $44+$ \\
\hline 247 & $\ldots$ do $\ldots . . . . . . . .$. & 67 & 30 & $36 ?$ \\
\hline 326 & Thonraston $\ldots \ldots \ldots \ldots \ldots \ldots \ldots$ & 79 & 27 & 14 \\
\hline 457 & 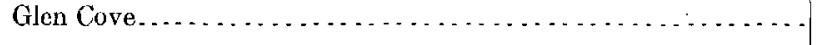 & 186 & $9+$ & 149 \\
\hline 459 & 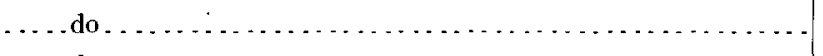 & 170 & 41 & 109 \\
\hline 460 & $\ldots$ do . . . . . . . . . . . . & 108 & $?$ & $90 \pm$ \\
\hline 465 & 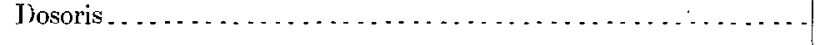 & 215 & $6+$ & $100 \pm$ \\
\hline 476 & Lattingtown $\ldots \ldots \ldots \ldots \ldots \ldots \ldots \ldots \ldots \ldots$ & 265 & 7 & 100 \\
\hline 477 & $\ldots$ do $\ldots \ldots$. & 110 & $?$ & $100 \pm$ \\
\hline 484 & $\therefore$ do....... & 108 & 0 & $108 \pm$ \\
\hline 485 & $\ldots$ do $\ldots \ldots \ldots . . . . . .$. & 110 & 0 & $100 \pm$ \\
\hline 564 & Mill Neck. . . . . . . . . . . & 330 & $?$ & $100 \pm$ \\
\hline 596 & Cold Spring Harbor . . . . . . . . . & 228 & 0 & 190 \\
\hline 601 & ....do. . . . . . . . & 195 & 0 & $195 \pm$ \\
\hline 613 & 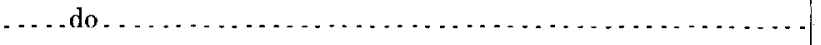 & 176 & 14 & 86 \\
\hline 624 & 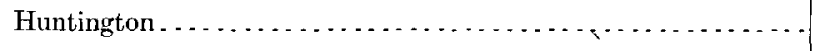 & 181 & 80 & $75 \div$ \\
\hline 628 & 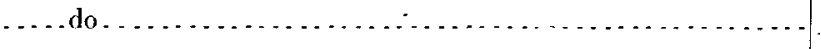 & 498 & 10 & 78 \\
\hline 629 & $\ldots$ do $\ldots \ldots \ldots \ldots \ldots \ldots \ldots$ & 97 & 25 & 60 \\
\hline 633 & 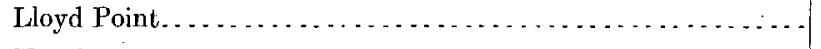 & 250 & $(0-$ & $95)$ \\
\hline 651 & 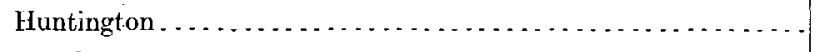 & 102 & $?$ & 102 \\
\hline 652 & 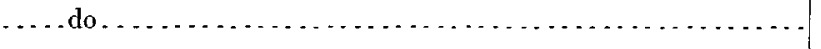 & 75 & 10 & 70 \\
\hline 654 & 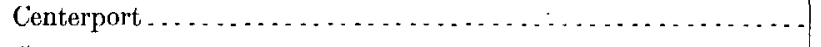 & 185 & $-\ldots .$. & 175 \\
\hline 659 & 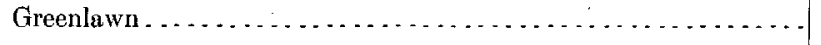 & 186 & 0 & $b 186$ \\
\hline 660 & 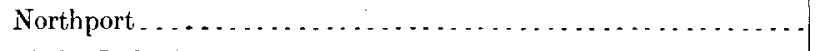 & 196 & 0 & 196 \\
\hline 666 & 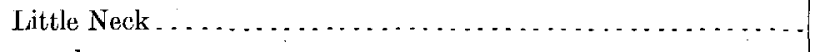 & 143 & $\ldots \ldots$ & 130 \\
\hline 667 & $\ldots$ do $\ldots \ldots$. & 127 & & $127+$ \\
\hline 683 & 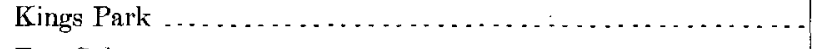 & 152 & 4 & 152 \\
\hline 686 & 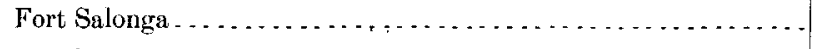 & 120 & ? & 115 \\
\hline 687 & 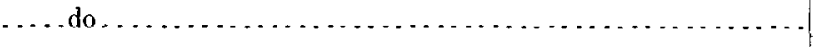 & 106 & $\underline{1}$ & $106 \pm$ \\
\hline 720 & Smithtown. . . . . . . . . . . . . . . . . . . . . . . & 212 & +0 & 130 \\
\hline 724 & 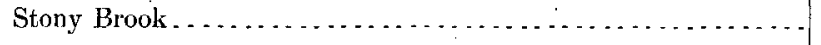 & 110 & 0 & 110 \\
\hline 750 & 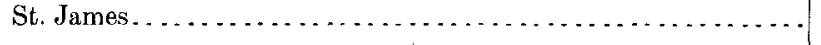 & 150 & $60 ?$ & 90 \\
\hline 751 & 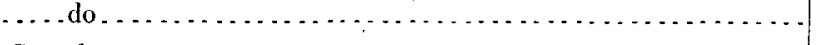 & 250 & 30 & $c 220 ?$ \\
\hline 763 & 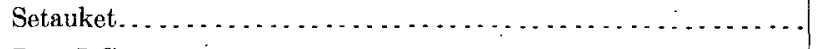 & 320 & 0 & 85 \\
\hline 811 & 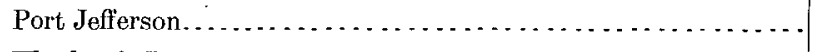 & 370 & $11 ?$ & $270+$ \\
\hline 825 & 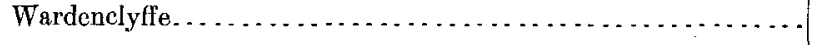 & 347 & $?$ & $135 \pm$ \\
\hline 892 & 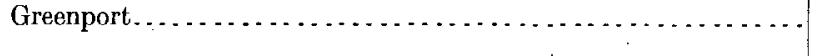 & 690 & 20 & 80 \\
\hline
\end{tabular}

a These numbers correspond with those used in Chapter IV, where additional details will be found. c Glacial, may be in part older than Tisbury. 
On the south shore the thick beds of sand with only a slight percentage of glacial material, which occur between the Sankaty clay and the surface Wisconsin gravel; are regarded as largely Tisbury.

\section{VINEYARD INTERVAL. ${ }^{a}$}

CHARAOTER OF SURFACE AT BEGINNING OF INTERVAL.

On Long Island the Tisbury deposits to a large extent buried the older topography. They continued the filling of the Sound Valley across western Long Island, which was begun in the Jameco epoch, and buried the deep valleys which had been developed in the northern portion of Long Island by streams flowing into the Sound River. It does not seem probable that the deposits extended entirely across the Sound, as they would have done had they been normal marine deposits.

MAJOR DRAINAGE.

With the retreat of the ice and the elevation of the land the rivers from the mainland discharged into the depression overlooked by the sharp edge. of the great Tisbury sand plain. The old channel across western Long Island having been completely filled by these deposits, the Housatonic must have discharged either through East River or to the east. The latter direction is indicated by the soundings in Long Island Sound. ${ }^{b}$ Dana has suggested that those soundings indicate that the river crossed the North Fluke near Mattituck. If such was its course, it probably continued southward, as indicated in Pl. VI, $D$. There is, however, no reason for regarding this course

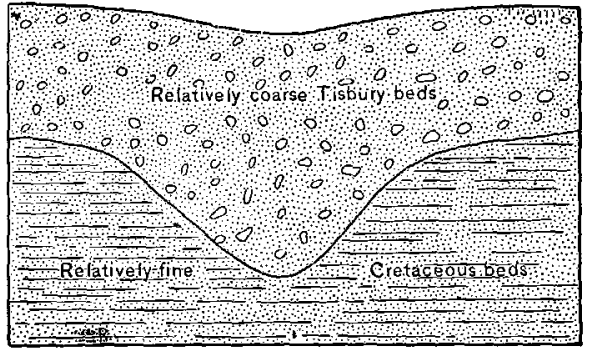

Fra. 19.-Diagram illustrating factors giving spring phenomena great power in reexcavating the north shore valleys. as any more probable than a continuation eastward to a juncture with the Connecticut.

The normal course for the Connecticut under these conditions would be between Plum and Fishers islands and Montauk Point and Block Island, and the present deep channel between these points is believed to be ultimately traceable to this cause. Moreover, the soundings of the Coast Survey show, at a depth which other considerations caused to be selected for the supposed shore line during this epoch, a distinct deltaform projection at the point where the Connecticut must have discharged (Pl. VI, D).

$$
\text { REEXCAVATION OF THE. NORTI SHORE VALLEYS. }
$$

With the establishment of these new drainage lines the reexcavation of the valleys on the north shore began along lines determined by the position of the buried valleys of the northward-flowing streams of the pre-Tisbury epoch. This reexcavation was greatly aided by the great porosity of the materials filling the valleys, which concentrated the underground waters in the older depressions and gave 


\section{UNDERGROUND WATER RESOURCES OF LONG ISLAND, NEW YORK.}

rise to large springs ${ }^{a}$ (fig. 19). According to this idea the deep recntrant valleys on the north shore represent only partly resurrected pre-Jameco valleys, whose upper portions are still partially buried and whose present heads represent the limit to which the. Tisbury sand and gravel has been removed, with perhaps some minor modifications produced by the Wisconsin ice.

\footnotetext{
LENGTH OF INTERVAL.
}

The amount of erosion represented is many times greater than that accomplished in post-Wisconsin time, though considerably less than that inferred to have been accomplished in the post-Mannetto or post-Isafayette.

\section{WISCONSIN EPOCH.}

CONDFIONS OF DETOSITION.

At the close of the long Vineyard erosion interval the ice again advanced, passed over the irregular remnants of the Tisbury beds, rounded out, but did not

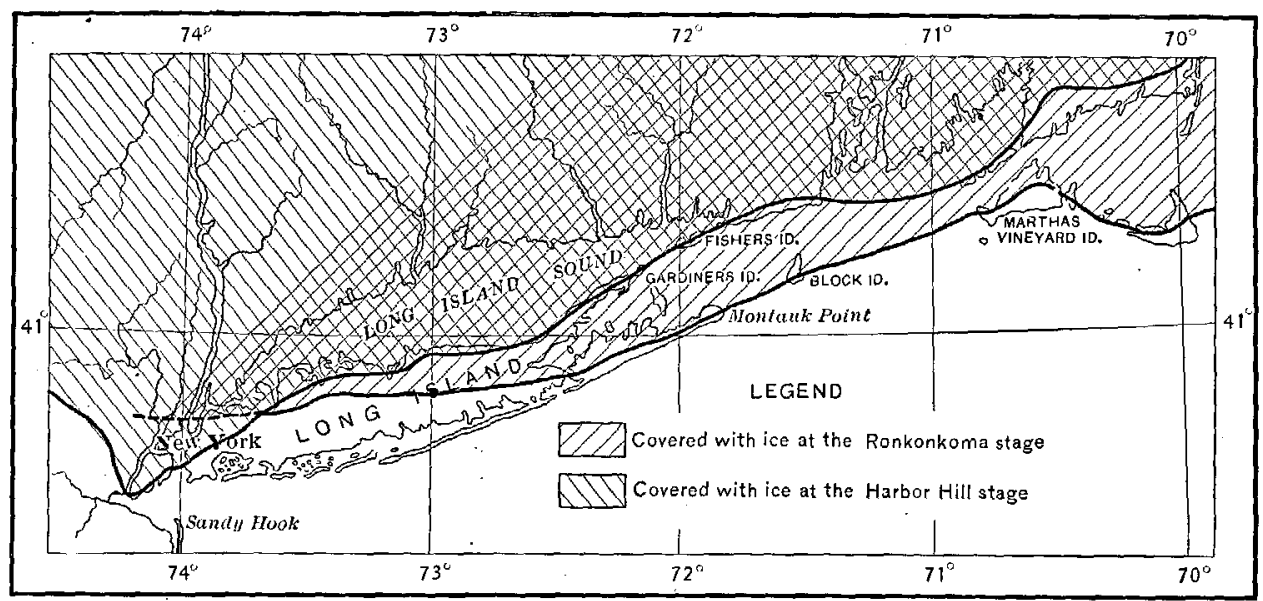

F1G. 20.-Sketeh map showing relative positions of the ice during the Ronkonkoma and Harbor Hill stages of the Wisconsin period.

greatly modify, the valleys redeveloped in the Vineyard interval, and extended southward to the remnants of the Perrineville Wold, page 31 (Pls. V and IX, $A$ ). As the Wisconsin deposits have not been greatly modified by erosion nor buried under nor commingled with younger deposits, the records of this ice advance are much more complete than those of the preceding periods. It is known that the ice advanced to a line roughly extending from Long Island City to Montauk Point, Block Island, Marthas Vineyard, and Nantucket (Ronkonkoma morair_d, fig. 20); that it then retreated and, the relative source of supply changing, advanced along a different line, passing the first advance in western Long Island, but not reaching it from Lake Success eastward (Harbor Hill moraine). ${ }^{b}$ The ice then retreated and the records of its minor halts have been found by Woodworth near Port Washington and College Point.

\footnotetext{
a On the effect of springs, see Mather, W. W., Geology of the first district, 1843, p. 33; Stone, Mon. U. S. Geol. Survey, vol. 34,1899, p. 19

b Woodworth, Bull. N. Y. State Mus., No..48, 1901, pp. 641,642.
} 


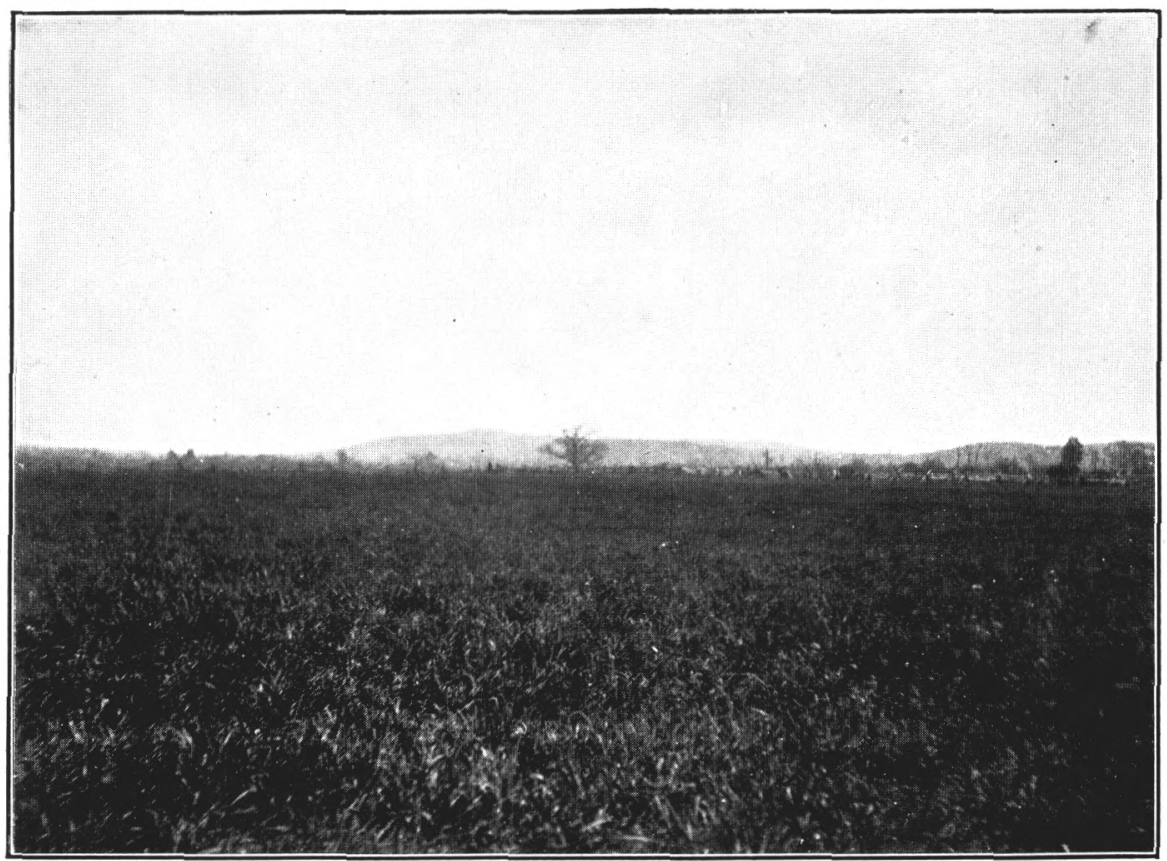

A. PORTION OF THE HARBOR HILL OUTWASH PLAIN OVER THE TISBURY TERRACE, SOUTH OF HUNTINGTON, N. Y.

The West and Half Holiow hills in the distance mark the southern limit of the ice and represent a portion of the Perrinevi!le Wold with a silight morainal covering.

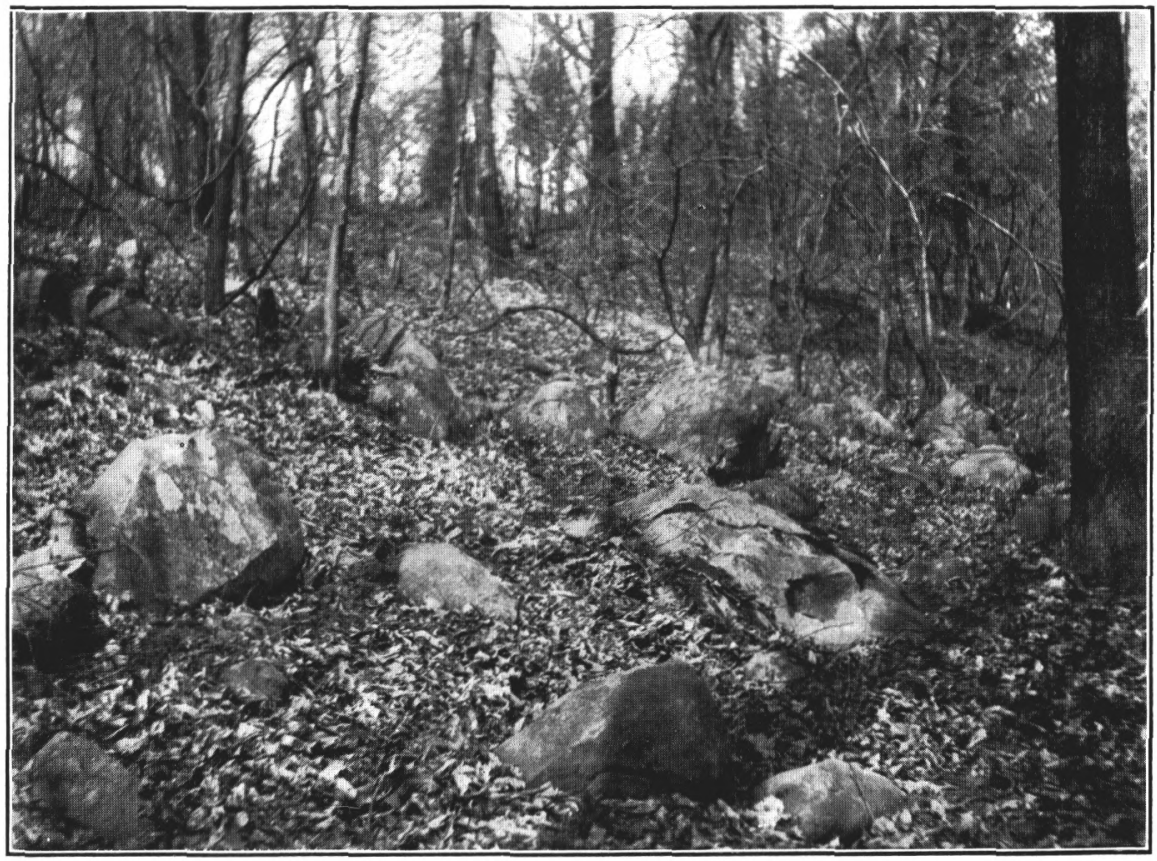

B. BOWLDERY PORTION OF. THE HARBOR HILL MORAINE NEAR CREEDMOOR, N. Y. 

CIARACTER OF DEPOSITS.

The Wisconsin deposits on Long Island do not differ in any respect from those on the adjoining mainland, which have been fully described by Salisbury in his report on the glacial geology of New Jersey." They show the same major divisions of till (unstratified drift or bowlder clay) and stratified drift, forming terminal moraines, till plains or ground moraines, outwash plains, kettle plains, deltas, etc. Terminal moraines (Pl. IX, $B$ ) represent more or less hilly accumulations formed at the end of an ice sheet during a halt; they are for the most part composed of till or unstratified material, but may under some circumstances show considerable stratification, when they become known as kames. Deposits which are formed under the ice, or when the ice is moving at such a uniform rate that it does not form a hilly accumulation in well-defined belts, are called till plains or ground moraines. When the ice is melting rapidly the outflowing water carries off a great amount of detrital material, which is spread out as alluvial fans, and when many streams are concerned in this action the adjoining fans coalesce and give rise to a comparatively level plain, called a sand or outwash plain, at the edge of which the more important fans produce a distinct lobate effect. If detached masses of ice are buried in this outwash plain, when the ice retreats these masses melt and produce a pitted or kettle plain. Deltas differ from sand plains in their more limited size and in the fact that they are formed in water by one major stream rather than by a great number of streams of about the same size.

The materials composing these several types on Long Island are largely derived from the local beds, for the most part from the Tisbury, and it is therefore not always possible to distinguish between the Tisbury and the reworked Tisbury belonging to the Wisconsin. The Wisconsin, however, as a rule contains a greater percentage of erratic material, shows decided morainic characteristics, and presents more or less pronounced topographic and stratigraphic distinctions.

Thickness.-The deposits of the Wisconsin, although widespread, are relatively thin. The till, which is regarded as its most characteristic deposit, has a thickness of 100 feet in places, and averages perhaps 10 to 20 feet. The extreme thinness of the Wisconsin can be well observed along the north shore, where the waves expose bluff sections. In the outwash plains the distinction is not so sharp, and considerable difficulty is experienced in drawing a line between the Tisbury and the Wisconsin outwash. If the more erratic portion of the plain is regarded as Wisconsin, the thickness of the deposits increases from only a few feet near Babylon to 192 feet at Ridgewood (fig. 10).

In the following table the more noteworthy occurrences have been brought together. Additional-data will be found in the table, page 118, and in the detailed well records, page 168 .

a Final Rept. State Geol. New Jersey, vol. 5, 1902. 
TABLE IV.-Thickness of Wisconsin deposits on Long Island.

\begin{tabular}{|c|c|c|c|c|c|c|}
\hline \multirow{2}{*}{ No. $a$} & \multirow{2}{*}{ Location. } & \multirow{2}{*}{$\begin{array}{l}\text { Pleistocene } \\
\text { (Recent to } \\
\text { Mannetto). }\end{array}$} & \multicolumn{2}{|c|}{ Wisconsin. } & \multirow{2}{*}{ Tisbury. } & \multirow{2}{*}{ Remarks. } \\
\hline & & & Till. & $\begin{array}{l}\text { Stratified } \\
\text { gravel. }\end{array}$ & & \\
\hline 3 & $\begin{array}{l}\text { Brooklyn (sewer tun- } \\
\text { nel) Sixty-fourth } \\
\text { and Sixty-second } \\
\text { streets. }\end{array}$ & $90+$ & 80 & $10 \pm$ & & \\
\hline 23 & Brooklyn ... . . . . & $135+$ & 45 & $90 \pm$ & 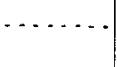 & $\begin{array}{l}\text { Stratified gravel may be in part } \\
\text { Tisbury. }\end{array}$ \\
\hline 41 & $\ldots$. . do $2 . . . .$. & $90-{ }_{-+}^{-} \cdot$ & 60 & $30 \pm$ & & Do. \\
\hline 42 & $\ldots$. do....... & $84+$ & 18 & $(?)$ & & \\
\hline 44 & . . . . do . . . . . . . & $60+$ & $60 \pm$ & $\cdots$ & & \\
\hline 46 & . . . do . ..... & $60 \uparrow^{-}$ & 21 & $39 \pm$ & & \\
\hline 35 & . . . . do. . . . . . & $275 ?$ & 23 & $(?)$ & & Sand from $23-105$ \\
\hline 32 & ... . do ......... & $105+$ & 0 & ..... & & All sand. \\
\hline 141 & Ridgewood...... & $284+-$ & (?) & $192 \pm$ & $\ldots$. & \\
\hline 137 & Spring Creek. ...... & $149+$ & 0 & $97 \pm$ & 29 & \\
\hline 201 & Jameco_. . . . . . . & $160+$ & 0 & 30 & 55 & \\
\hline 196 & Springfield...... & $180 \pm$ & 0 & 25 & 53 & \\
\hline 162 & Woodside........ & $138 ?$ & $38 \pm$ & $(?)$ & (?) & ' \\
\hline 326 & Thomaston........ & 42 & 27 & (?) & 15 & \\
\hline 369 & Manhasset Neck.... & (?) & 26 & & $\ldots$ & \\
\hline 368 & .... do.......... & (?) & 0 & (?) & $80 \pm$ & · \\
\hline 437 & Roslyn........... & 100 & 0 & (?) & $100 \pm$ & \\
\hline 435 & Wheatley Hills. ...... & 40 & 40 & $(?)$ & $(?)$ & \\
\hline & $(\ldots$. do $\ldots . . . . . .$. & 80 & 80 & 0 & (?) & \\
\hline 431 & $\left\{\begin{array}{l}\ldots \text { do } \ldots \ldots \ldots \\
\ldots \text { do } \ldots \ldots\end{array}\right.$ & $\begin{array}{c}149 \\
90+\end{array}$ & $\begin{array}{c}106 \pm \\
90\end{array}$ & 0 & $15 ?$ & . \\
\hline 751 & St. James.......... . & 250 & $30 \pm$ & 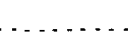 & ..... & Very few wolls report till in this \\
\hline 750 & $\ldots$. do. . . . . . . . & $150+$ & 60 & $(?)$ & $90-$ & region. \\
\hline 760 & Setauket......... & 62 & 25 & & $\ldots .$. & \\
\hline 765 & Port Jefferson . . . . . & $50+$ & 20 & & $30+$ & . \\
\hline 764 & $\ldots$. do........ & $40+$ & 20 & & $20 !^{-}$ & $\cdot$ \\
\hline 818 & Middle Island... & $39+$ & 39 & & $\ldots$. & \\
\hline 894 & East Marion.... . & $50+$ & 38 & & $12+$ & \\
\hline $8 \dot{8} 4$ & Shelter Island . . . & 53 & 12 & & $\cdots \cdot$ & \\
\hline 908 & ... . do ......... & 76 & 30 & & 46 & \\
\hline
\end{tabular}

a Numbers eorrespond to those used on Pl. XXIV and in Chapter IV, where additional data will be found.

DEVELOPMENT OF TOPOGRAPHIC FEATURES.

The effect of the Wisconsin deposits on the topography of Long Island is almost everywhere visible. It is shown in the many local details, which in the aggregate are so pronounced that they cause one to lose sight of the fact that the major topographic features are older and that the Wisconsin deposits have caused but surface 
changes in the topography of the island. Even had there been no Wisconsin ice and no terminal moraine Long Island would have existed and would have been roughly similar to the island of to-day. The island would have had a "backbone" and would have shown pronounced cliffs on the north shore, but many of the steep hills rising about 50 feet above the surrounding country would have been absent, as would the many lakes in funnel-shaped depressions and the immense bowlders which add so much to the picturesqueness of certain areas. These effects have been produced largely by transportation and deposition, though some features are traceable to erosion and folding.

Transportation and deposition.-The general effect of the Wisconsin epoch was to build up rather than to tear down. In some places it added materially to the relief, as in Brooklyn, which, without the moraine, would have been comparatively flat. In others, as in the West Hills, the older topography was so pronounced that it was not materially affected. The two lines of morainic hills, which have at times been regarded as the main skeleton of Long Island, are as a whole to be regarded as only surface deposits which are recognizable because of their peculiar minor topographic forms.

Associated with these morainic hills are kettle-shaped depressions, now the sites of many lakes, representing the positions of buried ice blocks which melted when the ice retreated. These depressions contain water when they satisfy either one of two conditions: (1) When they are lined with relatively impervious strata, which prevents the rapid outward passage of the water falling in them or draining from the adjacent hills, as Lake Success; and (2) when a portion of the depression lies below the main water table (pp. 61-63). In the latter case the level of the water represents the main ground-water table, and the character of the sides is therefore immaterial. Lake Ronkonkoma is an example of this class.

Erosion.-One of the most marked features of the southern plain are the dry stream channels slightly creasing it. These are now generally regarded as the work of glacial streams of late Wisconsin age. They are clearly not due to causes now in operation and contain streams only in their lower portions where the valleys cut the ground-water lable.

Folding.-The wrinkling of the beds on Long Island by the Wisconsin ice was slight compared with the Gay Head folding, from which, as a rule, it may be readily separated. The most evident wrinkle, and the one which is of greatest topographic importance, is a low ridge which extends from Far Rockaway to Lynbrook. On the one hand the Sankaty clay underlying it shows that it is a true fold (fig. 13), and on the other the coarse Tisbury gravel at the surface shows that the folding is post-Tisbury, for had a fold existed in Tisbury time this coarse gravel would have been deposited in the hollow rather than on the crest. The axis of this fold is, moreover, exactly parallel to the Wisconsin moraine to the north, all of which indicates that it is due to the weight of the Wisconsin ice. The Sankaty clay, with its underlying water-logged gravel, furnished the favorable conditions necessary for the production of a phenomenon of this sort.

The accompanying depression of Jamaica Bay is but slightly connected with this folding. It represents for the most part a partly filled portion of the old Sound River depression. 
POST-GLACIAL AND RECENT.

After the retreat of the Wisconsin ice the land stood somewhat above its present elevation. The only evidence indicating that this elevation was very considerable is that afforded by the close botanical affinities between the plants found in the sand hills of New Jersey, Long Island, and the New England islands. Hollick believes that the only explanation of this distribution is that since the Glacial epoch a land connection, broken only by the channels of the Hudson and Connecticut rivers, existed for a sufficient period to allow the migration of these plants. $^{a}$ 'This would involve an elevation of from 100 to 200 feet, and so recent an elevation should have left very pronounced channels on the sea bottom. Thus far no channels have been found which can be referred definitely to this epoch, and it is this lack of corroborative evidence that is the strongest argument against so high an elevation. There is, moreover, the natural question whether a land connection is really necessary to account for this distribution of the "pine-barren flora."

Another line of evidence pointing to a higher elevation, though not to the total amount, is offered by the drowned forests along the south shore and by the less conclusive though corroborative phenomena of barrier bars and receding sea cliffs. While buried vegetable deposits, barrier bars, and receding soa cliffs may, and often do, occur under conditions which do not indicate subsidence, the evidence at this point will bear no other interpretation. ${ }^{b}$ The tree stumps are not driftwood, but are clearly in the places where they grew. The swamp deposits are being exposed on the beach as the barrier bars migrate inland. Indian shell heaps or "kitchen middens" are found which are now covered at very high tide. Most of these data are available in the bays and marshes along the south shore, where the conditions were particularly favorable for the preservation of records of this sort, but even here the subsidence recorded is scarcely greater than 30 feet.

The work of Cook in New Jersey has led him to estimate the rate of subsidence at that point at about 2 feet per century, and a somewhat similar rate must affect Long Island.

\section{SUMMARY}

GEOLOGIC HISTORY.

Although Long Island is underlain by metamorphosed rocks which range from Archean to Silurian in age and which represent a long and complex history, its geologic history begins more properly with the Cretaceous deposits.

At this time the warping of the old land surface permitted a northward transgression of the sea, into which the rejuvenated streams carried the residual material formed in the preceding long period of erosion and weathering. In this region the basal Cretaceous beds are of the same age as the Raritan in New Jersey and belong to the upper Cretaceous; above these are more nearly normal sands and clays of Cretaceous age, the whole series having an aggregate thickness of 1,300

a Hollick, $\Lambda$ rthur, Plant distribution as a factor in the interpretation of geologic phenomena, with special reference to Long Island and vicinity: Trans. New York Acad. Sci., vol. 12, 1893, pp. 189-202.

${ }^{b}$ Lewis, F., Pop. Sei. Monthly, vol. 10, 1877, pp. 434-430. 
to 1,400 feet. These beds are correlated with the New Jersey formations in part by paleontologic and in part by stratigraphic evidence. The great greensand marl beds which occur in the upper part of the Cretaceous in New Jersey are absent on Long Island, their place being taken by fine sands with local clay beds, indicating a considerable change in the local conditions of deposition. These beds form the substructure of the island and are responsible for its major topographic features, the Pleistocene beds only mantling the older deposits.

During the greater part of the Eocene this part of the coastal plain was above water, but late in the Miocene it was again submerged and received a covering of the same beds which are now found along the New Jersey coast. On Long Island these beds have been almost entirely removed by erosion and are now recognized only in the top of the West Hills section. This distribution is similar to that found in New Jersey, of which Long Island is but the normal geologic continuation; and unless there is a much greater discordance in structure between the Miocene and underlying beds than is now known, the Tertiary can not occur on the north shore of Long Island and will be found only as elevated outliers, with the possible exception of a portion of the South Fluke.

After the early Pliocene erosion interval the Appomattox or Lafayette formation was spread as a littoral deposit over the coastal plain. Deposits of this age have not been recognized on Long Island, unless they are represented by the Mannetto, which is regarded as younger-probably Pleistocene. In the succeeding very long erosion interval the land stood higher than before and was more deeply eroded. The events of the early Pleistocene were very similar to those of the late Tertiary; the Mannetto, though containing compound crystalline pebbles, which have caused it to be referred to the Pleistocene, is apparently a littoral deposit, similar to the Lafayette, and the succeeding long erosion period resembles to a great degree the late Pliocene (post-Lafayette) erosion interval. On Long Island the results would have been essentially the same, whether there was one submergence and one erosion or two submergences (Lafayette and Mannetto) and two erosion periods. All of the beds were profoundly eroded, and in the gradual subsidence following this uplift a continental glacier advanced well toward the north shore of Long Island, and the streams issuing from it deposited great beds of gravel (Jameco) in the old Sound River Valley across western Long Island and over eastern Long Island and the New England islands. $\Lambda$ s the ice retreated and the submergence continued beds of sands and clay (Sankaty) were deposited around the nuclei of older uplands. In this epoch the land stood about 50 feet higher than at present, and the climatic conditions, as indicated by marine fossils, were much the same as to-day. A very extensive and important deglaciation is, therefore, represented.

With the return of the ice in the period of the Gay Head folding some of these . older beds were overridden and a wonderful series of superficial folds produced which involve alike the pre-Cretaceous beds, the Jameco gravel, and the Sankaty clay.

The tops of these folds in such exposed localities as Gardiners Island and the New England islands were then truncated by wave action, with the land about 100 feet below the present sea level. An estimate of the time involved in this 
wave cutting gives it a length of perhaps 40,000 years more than that which has elapsed since the Wisconsin.

The progressive subsidence continued throughout the next glacial epoch, the Tisbury, when the land was about 200 feet below the present sea level. In this epoch great outwash gravel beds were deposited horizontally on the truncated folds of the older deposits and around the islands of the older land.

In the Vineyard interval, when the Tisbury glacier had retreated, the lands stood perhaps 200 feet higher than to-day, and the older valleys were partially reexcavated. The erosion of this epoch, although very great when compared with that which has occurred in post-Wisconsin time, is very small when compared with that of the post-Mannetto or the post-Lafayette.

After this period of erosion and high elevation the Wisconsin glacier approached Long Island, and after an initial advance, when the ice reached a line extending from Long Island through Montauk Point to Block Island, Marthas Vineyard, and Nantucket, the ice retreated and returned again with the relative position of the ice front somewhat altered. In this readvance it passed the limit reached by the first advance in western Long Island, but did not reach so far south to the east (fig. 20).

In the comparatively short time which has elapsed since the retreat of the Wisconsin ice the changes have been almost entirely those produced by wind and wave action along exposed shores. The relative position of the land and sea uadoubtedly changed on the retreat of the ice, and while, according to certain peculiarities of the distribution of the flora, this change may have amounted to as much as 100 or 200 feet, there is no corroborative evidence of so high an elevation.

\section{TOPOGRAPHIC HISTORY.}

While the Atlantic coastal plain, of which Long Island forms a part, was subjected to some erosion during the Eocene, the elevation was either so slight that it left no pronounced record or the interval was so long that the country was essentially peneplained, and the beginnings of the present topography were not made until the post-Miocene uplift, when the streams emerging from the older land flowed directly across the coastal plain (Pl. VI, A). As the erosion progressed the thinner portions of the Miocene deposits near the Cretaceous contact were cut through, and the topography developed in this section began to show the effect of the differences in the hardness of the underlying strata. $\Lambda$ low, longitudinal valley, or vale, was developed from the softer basal Cretaceous layers, and a belt of hill land, or wold, cut by the narrow transverse valleys of the coastflowing streams, was formed from the overlying harder ones. To the south of Washington, where the cover of Miocene sediments was greater, the underlying Cretaceous was not reached, and the topography showed no distinctive features. In the Lafayette submergence, which followed, a littoral deposit was spread over the coastal plain, mantling the low topography developed in the post-Miocene. The narrow transverse valleys were obliterated more completely than the broad vale, and when the land was again elevated the rivers discharged into a longitudinal trough. Had there been no deflection the streams must have cut new 
channels through the barriers afforded by this ancestral Perrineville Wold (see p. 31) and by the more or less filled channels through it, but as there was a tilting to the south the rivers flowed down the partly filled Hightstown Vale until they found a partly filled break of a lower stream through which they could turn seaward. As the land rose higher and higher these streams trenched deeper and deeper, and at the end of the long late Pliocene (post-Lafayette) erosion interval the present topographic features of the coastal plain were well developed ( $\mathrm{Pl}$. VI, $B$ ). The Hightstown Vale was strongly marked, being more pronounced on the north than on the south, owing to the greater thickness of the Miocene deposits in the southern region. In it were found the northeast-southwest portions of the Potomac, Susquehanna, Delaware, and Sound rivers. Seaward of this vale was the range of hills now recognized as the Perrineville Wold, considerably dissected, but essentially continuous from southern New England to Virginia. The breaks in this range were of two kinds-those due to the present channels of the rivers where they turned seaward from the Hightstown Vale, and those possibly representing coastward channels of these streams in pre-Lafayette times before their deflection, wilich have persisted because of this slight initial advantage. Of such an ultimate origin may be the depression in the Perrineville Wold across New Jersey along Rancocas and Mullica rivers, and in Delaware and Máryland between Delaware and Susquehanna rivers.

As the subsidence which followed the late Pliocene (post-Lafayette) uplift progressed the Hightstown Vale became a coastal sound and the Perrineville Wold developed into a chain of islands. One of these was the first Long Island, and while it was somewhat different in shape from the present island, it showed many points of similarity. It was from this nucleus that the present island was developed.

In the Jameco, Sankaty, Gardiner, and Tisbury epochs the portion of the Sound Valley crossing western Long Island was largely filled, some of the beds were profoundly folded, the position of some of the more prominent points of the archipelago to the eastward changed, and a great deposit of gravel was laid down about the older nuclei. When the land was again elevated, Long Island showed more nearly its present outline. The Tisbury had filled in and rounded out the older topography and made a body of land somewhat larger than that of to-day, with a northward-facing scarp not far from the present bluff line. The short, deep valleys running northward from the crest of the wold were buried, and there were, therefore, no deep, reentrant bays or valleys such as now characterize this shore. The Connecticut, no longer able to discharge westward, cut a new channel directly seaward between Fishers and Plum islands on the one side and Block Island and Montauk Point on the other. The Housatonic probably flowed eastward and joined the Connecticut near Fishers Island.

As the elevation continued the excavation of valleys in the Tisbury began along lines determined by the preexisting valleys, in which, because of the difference in the porosity of the Tisbury gravel and the Cretaceous sands, the underground waters were concentrated. Tt is to this excavation, in which springs played a large part, that the present shape of the north shore valleys is in a large measure due. 
After this period of elevation the ice again advanced, and, by means of its relatively thin superficial deposits gave to Long Island its present glacial topography. The moraines were deposited without regard to previous topography, and therefore filled the Sound River Valley in Brooklyn, and to the east covered the older hills, giving rise to the erroneous correlation of the whole hill mass as moraine. In the outwash deposits accompanying these moraines, as well as in the moraines themselves, masses of ice were buried which, on the final retreat of the glacier, melted, and produced the many picturesque kettle holes which now dot the island. The channels across the southern plain were also produced at this time, and the shape of the north shore valleys was probably slightly modified. The changes since the retreat of the ice have been relatively slight and largely restricted to the shores; the waves have worn back the headlands, and the winds and tidal currents have carried this débris along the shore to form bars and spits, sometimes long, with but one end fixed, as the great barrier bar which extends from Montauk Point to Fire Island, sometimes fixed at both ends, as the bars which connect the former islands of Lloyd Neck, Eaton Neck, and Center Island, with the mainland. Behind these bars marshes have formed which, with the silt brought down by the streams, have been struggling to reclaim such areas from the sea. Along the beaches and in the areas laid bare of vegetation by man, or forest fires, the winds have taken up the loose sands and piled them into dunes. 


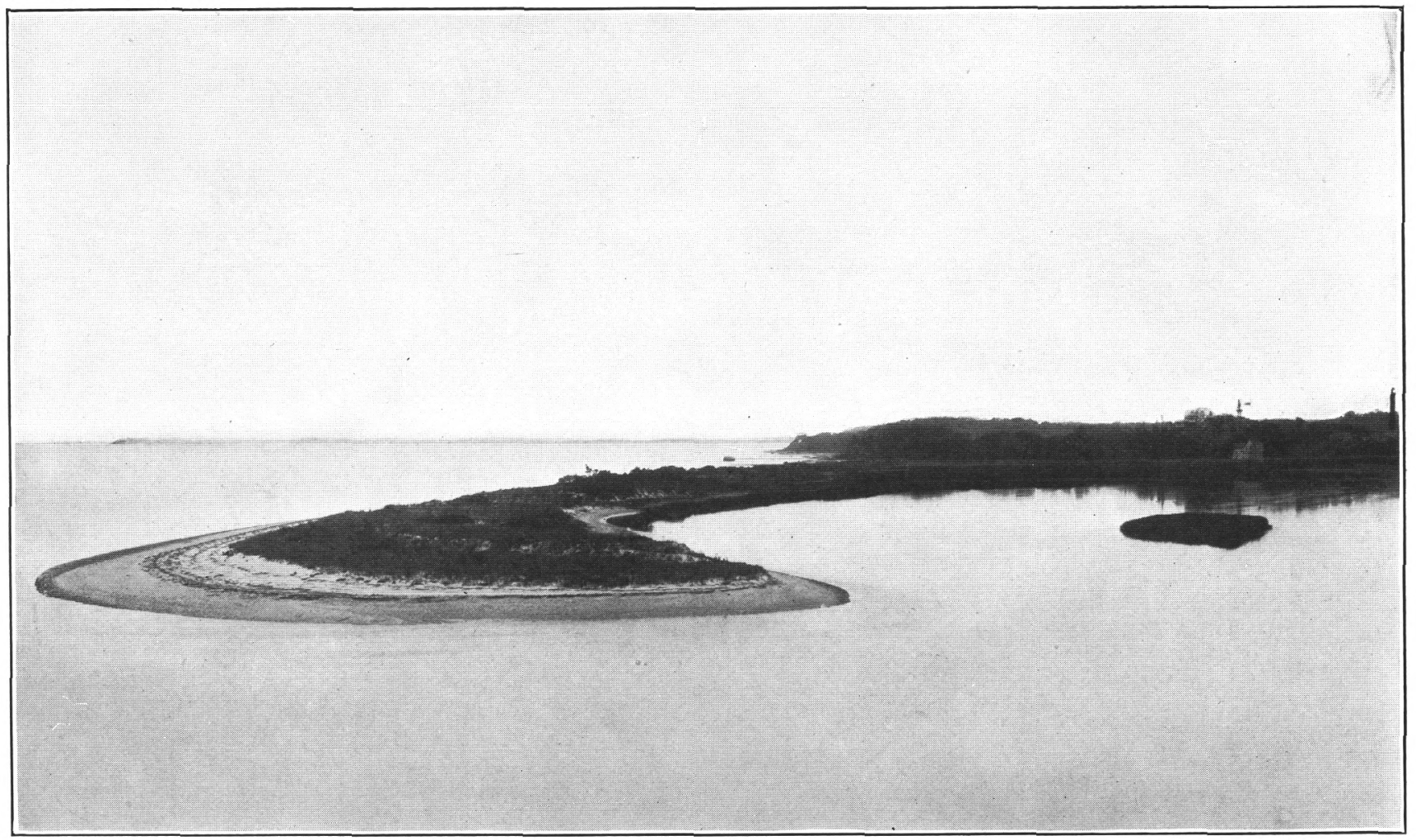

HOOKED SAND SPIT AT ENTRANCE TO SMITHTOWN HARBOR, LONG ISLAND.

An illustration of a recent change in the shore line. Photograph by Edward P. Buffet. 



\section{CHAPTER II. UNDERGROUND WATER CONDTTIONS OF LONG ISLAND.}

By A. C. Veatch.

GENERAL PRINCIPLES.

SOURCE OF UNDERGROUND WATER.

The water that falls on the land in part flows off on the surface and in part sinks into the ground. In both cases a portion is returned to the atmosphere by evaporation, and another portion is consumed by living organisms and in chemical work. The water which flows on the surface is called the run-off, though this term is used to include also the water which returns to the surface after a greater or less underground passage. 'The water which sinks into the ground through the interstices of the soil or rock, and furnishes the supply for springs and wells and in some cases for ponds and lakes, is called the ground water.

TRANSMISSION.

The "channels" through which this underground water moves are, with rare exceptions, the small spaces between the particles of which the rock is composed, as the sand of a sand bed or sandstone, or the gravel of a gravel bed or conglomerate; therefore, the coarser or more porous the bed the greater its water-carrying power. Water that travels through breaks in the rocks such as joint planes or fractures is rarely of very considerable economic importance and never, except in the case of limestones in which caverns have been developed, forms an underground stream in the usual sense. In the study of underground water it is therefore necessary at the outset to abandon the idea of underground streams resembling surface streams, and to conceive of the water as passing through the very small interstices of sand or gravel or other porous bed, rather than in great open channels or conduits.

The motion of underground water, like that of surface water, depends entirely on gravitation, and the rate of motion-or rapidity of flow--depends on two principal factors-slope and resistance. Surface waters are entirely unrestricted in one direction and their channels therefore readily adjust themselves to any amount of water, the only resistance being that of the bed and banks; underground waters, on the other hand, are carried in a "channel" composed of an infinite number of small openings, each of which offers a resistance that varies inversely as its size, the 
whole resistance being in a way the sum total of these separate resistances. It therefore happens that while surface water commonly moves at the rate of a few miles a day underground water moves at the rate of only a few feet.

\section{GROUND-WATER TABLE.}

The upper surface of the beds saturated by this percolating water is called the ground-water table. Its depth from the surface of the ground varies with

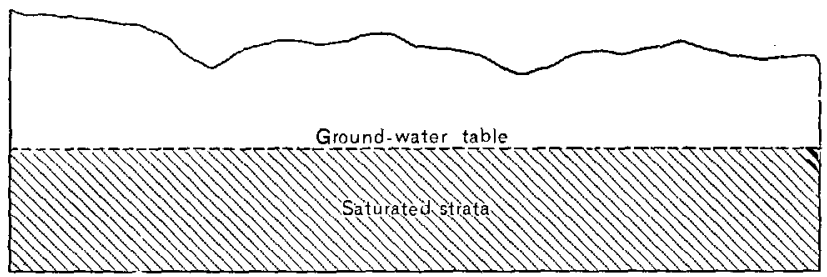

Fig. 21.-Diagram showing ground-water table unaffected by surface features. the character of the beds, whether relatively porous or impermeable; with the rainfall, whether heavy or light, and with the relief of the country. In regions of low rainfall and low relief the water table is very deep seated and relatively horizontal (fig. 21). In regions of greater rainfall and greater relief it is relatively near the surface, and may be directly affected by the tqpography. If the valleys cut the water table the ground water moves toward the valley, producing springs (fig. 22).

\section{REQUISITE CONDITIONS FOR FLOWING WELLS.}

Underground water in passing downward may go beneath a relatively impervious laycr which tends to confine it and produce a hydraulic head. In this respect underground waters differ from surface waters, which are always free on one side and can not, except where artificially confined, as by closed flumes, produce analogous phenomena. This natural confine-

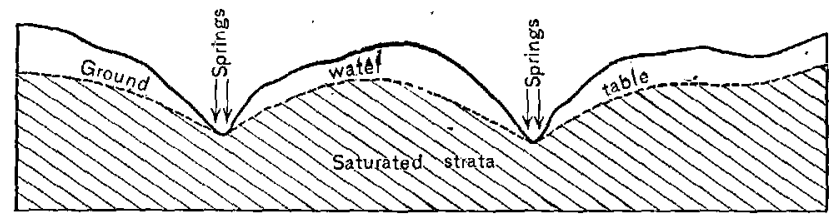

FIG. 22.-Diagram showing ground-water table cut by valleys. ment of the ground water causes water in wells to stand above the porous layer in which it is encountered, and is of vast economic importance, especially in arid regions where the water is very deep seated and has been transferred from a region of more bountiful rainfall.

In order that a well may flow, it is necessary that the following conditions be satisfied:

1. There should be sufficient rainfall.

2. There should be relatively porous beds suitably exposed to collect and transmit the water.

2. There should be less porous or relatively impervious layers so placed that they may confine the water collected.

4. The level of the ground water at the source should be at a sufficient height. about the mouth of the well to compensate for the loss of head due to resistance and leakage. 
The arrangement of the factors which produce a flow is by no means constant. These factors vary considerably from point to point, and relatively new combinations are to be constantly expected. Probably the commonest combination is that shown in the accompanying diagram (fig. 23). Here the confining beds are clay and the porous bed is a sand which dips regularly in the direction in which the

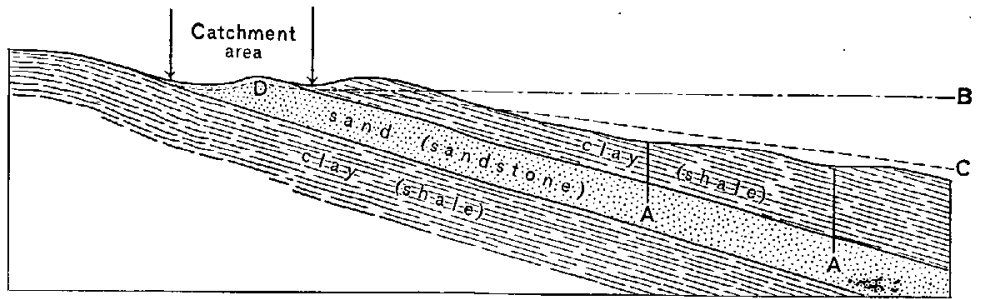

FIG. 23.--Diagram showing common arrangement of faccors producing artesian wells. A, Artesian wells; $B$, head of water if there be no loss by resistance or leakage; C, actual head or hydraulic gradient; $D$, ground-water table at outerop.

surface slopes. Water falling in the region marked "catchment area" sinks into the sands and supplies the artesian wells on lower ground:

While this arrangement of the factors may be taken as typical of a large class of artesian wells, and is, perhaps, the one most commonly expounded and understood, a radical rearrangement of the factors, such as is found in some wells on Long Island, will produce results depending on the same general principles.

\section{UNDERGROUND WATER CONDITIONS ON LONG ISLAND.}

\section{GEOLOGIO CONDITIONS.}

The geologic factors which affect the water supply of Long Island are graphically shown in the accompanying diagram (fig. 24), and may be briefly summarized as follows:

1. Above a rock floor which underlies the island at a greater or less depth, but which is of little importance except as a more or less complete ultimate barrier to the downward passage of water, Long Island is composed of a nucleus of Cretaceous beds. These are for the most part sand, but contain some discontinuous clay masses, and dip, except for minor disturbances produced by ice thrust, regularly southward.

2. Beds of glacial gravel deposited in an early ice advance surround this nucleus, except in a portion of the southern side of the island, which the older hill land protected from. direct currents and in other places where they have been removed by subsequent erosion. This formation, which has been called the Jameco gravel, is particularly well developed near the western end of the island, where it has partially filled a deep, broad valley in the older beds (fig. 10).

3. Over this gravel and around the edge of the Cretaceous beds is a layer of blue clay, the Sankaty - a deposit somewhat similar to, but of greater extent than the coastal marsh deposits of to-day, and at present situated from 50 to 100 feet below them.

4. Covering both the nucleus of Cretaceous beds and the younger blue clay, with its underlying early glacial gravel, are deposits of more recent ice 
advances-the Tisbury and Wisconsin. These are, for the most part, sand and gravels, though here and there are local beds of clayey material which, while they give rise to local water tables that may be of value for local wells, ponds, or springs, are of no general importance.

The more important results of these geologic conditions are:

1. The rain water sinks directly into the very porous surface gravels and produces, therefore, practically no run-off, except that supplied by springs. Since all streams are spring fed there is great difficulty in determining the exact limits of the watersheds, which depend on the relief of the ground-water table and only indirectly on the shape of the surface.

2. As the greater portion of the water of the island is under ground, and as the 25 to 30 per cent which normally returns to the surface is exposed for but a relatively short distance, the percentage of the total rainfall lost by evaporation

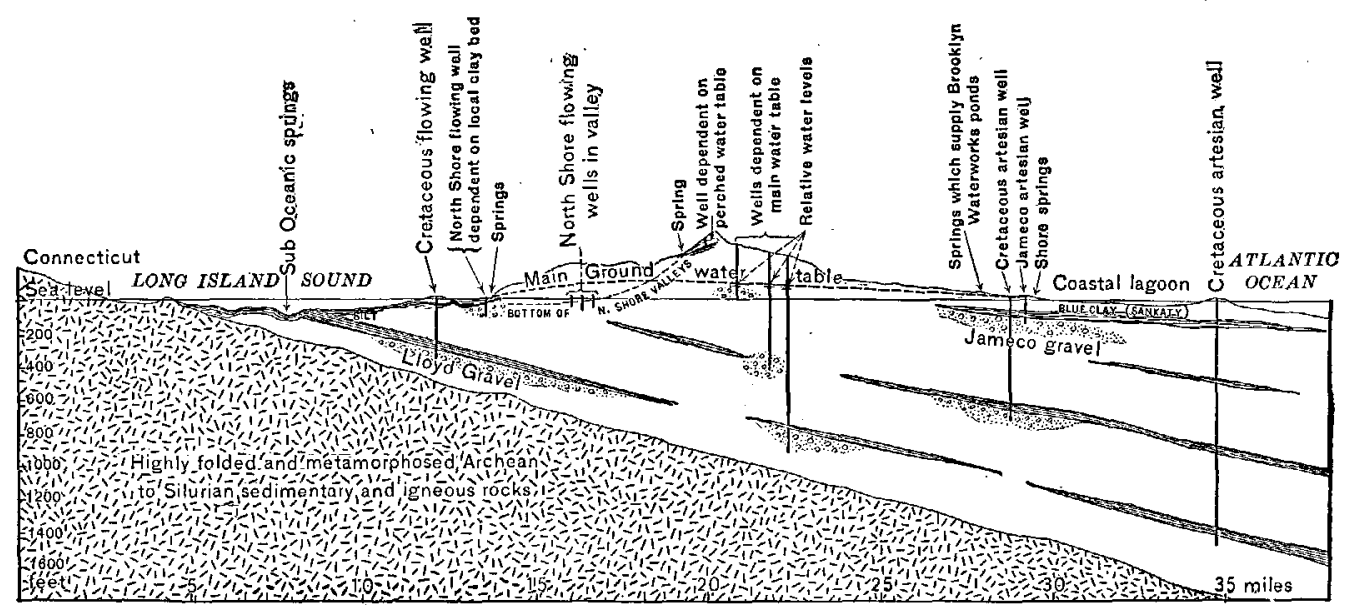

FIG. 24.-Diagrammatic cross section of Long Island, showing general water conditions and cause of flowing wells.

is abnormally small and the yield of this watershed, could all the water be economically obtained, would, therefore, be larger per square mile than in any adjoining areas.

3. As there is no uniform "blue-clay floor," or other extensive geologic barrier, a portion of the ground water passes coastward in the upper gravels and another portion, and by no means a negligible one, sinks, into the Jameco and Cretaceous sands and finally escapes in the form of suboceanic springs. This transmission of water is one of the more important factors of the underground conditions of Long Island. There is no geologic reason why a relatively important portion of the rainfall should not pass seaward in the beds below the surface gravel, and that this occurs has been proved by the many deep wells on the island and by the work of Prof. Charles S. Slichter, who has shown that there is a greater velocity beneath the bed of blue clay than in the surface gravel, page 102 . 
GROUND-WATER TABLES.

As all the water on the island is of ultimate ground-water origin, one of the most important points to be determined is the exact position of the ground-water table, since on it depends the stream flow, the depth to permanent water in wells, and the pressure in artesian, or flowing, wells. Were the island entirely homogeneous in composition there would be but one water table, which would be at ocean level on either side and would gradually rise toward the highlands in a curve entirely symmetrical with the surface, and at a depth determined by the porosity of the soil and the amount of rainfall. No wells, or springs, or ponds would be possible, except where this ground-water table was reached, and no water in any well would

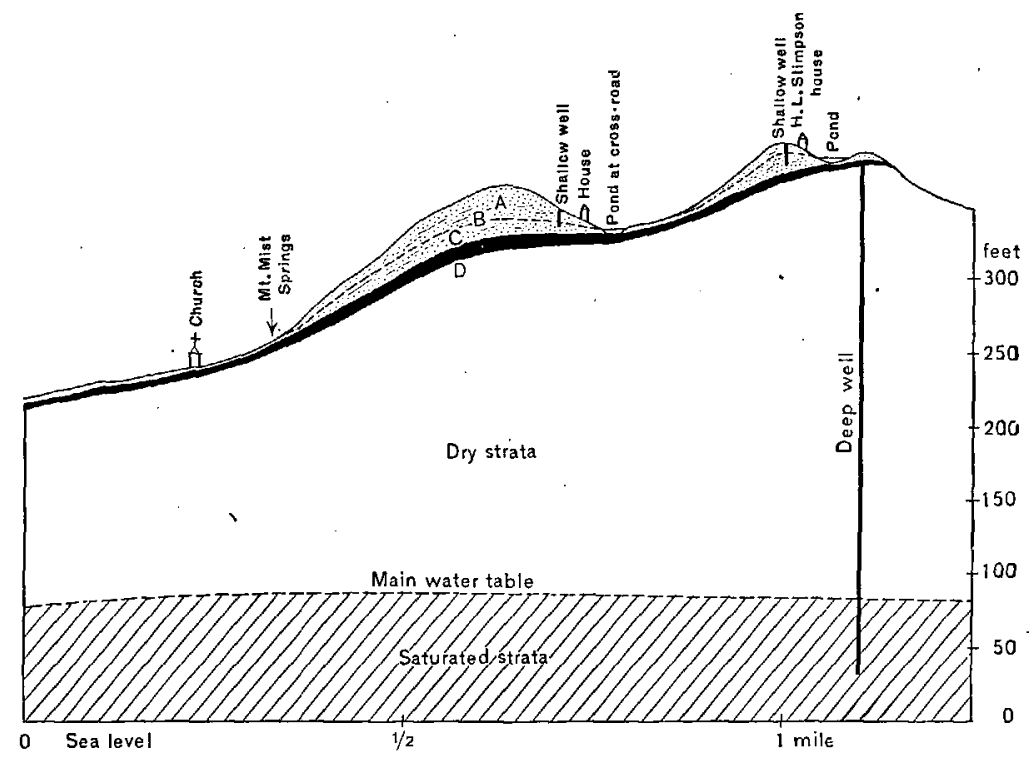

Fig. 25.-Diagram showing perched water table on north side of West Hills and source of Mountain Mist Springs.

A, unsaturated strata; B, perched water table; C, saturated strata; D, relatively impervious till

rise above the ground water at that point. There would, therefore, be no artesian wells.

As the island is not entirely homogeneous, the upper linit of the zone of complete saturation-that is, the main ground-water table, or "main spring," as it is locally called - is not entirely symmetrical with the curve of the surface, and there are, moreover, a number of more or less limited areas of saturated beds above the main one.

PERCIED GROUND-WATER TABLES.

These perched ground-water tables are for the most part confined to the moraine where local clay or other relatively impervious layers have arrested the flow of the underground water and prevented it from reaching the main groundwater table. One of the best examples of such a perched water table is found in the northern end of the West Hills, where a relatively impervious bed is furnished by the Wisconsin till (fig. 25). Other examples are shown in fig. 24 and Pl. XI. 
MAIN GROUND-WATER TABLE.

The general character and position of the main ground-water table is shown in Pls. XI and XII, which are based on the careful work during the summer of 1903 of the Long Island division of the New York commission on additional water supply. This work has developed the interesting point that while the slope of the groundwater table is in a general way the same as that of the surface, the divide of the ground water is farther to the north than the surface divide.

GPRINGS.

Whenever the main water table, or one of the perched water tables, is intercepted by the surface a spring is formed.

SPRINGS DEPENDENT UPON PERCHED WATER TABIES.

The water of springs dependent upon perched water tables penetrates the earth until it reaches a relatively impermeable stratum above which it collects,
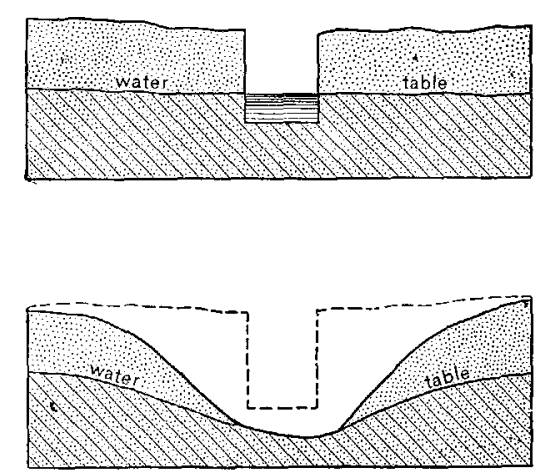

FiG. 26.-Diagram showing analogy between a well and a channcl that cuts the ground-water table. and along which it passes until it finds an outlet. Springs of this type are common wherever a perched water table occurs, and represent essentially the overflow of these underground basins. The much talked of springs that occur at the summits of hills or mountains are invariably of this class, and examination always shows that, though relatively at the top of the hill, there is always an appreciable area of higher ground above them which serves as a reservoir. The Mountain Mist Springs in the West Hills are of this type, and while they are situated at a height of about 280 feet above sea level, the hill behind them rises 140 feet higher, and there are several hundred acres of land to serve as a catchment area and reservoir (fig. 25). Springs of this type are found in many places along the north shore, and are particularly abundant where the fine Cretaceous beds are overlain by coarser Pleistocene gravels.

SPRINGS DEPENDENT UPON THE MAIN GROUND-WATER TABLE.

The water of springs formed by the cutting of the main water table escapes from the top of the water-logged beds, rather than at their base, as in the springs just discussed. The channels which cut this water table may be regarded as large wells, with one side open, into which the water is flowing and escaping (fig. 26). The old glacial channels across the southern plain invariably cut the ground-water table near their lower ends, and at the point where this occurs little streams start which grow very rapidly as the channel gets deeper into the saturated layers. A quantitative determination of this increase in Hempstead Brook was made by the engineers of the Brooklyn waterworks in 1895 . This valley, which was perfectly dry just above Hempstead village, showed an average dis- 


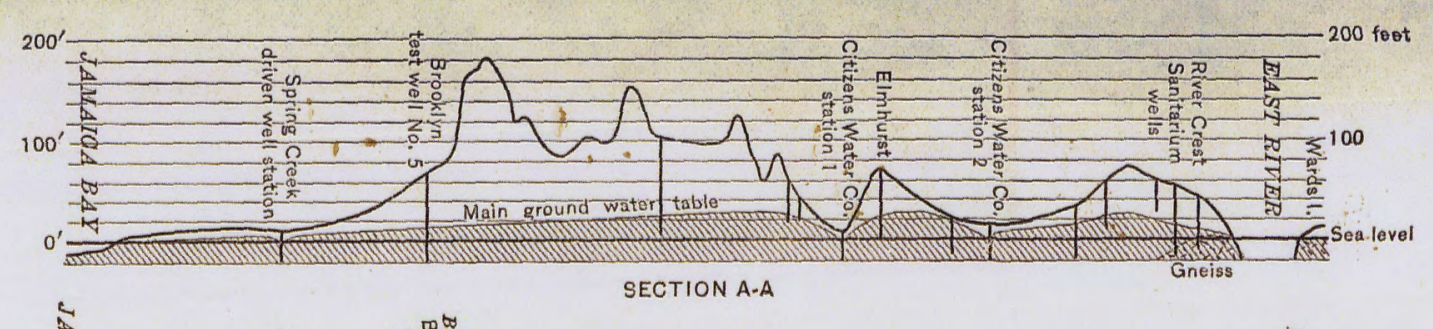

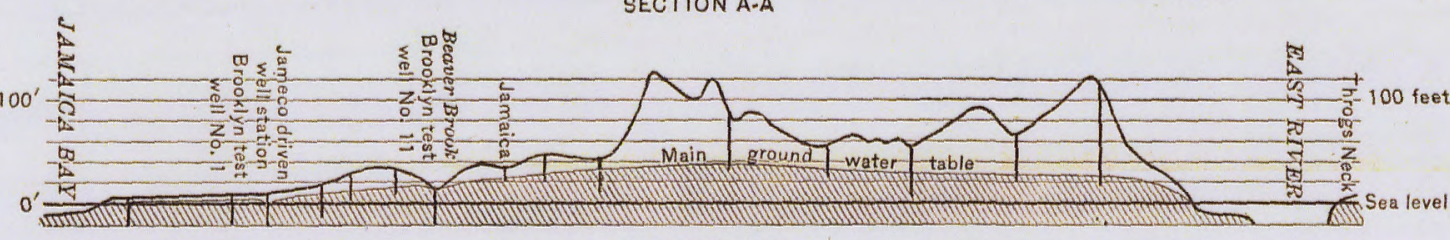
SECTION B-B
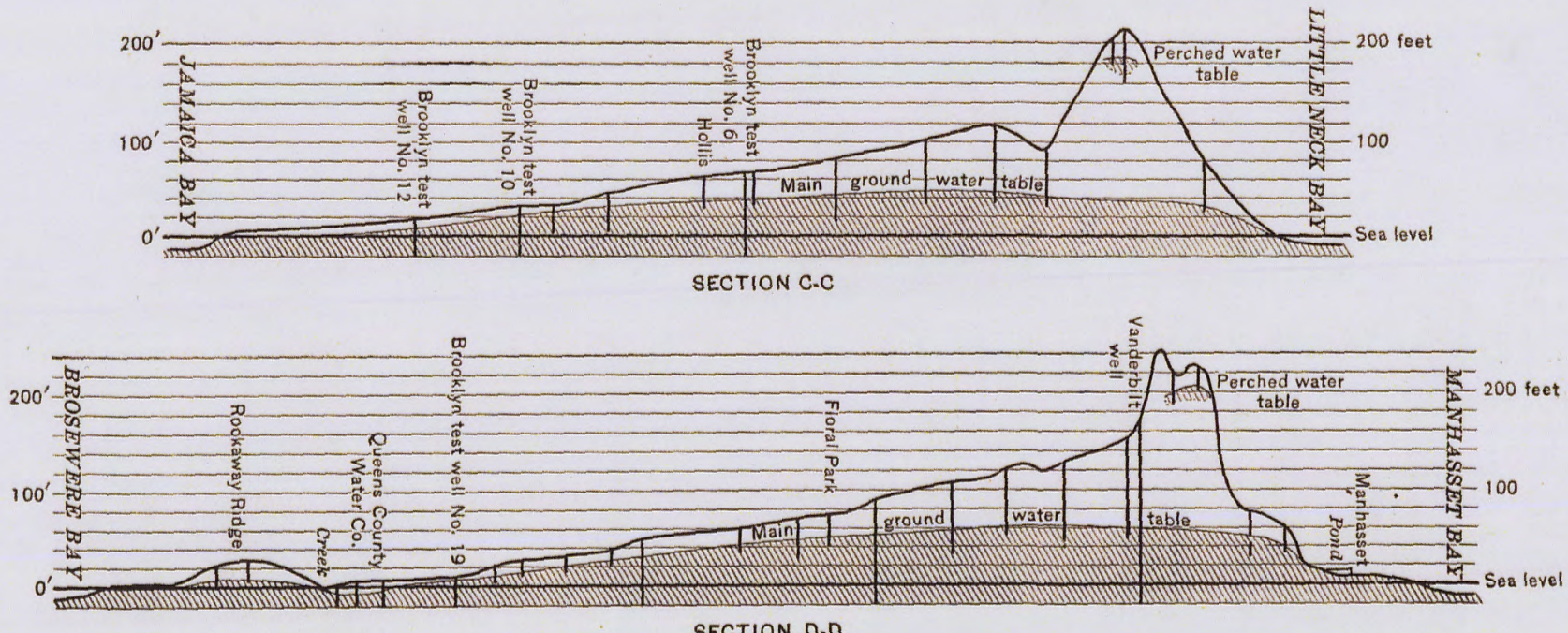
EETION D.D
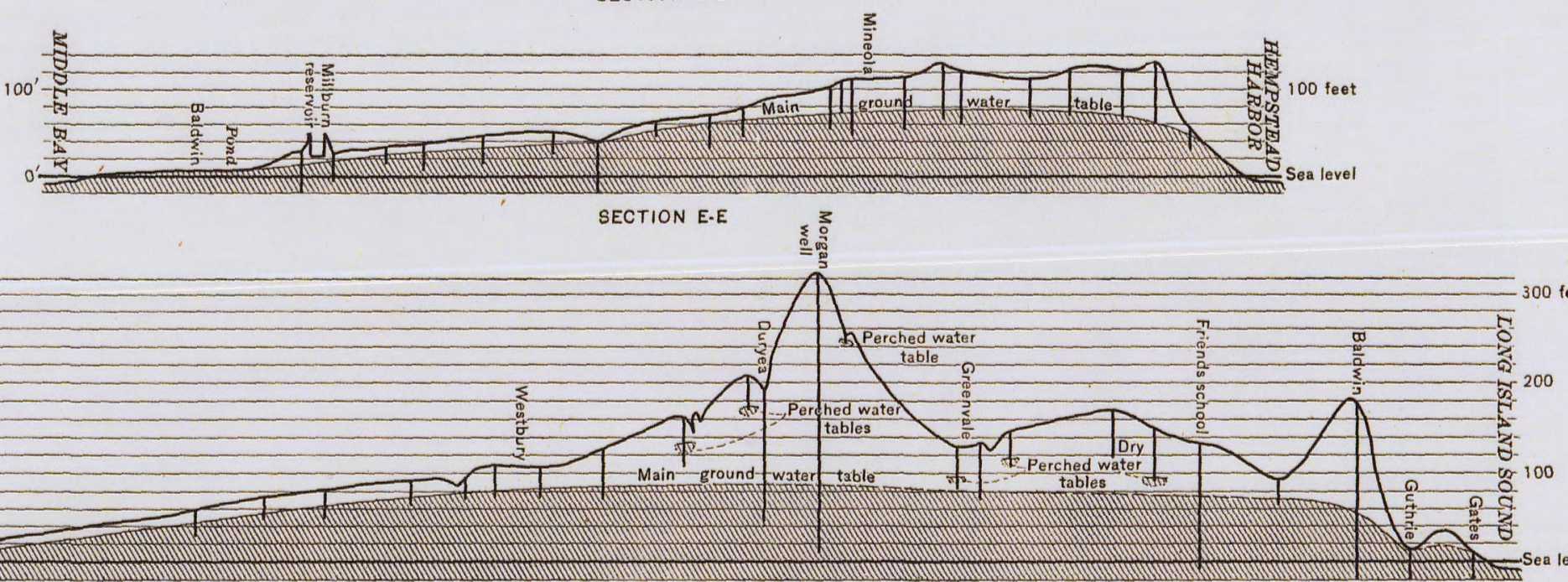

$100, \frac{0}{40}$

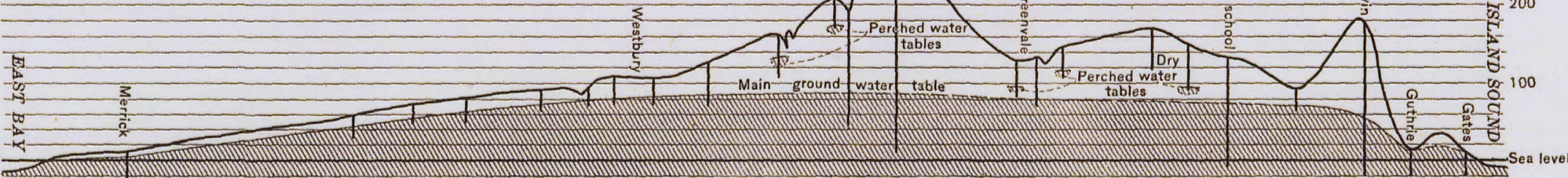

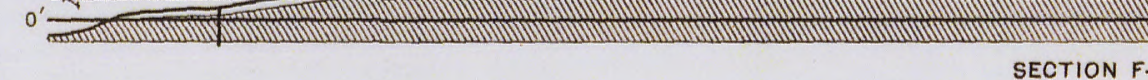

$\frac{x^{2}}{3}$

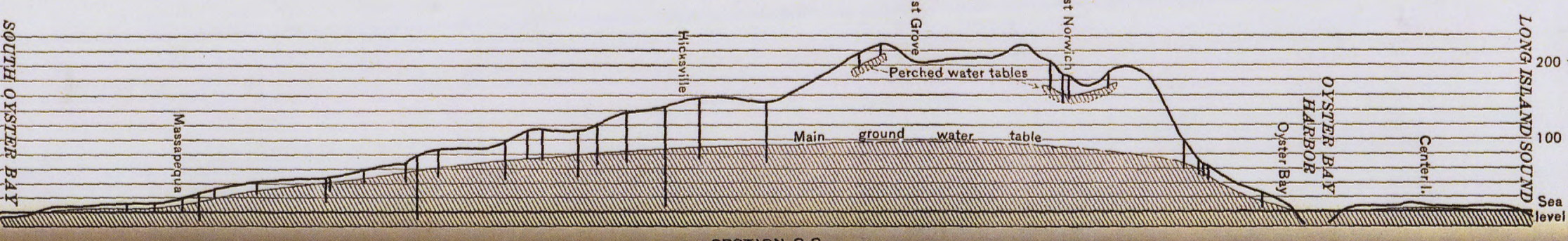

$100=\frac{3}{4}$ -

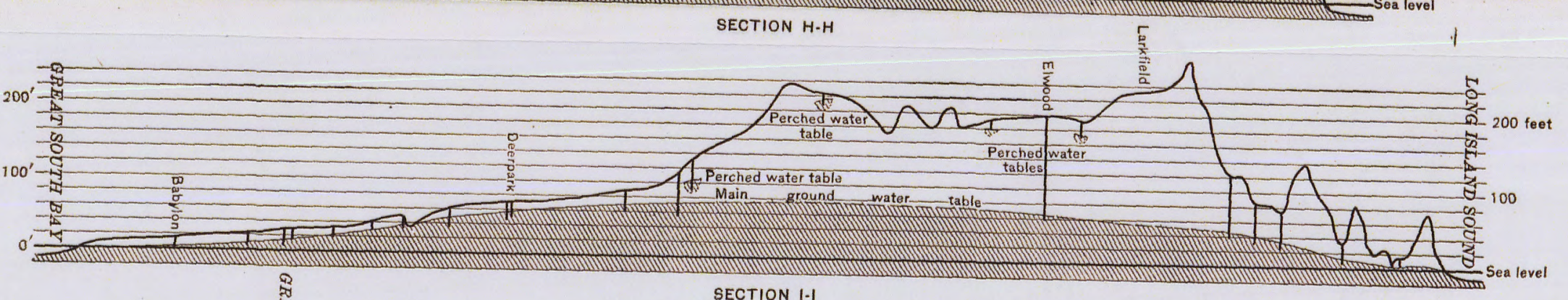

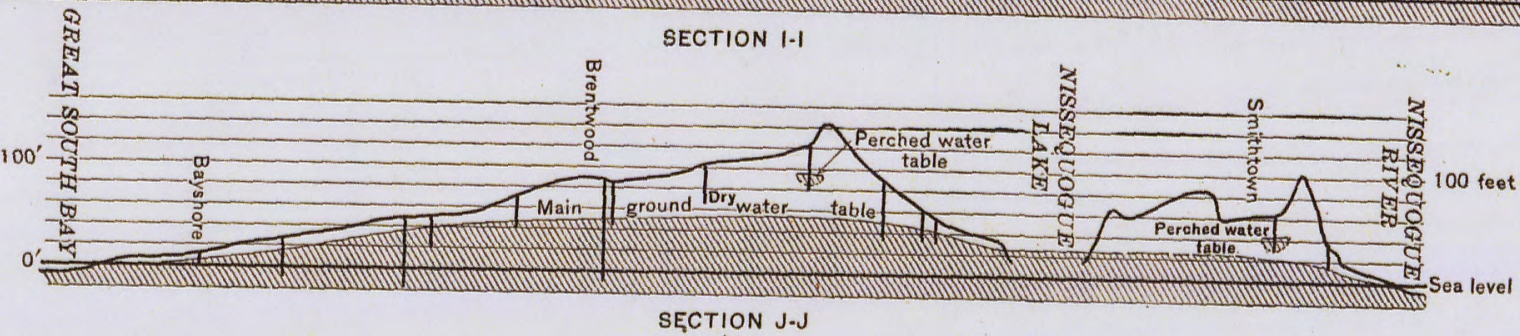

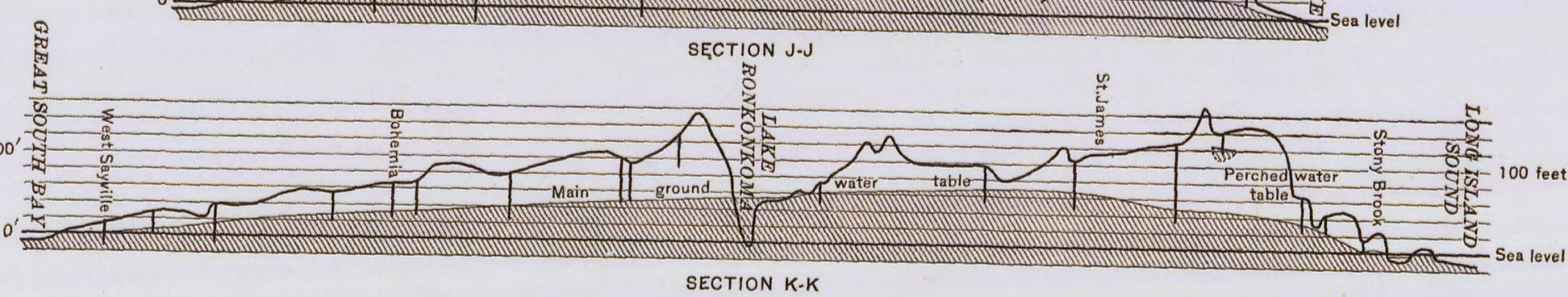
SECTION K-K

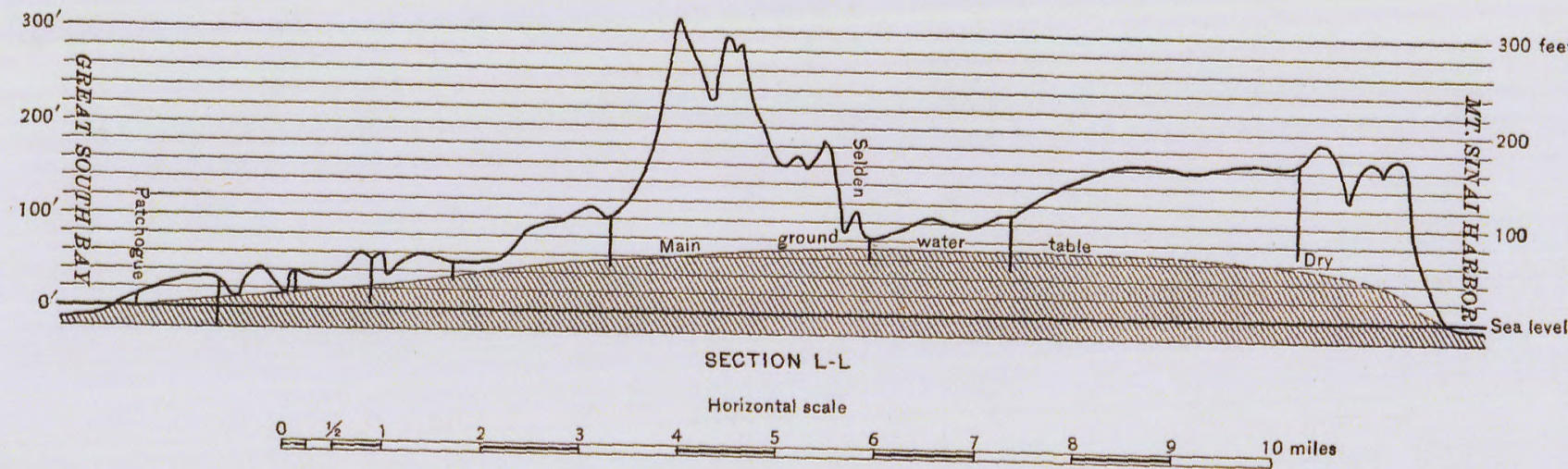



charge of 229,278 gallons per day at the Jackson street crossing and 675,907 gallons a mile lower down, near Mill road and Grove street, while at the efllux chamber at the end of the reservoir the discharge was $5,618,603$ gallons-an increase of five and a half million gallons ${ }^{a}$ in about 3 miles (fig. 27); and, as explained on page 62 , had there been no dam at this point the flow would have been much greater.

On the north shore the reentrant bays cut deep into the main water table, and large springs are abundant at, and near, high-tide level. Surveys made in the early fifties by Daniel Marsh, under the direction of Gen. W. B. Burnett, showed a spring discharge available for water supply amounting to $23,617,824$ gallons per day between Long Island City and Glen Cove. ${ }^{b}$

At the Fresh Pond pumping station (old Whitestone station) the spring flow amounts to 500,000 to 600,000 gallons per day, and the spring-fed pond at the Bayside (old Flushing) pumping station yields an average of $1,700,000$ gallons. A small spring area on the east side of Alley Creek, opposite the Bayside pumping station, and belonging to $\mathrm{Mr}$. William Corry, was gaged in September, 1903 , in connection with a study of the fluctuations of the wells of the Citizens Water Supply Company, and a yield of 365,000 gallons a day was indicated.

$$
\text { MTNERAL SPRINGS. }
$$

The well-known solvent power of water, especially when containing carbonic acid, causes it to dissolve whatever soluble salts are contained in the beds through which it passes. Thus, all springs and well waters contain a greater or less amount of mineral matter in solu-

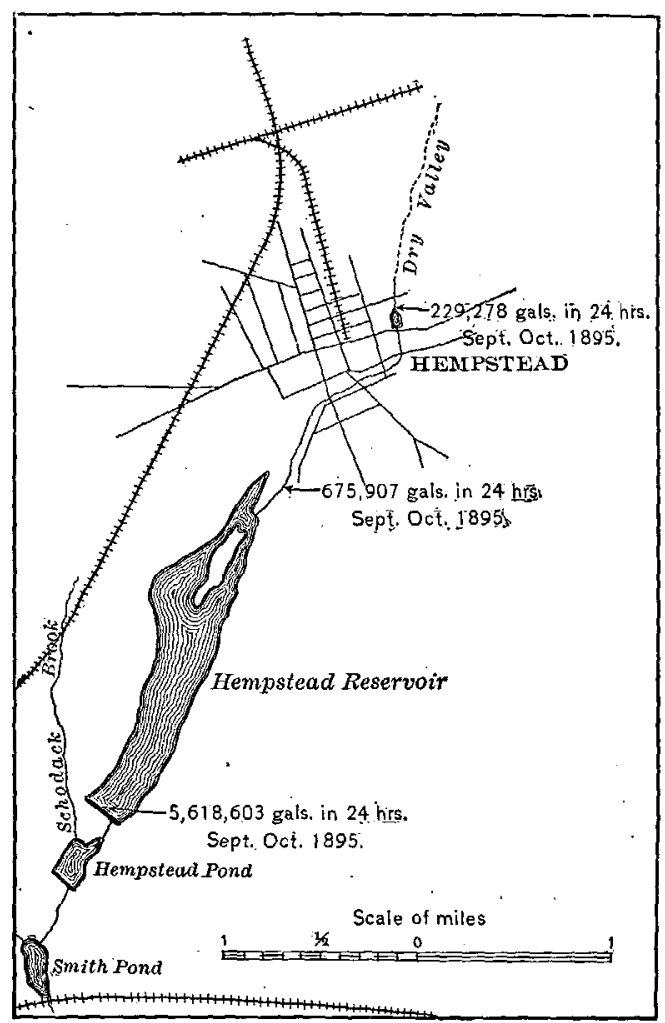

Fig. 27.-Sketch map showing increase in spring flow along Hempstead Brook. From data collected by the Brooklyn waterworks.

tion. Sometimes the ingredients have medicinal value, or the water is of so great relative purity that its use is recommended, and the springs are developed commercially. This forms "mineral water." So far as has been learned from a rather extensive inquiry, the waters of but four springs on Long Island have been put on the market, namely:

(643 c) The Colonial Spring, one-half mile west of Wyandanch.

(643) The Mo-mo-ne Spring, one-half mile northwest of Wyandanch.

(593) The Mountain Mist Spring, 2 miles south of Huntington station.

(226) Deep Glen Spring, $1 \frac{1}{2}$ miles northeast of Richmond Hill.

a History and Deseription of the Water Supply of the City of Brooklyn, 1896, p. 58 .

b Op. cit., p. 150 .

c These numbers correspond with those used in Chapter IV, where additional data will be found. 


\section{STREAMS}

ORIGIN.

As has already been indicated, the streams on Long Island are fed almost entirely by ground water. The valleys in which they flow were not formed by the present streams, but under conditions which existed in the Wisconsin and Vineyard epochs (pp. 43, 44), and the present streams may, almost without exception, be said to be the result, rather than the cause, of the valleys which they occupy. In other words, had not the channels across the southern plain been cut during the Wisconsin epoch, there would now either be no streams, or the streams would be of small magnitude, and the water which is now collected in them would appear as springs along the shores. The drainage areas of such streams depend entirely upon underground conditions, and, as was early appreciated in the study of this region, they can not be outlined with certainty from surface conditions. Another point of importance in such streams is that the flow is unusually uniform; the great beds of sand and gravel act as equalizing reservoirs in which the intermittent rainfall is stored and distributed throughout the year.

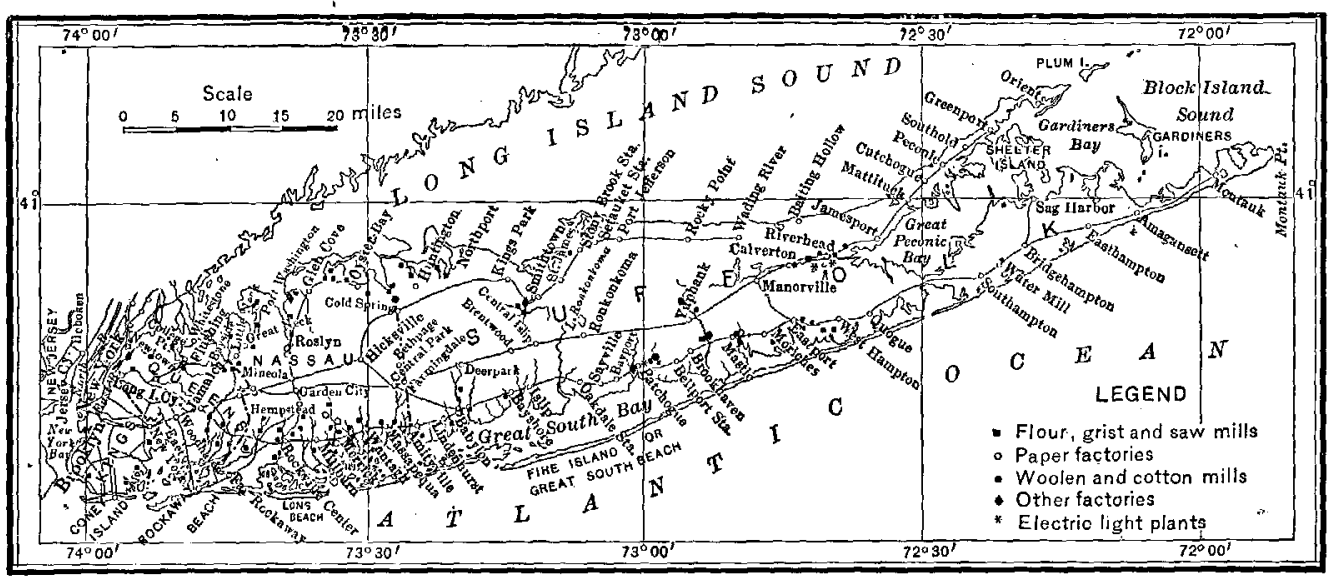

FIG. 28.-Sketch map of Long Island, showing distribution of water-power developments, 1800-1900.

WATER POWERS.

The comparatively steady flow of these short streams made them of considerable value for small water powers in the early history of the country, and one or more mills were erected along every important stream or branch (fig. 28). While a number of these were simply local grist or saw mills, requiring but a limited supply of water, a number of more pretentious mills were erected, among the more important of which the following may be mentioned: ${ }^{a}$

Jones \& Co., Woolen Factory, Cold Spring Harbor.

Patchogue Electric Light Company (new plant built which uses steam).

a Damerum, Wm., Map of the southern part of the State of New York, including Iong Island, the Sound, the State of Connecticut, part of the State of New Jersey, and islands adjacent, New York, 1815.

Burr, David H.; An atlas of the State of New York, containing a map of the State and of the several counties; projected and drawn under the superintendence and direction of Simeon De Witt, pursuant to an act of the legislature; also the physical geography of the State and of the several counties and statistical tables of the same, pp. 7-29, New York, 1829, 120 pp., 52 maps.

Smith, J. Calvin, Map of Long Island, with the environs of New York and the southern part of Conneeticut, New York, 1837 .

Beers, F. W., Atlas of Long Island, New York, 1873, 192 pp., 97 maps. 
Union Twine Mills, Patchogue. .

Patchogue Manufacturing Company (water and steam), Patchogue.

Swan River Cotton Mills, East Patchogue.

Patchogue Paper Mill Company (water and steam), $1 \frac{1}{2}$ miles north of Patchogue.

Perkins Brothers Woolen Mills, 1 mile west of Riverhead.

Riverhead Electric Light Company (water and steam), 1 mile west of Riverhead.

Tower Roller Mills, Riverhead (includes pumping plant of Riverhead Waterworks).

C. Hallett's Sons' Flour Mills and Electric Light Plant, Riverhead.

Jagger \& Luce's Flour Mill, Riverhead.

Phillips \& Company, Factory, Smithtown.

Paper mills were also operated at Roslyn (3), Meadow Brook (3), Merrick, Babylon, Moriches, Patchogue, and Riverhead.

No new projects are heard of and many of the old ones are falling into decay, but there seems to be a good opening for small developments for local electric lighting and power, especially at such favorable locations as Roslyn and Cold Spring Harbor.

\section{PONDS AND LAKES.}

Like the springs, the ponds and lakes of Long Island are of two classes, one dependent upon perched water tables or relatively impervious strata, the other on the main ground-water table, quite independent of impervious layers.

PONDS AND LAKES DEPENDENT ON PERched WATER TABLes.

To the first class belong almost all of the lakes and ponds situated in the more elevated portions of the island. The natural ones are for the most part due to. kettle holes made by the melting of blocks of ice detached from the glacier and buried during the last ice invasion, or to other irregularities of deposition by the glacier. When the sides of such depressions are of relatively impervious strata they collect the water falling in their limited drainage area, and form

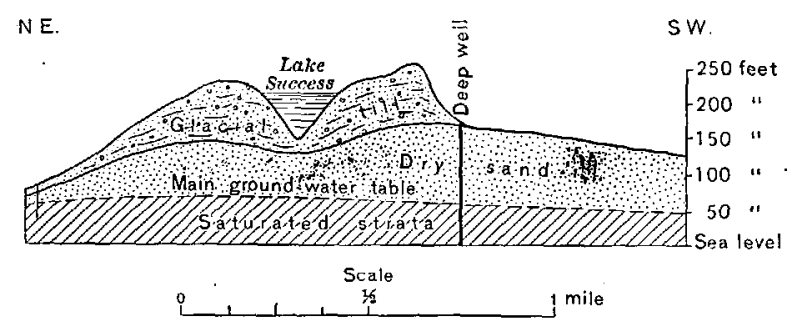

FIG. 29.-Take Success; an example of a kettle-hole lake depending on local impervious strata.

lakes or ponds. If the sides are

composed of pervious beds, these depressions. are dry, except where they extend below the main ground-water table. Ponds may be produced artificially by lining a depression or excavation with clay, and Mather states that at the time of his visit such artificial watering holes were a striking feature of the farming economy of the island."

The most striking example of a lake of this type is Lake Success, between Floral Park and Manhasset (fig. 29). It is situated high above the main water table and is clearly due to impervious beds in the moraine. Its. watershed is very limited, and as a source of water supply would be of small value. Such a lake could be drained very easily, since if a hole were drilled in the bottom the water would escape 
into the dry sands below. Other notable examples of the same type are the ponds shown in fig. 25. Whether or not such a pond has a surface outlet is determined by the relative importance of the following factors: (1) Size and condition of catchment area; (2) amount of rainfall; (3) amount of evaporation; and (4) permeability of the sides. .

PONDS DEPENDEN'T ON THE MAIN WATER TABLE.

As explained above, any depression, either natural or artificial, which cuts the main water table will tend to fill with water; if the depression is open at one

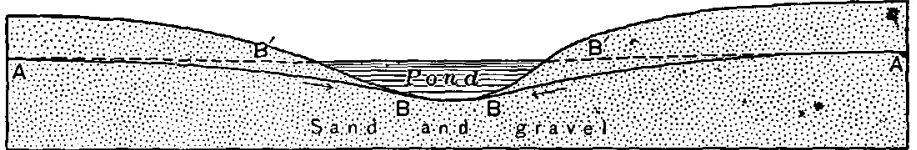

FIG. 30.-Diagram showing effect of a pond on the ground-water table and consequent deorease in spring flow on southern Long Island. A-B, initial position of ground-water table; $A-B^{\prime}$, resultant position of ground-water table. Arrows show direction of motion of ground-water. end it will form a springfed stream; if closed, a lake or pond; or, if still more closely inclosed, simply a well.

If a dam is thrown across a depression which cuts the main water table the effect is to obstruct the flow and to impond the water. As the flow in such a depression depends on the spring discharge, which in turn depends on the steepness of the slope of the ground-water table near it (A-B, fig. 30), it is evident - that a ponding of the water will decrease the steepness of the gradient and so reduce the spring flow. The crest flow of such a pond will, therefore, be much less than the normal flow of such a brook without a dam. Thus it has been found by the engineers of the Brooklyn waterworks that, under similar conditions, the Hempstead reservoir discharged 5,600,000 gallons per day when the water was maintained at a depth of 14.35 feet and 8,000,000 gallons when at 4 feet. ${ }^{a}$

If the water in a pond of this kind is raised above the level of the main water table in the adjacent divide (a condition which is possible because of the sloping nature of the groundwater table, the horizontal character of the surface of the pond, and the relatively rapid flow of the surface water) the ponded water will not

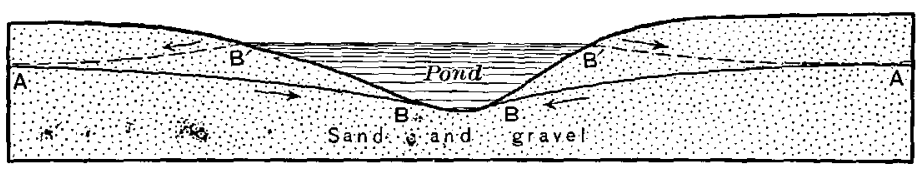

FIG, 31.-Dingram showing loss of water by leakage from pond whose surface is above the adjacent ground-water table.

only prevent a normal spring flow, but will flow out through the sides of the pond. (Fig. 31.) Such an outflow was clearly proved for the Hempstead reservoir by the engineers of the Brooklyn waterworks in 1878, when it was estimated that one million and a quarter gallons a day was transferred ${ }^{b}$ by ground flow from IIempstead reservoir to Schodack Brook. (Fig. 27.)

The effect of dams in the brooks of Lung Island is: (1) To raise the groundwater table; and (2) to very materially decrease the stream flow at the points where dams are erected.

In addition to the valleys which cut the main water table, and in which ponds are artificially constructed, a number of the large kettle holes extend below it, 
and therefore contain water. In such cases it is not necessary that the depression be lined with impervious beds, the sides may be entirely of sand and the depression still contain water. To this class belong all the large important lakes east of the West Hills, among the more important of which are Lake Ronkonkoma, Artist Lake, Long Pond (near Wading River), Deer Pond, Swan Pond, Great Pond, Big Fresh Pond, Poxabogue Pond, and Long Pond (near Sag Harbor). Lake Ronkonkoma may be taken as typical. Fig. 32 shows the essential difference between it and lakes of the Success type.

This difference is very important if these lakes are ever considered as sources for municipal or village water supplies, for while the yield of Lake Success would be relatively small, the yield of Lake Ronkonkoma would be large. Lake Success could be very easily pumped dry, but to dry Lake Ronkonkoma it would be necessary to remove a large part of the ground water above sea level from perhaps ouethird of Long Island. Its location near the center of the island, and its extreme depth, say 5 to 10 feet below sea level, make it an immense natural well of the utmost importance, and while the pop-

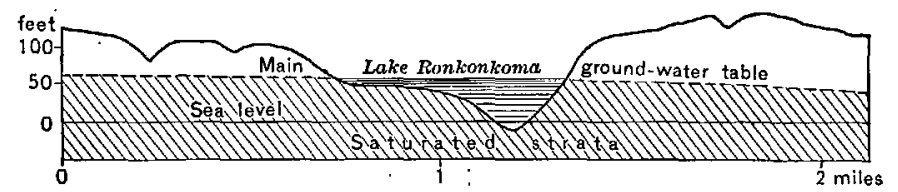

FIG. 32.-Lake Ronkonkoma; an example of a kettle-hole lakc depending on the main ground-water table.

ular idea that Lake Ronkonkoma is supplied by an underground stream from Connecticut or New England is entirely unfounded, the relation of the lake to the ground water of the island and its effective drainage area when lowered, say 50 feet below its present level, would give it a yield quite comparable to that which the believers in such an underground stream imagine for it. (See Pl. XI and fig. 32.)

\section{ARTESIAN AND DEEP WELLS.}

The discussion thus far has been confined almost wholly to phenomena such as ground-water tables, springs, streams, lakes, and ponds, which relate to surface waters. It has, however, been pointed out that this water is relatively free to pass downward, and that when it passes beneath a retaining layer a head sufficient to produce a flow may be developed. The nature of this retaining cover is purely relative. It must always be finer than the water-bearing stratum, but although the ideal retainer is a very fine clay, under certain conditions a flow may be obtained from a fairly porous sand above a coarse gravel.

SHALLOW NORTII-SHORE ARTESIAN WELLS.

Cause.-Flowing wells in which there is only a sand covering are found near the heads of many of the deep reentrant valleys on the north shore. In these valleys the slope of the water table is very great and the velocity of the ground water considerable. Many springs break out near water level, and often a pipe sunk entirely through sand to a depth ranging from 30 to 150 feet will furnish flowing water (see fig. 33). In these cases it is doubtless true that the layer which furnishes the flowing water is coarser than the overlying ones and affords a freer passage for the water. 
Such wells do not show many differences from the near-by springs. In the one a barrel is sunk 2 or 3 feet in the gravel and the water rises several inches above the surface. In the other a pipe 20 to 200 feet long is sunk entirely through sand and the water flows from it several feet above the surface.

Local clay beds are important in producing some of these flows, and in these cases the structure is essentially the same as that explained below for the Jameco gravels, which, in some of these north-shore wells, are doubtless the water-bearing horizon.

In most cases the water is from the Pleistocene gravels and the wells have yielded as high as 125 gallons per minute, natural flow. A flow of 50 gallons per minute is not uncommon (Pls. XIII, XIV).

Distribution.-While these wells are somewhat irregularly distributed, they are generally in the upper half of the steep-sided valleys or bays which characterize

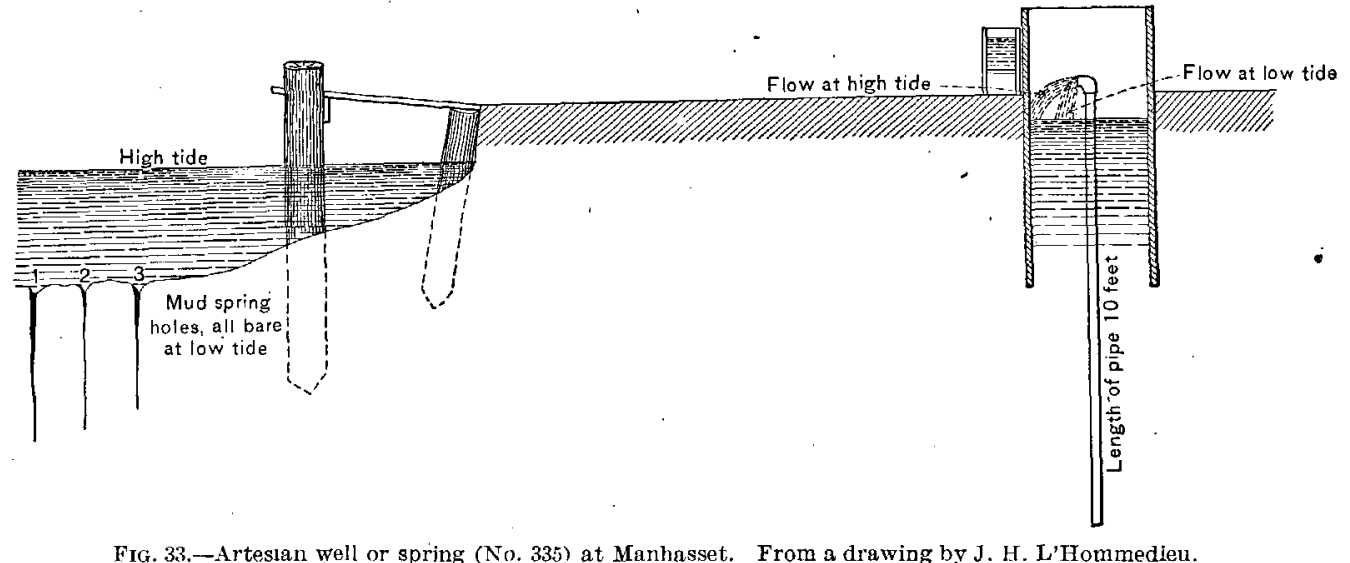

the region (Pl. XV). They seldom occur more than 10 to 20 feet above sea level, although flows have been obtained at an elevation of 35 to 50 feet at Dosoris (466), Huntington (626), and Glen Cove (455).

Predictions.-Many shallow, 50- to 200-foot artesian wells will doubtless be developed along the north shore during the next few years, and in prospecting for them the heads of steep hollows or the bottoms of steep banks should be chosen in preference to other sites, and the lower the elevation the greater will be the chances for obtaining a flow.

THE JAMECO ARTESIAN WELLS.

Cause.-The water passing under the blue clay (Sankaty), into the Jameco gravels (fig. 24), has a head dependent upon the height of the water table above the landward edge of the clay, and as the sand and gravel is fairly coarse and the loss by resistance not great, when a well is drilled only a few feet above tide level, the water from this gravel has a sufficient pressure to flow. In this case, although the water-bearing stratum has no outcrop and is not inclined, porous beds connect it with the surface, and the slope of the water table supplies the lack of slope of the strata. 
Distribution.-The head of water in the Jámeco gravels rarely exceeds 10 feet and flows can, therefore, not be expected much above this height. This basin is best developed in the region of the old valley and becomes of lesser importance in passing eastward because of the conditions which governed the deposition of the Jameco gravel (see p. 34). The coloring of the Jameco artesian area on $\mathrm{Pl}$. XV has therefore been discontinued near Babylon. At Riverhead the coarse gravels of this horizon again appear with a thin capping of clay, and yield as much as 130 gallons per minute, but the water is so chalybeate that it is necessary to obtain water from lower horizons. West of Jameco the artesian supply soon gives out because of leakage on the line where the Hudson has cut through the blue clay, and at the breaks in the clay layer at Barren Island and elsewhere.

Predictions.--The main outlines of this basin have been fully disclosed by the work of the Brooklyn waterworks. Along New York Bay no wells have reported potable water from this horizon and the limit of development must be drawn somewhere to the west of New Lots. On the south at Barren Island the blue clay is entirely absent, a fact which, it is believed, increases the danger of an influx of salt water from heavy pumping at the pumping stations to the north. This horizon may have an artesian value on the south side of the South Fluke, and near tide level wells 50 to 150 feet deep are likely to yield flows.

THE CRETACEOUS ARTESIAN WELLS.

Cause.-The water which sinks deep into the Cretaceous sands may pass under a clay sheet, and when this clay is penetrated at low points on the north and south shores, the head, which depends on the height of the water table above the landward edge of the particular clay layer in question, may, under favorable circumstances, be sufficient to produce a flow. The principal requisite in this case, in addition to those already mentioned, is that the gravel shall be of such a coarseness that the loss of head in transmission. from the edge of the clay bed may not be excessive. On the north shore the outlet of the gravel under the Sound should be more or less completely sealed by an impervious layer.

Distribution.--The principal bed of this character is the Lloyd sand (p. 19), the position of the surface of which is shown on Pl. XVI, from which the position of the bed at any point may be inferred. This horizon has been developed to a very considerable extent on the north shore and at one point on the south shore. The most important wells deriving water from it are the following:

TABLE V.-Wells in the Lloyd sand.

\begin{tabular}{|c|c|c|c|}
\hline No.a & Location and owner. & $\begin{array}{l}\text { Total } \\
\text { depth. }\end{array}$ & Remarks. \\
\hline 633 & Lloyd Neck; Dr. O. L. Jones..... & 248 & $\begin{array}{l}\text { Elevation approximately } 5 \text { feet above mean high tide. } \\
\text { Flows } 5 \text { gallons per minute at high tide. }\end{array}$ \\
\hline 620 & $\begin{array}{l}\text { Cold Spring Harbor; 'T. S. Wil- } \\
\text { liams. }\end{array}$ & 430 & $\begin{array}{l}\text { Elevation } 8 \text { feet above mean high tide. Flows } 12 \text { gallons } \\
\text { per minute. }\end{array}$ \\
\hline 559 & Center Island; C. W. Wetmore.... & 318 & $\begin{array}{l}\text { Elevation approximately } 5 \text { feet. Flows } 25 \text { gallons per } \\
\text { minute at high tide. }\end{array}$ \\
\hline
\end{tabular}

a Numbers correspond with those used in Chapter IV and on index map, Pl. XXIV. 
UNDERGROUND WATER RESOUROES OF LONG ISLAND, NEW YORK.

TABLE V.-Wells in the Iloyd sand-Continued.

\begin{tabular}{|c|c|c|c|}
\hline No. & Location and owner. & $\begin{array}{l}\text { Total } \\
\text { depth. }\end{array}$ & Remarks. \\
\hline 558 & Center Island; Colgate Hoyt .. & 320 & Elevation approximately 6 feet. Flows. \\
\hline 557 & - S. T. Shaw ............ & 292 & $\begin{array}{l}\text { Elevation approximately } 5 \text { feet. Flows } 5 \text { to } 6 \text { gallons } \\
\text { per minute at high tide, flows slightly at low tide. }\end{array}$ \\
\hline 556 & —— C.S. Sherman ..... & 351 & $\begin{array}{l}\text { Elevation approximately } 8 \text { feet. Flows } 30 \text { gallons per } \\
\text { minute at high tide, } 20 \text { at low tide. }\end{array}$ \\
\hline 555 & — G. M. Fletcher .... & 370 & $\begin{array}{l}\text { Elevation approximately } 10 \text { feet. Flows } 25 \text { to } 30 \mathrm{gal}- \\
\text { lons per minute at high tide. }\end{array}$ \\
\hline 554 & — G. C. Mackenzie ... & 379 & $\begin{array}{l}\text { Elevation approximately } 10 \text { fect. Flows } 75 \text { gallons per } \\
\text { minute at high tide, } 45 \text { at low tide. }\end{array}$ \\
\hline 553 & Oyster Bay; Emily Roosevelt. & 460 & $\begin{array}{l}\text { Flow was obtained at } 460 \text { feet, but well was abandoned } \\
\text { because of breaking of pipe. }\end{array}$ \\
\hline 560 & Bayville; Dr. O. I. Jones....... & 276 & Flows. \\
\hline 564 & Mill Neck; Irving Cox....... & 330 & Elevation about 12 feet. Flows 72 gallons per minute. \\
\hline 470 & Peacock Point; C. O. Gates. . & 230 & $\begin{array}{l}\text { Elevation approximately } 6 \text { feet. Flows } 30 \text { gallons per } \\
\text { minute. }\end{array}$ \\
\hline 472 & $\ldots$ - do... & 210 & $\begin{array}{l}\text { Elevation approximately } 15 \text { feet. Flowed when first } \\
\text { completed } 40 \text { gallons per minute. Is now being } \\
\text { pumped. }\end{array}$ \\
\hline 471 & _- ; do... & 225 & Flows 10 gallons per minute. \\
\hline 473 & — W. D. Guthric ............ & 340 & Elevation about 10 feet. Flows 10 gallons per minute. \\
\hline 317 & LakeSuccess; W.K.Vanderbilt, jr. & 755 & Pumps $300+$ gallons per minute. \\
\hline 130 & $\begin{array}{l}\text { Barren Island; Thomas F. White } \\
\text { Co. }\end{array}$ & 740 & $\begin{array}{l}\text { Elevation approximately } 7 \text { feet. Flows } 103 \text { gallons per } \\
\text { minute. }\end{array}$ \\
\hline 131 & $\begin{array}{l}\text { Barren Island; New York Sani- } \\
\text { tary Utilization Co. }\end{array}$ & 724 & Elevation $5-7$ feet. $\quad$ Flows 50 gallons per minute. \\
\hline 132 & - ; do . . . . . . . . . . . . . & 700 & $\begin{array}{l}\text { Elevation } 9 \text { feet. No flow. Pumps } 150,000 \text { gallons in } \\
24 \text { hours. }\end{array}$ \\
\hline
\end{tabular}

The many wells put down in the Cretaceous beds overlying this horizon have yielded very conflicting results. There scem, however, to be several water-bearing horizons of greater or less importance which can be made available, and which have been over'ooked in the earlier work because of the ease with which water could be obtained from the coarse Jameco gravels. A 10-inch well near Lynbrook, 504 feet deep, belonging to the Queens County Water Company, has been very carefully tested and found to yield 450,000 gallons per day. Flowing water has also been obtained at the following places and depths:

Flowing wells in the Cretaceous on Long Island other than those in the Lloyd sand.

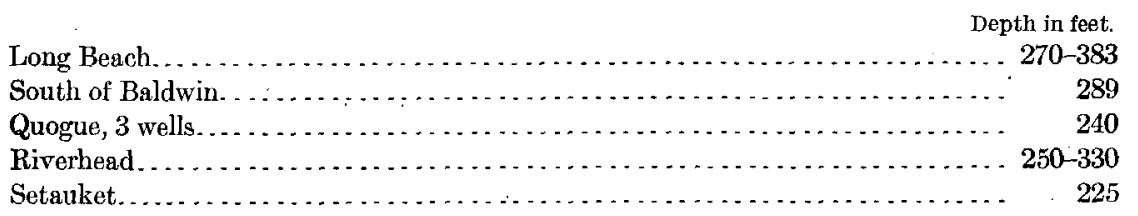

None of these wells has been carefully tested, and no definite data can be given regarding their capacity. 


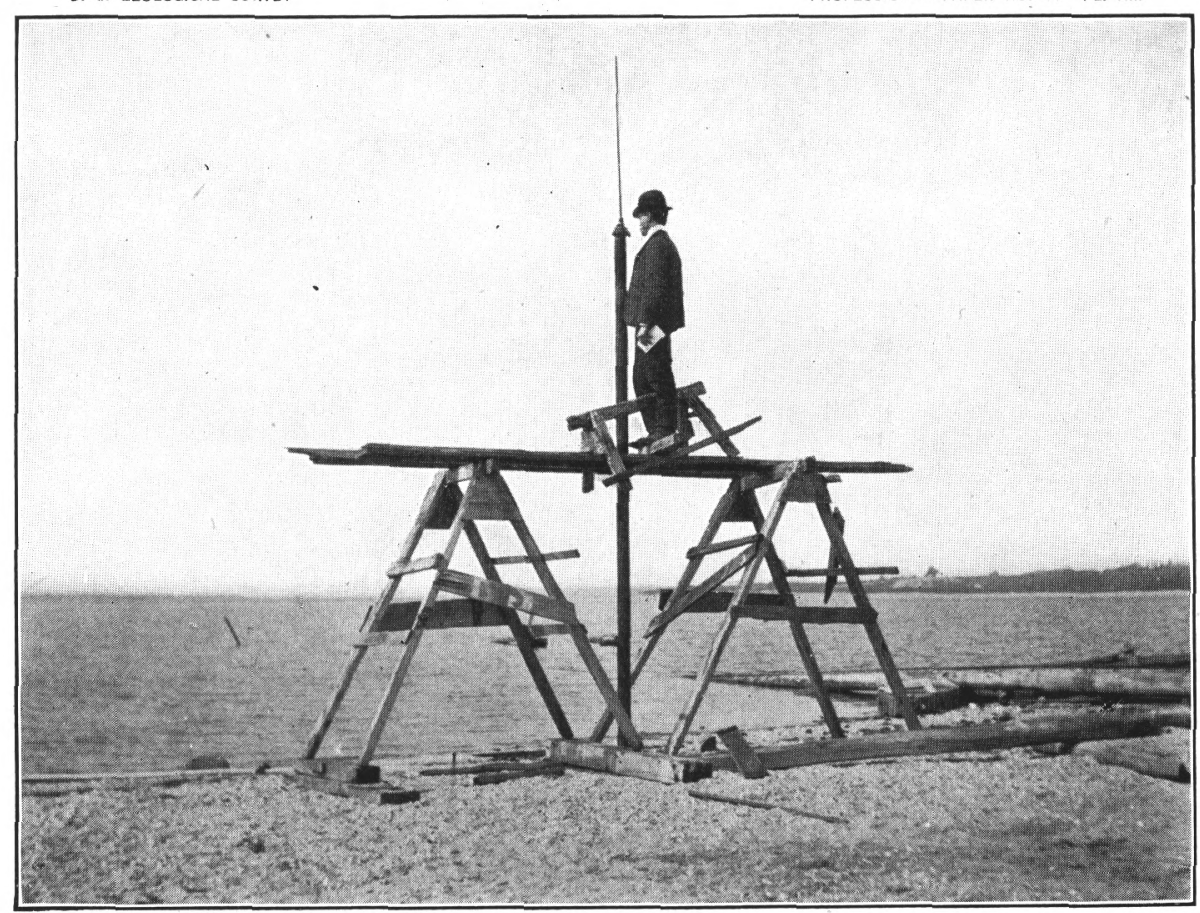

A. BURGESS WELL, OYSTER BAY

Water rises to within 1 foot of top of pipe. Rod projectıng above pipe is aluminum gage used in observations on tidal fluctuations.

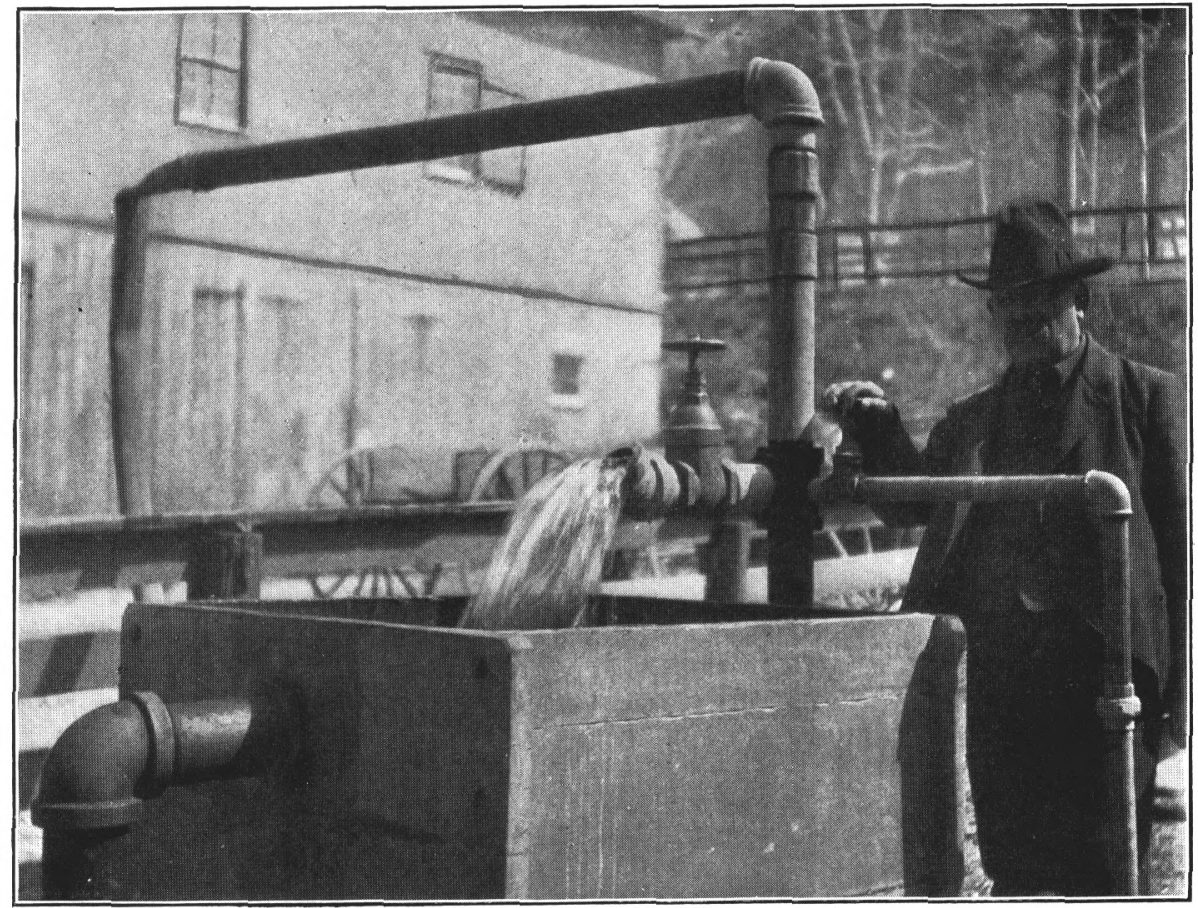

B. JONES WELL, COLD SPRING HARBOR.

Water flows freely over elbow in pipe. 



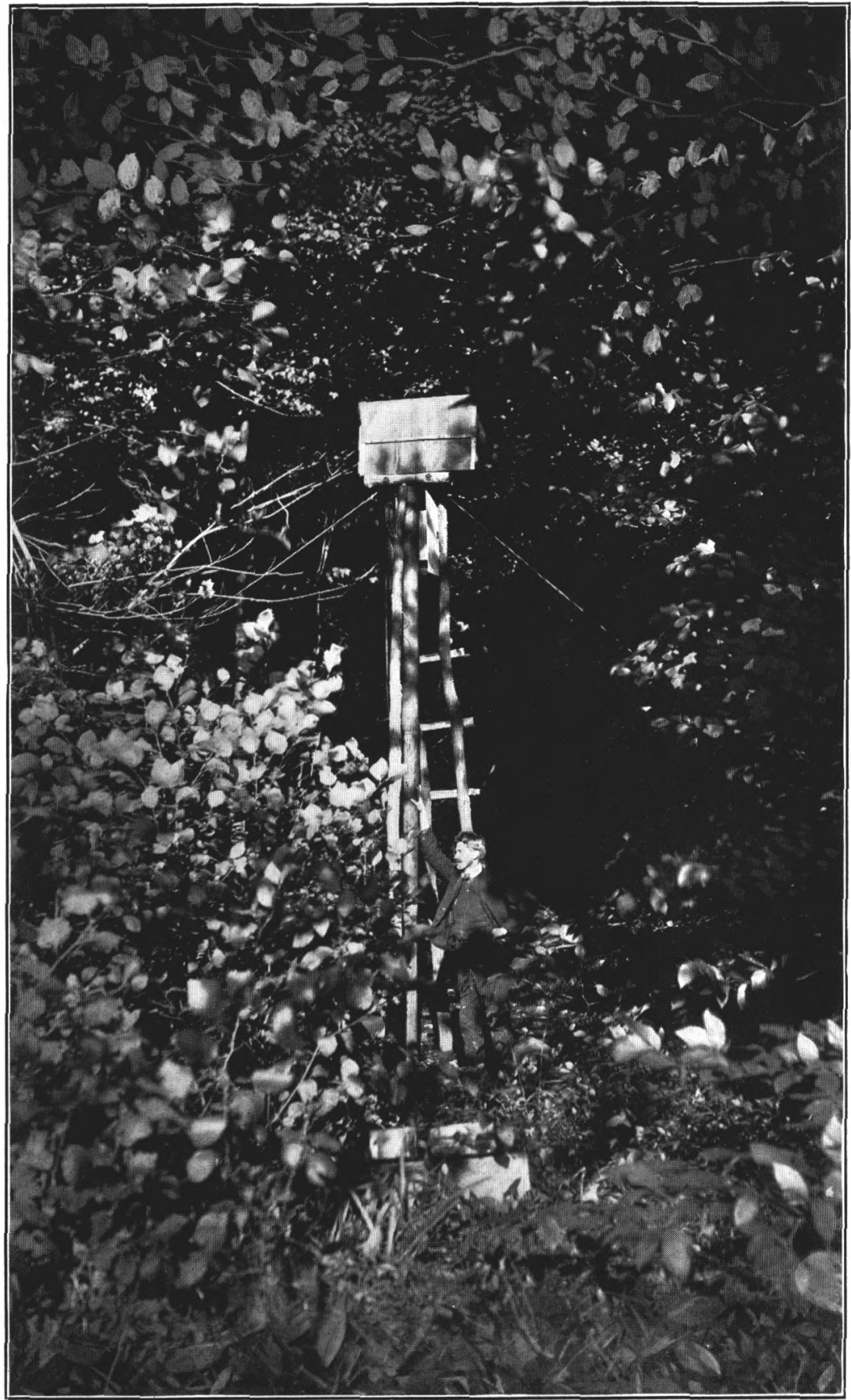

HEAD DEVELOPED IN A 40-FOOT ARTESIAN WELL BELONGING TO THE CITIZENS' WATER-SUPPLY COMPANY NEAR DOUGLASTON, N. Y.

Water rises to within 1.5 feet of top of pipe. Box contains automatic gage used in study of the tidal fluctuations in this well. 

Like the other artesian wells on Long Island, water from the Cretaceous horizons will seldom rise higher than 5 to 10 feet above sea level, and artesian wells are therefore restricted to the region of the shore.

Predictions.-The Lloyd gravel is the best-defined artesian horizon on the island and is believed to be remarkably persistent. It may be regarded as available south and east of a line connecting Bay Ridge and Willets Point to, perhaps, somewhat beyond Riverhead, and will furnish flowing water at elevations less than 5 to 10 feet above sea level. The importance of this horizon on the North and South flukes is uncertain because of the distance from the main uplands of the island. The South Fluke, however, is the more promising territory, because it is down the dip and has a greater area. The minor upper horizons are not so well known and their positions can not be definitely predicted.

REQUISITE CONDITIONS FOR SUCCESSFUL WELLS ON LONG ISLAND.

Were Long Island composed of entirely homogeneous porous materials it would be necessary to sink wells only slightly below the main ground-water table, a distance of 25 or 30 feet probably being all that would be required;in any case. The great irregularity of the formations, however, introduces a new factor. For a permanent well it is not only necessary to go to the main ground-water table, but to land the well in a coarse bed from which water will be given up freely. It is this point that makes well sinking on the island somewhat uncertain. In general it is not necessary to go far below the main water table (fig. 24), but in some cases, notably in the Wheatley Hills, the beds at the water table and for some distance below are so fine that they pass the strainers and fill the well with quicksand. In these cases it was necessary to drill until a coarser bed was reached, which in the Morgan well (431) was 100 feet and in the Duryea well (430) was about 140 feet below sea level, the main water table being in both cases about 85 feet above sea level. In the Vanderbilt well (317), although the main water table was encountered at about 50 feet above sea level, the well was pushed to a depth of 585 feet below sea level, completely penetrating the coarse Lloyd gravel, from which an abundant supply was obtained.

One very significant point in regard to these deep wells in the higher parts of the island is that the height to which the water will rise never exceeds the height of the main water table, and generally falls slightly below it. The point, then, in going deeper, except near the shores where artesian conditions are present, is not to get an increase in head, but to find a coarse layer which will readily yield water; in other words, to find an extensive natural horizontal strainer which will aid in separating the water from the adjacent fine sands.

\section{SOURCE OF THE UNDERGROUND WATER ON LONG ISLAND.}

The gradual decrease in head, with depth which is observable in deep wells in the center of the island, is an important matter in the consideration of the source of the water. Thus in the Vanderbilt well (317) while the main water table was encountered at 54 feet above sea level, the height to which the water rose from the Lloyd gravel was only 35 feet, a loss of 20 feet of head in about 550 feet of depth. This height is, moreover, greater than that to which the water will rise from the 
same horizon on the north and south shores. If the water came from below, as is very generally imagined, the pressure should decrease from bed rock upward for an appreciable distance, the pressure near bed rock being greater because of the loss in head in transmission through the sand; while if the water came from above it would be expected that the head would either remain the same or decrease with the depth. As it decreases it furnishes conclusive proof of the insular source of the water.

The geologic structure of the region (fig. 24), moreover, forbids the transportation of water from New England, except through bed rock, and the metamorphosed and highly folded character of these beds makes such transmission very doubtful. Early in the consideration of the possible reason for the deep flowing wells from the Lloyd gravel, after it had been found that the Cretaceous beds dip regularly southward, and before it was known that the clay beds were not continuous, it was suggested by Prof. W. H. Hobbs, of the University of Wisconsin, and Prof. H. E. Gregory, of Yale University, that if the faulted structure found in Connecticut continued under Long Island, and if the fault springs which are common in the former region were present, then the water furnished by these springs would be retained beneath the clay layer and give rise to an artesian condition. Fault springs, or natural artesian wells, produced under the proper conditions by the cutting of a porous water-bearing layer by a fault line, are comparatively simple phenomena, but the hypothesis that such springs occur under Long Island must rest on the assumption of a complexity of horizontal faulting of which there is no evidence. Moreover, the water obtained from these deep wells runs exceptionally low in chlorine, alkalinity, and hardness, while waters from the rock wells in the western part of the island and in neighboring regions of New York and Connecticut have, as a rule, a much higher mineral content.

'TARI.E VI.-Analyses showing difference between waters from the Lloyd sand and those from the rock wells of Connecticut.

[Parts per million.]

\begin{tabular}{|c|c|c|c|c|c|c|}
\hline Location & & Chlorine. & $\begin{array}{l}\text { Hard- } \\
\text { ness. }\end{array}$ & $\begin{array}{l}\text { Alka- } \\
\text { linity. }\end{array}$ & Analyst. & Remarks. \\
\hline $\begin{array}{l}\text { Center Island, Long } \\
(559) \text {. }\end{array}$ & Island & 3.54 & 20.0 & 19.0 & C. S. Slichter... & $\begin{array}{l}\text { Flowing well in Lloyd sand; } 318 \\
\text { feet deep. }\end{array}$ \\
\hline $\begin{array}{l}\text { Center Island, Long } \\
(556) \text {. }\end{array}$ & Island & 3.89 & 20.0 & 18.7 & ....do. & $\begin{array}{l}\text { Flowing well in I.loyd sand; } 351 \\
\text { feet deep. }\end{array}$ \\
\hline $\begin{array}{l}\text { Center Island, Long } \\
\text { (554). }\end{array}$ & Island & 4.25 & 20.0 & 20.0 & .... do. . & $\begin{array}{l}\text { Flowing well in Lloyd sand; } 378 \\
\text { feet deep. }\end{array}$ \\
\hline $\begin{array}{l}\text { Peacock Point, Long } \\
(470) \text {. }\end{array}$ & Island & 5.83 & 27.5 & .27 .2 & .... do. & $\begin{array}{l}\text { Flowing well in Lloyd sand; } 230 \\
\text { feet deep. }\end{array}$ \\
\hline $\begin{array}{l}\text { Lattingtown, Long } \\
(473) .\end{array}$ & Island & 4. 60 & 22.5 & 17.5 & .....do. & $\begin{array}{l}\text { Flowing well in Lloyd sand; } 342 \\
\text { feet deep. }\end{array}$ \\
\hline Long Island City (75).. & & $1,902.1$ & & & Jacob Blumer, Oet. 12,1888 & Well in rock; 275 feet deep. \\
\hline Connecticut: & & & & & & \\
\hline Greenwich.... & & 9.28 & 10. 7 & & H. T. Vulté. & Well in rock; 177 feet deep. \\
\hline Rowayton.... & & 32.00 & ...... & 22.0 & H. F. Smith....... & Well in rock; 395 feet deep. \\
\hline Norwalk..... & & 25.0 & 62.9 & & S. P. Wheeler..... & Artesian woll. \\
\hline Norwalk..... & & 20.0 & 60.0 & & ....do............ & Do. \\
\hline Norwaik . . . & & 5.6 & 45.7 & & ....do... & Do. \\
\hline Westport.. & & 12.0 & 74.3 & & .....do... & Do. \\
\hline Fairfield.... & & 31.0 & 121.0 & & & \\
\hline Fairficld. . & & 21.0 & 164.3 & & S. P. Wheeler... & Do. \\
\hline
\end{tabular}


TABLE VI.-Analyses showing difference between waters from the Lloyd sand and those from the rock wells of Connecticut-Continued.

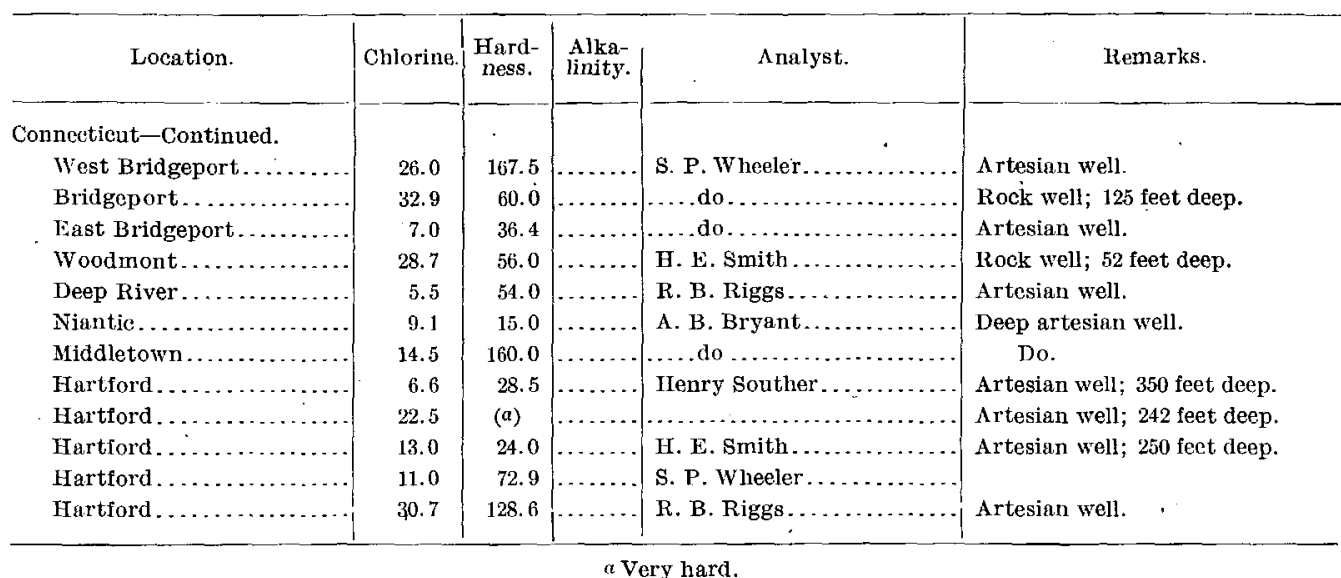

On the whole, there is absolutely no evidence of a Connecticut source for the underground water on Long Island. The water is derived entirely from rainfall on the island, and all the water phenomena observed can be directly traced to this source, except that a slight amount may be transmitted through the Lloyd gravel from New Jersey.

\section{OAUSES OF FLÚCTUATION OF THE GROUND-WATER TABLE. ${ }^{a}$}

The causes which produce fluctuations of the ground-water table on Long Island may be subdivided as follows:

A. Natural:

Rainfall.

Sympathetic tides.

Thermometric changes.

- Barometric changes.

B. Artificial :

Dams.

Pumping.

NATURAI, CAUSES OF FLUCTUATION.

Rainfall.- As rainfall is the source of ground water, it would seem self-evident that the ground-water level must vary directly with the rainfall, heavy rains raising it and long periods of drought lowering it. While this is true in a broad way, the relation between the rainfall and the changes in level of the ground-water table is not such a simple one as this statement might imply:

In the summer of 1903 the engineers of the Long Island division of the commission on additional water supply made daily observations on the water levels in wells in. many parts of Long Island and accumulated much definite data on this point. Fortunately the observations began just before the exceptional period of drought which extended from April 16 to June 7. The wells observed were

" Preliminary statement; a more complete report on the,observations on these fluctuations made during the summer of 1903 is now in press as Water-Supply and Irrigation Paper No. 155. 
largely open dug wells depending on the main ground-water level, which, as already indicated, rises from sea level on each side of the island to a summit somewhat north of the surface divide (p. 57).

From these records the typical hydrographs shown on Pl. XVII have been selected. This group of wells, with the possible exception of No. 6, are all south of the ground-water divide and in a region where the direction of underflow is southward.

In shallow wells near the south shore, such as Nos. 1 and 2, the relation between the rainfall and the fluctuation of the ground-water table is very apparent.

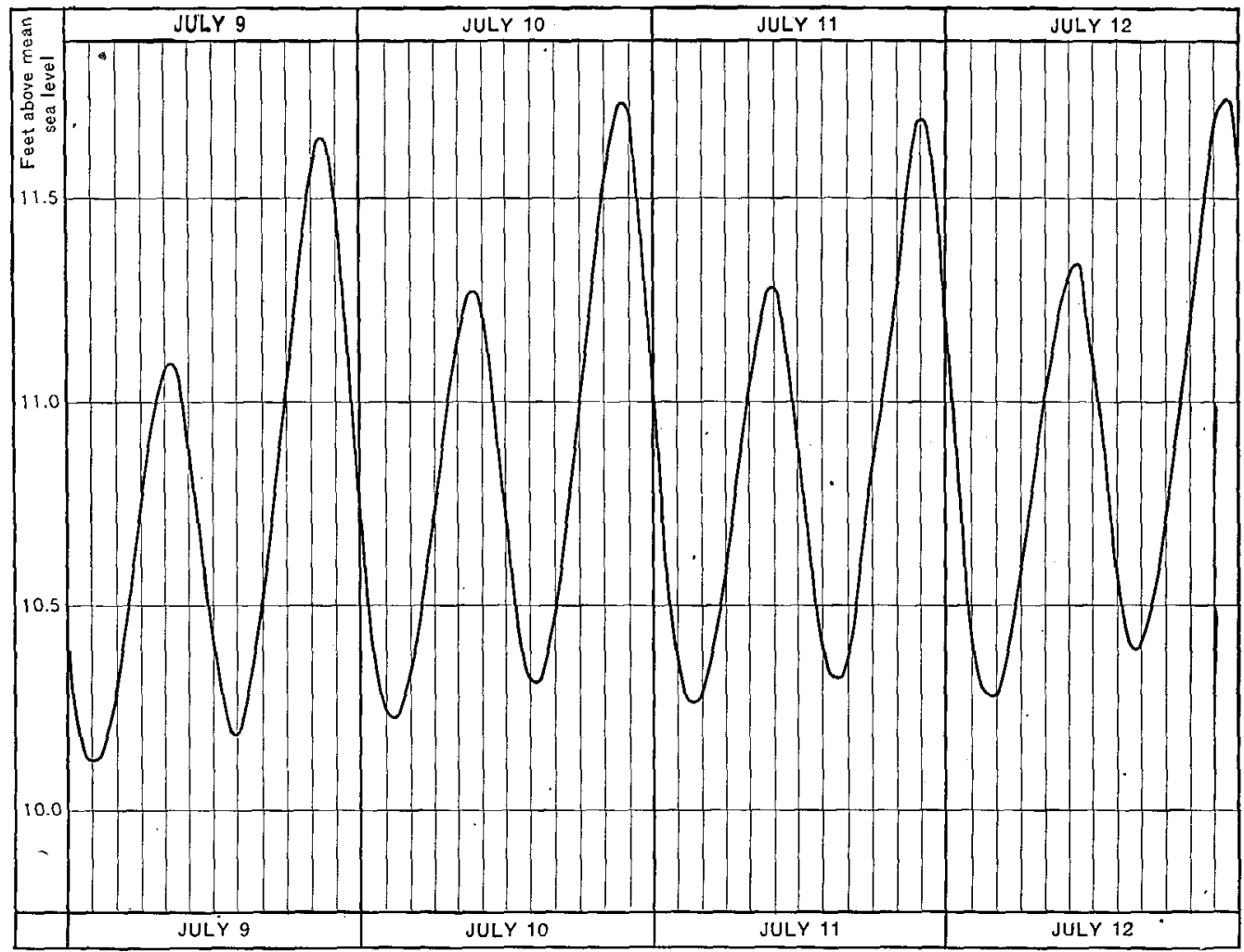

FIG. 34.-Autograph record of water level in a 386-foot well at Long Beach, N. Y., showing fluctuations due to tides. Record from a Means nilometer in charge of F. D. Rathbun, field assistant. Elevations indicated are approximate.

Five or six days after the heavy rains of April 14 and 15 the water, after rising for a few days, fell steadily through the period of dry weather. Three or four days after the rain of June 7 , which ended the drought, the water in both wells began to rise and continued to rise during the rainy weather which followed.

Farther inland, a gradual change is noted in the behavior of the surface of the ground-water, wells 7 or 8 miles from the shore, such as Nos. 3, 4, and 7, showing an entirely different curve. In these the water rose steadily during the drought and began to fall when the heavy rains commenced. In wells still farther inland, as Nos, 5, 6, and 8, the water rose steadily for the whole period shown. 


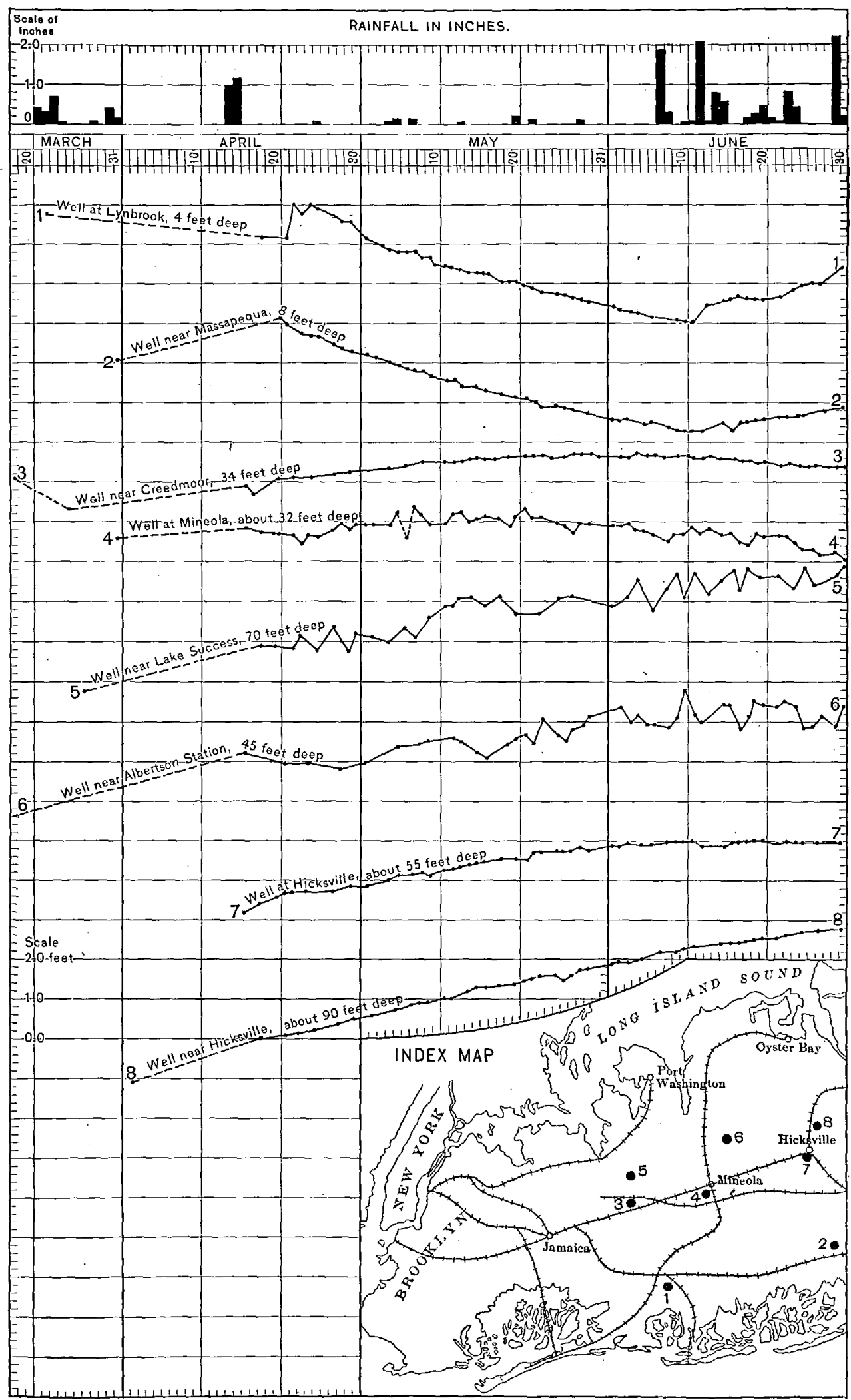

FLUCTUATIONS OF THE MAIN GROUND-WATER TABLE ON LONG ISLAND.

From observations of the Long Island division of the New York City commission on additional water supply, March-June, 1903. 

-well No. 8 actually rising over 2 feet during the greatest drought this section had ever experienced.

These curves indicate that the deeper the ground-water table and the farther it is from the shore (or the higher it is above sea level) the more slowly it-responds to the rainfail. The retardation is entirely out of proportion to the thickness of the unsaturated beds above the main water table. In the wells at Lynbrook and Massapequa, which are from 4 to 8 feet deep, rain water should, according to the rate of flow determined by laboratory tests, reach the ground-water table in a few minutes, yet the water table did not begin to rise until four or five days after the heavy rains. As the thickness of the unsaturated beds increases, this retardation is multiplied at an astonishing rate. Thus, while the 4 and 8 foot wells at Lynbrook and Massapequa began to fall seven days after the close of the rainy period in April, the 32-foot well at Mineola did not begin to fall until after thirty-five days, the 34-foot Creedmoor well after about fifty-five days, and the 55-foot Hicksville well after about sixty-five days, while the 70- and 90-foot wells at Lake Success and Hicksville showed no tendency to fall after seventy-five days, but were still rising from the effects of the March and April rains. In this delayed transmission the effects of single showers is almost wholly neutralized, the sand acting as so perfect an equalizer that only the mass results of long periods of rain or drought are indicated. The question involved here is apparently not so much how fast a constant stream of water under a given pressure will flow through a column of earth of a given height as how long it will take a given quantity of water precipitated on the top of this column during a relatively short time to entirely or almost entirely run out at its base.

Tides.-Nearly all the wells in the neighborhood of the shores, both shallow and deep, show a sympathetic vibration with the tides. The nature of this vibration and its clearly tidal character are shown in figs: 34 and 35 . Fig. 34 represents a 386-foot well at Long Beach and fig. 35 a 40 -foot well at Douglaston. This fluctuation is commonly
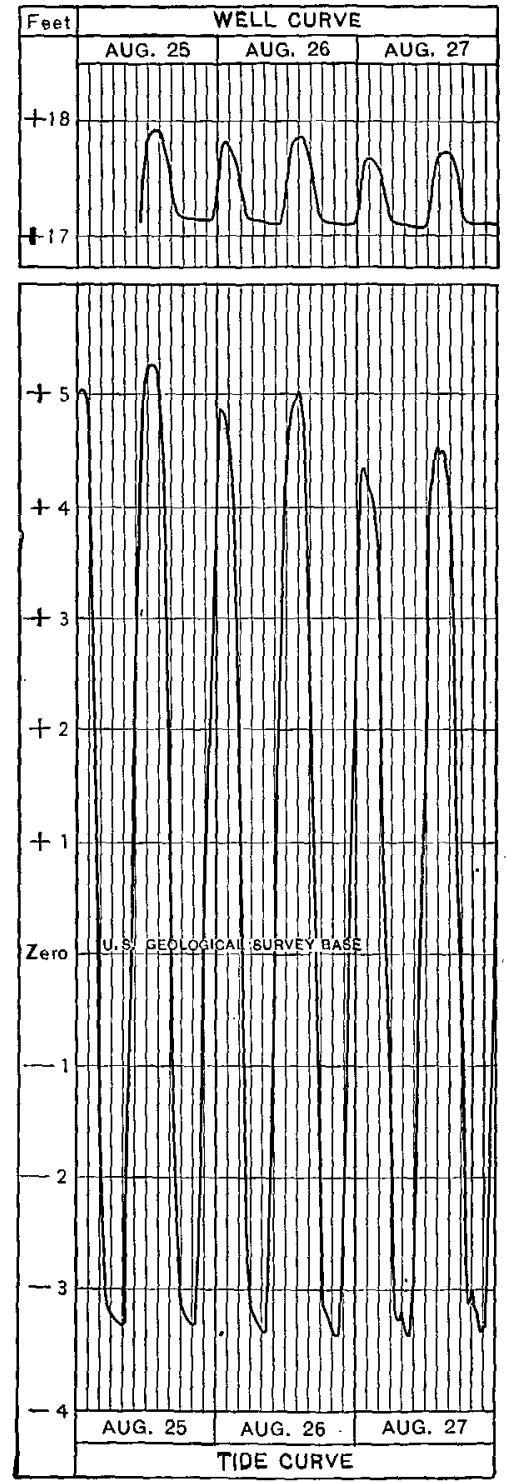

FiG. 35.-Record of water level in a 40 -foot well of the Citizens' Water Supply Company at Douglaston, $N, Y$, and tidal record in adjacent oreek. Record from Friez tide gages in charge of F. L. Whitney, field assistant. greatest at the shore and becomes less on passing inland, but this rule is by no means invariable, and many very peculiar local variations are found. 
The tidal curves in the wells are miniatures of those in the near-by body of water, but are generally somewhat behind them. Thus, at Oyster Bay, where the water is under sufficient hydraulic head to lift it considerably above the surface of the ground (see Pl. XIII, $A$ ), the tide in the Casino well, which is in the very edge of the water, is five to ten minutes behind that in the bay, while in the Underhill well, which is only 300 feet from the shore, it shows a lag of from sixty-five to seventy-five minutes.

While this great increase in the amount of lag in very small distances indicates that the factors concerned in the formation of these sympathetic tides are rather complex, it is not felt that the phenomenon necessarily involves a free outlet of the underground water into the ocean, as is very commonly held in this region. On the contrary, it is thought to be conceivable that the clay layers, rendered more or less sensitive by the water-logged artesian sands beneath them, may act

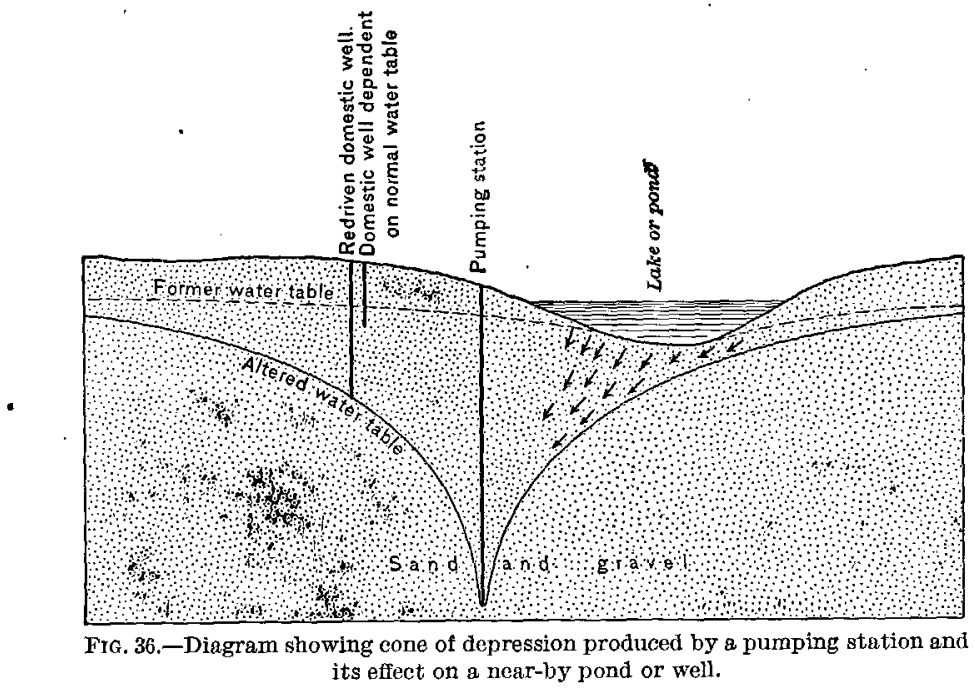
as large diaphragms and respond directly to the alternate loading and unloading caused by the flood and ebb tides.

Thermometric and barometric changes. - Selfrecording gages placed on the wells of the Queens County Water Company at Lynbrook (277) during the summer of 1903 showed very regular daily fluctuations of the groundwater table, which were clearly due neither to rainfall nor tidal action. A comparison of these curves with the thermograph and barograph records obtained at Floral Park and Brentwood by the commission on additional water supply (PI: XVIII) shows that the fluctuations closely correspond to the changes in temperature and only remotely to those of air pressure, except in the case of the 504-foot well. It was at first thought that the daily fluctuations in the temperature might produce minor barometric fluctuations and that the changes in the water level might be ultimately due to changes in air pressure, but a study of the data forces the conclusion that the normal fluctuation shown in curves 1 and 2 are directly due to temperature: Thus the important barometric depression indicated on July 26 produced no effect on the water level in the 14- and 72-foot wells, although. clearly noticeable in the 504-foot well. Even the sudden rise of the water, which occurred during the storm of July 30 and which has many aspects of being due to a change in air pressure, has a sharpness and definition not indicated by the barograph curve, although suggested by the thermograph curve. 
1903.

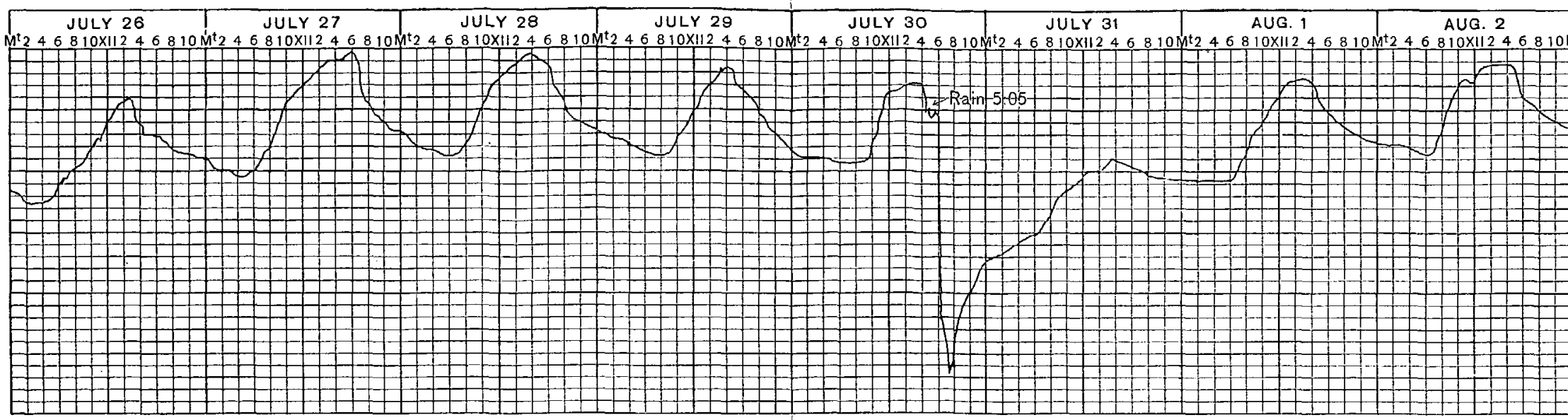

I

Autographic record of

fluctuations of

water surface

in 14-foot well at

Lynbrook.

New York

(inverted curve)

n

Autographic record of

fluctuations of

water surface

in 72 -foot well at

Lynbrook,

New York.

(inverted curve)

III

Autographic record of fluctuations of water surface

in 504.foot well at

Lynbrook,

New York.

(inverted curve)

Thermograph curve a Floral Park,

New York.

\section{晨}

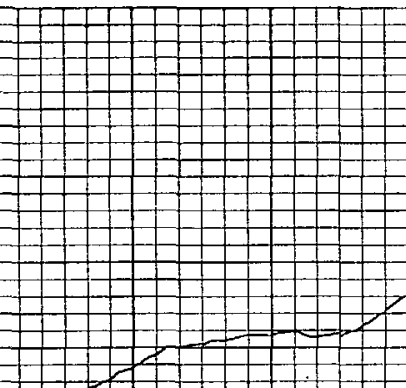

$+1+-1+1$

ㄴ

e
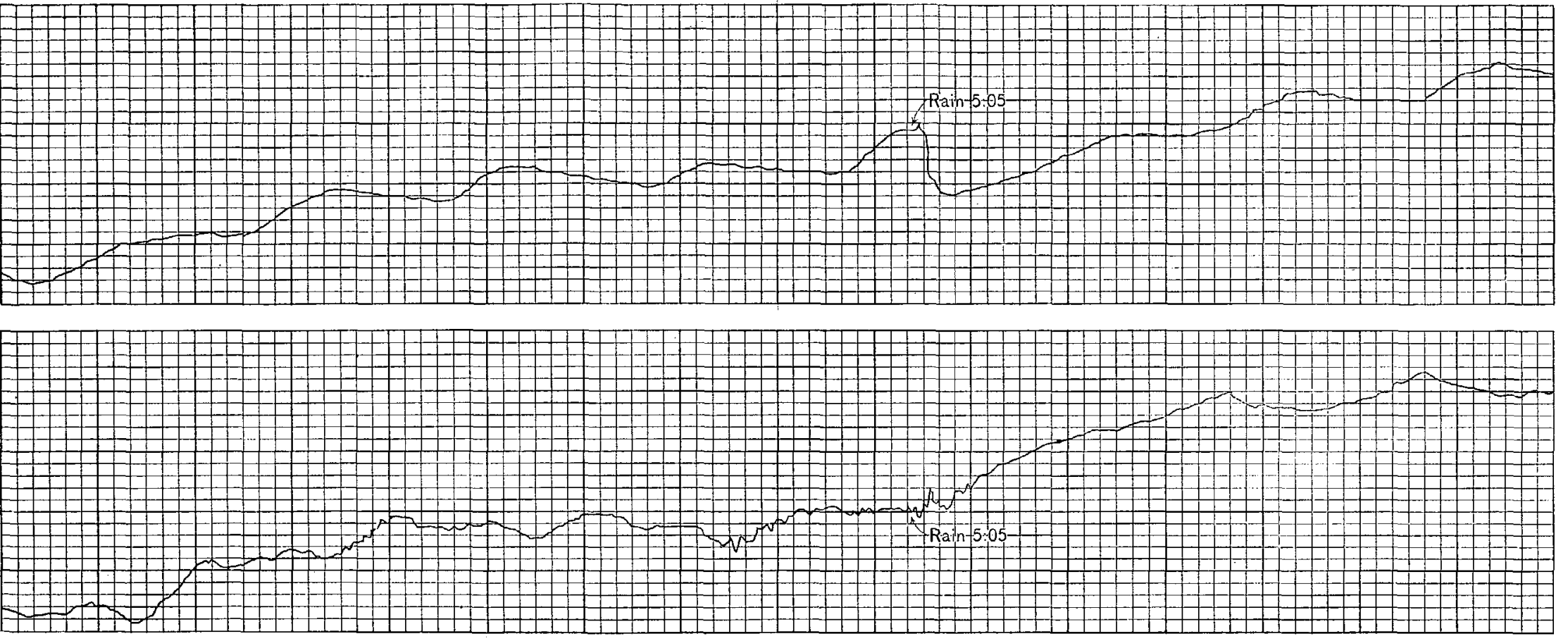

$+\mp+1$

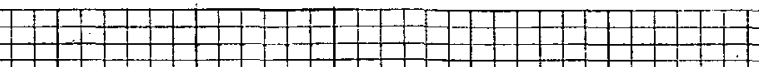

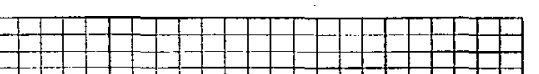

0

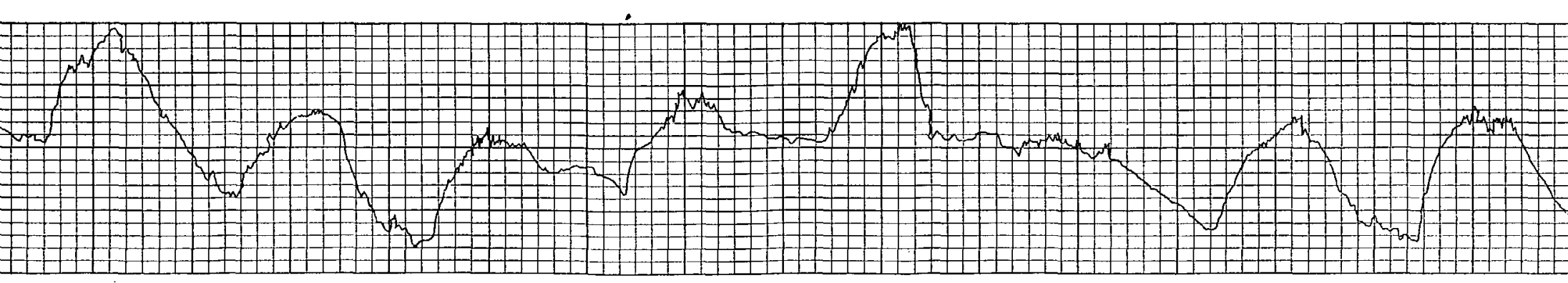



ARTIFICIAL CAUSES OF FLUGTUATION.

Dams.-The first important cause producing a change in the normal level of the ground-water table was the construction of dams for mill purposes. These, without exception, raised the ground-water table and decreased the spring flow in the valley above the points at which they were constructed (see p. 62). The crest flow in every case was less than the normal flow of the stream at the same point. The enlargement of these ponds for storage purposes by the Brooklyn waterworks but emphasized this condition.

Pumping.- - When pumping stations were established a diametrically opposite effect was produced. A pumping station instead of hindering the outward flow of the water helps it, and as the group of wells connected with a pumping station is usually restricted to a relatively small area, a more or less symmetrical cone of depression is produced with the group of the wells as a center. All wells, springs, and ponds which depend on this main water table and which are in the radius of the cone of depression are directly influenced. As a result preexisting wells have had to be driven to a depth slightly greater than that of the new water table (fig. 36), the spring flow is decreased, and adjacent ponds and marsh areas are more or less completely drained. Mr. L. B. Ward has compiled the following table showing the decrease in stream flow on southern Long Island between 1873 and 1899, which must be largely due to the effect of the pumping stations:

TABLE VII.-The effect of ground-water pumping in diminishing stream flow from 1873 to 1899 in the old watershed of the Brooklyn waterworks, comparing five-year periods.

[By L. B. Ward.]

\begin{tabular}{|c|c|c|c|c|c|c|c|c|c|c|c|c|}
\hline \multirow{3}{*}{ Period. } & \multirow{3}{*}{$\begin{array}{l}\text { Aver- } \\
\text { age } \\
\text { annual } \\
\text { rain- } \\
\text { fall. }\end{array}$} & \multirow{3}{*}{\multicolumn{2}{|c|}{$\begin{array}{c}\text { A verage annual } \\
\text { rainfall col- } \\
\text { lected, referred } \\
\text { to watershed } \\
\text { as a whole. }\end{array}$}} & \multirow{3}{*}{$\begin{array}{c}\text { Area } \\
\text { of } \\
\text { water- } \\
\text { shed. }\end{array}$} & \multicolumn{2}{|c|}{$\begin{array}{l}\text { Driven-well } \\
\text { supply. }\end{array}$} & \multicolumn{2}{|c|}{$\begin{array}{l}\text { Other pumped } \\
\text { sourees of } \\
\text { supply. }\end{array}$} & \multirow{3}{*}{$\begin{array}{c}\text { Daily } \\
\text { total per } \\
\text { square } \\
\text { mile } \\
\text { derived } \\
\text { from all } \\
\text { sources in } \\
\text { therater. } \\
\text { shed. }\end{array}$} & \multicolumn{3}{|c|}{$\begin{array}{l}\text { Water collected as stream } \\
\text { flow, referred to } 50 \text { square } \\
\text { miles of watershed. }\end{array}$} \\
\hline & & & & & Ex- & Daily per & $\underset{\text { Dressed }}{\mathbf{E x}}$ & Daily per & & Daily per & Expresse & $\begin{array}{l}\mathrm{d} \text { as rain- } \\
\mathrm{ll} \text {. }\end{array}$ \\
\hline & & & & & $\begin{array}{l}\text { as rain- } \\
\text { fall. }\end{array}$ & $\begin{array}{l}\text { square } \\
\text { mile. }\end{array}$ & $\begin{array}{c}\text { as rain } \\
\text { fall. }\end{array}$ & $\begin{array}{l}\text { square } \\
\text { mile. }\end{array}$ & & $\begin{array}{c}\text { square } \\
\text { mile. }\end{array}$ & Amount. & $\begin{array}{l}\text { Propor- } \\
\text { tion of } \\
\text { total. }\end{array}$ \\
\hline $1873-1877$. & $\begin{array}{c}\text { Inches. } \\
43.33\end{array}$ & $\begin{array}{l}\text { Per } \\
\text { cent. } \\
25.07\end{array}$ & $\begin{array}{c}\text { Inches. } \\
10.86\end{array}$ & $\begin{array}{l}\text { Square } \\
\text { miles. } \\
52.30\end{array}$ & $\begin{array}{c}\text { Inches. } \\
\text { (a) }\end{array}$ & $\begin{array}{l}\text { Gallons. } \\
\quad(\alpha)\end{array}$ & $\begin{array}{r}\text { Inches. } \\
0.18\end{array}$ & $\begin{array}{r}\text { Gallons. } \\
8.659\end{array}$ & $\begin{array}{r}\text { Gallons. } \\
517,206\end{array}$ & $\begin{array}{r}\text { Gallens. } \\
532.034\end{array}$ & $\begin{array}{r}\text { Inches. } \\
11.17\end{array}$ & Percent. \\
\hline $1878-1882$ & 41.58 & 29.60 & 12.31 & 55.14 & (a) & (a) & .99 & 47,063 & 585,978 & 594,310 & 12.48 & 30.02 \\
\hline $1883-1887$. & 43.30 & 31.60 & 13.68 & 64.42 & 2.95 & 140,392 & 2.30 & 109,041 & 651,506 & 518,071 & 10.88 & 25.13 \\
\hline $1889-1893 \ldots$ & 45.05 & 38.43 & 17.31 & 65.54 & 5.85 & 278,383 & 4.17 & 198,605 & 824,195 & 455,153 & 9.56 & 21.22 \\
\hline $1895-1899$. & 43.14 & 36.32 & 15.67 & 66.44 & 7.76 & 369,581 & 2.74 & 130,224 & 745,983 & 327,122 & 6.89 & 15.96 \\
\hline
\end{tabular}

a Began in 1883.

While a decrease in spring flow must follow any extensive method of removing the ground water in this region, it should be borne in mind that the cost of such a removal will probably be less than its collection from surface ponds and the subsequent filtration which must necessarily follow. A subterranean system will, moreover, result in the more or less complete reclamation of the swampy lands along many of the brooks.

The effect which the lowering of the ground-water table by a few feet in this region may have on farm products is not very clear. It is certainly true that 
plants thrive where the ground-water table is 25 to 100 . or more feet below the surface, and it is difficult to see how the lowering of the water table a few feet will very definitely affect farm products, except where it makes swamp land cultivable.

\section{BLOWING WELLS.}

Mr. William Jaegle, a well driller of Hicksville, reports a number of blowing wells about Woodbury $(519,588,589,590)$. These blow intermittently, generally before a storm, and are clearly very similar to the blowing wells reported from the Western States."

The cause of this blowing seems to a large extent to be due to changes in barometric pressure, an outflow of air occurring when the surface pressure is relatively low, and an inflow when it is relatively high.

A careful examination was made of the wells at Woodbury by Mr. R. D. Rathbun, field assistant, with a view to attaching a recording instrument and carefully studying this phenomenon, but the conditions were found not to be favorable.

\section{WATERWORKS.}

The porous nature of Long Island, which causes it to readily absorb, filter, and store the rain water, admirably fits it for furnishing large quantities of very pure water.

As has been pointed out, the total loss by evaporation is relatively small, and the run-off is almost wholly that supplied by springs. These short, steadyflowing, spring-fed streams, which were first utilized for small saw and grist mills, were the most natural source for water when the growing city of Brooklyn began to demand a water supply.

The original Brooklyn system, completed in 1862, derived its supply wholly from a number of surface streams between Brooklyn and Lynbrook, which were intercepted by a conduit in which the water flowed by gravity to Ridgewood, where it was lifted into reservoirs which supply a simple gravity system. As the demand increased, it became necessary to utilize other ponds and streams which were too low to flow naturally into the conduit, and in 1872 pumping stations were established at Watts Pond and Smiths Pond.

In the same year a private system supplied by springs was established at Sea Cliff. This was the first waterworks plant on Long Island after the Brooklyn system. In 1874 plants were completed by three villages: College Point, Flushing, and Long Island City; of these, the first two depended on spring and stream supply, - and the last on a single large well. This last was the first plant using the ground water as a source of supply. Garden City followed in a few years with a system depending on a single large well.

In 1880 the surface supply of the Brooklyn waterworks was supplemented by open-well stations at Springfield and Watts Pond, and in 1882 gang-well stations were established at Spring Creek and Baisleys. Since that time the

a Water-Sup. and Irr. Paper No. 67, U. S. Geol: Survey, 1902, pp. 72, 73; Nebraska Geol. Survey, vol. 1, 1903, pp. 93-97; Water-Sup. and Irr. Paper, No. 101, U. S. Geol. Survey, 1904, pp. 60-61. 
development of the ground water has been comparatively rapid; many local plants have been erected, which, with scarcely an exception, depend on wells. Of the plants of the five cities-Brooklyn, College Point, Flushing, Sea Cliff, and Northportwhich originally depended largely on surface water, the last three now depend wholly on wells. The plans for the change of the College Point (Fresh Meadow station) to a driven-well plant have been approved, and Brooklyn has so supplemented her supply by driven-well stations that at present only about two-thirds of the supply is derived from surface waters. At Sag Harbor it has been found advisable to abandon the wells, and the plant there is now the only one on the island wholly dependent on a surface supply.

The amount of water taken from Long Island for waterworks purposes during 1902 may be roughly estimated at $120,000,000$ gallons per day, of which $65,000,000$ was from springs or spring-fed streams and 55,000,000 was from wells. The Brooklyn Water Company consumed almost the whole of the surface water utilized and-slightly more, than 50 per cent of the well water.

The distribution of the various water systems on Long Island, the area covered by each, and the location of the pumping stations and other sources of supply, are shown on the accompanying map (Pl.XIX). Other data are presented in the following table and in the detailed records given on pages 116-337.

17116-No. $44-06-6$ 
TABLe VIII.-Waterworks

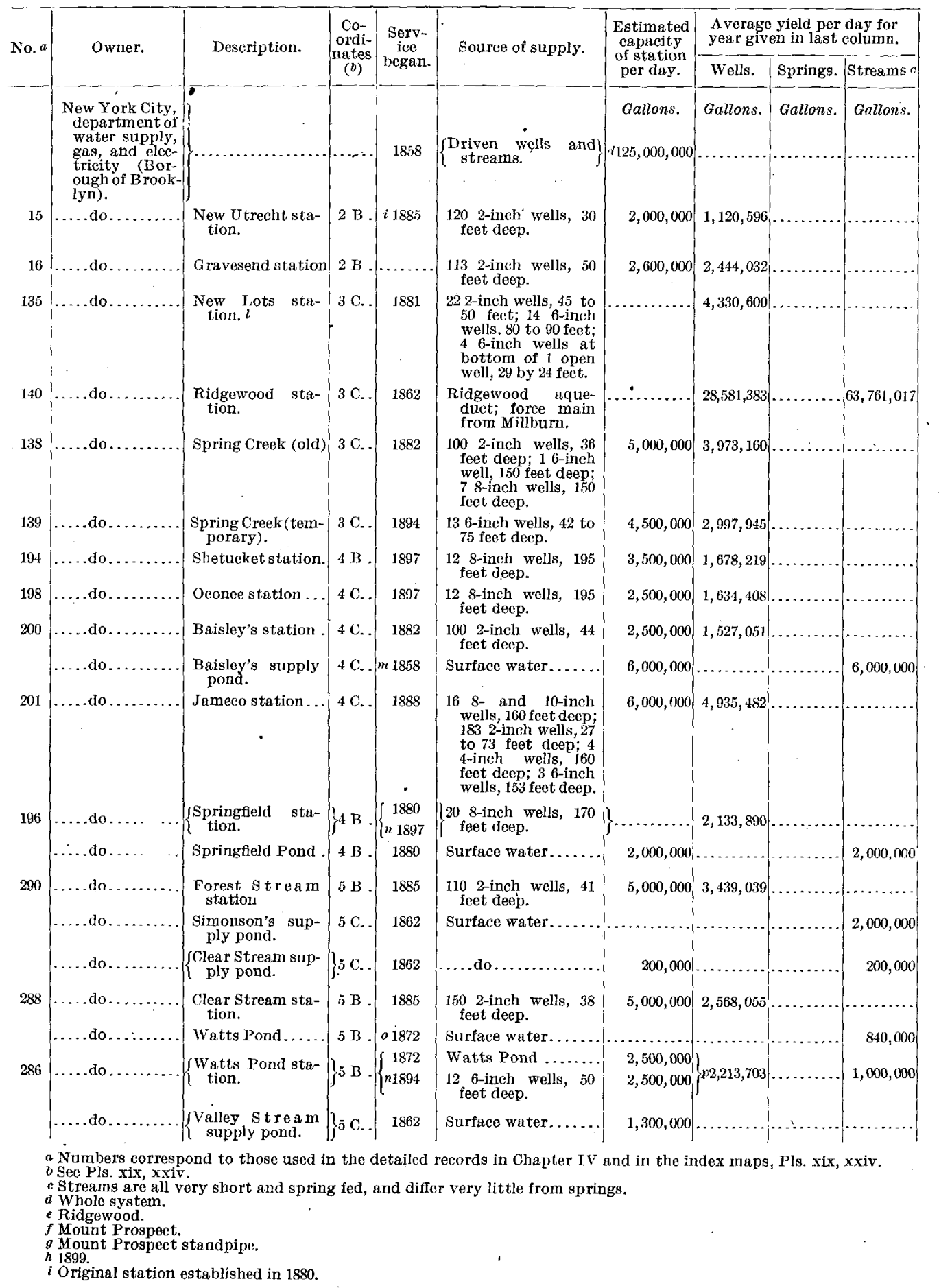


systems on Long Island.

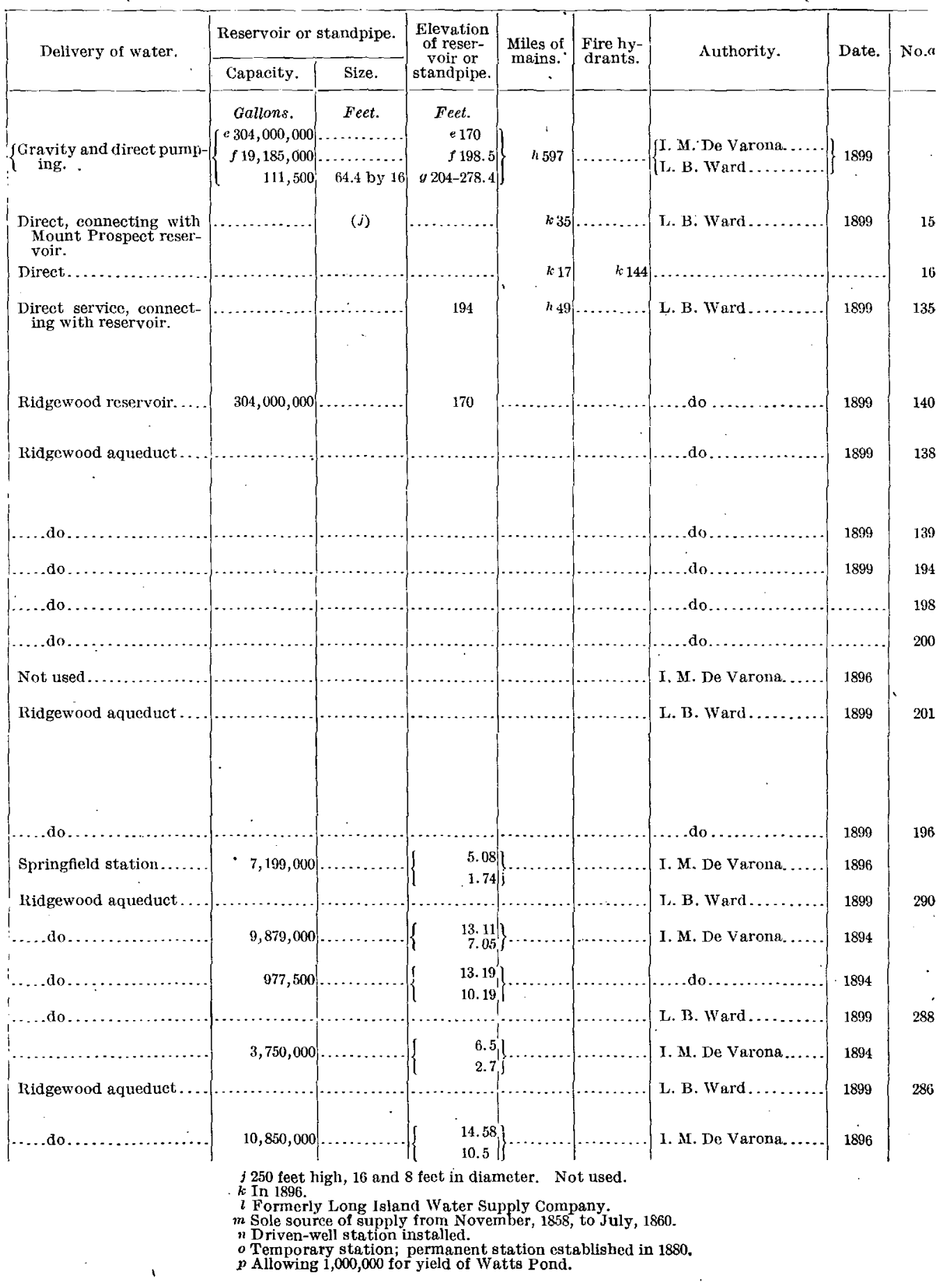


TABLE VIII.-Waterworks

\begin{tabular}{|c|c|c|c|c|c|c|c|c|c|}
\hline \multirow{2}{*}{ No. $a$} & \multirow{2}{*}{ Owner. } & \multirow{2}{*}{ Description. } & \multirow{2}{*}{$\left|\begin{array}{c}\text { Co- } \\
\text { ordi- } \\
\text { nates } \\
(b)\end{array}\right|$} & \multirow{2}{*}{$\begin{array}{l}\text { Serv- } \\
\text { ice } \\
\text { began. }\end{array}$} & \multirow{2}{*}{ Source of supply. } & \multirow{2}{*}{$\begin{array}{l}\text { Hstimated } \\
\text { capacity } \\
\text { of station } \\
\text { per day. }\end{array}$} & \multicolumn{3}{|c|}{$\begin{array}{l}\text { Average yield per day for } \\
\text { year given in last column. }\end{array}$} \\
\hline & & & & & & & Wells. & Springs. & Streams $c$ \\
\hline & $\begin{array}{l}\text { New York City, } \\
\text { department of } \\
\text { water supply, } \\
\text { gas, and elec- } \\
\text { tricity (Bor- } \\
\text { ough of Brook- } \\
\text { lyn). }\end{array}$ & Smiths Pond ..... & $6 \mathrm{~B}$. & 1872 & Surface water... & $\begin{array}{l}\text { Gallons. } \\
4,500,000\end{array}$ & Gallons. & Gallons. & Gallons. \\
\hline & . do.... & $\left\{\begin{array}{c}\text { Smiths Pond sta- } \\
\text { tion. }\end{array}\right.$ & \}$_{6 \mathrm{~B}}$ & 1872 & Smiths Pond ... & & & & $8,517,299$ \\
\hline & ..do. & $\left\{\begin{array}{c}\text { Pine's } \\
\text { pond. }\end{array}\right.$ & $36 \mathrm{c}$. & 1862 & Surface water... & 600,000 & & & \\
\hline & .... do. do. & $\begin{array}{l}\text { Schodack Brook. } \\
\text { Hempstead sup- } \\
\text { ply pond. }\end{array}$ & $6 \mathrm{C}$. & 1873 & . do .... & $1,000,000$ & & & \\
\hline & $\ldots$ do... & $\begin{array}{c}\text { Hempstead stor- } \\
\text { age reservoir. }\end{array}$ & $6 \mathrm{C}$. & & & $8,000,000$ & & & $8,000,000$ \\
\hline & ...do.... & Millburn reservoir & $6 \mathrm{~B}$ & & $\begin{array}{l}\text { Strearns, Millburn to } \\
\text { Massapequa. }\end{array}$ & & & & $30,450,000$ \\
\hline & . . do.. & $\left\{\begin{array}{l}\text { Millbum pump- } \\
\text { ing station. }\end{array}\right.$ & $66 \mathrm{~B}$ & & $\left\{\begin{array}{l}\text { Streams, Millburn to } \\
\text { Massapequa, und } \\
\text { driven-well sta- } \\
\text { tions given below. }\end{array}\right.$ & - & $3,114,739$ & & $36,974.474$ \\
\hline & $\ldots 10$. & $\left\{\begin{array}{c}\text { Millburn supply } \\
\text { pond. }\end{array}\right.$ & $6 \mathrm{~B}$ & 1892 & Surface .............. & & & & \\
\hline & . do... & $\left\{\begin{array}{cc}\text { East } & \text { Meadow } \\
\text { supply pond. }\end{array}\right\}$ & 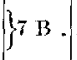 & & ... do. & & & & \\
\hline 487 & ..... do...... & Agawam station.. & $7 \mathrm{~B}$ & 1896 & $\begin{array}{l}32 \text { 6-inch wells, } 33 \text { to } \\
91 \text { feet deep. }\end{array}$ & $d 4,518,951$ & 520,305 & & \\
\hline 489 & . do & Merrick station. & $7 \mathrm{~B}$ & 1896 & $\begin{array}{l}624 \frac{1}{2} \text {-inch wells, } 40 \text { to } \\
100 \text { feet deep. }\end{array}$ & $d 4,693,432$ & 325,813 & & \\
\hline & ..... do. & $\left\{\begin{array}{c}\text { Matowa } \quad \text { (new } \\
\text { bridge) } \\
\text { pond. }\end{array}\right.$ & $7 \mathrm{BC}$ & 1892 & surface............. & & & & \\
\hline 491 & .....do.... & Matowa station. . & $7 \mathrm{~B}$ & 1896 & $\begin{array}{l}4641 \text {-inch wells, } 38 \text { to } \\
97 \text { feet deep. }\end{array}$ & $d 4,495,622$ & 890,939 & & \\
\hline & . do... & $\left\{\begin{array}{l}\text { Wantagh supply } \\
\text { pond. }\end{array}\right.$ & $7 \mathrm{C}$. & 1892 & Surface............ & & & & \\
\hline & $\ldots$ do... & $\left\{\begin{array}{l}\text { Seaman's supply } \\
\text { pond. }\end{array}\right.$ & \} 7 C.. & 1892 & $\ldots$.do. & & & & \\
\hline 493 & $\ldots$ do... & Wantagh station. & $7 \mathrm{C.}$. & 1896 & $\begin{array}{l}434 \text {-inch wells, } 24 \text { to } \\
89 \text { fcet deep; } 66- \\
\text { inch wells, } 92 \text { feet } \\
\text { deep. }\end{array}$ & $a 3,998,844$ & $1,37^{7} 682$ & & \\
\hline & -...do... & $\left\{\begin{array}{l}\text { Massapequa sup- } \\
\text { ply pond. }\end{array}\right.$ & $7 \mathrm{C}$. & 1893 & Surface............. & & & & \\
\hline 567 & .....do..... & $\begin{array}{l}\text { Massapequa sta- } \\
\text { tion. }\end{array}$ & $8 \mathrm{C.}$. & 1896 & $\begin{array}{l}534 \frac{1}{2} \text {-inch wells, } 37 \text { to } \\
106 \text { feet deep. }\end{array}$ & $d 5,373,196$ & (e) & & \\
\hline 81 & $\begin{array}{l}\text { New York City, } \\
\text { department of } \\
\text { water supply, } \\
\text { gas, and elec- } \\
\text { tricity (Iror- } \\
\text { o u g h of } \\
\text { Queens). }\end{array}$ & $\begin{array}{c}\text { Long Isiand City, } \\
\text { station No, } 1 .\end{array}$ & $2 \mathrm{C}$. & 1874 & $\begin{array}{l}76 \text {-inch welis, } 70 \text { feet } \\
\text { deep; } 1 \text { open well, } \\
47_{2}^{\frac{1}{2}} \text { fect diameter } \\
\text { by } 30 \text { feet deep. }\end{array}$ & & 682,800 & & \\
\hline 175 & ....do......... & $\begin{array}{l}\text { Long Island City, } \\
\text { station No. } 2 .\end{array}$ & $3 \mathrm{D}$ & 1886 & $\begin{array}{l}28 \text { 4-inch wells, } 15 \text { feet } \\
\text { deep; } 1 \text { i } 6 \text {-foot } \\
\text { well, } 22 \text { fcet deep. }\end{array}$ & & $f 803,000$ & & \\
\hline 99 & .....do.......... & $\begin{array}{l}\text { Long Island City, } \\
\text { station No. } 3 .\end{array}$ & $3 D$. & 1894 & $\begin{array}{l}\text { 12 4-inch wells, } 41 \text { feet } \\
\text { deep. }\end{array}$ & & 621,000 & & \\
\hline 231 & $\ldots \mathrm{do}$. & $\left\{\begin{array}{c}\text { Fresh Me a d ow } \\
\text { Station (for- } \\
\text { merly college } \\
\text { Point station). }\end{array}\right\}$ & $\int 4 \mathrm{C}$. & 1874 & Springs ........ & $2,500,000$ & & $\begin{array}{c}970,789 \\
622,700\end{array}$ & \\
\hline
\end{tabular}

a Numbers correspond to those used in the detailed records in Chapter IV and.in the index maps, Pls. xix, xxiv. $u$ See Pls. xix, xxiv.

$c$ Streams are very short and spring fed, and differ very little from springs.

d Average daily yield for test of July-December, 1896. 
systems on Long Island-Continued.

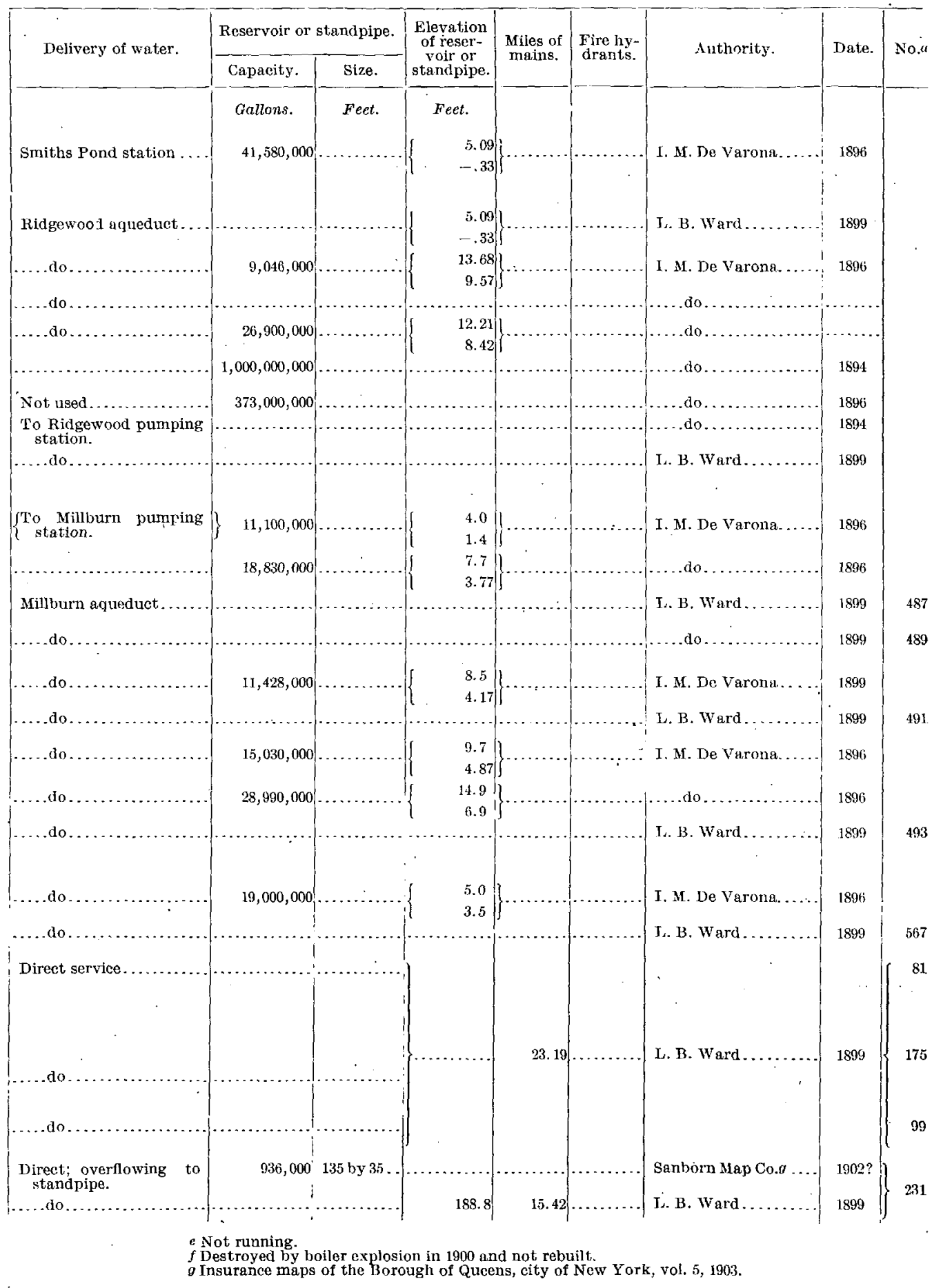


TABLE VIII.-Watemworks

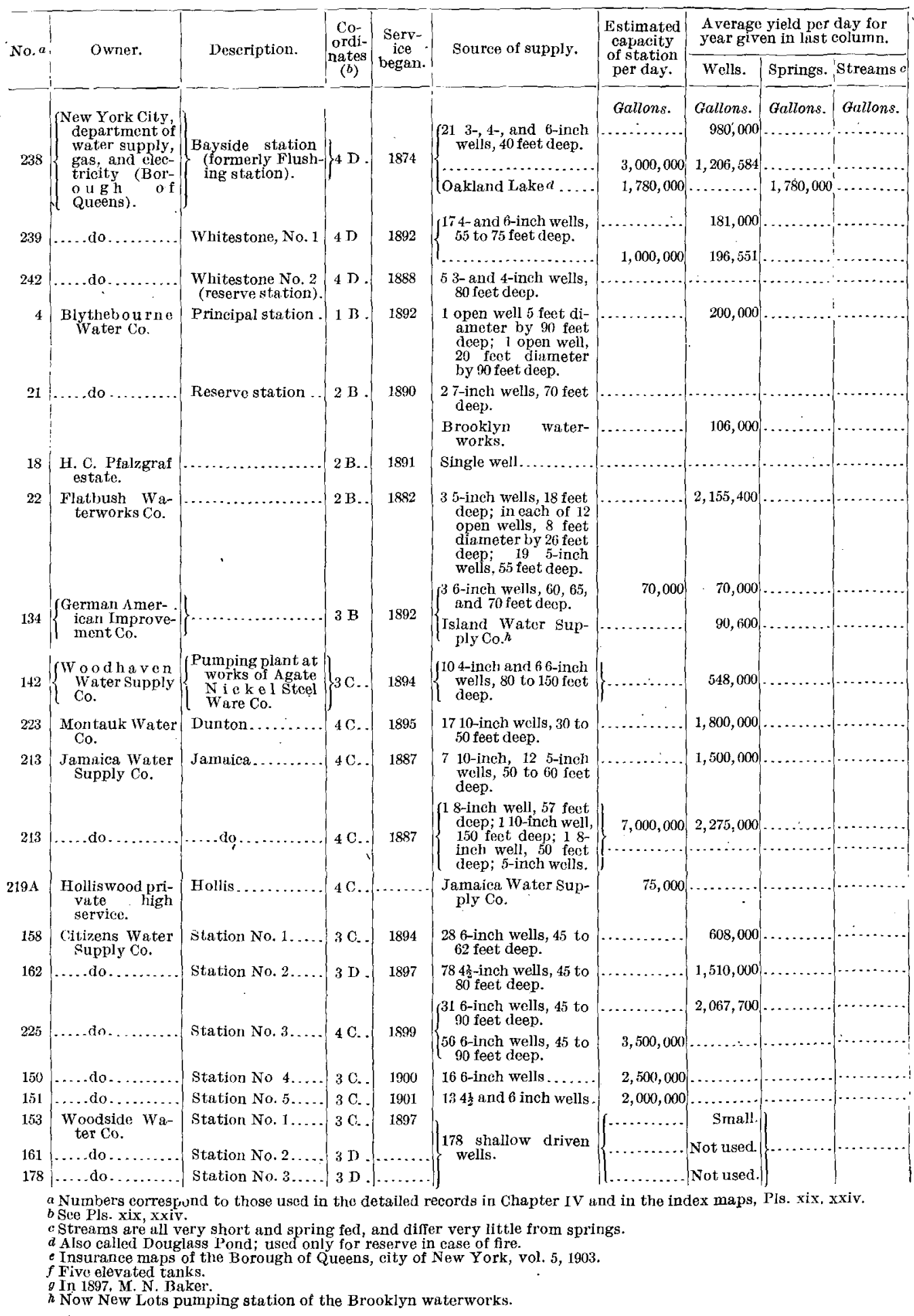


systems on Long Island-Continued.

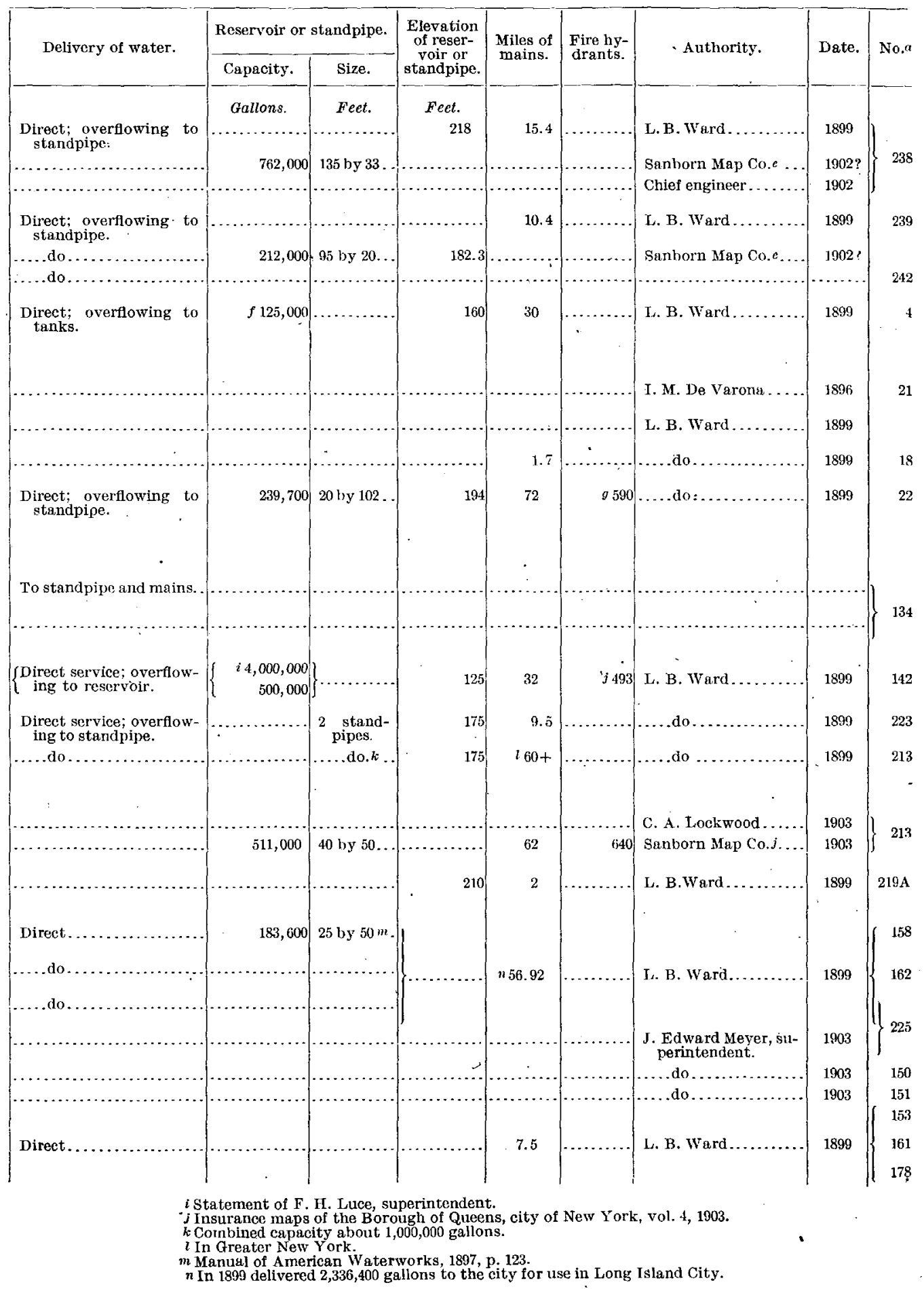


TABLE III.-Waterworks

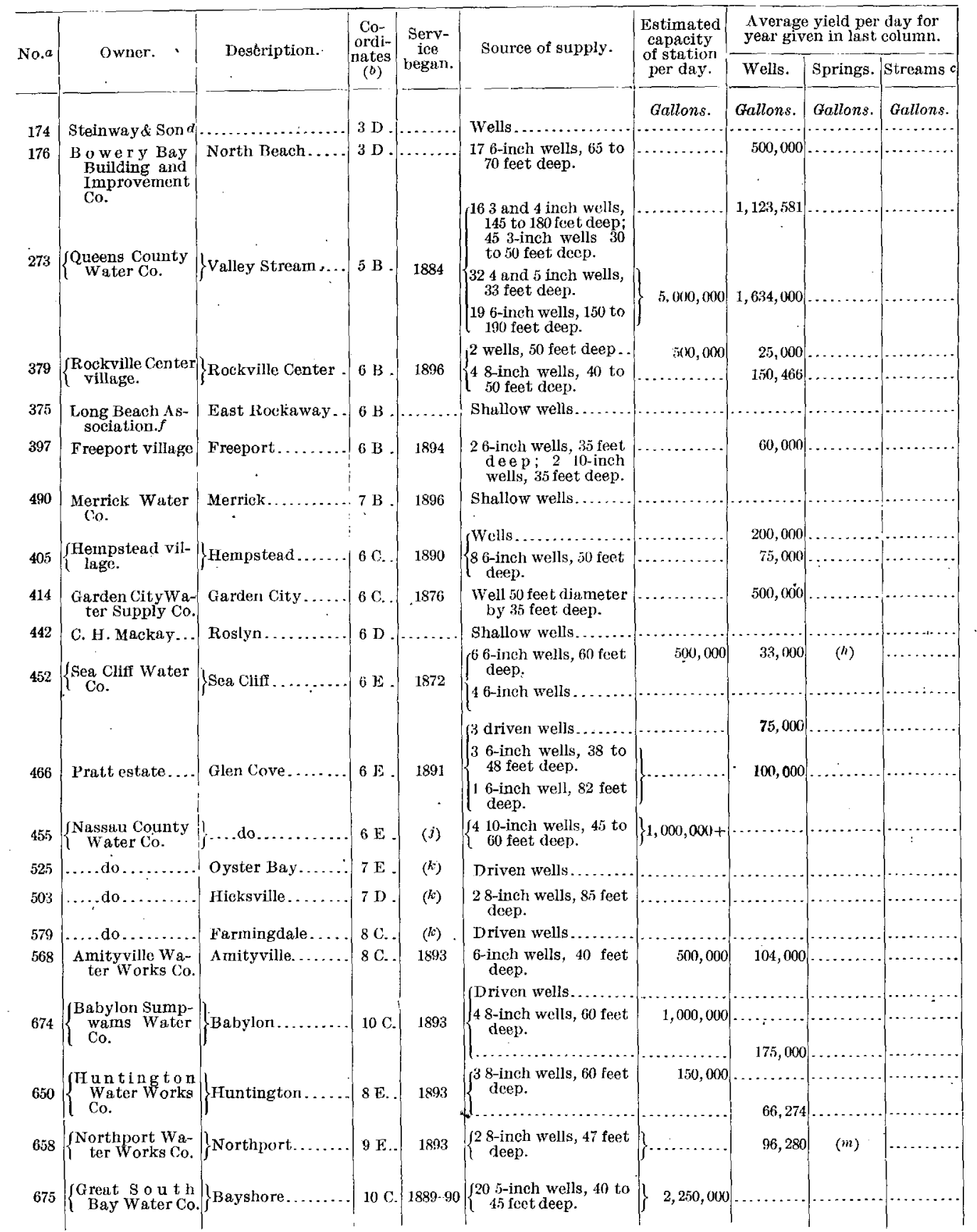

a Numbers correspond to those used in the detailed records in Chapter IV and in the index maps, PIs. xix, xxiv. $b$ See Pls. Xix, xxiv.

$c$ Streams are very short and spring fed, and differ very little from springs.

dA small private plant supplying houses in the vicinity of the Steinway piano factory.

$e$ Two standpipes.

$f$ Private plant supplying Long Beach.

g Manual of American Waterworks, 1897 
systems on Long Island-Continued.

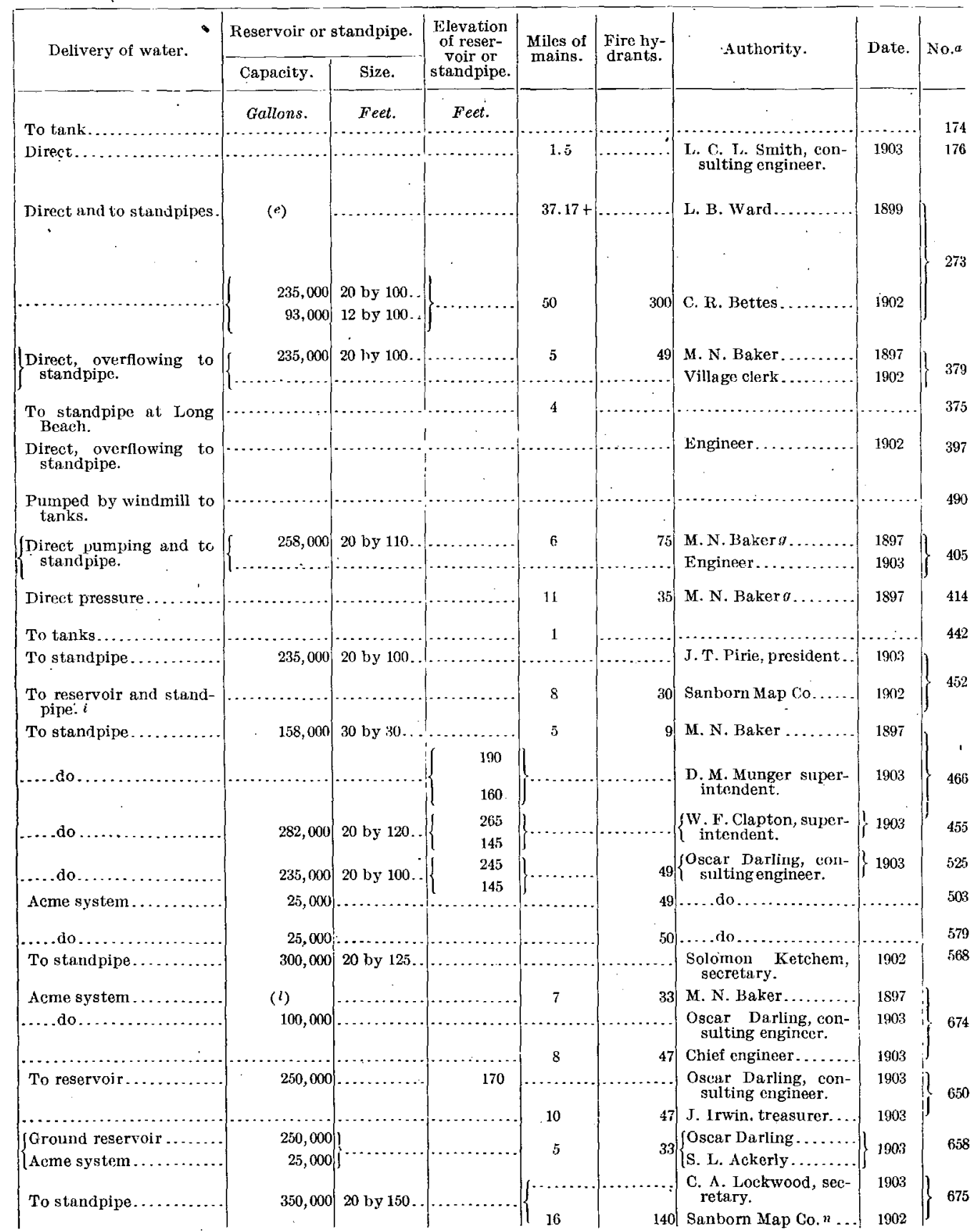

$h$ Originally supplied by springs.

$i$ Reservoir, 120,000 gallons; elevation, 175 feet; standpipe, 235,000 gallons; elevation, 250 feet.

$j$ Construction well advanced in September, 1903 .

$k$ Under construction September, 1903.

$i$ Two 7,000-gallon tanks.

$m$ The springs which formerly supolied this plant were abandoned in 1903 .

$n$ Maps of Bayshore and Islip. 
84 UNDERGROUND WATER RESOUROES OF LONG ISLAND, NEW YORK.

TABLE III-Watemorks

\begin{tabular}{|c|c|c|c|c|c|c|c|c|c|}
\hline \multirow{2}{*}{ No.a } & \multirow{2}{*}{ Owner. } & \multirow{2}{*}{ Description. } & \multirow{2}{*}{$\begin{array}{c}\text { Co- } \\
\text { ordi- } \\
\text { nates } \\
(b)\end{array}$} & \multirow{2}{*}{$\begin{array}{l}\text { Serv- } \\
\text { ice } \\
\text { began. }\end{array}$} & \multirow{2}{*}{ Source of supply. } & \multirow{2}{*}{$\begin{array}{c}\text { Estirnated } \\
\text { capacity } \\
\text { of station } \\
\text { per day. }\end{array}$} & \multicolumn{3}{|c|}{$\begin{array}{l}\text { Average yield per day for } \\
\text { year given in last colimn. }\end{array}$} \\
\hline & & & & & & & Wells. & Springs. & Streams $c$ \\
\hline & & & & & $\cdot$ & Gallons. & Gallons. & Gallons. & Gallons. \\
\hline 777 & $\begin{array}{c}\text { Great South } \\
\text { Bay WaterCo.d }\end{array}$ & Patehogue : - & $13 \mathrm{D}$ & 1887 & & & & & \\
\hline 803 & $\begin{array}{l}\text { Port Jefferson } \\
\text { Water Co. }\end{array}$ & Port Jefferson. & $11 \mathrm{~F}$ & 1898 & $\begin{array}{l}\text { 26-inch wells, } 54 \text { feet } \\
\text { deep. }\end{array}$ & 200,000 & 80,000 & & \\
\hline 863 & $\left\{\begin{array}{c}\text { Riverhead Wa- } \\
\text { ter Works. }\end{array}\right.$ & Riverhead:.. & $18 \mathrm{E}$ & 1892 & $\left\{\begin{array}{l}1 \text { 8-inch well, } 225 \text { foet } \\
\text { deep; } 16 \text {-inch well, } \\
305 \text { feet deep.e }\end{array}\right.$ & & 6,000 & & \\
\hline $86 t$ & $\begin{array}{l}\text { Quantuck Wa- } \\
\text { ter Co. }\end{array}$ & Quogue.. & $18 \mathrm{D}$ & g 1903 & $\begin{array}{l}68 \text {-inch wells, } 40 \text { feet } \\
\text { deep. }\end{array}$ & 500,000 & & & \\
\hline 879 & $\begin{array}{l}\text { Southampton } \\
\text { Waterworks } \\
\text { Co. }\end{array}$ & Southampton - & $21 \mathrm{E}$ & 1894 & $\begin{array}{l}\text { 36-inch wells, } 80 \text { feet } \\
\text { deep. }\end{array}$ & $1,000,000$ & 340,500 & & \\
\hline 910 & $\begin{array}{l}\text { Iasthampton } \\
\text { IIome Water } \\
\text { Co. }\end{array}$ & Easthampton. & $23 \mathrm{~F}$ & 1899 & $\begin{array}{l}\text { 34-ineh wells, } 70 \text { to } 75 \\
\text { feet deep. }\end{array}$ & 250,000 & i 250,000 & & \\
\hline 903 & $\underset{\text { Sag I arbor }}{\text { Water }}$ & Sag Harbor ... & $22 \mathrm{~F}$ & 1889 & Ligonee Brook ...... & & $(j)$ & & 150,000 \\
\hline 889 & $\begin{array}{l}\text { Shelter Island } \\
\text { IIeights Asso- } \\
\text { ciation. }\end{array}$ & Shelter Island. & $21 \mathrm{H}$ & . & $\begin{array}{l}\text { Open well, } 21 \text { feet } \\
\text { deep, with } 6 \text {-inch } \\
\text { pipe to a depth of } \\
33 \text { feet. }\end{array}$ & 120,000 & $i 96,000$ & & \\
\hline 890 & $\begin{array}{l}\text { Manhassot } \\
\text { House. }\end{array}$ & ....do..... & $21 \mathrm{H}$ & & Group of 18 wells... & & $(l)$ & & \\
\hline 892. & $\begin{array}{l}\text { Greenport vil- } \\
\text { lage. }\end{array}$ & Greenport. & $21 \mathrm{H}$ & $m 1889$ & $\begin{array}{l}\text { 96-inch wells, } 28 \text { to } 48 \\
\text { feet deep. }\end{array}$ & & & & \\
\hline
\end{tabular}

a Numbers correspond to those used in the detailed records in Chapter IV and in the index maps, Pls. xix, xxiv.

c See Pls. xix, xxiv.

dUntil 1894 the Suffolk County Water Company.

e Statement of driller, N. W. Davis.

$f$ Pumped by water power.

o June 11903 
systems on Long Island-Continued.

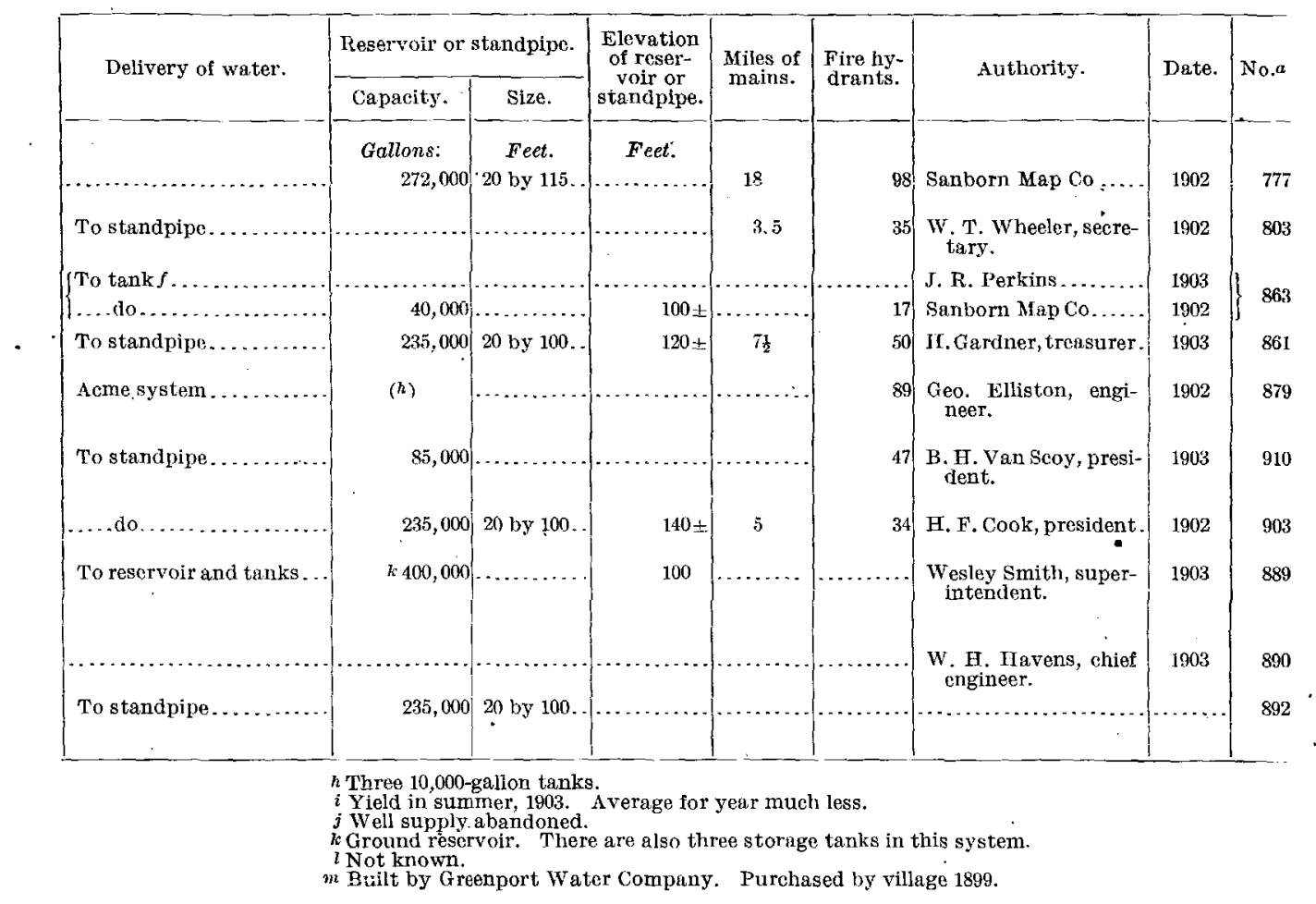




\title{
CHAPTER III.
}

\author{
MEASUREMEN'TS OF THE RA'TE OF UNDERFLOW ON LONG ISLAND. * \\ Bỳ Charles S. Stiohter. \\ DISTRICT INVESTIGATED.
}

The following determinations of ground-water velocities were made along the south side of Long Island, between the villages of Freeport and Massapequa. These places are located about 6 miles apart on the Montauk division of the Long Island Railroad, which between these points runs nearly east and west about 1 mile north of the edge of the extensive salt marshes which border the Atlantic Ocean. (See fig. 37.)

Freeport is about 24 miles from Brooklyn Bridge, and Massapequa, 6 miles east of Freeport, is within 2 miles of the western line of Suffolk County.

Within the 6-mile stretch above mentioned the city of Brooklyn has 5 pumping stations, drawing water from extensive batteries of driven wells. The names of these stations, from the west, are: Agawam, Merrick, Matowa, Wantagh, and Massapequa. A brick conduit on the north side of the right of way of the Long Island Railroad receives the water from the pumping station and carries it by gravity to a pumping station at Millburn, just west of Freeport, where an additional lift sends it into the city of Brooklyn.

Within the 6 miles from Freeport to Massapequa the conduit crosses several small surface streams, four of which have been ponded and their waters gated into the conduit. 'These surface waters flow into the conduit the year round, the driven wells constituting an auxiliary supply for the summer months, the period of use extending usually from July to December, but varying with the rainfall and other climatic conditions.

The particular district under discussion was selected as the object of study because, first, the region seemed typical of conditions on the south side of the island, and second, because the ground water was substantially in normal condition, owing to the fact that the driven-well plants had not been operated since the previous December. The purpose of the work was to determine the principal facts concerning the underground drainage of the island, so that a preliminary basis might be established from which an estimate of the amount of ground waters available for municipal supply could be made.

The determination of ground-water velocities was made at certain selected stations or localities, following in general an east-west line. The stations were 
restricted for the most part to the highways or other public lands, but this fact did not interfere materially with the selection of the best sites for the work. One set of stations was placed south of the railroad and just north of the line of wells of the driven-well stations, it being considered of importance to measure velocities in the immediate neighborhood of the pumping plants both before and after pumping had commenced. Other stations were located north of the railroad and conduit, out of range of any extensive influence of the pumping plants.

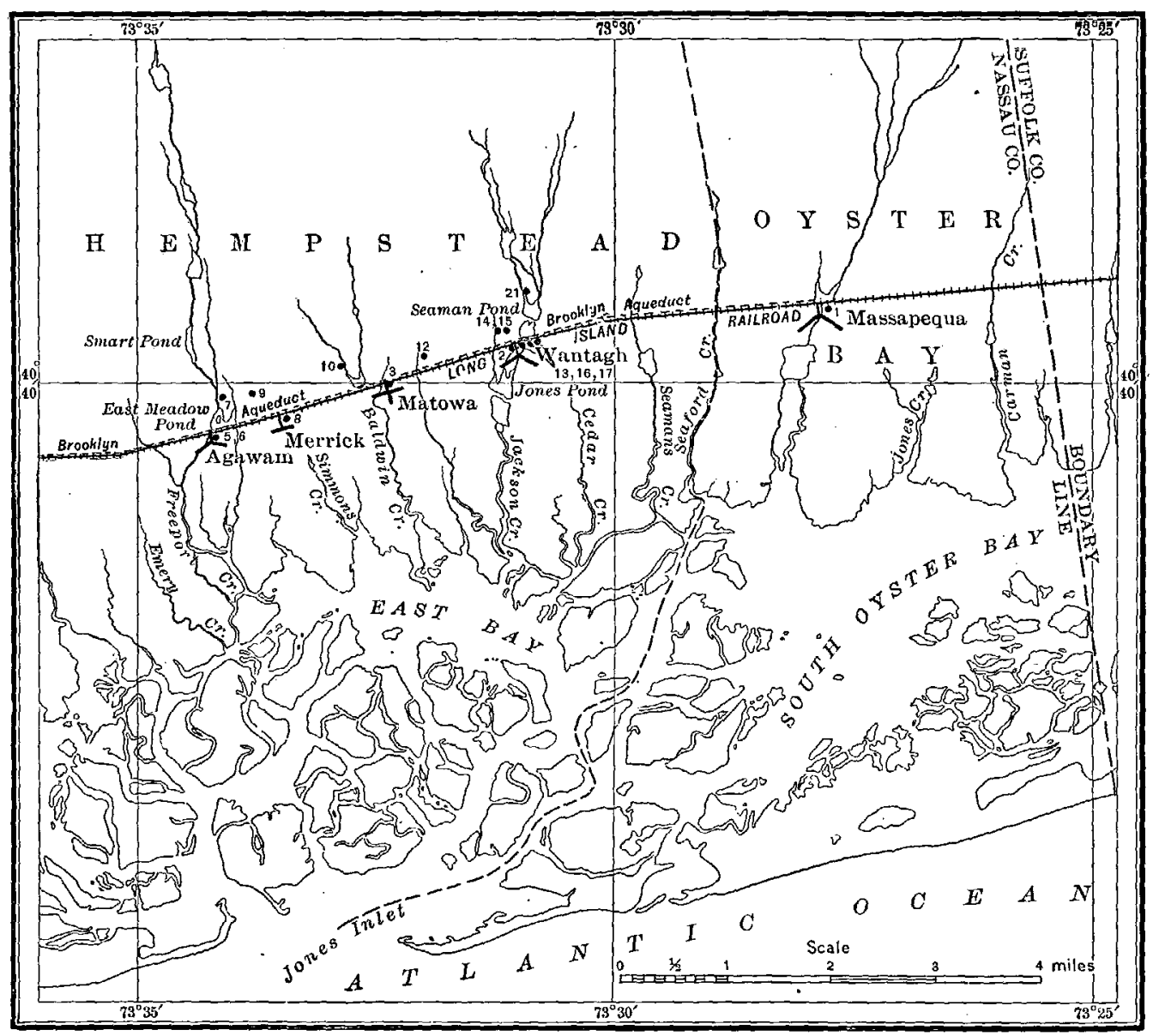

FIG. 37,-Map of southern Isong Island; showing location of underflow stations at which determinations of the rate of flow of underground water were made.

Measurements were made by the electrical method described by the writer in Engineering News for February 20, 1902, and in Water-Supply and Irrigation Paper No. 67 of the United States Geological Survey.

The test wells were driven by the commission on additional water supply, and the measurements were in charge of the writer and of Mr. Henry C. Wolff. 
APPARATUS USED.

The apparatus used comprised a series of test wells and various electrical devices for ascertaining the conditions that obtained in them.

TEST WELLS.

Test wells may be common $1 \frac{1}{1}$-inch or 2 -inch drive wells if the soil and waterbearing material is easily penetrated and if the depths desired to be reached do not exceed 30 or 40 feet; for greater depths and more difficult materials wells of heavier construction are necessary. The test wells put down by the commission on additional water supply for Greater New York in 1903 for the work described herewith are suitable for ordinary conditions as met with in the eastern part of the United States or in any place where the gravels are not too coarse or too compact. In them there was used full-weight standard wrought-iron 2-inch pipe

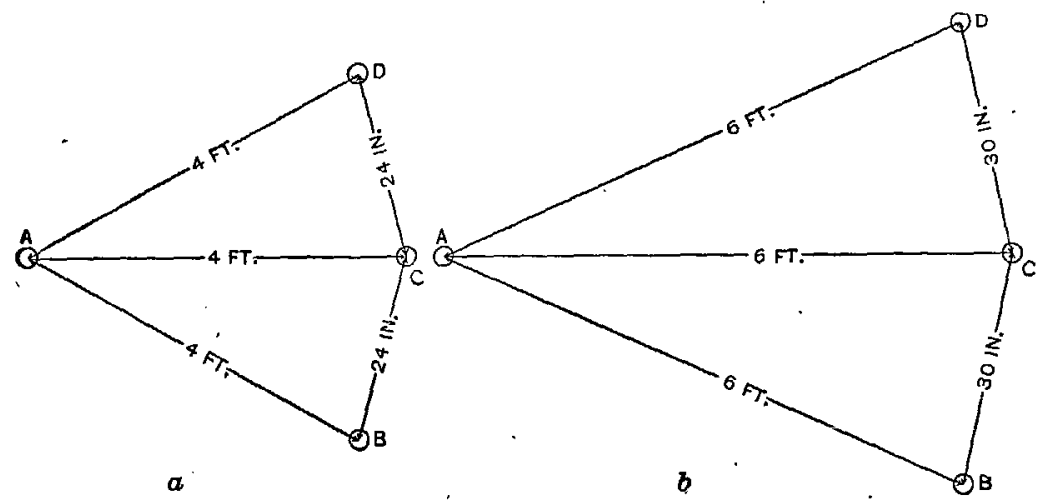

FIa. 38.-Plan of arrangement of test wells used in determining the veloeity and direction of motion of ground waters. $\mathrm{A}, \mathrm{B}, \mathrm{C}, \mathrm{D}$ are the test wells. The direction $\mathrm{A} \mathrm{C}$ is the direction of probable motion of the ground waters. The dimensions given in plan (a) are suitable for depths up to about 25 or 30 feet; those in plan $(b)$ for depths up to about 75 feet. For greater depths the distances A B, A C, A D, should be increased to 9 or 10 feet and the distances B C and C D to 4 feet. The well $A$ is the "salt well" or well in which the electrolyte is placed.

in lengths of 6 or 7 feet, with long threads ( $1 \frac{1}{2}$-inch) and heavy wrought nipples which could be screwed up until the ends of the pipe abutted.

The well points were 4 -foot standard brass jacket points, No. 60 gauze. For wells no deeper than 30 feet closed-end points were driven, but for deeper work open-end points were used. The test wells were driven in place by use of a ram from 150 to 250 pounds in weight, simultaneously hydraulicking a passage for the pipe with water jet in $\frac{3}{4}$-inch standard wash pipe. In fine material there were coupled ahead of the open-end well point 3 or 4 feet of pipe carrying a shoe coupling, so that the sand in running in through the open end of the pipe would not rise above the bottom of the screen inside of the finished well.

The test wells were grouped as shown in figure 38 .

In case the wells are not driven deeper than 25 feet, an "upstream" or "salt" well, $A$, is located, and three other wells, $B, C$, and D, are driven at a distance of 4 feet from $\mathrm{A}$, the distance between $\mathrm{B}$ and $\mathrm{C}$ and between $\mathrm{C}$ and $\mathrm{D}$ being about 2 feet. The well $\mathrm{C}$ is located so that the line from $\Lambda$ to $\mathrm{C}$ will coincide with the 
probable direction of the expected ground-water movement. This direction should coincide, of course, with the local slope of the water plane. For deeper work the wells should be located farther apart, as shown in the right portion of figure 38 . For depths exceeding 75 feet, a radius of 8 or 9 feet and chords of 4 feet should be used, the general requirement being that the wells should be as close together as possible, so as to cut down to a minimum the time required for

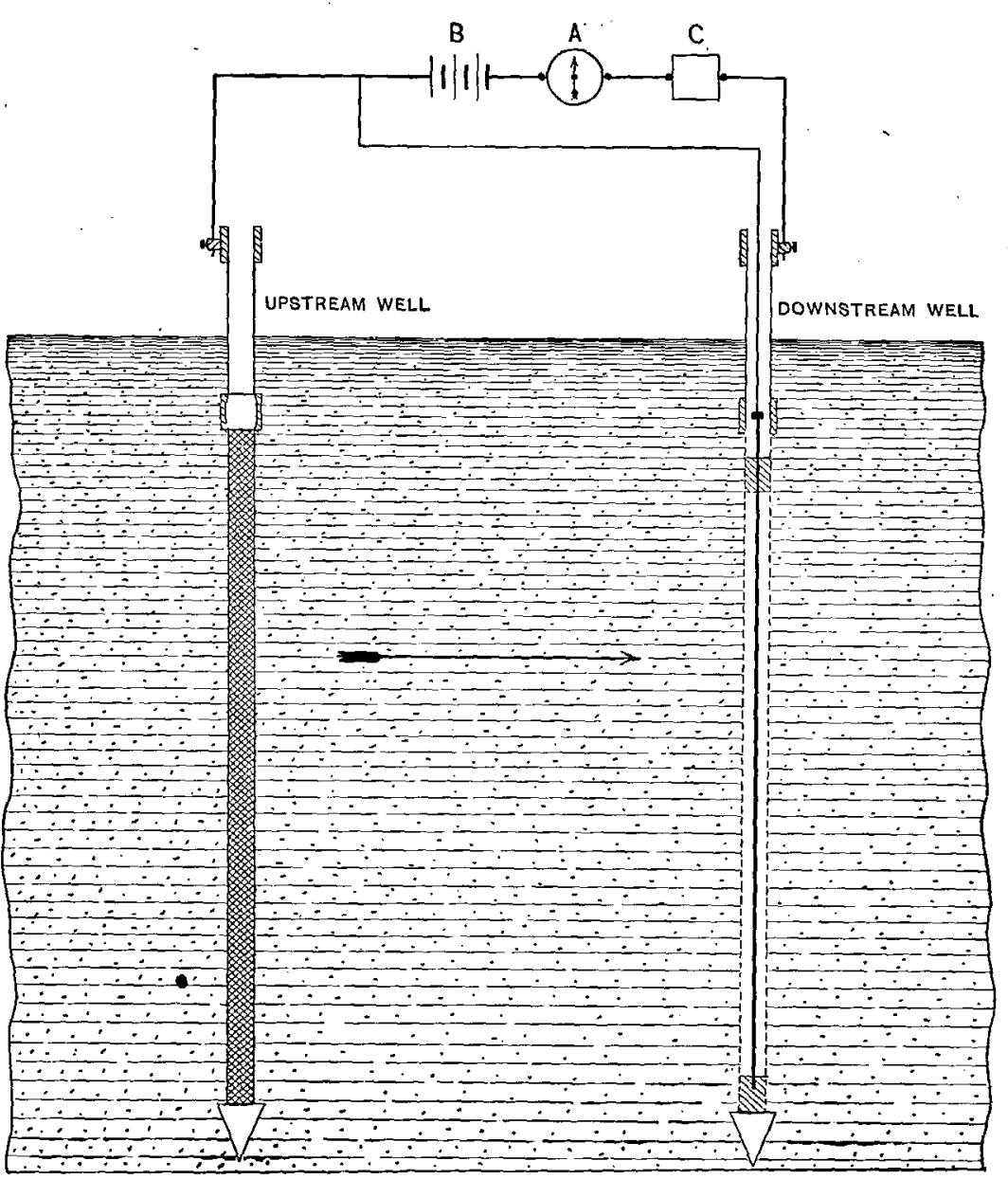

FIG. 39.-Diagram showing electrical method of determining the velocity of ground water. The ground water is supposed to be moving in the direction of the arrow. The upstream well is charged with an electrolyte. The gradual motion of the ground water toward the lower well and its final arrival at that well are registered by the ammeter $A$. $B$ is the battery and $\mathrm{C}$ a commutator clock which is used when $\mathrm{A}$ is a recording ammeter

a single measurement, but not so close that important errors are liable to be introduced from the inability to drive the wells perfectly straight and plumb. On this account, the deeper the wells the farther apart they should be placed. The angles $\mathrm{B} A \mathrm{C}$ and $\mathrm{C} \mathrm{A} \mathrm{D}$ should not exceed $30^{\circ}$.

Electrical connection is made with the casing of each test well by means of a drilled coupling carrying a binding post. Each of the downstream wells, B, C, D, 
contains within the well point or screen section an electrode consisting of a nickeled brass rod three-eighths inch by 4 feet, insulated from the casing by wooden spools. This electrode communicates with the surface by means of a rubber-covered copper wire. Fig. 39 illustrates the arrangement of electric circuits between the upstream well and one of the downstream wells. An electrode is shown in Pl. XX Each of the downstream wells is connected to the upstream well in the manner shown in that plate.

FORMS OF METERS.

The meters used were of two types: (1) Direct reading or hand, which required the personal presence of the operator every hour for reading, and (2) self-recording, which required attention but once a day.

DIREOT-READING METERS.

A photograph of the direct-reading underflow meter is shown in PI. XXI, $A$. Six standard dry cells are contained in the bottom of the box, their poles being connected to the 6 switches shown at the rear of the case. By means of these switches any number of the 6 cells may be thrown into the circuit in series. One side of the circuit terminates in 8 press keys, shown at the left end of the box. The other side of the circuit passes through an ammeter shown in the center of the box, to 2 three-way switches at right end of the box. Four of the binding posts at the left end of the box are connected to the casing of well $A$, and to the three electrodes of wells $\mathrm{B}, \mathrm{C}$, and $\mathrm{D}$, in order. The binding posts at the right end of the box are connected to the casings of wells $\mathrm{B}, \mathrm{C}$, and $\mathrm{D}$. There are enough binding posts so that two different groups of wells can be connected to the same instrument. When the three-way switch occupies the position shown in photograph, pressing the first key at left end of box will cause the ammeter to show the amount of current between casing of well $A$ and casing of well $B$. When the next key is pressed the ammeter will indicate the current between the casing of well $B$ and the electrode contained within it. In one case the current is conducted between the two well casings by means of the ground water in the soil; in the second case by means of the water within well B. By putting the three-way switch ir second position and pressing the first and the third keys in turn, similar readings can be had for the current between casings $\mathrm{A}$ and $\mathrm{C}$, and between casing $\mathrm{C}$ and its internal electrode. Similarly with the switch in the third position readings are taken by pressing the first and the fourth keys. The results may be entered in a notebook, as shown in Table IX, p. 95.

The electrolyte does not appear at one of the downstream wells with very great abruptness, but its appearance there is somewhat gradual, as shown in the curves in figs. 40 and 41 . The time required for the electrolyte to reach its maximum strength in one of the downstream wells (and, hence, for the current to reach its maximum value) may vary from a few minutes in a case of high ground-water. velocity to several hours in a case of low velocity. The writer formerly supposed that the gradual appearance of the electrolyte at the downstream well was largely due to the diffusion of the dissolved salt, but it is now evident that diffusion plays but a small part in the result. The principal cause of the phenomenon is now 
known to be the fact that the central thread of water in each capillary pore of the soil moves faster than the water at the walls of the capillary pore, just as the water near the central line of a river channel usually flows fastei than the water near the banks. For this reason, if the water of a river suddenly be made muddy at a certain upstream point, the muddy character of the water at a downstream point will appear somewhat gradually, being first brought down by the rapidły

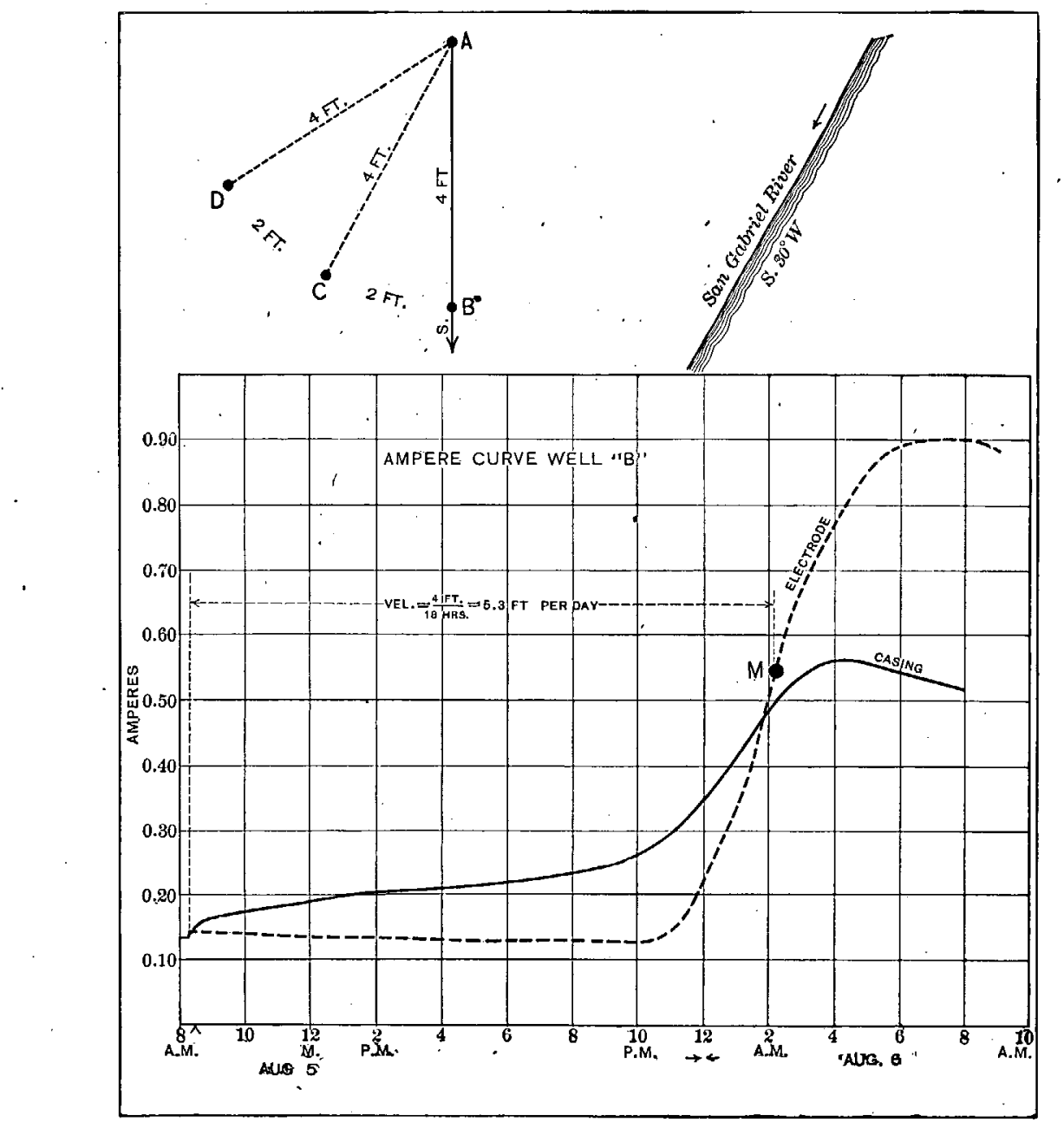

FIG. 40.-Curves showing electric current between casing of well A and casing of well $B$ (heavy curves), and between casing of well $B$ and its internal electrode (dotted curve) at station No. 5, San Gabriel River, California. These curves illustrate results made with the hand form of apparatus.

moving water in the center of the channel, and later by the more slowly moving water near the banks. The effect of the analogous gradual rise in the electrolyte in the downstream well requires us" to select the "point of inflection" of the curve of electric current as the proper point to determine the true time at which the 17116-No. 44-06-7 
arrival of the electrolyte should be counted. This point is designated by the letter " $M$ " in figs. 40 and 41.

Owing to the repeated branching and subdivision of the capillary pores around the grains of the sand or gravel, the stream of electrolyte issuing from the well will gradually broaden as it passes downstream. The actual width of this charged water varies somewhat with the velocity of the ground water, but in no case is the rate of the divergence very great. Figures 42 and 43 show some actual determinations of the spread of the electrolyte around a well in a coarse sand, in one case the ground water moving 12 feet per day, and in the other case moving 23 feet per day. Samples of ground water were taken from small test wells placed only 6 inches apart, and the amount of salt or electrolyte was determined chem-

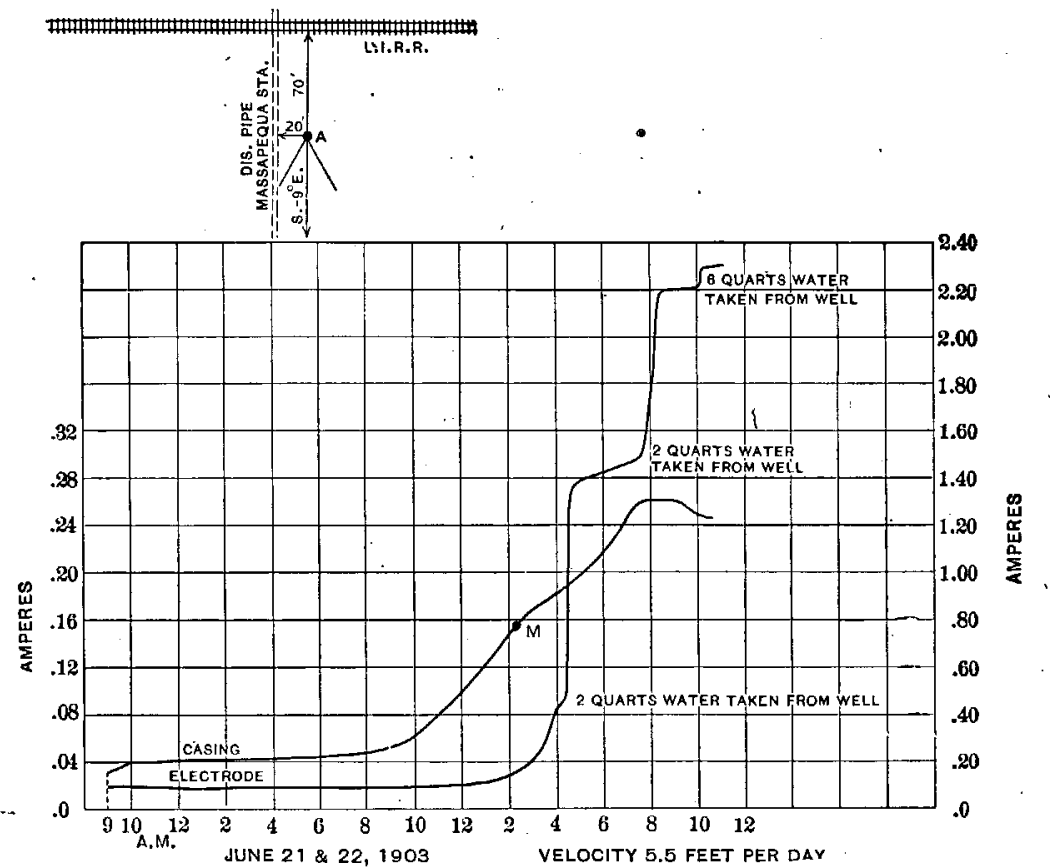

FIG. 41.-Curves showing possibility of using direct-reading apparatus when well points are not used. The casing in this instance consisted of common black 2-inch pipe, with a few small holes in bottom section. The "casing" curve must be relied upon for determining velocity. The "electrode" curve was obtained by drawing water from well $\mathrm{C}$, as shown on diagram, the charged water penetrating the well through small holes and the open end of well.

This diagram shows the velocity and direction of flow of underground water at Massapequa, L. I., Station No. I. Velocity 5.5 feet a day, S. $10^{\circ} \mathrm{E}$.

ically. The amount at any point is indicated by the area of the circles shown in the diagrams. It will be seen that the salt barely showed itself at a distance of 3 inches upstream from the well. Three feet downstream from the well the width of the salt stream was about 3 feet in the first case and about 2 feet in the other case.

Application of principles.- - It is possible to dispense with the circuits from the casing of well $A$ to those of the other wells, as the short circuit between the well and the electrode forms the best possible indication of the arrival of the electrolyte at 
U. S. GEOLOGICAL SURVEY

PROFESSIONAL PAPER NO. 44 PL. $X X$

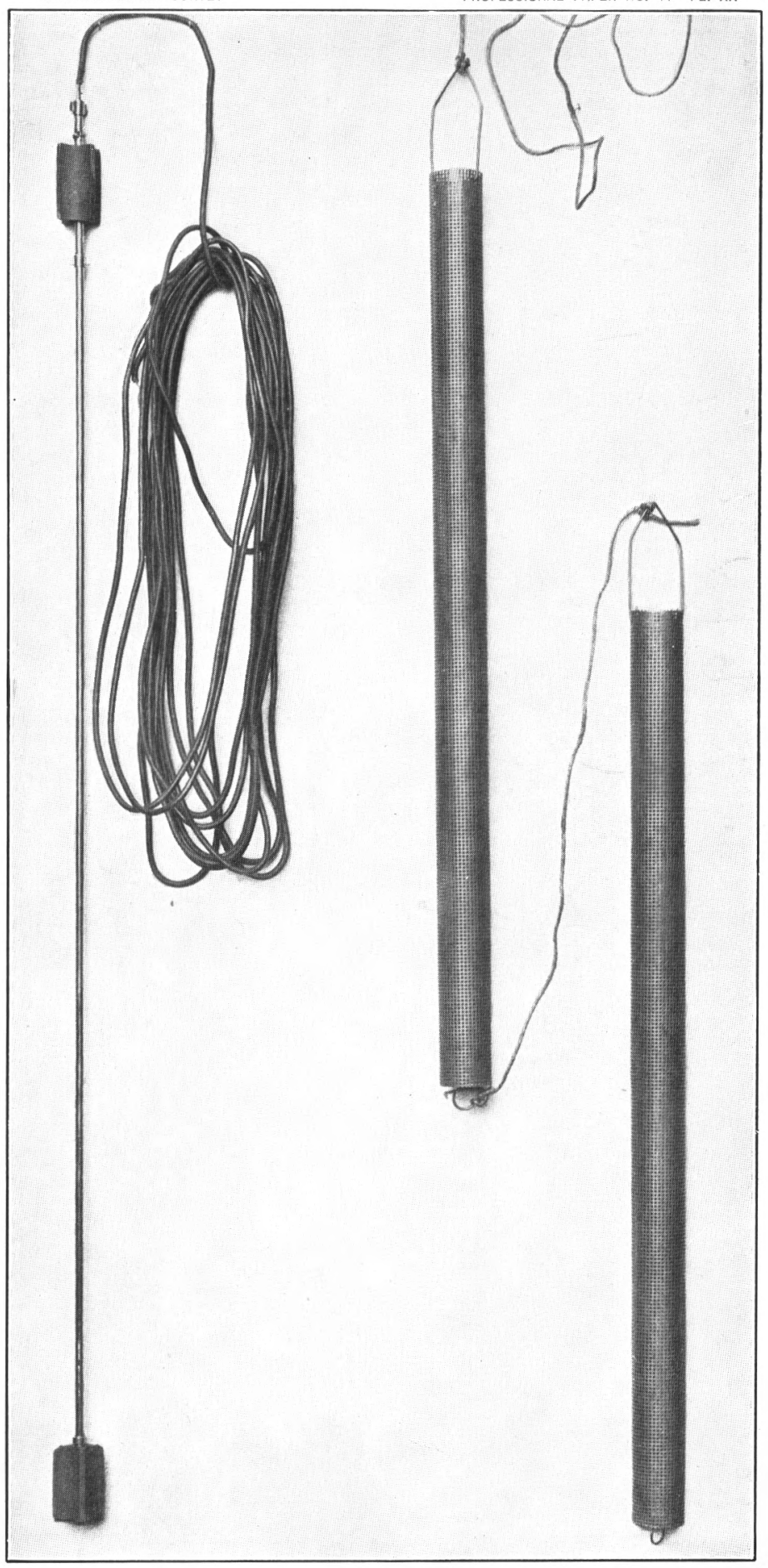

ELECTRODE AND PERFORATED BRASS BUCKETS USED IN CHARGING WELLS. 



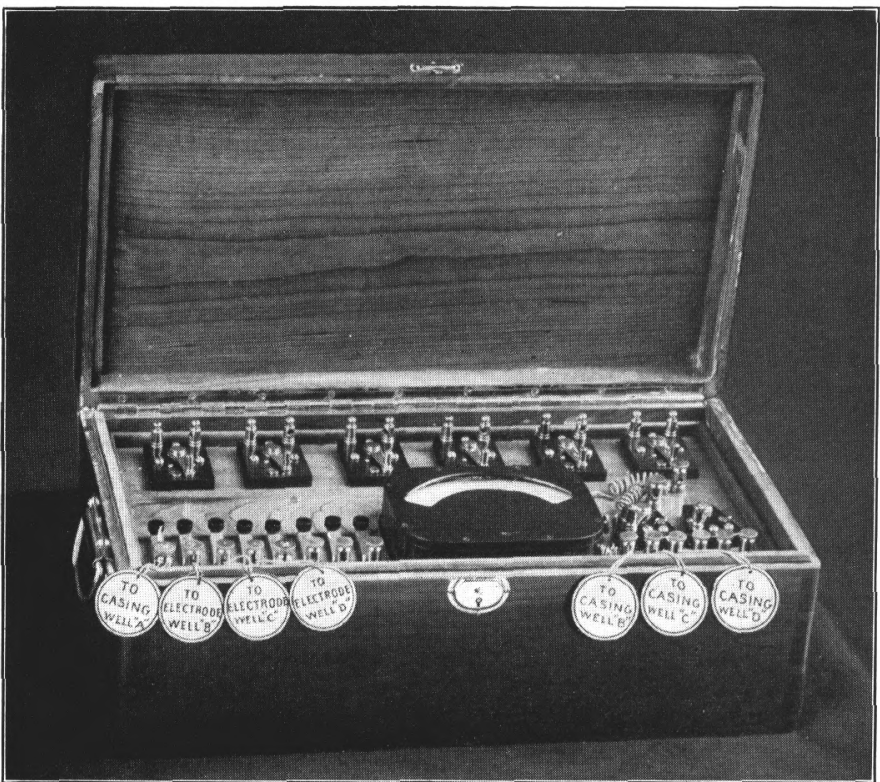

A. UNDERFLOW METER, SHOWING CONNECTIONS WHEN USED AS DIRECT-READING APPARATUS.

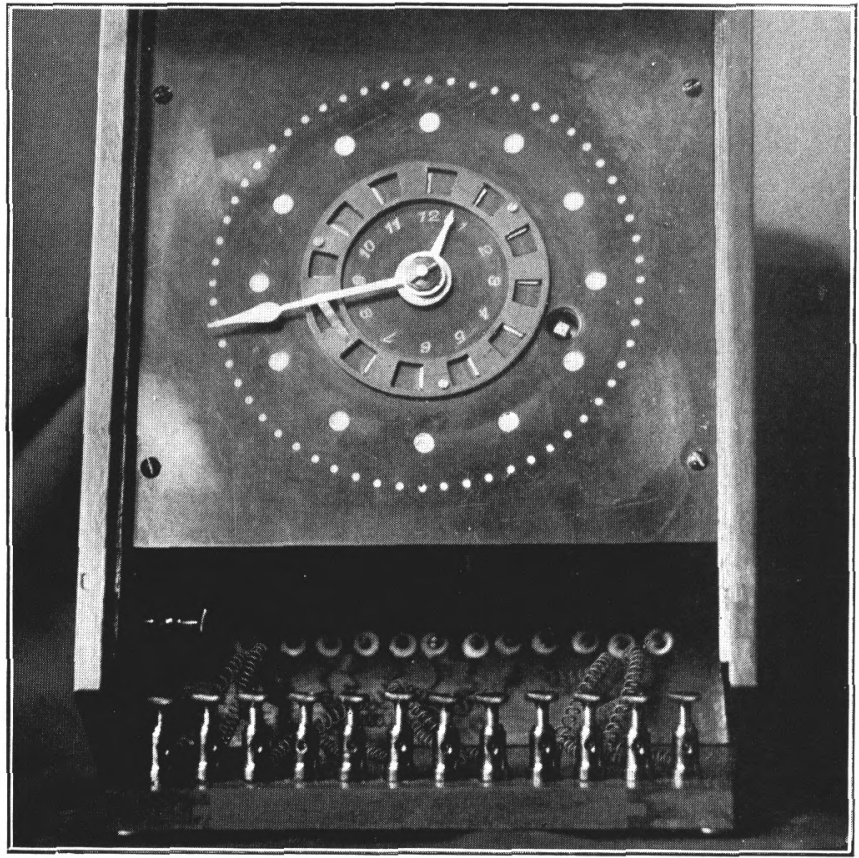

B. COMMUTATOR CLOCK FOR USE WITH RECORDING AMMETER. 

the downstream well. For cases in which the velocity of ground water is high the , circuit to well $\mathrm{A}$ is practically of no value, but for slow motions this circuit shows a rising current before the arrival of the electrolyte at the lower well, often giving indications of much value to the observer.

The method can be used quite successfully even though nothing but common

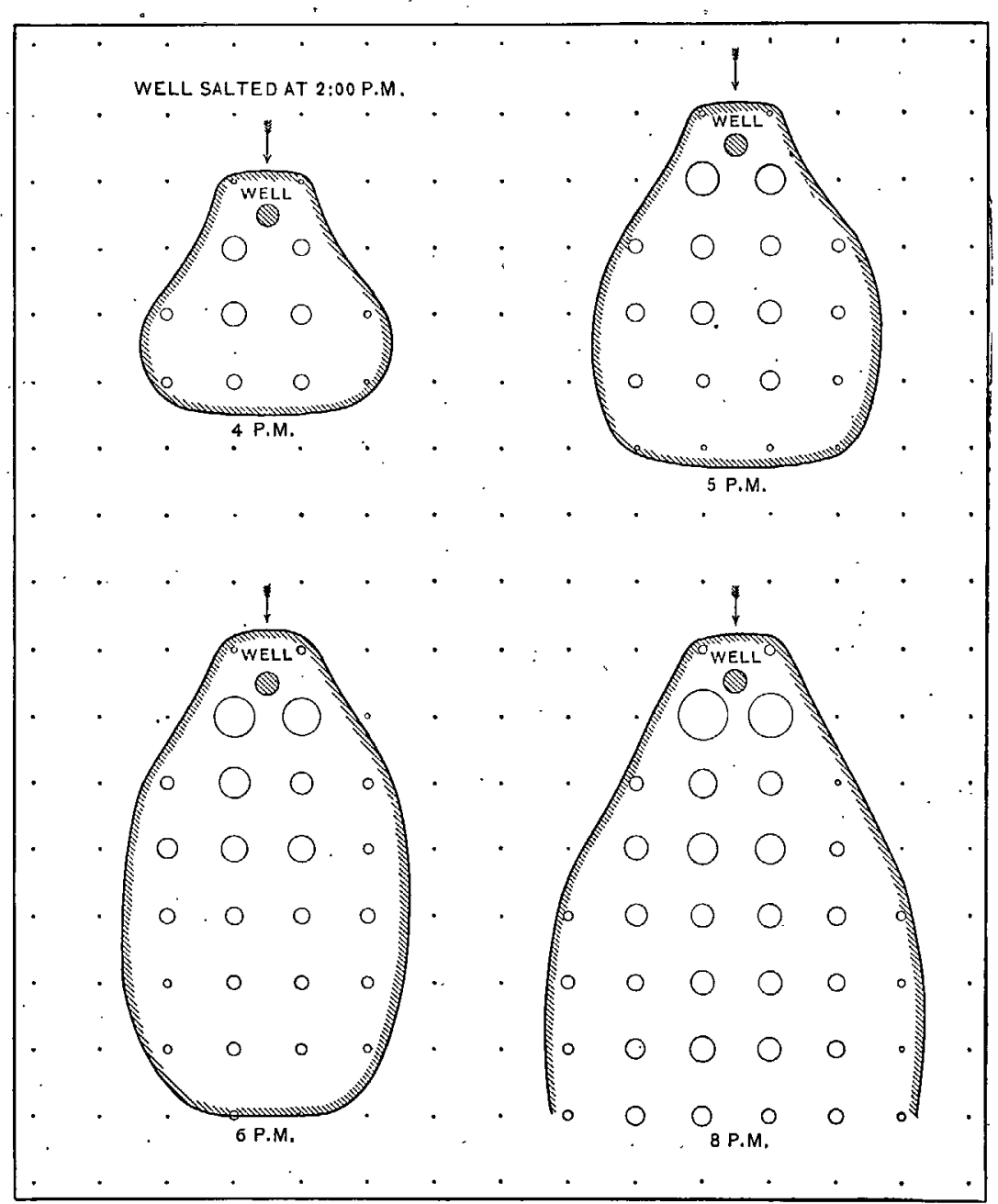

FIG. 42.-Diagram showing the manner in which the electrolyte spreads in passing downstream with the ground water. The shaded circle shows the location of the salted well, and samples were taken from the sand at the corners of fi-inch squares, shown by dots in the diagram. The areas of the circles are proportional to the strength of the electrolyte found at their centers. The rough outline indicates the area covered by the charged water at the times specified. The velocity of the ground water (in the direction of the arrows) was 12 feet a day. It can be seen that the electrolyte barcly reached a distance of 3 inches against the direction of flow.

pipe be used for the wells. In this case, however, the absence of screen or perforations in the wells renders the internal electrodes useless, and one must depend upon the circuit from well casing of the upstream well to well casing of downstream well. 
The results in the table on page 95 and fig. 41 present such a case. In this case the wells were not provided with well points, but merely possessed a 4 -foot length of pipe, provided with 4 or 5 holes on opposite sides of the pipe containing small $\frac{1}{2}$-inch washer screens. These few openings are not sufficient to permit the electrolyte to freely enter the well, so that readings between casings were relied

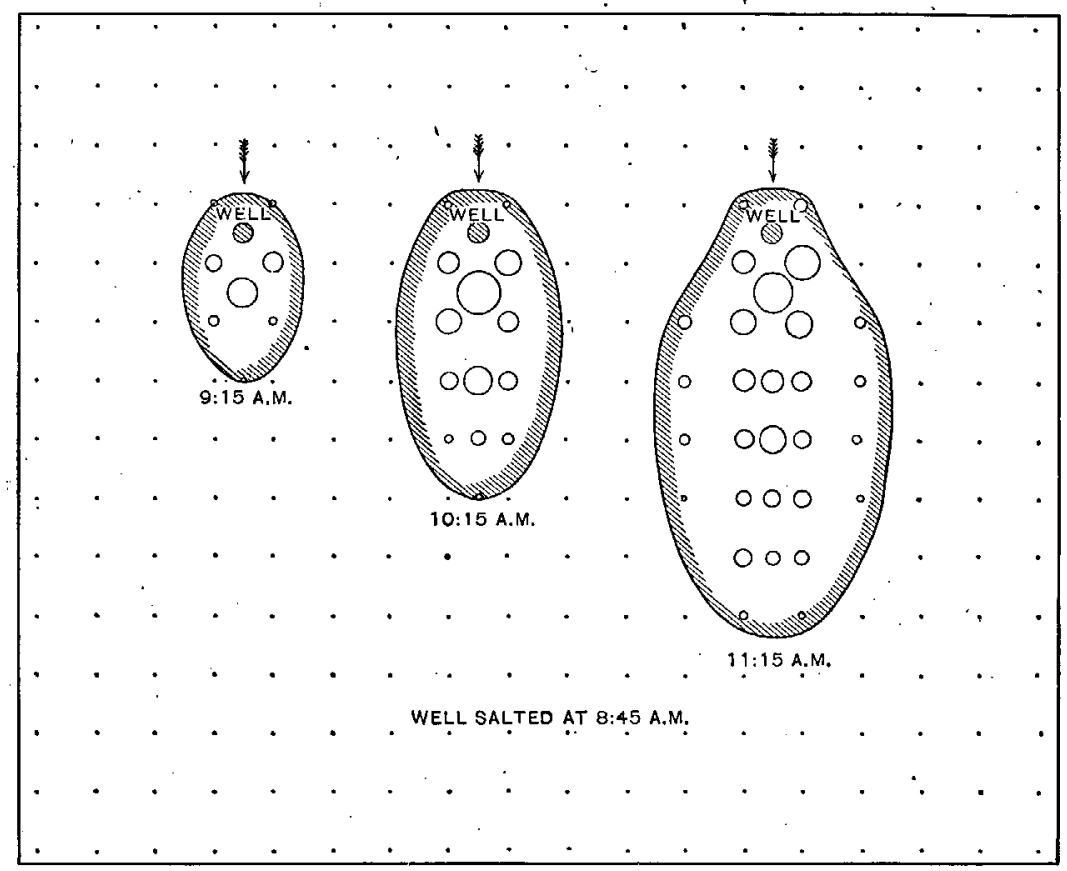

FIG. 43.-Diagram showing spread of electrolyte from a well with ground water moving about twice as fast as in fig. 42 , or 22.9 feet a day. The electrolyte spreads less rapidly for the higher velocity, as is shown at a glance.

upon for results. As a matter of fact, enough of the electrolyte did get into the well to give small increased readings, but in order to secure the electrode readings given in the table, water was removed from the downstream wells by a small bucket holding about 6 ounces, so as to force a quantity of the water surrounding the well into the perforated sections. 
TABLE IX-Station No. 1, Massapequa, Long Island, June 21 and 22, 1903. FIELD RECORD OF ELECTRIC CURRENT READINGS IN AMPERES, OBTAINED WITH DIRECT READING UNDERFLOW METER.

\begin{tabular}{|c|c|c|c|c|c|c|}
\hline . Time. & Casing B. & Electrode B. & Casing c. & Electrode C. & Casing D. & Electrode D. \\
\hline \multicolumn{7}{|l|}{ June 21 ; ค. $\mathrm{m}$ : } \\
\hline 8.45 . & 0.03 & 0.08 & 0.03 & 0.10 & 0.03 & 0.09 \\
\hline $9 a \ldots$ & & $\cdots \cdot$ & $\ldots$. & $\ldots$ & $\ldots .$. & $\ldots \ldots$ \\
\hline $9.30 \mathrm{~b} \ldots \ldots$ & .04 & .08 & .04 & 095 & .036 & .088 \\
\hline $10 \ldots \ldots$ & .04 & .079 & .039 & .092 & .036 & .088 \\
\hline $10.30 \ldots$. & .04 & .079 & .04 & .097 & .039 & .087 \\
\hline $11 \ldots \ldots \ldots \ldots \ldots \ldots \ldots$ & .04 & .079 & .04 & .095 & .059 & .087 \\
\hline $11.30 \ldots \ldots \ldots \ldots \ldots \ldots$ & .04 & .079 & .04 & .091 & .039 & .087 \\
\hline $12 \ldots \ldots \ldots \ldots$ & .041 & $: 079$ & .04 & .092 & .040 & .087 \\
\hline \multicolumn{7}{|l|}{ June $21, \mathrm{p} \cdot \mathrm{m}$ : } \\
\hline $1 \ldots \ldots$ & .042 & .079 & .04 & .090 & .040 & .088 \\
\hline $1.30 \ldots$ & .042 & .079 & .04 & .092 & .040 & .088 \\
\hline $2 \ldots \ldots$ & .043 & .079 & $.04^{\circ}$ & .092 & .040 & .089 \\
\hline $2.30 \ldots$ & $.0 \dot{4} 3$ & .078 & .041 & .094 & .040 & .088 \\
\hline $3 b \ldots \ldots$ & .043 & .078 & .041. & .094 & .040 & .090 \\
\hline $3.30 \ldots$ & .043 & .078 & .040 & .094 & .041 & .090 \\
\hline $4 \ldots .$. & .043 & .078 & .042 & .094 & .041 & .090 \\
\hline $4.30 \ldots$ & .043 & .078 & .042 & .095 & .041 & .090 \\
\hline $5 \ldots \ldots$ & .043 & .078 & .042 & .096 & .041 & .090 \\
\hline $5.30 \ldots$ & .045 & .078 & .043 & .096 & .041 & .090 \\
\hline $6.30 \ldots$ & .045 & .078 & .043 & .097 & .042 & .091 \\
\hline $7 \ldots \ldots$ & .045 & .078 & .046 & .099 & .041 & .091 \\
\hline $7.30^{b} \ldots$. & .045 & .078 & .046 & .099 & .041 & .090 \\
\hline $8 \ldots \ldots$ & .045 & .080 & .048 & .099 & .042 & .093 \\
\hline $8.30 \ldots$ & .049 & .080 & .049 & .100 & .043 & .094 \\
\hline $9 \ldots \ldots \ldots \ldots$ & .048 & .079 & .050 & .100 & .043 . & .094 \\
\hline $10.30 \ldots \ldots \ldots \ldots \ldots \ldots$ & .050 & .079 & .070 & .101 & .045 & .095 \\
\hline $12 \ldots \ldots \ldots \ldots \ldots \ldots \ldots$ & .050 & .079 & .095 & .106 & .047 & .095 \\
\hline
\end{tabular}

a 10 pounds of sal ammoniac placed in well A.

b 2 pounds of sal ammoniac placed in well $\mathrm{A}$. 
TARLE IX.-Station No. 1, Massapequa, Long Island, June 21 and 22, 1908-Continned.

FIELD RECORD OF ELECTRIC CURRENT READINGS IN AMPERES, OBTAINED WITH DIRECT READING UNDERFLOW METFR-Continued.

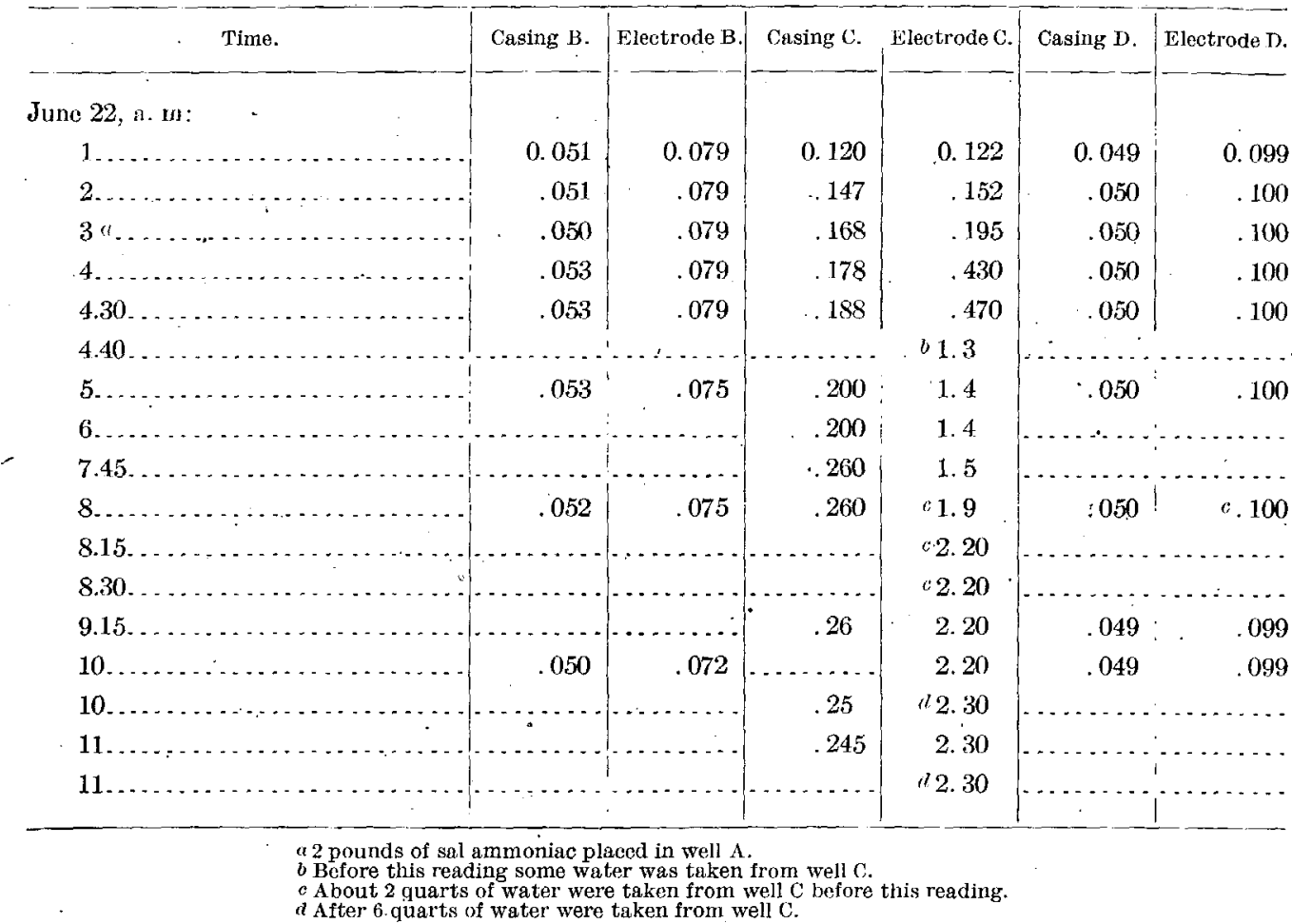

In cases where good well points are used the ground water charged with the electrolyte finds its way gradually and naturally into the well. The well point should be clean enough to allow as free passage into the well as through the soil itself. Second-hand points used for this purpose may show a marked lag in the entry of the electrolyte. By comparing the curves for station No. 1 (fig. 41) with those of stations No. 6 (fig. 46) and No. 21 (fig. 57), where good well points were used, the lag caused by insufficient perforations in the well of station No. 1 is brought out very clearly.

Granulated sal ammoniac is used in well $\mathrm{A}$, a single charge varying from 4 to 10 pounds. If common pipe without points or screens is used for the wells, so that internal electrodes must be dispensed with, about 2 pounds should be used every hour. The dry salt should not be poured directly into the well, but should be lowered in perforated buckets, shown in Pl. XX. These buckets are $1 \frac{3}{4}$ by 30 inches and hold about 2 pounds of the salt. Two of these buckets may be tied one above the other for the initial change, followed by two more in ten or twenty minutes.

If the wells are not too deep, the sal ammoniac may be introduced into the well in the form of a solution. A common bucketful of saturated solution is suffi- 
cient. There is an uncertainty in introducing the sal ammoniac in solution in deep wells, as the time required for the solution to sink to the bottom of the well may be considerable.

The ammeter used in the work has two scales, one reading from 0 to 1.5 amperes and the other from 0 to 5 amperes. With a given number of cells, the amount of current between the upstream and downstream wells will depend, of course, upon several factors, such as the depth of the wells and their distance apart; but more especially upon the amount of dissolved mineral matter in the ground water. The initial strength of the current can be readily adjusted, however, after the wells have been connected with the instruments, by turning on or off some of the battery cells by means of the switches at the rear of the box. A good rule is to use enough cells to make the initial current, if practicable, about one-tenth of an ampere.

SELF-RECORDING METER.

In the second form of underflow meter, self-recording instruments are used so as to do away with the tedious work of taking the frequent observations day and night, required when direct-reading instruments are used. The arrangement of the apparatus is not materially different from that described above. In the place of the direct-reading ammeter a special recording ammeter is used, of range 0 to 2 amperes. It has been found practicable, although a matter of no small difficulty, to construct an instrument of this low range sufficiently portable for field use and not too delicate for the purpose for which it is intended. The ammeter has a resistance of about $1.6 \mathrm{ohms}$ and is provided with an oil dash pot to dampen swing of arm carrying the recording pen. The instruments were manufactured by the Bristol Company; they have gone through hard usage in the field without breakage or mishap. The portability of the instruments will be materially increased by changes in design which are now being made.

The methods of wiring the wells when the recording instruments are used is slightly changed. In this case one side of the battery circuit is connected to casing of well $\mathrm{A}$ and to all of the electrodes of wells B, C, and D. The other side of the battery is run through the recording ammeter to a commutator clock, which, once every hour, makes a contact and completes the circuits, one after the other, to a series of binding posts. One of these binding posts is connected to the casing of well $\mathrm{B}$, one to the casing of well $\mathrm{C}$, and one to the casing of well $\mathrm{D}$. The time of contact is ten seconds, which gives the pen abundant time to reach its proper position and to properly ink its record.

Pls. XXI, $B$, and XXII, $A$, show two commutator clocks made for this purpose by the instrument maker of the college of engineering, University of Wisconsin. The clock movement is a standard movement of fair grade, costing about $\$ 5$. It can readily be taken from the case for cleaning or oiling and as quickly replaced. A seven-day marine movement with powerful springs is best for this purpose.

It will be seen from the method of wiring the wells that the record will show the sum of the current between well $A$ and well $B$ added to the current between 
the casing of well $\mathrm{B}$ and its electrode. The removal of the connection to well $\mathrm{A}$ would permit the record to show the current between the casing of a downstream well and its electrode, but the connection to the upstream well involves no additional trouble and occasionally its indications are of much service, especially if the velocities are low.

All of the instruments above mentioned can be placed in a common box, 16 by 22 by 36 inches, covered with tar paper and locked up. PI. XXII, $B$, shows a photograph of the instruments thus arranged. The shelf contains the recording ammeter (shown at left of cut) and the commutator clock (shown at right of cut).

The contacts of the commutator clock are arranged about five minutes apart, so that the record made for the wells will appear on the chart as a group of lines,

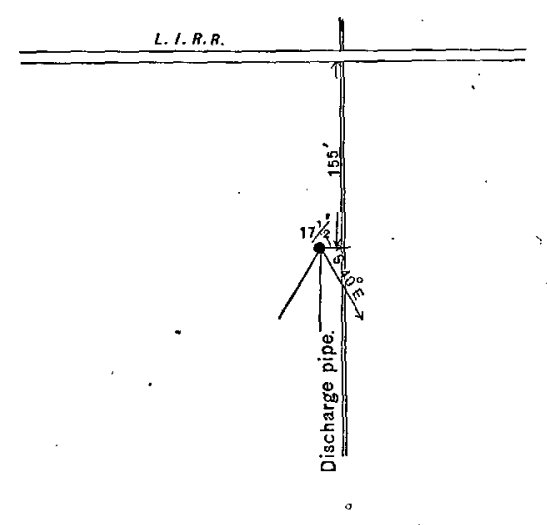
one for each downstream well, of length corresponding to the strength of the current. The increasing current corresponding to one of the wells will finally be indicated by the lengthening of the record lines for that well. This can be seen by consulting the photographs of records shown in Pl, XXIII. Lightgreen ink is used for record charts and red ink in the recording pen, so that record lines can be distinguished when superimposed upon the lines of the chart. A special chart has been designed for this work, and is furnished by the Bristol Company as Chart ' 458 .

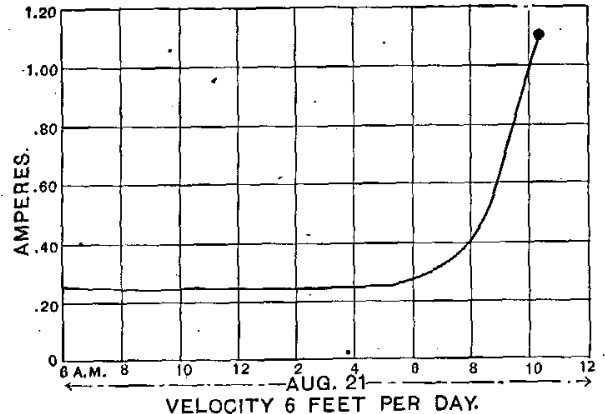

FIG. 44.-Diagram showing velocity and direction of. flow of underground water at Wantagh pumping station:

The recording instruments in use have given perfect satisfaction, and the method is a great improvement in accuracy and convenience over the direct-reading method. The highest as well as the lowest groundwater velocities yet found have been successfully measured by the recording instruments. By using one or two additional dry cells the instrument is quite as sensitive as the direct-reading type.

In using the recording instruments but a single charge of salt need be placed in the upstream well. If the wells are deep it is important to use enough salt solution to be sure that the salt reaches as far down as the screen of the well point immediately after the solution is poured into the well. A gallon of solution will fill about 6 feet of 2 -inch wrought-iron pipe, so that 10 gallons of solution should be used if well is 60 feet deep. If the proper amount of solution be not used it will take an appreciable time for the solution to reach the bottom of the well by convection currents and the results will be vitiated to that extent. As before stated, it is preferable to introduce into the well granulated sal ammoniac contained in a suitable bucket, in case the depth of the well renders the use of a solution uncertain. 


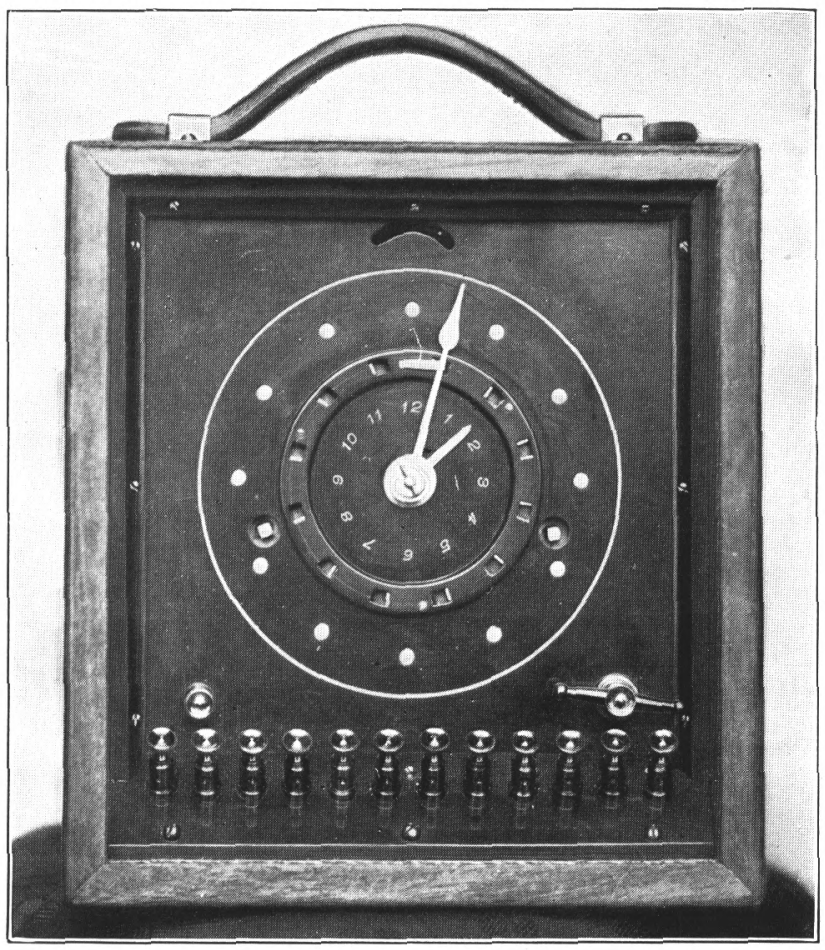

A. COMMUTATOR CLOCK FOR USE WITH RECORDING AMMETER.

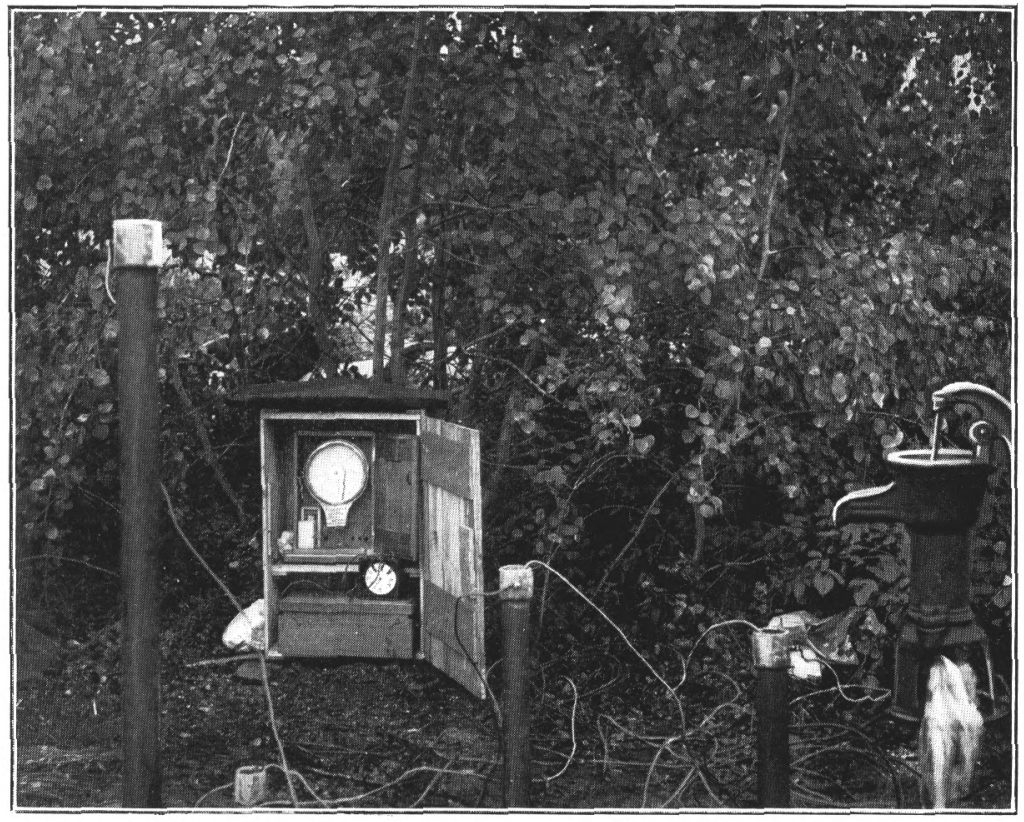

B. RECORDING AMMETER, COMMUTATOR CLOCK, AND BATTERY BOX IN USE IN THE FIELD, 

PRINCIPLES INVOLVED.

The principles involved in the working of the apparatus are quite simple. The upstream well $\mathrm{A}$ is charged with a strong electrolyte, such as sal ammoniac, which-passes downstream with the moving ground water, rendering the ground water a good electrolytic conductor of electricity. If the ground water moves in the direction of one of the lower wells, B, C, D, etc., the electric current between $\mathrm{A}$ and $\mathrm{B}, \mathrm{A}$ and $\mathrm{C}$, or $\mathrm{A}$ and $\mathrm{D}$ will gradually rise, mounting rapidly when the

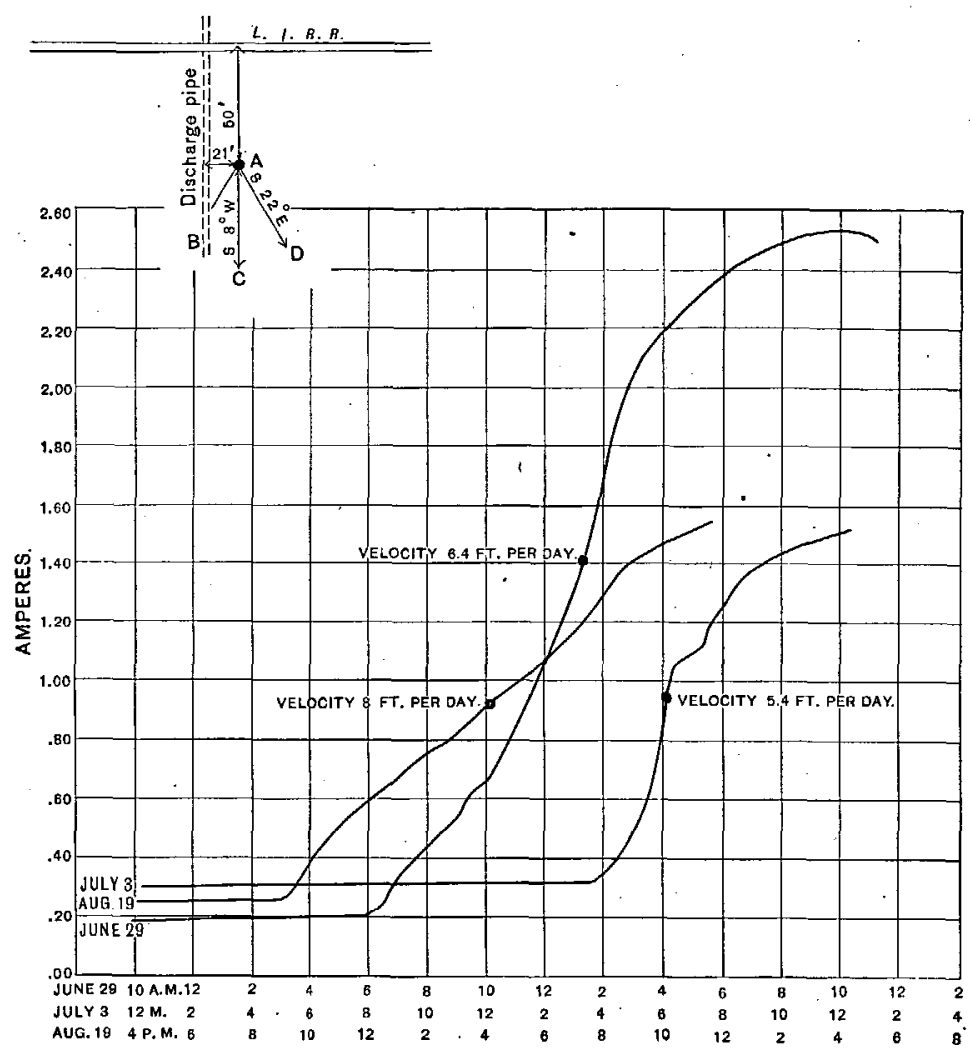

FỊG. 45.-Diagram showing velocity and direction of flow of underground water at $A$ gawam pumping station (Station 5).

electrolyte begins to touch one of the lower wells. When the electrolyte finally reaches and enters one of the wells $B, C, D$, it forms a short circuit between the casing of the well and the internal electrode, causing an abrupt rise in the electric current. The result can be easily understood by consulting fig. 40 , in which the current is depicted graphically.

The time which elapses from the charging of the well $A$, to the arrival of the electrolyte at the lower well, gives the time necessary for the ground water to cover the distance between these two wells. Hence, if the distance between the wells be. divided by this elapsed time, the result will be the velocity of the ground water. 


\section{RESULTS AND CONCLUSIONS.}

\section{EXISTENCE OF UNDERFLOW.}

The 6-mile line from Freeport to Massapequa is, as has been stated, about 1 mile distant from the edge of the tidal marshes bordering the Atlantic Ocean. North of this line for a distance of 9 or 10 miles the natural surface drainage of the land is toward the south, the slope for nearly 8 miles of the distance being almost exactly 15 feet to the mile. This drainage plain is not only very flat and unbroken, but the surface conditions are exceedingly favorable for the absorption of a large percentage of the rainfall. The soil for the most part is coarse and sandy and
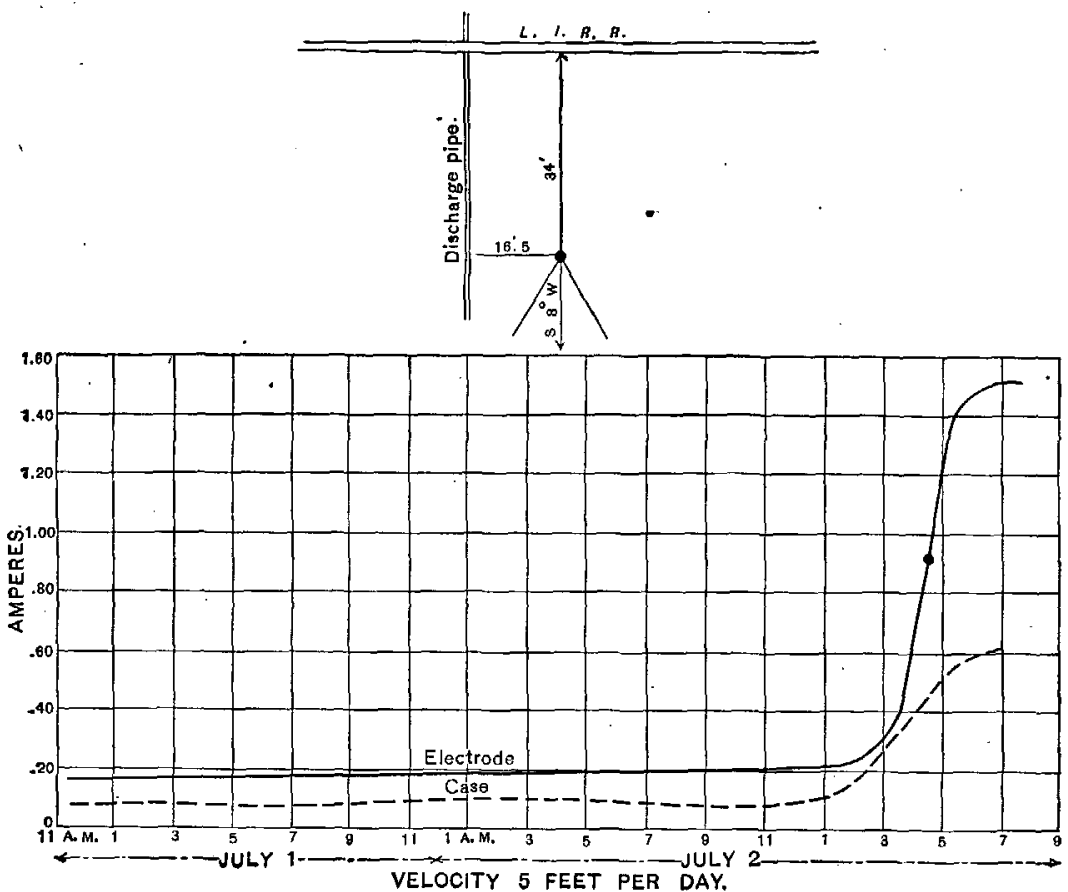

FIG. 46.-Diugram showing velocity and direction of flow of underground water at Agawam pumping station (Station 6).

very porous. The slope of the water plane is somewhat less than that of the surface of the land, being approximately 10 or 12 feet to the mile. The underground drainage is in general toward the south, the main east-west underground watershed probably coinciding within a mile or two with the surface watershed. The average rainfall is about 44 inches, a very large share of which enters the ground.

In the localities where the test wells were bored the material for the first 30 to 40 feet was yellow sand and gravel, quite clean and uniform, but growing finer with the depth. The first 20 feet below the water plane seemed in every case to be of high transmission capacity, and the material below this level was usually of increasing fineness, finally changing into a fine, dark-colored, micaceous sand. At a depth of from 40 to 60 feet a compact layer of clayey and bog-like material was 


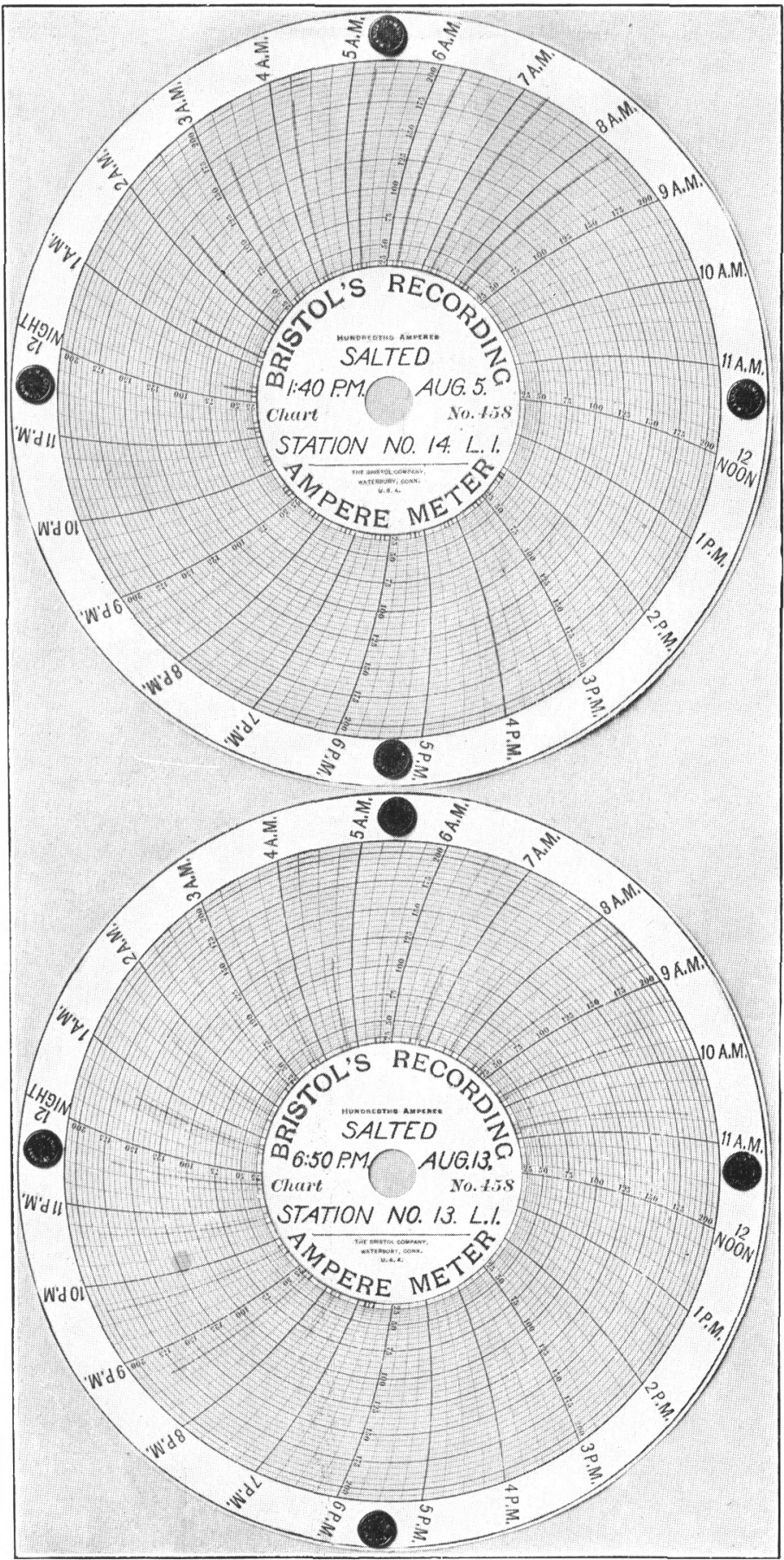

CHARTS MADE BY RECORDING AMMETER. 

often met with, and in driving the test wells into and through this layer the water rose continuously in the wells until a marked artesian head was developed. Immediately below this compact layer good sands were again encountered.

In the report on New York's water supply made by John R. Freeman in the year 1900 it is stated as probable that the layer of clayey material referred to above is distributed as a wide and practically unbroken sheet 40 to 60 feet beneath the surface of the south-sloping drainage plain of the island.

One of the objects of the measurement of ground-water velocities was to determine whether or not there was a considerable southerly movement to this water in the sands and gravels above the supposed clay sheet and to determine the order of magnitude of such a movement if it existed. Whenever there
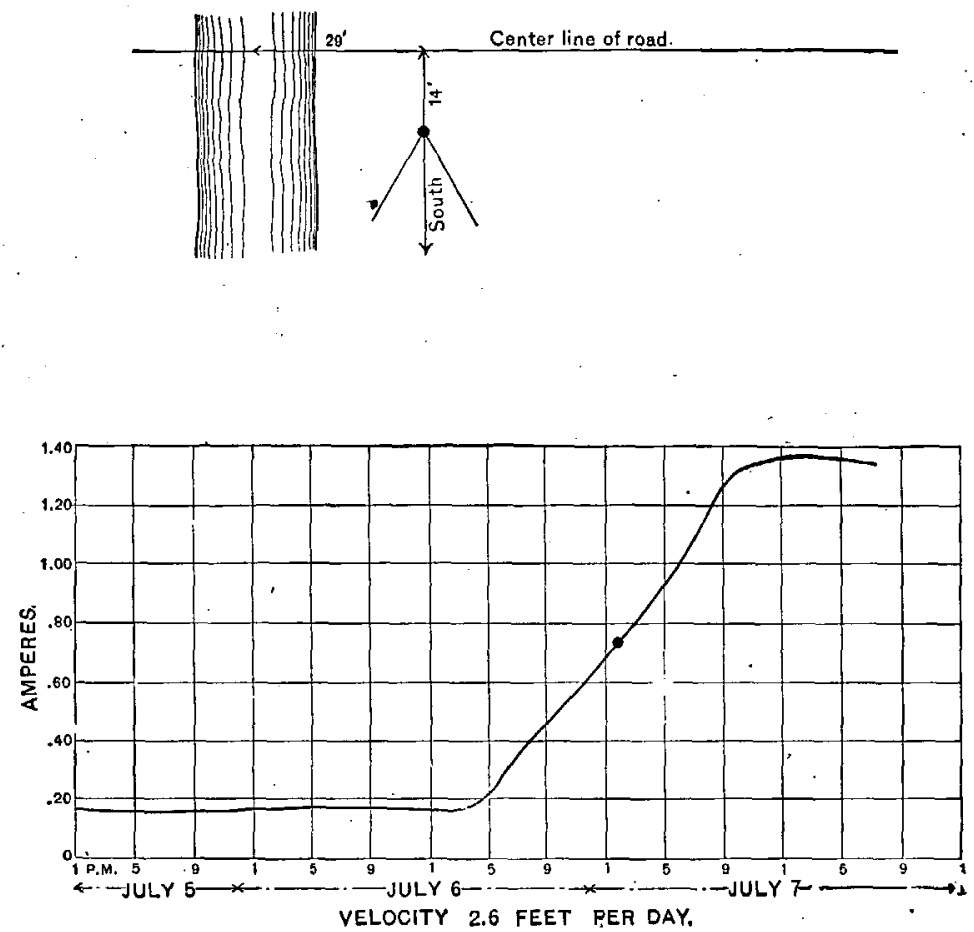

FIG. 47,-Diagram showing direction and velocity of fow of underground water at East Meadow Brook and Babylon road (Station 7).

exists in any drainage area a body of ground water which does not escape into the beds of surface streams as seepage water but continues seaward through the sands and gravels independent of the surface streams, this moving sheet of water is known as the underflow. One of the problems was, therefore, to determine whether or not a true underflow existed in this part of Long Island, and to learn something of its magnitude if it was found to exist. Another problem was to discover, if practicable, if any part of the underground drainage existed below the bed of clay; in other words, it was sought to determine whether the underground drainage consisted only of a surface zone of flow, or whether a deeper zone of flow-or possibly several deeper zones-were also present. 
In respect to the first problem above mentioned - the existence of an underflowthere can be no question but that a true underflow of considerable importance exists within a depth below the surface of from 40 to 50 feet. In practically all of the stations established a good movement was found to exist, having a strong southerly component, in many cases surprisingly free from the influence of neighboring surface streams. The velocity near the surface-from 16 to 24 feet below the water plane-ran as high as 5 to 12 feet per day. At greater depths the velocities ran much less; at two stations, at depths of 30 and 42 feet, the velocities were each about 15 inches per day. At station No. 9 the sand was so fine at a depth of 45 feet
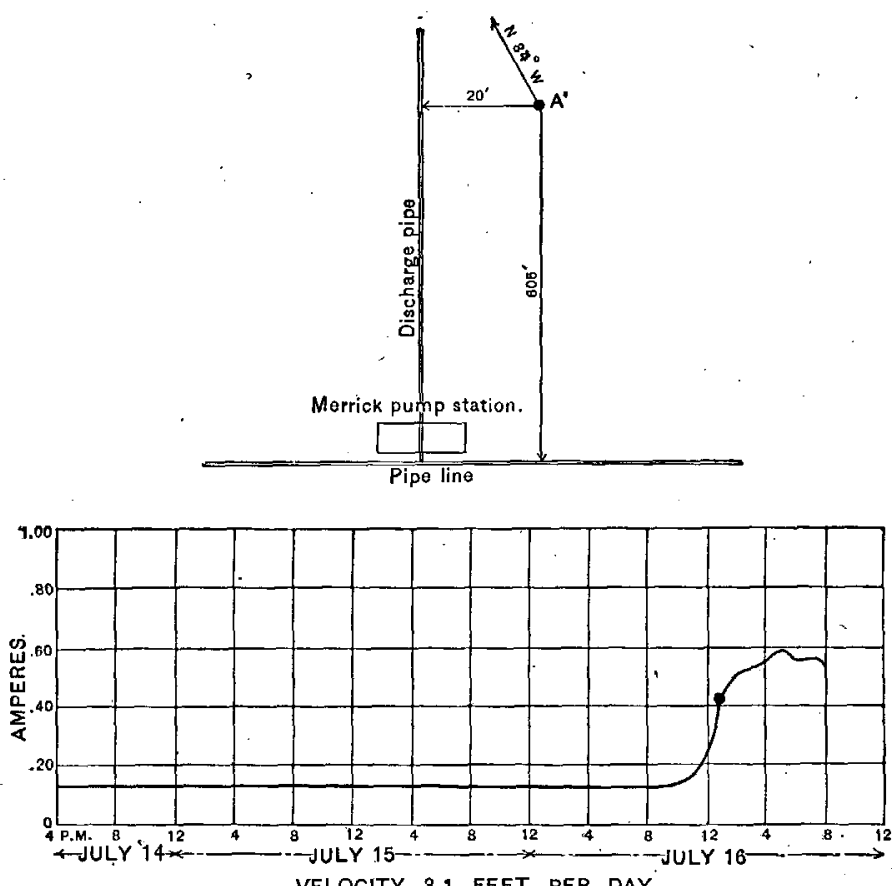

FIG. 48.-Diagram showing velocity and direction of flow of underground water near Merrick pumping station (Station 8).

that it could not be prevented from running into the bottom of the well above the top of the screen so that the wells could not be used.

The existence of a deep zone of flow was also established. At station No. 15 clay was encountered at a depth of about 44 , feet. These wells were driven to a depth of about 62 feet, when an artesian head of about 30 inches developed. A measurement was then made, the screens on the wells being just below the impervious layer. A velocity of 6 feet per day was found to exist, in a direction about $10^{\circ}$ west of south. The rate of flow at the same point just above the clay was only 18 inches per day, so that a true deep zone of flow undoubtedly exists at this point. This result, although very important, was not a surprise, as it had already been quite well established by the work of Mr. A. C. Veatch, of the United States Geological Survey. and others, that the clay layer, formerly supposed to be of 
wide expanse and quite unbroken, is, as a matter of fact, absent over considerable areas of the island, so that no reason exists why a part of the underground drainage should not exist below this impervious bed.

The surface zone of flow of the underground waters is probably divided into a number of drainage areas, although it is exceedingly doubtful if the underground drainage basins coincide very closely with the drainage areas of the surface streams. In general, the velocities seemed to increase from west to east, the lowest velocities, however, being in a middle area, where the yellow gravels contain a quantity of

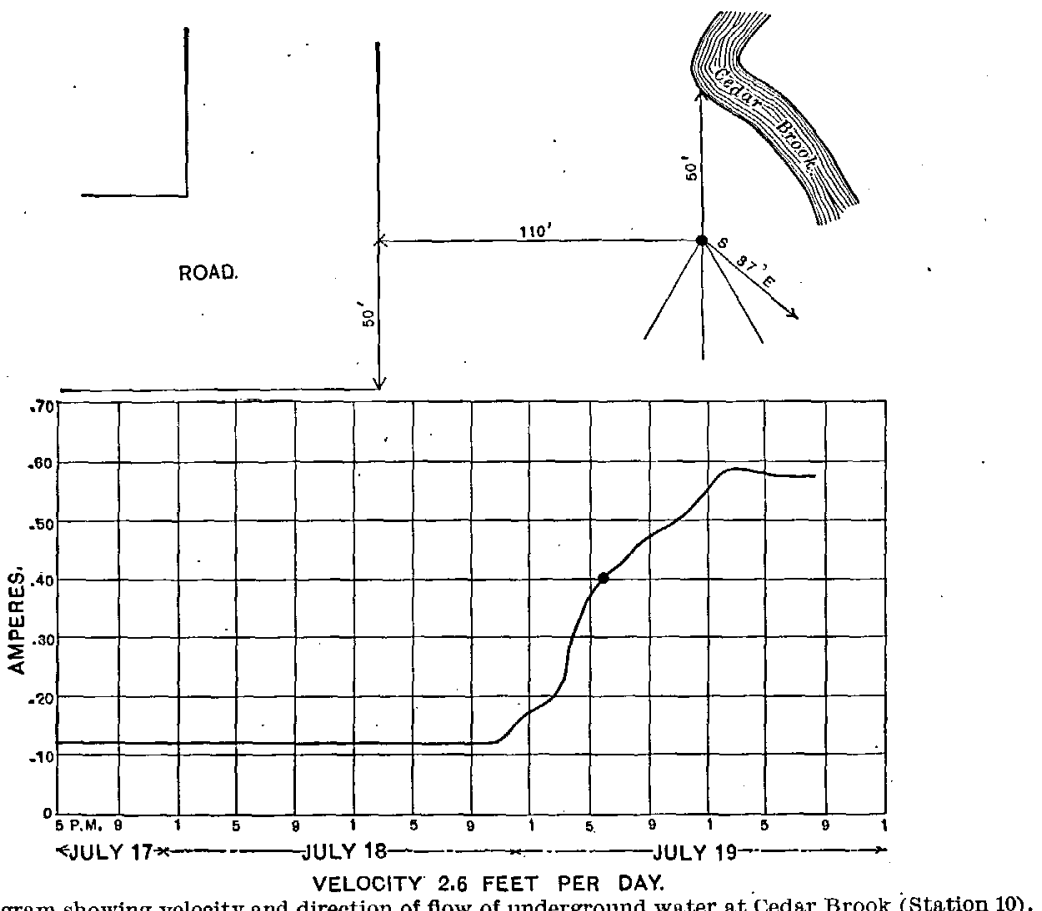

Frg. 49.-Diagram showing velocity and direction of flow of underground water at Cedar Brook (Station 10).

fine, clay-like silt. The Wantagh area seemed to have the largest underflow. It would be exceedingly interesting to have series of measurements extended eastward into Suffolk County. By increasing somewhat the number of stations in the area already covered and comparing with results from drainage areas in Suffolk County, a comparative study of underground drainage systems would result which ought to have much value in planning new sources of supply for Brooklyn.

The details of the measurements are given in the reports on individual stations contained in the following table. The locations of the stations are shown in fig. 37 (p. 87), and the curves of electrical current for various stations are given in fig. 41 and figs. 44 to 57. 
TABLE X.-linderflow measurements on Long Island.

\begin{tabular}{|c|c|c|c|c|c|}
\hline $\begin{array}{l}\text { Number of } \\
\text { station. }\end{array}$ & $\begin{array}{l}\text { Velocity of } \\
\text { ground } \\
\text { water per } \\
\text { day. }\end{array}$ & Direction. & Date, 1903. & $\begin{array}{c}\text { Depth of } \\
\text { wells below } \\
\text { water plane. }\end{array}$ & Kind of point. \\
\hline & Feet. & & & Feet. & \\
\hline $1 \ldots \ldots$ & 5.5 & S. $10^{\circ} \mathrm{E}$. & June $21 \ldots . . . . .$. & 22 & Perforated pipe. \\
\hline $2 \ldots \ldots$. & $<2.0$. & & June $24 \ldots . . . .$. & 22 & Do. \\
\hline $2 x \ldots$ & 6.0 & S. $40^{\circ} \mathrm{E}$. & August $21 \ldots . .$. & 22 & Do. \\
\hline 3. & $<2.0$ & & June $26 \ldots \ldots \ldots$ & 22 & Do. \\
\hline $4 \ldots \ldots$ & $<2.0$ & $\ldots \ldots \ldots$ & June $27 \ldots \ldots . .$. & 22 & Do. \\
\hline $5 \ldots$. & 6.4 & S. $8^{\circ} \mathrm{W}$ & June $29 \ldots \ldots$ & 22 & Common point. \\
\hline $5 x_{-}$ & 5.4 & S. $8^{\circ} \mathrm{W} \ldots$ & July $3,4 \ldots \ldots$. & 22 & Do. \\
\hline $5 y-$ & 8.0 & S. $22^{\circ} \mathrm{F}$. & August $19 \ldots \ldots$ & 22 & Do. \\
\hline $6 \ldots$ & 5.0 & S. $8^{\circ} \mathrm{W}$. & July $1,2 \ldots$. & 34 & Do. \\
\hline $7 \ldots$ & 2.6 & $S_{\ldots} \ldots \ldots$ & July $5,6 \ldots \ldots$. . . & 20 & Do. \\
\hline $8 \ldots$ & .0 & S........ & July $9,11,11 \ldots \ldots$ & 21.6 & Open-end point. \\
\hline $8^{\prime} \ldots$ & 3.1 & N. $34^{\circ} \mathrm{W}$ & July $14,15,16,17 \ldots$ & 21.6 & Do. \\
\hline 10. & 2.6 & S. $37^{\circ} \mathrm{L}$ & July $17,18,19,20 \ldots$ & .28 .0 & Common point. \\
\hline 11. & .0 & & July $27-A$ ugust $8 . \therefore$ & 22.0 & Do. \\
\hline $12 \ldots \ldots$ & 1.07 & S. $3^{\circ} \mathrm{E}$. & July 27-August 1... & 27.0 & Open-end point. \\
\hline $13 .$. & 96.00 & S...... & August $3,4 \ldots \ldots$. & 16.0 & Common point. \\
\hline 13. & 6.90 & S..... & August $3,4 \ldots \ldots$ & 16.0 & Do. \\
\hline 14. & 9.30 & S...... & August $5,8 \ldots \ldots \ldots$ & 17.0 & Do. \\
\hline $15 \ldots$. & 1.53 & S...... & August $6,7,8,9,10$. & 42.0 & Open-end point. \\
\hline $15 x \ldots$ & 6.00 & S. $15^{\circ} \mathrm{W}$ & August $17,18,19 \ldots$ & 62.5 & Do. \\
\hline $16 \ldots$ & .00 & S. $30^{\circ} \mathrm{E}$ & August $10,11 \ldots$. & 16.0 & Common point. \\
\hline $16 x \ldots$. & 77.00 & S. $60^{\circ}$ E. . & August $13,14 \ldots \ldots$ & 16.0 & Do. \\
\hline $16 x \ldots .$. & 11.60 & S. $60^{\circ} \mathrm{E}$. & August $13,14 \ldots$ & 16.0 & Do. \\
\hline $17 \ldots \ldots$ & 10.60 & S. $30^{\circ} \mathrm{W}$. & August $12,13 \ldots$. & 20.0 & Do. \\
\hline $18 \ldots . . .$. & $<1.00$ & S........ & August $15-21 \ldots$ & 62.0 & Open-end point. \\
\hline $21 \ldots \ldots$. & 21.30 & S. $50^{\circ}$ E... & August $18,19 \ldots \ldots$ & 16.5 & Common point. \\
\hline $22 \ldots$ & 5.60 & S. $30^{\circ} \mathbf{E} \ldots$ & August $20,21 \ldots .$. & 16.0 & Do \\
\hline
\end{tabular}

DFFECT OF THE RAINFALL ON RATE OF MOTION OF GROUND WATER.

An excellent opportunity was presented at onc of the stations for noting the influence of a heavy rain upon the velocity of ground waters.

At station No. 5, at Agawam pumping station (see figs. 45 and 58), the upstream well A was salted at 9.45 a. m., June 27, 1903. Between 9 a. m. and 1 p. m. nearly 3 inches of rain fell, so that the heavy precipitation coincided with the early part of the ground-water measurements. The velocity found was 6.4 feet per day. On. July 3 the experiment was repeated, there being no rain in the intervening time. The velocity found in the second trial was 5.4 feet per day. The change in velocity was undoubtedly due to the enormous rainfall during the first experiment. Part of the high velocity during the rainstorm may be attrib- 
uted to the effect of the low barometer accompanying the storm, but part of it should be assigned to the increased head of ground-water pressure caused by the heavy rainfall upon the receiving area. As I have shown in another place, " ground, waters move very much as electricity is conducted in a good conductor, the most striking quality in ground-water motion being an almost complete absence of true inertia. The motion of a mass of ground water, even for the highest velocities, is so slow that the resistance presented by the inertia of the ground water to an accelerating force is almost nothing when compared with the component of the retarding force, consisting of the capillary resistance in the small pores of the sand

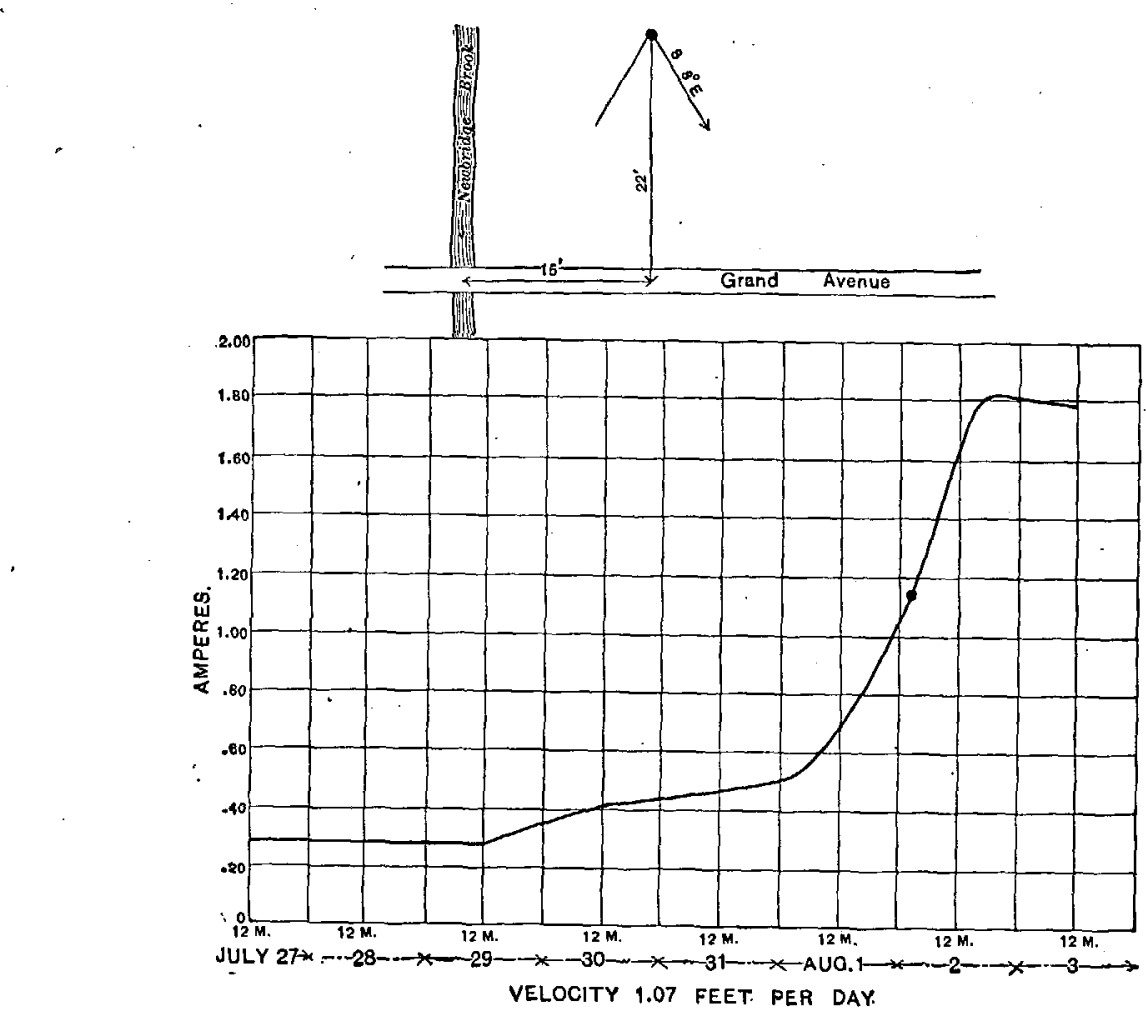

FIG. 50.-Diagram showing velocity and direction of flow of underground water at Grand avenue and Newbridge Brook (station 12).

or gravel. Actual computation will show that in a uniform sand of diameter of grain of one-half millimeter the ground water will reach within 1 per cent of its final maximum velocity by a sudden application of pressure or head in approximately thirty seconds of time. This surprising result of the theory of ground-water motions receives'a very striking verification in the increase in velocity noted during the rain storm as described above.

These results have important bearings on our knowledge of ground-water phenomena in the neighborhood of a well. They indicate that the velocity of the

a Slichter, C. S., Theoretical investigation of motion of ground waters: Nineteenth Ann. Rept. U. S. Geol. Survey, pt. 2, 1899, p. 331 
ground waters in the neighborhood of a well reaches a maximum value soon after pumping is commenced. The gradual formation of the cone of depression near the well shows that there must be a progressive augmentation to the initial velocity of the ground waters toward the well. Nevertheless, the rate of depression of the water table is so slow that the ground-water motion established soon after the pumping has begun is substantially the same as after prolonged pumping. These remarks have their most important bearing upon the phenomena of the mutual interference of wells. The interference of one well with the supply of a neighboring well is thus seen to come into existence almost instantaneously and need not wait for the establishment of a cone of depression of large area. The phenomena of the cone of depression have much to do with the permanent supply of the well, but have
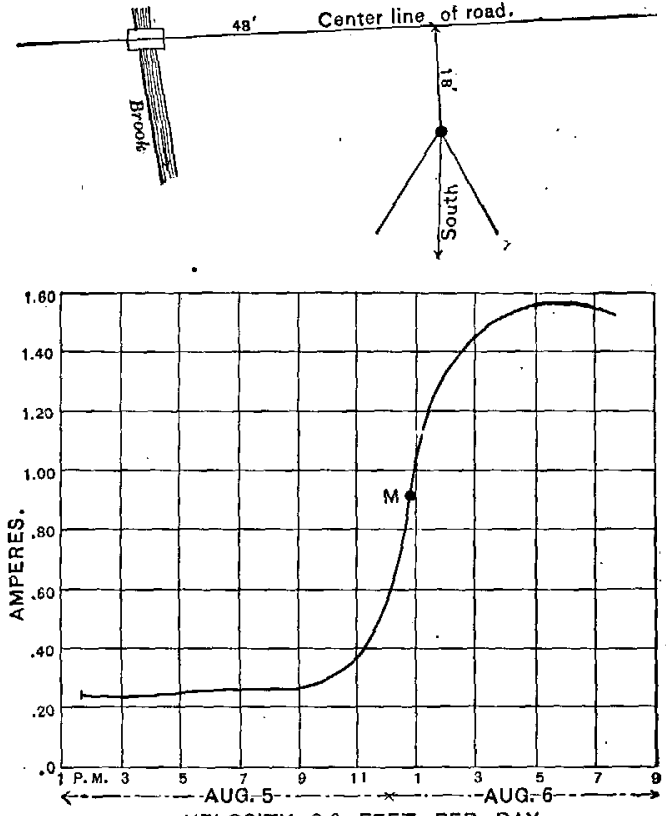

VELOCITY 8.6 FEET PER DAY.

Fig. 51.-Diagram showing velocity and direction of flow of underground water at Bellevue road (station 14). slight bearing upon the proper spacing of the wells or the percentage of interference of one well with another.

EFFECT OF SEEPAGE WATERS FROM PONDS AND RESERVOIRS ON RATE OF MOTION OF GROUND WATER.

Some unusually good opportunities occurred during the work on Long Island of determining the rate of seepage below the impounding dams of some of the storage ponds which the Brooklyn waterworks has established north of the conduit line referred to in the opening pages of this chapter. The batteries of driven wells which have been placed a few hundred feet south of nearly all of these ponds were quiescent during the summer of 1903 , as the heavy rains furnished a sufficient quantity of surface water, and the auxiliary supply from the wells was not drawn upon, as usual, during July and August. At station No. 5, below East Meadow Pond and somewhat within its line of seepage (see fig. 58), the normal velocity of the ground water is 5.4 feet per day. At station No. 7, just north of the pond, the velocity was 2.6 feet per day. It seems clear that the natural velocity at these points, if the influence of the dam and pond were removed, would be about 4 feet per day. The velocity at station No. 6, located but a few feet from No. 5, at a depth of 34 feet, was 5 feet per day, as compared with 5.4 feet per day at a depth of 22 feet. The dam has the effect of making the water table nearly level in the immediate neighborhood of the pond, and also of greatly augmenting the slope of the water table for a short distance below the pond. The lower velocity above the pond and the higher velocity below the pond correspond with these facts. When there was no flow over the waste weir of the dam I measured the flow of the small 
stream which rises below the dam at the bridge marked "A" in fig. 58 . On July 10 this flow was 1.2 second-feet, practically all of which represented seepage water from the reservoir.

This amount, 1.2 second-feet, or 103,680 cubic feet per day, represents the amount of water that would flow through a bed of sand 30 feet deep and 1,000 feet wide at a velocity of 1 foot per day, the porosity of the sand being supposed equal to one-third. The normal velocity of the ground water is augmented, as shown by the measurement quoted above, by somewhat more than 1 foot per day. The width of the lower end of the pond, or the length of the earthen dam, is about 1,400 feet; basing the estimate on this minimum length and on a minimum depth of 30 feet, and augmented velocity of 1 foot per day, gives a minimum esti-
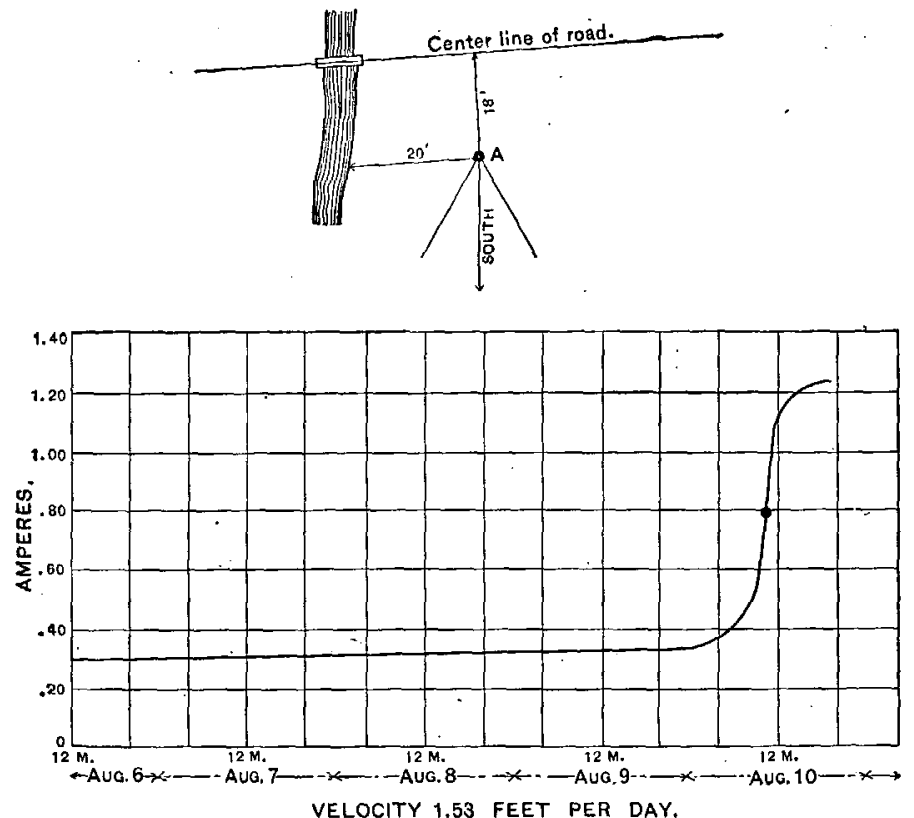

Fig. 52.-Diagram showing velocity and direction of flow of underground water at Bellevue road (station 15).

mate of the seepage from the dam of 1.6 second-feet; since 1.2 feet are known to actually come to the surface to feed the stream below the dam, it is evident that this estimate of seepage is a minimum. It seems evident that a considerable volume of seepage water could be recovered, without seriously lowering the water plane, by extending the line of driven wells to the east of the present terminus a distance of 600 or 700 feet.

A test well was driven in the lower south end of East Meadow Pond to a depth of 10 feet to determine the pressure gradient of ground water beneath the surface of the pond. The water in this test well stood about 1 foot lower than the water in the pond itself, showing a slope of the water plane, or a hydraulic gradient, of 7 feet to a mile. These facts are shown in fig. 59 (p. 113). 
The gradient of the water plane below the dam-that is, between the dam and station No. 5-was 17 feet to the mile, so that the velocities to be compared are:

Pressure gradients and velocities above and below East Meadow Pond, Long Island.

\begin{tabular}{|c|r|r|}
\hline Station. & $\begin{array}{c}\text { Gradient of } \\
\text { water piane } \\
\text { per mile. }\end{array}$ & $\begin{array}{c}\text { Velocity of } \\
\text { ground water } \\
\text { per day. }\end{array}$ \\
\hline No. 7, above pond........... & Feet. & Feet. \\
No. 5, below pond.......... & 7 & 2.6 \\
\hline
\end{tabular}

These results check very favorably, especially when it is considered that the, gradient above or north of station No. 7 was probably 10 or 12 feet per mile, which
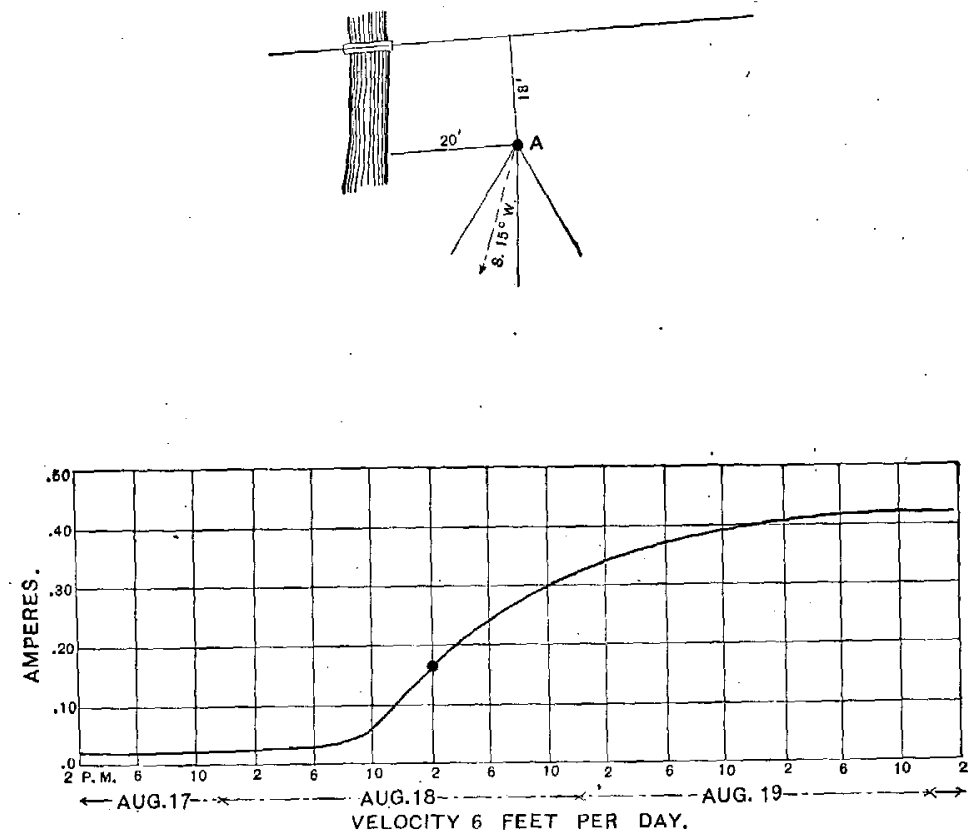

FIG. 53.-Diagram showing velocity and direction of flow of underground water at Bellevue road (station $15 \mathrm{x}$ ).

would make the effective gradient at this station somewhat greater than 7 feet per mile.

Very striking results were obtained below the dam at the Wantagh Pond, where measurements were undertaken especially to determine the rate of seepage. The dam of the Wantagh Pond runs parallel to the right of way of the Long Island Railroad about 75 feet north of the latter, and has an extreme length of 500 or 600 feet. About 150 feet south of the railroad, downstream from the reservoir, the city of Brooklyn began in the summer of 1903 the construction of an infiltration gallery, consisting of a line of 36 -inch double-strength tile laid at a depth of 16 feet below the water plane. It is purposed to extend this gallery for a mile east and west from the Wantagh pumping station. Stations Nos. 13, 16, and 17 were 
established for the purpose of measuring the normal ground-water velocities at the depth (16 feet) of the purposed gallery. Two of these stations are immediately south of the pond and in the apparent direct line of seepage, while station No. 17 is located slightly to the east of the edge of the pond, and, as seems evident from fig. 60, just on the edge of the main influence of seepage from the ponds. The seepage velocities at stations No. 13 and 16 turned out to be enormous, the velocity at No. 13 being 96 feet per day, S., while at station No. 16 it was 77 feet per day, about $\mathrm{S} .30^{\circ} \mathrm{E}$., the deflection being toward the neighboring stream as shown in fig. 60 . These velocities are the highest the writer has determined, and may be regarded as record-making rates for the horizontal motion of ground waters. Both measurements were made with the recording instruments; by consulting the curves in figs. 54, 55, and 56 it will be noted that each curve has two maximum points, which must correspond to the velocities in two distinct layers of gravel. The secondary velocity for station No. 13 was 7.4 feet per day and for station No. 16, 11.3 feet per day. A very striking verification of the fact that the high movements here found were due to the escape of water from the pond will be noted when the temperatures of the waters in the wells of these stations are compared with the temperatures of the water in the pond and the water in wells outside of the influence of seepage from the pond. Practically all water from wells on Long Island has a temperature between $58^{\circ}$ and $60^{\circ} \mathrm{F}$. In the present case, the temperature of water drawn from $\mathrm{H}$. A.

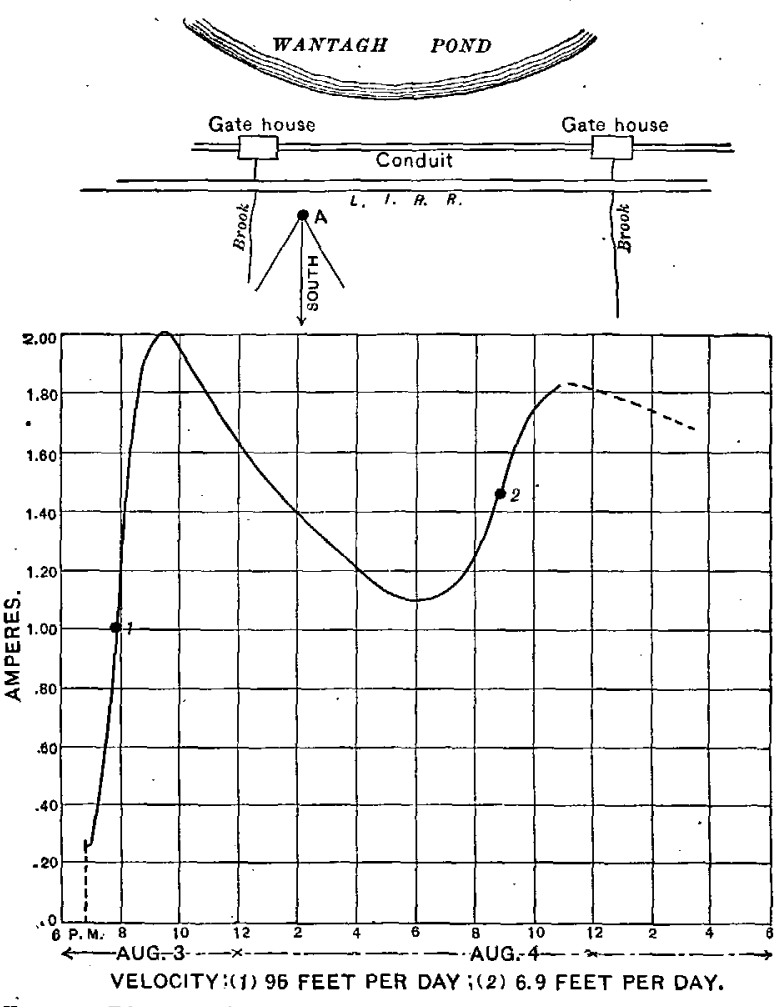

FIG. 54.-Diagram showing velocity and direction of flow of underground water south of Wantagh Pond at station 13.

Russell's well, 22 feet deep, located just west of the Wantagh Pond (see fig. 60), was $59^{\circ} \mathrm{F}$. on August 8, 1903, while the temperature of water from well $\mathrm{D}$, of station No. 17, just east and slightly below the pond, was $61.2^{\circ} \mathrm{F}$. on August 11, 1903. This well was 20 feet deep, the bottom being at the same depth as the wells of stations Nos. 13 and 16 . The temperature of water in the pond varies more or less, especially the temperature of the surface layer. The temperature of the pond water on August 8, a cloudy day, was $72.5^{\circ} \mathrm{F}$., and on July 30, a sunny day, $80^{\circ} \mathrm{F}$. The temperature of water from the wells of station No. 13 was $65.8^{\circ} \mathrm{F}$. on July 30 , and that from the wells of station No. 16 on August 8 was $69.5^{\circ} \mathrm{F}$. These high temperatures at stations Nos. 13 and 16 show that a large portion of the moving ground water must come directly from the pond, and 
that the rate of motion is so great that the ground water has not time to be reduced to the normal temperature of the ground.

At station No. 17 the water had a velocity of 10.6 feet per day in a direction S. $30^{\circ} \mathrm{W}$, and a temperature of $61.5^{\circ} \mathrm{F}$. The ground water at this point is probably not entirely free from the seepage water from the pond. The direction of flow, the velocity, and the temperature of the water all indicate, however, that a considerable part of the water is the natural underflow which at this point is diverted toward the lowland occupied by the streams below the pond.

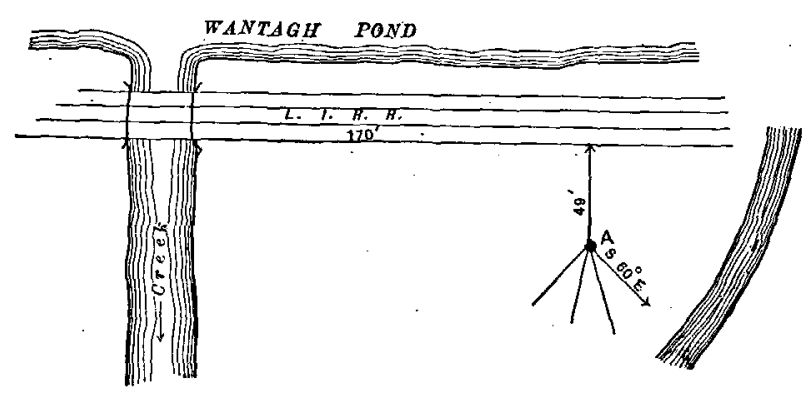

There can be no doubt but that the proposed infiltration gallery will intercept a large amount of seepage water from the pond which at present runs entirely to waste. The amount of seepage in the first 16 feet of depth is probably somewhat less than 3 second-feet per 1,000 feet of length of cross section, or about 2 million' gallons per twenty-four hours.

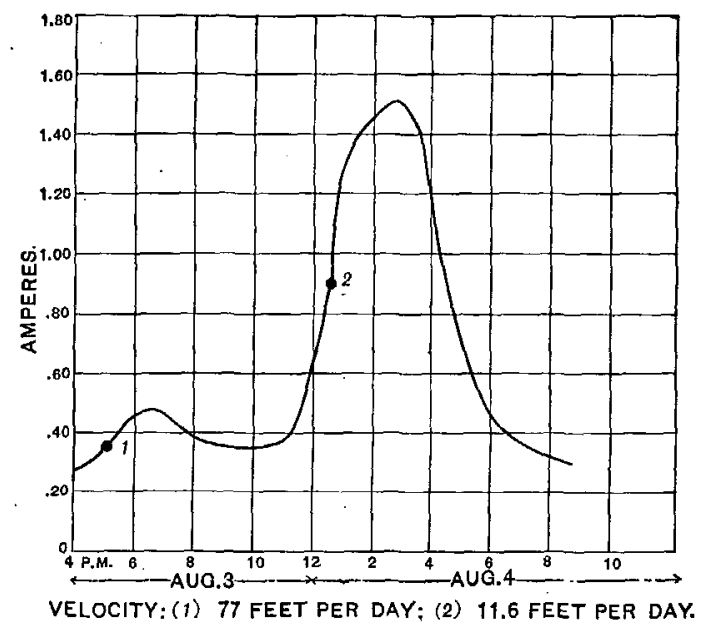

FIG. 55.-Diagram showing volocity and direction of flow of underground water at Wantagh Pond (station 16x)

At station No. 21, located just above the Wantagh. Pond, the velocity at a depth of 17 feet was 21.3 feet per day in a direction $60^{\circ}$ east of south. This station is near the west bank of the main brook that feeds the pond, and the greater share of the ground water at this point percolates into the bed of the stream. The true underflow at this point can be found by taking the southerly component of this velocity, which gives 10.6 feet per day. The temperature of the ground water at this point was $58^{\circ} \mathrm{F}$.

The increase of underflow rate at the Wantagh Pond from 10.6 feet per day to 96 and 77 feet per day, as compared with velocities above and below East Meadow Pond of 2.6 and 5.3 feet per day, respectively, are easily understood whon the material constituting the bottom of the ponds is inspected. The material of the bed of the pond at Agawam is good, the soil being fine and compact, while at Wantagh the bottom of the pond is very sandy, in some places having a closer resemblance to a filter bed than to a puddled floor. 
EFFECT OF PUMPING ON RATE OF MOTION OF GROUND WATER.

Through the courtesy of Mr. I. M. De Varona, an excellent opportunity was furnished the writer of making some observations upon the influence of pumping upon the normal rate of motion of ground water in the neighborhood of some of the Brooklyn driven-well stations. For this special purpose, the pumping stations at Agawam and Wantagh, which had been idle since December, 1902, were started up for two days each in August, 1902. Agawam was operated continuously from 7 a. m., August 19, to 7 a. m., August 21, and Wantagh was operated from 7 a. m., August 22, to 7 a. m., August 24. At the Agawam station observations were made at station No. 5 , by means of the recording instrument. Well A was charged at 4 p. m., August 19, or after nine hours of continuous pumping, an interval supposed to be sufficient for the establishment of the maximum rate of flow of the ground water, although, of course, the cone of depression near the wells would still be changing quite rapidly.

Station No. 5 is 30 feet north of the intersection of the chief suction mains communicating with the line of driven wells and 12 feet east of the central discharge main (see fig. 58). The depth of the test wells was 22 feet, while the depth of the 30 supply wells of the Agawam station system varies from 30 to
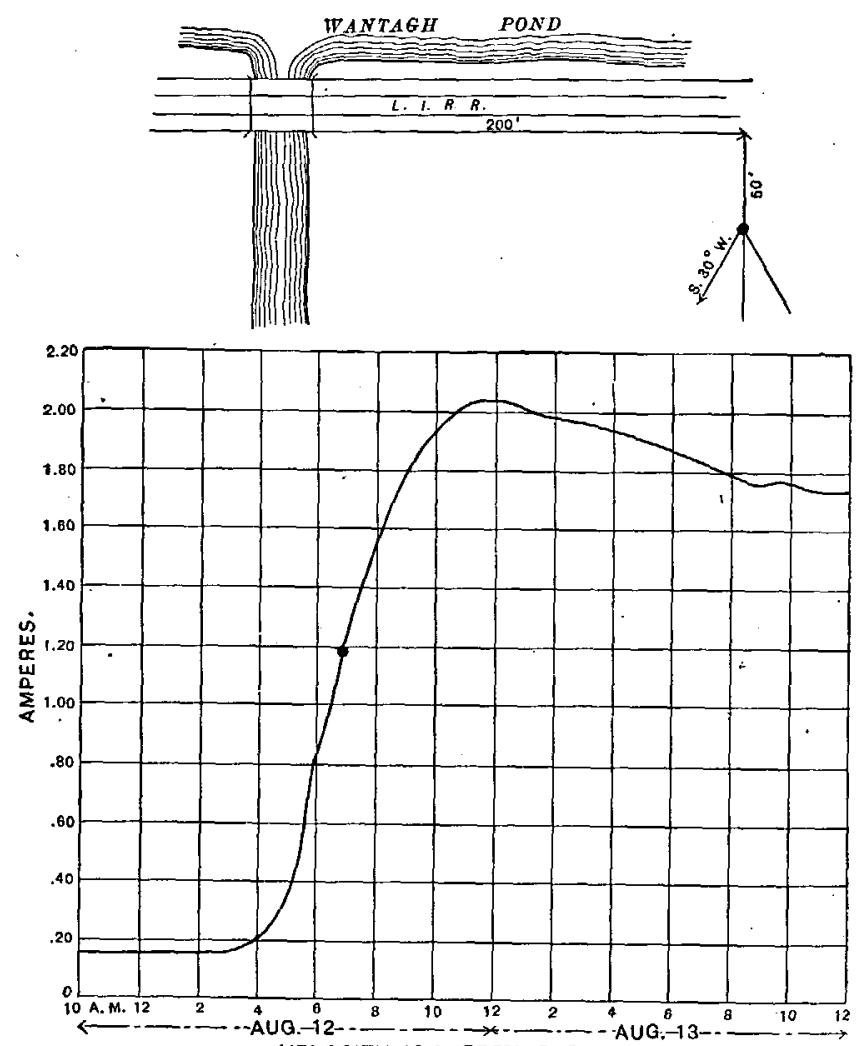

VELOCITY 10.6 FEET PER DAY.

FIG. 56.-Dingram showing velocity and direction of flow of underground water at Wantagh Pond (station 17).

105 feet, the wells being arranged at intervals of 50 feet along two suction mains, each 750 feet long.

The rate of pumping during the 48-hour test was very uniform, this average being 2,250,000 gallons per twenty-four hours. The vacuum at the pump was maintained at 24 inches, while that at the first well east of the engine house was 23.2 inches. The charge of the centrifugal pump was dropped from $4 \mathrm{p}$. m. to 4.40 p. m. August 19, during which time the vacuum fell to 7 inches. This was the only interruption during the test. 
The velocity determined at station No. 5 during the test was 8 feet per day, in a direction $\mathrm{S} .22^{\circ} \mathrm{E}$. The normal velocity at this station is 5.4 feet per day, $\mathrm{S}$. $8^{\circ} \mathrm{W}$., so that the influence of the pumping was to increase the velocity by 2.6 feet per day, or an increase of about 50 per cent. The actual velocity found and the percentage of increase are both very moderate, and indicate that the pumping station is not making an unreasonable draft upon the ground-water supply at this point.

The 30 wells of the Agawam supply station have screens each 10 feet long, or altogether about 730 square feet of screen. The maximum velocity of ground water as it enters these screens must be at the rate of 1,230 feet per day, since the

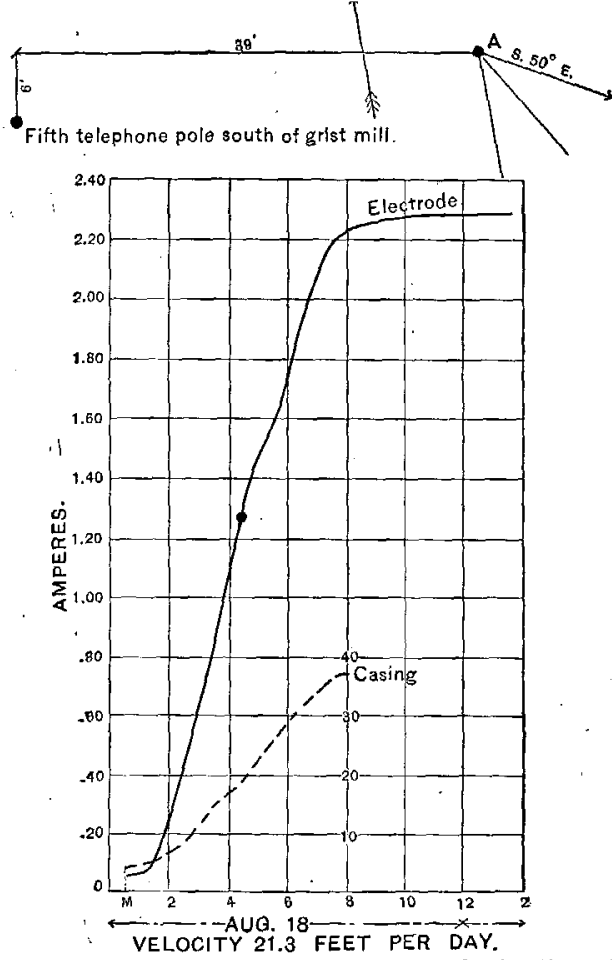

FIG. 57,-Diagram showing velocity and dircction of flow of underground water above Wantagh Pond at station 21 . actual pumpage was $2,250,000$ gallons or 300,000 cubic feet per twenty-four hours. The mean velocity in the area, 10 by 1,500 feet cross section, immediately drawn upon by the wells (the supply wells covering an expanse of about 1,500 feet) was about 30 feet per day. The reduction of this rate to 2.6 feet per day represents a ratio of reduction of 11 to 1 , which could be taken care of by a depth of 110 feet in the water-bearing gravels, without going outside of the 1,500-foot east and west line of the driven wells.

To put this in another way: The daily pumpage of 300,000 cubic feet of water could be supplied by the normal rate of motion of the ground water at this point (5.4 feet per day) through a cross section of 510,000 square feet, or, say, 100 feet deep by 1 mile wide. To supply this amount of water, if removed from the ground on each of the 365 days in a year, would require 1 foot of rainfall on 12 square miles of catchment area, or 18 inches of rainfall on 8 square miles of catchment area. Since the watershed is at least 12 miles north of the station, there is ample area to supply this amount of ground water, and the rate of removal at the Agawam station must, therefore, be regarded as moderate.

The observations at Wantagh pumping station were made on August 21 and 22. The pumping at this station began at 7 a. m., August 21 , and continued forty-eight hours at the uniform rate of 4,366,000 gallons per twenty-four hours. The water at this station is drawn from 48 driven wells, arranged on three lines of suction mains, as shown in fig. 60. The east-west expanse of the two chief lines of wells is about 1,500 feet. The wells of this station are of two different typesshallow wells of depth of about 24 feet; and deeper wells, extending below an impervious bed to depths of from 60 to 112 feet. These latter wells have an artesian 
head of 3 or 4 feet, and when the pumping plant is idle the water from the deep wells flows into the suction main and into the shallow wells, from the latter of which it escapes into the sands and gravels of the upper zone of flow, raising abnormally the zone of saturation.

An attempt was made on June 24 to measure the rate of motion of the ground water at station No. 2, situated 17 feet west of the chief discharge pipe, and 300 feet north of the intersection of the main suction pipes from the driven wells, as shown in fig. 60 . The attempted measurement was a failure, it not being known

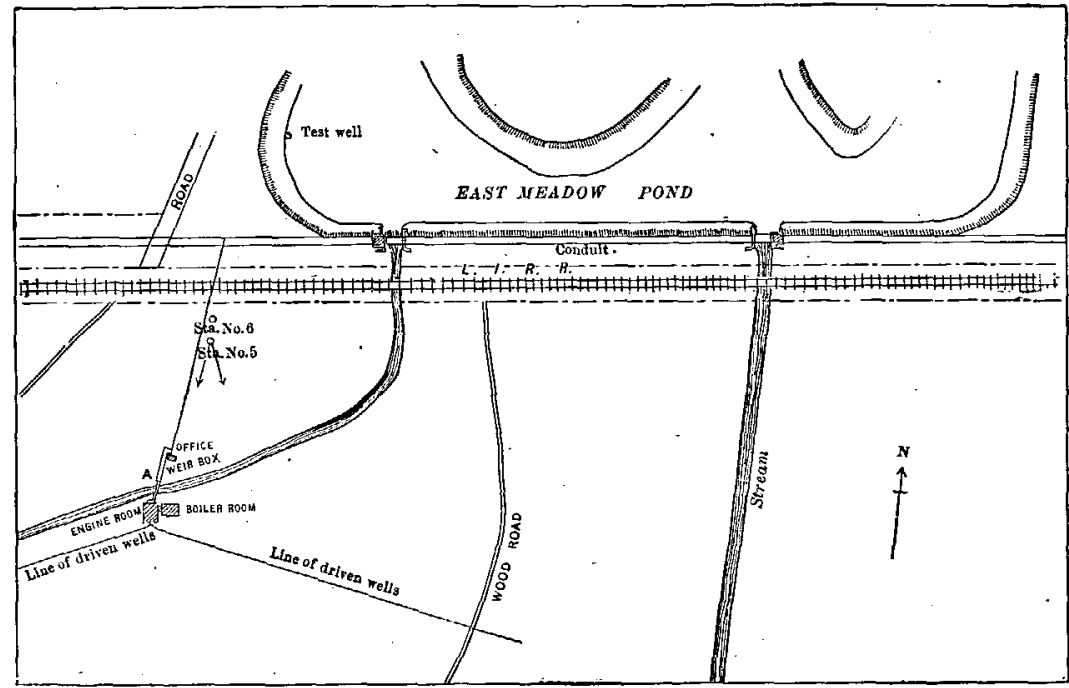

Fig. 58.-Map showing locations of stations 5 and 6 with reference to Aguwam pumping station and East Meadow Pond.

at the time that the discharge from the numerous artesian wells was entering the surface layers of gravels and hence interfering with the normal flow in these gravels. The ground water at station No. 7 was, on account of this situation, either entirely stationary or moving slightly toward the north. On August 21, well $A$, of station No. 2, was charged at 6 p. m., after eleven hours of continuous

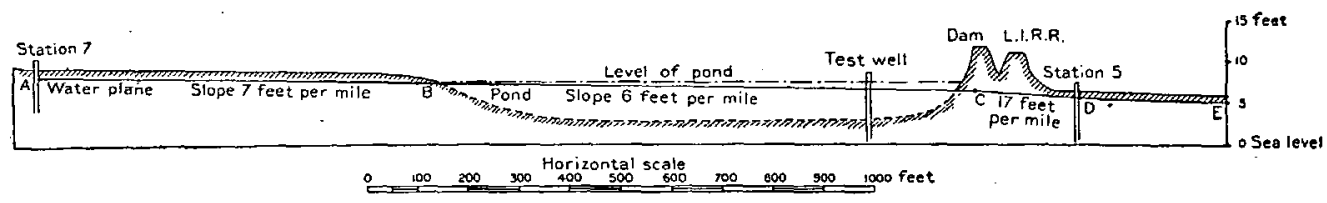

Fig. 59.-Vertical sections through stations 5 and 7 and test wells in Agawam Pond, shown in fig. 58

pumping from the driven wells. The velocity of the ground water observed was at the rate of 6 feet per day in a direction S. $10^{\circ} \mathrm{E}$. As this station is distant only 300 feet from the lines of driven wells, it is evident that the withdrawal of $4,366,000$ gallons or 582,000 cubic feet per twenty-four hours has not an excessive influence on the normal rate of motion of the ground water. The results at Wantagh compare very well with the results at Agawam and indicate that the driven-well plants have not exhausted the possibilities of ground-water developments. 
The writer uses the term "specific capacity" to designate the numerical expression of the readiness with which a well furnishes water to the pump. ${ }^{a}$ This quantity can be obtained by dividing the yield of a well by the amount that the water is lowered in the well. Thus, in the case of the Agawam wells, the discharge was 1,560 gallons per minute under a vacuum of 23.2 inches of mercury at the first well east of the engine house. This vacuum corresponds to a head of 26 feet of water, but the water in the wells was lowered only 20 feet by the pump. The specific capacity of the group of wells was therefore 78 gallons per minute. The area of all strainer surface in the wells was 730 square feet. From these data it can be readily estimated that the specific capacity of the Agawam wells was 0.11

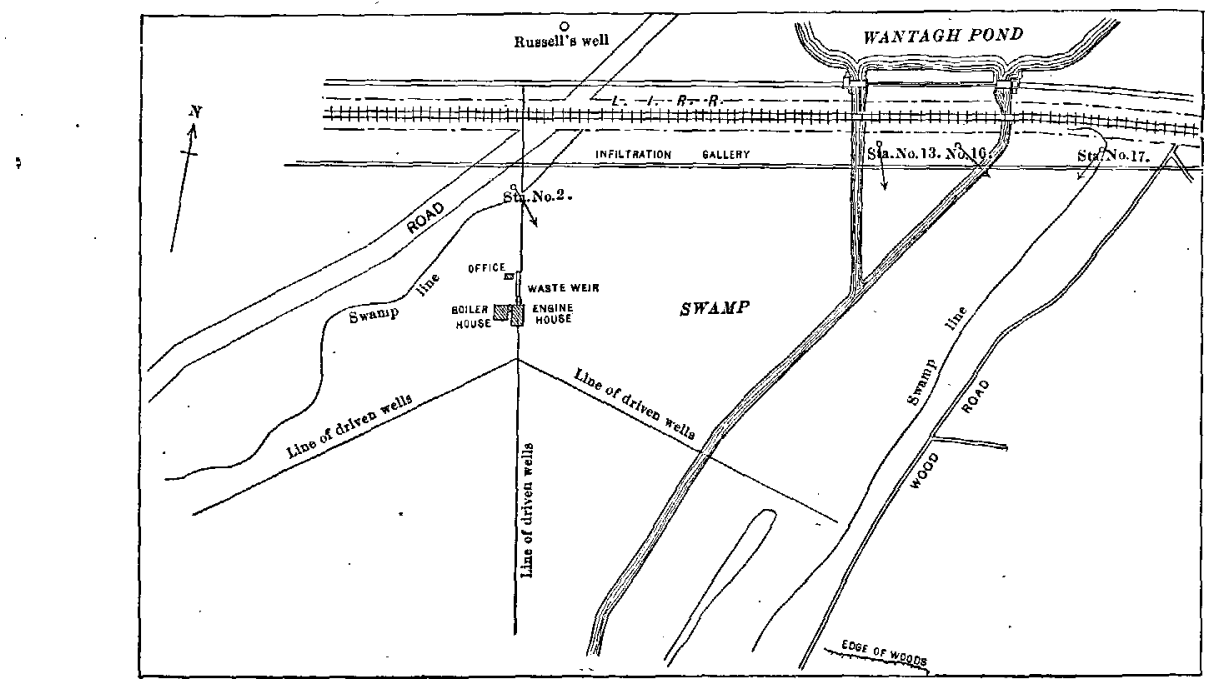

FIG. 60.--Map showing locations of stations 2, 13, 16, and 17, near Wantagh pumping sta.tion and Wantagh Pond.

gallon per square foot of well strainer under 1 foot head. This is a numerical expression of the degree of coarseness of the material in which the well is placed, combined, of course, with any resistance offered to the intake of water by the well strainer itself. At Wantagh station the discharge of 3,030 gallons per minute took place under a vacuum of 15.3 inches of mercury at the wells. The average head under which the water entered the wells was equivalent to 17.4 feet of water, from which the specific capacity is estimated to be 176 gallons per minute. The total strainer surface on the wells of this group amounts to 1,170 square feet, from which we conclude that the specific capacity per square foot of well strainer is 0.15 gallon per minute. This is nearly 40 per cent higher than at the Agawam wells. With carefully constructed wells of large diameter a minimum specific capacity of 0.15 gallon per minute per square foot of strainer can be depended upon for all wells in the Long Island watershed if properly designed strainers be used. 
CONCLUSION.

The very evident conclusion from observations on Long Island is that large amounts of ground water can still be obtained along the south shore of the island, espécially if deep wells of large diameter can be successfully bored. The writer has already called attention to the possibility of constructing 12-inch wells of the California or "stovepipe" type in the unconsolidated material met with to considerable depths on Long Island ${ }^{a}$ Such wells, several hundred feet in depth, with perforations opposite the best water-bearing material, would utilize a large part of the underflow which now escapes to the sea. The practicability and success of such wells in this locality seem very probable, but the actual construction of a test well is the only way of arriving at an entirely satisfactory conclusion.

a Slichter, C. S., The California or "stovepipe" method of well construction for water supply: Eng. News, Nov. 12, 1903, p. 429. 


\section{CHAPTER IV. \\ WELL RECORDS ON LONG ISLAND.}

Compiled by A. C. Veatci and Isaiah Bowman.

INTRODUCTION.

The presentation in a compact form of the data and detailed well records collected during the work on Long Island has proved a considerable problem. Presented in the text in connection with the geologic discussion, they furnish the necessary proof of many of the statements there made but so encumber the text that the mind loses itself in the mass of detail. Recourse has therefore been had to the presentation of all the well data in a compact table with notes giving such additional information as may be available. The arbitrary numbers assigned to the wells in the table correspond to those used in the index map (Pl. XXIV) and through the text in Chapters I, II, and V.. While an attempt has been made to indicate the geologic subdivisions in some of the records for a critical discussion of their geologic bearing, the reader is referred to the paper on the geology of Long Island, which will be published in a short time.

\section{ACKNOWLEDGMENTS.}

Thanks are due to Mr. I. M. De Varona, chief engineer of the Borough of Brooklyn, for access to some of the invaluable records collected by his department; to Mr. L. C. L. Smith, engineer in charge of the Borough of Queens, for many kindnesses and suggestions regarding that borough; to $\mathrm{Mr}$. Cord Meyer and $\mathrm{Mr}$. Edward Meyer, of the Citizens' Water Supply Company, and to Mr. Franklin B. Lord and Charles R. Bettes, of the Queens County Water Company, for much assistance in the study of the fluctuations of well waters.

From the commission on additional water supply, samples were received from the many shallow wells which they put down in their study of the position of the ground-water table. Descriptions of these samples will be found in the descriptive notes following and the resul ts of the sizing and filtration tests in Chapter V.

The well drillers on the island almost without exception rendered valuable assistance, and it is a great pleasure to acknowledge aid from the following sources:

Samuel H. Allen, well driver, 513 Broadway, Long Island City, N. Y.

Arthur \& 'Tuthill, well drivers, Cutchogue, N. Y.

Gilbert Baldwin, well driver, Woodmere, N. Y. 
William H. Beers, well driver, Wading River, N. Y.

Ralph B. Carter Company, artesian-well contractors, 47 Dey street, New York City.

Cole Brothers, artesian-well contractors, 102 Fulton street, New York City.

P. II. \& J. Conlan, artesian-well contractors, 253 Lafayette street, Newark, N. J.

Chester D. Corwin, artesian-well contractor, 198 Seventh avenue, New York City.

C. H. Danis, artesian-well driller, Cold Spring Harbor, N. Y.

N. W. Davis, artesian-well driller, Port Jefferson, N. Y.

Dollard Brothers, artesian-well drillers, Babylon, N. Y.

H. J. Dubois, artesian-well driller, Huntington, N. Y.

L. J. Dubois, artesian-well driller, Glen Cove, N. Y.

J. Elliott, tile wells, Pinelawn, N. Y.

John Fisher, well driller, Westbury, N. Y.

I. H. Ford, artesian-well contractor, 102 Fulton street, New York City.

C. L. Grant, artesian-well contractor, Hartford, Conn.

Elisha Gregory, artesian-well contractor, 123 Liberty street, New York City.

Paul Haller, well driver, Cedarhurst, N. Y.

W. J. Hancock, well driver, Baldwin, N. Y.

Thomas B. FIarper, artesian-well contractor, Jenkinstown, Pa.

John Heerdegen, 44-46 Broadway, New York City.

J. H. Herbert, tubular wells, Floral Park, N. Y.

Hudson Engineer and Contracting Company, water supply engineers, 92 William street, New York City.

E. K. Hutchinson, artesian-well driller, Oyster Bay, N. Y.

W. C. Jeagle, artesian-well driller, Hicksville, N. Y.

Isaac Kasteard, well digger, Port Washington, N. Y.

Thomas J. Kirk, well driver, Patchogue, N. Y.

J. W. Nichols, well driver, Manorville, N. Y.

R, F. Nichols, well driller, Oyster Bay, N. Y.

J. M. Peler, well driver, Manhasset, N. Y.

Phillips \& Worthington, artesian-well contractors, 136 Liberty street, New York City.

Pierce Well Engineering and Supply Company, artesian-well contractors, 136 Liberty street, New York City.

Charles E. Price, artesian-well driller, Sinithtown Branch, N. Y.

O. W. Quinn, well driller, 257 Seventh avenue, New York City.

J. B. Redwood, well digger, Smithtown, N. Y.

Robinson Brothers, well drivers, Center Moriches, N. Y.

T. B. Rogers, artesian-well driller, Stonybrook, N. Y.

Rust Well Machincry Company, artesian-well contractors, Ithaca, N. Y.

A. O. Ryder, well digger, 227 Franklin place, Flushing, N. Y.

George Schmidt, well driller, East -Williston, N. Y.

Ed. Schmidt, well driver, Mineola, N. Y.

Harry Strausbinger, well digger, Shelter Island, N. Y.

H. S. Stewart, well contractor, 354 South Highland avenue, East End, Pittsburg, Pa.

Stotthoff Brothers, artesian-well contractors, Flemington, N. Y.

Sweeney \& Gray, consulting engineers and well drillers, 81-85 Sixth street, Long Island City.

John Tart, driller, with Hudson Engineering and Contracting Company, 92 William street, New York City.

S. E. Terry, well borer, Holtsville, N. Y.

Andrew Vandewater, well digger, Hempstead, N. Y.

A. J. Velsor, well digger, Fort Salonga, N. Y.

Lawrence Verdon, well driller, Far Rockaway, N. Y., with Queens County Water Company.

F. K. Walsh, artesian-well driller, Woodmere, N. Y.

Frank Wankel, well driller, 535 Himrod street, Brooklyn, N. Y., with Hudson Engineering and Contract-

ing Company.

Alfred Wisson, well driller, Old Westbury, N. Y.

W. V. Young, artesian-well driller, Baiting Hollow, N. Y. 


\begin{tabular}{|c|c|c|c|c|c|}
\hline No. & Loeation. & $\begin{array}{l}\text { Coordi- } \\
\text { nates. }\end{array}$ & Owner. & Driller. & Authority. \\
\hline$*_{1}$ & Hoffmann Island. . & $1 \Lambda$. & $\begin{array}{l}\text { New York Quarantine Sta- } \\
\text { tion. }\end{array}$ & Elisha Gregory.. & Elisha Gregory...... \\
\hline$* 2$ & Fort Tafayette.. & $1 \mathrm{~B}$. & 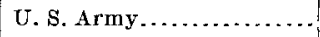 & & E. Lewis . . . . . \\
\hline$* 3$ & Bay Ridge...... & $1 \mathrm{~B}$. & $\begin{array}{l}\text { Brooklyn sewer depart- } \\
\text { ment. }\end{array}$ & & J. C. Meem, engineer. . \\
\hline$* 4$ & Bay Ridge Park. & $1 \mathrm{~B}$. & Blythebourne water Co... & & L. B. Ward $b \ldots$ \\
\hline$* 5$ & Brooklyn... & $1 \mathrm{~B}$. & Brooklyn Rapid Transit Co & Elisha Gregory .... & Brooklyn Rapid Transit Co \\
\hline 6 & ....do..... & $1 \mathrm{C}$. & Milliken Bros............ & Milliken Bros... & Mulliken Bros............. \\
\hline *7 & $\ldots$... do. & $1 \mathrm{C}$. & Barrett Manufacturing Co. & & Barrett Manufacturing Co. \\
\hline 8 & ....do.......... & $1 \mathrm{C}$. & P. H. Gill \& Sons.......... & & P. II. Gill \& Sons......... . \\
\hline 9 & .... do...... & $1 \mathrm{C.}$ & Crescent Chemical Co... & & Crescent Chemical Co. .... \\
\hline *10 & Governors Island. & $1 \mathrm{C}$. & U.S. Army... & P. H. and J. Conlan.... & Elisha Gregory .......... \\
\hline$* 11$ & Ellis Island....... & 10. & & $\begin{array}{l}\text { Pierce Well Engineering } \\
\text { Co. }\end{array}$ & Pierce Well Fngineering Co. \\
\hline$* 12$ & B́rooklyn.. & $1 \mathrm{C}$. & Long Island R. R.. & & C. M. Jacobs, engineer.... \\
\hline$* 13$ & New York-Brooklyn.. & $\begin{cases}1 & \mathrm{C} \\
2 \mathrm{C}\end{cases}$ & Rapid Transit.......... & & Chicf engineer.... \\
\hline 14 & Manhattan Beach...... & $2 \mathrm{~A}$. & Manhattan Beach IIotel.... & Dollard Bros.... & Dollard Bros.... \\
\hline 15 & $\begin{array}{l}\text { New Utrecht pumping } \\
\text { station. }\end{array}$ & $2 \mathrm{~B}$. & Brooklyn waterworks...... & 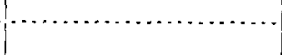 & L. B. Ward $b \ldots$. \\
\hline$* 16$ & $\begin{array}{l}\text { Gravesend pumping sta- } \\
\text { tion. }\end{array}$ & $2 \mathrm{~B}$. & $\ldots$. do... & W. D. Andrews \& Bro. . & $\ldots \ldots$ do $b, \ldots \ldots \ldots \ldots \ldots$ \\
\hline 17 & Gravesend.............. & $2 \mathrm{~B}$. & Brooklyn Borough Gas Co. & & Brooklyn Borough Gas Co. \\
\hline$* 18$ & Mapleton........ & $2 \mathrm{~B}$. & Pfalzgraf estate............ & & 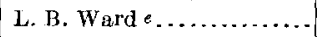 \\
\hline$* 19$ & Borough Park.... & $2 \mathrm{~B}$. & (West Brooklyn Water Co.) & & I. M. De Varona $f \ldots \ldots \ldots$ \\
\hline$*_{20}$ & West Brooklyn. . & $2 \mathrm{~B}$. & ....do... & & $\ldots$. do.g... \\
\hline 21 & Blythebourne... & $2 \mathrm{~B}$. & Blythebourne Water Co. & & ....do.f... \\
\hline 22 & Flatbush . . ............ & $2 \mathrm{~B}$. & Flatbush Water Co... & & L. B. Ward... \\
\hline$* 23$ & $\begin{array}{l}\text { Brooklyn: } \\
\text { 8th thenue and 18th } \\
\text { street. }\end{array}$ & $2 \mathrm{~B}$. & The Maltine Co... & Foster Pump Works. & The MaItine Co.... \\
\hline 24 & $\begin{array}{l}\text { 12th street and Go- } \\
\text { wanus Canal. }\end{array}$ & $2 \mathrm{C}$. & Brooklyn Union Gas Co.. & & Brooklyn Union Gas Co... \\
\hline$* 25$ & $\begin{array}{l}\text { 9th street and Go- } \\
\text { wanus Canal. }\end{array}$ & $2 \mathrm{C}$. & Tartar Chemical Co........ & Elisha Gregory ..... & Elisha Gregory ...... \\
\hline 26 & Hoyt and 5 th avenue & $2 \mathrm{C} .$. & Brooklyn Union Gas Co.... & & Brooklyn Union Gas Co.... \\
\hline 27 & $\begin{array}{l}\text { 3d avenue and } \mathbf{3 d} \\
\text { street. }\end{array}$ & $2 \mathrm{C} \ldots$ & Transit Development Co... & $\ldots \ldots \ldots+\ldots$ & $\begin{array}{l}\text { Samples in office of Transit } \\
\text { Development Co. }\end{array}$ \\
\hline$* 28$ & $\begin{array}{l}\text { 3d avenue, between } \\
\text { Degraw and Doug- } \\
\text { lass streets. }\end{array}$ & $2 \mathrm{C} \ldots$ & Brooklyn Union Gas Co... & & Brooklyn Union Gas Co... \\
\hline$* 29$ & $\begin{array}{l}\text { Dean street, near } \\
\text { Vanderbilt avenue. }\end{array}$ & $2 \mathrm{C} \ldots$ & Humbert \& Andrews...... & Chester D. Corwin. : & Chester D. Corwin ......... . \\
\hline$* 30$ & $\begin{array}{l}\text { St. Marks and Grand } \\
\text { avenues. }\end{array}$ & & Knox Hat Co....... & Elisha Gregory..... & Elisha Gregory...... \\
\hline$* 31$ & $\begin{array}{l}\text { Lewis and De Kalb } \\
\text { avenues. }\end{array}$ & $2 \mathrm{C} . \mathrm{.}$ & Borden Condensed Milk Co. & Chester D. Corwin. . & Chester D. Corwin.... \\
\hline & $\begin{array}{l}\text { * For additional dat } \\
\text { a Yield from a single } \\
\text { b Merchants' Associ } \\
\text { c Average per well fc } \\
\text { d See Table VIII. }\end{array}$ & 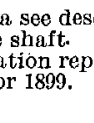 & & & 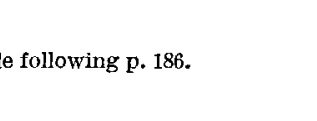 \\
\hline
\end{tabular}




\section{REPRESENTATIVE WELLS.}

wells on Long Island.

\begin{tabular}{|c|c|c|c|c|c|c|c|}
\hline $\begin{array}{l}\text { Diameter } \\
\text { of well. }\end{array}$ & $\begin{array}{l}\text { Depth of } \\
\text { of well. }\end{array}$ & $\begin{array}{l}\text { Depth of } \\
\text { principal } \\
\text { water } \\
\text { supply. }\end{array}$ & $\left\{\begin{array}{c}\text { Height of } \\
\text { water } \\
\text { above }(+) \\
\text { or } \\
\text { below }(-) \\
\text { ground } \\
\text { level. }\end{array}\right.$ & $\begin{array}{c}\text { Yield } \\
\text { per } \\
\text { minute. }\end{array}$ & $\begin{array}{l}\text { Geologic horizon of } \\
\text { water-bearing strata. }\end{array}$ & Remarks. & No. \\
\hline Inches. & Feet. & Feet. & Feet. & Gallons. & & & \\
\hline & $\begin{array}{r}1,000 \\
53\end{array}$ & $7 \overline{50}-1,000$ & …..... & 33 & Cambro-Silurian (?) . - & $\begin{array}{l}\text { Rock encountered at a depth of } 450 \text { feet. } \\
\text { Foundation test borings . . . . . . . . . . . }\end{array}$ & 1 \\
\hline & $40-90$ & & ...... & a $520-695$ & Wisconsin ....... & Sewer tunnel.................... & 3 \\
\hline 60 & 90 & & & c 139 & & See Table VIII. & 4 \\
\hline 240 & 90 & & & & & & \\
\hline $8-6$ & 1,503 & & & & Cambro-Silurian. . & Salt water $\ldots \ldots \ldots \ldots \ldots \ldots \ldots \ldots$ & 5 \\
\hline $2 \frac{1}{2}$ & 65 & 65 & -10 & 100 & Wisconsin ........ & Hard water; used only for cooling $\ldots . . . .$. & 6 \\
\hline 8 & 50 & 50 & -10 & & $\ldots$. do $\ldots . . . . .$. & Used for cooling purposes only ........... & 7 \\
\hline $4-2$ & 50 & $\cdots$ & -35 & $\cdots$ & $\ldots$. do........... & Water salty and hard $\ldots \ldots \ldots \ldots \ldots \ldots \ldots$ & 8 \\
\hline 6 & 56 & 52 & -15 & 40 & $\ldots$. do $\ldots . . . .$. & $\begin{array}{l}\text { Slightly brackish; not used for boiler or } \\
\text { drinking. }\end{array}$ & 9 \\
\hline $12-8$ & $1,822.5$ & 1,715 & Flows. & 15 & Cambro-Silurian. . & 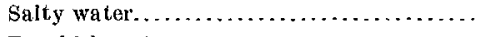 & 10 \\
\hline 8 & 1,400 & & ......... & & $\ldots \ldots \ldots \ldots \ldots \ldots \ldots$ & 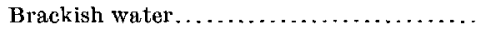 & 11 \\
\hline & 120 . & & & & & Test boring. . & 12 \\
\hline & $12-102$ & & & & $\left\{\begin{array}{l}\text { Pleistocene; Cambro- } \\
\text { Silurian. }\end{array}\right.$ & ... do $\ldots . . .$. & 13 \\
\hline & 40 & & -10 & ........... & Pleistocene...... & Coarse sand and gravel.............. & 14 \\
\hline 2 & -30 & & -3.6 & . $c 6.5$ & Wisconsin....... & Group of 120 driven wells . ............. & $d 15$ \\
\hline 2 & 50 & & -17.4 & c 15 & ....do.. & Group of 113 driven wells.... & $d 16$ \\
\hline & 14 & & & & & Blue clay at a depth of 14 feet............. & 17 \\
\hline & & & & & & Single well used for local water supply ..... & $d 18$ \\
\hline 8 & & & & & & $\begin{array}{l}\text { Reserve station of old West Brooklyn } \\
\text { Water Co. }\end{array}$ & 19 \\
\hline 96 & 65 & & -60 & & & $\begin{array}{l}\text { Principal station of old West Brooklyn } \\
\text { Water Co. }\end{array}$ & 20 \\
\hline 7 & 60 & & & & Wisconsin. & Reserve station; not used $\ldots . \ldots \ldots \ldots \ldots$. & $d 21$ \\
\hline $96-5$ & 44 & & & & & , & $i 22$ \\
\hline 5 & 55 & & -... & c 27 & Wisconsin .. & Group of 55 wells $\ldots \ldots \ldots \ldots \ldots \ldots \ldots$ & $a_{2 Z}$ \\
\hline $96-8$ & 177.5 & $|157.5-177.5|$ & -157.5 & 500 & Tisbury? ... & $\begin{array}{l}\text { Used for cooling and manufacturing; } \\
\text { slightly hard. }\end{array}$ & 23 \\
\hline 6 & 65 & & …. & $i 100$ & & 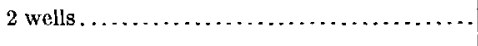 & 24 \\
\hline 8 & 143 & 140 & -6 & $i 1,200$ & Jameco?... & 2 wells; water not used for boilers. . . . . . . . & 25 \\
\hline $4 \frac{1}{2}$ & 40 & & $\cdots$ & $\mathbf{t} 20$ & & Group of 5 wells $\ldots \ldots \ldots \ldots \ldots \ldots \ldots$ & 26 \\
\hline 2 & $30-50$ & & ......... & $\cdots$ & $\ldots \ldots \ldots \ldots \ldots$ & Test borings for foundations............. & 27 \\
\hline $6-4 \frac{1}{2}$ & $72-90$ & & $\ldots \ldots$. & $i 38$ & Jameco?... & Group of 11 wells..... & 28 \\
\hline 6 & 98 & $81-98$ & $-7 \bar{j}$ & $40+$ & Tisbury?.. & & 29 \\
\hline 10 & 331 & 217 & & & Jameco.... & Bed rock at 331 feet. ........ & 30 \\
\hline$\cdot 8$ & 101 & & -67 & 100 & -Tisbury & ..... & 31 \\
\hline
\end{tabular}

¿Op.cit., p. 181.

$f$ History and Description of the Water Supply of Brooklyn, 1896, p. 139.

g. Op. cit., p. J38.
$i$ A verage of each well. 
TABLE XI.-Representative wells

\begin{tabular}{|c|c|c|c|c|c|}
\hline No. & Location. & $\begin{array}{l}\text { Coordi- } \\
\text { nates. }\end{array}$ & Owner. & Driller. & Authority. \\
\hline 32 & $\begin{array}{l}\text { Brooklyn-Continued. } \\
\text { Pulaski street and } \\
\text { Iewis avenue. }\end{array}$ & $2 \mathrm{C}$. & H: B. Scharmann \& Sons... & & H. B. Scharmann... \\
\hline$* 33$ & 254 Hart street...... & $2 \mathrm{C} \ldots$ & Excelsior Brewing Co. & Elisha Gregory ........ & Elishe Gregory $\ldots \ldots \ldots \ldots$ \\
\hline 34 & $\begin{array}{l}\text { Central avenue and } \\
\text { Grove street. }\end{array}$ & $2 \mathrm{c} \ldots$ & Jos. Epping. . .......... & $\begin{array}{l}\text { Picrce Well Engineer- } \\
\text { ing Co. }\end{array}$ & Pierce Well Engineering Co. \\
\hline$* 35$ & $\begin{array}{c}\text { Forest street and } \\
\text { Evergreen avenue. }\end{array}$ & $2 \mathrm{C}$. & $\begin{array}{l}\text { S. Liebmann Sons Brewing } \\
\text { Co. }\end{array}$ & & Alfred Liebmann.... \\
\hline 36 & $\begin{array}{l}\text { Noll and Bremen } \\
\text { streets. }\end{array}$ & $2 \mathrm{C} \ldots$. & Obermeyer \& Liebmann. .. . & & E. Obermeyer...... \\
\hline *37 & $\begin{array}{l}\text { Bartlett stroet and } \\
\text { Harrison avenue. }\end{array}$ & $2 \mathrm{C}$ & Pfeizer Chemical co.. & C. D. Corwin ........... & C. J. Corwin.. \\
\hline *38 & $\begin{array}{l}\text { Bartlett street and } \\
\text { Flushing avenue. }\end{array}$ & 20 & ....do. . & Phillips \& Worthington. & Phillips \& Worthington... \\
\hline 39 & $\begin{array}{l}\text { Flushing and Frank- } \\
\text { lin a.venues. }\end{array}$ & $2 \mathrm{C}$. & Malcom Brewing Co ..... & Stothoff Bros... & Stotthoff Bros.a... \\
\hline *40 & 20-34 Ryerson street. & $2 \mathrm{C}$. & Merger \& Thrall (?) ....... & C. D. Corwin.... & C. D. Corwip..... \\
\hline$*_{4} 1$ & 163 Carlton avenue.. & $2 \mathrm{C} \ldots$ & Walter M. Debevoise....... & I. H. Ford ......... & I. H. Ford ............. \\
\hline$* 42$ & $\begin{array}{l}\text { Between Wallabout } \\
\text { and Gowanus. }\end{array}$ & $20 \ldots$ & $(-$ Johnson $) \ldots . .$. & $\ldots \ldots \ldots \ldots$ & W. W. Mather $b$... \\
\hline$* 43$ & $\begin{array}{l}\text { Clarke, Willow, and } \\
\text { Pineapple streets. }\end{array}$ & $2 \mathrm{C}$. & & & Arthur Holiick o...... \\
\hline 44 & 66 Water street..... & $2 \mathrm{C}$. & Sweeney Manufacturìg $\mathrm{Co}_{-}$ & I. H. Ford.... & T. H. Ford....$\ldots \ldots \ldots \ldots$ \\
\hline$* 45$ & Brooklyn Bridge... & $2 \mathrm{C}$. & & & $\begin{array}{l}\text { Iong Island Historical So- } \\
\text { ciety. }\end{array}$ \\
\hline$* 46$ & $\begin{array}{l}\text { Pearl and Front } \\
\text { streets. }\end{array}$ & $2 \mathrm{C}$. & Jones Bros... & & \\
\hline$* 47$ & $50 \mathrm{Jay}$ street.. & $2 \mathrm{C}$ & John W. Masury \& Son . & $\left\{\begin{array}{l}\text { Pierce Well Engineering } \\
\text { Co. }\end{array}\right.$ & $\begin{array}{l}\text { Pierce Well Engineering Co. } \\
\text { John W. Masury \& Son.... }\end{array}$ \\
\hline$* 48$ & John and Jay streets. & $2 \mathrm{C} \ldots$ & 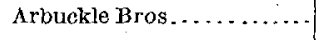 & H. S. Stewart..... & H. S. Stewart..... \\
\hline 49 & $\begin{array}{l}\text { B ridge and Ply- } \\
\text { mouth strets. }\end{array}$ & $2 \mathrm{C}$. & $\begin{array}{l}\text { Howard \& Fuller Brewing } \\
\text { Co. }\end{array}$ & & Howard \& Fuller...... \\
\hline 50 & Brooklyn Navy-Yard & $2 \mathrm{C}$. & 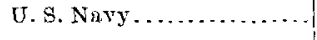 & & Civil engineer of navs-yard. \\
\hline$* 51$ & BrooklynNavy-Yard & $2 \mathrm{C} \ldots$ & ....do.... & F. Wankel.... & F. Wankel........... \\
\hline$*_{52}$ & Brooklyn Navy-Y $z$ rd & $2 \mathrm{C}$. & $\ldots$...do........ & .....do.... & ....do..... \\
\hline *53 & Brooklyn Navy-Yard & $2 \mathrm{C} \ldots$ & ....do................ & .... do................ & ... do $\ldots$. . . . \\
\hline *54 & Brooklyn Navy-Yard & $2 \mathrm{C} \ldots$ & ....do... & & Civil engineer of navy-yard. \\
\hline$* 55$ & 556 Kent avenue... & $2 \mathrm{C}$. & Brookiyn Union Gus Co. & & Brooklyn Union Gas Co... \\
\hline 56 & $\begin{array}{l}\text { Keap s treet and } \\
\text { Kent avenue. }\end{array}$ & $2 \mathrm{C}$. & Chrome Steel Works... & & T. I. Jones, treasurer... \\
\hline 57 & $\begin{array}{l}\text { Harrison and Broad- } \\
\text { way streets. }\end{array}$ & $2 \ldots$ & The J. H. Shults Co... & & The J. H. Shults Co......... \\
\hline 58 & $\begin{array}{l}\text { Leonard and Meser- } \\
\text { ole streets. }\end{array}$ & $20 \ldots$ & Burger Brewing Co... & & I. G. Burger ............... \\
\hline 59 & $\begin{array}{l}\text { Meserole and Hum- } \\
\text { bolt streets. }\end{array}$ & $2 \mathrm{C}$. & Congress Brewing Co... & & James D. Long, manager.... \\
\hline$* 60$ & $\begin{array}{l}\text { Bushwick and Mes- } \\
\text { erole avenues. }\end{array}$ & & Eastern Brewing Co.... & Chester D. Corwin.. & Choster D. Corwin .......... \\
\hline 61 & $\begin{array}{l}\text { White and Boerum } \\
\text { streets. }\end{array}$ & $2 \mathrm{C}$. & F. H. Klabfleisch Co. & & F. I. Klabfeiseh Co. \\
\hline
\end{tabular}

*For additional data see deseriptive notes, pp. 168 et seq. 
on Long Island-Continued.

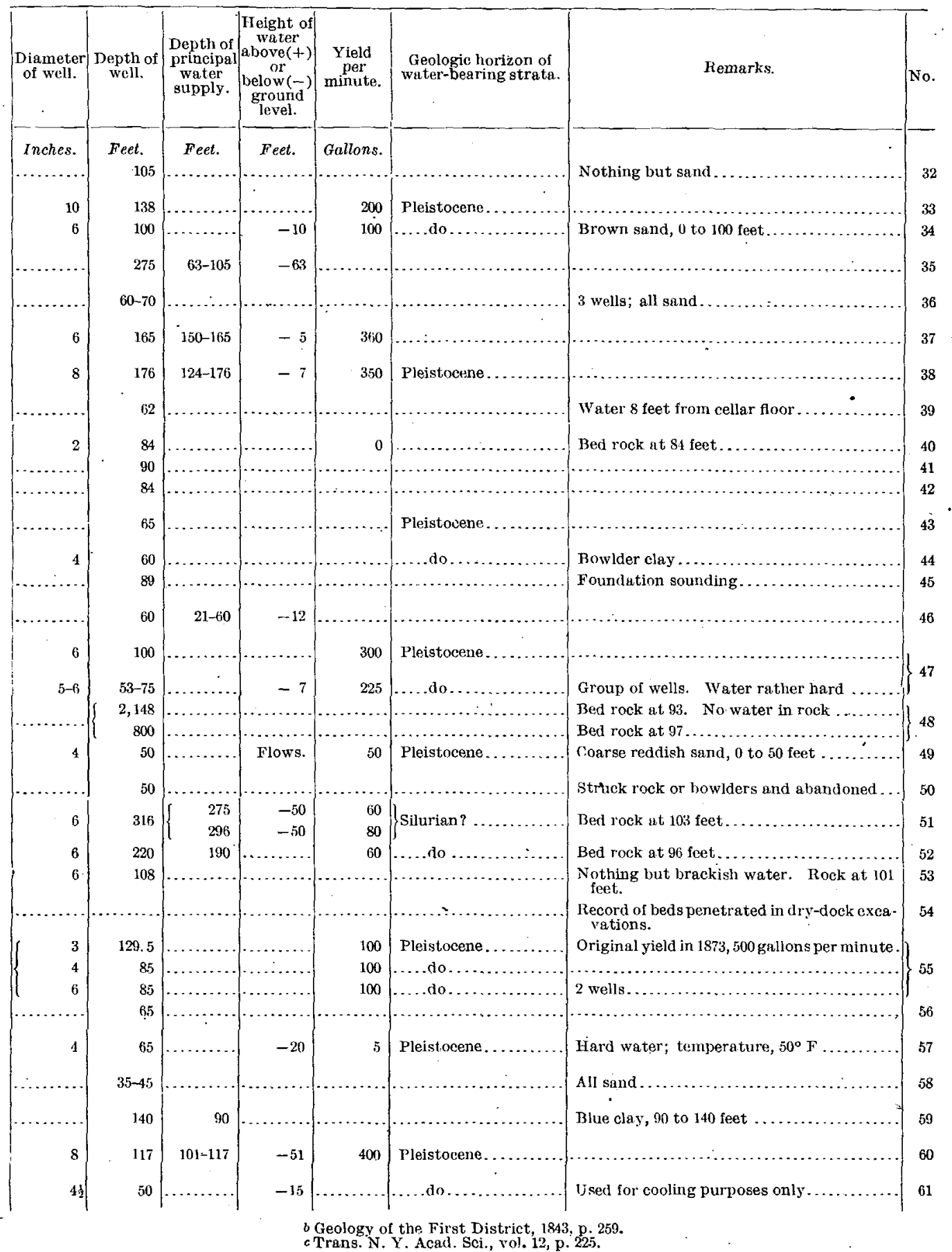


TABLE XI.-Representative wells

\begin{tabular}{|c|c|c|c|c|c|}
\hline No. & Loeation. & $\begin{array}{l}\text { Coordi- } \\
\text { nates. }\end{array}$ & Owner. & Driller. . & Authority. \\
\hline$* 2$ & $\left\{\begin{array}{l}\text { Brooklyn-Continued. } \\
\text { Ten Fyck street, } \\
\text { between Bushwick } \\
\text { and Florence }\end{array}\right.$ & $2 \mathrm{C}$. & N. Seitz's Sons.... & $\left\{\begin{array}{l}\text { Phillips \& Worthington } \\
\text { I. H. Ford..................... }\end{array}\right.$ & $\begin{array}{l}\text { Phillips \& Worthington... } \\
\text { I. H. Ford ................. }\end{array}$ \\
\hline 63 & $\begin{array}{l}\text { Montrose and - Sen- } \\
\text { eca streets. }\end{array}$ & $2 \mathrm{C}$. & Robinson Bros... & & Robinson Bros......... \\
\hline$*_{64}$ & $\begin{array}{l}\text { Maspeth and Gardi- } \\
\text { ner avenues. }\end{array}$ & $2 \mathrm{C}$. & Peter Cooper Glue Co. & & Peter Cooper Glue Co... \\
\hline$* 65$ & $\left\{\begin{array}{c}\text { Porter and Maspeth } \\
\text { avenues. }\end{array}\right.$ & $2 \mathrm{C}$. & Brooklyn Union Gas Co.. & & \\
\hline$* 66$ & $\begin{array}{l}\text { Meeker and Kings- } \\
\text { land avenues. }\end{array}$ & $2 \mathrm{C}$ & $\begin{array}{l}\text { Neptune Consumers Ice } \\
\text { Co. }\end{array}$ & I. H. Ford . . . . & C. Harty, foreman......... \\
\hline$* 67$ & $\begin{array}{l}\text { Meeker avenue, be- } \\
\text { tween North } \\
\text { Moore and Moni- } \\
\text { tor streets. }\end{array}$ & $2 \mathrm{C}$ & & Chester D. Corwin .. & Chester D. Corwin. . \\
\hline 68 & $\begin{array}{l}\text { Wythe and Metro- } \\
\text { politan avenues. }\end{array}$ & $2 \mathrm{C}$. & Streeter \& Dennison... & & Streeter \& Dennison..... \\
\hline 69 & $\begin{array}{l}\text { Kent avenue and } \\
\text { North 12th street. }\end{array}$ & $2 \mathrm{C} .$. & Brooklyn Union Gas Co.. & & Brooklyn Union Gas Co. \\
\hline 70 & $\begin{array}{l}\text { 110-11.8 North 1]th } \\
\text { street. }\end{array}$ & $2 \mathrm{C}$. & Hecla Iron Works.... & & Hecla Iron Works.... \\
\hline$* 71$ & $\begin{array}{l}99-117 \text { North } 11 \text { th } \\
\text { street. }\end{array}$ & 20. & $\begin{array}{l}\text { Now York Quinine and } \\
\text { Chemical Co. }\end{array}$ & $\begin{array}{l}\text { The Rust Well Ma- } \\
\text { chinery Co. }\end{array}$ & $\begin{array}{l}\text { New York Quinine and } \\
\text { Chemical Co. }\end{array}$ \\
\hline$* 72$ & $\begin{array}{l}\text { Kent and } 12 \text { th } \\
\text { streets. }\end{array}$ & $2 \mathrm{C}$. & Standard Oil Co............ & & H. L. Pratt $\ldots \ldots \ldots \ldots \ldots$ \\
\hline 73 & $\left\{\begin{array}{c}\text { Noble and West } \\
\text { streets. }\end{array}\right.$ & $2 \mathrm{C}$ & $\left\{\begin{array}{c}\text { American Cordage an } \mathrm{d} \\
\text { Manufacturing Co. }\end{array}\right.$ & $\left\{\begin{array}{l}\text { P. FI. \& J. Conlan ...... } \\
\text { Flisha Gregory ......... }\end{array}\right.$ & $\begin{array}{l}\text { P. H. \& J. Conlan } a \ldots \ldots \\
\text { Elisha Gregory .......... }\end{array}$ \\
\hline 74 & $\begin{array}{l}\text { Fly Island, New- } \\
\text { town Creek. } \\
\text { Long Island City: }\end{array}$ & $2 \mathrm{C}$. & Empire Oil Refinery ........ & 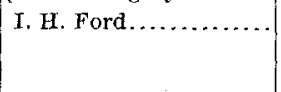 & I. H. Ford............. \\
\hline$* 5$ & Blissville.. & $2 \mathrm{C}$. & $\left\{\begin{array}{c}\text { Fleischmann Manufactur- } \\
\text { ing Co. }\end{array}\right.$ & & Jacob Blumer, chemist. . \\
\hline$* 76$ & Blissville... & $2 \mathrm{C}$. & Standard Oil Co.............. & & $\begin{array}{l}\text { Chas. O'Conner, superin- } \\
\text { tendent. }\end{array}$ \\
\hline 77 & Laurel Hill.. & $2 \mathrm{C}$. & Nichols Chemical Co.... & $\left\{\begin{array}{l}\text { P. H. \& J. Conlan ...... } \\
\text { Nichols Chemical Co }\end{array}\right.$ & P. H. \& J, Conlan C......... \\
\hline 78 & Laurel Hill. . ....... & $2 \mathrm{C}$ & General Chemical Co... & General Chemical co... & General Chemical Co....... \\
\hline$*_{79}$ & $\begin{array}{l}\text { New Calvary Ceme- } \\
\text { tery: }\end{array}$ & $20 \ldots$ & Calvary Cemetery........ & $\begin{array}{l}\text { Pierce Well Engineering } \\
\text { Co. }\end{array}$ & E. Lewis $d . \ldots \ldots \ldots \ldots$ \\
\hline$* 80$ & & $2 \mathrm{C}$. & & Commission ............ & Commission... \\
\hline 81 & Van Dam street. & $2 \mathrm{C}$. & $\left\{\begin{array}{c}\text { Department Water Supply, } \\
\text { gas and electricity. }\end{array}\right.$ & & I. B. Ward e.............. \\
\hline$* 82$ & & $2 \mathrm{C}$ & Flower estate.............. & $\begin{array}{l}\text { Pierce Well Engineering } \\
\text { Co. }\end{array}$ & Chas. D. Pierce, manager ... \\
\hline$* 83$ & & $2 \mathrm{C} \ldots$ & & Commission .............. & Commission $: . . . \ldots \ldots \ldots \ldots$ \\
\hline 81 & & $2 \mathrm{C} . \ldots$ & Flower estate............ & $\begin{array}{l}\text { Pierce Well Engineering } \\
\text { Co. }\end{array}$ & Pierce Well Fngineering co. \\
\hline$* 85$ & $\begin{array}{l}\text { Manhattan Bor- } \\
\text { ough to Thom- } \\
\text { son street. }\end{array}$ & $2 \mathrm{C}$. & $\begin{array}{l}\text { Pennsylvunia, New York } \\
\text { and Long Island R. R. }\end{array}$ & & Chief engineer... \\
\hline$* 86$ & Ncar depot..... & $2 \mathrm{C} .$. & Westinghouse Elentric Co... & W. F. Dohrman.... & W. E. Dohrman.... \\
\hline$* 87$ & 6th and West ....... & $2 \mathrm{C} \ldots$ & A.\& s. B. Coyson........... & Sweeney \& Gray ....... & Swceney \& Gray.......... \\
\hline$* 88$ & Vernon avenue..... & $2 \mathrm{C} \ldots$ & Jas. Gillis \& Sons......... & $\begin{array}{l}\text { Pierce Well Engineering } \\
\text { Co. }\end{array}$ & Pierce Well Engineering Co. \\
\hline \multicolumn{6}{|c|}{$\begin{array}{l}\text { * For additional data see descriptive notes, pp. } 168 \mathrm{et} \mathrm{seq} . \\
\text { a Ann. Rept. Geol. Survey New Jersey for } 1900,1901, \mathrm{p} .156 . \\
\text { h Originaliy -5. } \\
\text { c Ann. Rept. Geol. Survey New Jersey for } 1897,1898, \mathrm{p} .284\end{array}$} \\
\hline
\end{tabular}


on Long Island-Continued.

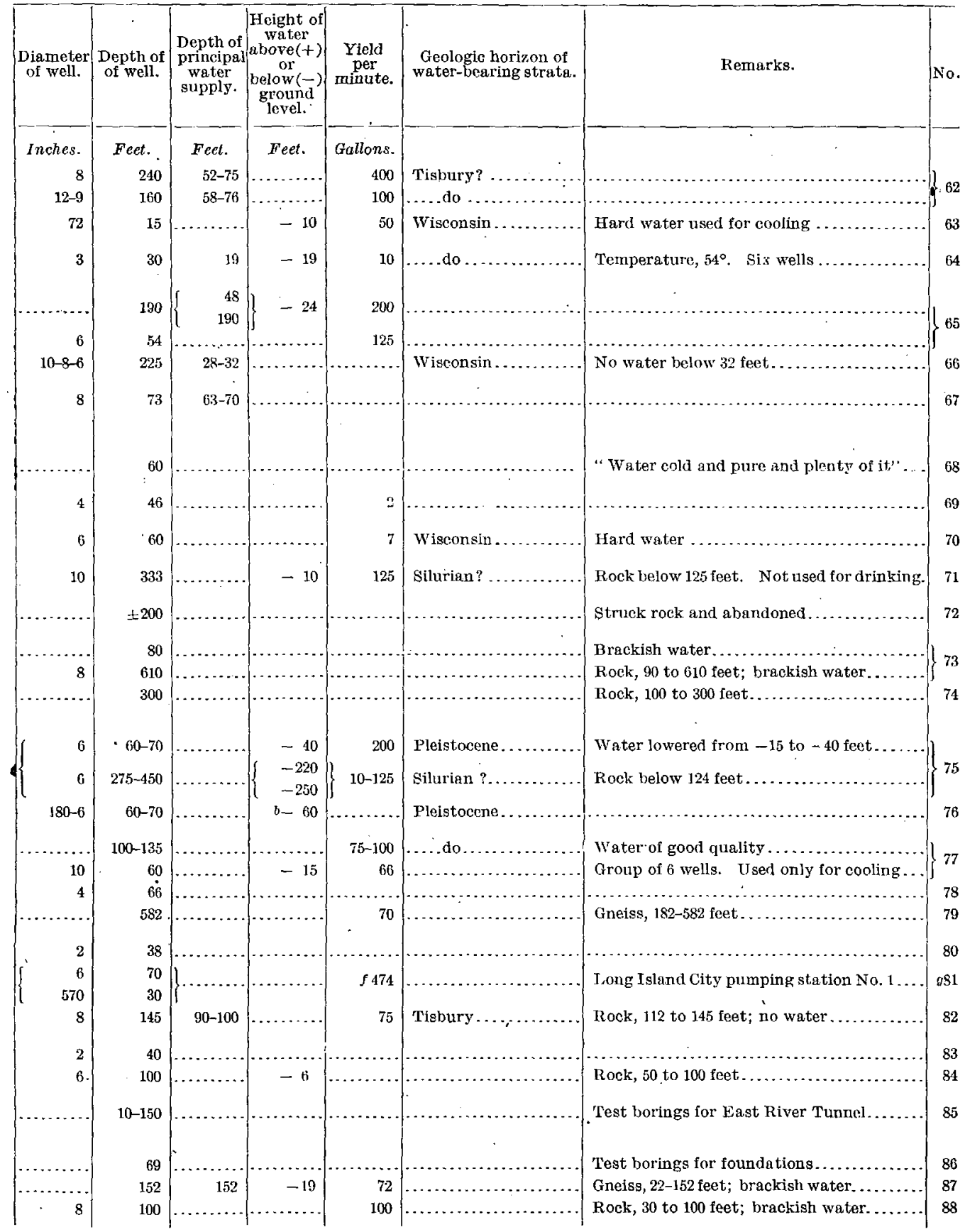

d Annals N. Y. Acad. Sei., vol. 3, p. 376; Bull. U. S. Geol. Survey No. 138, p. 34

e Merchants' Association report on water supply of the city of New York, 1900, table following p. 186. $f$ A verage from station for 1899 .

0 See Table VIII.

17116-No. 44-06-- 9 


\section{UNDERGROUND WATER RESOURCES OF LONG ISLAND, NEW YOKK.}

TABLE XI.-Representative wells

\begin{tabular}{|c|c|c|c|c|c|}
\hline No. & Loeation. & $\begin{array}{l}\text { Coordi- } \\
\text { nates. }\end{array}$ & Owner. & Driller. & Authority. \\
\hline *89 & $\begin{array}{l}\text { Long Island City-Con. } \\
\text { Vernon and Nott }\end{array}$ & 20. & C. A. Willey \& $\mathrm{Co}$.. & Sweeney \& Gray... & Sweeney \& Gray... \\
\hline 90 & $\begin{array}{l}\text { Skillman avenue atid } \\
\text { School street. }\end{array}$ & $2 \mathrm{C} \ldots$ & Mrs. Mary Ryan.. & & S. H. Allen... \\
\hline$* 91$ & $\begin{array}{l}\text { Skillman avenue and } \\
\text { School street. }\end{array}$ & $2 \mathrm{C} \ldots$ & Bragnaw estate. . & S. H. Allen, foreman. & .....do... \\
\hline *92 & 596 Jackson avenue. & $2 \mathrm{D} \ldots$ & Gus. Steinhert. . & Sweeney \& Gray.. & Sweeney \& Gray... \\
\hline$* 93$ & $\begin{array}{l}\text { Jackson a venue } \\
\text { und Hill street. }\end{array}$ & $2 \mathrm{D} \ldots$ & Long Island R. R... & & Long Island R.R.. \\
\hline *94 & $\begin{array}{l}\text { Long Island R. R. } \\
\text { und Remsen } \\
\text { street. }\end{array}$ & $2 \mathrm{D} .$. & Westcott Express Co. & S. H. Allen.. & S. H. Allen.. \\
\hline$* 95$ & $\begin{array}{l}\text { Millersljurg avenue } \\
\text { and Moore street. }\end{array}$ & $2 \mathrm{D} \ldots$ & - Smith... & ....do $\ldots . . . .$. & ....do do........... \\
\hline$* 96$ & $\begin{array}{l}\text { Buckley and Mid- } \\
\text { dleburg. }\end{array}$ & $2 \mathrm{D} \ldots$ & & Sweeney \& Gray. & Sweeney \& Gray.. \\
\hline 97 & & $2 \mathrm{D} \ldots$ & Ed. O'Kiefe.. & S. H. Allen.. & S. H. Allen. \\
\hline *98 & $\begin{array}{l}\text { Long Island R. R. } \\
\text { and Lowery street. }\end{array}$ & $l_{2} \mathrm{D}$ & Consumers Hygeia Ice Co. & $\left\{\begin{array}{l}\ldots \text { do ................ } \\
\text { Sweeney \& Gray. }\end{array}\right.$ & Sweeney \& (tray.. \\
\hline$* 99$ & $\begin{array}{l}\text { Long lsland } R \text {. R. } \\
\text { and Grove street. }\end{array}$ & & $\left\{\begin{array}{l}\text { Department water supply, } \\
\text { gas, and electricity. }\end{array}\right.$ & $\left\{\begin{array}{l}\cdots, \ldots \ldots \ldots \\
\text { Commission }\end{array}\right.$ & $\begin{array}{l}\text { L. B. Ward } b . . . \\
\text { A. S. Farmer ..... }\end{array}$ \\
\hline$* 100$ & $\begin{array}{l}\text { Steinway avenue } \\
\text { between Pierce } \\
\text { and Graham. }\end{array}$ & $2 \mathrm{D}$. & & S. II. Allen... & S. Н. Allen .............. \\
\hline$*^{* 101}$ & $\begin{array}{l}\text { 5th avenue between } \\
\text { Pierce and Gra- } \\
\text { ham. }\end{array}$ & $2 \mathrm{D} \ldots$ & & .....do.. & $\ldots$ do $\ldots$ \\
\hline$*_{102}$ & $\begin{array}{l}\text { Washington and } \\
\text { 4th avenues. }\end{array}$ & $2 \mathrm{D}$. & Frank Froellich. & $\ldots$. do ..... & $\ldots$...do... \\
\hline 103 & $\begin{array}{l}\text { 2d avenue between } \\
\text { Pierce and Wash- } \\
\text { ington avenues. }\end{array}$ & $2 \mathrm{D} \ldots$ & Martin Hummel... & .....do..... & $\ldots$. do $\ldots . .$. \\
\hline$* 04$ & $\begin{array}{l}\text { Pierce avenue and } \\
\text { Crescent street. }\end{array}$ & $2 \mathrm{D} \ldots$ & Mrs. Wonder...... & .....do........ & ....do... \\
\hline *105 & $\begin{array}{l}\text { Williams street and } \\
\text { Beebe avenue. }\end{array}$ & $2 \mathrm{D} \ldots$ & W. J. Matherson \& Co.... & C. C. Vermenle.. & W. J. Matherson \& Co. \\
\hline *106 & $\begin{array}{l}\text { Ely between Payn- } \\
\text { tar and Beebe } \\
\text { avenues. }\end{array}$ & $2 \mathrm{D}$ & & S. H. Allen... & S. H. AlJen .. \\
\hline 107 & $\begin{array}{c}\text { Hancock avenue near } \\
\text { Bodine street. }\end{array}$ & $2 \mathrm{D}$ & Emken Chernical Co.. & Sweeney \& Gray... & Sweeney \& Gray.. \\
\hline 108 & 337 Vernon avenue. & $2 \mathrm{D} \ldots$ & Young \& Metzner. & .... do. . & .....do. \\
\hline 109 & $\begin{array}{l}\text { Vernon and Harris } \\
\text { avenues. }\end{array}$ & $2 \mathrm{D} \ldots$ & D. G. Morrison... & $\ldots$...do... & .....do... \\
\hline$* 110$ & 401 Vernon avenuc. & $2 \mathrm{D} \ldots$ & $\begin{array}{l}\text { New York Architectural } \\
\text { Terra Cotta Co. }\end{array}$ & ....do.. & $\ldots$...do... \\
\hline 111 & Vernon avenue ..... & $2 \mathrm{D} \ldots$ & $\begin{array}{l}\text { John Good Cordage and } \\
\text { Machine Co..................... }\end{array}$ & $\begin{array}{l}\text { Pierce Well Engineering } \\
\text { Co. }\end{array}$ & Pierce Wéll Engincering $c o$ \\
\hline 112 & Vernon avenue .... & $2 \mathrm{D} \ldots$ & Young Bag Co......... & ....do do..... & $\ldots$.do.... \\
\hline 113 & Vernon avenue ..... & $20 \ldots$ & East River Gas Co.......... & $\ldots \ldots$ do $\ldots$..... & ....do .............. \\
\hline 114 & 725 Vernon avenue. . & $2 \mathrm{D} \ldots$ & Witherspoon \& Son..... & Sweeney \& Gray .. & Sweeney \& Gray... \\
\hline$*_{115}$ & & $2 \mathrm{D}$. & New York Asbestos Co.. & F. W. Miller... & \\
\hline$*_{116}$ & $\begin{array}{l}\text { Broadw a y and } \\
\text { Academy street. }\end{array}$ & $2 \mathrm{D}$ & & Sweeney \& Gray. & Sweeney \& Gray ..... \\
\hline${ }^{* 117}$ & $\begin{array}{l}\text { 9th and Jamaica } \\
\text { avenues. }\end{array}$ & $2 \mathrm{D} \ldots$. & Rudolph Harek. & S. H. Allen.. & S. H. Allen. \\
\hline
\end{tabular}


on Long Island-Continued.

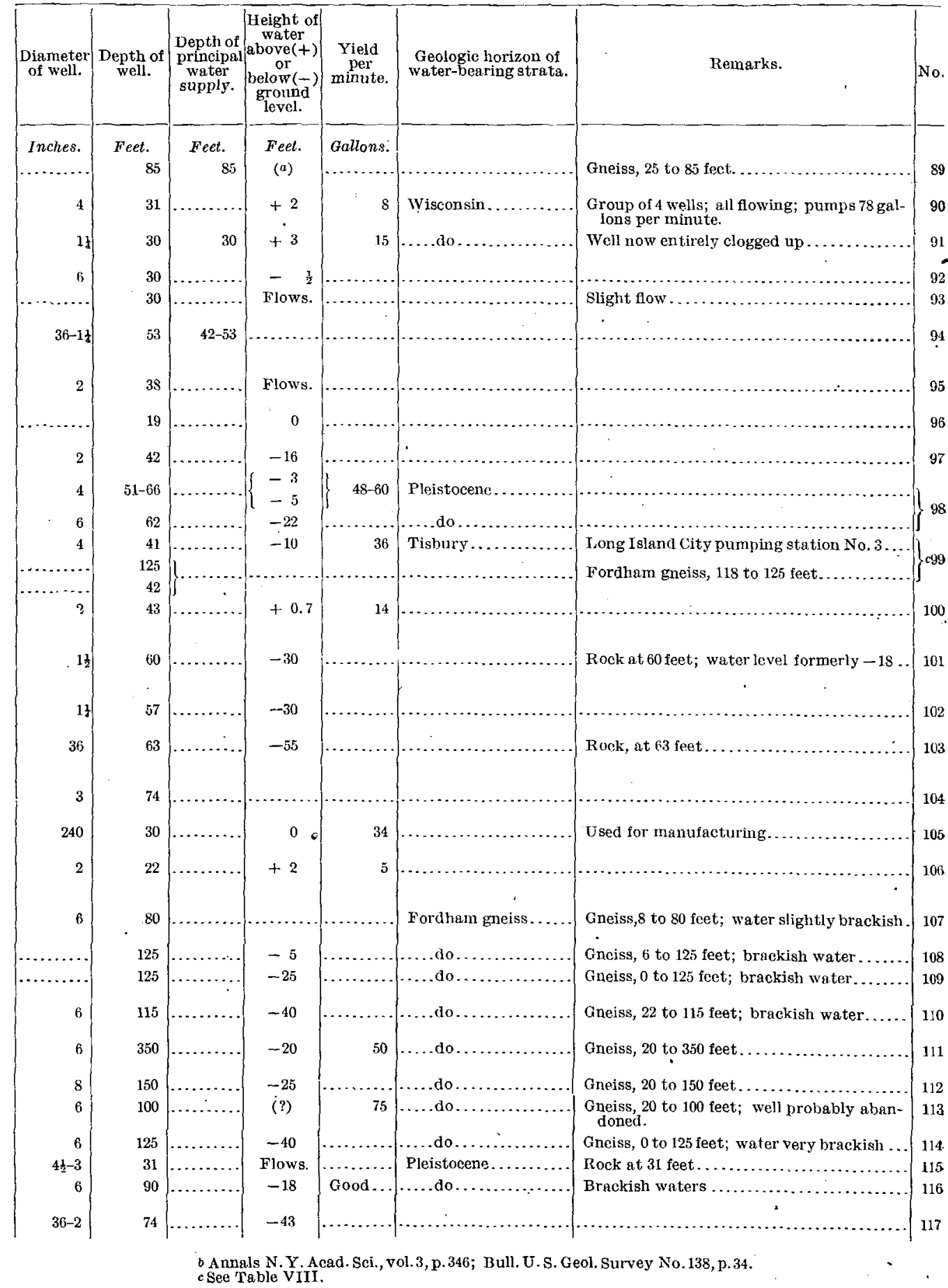




\section{UNDERGROUND WATER RESOURCES OF LONG ISLAND, NEW YORK.}

TABLE XI.--Representative wells

\begin{tabular}{|c|c|c|c|c|c|}
\hline No. & Location. & $\begin{array}{l}\text { Coordi- } \\
\text { nates. }\end{array}$ & Owner. & Driller. & Authority. \\
\hline$* 118$ & $\begin{array}{l}\text { Long Istand City-Con. } \\
408 \text { 9th a venue...... }\end{array}$ & $2 \mathrm{D} \ldots$ & & S. H. Allen. & S. H. Allen ... \\
\hline *119 & $\begin{array}{l}\text { Steinway and } J^{a-} \\
\text { maica avenues. }\end{array}$ & $2 \mathrm{D}$ & Dr. Harnier....... & $\ldots$.. do $\ldots=\ldots$ & $\ldots$ do........... \\
\hline$* 120$ & $\begin{array}{c}\text { Albert street and } \\
\text { Jamaica avenue. }\end{array}$ & $2 \mathrm{D}$. & Chas. Bickerman.... & $\ldots$ do........ & ....do................ \\
\hline$* 121$ & $\begin{array}{l}\text { 12th between Broad- } \\
\text { way and Jamaica } \\
\text { avenue. }\end{array}$ & $2 \mathrm{D}$ & & do.. & ....do.... \\
\hline$* 122$ & $\begin{array}{l}\text { Grand street and } \\
3 d \text { avenue. }\end{array}$ & $2 \mathrm{D}$. & Commission.... & & Commission..... \\
\hline * 233 & $\begin{array}{l}\text { Fim street and } \\
\text { Hopkins avenue. }\end{array}$ & $2 \mathrm{D} \ldots$ & Mrs. Fleming... & S. H. Allen... & S. H. Allen....... \\
\hline 124 & $\begin{array}{l}\text { Fulton and Halsey } \\
\text { streets. }\end{array}$ & $2 \mathrm{D} \ldots$ & Ward's ship yards.... & ... do .............. & 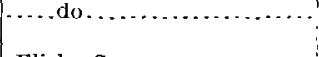 \\
\hline$* 125$ & $\begin{array}{l}\text { Munson and Or- } \\
\text { chard streets. }\end{array}$ & $2 \mathrm{DA}$ & Astoria Steel Co... & Elisha Gregory... & Elisha Gregory .............. \\
\hline$*_{\mathrm{L} 26}$ & $\begin{array}{l}\text { Steinway a venuc } \\
\text { and River road. }\end{array}$ & $2 \mathrm{D}$ & Consolidated Gas $\mathrm{Co}$. & & Phillips \& Worthington..... \\
\hline 127 & $\begin{array}{l}\text { Woolsey and Van } \\
\text { Alst avenues. }\end{array}$ & $2 \mathrm{D}$. & Newwitter \& Migel . . & & S. F. Allen ............. \\
\hline${ }^{*}+28$ & Barren Island......... & $\begin{array}{l}2 \mathrm{D} \\
3 \mathrm{~A}\end{array}$ & & S. F. Allen ............ & 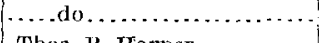 \\
\hline${ }^{* 1} 130$ & 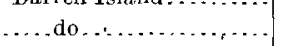 & $\begin{array}{l}3 \mathrm{~A} \ldots \\
3 \mathrm{~A}, \mathrm{C}\end{array}$ & $\begin{array}{l}\text { - MeKievery ............ } \\
\text { Thos. F. White Co . . . . }\end{array}$ & 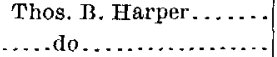 & $\begin{array}{l}\text { Thos. B. Harper ........... } \\
\text { Lewis Woolman } a . . . . . .\end{array}$ \\
\hline$* 131$ & 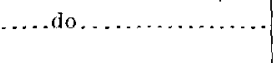 & $3 B \ldots$ & $\begin{array}{l}\text { New York Sanitary Utiliza- } \\
\text { tion Co. }\end{array}$ & ... do ................ & ....do. $a \ldots \ldots \ldots \ldots$ \\
\hline$* 132$ & ..... do & $3 \mathrm{~B} \ldots$ & 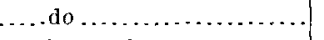 & Chester D. Corwin... & Chester D, Corwin......... \\
\hline$*^{*} 133$ & $\begin{array}{l}\text { Crook Island............ } \\
\text { East New York: }\end{array}$ & 3 B... & White Lead Co............. & ..... do .................. & , . do do ..................... \\
\hline$k^{*} 134$ & $\left\{\begin{array}{c}\text { Pennsylvania a ind } \\
\text { Stanley avenues. }\end{array}\right.$ & $3 \mathrm{~B} \ldots$ & $\left\{\begin{array}{l}\text { German American Improve- } \\
\text { ment Co. }\end{array}\right.$ & 1 & L. B. Ward . . . . . . . . \\
\hline *135 & $\begin{array}{l}\text { New I, ots road and } \\
\text { Fountain avenue. }\end{array}$ & $\int_{3} \mathrm{c}$. & $\left\{\begin{array}{l}\text { Department water supply, } \\
\text { gas, and electricity. } \\
\text {. }\end{array}\right.$ & & 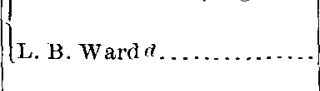 \\
\hline$* 136$ & Brooklyn Aqueduct & $30 \ldots$ & .....do...... & $\cdots+\cdots$ & I. M. De Varona $f$............. \\
\hline$* 137$ & Brooklyn Aqueduct & $3 \mathrm{C} \ldots$ & $\ldots$ do $\ldots$... & 1 & $\mid \begin{array}{c}\ldots \text { do. } \\
\ldots \ldots \text { do } \\
\ldots \ldots\end{array}$ \\
\hline$* 138$ & $\left\{\begin{array}{c}\text { Old Spring Creek } \\
\text { pumping station. } \\
\cdot\end{array}\right.$ & $3 \mathrm{C}$ & ...do... & W. D. Androws \& Bro.. & $\begin{array}{l}\text { L. B. Ward } d \ldots \ldots \ldots \\
\text { W.D. Andrews \& Bro..... }\end{array}$ \\
\hline *139 & $\begin{array}{c}\text { Temporary spring } \\
\text { Creek pumping } \\
\text { station. }\end{array}$ & 30 & ..do.. & & 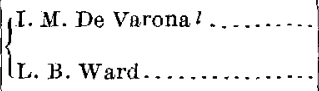 \\
\hline 140 & $\begin{array}{l}\text { Ridgewood pump- } \\
\text { ing station. }\end{array}$ & $3 \mathrm{C}$. & ...do...... & & I. M. De Varona $n$........... \\
\hline$* 141$ & $\begin{array}{l}\text { Jamaicu a vemue and } \\
\text { Aqueduct. }\end{array}$ & & …. do . & & .00 .8 \\
\hline$* 142$ & Woodhaven & $3 \mathrm{C} \ldots$ & $\left\{\begin{array}{c}\text { Woodhaven Water Supply } \\
\text { Co. }\end{array}\right.$ & & L. B. Ward $d \ldots$ \\
\hline
\end{tabular}

*For additional data see descriptive notes, pp. 168 et seq.

a Ann. Rept. New Jersey Geol. Survey for 1896, pp. 155-156.

$b$ Average per well for 1899 .

c Seo Table VIII.

'Merchants' $\Lambda$ ssociation report on water supply of the city of New York, 1900, table facing p. 186,

e Report of P. H. \& J. Conlan, drillers; Ann. Rept. Geol. Survey New Jersey for 1898, p. 142

$f$ Ann. Rept. Dept. City Work», Brooklyn, 1896, p. 298.

f Anistory and Description of the Brooklyn.Waterworks, 1896, pl. 40.
h Op. cit., p. 20 . 
on Long Island-Continued.

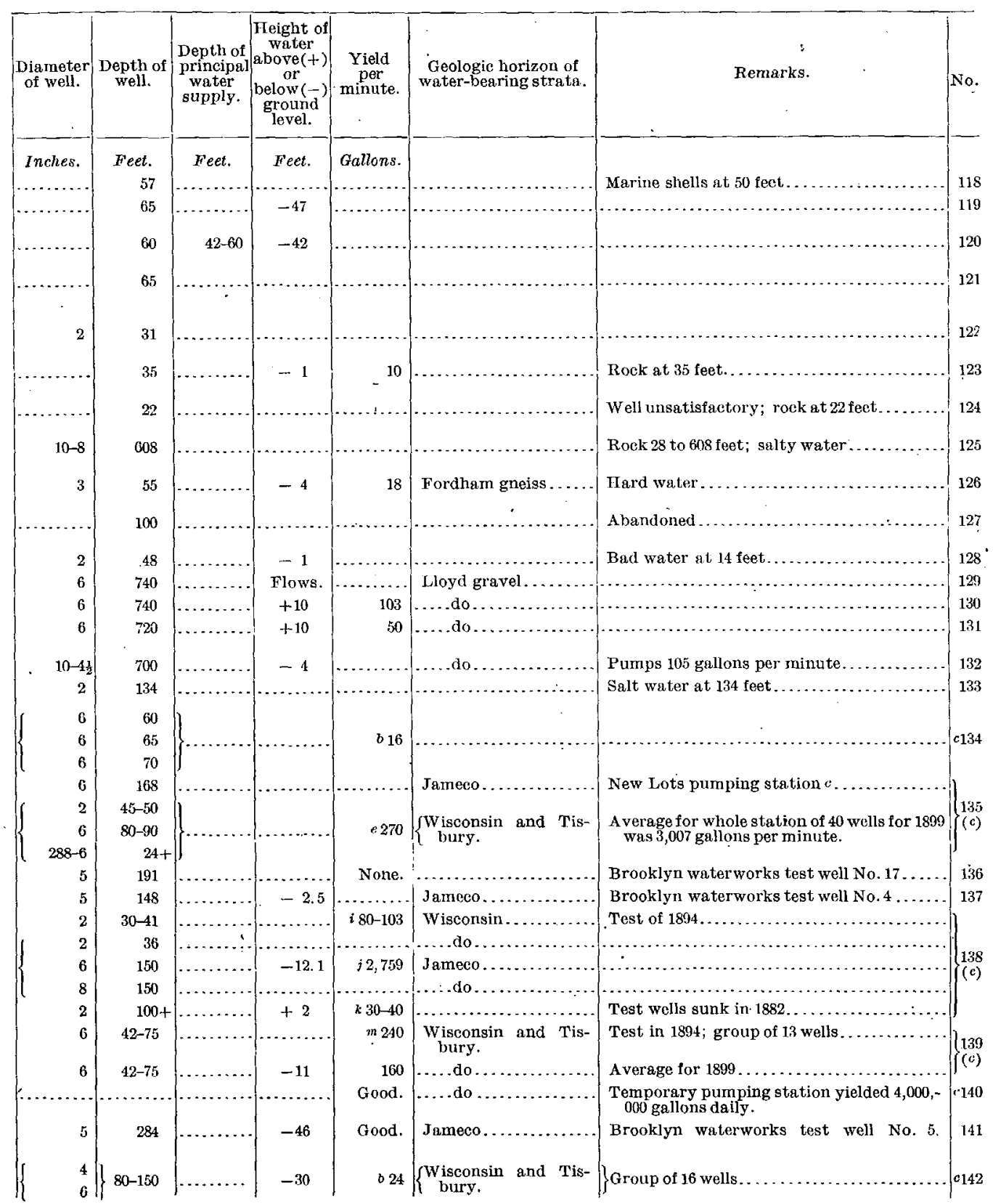

$i$ Tests of separate wells. Average per well for test of whole plant at same time $30+$ gallons.

$j$ A verage for whole station of 108 wells for 1899 .

$k$ With a hand pump.

$l$ History and Description of the Brooklyn Waterworks, 1896, p. 21

$m$ Average yield in 1899 was 160 gallons.

$n$ Op. cit., p. 100 . 


\section{UNDERGROUND WATER RESOURCES OF LONG ISLAND, NEW YORK.}

TABLE XI.--Representative wells

\begin{tabular}{|c|c|c|c|c|c|}
\hline No. & Location. & $\begin{array}{l}\text { Coordi- } \\
\text { nates. }\end{array}$ & Owner. & Driller. & Authority. \\
\hline-7 & & & & & \\
\hline *143 & Woodhaven... & $3 \mathrm{C}$. & $\begin{array}{l}\text { Lalance \& Grosjean Manu- } \\
\text { facturing Co. }\end{array}$ & & John Bryson $a \ldots . . . .$. \\
\hline *144 & Union Place..... & $3 \mathrm{C} \ldots$ & Commission............... & & Commission .......... \\
\hline *145 & Glendale........... & $3 \mathrm{C} \ldots$ & 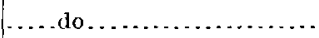 & & ....do................. \\
\hline 146 & Evergreen....... & $30 \ldots$ & The Frunk Brewery . .... & & The Frank Brewery .... \\
\hline$* 147$ & Metropolitan..... & $3 \mathrm{C} \ldots$ & Montauk Brewing Co... & & Montauk Brewing Co... \\
\hline$*_{148}$ & Middle Village.... & $3 \mathrm{C} \ldots$ & Commission........ & & Commission ............. \\
\hline$* 149$ & Middle village......... & $3 \mathrm{C} \ldots$ & H. Bottjer................. & Ed. Schmidt.......... & Ed. Schmidt............ \\
\hline 150 & Flushing Creek..... & $3 \mathrm{C} \ldots$ & Citizens' Water Supply Co. & 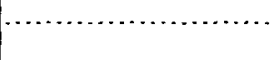 & $\begin{array}{l}\text { J. Edward Meyer, superin- } \\
\text { tendent. }\end{array}$ \\
\hline *151 & Flushing Creek.... & $3 \mathrm{C} \ldots$ & $\ldots$. do........ & & ...do......... \\
\hline$* 152$ & Flushing Creek..... & $3 \mathrm{C} \ldots$. & Commission......... & & Commission... \\
\hline$* 153$ & Maspeth............. & $3 \mathrm{C} \ldots$ & Woodside Water Co....... & & $\ldots \ldots \ldots \ldots \ldots$ \\
\hline *154 & New Calvary Cometery & $3 \mathrm{C} \ldots$ & New Calvary Cemetęry..... & S. H. Allen...... & S. H. Allen........... \\
\hline$* 155$ & New Calvary Cemetery. & $3 \mathrm{C} \ldots$ & 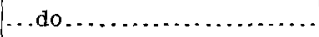 & $\ldots . d p \ldots . . .$. & $\ldots$.do $\ldots . . . . . .$. \\
\hline$* 156$ & New Calvary Cemetery. & $3 \mathrm{C} \ldots$ & ...do......................... & $\ldots \ldots$ do $\ldots \ldots \ldots \ldots \ldots$ & $\ldots \ldots$ do $\ldots \ldots \ldots \ldots \ldots \ldots$ \\
\hline$* 157$ & Newtown .............. & $3 \mathrm{C} \ldots$ & 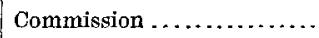 & & Commission . . . . . . . . . . \\
\hline 158 & Newtown............. & $3 \mathrm{C} \ldots$ & Citizens' Water Supply Co.. & & L. B. Ward............. \\
\hline$* 159$ & Elmhurst. . . . . . . . . . & $3 \mathrm{C} \ldots$. & 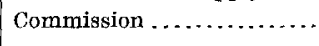 & 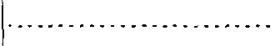 & Commission ............. \\
\hline$* 160$ & Woodside .......... & $3 \mathrm{D} \ldots$ & $\left\{\begin{array}{l}\text { New York and Queens } \\
\text { County } R . \mathrm{R} .\end{array}\right.$ & S. H. Allen. & s. H. Allen........ \\
\hline$* 161$ & Do $\ldots \ldots$ & $3 \mathrm{D} \ldots$ & Woodside Water Co... & & \\
\hline$* 162$ & $\begin{array}{l}\text { Do.............. } \\
\text { Long Island City: }\end{array}$ & $3 \mathrm{D}$. & Citizens' Watẹr Supply Co. & $\left\{\begin{array}{l}\text { Stotthoff Bros } \\
\text { Stan }\end{array}\right.$ & $\begin{array}{l}\text { L. B. Ward............. } \\
\text { Lewis Woolman } f \ldots \ldots\end{array}$ \\
\hline$* 163$ & $\begin{array}{l}\text { Albert street near } \\
\text { Grand avenue. }\end{array}$ & $3 \mathrm{D} \ldots$ & I. Isenburg.. & S. If. Allen......... & S. H. Allen...... \\
\hline *164 & $\begin{array}{l}\text { Grand and } 9 \text { th àve- } \\
\text { nues. }\end{array}$ & $3 \mathrm{D}$. & & . do. & ....do... \\
\hline *165 & $\begin{array}{l}\text { 13th avenue near } \\
\text { Vandevente: }\end{array}$ & $3 \mathrm{D}$ & & ...do.. & .....do.... \\
\hline$* 166$ & $\begin{array}{l}\text { Bowery Bay road } \\
\text { near Flushing } \\
\text { avenue. }\end{array}$ & $30 .$. & Commission... & & Commission.... \\
\hline$* 167$ & $\begin{array}{l}\text { Albert street and } \\
\text { Ditmars avenue. }\end{array}$ & $3 \mathrm{D} \ldots$ & Commission........... & & .... do.......... \\
\hline$* 168$ & Steinway avenue... & $3 \mathrm{D} \ldots$ & Astoria Silk Works .... & S. H. Allen..... & S. H. Allen...... \\
\hline$* 169$ & $\begin{array}{l}\text { Potter avenue near } \\
\text { Park place }\end{array}$ & $3 \mathrm{D} \ldots$ & - Dillman........... & $\ldots \ldots$ do......... & $\ldots$. do $\ldots . . . . .$. \\
\hline$* 170$ & $\begin{array}{l}\text { Merchantstreet and } \\
\text { Ditmars avenue. }\end{array}$ & $30 \ldots$ & Rivercrest Sanitarium.. & ....do... & .... do.... \\
\hline$* 171$ & $\begin{array}{l}\text { Near Merohant } \\
\text { street and Dit- } \\
\text { mars avenue. }\end{array}$ & $3 \mathrm{D} \ldots$ & .... do do...... & .... do .... & ....do.......... \\
\hline$* 172$ & $\begin{array}{l}\text { Crescent street near } \\
\text { Ditmars avenue. }\end{array}$ & $3 \mathrm{D} \ldots$ & ....do........... & $\ldots$... do... & ....do..... \\
\hline$*_{173}$ & $\begin{array}{l}\text { Lawrence street and } \\
\text { Wolcott avenue. }\end{array}$ & $3 \mathrm{D} \ldots$ & Commission..... & & Commission ............. \\
\hline 174 & Bowery Bay ....... & $\mid 3 \mathrm{D} \ldots$ & Steinway \& Son ........... & $\begin{array}{l}\text { Pierce Well Engineering } \\
\text { Co. }\end{array}$ & Pierce Well Engineering Co. \\
\hline & (1) & \multicolumn{4}{|c|}{$\begin{array}{l}\text { *For additional data see deseriptive notes, pp. } 168 \text { et seq. } \\
\text { a Am. Geol., vol. } 2 \text {, } 1888 \text {, pp. } 136-137 \text {; vol. } 3,1889 \text {, pp. } 218-219 \text {. } \\
\text { b Estimate for whole station. } \\
\text { o Sec Table VIII. }\end{array}$} \\
\hline
\end{tabular}


on Long Island-Continued.

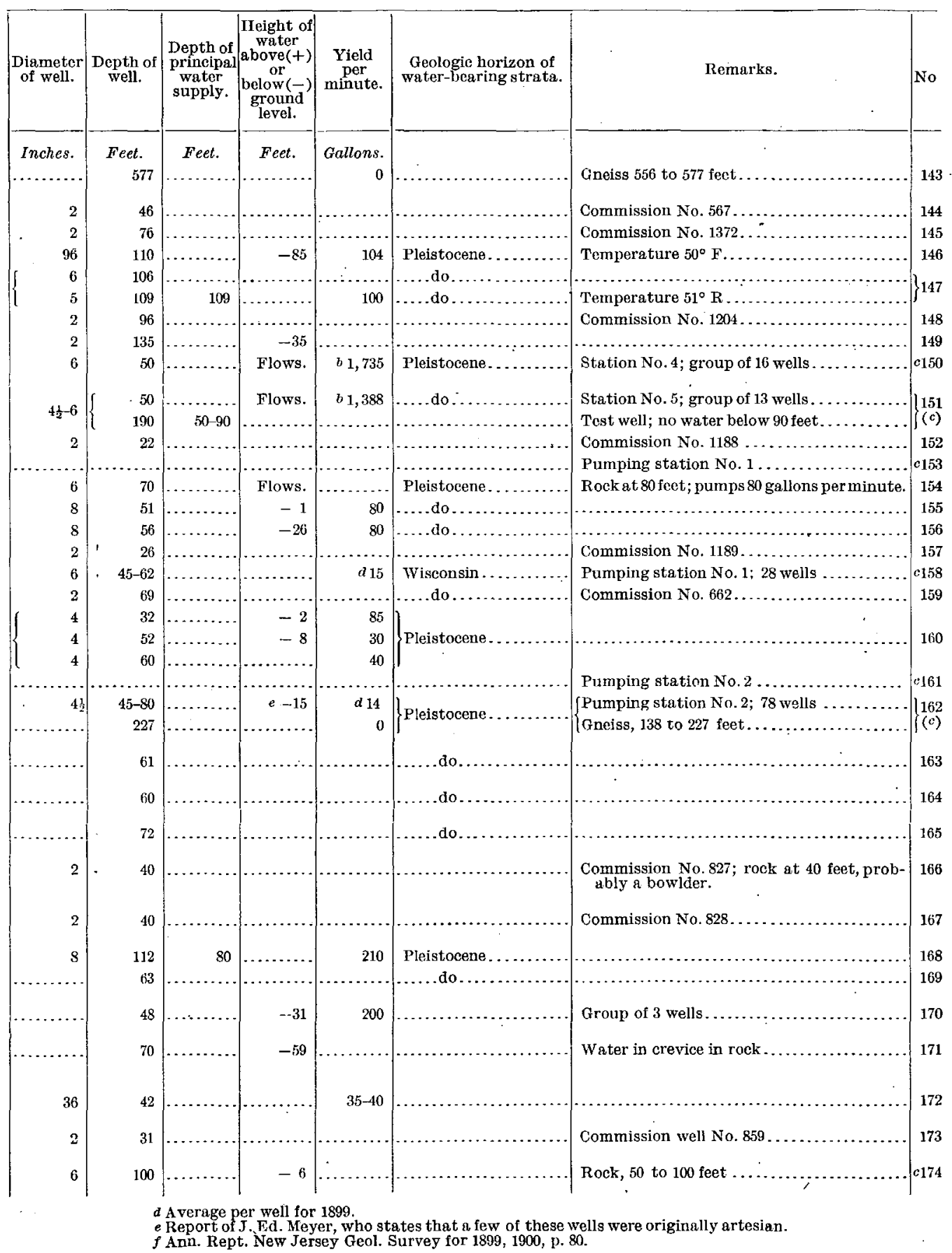


TABLE XI.---Representative wells

\begin{tabular}{|c|c|c|c|c|c|}
\hline No. & Location & $\begin{array}{l}\text { Coordi- } \\
\text { nates. }\end{array}$ & Owner. & . Driller. & Authority. \\
\hline 175 & $\left\{\begin{array}{c}\text { Long Island City-Con. } \\
\text { Cabinet and Bowery } \\
\text { Bay road. }\end{array}\right.$ & \} $3 .$. & $\left\{\begin{array}{c}\text { Department water supply, } \\
\text { gas, and electricity. }\end{array}\right.$ & \}$\ldots \ldots \ldots \ldots \ldots$ & L. B. Ward.......... \\
\hline$* 176$ & North Beach...... & $3 \mathrm{D} \ldots$ & $\begin{array}{l}\text { Bowery Bay Building and } \\
\text { Improvement Association. }\end{array}$ & Sweeney \& Gray... & $\begin{array}{l}\text { L. C. L. Smith, consulting } \\
\text { engineer. }\end{array}$ \\
\hline *177 & North Beach....... & $3 \mathrm{D} \ldots$ & $\ldots \ldots \ldots \ldots \ldots \ldots \ldots \ldots \ldots \ldots \ldots$, & & \\
\hline$* 178$ & $\begin{array}{l}\text { Flushing and Ehret } \\
\text { avenues. }\end{array}$ & $3 \mathrm{D} \ldots$ & Woodside Water Co... & & (n................. \\
\hline$* 179$ & $\begin{array}{l}\text { Trains Meadow and } \\
\text { Highway roads. }\end{array}$ & $3 \mathrm{D}$ & Commission. . & & Commission... \\
\hline$* 180$ & $\begin{array}{l}\text { Trains Meadow } \\
\text { road near Jack- } \\
\text { son avenue. }\end{array}$ & $3 \mathrm{D}$. & .... do. & & .....do... \\
\hline *181 & $\begin{array}{c}\text { Junctionavenueand } \\
\text { Strongs Lane. }\end{array}$ & $3 \mathrm{D}$. & .... do...... & & ....do.................. \\
\hline$* 182$ & College Point........... & 3D... & India Rubber Comb Co..... & & A.D. Schlissinger, president \\
\hline 183 & $\ldots$. do............. & $3 \mathrm{D} \ldots$ & —. Stonebanks........... & n................... & A. D. Sehlissinger........... \\
\hline$* 184$ & ....do......... & $3 \mathrm{D} \ldots$ & American Hard Rubber Co. & Chester D. Corwin ..... & Chester D. Corwin........ \\
\hline 185 & ...do................. & $3 \mathrm{D} \ldots$ & $\ldots \ldots \ldots \ldots \ldots \ldots \ldots \ldots \ldots \ldots \ldots \ldots$, & 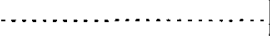 & A. D. Schlissinger......... \\
\hline$* 186$ & Tallman Island........ & $3 \mathrm{D} \ldots$ & Long Island R. R......... & & $\begin{array}{l}\text { C. M. Jacobs, consulting } \\
\text { engineer. }\end{array}$ \\
\hline$* 187$ & Far Rockaway. & $4 \mathrm{~B} .$. & Jas. Caffery........ & Lawrence Verdon...... & Law rence Verdon .......... \\
\hline *188 & .....do... & $4 \mathrm{~B} \ldots$ & B. L. Carroll ............ & Gilbert Baldwin ........ & Gilbert Baldwin ......... \\
\hline$* 189$ & $\ldots$. do. & $4 B \ldots$ & Jas. Caffery .............. & F. K. Walsh........ & F. K. Walsh......... \\
\hline *190 & …do.................... & $4 \mathrm{~B} \ldots$ & Long Island $\mathrm{R} . \mathrm{R} . . . \ldots$. & (n) & Long Island R. R . . . . . \\
\hline$* 191$ & $\ldots \ldots$ do $\ldots \ldots \ldots \ldots \ldots$ & $4 \mathrm{~B}$. & Queens County water Co.. & C. A. Lockwood........ & C. A. Loekwood ......... \\
\hline 192 & Nigger Point.... & $4 \mathrm{~B} \ldots$ & Idlowild Hotel............ & 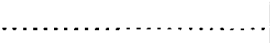 & Theo. R. Chapman.... \\
\hline$* 193$ & $\ldots \ldots \ldots \ldots \ldots \ldots \ldots$, & $4 \mathrm{~B} .$. & Theo. $\mathbf{R}$ Chapman......... & 'Theo. R. Chapman ..... & ....do do................... \\
\hline 194 & $\begin{array}{l}\text { Shetucket pumping sta- } \\
\text { tion. }\end{array}$ & $4 \mathrm{~B} \ldots$ & $\begin{array}{l}\text { Department water supply, } \\
\text { gas, and electricity. }\end{array}$ & & L. B. Ward . . . . . . \\
\hline *195 & $\ldots \ldots$ do $. . . \ldots \ldots \ldots . . .$. & $4 \mathrm{~B} \ldots$ & .....do....... & & I. M. De Varona........... \\
\hline$* 196$ & $\left\{\begin{array}{c}\text { Springfield pumping } \\
\text { station. }\end{array}\right.$ & $4 \mathrm{~B}$ & ... do. & 1 & $\left\{\begin{array}{l}\text { L. B. Ward .......... } \\
\text { Peter C. Jacobsen } c_{\ldots} \ldots\end{array}\right.$ \\
\hline$* 197$ & $\begin{array}{l}\text { Near Springfield pump- } \\
\text { ing station. }\end{array}$ & $4 \mathrm{~B}$. & ..... do.... & $\ldots \ldots \ldots \ldots \ldots$ & I. M. De Varona....... \\
\hline 198 & Oconee pumping station & $4 \mathrm{C} \ldots$ & .... do..... & & L. B. Ward................ \\
\hline *199 & $\begin{array}{l}\text { Near Oconee pumping } \\
\text { station. }\end{array}$ & $4 \mathrm{C} \ldots$ & $\ldots \ldots$ do $\ldots \ldots \ldots \ldots . .$. & 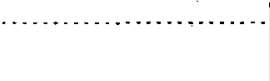 & $\begin{array}{l}\text { I. M. De Varona . . . . . . . . } \\
\text { W. D. Andrews \& Bro.... }\end{array}$ \\
\hline *200 & $\left\{\begin{array}{l}\text { Baisley's pumping sta- } \\
\text { tion. }\end{array}\right.$ & $4 \mathrm{C}$. & ..... do..... & W. D. Andrews \& Bro. & $\left\{\begin{array}{l}\text { I. M. De Varona } \ldots \ldots \ldots \\
\text { L. B. Ward................. } \\
\text { I. M. De Varona } \ldots \ldots \ldots\end{array}\right.$ \\
\hline *201 & $\left\{\begin{array}{c}\text { Jameco pumping sta- } \\
\text { tion. }\end{array}\right.$ & \} $4 \mathrm{C}$. & $\ldots$ do... & ..... do .... & \\
\hline & & & & & I. B. Ward .......... \\
\hline *202 & $\begin{array}{l}\text { Aqueduct and Cornell } \\
\text { Creek. }\end{array}$ & $4 \mathrm{C} \ldots$ & .....do... & & I. M. De Varona............ \\
\hline *203 & $\begin{array}{l}\text { Aqueduct and Rocka- } \\
\text { way road. }\end{array}$ & $4 \mathrm{C} \ldots$ & ...do... & & ... do.. \\
\hline
\end{tabular}

* For additional data see descriptive notes, pp. 168 et seq. a Average yield to pumps per minute from whole station, 1899.

c Reports to Chicf Engineer I. M. De Varona. 
on Long Island-Continued.

\begin{tabular}{|c|c|c|c|c|c|c|c|}
\hline $\begin{array}{l}\text { Diameter } \\
\text { of well. }\end{array}$ & $\begin{array}{l}\text { Depth of } \\
\text { of well. }\end{array}$ & $\begin{array}{l}\text { Depth of } \\
\text { prineipas } \\
\text { water } \\
\text { supply. }\end{array}$ & $\begin{array}{c}\text { Height of } \\
\text { water } \\
\text { above }(+) \\
\text { or } \\
\text { below }(-) \\
\text { ground } \\
\text { level. }\end{array}$ & $\begin{array}{c}\text { Yield } \\
\text { per } \\
\text { minute. }\end{array}$ & $\begin{array}{c}\text { Geologic horizon of } \\
\text { water-bearing strata. }\end{array}$ & Remarks. & No \\
\hline Inches. & Feet. & Feet. & Feet. & Gallons. & & & \\
\hline & 45 & & Flows. & $a 557$ & Pleistocene.. & Long Island City station No. 2; 29 wells. & bọt \\
\hline 192 & 22 & & 1. Thes. & W & & & \\
\hline 6 & 70 & & -12 & 20 & .....do. & Group of 17 wells .......... & 6176 \\
\hline 6 & $40-50$ & & & & & Group of 3 wells........ & 177 \\
\hline & & & & & & Station No. 3 ; not used . . . . . . . . . . & 10178 \\
\hline 2 & 28.5 & & & & & Commission No. $767 \ldots \ldots \ldots$. & 179 \\
\hline 2 & 31 & & & & & Commission No. $762 \ldots$. & 180 \\
\hline 2 & 53 & & & & & Commission No. $768, \ldots \ldots \ldots$ & 181 \\
\hline & 35 & & & & & 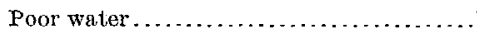 & 182 \\
\hline 26 & 28 & & & & & Lignitized wood at 28 feet.......... & 183 \\
\hline 2 & 86 & & & 50 & Pleistocene & 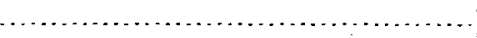 & 184 \\
\hline & 60 & & & 0 & & Bluo clay, 0-60 feet $\ldots \ldots \ldots \ldots \ldots . .$. & 185 \\
\hline & 149 & & & -0 & & Fordham gneiss, 110 to 149 feet.... & 186 \\
\hline 6 & 112 & & Fiows. & & & & 187 \\
\hline 6 & 190 & $150-190$ & Flows. & & Jameco?. & Salt water ........... & 188 \\
\hline 6 & 90 & 90 & 0 & ......... & Jameco.. & Fresh water............................ & \\
\hline & 30 & $20-30$ & -20 & Good. & Tisbury & & I 89 \\
\hline & $20-30$ & & & & & & 190 \\
\hline 5 & 50 & & & & Tisbury.. & Abandoned station........... & 191 \\
\hline 5 & 200 & 200 & & & Jameco..... & Brackish water.................. & \\
\hline 2 & 200 & 200 & Flows. & & .... do..... & 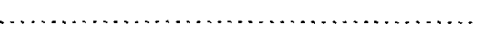 & 192 \\
\hline 8 & 203 & 202 & Flows. & $\therefore \cdots$ & .... do... & $\ldots \ldots \ldots \ldots \ldots \ldots \ldots$ & 193 \\
\hline 8 & 180 & $\cdots \cdot$ & -10.3 & $f 97$ & .....do... & Group of 12 wells ....... & $b 194$ \\
\hline 5 & 154 & $346-154$ & $\begin{array}{l}-10.7 \\
-16.7\end{array}$ & & .... do. & Brooklyn test well No. $16 \ldots$. & 195 \\
\hline 8 & 170 & .......... & -9.6 & f 74 & .... do.. & Group of 20 wells $\ldots \ldots \ldots \ldots \ldots \ldots \ldots \ldots$ & \\
\hline 8 & $156-207$ & $117-207$ & Flows. & $15-25$ & ....do. & $\begin{array}{l}\text { Well No. } 15 \text { yielded on test } 694 \text { gallons a } \\
\text { minute, lowering water to about }-14 \text { feet. }\end{array}$ & $(b)$ \\
\hline 5 & 271 & & & $(d)$ & & Brooklyn test well No. $9 \ldots \ldots \ldots \ldots \ldots$ & 197 \\
\hline 8 & $19 \overline{5}$ & ... & +.8 & $f 95$ & Jameco . & Group of 12 wells; pumps ..... & $b 198$ \\
\hline 5 & 192 & $185-192$ & $\ldots \ldots$. & & .... do... & Brooklyn test well No. $18 \ldots \ldots \ldots \ldots$. & 199 \\
\hline 2 & +100 & .......... & +3 & ...... & $\ldots .$. do..... & Test well of $1884 \ldots \ldots \ldots \ldots \ldots \ldots \ldots$ & \\
\hline 2 & $28-65$ & $\ldots \ldots \ldots$ & -10.6 & $18-42$ & Wisconsin. & 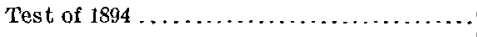 & 200 \\
\hline 2 & 44 & …..... & -11.7 & 10 & $\ldots$. do.... & Average for $1899 \ldots \ldots \ldots \ldots$. & $(b)$ \\
\hline 2 & 200 & $140-170$ & Flows. & Smail. & $\ldots \ldots \ldots$ & Test well of $1894 \ldots \ldots \ldots \ldots \ldots \ldots \ldots \ldots$ & \\
\hline 4 & 150 & $\ldots \ldots \ldots$ & $e+7$ & 90 & Jameco. & Pumps 280 gallons per minute $\ldots . . \ldots \ldots$ & \\
\hline 6 & 155 & & ….... & 100 & ....do... & Purnps 300 gallons per minute .......... & \\
\hline 6 & $\cdot 151$ & ........ & +11 & 475 & .....do... & Purnps 500 gallons per minute ......... & \\
\hline 10 & 230 & 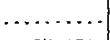 & .......... & … & ............ & Andrews's well; abandoned ........ & $\left\{\begin{array}{l}201 \\
(b)\end{array}\right.$ \\
\hline 8 & 151 & $137-151$ & Flows. & 154 & Jameco . . & 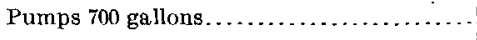 & \\
\hline 2 & $23-73$ & & & & & Groups of 207 wells. . & \\
\hline $4-10$ & 160 & $\ldots \ldots$. & -3.4 & & & & \\
\hline 5 & 156 & $141-156$ & +1 & & Jameco. & $\begin{array}{l}\text { Brooklyn test well No.1; flows } 30 \text { gallons } \\
\text { per minute. }\end{array}$ & 202 \\
\hline 5 & 257 & $146-162$ & +2 & & .... do. & $\begin{array}{l}\text { Brooklyn test well No.2; flows } 5 \text { gallons } \\
\text { per minute. }\end{array}$ & 203 \\
\hline
\end{tabular}

d No water was obtained below surface gravel.

e Letter from $W$. D. And rews \& Bro.
$f$ Average yield to pumps per well per minute for 1899 
TABLE XI.-Representative wells

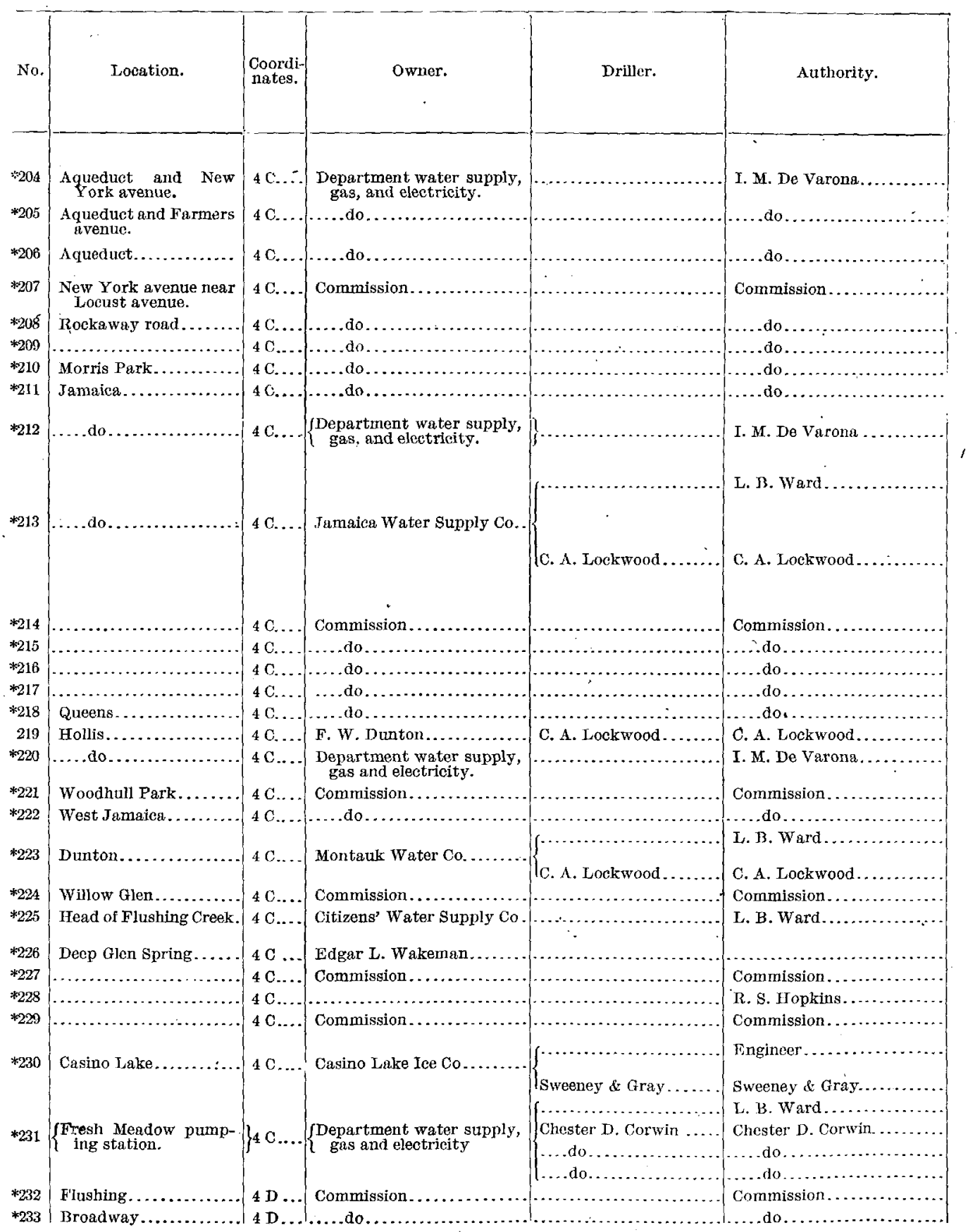

*For additional data see deseriptive notes, pp. 168 et seq.

$a$ With hand pump from water bearing stratım between 176 and 182 feot.

$b$ Average of whole station for 1899 . 
on Long Island--Continued.

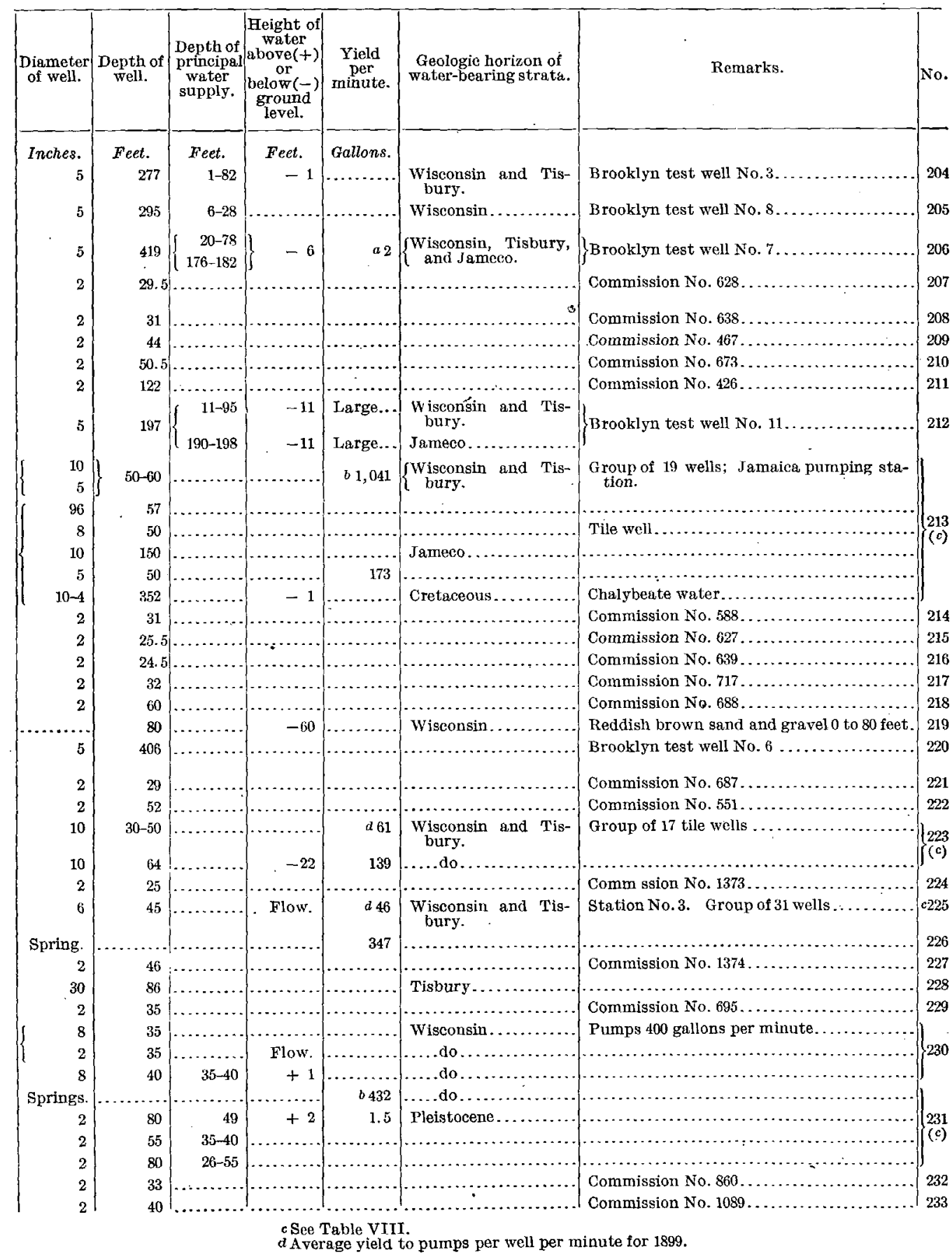




\section{UNDERGROUND WATER RESOURCES OF LONG ISLAND, NEW YORK.}

TABLE XI.-Representative wells

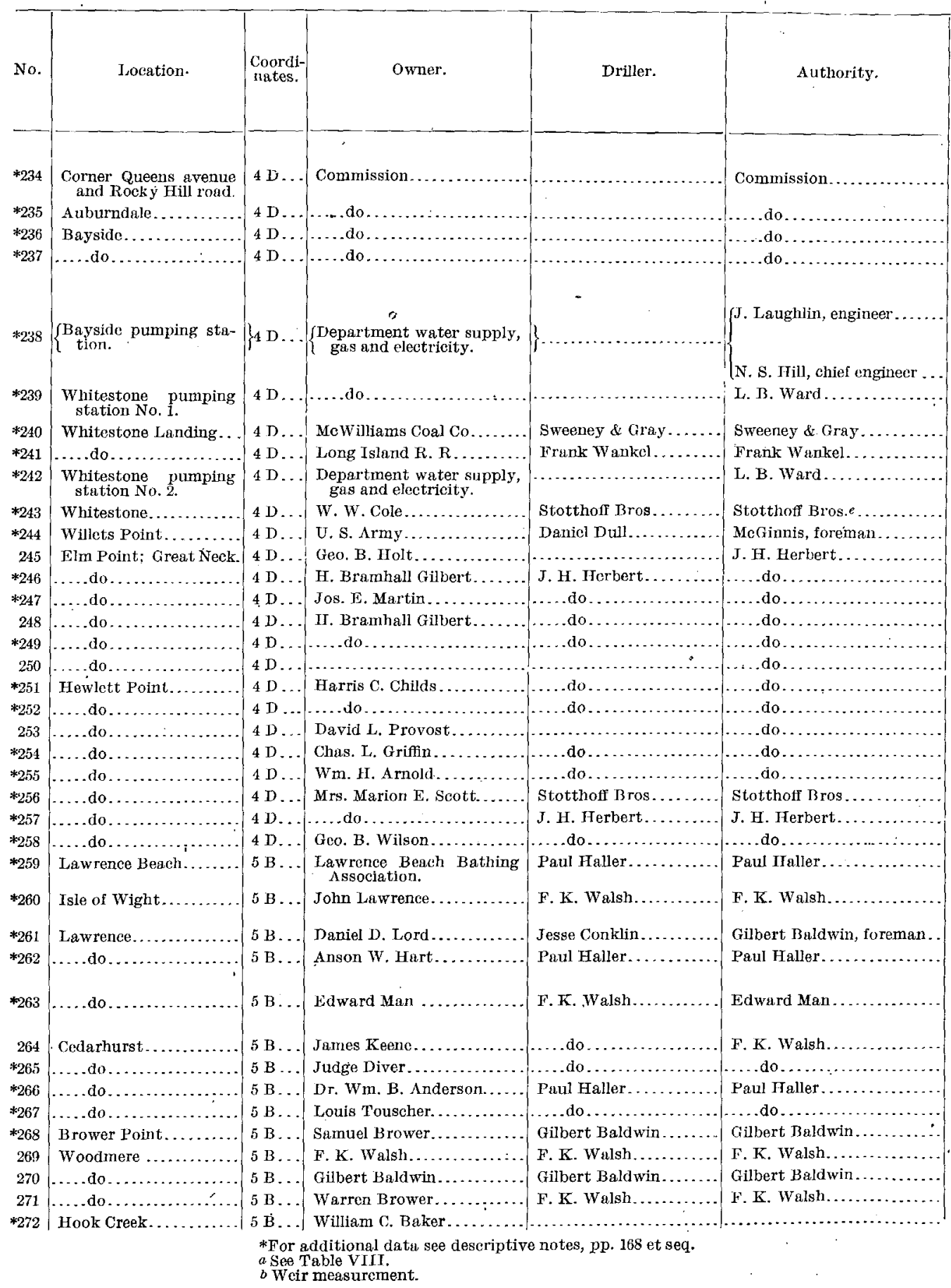


on Long Island-Continued.

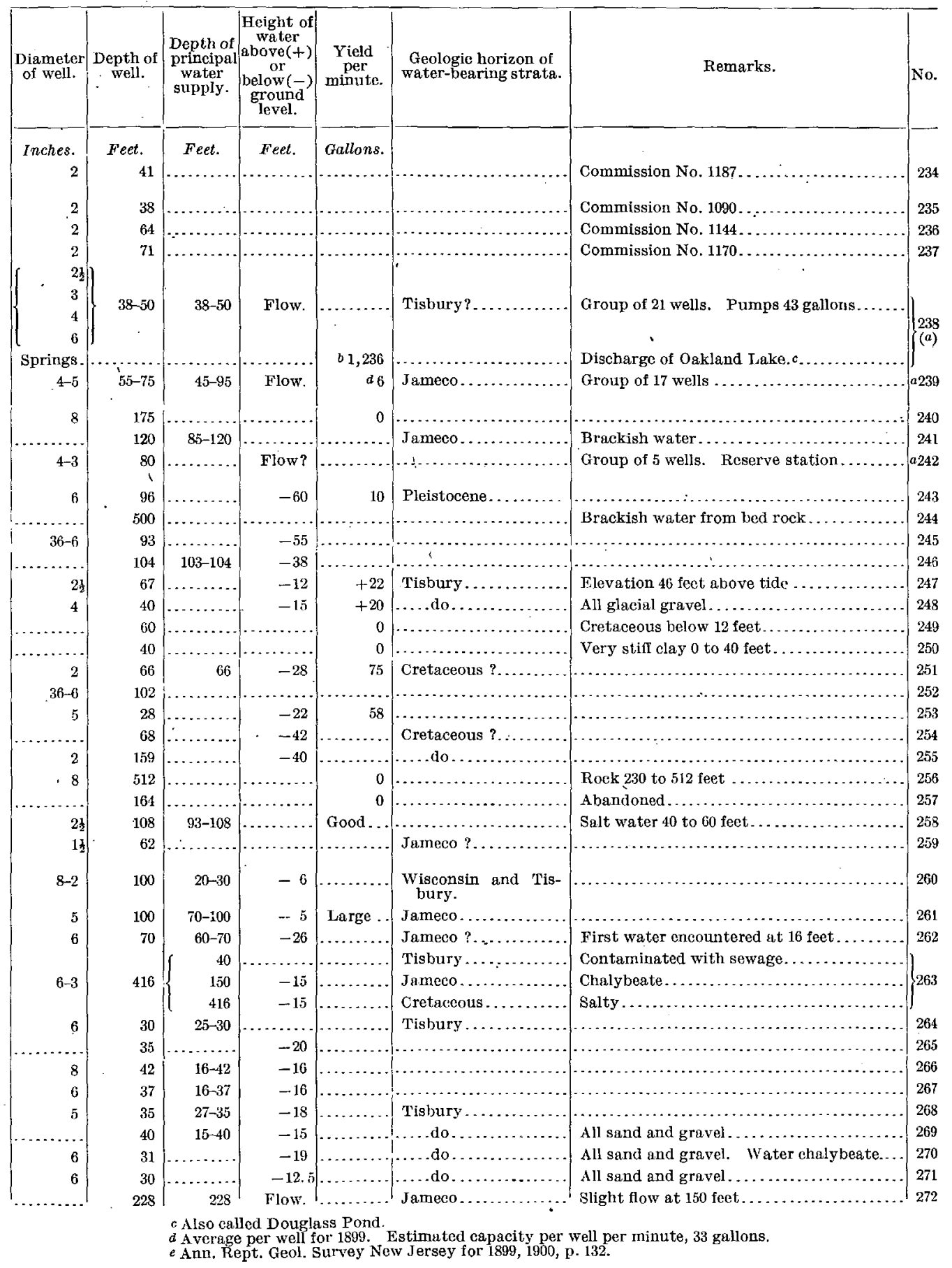




\section{UNDERGROUND WATER RESOURCES OF LONG ISLAND, NEW YORK.}

TABLE XI.-Representative wells

\begin{tabular}{|c|c|c|c|c|c|}
\hline No. & Location. & $\begin{array}{l}\text { Coordi- } \\
\text { nates. }\end{array}$ & Owner. & Driller. & Authority. \\
\hline$* 273$ & $\left\{\begin{array}{l}\text { Queens County Water } \\
\text { Co. pumping sta- } \\
\text { tions. }\end{array}\right.$ & $5 \mathrm{~B} \ldots$ & Queens County Water Co... & & $\left\{\begin{array}{l}\text { Chas. R. Bettes, chief engi- } \\
\text { neer. }\end{array}\right.$ \\
\hline$* 274$ & Hewlett. & $5 \mathrm{~B}$. & Jirden Abrames....... & F. K. Walsh.. & F. K. Walsh... \\
\hline$*_{275}$ & Lynbrook.... & $5 \mathrm{~B}$. & Mirs. Julia Flower..... & .... do.......... & ....do........... \\
\hline 276 & ....do.................. & $5 \mathrm{~B} .$. & T. J. Simpson Co...... & Chas. A. Fass.... & Chas. A. Fass............ \\
\hline$* 277$ & ..... do. & $5 \mathrm{~B}$. & Queens County Water Co... & & $\left\{\begin{array}{c}\text { Franklin } \\
\text { dent. }\end{array}\right.$ \\
\hline$* 278$ & Brooklyn Aqueduct.. & $5 \mathrm{~B}$. & $\begin{array}{l}\text { Department water supply, } \\
\text { gas and electricity. }\end{array}$ & & I. M. De Varona ......... \\
\hline$*_{229}$ & $\ldots \ldots$ do $\ldots \ldots \ldots \ldots \ldots \ldots$ & $5 \mathrm{~B}$. & 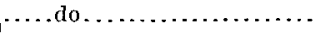 & ...... & .....do... \\
\hline$* 280$ & ....do $\ldots \ldots \ldots \ldots \ldots \ldots$ & $5 \mathrm{~B}$. & $\ldots$ do $\ldots \ldots \ldots \ldots$ & & ..... do ........... \\
\hline *281 & ....do................. & $5 \mathrm{~B} \ldots$ & .....do . . . . & & .... do .... \\
\hline$*_{282}$ & ....do.............. & $5 \mathrm{~B} .$. & .....do....... & & $\ldots$ do $\ldots \ldots \ldots \ldots \ldots$ \\
\hline *283 & Valley stream.......... & $5 \mathrm{~B} .$. & C. Schreiber. ......... & Gilbert Baldwin.. & Gilbert Baldwin.......... \\
\hline$*_{284}$ & $\ldots$ do $\ldots \ldots \ldots \ldots \ldots$ & $5 \mathrm{~B}$. & Long Island R. R.......... & . & \\
\hline *285 & Brooklyn Aqueduct.... & $5 \mathrm{~B} \ldots$ & $\begin{array}{l}\text { Department water supply, } \\
\text { gas, and electricity. }\end{array}$ & & I. M. De Varona ..... \\
\hline$*_{286}$ & $\begin{array}{l}\text { Watts Pond pumping } \\
\text { station. }\end{array}$ & 5 B.. & 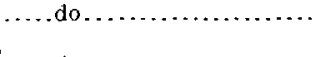 & J. Edwards \& Co... & .....do... \\
\hline$* 287$ & ..... do ................. & $5 \mathrm{~B}$. & ....do... & & ......do. \\
\hline$* 288$ & $\left\{\begin{array}{l}\text { Clear Stream pumping } \\
\text { station. }\end{array}\right.$ & $5 \mathrm{~B}$ & ....do.. & 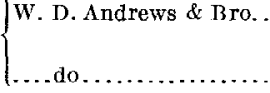 & $\begin{array}{l}\text { L. B. Ward.......................... } \\
\text { W. D. Andrews \& Bro .... }\end{array}$ \\
\hline$* 289$ & .....do... & $5 \mathrm{~B} .$. & .....do............... & W. D. Andrews \& Bro & $\left\{\begin{array}{l}\text { I. M. De Varona } \\
\{\ldots \text { do } \ldots \ldots \\
\text { L. B. Ward } \ldots \ldots \ldots\end{array}\right.$ \\
\hline$* 290$ & $\left\{\begin{array}{c}\text { Forest Stream pump- } \\
\text { ing station. }\end{array}\right.$ & $5 \mathrm{~B} \ldots$ & ....do.... & & W. D. Andrews \& Bro.. \\
\hline & & & & $\begin{array}{l}\text { Phillips and Worthing- } \\
\text { ton. }\end{array}$ & Phillips and worthington. \\
\hline$*_{291}$ & Brooklyn Aqueduet... & $5 \mathrm{C} \ldots$ & ....do... & & I. M. De Varona.... \\
\hline$*_{292}$ & .....do ................. & $5 \mathrm{c} . .$. & ....do... & & ....do............. \\
\hline *293 & .....do .................. & $5 \mathrm{C} \ldots$ & $\ldots$. do . . ...... & & ....do.............. \\
\hline *294 & Rosedale........... & $5 \mathrm{c} \ldots$ & Commission......... & & Commission...... \\
\hline$*_{295}$ & Springfield............. & $5 \mathrm{C} \ldots$ & $\begin{array}{l}\text { Department water supply, } \\
\text { gas, and clectricity. }\end{array}$ & & 1. M. De Varona ... \\
\hline$* 296$ & Fosters Meadows....... & $5 \mathrm{C} \ldots$ & Commission................. & & Commission.. \\
\hline *297 & $\begin{array}{l}1 \text { mile north of Valley } \\
\text { Stream. }\end{array}$ & $5 \mathrm{C} . .$. & .....do............. & & .....do............. \\
\hline *298 & $\begin{array}{l}2 \text { miles north of Valley } \\
\text { Stream. }\end{array}$ & $5 \mathrm{C} \ldots$ & $\ldots \ldots$ do $\ldots \ldots \ldots$ & & .....do.. \\
\hline *299 & & $5 \mathrm{C}$. & $\ldots$ do $\ldots \ldots$ & & $\ldots$...do... \\
\hline$* 300$ & ...... & $5 \mathrm{C} \ldots$ & $\ldots .$. do $\ldots . .$. & & ..... do $\ldots$ \\
\hline *301 & (............... & $5 \mathrm{C} \ldots$ & .... do . . ..... & & ..... do $\ldots . . . . .$. \\
\hline$* 302$ & Elmont............ & $5 \mathrm{C} \ldots$ & ....do............... & & 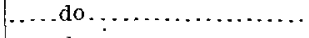 \\
\hline *303 & Floral Park...... & $5 \mathrm{C} \ldots$ & $\ldots \ldots$ do $\ldots . . . \ldots \ldots \ldots$ & & .... do \\
\hline *304 & $\cdots \cdot$ & $5 \mathrm{C} \ldots$ & $\cdots .00$ & & ...do. \\
\hline
\end{tabular}


on Long Island-Continued.

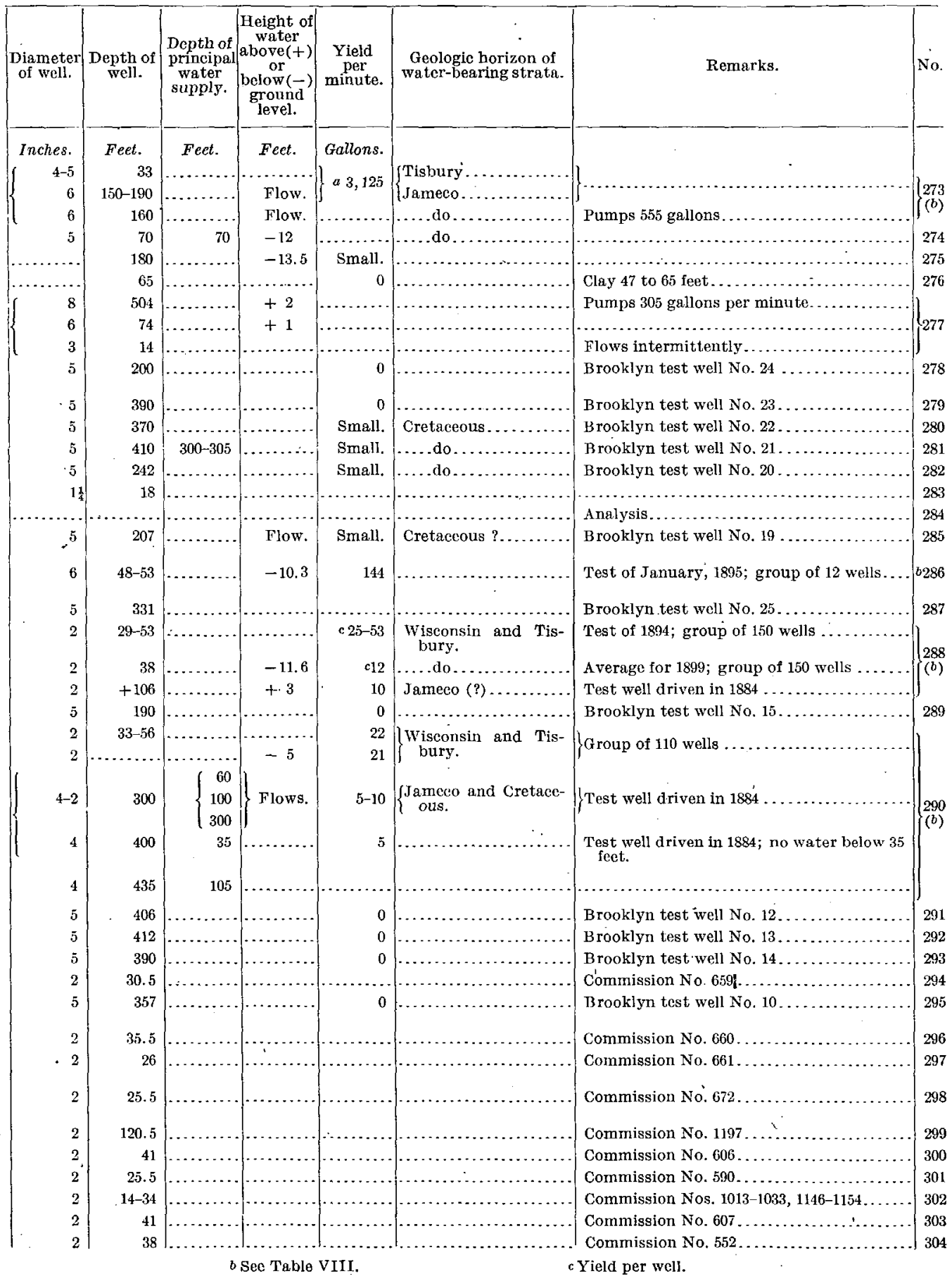




\section{- 138 UNDERGROUND WATER RESOUROES OF LONG ISLAND, NEW YORK.}

TABLE XI.-Representative wells

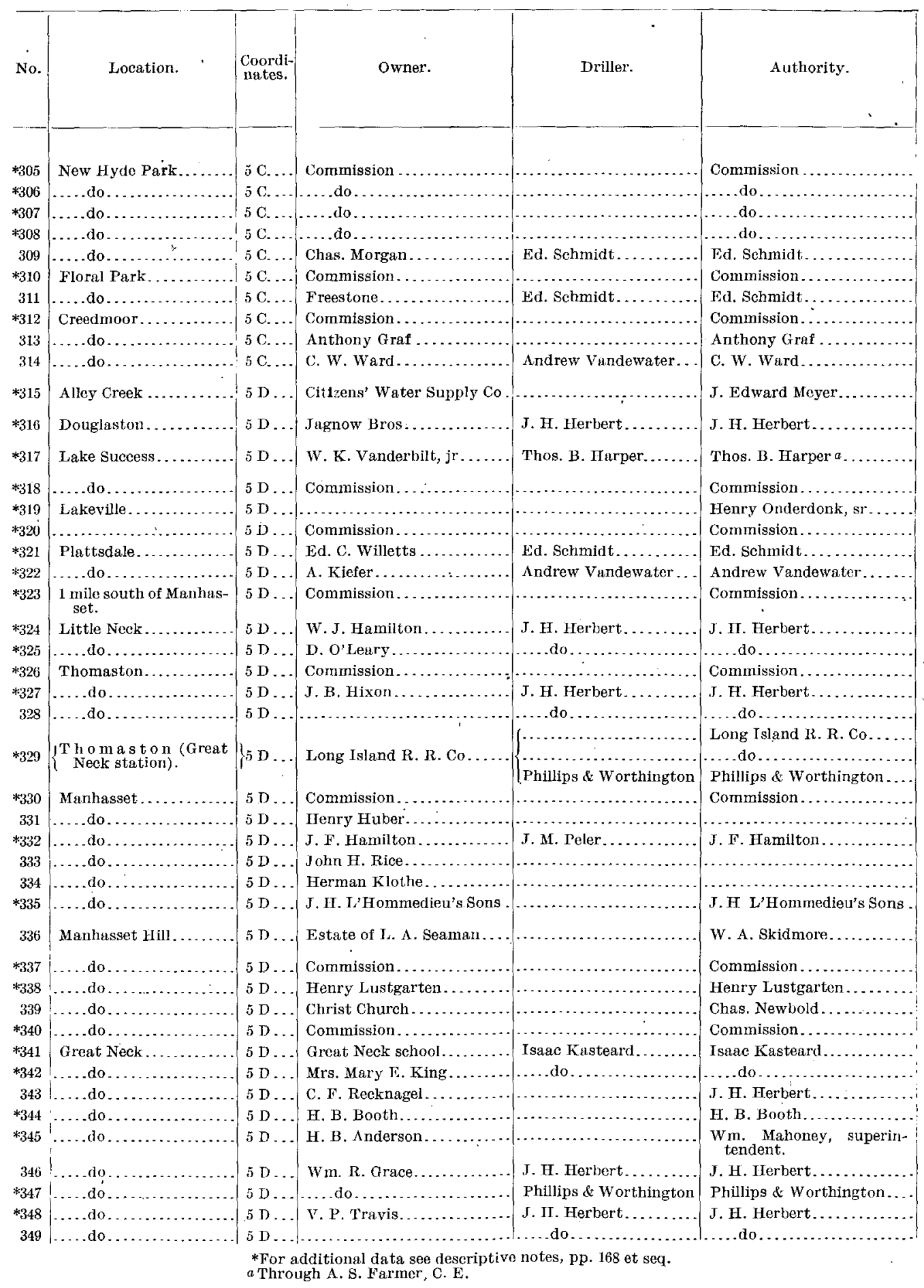


on Long Island-Continued.

\begin{tabular}{|c|c|c|c|c|c|c|c|}
\hline $\begin{array}{l}\text { Diameter } \\
\text { of well. }\end{array}$ & $\begin{array}{c}\text { Depth of } \\
\text { well. }\end{array}$ & $\begin{array}{c}\text { Depth of } \\
\text { primeipal } \\
\text { water } \\
\text { supply. }\end{array}$ & $\left|\begin{array}{c}\text { Height of } \\
\text { water } \\
\text { above }(+) \\
\text { or } \\
\text { below }(- \\
\text { ground } \\
\text { level. }\end{array}\right|$ & $\begin{array}{c}\text { Yield } \\
\text { per } \\
\text { minute. }\end{array}$ & $\begin{array}{c}\text { Geologic horizon of } \\
\text { water-bearing strata. }\end{array}$ & Remarks. & No. \\
\hline Inches. & Feet. & Feet. & Feet. & Gallons. & & & \\
\hline 2 & 32.5 & & & & & Commission No. 553 . & 305 \\
\hline 2 & 56 & & & & & Commission No. $740 \ldots$ & 306 \\
\hline 2 & 74 & & …... & & 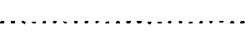 & Commission No, $741 \ldots \ldots \ldots \ldots \ldots$. & 307 \\
\hline 2 & 66.5 & & …... & & & Commission No. $907 \ldots \ldots \ldots$. . & 308 \\
\hline $1 \frac{1}{2}$ & 37 & $35-37$ & -35 & & & Bowlders, 35 to 37 feet....... & 309 \\
\hline 2 & 106 。 & & & & & Commission No. $829 \ldots \ldots$. . . & 310 \\
\hline $1 \frac{1}{2}$ & 40 & ....... & ….... & . & $\cdots \cdots \cdots$ & Coarse white sand, 28 to 40 feet.......... & 311 \\
\hline 2 & 56 & ....... & $\cdots \cdots \cdots$ & & (n)......... & Commission No, $619, \ldots \ldots \ldots \ldots \ldots$ & 312 \\
\hline 33 & 60 & $55-60$ & -55 & & $W$ isconsin........ & $\ldots \ldots \ldots \ldots \ldots \ldots \ldots \ldots \ldots \ldots$ & 313 \\
\hline 48 & 70 & $62-70$ & -62 & n........ & $\ldots$ do........... & $\ldots \ldots \ldots \ldots \ldots \ldots \ldots \ldots$ & 314 \\
\hline $4 \frac{1}{2}$ & $32-42$ & & $\left\{\begin{array}{l}-2 \\
+8\end{array}\right.$ & $\begin{array}{r}\text { Flow. } \\
0-50\end{array}$ & & Group of 8 wells; 6 flowing.. & 315 \\
\hline b & 127 & & ….... & $\cdots \cdot$ & Tisbury?.. & & 316 \\
\hline & 755 & 191 & -116 & 40 & Cretaceous....... & $\therefore$ & 317 \\
\hline $8-4 \frac{1}{2}$ & 100 & $700-750$ & -135 & +300 & Lloyd gravel... & & \\
\hline 2 & 35 & & & & & Commission No. $864 \ldots \ldots \ldots$ & 318 \\
\hline 36 & 140 & & & & & (n) & 319 \\
\hline 2 & 45 & & ….... & & $\cdots \ldots \ldots \ldots \ldots$ & Commission No. $776 \ldots \ldots \ldots \ldots \ldots \ldots . . . . .$. & 320 \\
\hline & 37 & & -22 & & Wisconsin.... & Depends on perched water table....... & 321 \\
\hline 36 & 116 & & 114 & & Cretaceous?..... & $\cdots, \ldots, \ldots, \ldots, \ldots, \ldots, \ldots$, & 322 \\
\hline 2 & 48.5 & & ….... & $\cdots \cdots$ & $\ldots \ldots \ldots \ldots$ & Commission No. $956 \ldots \ldots \ldots$ & 323 \\
\hline $2 \frac{1}{2}$ & 136 & & & & Cretaceous. & & 324 \\
\hline $2 \frac{1}{2}$ & 142 & & & & ....do..... & & 325 \\
\hline 2 & 79 & & $\ldots \ldots$ & & $\ldots$... do... & Commission No. $1191 \ldots \ldots \ldots$. & 326 \\
\hline $2 k$ & 93 & $\cdots$ & -87 & & ....do... & & 327 \\
\hline 21 & 87 & ..... & …..... & 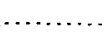 & $\ldots$. do.... & $\ldots \ldots \ldots \ldots \ldots \ldots \ldots \ldots \ldots \ldots \ldots$ & 328 \\
\hline 6 & 96 . & ....... & ........ & +30 & …........ & Well 200 feet from station............ & \\
\hline & 117 & 117 & $b-10$ & $15 \dot{0}$ & .... & 2 wells one-fourth mile apart $\ldots \ldots \ldots$ & 329 \\
\hline 6 & $112^{\circ}$ & $93-112$ & -53 & 100 & …... & 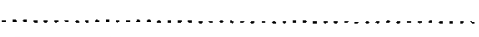 & \\
\hline 2 & 25 & $\cdots \cdots$ & …........ & & …. & Commission No. $957, \ldots \ldots \ldots \ldots \ldots$ & 330 \\
\hline 2 & 30 & & Flows. & & …...... & 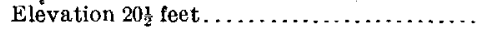 & 331 \\
\hline 2 & 78 & & Flows. & & $\cdots \cdots$ & (n, & 332 \\
\hline$\frac{1}{2}$ & 37 & & Flows. & & & 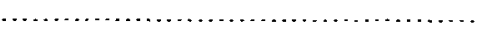 & 333 \\
\hline$\frac{1}{2}$ & 35 & $\cdots$ & Flows. & 2 & … & . $\therefore \ldots \ldots \ldots$ & 334 \\
\hline 1 & 10 & $\cdots$ & Flows. & & & & 335 \\
\hline 34 & 86 & $\begin{array}{l}40 \\
80\end{array}$ & -80 & & & & 336 \\
\hline 2 & 28 & & & & & Commission No. $1190 \ldots$ & 337 \\
\hline $34-2$ & 122 & & -81 & & & (n..., & 338 \\
\hline 36 & 108 & ...... & -103 & & n................. & Temperature about $50^{\circ} \mathrm{F} \ldots \ldots \ldots \ldots$ & 339 \\
\hline 2 & 87.5 & & ….... & & Cretaceous.......... & Commission No. $963 \ldots \ldots \ldots \ldots \ldots \ldots$ & 340 \\
\hline & 52 & $\ldots \ldots \ldots$ & -30 & & $\because \ldots \ldots \ldots \ldots \ldots \ldots$ & 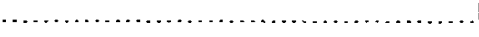 & 341 \\
\hline & 52 & n......... & -48 & & Tisbury .......... & (n) & 342 \\
\hline & 96 & 92 & …... & & n........ & Blue clay 0 to 92 feet $\ldots \ldots \ldots \ldots \ldots \ldots$ & 343 \\
\hline & 240 & -240 & ....... & & $\ldots, \ldots$ & 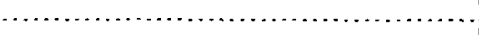 & 344 \\
\hline & 237 & 237 & $\cdots \ldots$. & (......... & $\ldots \ldots \ldots \ldots \ldots \ldots \ldots$ & 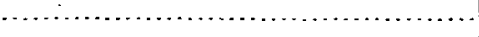 & 345 \\
\hline & 86 & & -74 & & & Well is near barn.... & 346 \\
\hline 6 & 104 & & …..... & 60 & Tisbury .... . & 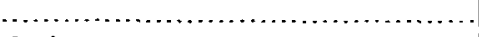 & 347 \\
\hline 2 & 119 & & -77 & 500 & Tisbury?........... & Surface water at 24 feet........ & 348 \\
\hline & 32 & ... & -8 & 500 & Wisconsin........ & Elevation about 95 reet........ & 349 \\
\hline
\end{tabular}

$17116--$ No. $44-06--10$ 
TABLE XI.--Representative wells

\begin{tabular}{|c|c|c|c|c|c|}
\hline No. & Location. & $\begin{array}{l}\text { Coordi- } \\
\text { nates. }\end{array}$ & Owner. & Driller. & Authority. \\
\hline *350 & & $5 \mathrm{D} \ldots$ & Robert Cox .... & J. H. Herbert... & J. H. Herbert .... \\
\hline$* 351$ & Plandome Mills..... & $5 \mathrm{D} \ldots$ & Robert Seizer.... & Geo. Schmidt... & Geo. Schmidt..... \\
\hline$* 352$ & Port Washington. . . & $5 \mathrm{D}$. & Chas. Vanderbilt. & Isaac Kasteard. & Isaac Kasteard. . \\
\hline 353 & $\ldots$ do .............. & $5 \mathrm{D} \ldots$ & J. Reed........... & ....do........ & .... do........ \\
\hline *354 & $\ldots$. do... & $5 \mathrm{D} \ldots$ & Commission......... & 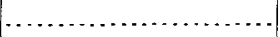 & Commission..... \\
\hline 355 & .....do... & $5 \mathrm{D} \ldots$ & Howard Place........ & Isaac Kasteard. & Isaac Kusteard.. \\
\hline 356 & .....do... & $5 \mathrm{D} .$. & Stephen Kimmerly... & $\ldots$.... do $\ldots \ldots \ldots$ & .....do............ \\
\hline *357 & $\ldots$...do.... & $5 \mathrm{D} \ldots$ & Theo. Valentine... & $\ldots$ do $\ldots \ldots \ldots$ & .....do...... \\
\hline *358 & ....do..... & $5 \mathrm{D} \ldots$ & N. H. Jacobs..... & ....do........... & ....do \\
\hline 359 & .....do.. & $5 \mathrm{D} \ldots$ & Lorenzo Smull... & ….............. & Lorenzo Smull.: \\
\hline *360 & .....do $\ldots$ & $5 \mathrm{D} .$. & Thos. E. Webb... & Geo. Schmidt... & Thos. E. Webb.. \\
\hline *361 & $\ldots . \mathrm{do} \ldots$ & $5 \mathrm{D} \ldots$ & Isaac Kasteard.... & Isaac Kasteard. & Isauc Kasteard.. \\
\hline *362 & $\ldots .$. do $\ldots . . . . . .$. & $5 \mathrm{D} \ldots$ & Long Island R. R.. & ……… & Long Island R. R. \\
\hline *363 & .....do......... & $5 \mathrm{E} \ldots$ & Frank Vanoski.... & Isaac Kasteard.. & Isaac Kasteard... \\
\hline *364 & .....do... & $5 \mathrm{E} \ldots$ & Chas. H. Mason... & & Lorenzo Smull... \\
\hline *365 & ....do... & $5 \mathrm{E} \ldots$ & Catholic ehureh.. & Isaac Kasteard. & Isane Kasteard.. \\
\hline *366 & .....do........... & $5 \mathrm{E} \ldots$ & Dodge estate........... & ....do............. & $\ldots \ldots$ do $\ldots \ldots \ldots \ldots \ldots$ \\
\hline 367 & Barker Point.... & $5 \mathrm{E} \ldots$ & W. De Forest Wright.. & & $\begin{array}{l}\text { Osear Darling, consulting } \\
\text { ongineer. }\end{array}$ \\
\hline *368 & Sands Point... & $5 \mathrm{E}_{-}, \ldots$ & Geo, Zabriskic.... & Geo. Schmidt.... & Geo. Schmidt ............... \\
\hline$* 369$ & Castle Gould.. & $5 \mathrm{~N} \ldots$ & Howard Gould... & Isaac Kasteard.. & Isaac Kasteard... \\
\hline$* 370$ & ......do... & $5 \mathrm{E} \ldots$ & .....do....... & .C. II. Danis..... & C. H. Danis........ \\
\hline$* 371$ & .....do. & $5 \mathrm{E}$. & ....do... & $\left\{\begin{array}{c}\mathrm{H} \text { ud s o o n Engineering } \\
\text { and Contracting Co. }\end{array}\right.$ & J. D. Kilpatrick $a$. \\
\hline$* 372$ & ….............. & $5 \mathrm{E} \ldots$ & Bourke Cockran............. & C. H. Danis........... & C. H. Danis....... \\
\hline$* 373$ & Long Beach... & $6 \mathrm{~B} \ldots$ & Long Beach Association.... & Wm. C. Jaegle.... & Wm. C. Jaegle..... \\
\hline$* 374$ & (. & & & & \\
\hline$* 374$ & Barnum Island... & $6 \mathrm{~B} .$. & Hempstead Poor House.. & Theo. A. Carmen. & E. Lewis, jr., Theo. Carmen: \\
\hline$* 375 \mathrm{~A}$ & $\begin{array}{l}\text { East Roekaway ... } \\
\ldots \ldots \text { do ................ }\end{array}$ & $6 \mathrm{~B} \ldots$ & Long Beach Association. & & \\
\hline$* 376$ & Rockville Center... & $\begin{array}{l}6 \mathrm{~B} \ldots \\
6 \mathrm{~B} \ldots\end{array}$ & $\begin{array}{l}\text { J. H. Clark. } \\
\text { গ. M. Smith . } \ldots \ldots \ldots \ldots\end{array}$ & $\begin{array}{l}\text { Chas. A. Fass.... } \\
\text { E. E. MeCarten.. }\end{array}$ & Chas. A: Fass ................ \\
\hline *377 & Smith Pond...... & $6 \mathrm{~B} \ldots$ & $\left\{\begin{array}{l}\text { Department water supply } \\
\text { gas and electricity. }\end{array}\right.$ & & I. M. De Varona .... \\
\hline *378 & Rockville Center. . & $6 \mathrm{~B} \ldots$ & Commission .............. & & Commission.. \\
\hline$* 379$ & ....do do............. & $6 \mathrm{~B} \ldots$ & $\begin{array}{c}\text { Rockville Center water- } \\
\text { works. }\end{array}$ & F. K. Walsh.. & Village clerk... \\
\hline$* 380$ & $\ldots \ldots$ do $\ldots . . . . . . . .$. & $6 \mathrm{~B} \ldots$ & Commission........... & & Commission.. \\
\hline$* 381$ & Millburn Reservoir.. & $6 \mathrm{~B} \ldots$ & ......do........ & & ....do ......... \\
\hline *382 & .....do................ & $6 \mathrm{~B}$. & .....do.... & & $\ldots . . . d o \ldots \ldots$ \\
\hline$* 383$ & .....do $\ldots \ldots \ldots \ldots$ & $6 \mathrm{~B}$. & ....do... & & ....do....... \\
\hline$* 384$ & .... do............ & $6 \mathrm{~B}$. & ....do.. & & ....do... \\
\hline *385 & .....do $\ldots \ldots \ldots$ & B B.. & ....do... & & .....do... \\
\hline *386 & .....do.. & $6 \mathrm{~B}$. & ....do.. & & .....do.. \\
\hline$* 387$ & ....do............. & $6 \mathrm{~B} \ldots$ & $\ldots$. do........ & & .....do.. \\
\hline *388 & .....do.............. & $6 \mathrm{~B}:$ & .....do.... & $\cdots$ & .....do....... \\
\hline *389 & ....do...... & $\begin{array}{ll}6 \mathrm{~B} \\
6 \mathrm{~B}\end{array}$ & …do. & & .....do... \\
\hline *300 & .... do...... & $\begin{array}{ll}6 & B\end{array}$ & $\begin{array}{l}\text { (...do... } \\
\text { …do... }\end{array}$ & & ....do..... \\
\hline$* 392$ & .....do................. & $6 \mathrm{~B} \ldots$ & . . do & {$[\ldots, \ldots, \ldots, \ldots$,} & ......do \\
\hline
\end{tabular}


on Long Island-Continued.

\begin{tabular}{|c|c|c|c|c|c|c|c|}
\hline $\begin{array}{l}\text { Diameter } \\
\text { of well. }\end{array}$ & $\begin{array}{l}\text { Depth of } \\
\text { well. }\end{array}$ & $\mid \begin{array}{l}\text { Depth of } \\
\text { priucipal } \\
\text { water } \\
\text { supply. }\end{array}$ & $\begin{array}{c}\text { Eeight of } \\
\text { water } \\
\text { above }(+) \\
\text { or } \\
\text { below }(-) \\
\text { ground } \\
\text { level. }\end{array}$ & $\begin{array}{c}\text { Yield } \\
\text { per } \\
\text { minute. }\end{array}$ & $\begin{array}{c}\text { Geologic horizon of } \\
\text { water-bearing strata. }\end{array}$ & Remarks. & No. \\
\hline Inches. & $\begin{array}{c}\text { Feet. } \\
107\end{array}$ & Feet. & $\begin{array}{l}\text { Feet. } \\
\text { - }\end{array}$ & Gallons. & Cretaccous. & & 350 \\
\hline 3 & 113 & $\left\{\begin{array}{r}21 \\
100-113\end{array}\right.$ & $\begin{array}{l}-17 \\
-80\end{array}$ & 166 & Jameco?.. & & 351 \\
\hline 32 & 80 & $\cdots$ & -76 & & & & 352 \\
\hline 32 & 76 & & -72 & & Tisbury .... & Sand 0 to 76 feet..... & 353 \\
\hline 2 & 87 & & $\ldots \ldots$. & & & Commission No. 1143..... & 354 \\
\hline 32 & 69 & & -65 & & & 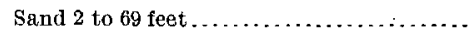 & 355 \\
\hline 32 & 46 & & -42 & & -... & White and yellow sand 0 to 46 feet.... & $35 \%$ \\
\hline 32 & 129 & & -125 & & & & 357 \\
\hline 32 & 35 & 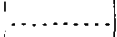 & -31 & ...... & $\ldots \ldots \ldots \ldots$ & .... & 358 \\
\hline 3 & .55 & i.......... & -41 & +12 & Tisbury?... & White sand and gravel 42 to 55 fect.... & 359 \\
\hline 4 & 206.5 & 205.6 & -71 & Small. & Cretaceous...... & . & 360 \\
\hline 32 & 69 & $\cdots$ & -65 & & 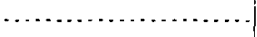 & & 361 \\
\hline & $60-70$ & & ..... & & $\ldots \ldots \ldots \ldots \ldots$ & & 362 \\
\hline 32 & 46 & & -42 & & Cretaceous?.. & & 363 \\
\hline $30-6$ & 83 & $\ldots \ldots \ldots$ & -50 & Large. & $\ldots \ldots \ldots \ldots \ldots \ldots \ldots$ & 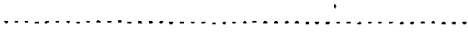 & 364 \\
\hline 32 & 54 & $\ldots \ldots \ldots$ & -50 & & Tisbury ........ & ….............. & 36.5 \\
\hline ..... & 91 & & ....... & Small. & $\ldots \ldots \ldots \ldots$ & 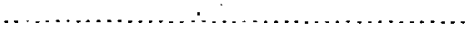 & 366 \\
\hline 3 & 30 & .... & -20 & & .... & $\begin{array}{l}\text { "Coarse sandy gravel, with water of great } \\
\text { pturity." }\end{array}$ & $\dot{3} 67$ \\
\hline 6 & 250 & & ...... & & & Rock at 250 feet.$\ldots \ldots \ldots \ldots \ldots \ldots \ldots$ & 368 \\
\hline 32 & 88 & 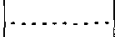 & -84 & & $\ldots \ldots \ldots \ldots \ldots$ & , & 369 \\
\hline 6 & 65 & ....... & -20 & Large. & Tisbury ......... & 2 wells $\ldots \ldots \ldots \ldots \ldots \ldots \ldots \ldots \ldots \ldots \ldots$ & 370 \\
\hline $6-4$ & 300 & & -20 & & Cretaceous .... & Abandoned $\ldots . . . \ldots \ldots$ & 370 \\
\hline & 169 & & ….... & 30 & ....do........... & $\ldots \ldots \ldots \ldots \ldots \ldots \ldots \ldots$ & \\
\hline & 120 & & ........ & 102 & ....do. . ...... & n..., & 371 \\
\hline & 354 & 220 & Flows. & Sinall. & . do................. & Pipe ologs with quicksand $\ldots \ldots \ldots \ldots \ldots$ & 372 \\
\hline 6 & 386 & $\left\{\begin{array}{l}270 \\
340 \\
383\end{array}\right.$ & +6 & 5 & .....do.......... & Water chalybeate.... & 373 \\
\hline & 383 & $\begin{array}{c}123 \\
\text { Shallow. }\end{array}$ & & & Jameco..... & $\begin{array}{l}\text { Well abandoned } \ldots \ldots \ldots \ldots \ldots \ldots \ldots \\
\text { Pumping station for Long Beach. }\end{array}$ & $\begin{array}{r}374 \\
b 375\end{array}$ \\
\hline 4 & 27 & & & & Tisbury ..... & & $375 \mathrm{~A}$ \\
\hline & 18 & ......... & -17 & & ....do............ & 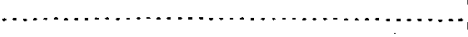 & 376 \\
\hline 5 & 587 & $\left\{\begin{array}{r}40-45 \\
578-587\end{array}\right.$ & Flows. & 5 & Cretaceous.. & Brooklyn test well No. $26 \ldots \ldots$..... & 377 \\
\hline 2 & 74 & & & & & Commission No. 605........... & 378 \\
\hline 8 & $40-50$ & & -8 & $a 26$ & Tisbury ......... & Group of 4 wells $\ldots \ldots \ldots \ldots \ldots \ldots \ldots \ldots$ & $b 379$ \\
\hline 2 & 24 & & & & & Commission No. $844 .$. & 380 \\
\hline 2 & 38 & & & & & Commission No. $697 \ldots \ldots \ldots \ldots$ & 381 \\
\hline 2 & 97 & & & & & Commission No. $658 . . . . . . . . .$. & 382 \\
\hline 2 & 31 & & & & & Commission No. $641 \ldots \ldots \ldots \ldots \ldots \ldots \ldots$ & 383 \\
\hline 2 & 32 & & & & & Commission No. $640, \ldots \ldots \ldots \ldots \ldots \ldots . . . \ldots$ & 384 \\
\hline 2 & 31 & & & & & Commission No. $630, \ldots \ldots \ldots \ldots \ldots \ldots$ & 385 \\
\hline 2 & 25.5 & & & & & Commission No. $615 \ldots \ldots \ldots \ldots \ldots \ldots \ldots$ & 386 . \\
\hline 2 & 25.6 & & & & & Commission No. $629 \ldots \ldots \ldots \ldots \ldots \ldots \ldots \ldots$ & $387^{\circ}$ \\
\hline 2 & 25 & & & & $\ldots \therefore \ldots$ & Commission No. $616 \ldots \ldots \ldots \ldots \ldots \ldots \ldots$ & 388 \\
\hline 2 & 25.5 & & & & & Commission No. $623 . \ldots \ldots \ldots \ldots \ldots \ldots \ldots$ & 389 \\
\hline 2 & 29.8 & & & & & Commission No. $617 \ldots \ldots \ldots \ldots \ldots \ldots \ldots$ & 390 \\
\hline 2 & 32.3 & & & & & Commission No. $618 \ldots \ldots \ldots \ldots \ldots \ldots \ldots \ldots \ldots \ldots$ & 391 \\
\hline 2 & 31.7 & . & & & n...................... & Commission No. $622 \ldots \ldots \ldots \ldots \ldots \ldots \ldots$ & 392 \\
\hline
\end{tabular}


TABLE XI.-Representative wells

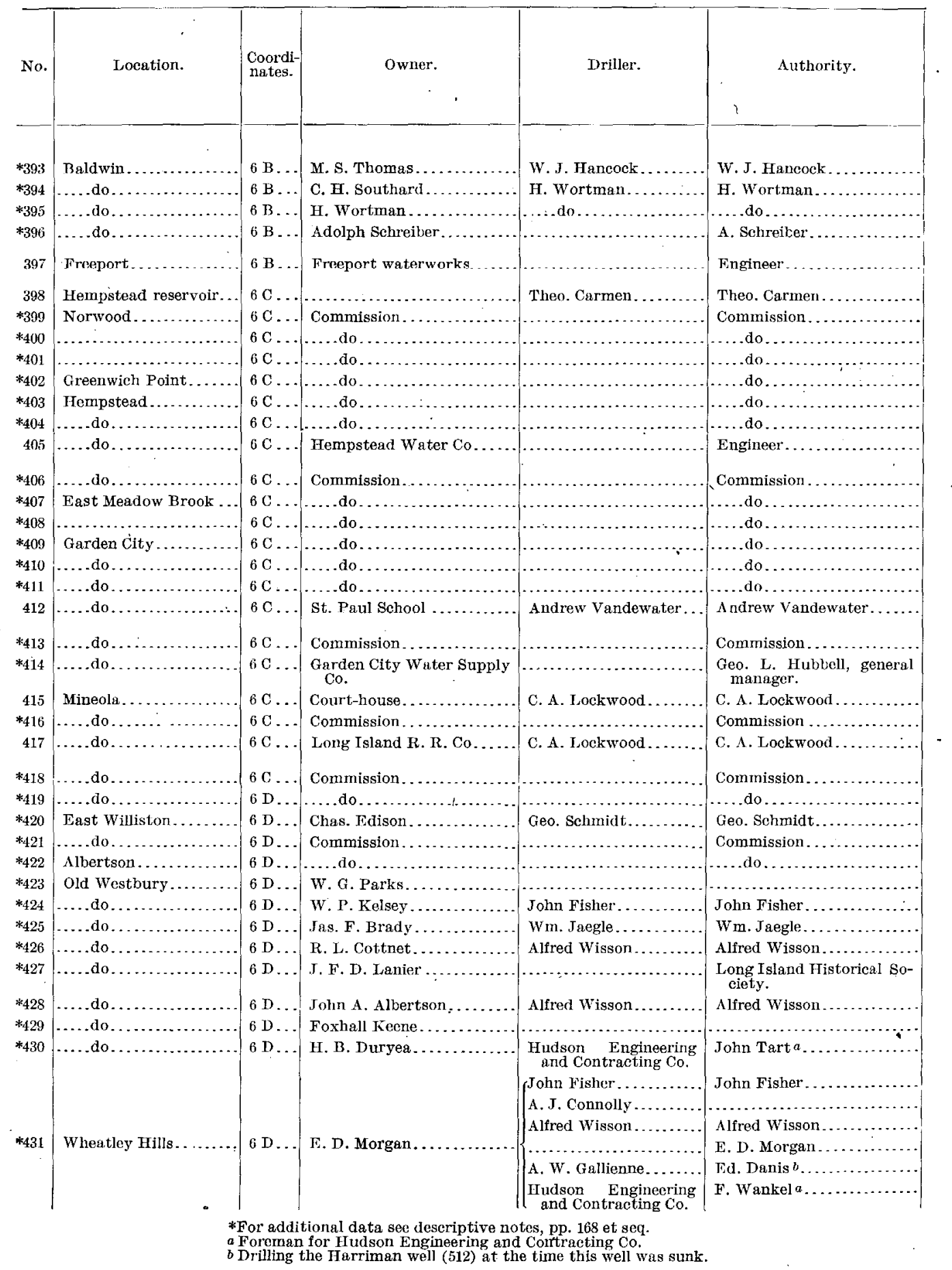


on Long Island-Continued.

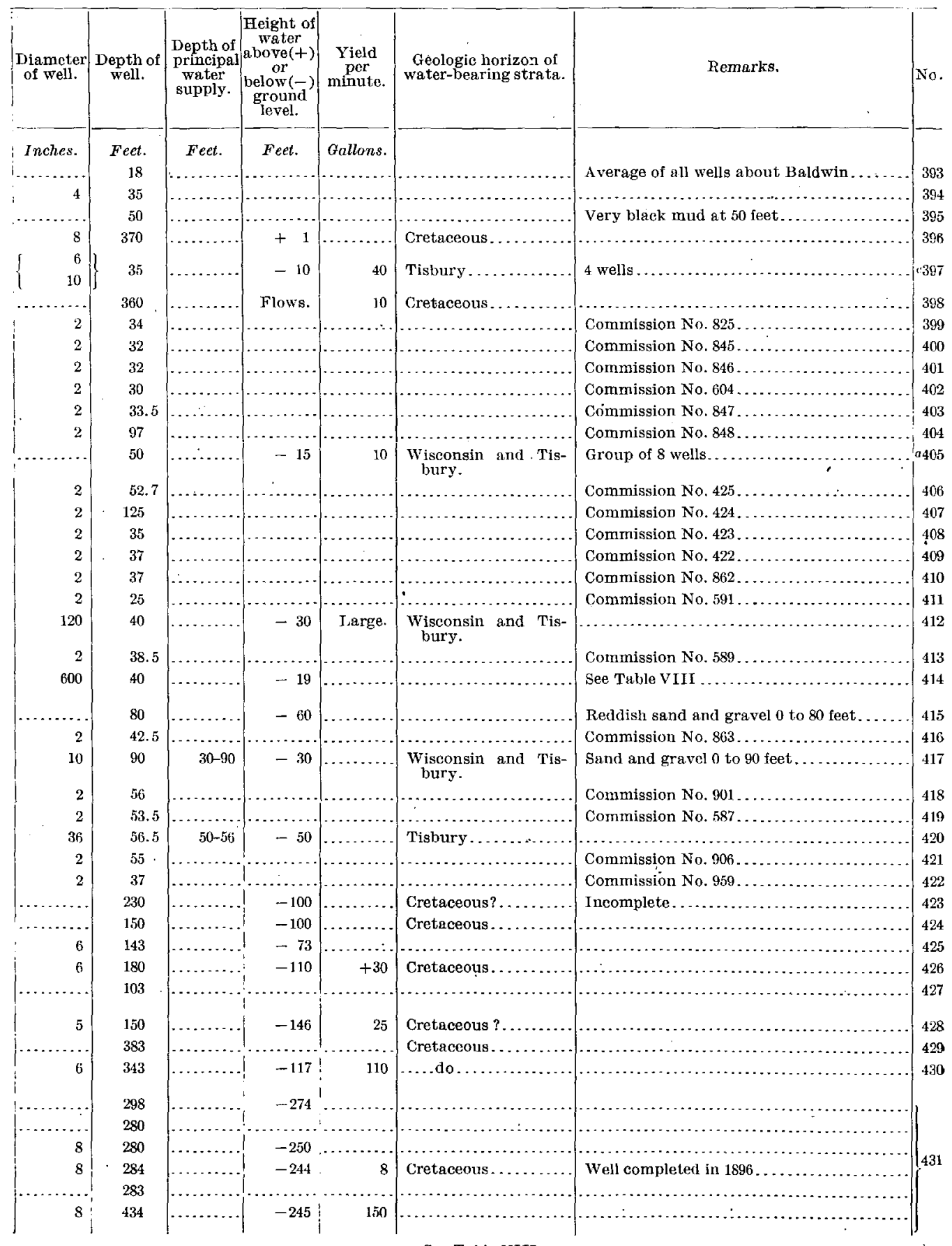

c See Table VIII. 
TABLE XI.--Representative wells

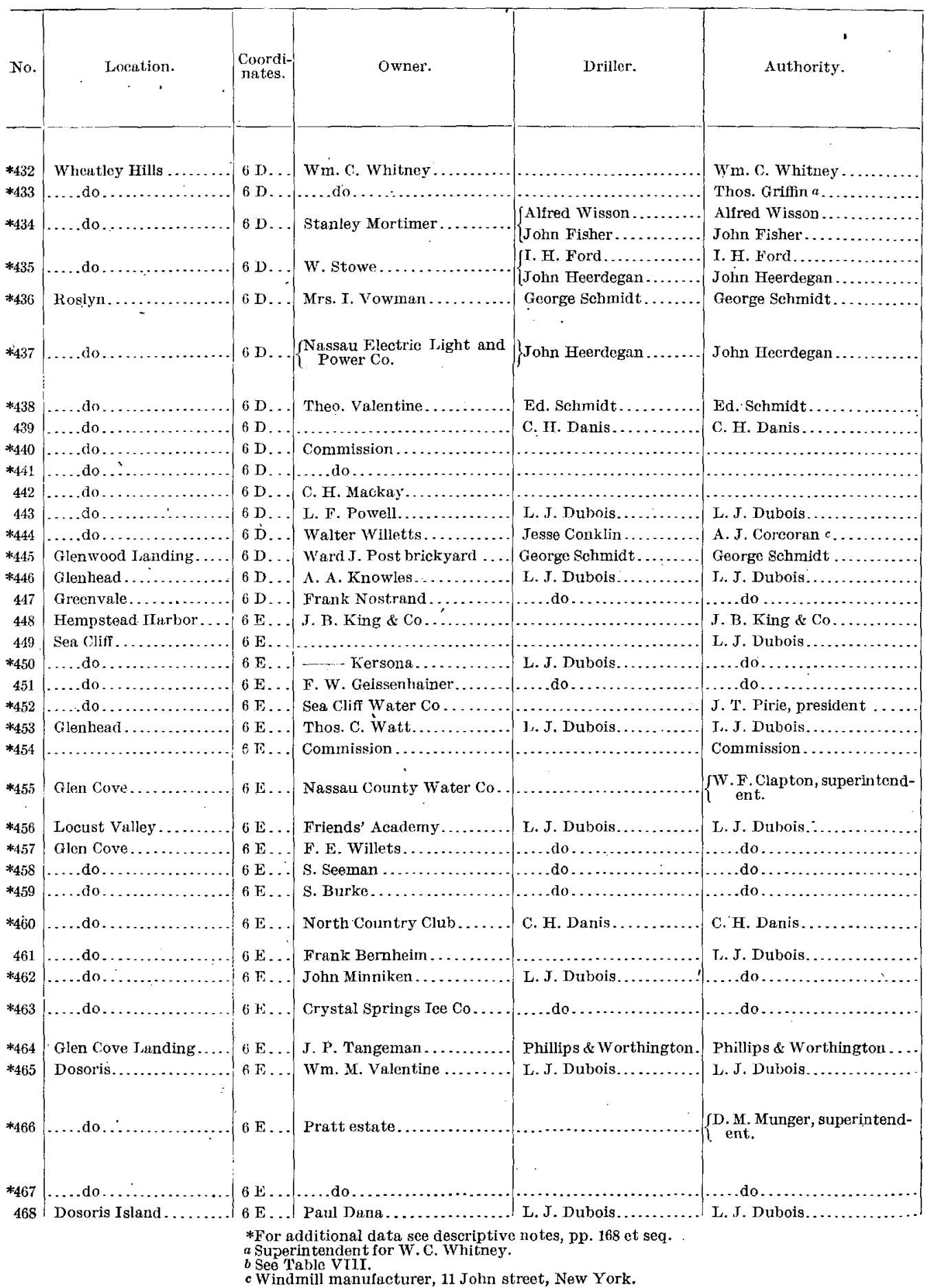


on Long Island-Continued.

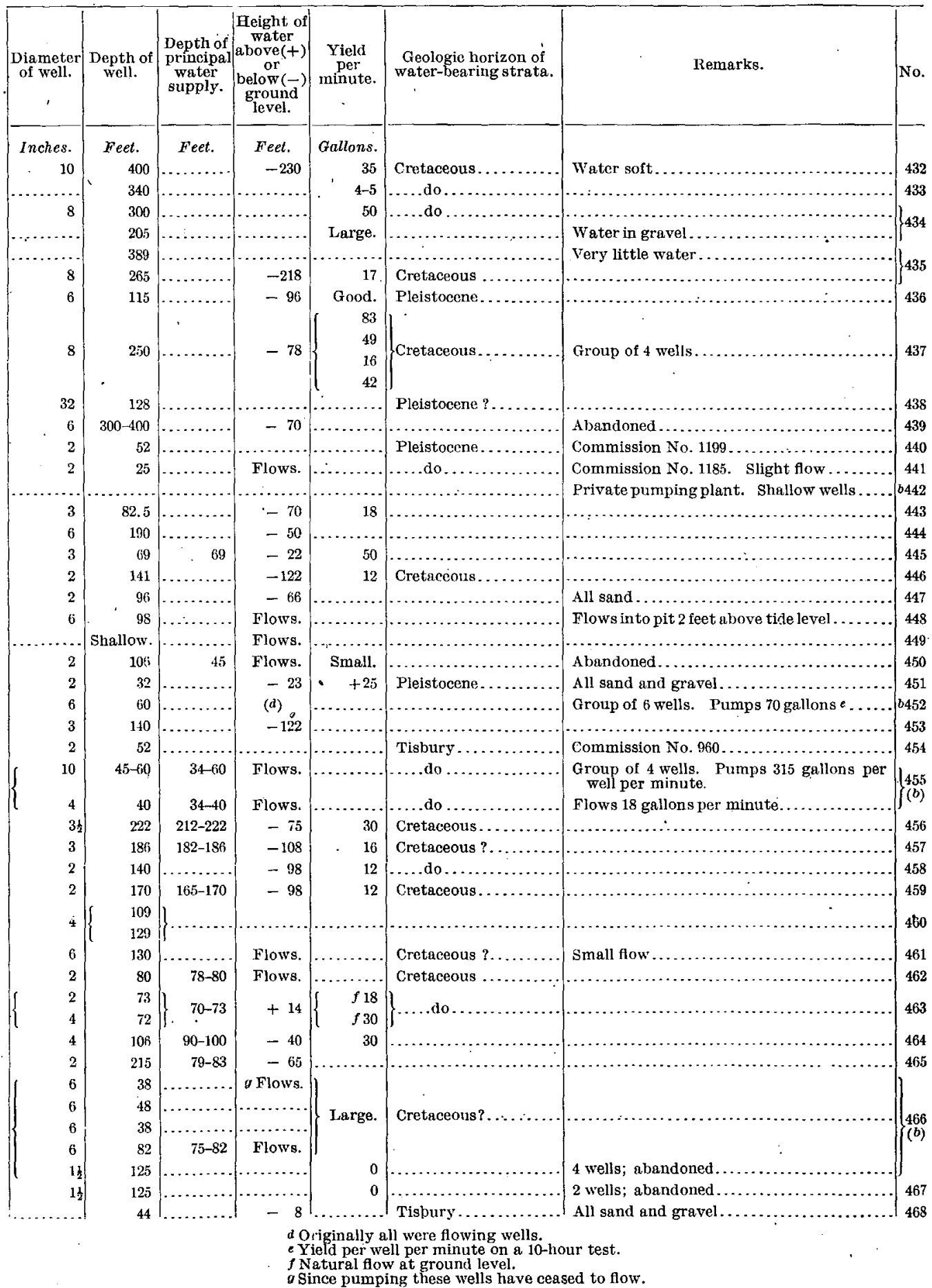


146 UNDERGROUND WATER RESOUROES OF LONG ISLAND, NEW YORK.

TABLE XI.-Representative wells

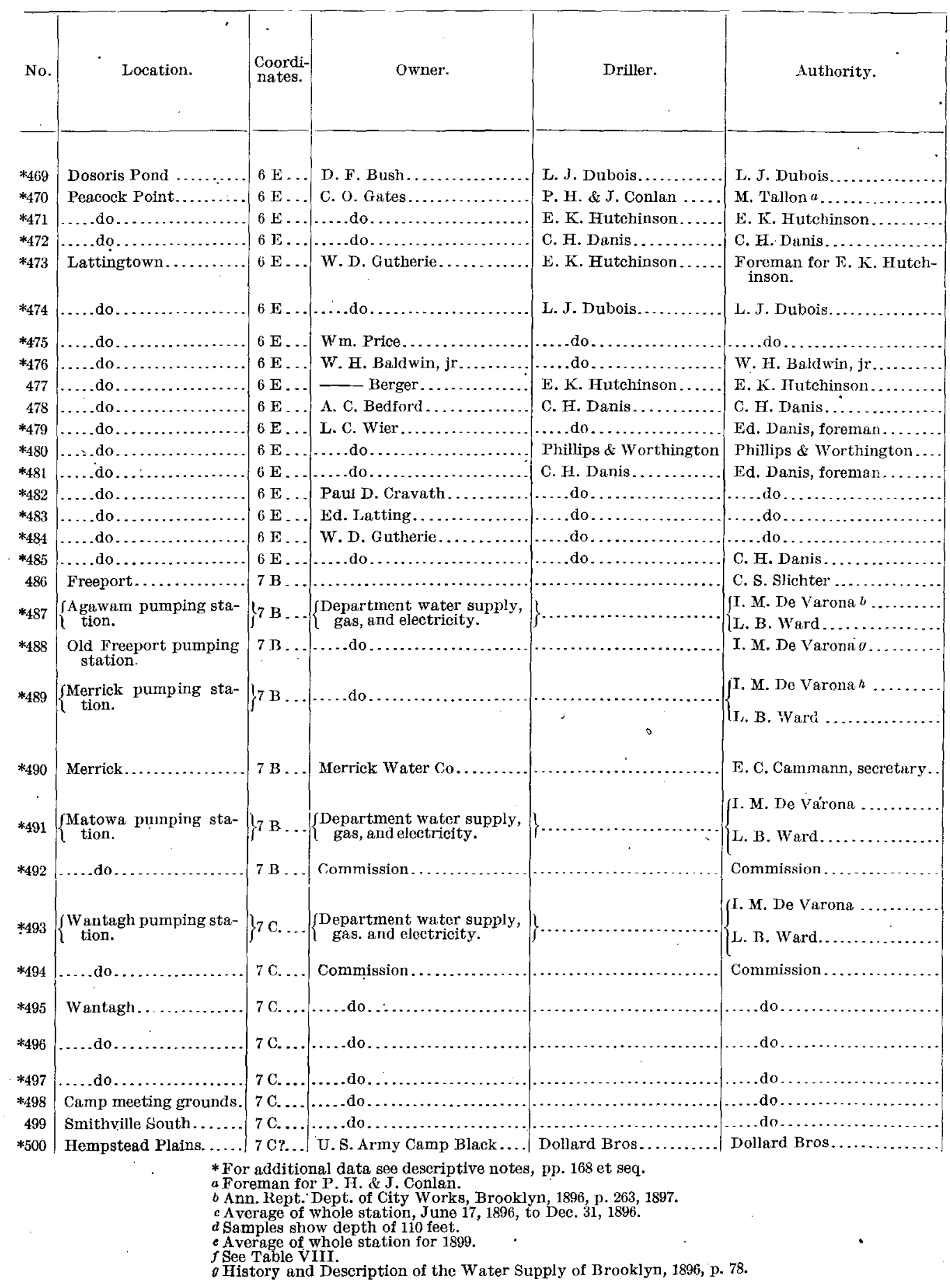


on Long Island-Continued.

\begin{tabular}{|c|c|c|c|c|c|c|c|}
\hline $\begin{array}{l}\text { Diameter } \\
\text { of well. }\end{array}$ & $\begin{array}{l}\text { Depth of } \\
\text { well. }\end{array}$ & $\begin{array}{l}\text { Depth of } \\
\text { principal } \\
\text { water } \\
\text { supply. }\end{array}$ & $\mid \begin{array}{c}\text { Height of } \\
\text { water } \\
\text { above( }(+) \\
\text { or } \\
\text { below (-) } \\
\text { ground } \\
\text { level. }\end{array}$ & $\begin{array}{l}\text { Supply } \\
\text { per } \\
\text { minute. }\end{array}$ & $\begin{array}{l}\text { Geologic horizon of } \\
\text { water-bearing strata. }\end{array}$ & Remarks. & $\mathrm{No}$ \\
\hline Inches. & Feet. & Feet. & Feet. & Gallons. & & . & \\
\hline 3 & 97 & $95-97$ & +6 & & Jameco?.... & Flows 30 gallons per minute..... & 469 \\
\hline 6 & 230 & 230 & Flows. & 30 & Lloyd sand. & & 470 \\
\hline 6 & 225 & 225 & Flows. & 5 & $\ldots$ do $\ldots . .$. & . & 471 \\
\hline ....... & 210 & ....... & Flows. & .... & $\ldots$ do ....... & & 472 \\
\hline 6 & 342 & $260-342$ & Flows. & 10 & $\ldots$. do...... & 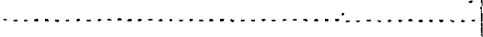 & 473 \\
\hline 2 & 92 & $\begin{array}{l}13 \\
25\end{array}$ & $\begin{array}{l}+2 \\
+2.5\end{array}$ & & Pleistocene. & Test wells . . . . . & 474 \\
\hline 2 & 162 & 162 & -90 & +12 & Cretuceous. . & & 475 \\
\hline $2 \frac{1}{3}$ & 265 & $260-265$ & -125 & +10 & ....do............. & 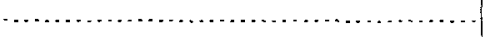 & 476 \\
\hline $2 \frac{1}{2}$ & 110 & & -94 & & & Reported as all sand and gravel... & 477 \\
\hline & 148 & & -110 & 25 & & & 478 \\
\hline & 132 & & -114 & +25 & & & 479 \\
\hline 6 & 123.5 & & -93.5 & +40 & ....... & …… & \\
\hline 6 & 91.9 & & $\cdot-76$ & +25 & Pleistocene ?.... & $\ldots \ldots$ & 481 \\
\hline 4 & 105 & & -80 & & & & 482 \\
\hline 4 & 138 & & ...... & & & . & 483 \\
\hline 3 & 108 & & -70 & $\ldots$. & $\ldots \ldots \ldots \ldots$ & 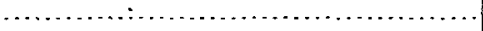 & 484 \\
\hline 3 & 144 & & -60 & +25 & & 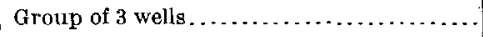 & 485 \\
\hline & 60 & & Flows. & & 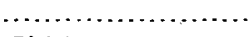 & n. & 486 \\
\hline & $\ldots \ldots$ & & $\ldots \ldots \ldots$ & c 3,131 & Pleistocene....... & $\ldots \ldots \ldots \ldots \ldots \ldots \ldots \ldots$ & \\
\hline 6 & d 33-91 & & 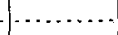 & $e 361$ & Tisbury; Jameco? . & Group of 32 wells..... & $\int(f)$ \\
\hline $4 \frac{1}{2}-6$ & 37 & & & & Pleistocene.......... & $\begin{array}{l}\text { Group of } 40 \text { wells; abandoned because of ex- } \\
\text { cess of chlorine. }\end{array}$ & 488 \\
\hline $4 \frac{1}{2}-6$ & $\left\{\begin{array}{r}45 \\
106-109\end{array}\right.$ & & & $i 3,259$ & Tisbury; Jameco? ... & Group of 62 wells $\ldots \ldots \ldots \ldots \ldots \ldots \ldots \ldots$ & \\
\hline 412 & $40-110$ & & & $e 378$ & & $\ldots .$. do $\ldots . . . .$. & \\
\hline & $\left\{\begin{array}{r}30-40 \\
83\end{array}\right.$ & $6-35$ & $\begin{array}{l}-4 \\
-8\end{array}$ & 5 & Pleistocene. & $\begin{array}{l}\text { Group of } 8 \text { wells............... } \\
\text { No water below } 40 \text { feet......... }\end{array}$ & \\
\hline $4 \frac{1}{2}$ & $38-97$ & & $\left\{\begin{array}{l}-6 \\
k \text { Flow. }\end{array}\right.$ & $\begin{array}{r}j 3,122 \\
e 618\end{array}$ & $\left\{\begin{array}{l}\text { Tisbury } \ldots \\
\text { Jameco ? }\end{array}\right.$ & 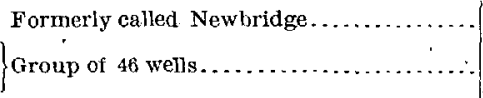 & $\left\{\begin{array}{l}491 \\
(f)\end{array}\right.$ \\
\hline 2 & 20 & & & & ........... & $\begin{array}{l}\text { Commission No. 1161. Slichter underflow } \\
\text { station No.3. }\end{array}$ & 492 \\
\hline $4 \frac{1}{2}-6$ & $24-92$ & & $\left\{\begin{array}{c}-2.7 \\
k \text { Flow }\end{array}\right.$ & & $\begin{array}{l}\text { Tisbury.... } \\
\text { Jameco } \ldots\end{array}$ & Group of 49 wells..... & $\left\{\begin{array}{l}493 \\
(f)\end{array}\right.$ \\
\hline 2 & 20 & & & & & $\begin{array}{l}\text { Slichter underflow station No. 2; commis- } \\
\text { sion No. 1176. }\end{array}$ & 494 \\
\hline 2 & 71 & & m. Flows. & & & $\begin{array}{l}\text { Commission No. 1272, Slichter underfow } \\
\text { station No. } 2 \text {. }\end{array}$ & 495 \\
\hline 2 & 83 & & $m$ Flows. & & & $\begin{array}{l}\text { Commission No. 1293. Slichter underflow } \\
\text { station No. 15. }\end{array}$ & 496 \\
\hline 2 & 13 & & & & & Commission No. $1356 \ldots \ldots \ldots \ldots \ldots$ & 497 \\
\hline 2 & 17 & & & & & Commission No. $1357 \ldots \ldots \ldots \ldots \ldots \ldots \ldots$ & 498 \\
\hline 2 & 14 & & $\cdots$ & & $\cdots \cdots$ & Commission No. $1375, \ldots \ldots \ldots \ldots \ldots$ & 499 \\
\hline
\end{tabular}

h Ann. Rept. Dept. of City Works, Brooklyn, 1896, p. 266. 1897; History and Description of the Water Supply of Brooklyn, 1896 , 1 . 79

i Average of whole station, Jan. 23,1896 , to Dec. $31,1896$.

1 Average of whole station, Sept. 23,1896 , to Dec. 31,1896

$k$ Deep wells only

, to Dec. 31, 1896.

$m$ Flow began at 62 feet. 


\section{UNDERGROUND WATER REȘOUROES OF LONG ISLAND, NEW YORK.}

TABLE XI.-Representative wells

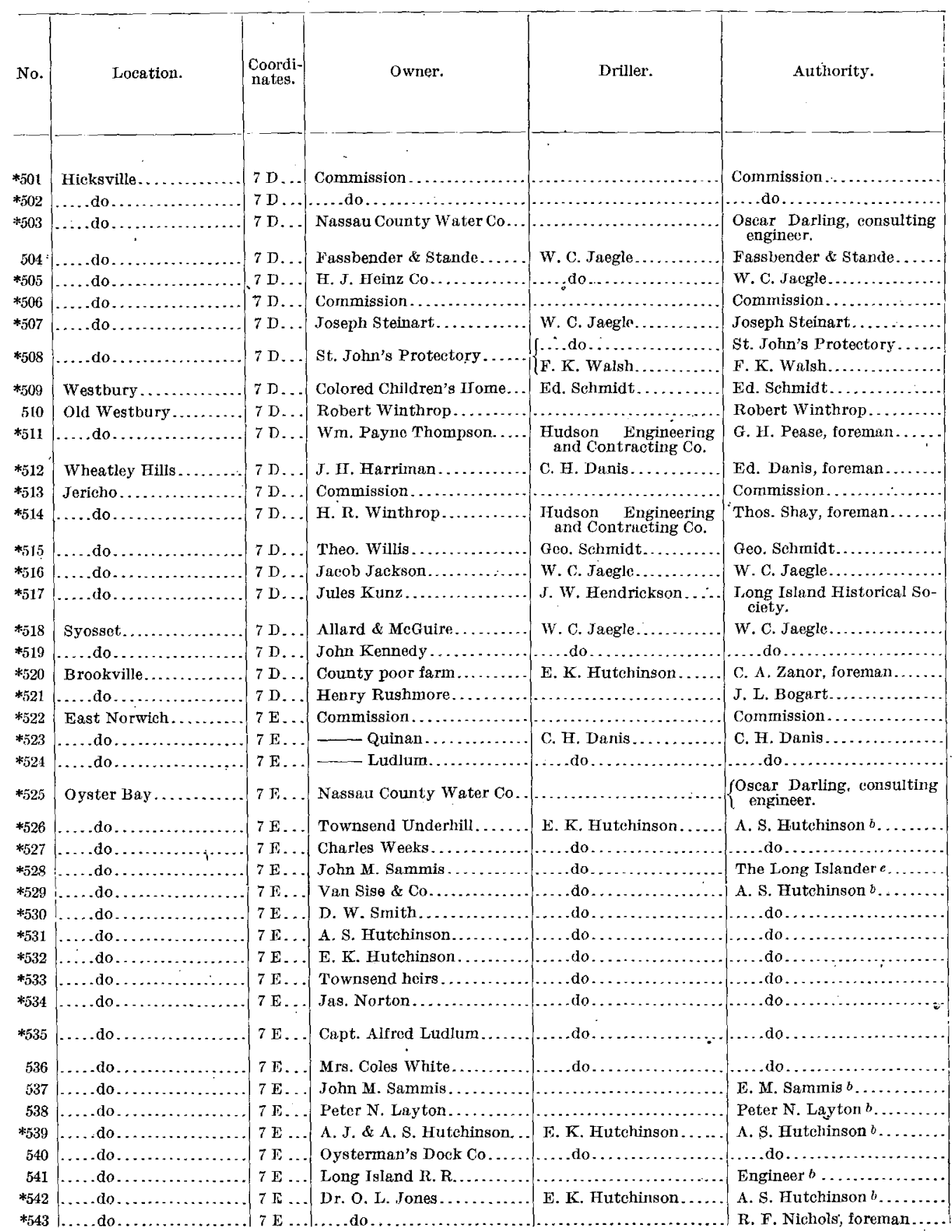

*For additional data see descriptive notes, pp. 168 et seq.

o Records transmitted to the Survey by Mr. W. H. C. Pynchon, civil engineer and geologist, Oyster Bay, N. Y. 
on Long Island-Continued.

\begin{tabular}{|c|c|c|c|c|c|c|c|}
\hline $\begin{array}{l}\text { Diameter } \\
\text { of well. }\end{array}$ & $\begin{array}{c}\text { Depth of } \\
\text { well. }\end{array}$ & $\begin{array}{l}\text { Depth of } \\
\text { principal } \\
\text { water } \\
\text { supply. }\end{array}$ & $\left|\begin{array}{c}\text { Height of } \\
\text { water } \\
\text { above }(+) \\
\text { or } \\
\text { below }(-) \\
\text { ground } \\
\text { level. }\end{array}\right|$ & $\begin{array}{c}\text { Supply } \\
\text { per } \\
\text { minute. }\end{array}$ & $\begin{array}{l}\text { Geologic horizon of } \\
\text { water-bearing strata. }\end{array}$ & Remarks. & No. \\
\hline Inches. & Feet. & Feet. & Feet. & Gallons. & & & \\
\hline & 56 & & & & & Commission No. $909 \ldots$ & 501 \\
\hline 2 & 135.5 & 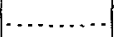 & . & & & Commission No. 955.:. & 502 \\
\hline 8 & 85 & $63-85$ & -63 & ......... & Tisbury ..... & 2 wells . . . . . . . . . . . . . & $a 503$ \\
\hline 6 & 79 & & -71 & Large. & Tisbury? . & & 504 \\
\hline $42-4$ & 90 & & -68 & 4 & Cretaceous. & $\ldots \ldots \ldots \ldots \ldots \ldots \ldots \ldots \ldots$ & 505 \\
\hline 2 & 80.5 & 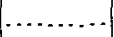 & ....... & & & Commission No. $1142 \ldots$ & 506 \\
\hline 6 & 150 & $130-150$ & -75 & $\cdots \cdots$ & & 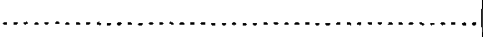 & 507 \\
\hline 6 & 73 & & -61 & 35 & & & 508 \\
\hline 6 & 80 & & -60 & . & & & 508 \\
\hline 36 & 60 & & -35 & Small. & & & 509 \\
\hline 10 & 377 & $\ldots \ldots \ldots$ & -200 & 25 & Cretaceous... & Water slightly hard:... & 510 \\
\hline & 209 & $195-205$ & ......... & 60 & .... do..... & & 511 \\
\hline -.. & 220 & $200-220$ & -180 & +25 & $\ldots$ do:... & ( $\ldots \ldots \ldots \ldots \ldots \ldots \ldots \ldots \ldots \ldots \ldots \ldots$, & 512 \\
\hline 2 & .60 & & & & ........... & Commission No. $1193 \ldots \ldots \ldots$. . & 513 \\
\hline 3 & 183 & $150-183$ & -150 & & Cretaceous.. & 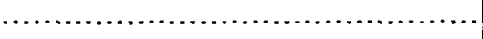 & 514 \\
\hline 6 & 175 & & -160 & & Cretaceous?. & & 551 \\
\hline $36-3$ & 168 & & -165 & & & Originally reported 210 feet deep........... & 516 \\
\hline 36 & 147.5 & & & & & 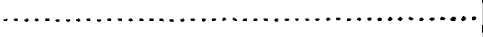 & 517 \\
\hline & 53 & $47-50$ & & Large. & & & 518 \\
\hline & +150 & & & & & Well "blows" at a depth of 150 feet........ & 519 \\
\hline & 278 & & -105 & (n........ & & 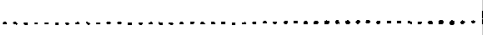 & 520 \\
\hline$\ldots$ & 396 & & 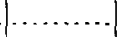 & 0 & & 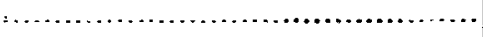 & 521 \\
\hline 2 & 23 & & & & & Commission No. $1192 \ldots \ldots \ldots \ldots \ldots$. & 522 \\
\hline 3 & 224 & & $\cdots \cdots \cdot$ & -........ & … & & 523 \\
\hline 3 & 149 & ...... & -213 & & & n.................... & 524 \\
\hline $1 \frac{1}{2}$ & 160 & $4-10$ & $\cdots$ & & & Abandoned.............. & 525 \\
\hline 4 & 35 & $10-30$ & 0 & $\ldots$ & $\ldots$ & Group of wells . ........ & \\
\hline 3 & 165 & 162 & +13 & ed 100 & Jameco? . & 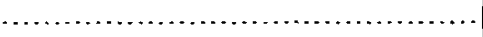 & 526 \\
\hline 2 & 110 & $90-110$ & Flows. & $c 20$ & $\ldots$.do $\ldots \ldots \ldots . .$. & 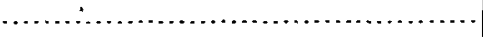 & 527 \\
\hline 2 & 140 & $\cdots \cdots$ & Flows. & ..... & ...... & Snouder's pharmacy................ & 528 \\
\hline 2 & 56 & $53-57$ & +1.5 & c 3 & Jameco? . & Original flow 9 gallons $\ldots \ldots \ldots \ldots \ldots \ldots \ldots$ & 529 \\
\hline 2 & 65 & 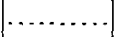 & Flows. & $c 5$ & $\ldots$. do... & Original flow 15 gallons...................... & 530 \\
\hline 2 & $30-160$ & & +6 & $c 8.5$ & $\ldots$...do... & 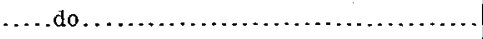 & 531 \\
\hline 2 & 83 & & .......... & cf 21 & $\ldots$. do.... & $\ldots \ldots \ldots \ldots \ldots \ldots \ldots \ldots \ldots, \ldots$ & 532 \\
\hline 2 & 133 & & Flows. & $c f 15$ & .....do..... & 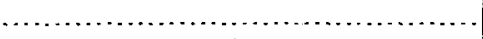 & 533 \\
\hline 2 & $65-70$ & & ......... & $c 4$ & .... do... & Original flow 10 gallons...$\ldots \ldots \ldots \ldots$ & 534 \\
\hline 2 & 82 & & +2.3 & $c 2$ & ....do.... & Original flow 9 to 10 gallons $\ldots . . . .$. & 535 \\
\hline 3 & 118 & & +7 & $c 30$ & .... do.... & Original flow 36 gallons............. & \}$^{335}$ \\
\hline 2 & 115 & & +1.5 & $c 7.5$ & $\ldots$.... do..... & 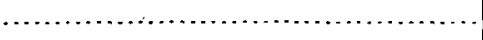 & 536 \\
\hline 3 & 60 & ....... & +2 & $c 5.5$ & ..... do.... & 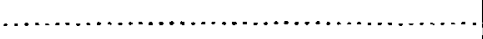 & 537 \\
\hline $1 \frac{1}{2}$ & 48 & $\cdots \cdots \cdots$ & +.5 & $c 1$ & $\ldots$ do ... & Ceases to flow at low tide............ & 538 \\
\hline 5 & 190 & 185 & +17 & c 70 & ...do.... & 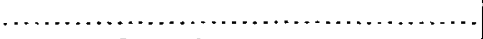 & 539 \\
\hline 2 & 36 & & Flows. & $4-5$ & ( & Does not flow at low tide................ & 540 \\
\hline 4 & 20 & & -10 & +66 & & 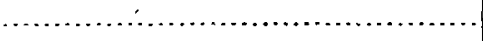 & 541 \\
\hline 2 & 126 & & +9 & 18 & Jameco? . . & & 542 \\
\hline 4 & 220 & & $\mid \ldots \ldots \ldots . .$. & $g 26.5$ & .....do.... & & 543 \\
\hline
\end{tabular}

$c$ Rate of flow varies with the tide.

$d$ Flow at ground level. At +17 feet furnishes 5 gallons per minute.

e Huntington, N.Y., June 15, 1895.

$f$ Initial flow.

$g$ Flow at low tide July 30, 1903. 
150 UNDERGROUND WATER RESOURCES. OF LONG ISLAND, NEW YORK.

TABLE XI.-Representative wells

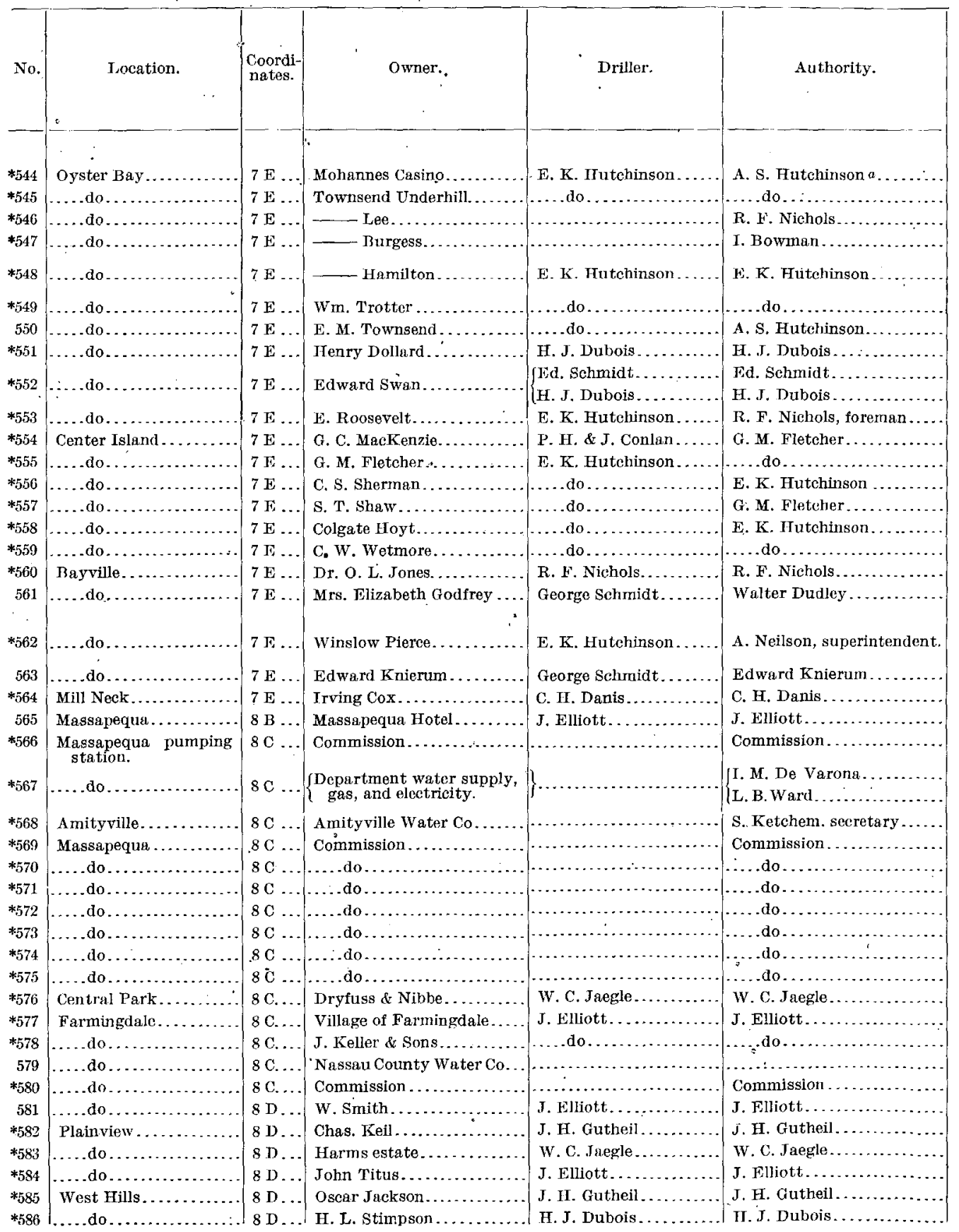

* For additional data see descriptive notes, pp. $168 \mathrm{et}$ seq.
a Records transmitted to the Survey by Mr. W. H. C. Pynchon, civil engineer and geologist, Oyster Bay, N. Y.

$a$ Flow varies with the tide.

$b$ Flow varies with the tide
$c$ Depth July, 1903, 188.3. 
on Long Island-Continued.

\begin{tabular}{|c|c|c|c|c|c|c|c|}
\hline $\begin{array}{l}\text { Diam- } \\
\text { eter of } \\
\text { well. }\end{array}$ & $\begin{array}{l}\text { Depth of } \\
\text { well. }\end{array}$ & $\begin{array}{c}\text { Depth of } \\
\text { principal } \\
\text { water } \\
\text { supply. } \\
\text {. }\end{array}$ & $\mid \begin{array}{c}\text { Height of } \\
\text { water } \\
\text { above }(+) \\
\text { or } \\
\text { below }(-) \\
\text { ground } \\
\text { level. }\end{array}$ & $\begin{array}{l}\text { Supply } \\
\text { per } \\
\text { minute. }\end{array}$ & $\begin{array}{l}\text { Geologic horizon of } \\
\text { water-bearing strata. }\end{array}$ & $\begin{array}{l}. \\
+ \\
\end{array}$ & No. \\
\hline Inches. & Feet. & Feet. & Feet. - & Gallons. & & & \\
\hline 3 & 99 & & $\cdots \ldots$. & $b 20-100$ & Jameco?.... & & 544 \\
\hline 2 & 107 & & +2 & $b 18$ & $\ldots \ldots$ do $\ldots . .$. & $\cdots \ldots \ldots, \ldots, \ldots, \ldots$, & 545 \\
\hline 3 & $c 200$ & & Flows. & $b 10$ & $\ldots$. do...... & & $\tilde{5} 46$ \\
\hline 2 & 155.5 & ....... & Flows. & b? 25 & ....do....... & n.......... & 547 \\
\hline$\cdot$ & 227 & 130 & & & & & 548 \\
\hline & 105 & 105 & Flows. & & & & 舟 \\
\hline 3 & 90 & & Flows. & $b 100$ & Jameco?. . & & 549 \\
\hline 3 & 77 & .... & Flows. & $b 100$ & .... do. . . . . & $\cdots \cdots, \ldots, \ldots, \ldots$ & 550 \\
\hline $2 \frac{1}{2}$ & 259 & 259 & -1 & ........... & Cretaceous... & Well flows into pit... & 551 \\
\hline 4 & 60 & & -3 & (........... & & & 552 \\
\hline 2 & 212 & $\cdots$ & +13 & 65 & 1 & & 396 \\
\hline & 465 & 465 & Flows. & $\cdots \ldots \ldots$ & Lloyd sand. & Abandoned because of breaking of pipe.. & 503 \\
\hline & 378 & $\cdots$ & Flows. & $845-75$ & $\ldots$. do $\ldots . . .$. & & 554 \\
\hline & 370 & 360 & Flows. & b 30 & ....do....... & & 555 \\
\hline & 351 & & Flows. & $620-30$ & ....do............ & 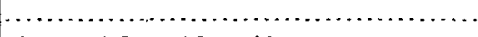 & 556 \\
\hline & 292 & & Flows. & $5-6$ & Cretaceous . . . . . . & Does not flow at low tide....... & 557 \\
\hline & 320 & $300-320$ & Flows. & 50 & Lloyd sand...... & 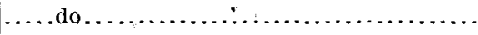 & 558 \\
\hline & 318 & $300-318$ & Flows. & 25 & $\ldots$.do $\ldots \ldots \ldots \ldots$ & $\ldots, \ldots \ldots \ldots, \ldots, \ldots, \ldots$ & 559 \\
\hline & 295 & & Flows. & 8 & Lloyd sand.... & 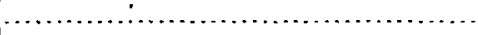 & 560 \\
\hline 84 & 23 & & $-17 \frac{1}{2}$ & 83 & Pleistocene... & $\begin{array}{l}\text { Private water supply system supplying } \\
\text { Pine Island Park and vicinity. }\end{array}$ & 561 \\
\hline $60-6$ & 40 & & -27 & 25 & .....do...... & & 562 \\
\hline 6 & 80 & & ...... & +25 & $\ldots$. do...... & & \\
\hline 6 & 45 & $\ldots \ldots \ldots$ & -39 & Large. & $\ldots$. do........... & $\cdots$ & 563 \\
\hline 3 & 330 & $300-330$ & - $\cdots \cdots$ & $b 120$ & Lloyd sand.. & ….... & 564 \\
\hline 8 & 27 & $\ldots \ldots \ldots$ & -22 & $\ldots \ldots \ldots$ & Pleis tocene.. & 2 tile wells $\ldots \ldots \ldots \ldots \ldots \ldots \ldots \ldots \ldots \ldots \ldots$ & 565 \\
\hline 2 & 24 & & & & $\ldots \ldots \ldots+\ldots, \ldots$ & $\begin{array}{l}\text { Commission No. } 1173 \text {. Slichter underfow } \\
\text { station No. 1. }\end{array}$ & 566 \\
\hline & $37.5-106$ & & $\begin{array}{l}\text { e....... } \\
\text { e Flow, }\end{array}$ & d 3,731 & Tisbury, Jameco?.. & Group of 106 wells.... & $\int(f)$ \\
\hline 6 & 40 & & -12 & 072 & Tisbury............ & $\ldots \ldots \ldots \ldots \ldots \ldots \ldots$ & 5568 \\
\hline 2 & 31 & & ...... & & $\cdots \ldots \ldots \ldots \ldots$ & Commission No. $849, \ldots \ldots \ldots$. & 569 \\
\hline 2 & 18 & & $\cdots$ & & ... & Commission No. $1354 \ldots \ldots \ldots \ldots \ldots \ldots$ & 570 \\
\hline 2 & 31 & & & & & Commission No. $720 . \ldots \ldots \ldots \ldots \ldots$. & 571 \\
\hline 2 & 35.5 & & $\cdots$ & & $\cdots \cdot$ & Commission No. $696 \ldots, \ldots \ldots \ldots$ & 572 \\
\hline 2 & 25 & & ..... & & $\ldots \ldots \ldots \ldots \ldots$ & Commission No. $858 \ldots \ldots \ldots \ldots \ldots \ldots$ & 573 \\
\hline 2 & 85 & & & & & Commission No. $865 \ldots \ldots \ldots \ldots \ldots \ldots$ & 574 \\
\hline 2 & 41.5 & & …. & & $\ldots \ldots \ldots \ldots$ & Commission No. $908 \ldots \ldots \ldots \ldots \ldots \ldots . . . . . .$. & 575 \\
\hline & 55 & $\cdots$ & -28 & & $\ldots \ldots \ldots \ldots, \ldots, \ldots$ & 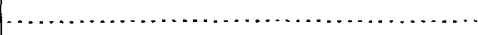 & 576 \\
\hline 20 & $34-36$ & & -16 & Large. & Pleistocene?....... & 4 tile wells used for fire protection ....... & 577 \\
\hline 12 & 40 & $\cdots$ & -28 & Large. & 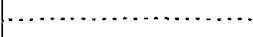 & Tile well $\ldots \ldots \ldots \ldots \ldots \ldots \ldots \ldots \ldots \ldots \ldots$ & 578 \\
\hline & & & …... & & 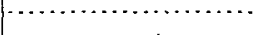 & Commion 771 & 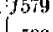 \\
\hline 2 & 21 & & $\cdots \cdots$ & $\cdots$ & 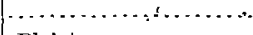 & Commission No. $771 .$. & 580 \\
\hline 8 & 34 & $\ldots \ldots \ldots$ & -25 & $\ldots \ldots \ldots$ & Pleistocene.......... & $\cdots \cdots \cdots$ & 581 \\
\hline $36-1 \frac{1}{4}$ & 111 & & $\cdots \cdots \cdot$ & Good. & $\ldots \ldots \ldots \ldots \ldots$ & $\ldots . .$. & 582 \\
\hline ...... & 65 & & -61 & $\cdots \cdots$ & & & 583 \\
\hline 8 & 70 & & ....... & & & & 584 \\
\hline $36-1 \frac{1}{4}$ & 141.5 & & n..... & & $\cdots \cdots \cdots, \ldots, \cdots, \cdots$ & $\ldots \ldots \ldots \ldots \ldots$ & 585 \\
\hline 21 & 343 & & -294 & 7 & Cretaccous.......... & $\ldots \ldots \ldots \ldots$ & 586 \\
\hline & & & $\begin{array}{l}d \text { Averag } \\
\text { e Only th } \\
f \text { See Tal } \\
\text { A Averag }\end{array}$ & $\begin{array}{l}\text { e pumped } \\
\text { ie deeper } \\
\text { ole VIII. } \\
\text { e for } 1902 \text {. }\end{array}$ & $\begin{array}{l}\text { from A pril } 13,1896 \text {, to } \\
\text { ells flow. }\end{array}$ & ecember 31,1896 . & . \\
\hline
\end{tabular}


152. UNDERGROUND WATER RESOURCES OF LONG ISLAND, NEW YORK.

TABLE XI.-Representative wells

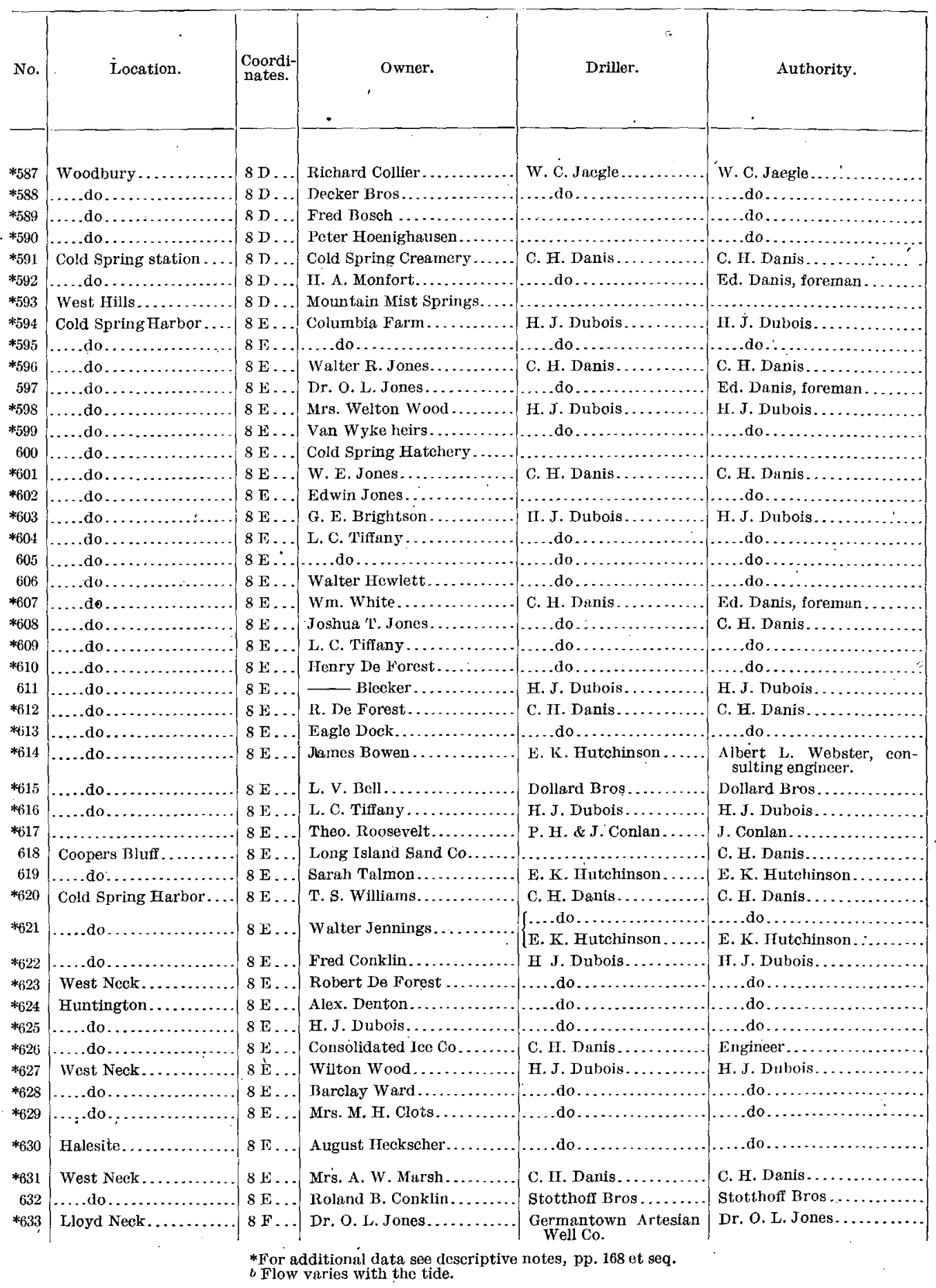


on Long Island-Continued.

\begin{tabular}{|c|c|c|c|c|c|c|c|}
\hline $\begin{array}{l}\text { Diameter } \\
\text { of well. }\end{array}$ & $\begin{array}{c}\text { Depth of } \\
\text { well. }\end{array}$ & $\begin{array}{l}\text { Depth of } \\
\text { principal } \\
\text { water } \\
\text { supply. }\end{array}$ & $\left|\begin{array}{c}\text { Height of } \\
\text { water } \\
\text { above }(+) \\
\text { or } \\
\text { below }(-) \\
\text { ground } \\
\text { level. }\end{array}\right|$ & $\begin{array}{c}\text { Supply } \\
\text { per } \\
\text { minute }\end{array}$ & $\begin{array}{l}\text { Geologic horizon of } \\
\text { water-bearing strata. }\end{array}$ & Remarks. & No. \\
\hline $\begin{array}{r}\text { Inches. } \\
6\end{array}$ & $\begin{array}{c}\text { Feet. } \\
144\end{array}$ & $\begin{array}{c}\text { Feet. } \\
138-1+4\end{array}$ & $\begin{array}{l}\text { Feet. } \\
-100\end{array}$ & $\begin{array}{l}\text { Gallons. } \\
\quad+10\end{array}$ & .... do..... & & 587 \\
\hline $6-4$ & 185 & & & & $\ldots \ldots \ldots$ & Well blows at 120 to 150 feet. & 588 \\
\hline & $\cdots \cdot \cdot$ & & & & & Blowing well $\ldots \ldots \ldots \ldots \ldots$ & 589 \\
\hline & 200 & & $\cdots \cdots$ & $\cdots$ & 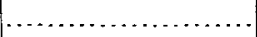 & $\ldots$ do............ & 590 \\
\hline$\ldots$ & 96 & & $\ldots \ldots$. & $\ldots \ldots \ldots$ & Cretaceous.... & 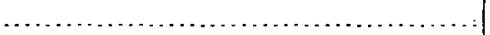 & 591 \\
\hline 4 & 195 & & -135 & 25 & ....dó......... & & 592 \\
\hline Spring.- & .... & & …. & .......... & $\ldots \ldots \ldots \ldots \ldots$ & n. & 593 \\
\hline 4 & 235 & & -200 & Small. & Cretaceous.... & & 594 \\
\hline 4 & 295 & $\ldots \ldots \ldots$ & $\ldots \ldots \ldots$ & Small. & $\ldots$. do....... & 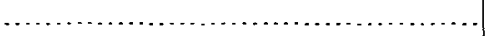 & 595 \\
\hline 2 & 228 & $\ldots \ldots \ldots$ & -198 & 6 & $\ldots$......... & & 596 \\
\hline 4 & 260 & $232-260$ & -232 & 25 & & & 597 \\
\hline 2 & 163 & & -5 & & $\ldots \ldots \ldots$ & & 598 \\
\hline$\ldots \ldots \ldots$ & 150 & 150 & -20 & $\ldots$. & Cretaceous... & .. & 599 \\
\hline Spring.. & $\ldots$. & & $\ldots \ldots \ldots$ & 150 & $\ldots \ldots \ldots \ldots \ldots$ & & 600 \\
\hline 3 & 195 & $\ldots \ldots$ & +2 & $b 7.5$ & Pleistocene..... & $\ldots \ldots \ldots \ldots \ldots \ldots$ & 601 \\
\hline $1 \frac{1}{4}$ & 49 & 49 & $\ldots \ldots$ & $b 5$ & .... do . . . . . . . & No flow at low tide.. & 602 \\
\hline & 177 & $170-177$ & -117 & & & & 603 \\
\hline 6 & 243 & $235-243$ & $\ldots \ldots$ & +30 & (2. & & 604 \\
\hline 3 & 256 & $200-256$ & -150 & ...... & $\ldots \ldots \ldots \ldots \ldots$ & 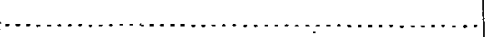 & 605 \\
\hline & 51 & ......... & -4 & ........ & Pleistocenc.......... & Flevation about 20 feet above tide ........ & 606 \\
\hline 3 & 179 & 179 & Flows. & b 33 & & & 607 \\
\hline 3 & 70 & $66-70$ & Flows. & $b 60$ & …... & 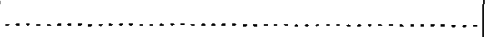 & 608 \\
\hline & 77 & $58-77$ & Flows. & b 75 & …. & Initial flow estimated 125 gallons...... & 609 \\
\hline & 165 & $\ldots \ldots \ldots$ & 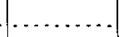 & ........ & $\ldots \ldots$. & & 610 \\
\hline 4 & 45 & $35-45$ & Flows. & $\ldots \ldots$. & $\ldots \ldots \ldots \ldots \ldots$ & $-\ldots \ldots$ & 611 \\
\hline 6 & 184 & $177-184$ & Flows. & $b 45-65$ & Cretaceous. . . . . . & & 612 \\
\hline 3 & 176 & & & $3-5$ & Pleistocene... & & 613 \\
\hline 6 & 58 & & ...... & $16-50$ & $\therefore \ldots \ldots \ldots \ldots \ldots \ldots \ldots$, & $\ldots \ldots \ldots \ldots \ldots \ldots$ & 614 \\
\hline & 65 & & -50 & & & & 615 \\
\hline 8 & $\ldots$ & & -15 & ... & ... & Elevation, 40 feet above tide....... . & 616 \\
\hline 4 & 140 & & -125 & 25 & & 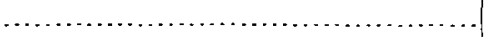 & 617 \\
\hline 4 & 248 & $\cdots$ & $\cdots \cdots$ & Targe. & $\ldots \ldots$. & Salt water $\ldots . . . \ldots \ldots \ldots \ldots \ldots \ldots \ldots \ldots \ldots \ldots$ & 618 \\
\hline 2 & 75 & $\ldots \ldots \ldots$ & $\ldots \ldots \ldots$ & 16 & $\cdots \ldots \ldots \ldots \ldots$ & 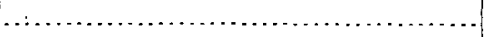 & 619 \\
\hline & 398 & $394-398$ & Flows. & 10 & Lloyd sand .......... & $\ldots \ldots \ldots \ldots \ldots \ldots \ldots$ & 620 \\
\hline 3 & 76-92 & & $c$ Flows. & a $18-20$ & -....... & 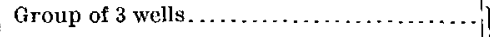 & 621 \\
\hline 6 & 246 & $\cdots$ & $\ldots \ldots \ldots$ & d 50 & $\therefore \ldots$. & $\ldots$ do $\ldots \ldots \ldots \ldots \ldots \ldots \ldots \ldots \ldots \ldots \ldots$ & $3^{621}$ \\
\hline & 68 & $\ldots \ldots \ldots$ & -18 & $\cdots \ldots$. & $\ldots \ldots \ldots \ldots \ldots \ldots$ & $-\ldots \ldots \ldots \ldots \ldots \ldots \ldots$ & 622 \\
\hline 2 & 90 & $\ldots \ldots$ & -25 & $\ldots \ldots \ldots$ & $\ldots \ldots \ldots \ldots \ldots \ldots \ldots$ & $\ldots \ldots \ldots \ldots \ldots \ldots \ldots \ldots$ & 623 \\
\hline & 181 & $\ldots \ldots \ldots$ & -152 & +10 & $\ldots \ldots \ldots \ldots \ldots$. & & 624 \\
\hline 3 & 264 & $255-264$ & -144 & ...... & Cretaceous?.... & & 625 \\
\hline 6 & \pm 60 & $\ldots \ldots \ldots$ & Flows. & $5-10$ & Pleistocene.... & Pumps 80 gallons.... & 626 \\
\hline $3 \frac{1}{2}$ & 166 & & -40 & $\ldots \ldots \ldots$ & & & 627 \\
\hline 3 & $\cdot 499$ & & ....... & 0 & & & 628 \\
\hline 2 & . 97 & & -35 & $\cdots \cdot$ & & & 629 \\
\hline 4 & 142 & & & $e 10$ & Gret & & 630 \\
\hline 5 & 147 & & $\therefore . .$. & e 18 & & & 0000 \\
\hline $36-4$ & 131 & & $\cdots \cdots$ & .... & & & 631 \\
\hline & 56 & & -32 & 25 & Cretaceous........... & & 632 \\
\hline 8 & 248.5 & $\ldots \ldots \ldots$ & Flows. & 5 & Lloyd sand .......... & 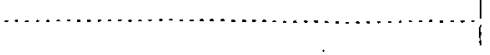 & 633 \\
\hline \multicolumn{8}{|c|}{$\begin{array}{l}\text { c At extremely high tide. } \\
\text { d Each well. } \\
\text { e Flows into underground cistern } 17 \text { feet below the surface. }\end{array}$} \\
\hline
\end{tabular}


UNDERGROUND WATER RESOUROES OF LONG ISLAND, NEW YORK.

TABLE XI.-Representative wells

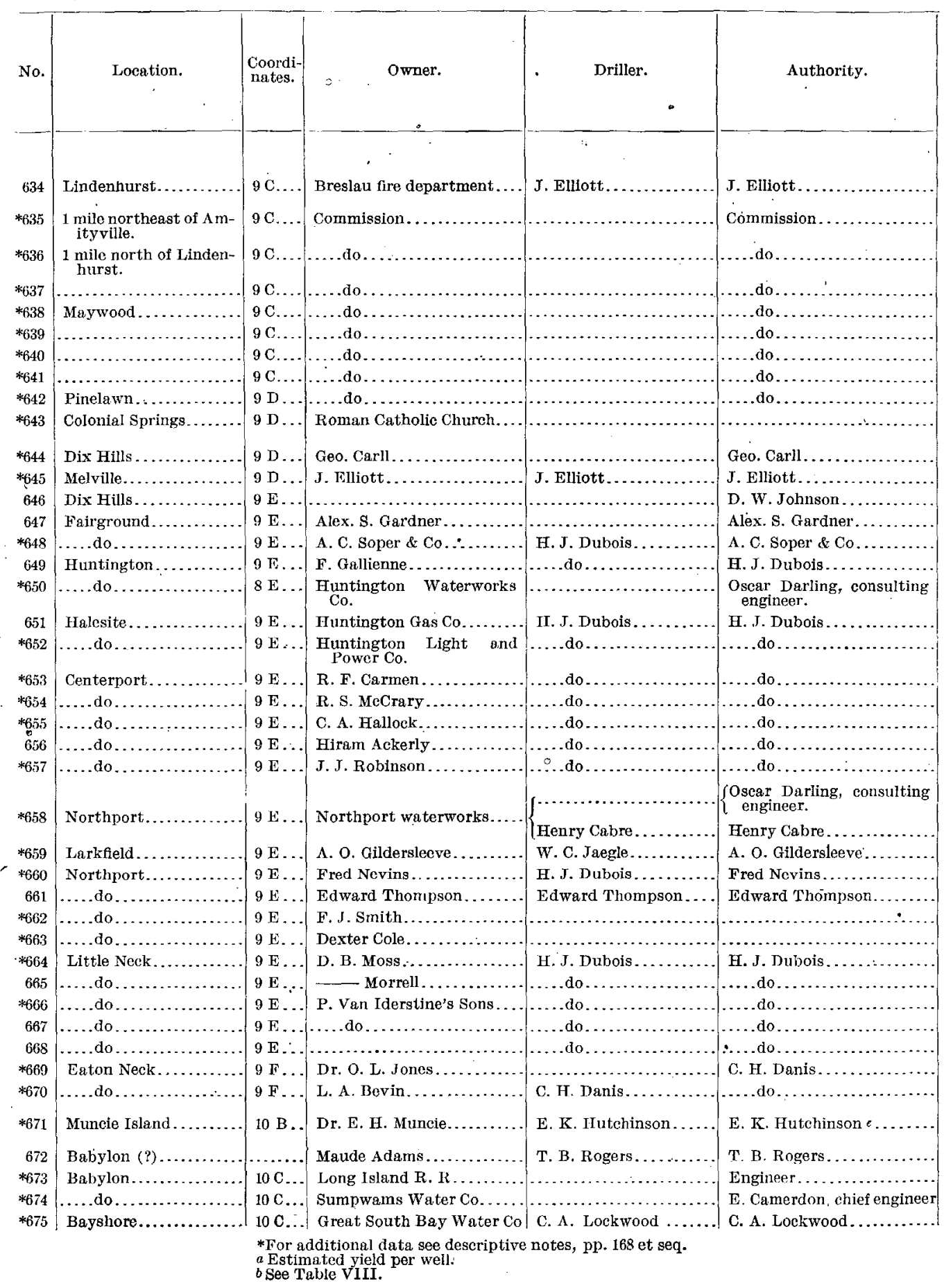


on Long Island-Continued.

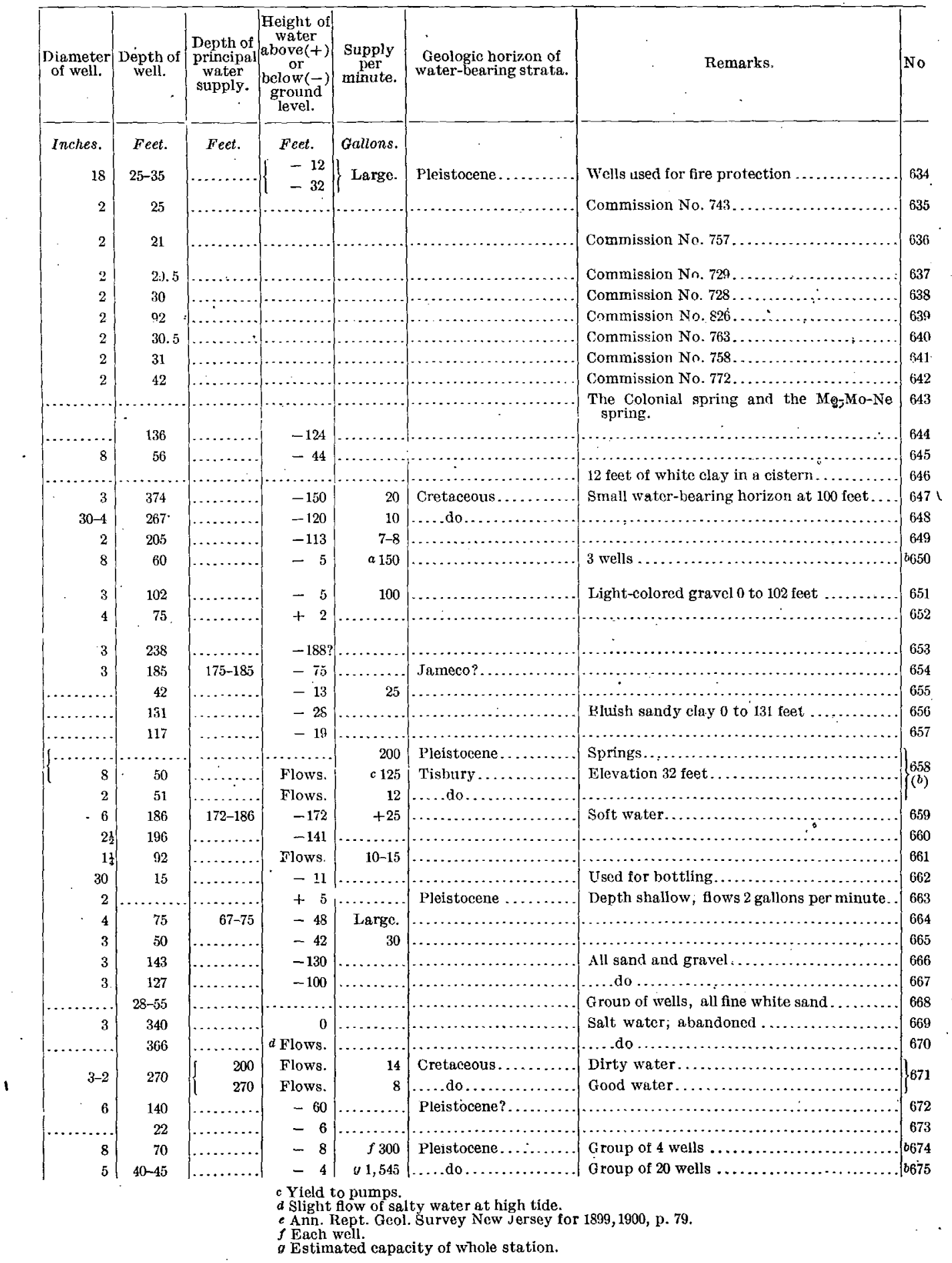

17116-No. 44-06-11 
TABLE XI.--Representative wells

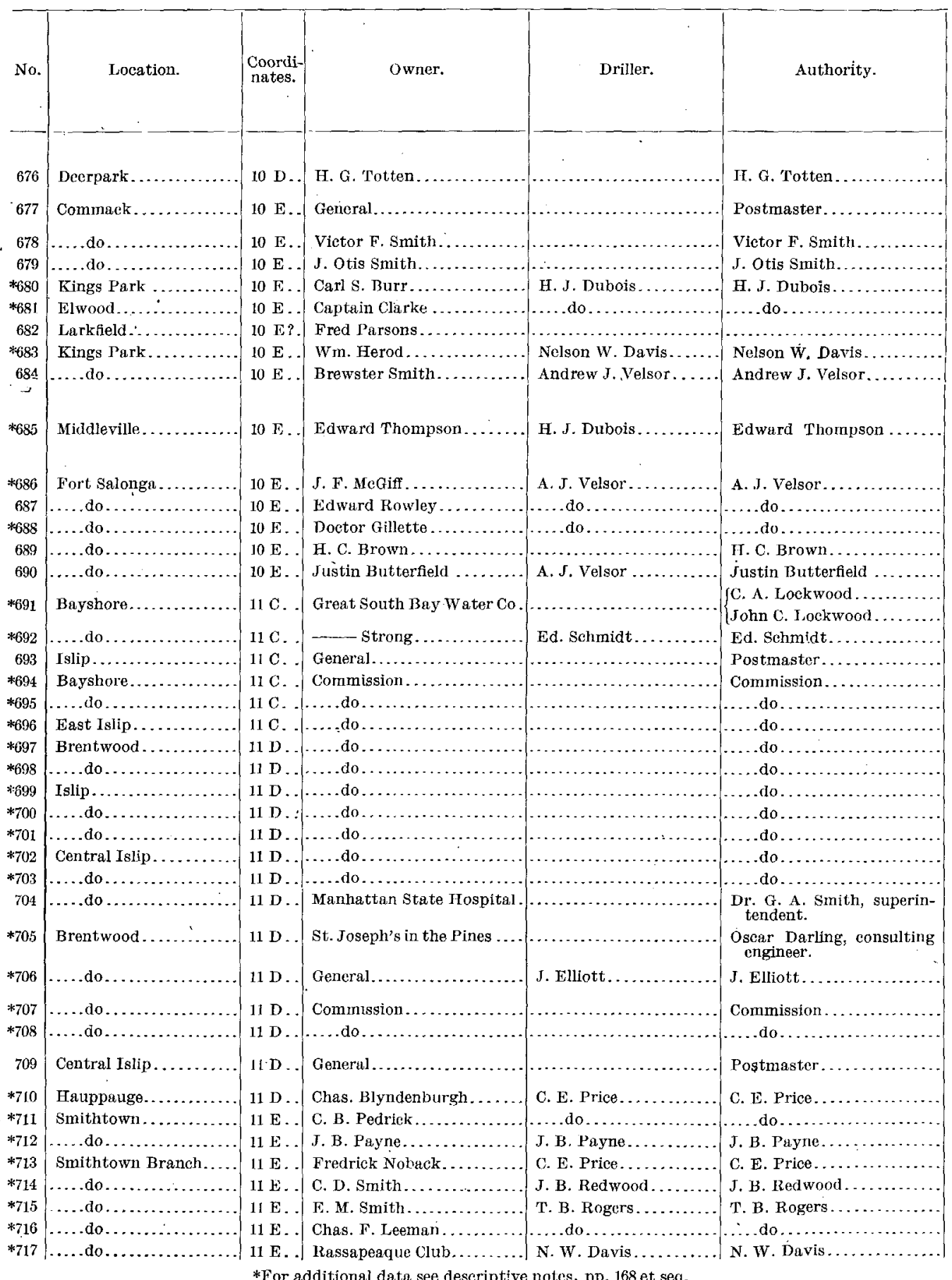


on Long Island-Continued.

\begin{tabular}{|c|c|c|c|c|c|c|c|}
\hline $\begin{array}{l}\text { Diameter } \\
\text { of well. }\end{array}$ & $\underset{\text { well. }}{\text { Depth of }}$ & $\begin{array}{c}\text { Depth of } \\
\text { principal } \\
\text { water } \\
\text { supply. }\end{array}$ & $\left|\begin{array}{c}\text { Height of } \\
\text { water } \\
\text { above }(+) \\
\text { or } \\
\text { below }(-) \\
\text { ground } \\
\text { level. }\end{array}\right|$ & $\begin{array}{c}\text { Supply } \\
\text { per } \\
\text { minute. }\end{array}$ & $\begin{array}{c}\text { Geologic horizon of } \\
\text { water-bearing strata. }\end{array}$ & Remarks. & No. \\
\hline Inches. & Feet. & Feet. & Feet. & Gallons. & & & \\
\hline 30 & $\begin{array}{r}43 \\
15-75\end{array}$ & $\left\{\begin{array}{c}15-30 \\
\{\end{array}\right.$ & -37 & & $\cdots \cdots \cdots \cdots$ & , $\cdots \cdots+\cdots \cdots+\cdots$ & 670 \\
\hline 36 & 96 & $\mid \begin{array}{r}\quad 50-75 \\
\ldots \ldots\end{array}$ & -93 & & & Soft water......... & 678 \\
\hline $36-6$ & 110 & & -95 & & $\ldots \ldots \ldots \ldots \ldots$ & $\ldots$. do .............. & 679 \\
\hline $36-3$ & 142 & $138-142$ & $\ldots \ldots$ & Large. & Cretaceous.. & & 680 \\
\hline $36-3$ & 170 & & $\ldots \ldots$ & Small. & & & 681 \\
\hline 72 & 136 & & -100 & & .... & & 682 \\
\hline 6 & 152 & & -142 & & Tisbury .... & & 683 \\
\hline 30 & 153 & $\ldots \ldots \ldots$ & -148 & & $\ldots \ldots \ldots \ldots$ & All sand and gravel... & 684 \\
\hline 14 & 33 & $\ldots \ldots \ldots \ldots$ & Flows. & ..... & l. & & \\
\hline 6 & 33 & ......... & Flows. & 50 & & & 685 \\
\hline 6 & 162 & & Flows. & 10 & & & 085 \\
\hline $6-11$ & 45 & …... & Flows. & & & & \\
\hline $36-4$ & 120 & 120 & $\rightarrow 110$ & & & & 686 \\
\hline 33 & 106 & $\ldots \ldots \ldots$ & -100 & & -...... & All sand and gravel... & 687 \\
\hline & 73 & & -66 & & & & 688 \\
\hline$\ldots$. & 116 & & …... & Small. & & & 689 \\
\hline 48 & 106 & & -100 & 4 & .............. & & 600 \\
\hline 5 & 60 & ............ & ......... & $\cdots \cdots$ & Pleistocene... & Group of wells; abandoned.. & 697 \\
\hline & +262 & 262 & Flows. & 49.5 & Cretaceous.......... & Abandoned...$\ldots \ldots \ldots \ldots$ & 691 \\
\hline 2 & 67 & $\ldots \ldots \ldots$ & -7 & & Pleistocene......... & & 692 \\
\hline & a $12-15$ & & $a-8$ & & $\ldots \ldots \ldots \ldots \ldots$ & $\therefore \ldots \ldots \ldots \ldots \ldots \ldots$ & 693 \\
\hline 2 & 102.5 & & c........ & & & Commission No. $861 \ldots$ & 694 \\
\hline 2 & 36 & & ......... & & & Commission No. $843 \ldots \ldots$. . & 695 \\
\hline 2 & 30 & & $\ldots \ldots$ & $\therefore$ & $\ldots$. & Commission No. $1086 \ldots \ldots$. . . & 696 \\
\hline 2 & 30 & & ....... & & (2. & Commission No. $1087 \ldots \ldots$. . . & 697 \\
\hline 2 & 30.5 & & . & & $\cdots \cdots \cdots \cdots_{r}$ & Commission No. $1088 \ldots \ldots$. & 698 \\
\hline 2 & 40.5 & & ... & & & Commission No. $842, \ldots$. & 699 \\
\hline 2 & 41 & & $\ldots \ldots$ & & & Commission No. $830 \ldots \ldots$. & 700 \\
\hline 2 & 35 & & ........ & & & Commission No. $1186 \ldots \ldots \ldots \ldots \ldots$ & 701 \\
\hline 2 & 25 & & ….... & & $\ldots \ldots \ldots \ldots \ldots$ & Commission No. $1194 \ldots \ldots \ldots \ldots$. . . . & 702 \\
\hline 2 & 35 & & ........ & & $\ldots \ldots \ldots \ldots$, & Commission No. $1183 \ldots \ldots \ldots \ldots \ldots$ & 703 \\
\hline 2 & 40 & $\ldots \ldots \ldots$ & -21 & $b+125$ & Pleistocene. .......... & Group of 17 wells $\ldots \ldots \ldots \ldots \ldots \ldots \ldots$ & 704 \\
\hline 8 & 52 & & -25 & 150 & Pleistocene ?.... & & 705 \\
\hline & $50-80$ & & $\left\{\begin{array}{l}-28 \\
-52\end{array}\right.$ & & Pleistocene... & , & 706 \\
\hline 2 & 103 & & …... & & & Commission No. $1141 \ldots$ & 707 \\
\hline 2 & 44 & & $\cdots \cdots \cdots$ & & (n) & Commission No. $1195 \ldots \ldots \ldots \ldots \ldots \ldots \ldots$ & 708 \\
\hline & $50-60$ & & $\left\{\begin{array}{l}-45 \\
-50\end{array}\right.$ & \} . & Pleistocene?..... & & 709 \\
\hline & 49.5 & $\ldots \ldots \ldots$ & -43 & & & & 710 \\
\hline & 168 & $165-168$ & $\cdots \cdots$ & & Pleis tocene. . & $\mid \cdots \ldots \ldots, \ldots$ & 711 \\
\hline 2 & 127 & $\ldots \ldots \ldots$ & -30 & & …........... & Soft water; incomplete..... & 712 \\
\hline $36-2$ & 1.25 & 125 & -50 & & Pleistoccne........... & 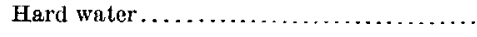 & 713 \\
\hline .......... & 95 & ........... & -84 & $\cdots \cdots$ & 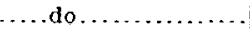 & $\ldots \ldots \ldots \ldots \ldots \ldots \ldots \ldots \ldots \ldots \ldots \ldots \ldots$ & 714 \\
\hline 6 & 100 & $\therefore \ldots \ldots$ & -85 & & & & 715 \\
\hline$\cdots \cdots \cdot$ & 160 & & a....... & & & & 716 \\
\hline 6 & 18 & Elows. & 60 & & & & 717 \\
\hline
\end{tabular}

a Average for this vicinity.

$\checkmark$ For whole system. 


\section{UNDERGROUND WATER RESOURCES OF LONG ISLAND, NEW YORK.}

TABLE XI.-Representative wells

\begin{tabular}{|c|c|}
\hline No. & Location. \\
\hline *718 & King's Park... \\
\hline$* 719$ & $\ldots$ do $\ldots . . .$. \\
\hline 720 & Nisscquogue River. \\
\hline$* 21$ & $\ldots \ldots$ do $\ldots \ldots \ldots$. \\
\hline$* 722$ & $\ldots \ldots$ do $\ldots . . . . . . .$. \\
\hline$* 723$ & Stony Brook Harbor. \\
\hline$* 724$ & ....do............. \\
\hline 725 & Oakdale..... \\
\hline 726 & $\ldots . . d o \ldots . .$. \\
\hline$* 727$ & $\ldots$. do....... \\
\hline 728 & West Sayville. \\
\hline$* 729$ & Sayville...... \\
\hline$* 730$ & Ronkonkoma.. \\
\hline$* 731$ & .... do......... \\
\hline$* 732$ & .....do...... \\
\hline 733 & ....do.......... \\
\hline$*_{734}$ & ....do do .............. \\
\hline 735 & Iake Ronkonkoma. \\
\hline$* 736$ & $\ldots$. do.......... \\
\hline$* 737$ & .....do... \\
\hline$* 738$ & ....do... \\
\hline$* 739$ & ..... do.... \\
\hline$* 740$ & .....do..... \\
\hline$* 741$ & $\ldots$ do....... \\
\hline 742 & ....do....... \\
\hline$* 743$ & ... . do....... \\
\hline$* 744$ & Lake Grove... \\
\hline$* 745$ & $\ldots$. do ........ \\
\hline$* 746$ & . . . do.... \\
\hline 747 & .....do.... \\
\hline 748 & .....do....... \\
\hline$* 749$ & St. James... \\
\hline$* 750$ & ....do....... \\
\hline$* 751$ & .....do...... \\
\hline$* 752$ & I....do.... \\
\hline$* 753$ & !....do.... \\
\hline$* 754$ & -....do....... \\
\hline *755 & .... do....... \\
\hline$* 756$ & ....d do ........... \\
\hline$* 757$ & Stony Brook..... \\
\hline 758 & ....do........... \\
\hline$* 759$ & $\ldots$. do $\ldots . . . . . . .$. \\
\hline$* 760$ & Setauket......... \\
\hline$* 761$ & ....do............ \\
\hline$* 762$ & $\ldots$. do ............. \\
\hline$* 763$ & $\ldots$. do.......... \\
\hline$* 764$ & .... do . . . . . \\
\hline$* 765$ & .....do... \\
\hline
\end{tabular}

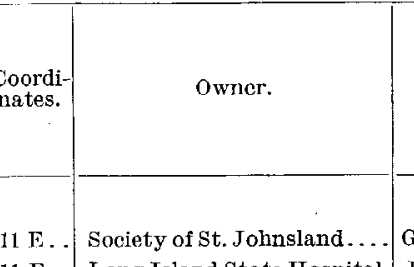

George Schmidt.........

11 E .. Long Island State IIospital ${ }_{\text {and Contracting Co. }}^{\text {Hudson }}$

$11 \mathrm{E}$. W. W. Konyon.

11 E... W. J. Matherson.

$11 \mathrm{E}$. L. Harris.

11 E... R. H. smith.

$11 \mathrm{E}$. H. W. Rebotul...

$12 \mathrm{C}$. W. K. Vanderbilt

$12 \mathrm{C}$. F. G. Boume.

$12 \mathrm{C} .$.

$12 \mathrm{C}$

$12 \mathrm{C}$

$12 \mathrm{D}$

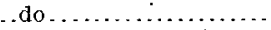

$12 \mathrm{D} . .$. . do . . . . . . . .

$12 \mathrm{D}$. . General.

12 D . John Klaiber.

12 D . F. G. Hallock

12 D. . Wm. Ralston

$12 \mathrm{D}$. J. Weber.

12 D. . George E. Plunkett

12 D. . R. W. Newton...

12 D.. W. Imhauser estate

12 D. . Nelson Newton.

$12 \mathrm{D} .$. E. Hollis Newton.

12 D. . W. H. Wamer.

12 E .. John Morrissey

$12 \mathrm{E}$.. Irving Overton

12 E.. Dr. Monecke. .

12 E. . M. A. Metzner .

12 E . B. Franklin Hallock,

$12 \mathrm{E}$.. Commission.

$12 \mathrm{E}$. Father Ducey

$12 \mathrm{E} .$. Jerome Saxe.

12 E. D. Emmett.

$12 \mathrm{E}$. ...... do..

$12 \mathrm{E} \ldots . .$. do ......

12 E. . .... do. .

$12 \mathrm{~F}$. Chas. T. Darling.

$12 \mathrm{~F}$. W $\mathrm{W}$. Shipman estate

12 F .. George Frland, sr..

$12 \mathrm{~F}$. . Woodhull Rowland.

$12 \mathrm{~F}$. . Wm. Clarke.

12 F. . Ioward Wallace.

12 F.. Wilmot T. Cox.

$12 \mathrm{~F}$. Nort House.

$12 \mathrm{~F}$. . Chas. Benner.

*For additional data see descriptive notes, pp. 168 et seq.

Nelson W. Davis
H. J. Dubois.

C. H. Danis .

T. B. Rogers.

…do.............................

J. Elliott.

Theo. J. Kirk

S. E. Terry

Arthur \& Tuthill

WIn. Ralston.

Arthur \& Tuthill

....do.......

T. B. Rogers........... T. B. Rogers

Arthur \& Tuthill

Wm. Ralston

Arthur \& Tuthill

S. E. Terry

..do...

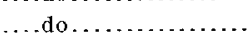

T. B. Rogers ............

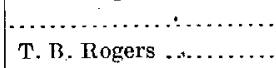

....do.

.... do....

Plat Gildersleeve.

T. B. Rogers

.... do...

.... do . . . . . . . . . . . .

....do ..............

... do...
Authority.

Society of St. Johnsland...

Long Island State Hospital.

H. J. Dubois

C. H. Danis.

.....do..

J. Elliot

.....do..............

Theo. J. Kirk . . . . . . . .

Postmaster.

Commission ................

... do .....................

....do.

Postmaster

S. E. Terry

W. T. Arthur

Wm, Ralston

W. T. Arthur

W. T. Arthur

Wm. Ralston.

E. Hollis Newton

W. T. Arthur

S. E. Terry.

...do....................

B. Franklin Hallock.

...do.

Commission.

T. B. Rogers

....do.

do.

A. J. Velsor

Commission. ............

Chas. T. Darling . . . . . .

....do . . . . . . . . . . . . . . .

T. B. Rogers.

.....do...

do....................

Wilmot T. Cox

Nelson W. Davis .

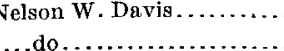


on Long Island-Continued.

\begin{tabular}{|c|c|c|c|c|c|c|c|}
\hline $\begin{array}{l}\text { Diameter } \\
\text { of well. }\end{array}$ & $\begin{array}{l}\text { Depth of } \\
\text { well. }\end{array}$ & \begin{tabular}{|} 
Depth of \\
principal \\
water \\
supply.
\end{tabular} & $\begin{array}{c}\text { Height of } \\
\text { water } \\
\text { above( }+) \\
\text { or } \\
\text { below(-) } \\
\text { ground } \\
\text { level. }\end{array}$ & $\begin{array}{c}\text { Supply } \\
\text { per } \\
\text { minute. }\end{array}$ & $\begin{array}{c}\text { Geologic horizon of } \\
\text { water-bearing strata. }\end{array}$ & Remarks. & No. \\
\hline $\begin{array}{r}\text { Inches. } \\
6\end{array}$ & $\begin{array}{r}\text { Feet. } \\
68\end{array}$ & $\begin{array}{l}\text { Feet. } \\
\ldots \ldots .\end{array}$ & $\begin{array}{l}\text { Feet. } \\
-55\end{array}$ & $\begin{array}{l}\text { Gallons. } \\
\ldots \ldots \text {. }\end{array}$ & & Hard water ..... & 718 \\
\hline 4 & $\begin{array}{r}90 \\
212\end{array}$ & $\begin{array}{r}40 \\
196-212\end{array}$ & $\begin{array}{l}\text { a Flow. } \\
-114\end{array}$ & b 625 & Pleistocene. . & $\begin{array}{l}\text { Group of } 12 \text { wells. Water hard and salty } \\
\text { Soft water. }\end{array}$ & 719 \\
\hline $\begin{array}{l}4 \\
3\end{array}$ & $\begin{array}{l}212 \\
140\end{array}$ & $\begin{array}{c}150-212 \\
\ldots \ldots \ldots\end{array}$ & $\begin{array}{r}-114 \\
-\quad 8\end{array}$ & & Cretaceous?. & sо1 & 720 \\
\hline & 100 & & -80 & & Tisbury ...... & All sand.......... & 722 \\
\hline$\cdots$ & 117 & & -60 & & Tisbury ?... & 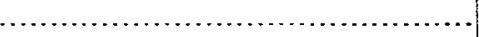 & 723 \\
\hline & 110 & & -80 & & $\ldots$........ & All sand $\ldots \ldots \ldots \ldots \ldots$ & 724 \\
\hline 12 & 50 & & -10 & & Pleistocene.. & 2 tile wells..................... & 725 \\
\hline 12 & 40 & & -5 & & .... do....... & 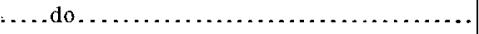 & 726 \\
\hline 2 & 170 & & $\cdots \cdots$ & & & Water unsatisfactory ................... & 727 \\
\hline & $10-50$ & & $\left\{\begin{array}{l}-12 \\
-20\end{array}\right.$ & & & $\cdot$ & 728 \\
\hline 2 & 45 & & $\ldots \ldots$ & & & Commission No. $1198 \ldots \ldots \ldots \ldots \ldots \ldots . . . . . .$. & 729 \\
\hline 2 & 62 & & $\ldots \ldots$ & & & Commission No. $1196 . \ldots \ldots \ldots \ldots \ldots \ldots \ldots$ & 730 \\
\hline 2 & 56 & & ...... & & & Commission No. $1200 \ldots \ldots \ldots \ldots \ldots \ldots \ldots$ & 731 \\
\hline 2 & 25 & & ...... & & & Commission No. $1202 \ldots \ldots \ldots \ldots \ldots \ldots$ & 732 \\
\hline & $60-90$ & $\ldots \ldots \ldots$ & -65 & & $\ldots \ldots \ldots \ldots \ldots$ & & 733 \\
\hline 8 & 81 & $63-81$ & -63 & & Pleis tocene ?. & n...................... & 734 \\
\hline $1 \frac{1}{2}$ & 73 & $63-73$ & -63 & & $\ldots \ldots \ldots+1$ & All white sand .......... & 735 \\
\hline 36 & 54 & ....... & -50 & & Pleistocene. . & 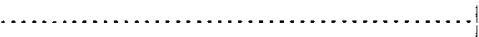 & 736 \\
\hline $1 \frac{1}{2}$ & 117 & $\begin{array}{r}32 \\
-112\end{array}$ & -31 & & $\ldots$. do $\ldots . .$. & Soft water... & 737 \\
\hline $36-1 \frac{1}{2}$ & 70 & & -62 & & & & 738 \\
\hline 6 & 60 & & -48 & & Pleis toecne. . & $\cdots$ & 739 \\
\hline $1 \frac{1}{2}$ & 75 & & -8 & & Pleis tocene ?.. & $\ldots \ldots \ldots$. & 740 \\
\hline 36 & 33 & & -30 & & & n............... & 741 \\
\hline 40 & 27 & & -23 & 5 & & Hard water..$\ldots \ldots \ldots \ldots \ldots \ldots \ldots \ldots \ldots \ldots \ldots$ & 742 \\
\hline 1) & 47 & & -35 & & & Water used for local irrigation............. & 743 \\
\hline 8 & 86 & & -72 & & & & 744 \\
\hline 8 & 58 & & -52 & & & & 745 \\
\hline$s$ & 24 & & -17 & & & Clay 8 to 21 feet................... & 746 \\
\hline & $\cdots$ & & $\ldots .$. & & & G roup of 4 wells used for irrigation & 747 \\
\hline 2 & 38 & $\ldots \ldots$ & -28 & & & & 748 \\
\hline 2 & 59 & & $\ldots \ldots$ & & $\ldots \ldots \ldots \ldots$ & Commission No. $1205 . \ldots \ldots \ldots$ & 749 \\
\hline 6 & 150 & & -132 & Large. & Pleis tocene...... & $\ldots \ldots \ldots$ & 750 \\
\hline 6 & 250 & & $-208 ?$ & & Tisbury .......... & & 751 \\
\hline 6 & 300 & & -90 & & & & 752 \\
\hline 6 & 97 & & -83 & & Tisbury..... & 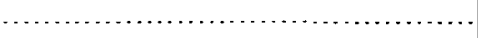 & 753 \\
\hline 36 & 160 & & -156 & & $\ldots \ldots \ldots \ldots \ldots$ & (n. & 754 \\
\hline 2 & 90 & $\therefore$ & $\ldots \ldots$ & & 'Tisbury ............ & Commission No. $1206 \ldots \ldots \ldots \ldots \ldots$ & 755 \\
\hline 2 & 70 & ........... & $\ldots \ldots$ & & $\ldots$. do $\ldots . . . . . . .$. & Commission No. $1236 \ldots \ldots \ldots \ldots \ldots$ & 756 \\
\hline $30-6$ & 123 & $88.5-123$ & -88.5 & & $\ldots$ do $\ldots . . . . . . .$. & 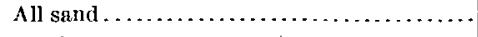 & 757 \\
\hline 36 & 90 & & $\ldots \ldots \ldots$ & & $\ldots$. do............. & 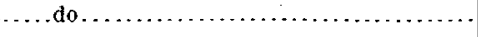 & 758 \\
\hline 6 & 107 & & & & n......... & ................ & 759 \\
\hline 6 & 252 & & ...... & & Cretaceous. . & $\ldots \ldots \ldots \ldots$ & 760 \\
\hline 6 & 90 & & -75 & & ................ & n............. & 761. \\
\hline 6 & 70 & & -50 & …... & Tisbury ............ & 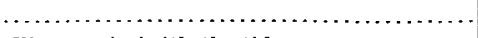 & 762 \\
\hline 6 & 320 & & Flows. & +18 & Cretaceous......... & Flow varies with the tide.......... & 763 \\
\hline 2 & 40 & & -24 & & Pleistocene?.... & (n, & 764 \\
\hline 3 & 50 & $\mid \ldots \ldots \ldots$ & -44 & . & ....do. do.......... & (n, & 765 \\
\hline
\end{tabular}


UNDERGROUND WATER RESOURCES OF LONG ISLAND, NEW YORK.

TABLE XI.-Representative wells

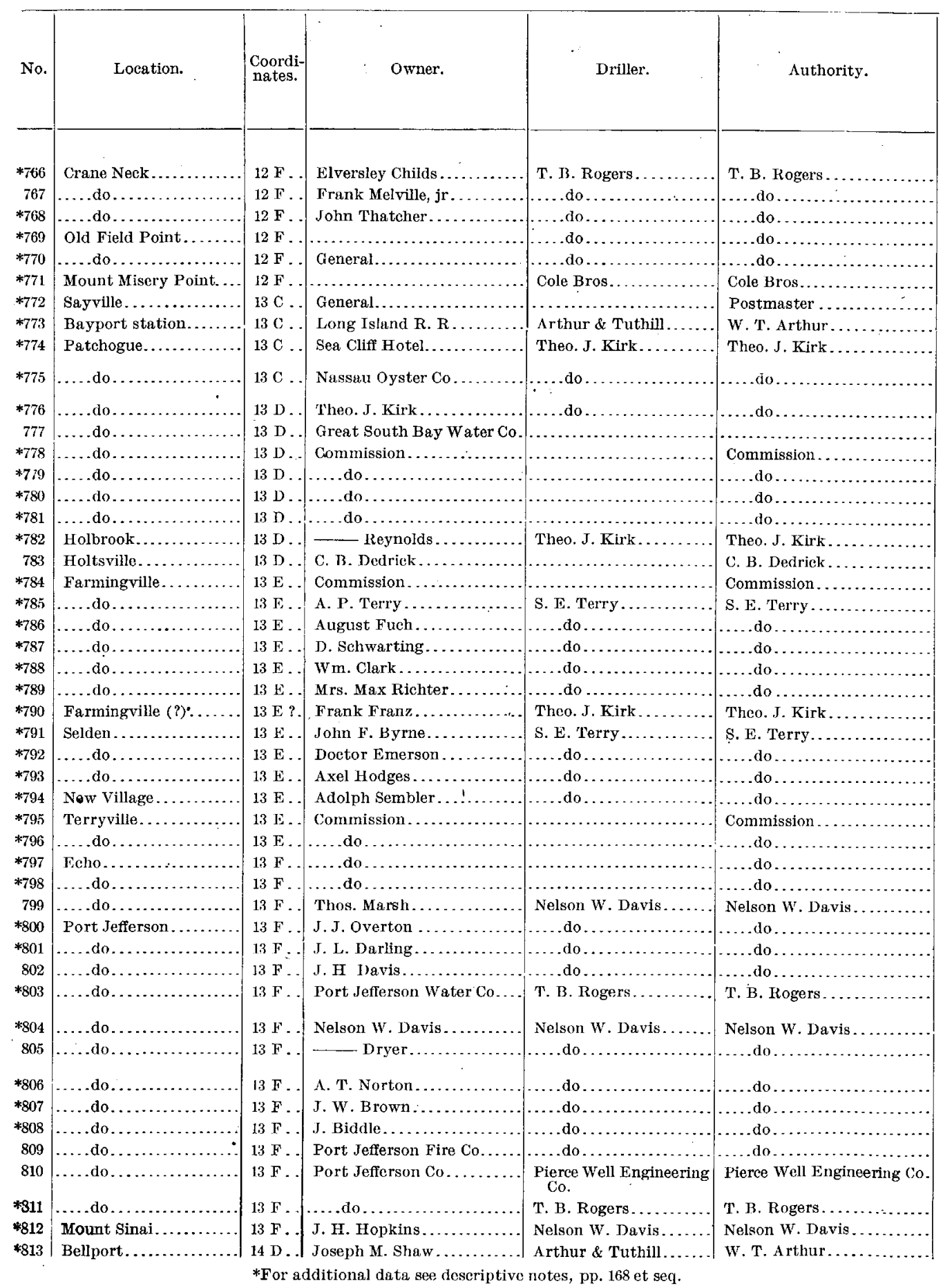


on Long Island-Continued.

\begin{tabular}{|c|c|c|c|c|c|c|c|}
\hline $\begin{array}{l}\text { Diameter } \\
\text { of well. }\end{array}$ & $\begin{array}{l}\text { Depth of } \\
\text { weil. }\end{array}$ & $\begin{array}{l}\text { Depth of } \\
\text { principal } \\
\text { water } \\
\text { supply. }\end{array}$ & $\mid \begin{array}{c}\text { Height of } \\
\text { water } \\
\text { above }(+) \\
\text { or } \\
\text { below }(-) \\
\text { ground } \\
\text { level. }\end{array}$ & $\begin{array}{c}\text { Supply } \\
\text { per } \\
\text { minute. }\end{array}$ & $\begin{array}{l}\text { Geologic horizon of } \\
\text { water-bearing strata. }\end{array}$ & Remarks. & No. \\
\hline Inches. & Feet. & Feet. & Feet. & Gallons. & & & \\
\hline & 38 & & $-12 ?$ & & Pleistocene.. & Salt water............. & 766 \\
\hline 6 & 80 & & $\ldots \ldots$. & & Tisbury?......... & All sand and gravel............... & 767 \\
\hline 6 & 65 & & -30 & +15 & & & 768 \\
\hline 6 & 50 & & & & & Salt water......... & 769 \\
\hline $1 \frac{1}{2}$ & 43 & 36 & . & & & Fresh water ........... & 770 \\
\hline $8-6$ & 275 & & ....... & & ........... & Well abandoned........... & $\bar{i} \overline{1}$ \\
\hline$\cdots$. & $40-45$ & & -30 & & Pleistocene...... & (5) & 772 \\
\hline $1 \frac{1}{4}$ & 28 & & ....... & & 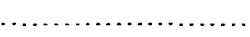 & $\cdots \ldots \ldots \ldots$ & 773 \\
\hline & 8 & & - $\cdots \cdot$ & & & $\ldots \ldots \ldots \ldots \ldots$ & 774 \\
\hline 2 & 72 & & ….. & & & Black water; abandoned.. & 775 \\
\hline 2 & 19 & & -17 & & & Good water........................... & 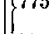 \\
\hline 2 & 28 & & -18 & & Pleistocene... & $\ldots \ldots \ldots \ldots \ldots \ldots \ldots \ldots \ldots \ldots \ldots, \ldots$, & 776 \\
\hline & Shallow. & & & & & 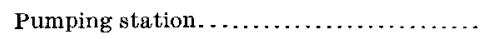 & $a 777$ \\
\hline 2 & 150 & & & & & Commission No. $1145 \ldots \ldots \ldots \ldots \ldots \ldots$ & 778 \\
\hline 2 & 50 & & ....... & & & Commission No. $1184 \ldots \ldots \ldots \ldots \ldots \ldots$ & 779 \\
\hline 2 & 50 & & $\ldots .$. & & & Commission No. $1007 \ldots \ldots \ldots \ldots \ldots$ & 780 \\
\hline 2 & 51 & & ....... & & $\ldots \ldots$. & Commission No. $1169 \ldots \ldots \ldots \ldots \ldots \ldots$ & 781 \\
\hline $1 \frac{1}{4}$ & 90 & & -80 & & Tisbury?... & ${ }^{\prime} \ldots \ldots \ldots \ldots \ldots \ldots \ldots \ldots \ldots \ldots \ldots \ldots \ldots$ & 782 \\
\hline 36 & 46 & & -40 & & $\ldots \ldots \ldots \ldots$ & $\ldots \ldots \ldots \ldots \ldots \ldots \ldots \ldots \ldots \ldots \ldots \ldots \ldots \ldots$ & 783 \\
\hline 2 & 50 & & …… & & $\cdots \ldots$. & Commission No. 1008. & 784 \\
\hline 12 & 110 & & ....... & & Pleistocene....... & $\ldots \ldots \ldots \ldots \ldots \ldots \ldots \ldots$ & 785 \\
\hline 8 & 70 & & -65 & & $\ldots$.......... & & 786 \\
\hline 8. & 27 & & -22 & & $\ldots$. .do.......... & $\ldots \ldots \ldots \ldots \ldots \ldots \ldots \ldots, \ldots \ldots \ldots, \ldots$, & 787 \\
\hline 8 & 59 & & -52 & & $\ldots$ do ......... & …… & 788 \\
\hline 8 & 60 & & -54 & & & $\ldots .$. & 789 \\
\hline 2 & 80 & & -70 & & & & 790 \\
\hline 8 & 64 & & $\ldots \ldots$. & & & & 791 \\
\hline 8 & 59 & & -54 & & & $\ldots:$ & 792 \\
\hline 8 & 38 & & -26 & & & & 793 \\
\hline 8 & 38 & & -32 & & & & 794 \\
\hline 2 & $70^{\circ}$ & & ....... & & $\ldots \ldots \ldots$ & Commission No. $1237 \ldots \ldots \ldots \ldots \ldots$ & 795 \\
\hline 2 & 59 & & ( & & Tisbury?..... & Commission No. $1214 \ldots \ldots \ldots \ldots \ldots \ldots$ & 796 \\
\hline 2 & 85 & & 刑 & & $\bullet$ & Commission No. $1233 \ldots \ldots \ldots \ldots \ldots \ldots$ & 797 \\
\hline 2 & 90 & & $\cdots \cdots$ & & & Commission No $1215 \ldots \ldots \ldots \ldots \ldots \ldots$ & 798 \\
\hline & 150 & & $-14 \overline{5}$ & Large. & ( & $\ldots \ldots \ldots \ldots \ldots \ldots \ldots \ldots \ldots \ldots \ldots \ldots \ldots, \ldots$ & 799 \\
\hline 4 & 20 & & Flows. & & & & 800 \\
\hline 3 & 96 & & -40 & & & & 801 \\
\hline 2 & \pm 25 & $\cdots$ & Flows. & 5 & $\ldots \ldots \ldots \ldots \ldots$ & 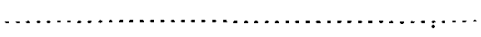 & 802 \\
\hline 6 & 54 & $\cdots \cdot$ & Flows. & & Pleistocene....... & $\begin{array}{l}2 \text { wells. Pumps } 133 \text { gallons per well per } \\
\text { minute. }\end{array}$ & 803 \\
\hline 6 & 75.5 & & Flows. & 25 & $\ldots$....... & ……… & 804 \\
\hline $1 \frac{1}{2}$ & 60 & & Flows. & & & $\begin{array}{l}\text { This well ceased to flow when No. } 804 \text { was } \\
\text { completed. }\end{array}$ & 805 \\
\hline $1 \frac{1}{2}$ & 140 & 140 & & & & & 806 \\
\hline 2 & 90 & & -70 & & & & 807 \\
\hline 3 & 120 & & -110 & .... & n.............. & $\ldots \ldots \ldots \ldots \ldots, \ldots \ldots \ldots \ldots$ & 808 \\
\hline$\ldots$ & Shallow. & & $\ldots . .$. & 100 & Pleistocene...... & Wells for fire protection $\ldots \ldots \ldots \ldots \ldots \ldots$ & 809 \\
\hline 2 & 35 & & Flows. & & & $\begin{array}{l}\text { Temperature } 58^{\circ} \text { F.; pumps } 42 \text { gallons per } \\
\text { minute. }\end{array}$ & 810 \\
\hline & 370 & & ….... & Small. & Cretaceous... & 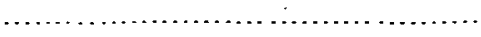 & 811 \\
\hline $2 \frac{1}{2}$ & 95 & & -88 & Good. & $\ldots \ldots \ldots \ldots \ldots \ldots \ldots$ & $\ldots .$. & 812 \\
\hline $1 \frac{1}{2}$ & 45 & 烈 & -20 & & $\ldots \ldots \ldots+\ldots, \ldots, \ldots$ & . $\ldots \ldots \ldots \ldots \ldots \ldots$ & 813 \\
\hline
\end{tabular}




\section{UNDERGROUND WATER RESOUROES OF LONG ISLAND, NEW YORK.}

TABLe XI.-Representative wells

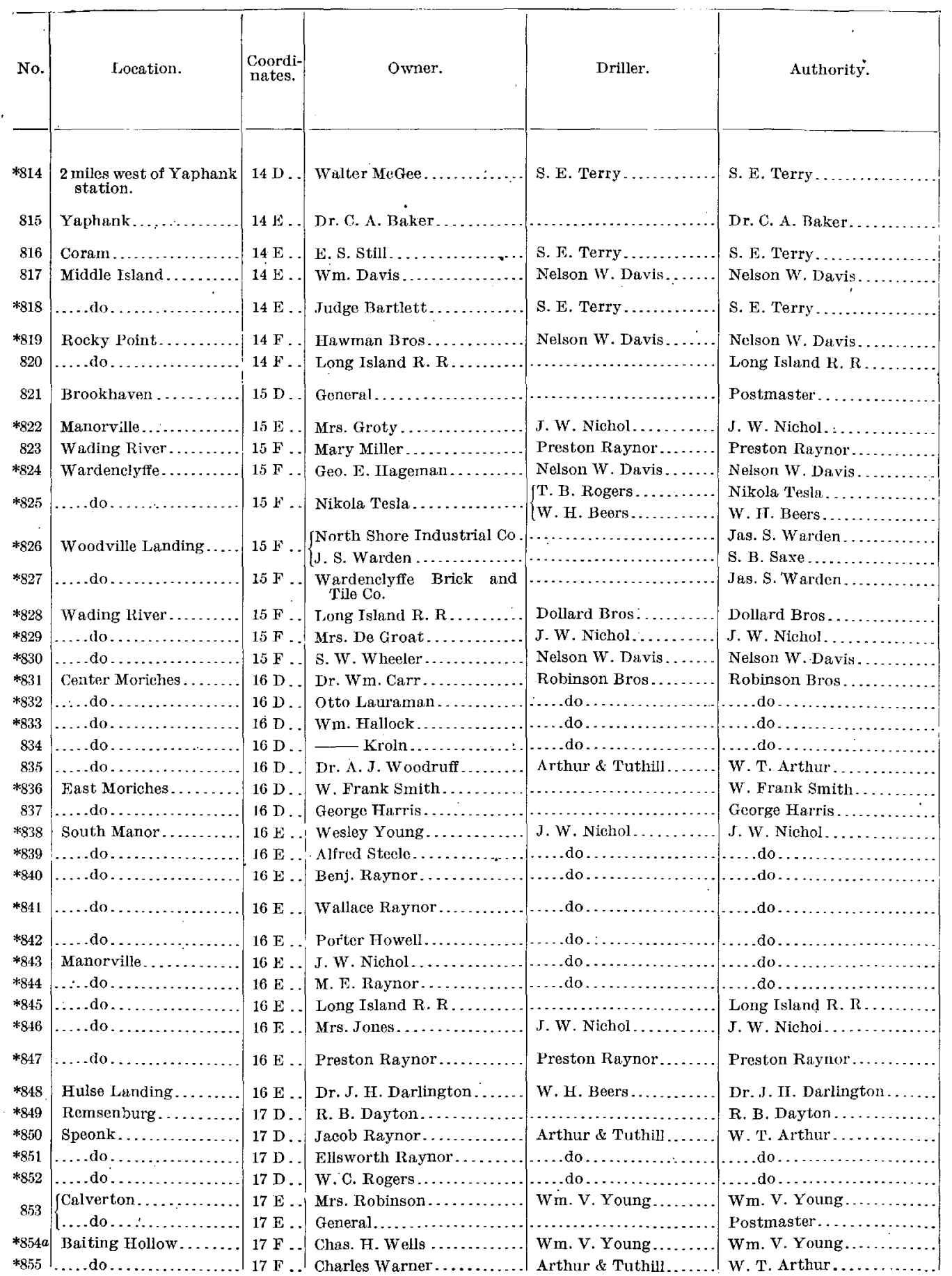

*For additional data see descriptive notes, pp. 168 et seq. 
on Long Island - Continued.

\begin{tabular}{|c|c|c|c|c|c|c|c|}
\hline $\begin{array}{l}\text { Diameter } \\
\text { of well. }\end{array}$ & $\begin{array}{c}\text { Depth of } \\
\text { well. }\end{array}$ & $\begin{array}{l}\text { Depth of } \\
\text { principal } \\
\text { water } \\
\text { supply. }\end{array}$ & $\begin{array}{c}\text { Height of } \\
\text { water } \\
\text { above }(+) \\
\text { or } \\
\text { below }(-) \\
\text { ground } \\
\text { level. }\end{array}$ & $\begin{array}{l}\text { Supply } \\
\text { per } \\
\text { minute. }\end{array}$ & $\begin{array}{l}\text { Geologic horizon of } \\
\text { water-bearing strata. }\end{array}$ & Remarks. & No. \\
\hline $\begin{array}{r}\text { Inches. } \\
8\end{array}$ & $\begin{array}{r}\text { Feet. } \\
68\end{array}$ & Feet. & $\begin{array}{l}\text { Feet. } \\
-62\end{array}$ & Gallons. & & & 814 \\
\hline 36 & $18-24$ & & $\{-16$ & & & & 815 \\
\hline $\begin{array}{l}1+1 \\
8\end{array}$ & 33 & & $(-18$ & & Wiseonsin. & All morainal material. & 816 \\
\hline 4 & 62 & & -12 & & & Medium white sand 0 to 62 feet..... & 817 \\
\hline 8 & 39 & $\begin{array}{r}8 \\
32\end{array}$ & -30 & & Wisconsin... & & 818 \\
\hline 4 & 128 & .... & -121 & $12-15$ & Tisbury?.. & $\cdots$ & 819 \\
\hline 4 & 120 & & --106 & & ......... & $\ldots \ldots \ldots \ldots$. & 820 \\
\hline & $14-20$ & & $\begin{array}{l}-8 \\
-10\end{array}$ & & & & 821 \\
\hline $1 \frac{1}{4}$ & 29 & 29 & -21 & & & Clay 3 to 29 feet..... & 822 \\
\hline 2 & 50 & & ......... & & Tisbury? ..... & All sand and gravel............. & 823 \\
\hline 3 & 123 & & -113 & & $\ldots \ldots \ldots \ldots . .$. & 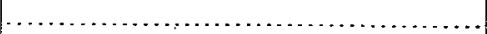 & 824 \\
\hline 8 & 166 & … & -110 & 15 & 'Iisbury ...... & Water pure and soit.......... & 805 \\
\hline$\cdots \cdots$ & 347 & & ....... & & $\ldots \ldots \ldots \ldots$ & 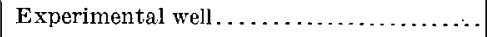 & 020 \\
\hline $60-8$ & 94 & $\cdots$ & -57 & & Tisbury & & 826 \\
\hline ….. & 90 & & -50 & & Tisbury . & & 820 \\
\hline $48-3$ & 57 & & -4 & $\cdots \therefore$ & Jameco?... & Clay from 0 to 47 feet... & 827 \\
\hline & 110 & & -80 & & & & 828 \\
\hline 11 & 38 & & - & 0 & $\ldots \ldots . . .6$ & Abandoned $\ldots \ldots \ldots \ldots \ldots \ldots$ & 829 \\
\hline 3 & 68 & & -28 & & Tisbury .... & $\mid \ldots \ldots \ldots \ldots \ldots \ldots$ & 830 \\
\hline 3 & 20 & & $\ldots \ldots$ & & Pleistocene...... & (n............ & 831 \\
\hline 17 & 34 & & $\ldots \ldots \ldots$ & & .... do ....... & $\ldots \ldots \ldots \ldots \ldots$ & 832 \\
\hline $1 \frac{1}{2}$ & 20 & & ......... & & $\ldots$. do ........ & …… & 833 \\
\hline 3 & 67 & & ......... & & ... do do..... & All sand and gravel........ & 834 \\
\hline $1 \frac{1}{4}$ & 26 & & -10 & & .....do....... & 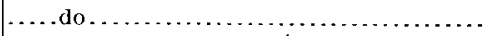 & 835 \\
\hline 3 & 33 & & -28 & & .....do....... & 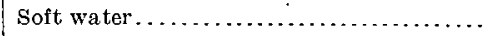 & 836 \\
\hline 28 & 60 & & $-56.5 !$ & & ....do..... & $\ldots \ldots$ do $\ldots \ldots \ldots \ldots \ldots \ldots \ldots \ldots \ldots \ldots \ldots \ldots$ & 837 \\
\hline 11 & 22 & $\ldots \ldots \ldots$ & -18 & & …........ & Sand and stones 2 to 22 feet............. & 838 \\
\hline $1 \frac{1}{4}$ & 15 & ........... & -12 & & $\ldots \ldots: \ldots \ldots$ & 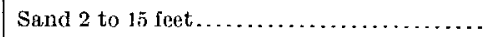 & 839 \\
\hline $1 \frac{1}{4}$ & 24 & . . . . . & -22 & & $\ldots \ldots \ldots \ldots$. & Clay 22 to $24+$ feet................. & 840 \\
\hline $1 \frac{1}{2}$ & 36 & +36 & $\left\{\begin{array}{l}-5 \\
-10\end{array}\right.$ & & Pleistocene?.. & Clay 3 to 15,19 to 36 feet................ & 841 \\
\hline 3. & 20 & & -15 & & .....do....... & $\ldots \ldots \ldots \ldots \ldots$. & 842 \\
\hline $1 \frac{1}{4}$ & 12 & .......... & -9 & & $\ldots$. do . . . . . . . & Clay 6 to 7 feet...... & 843 \\
\hline $1 \frac{1}{4}$ & 15 & ...... & -12 & & ....do...... & $\ldots \ldots \ldots \ldots \ldots \ldots$ & 844 \\
\hline & … & & $\cdot \ldots \ldots \ldots$ & & $\ldots \ldots \ldots \ldots \ldots \ldots$ & & 845 \\
\hline $1 \frac{\pi}{2}$ & 42 & .......... & ............. & & Pleistocene?... & 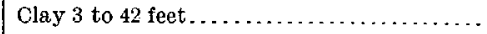 & 846 \\
\hline 11 & 42 & & .......... & & & 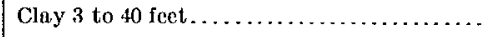 & 847 \\
\hline $1+\frac{1}{4}$ & 32 & & .......... & & & Clay 12 to 28 feet. . . . . . . . & 848 \\
\hline 3 & 92 & 92 & -87 & Large.' & $\ldots \ldots \ldots \ldots \ldots$ & 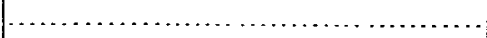 & 848 \\
\hline $1 \frac{1}{2}$ & 25 & $10-25$ & -10 & 20 & Pleistocene......... & $\ldots \ldots \ldots \ldots \ldots \ldots \ldots$ & 849 \\
\hline $1 \frac{1}{4}$ & 29 & $\ldots \ldots \ldots$ & -21 & & $\ldots$.do $\ldots \ldots \ldots \ldots \ldots$ & 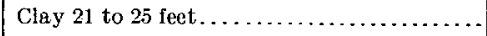 & 850 \\
\hline $1 \frac{1}{4}$ & 26 & & ...... & & $\ldots \ldots \ldots \ldots \ldots \ldots \ldots$ & Clay 18 to 20 feet. . . . . . . . . . . . . . . & 851 \\
\hline 11 & 26 & $\ldots \ldots \ldots$. & -20 & & Pleistocene......... & 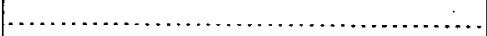 & 852 \\
\hline & 65 & & -54 & & Pleistocene?. & 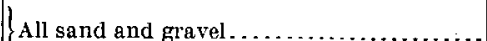 & 853 \\
\hline & $20-50$ & & -20 & & & & \\
\hline 3 & 105 & & -94 & & & & 854 \\
\hline $2 \frac{1}{2}$ & 99 & $\ldots$. & -90 & & . & . & 855 \\
\hline
\end{tabular}


TABLE XI.-Representative weills

\begin{tabular}{|c|c|c|c|c|c|}
\hline No. & Location. & $\begin{array}{l}\text { Coordi- } \\
\text { nates. }\end{array}$ & Owner. & Driller. & Authority. \\
\hline-4856 & Baiting Hollow.. & $17 \mathrm{~F}$. & Howell Sandford... & Arthur \& Tuthill. . & W. T. Arthur........ \\
\hline$* 856 \Lambda$ & Centerville........... & $17 \mathrm{~F}$. & Sydney Shaw..... & S. E. Terry ....... & S. E. Terry ............. \\
\hline$* 857$ & West Hampton Beach. & $18 \mathrm{D} .$. & Augustus Zabriskie .. & Arthur \& Tuthill ...... & W. T. Arthur . . . . . . . . \\
\hline$* 858$ & Quogue Beach....... & $18 \mathrm{D} \ldots$ & Hallock \& small.......... & Nelson W. Davis...... & NeIson W. Davis........ \\
\hline$* 859$ & Quogue......... & $18 \mathrm{DL}$ & Asha B. Hallock........... & $\ldots$ do ............. & Asha B. Hallock...... \\
\hline$* 860$ & .... do. . . & $18 \mathrm{D}$. & J. Wendell. ........... & Cóle Bros.......... & Cole Bros. . . . . . . . . . \\
\hline$* 861$ & ....do........... & $18 \mathrm{D} .$. & Quantuck Water Co...... & & Henry Gardner, treasure:' \\
\hline 862 & Quoguc Station. & $18 \mathrm{E}$. & Long Island R. R......... & 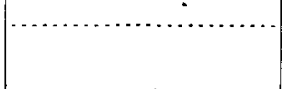 & $\begin{array}{l}\text { Long Island R. K........ } \\
\text { Nelson W. Davis........... }\end{array}$ \\
\hline$* 863$ & Riverhead. . & $18 \mathrm{E}$ & Riverhead Waterworks... & Nelson W. Davis.. & John R. Perkins... \\
\hline$* 864$ & .....do........ & $18 \mathrm{E} .$. & Yetter \& Moore.... . & W. V. Young....... & W. V. Young........... \\
\hline 865 & Northville..... & $18 \mathrm{~F} \ldots$ & Chas. Wells........ & A. 0. Ryder...... & A. O. Ryder.......... \\
\hline 866 & Cum City ......... & $18-19 \mathrm{~F}$ & Nicholas Brown.... & $\ldots$ do........ & ....do do.............. \\
\hline 867 & Good Ground.. & $19 \mathrm{~F}$. & General......... & & s. L. Squires............ \\
\hline 868 & ... do do........ & $19 \mathrm{E}$ & Gilsey estate ....... & J. Elliott........ & J. Flliott............. \\
\hline$* 869$ & Jamesport. . & $19 \mathrm{~F} .$. & Capt. Jas. Downs.... & W. v. Young... & W. V. Young................ \\
\hline$* 870$ & ....do...... & $19 \mathrm{~F}$. & John J. McLaughlin. . . & & $\begin{array}{c}\text { Chas. Darling, consulting } \\
\text { engineer. }\end{array}$ \\
\hline 871 & Mattituck & $19 \mathrm{~F} ? .$. & F. M. Jupton................ & & .... do $\ldots \ldots \ldots \ldots \ldots \ldots$ \\
\hline$* 872$ & (....do........ & $19 \mathrm{~F} \ldots$ & Long Island R. R.... . & $\ldots \ldots \ldots \ldots \ldots \ldots$ & Long I sland $\mathrm{s} . \mathrm{R} \ldots \ldots$ \\
\hline 873 & $\ldots$ do. & $19 \mathrm{~F}-\mathrm{G}$ & General............... & & Dr. E. K. Morton . . . . . . . \\
\hline$* 874$ & Shinnecock Hills.... & $20 \mathrm{E} \ldots$ & 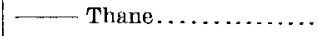 & Chester D. Corwin... & Chester D. Corwin.... \\
\hline$* 875$ & North Sea........... & $20-21 \mathrm{~F}$ & Chas. W. Payne......... . . & W. 'T' Arthur. ......... & W. T. Arthur..... \\
\hline 876 & Now Suffolk......... & $20 \mathrm{~F}$. & Donald Goldsmith ..... & Arthur \& Tuthill...... & ....do $\ldots \ldots \ldots \ldots \ldots$ \\
\hline$* 877$ & ....do.............. & $20 \mathrm{~F} \ldots$ & - Reid ............ & .... do $\ldots . . . . . . . . .$. & ....do...................... \\
\hline 878 & Southold.......... & $20-21 \mathrm{G}$ & ................... & Nelson W. Davis....... & Nelson W. Davis . . . . . . . . . \\
\hline 879 & Southampton . & $21 \mathrm{E}$ & Southampton Water Co... & & $\left\{\begin{array}{l}\text { Oscur Darling, consulting } \\
\text { engineer. } \\
\text { Geo. Elliston, engineer ... }\end{array}\right.$ \\
\hline$* 80$ & Hampton Park.. & $21 \mathrm{E}$. & Mrs. S. F. McDonald....... & Arthur \& Tuthill... & W. T. Arthur............... \\
\hline$* 881$ & $\ldots$. do........... & $21 \mathrm{E}$. & Edward G. Whittaker...... & .....do............. & $\ldots \ldots$ do $\ldots \ldots \ldots \ldots \ldots \ldots$. \\
\hline$* 881 \mathrm{~A}$ & Water Mill..... & $21 \mathbf{E}$ & General................... & $\ldots \ldots \ldots \ldots \ldots \ldots \ldots$ & Frederick H. Rose........ \\
\hline$* 882$ & Shelter Island.. & $21 \mathrm{G} .$. & John F. Becker.... & Harry Strausbinger .... & Harry Strausbinger........ \\
\hline$* 883$ & .....do......... & $21 \mathrm{G} .$. & — Ulmer....... & $\ldots$. do . . .............. & ....do.................... \\
\hline *884 & ....do............ & $21 \mathrm{G} \ldots$ & John Weber............ & ..... do . ........... & .... do .................. \\
\hline$* 885$ & $\mid \ldots .$. do............. & $21 \mathrm{G}$. & J. N. Stearns.......... & A. O. Ryde: ....... & A. O. Ryder............. \\
\hline 886 & $\ldots$ do $\ldots . . . . .$. & $21 \mathrm{G} \ldots$ & Mrs. Post.................. & Nelson W. Davis....... & Nelson W. Davis.......... \\
\hline 887 & ....do............ & $21 \mathrm{G}$. & Capt. Max Walthers...... & A. O. Ryder........... & A. O. Ryder................ \\
\hline$* 888$ & $\ldots$. do........... & $21 \mathrm{G}$. & 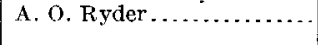 & 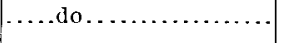 & 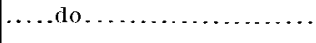 \\
\hline *889 & Shelter Island Heights. & $21 \mathrm{H}$. & $\left\{\begin{array}{l}\text { Shelter Island Heights As- } \\
\text { sociation. }\end{array}\right.$ & & $\left\{\begin{array}{l}\text { Wesley Smith, superintend- } \\
\text { ent. }\end{array}\right.$ \\
\hline$* 890$ & Shelter Island.. & $21 \mathrm{H}$. & Manhanset House.. & & $\begin{array}{l}\text { W. H. Havens, chief engi- } \\
\text { neer. }\end{array}$ \\
\hline 891 & Greenport.... & $21 \mathrm{H}$. & J. Madison Wells.. & A. 0 . Ryder & A. o. Ryder............... \\
\hline
\end{tabular}


on Long Island-Continued.

\begin{tabular}{|c|c|c|c|c|c|c|c|}
\hline $\begin{array}{l}\text { Diameter } \\
\text { of well. }\end{array}$ & $\begin{array}{c}\text { Depth of } \\
\text { well. }\end{array}$ & $\begin{array}{c}\text { Depth of } \\
\text { principal } \\
\text { water } \\
\text { supply. }\end{array}$ & \begin{tabular}{|} 
Height of \\
water \\
above $(+)$ \\
or \\
below $(-)$ \\
ground \\
level.
\end{tabular} & $\begin{array}{c}\text { Supply } \\
\text { per } \\
\text { minute. }\end{array}$ & $\begin{array}{c}\text { Geologic horizon of } \\
\text { water-bearing strata. }\end{array}$ & Remarks. & No. \\
\hline Inches. & Feet. & Feet. & Feet. & Gallons. & & & \\
\hline 2눈 & 104 & .......... & -96 & $\ldots \ldots \ldots$ & & ................. & 856 \\
\hline $12-3$ & 109 & $\cdots \cdot$ & -92 & $\cdots \cdots$ & $\cdots \ldots \ldots \ldots$ & $\ldots \ldots, \ldots, \ldots, \ldots, \ldots$, & $856 \mathrm{~A}$ \\
\hline $2-1 \frac{1}{2}$ & 20 & & - $\cdots \cdots \cdot$ & $\cdots .$. & Pleistocene. & Fluctuates with the tide .... & 857 \\
\hline & 225 & 225 & +12 & $\ldots$ & Cretaceous. & Flows 16 gallons per minute... & 858 \\
\hline & 277 & & +3 & $\ldots$. & $\ldots$. do ..... & Flows 1 gallon per minute..... & 859 \\
\hline 8 & 277 & & Flows. & $I-2$ & ..... do.... & 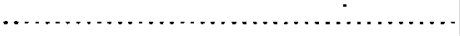 & 860 \\
\hline 8 & 40 & & -4 & $a 347$ & ......... & Group of 6 wells $b \ldots$ & $b 861$ \\
\hline 4 & $42-46$ & $\cdots$ & -30 & & …. & 2 wells............ & 862 \\
\hline 8 & 225 & & Flows. & & & $(b) \ldots \ldots$ & \\
\hline 6 & 305 & & Flows. & & & 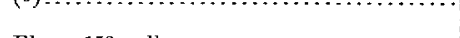 & \\
\hline & & 83 & Flows. & & & Flows 150 gallons.. & 863 \\
\hline & & 225 & Flows. & & & Pumps 133 gallons. . & \\
\hline & 16 & & -5 & & & & 864 \\
\hline & 150 & & -135 & & Pleistocene? & All coarse white sand.. & 865 \\
\hline & 35 & & -30 & & Pleistocene... & All medium red sand... & 866 \\
\hline & $15-90$ & & $\left\{\begin{array}{r}c-40 \\
-50\end{array}\right.$ & Fair. & ...do........ & & 867 \\
\hline 8 & 32 & & -22 & & ....do. & Ali light-colored sand and gravel... & 868 \\
\hline & 45 & & -6 & & & $\ldots \ldots \ldots \ldots \ldots, \ldots$ & 869 \\
\hline $\begin{array}{l}2 \\
4\end{array}$ & 70 & $60-70$ & -7 & $30-40$ & & All sand... & 870 \\
\hline 4 & 30 & & -16 & 50 & Pleistocene. & $\ldots$. do... & 871 \\
\hline & 20 & & $\cdots \cdots \cdots$ & $\cdots \cdots$ & $\cdots \ldots \ldots \ldots \ldots$ & $\ldots \ldots \ldots \ldots \ldots \ldots \ldots$ & .872 \\
\hline & $12-90$ & & $\left\{\begin{array}{l}-12 \\
-90\end{array}\right.$ & Good. & Pleistocenc. & & 873 \\
\hline & 35 & & ….... & & ....do. & & 874 \\
\hline 13 & 25 & & …... & & ....do... & & 875 \\
\hline & 28.5 & & -25 & & & All white sand.... & 876 \\
\hline $1 \frac{1}{4}$ & 88 & & $\ldots \ldots \cdots \cdots$ & 0 & $\cdots$ & Clay 4 to 88 feet..... & 877 \\
\hline & 70 & & 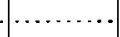 & & $\cdots$ & Clay 40 to 60 feet.... & 878 \\
\hline & so & & $-2 \bar{r}$ & & $\ldots \ldots \ldots+\cdots$, & (n) & 879 \\
\hline$\varepsilon$ & 80 & & -35 & $d 683$ & Pleistocene. & Group of 3 wells.: & (b) \\
\hline $1+$ & 80 & & ….... & Small. & $\ldots \ldots \ldots \ldots$ & Clay 34 to 80 feet.... & 880 \\
\hline .3 & 111 & & -100 & & Jameco?........ & Clay 2 to 82 feet.... & 881 \\
\hline & & & $\ldots .$. & & Pleistocen & $\ldots \ldots \ldots \ldots \ldots \ldots \ldots$ & $881 \mathrm{~A}$ \\
\hline 361 & 52 & & $\cdots$ & & $\ldots \ldots \ldots$ & $\ldots$ & .882 \\
\hline 36 & 43 & & & & Pleistocene. & & 883 \\
\hline & 53 & & …‥ & & ....do....... & ........... & 884 \\
\hline & 35 & & -30 & ..... & Sankaty........ & $\ldots \ldots \ldots \ldots \ldots \ldots \ldots \ldots \ldots \ldots$ & 885 \\
\hline & 60 & & -50 & & Tisbury ............. & All sand and gravel. . . . . . . . . . . . . . & 886 \\
\hline$\ldots{ }^{\prime}$ & 73 & & -68 & & Tisbury?........ & 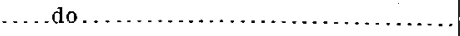 & 887 \\
\hline$\cdots \cdots$ & 62 & & -50 & .......... & Sankat. .......... & (n) & 888 \\
\hline $36-6$ & 23 & & -18 & 100 & Tisbury ............ & … & 889 \\
\hline$\cdots \cdots$ & 60 & & & $\mathbf{0}$ & & & (b) \\
\hline 36 & $\begin{array}{l}36 \\
65\end{array}$ & & $\begin{array}{l}\cdots \cdots \\
-32\end{array}$ & & Tisbury...... & Group of 18 wells & J. \\
\hline & 45 & -45 & -45 & & Jameco? . . . . . . . . & 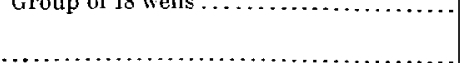 & 8890 \\
\hline & a Estimat & & $b$ See Tal & ble VIII. & c Average. & $d$ Test for whole station of 3 wells. & 091 \\
\hline
\end{tabular}



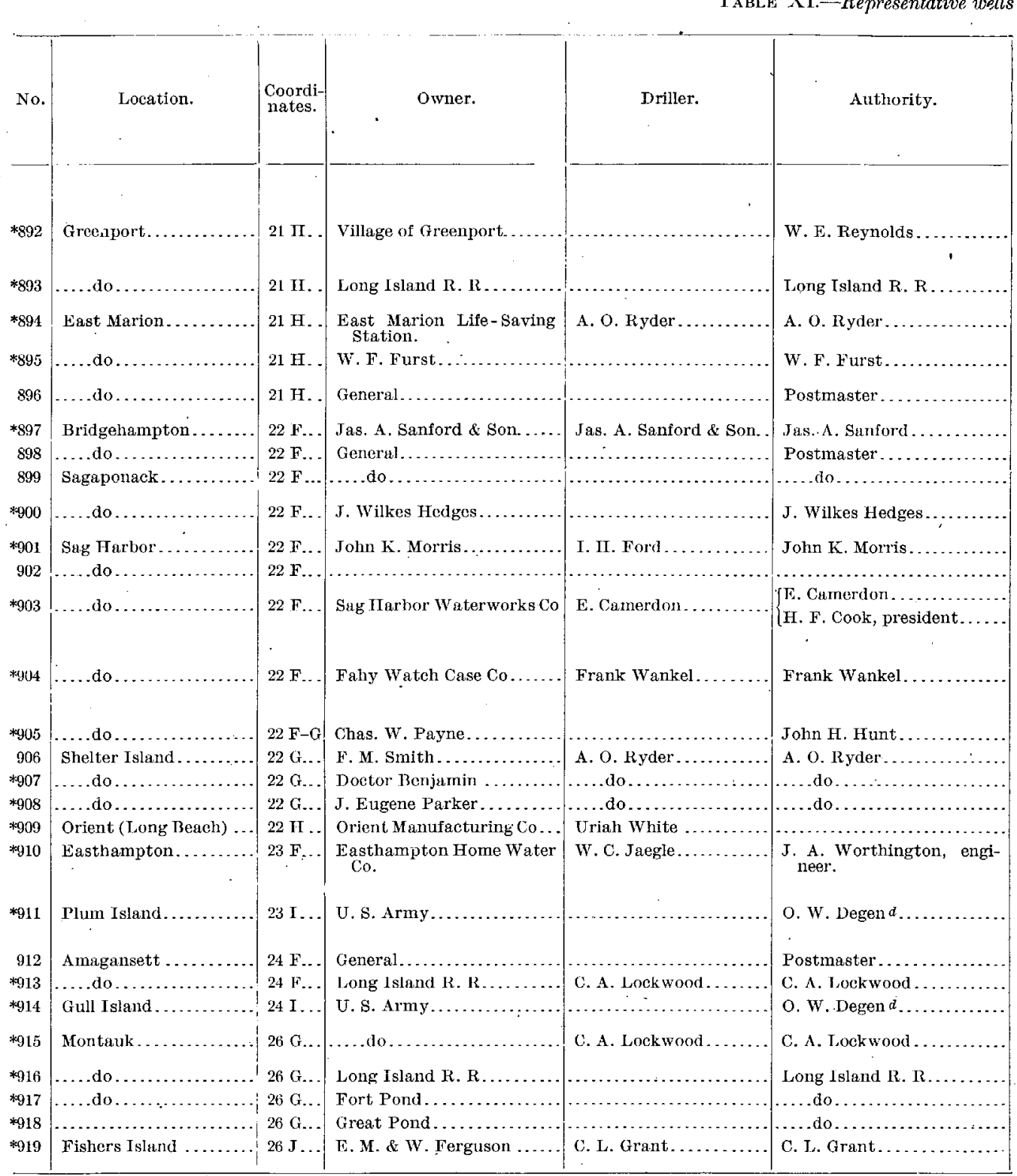

* For additional data see descriptive notes, pp, 168 et seq. a Reported test of first 4 wells. 
on Long Island-Continued.

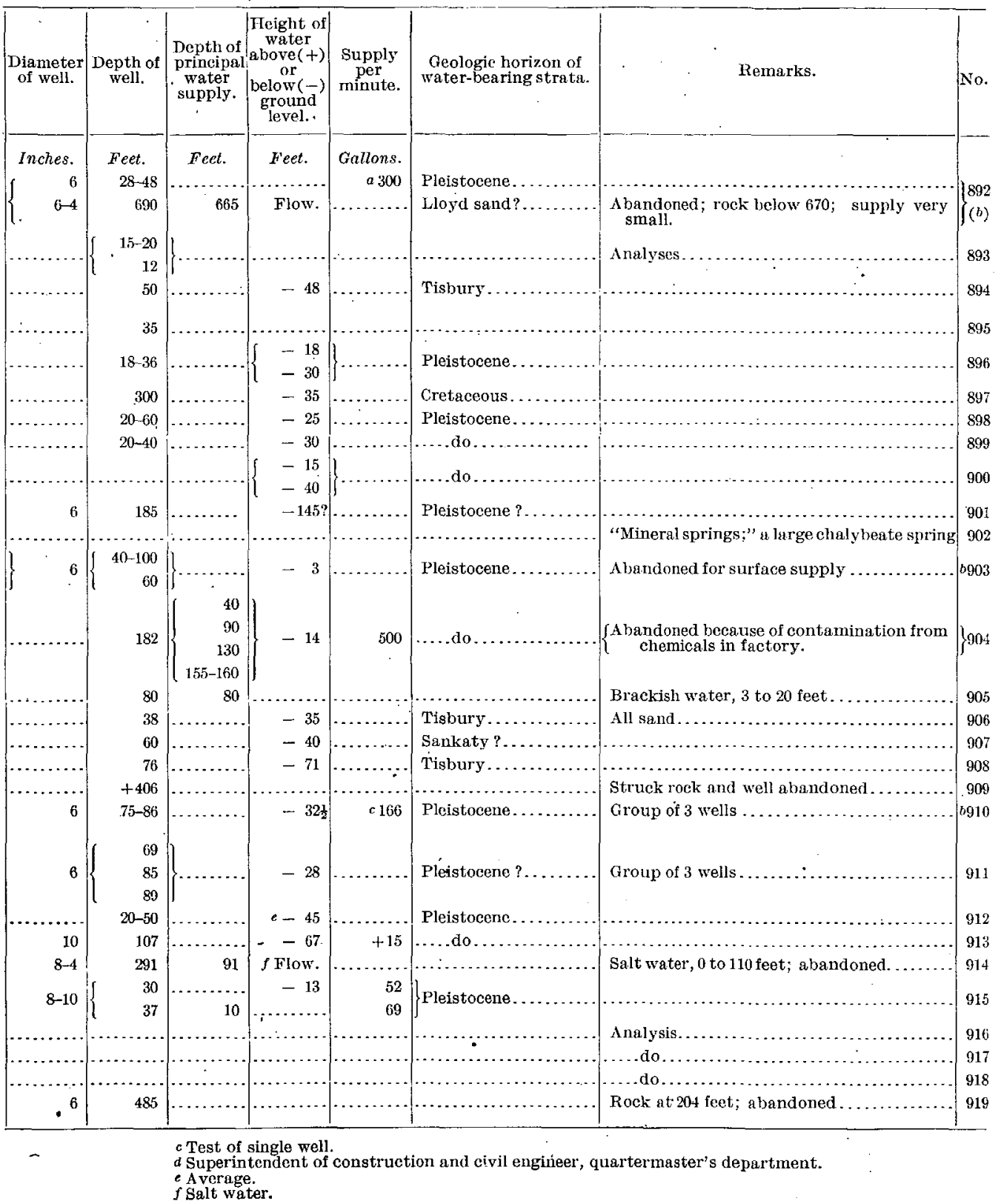




\section{UNDERGROUND WATER RESOURCES OF LONG ISLAND, NEW YORK.}

\section{DESCRIPTIVE NOTES.}

[Numbers in black-faced type correspond to those used in the table preceding.]

1. The sample from this well preserved in Mr. Gregory's officc, marked "Hoffmans Island 210 feet," is a dark, bluish-gray sand, apparently glacial.

Record of quarantine station well, Ioffmann Island, New York.

1. Sand, clay, and gravel, with salt water.

Feet.

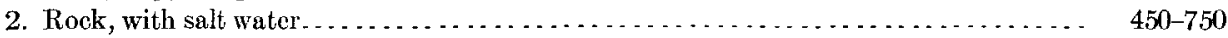

3. Rock, with fresh water. . . . . . . . . . . . . . . . . . . . . . . . . . . . . . . . . $750-1,000$

2. In 1867 John Nadir, United States engineer at Fort Hamilton, carefully examined the underlying formations at Fort Lafayette, making borings 800 to 1,000 feet from the shore. These borings showed the following section: $a$

Generalized record of United States Army test borings at Fort Lafayette, N. Y.

1. Coarse sand and gravel, with a few broken shells ....................... Feet.

2. Decayed marsh or meadow mud with diatomacer and spicule of sponges and shells . . . . . . 20-23

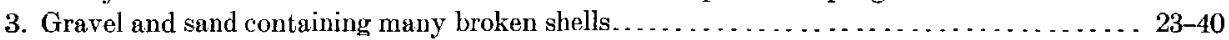

4. Mud, quite compact, which appears to have been a marsh with scanty vegetation, rather than a meadow. In this formation a great number of shells were found which were identified as Nassa obsoleta, Anomia ephippium, Mya arenaria, Crepidula fornicata, Solen ensis, Mytilus edulis. . . . . . . . . . . . . . . . . . . . . . . . . . . . . . . 40-53

3. See plan and cross section of south Brooklyn tunnel, by Isaiah Bowman, from notes furnished by J. C. Neem, civil engineer (PI. XXV). Mr. Meem states that in order to keep the tunnel dry 750,000 to $1,000,000$ gallons per day were pumped from each of the seven shalts.

4. Mr. L. B. Ward gives the following data: $b$ "This company has no municipal contract. Its area of operation comprises Blythebourne and Borough Park tracts, situated in the Thirtieth Ward. The supply is pumped from open wells at a depth of 80 feet. The works consist of 1 principal pumping station, and 1 reserve station, also 5 elevated tanks (wooden structures) of 25,000 gallons cach. Daily pumpage 200,000 gallons. An average of 106,000 gallons per day is also received from the city."

5. Mr. J. C. Breckenridge, general manager of the Brooklyn Rapid Transit Company, in a letter dated April 29, 1901, gives the following data regarding this well: "Well was put down 1,503 feet; 8 inches in diameter to 1,000 feet, and 6 inches below that point. It was never pumped to determine the yield, as the water always tested salty and unfit for boiler use. The original plan was to go down to a fissure in the bed rock where it was supposed a stream of running water suitable for boiler use could be found. The nature of the material penetrated was as follows:

Record of Brooklyn Rapid Transit Company's well at Brooklyn, N. Y.

Wisconsin and Tisbury: Feet.

1. Sand

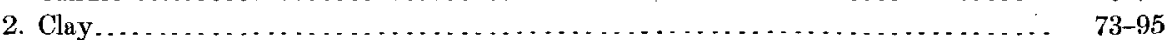

Sankaty:

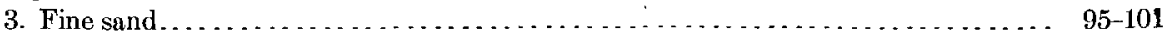

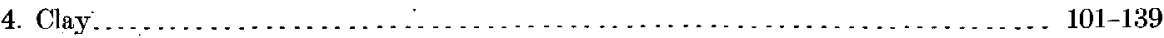

Jameco:

5. "Hard pan," with small stones, black, and varying in size. . . . . . . . . . . . . . . 139-169

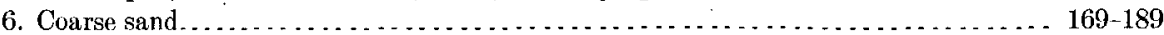

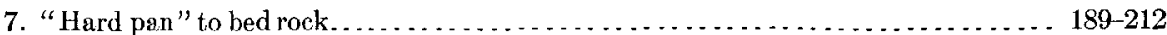

"At 140 feet no clay, struck a bowlder and were obliged to shoot the well to get it out of the way, as it jammed the drilling at the end of casing. At 292 feet a sand pocket was struck. When the sand had been pumped out the cavity was filled with cement and the drilling continued. Work was started on August 31, 1897 and stopped December $21,1898 . "$

a Am. Nat., vol. 2, 1869 , p. 335.

o Merchants' Association report on water supply of the city of New York, 1900, p. 181 


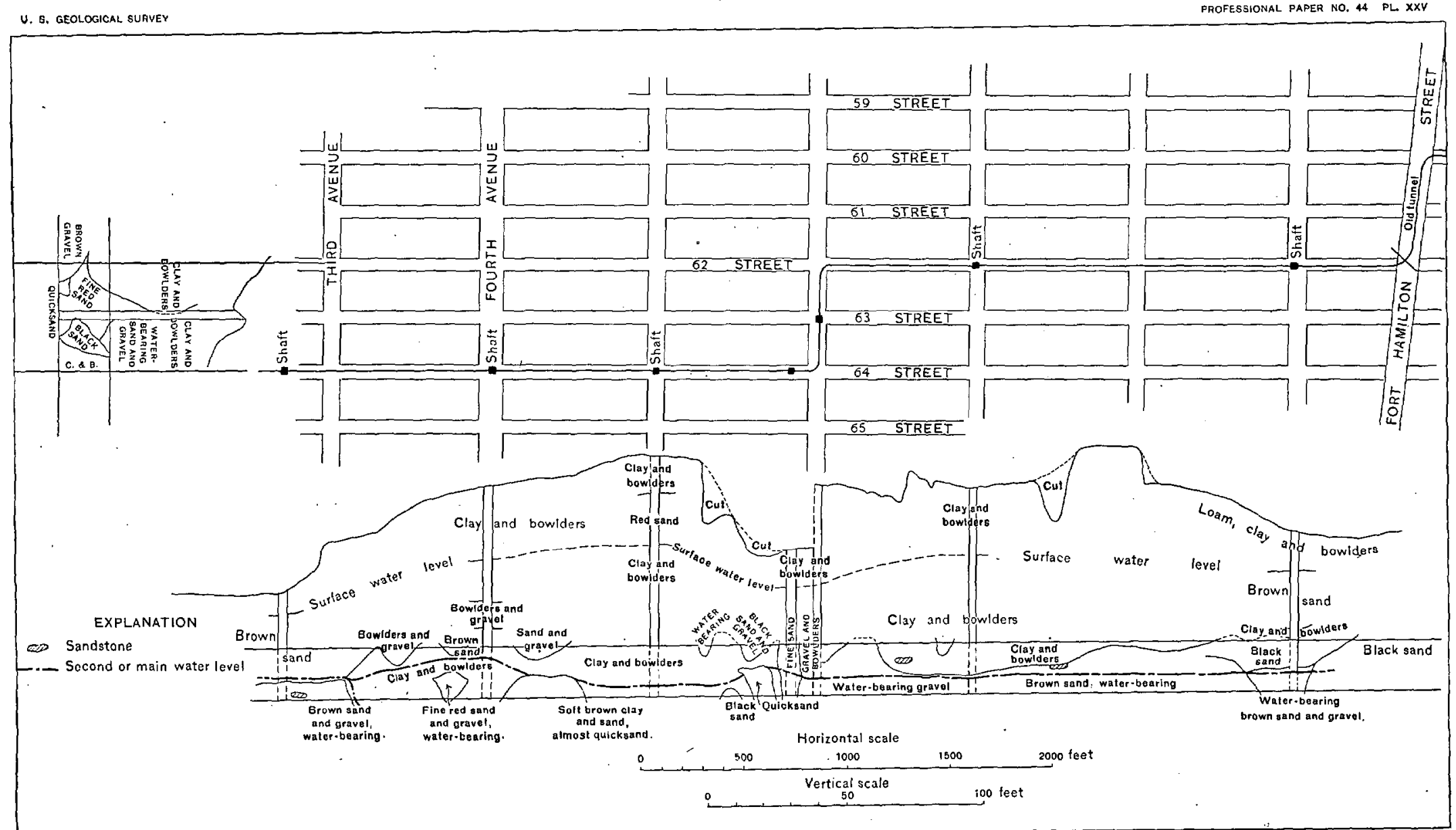

PLAN AND LONGITUDINAL SECTION OF STRATA ENCOUNTERED IN THE SOUTH BROOKLYN SEWER TUNNEL. By Isaiah Bowman, from personal examination and notes furnished by J. C. Meem, C. E. 



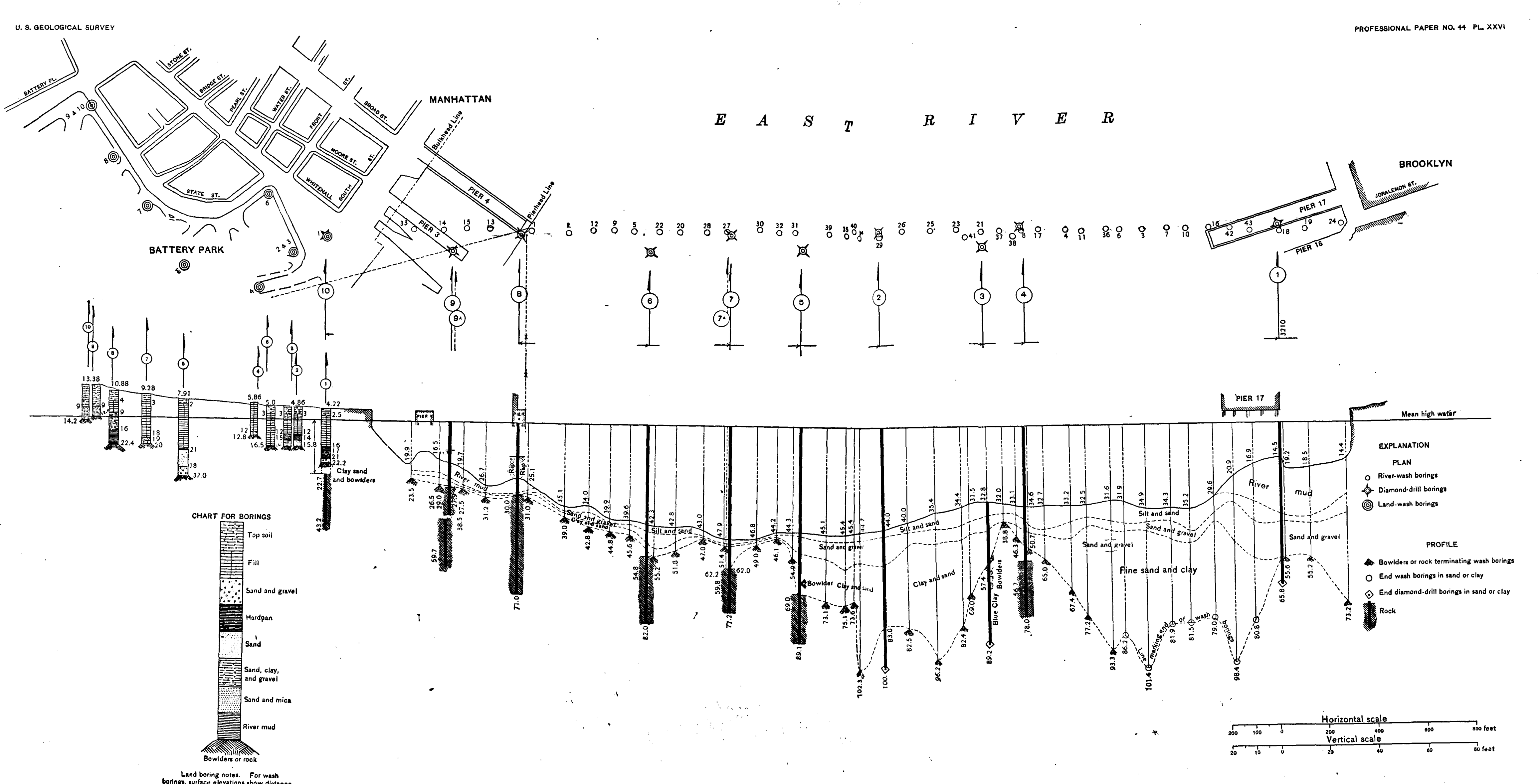





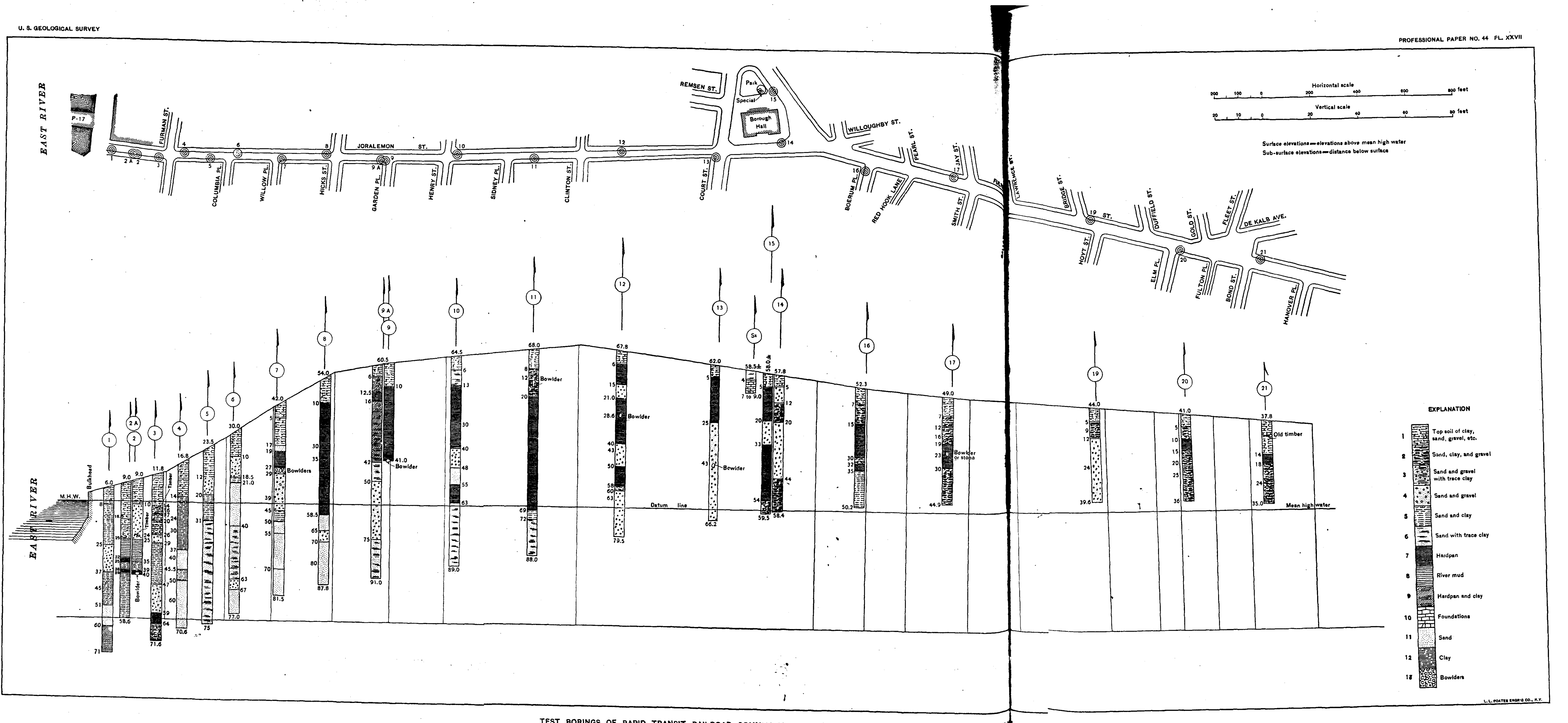

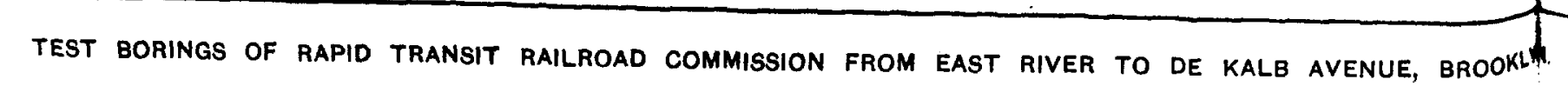




7. This well is about 10 feet above low tide and was completed in November, 1903. It is entirely in sand and gravel. At 50 feet clay was encountered, below which the driller stated it was useless to look for water in this vicinity. The clay suggests the Sankaty, and it is supposed that the underlying Jameco does not yield potable water at this point, because of the removal of the clay covering in the upper bay.

10. Q. M. Gen. C. F. Humphrey reports: At Governors Island an 8-inch well was recently sunk to a depth of 1,822 feet 6 inches. At 1,175 feet a flow of 15 gallons per minute was obtained. By torpedoing the well the flow was increased to aboul 18 gallons per minute. The water was salty and chemical analysis pronounced it unfit for drinking purposes.

The following samples have been received from this well:

Record of United States Army well on Governors Island, New York.

Feet.

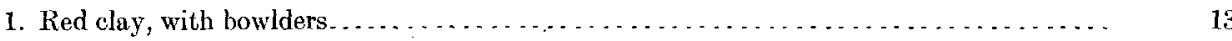

2-4. Red clay; no bowlders........................................ $44-55$

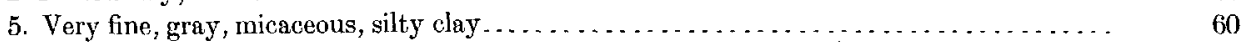

6. Dark multicolored gravel, with fragments of Recent shells.....................

7-8. Disintegrated micaceous rock, with fragments of Recent shells ... . . . . . . . . . . . . $\quad 73-87$

9. Highly micaceous schist or diorite, thought by Mr. E. C. Eckel, of this Survey, to resemble the Harrison diorite . . . . . . . . . . . . . . . . . . . . . . . . . . . . . . . . . 87-1,700

1. Record of well on Ellis Island, New York.

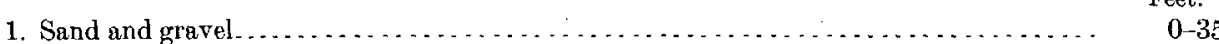

2. Rock; trap and gneiss......................................... $35-1,400$

12. Samples and record in the Long Island Historical Museum show:

Record of Long Island Railroad well in Brooklyn, N.Y.

1. Sand, gravel, clay, etc -

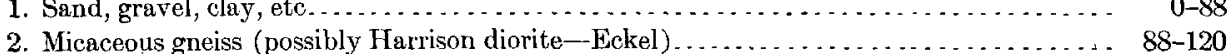

13. See Pls. XXVI, XXVII.

16. The following analysis has been made by the Brooklyn health department:

Analysis of well water at Gravesend pumping station.

Total solids. . . . . . . . . . . . . . . . . . . . . . . . . . . . . . . . . 127.00

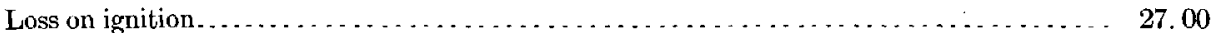

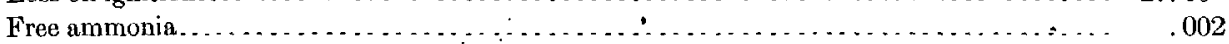

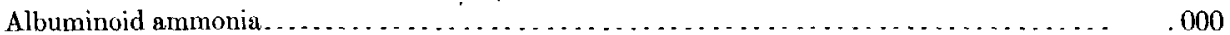

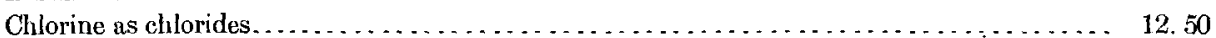

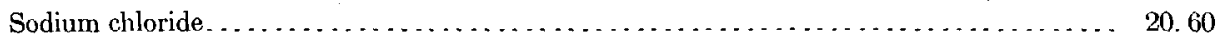

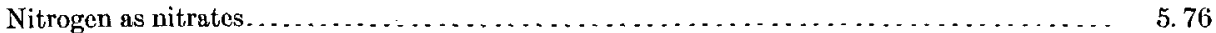

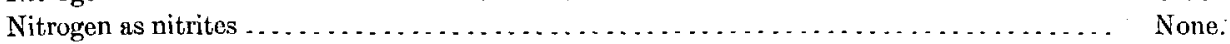

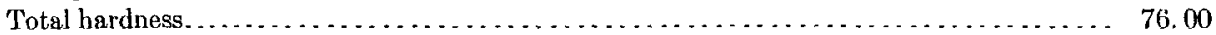

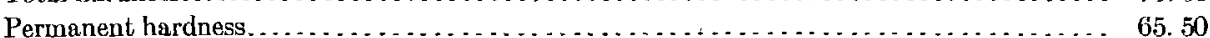

18. Mr. L. B. Ward gives the following data regarding this company: "This tract of 90 acres, located in the Thirticth Ward, between Fifteenth and Eighteenth avenues, and Fifty-third and Sixtieth streets, has an independent water service, with 1.7 mile of distributing pipes and one pumping station located at Seventeenth avenue and Sixtieth street, supplied from a single well."

23. Temperature $52^{\circ}$ to $53^{\circ}$. Water used for cooling and manufacturing.

$$
17116--N_{0} .44-06-12
$$


Sanitary analysis of water from well at Eighth avenue and Eighteenth street, Brooklyn.

[Analyst not reported.]

Parts per million.

Total solids. . . . . . . . . . . . . . . . . . . . . . . . . . . . . . . . . . . 376.04

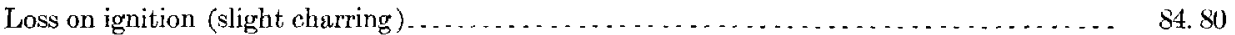

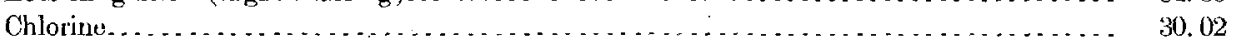

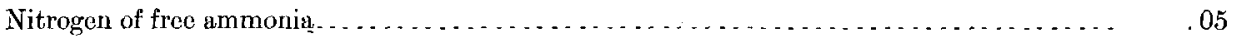

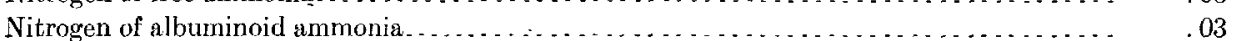

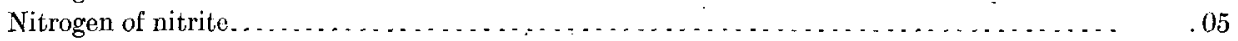

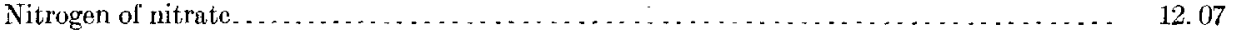

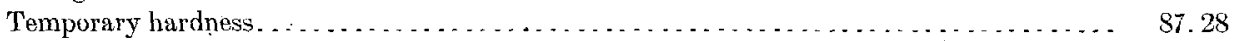

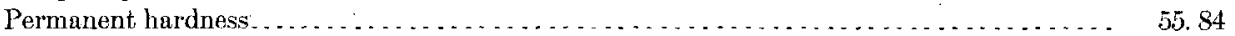

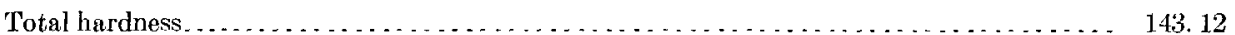

Iron. . . . . . . . . . . . . . . . . . . . . . . . . . . . . . . . Very faint trace.

Samples received from Mr. R. A. Ward, treasurer, show the following section:

Wisconsin till:

Record of well at Eighth avenue and Eighteenth street, Brooklyn.

1. Reddish bowlder clay . . . Feet

2. Fine to coarse silty sand with a little gravel. . . . . . . . . . . . . . . . . . . . .

3. Same, but much cleaner; note on samples says, "Struck first water, which yielded 3 gallons per minute",

Wisconsin and Tisbury:

4-5. Reddish-brown bowlder clay . . . . . . . . . . . . . . . . . . . . . . . . $35-45$

6-14. Clean, dark-colored, reddish-brown glacial sand and gravel . . . . . . . . . . . . $55-135$

25. Sample preserved in Mr. Gregory's office dated April 24, 1894, and marked "141 feet; 46 gallons per minute" is a mixture of sand and coarse gravel with much glacial material. It is believed to represent the Jameco gravels. The Tartar Chemical Company report that the water falls to 14 feet when the well is pumped. Temperature $54^{\circ}$.

Analysis of unfltered well water from Ninth street and Gowanus Canal, Brooklyn.

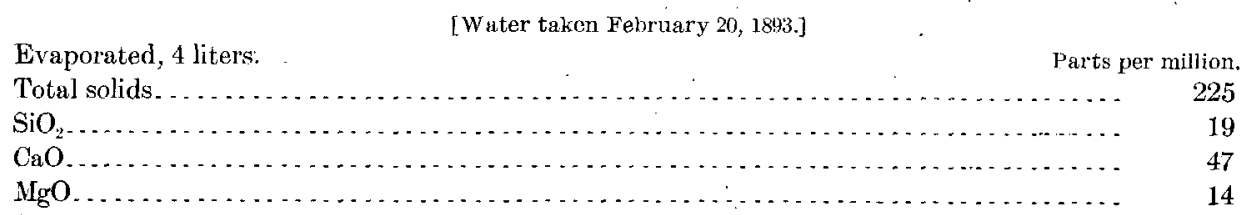

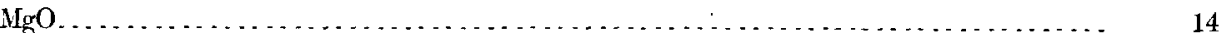

Traces of $\mathrm{FeAl}_{2} \mathrm{O}_{3}$.

Analysis of filtered well water from Ninth street and Gowanus Canal, Brooklyn.

Evaporated, 7 liters.

[Water taken February 23, 1893.]

Parts per million.

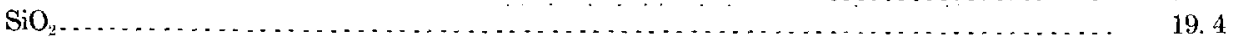

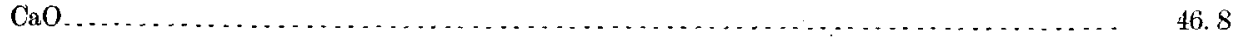

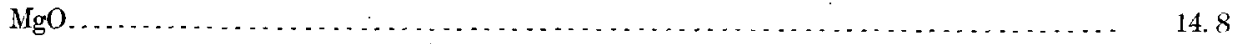


Analysis of well water from Ninth street and Gowanus Canal, Brooklyn.

[Analysis by Charles L. Bauer, Springfield, Ohio, September 26, 1896.]

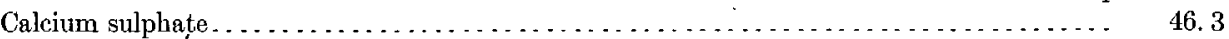

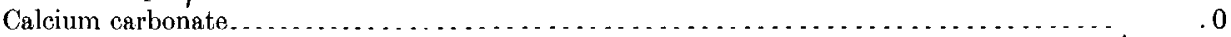

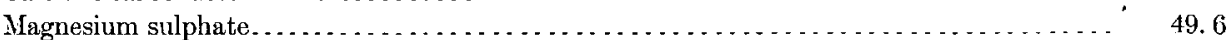

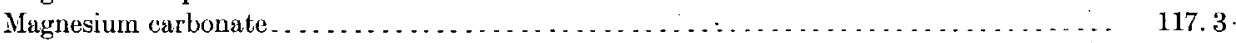

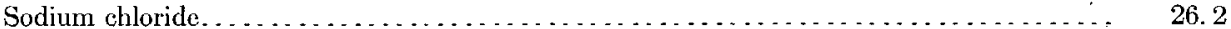

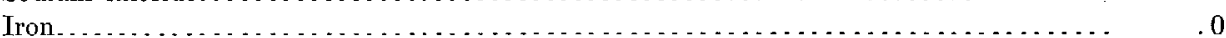

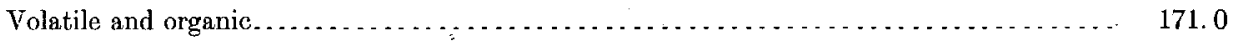

Total solids. ......................................... 410.4

Remarks: Odorless and clear.

Analysis of well water from Ninth street and Gowanus Canal, Brooklyn.

Analysis by bureau of chemistry, board of he§lth, Brooklyn, September 16, 1897; G. J. Volckening, chief chemist; II. W. Walker, assistant chemist.]

Chlorine in chlorides

Parts per million.

Equivalent to sodium chloride . . . . . . . . . . . . . . . . . . . . . . . . . . . 77.50

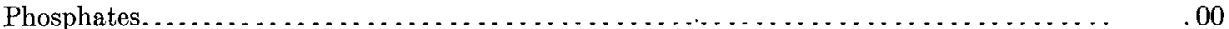

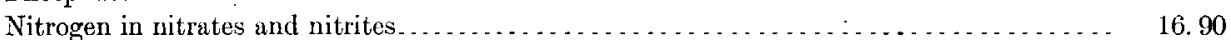

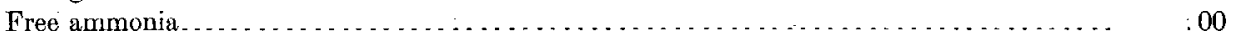

Albuminoid ammonia. . . . . . . . . . . . . . . . . . . . . . . . . . . . . . . . . . . . . . . 00

Hardness equivalent to carbonate of lime (before boiling) $\ldots \ldots \ldots \ldots \ldots \ldots \ldots \ldots \ldots \ldots$

Hardness equivalent to carbonate of lime (after boiling) . . . . . . . . . . . . . . . . . 197.5

Organic and volatile. . . . . . . . . . . . . . . . . . . . . . . . . . . . . . . . . . . . 145.3

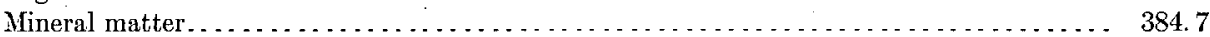

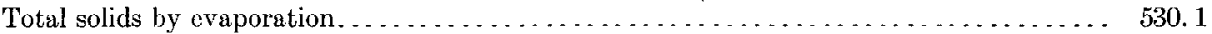

Analysis of well water from Ninth street and Gowanus Canal, Brooklyn.

[Analysis by Pittsburg Filter Manufacturing Company, Pittsburg, Maroh 20, 1903; F. T. Asehman, chemist.]

Sodium chloride

Parts per million.

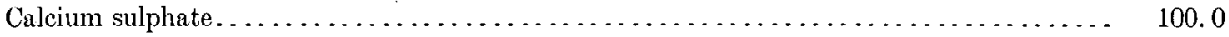

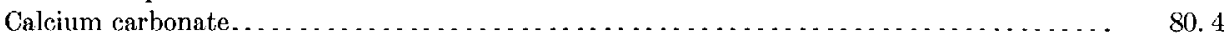

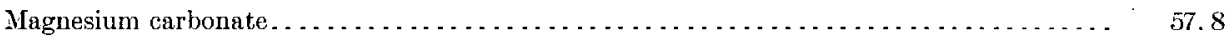

Iron and aluminum oxides. . . . . . . . . . . . . . . . . . . . . . . . . . . . . . . . 5.8

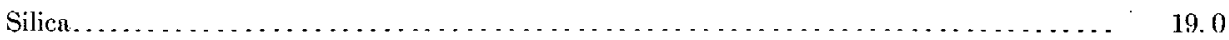

Organic and volatile matters . . . . . . . . . . . . . . . . . . . . . . . . . . . . .

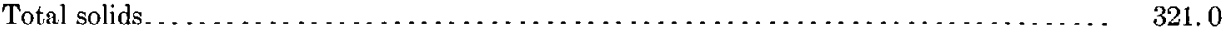

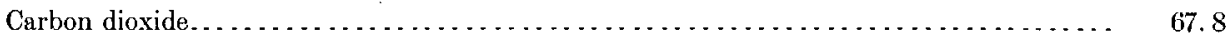

Sample clear.

27. Seventeen test borings were sunk at this point: No. 1, 110 feet north of Third avenue and Third street; Nos. 2-9, at intervals of 50 feet west; Nos. 10-16, bordering Third street, at intervals of 50 feet east toward Third avenue; No. 17, opposite No. 2. The following sections may be taken as typical:

Record of Transit Development Company's test boring No. 3 near Third avenue and Third street, Brooklyn.

1. Light-yellow sand filling.

Feet.

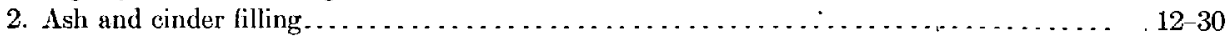

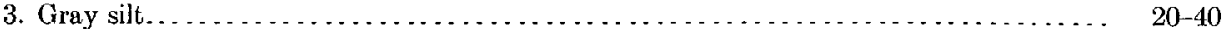

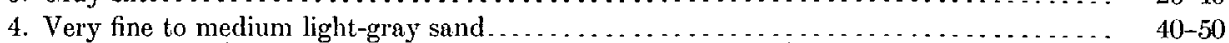




\section{UNDERGROUND WATER RESOURCES OF LONG ISLAND, NEW YORK.}

Record of Transit Development Company's test boring No. 9 near Third avenue and Third street, Brooklyn.

1. Ash filling

Feet.

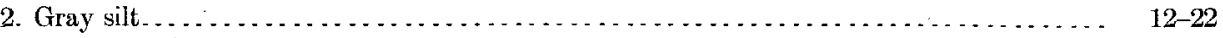

3. Medium light-gray sand containing muscovite and a considerablc percentage of erratic

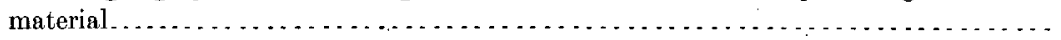

4. Light-brown sand, gradually becoming coarser and with an increasing percentage of erratic material. . . . . . . . . . . . . . . . . . . . . . . . . . . . . . . . . . . . . . .

Record of Transit Development Company's test boring No. 13 near Third avenue and Third street, Brooklyn.

1. Ash filling.

Feet.

2. Gray silt..................................................... $7-15$

3. Coarse light-gray sand with a high percentage of erratics. . . . . . . . . . . . . . .

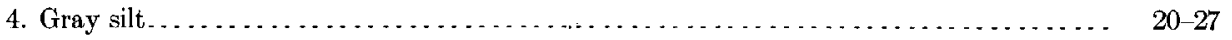

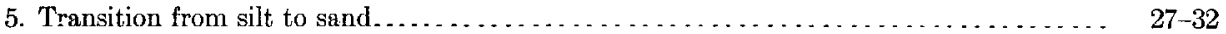

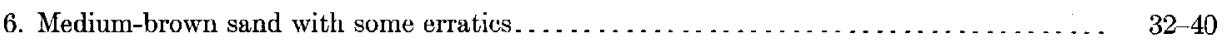

Record of Transit Development Company's test boring No. 16 near Third avenue and Third street, Brooklyn.

1. Light-brown sand with erratic material between 5 and 10 , and fine sand between 10

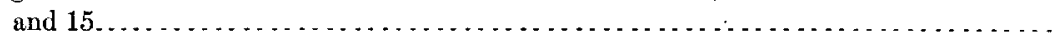

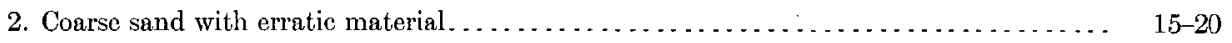

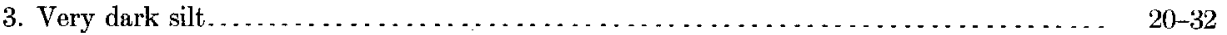

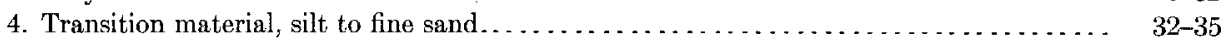

5. Fine to medium dark-gray sand with a considerable amount of erratic material ...... . 35-42

28. Record of well on Third avenue between Degraw and Douglas streets, Brooklyn.

Recent:

1. Filled ground

Feet.

Recent?

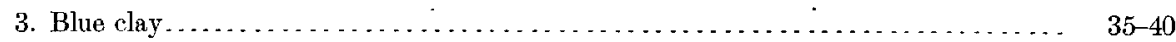

Wisconsin and Tisbury?:

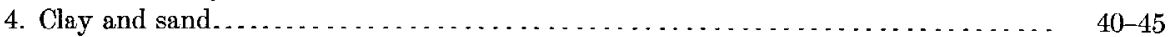

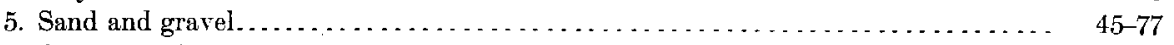

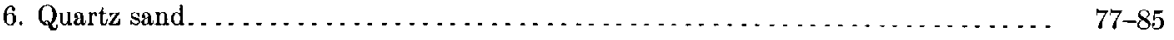

'29. Record of well on Dean street near Vanderbilt avenue, Brooklyn.

Wisconsin:

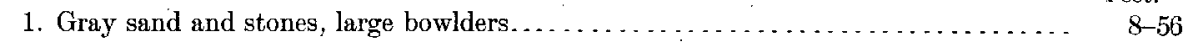

Wisconsin and Tisbury?:

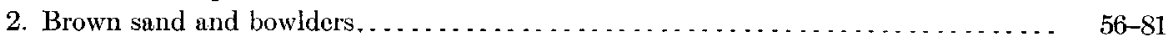

3. Coarse brown sand (water at 81 feet) ............. . . . . . . . . . . . . . 81-98

Mr. Corwin adds: "Nearly always we get water in brown sand-pepper and salt mixture-sometimes in yellow coarse sand; never, or hardly ever, in white sand."

30. A sample from this well preserved by Mr. Gregory, and marked 217 feet, is a coarse, multicolored, glacial gravel, similar to the Jameco gravel in the Brooklyn test wells.

Record of well at St. Marks and Grand avenues, Brooklyn.

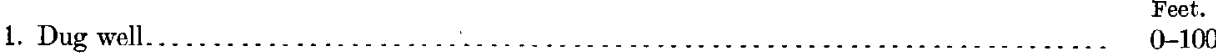

2. Sand, gravel, and clay . . . . . . . . . . . . . . . . . . . . . . . . . . . . . . . . . . . . $100-331$

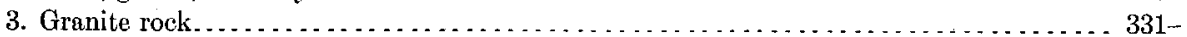


31.

Record of well at Lewrs and De Kalb avenues, Brooklyn

1 Dug well.... . . . . . . . . . . . . . . . . . . . . . . . 0 0 63

Tisbury?

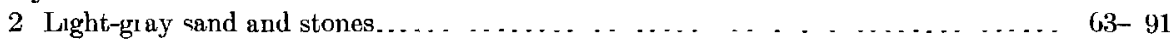

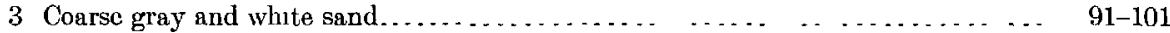

33. A sample preserved by $\mathrm{Mf}_{1}$ Gregory, marked "125-138 feet," is a highly erratie glacial gravel

35.

Record of well at Forest street and Evergreen avenue, Brooklyn

Wisconsin

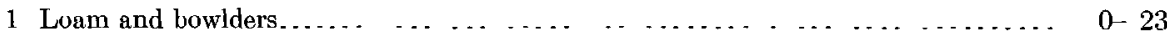

Wisconsin and Tisbury

2 Yellow gravel and sand . . . . . . . . . . . . . . . . . . . . . . . . . 23-63

3 Yellow gravel with water.. . . . . . . . . . . . . . . . . . . . . . . . . . . 63-105

Sankaty or Cretaceous

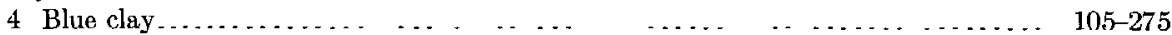

37.

Record of well at Bartlett street and Marrson avenue, Brooklyn

Tisbury?

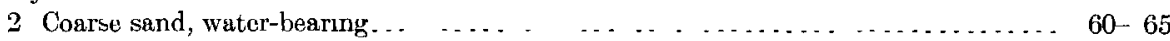
Sankaty

3 Red clay with an occasional large bowlder..................... 65-150 Jameco

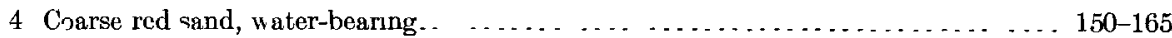

38. Record of well at Bartlett street and Flushing avenue, Brooklyn

Recent

1 Miscellaneous fillıng down to bottom of old creek. ..................... 0 . 6

Wisconsin and Tisbury

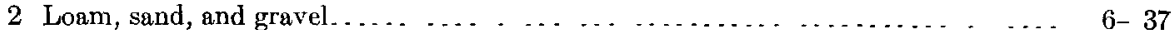

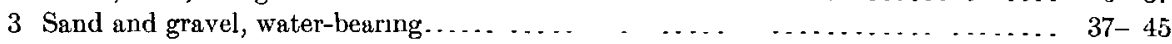

Sankaty

4 Interstratifications of clay and fine sand and gravel..... . . . . . . . . . . . . 45-139 Jameco

5 Water-bearing stratum of coarse yellow sand........................ 139-176

The samples of the material encountered in this well, which were obtaned through the courtesy of Mr

E L Heusner, chief engineer, show the following section

Recent

Record of well at Bartlett street and Flusheng avenue, Brooklyn

Filled ground

2 Black marsh mud.... . . ... . . . . ...................... 8 9

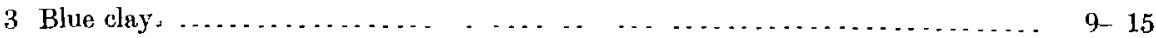

Wisconsin and Tishury?

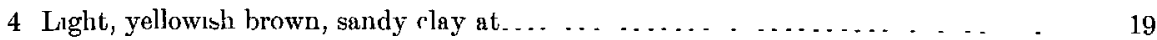

5 Blush gray, rather pure, clay at... ............................. 26

6 Highly erratıc glacial gravel. ...... ......................... $31-36$

7-9 Medium sand, the particles being uniform in size, the composition very similar to

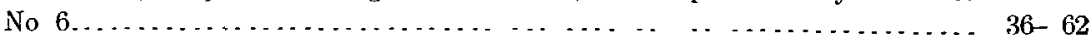

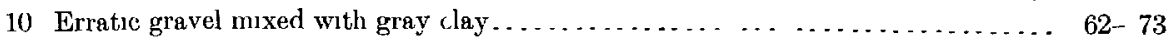

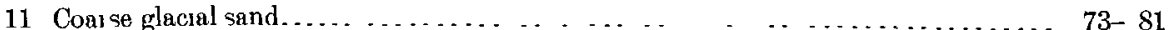
Sankaty

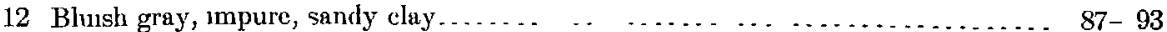


Sankaty-Continued.

13. Yellowish gray sand mixed with clay.

14. Clean, light-brown, medium, erratic sand ....................... . 108-122

15. Bluish gray sandy clay . . . . . . . . . . . . . . . . . . . . . . . . . . . . . . . . . . . . . 122-124

\section{Jameco:}

16. Yellowish brown medium sand, slightly clayey . . . . . . . . . . . . . . . . . . . . . 124-127

17. Yellowish brown, coarse, clayey sand . . . . . . . . . . . . . . . . . . . . . . 127-134

18-19. Very coarse slightly clayey sand, having a dark-yellow color............. . 134-145

20-22. Reddish yellow, extremoly coarse sand with erratic material as in the preced-

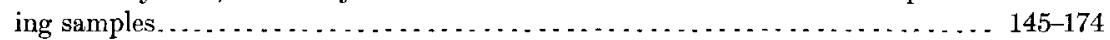

23. Similar to the preceding, but much coarser and with erratic material more abundant .... . . . . . . . . . . . . . . . . . . . . . . . . . . . . . . . . . . 174-175

Mr. Heusner states that the first wells which were used on this property by the chemical company were 30 feet deep. These wells were successively deepened to 40, 50, and 70 feet, and finally it became necessary to sink the deep wells above described. The water from the deep wells rises to within 7 or 8 feet of the surface, or to the surface of the original ground before this section was built up by filling. The capacity of this well is 500,000 gallons in twenty-four hours, the well being pumped steadily the year round, night and day.

40. Record of well at 20 to 34 Ryerson street, Brooklyn.

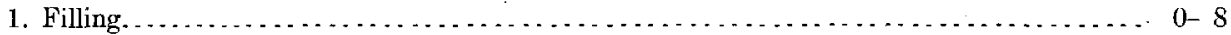

2. Sand, stones, and little clay.................................... $8-32$

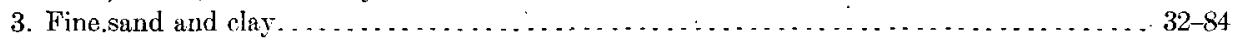

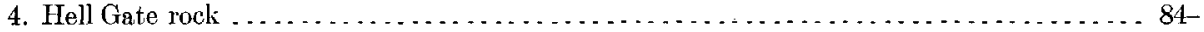

41. Record of well at 163 Carlton avenue, Brooklyn.

Wisconsin: Feet.

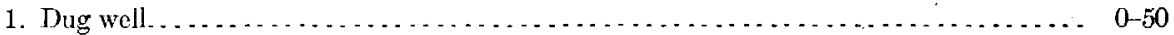

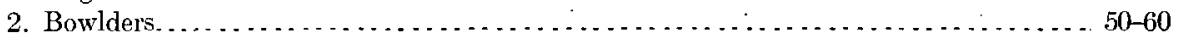

Wisconsin and Tisbury: $\quad$ ․ $\quad$. . . 60

42. Mather gives the following section of a well sunk for Mr. Johnson in Brooklyn between "Wallabout and Guanus" in April, 1811:

Record-of well between Wallabout and Gowanus, Brooklyn.

Feet:

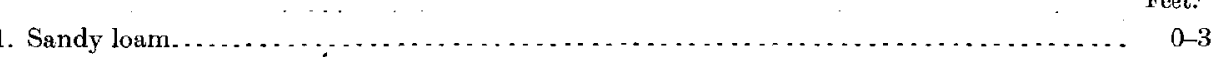

2. Hard concreted clay, sand, and stones colored with iron and requiring a pick to dig; com-

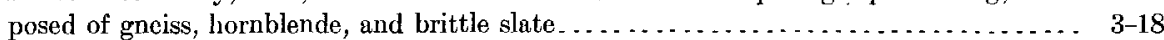

3. Loose gravel and grayish sand, with thin streaks of gravel, the gravel of quartz, bassanite,

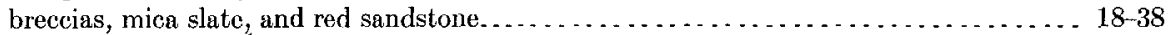

4. Alternating layers of 2 - or 3 feet of sand and gravel, containing coarse green soapstone in

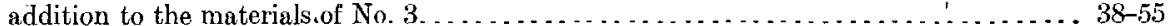

5. Sand and gravel in alternating layers; the gravel beds contain sea shelis, mostly clams and oysters, but the sand none. . ........................................ $55-84$

43. According to Hollick, " this is the location of the well from which the Exogyra costala, reported by Redfield $b$ and Cozzens, ${ }^{c}$ was taken at a depth of 65 feet.

45. A drawing in the museum of the Long Island Historical Society, by C. M. Jacobs, consulting engineer, gives the following section at the east tower of the Brooklyn Bridge:

a Trans. New York Acad. of Sci., vol. 12, 1893, p. 225

$b$ Am. Jour. Sci., 1st ser., vol. 45, 1843, p. 156 .

$c_{\Lambda}$ Geological History of Manhattän Island, 1843, p. 51. 
Record of excavation for east tower of Brooklyn Bridge, Brooklyn.

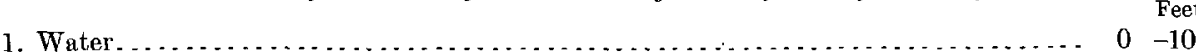

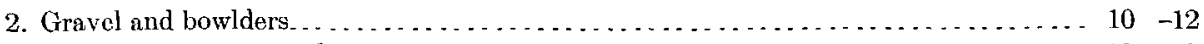

3. Hardpan; concrete and serpentine rock . . . . . . . . . . . . . . . . . . . . . . . . 12 . 23.6

4. Bowlders and sand; a trap bowlder. . . . . . . . . . . . . . . . . . . . . . . . . . 23.6

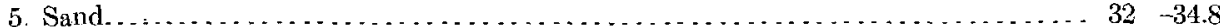

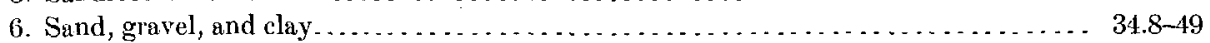

7. Reddish clay ................................................. $49-50$

8. Very compact sand, gravel, and clay, mixed with trap. . . . . . . . . . . . . . . $50 \quad-89$

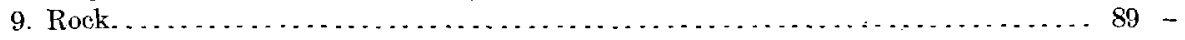

46. Record of well at Pear and Front streets, Brooklyn.

Wisconsin and Tisbury: Feet.

1. 'Sand and bowlders.................................... $0-21$

2. Coarse brown sand .......................................... 21-60

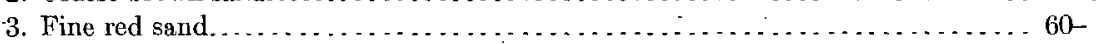

47. Mr. Ingalls, of John W. Masury \& Son, reports two 5-inch and five 6-inch wells drilled between 1877 and 1902. He gives the following description of the locality: "After a few feet of loose earth there is clay, very hard, from 27 to 33 feet; where we get gravel and clay to 40 feet. The lower clay and gravel are filled with hard bowlders (probably glacial, as every well in this end of Long Island has shown these to be widely distributed). Below 40 feet the sand becomes finer and is water bearing. Our wells give a good supply at 53 feet, which is not much increased at 75 . Below 50 feet is clear fine sand, with bowlders in some places, extending to about 90 feet, where hard blue clay is reached.

"An interesting feature of our wells is the rapid corrosion of brass strainers. The metal loses the zinc. A corroded and useless strainer showed 65 per cent copper and 35 per cent zine in the perfect spots and over 99 per cent copper in the corroded parts, which were chiefly at the bottom, the top being in perfect condition. The water is not acid except with $\mathrm{CO}_{2}$, and the prevailing opinion is that the action is electrolytic, though this has not been proved.

"The 75-foot well has a casing of 59 feet of 8-inch pipe, with a 16-foot brass strainer, 6 inches in diameter, extending below and connected with a 6 -inch iron pipe inside the casing. This well when first completed gave 225 gallons per minute with a centrifugal test pump.

"The supply is all right, but the strainers give out, the pump fills with sand, and we have to keep putting down new wells and strainers cvery ycar or two.

"Changing the position of the wells only 25 or 50 feet seems to make a difference in durability. Water is good, but hard. "It is used principally for cooling purposes."

Mr. Jamieson, of Arbuckle Brothers, reports that a sample taken from this well November, 1899 , showed 1.662.5 parts of chlorine per million.

48. Mr. H. S. Stewart reports: "Well No. 1 was about 800 feet deep. We struck what I would call trap rock at 97 feet-until that depth it was quicksand or gravel and bowlders. We shut that off with 18-inch pipe. From 97. feet to 800 feet it was trap rock standing on edge all the way and full of crevices, making it very hard to keep a straight hole. We abandoned that well at about 800 leet, and started No. 2 about 500 feet away. We encountere the same formation in this well to a depth of about, 93 feet and then struck the same kind of 'trap rock, which continued for about 800 feet. Below this the rock lay level and we had no more trouble in keeping a straight hole. This rock was granite, some dark and some red. It would change in color sometimes in 20 feet and sometimes run in the same color for 30 feet. This well was drilled to a depth of 2,148 feet. There was water in the gravel above the trap rock, but it was not the quality of water wanted. We cased it off and went on down. There was no water in the granite nor trap rock-it was too hard to contain water. The well was then abandoned at Mr. Arbuckle's request."

Water from a depth of 51 feet showed 560 parts of chlorine per million. 


\section{UNDERGROUND WATER RESOURCES OF LONG ISLAND, NEW YORK.}

51. Record of well at Brooklyn Navy-Yard, Brooklyn.

1. Filled ground

Feet.

2. Stiff blue clay.

$0-15$

3. Hardpan ...

4. Blue clay and gravel

5. Hard pan.

$15-24$

6. Reddish blue clay

7. Sand.

8. Blue clay.

9. Gravel and hardpan

10. Stiff blue clay.

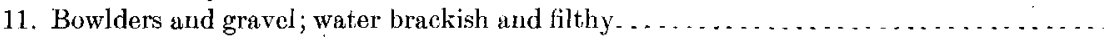

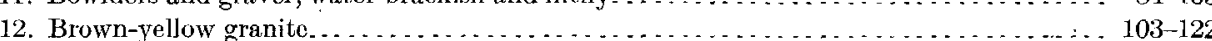

13. White granite . . . . . . . . . . . . . . . . . . . . . . . . . . . . . . . . . . . . . 122-129

14. Gray granite. ............................................. 129-144

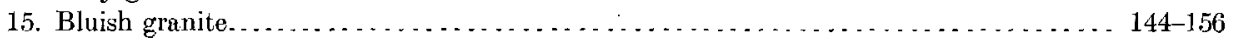

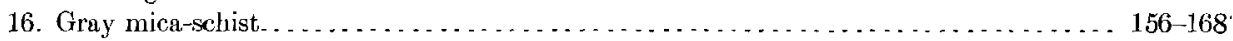

17. Darker schist . . . . . . . . . . . . . . . . . . . . . . . . . . . . . . . . . 168-172

18. White schist............................................... 172-185

19. Black schist . . . . . . . . . . . . . . . . . . . . . . . . . . . . . . . . . . . . . . 185-227

. 20. White granite ..... . . . . . . . . . . . . . . . . . . . . . . . . . . . . . . . 227-246

21. Gray granite . . . . . . . . . . . . . . . . . . . . . . . . . . . . . . . . . . . 246-259

22. White granite, yielding good clear water, which dissolved scale in boilers, and contained

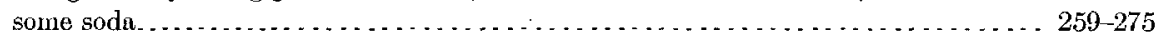

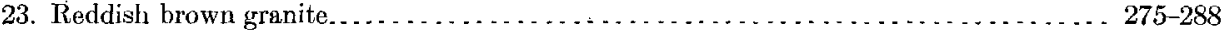

24. Black granite, more water. . . . . . . . . . . . . . . . . . . . . . . . . . . . . . . . 28 28 296

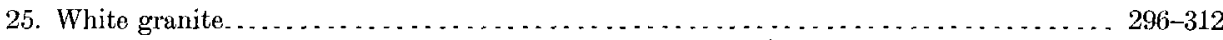

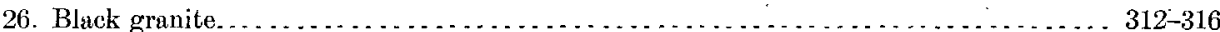

Mr. Wankel adds: "All the water above the rock is of very poor quality. The water from the rock rosc to within 50 feet of the surface. A supply of 60 gallons was obtained at, 275 feet and 80 gallons at 296 . This well was put down in the granite building which inclosed the large pumping engine."

The civil engineer at the navy-yard reports in a letter transmitted by the Secretary of the Navy: "At a point marked 2 on the map a well was driven to a depth now unknown, but from such sources as are now available, this depth is supposed to have been about 120 feet. The water was found to be brackish and the well was abandoned." From the location given, this is clearly the well described by Mr. Wankel.

52. Mr. Wankel says: "This well furnished about 60 gallous per minute of very good clear water from the rock at about 190 feet. It dissolved scale in boilers, and contained some soda and carbonic-acid gas."

Record of well at Brooklyn Navy-Yard, Brooklyn.

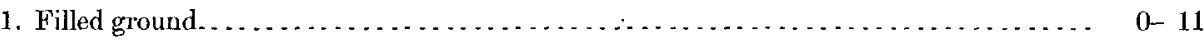

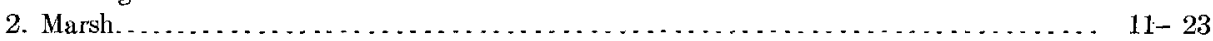

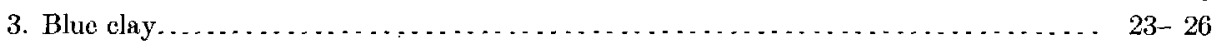

4. Fine white sand............................................... 26-29

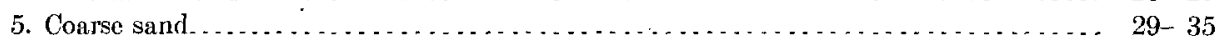

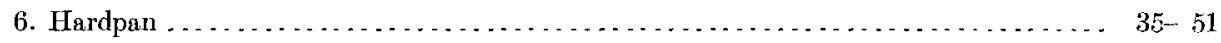

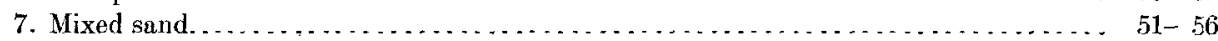

8. Yellow water sand............................................. $56-62$

9. Brown water sand............................................. $62-74$

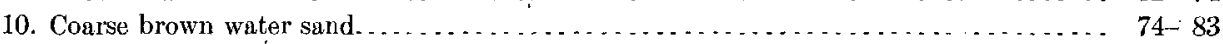

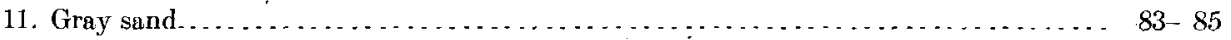

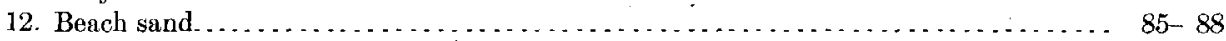

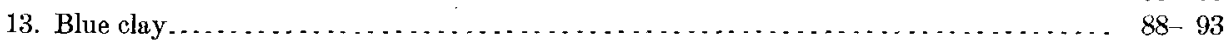

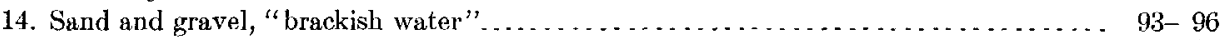

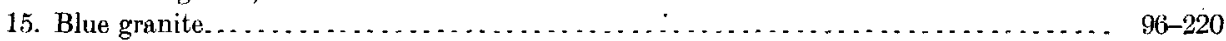


The civil engineer of the navy-yard reports: "Only one well was a success. It still exists and it is stated that the depth is about 216 feet, and that rock was struck at a depth of 96 feet. From measurements made recently the depth of this well is found to be 205.6 feet from the top of the casing."

53.

Record of well at Brooklyn Navy-Yard, Brooklyn.

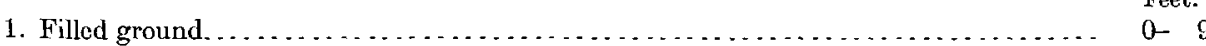

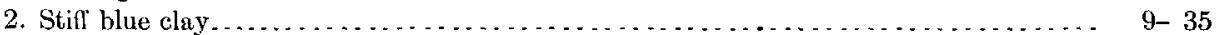

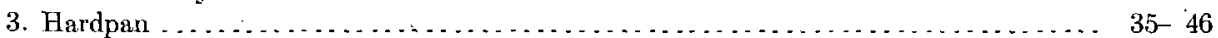

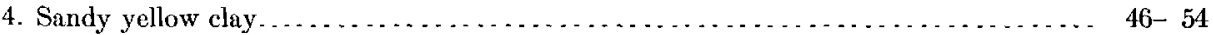

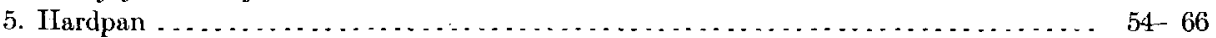

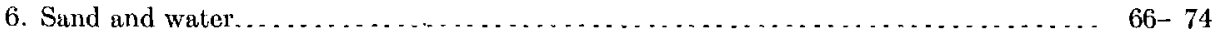

7. Hardpan ..................................................... $74-80$

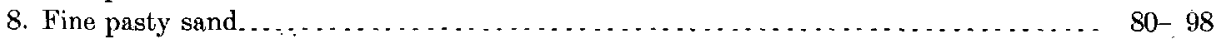

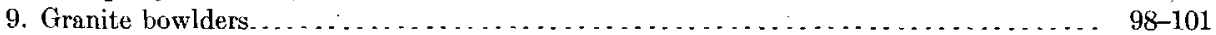

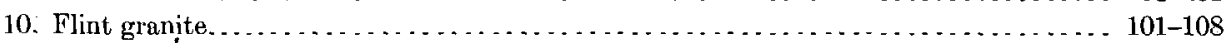

54. The various depths given in the following record are referred to the top of the coping of dry docks 2 and 3 :

Record of well at Brooklyn Navy-Yard, Brooklyn.

Feet

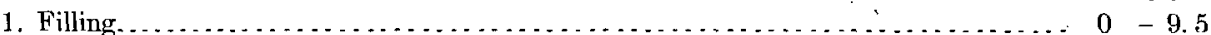

2. Bluish clay-like materials mixed with shells ....................... 9.5 5

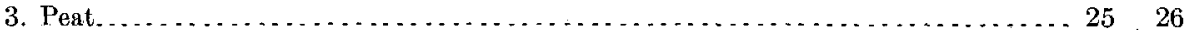

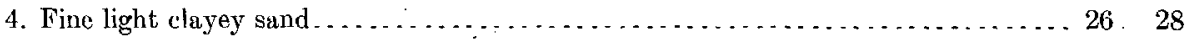

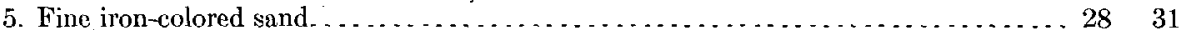

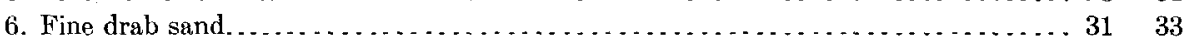

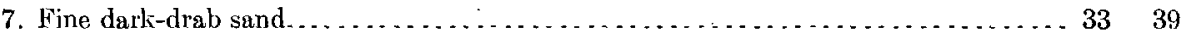

55. The original record of this well, published by E. Lewis, jr., in the Popular Science Monthly; volume 10,1877 , page 443 , is as follows:

Record of well at 556 Kent avenue, Brooklyn:

1. Surface gravel

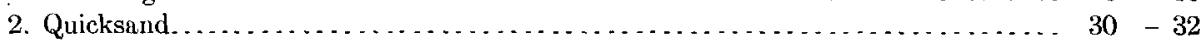

3. Bowlder drift. . . . . . . . . . . . . . . . . . . . . . . . . . . . . . . . . . 32 .

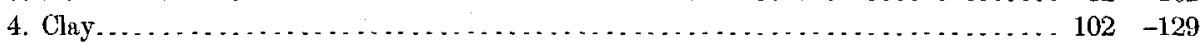

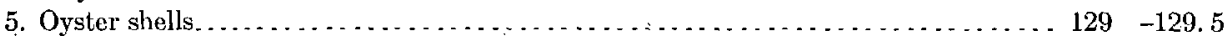

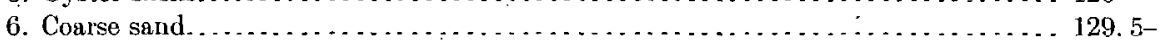

Samples preserved in the museum of the Long Island Historical Society show the following section:

$$
\text { Record of well at } 556 \text { Kent avenue, Brooklyn. }
$$

1. Bowlder clay - - -

2. Water-worn fragments of snells apparently Recent from a layer at a depth of . . . . . . 129.5

3. Medium white sand, not clearly glacial. Depth not given, marked "water-bearing stratum."

An error has apparently been made in transcribing the record, which is published by Merrill (Annals N. Y. Acad. Sci., vol. 3, p. 346) and reprinted by Darton (Bull. U. S. Geol. Survey Fio. 138, 1896, p. 34).

Mr. Fred S. Benson, chief engineer of the eastern division of the Brooklyn Union Gas Company, reports inder date of November 30, 1903: "The well you refer to as being 129 feet 6 inches deep was put down by the Nassau Guslight Company in 1873 or 1874 . The well was located at Kent avenue and Cross street, Brooklyn. Its yield when first tested was 500 gallons per minute. We have since put down two other pipes in the same excavation, but the quantity of water has diminished yearly. I might add that the pipes have been drawn up to a depth of 85 feet from the ground level, that being the depth from which the maximum quantity was obtained in 1902." 


\section{UNDERGROUND WATER RESOURCES OF LONG ISLAND, NEW YORK.}

60. Mr. C. D. Corwin reports the following section:

Record of well at Bushwick and Meserole avenues, Brooklyn.

1. Yellow clay and stones . . . . . . . . . . . . . . . . . . . . . . . . . . . . . . . . . . 049

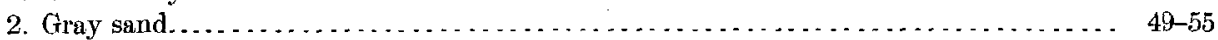

3. Fine sand and mica .............................................. $55-63$

4. Yellow clay; with quartzite, slate, conglomerate, and feldspar pebbles . . . . . . . . . 63-101

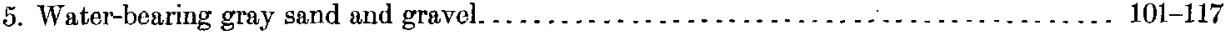

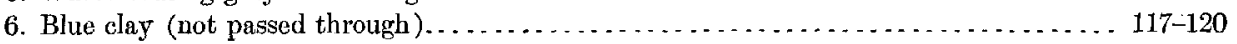

62. Phillips and Worthington report the following section:

Record of well on Ten Eyck street, between Bushwick avenue and Florence street, Brooklyn.

Wisconsin: $\quad$ Fect.

1. Interlying strata of clay, sand, and gravel (very heavily bedded with bowlders) .. $\quad 0-52$ Tisbury?:

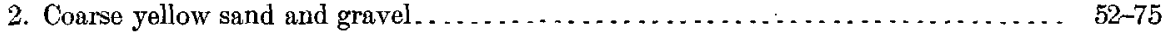

Sankaty?:

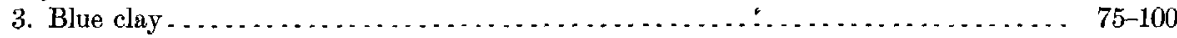

Jameco? and Cretaceous:

4. Beach sand..

This well was abandoned and a new one sunk, which obtained its supply from the water-bearing strata between 52 and 75 feet.

Mr. I. H. Ford gives the following section:

Record of well on Ten Eyck street, between Bushwick avenue and Florence street, Brooklyn:

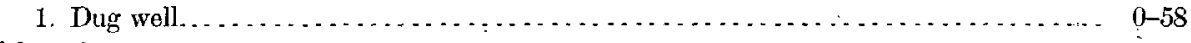

Tisbury?:

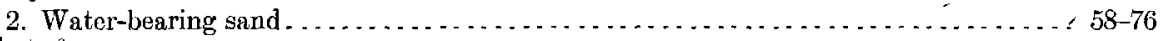

Sankaty?:

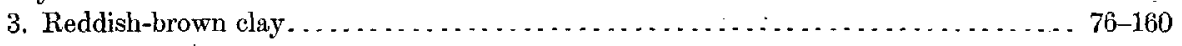

64. These wells are entirely in sand; there is plenty of water, but if too much is drawn, salt water from Newtown Creek comes in. Analysis of the water shows 1,000 parts of chlorine per million.

65. A test.well at this point gave the following section:

Wisconsin:

Record of well at Porter and Maspeth avenues, Brooklyn.

1. Stones and rough material; no sand ............................. ${ }_{0-12}$

Wisconsin and Tisbury?:

2. Loam, sand, etc............................................ $12-48$

Sankaty?:

3. Clay having a blue color . . . . . . . . . . . . . . . . . . . . . . . . . . . . . . . . $48-190$ Jameco?:

4. Water-bearing gravel. . . . . . . . . . . . . . . . . . . . . . . . . . . . . 190

A good supply of water is reported from layer 4, but the wells at this point are completed in layer 2 .

66. Mr. C. Harty, foremian for I. H. Ford, has kindly furnished the following data of the deep test wells at this point: Diameter, 10 inches, $0-137$ feet; 8 inches, $137-200 ; 6$ inches, 200-225.

$$
\text { Record of well at Meeker and Kingsland avenues, Brooklyn. }
$$

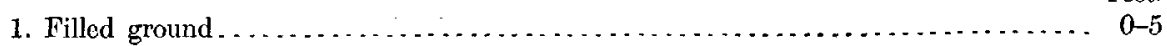

Wisconsin:

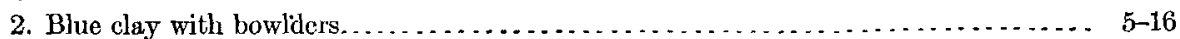

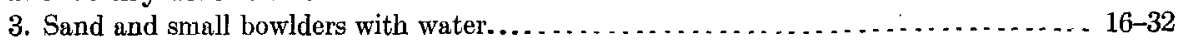


Cretaceous ?:

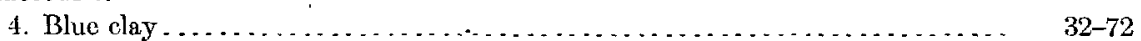

5. Light-gray clay ....................................... $72-180$

Cretaceous:

6. Sand--not water bearing................................... 180-180.5

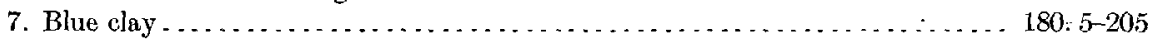

8. Light-greenish clay, passing into dark-greenish clay containing small concretionary masses.......................................... 205-215

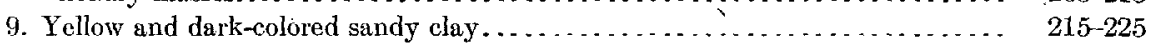

Pre-Cretaceous:

10. Rock, mica-schist . . . . . . . . . . . . . . . . . . . . . . . . . 225

This well was abandoned and a shallow well sunk near it, which obtained water from the glacial gravels between 28 and 32 feet. The section of this shallower well is:

Wisconsin:

Record of well at Meeker and Kingsland avenues, Brooklyn.

1. Blue clay; no stone. . . . . . . . . . . . . . . . . . . . . . . . . . . . . . . . 0 .28

2. Reddish-brown glacial sand and gravel. . . . . . . . . . . . . . . . . . . . . . . . . . 28 28

Cretaceous?:

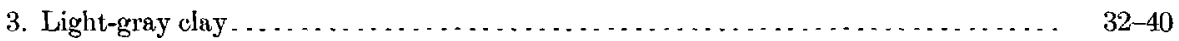

67. Record of well on Meeker avenue, between North Moore and Monitor streets, Brooklyn.

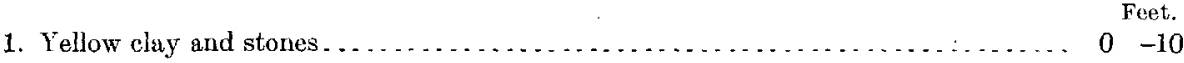

2. Gray sand and stones. . . . . . . . . . . . . . . . . . . . . . . . . . . . . 10.18

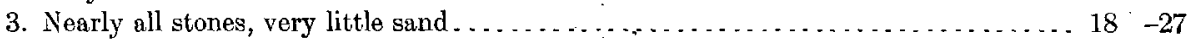

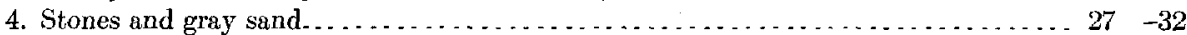

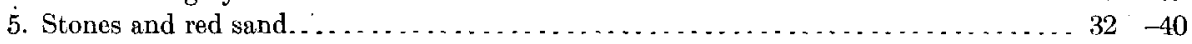

6. Fine gray sand. . . . . . . . . . . . . . . . . . . . . . . . . . . . . . . . . . . 40.43

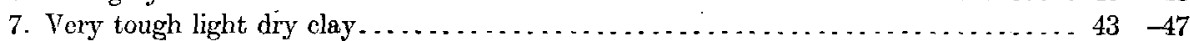

8. Fine sand with conglomerate, quartz, feldspar, and jasper pebbles. . . . . . . . . . $47 \quad-54$

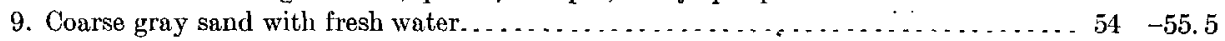

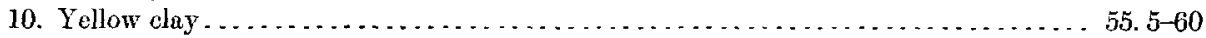

11. Blue clay . . . . . . . . . . . . . . . . . . . . . . . . . . . . . . . . . . . . . . . . . 60.63

12. Gray sand and gravel; good water-bearing stratum. $\ldots \ldots \ldots \ldots \ldots \ldots \ldots \ldots \ldots \ldots \ldots$

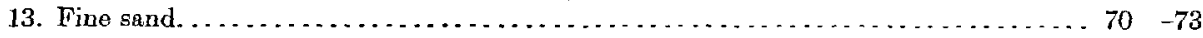

71. Mr.F.P. Rust, manager of the Rust Well Machinery Company, gives the following record of this well:

Record of well at 99 to 117 North Eleventh street, Brooklyn.

Wisconsin and Tisbury:

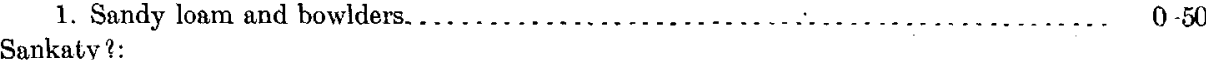

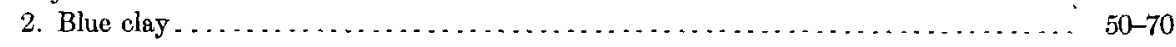

Jameco?:

3. Gravel and bowlders............................................ $70-100$

Cretaceous?:

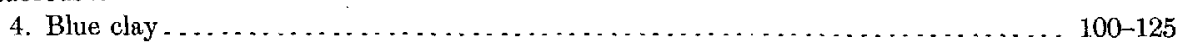

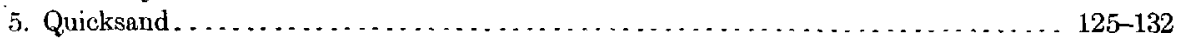

Pre-Cretaceous:

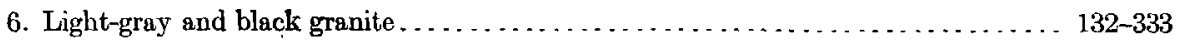

The New York Quinine Chemical Company report a yield of 7,500 gallons per hour. The water level lowers 5 feet on pumping eight hours; temperature of water $65^{\circ} \mathrm{F}$; it contains much lime and magnesia and is not used for drinking. 


\section{UNDERGROUND WATER RESOURCES OF LONG ISLAND, NEW YORK.}

72. Mr. H. L. Pratt of the Standard Oil Company gives the following information: "About twenty-five years ago an attempt was made to drive a well at this point, but after going to the depth of 200 feet without getting any water the woll was abandoned on account of a ledge of rock."

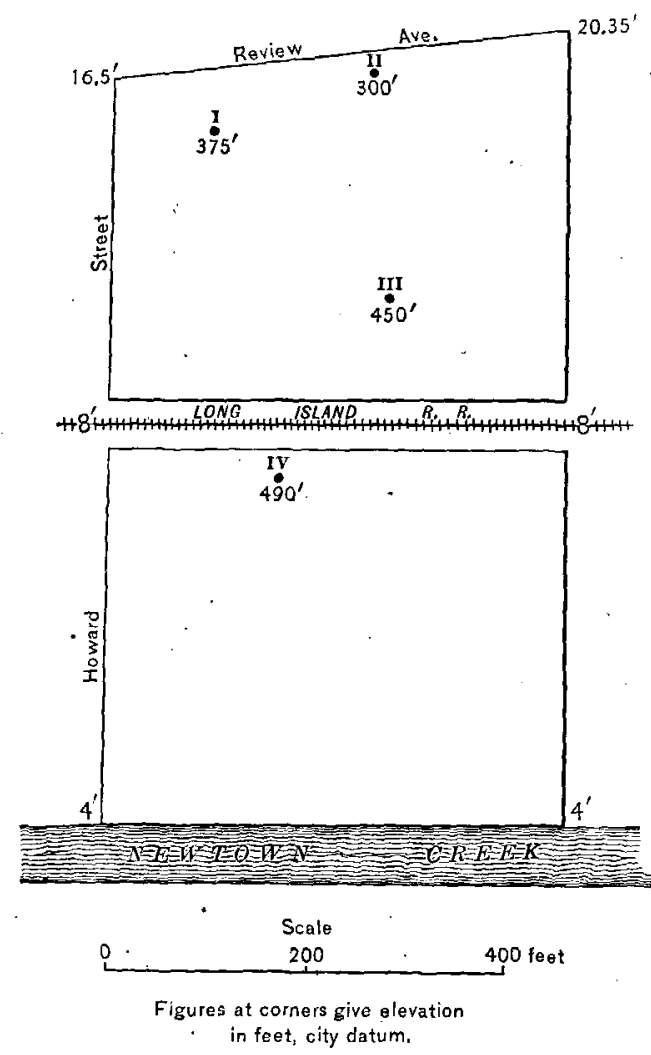

FIG. 61.-Sketch map showing location of deep wells of the Fleischmann Manufacturing Company at Long Island city.
75. The following data have been furnished by Mr. Jacob Blumer:

"We drove at least 25 shallow wells to a depth of from 60 to 70 feet. In the depth mentioned, we went through sand and gravel only and never struck rock. All the wells fumished water, but of late years the levels became lower. Twenty yours ago the levels stood 15 to 16 feet from the surface, and in the last wells, made in 1897, it was as low as 38 to 40 feet. As for the amount of water furnished, I can only give you figures for the three wells which were made in 1897. These were made by sinking a pit 7 by 9 feet to a depth of 36 or 40 feet, and then we drove 20 to 25 feet of 6-inch pipe with a perforated strainer at the end through sand and gravel. The pump was at the bottom of the pit and cach well was good for 200 gallons per minute. The water in these wells was clear and cold, but exposed to the air became yellowish. Hydroxide of iron presipitated in the water; this was quite troublesome in our pipe lines. After about three years a 3 -inch pipe would be nearly filled up solid with the precipitate.

"We also made four deep wells, the locations of which are shown on the accompanying diagram [fig. 61]. In these we went through 70 to 80 feet of sand and gravel, and in all of them struck rock at 124 feet; the rock was porous but hard. In all wells we found plenty of petrified wood; some black, some yellowish white, like hard maple, and in some of the shallower wells we found petrified fish roe.

"No. 1 deep well was originally 275 feet deep, and yielded 80 to 100 gallons per minute. After No. 3 well was made and operated, the yield of No. 1 diminished; then we drilled to 375 feet, but did not get the water back, and in a few months it had gone out entirely and the well was abandoned.

"No. 2 deep well was 300 feet deep, and yielded 120 to 125 gallons per minute, and kept it up until we closed the works. Level of water in the well when not in operation was 220 feet from the surface.

"No. 3 deep well was 450 feet deep; it yielded 125 gallons as long as we were running the place; level was 250 feet from surface.

"No. 4 deep well was 490 fect deep; it yielded about 10 gallons per minute and was abandoned. The water from all the deep wells was a little brackish-the amount of chloride increasing continuously. I . can give you the amount of chlorine for No. 1 well for a period of over three years." 
Chlorine in water of Fleischmann deep well No. 1 at Blissville, Long Island City.

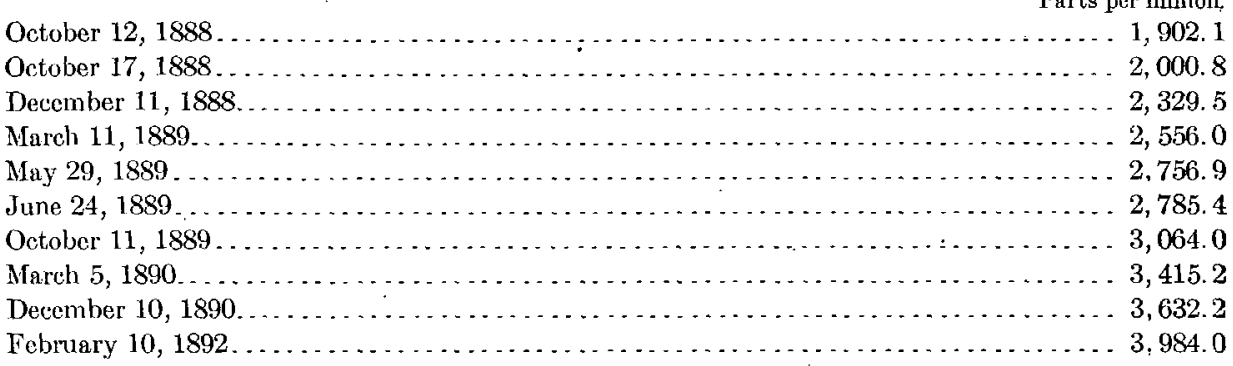

76. A large well at this point, 25 feet in diameter and 50 feet deep, has a number of 6 -inch points driven in the bottom of it to a depth of 60 to 70 feet; the water level was originally 5 or 6 feet from the surface of the ground, but is now 60 feet, and the large well, or pit, has been deepened from time to time as the waterlevel lowered. In the spring of 1903 the large plants across Newtown Creek which have wells in the same stratum closed down, and the water is rapidly rising in the Standard Oil Company well, and threatens to drown out the pumps which are placed in the bottom of the large well. The water is "everything that is bad;" it is used for condensing purposes only.

79. The original record by Lewis is as follows:

Record of uell at New Calvary Cemetery, Long Island City.

Pleistocene: Feet.

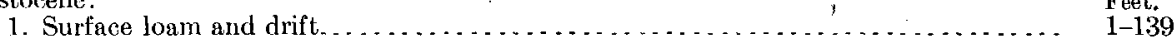

Raritun:

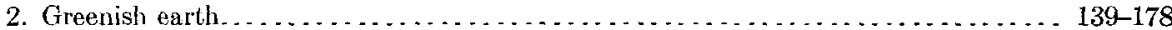

3. White clay with red streaks .........

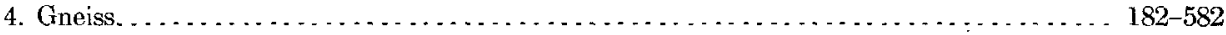

Darton reports the water soft, with only a little lime, magnesia, and chlorine. In the museum of the Long Island Historical Society, the following samples are preserved: (1) Green sandy clay, marked: " 39 feet thick at a depih of 139 feet:" (2) mottled red and white clay, "200-204." Of the green sandy clay Merrill says: "The greenish earth was found to be ferruginous, and on treatment with hydrochloric acid left a residue which, under the microscope, was seen to consist of fragments of kaolinized feldspar, with occasional grains of coarse sand."

80.

Record of commission's test well, Long Island City.

Wisconsin:

Feet.

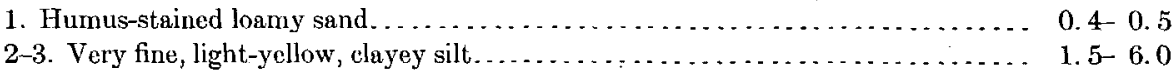

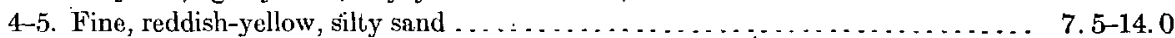

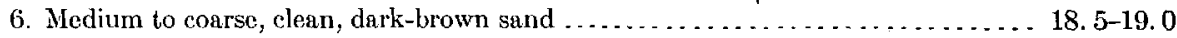

7. Fine, reddish-yellow, silty sand ............................... 20.5-21.5

8-10. Dark, grayish-brown sand to small gravel .................... 27.5-38

All samples have at decidedly glacial appearance.

Record of Flower estate well, Long Island City.

Wisconsin :

Feet.

$0-90$

1. Sandy clay

Tisbury? :

2. Coarse sand, full of water

$90-100$

Cretaceous?

3. Clay.

$100-112$

4. Rock 112-145 
83.

Record of commission's test well., Long Island City.

1. Humus-stained sandy loam .................................

2. Yellow sandy loam . . . . . . . . . . . . . . . . . . .

3-10. Fine to medium, reddish-yellow, glacial sand with a little silt. $\ldots \ldots \ldots \ldots \ldots \ldots 8-40.0$

85. See fig. 62 and Pls. XXVIII-XXXIII.

86. A number of test borings were put down at this point for foundations for an electrical plant. In one well marine shells were found at a depth of about $60 \mathrm{feet}$; in another water was encountered which flowed 1 foot above the surface; bẹd rock was reached at different depths, the greatest being 69 feet.

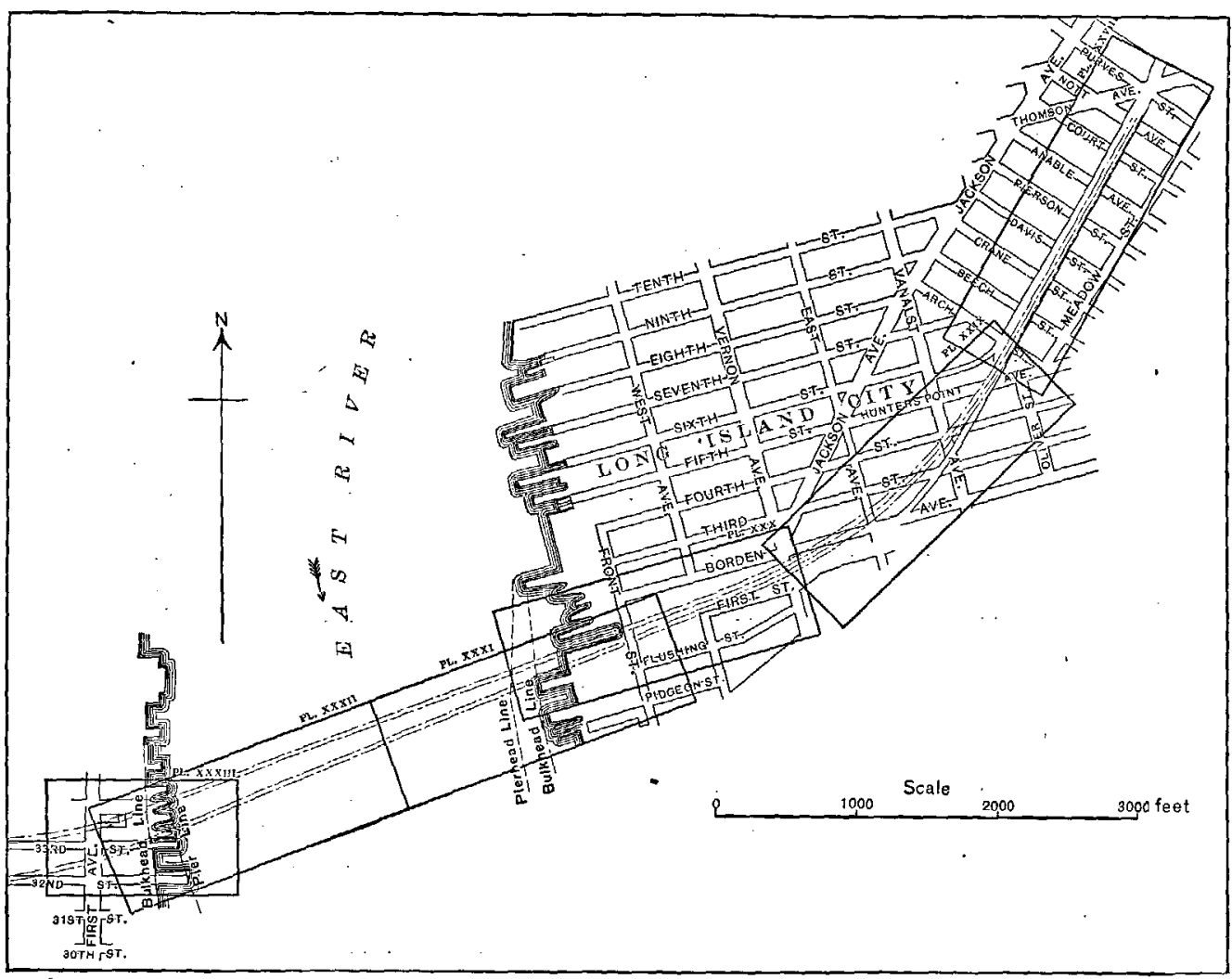

FIG. 62.-Index map showing location of borings represented on Pls. XXVIII-XXXIII.

87. Record of well at Sixth street and West avenue, Long Island City.

1 Ash and cinder filling-.... Feet.

. . . . . . . .

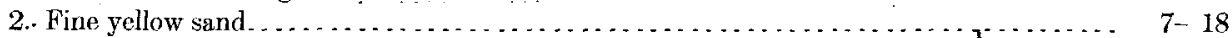

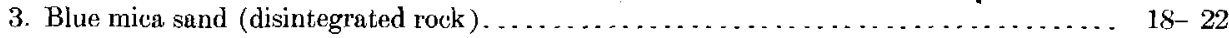

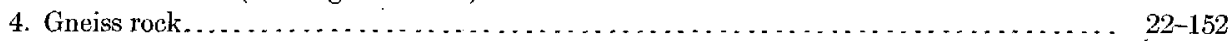

Mr. Sweeney reports that the rock in this well dipped at rather a high angle, and that it was very difficult to enter the pipe in the rock. At the last depth given a supply of brackish water was found in a crevice. The granite was of unequal hardness.

88. Water is only slightly brackish, is excellent for boiler use and gang purposes, and contains lime, magnesia, and salt. 

89. The elevation of this well is about 8 feet above mean tide.

$$
\text { Record of well at Vernon and Noit avenues, Long Island City. }
$$

1. Ash and sand filling. ... . . . . . . . . . . . . . . . . . . . . . . . . . . . . . . . .

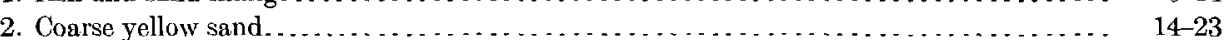

3. Blue sand (disintegrated rock) . . . . . . . . . . . . . . . . . . . . . . . . . $23-25$

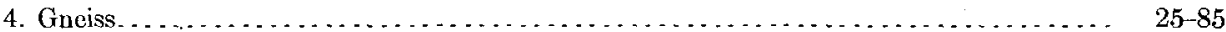

91. This well, which is about 5 feet above the adjacent sea marsh, is reported to have passed through nothing but gravel, but a near-by well struck beds of blue clay with but 1 or 2 feet of gravel. Both were test wells put down by the water department of Long Island City.

92.

Record of well at 596 Jackson avenue, Long Island City.

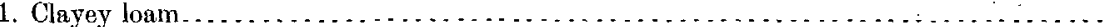

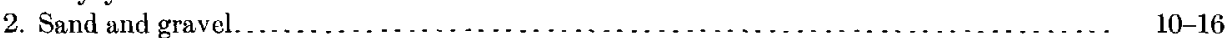

3. Layer of stones averaging about half the size of paving stones. . . . . . . . . . . . $16-19$

4. White clay . . . . . . . . . . . . . . . . . . . . . . . . . . . . . . . . . . . . . . . . 19

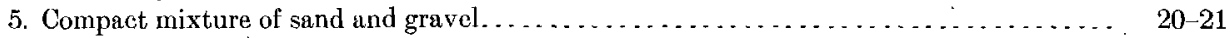

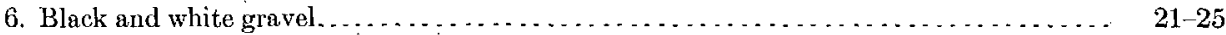

93. A group of 20 or 30 wells, of which several are flowing; they are so connected that it is impossible to tell which is and which is not flowing. The one nearest the branch is reported to have originally flowed 18 gallons per minute; it flowed July 19, 1903, about one-half gallon per minute from the pipe and 1 or 2 gallons on the outside of the pipe. The Long Island Railroad Company reports one well flowing slightly, but readily pumped down. The water is excellent, but the supply not great.

Analysis of water from well at Jackson avenue and Fill street, Long Island City.

Parts

per' million.

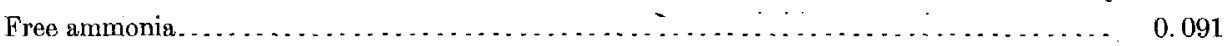

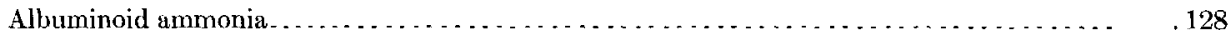

Oxygen consumed. . . . . . . . . . . . . . . . . . . . . . . . . . . . . . . . . . . . 842

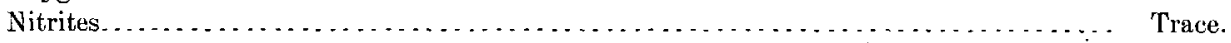

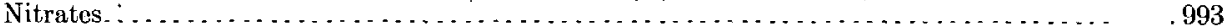

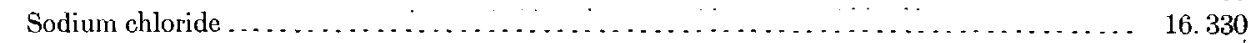

Hardness. . . . . . . . . . . . . . . . . . . . . . . . . . . . . . . . . . . . 120

Permanent hardness. . . . . . . . . . . . . . . . . . . . . . . . . . . . . . . . 50

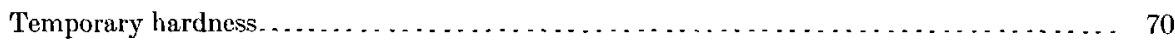

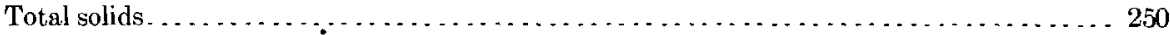

In the near-by well the following section was encountered by Mr. S. H. Allen:

Record of well at Jackson avenue and Hill street, Long Island City.

1. Blue clay...

F. Feet.

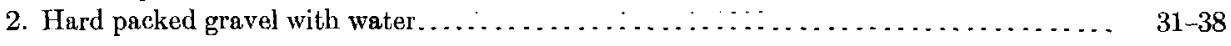

94. Record of well at Long Island Railroad and Remsen street, Long Island City.

1. Bowlders and loam.

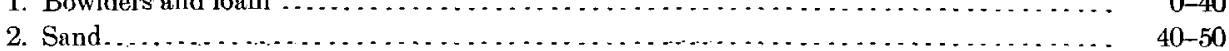

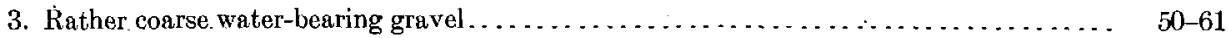

95. Mr. Allen states that the well at this point will flow when the near-by waterworks station is not pumping; when it is pumping its maximum capacity the well will lower about 15 feet; it is also affected by the pumping at the ice factory near Jackson and Steinway avenues (No. 98). 


\section{UNDERGROUND WATER RESOURCES OF LONG ISLAND, NEW YORK.}

96.

Record of well at Buckley street and Middleburg avenue, Long'Island City.

1. Sand.

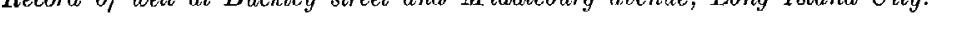

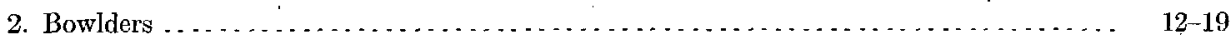

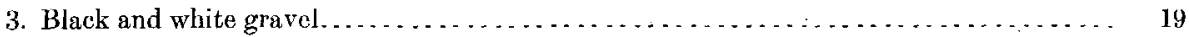

The bowlders in stratum 2 were so large and numerous at 12 feet that a hole 10 feet square was dug and the bowlders removed; the bowlders varied from 8 to 10 feet in diameter.

98. Mr. S. H. Allen furnishes the following data regarding the six wells which he completed at this point:

Well No. 1: Depth, 66 feet; diameter, 4 inches; depth to water, 3 feet; tested, 48 gallons per minute, full capacity not reached.

Record of well No. 1 at Long Island Railroad and Lowery street, Long Island City.

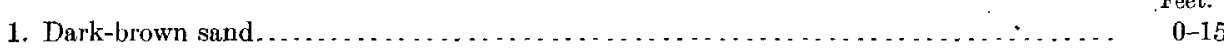

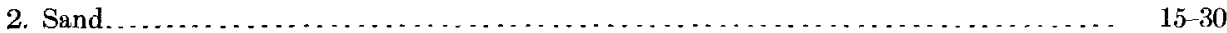

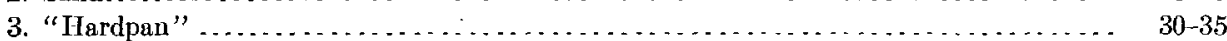

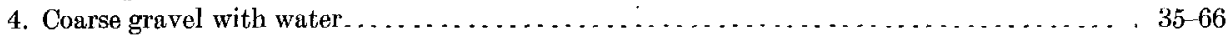

Well No. 2: Depth, 97 feet; diameter, 2 inches; depth to water, 5 feet; capacity, 160 gallons per minute. With direct suction the water lowers to 17.9 feet, but will not lower farther.

Well No. 3: Depth, 51 feet; diameter, 4 inches; capacity; 60 gallons per minute.

Well No. 4: Depth, 54 feet; diameter, 2 inches; capacity, 60 gallons per minute.

Well No. 5: Depth, 55 fect; diameter, 3 inches; capacity, unsatisfactory.

Well No. 6: Depth, 66 feet; diameter, 3 inches; capacity, 60 gallons.

Sweeney \& Gray completed threc wells at this place and report the following typical section:

\section{Record of well at Long Island Railroad and Lowery street, Long Island City.}

1. Medium red sand

Mixture of red, blue, and white clays $\ldots . . . . . .$.

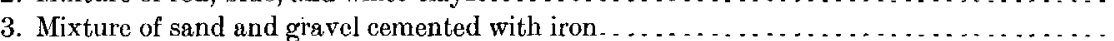

4. Fine bluc, water-bearing sand. . . . . . . . . . . . . . . . . . . . . . . . . . . .

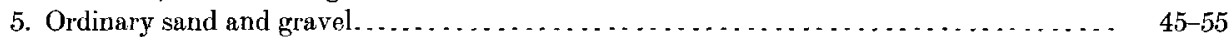

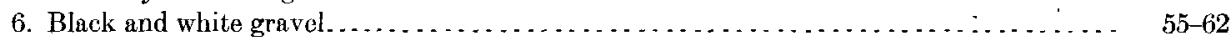

99. For records of original wells put down at this station see Pl. XXXIV, which was prepared by Mr. A.S. Farmer from samples. Mr. Farmer has also furnished the following record and samples of the test boring made in connection with a new series of wells which was completed at this point in the spring of 1904:

Record of wells at Long Island Railroad and Grove street, Loing Island City.

Recent:

1. Marsh deposits

Wisconsin:

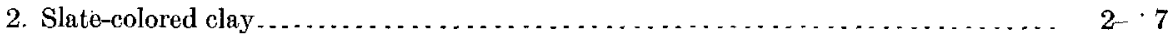

3. Sand and clay cemented into hardpan ........................... $7-32$

4. Water-bearing sand; not a very good water stratum.................. $32-33$

Wisconsin or Tisbury:

5. Sand and gravel up to one-half inch in diameter cemented into hardpan; very hard to drill. .......................................... $33-55$

6. Reddish brown sand mixed with small gravel ..................... $55-58$ Cretaceous (Raritan):

7. Sand and clay of greenish color; easy to drill; color changing to gray at $85 \ldots \ldots \ldots$. $55-85$

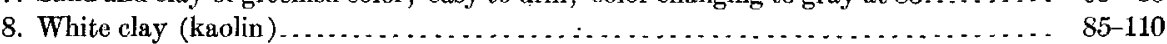

9. White and greonish clay (not greensand) evidently a rock-weathering product... . . 110-118 Fordham?

10. Gneiss 


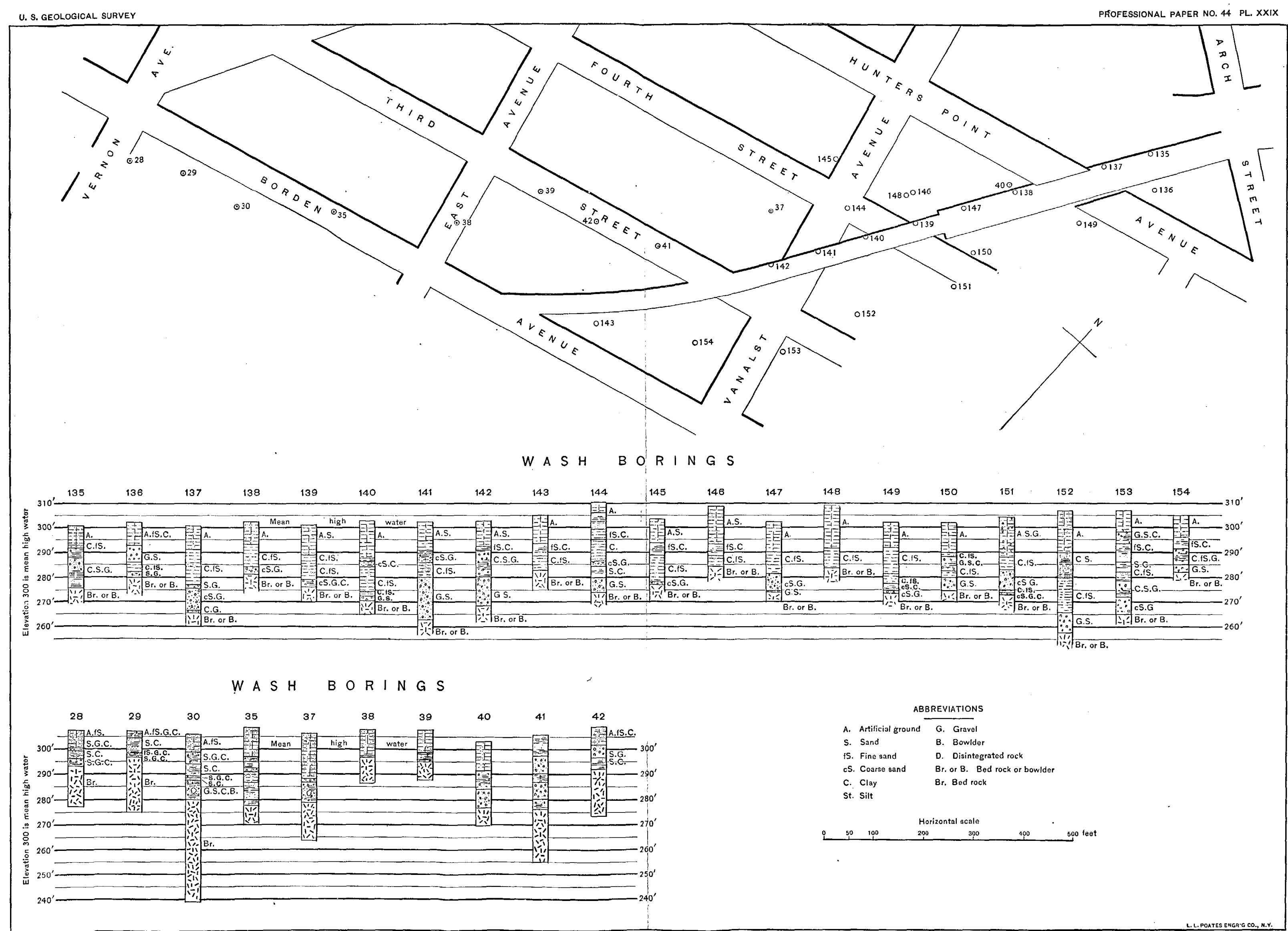

MAP AND DIAGRAM OF BORINGS FOR PENNSYLVANIA, NEW YORK AND LONG ISLAND RAILROAD TUNNEL; ARCH STREET TO VERNON AVENUE, LONG ISLAND CITY. 



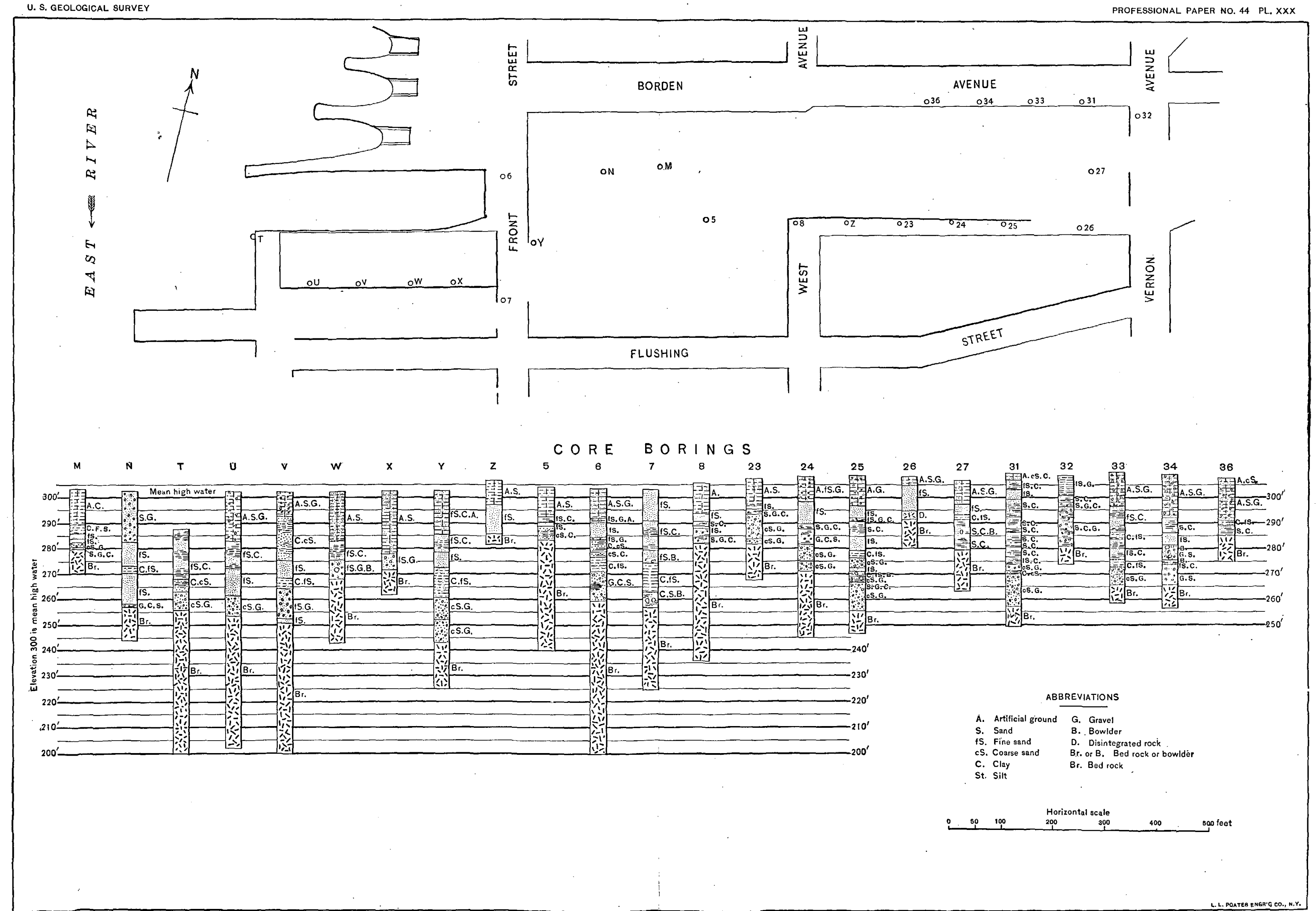

MAP AND DIAGRAM OF BORINGS FOR PENNSYLVANIA, NEW YORK AND LONG ISLAND RAILROAD TUNNEL; VERNON AVENUE TO EAST RIVER, LONG ISLAND CITY. 



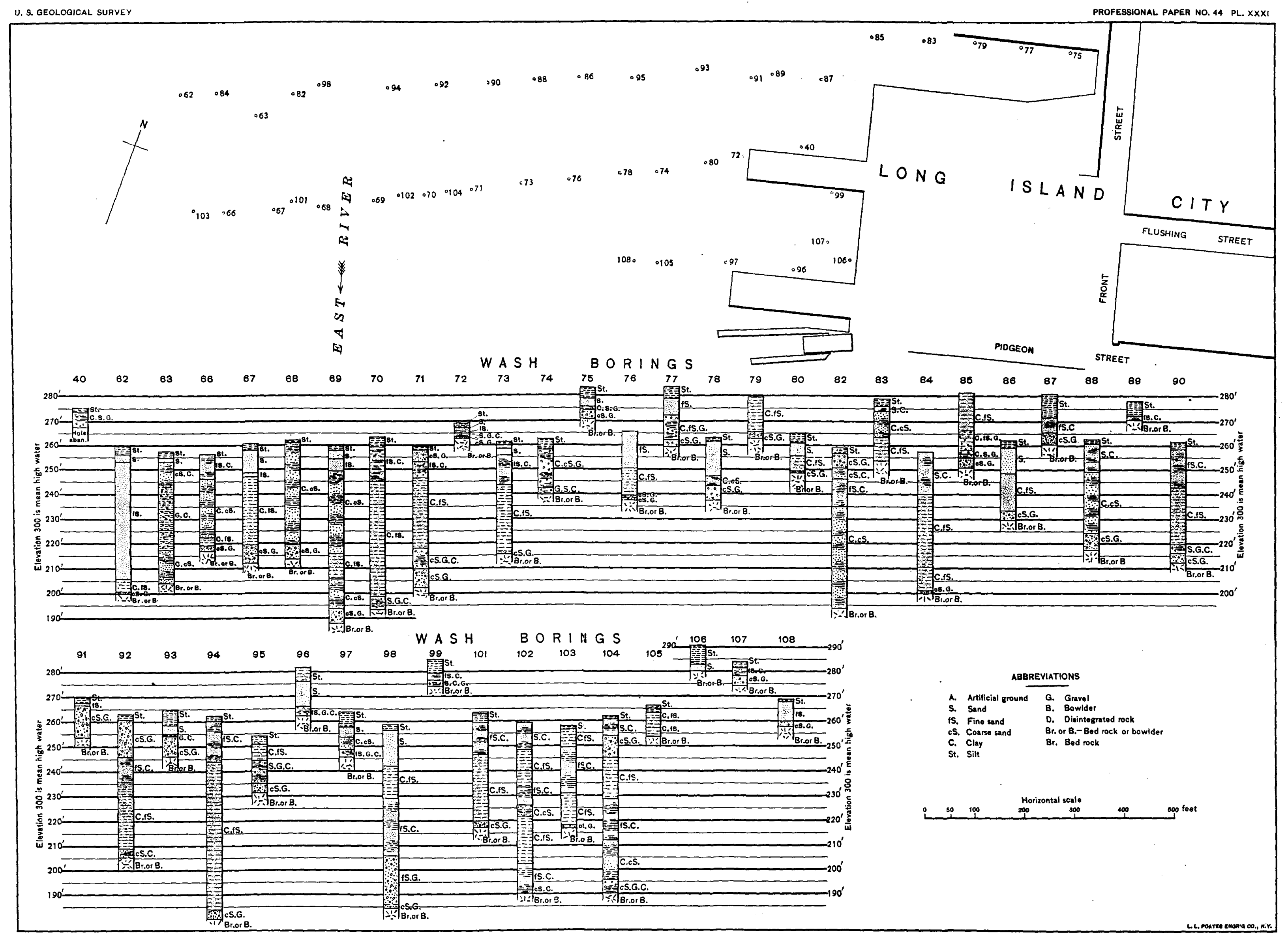

MAP AND DIAGRAM OF BORINGS FOR PENNSYLVANIA, NEW YORK AND LONG ISLAND RAILROAD TUNNEL; EASTERN HALF OF EAST RIVER. 

A well put down by the commission on additional water supply about 60 feet west of the pumping station showed the following section:

Record of commission's test well at Long Island Railroad and Grove street, Long Island City.

Wisconsin:

3. Black bumus-stained clay -

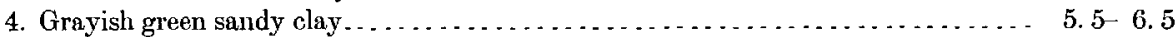

5. Multicolored fine silt to medium sand. ......................... 9. 5-10.5

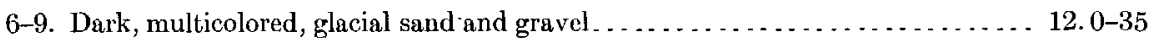

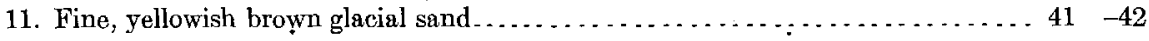

100. Record of well on Steinway avenue, between Pierce and Graham avenues, Brooklyn.

2 Blue clay; no bowlders $2 . . .28$

3. Quicksand with black water.......................................... $30-38$

4. Hardpan ... . . . . . . . . . . . . . . . . . . . . . . . .

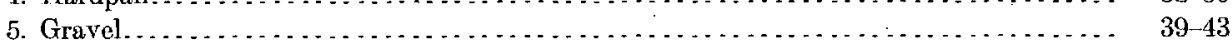

101. Mr. Allen sunk five wells on the east side of Fifth avenue between Pierce and Graham streets to an average depth of 32 feet; one he sunk to a depth of 60 feet and reached rock without getting a second water-bearing stratum. Water in these wells has been lowered from 18 feet below the surface to 30 feet below the surface by the pumping of the ice-factory well; and the wells have been driven 5 feet deeper, or to 37 feet. A well just across the block, on Fourth avenue, belonging to Mr. Vanderhyde, reached rock at 58 feet; water was found on the rock.

102. Record of well at Washington and Fourth avenues, Long Island City.

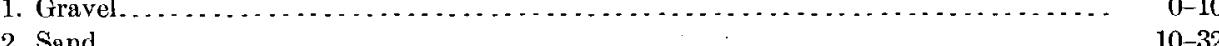

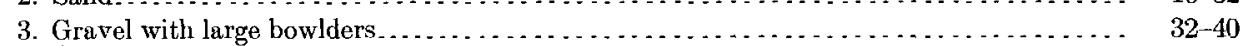

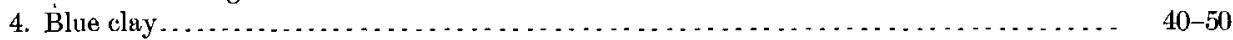

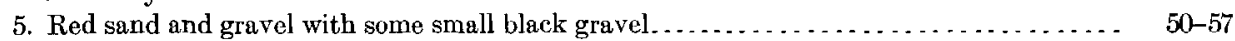

104. Record of well at Pierce avenue and Crescent street, Long Island City.

1. Gravel and bluish rock ... Feet.

2. Blue clay $\ldots$

3. Gravel... . . . . . . . .

105. Well is pumped empty and then allowed to fill; water is used for manufacturing purposes, and not for drinking.

Sanitary analysis of water of well at Williams and Beebe avenues, Long Island City.

[By E. H. Richards.]

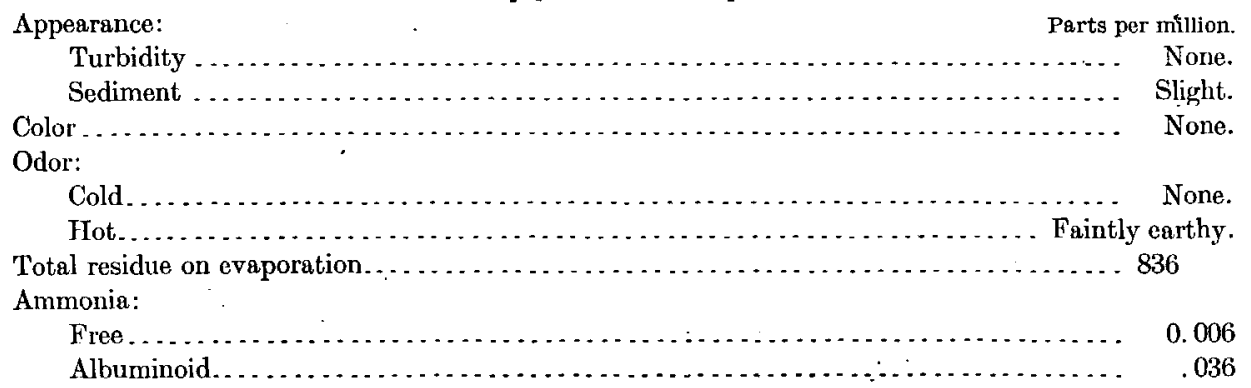

17116-No. 44-06- 13 
Chlorine Parts per million.

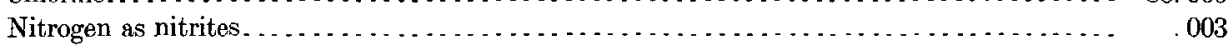

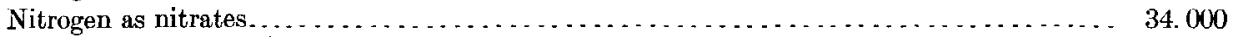

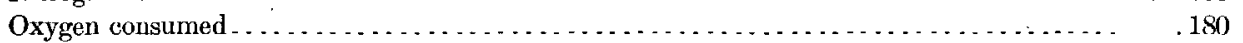

I am afraid it is a case of the border line. I do not know why the nitrates should be so high, unless there is some contamination.-E. H. Richards, May $22,1900$.

106. Ir the spring of $1903 \mathrm{Mr}$. Allen completed three wells in the kitchens of new houses on the west side of Ely street between Paynter and Beebe streets, which afforded the following section:

Records of wells on Ely avenue between Payntar and Beebe avenues, Long Island City.

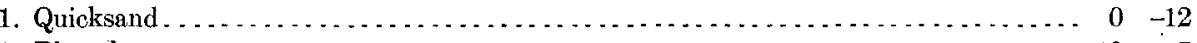

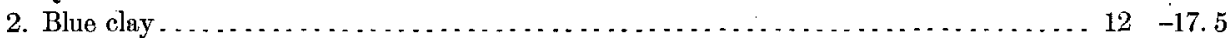

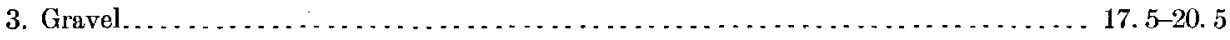

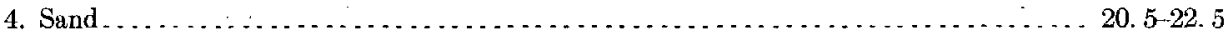

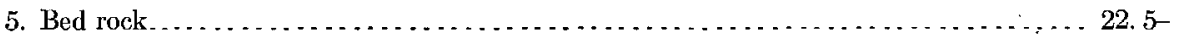

The well in the house nearest Paynter street flows about 5 gallons per minute 2 feet above the ground; the others flow, but a less amount. On the corner of Beebe and Paynter streets, just northwest of the ast of these houses, is an old factory with a well about 20 feet deep which reached bed rock and furnished flowing water. This well has now stopped flowing, because of the construction of the sewer along Beebe street which drains the water from it. The elevation at the corner of Ely and Paynter streets is 9 feet, city daturn.

1 10. The water of this well is extremely brackish and can not be used in boilers; it is used for mixing clays. The elevation of the grade line, corner of Wallach and Vernon avenues, is 14.89 feet, city datum.

Record of well at 401 Vernon avenue, Long Island City.

2. Coarse red sand

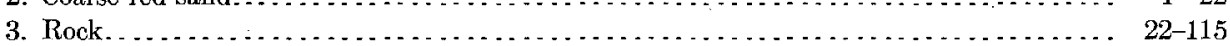

15. These wells are reported as being about one-half mile north of bridge No. 4 (Blackwells Island bridge). There are two 3 -inch wells, and two $4 \frac{1}{2}$-inch wells.

$$
\text { Record of well near Blackwells Island bridge, Long Island City. }
$$

1. Marsh mud.

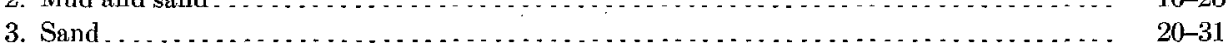

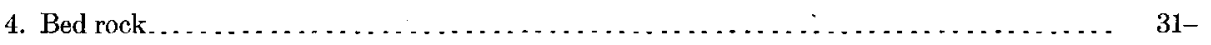

116. Record of well at Broadway and Academy street, Long Island City.

1. Heavy, coarse, building sand, with bowlders of various sizes . . Feet.

2. Very large bowlders packed closely together, many weighing a ton or more $\ldots \ldots \ldots \ldots . \quad 35-40$

3. Coarse gravel containing stones 4 to 6 inches in diameter..................... 40.50

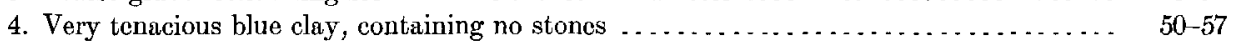

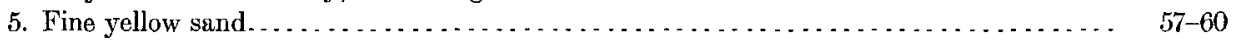

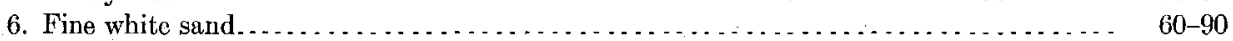

The bowlders in stratum 2 so hindered the sinking of the well that it was necessary to dig a hole 10 feet square and blast them out. At 90 feet a large supply of brackish water was obtained.

$11 \%$

$$
\text { Record of well at Ninth and Jamaica avenues, Long Island City. }
$$

1. Stratified sand and gravel

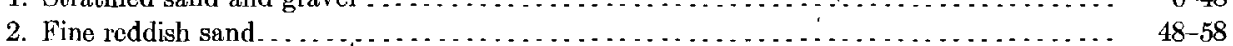

3. Red sand and gravel .... 


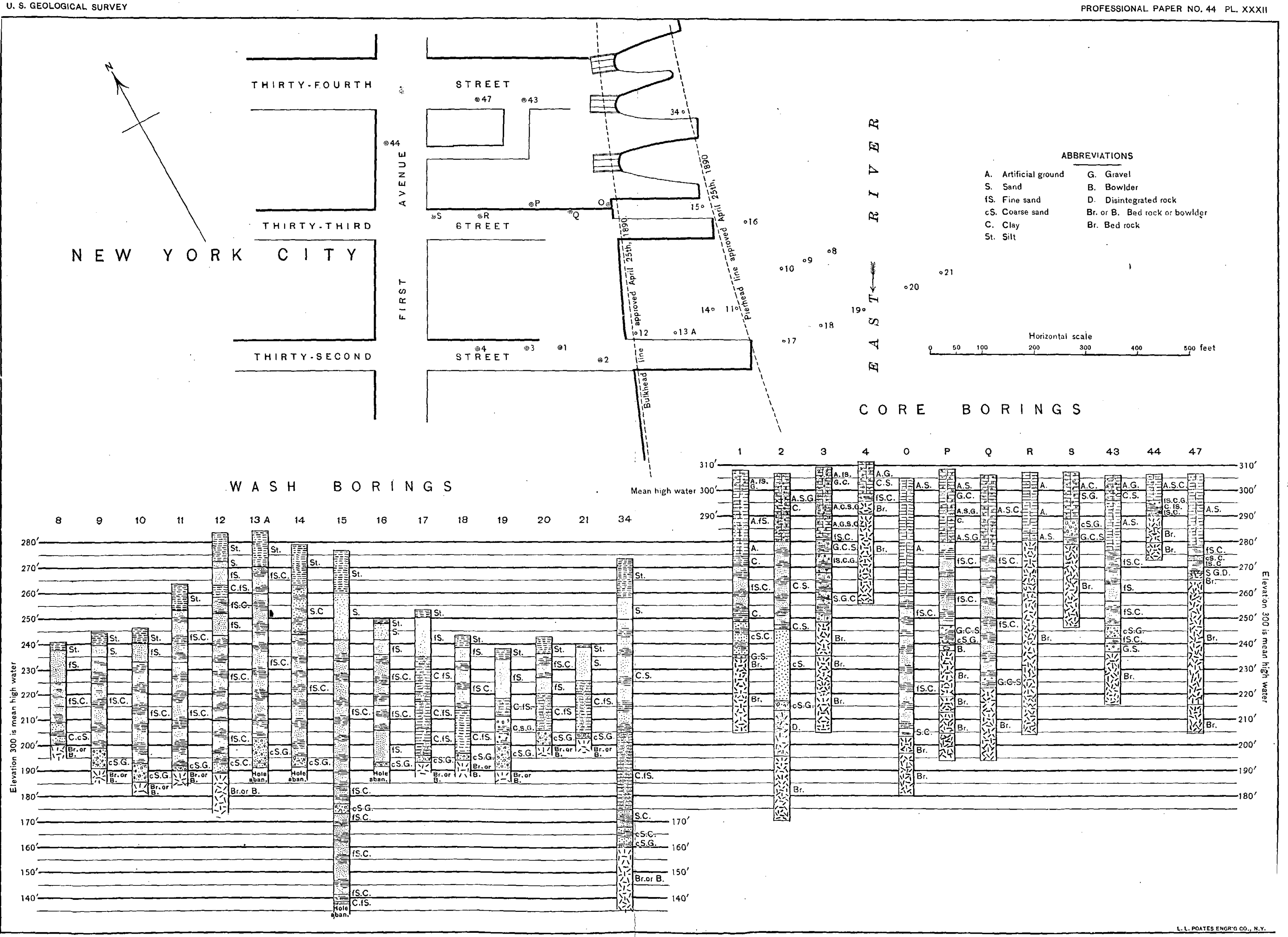

MAP AND DIAGRAM OF BORINGS FOR PENNSYLVANIA, NEW YORK AND LONG ISLAND RAILROAD TUNNEL; WESTERN HALF OF EAST RIVER. 



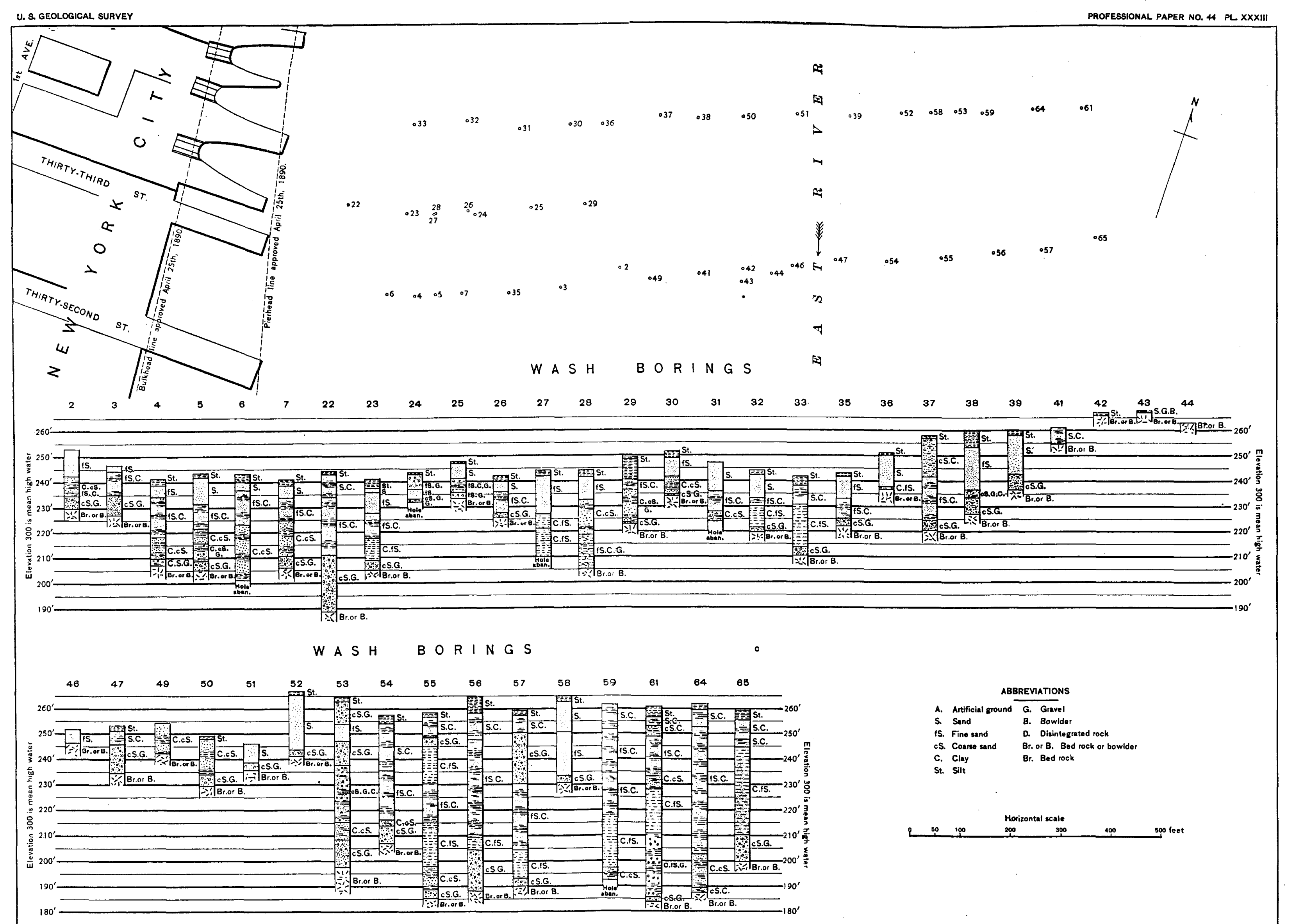



In this well the first water was encountered at 32 feet; below this was 7 feet of clayey sand and a second layer of clay and gravel.

118. Record of well at 408 Ninth avenue, Long Island City.

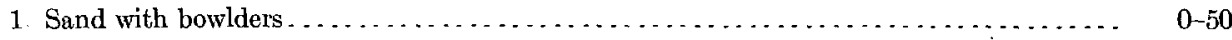

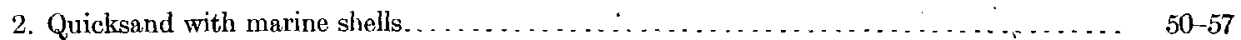

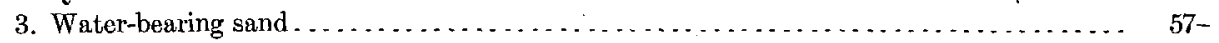

4. Very hard layer of red sand and gravel.

19. Record of well at Steinway and Jamaica avenues, Long Island City.

1. Glacial gravel with bowlders

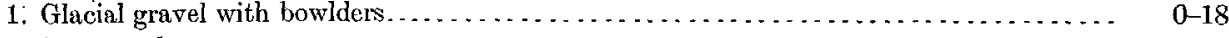

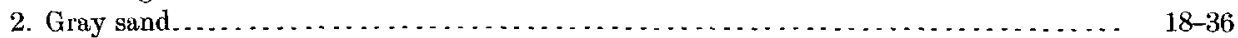

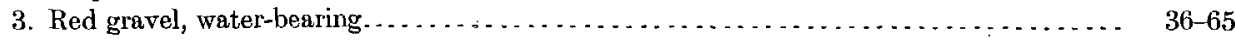

120. Record of well at Albert street and Jamaica avenue, Long Island City.

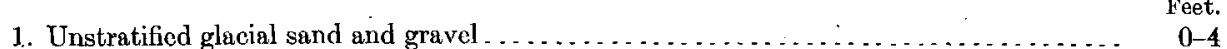

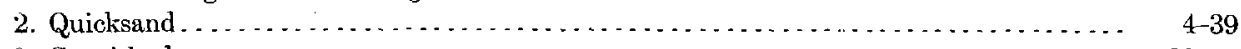

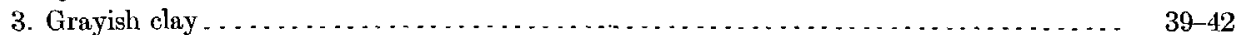

4. Red sand and gravel, water-bearing . . . . . . . . . . . . . $\ldots \ldots \ldots \ldots \ldots \ldots \ldots \ldots$

121. Record of well on Twelfth street between Broadway and Jamaica avenue, Long Island City.

1. Unstratified sand and bowlders . . . . . . . . . . . . .

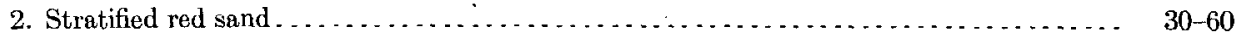

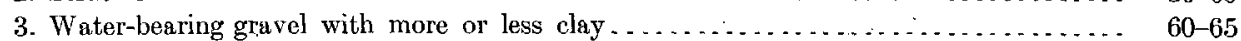

4. Black clay. . . . . . . . . . . . . .

Mr. Allen reports that in this vicinity the water-bearing gravel lying between the stratified sand and black clay ranges in thickness from 0 to 23 feet.

122. . Record of well at Grand street and Third avenue, Long Isiand City.

1. Humus-stained sandy loam.............. Feet.

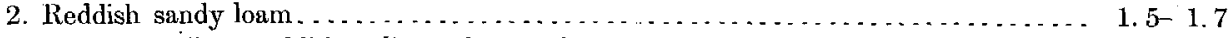

3. Fine to medium reddish yellow silty sand $\ldots \ldots \ldots \ldots \ldots \ldots \ldots \ldots \ldots \ldots \ldots, 6,0,7.0$

4-6. Dark brownish gray multicolored glacial sand and gravel... . . . . . . . . 11.523 .0

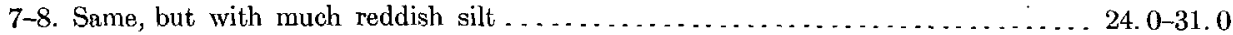

123. Mr. Allen reports that this well is in an area which is about a block and a half square, in which it is quite easy to get water; outside of this local basin it is much more difficult.

125. Surface water was shut out at 225 feet, and the well tested at 352 and 608 ; both tests gave salty water.

126. No water encountered until 43 feet, where it was found in a crevice of the rock, and came up to within 4 feet of the surface. Water contains too much lime for boiler use. Well pumps 18 gallons a minute at suction limit.

Record of well at Steinway avenue and River road, Long Island City.

1. Yellow bowldery clay.

2. Quicksand (very fine, clean sand-no mica) ...................... 24-37

3. Coarse, white gravel and beach sand . . . . . . . . . . . . . . . . . . . . . $37-42$

4. Conglomerate rock ("like the rock at Scranton, Pa., just above the hard coal") ... $42-45$

5. Gray gneiss. . . . . . . . . . . . . . . . . . .

128. Impotable water is reported at 14 feet; good water at 48 feet.

129. Mr. Harper states that the record of material penetrated in this well is exactly the same as in the other wells, which he put down on Barren Island. (See Nos. 130 and 131.) 


\section{UNDERGROUND WATER RESOURCES OF LONG ISLAND, NEW YORK.}

130. The following section has been prepared by Mr. Lewis Woolman from samples furnished by Mr. Thomas B. Harper: $a$

Record of T. F. White Company's well on Barren Island, New York.

Pleistocene:

Fect.

$0-70$

1. Interval; no specimens . . . . . . . . . . . . . . . . . . . . . . . . . . . . . . .

2. Brownish sands, sometimes slightly yellowish and sometimes slightly reddish in

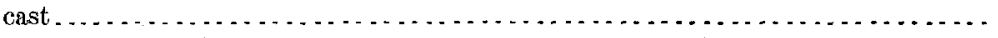

3. Reddish-brown and yellowish-brown sands, same as next above, with the addition

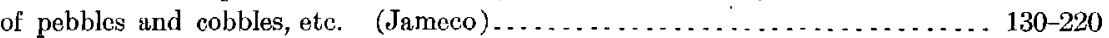

Cretaceous:

4. Whitish sands............................................. 220 . 240

5. Brown sands. . . . . . . . . . . . . . . . . . . . . . . . . . . . . . . . . . . . 240 260 260

6. Bluish-white sands with some lignite throughout $\ldots \ldots \ldots \ldots \ldots \ldots \ldots \ldots \ldots$. . . . . . . . . . .

7. Dark, micaceous sandy clay, no lignite . . . . . . . . . . . . . . . . . . . . . . . . . 500-690

8. Yellowish-white, water-bearing sand, coarse at 700 to 720 (Lloyd sand) . . . . . 690-740

131. The following samples were furnished by Mr. Thomas B. Harper to the New Jersey Geological Survey: $b$

Record of Sanitary Utilization Company's well on Barren Island, New York.

Pleistocene:

1. Whitish sand for some distance down from the surface; heavy gravels and cob-

bles at . . . . . . . . . . . . .

3. "Reddish" (?) sand.

4. Dark-colored conglomerate, quartz grains and pebbles size of mustard seed to

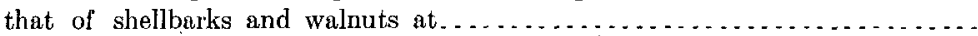

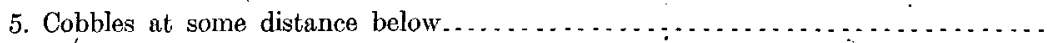
Cretaceous:

6. Whitish sand.

7. "Cemented material" of feldspar and quartz ....................... 495-500

8. Bluish soft marl (?) ........................................ $500-560$

9. Alterations of sands and clays, each 15 to 20 feet thick.............. 560-660

10. Red clay at................................................ 706

11. Yellowish-white coarse sand and fine gravel, water bearing (Lloyd sand) .... 712-720

12. Whitish clay, prospected 4 feet, or from . . . . . . . . . . . . . . . . . . . . . 720-724

132. 'The following section was reported by Mr. Chester D. Corwin:

Record of Sanitary Utilization Company's well on Barren 1sland, New York.

1. Medium finc gray sand ...........

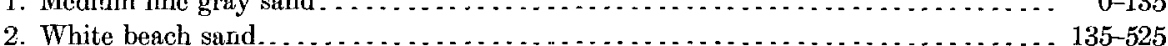

3. Hardpan, clay and stones; clay and gravel-like cement; color between gray and brown ................................................. 525-530

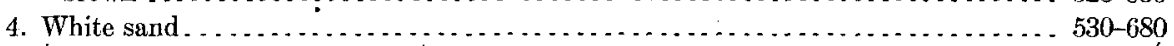

5. Light-gray medium gravel; good water-bearing stratum ............... 680-700

133. Record of White Lead Company's well on Crook Island, New York.

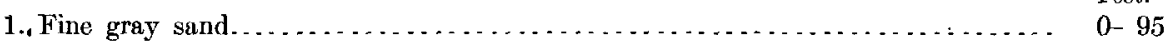

2. Brown médium sand......................................... 95-115

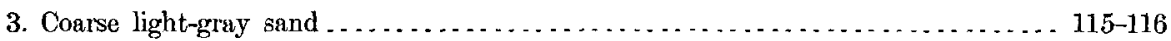

4. Brown medium sand (similar to No. 2) ........................... 116-130

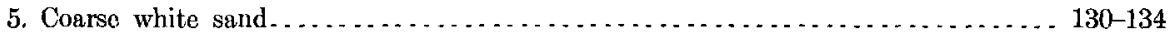

b Op. cit., p. 155 . 


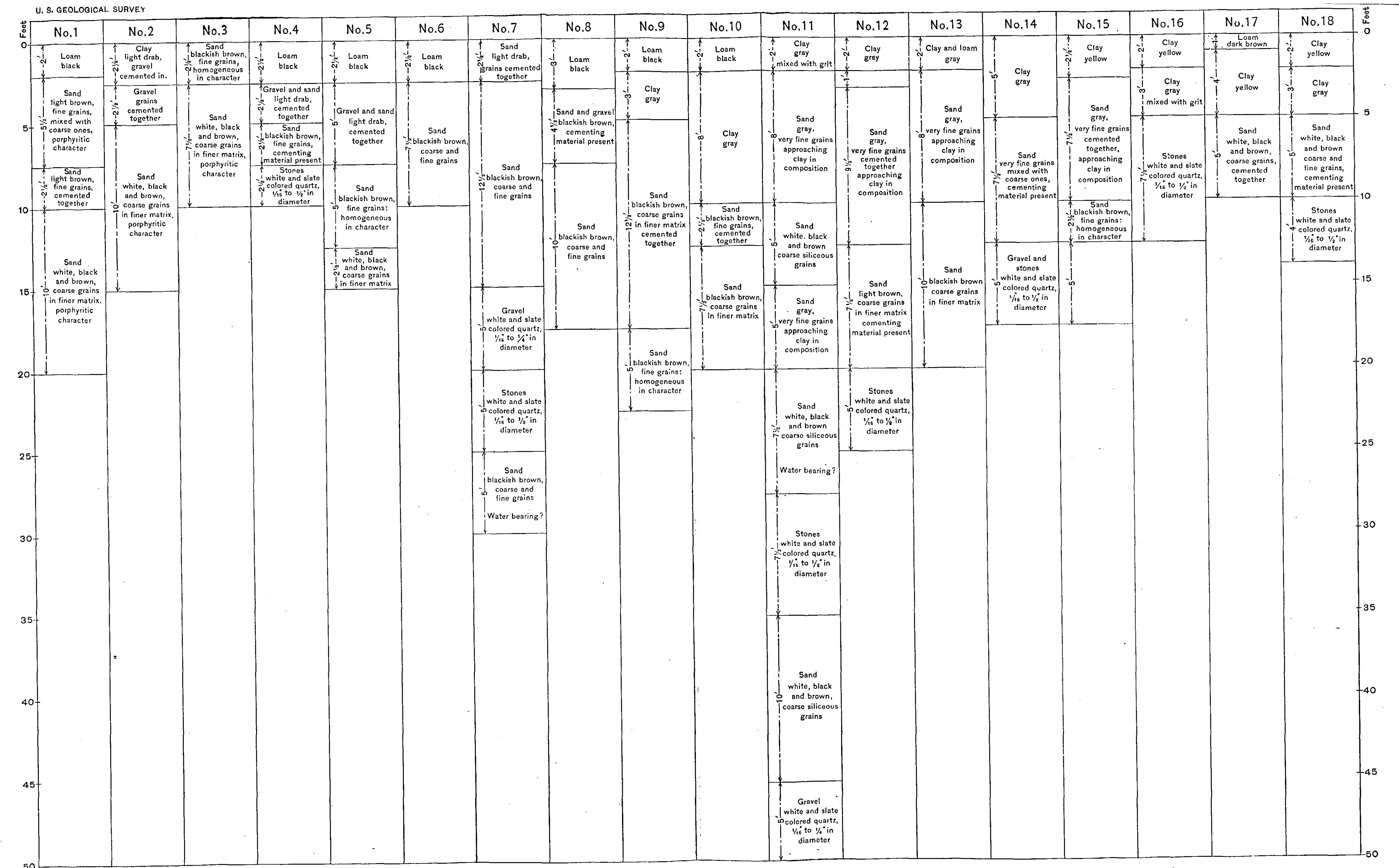



134. Mr. L. B. Ward gives the following data: "This company operates under the franehise of the Long Island Water Supply Company in the Twenty-sixth Ward, where its property is situated. It pumps 70,000 gallons of water daily from driven wells for the supply of houses built on its tract No. 1, and takes 90,600 gallons additional from the Long Island Water Supply Company for use in tract No. 2. The plant consists of a pumping station and a standpipe. It supplies 176 houses on tract No. 1 and 275 houses on tract No. 2."

135. Mr. Robert Van Buren, of the department of water supply, Borough of Brooklyn, has kindly furnished samples from the deep wells put down at New Lots in 1903. From these the following section has been compiled (see fig. 10):

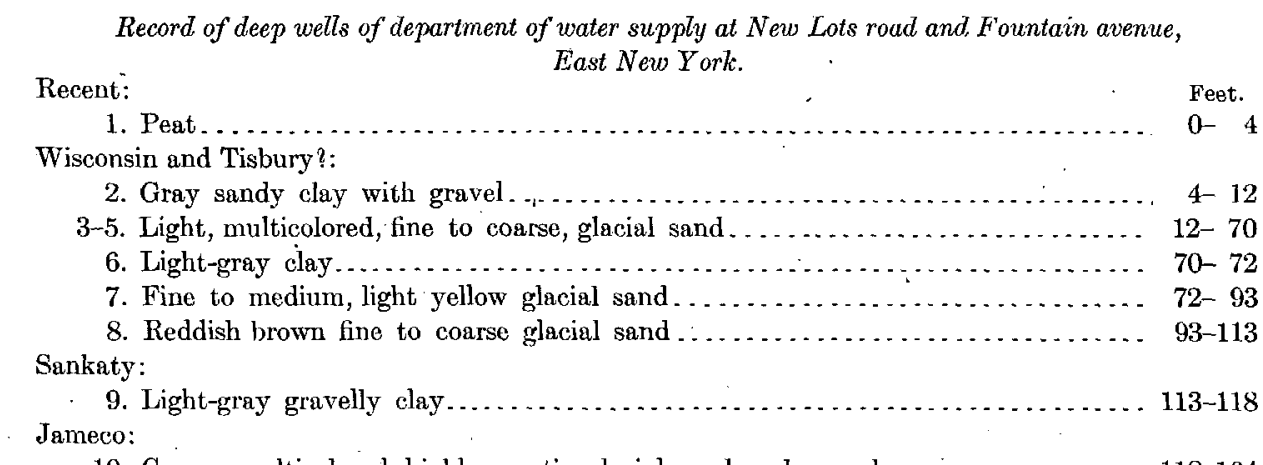

10. Coarse, multicolored, highly erratic glacial sand and gravel. ............. 118-164

Messrs. P. H. \& J. Conlan reported to the Geological Survey of New Jersey $a$ in 1896 the following: "The greatest yield and the best quality of water for the Long Island Water Supply Company were found at East New York, where it is all gravel and coarse sand. 'The yield was about two and one-half million gallons per day from six 8-inch wells that run from 65 to 95 leet deep."

In 1898 the same firm reported:" "We have erected a pumping plant for the Iong Island Water Supply Company in Brooklyn, N. Y. We put down five wells averaging a depth of 80 feet. Supply collectively was $2,000,000$ gallons per day of 24 hours. The strata were:

\section{Record of wells of department of water supply at New Lots road and Fountain avenue,} East New York.

1. Lroam. . . . . . . . . . . . . . . .

2. Fine sand.... $4-10$

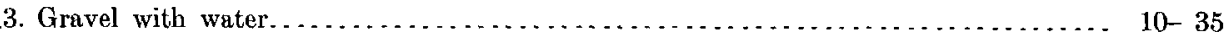

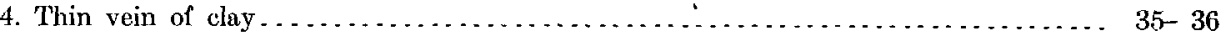

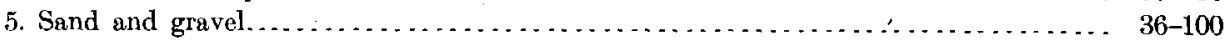

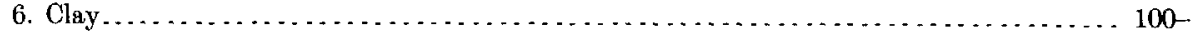

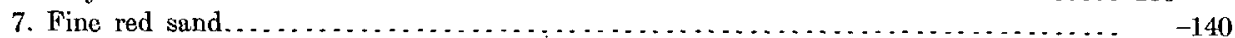

"We went to 140 feet with one well, but got no water. It was fine red sand with much iron and no gravel, and we went no deeper. The levels of the wells are about high-tide level; a very high tide breaks up, so that they are all connected at tide level, but the water is fresh and good for use, but a little hard."

136. The following section has been prepared from the samples preserved in the office of the department of water supply in the nunicipal building, Brooklyn (see fig. 10):

Wisconsin:

Record of Brooklyn test well, No. 17 .

2. Light, reddish-brown, fine to conroi specked sond. Tisbury:

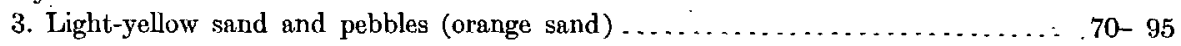

a Ann. Rept, Geol. Survey New Jersey for 1896, 1897,-p. 186. b Ann. Rept. Geol, Survey New Jersey for 1898, 1899, p. 142. 
Sankaty:

4. Dark-gray clay with vegetable matter (swamp deposit) .............. 95-106

Sankaty?

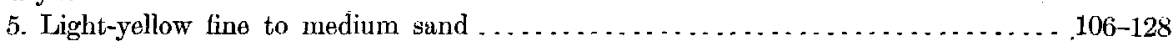

Jameco:

6. Fine, dark, reddish-brown sand, glacial. . . . . . . . . . . . . . . . . . . . 128-140

7. Very coarse multicolored sand . . . . . . . . . . . . . . . . . . . . . . . . . 140 150

8. Coarse multicolored gravel, with a very small percentage of quartz........ 150-170

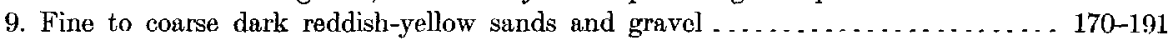

The elevation of the surface at this point is 10.6 feet above the Brooklyn base.

137. The following records have been prepared from the samples preserved in the offlce of the department of water supply, municipal building, Brooklyn (see fig. 10):

Wisconsin:

$$
\text { Record of Brooklyn test well, No. } 4 \text {. }
$$

1. Dark, humus-stained surface, sandy loam .... .

2. Clean reddish-brown sands and gravels of glacial origin $\ldots \ldots$ 'Tisbury:

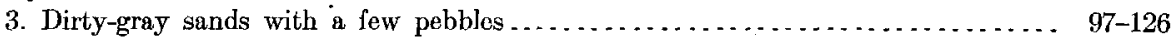
Sankaty:

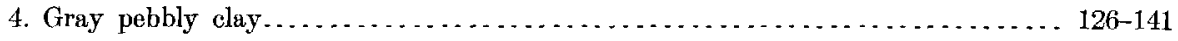

Jameco:

5. Coarse multicolored gravel with a very few quartz pebbles.

Mr. De Varona reports: "Water level above the blue clay is about 16 feet below the surface of the ground, and below the clay it is about 2.5 feet below the surface." $a$

Analysis of water of Brooklyn test well, $N \mathrm{~N}^{\prime} .4{ }^{\circ}$

Parts per million.

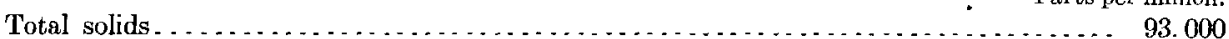

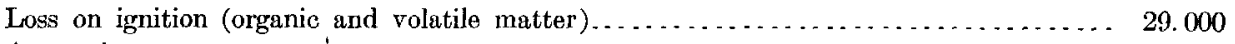

Ammonia:

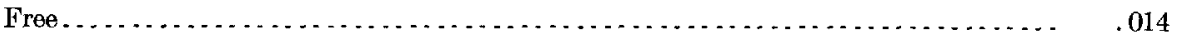

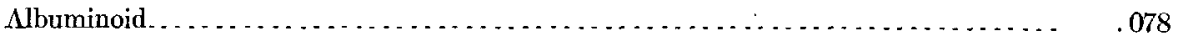

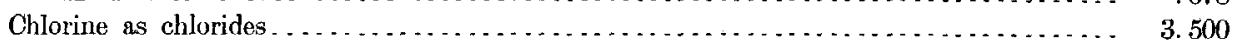

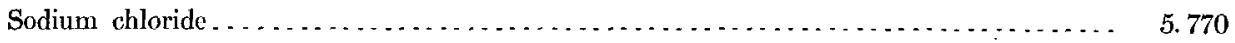

Nitrogen as nitrates. . . . . . . . . . . . . . . . . . . . . . . . . . . . . . . . . . . . . . 422

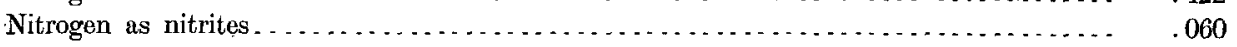

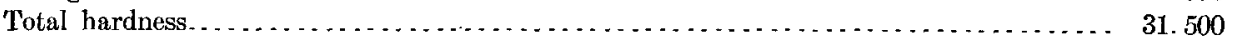

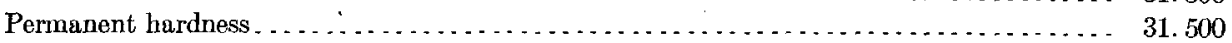

138. The following section has been prepared from the samples preserved by the Brooklyn department of water supply:

Record of wells at Old Spring Creek pumping station.

\begin{tabular}{|c|c|c|c|c|c|c|}
\hline & Well number. . . . . . . . . . . . . & $1 \mathrm{~A}$. & $2 A$. & $3 \Lambda$. & $4 A$. & $5 \Lambda$. \\
\hline Recent............... & 1. Peat $\ldots \ldots \ldots \ldots \ldots \ldots \ldots \ldots \ldots \ldots \ldots \ldots \ldots \ldots \ldots \ldots \ldots \ldots$ & $0-3$ & $0-4$ & $0-4$ & $0-2$ & $0-2$ \\
\hline Wiscon $\sin$ and Tisbury.. & $\begin{array}{l}\text { 2. Fine to medium yellow to reddish yellow } \\
\text { sand with some gravel. }\end{array}$ & $3-125$ & $4-127$ & $4-127$ & $2-126$ & $2-124$ \\
\hline Sankaty.... & 3. Gray gravelly clay $\ldots \ldots \ldots \ldots \ldots \ldots \ldots \ldots$ & $125 \cdot 133$ & $12 \bar{\gamma}-137$ & $127-136$ & $126-136$ & $124-134$ \\
\hline Jameco........ & $\begin{array}{l}\text { 4. Multicolored sand and gravel with rela- } \\
\text { tively small percentage of quartz. }\end{array}$ & $133-158$ & $137-151$ & $136-153$ & $136-153$ & $134-151$ \\
\hline
\end{tabular}

a Ann. Rept. Commr. City Works of Brooklyn for 1895, 1896, p. 346.

$b$ Analysis by the Brooklyn health department, op. cit., pp. 140, 142 
Messrs. W. D. Andrews \& Brother, who put in the original plant at this point, report under date of March 8, 1895: "At Spring Creek and Baisley trial tubes and wells were driven to a depth of 100 feet or more, from which water flowed, and would rise 2 or 3 feet above the surface, if confined in a tube. By hand pumping these 2-inch wells would yield 30 to 40 gallons per minute."

Analysis of water from shallow driven well plant at Spring Creek pumping station.

[By Brooklyn health department.]

Parts per million.

Total solids . . . . . . . . . . . . . . . . . . . . . . . . . . . . . . . . . . . . . . . . . . . 194. 429

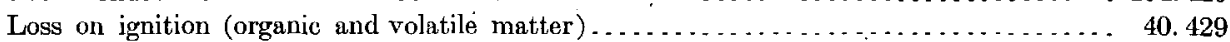

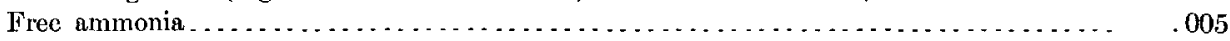

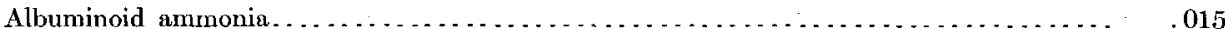

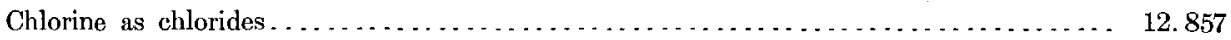

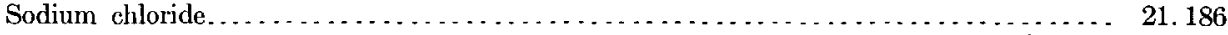

Nitrogen as nitrates. . . . . . . . . . . . . . . . . . . . . . . . . . . . . . . . 4.510

Nitrogen as nitrites . . . . . . . . . . . . . . . . . . . . . . . . . . . . . . . None.

Total hardness. . . . . . . . . . . . . . . . . . . . . . . . . . . . . . . . . . . . . . . . 110.214

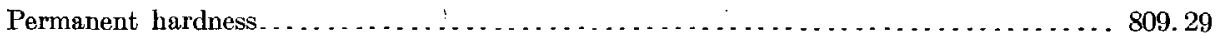

139. The following analysis was furnished by Mr. I. M. De Varona:

Analysis of water from well at temporary Sming Creek pumping station.

[Analysis by Brooklyn health department.]

Parts per million.

Total solids

223. 500

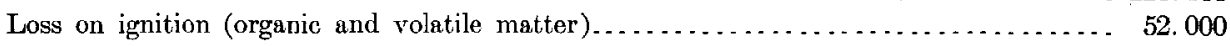

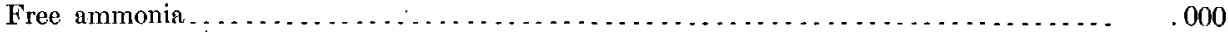

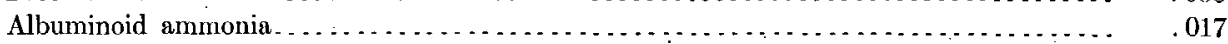

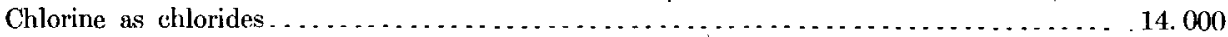

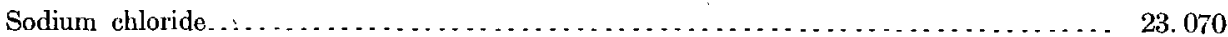

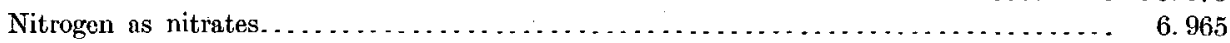

Nitrogen as nitrites . . . . . . . .

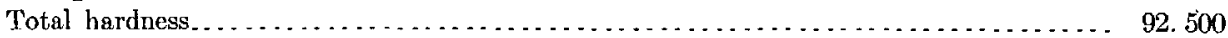

Permanent hardness............................................. 91.000

141. Section from samples preserved by the Brooklyn water department (see fig. 10):

Record of Brooklyn test well No. 5 .

Wisconsin: Feet.

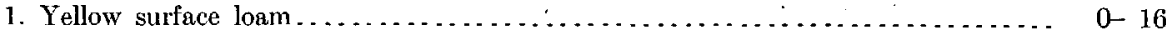

2. Reddish brown multicolored sands and gravel of glacial origin........... 16-192

Sankaty:

3. Gray clay ............................................... 192-200

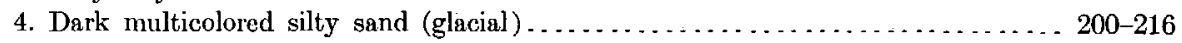

5. Gray silty clay............................................ 216-281 Jameco:

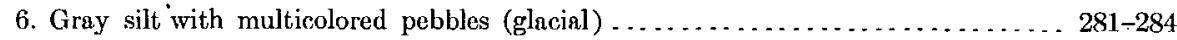

Accompanying the samples preserved in the glass tube is a sample in an envelope, marked "Test well No. 5, below clay, received August 21, 1895." This sample consists of large dark-colored pebbles, only about one-fourth of which are quartz. Mr. I. M. De Varona adds: "When the pipe was down about 284 feet the water level was about 46 feet below the surface." Elevation of ground 61.8 feet, Brooklyn base. 
Total solids

Loss on ignition (organic and volatile matter)....

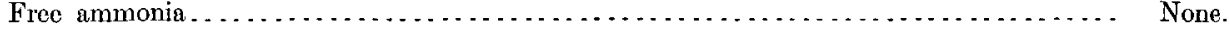

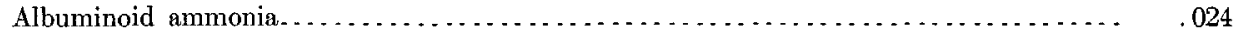

Chlorine as chlorides. . . . . . . .

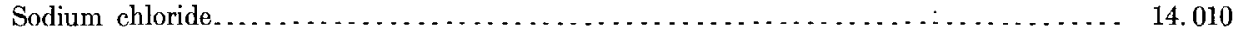

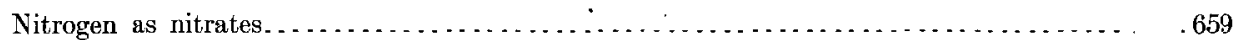

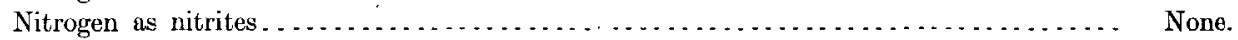

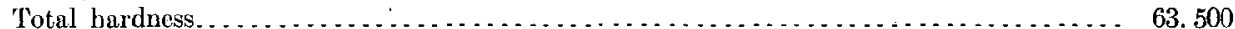

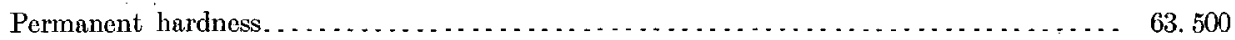

142. The wells of this company are arranged in two groups about one-half mile apart, the northern one consisting of 4 wells and the southern one of 12 . The pumping station is located about midway between them, in the factory of the Agate-Nickel Steel Ware Company.

Section from samples preserved in the office of the Agate-Nickel Steel Ware Company:

Record of Woodhaven Water Supply Company's well near Woodhaven.

Wisconsin and Tisbury?:

1. Light-brown, medium, glacial sand

$2-3$. Coarse glacial sand and gravel, containing a large percentage of granitic and

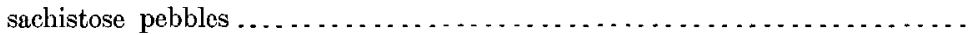

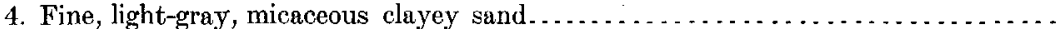

5-8. Brown glacial sand and gravel.

The whole section is pronouncedly glacial, with the highest percentage of erratic material between 16 and 31 feet. An analysis of this water, made November 28, 1902, gave the following results:

Analysis of water from Woodhaven Water Supply Company's well near Woodhaven.

Appearance, etc., clear pale brownish yellow.

Odor (heated to $100^{\circ} \mathrm{F}$.), faint earthy.

Chlorine in chlorides

Parts per million.

. 11.5

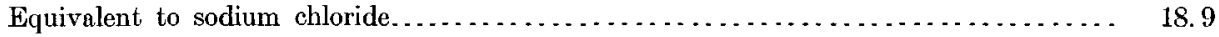

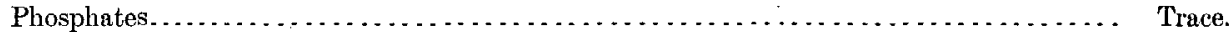

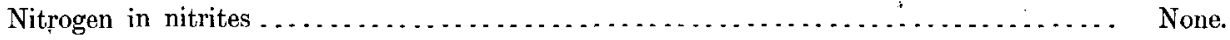

Nitrogen in nitrates................................................ 2.1

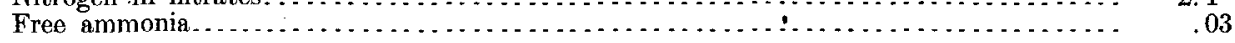

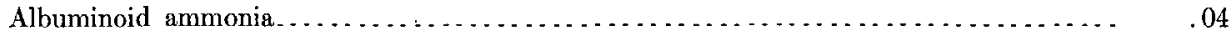

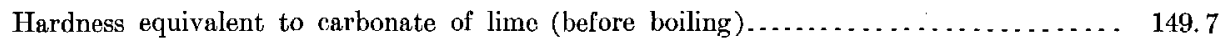

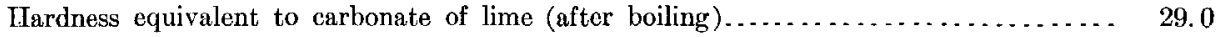

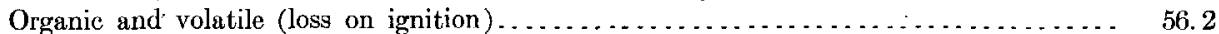

Mineral matter (nonvolatile) .......................................... 146.1

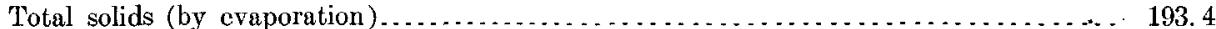

143. The record of the deep well at this point has been published by Messrs. Bryson, $\alpha$ Lewis, $b$ Darton, $c$ and Woolman.d A complete set of samples presented by Mr. F.H. Luce, superintendent of the Woodhaven Water Supply Company, which are preserved in the museum of the Long Island Historical Society, show the following section:

a Am. Geologist, vol. 3, pp. 214, 1889

$b$ Am. Jour. Sci., 3d ser., vol. 37, p. 233, 1889.

c Bull. U. S. Geol. Survey No. 138, 1896, pp. 31-32,

d Ann. Rept. Geol. Survey New Jersey, for 1896, 1897, pp. 158-160. 
Record of well of Lalance \& Grosjean Manufacturing Company near Woodhaven.

Pleistocene:

1. Reddish-yellow glacial sands and gravels $a$

Cretaceous:

2. Dark laminated clay with some quartz pebbles...................... 213-358

3. Gray clayey sand with lignite. ................................... 358-430

4. Very dark sandy clay ..................................... $430-436$

5. Fine white sand ............................................ 436-443

6. Very dark-gray dirty sand.................................... $443-456$

7. Medium white sand; with small quartz pebbles...................... 456-460

8. Very dark clayey sand . . . . . . . . . . . . . . . . . . . . . . . . . . . . . . 460-475

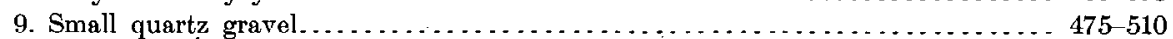

10. Fine to medium, dirty, clayey sand..............

11. Dark, sandy, laminated clay, with quartz pebbles. . . . . . . . . . . . . . . . 515-518

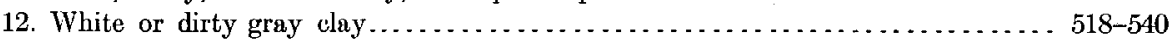

13. Dirty gray medium sand................................. $540-556$

Pre-Cretaceous:

14. Rock. . . . . . . . . . . . . . . . . . . . . . . . . . . . . . . . $556-570$

144.

Record of commission's test well near Union Plece.

Wisconsin and Tisbury?:

1. Surface dark-yellow sandy loam.

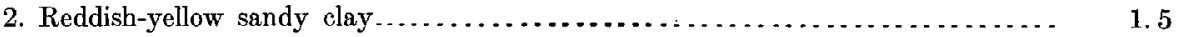

3. Dark-yellow sand and small gravel.................

4-5. Sand and coarse gravel, with much erratic material. . . . . . . . . . . . 10-15

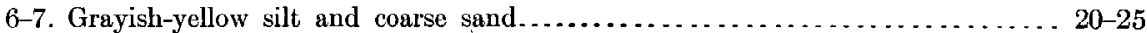

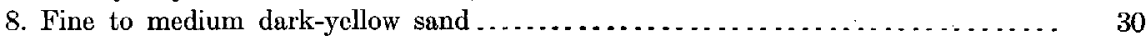

9. Coarse, dark-gray, multicolored sand, with much erratic material. . . . . . . . 32-32.5

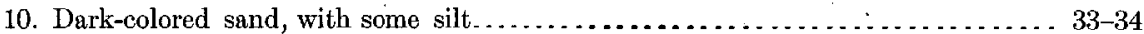

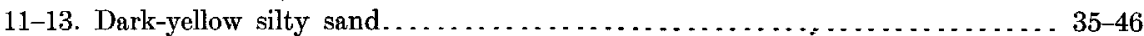

This whole section is apparently outwash glacial gravel.

145. Record of commission's test.well near Glendale.

Wisconsin and Tisbury?: Feet.

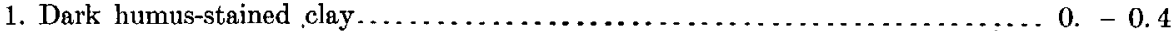

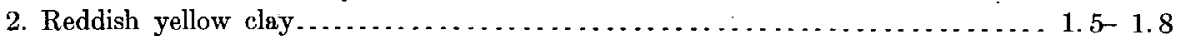

3-5. Reddish yellow silty sand............................. 6.18

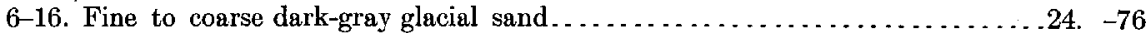

147. Analysis of water of Montauk Brewing Company's well near Metropolitan. [By H. W. Walker.]

Appearance clear.

Color normal. $\quad$ Parts per million.

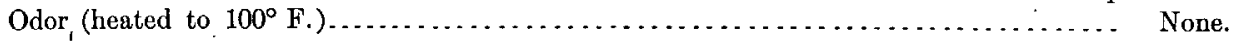

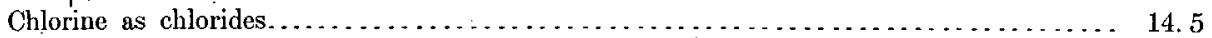

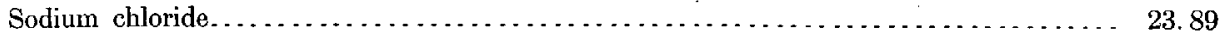

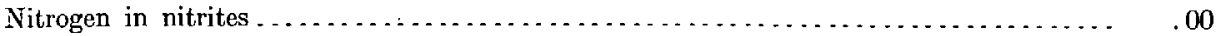

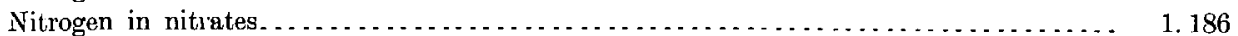

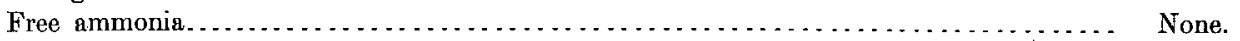

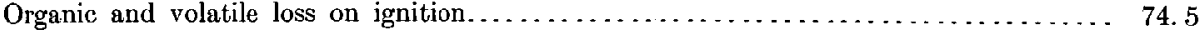

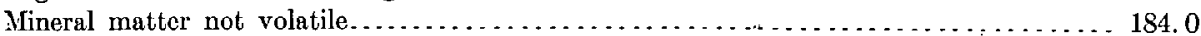

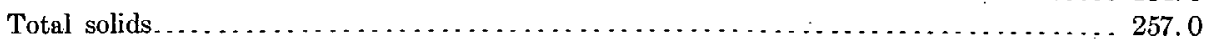

Mr. H. W. Walker, of the Brooklyn city health department, says, August 8, 1903: "This water is of bright and sparkling appearance, and the analysis indicates a high degree of purity."

a In the samples the sand ends at 153 feet, but as both the Lewis and Bryson records carry it to 213 feet. it has been so placed in this record. 


\section{UNDERGROUND WATER RESUIIRCES OF LONG ISTAND, NEW YORK.}

148.

Record of commission's test well near Middle Village.

Wisconsin and Tisbury?

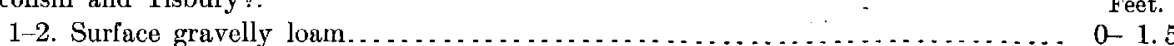

3-5. Yellowish clayey sand........................................ $5-16$

6. Fine to medium, dark, multicolored sand .......................... 19-20

7. Dark yellowish brown, clayey sand, glacial . . . . . . . . . . . . . . . . . . . . 21-22

8. Dark, multicolored, fine sand to coarse glacial gravel.................. 22-23

9-11. Dark, multicolored, clayey sand and gravel, glacial. . . . . . . . . . . . . . . 30-37

12-20. Dark, multicolored, fine to coarse glacial sand................... $44-96$

See Table XIII.

149. $\quad$ Record of II. Bottjer's well near Middle Village:

Wisconsin and Tisbury?: , Feet.

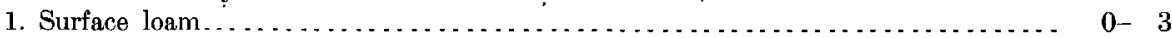

2. Stones and clay "hard pan," with occasional streaks of water-bearing sand and

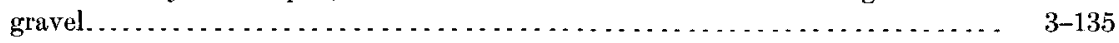

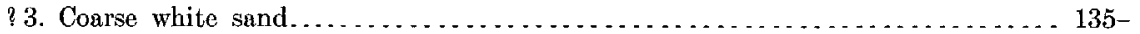

151. A test well put down at station No. 5 gave the following section (see fig. 13):

Record of well of Citizens' Water Supply Company at Station No. 5, near Flushing Creek.

Wisconsin and Tisbury:

1. Reddish-brown sand and fine gravel ............... 0 . 60

Tisbury? :

2. Coarse reddish-brown gravel. ............................... $50-90$

Sankaty?:

3. Blue stony clay .......................................... $90-190$

152.

Record of commission's test well near Flushing Creek.

Wisconsin:

1-2. Surface; sandy loam.

Feet.

$0-2$

3-4. Reddish-brown clayey sand.................................. $4-11$

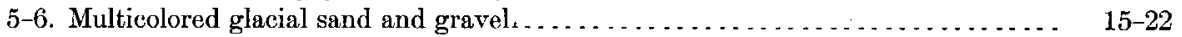

153. Mr. L. B. Ward gives the following data: "The works of this company are located in the Second Ward of Queens Borough and were erected to supply Long Island City. They consist of three pumping stations, each containing one pumping engine, also 178 driven wells, and 7.5 miles of 12 -inch and 16-inch pipe in three force mains laid to connect with the Long Island City distribution system. The pumps and pump houses are of a provisional character and the works are idle except for the formal operation of ono small pump."

154.

Record of well at New Calvary Cemetery, Long Island City.

1. Black mud. .

Feet.

2. Bluc clay and small blue rock ............................ $22-70$

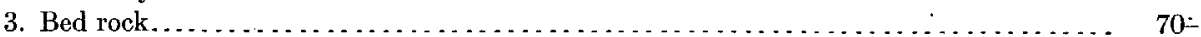

155. Record of well at New Calvary Cemetery, Long Island City.

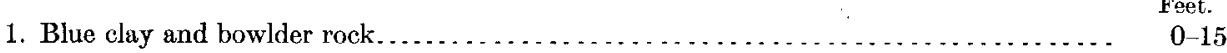

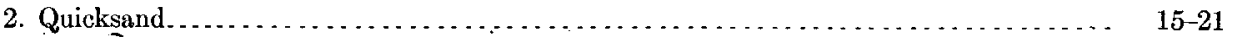

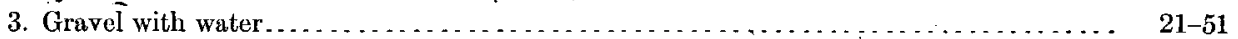

156. Record of well at New Calvary Cemetery, Long Island City.

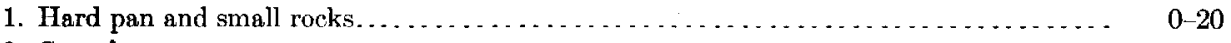

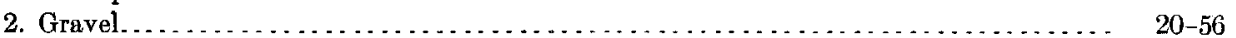


157.

Record of commission's test well near Newtown.

Wisconsin:

Feet.

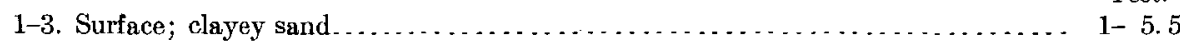

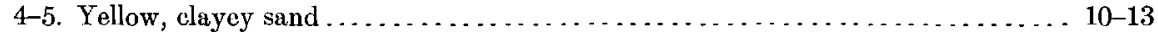

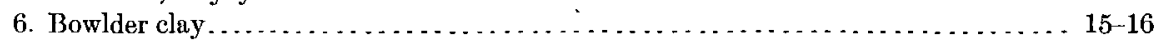

7-a. Dark multicolored sand and gravel, increasing in coarseness with depth....... 18-26

159.

Record of commission's test well near Elmhurst.

Wisconsin:

1-5. Yellow loam with gravel................................ 0 - 5

6-12. Fine to coarse, dark, multicolored sand with some gravel; "hardpan, very

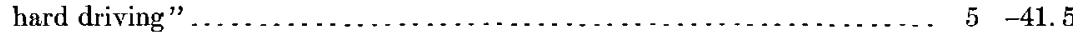

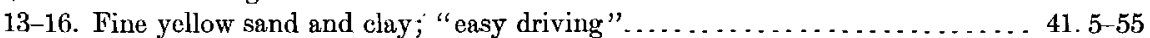

17-18. Dark multicolored sand and gravel; "hardpan"............... $55-65$

Bowlder or ledge at....................................... 69

See Table XII.

160. The difference in elevation between these wells is very slight. Well No. 1 , if anything, is on higher ground than wells Nos. 2 and 3 . There is apparently a very rapid and irregular variation in the water table.

161. See No. 153 .

162. Messrs. Stotthoff Brothers report $a$ the following data to the New Jersey Geological Survey (see fig. 13):

Wisconsin:

Record of Citizens' Water Supply Company's well near Woodside.

1. Earth, clay, and bowlders..................................... $0-38$

Jameco and Cretaceous?

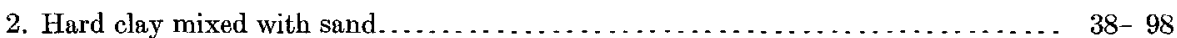

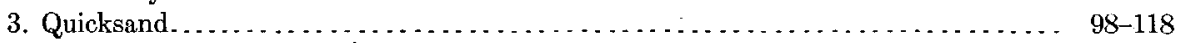

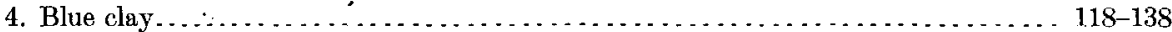

Fordham gneiss:

5. Rock, "gneiss layer," etc..................................... 138-227

163. Mr. Allen reports that the bowlder in the following section was blasted and that water-bearing gravel was found immediately below it. When he penetrated the gravel 3 feet the water rose in the pipe 15 feet, or 4 feet above the bowlder.

Record of I. Isenburg's well on Albert street near Grand avenue, Long Island City.

1. Sand with bowlders.

Feet.

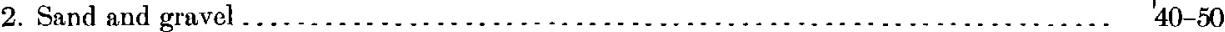

3. Large bowlder. . . . . . . . . . . . . . . . . . . . . . . . . . . . . . . . $50-58$

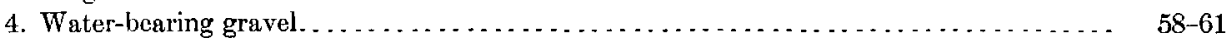

164. Mr. S. H. Allen states that most of the wells in this vicinity are about 60 feet deep and that he encountered two water-bearing layers separated more or less by a bed of sandy clay or clayey sand. The water in the upper layer has no pressure, while that from the lower often has pressure enough to rise 5 or 10 feet. The water from the lower layer is considered better both in quantity and quality.

165. Record of well on Thirteenth avenue near Vandeventer avenue, Long Island City.

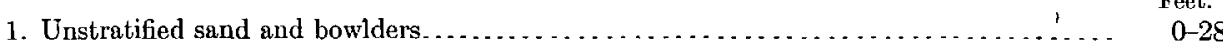

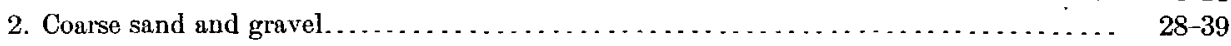

3. Hard sand with bowlders........................................ $39-46$

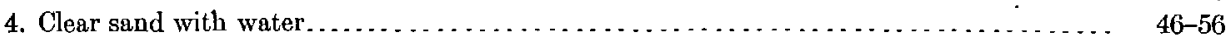

5. Lairge bowlders...................................................

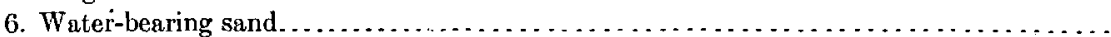

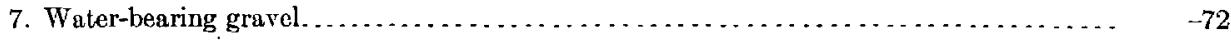

a Ann. Rept. Geol. Survey New Jersey for 1899, 1900, p. 80. 


\section{UNDERGROUND WATER RESODRCES OF LONG ISLAND, NFW YORK}

166. Record of commission's well on Bowery Bay road near Flushing avenue, Long Island City.

Wisconsin:

Feet.

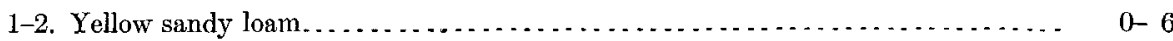

4. Very fine dark-gray sand and clay ................................... 10-11

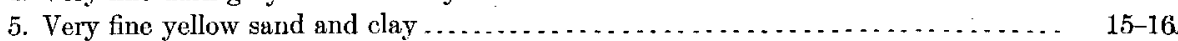

6-11. Fine to coarse, multicolored glacial sand and gravel $\ldots \ldots \ldots \ldots \ldots \ldots \ldots$

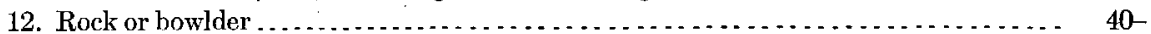

Sec Table XII.

167. Record of well at Albert street and Ditmars avenue, Long Island City.

Wisconsin:

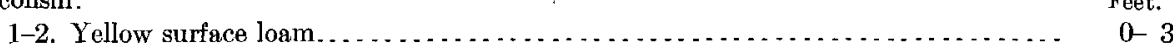

3-5. Fine, yellow, silty sand and gravel.............................. $5-16$

6-10. Clean, coarse, multicolored glacial sand and gravel ................... 20.39

11. Rock or bowlder. . . . . . . . . . . . . . . . . . . . . . . . . . . . . . . . . . . . . $39-40$

168. Mr. S. H. Allen completed a well at this place 79 feet 9 inches deep, which yiclded 210 gallons per minute. This well ended on what appeared to be bed rock. Later the well was deepened, and after drilling 32 feet the rock was penetrated and quicksand found. It was found impossible to obtain water from this quicksand and a new well was drilled to the original depth.

Record of Astoria Silk Works' well on Steinway avenue, near Ditmars avenue, Long Island City.

1. Tardpen Feet.

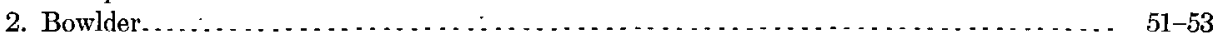

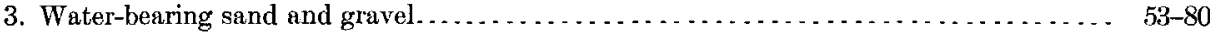

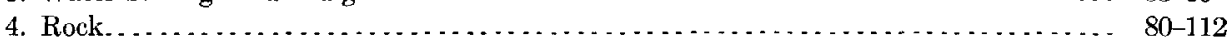

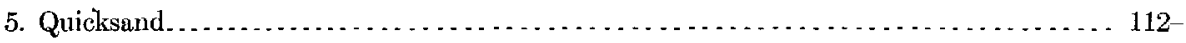

169. Record of well on Potter avenue near Park Place, Ling Island City.

1. Solid stone and hard pressed gravel .

2. Blue clay and hard pressed gravel................. $40-40$

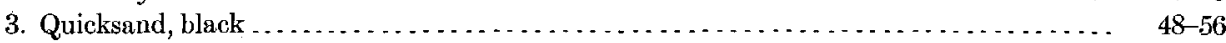

4. Micaceous sand with water; took quite a time to clear. . . . . . . . . . . . . . . . . 56 . $56-63$

170. Record of well at Merchant street and Ditmars avenue, Long Island City.

1. Sand and bowlders.

Feet.

(1)

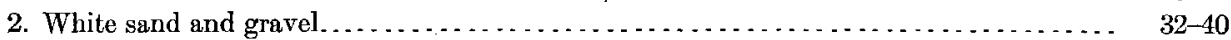

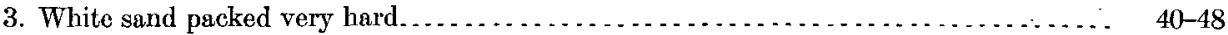

171.' Record of well near Merchant street, and Ditmars avenue, Long 1sland City.

1. Sand and gravel

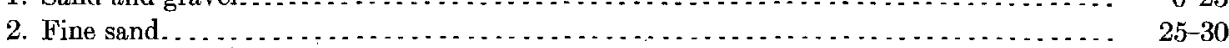

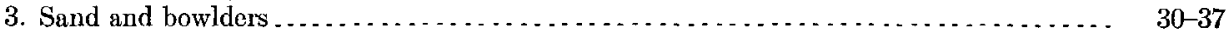

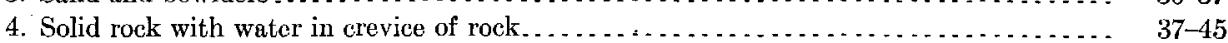

172. Record of well at Crescent street and Ditmars avenue, Long Island City.

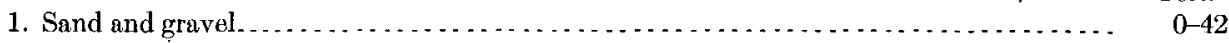

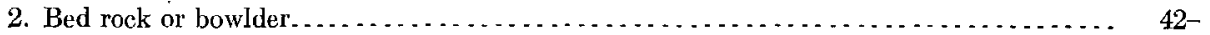


173. Record of commission's test well at Lawrence street and Wolcott avenue, Long Island City.

Pleistocene:

Feet.

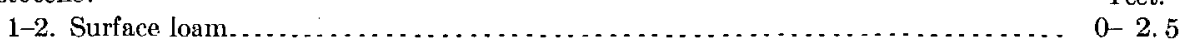

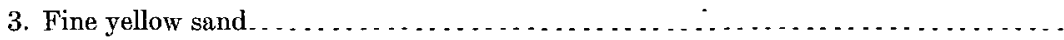

4. Small gravel of a dark mud color . . . . . . . . . . . . . . . . . . . . . . . . .

5. Yellow to dark-brown rock flour formed from drilling in bowlder. . . . . . . . 12-12.5

6. Multicolored glacial sand and gravel ............................ 14-31.

See Table XII.

176. Mr. L. C. L. Smith, consulting engireer, reports that there are 17 wells at this station which pass through the following material:

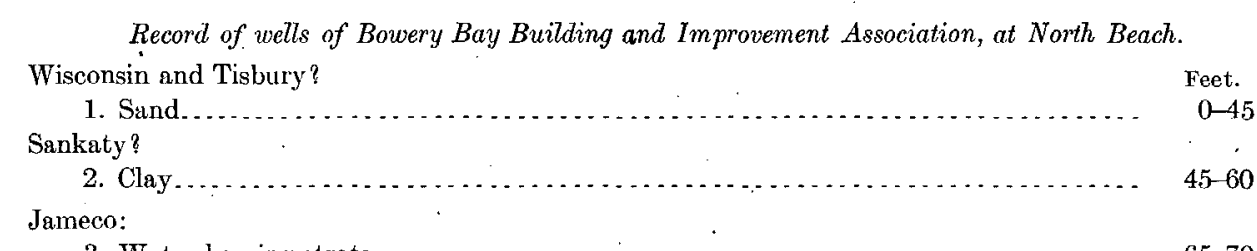

3. Water-bearing strata........
Sweeney \& Gray, drillers, report the following section:
Récord of wells of Bowery Bay Building and Improvement Association at North Beach.

Wisconsin to Tisbury?

1. Sandy top soil varying in color from white to yellow

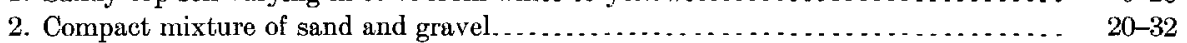

Sankaty?

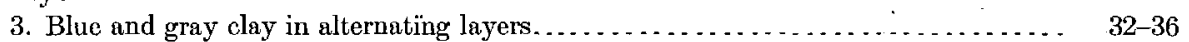
Jameco?

4. Very coarse sand and gravel in alternate layers. ....................... $36-82$

177. This is the locality from which the wells described by Darton as "Bowery Bay: 110 feet deep; 6 inches in diameter; one flowed 50 gallons," were reported. It seems that several parties attempted wells at this point, but that no results were obtained until after this information had been given the Survey, when three 6-inch wells were put down in a near-by hollow to a depth of 40 or 50 feet, the present water supply being derived from these. Mr. I. H. Ford states that the first well was sunk to a depth of 400 or 500 feet, but no further data has been obtained regarding it.

178. See No. 153.

179. Record of commission's well at Trains Meadow and Highway roads, Long Island City. Recent: Feet.

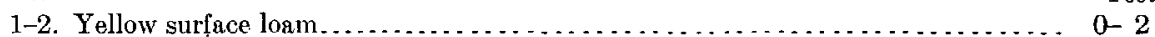

3. Dark clay with decayed glacial pebbles and peat.................... $5-6$ Wisconsin:

4. Very fine, grayish or reddish brown, clayey sand, glacial 10-28. 5

180. Record of commission's well on Trains Meadow road near Jackson avenue, Long Istand City. Wisconsin: Feet.

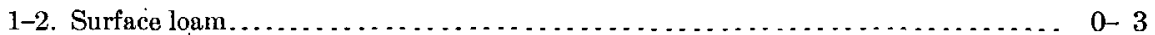

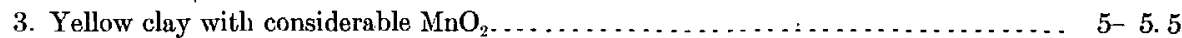

4-5. Fine, daik-colored, micaceous clayey sand ........................ 10-13.5

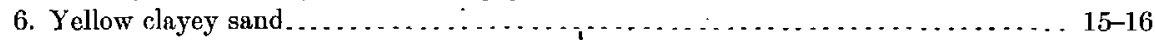

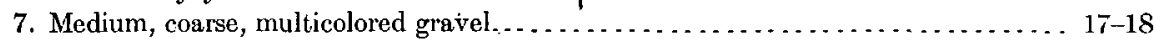

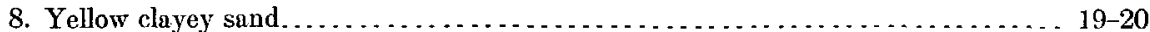

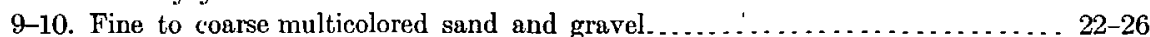

11. Dark reddish speckled sand suggesting disintegrated Triassic sandstone..... $30-31$

See Table XII. 
181. Record of commission's test well at Junction avenue and Strongs lane, Long Island City.

Wisconsin:

1-2. Missing.

Feet.

3. Light-yellow clayey loam with pebbles, "bowlder clay" $\ldots \ldots \ldots \ldots \ldots \ldots \ldots 7$. 8

4-7. Reddish yellow, fine to medium, silty sand...................... 14. $0-30.5$

8-11. Dark multicolored glacial sand and gravel ...... . . . . . . . . . . $36.5-53.0$

182. A. D. Schlissinger, president of the India Rubber Comb Company, reports as follows:

Record of India Rubber Comb Company's well near College Point.

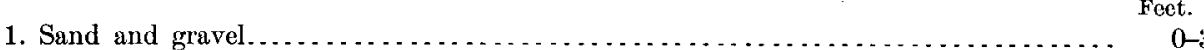

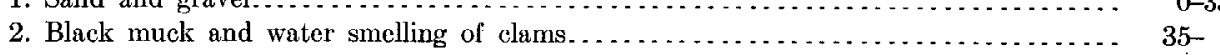

184. C. D. Corwin reports the following section from this well:

Record of well of American Hard Rubber Company, near College Point.

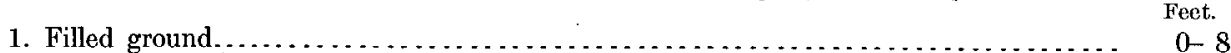

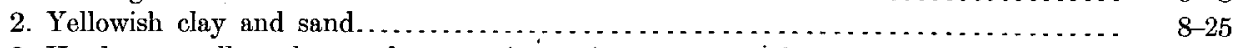

3. Hard pan, yellow clay, and stones, impervious to water; like macadamized roads... 25-60

4. Water-bearing gravel and light-brown coarse sand .................... $60-70$

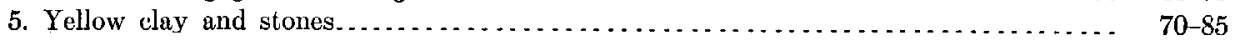

186. A sketch in the museum of the Long Island Historical Society by Mr. C. M. Jacobs, consulting engineer, gives the following section of the test well at this point:

Pleistocene:

Record of railroad test boring on Tallman Island, New York.

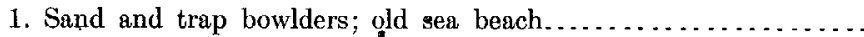

2. Yellow quartz sand

3. Quartz gravel and bowlders................................. $30-31.5$

4. Yellow quartz sand, medium fine ............................ 31.5-50

5. Trap bowlders, quartz sand, and gravel; regular glacial drift.......... $50-57$

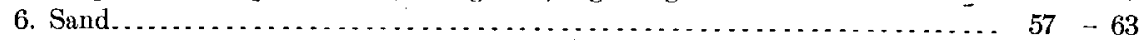

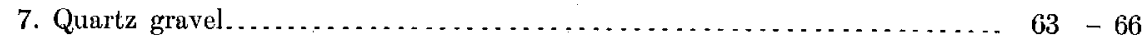

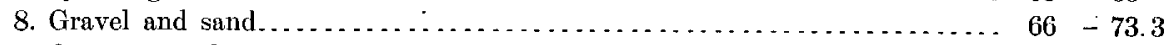

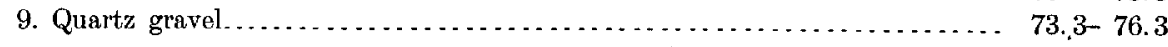

Cretaceous:

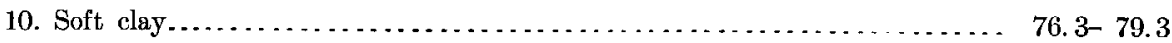

11. Lignite intermixed with clay bands......................... $79.3-91.7$

12. Streaked red and white clay; hard, bored out as a solid core ......... 91.7-110.4 Fordham:

13. Soft, white micaceous "sandstone," the upper part of which was so soft as to wash to powder under diamond drill; below it gradually became less micaceous and harder, the lower part coming out as a solid core ...... 110. 4-159

The core mentioned in No. 13 is regarded by Mr. Eckel as quartzitic Fordham.

187. Lawrence Verdon says: "Stopped at 112, as I could get the well no farther."

Tisbury:

$$
\text { Record of James Caffery's well near Far Rockaway. }
$$
Sankaty:

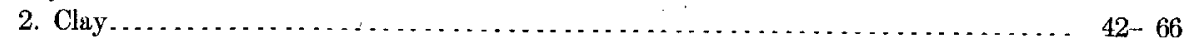

Jameco:

3. Black sand with water which looked and tasted good................ $66-88$

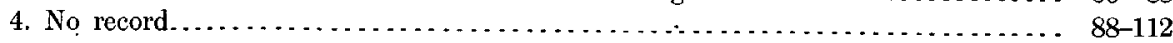


188. This well was put down by Mr. Gilbert Baldwin under the direction of Mr. Jesse Conklin. Mr. 'Conklin, under date of April 25, 1895, gives the following: "At Far Rockaway, about one-fourth mile from the ocean I drove a well 210 feet. I found water at 15 feet from the surface and got a good supply. I drove 180 feet through beach sand and gravel. At 195 feet struck petrified wood. Last 15 feet was clear white gravel, with a very good supply of water of about 40 gallons." From Mr. Baldwin it is learned that this water was so salty that the well was abandoned. The record, according to Mr. Baldwin, is as follows:

Tisbury:

Record of B. L. Carroll's well near Far Rockaway.

1. Fine beach sand

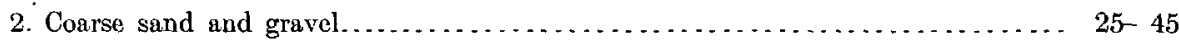

Sankaty:

3. Blue clay; no stones.......................................... $45-65$

Jameco and Cretaceous?:

4. Fine gravel and sand with brackish water (this layer furnished but a small quan-

tity of water) ............................................ $65-180$

5 . Coarse gravel with a vigorous supply of salty water.................. 180-190

The second well was drilled at a distance of about 400 feet, and $\mathrm{Mr}$. Carroll reports the following section:

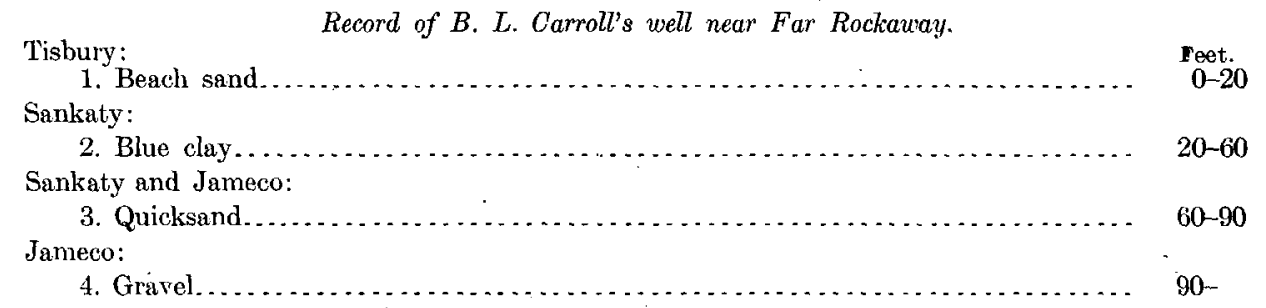

The water from this layer was of sufficiently good quality for ordinary use. Analysis showed a large amount of chlorine, but this was not sufficient to be perceptible to the taste.

189.

Record of James Caffery's well near Far Rockaway.

1. Ordinary soil sandy loam

2. Fine sand with no gravel except in streaks.......................... $2-30$

Mr. Walsh reports that the material was so fine that he used a Cook strainer to prevent tbe sand from entering the tube and clogging the well. He adds that in general the water on Rockaway Ridge occurs from 12 to 18 feet below the surface, and that the water near the conter of the ridge is better in quality than that near the margin. At the edge of the meadows there is a fine nonwater-bearing sand.

190. The following analysis is reported by the Long Island Railroad Company:

Analysis of water from railroad well at Far Rockaway.

Parts per million.

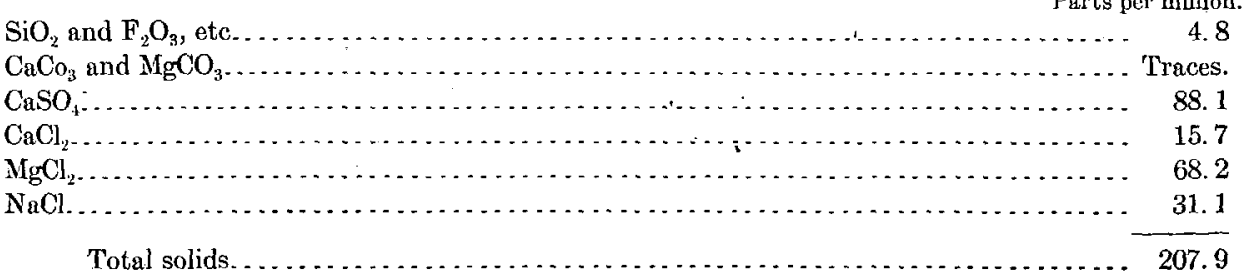

A corrosive water at 200 pounds pressure. 


\section{0 . UNDERGROUND WATER RESOUROES OF LONG ISLAND, NEW YORK.}

191. This was the site of the first plant of the Queens County Water Company. Mr. C. A. Lockwood, who put in the wells, reports that there were twenty 5 -inch wells, 50 feet dcep. These were entirely in lightbrown sand and gravel. As these wells did not yield a sufficient supply two deep wells were sunk to a depth of 200 feet, but in both brackish water was cncountered and they were abandoned.

The section reported is as follows:

Tisbury:

Record of well of Queens County Water Company near Far Rockaway.

1. Light-brown sand and gravel similar to the rest of the Rockaway Ridge material 0 - 60 Sankaty:

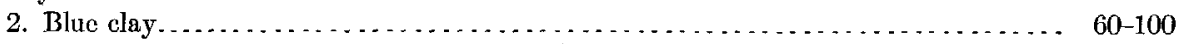

Jameco and Cretaceous?:

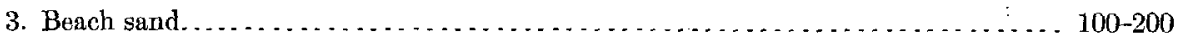

The above record is for the well nearest the bay north of Far Rockaway; the one farther south near the railroad station contained clay from 60 to 78 feet.

193. Record of T. R. Chapman's well on Hooks Creek.

Wisconsin and Tisbury:

1. Sand.

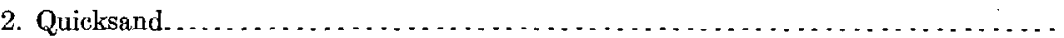

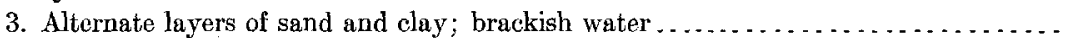
$0-$ Sankaty:

4. Dark-colored clay. . . . . . . . . . . . . . . . . . . . . . . . . . . . . . . . . . . . . . 140-200

5. Very hard clay; required 120 blows from 1 -ton hammer to drill 1 inch. . . . . . . 200-202 Jameco:

6. Gravel with artesian water. .................................. 202-203

Water at first flowed a good stream several fect above the surface, but the yield is now much less.

195. The following record has been prepared from samples preserved by the department of water supply in the municipal building, Brooklyn (see fig. 10):

Wisconsin:

Record of Brooklyn test well No. 16 at Shetuckei pumping station.

2. Fine to coarse, light, yellowish white, speckled sand $\ldots \ldots \ldots \ldots \ldots \ldots \ldots \ldots . . .20$

Tisbury:

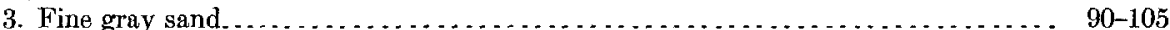

Sankaty:

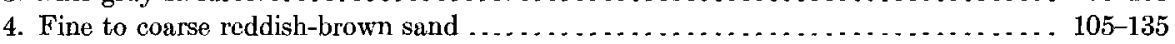

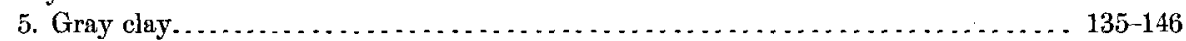
Jameco:

6. Dark multicolored sand and gravel with some clay (glacial) $146-154$

Elevation of ground, 12.7 feet Brooklyn basc; water was found below the blue clay, and water level was originally 9 feet from the surface. The elevation of the water in this test well ranged from 11 to 17 feet below the surface in 1901 . 
196. The following records have been compiled from the manuscript. reports of Mr. Peter C. Jacobson which were kindly placed at our disposal by chief engineer I. M. De Varona (see fig. 10):

Records of wells at Springfield pumping station.

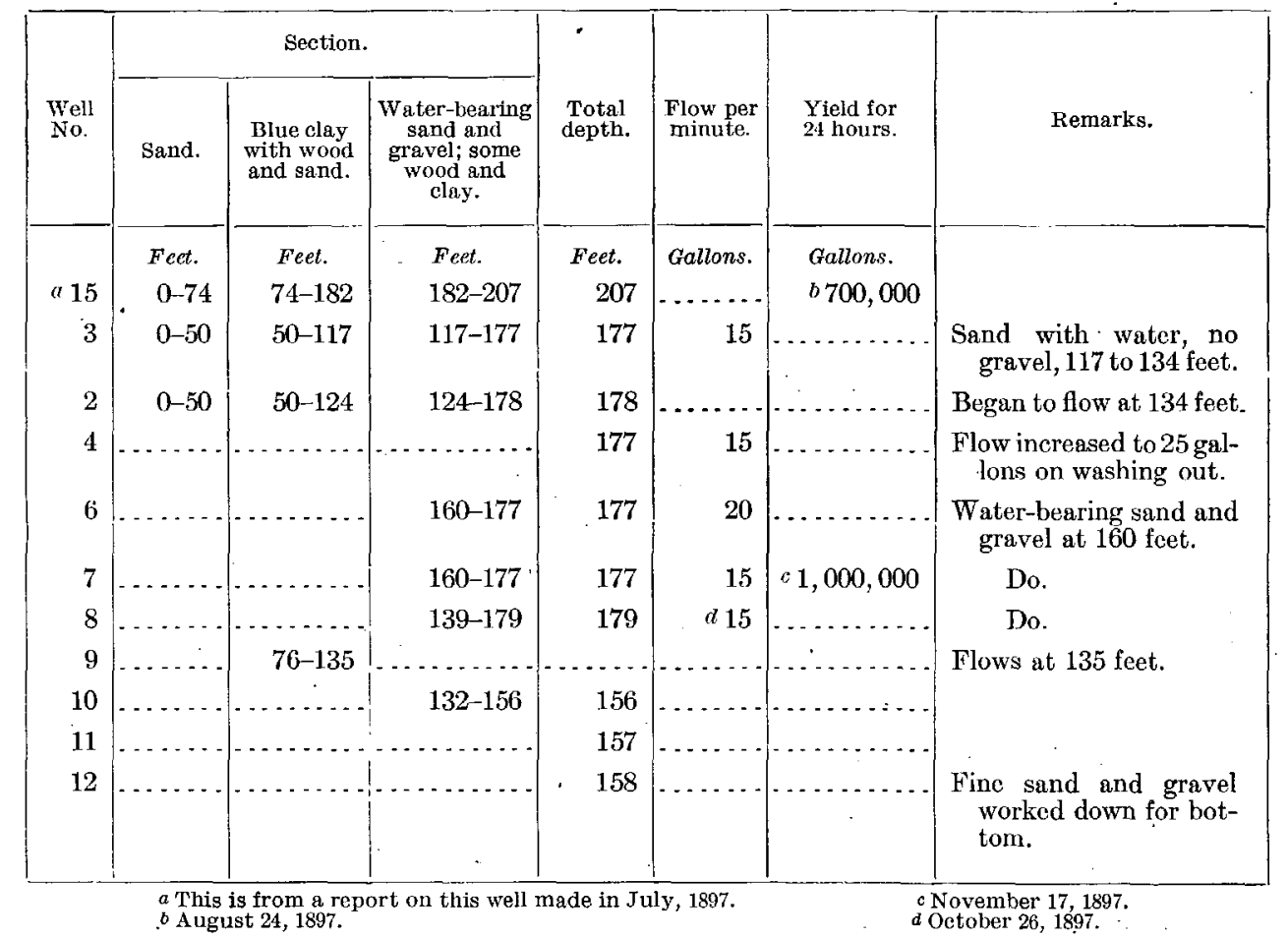

In the report for' June 7,1897 , the following record is given, apparently referring to well No. 15 :

Wisconsin:

Record of well 15 at Springfield pumping station.

Tisbury:

1. Sharp water-bearing sand

2. Fine hard packed sand with very little water.

$0-25$

Sankaty:

3. Blue clay with sand and gravel

Jameco:

4. Small gravel and sand with a large percentage of carbonized wood; water bearing; water level 6 inches above surface; will yicld with hand pump 75 gallons per minute; pumping with hand pump lowers it 6 feet; when pumping is stopped

the level of 6 feet is recovered in $\frac{1}{2}$ minute.......................... 123-129

5. Sand of various fineness containing carbonized wood and clay.............. 129-158

6. Gravel, sand, and a little clay mixed; water bearing. ................... 158-178

On June 14 it was stated that the flow of well 15 had increased to 9 gallons per minute and that its pumping capacity was almost a million gallons a day.

As no samples from these wells were preserved and as the data are very meager and somewhat confusing, it is not possible to arrive at a very satisfactory conclusion regarding the exact structure at this point. From the Jocation of the wells and from the data furnished by adjoining wells it is felt that the water-bearing sands and gravels are, in part at least, Jameco, and the blue clay, Sankaty. The locality is very near the eastern edge of the old Sound River Valley, and the irregularity of the lower part of this section is doubtless due to

$$
\text { 17116-No. 44-06-14 }
$$


the unevenness of the old land surface and the redeposition of the pre-Pleistocene materials. The statement of the inspector that the water-bearing stratum grows finer and the gravel less toward the west seems to indicate a rise in the old surface in that direction, as indicated in fig. 10. Toward the east it is known from the samples of well No. 197 that the pre-Pleistocene beds are very near the surface. All the data at hand point to the conclusion that the development at this place is in a small valley in the older beds.

\section{Analysis of Springfield Pond pump well at Springfield.}

[Surface water; analysis by Brooklyn health department.]

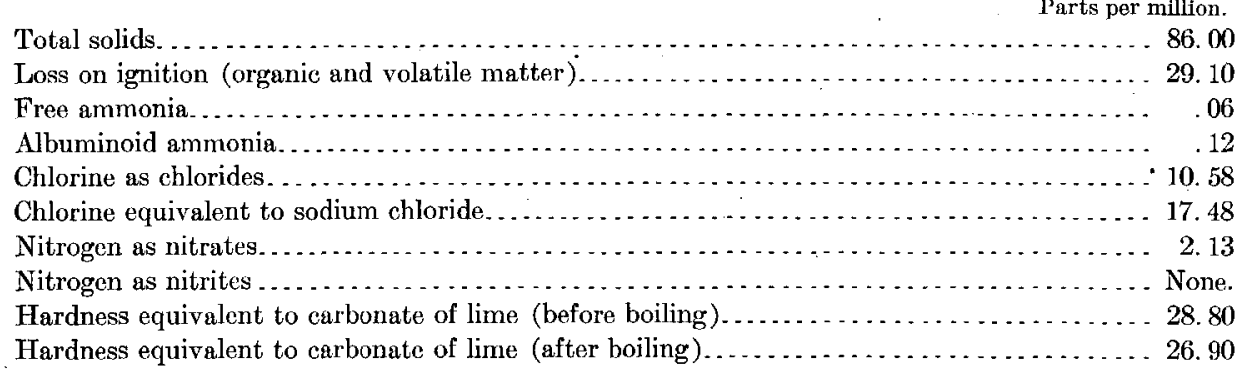

197. The following record has been prepared from the samples preserved by the Brooklyn waterworks in the municipal building, Brooklyn (see fig. 10) :

Wisconsin:

\section{Record of well near Springfield pumping station.}

1. Fine to medium, light, reddish-yellow sands. . . . . . . . . . . . . . . $0.0_{-3}$

2. Same, but a little lighter................................ $33 \quad 39$

3. Light, brownish-yellow, fine to medium sands.................. $39-54$

Tisbury:

4. Bright-yellow silt (looks like surface loam) $\ldots \ldots \ldots \ldots \ldots \ldots \ldots \ldots \ldots \ldots \ldots$

5. Fine olive-yellow sand.............................. $56 \quad 59$

6. Bright-orange fine to coarse sand........................... $59-77$

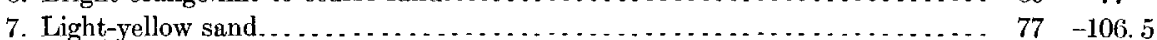
Jameco?:

8. Fine steel-gray sand with quartz, jasper, and ferruginous sandstone pebbles . . 106. 5-109. 5 Cretaccous:

9. Very dark-blue elay (different from clay above the glacial gravels) . . . . . . . 109. 5-130

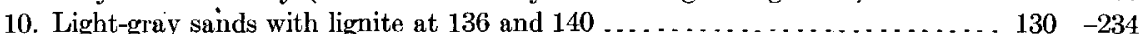

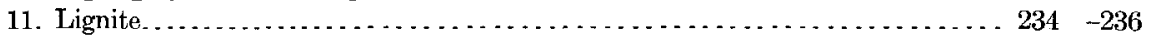

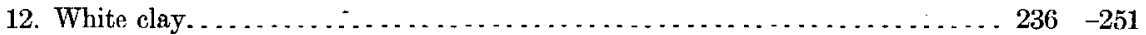

13. Dark-blue clay........................................... $251-258$

14. Fine gray sand ............................................. $258-271$

In addition to the samples preserved in the glass tube, there are a number of samples in cans which may be described as follows: 107-110, several large quartz, jasper, and ferrüginous sandstone pebbles; 110-125, lignite and gray clay; 125, lignite and pyrite; "130, specimen found in white sand October 25, 1895"-large pieces of lignitized wood, evidently part of a tree. Elevation of surface is 10.3 feet, Brooklyn base.

199. Section prepared from samples preserved by the Brooklyn water department, in the municipal building, Brooklyn:

$$
\text { Record of test well No. 18, near Oconee pumping station. }
$$

Wisconsin:

1. Dark reddish-brown loam.

Feet. $0-8$

Wisconsin and Tisbury:

2. Fine to coarse, light, reddish-yellow sand.

$9-56$

Tisbury:

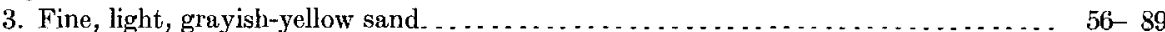

4. Dark, medium, reddish-brown sand................................ $89-115$ 
Sankaty:

Feet.

5. Fine gray clay. $115-185$

Jameco:

6. Dark, multicolored, very coarse sand (glacial)

$185-192$

Elevation of surface, 10.3 feet; average height of water in December, 1901, 17 feet from the surface; in November of the same year, 16.2 .

200. Section prepared from samples preserved by the Brooklyn water department, in the municipal building, Brooklyn (see fig. 10):

Record of test well at Baisley's pumping station.

Wisconsin:

1. Yellowish sand and gravel

Feet.

2. Fine yellow sand

$0-21.5$

3. Coarser yellowish sand.

$21.5-34$

Tisbury:

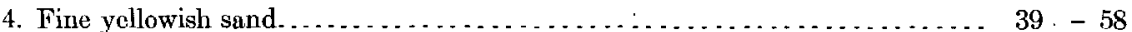

5. Gray sand and gravel................................. $58 \quad 77.5$

6. Gray sand......................................... $77.5-97.5$

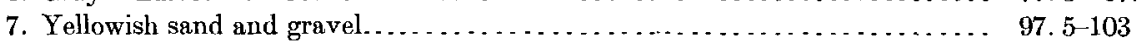

8. Yellowish sand, gravel, and clay .......................... $103-106$

Sankaty:

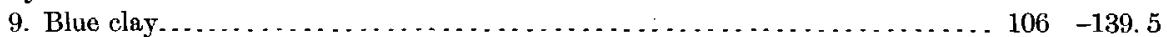

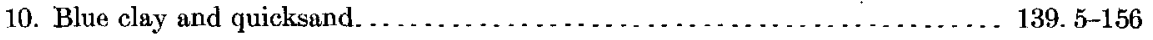
Jameco:

11. Black sand and gravel. ................................. $156-166$

12. Black sand............................................... $166 \quad-174$

13. Finer black sand. ....................................... $174-200$

Elevation of surface, 6.7 feet; see report of Andrews \& Bro., under No. 138.

Analysis of water from test well at Baisley's pumping station.

[By Brooklyn health department.]

Parts per million.

Total solids.

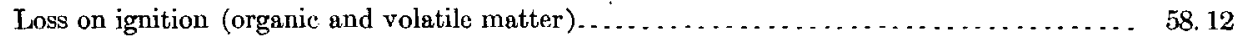

Free ammonia . . . . . . . . . . . . . . . . 06

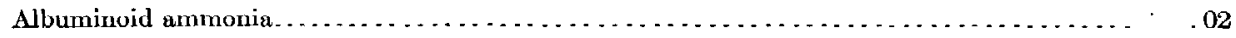

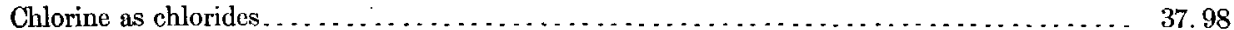

Chlorine equivalent to sodium chloride................................. 62.61

Nitrogen as nitrates. ... . . . . . . . . . . . . . . . . . . . . . . . . . . . . . . . . . 39

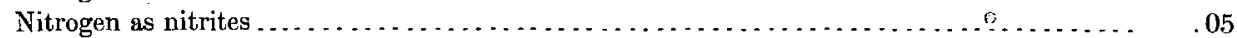

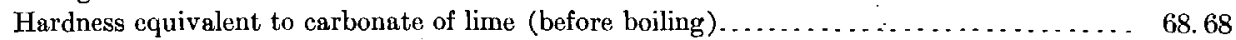

Hardness equivalent to carbonate of lime (after boiling) . . . . . . . . . . . . . . . . . 61.37 


\section{UNDERGROUND WATER RESOURCES OF LONG ISLAND, NEW YORK.}

201. The following summary of the material penetrated at the Jameco pumping station has been prepared from the samples preserved by the Brooklyn waterworks (see fig. 10):

Records of wells at Jameco pumping station.

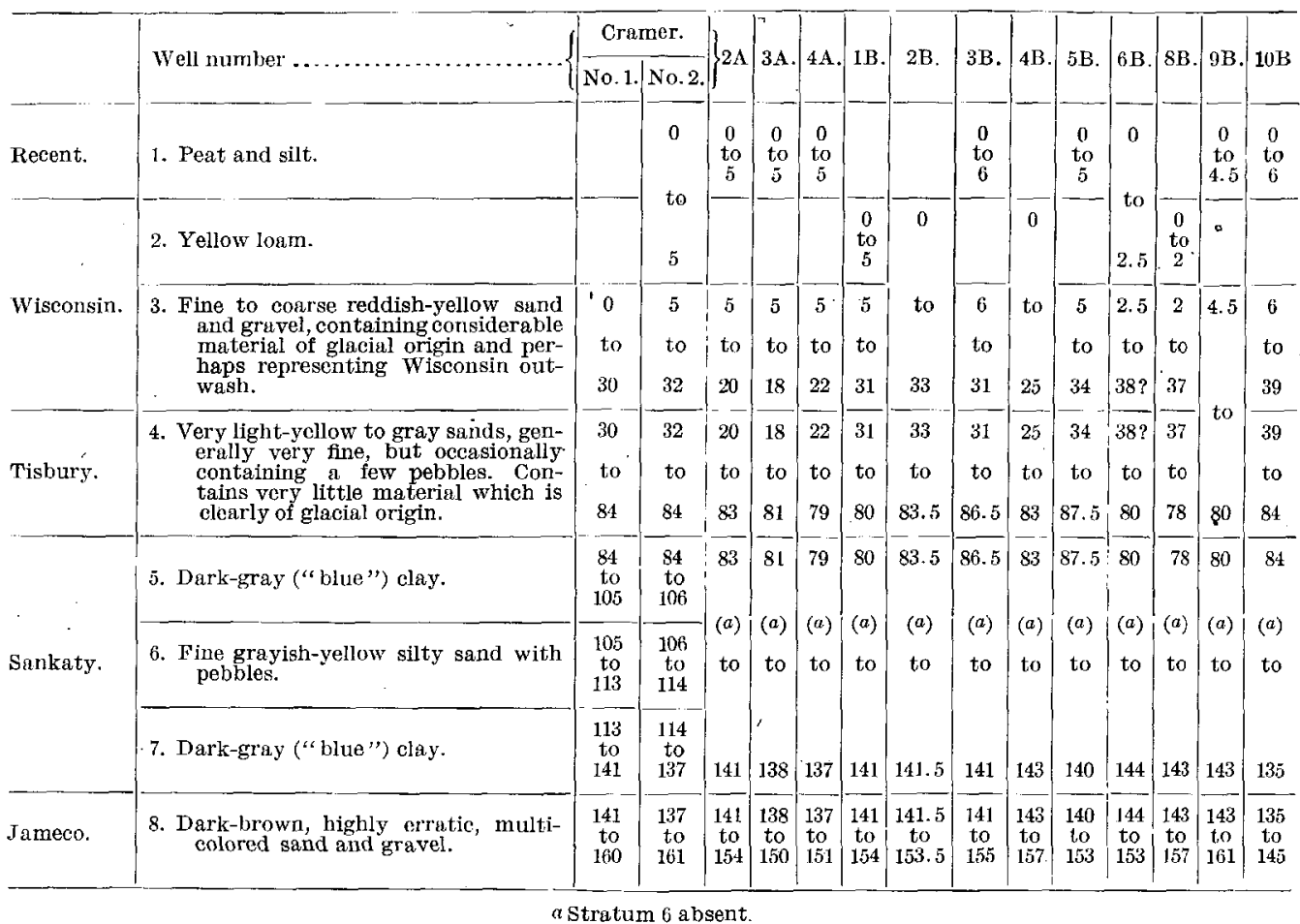

The 183 shallow-driven wells which originally constituted this station were supplemented by 7 deep wells. Data regarding these is presented by Chief Engineer I. M. De Varona in the following table:

Records of deep wells at Jameco pumping station.

\begin{tabular}{|c|c|c|c|c|c|c|}
\hline $\begin{array}{l}\text { No. of } \\
\text { well. }\end{array}$ & $\begin{array}{l}\text { Size of } \\
\text { well. }\end{array}$ & $\begin{array}{l}\text { Size of } \\
\text { suction. }\end{array}$ & $\begin{array}{l}\text { When } \\
\text { com- } \\
\text { pleted. }\end{array}$ & $\begin{array}{l}\text { Depth } \\
\text { driven. }\end{array}$ & $\begin{array}{l}\text { latate of nor- } \\
\text { mal flow per } 24 \\
\text { hours. }\end{array}$ & $\begin{array}{l}\text { Yield per } 24 \\
\text { hours, when } \\
\text { pumped. }\end{array}$ \\
\hline 186 & $\begin{array}{c}\text { Inches. } \\
4\end{array}$ & $\begin{array}{c}\text { Inches. } \\
2\end{array}$ & 1891 & $\begin{array}{ll}\text { Ft. } & \text { in. } \\
165 & 0\end{array}$ & $\begin{array}{l}\text { Gallons. } \\
30,240\end{array}$ & $\begin{array}{l}\text { Gallons. } \\
172,800\end{array}$ \\
\hline 185 & 4 & $2 \frac{1}{2}$ & 1892 & 163 & 34,560 & 158,400 \\
\hline 100 & 4 & $2 \frac{1}{2}$ & 1892 & $150 \quad 4$ & 129,600 & 403,200 \\
\hline & 4 & $2 \frac{1}{2}$ & 1893 & $157 \quad 4 \frac{1}{2}$ & 34,560 & 504,000 \\
\hline & 6 & $2 \frac{1}{2}$ & 1893 & $151 \quad 4 \frac{1}{2}$ & 684,000 & 720,000 \\
\hline & 6 & $4 \frac{1}{2}$ & 1893 & 1549 & 144,000 & 432,000 \\
\hline & 6 & $4 \frac{1}{2}$ & 1893 & 150,10 & 201,600 & 864,000 \\
\hline
\end{tabular}

In 1894 wells No. 100 and 186 were pulled up, cleaned, and redriven to depths of 157 fcet 8 inches and 160 feet 7 inches, respectively. After being cleaned the normal flow of well No. 100 was 4,320 gallons per day, and with a pump it yielded 20,160 gallons; well No. 186 flowed 5,760 gallons per day, which was increased to 60,480 gallons by pumping. No. 185 was tested without cleaning, and flowed 20,160 gallons, and with a pump yielded 90,000 gallons per day of twenty-four hours. 


\section{DESCRIPTIVE NOTES ON WELLS.}

The results from these wells were so satisfactory that arrangements were made with Messrs. Andrews \& Bro. to construct additional wells. Four 8-inch wells completed late in 1894 gave the following results:

Records of Andrews deep wells at Jameco pumping station.

\begin{tabular}{|c|c|c|c|c|}
\hline $\begin{array}{l}\text { No. of } \\
\text { well. }\end{array}$ & $\begin{array}{l}\text { Thickness of } \\
\text { sand stratum. }\end{array}$ & $\begin{array}{l}\text { Thickness of } \\
\text { clay stratum. }\end{array}$ & $\begin{array}{l}\text { Length of pipe } \\
\text { in water-bear } \\
\text { ing stratum. }\end{array}$ & $\begin{array}{l}\text { Normal yield } \\
\text { per } 24 \text { hours. }\end{array}$ \\
\hline $1 \mathrm{~A}$ & $\begin{array}{cc}\text { Ft. } & \text { in. } \\
82 & 0\end{array}$ & $\begin{array}{cc}\text { st. } & \text { in. } \\
55 & 6\end{array}$ & $\begin{array}{rr}\text { Ft. } & \text { in. } \\
10 & 4\end{array}$ & $\begin{array}{l}\text { Gallons. } \\
201,000\end{array}$ \\
\hline $2 \mathrm{~A}$ & $83 \quad 0$ & $59 \quad 4$ & $115 \frac{1}{2}$ & 144,000 \\
\hline $3 \mathrm{~A}$ & $81^{\circ} \quad 6$ & $57 \quad 9 \frac{1}{2}$ & 11 & 159,000 \\
\hline $4 \mathrm{~A}$ & $78 \quad 10$ & 58 & $12 \quad 7 \frac{1}{2}$ & 222,000 \\
\hline
\end{tabular}

In January, 1895, a test was made of these wells extending over a period of twelve days, during which time the wells were run under various combinations, from singly to all four together; the gaging showed an average daily delivery of over $1,000,000$ gallons when one well was being pumped, and $3,500,000$ gallons with the four wells connected. During the period of observations the elevation of the underground water at the 2-inch test wells, Nos. 8 and 9, at Jameco (each of which was about 140 feet deep), and the deep test well at Baisley's station (No. 200), about one-half mile distant, was noted. The lowering of the water at the station was approximately 5 feet when $1,000,000$ gallons were being pumped, and 10 feet when the delivery was 3,500,000 gallons. The greatest lowering shown at Baisley's deep test well was slightly over 4 feet. The effect of the rise and the fall of the tide on the level of the ground water could not be taken into account at the time in determining the lowering of the water.

Early in 1895 Mr. C. P. Cramer, of Paterson, N. J., completed a 10-inch well 160 feet deep, which flowed 150,000 gallons in twenty-four hours. A test of the four 8-inch Andrews wells (Nos. $1 \Lambda, 2 \mathrm{~A}, 3 \mathrm{~A}$, and $4 \mathrm{~A}$ ) and the 10-inch Cramer well (No. 5A), was made from December 9 to 28,1895 , the wells being run singly and in groups of from 2 to 5 . Elevations of the deep underground water level were taken at the Jameco test wells Nos. 8 and 9, at the 5-inch test wells Nos. 1, 2, 4, 5, 7, and 11, and at Baisley's deep test well. The average daily yield per well was approximately $1,000,000$ gallons, with nearly a pro rata increase for each well connected, making the total yield about 5,000,000 gallons. During the test, lasting twenty days, the total amount pumped was $61,239,555$ gallons, and when pumping the maximum of $5,000,000$ gallons daily the greatest. lowering of water at Jameco was slightly over 14 feet.

The greatest lowering of water in the deep test wells during the above test is given as follows:

Depth to which water level in neighboring deep test wells was lowered by pumping at Jameco station, December 9-28, 1895 .

Jameco test well No. 8

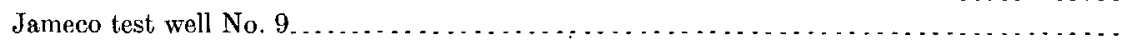

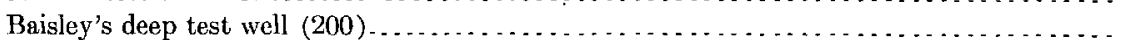

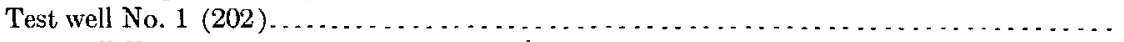

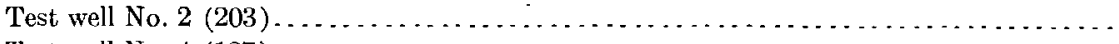

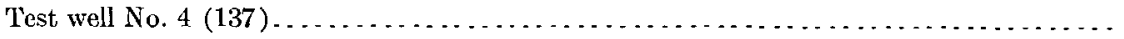

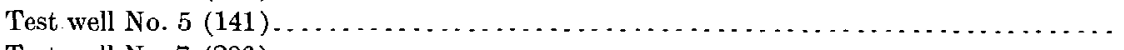

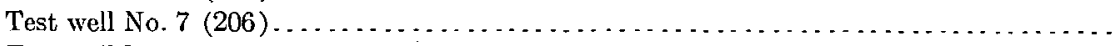

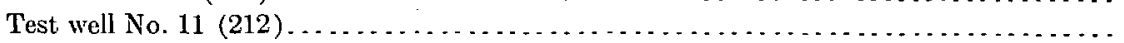

The locations of these wells are shown on PI. XXIV. 
Analyses of waters from wells at Jameco pumping station.

[By the Brooklyn health departmènt.a Parts per million.]

\begin{tabular}{|c|c|c|c|c|c|}
\hline & $\begin{array}{c}\text { Old } \\
\text { driven } \\
\text { wells } \\
\text { (shal- } \\
\text { low). }\end{array}$ & $\begin{array}{c}\text { Old } \\
\text { driven } \\
\text { wells } \\
\text { (deep). }\end{array}$ & $\begin{array}{l}\text { No. 2A } \\
\text { (An- } \\
\text { drews). }\end{array}$ & $\begin{array}{l}\text { No. 4A } \\
\text { (An- } \\
\text { drews). }\end{array}$ & $\begin{array}{c}\text { No. 5A } \\
\text { (Cramer). }\end{array}$ \\
\hline Number of analyses. : & 2 & 1 & 4 & 3 & 1 \\
\hline Total solids........ & 174.50 & 125.00 & 119.25 & 123.66 & 138.00 \\
\hline $\begin{array}{l}\text { Loss on ignition (organic and } \\
\text { volatile matter) }\end{array}$ & 44.50 & 20.00 & 15.50 & 19.33 & 35.00 \\
\hline Mineral matter........ & 130.00 & 105.00 & 103. 75 & 104.33 & 103. 00 \\
\hline Free ammonia.......... & .48 & .78 & .77 & .61 & 1.04 \\
\hline Albuminoid ammonia... & .14 & .15 & .07 & .7 & .00 \\
\hline Chlorine as chlorides... & 32.50 & 4. 50 & 9.37 & 6.66 & 6.00 \\
\hline Sodium chloride..... & 53.56 & 7. 42 & 15.44 & 10.98 & 9. 89 \\
\hline Nitrogen as nitrates... . & .42 & .71 & .31 & .34 & .00 \\
\hline Nitrogen as nitrites. . . . . & None. & None. & None. & None. & None. \\
\hline Total hardness. ......... . & 50.75 & 92.00 & 70.00 & 72.83 & 60.50 \\
\hline Permanent hardness. . ... . . & 50.75 & 87.00 & 40.25 & 69.16 & 60.50 \\
\hline
\end{tabular}

a Ann. Rept. Comm. City Works, Brooklyn, 1895, pp. 139, 141

A letter from W. D. Andrews \& Bro., dated May 8, 1895, gives the following: "In 1890 at Jameeo Park we, on our own account, sunk test wells 4,5 , and 6 inches in diameter. From veins of water varying in depth from 30 to 160 feet the water rose 10 feet above the surface. The natural flow from one 4 -inch open-cnded pipe was 90 gallons per minute. Another 6 -inch tube delivered at the ground level 500 gallons per minute and rose inside of the tube 11 feet above the surface. During Major Boody's term we made several 6-inch wells at Jameco station having an average depth of 150 feet and a natural flow at the surface of 120 to 180 gallons per minute."

202. The following section has been prepared from samples preserved by the department of water supply, municipal building, Brooklyn:

Record of Brooklyn test well No. 1, Brooklyn aqueduct and Cornell Creek.

Wisconsin:

1. Light yellowish sands and gravel, glacial. . . . . . . . . . . . . . . .

Tisbury:

2. Fine, yellowish-gray, "pepper and salt" sand.................... 54-62

3. Fine yellowish-white sand...................................... $62-75$

4. Grayish white silty sand and gravel (very few glacial pebbles) .............. . $75-89$ Sunkaty:

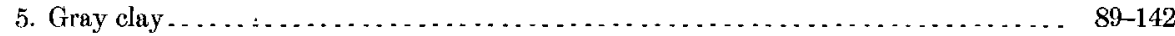

Jameco:

6. Dark multicolored sands and gravel............................. 142-156

"When the well casing was worked down to the surface of the ground the flow was 30 gallons per minute. The normal level of the water in the strata below the clay bed was 0.75 foot above the surface of the ground." a

a Ann. Rept. Dept. City Works, Brooklyn, for $1895,1896, p_{\diamond} 343$. 
Analysis of water from Brooklyn test well No. 1, Brooklyn aqueduct and Cornell Creek.

[By Brooklyn health department.]

Total solids

Parts per million.

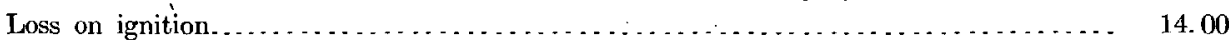

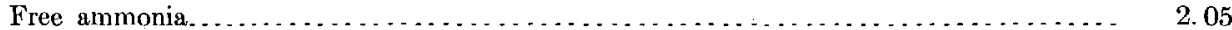

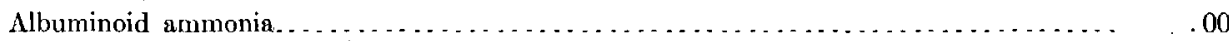

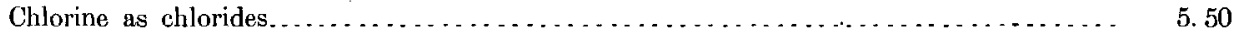

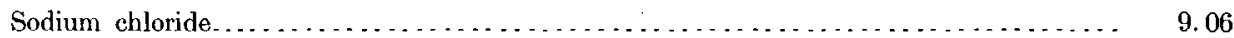

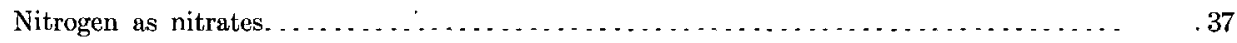

Nitrogen as nitrites. . . . . . . . . . . . . . . . . . . . . . . . . . . . . . . . . . None

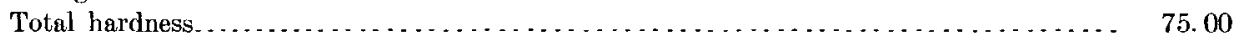

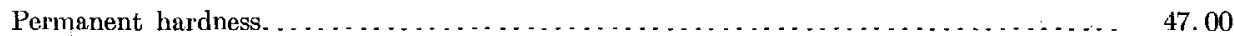

203. The following section has been prepared from the samples preserved by the Brooklyn water department (see fig. 10):

Record of Brooklyn test well No. 2, Brooklyn aqueduct and Rockaway road.

Wisconsin:

Tisbury:

2. Fine light, yellowish gray, "pepper and salt" sand .................. 19-43

3. Fine, darker, yellowish gray sand; some pebbles near bottom of layer....... 43- 72

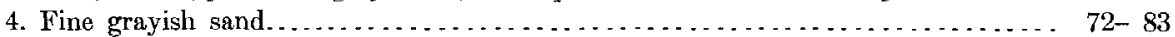

Sankaty:

5. Dark-gray silty clay...................................... $83-140$

6. Very fine, dark-gray, sandy silt. . . . . . . . . . . . . . . . . . . . . . . Jameco:

7. Dark multicolored sands and gravels; only a small percentage of quartz (pro-

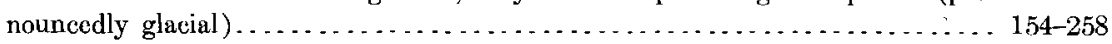

"At a' depth of 169 feet the water rose in the well to within 18 inches of the surface. When the pipe was down to 239 feet, the top of the pipe being 2.25 feet . below the surface of ground, the flow was 5 gallons per minute." Elevation 7.4 feet, Brooklyn base.

Analysis of water from Brooklyn test well No. 2, Brooklyn aqueduct and Rockaway road.

[By Brooklyn health department.]

Total solids.

Parts per million.

.

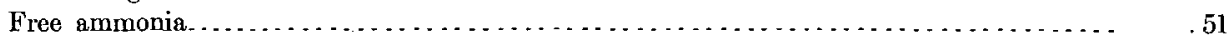

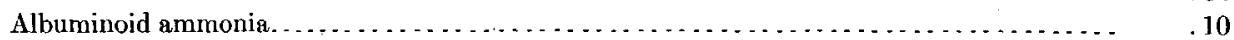

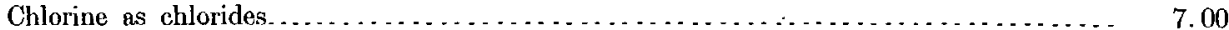

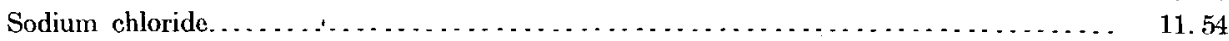

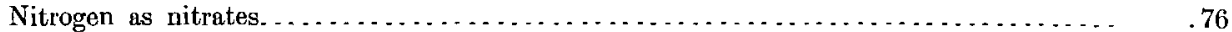

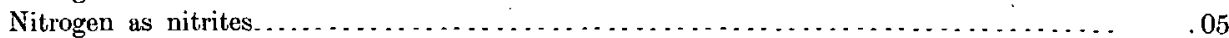

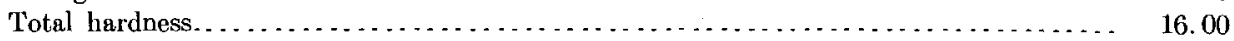

Permanent hardness. . . . . . . . . . . . . . . . . . . . . . . . . . . . . . . . . . 16.00

204. The following section has been prepared from the samples preserved by the Brooklyn water department (see fig. 10): 
Record of Brooklyn test well No. 3, Brooklyn aqueduct and New York avenue.

Wisconsin:

1. Reddish yellow silty sand and gravel........................ 0 . 9

Wisconsin and Tisbury:

2. Fine to coarse reddish yellow sand with pebbles in lower portion (glacial).... 9-45 Tisbury:

3. Fine light-yellow sand..................................... $45-86$

Sankaty:

4. Dark-gray, silty, lead-colored clay ............................... . $86-139$

5. Very fine, dark-gray, silty sand. . . . . . . . . . . . . . . . . . . . . . 139-158

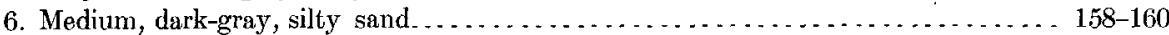

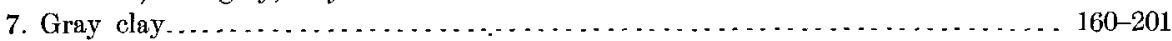

Jameco:

8. Dark, multicolored, silty, fine to coarse sand (glacial)

$201-277$

Elevation 9.8 fect, Brooklyn base.

In addition to the samples preserved in the glass tube a number were found in a can marked "Third, 5-inch test well;" they are as follows: "69 feet clay," light-gray silty clay; " 72 feet wood," small pieces of peat, evidently a swamp deposit; " 140 to 158 feet wood," fragments of lignitized driftwood; " 161 to 202 feet wood," lignitized pieces of driftwood. "No water was found in the strata below the clay bed."

205. The following section has been prepared from the samples preserved by the Brooklyn water department (see fig. 10):

Record of Brooklyn test well No. 8, Brooklyn aqueduct and Farmers avenue.

Wisconsin:

Tisbury:

2. Light, brownish yellow, fine to coarse sand..................... $27-59$

3. Fine speckled gray sand ................................... $59-72$

Sankaty:

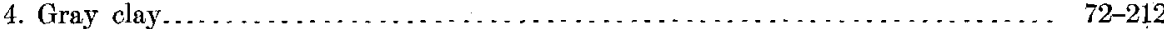

Jameco:

5. Dark, multicolored, fine to medium, dirty glacial sand (same as 8 in well 204)... 212-260

Cretaceous:

6. White micaccous sand.

$260-293$

Elevation 10 feet, Brooklyn base.

The following samples were preserved in can marked "Eighth, 5-inch test well:" " 59.7 to 72.3 feet, specimens found in gray sand October 7, 1895"--water rolled twigs (only slightly lignitized), water rolled pieces of lignite, and large flakes of muscovite; " 258 to 275 feet, specimens found in sharp white sand October 14, 1895," fragments of lignitized wood; " 258 to 275 feet," several small pieces of yellow amber, and a piece as large as a pigeon's egg of yellow gum. No water was found in the strata below the clay bed.

206. The following section has been prepared from the samples preserved by the Brooklyn water department (see fig. 10):

Record of Brooklyn test well No. 7, Brooklyn aqueduct, northwest of Springfield pumping station.

Wisconsin:

Feet.

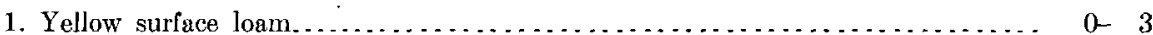

Tisbury:

2. Light, multicolored, clean, fine to coarse sand.................... $3-20$

3. Clean, reddish yellow, fine to coarse sand......................... $20-32$

4. Dirty yellowish-white, medium, "pepper and salt" sand............... 32-43

5. Fine to coarse, dark, yellowish gray sand......................... $43-65$ 
Sankaty: - . Feet.

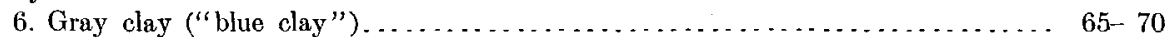

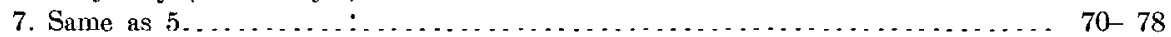

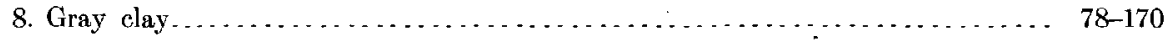
Jameco:

9. Reddish yellow multicolored sand and pebbles (glacial) $\ldots \ldots \ldots \ldots \ldots \ldots \ldots \ldots$. $\ldots \ldots$. $\ldots \ldots 3$

Cretaceous:

10. Fine to coarse white sand with a few slightly darker quartz pebbles below . . 183-420

The following samples are preserved in cans:

" 90 to 95 feet," pieces of gray clay with vegetable matter, apparently marsh or swamp deposit.

" 88 feet, drilled through something hard for about a foot, presumably a log, as these fragments of wood were washed up." "These fragments of wood" prove to be pieces of peat made up of parts of many small plants closely compacted.

"230 feet," large pebbles of rose quartz, much disintegrated felspathic rock, black chert, banded limestone, ferruginous sandstone, conglomerate, iron pyrite, and lignite.

"Contained in gravel washed up from a depth of 171 feet"--fragments of soft red Newark sandstone.

"Pieces of wood washed up from a depth of 196 feet September 6, 1895"-lignitized wood, evidently parts of a $\log$.

As the material in the tube from 183 to 420 is clearly not glacial, the sample from 230 shows some disagreement. According to the tube samples the glacial material ended at 182 feet, while according to the samples in the cans it extends to at least 230 feet.

Elevation of surface, 10 feet Brooklyn base. "No water was found in the strata below the clay bed."

207. Record of commission's test well near New York and Locust avenues, south of Jamaica.

Wisconsin:

Feet.

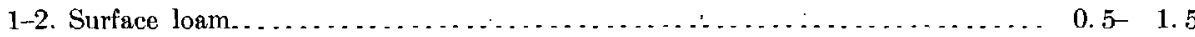

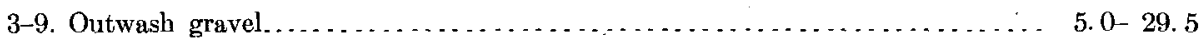

See Table XII.

208.

Record of commission's test well on Rockaway road.

Wisconsin: Feet. 1- 2. Yellow surface loam . . . . . . . . . . . . . . . . . . .

3-10. Outwash gravel with quite a considerable percentage of erratic material... 5- 31 See Table XII.

209. Record of commission's test well, 2 miles south of Dunton.

Wisconsin:

Feet.

$0-2$

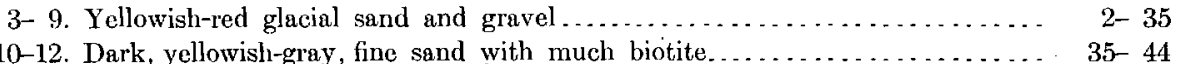

210.

Record of commission's well near Morris Park.

Wisconsin and Tisbury :

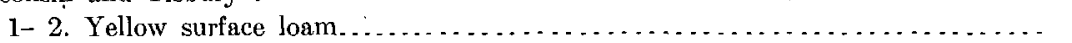

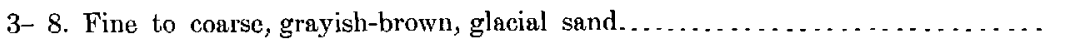

9-12. Dark steel-gray sand (glacial)

Feet.

$0-1$

$5-31$

$31-50.5$

211.

Record of commission's test well near Jamaica.

Wisconsin:

1- 3. Filled ground

Feet.

$0-4$

4-12. Dark-gray fine sand and gravel with much biotite and erratic material.... 4 4 41

13. Small, multicolored, glacial gravel with much erratic material......... $41-43$ 
Tisbury:

14-27. Dark-gray line to medium sand.

28-30. Medium to coarse light-yellow sand with a very small percentage of glacial material

Sankaty:

31-33. Blue sandy clay

212. The following section has been prepared from the samples preserved by the Brooklyn water department (see fig. 13):

Wisconsin:

$$
\text { Record of Brooklyn test well No. 11, near Jamaica. }
$$

to coarse light sand with son

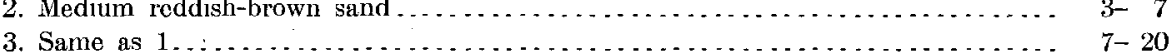

Tisbury:

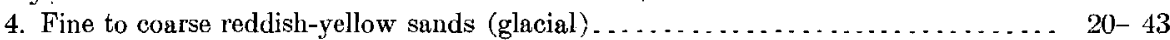

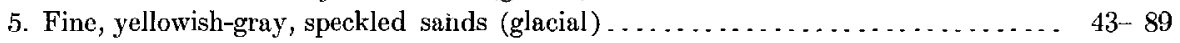

6. Coarser yellowish gray sand with gravel......................... $89-95$ Sankaty:

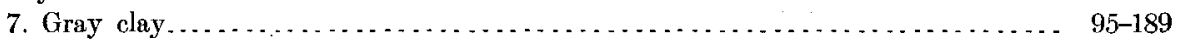

Jameco:

8. Dark multicolored fine to coarse sand (glacial)

$189-200$

Elevation of surface, 19.2 feet. Between 190 and 198 feet below the surface large quantities of water were found.

213. Mr. C. A. Lockwood has kindly furnished the following record of a deep well puit down at the pumping station of the Jamaica Water Supply Company (see fig. 13):

Record of well at pumping station of Jamaica Water Supply Company, Jamaica.

\begin{tabular}{|c|c|c|}
\hline isconsin and Tisbury: & & \\
\hline 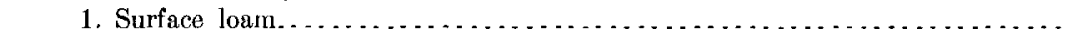 & & \\
\hline 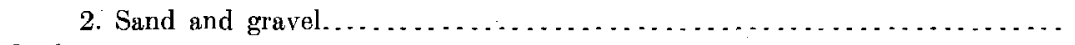 & & $5-60$ \\
\hline ankaty: & & \\
\hline 3. Blue clay... & 60 & -104 \\
\hline imeco: & & \\
\hline 4. Coarse sand and reddish gravel. . & 104 & -120 \\
\hline retaceous: & & \\
\hline 5. Blue clay like $t$ & 120 & -140 \\
\hline hter in color than preceding...... & 140 & -156 \\
\hline strata 3 and $5 \ldots \ldots \ldots \ldots \ldots \ldots \ldots \ldots \ldots \ldots \ldots$ & 156 & -175 \\
\hline 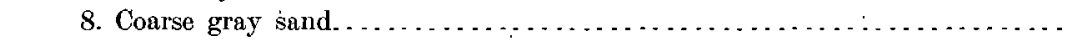 & 175 & -235 \\
\hline 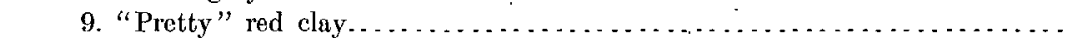 & 235 & -239 \\
\hline & 239 & -240 \\
\hline 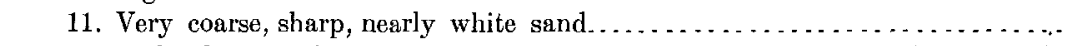 & 240 & - \\
\hline 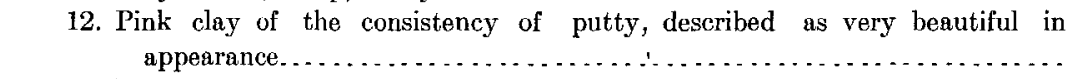 & & 241 \\
\hline 13. Lign & 241 & -242 \\
\hline 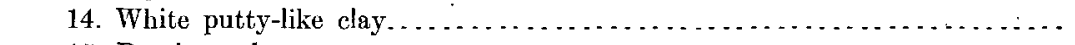 & 242 & -243 \\
\hline 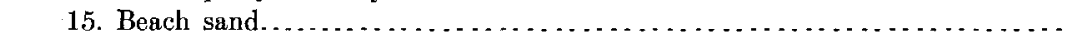 & 243 & -352 \\
\hline
\end{tabular}

Near this well another well was put down to a depth of 330 feet, when work was discontinued because of the great amount of lignite encountered. The first clay bed. in the second well was of somewhat less thickness than in the first. Clam shells are reported at various depths. The water in this well contains considerable quantities of iron. 
Other wells at the Jamaica pumping station are as follows: One 8-foot brick-curb well 57 feet deep; one 8 -inch tile well 50 feet deep; one 10 -inch well 150 feet deep. The material above the first layer of clay in these wells varies in different localities from sand and gravel to a red or gray sand and in some places to quicksand.

Mr. Lockwood reports that the capacity of the entire series of wells is $7,000,000$ gallons a day, but that only $3,000,000$ gallons a day are actually pumped during the summer months, and that the average for the year is from $2,275,000$ to $2,500,000$ gallons a day. 'The smallest wells at the station are 5 inches in diameter and out of a single one of these 250,000 gallons a day is pumped.

In 1886 if the 10-inch and 5-inch wells at the station were allowed to remain without pumping, it took the water five seconds to recover its natural level. In $1903 \mathrm{it} \mathrm{took} \mathrm{four} \mathrm{and} \mathrm{one-half} \mathrm{minutes} \mathrm{to} \mathrm{recover.} \mathrm{In} \mathrm{the}$ interval of seventeen years the water level has been lowered about 1 foot.

Analysis of well water from pumping station of Jamaica Water Supply Company, Jamaica.

[By Brooklyn health department, July 31, 1903. Analyst, Richard J. Reilly, assistant chemist.]

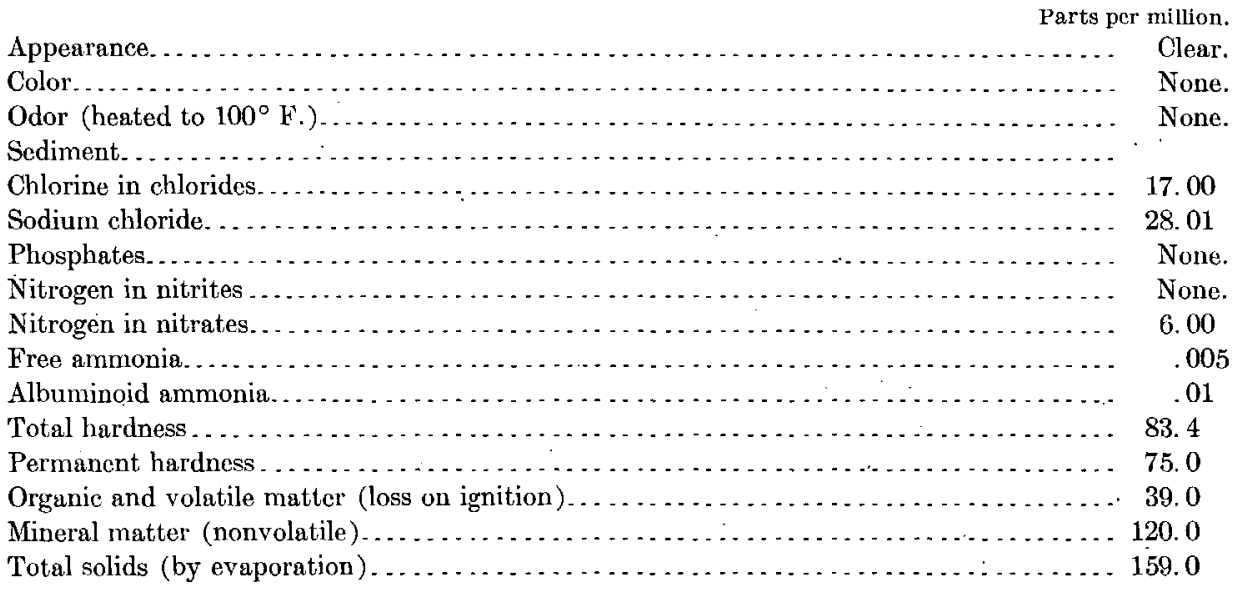

Analysis of well water from pumping station of Jamaica Water Supply Company, Jamaica.

[By Long Island Railroad Company, May, 1897.]

Parts per million.

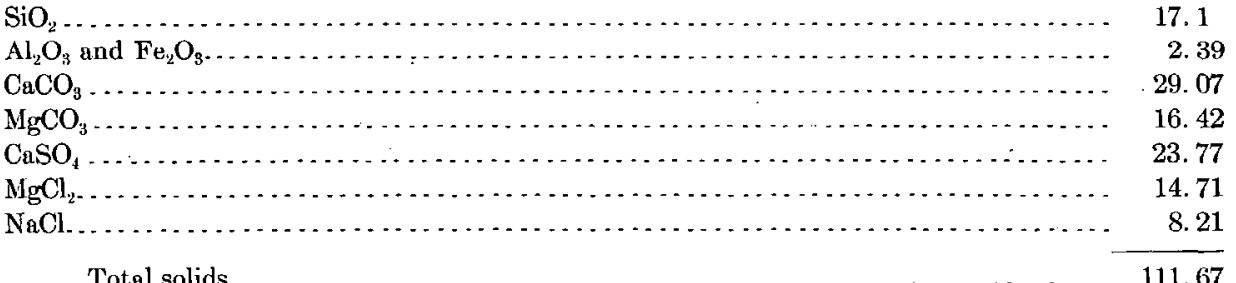

Total solids. . . . . . . . . . . . . . . . . . . . . . . . . . . . . . . . . . . 111.67

214.

Record of commission's test well near Jamaica.

Wisconsin:

Fect.

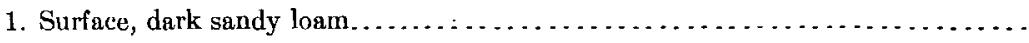

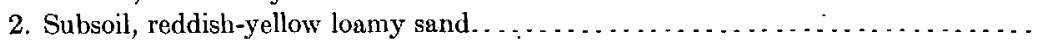

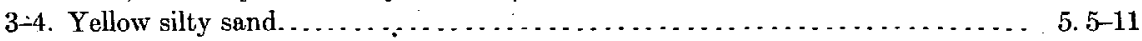

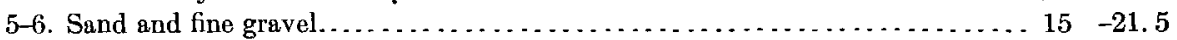

7-8. Sand bucket sample shows sand with a considerable percentage of fine gravel, and a wash sample shows reddish yellow sand. 


\section{UNDERGROUND WATER RESOURCES OF LONG ISLAND, NEW YORK.}

215.

Record of commission's test well near Jamaica.

Wisconsin:

1-2. Surface loam . . . . . . . . . . . 0 -

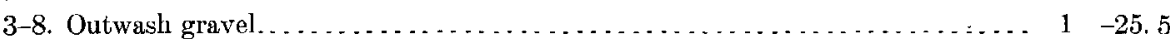

See Table XII

217.

Record of commission's test well near Springland.

Wisconsin.

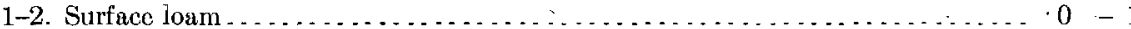

3-8. Outwash sand and gravel with much biotite. . . . . . . . . . . . . . . . . . . . $3 \quad-24.5$

See Table XII.

216.

Record of commission's test well near Jamaica.

Wisconsin:

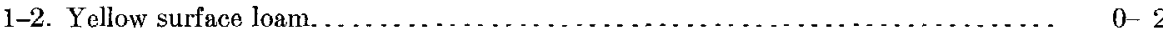

3-5. Outwash material increasing in coarseness with depth............. $5-16$

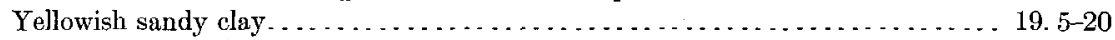

6-8. Reddish-brown outwash sand and gravel . . . . . . . . . . . . . . . . . . . . . . . $21 \quad 32$ See Table XII.

218.

Wisconsin and Tisbury?

Record of commission's test well near Queens.

Yellow loamy sand........................ 5-5.5

4-5. Light, grayish yellow, outwash sand and gravel . . . . . . . . . . . . . . . 10-16

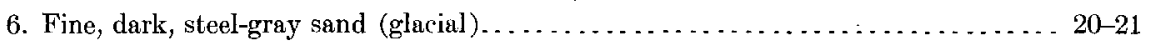

7-11. Light, grayish yellow, outwash sand and gravel. . . . . . . . . . . . . 26-60

Sec Table XII.

219A. This is a small private, high-service system, which draws its water from the mains of the Jamaica Water Supply Company and supplies an area of about 195 acres.

220. The following section has been prepared from the samples preserved by the Brooklyn water department:

Wisconsin:

Record of Brooklyn test well No. 7, near Hollis.

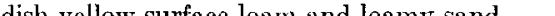

Wisconsin and Tisbury:

2. Light, reddish yellow, multicolored sands and gravel (glacial)

$0-15$ Tisbury:

3. Medium-light grayish yellow sand . . . . . . . . . . . . . . . . . . . $69-77$

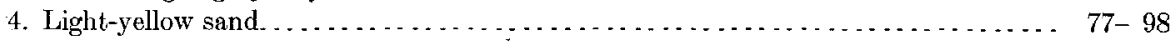

Cretaceous?:

5. Very fine, gray, silty clay ("blue clay") . . . . . . . . . . . . . . . . . . . . 98-103

Cretaceous:

6. Reddish yellow sand and gravel, with muscovite.................... 103-117

7. Light, yellowish white, medium sands . . . . . . . . . . . . . . . . . . . . . . . 117-144

8. Darker yellowish white sands. . . . . . . . . . . . . . . . . . . . . . . . . . . . 144-157

9. Light, yellowish white, fine to medium sands. . . . . . . . . . . . . . . . . . $157-217$

10. White quartz pebbles. . . . . . . . . . . . . . . . . . . . . . . . . . . . . . . 217-224

11. Fine to coarse, light, ycllowish sands . . . . . . . . . . . . . . . . . . . . . . . . . 224-294

12. F ne pink sands. . . . . . . . . . . . . . . . . . . . . . . . . . . . . . . . . . . . . . 294-297

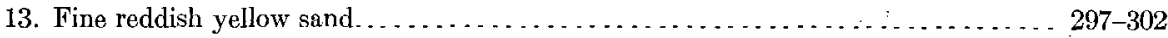

14. Dark blue-gray clay . . . . . . . . . . . . . . . . . . . . . . . . . . . . . . . . . . . . . 302-319

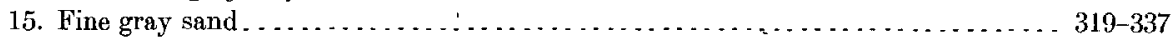

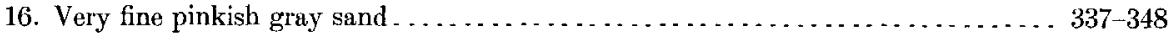

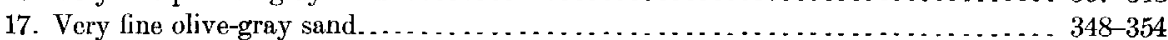

18. Alternate layers of very fine and fine pinkish gray sand........ . . . . . . . $354-369$ 
Cretaceous-Continued.

Feet.

19. Fine light-gray sand

369-401

20. Medium dark-gray sand. ... . . . . . . . . . . . . . . . . . . . . . . . . . . . . 401-403

21. Very fine very dark-gray sand. 403-407

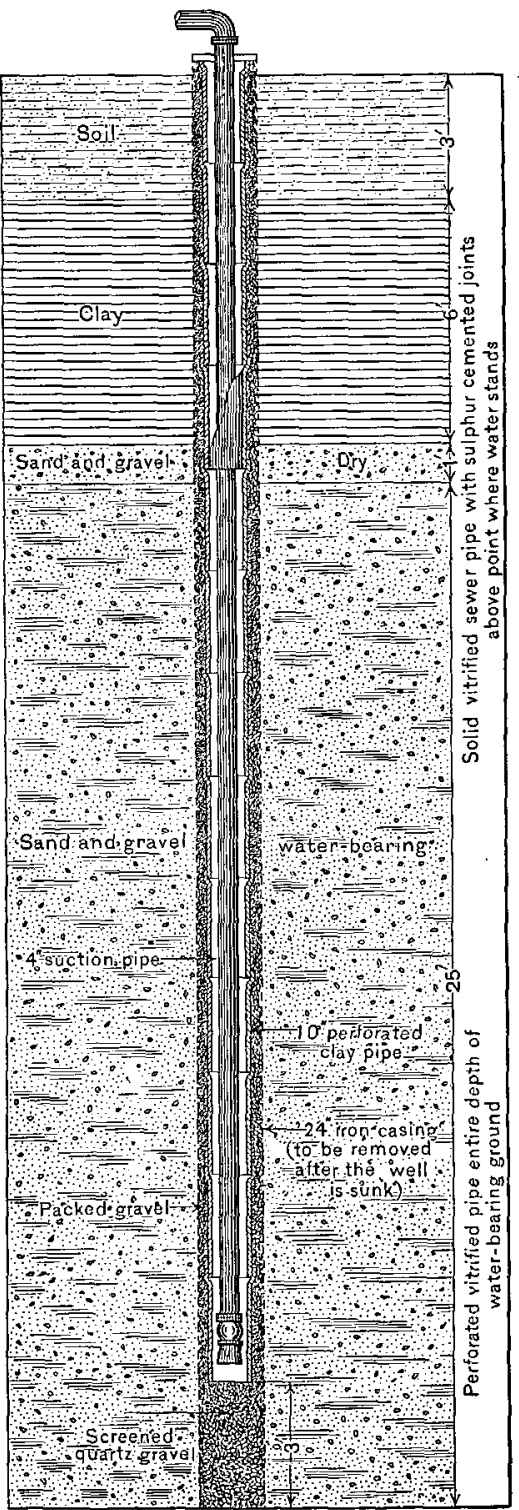

F'ig. 63.-Type of well used at the Montauk waterworks plant at Dunton, N. Y.

Elevation, 58.6 feet, Brooklyn base. The samples below No .7 all have cons derable muscovite and resemble the yellow Cretaceous sands of the old Westbury section (well No. 430) and the Melville section in the West Hills.

221. Record of commission's test well near Woodhull Park.

Wisconsin:

Feet.

1-2. Yellow surface loam ........... 0 - 5.2

3. Very fine dark-gray clayey sand ..... 10-11

4-8. Highly erratic outwash sand and gravel.................... 15-29

See Table XII.

222. Record of commission's test well near West Jamaica.

Wisconsin and Tisbury?: Feet.

1. Surface sandy loam . . . . . . . . . . . . $0-2$

2-4. Reddish brown fine to coarse glacial sand ................... $2-20$

5-9. Medium gray sand with much biotite _ 20-52

223. The plant of the Montauk Water Company, situated at Dunton, consists of eighteen 10 -inch tile wells having an average depth of 50 feet. The type of the well and the character of strata penetrated is shown in the accompanying figure (fig. 63). Mr. C. A. Lockwood gives the following section of a well completed by him at this point:

Record of Montauk Water Company's well at Dunton.

Wisconsin and Tisbury?:

2. Blue clay . . . . . . . . . . . . . . . $8-24$

3. Coarse gray sand and gravel ...... 24-64

The following analyses were reported by the Long Island Railroad Company, April, 1897, and September, 1901, respectively: Analyses of water from Montauk Water Company's well at Dunton. Parts per million.

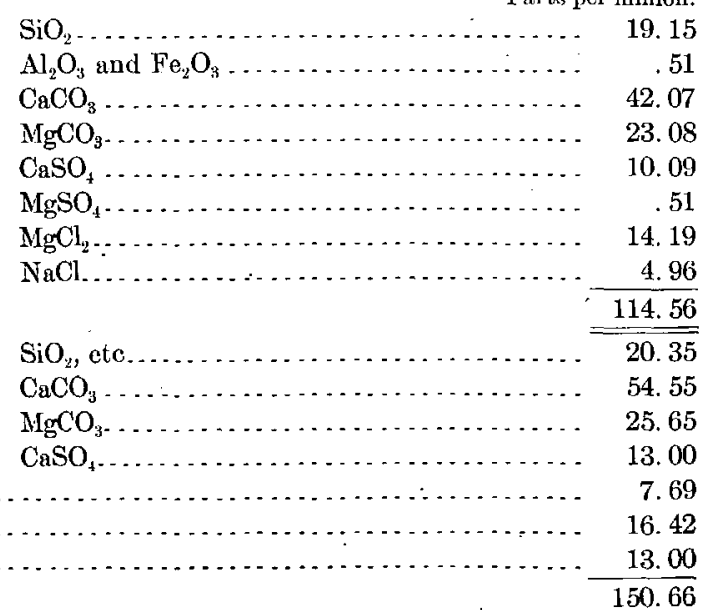

An excellent boiler water, but forms some scale. 


\section{UNDERGROUND WATER RESOUROES OF LONG ISLAND, NEW YORK.}

Analysis of water from Montauk Water Company's well at Dunton.

[Analyst, H. B. Hodges.]

Parts per million

Silica oxide of iron and alumina.

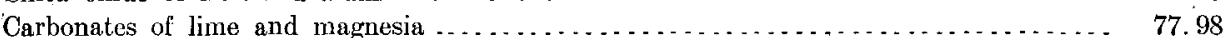

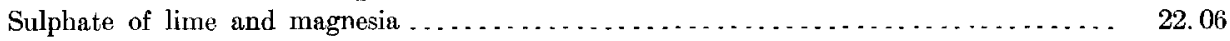

Chlorides .....

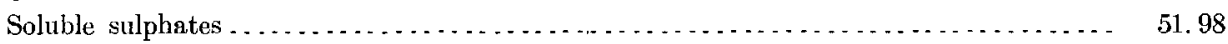

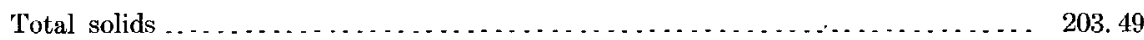

224. Record of commission's test well near Willow Glen.

Wisconsin: $\quad$ Feet.

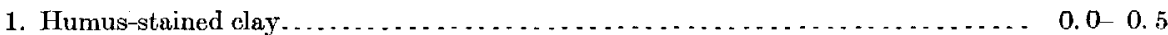

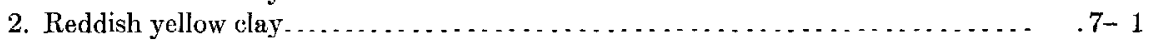

3-4. Very fine, reddish-yellow, clayey sand......................... $7 \quad-14$

$5-6$. Very fine, dark-gray, glacial sand. . . . . . . . . . . . . . . . . . . . 19 -25

225. The average section at this point is reported as follows:

Record of wells of Citizens' Water Supply Company at head of Flushing Creek.

Wisconsin and Tisbury?: $\quad$ Feet

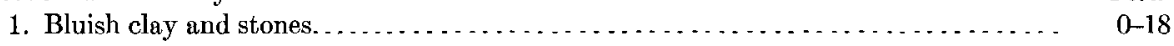

2. Coarse brown sand and gravel................................. 18-45

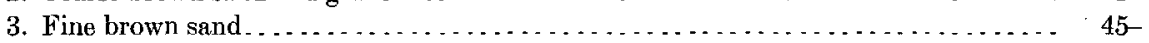

Water below 50 feet is poor.

226. Mr. Edgar L. Wakeman, proprietor of the Deep Glen Spring, reports that in 1903 between 2,000 and 2,500 gallons of this spring water were placed on the market every week, having a value of from $\$ 200$ to $\$ 250$. Analysis of water of Deep Glen Spring, near Flushing.

Sodium chloride . . . . . . . . . . . . . . . . . . . . . . . . . . . . . . . . . . . . . . . . . . 26. 3940

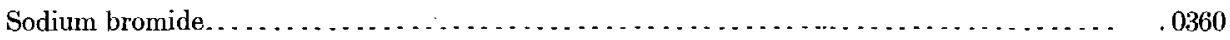

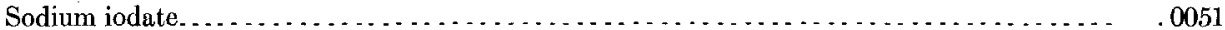

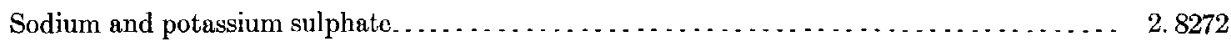

Sodium carbonate . . . . . . . . . . . . . . . . . . . . . . . . . . . . . . . . . . . . . 5040

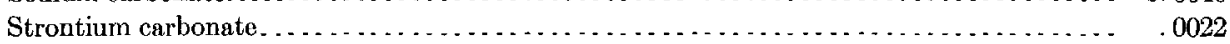

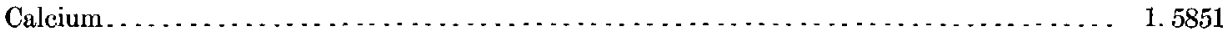

Magnesium.......................................................... ${ }^{5147}$

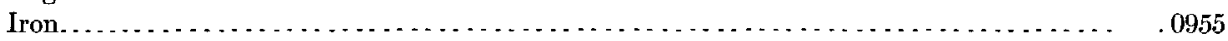

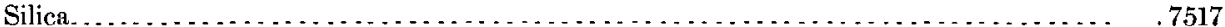

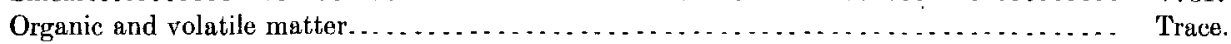

227. Record of commission's test well north of Jamaica.

Wisconsin:

1. Black, humus-stained, gravelly loam........................... $0-0.5$

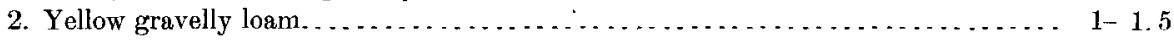

3-4. Reddish yellow clayey sand.................................. 5-11

5-8. Dark, grayish brown, fine to medium sand .......................... 15-31

9-11. Dark, multicolored, glacial sand and gravel . . . . . . . . . . . . . . . . . . $35-46$

228. Record of well between Queens and Bayside.

Wisconsin and Tisbury:

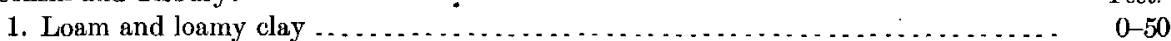

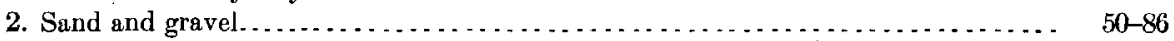


229.

Record of commission's test well near Flushing.

Wisconsin:

1-8. Ycllowish gray sand of probable outwash origin $\ldots \ldots \ldots \ldots \ldots \ldots \ldots \ldots \ldots \ldots \ldots . . . \ldots 1$

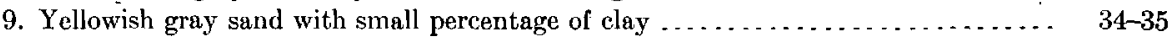

See Table XII.

230. This well flows 12 or 14 inches above the top of the ground. It is just below the dam of the ice pond, and Mr. Sweeney believes that this is possibly responsible for the head.

Record of well of Casino Lake Ice Company at Casino Lake, near Flushing.

1. Black mud................................................. 5

2. Compact mixture of sand and gravel............................. $5-13$

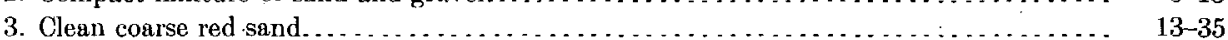

4. Pure-white quartz gravel. ...................................... $35-40$

231. This is the old College Point municipal plant, which was built in $1874-75$ at the Kassena spring south of Flushing. ${ }^{*}$ It has now been decided to replace or supplement the spring supply by driven wells.

The following sections of 3 test wells are reported by Mr. C. D. Corwin:

Record of test well No. 1, Fresh Meadow pumping station, south of Flushing.

Feet.

$0.0-2.6$

1. Black silty mud.

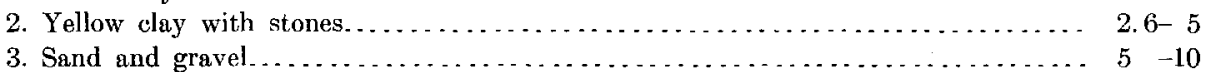

3. Sand and gravel $\ldots \ldots \ldots \ldots \ldots \ldots \ldots \ldots \ldots \ldots \ldots \ldots \ldots \ldots \ldots \ldots \ldots \ldots \ldots$
4. Yellow sand.

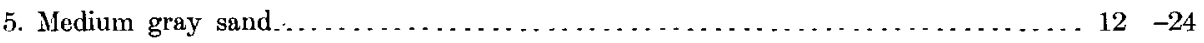

6. Fine yellow sand . . . . . . . . . . . . . . . . . . . . . . . . . . . . . . . . . . . $24 \quad 26$

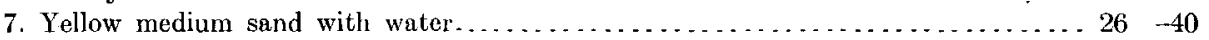

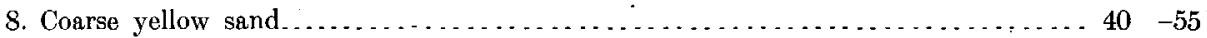

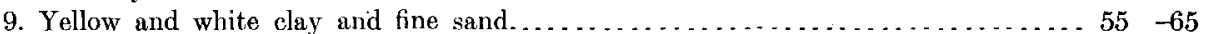

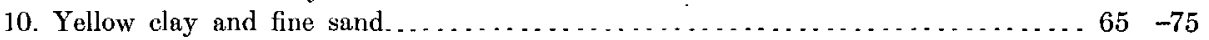

11. Fine white sand; flowed slightly............................... $75 \quad-80$

At 49 feet flowed $1 \frac{1}{2}$ gallons per minute 24 inches above ground. Brook is 9 inches higher than pond. Temperature of water of well, $56^{\circ}$; of pond, $44^{\circ}$.

Mr. Corwin has furnished the following samples from this well:

Record of test well No. 1, Fresh Meadow pumping station, south of Flushing.

Wisconsin or Tisbury:

1. Clean, orange-colored, quartz sand and small gravel, with considerable percentage

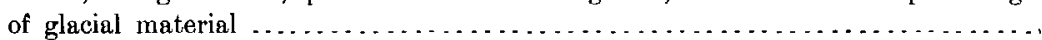

Mannetto or Cretaceous:

2-3. Very coarse orange sand and small gravel; quartz with a small percentage of decayed white chert, which suggests Cretaceous or Mannetto..................

Cretaceous?:

4. Medium white quartz sand, with much muscovite...................

Feet.

Record of test well No. Q, Fresh Meadow pumping station, south of Flushing.

i. Black silty mud.

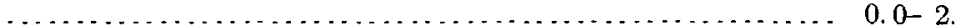

2. Yellow clay with stones.......................................... 5

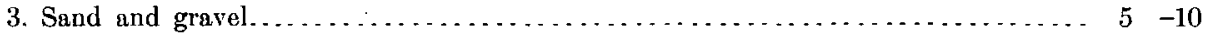

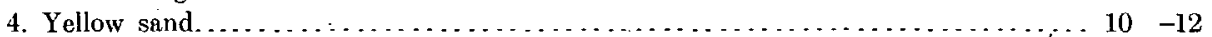

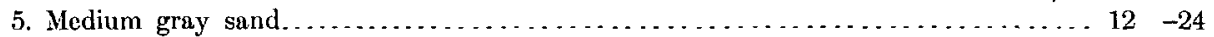

6. Fine yellow sand . . . . . . . . . . . . . . . . . . . . . . . . . . . . . . . . . . $24 \quad 26$

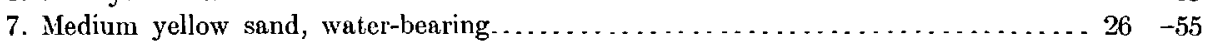

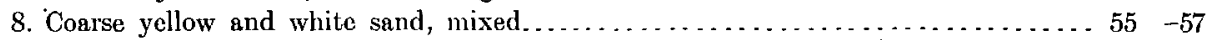

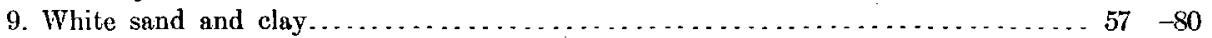


Record of test well No. 3, Fresh Meadow pumping station, south of Flushing.

1. Gray sand with stones. . . . . . . . . . . . .

2. Hardpan; clay and stones. . . . . . . . . . . . . . . .

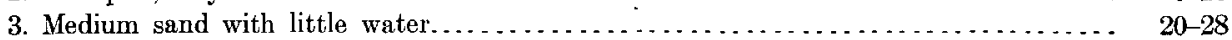

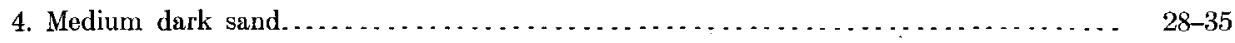

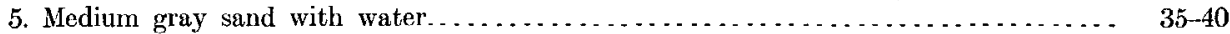

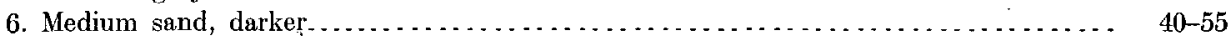

23i. . Record of commission's test well near Flushing.

Wisconsin: $\quad$ Fect.

1-2. Surface loam.............................................. $0-3.5$

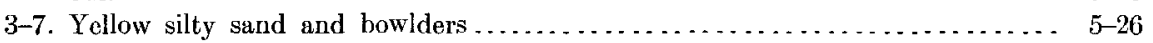

8-9. Fine sand to small gravel, dark, multicolored.................... $30-33$

233.

Record of commission's test well near Broadway.

Wisconsin:

Feet.

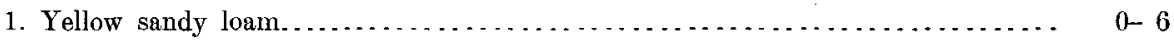

4. Fine, dark yellowish, clayey, silty sand.......................... 10-11

5-6. Dark multicolored sand and gravel; large percentage of erraties......... 14-20

7-11. Dark, yellowish brown, fine to medium sand with considerable mica....... 21-40

234. Record of commission's test well at Queens avenue and Rocky Hill road.

Wisconsin:

1-2. Dark loamy sand and gravel . . . . . . . . . . . . . . . . . . . . . . .

3-8. Glacial sand and gravel with a very large percentage of fresh glacial material. . 5-30 Wisconsin and Tisbury:

9. Dark, reddish brown, fine to coarse micaceous sand (apparently glacial) ...... 33-34 Tisbury:

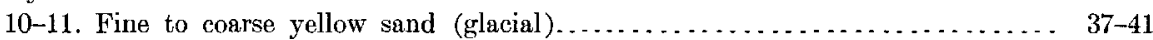

235. Record of commission's test well near Auburndale.

Wisconsin:

1-3. Yellow loamy sand.................................... $0-6$

4-8. Dark yellowish brown sand and gravel of glacial origin............... 10-27

9. Dark silty sand formed from drilling in rock....................... 28-28.5

10-11. Multicolored, glacial, gravel till ............................. $30-38$

See Table XII.

236.

Record of commission's test well near Bayside.

Wisconsin:

Feet.

1. Yellowish brown surface loam.

$0.5-1$

2-3. Reddish loamy sand

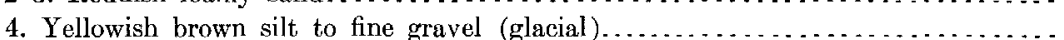

$2-6$

5. Yellowish clayey sand............................................ $15-16$

6. Black clayey sand. . . . . . . . . . . . . . . . . . . . . . . . . .

Wisconsin and Tisbury:

7-14. Dark reddish brown sand and gravel (pronouncedly glacial)........... 19-55 Tisbury:

15. Light, reddish yellow, medium sand........................ $56-57$

16. Grayısh white sand and gravel with a very small percentage of glacial material . $\quad 60-61$ Cretaceous?

17. Medium grayish yellow sand with muscovite (probably not glacial) ........ 63-64 
237.

Record of commission's test well near Bayside.

Wisconsin:

Feet.

1-2. Yellow sandy clay . ...............

3-4. Yellow clayey sand with some pebbles............

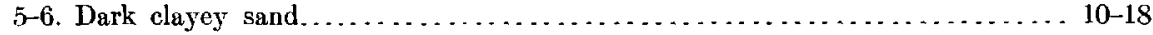

7-10. Mottled sand and gravel (pronouncedly glacial) .................. 20-29.5

11. Multicolored sand and gravel similar to that found below the blue clay on the

south shore ............................................ 35-36

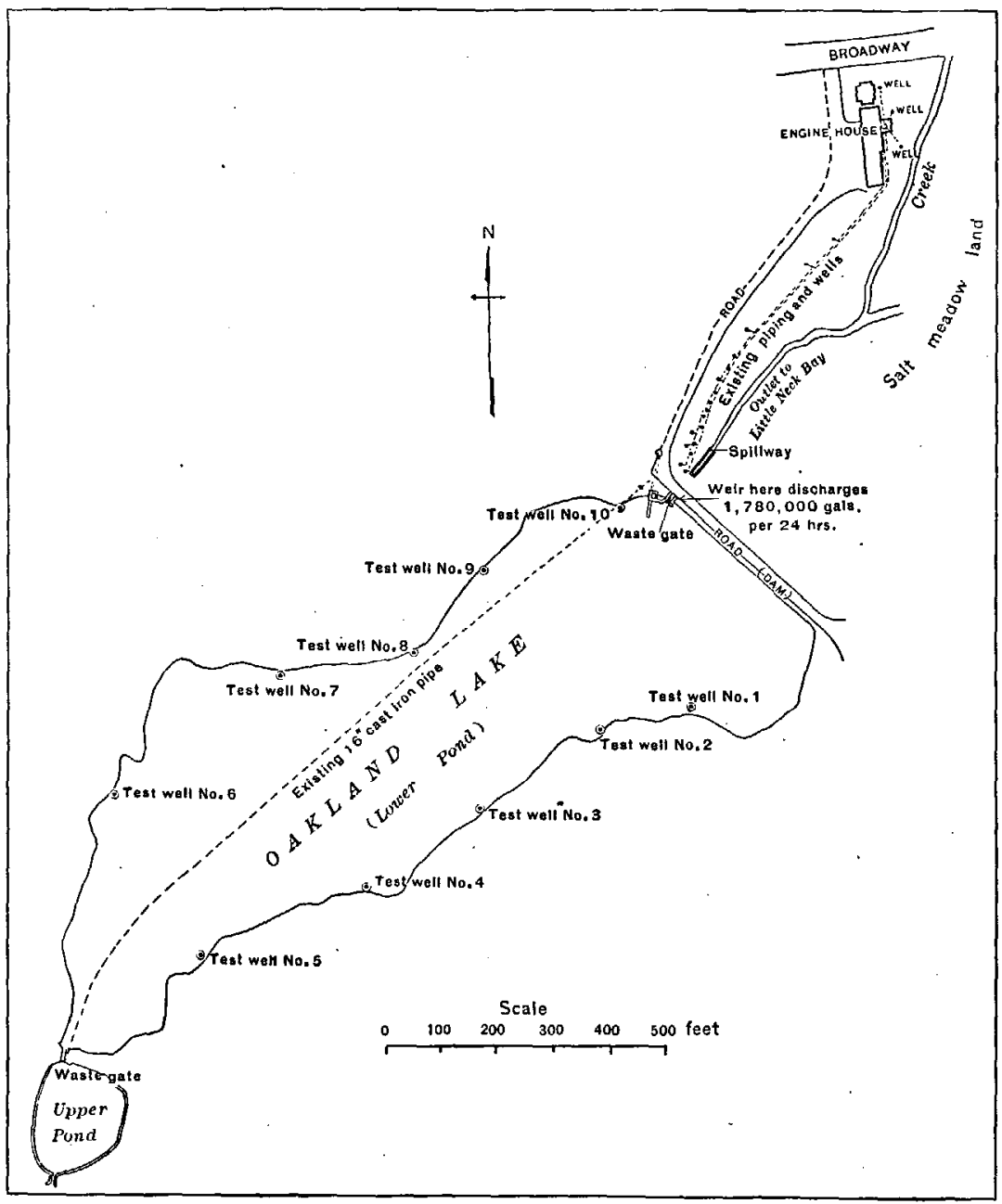

Fig. 64.-Sketch map showing location of test borings at Bayside pumping station.

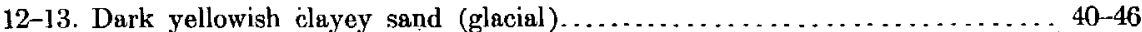

14-16. Dark, multicolored, fine to coarse sand (glacial) ................ $49-65$ Tisbury:

17. Fine to coarse yellow sand with very little glacial material $\ldots \ldots \ldots \ldots \ldots \ldots 6-66$ Tisbury?:

18. Yellow sand and small gravel with many fragments of ferruginous concretions. . 70-71

$17116-$ No. $44-06-15$ 


\section{UNDERGROUND WATER RESOURCES OF LONG ISLAND, NEW YORK.}

239. The 21 wells which now supply the Bayside pumping station are all finished in glacial sand and gravel of Wisconsin or Tisbury age. The engineer at the station reports that the wells will begin flowing about five hours after pumping is stopped. Ten test wells were put down several years ago around the edge of Oakland Lake just above the pumping station; the material penetrated is shown in the following table, which was prepared from the samples by Mr. Alexander S. Farmer. The location of the wells is shown on the accompanying sketch map, fig. 64 .

Description of samples from test borings at Bayside pumping station.

[By Alexander S. Farmer.]

\begin{tabular}{|c|c|c|c|c|}
\hline \multirow{2}{*}{$\begin{array}{l}\text { Test } \\
\text { boring } \\
\text { No. }\end{array}$} & \multicolumn{4}{|c|}{ Composition of soil at a depth of- } \\
\hline & 5 feet. & 10 foet. & 15 feet. & 20 feet. \\
\hline 1 & $\begin{array}{l}\text { Sand, brown, mixed with } \\
\text { black; fine quartz grains. }\end{array}$ & $\begin{array}{l}\text { Sand, brown ish white, } \\
\text { mixed with black; coarse } \\
\text { and fine quartz grains. }\end{array}$ & $\begin{array}{l}\text { Sund, b rown ish white, } \\
\text { mixed with a little black; } \\
\text { coarse grains, homogrne- } \\
\text { ous in character. Water } \\
\text { bearing? }\end{array}$ & $\begin{array}{l}\text { Sand, white with brown- } \\
\text { ish tinge; fine quartz } \\
\text { grains, homogeneous in } \\
\text { character. }\end{array}$ \\
\hline 2 & $\begin{array}{l}\text { Sand, brown, mixed with } \\
\text { black; coarse and fine } \\
\text { quartz grains. }\end{array}$ & $\begin{array}{l}\text { Sand, brown, mixed with } \\
\text { black; coarse and fine } \\
\text { quartz grains. }\end{array}$ & $\begin{array}{l}\text { Sand, white with brownish } \\
\text { tinge; very fine quartz } \\
\text { grains, homogeneous in } \\
\text { character. }\end{array}$ & $\begin{array}{l}\text { Sand, white with brown- } \\
\text { ish tinge; very fine } \\
\text { quartz grains, homoge- } \\
\text { neous in character. }\end{array}$ \\
\hline 3 & $\begin{array}{l}\text { Sand, brown; coarse grains } \\
\text { 16 to } 1 \text { inch in diameter in } \\
\text { a matrix of finer quartz } \\
\text { grains: porphyritic in } \\
\text { chr acter. Water bear- } \\
\text { ing? }\end{array}$ & $\begin{array}{l}\text { Sand, brown; coarse quartz } \\
\text { grains } \frac{1}{16} \text { tc } \frac{1}{4} \text { inch in di- } \\
\text { ameter in a matrix of } \\
\text { finer grains: porphyritic } \\
\text { in char acter. Water } \\
\text { bearing? }\end{array}$ & $\begin{array}{l}\text { Sand, light brown; fine } \\
\text { quartz grains mixed with } \\
\text { some coarse ones. }\end{array}$ & $\begin{array}{l}\text { Sand, light brown; fine } \\
\text { q ta } \mathrm{rtz} \text { grains mixed } \\
\text { with some coarse ones. }\end{array}$ \\
\hline 4 & $\begin{array}{l}\text { Sand, brown; coarse grains } \\
\text { in finer matrix. }\end{array}$ & $\begin{array}{l}\text { Sand, brown; coarse grains } \\
\text { in finer matrix. }\end{array}$ & Clay, white................ & Clay, white. \\
\hline 5 & $\begin{array}{l}\text { Sand, light brown; coarse } \\
\text { grains in fincr matrix; } \\
\text { porphyritic in character. }\end{array}$ & $\begin{array}{l}\text { Sand, light brown; coarse } \\
\text { grains in finer matrix; } \\
\text { porphyritic in character. }\end{array}$ & $\begin{array}{l}\text { Small gravel } \frac{1}{8} \text { to inch in } \\
\text { diameter, cemented in } \\
\text { brownish-white clay ma- } \\
\text { trix; porphyritic struc- } \\
\text { ture. }\end{array}$ & $\begin{array}{l}\text { Sand, cemented in slate- } \\
\text { colored clay matrix. }\end{array}$ \\
\hline 6 & $\begin{array}{l}\text { Sand, light brown; coarse } \\
\text { grains. Water bearing? }\end{array}$ & $\begin{array}{l}\text { Sand, light brown; coarse } \\
\text { grains, } 1 \text { to to inch diam- } \\
\text { eter, mixed with finer } \\
\text { ones; porphyritic charac- } \\
\text { ter. Water hearing? }\end{array}$ & $\begin{array}{l}\text { Sand, light brown; coarse } \\
\text { grain , 1, to } \frac{1}{ \pm} \text { inch diam- } \\
\text { eter, mixed with finer } \\
\text { ones; porphyritic charac- } \\
\text { ter. Water bearing? }\end{array}$ & $\begin{array}{l}\text { Sand, light brown; coarse } \\
\text { grains, it to } \frac{1}{4} \text { inch diam- } \\
\text { eter, mixed with finer } \\
\text { ones; porphyritic char- } \\
\text { acter, Water bearing? }\end{array}$ \\
\hline 7 & $\begin{array}{l}\text { Sand, brown; coarse grains } \\
\text { containing some mica; } \\
\text { bomogeneous in "charac- } \\
\text { ter. }\end{array}$ & $\begin{array}{l}\text { Sand, light brown; con- } \\
\text { tains some black; very } \\
\text { fine grains: mica present; } \\
\text { homogeneous in charac- } \\
\text { ter. }\end{array}$ & $\begin{array}{l}\text { Sand, light brown: coarse } \\
\text { grains in finer matrix; } \\
\text { micapresent; porphyritic } \\
\text { in e ha a cter. Water } \\
\text { bearing? }\end{array}$ & $\begin{array}{l}\text { Sand, light brown; fine } \\
\text { grains; mica present; } \\
\text { homogeneous in charac- } \\
\text { ter. }\end{array}$ \\
\hline 8 & $\begin{array}{l}\text { Sand, light brown; coarse } \\
\text { grains in finer matrix. }\end{array}$ & $\begin{array}{l}\text { Sand, light brown: coarse } \\
\text { grains in finer matrix. } \\
\text { Water bearing? }\end{array}$ & $\begin{array}{l}\text { Sand, light brown; coarse } \\
\text { grains in finer matrix. } \\
\text { Water bearing? }\end{array}$ & $\begin{array}{l}\text { Sand, light brown; coarse } \\
\text { grains in finer matrix. } \\
\text { Water bearing? }\end{array}$ \\
\hline 9 & $\begin{array}{l}\text { Sand, Jight brown; coarse } \\
\text { grains, is to } \frac{3}{6} \text { inch diam- } \\
\text { eter, in ther matrix; mica } \\
\text { present; p o r p y y ritic } \\
\text { character. Water bear- } \\
\text { ing? }\end{array}$ & $\begin{array}{l}\text { Sand, light brown; coarse } \\
\text { grains, th to } x^{3} \text { inch diam- } \\
\text { eter, in finer matrix; mica } \\
\text { present; p orph y itic } \\
\text { character. Water bear- } \\
\text { ing? }\end{array}$ & $\begin{array}{l}\text { Sand, light brown; coarse } \\
\text { grains, } \frac{1}{8} \text { to } \text { ponch indiam- }^{3} \text { inch, in finer matrix; mica } \\
\text { present; p orphy ritic } \\
\text { character. Water bear- } \\
\text { ing? }\end{array}$ & $\begin{array}{l}\text { Sand, light brown: fine } \\
\text { grains, homogeneous in } \\
\text { efuracter. }\end{array}$ \\
\hline 10 & $\begin{array}{l}\text { Sand, buff colored; fine } \\
\text { grains approaching clay } \\
\text { in composition. }\end{array}$ & $\begin{array}{l}\text { Sand, buff colored; fino } \\
\text { grains approaching clay } \\
\text { in composition. }\end{array}$ & $\begin{array}{l}\text { Sand, light brown; fine } \\
\text { grains, homogeneous in } \\
\text { character. }\end{array}$ & Do. \\
\hline \multirow{2}{*}{$\begin{array}{l}\text { Test } \\
\text { boring } \\
\text { No. }\end{array}$} & \multicolumn{4}{|c|}{ Composition of soil at a depth of- } \\
\hline & 25 feet. & 30 feet. & 35 feet. & 40 feet. \\
\hline 1 & $\begin{array}{l}\text { Sand, white with brownish } \\
\text { tinge; very fine quartz } \\
\text { grains, homogeneous in } \\
\text { character. }\end{array}$ & $\begin{array}{l}\text { Sand, almost white; very } \\
\text { fne quartz grains, homo- } \\
\text { geneous in character. }\end{array}$ & $\begin{array}{l}\text { Sand, almost white; very } \\
\text { fine quartz grains, homo- } \\
\text { geneous in character. }\end{array}$ & $\begin{array}{l}\text { Sand, white with brown- } \\
\text { ish tinge; course quart' } \\
\text { grains, y to. } \text { I }^{3} \text { inch in } \\
\text { diameter, mixed with } \\
\text { coarse and fine grains; } \\
\text { porphyritic in eharac- } \\
\text { ter. Water bearing? }\end{array}$ \\
\hline 2 & $\begin{array}{l}\text { Sand, white with brownish } \\
\text { tinge; fine quartz grains, } \\
\text { homogeneous in charae- } \\
\text { ter. }\end{array}$ & $\begin{array}{l}\text { Sand, brown is h white; } \\
\text { coarse quartz grains, } \\
\text { homogeneous in charac- } \\
\text { ter. Water bearing? }\end{array}$ & $\begin{array}{l}\text { Sand, brownish white; } \\
\text { coarse and fine quartz } \\
\text { grains. }\end{array}$ & $\begin{array}{l}\text { Sand, light brown; very } \\
\text { fine quartz grains, ho- } \\
\text { mogeneous in character. }\end{array}$ \\
\hline
\end{tabular}


Description of samples from test borings at Bayside pumping station-Continued.

\begin{tabular}{|c|c|c|c|c|}
\hline \multirow{2}{*}{$\begin{array}{l}\text { Test } \\
\text { boring } \\
\text { No. }\end{array}$} & \multicolumn{4}{|c|}{ Composition of soil at a depth of- } \\
\hline & 25 leet. & 30 feet. & 35 feet. & 40 feet. \\
\hline 3 & $\begin{array}{l}\text { Sand, light brown; very } \\
\text { fne quartz grains, homo- } \\
\text { geneous in character. }\end{array}$ & $\begin{array}{l}\text { Sand, brown; coarse quartz } \\
\text { grains, homogeneous in } \\
\text { character. Water bear- } \\
\text { ing? }\end{array}$ & $\begin{array}{l}\text { Gravel, brown; grains aver- } \\
\text { aging is inch in diameter, } \\
\text { homogeneous in charac- } \\
\text { ter. Water bearing? }\end{array}$ & $\begin{array}{l}\text { Sand, light brown; very } \\
\text { fine grains mixed with } \\
\text { coarse ones. }\end{array}$ \\
\hline 4 & $\begin{array}{l}\text { Sand, light brown; very } \\
\text { fine grains, homogeneous } \\
\text { in character. }\end{array}$ & $\begin{array}{l}\text { Sand, very light brown; } \\
\text { very fine grains, homo- } \\
\text { geneous in character. }\end{array}$ & $\begin{array}{l}\text { Gravel, light brown; grains } \\
\text { averaging } 16 \text { inch in di- } \\
\text { ameter, homogeneous in } \\
\text { character. Water bear- } \\
\text { ing? }\end{array}$ & $\begin{array}{l}\text { Sand, light brown; fine } \\
\text { grains inixed with coarse } \\
\text { ones. }\end{array}$ \\
\hline 5 & $\begin{array}{l}\text { Sand, cemented in slate- } \\
\text { colored clay matrix. }\end{array}$ & $\begin{array}{l}\text { Sand, light brown; coarse } \\
\text { grains, homogeneous in } \\
\text { character, Water bear- } \\
\text { ing? }\end{array}$ & $\begin{array}{l}\text { Sand, brown is h whitc; } \\
\text { very fine grains; resem- } \\
\text { bles sea, sand; homogene- } \\
\text { ous in character. }\end{array}$ & $\begin{array}{l}\text { Sand, light brown; very } \\
\text { fine grains, homogene- } \\
\text { ous in character. }\end{array}$ \\
\hline 6 & $\begin{array}{l}\text { Sand, light brown; coarse } \\
\text { grains, is to } 1 \text { inch diam- } \\
\text { eter, mixed with finer } \\
\text { ones; porphyritic charac- } \\
\text { ter. Water bearing? }\end{array}$ & $\begin{array}{l}\text { Sand, light brown; coarse } \\
\text { grains, 1b to } \frac{1}{1} \text { inch diam- } \\
\text { eter, mixed with finer } \\
\text { ones; porphyritic in char- } \\
\text { acter. Water bearing? }\end{array}$ & $\begin{array}{l}\text { Sand, light brown; coarse } \\
\text { grains, } 1 \text { to to inch diam- } \\
\text { eter, mixed with finer } \\
\text { ones; porphyritic in char- } \\
\text { acter water bearing? }\end{array}$ & $\begin{array}{l}\text { Sand, light brown; fine } \\
\text { grains mixed with coarse } \\
\text { ones. }\end{array}$ \\
\hline 7 & $\begin{array}{l}\text { Grains averaging }{ }_{1}^{1} \text { inch in } \\
\text { diameter, cemented to } \\
\text { some extent in grayish- } \\
\text { white clay matrix. }\end{array}$ & Clay, grayish white....... & $\begin{array}{l}\text { Sand, light brown; fine } \\
\text { grains, homogeneous in } \\
\text { character. }\end{array}$ & $\begin{array}{l}\text { Sand, light brown; fine } \\
\text { grains, homogeneous in. } \\
\text { character. }\end{array}$ \\
\hline 8 & $\begin{array}{l}\text { Sand, light brown; coarse } \\
\text { grains in finer matrix. } \\
\text { Water bearing? }\end{array}$ & $\begin{array}{l}\text { Sand, light brown; coarse } \\
\text { grains in finer matrix. } \\
\text { Water bearing? }\end{array}$ & $\begin{array}{l}\text { Sand, light colored; very } \\
\text { fine grains approaching } \\
\text { clay in composition. }\end{array}$ & $\begin{array}{l}\text { Sand, light colored; very } \\
\text { fine grains, homogene- } \\
\text { ous in character. }\end{array}$ \\
\hline 0 & $\begin{array}{l}\text { Sand, light brown; fine } \\
\text { grains, homogencous in } \\
\text { character. }\end{array}$ & $\begin{array}{l}\text { Sand, light brown; coarse } \\
\text { grains, is inch diameter, } \\
\text { in finer matrix. Water } \\
\text { bearing? }\end{array}$ & $\begin{array}{l}\text { Sand, gray; large grains, } \\
\text { inch diameter, in finer } \\
\text { matrix; porphyritic in } \\
\text { character. Water bear- } \\
\text { ing? }\end{array}$ & $\begin{array}{l}\text { Sand, light brown; coarse } \\
\text { grains in finer matrix. } \\
\text { Water bearing? }\end{array}$ \\
\hline 10 & $\begin{array}{l}\text { Sand, b la ck is h brown; } \\
\text { coarse grains, homogene- } \\
\text { ous in eharacter. Water } \\
\text { bearing? }\end{array}$ & $\begin{array}{l}\text { Sand, white; very fine } \\
\text { grains containing coarse } \\
\text { ones; resembles sea sand. }\end{array}$ & $\begin{array}{l}\text { Sand, light brown; coarse } \\
\text { grains in finer matrix. }\end{array}$ & $\begin{array}{l}\text { Sand, light brown; fine } \\
\text { grains, homogeneous in } \\
\text { character. }\end{array}$ \\
\hline \multirow{2}{*}{$\begin{array}{l}\text { Test } \\
\text { boring } \\
\text { No. }\end{array}$} & \multicolumn{4}{|c|}{ Composition of soil at a depth of - } \\
\hline & 45 feet. & 50 feet. & 55 fcet. & 60 reet. \\
\hline 1 & $\begin{array}{l}\text { Sand, brown ish whice; } \\
\text { coarse, differentiating to } \\
\text { fine quartz grains. Wa- } \\
\text { ter bearing? }\end{array}$ & $\begin{array}{l}\text { Sand, brown is h white; } \\
\text { coar se quartz grains, } \\
\text { homogeneous in charac- } \\
\text { ter. Water bearing? }\end{array}$ & Clay, yellowish white. & Clay, yellowish white. \\
\hline 2 & $\begin{array}{l}\text { Sand, light brown; very } \\
\text { fine quartz grains, homo- } \\
\text { geneous in character. }\end{array}$ & $\begin{array}{l}\text { Sand, light brown; very } \\
\text { fine quartz grains, homo- } \\
\text { geneous in character. }\end{array}$ & $\begin{array}{l}\text { Sand, light brown; very } \\
\text { fine quartz grains, homo- } \\
\text { geneous in character. }\end{array}$ & $\begin{array}{l}\text { Sand, light brown; very } \\
\text { fine quartz grains, bo- } \\
\text { mogeneous in character. }\end{array}$ \\
\hline 3 & $\begin{array}{l}\text { Sand, light brown; very } \\
\text { fine grains mixed with } \\
\text { coarse ones. }\end{array}$ & $\begin{array}{l}\text { Sand, light brown; very } \\
\text { fine grains mixed with } \\
\text { coarse ones. }\end{array}$ & $\begin{array}{l}\text { Sand, light brown; coarse } \\
\text { grains in finer matrix. }\end{array}$ & $\begin{array}{l}\text { Sand, very light brown; } \\
\text { fine grains mixed with } \\
\text { coarse ones. }\end{array}$ \\
\hline 4 & Clay, yellowish white...... & Clay, ycllowish white....... & Clay, yellowish white...... & Clay, yellowish white. \\
\hline 5 & $\begin{array}{l}\text { Sand, light browh; very } \\
\text { fine grains, homogeneons } \\
\text { in character. }\end{array}$ & $\begin{array}{l}\text { Sand, light brown; very } \\
\text { fine grains, homogeneous } \\
\text { in character. }\end{array}$ & $\begin{array}{l}\text { Sand, light brown; fine } \\
\text { grains mixed with coarse } \\
\text { ones. }\end{array}$ & $\begin{array}{l}\text { Sand, light brown; fine } \\
\text { grains mixed with } \\
\text { coarse ones. }\end{array}$ \\
\hline 6 & $\begin{array}{l}\text { Sand, light brown; fine } \\
\text { grains mixed with coarse } \\
\text { ones. }\end{array}$ & $\begin{array}{l}\text { Sand, light brown; fine } \\
\text { grains mixed with coarse } \\
\text { ones. }\end{array}$ & ....do................... & Do. \\
\hline 7 & $\begin{array}{l}\text { Sand, light brown; fine } \\
\text { grains, homogeneous in } \\
\text { character. }\end{array}$ & Clay, grayish white........ & Clay, light drab.... & Clay, light drab. \\
\hline 8 & $\begin{array}{l}\text { Sand, almost white; resem- } \\
\text { bles sea sand; much mica } \\
\text { present. }\end{array}$ & $\begin{array}{l}\text { Sand, grayish white; very } \\
\text { fine grains: on the border } \\
\text { line between clay and } \\
\text { sand. }\end{array}$ & .....do. & Do. \\
\hline 9 & $\begin{array}{l}\text { Sand, light brown; coarse } \\
\text { grains in finer matrix. } \\
\text { Water bearing? }\end{array}$ & $\begin{array}{l}\text { Sand, light colored; fine } \\
\text { grains containing some } \\
\text { coarse nnes. }\end{array}$ & $\begin{array}{l}\text { Sand, grayish white; al- } \\
\text { most clay; impalpable } \\
\text { character. }\end{array}$ & $\begin{array}{l}\text { Sand, grayish white; al- } \\
\text { most clay; impalpable } \\
\text { character. }\end{array}$ \\
\hline 10 & $\begin{array}{l}\text { Sand, light brown; fine } \\
\text { grains, homogeneous in } \\
\text { character. }\end{array}$ & Clay, grayish white....... & Clay, grayish white...... & Clay, grayish white. \\
\hline
\end{tabular}


239. The following section has been prepared by Mr. Alexander S. Farmer:

Record of well at pumping station No. 1, Whitestone.

Wisconsin and Tisbury:

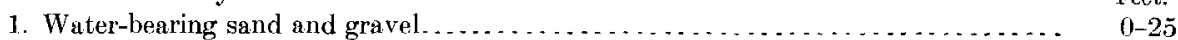

Sankaty:

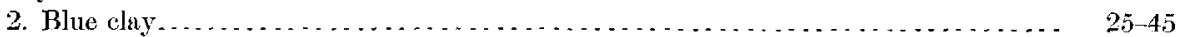

Jameco:

3. Water-bearing glacial sand and gravel . . . . . . . . . . . . . . . . . . . . . 45

Cretaceous?:

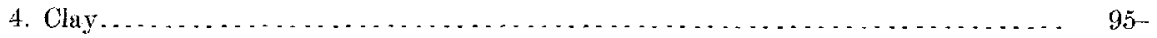

240. This well was driven in the bay 100 feet from the shore; at high tide it is covered with from 12 to 14 feet of water. Recent:

Record of well of MeWilliams Coal Company near Whitestone Landing.

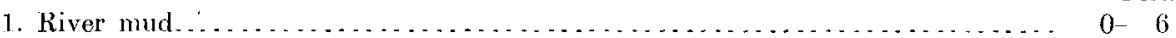

Cretaceous:

2. Blue, white, and red clay, arranged in alternate layers, but containing no sand

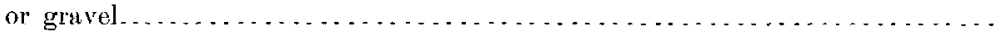

$6-175$

24 L. Four test wells were put down to deptls ranging from 90 to $120 \mathrm{feet}$; in all of them the water was found to be brackish, and the wells were abandoned. The tops of the wells are about 15 feet above high tide level, and the water in them fluctuates with the tide, to an amount thought by the driller to be almost equal to that in the bay; they are situated about 400 feet from the water's edge.

Recent:

Record of railroad wells near Whitestone Landing.

Tisbury:

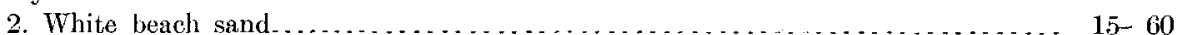

Sankaty:

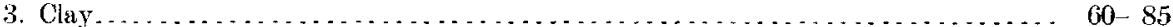

Jameco:

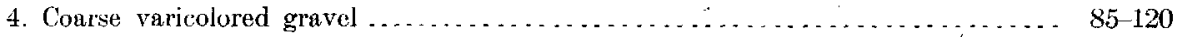

242. This was formerly a private plant from which the water was pumped into a ground reservoir on the hill behind it. It was later acquired by the city, and is now used only as a reserve station.

243. Stotthof Brothers report the following section for this well:

Record of W.W. Cole's well near Whitestone.

1. Dug well .

Sankaty?

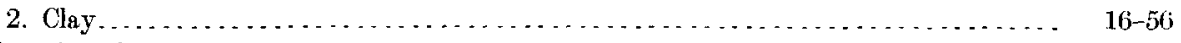

Saukaty? and Jameco?:

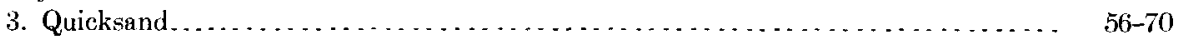

Jameco:

4. Vedium, coarse, water-bearing gravel. .......................... $70-96$

244. No definite information has been obtained regarding the deep well at this point, other than that it is about 500 feet deep. The depth to bed rock, which is an interezting point, because this well is in the line of the old Sound River Valley, is likewise not obtainable. 'It was reported from one source as being about 10 feet above sea level, but Maj. Edward Burr, of the Corps of Engineers, reports that the excavations at this point have not shown rock at such a height. 
246.

Wisconsin:

Record of H. B. Gilbert's well near Great Neck, Elm Point.

Tisbury:

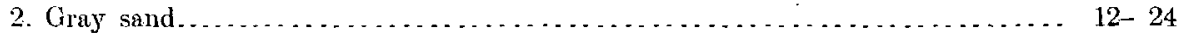

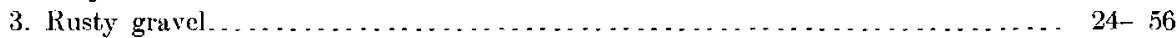

Tisbury?:

4. Fine canary-colored sand ................................. $56-66$

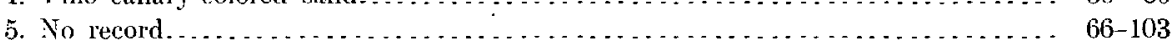

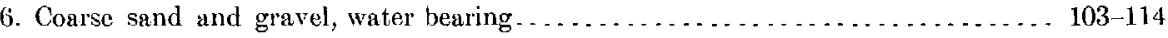

A near-by dug well encountered water at 26 feet, evidently in layer No. 3. This dug well goes dry in dry seasons.

247.

Record of J. E. Martin's well near Great Neck, Elm Point.

Wisconsin:

Feet.

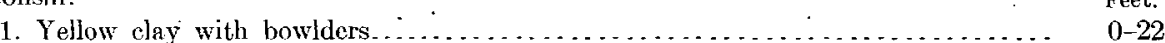

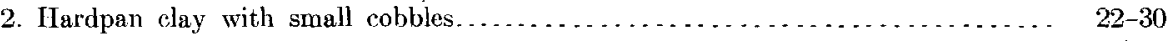
Tisbury:

3. Gray sand, passing below into gravel about the size of shelled corn . . . . . . . 30-67

249. Mr. J. H. Herbert has kindly furnished the following samples from this well:

Wisconsin:

Record of H. B. Gilbert's well near Great Neck, Elm Point.

1-2. Red sandy clay (glacial)

Cretaceous:

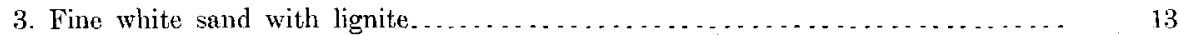

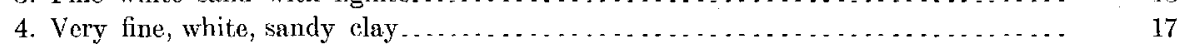

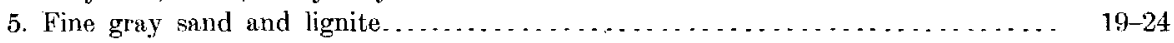

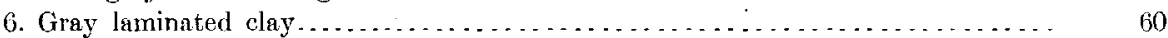

The well was abandoned at this point and a new well (248) sunk at a distance of about 300 yards, where a good supply was obtained in glacial gravel.

251. The driller reports a dry hole at 65 feet, and water at 66 . The well is about 6 feet above high tide noar the beach, and it is stated that a float placed in this well did not fluctuate with the tide.

Wisconsin :

Record of H. O. Ohilds's well near Hewlett Point.

1. Surface loamy sand : Feet.

Wisconsin and Cretaceous?

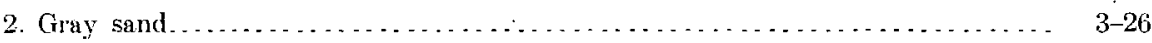

Cretaceous?
3. Blue clay.
$26-29$

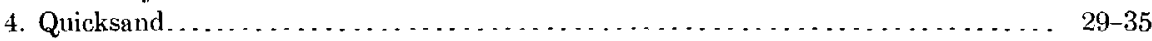
5. Coarse gray sand, dry ... . . . . . . . . . . . . . . . . . . . . . . .

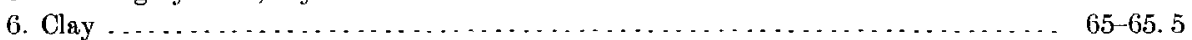

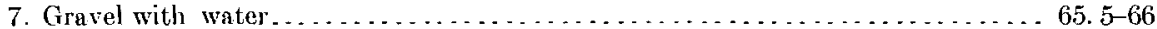

252. Mr. Herbert reports having sunk a 6 -inch pipe 40 feet in the bottom of a 52 -foot dug well; he then encountered hard rock (probably a bowlder) and discontinued the work. The material penetrated was all quicksand and gray beach sand.

254. It is stated that Mr. Griffin had 17 wells put down at his place without success; one on the edge of the beach; about 200 yards north of well No. 251, is reported to have been 90 feet deep and to have found no water. The well completed by $\mathrm{Mr}$. Herbert furnishes a good supply of water. The material encountered in this well is as follows: 
Record of C. L. Griffin's well near Hewlett Point.

1. Top soil

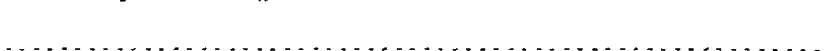

2. Blue clay.

Feet.

$0-3$

4. Quicksand . . . . . . . . . . . . . . . . $3-26$

5. Light-gray hardpan..

6. Gray gravel.

$26-$

255.

Record of W. H. Arnold's well near Hewlett Point.

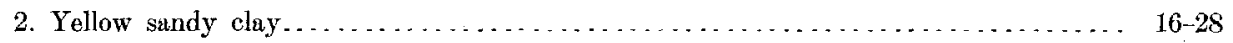

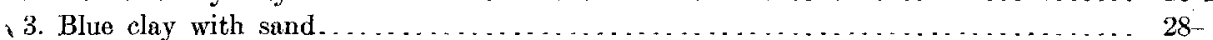

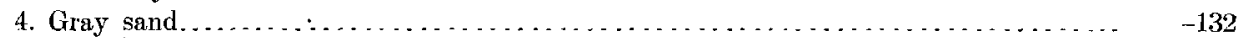

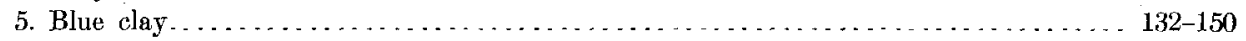

6. Gray gravel. . . . . . . . . . . . . . . . . . . . . . . . . . . . . . $150-159$

256. Stothoff Brothers, in letter dated April 30,1903 , report the following: "The well is 512 feet deep, and 8 inches in diameter; the first 90 feet light gray sand with coarse seams, 5 to 20 feet apart, and from 6 inches to 2 feet thick; there being enough clay to hold the sand so that it would hold itself while driving the pipe; the next 140 feet fine gray sand and quicksand very fine and uniform to top of rock; no water. Rock from 230 to 512 feet, soft gray granite and mica veins, same character as is found throughout Westchester County, N. Y."

257.

Record of Mrs. M. E. Scott's well at Hewlett Point.

1. Dug well

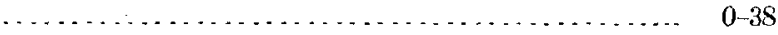

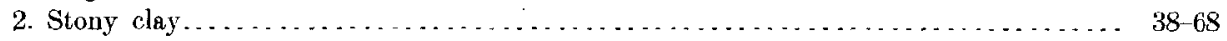

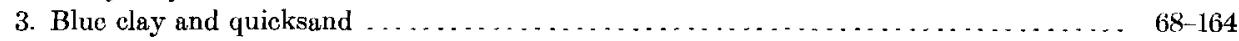

The well was abandoned at 164 feet.

258. Record of G.,B. Wilson's well near Hewlett Point.

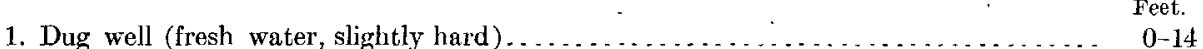

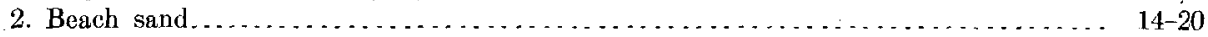

3. Light-colored clay with stone... . . . . . . . . . . . . . . . . . . . . . . 20 20 30

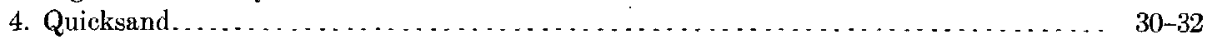

5. Stony clay . . . . . . . . . . . . . . . . . . . . . . . . . . . . . . $32-36$

6. Coarse gray sand containing salt water at 46 feet, and brackish water at 59 feet... $36-59$

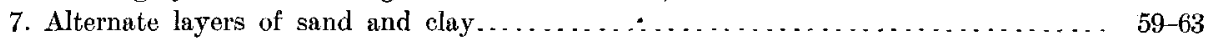

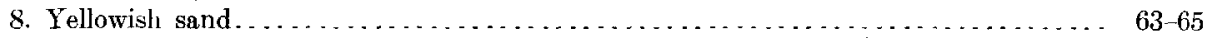

9. Blue clay . . . . . . . .

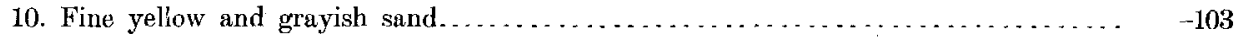

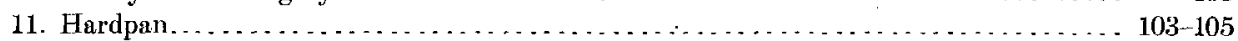

12. Yellow gravel with fresh water................................ 105-108

259. Record of well of Lawrence Beach Bathing Association, at Lawrence Beach.

Recent to Tisbury:

Sankaty:

Jameco:

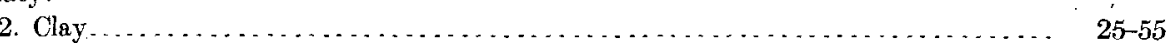

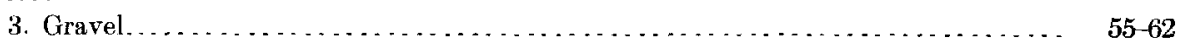

260. Record of John Lawrence's well, on Isle of Wigh', New York.

Recent and Tisbury:

1. Fine beach sand and clay mixed

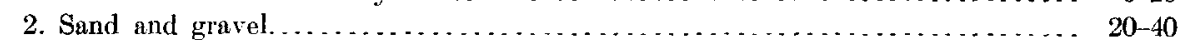

Sankaty:

3. Sand and clay . . . . . . . . . . . . . . . 40.100

The well was completed at 30 feet, this being the best gravel layer encountered. 
261. Mr. Gilbert Baldwin, who was in charge of the sinking of this well, gives the following record:

Tisbury:

$$
\text { Record of D. D. Lord's well near Laurence. }
$$

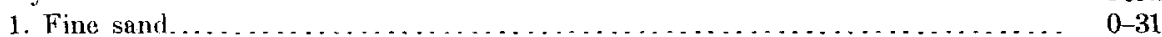

Sankaty:

2. Clay, containing few small stones ........................... $35-50$ Jameco:

3. Sand, containing shells, like clant and oyster shells ................ $50-70$

4. Coarse sand changing to gravel. ............................. $70-100$

The contractor, Mr. Jesse Conklin, under date of April 25, 1895, gives the following data: "At Lawrence I drove a 6-inch well 107 feet; I struck water at 5 feet; drove 25 feet in water and got a good supply; struck blue clay at 30 feet; drove 25 feet through it and struck fine sand and some oyster shells, continuing 30 feet; at 90 feet I struck white gravel, drove 17 feet in this and got an unlimited supply of water."

262. Record of A. W. Hart's well near Lawrence.

Tisbury:

1. Yellow sand and gravel........... 0 -40

Sankaty and Jameco:

2. Grayish clay, no pebbles . . . . . . . . $40-60$

3. Coarse white sand mixed with a little elay (some oyster shells found in this sand) . $\quad 60-70$

26:3. Mr. Edward Man gives the following data regarding this well: "At a depth of 416 feet I struck a plentiful supply of bright clear water, which, however, was exceedingly salt. It contained quite a large amount of iron, and had a slight odor of sulphureted hydrogen. In my opinion this water contained considerably more salt than the ocean itself. The water rose in the pipe to within about 15 feet of the surface.

"In driving the well I encountered a water-bearing layer at about 40 feet and another at about 150 feet; these I should judge to have been about 3 feet in thickness. The first layer yielded a bright, clear, fresh water, pleasant to the taste, and apparently free from any iron, but was unfortunately found, after being used for several years, to be 'contaminated with sewage to a marked

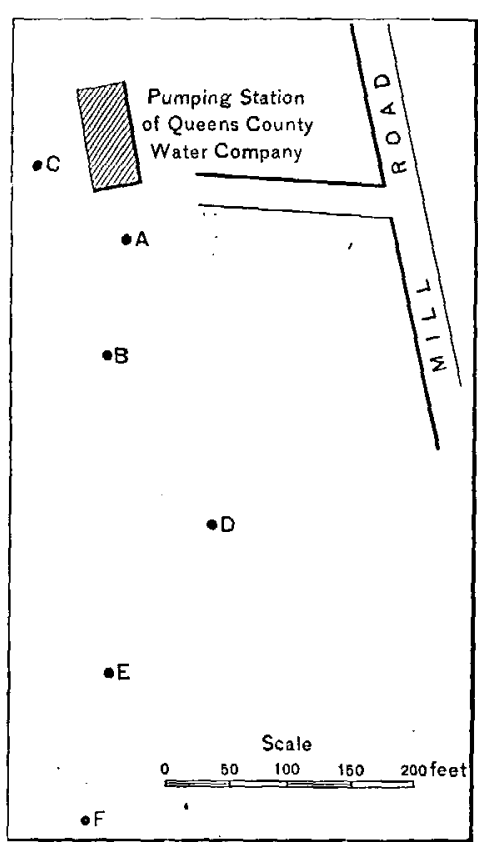

FIG. 65.-.Sketch map giving locations of wells of the Queens County Water Company shown in fig. 66 . degree,' according to the report of Mr. Vulte, Professor Chandler's assistant at Columbia College.

"At 150 feet there was another water-bearing layer, which yielded a plentiful supply of bright clear water, but as soon as the water was exposed to the air, the iron in it seemed to be chemically changed by the light and air and the water became quite brown, so that it could not be used for washing. This water when filtered through a Gate City stone filter was entirely free from any appearance or taste of iron, so that I think the iron in it was not in solution but in suspension."

This is the well reported by Darton as "Lawrence: Depth 205 feet; capacity 35 gallons; water layer at 40 feet." It has since been deepened.

265. Mr. Walsh reports that in the vicinity of Cedarhurst he usually encounters streaks of hard pan 6 inches thick at 22 or 25 feet below the surface. The hard pan is described as a mixture of brown clay and coarse gravel, packed closely together and cemented with iron. . 
Record of Judge Diver's well near Cedarhurst.

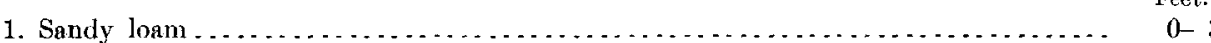

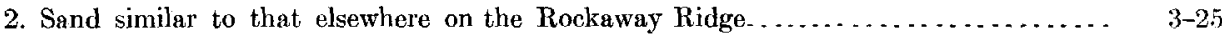

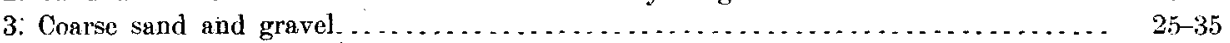

266. $\quad$ Record of Dr. Anderson's well near Cedarhurst.

Tisbury:

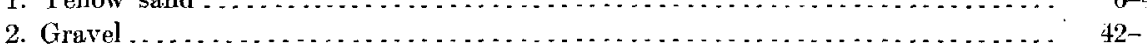

267.

Record of Louis Touscher's well near Cedarhurst.

Tisbury:

Feet.

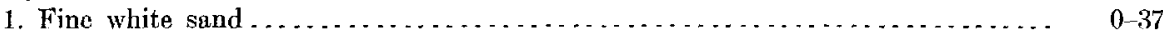

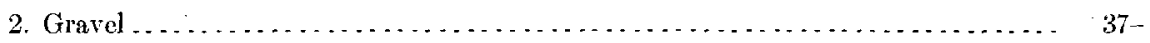

268. $\quad$. Record of Samuel Brower's well near Brower Point.

Tisbury:

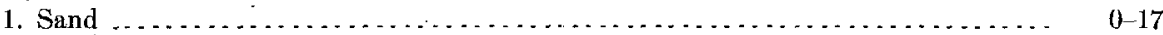

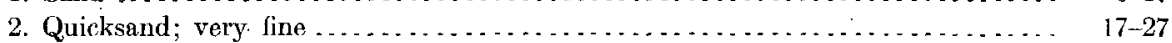

3. Sand and gravel; coarseness increasing with depth. ................ $27-35$

272. Mr. Jaegle reports that the marsh deposits in this well were about 10 feet thick, below which there was 15 fect of fine dark-colored sand, the remainder of the well being through an alternation of lead-colored sand and clays. A small flow was obtained at 150 fcet; at 228 feet a coarse gravel was encountered, from which a good supply of pure water was obtained, flowing 3 feet above the surface of the meadow. A sample of the water-bearing sand from $\boldsymbol{a}$ depth of 228 fect, presented by Mr. Jaegle, is composed of small white quartz pebbles, with a very considerable percentage of erratic material.

273. Mr. Charles R. Bettes, chief engineer, reports that there were in use at this station in the summer of 1903 thirty-two 4- and 5- inch wells 33 feet deep and nineteen 6-inch wells 150 to 190 fect deep. The average daily pumping in 1902 was $1,634,000$ gallons, the minimum 850,000 gallons, and the maximum $4,500,000$ gallons. One of the now 6 -inch test wells completed in 1903 tested 800,000 gallons per day. The water is pumped from the wells to a filter which removes the exeess of iron, and is then pumped into mains.

Samples obtained from one of the 6-inch wells drilled during the summer of 1903 show the following section:

Record of well at Queens County pumping station.

Wisconsin:

1. Gray silty sand and gravel, with a large percentage of biotite.......... $6-10$

Tisbury:

2-3. White to light-yellow quartz sand and gravel, with only a small percentage of Sankaty?: glacial material.

$10-32$

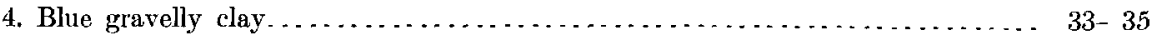

Sankaty:

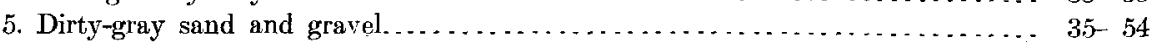

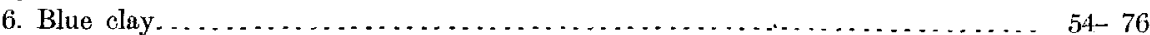

7-10. Fine, gray, pepper-and-salt sand, composed of a mixture of white quartz and green sand, weathering to a reddish yellow................... $76-95$

Sankaty and Jameco:

11-12. Light yellowish white sand and gravel; no greensand . . . . . . . . . . 95-100

13. Lignite . . . . . . . . . . . . . . . . . . . . . . . . . . . . . . . . . . . .

14. Fine pepper-and-salt sand, composed of mixture of white quartz and greensand, containing a little gray clay. 


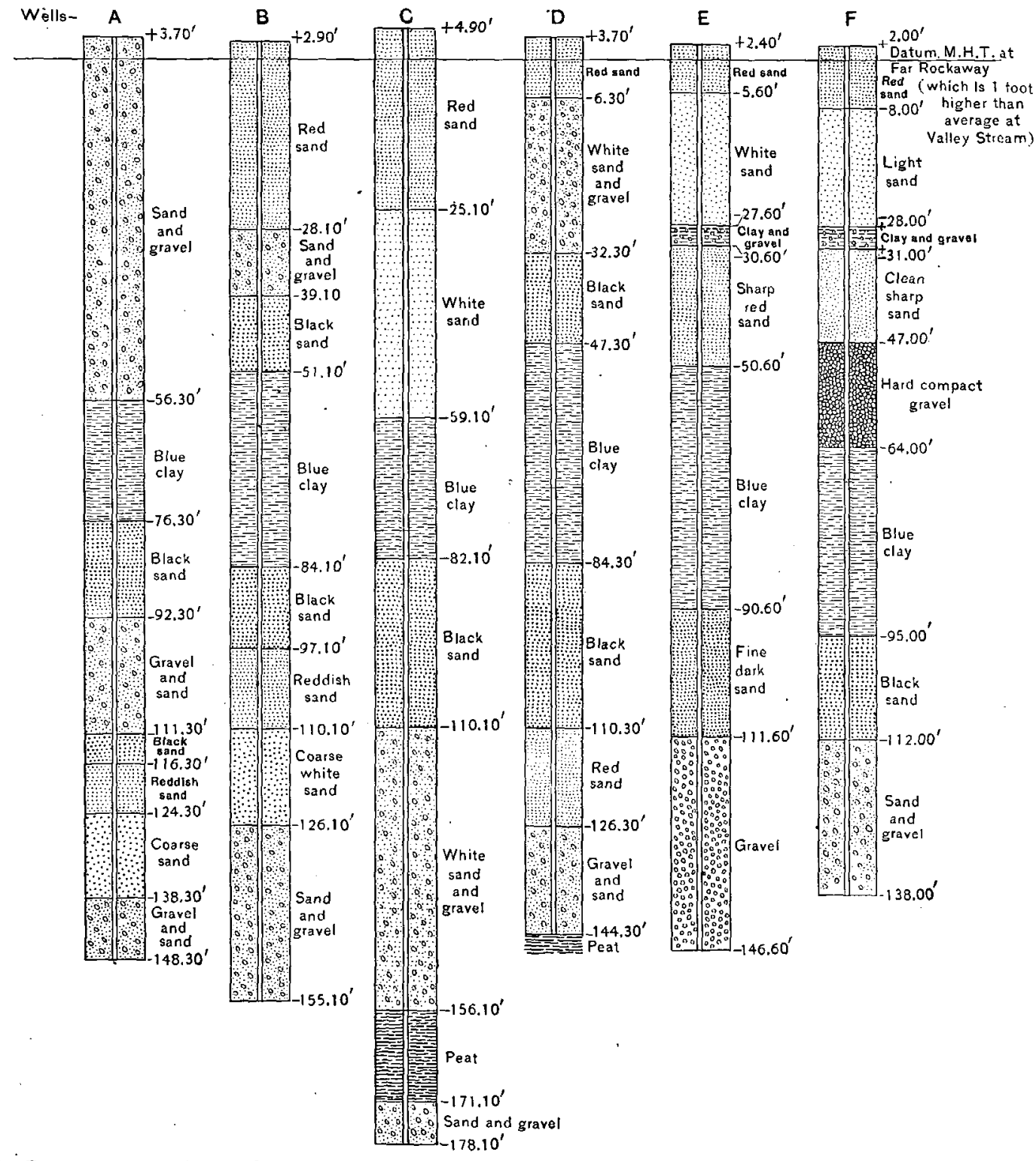

Fig. 66.-Sections of wells of the Queens County Water Company, by Charles R. Bettes, chief engincer. (For locations of wells see fig. 65. ) 
Jameco:

15-19. Light-colored, coarse sand and gravel containing a considerable percentage

Feet. of erratic material.

Records of the first five wells are shown in fig. 66; for general relations see fig. 13 .

\section{Analysis of water from Queens County pumping station.}

[Analysis by C. F. Chandler, September 17, 1902.]

Chlorine in chlorides . . . . Parts per million

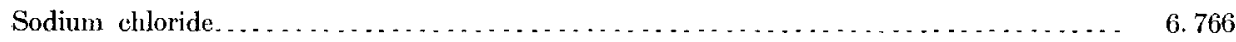

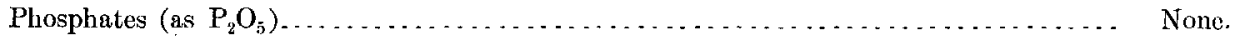

Nitrogen in nitrites. . . . . . . . . . . . . . . . .

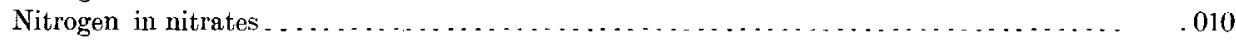

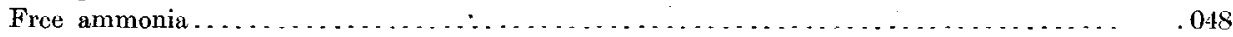

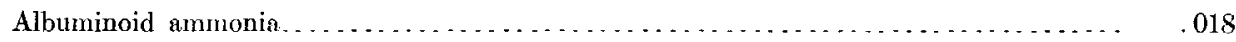

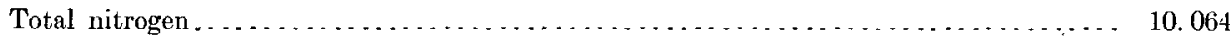

Hardness:

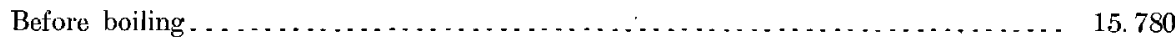

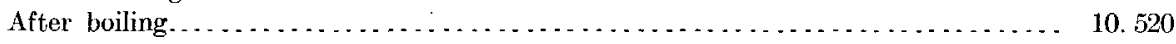

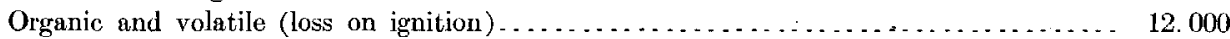

Mineral matter (nonvolatile) $\mathrm{CO}_{2}$ restored with ammonium cárbonate . . . . . . . . 34.500

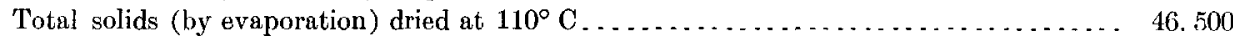

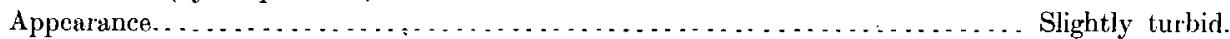

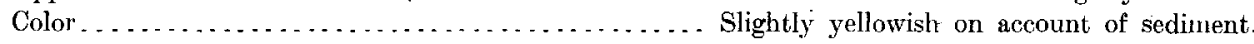

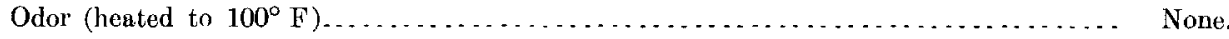

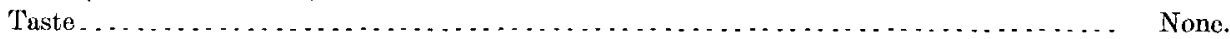

The Long Island Railroad Company report the following analysis of water taken from the mains of thr: Queens County Water Company at Rockaway Beach, May, 1897:

-Analysis of water of Queens County Water Company at Rockaway Beach.

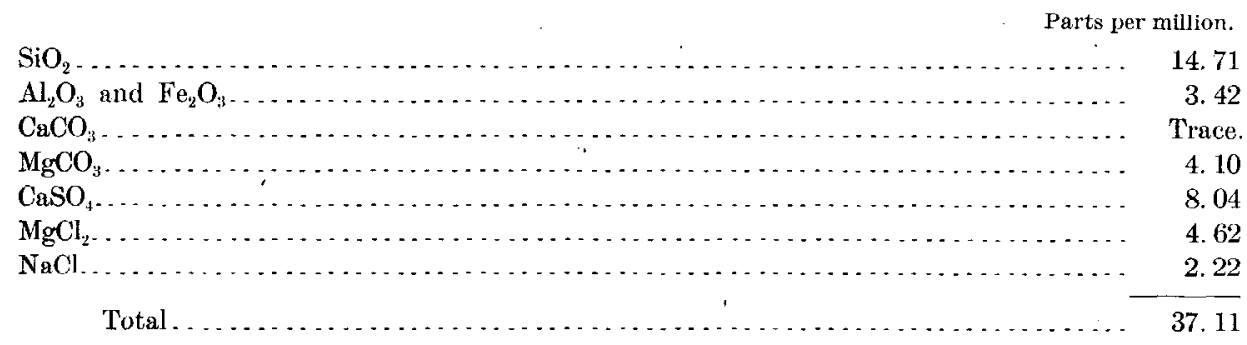

27 4. Mr. Walsh reports that at a depth of 50 feet layers of lignite and mud were encountered, in which were found "snail shells, skimmer shells, and razor shells.". (See fig. 13.)

Tisbury:

Record of well near Hewlett.

1. Sand and gravel similar to material elsewhere on Rockaway Ridge

Feet.

0-13

Sankaty:

2. Blue clay.

Sankaty and Jameco:

3. Fine sand with no available water.

$13-21$

4. Good water-bearing sand. 
275.

Record of Ars. Julia Flower's well near Lynbrook.

Tisbury:

Feet:

1. Brown to red sand and gravel............................ 0 . 13.5

Sankaty and Cretaceous:

2. Perfectly dry blue clay; no stones............................ 17-80

3. White clay, which became creamy under the action of the wash pipe...... $80-82$

4. White sand.............. $82-90$

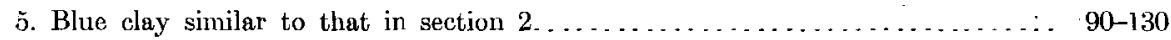

6. Fine sand, somewhat clayey from" clay above.................... 130-135

7. Sand, changing gradually to white gravel. (This layer was water bearing, but

the water had a puckerish taste, like alum.) . . . . . . . . . . . . . . 135-155

$8 . \Lambda$ blue dry clay, similar to that in sections 2 and $5 \ldots \ldots \ldots \ldots \ldots \ldots \ldots .155-180$

27 7. Through the kindness of Mr. Franklin B. Lord, president of the Queens County Water Company, and Mr. Chas. R. Bettes, chief engineer, self-recording gages were placed on three wells at this point: One 504 feet deep, another 74, and the third 14 feet deep. A portion of the results of this work is shown in PI. XVIII; a detailed report may be expected later. Mr. Lord reports that in 1903 the deep well was pumped at the rate of from 36,000 to 44,000 gallons for twenty-four hours, for a period of twenty-four and one-fourth hours, with three stops of fifteen minutes each. This reduced the level of the water 3.92 feet; it returned to its normal level in seventy minutes after the pumping was stopped. During this test the level of the water in the 74-foot well was not reduced, and the 504-foot well was not affected by the pumping of the shallower well. On February 13,1903, the 74-foot well was given a five-hour test, and the level of the water was reduced more than 22 feet; it regained its normal level in eighteen minutes. In December, 1903, a new well was started at this place from which $\mathrm{Mr}$. Bettes has furnished the following samples:

T.sbury:

Record of Queens County Water Company's well at Lynbrook.

Coarse yellow quartz sand; no erratic material:- .

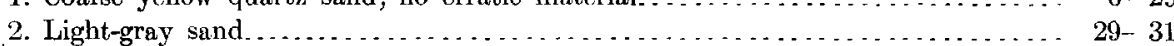

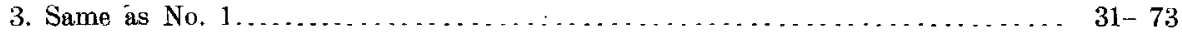

Cretaceous?

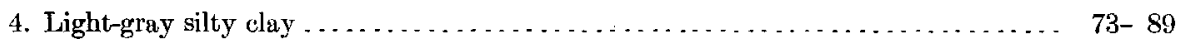

5. Light-yellow medium sand; no erratic material................... $89-150$

Cretaceous:

6. Fine to medium gray, lignitic sand . . . . . . . . . . . . . . . . . . . . 150-158

7. Very fine black, micaceous, lignitiferous silt . . . . . . . . . . . . . . . . . . . . . . . . 158-200

8-9. Very fine, dark-colored, lignitiferous sand. . . . . . . . . . . . . . . . . . 200-228

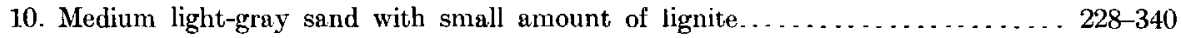

11. Dark-colored, lignitiferous, silty clay. . . . . . . . . . . . . . . . . . . . . 340-363

12. Medium dirty-yellow sand, lignitic.............................. 363-403

13. Medium to coarse gray sand ................................. $403-536$ 
Analysis of water from wells of Queens County Water Company at Lynbrook. [By F. C. Chandler, February 25, 1903. Parts per million.]

\begin{tabular}{|c|c|c|}
\hline & $\begin{array}{l}\text { 504-foot } \\
\text { well. }\end{array}$ & 72-foot well. \\
\hline Appearance. . . . & Clear. & Faint milkiness. \\
\hline Color...$\ldots \ldots \ldots$. & None. & None when filtered. \\
\hline Odor (heated to $100^{\circ} \mathrm{F}$.) .... & None. & None. \\
\hline Taste $\ldots \ldots \ldots \ldots \ldots \ldots \ldots \ldots$ & None. & None. \\
\hline Chlorine in chlorides. . & 3.000 & 9.000 \\
\hline Sodium chloride................. & 4.950 & 14.851 \\
\hline Phosphates (as $\mathrm{P}_{2} \mathrm{O}_{5}$ ) $\ldots \ldots \ldots \ldots \ldots \ldots \ldots$ & None. & None. \\
\hline Nitrogen in nitrites . . . . . . . . . . . & None. & None. \\
\hline Nitrogen in nitrates. ....... & .014 & .562 \\
\hline Free ammonia. ............ & .022 & .016 \\
\hline Albuminoid ammonia............... & .026 & .006 \\
\hline 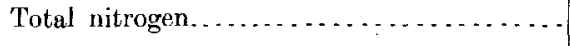 & .053 & .580 \\
\hline \multicolumn{3}{|l|}{ Hardness: } \\
\hline Before boiling..... & 8.855 & 13. 915 \\
\hline After boiling ................. & 3.795 & 8.855 \\
\hline Organic and volatile (loss on ignition)..... & 2.000 & 5.000 \\
\hline $\begin{array}{l}\text { Mineral matter (nonvolatile), } \mathrm{CO}_{2}, \text { restored } \\
\text { with ammonium carbonate............ }\end{array}$ & 13.500 & 40.500 \\
\hline Total solids (by evaporation) dried at $110^{\circ} \mathrm{C}$. & 15. 500 & 45.500 \\
\hline
\end{tabular}

278. The following record is taken from a blueprint kindly furnished by Chief Engineer I. M. De Varona:

Oet.

$0-4$

2. Yellowish sand, water bearing................................... $4-20$

3. Gray sand, water bearing...................................... $20-28$

4. Gray sand with little gravel, water bearing. . . . . . . . . . . . . . . . . $28-36$

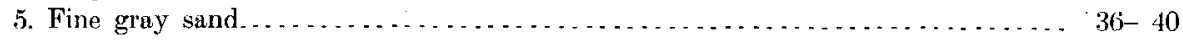

6. Yellowish sand and gravel..................................... 40.44

7. Yellowish sand and gravel; traces of clay ................... $44-58$

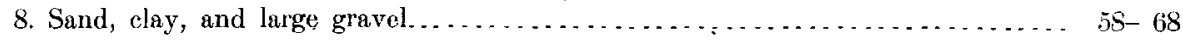
Tisbury? :

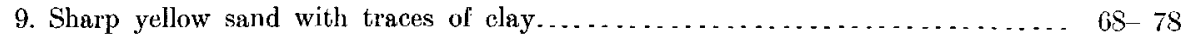

Cretaceous? :

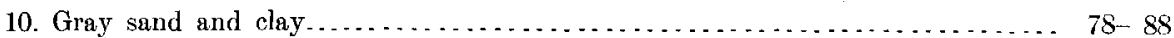

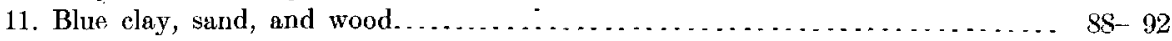

12. Yellowish sand and clay ................................... 92-98

13. White sand, wood, and clay................................ $98-108$ Cretaccous:

14. Gray sand, wood, and clay ................................... 108 128

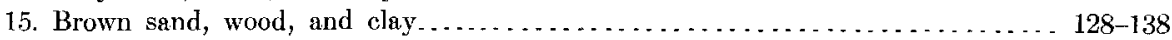

16. White sand with traces of clay . . . . . . . . . . . . . . . . . . . . . . . . 138-160

17. White sand with wood and clay . . . . . . . . . . . . . . . . . . . . . .

Elevation of surface, 16.0 feet. 
279. The following record is taken from a blueprint kindly furnished by Chief Engineer I. M. De Varona:

Record of Brooklyn test well No. 2S.

Wisconsin and Tisbury:

1. Tellow sand. ......................................... 8

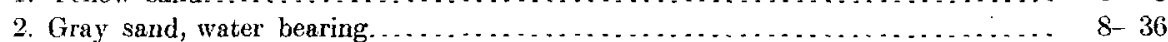

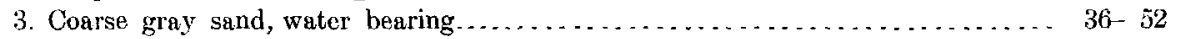

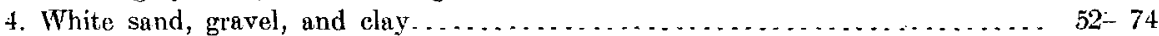

Transition:

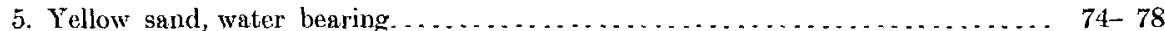

Cretaceous?:

6. Clay, sand, and gravel ................ 78-100

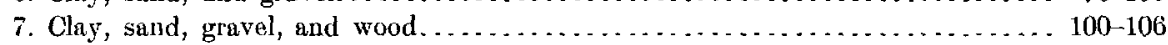

8. White sand, clay, and wood .................................. 106-130 Cretaceous:

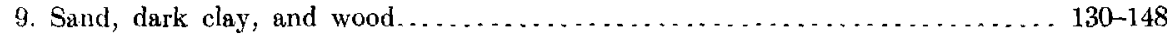

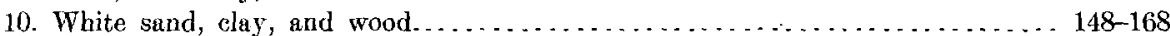

11. Sand, gravel, wood, and blue elay......................... 168-172

12. Blue clay. . . . . . . . . . . . . . . . . . . . . . . . . . . . . . . . . . . . . . 172-185

13. Sandstone, iron ore, and wood embedded in black clay . . . . . . . . . . . 185-198

14. Wood and black clay ................ 198-202

15. Fine white sand, wood, and clay . . . . . . . . . . . . . . . . . . . . . . $202-220$

16. Sand, wood, and blue clay .................................. 220-247

17. Blue clay and iron ore ...................................... 247-260

18. Sand, wood, and clay........................................ 260-276

19. Sand, wood, clay, and iron ore................................... 276-282

20. Sand, clay, and wood.................................... 282-296

21. Hard pan; iron. . . . . . . . . . . . . . . . . . . . . . . . . . . . . . . . . 296-298

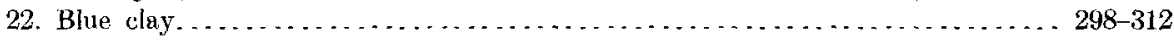

23. Sand, wood, and clay . . . . . . . . . . . . . . . . . . . . . . . . . . . . . $312-367$

24. Clay with a little sand . . . . . . . . . . . . . . . . . . . . . . . . . . . . $367-374$

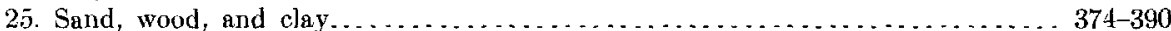

Elevation of surface, 16.7 feet.

280. The following record is taken from a blueprint kindly furnished by Chief Engineer I. M. De Varona:

Wisconsin and Tisbury:

Record of Brooklyn test well No. 22.

1. Yellow sand.

Feet.

2. Sharp grayish sand, water bearing. ...

3. Coarse, grayish sand, with gravel, water bearing ..................... 24-36

4. Same sand; larger gravel, water bearing........................... $36-44$

5. Fine grayish sand, water bearing. .............................. $44-56$

6. Gravel, sand, and clay....................................... $56-67$

Wisconsin and Tisbury? :

7. Sharp, yellow sand, water bearing......................... $67-82$

8. Fine grayish sand......................................... $82-90$

9. Fine grayish sand with gravel, wood, and clay.................... $90-100$

Tisbury and Cretaccous?

10. Fine grayish sand with larger gravel, wood, and clay............... 100-107

Cretaceous:

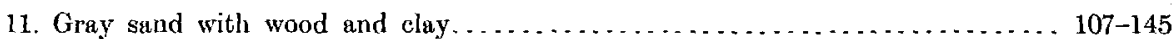

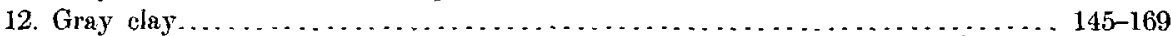

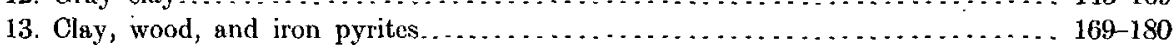

14. Sand, gravel, clay, wood, and iron pyrites........................ 180-190

15. Fine grayish sand, clay, and wood............................. 190-220 
Cretaceous-Continued. Feet.

16. Fine sand, clay, wood, and iron pyrites. ...................... 220-276

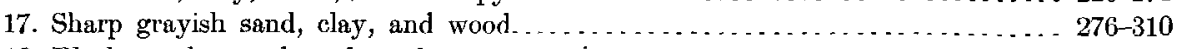

18. Black muck, wood, and sand . . . . . . . . . . . . . . . . . . . . . . . . 310-324

19. Light-colored gray sand, wood, and traces of clay . . . . . . . . . . . . . 324-327

20. White clay, wood, sand, and gravel. . . . . . . . . . . . . . . . . . . . $327-333$

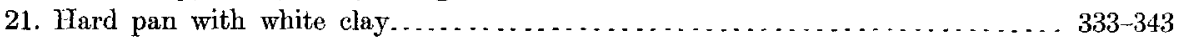

22. Sharp grayish sand, wood, and traces of clay . . . . . . . . . . . . . . . . 343-347

23. Same sand; slightly water bearing. . . . . . . . . . . . . . . . . . . . . . . . . . 347-356

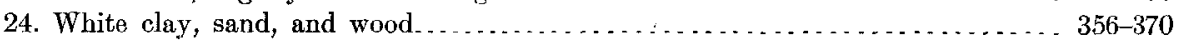

Elcvation of surface, 17.4 feet.

281. As no samples were preserved from test well No. 21 , the following record is taken from the reports of the inspector: $a$

Wisconsin and Tisbury:

\section{Record of Brooklyn test well No. 21 .}
1. Yellow sand
Feet.
2. Fine yellowish sand ........................... $8-28$
3. Fine yellowish sand with a large quantity of mica scales ............ 28-36
4. Coarser yellowish sand....................................... 36- 50

Wisconsin and Tisbury?:

5. Finer yellowish sand with small gravel........................ $50-65$

6. Fine white sand with large gravel. .............................. $65-69$

7. Fine white sand with wood and traces of clay ..................... $69-80$ Tisbury and Cretaceous?:

8. Fine brownish sand with wood and traces of clay ................... $80-85$ Cretaceous:

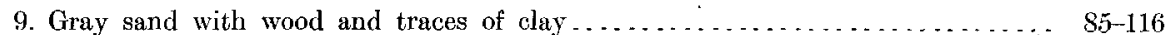

10. Fine gray sand with wood and traces of clay $\ldots \ldots \ldots \ldots \ldots \ldots \ldots \ldots \ldots . .116-195$

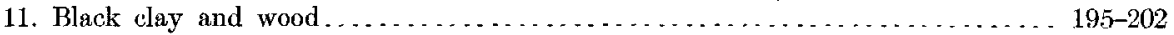

12. Gray sand with wood and traces of clay . . . . . . . . . . . . . . . . . . . 202-225

13. Gray clay with wood and hardpan. . . . . . . . . . . . . . . . . . . . . 225 236

14. Gray sand with traces of wood and clay ........ . . . . . . . . . . . . . 236-240

15. Gray quicksand with traces of wood and clay . . . . . . . . . . . . . . . 240-248

16. Gray clay ................................................ 248 . 262

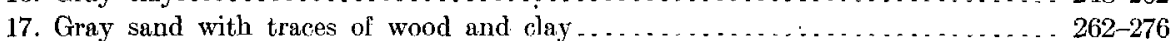

18. Gray sand with wood, clay, and hardpan...................... 276-282

19. Fine gray sand and clay. . . . . . . . . . . . . . . . . . . . . . . . . . . . . 282-290

20. Sharp grayish sand with traces of wood and clay, slightly water bearing. . . . 290-295

21. Sharp grayish sand with wood and traces of clay . . . . . . . . . . . . . 295-345

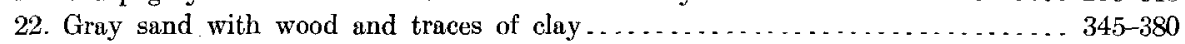

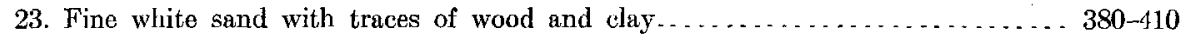

Elevation of surface 17.8 feet.

282. As no samples were preserved from test well No. 20, the following record is taken from the report of the inspector: $b$

Wisconsin and Tisbury:

Record of Brooklyn test well No. 20 .

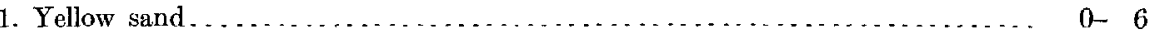

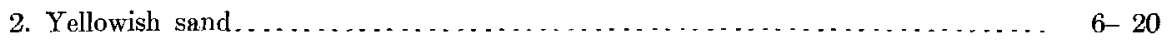

3. Yellowish sand with a little gravel ........................... 20 26

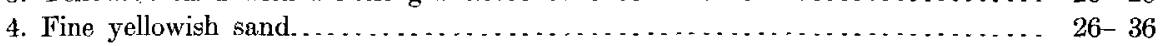

5. Small light-colored gravel with gray sand...................... $36-78$

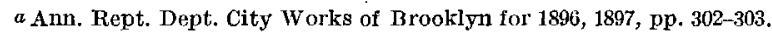
$b$ Ibid., p. 301. 
Cretaceous? :

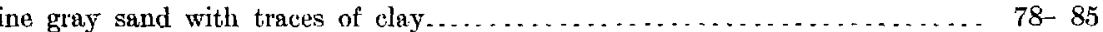

Cretaceous:

7. Gray clay and gravel. . . . . . . . . . . . . . . . . . . . . . . . . . $85-90$

8. Gray sand with clay and wood. . . . . . . . . . . . . . . . . . . . . . . 50.100

9. Gray sand with clay, wood, and gravel ... . . . . . . . . . . . . . . . . 10 10 110

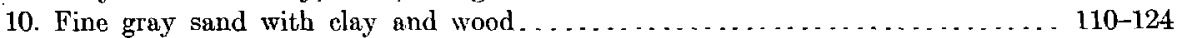

11. Fine gray sand with clay, wood, and gravel. . . . . . . . . . . . . . . . . . . 124-130

12. Gray sand with clay and wood: ............................. 130 148

13. Darker gray sharp sand with a little wood . . . . . . . . . . . . . . . . . . . 148-152

14. Dark-gray clay with wood and gravel. . . . . . . . . . . . . . . . . . . . . 152-154

15. Light-gray sand with wood and traces of clay $\ldots \ldots \ldots \ldots \ldots \ldots \ldots \ldots \ldots \ldots$. . . . . . . . . 172

16. Finer light-gray sand with wood and clay $\ldots \ldots \ldots \ldots \ldots \ldots \ldots \ldots \ldots \ldots \ldots \ldots \ldots$

17. Fine white sand with wood and clay ............................ 178-212

18. Sharp light-gray sand with wood and clay (contains water, but not enough for pumping) . . . . . . . . . . . . . . . . . . . . . . . . . . . . 2 212 225

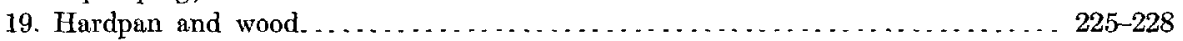

20. Gray sand with clay and wood (contains water but not enough for pumping). 228-242 Elevation of surface 14.6 feet.

283.

Record of C.Schreiber's well at Valley Stream.

Mr. Baldwin says that the description of this well will apply to all the wells in the vicinity of Valley Stream. In. some places it is 2 or 3 feet farther to the water, but there is no change in the material.

284. The Long Island Railroad Company gives the following part analysis of its shallow dug well at this point:

$$
\text { Analysis of railroad well at Valley Stream. }
$$

Total solids

Parts per million

285. The following section has been prepared from samples preserved by the Brooklyn water department (see fig. 10):

Wisconsin:

Record of Brooklyn test well No. 19.

Transition:

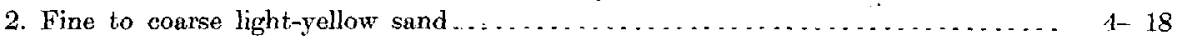

Tisbury:

3. Light-gray and grayish-yellow sands and gravel, probably glacial.......... 18 - 72 Sankaty?:

4. Yellowish gray clay

Cretaceous:

5. Dark-gray fine to medium sand, with lignite................... 95-132

6. Black elay with lignite. . . . . . . . . . . . . . . . . . . . . . . . . . . 132-140

7. Grayish-white clay ... . . . . . . . . . . . . . . . . . . . . . . . . . . . . 140-150

8. Medium gray sand with lignite ... . . . . . . . . . . . . . . . . . . . . . . . 150-208

"At extreme depth was found to be slightly water bearing; very small flow." Elevation of surface, 9.4 feet, Brooklyn base.

286. The following analysis has been made by the Brooklyn health department:

Analysis of water from wells at Watt's Pond pumping station.

Total solids.

Loss on ignition.

Parts per million.

Free ammonia. 63.25

Albuminoid ammonia.

15. 15

.06

.04 
Chlorine as chlorides

Parts per million.

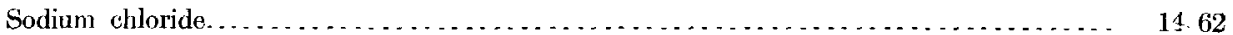

Nitrogen as nitrates. . . . . . . . . . . . . . . . . . . . . . . . . . . . . . . . . . .

Nitrogen as nitrites . . . . . . . . . . . .

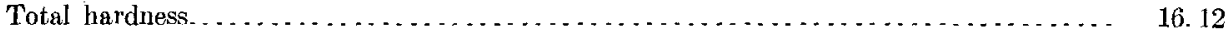

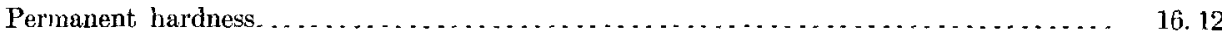

This is the average of 4 analyses.

287. The following section has been taken from a blueprint kindly furnished by Chief Engineer I. M. De Varona; no samples were preserved from this well (see fig. 10):

Wisconsin

Record of Brooklyn test well No. 25, at Watt's Pond pumping station.

1. Top soil

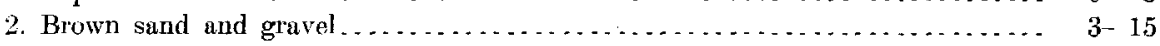

Tisbury:

3. Yellowish fine sand. . . . . . . . . . . . . . . . .

Transition:

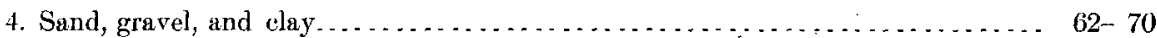
Sankaty?:

5. Clay and gravel. ........................................... $70-95$ Jameco?:

6. Fine gray sand; traces of clay and wood. ......................... $95-118$

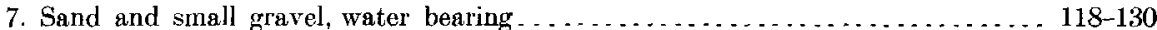
Cretaceous:

8. Black clay, gravel, and wood . . . . . . . . . . . . . . . . . . . . . . . . . . . . . . . 130-148

9. Blue clay, gravel, and wood . . . . . . . . . . . . . . . . . . . . . . . . . . 148-157

10. Sand, wood, and clay . . . . . . . . . . . . . . . . . . . . . . . . . . . $157-160$

11. Small gravel, wood, and clay .................................... 160-168

12. Sand, wood, and clay ...................................... 168-176

13. Sand, wood, and clay, water-bearing ............................. 176-184

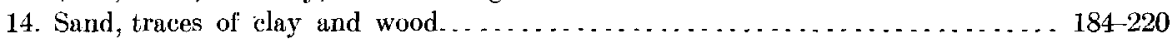

15. Sharp sand, clay and wood, water-bearing ....................... 220-235

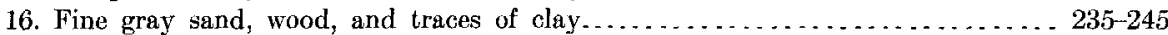

17. Sharp, gray sand, clay, and wood, water-bearing . . . . . . . . . . . . . . . . 245-284

18. Sand, small gravel, clay, and wood, water-bearing . . . . . . . . . . . . . . . . . . 284-296

19. Whitish clay, sand, and wood . . . . . . . . . . . . . . . . . . . . . . . . 296-302

20. Small gravel, wood, and clay, water-bearing ...................... $302-331$ Elevation of surface, 8.2 feet.

288. See under No. 290. The following analysis has been made by the Brooklyn health department: Analysis of water from Clear Stream pumping station.

Total solids.

Parts per million.

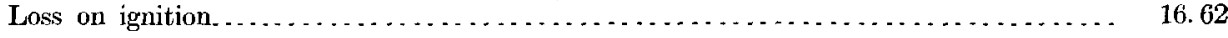

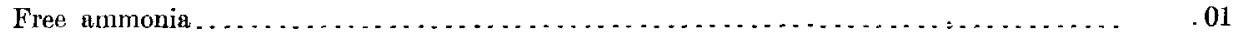

Albuminoid ammonia. . . . . . . . . . . . . . . 02

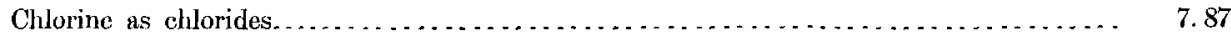

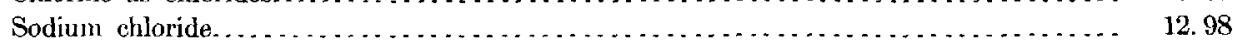

Nitrogen as nitrates. . . . . . . . . . . . . . . . . 51

Nitrogen as nitrites. ... . . . . . . . .

Total hardness. . . . . . . . . . . . . . . . . . . . . . . .

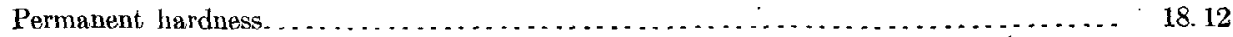

This is an average of 8 analyses. 
289. The following record has been prepared from samples preserved by the Brooklyn department of water supply (see fig. 10):

Wisconsin:

Record of well at Clear Stream pumping station. Transition:

2. Light-yellow medium sand. $\ldots \ldots \ldots \ldots \ldots \ldots \ldots \ldots \ldots \ldots \ldots \ldots \ldots \ldots \ldots, 6,35$

Tisbury:

3. Darker, yellowish-brown, fine to medium sand................... $35-44$

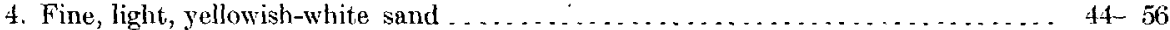

Cretaceous:

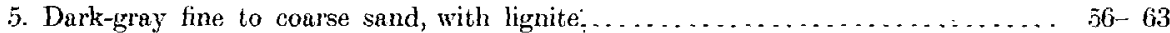

6. Very dark-gray clay (unlike clay above old glacial beds; resembles clay in No. 197) . 63-87

7 . Fine to medium gray sands ............................... 87-125

8. Gray sand and lignite or peat. . . . . . . . . . . . . . . . . . . . . . . . . $12 \%$

9 . Fine to medium gray sand. . . . . . . . . . . . . . . . . . . . . . $130-190$

Elevation of surface, 13.6 feet. "No water was found in this well."

290. W. D. Andrews \& Brother, under date of May 8, 1895, report: "In 1894 we completed for the city of Brooklyn a second contract for two tubular gang-well plants, with a capacity of $5,000,000$ gallons each, one plant being located at the Forest, Stream and the other at the Clear Stream station. *** Wo struck veins of water at these two stations, at and beyond 106 feet in depth, that flowed 10 gallons per minute at the surface from a 2-inch tube, and would rise in a pipe 3 feet above, while the water levels in the nuxiliary tubes of the gang wells were several feet below this surface (lowered to that depth by continuous pumping of double the quantity of water required by our contract obligations). Within 60 feet of water veins that would flow 5 to 10 gallons per minute, through 2 -inch tubes, from depths of 60,100 , and 300 feet, and yield by hand pumping 30 to 50 gallons from any one of the depths named, we sunk a 4-inch tube 400 feet, and the only water found was at about 35 feet, which did not rise above the level at which it was first encountered, nor yield, by hand pumping, above 5 gallons per minute.

Phillips \& Worthington report the following section of a test well at this point (sec fig. 10):

Record of test well at Forest Stream pumping station.

Wisconsin and Tisbury: Feet.

1. Stratified clays and sands, with underlying strata containing water........ $0-100$

Jameco?:

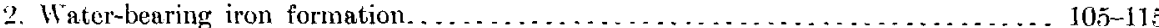

Cretaceous:

3. Hard white sticky clay . . . . . . . . . . . . . . . . . . . . . . . . . . . . $115-260$

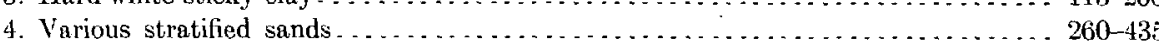

It is quite probable that the water-bearing formation from 105 to 115 is Jameco. The "hard white sticky clay" is probably the same as the fine white or gray lignitiferous sands found in the Brooklyn waterworks test wells.

Mr. De Varona reports that the water from the deep test well at Forest Stream station is so impregnated with sulphureted hydrogen as to be unfit for use."

'The following analysis has been.made by the Brooklyn health department:

Analysis of water from Forest Stream pumping station.

Parts per million

Total solids. ........

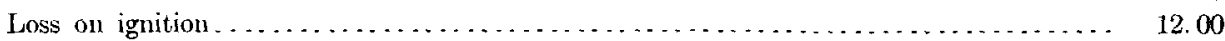

Free ammonia . . . . . . . . . . . .

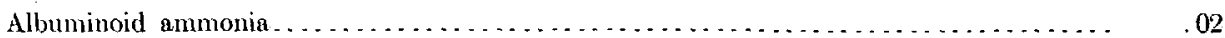

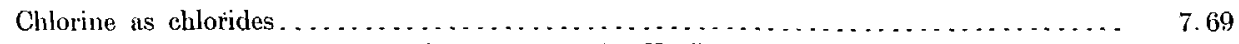
a History and Description of the Brooklyn Waterworks, 1896, p. 16.

$17116-$ No. 44-06-16 


\section{UNDERGROUND WATER RESOURCES OF LONG ISLAND, NEW YORK.}

Parts per million.

(2.

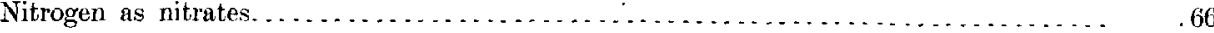

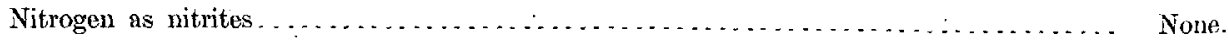

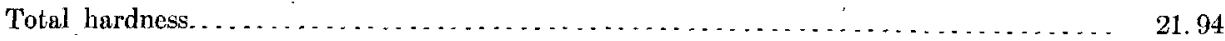

Permanent hardness.............................................. 19.44

This is the average of 8 analyses.

291. The following record has been prepared from the samples preserved by the Brooklyn water department (see figs. 10, 13):

Wisconsin:

Record of Brooklyn test well No. 12.

1. Yellowish surface loam.................................

2. Fine to coarse light yellowish brown speckled sunds............., $5-30$ Tisbury:

3. Medium to coarse light yellowish, white sand .................. $30-46$

4. Medium yellow sand with quartz pebbles below. . . . . . . . . . . . . $46-63$

5. Yellowish gray clay with quartz pebbles . . . . . . . . . . . . . . . . 63 - 66

6. Medium light-yellow sand ............................... 66 . Sankaty:

7. Light, reddish yellow, medium sands....................... 73 - 98 Jameco:

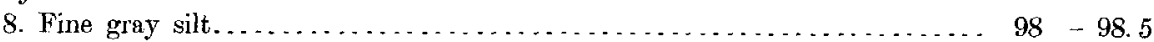

9. Dark, multicolored, dirty sands (old glacial) . . . . . . . . . . . . . . . 98. 5-138

Transition:

10. Transition. . . . . . . . . . . . . . . . . . . . . . . . . . . . $138 \quad-145$

Cretaceous:

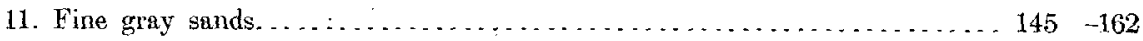

12. Fine, dark-gray, clayey silt. . . . . . . . . . . . . . . . . . . . . . . . . $162 \quad-172$

13. Gray sand with occasional quartz pebbles and pieces of lignitized wood. Wood

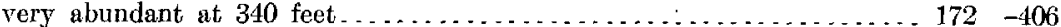

Elevation of surface 18 feet. "No water was found in the strata below the clay bed."

292. The following record has been prepared from the samples preserved in the municipal building, Brooklyn (see fig. 10):

Wisconsin:

Record of Brooklyn test well No. 13

1. Surfuce yellow loam .

2. Fine to medium reddish-yellow sands ......................... $8-32$

Transition:

3. Medium yollow sand, speckled with black ..................... 32-58 Tisbury:

4. Yellowish-white sund and gravel. No glacial pebbles. . . . . . . . . . . . 58-70 Jameco:

5. Fine, reddish yellow, silty sand becoming coarser below, and containing good sized pebbles; many erratics . . . . . . . . . . . . . . . . . . . . . . . . . . . $70-102$

Cretaceous:

6. Gray clay . . . . . . . . . . . . . . . . . . . . . . . . . . . . . $102-105$

7. Very dark clay, lignite, and pebbles . . . . . . . . . . . . . . . . . . . $105-112$

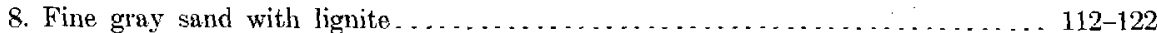

9. Gray clay . . . . . . . . . . . . . . . . . . . . . . . . . . . . . . . . . 122-130

10. Fine dark-gray sand ................................... 130-175

11. Very coarse gray sand and small pebbles. . . . . . . . . . . . . . . . . . .

12. Fine dark-gray sand with occasional quartz pebbles and lignite. . . . . . . . . 190-412

Elevation 21.5 feet, Brooklyn base. 
The presence of reddish yellow silty sand containing a considerable percentage of the compound pebbles which ordinarily characterize the glacial deposits, and which is here not separated by a clay bed from the overlying yellow sands with no glacial material, is unique in this section and doubtless represents a Jameco deposit, which has either never been covered by Sankaty clay or from which the clay has been removed by erosion.

293. The following section has been prepared from the samples preserved by the Brooklyn water department (see fig. 10):

Wisconsin: Record of Brooklyn test well No. 14.

Transition :

0

2. Medium light-yellow speckled sand $\ldots \ldots \ldots \ldots \ldots \ldots \ldots \ldots \ldots \ldots, 6,50$

Tisbury:

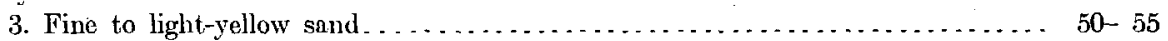

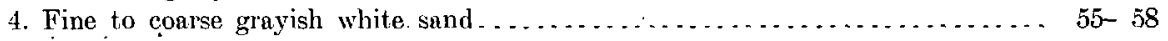

Cretaceous:

5: Very light-yellow silt, looks like loess . . . . . . . . . . . . . . . . . . 58,62

6. Fine sand to coarse gravel, with many pieces of ferruginous concretion...... 62- 72

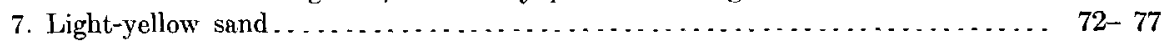

8. Yellowish white sand and gravel............................ $77-92$

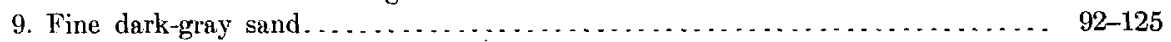

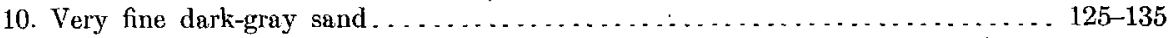

11. Grayish white fine to medium sands, with lignite at 181 and at 244 feet.... 135-328

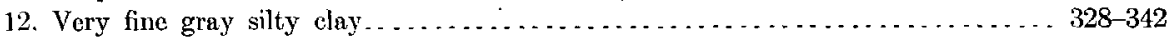

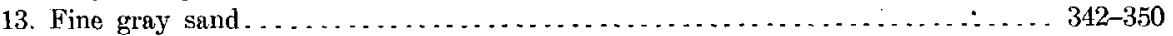

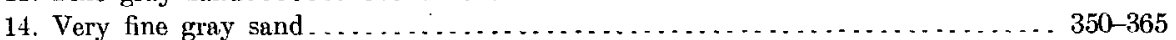

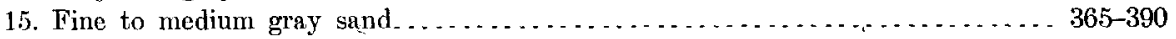

Elevation of surface, 16.7 feet. "No water found in this well."

294. Record of commission's well near Rosedale.

Wisconsin: $\quad$ Feet.

1-2. Surface loam ............................................. $0-1$

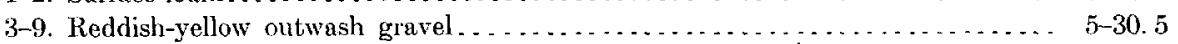

Sce Table XII.

295. The following section has been prepared from the samples preserved by the Brooklyn water department:

Wisconsin:

Record of Brooklyn test well No. 10, near Springfield.

1. Yellow sùrface loam . . . . . . . . . . . . . . . . . . . . . . . . . . . . . . 0 .

2. Fine to coarse yellow sands and gravel (glacial) $\ldots \ldots \ldots \ldots \ldots \ldots \ldots \ldots .2,40$

Tisbury:

3. Medium bright-yellow sands, probably glacial. . . . . . . . . . . . . . . . $40-54$

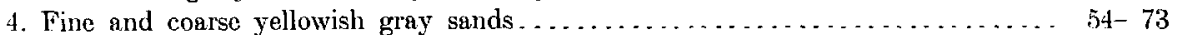

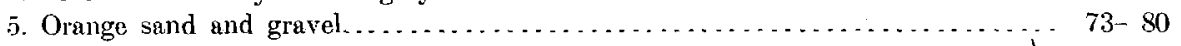

Transition:

6. Gray sand with much lignite. ............................... $80-89$

Sankaty?:

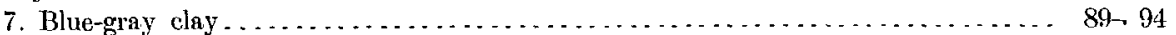

Cretaceous:

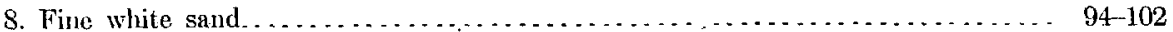

9. Fine gray sands with lignitized wood, well marked at $110-112,139,177-180$,

- $199-200,219,229,235,241-242,250-252,295,306$ feet................. 102-357

All trace of glacial material ceases at 54 feet, and in the examination of samples this point was selected for the line between the Pleistocene and pre-Pleistocene deposits. The yellow gravels, however, suggest the Far Rockaway material, and the blue-gray clay the Sankaty. Elevation of surface, 27 feet. "No water was found in the strata below the blue clay bed." 
296.

Record of commission's test well near Fosters Meadows.

Wisconsin and Tisbury? Feet.

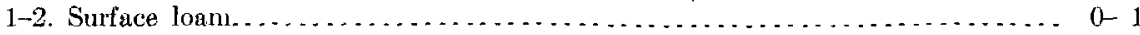

3-10. Reddish-brown outwash sand, with very little gravel. . . . . . . . . . . . . 5-35.5 See Table XII.

\section{$29 \%$.}

Record of commission's test well 1 mile north of Valley Stream.

Recent?

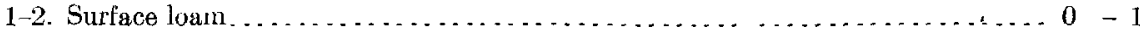

3-4. Black sand; considerable percentage of $\mathrm{MnO}_{2}$ (swamp deposit) . . . . . . . 2.5-5.5

Wisconsin and Tisbury:

$5-8$. Light yellow sand . . . . . . . . . . . . . . . . . . . . . . . . . . . . . . 9.5-26

There is very little glacial material in the two lower samples.

\section{8.}

Record of commission's test well 2 miles north of Valley Stream.

Wisconsin:

$1-2$. Yellow surface loam.................................... 0.1

$3-6$. Outwash sand, reddish brown. . . . . . . . . . . . . . . . . . . . . . . . $5-21$

Tisbury?

7. Light-yellow sand (nothing recognizably glacial) . . . . . . . . . . . . . 25-25.5

299. Record of commission's test well between Valley Stream and Floral Park.

Wisconsin: $\quad$. Feet.

1-2. Surface loam. . . . . . . . . . . . . . . . . . . . . . . . . . . . . 0.7

3-9. Reddish brown silty sand and gravel (considerable glacial material).... $6 \quad 6 \quad 36$

Tisbury:

10-16. Lighter, brownish yellow, medium sand, doubtfully glacial; no sharp line

can be drawn between this material and that either above or below . . . . $42 \quad-73$

Cretaceous:

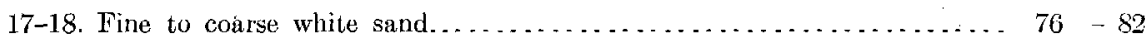

19-21. White sand and gravel (not recognizably glacial) .............. 84 . 96

22-23. Yellowish white sand with a little clay ...................... 96.5-98.5

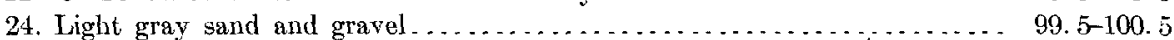

25. Very line, dark-yellow, clayey sand. . . . . . . . . . . . . . . . . . . . . . . . . 101. 7-102

26. Very fine, light grayish white sand, with much silvery white muscovite... 105. 5-106.5

28. Very light, grayish yellow, clayey sand . . . . . . . . . . . . . . . . . . 106.6-107.1

29. Very fine white sand with muscovite and a little lignite. . . . . . . . . 107, 5-108

30. Very fine clayey sand with lignite and muscovite, yellowish brown...... $108 \quad-109$

31. Fine grayish sand with muscovite and lignite $\ldots \ldots \ldots \ldots \ldots \ldots \ldots \ldots \ldots \ldots . \ldots 110.111$

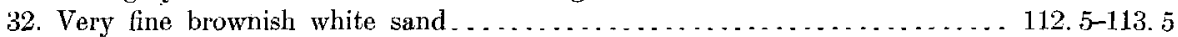

33. Very dark grayishs and, with muscovite. . . . . . . . . . . . . . . . . 114.7-115.5

34. Black sandy clay with lignite . . . . . . . . . . . . . . . . . . . . 115.5-116.5

35. Very black sandy clay, with FeS . . . . . . . . . . . . . . . . . . $120 \quad-120.5$

300. Record of commission's test well $\mathscr{2}$ miles southeast of Queens.

Wisconsin and Tisbury?: - Fect.

1-12. Reddish-yellow glacial sands and gravel, with much biotite............. 0 . . .

301. Record of commission's test well 1 mile south of Queens.

Wisconsin and Tisbury?:

1. Dark sandy loam.

2. Subsoil sandy loam.

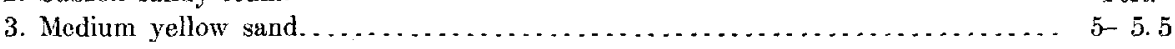

4. Sand with fine gravel; considerable erratic material . . . . . . . . . . . . . . . . . . 10-11

5. Wash sample shows fine grayish sand, while the sand-bucket sample shows a large percentage of gravel. . . . . . . . . . . . . . . . . . . . . . . . . . . . 15-15.5

6. Grayish-yellow sand (sand-bucket and wash samples very nearly the same) . . . . . 20-21

7. Same, except that sand-bucket sample shows some gravel. . . . . . . . . . . . 25-25.5 
302. A number of shallow wells were put down at this point by the commission on additional water supply for pollution tests, by Mr. George Whipple, of the Mount Prospect laboratory. The material penetrated was entirely glacial outwash sand and gravel.

303. Record of commission's test well near Floral Park.

Wisconsin and Tisbury: Fcet.

1. Surface, dark sandy loan...................................

2. Lighter loamy sand, some gravel........................ 5

3. Coarse yellow sand. . . . . . . . . . . . . . . . . . . . . . . .

4. Medium grayish-yellow sand with some small gravel. . . . . . . . . . . . . . 10-10. 5

5. Same, with more gravel.................................... 15-16

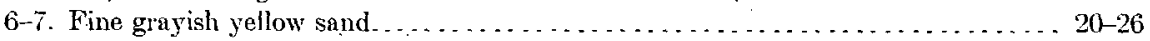

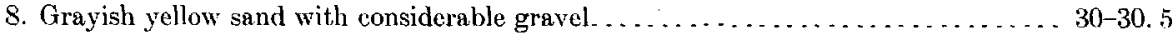

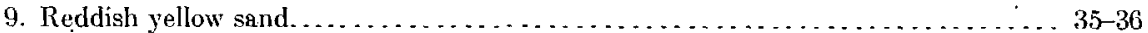

10. Same, but with more gravel . . . . . . . . . . . . . . . . . . . . . . . . . . . 40-41

The whole section is apparently outwash gravel. See Tables XII and XIII.

304. Record of commission's test well, 2 miles south of New Hyde Park.

Wisconsin and Tisbury?:

1-2. Yellow loamy sand.

Feet.

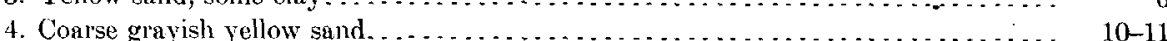

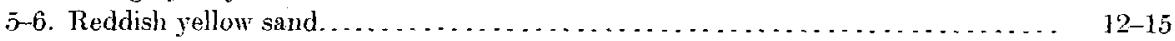

7-8. Coarse yellow sand to fine gravel, with some erratics . . . . . . . . . . . . .

9-10. Grayish yellow sand, with much biotite ....................... $29-36$

11. Coarse grayish yellow sand. . . . . . . . . . . . . . . . . . . . . . . . . . . 38

305.

Record of commission's test well near New Hyde Park.

Wisconsin:

Feet.

1. Black sandy loam..................................... 0 . 5

2. Reddish yellow elayey sand. ..................................... 4

3. Reddish yellow sandy clay ................................. 8 9

4. Reddish brown very fine to coarse sand, with much mica and erratics......... 14-15

5. Gravel up to three-eighths inch in diameter; some erratics................. 19.5

Tisbury:

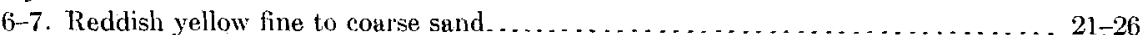

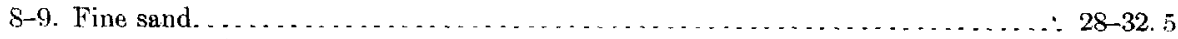

306.

Record of commission's test well near New Hyde Park.

Wisconsin:

Feet.

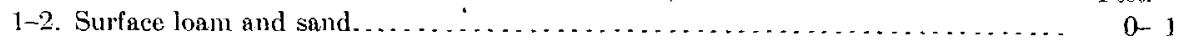

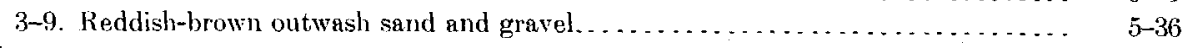
Tisbury:

10-13. Fine to coarse grayish sand, clearly glacial, but differing in appearance from that just above it

307.

Record of commission's test well near New Hyde Park.

Wisconsin:

1-2. Surface, loamy sand...............................

3-5. Light reddish yellow outwash sand and gravel $\ldots \ldots \ldots \ldots \ldots \ldots \ldots \ldots \ldots \ldots . . . \ldots$

$5 \frac{1}{2}$. Dark-gray outwash sand and gravel . . . . . . . . . . . . . . . . . . . . . . 17-17.3

6-7. Reddish brown silt to coarse sand (glacial outwash) . . . . . . . . . . . . . . . . 20-20

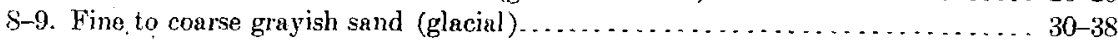

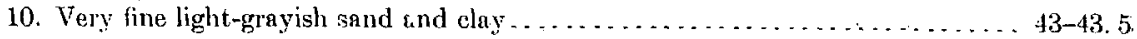
Transition:

11-16. Grayish brown outwash sand and gravel...................... $45-71$ Tisbury:

17. Coarse light-yellow sand, with a much smaller percentage of glacial material than in samples above. 


\section{UNDERGROUND WATER RESOUROES OF LONG ISLAND, NEW YORK.}

308.

Record of commission's test well near New Hyde Park.

Wisconsin:

Feet.

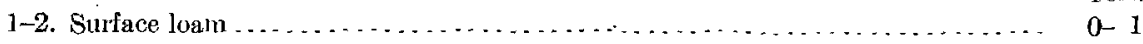

3-17. Dark-gray outwash sand and gravel; very large percentage of erratic materizl _ 2.5-66.5 See Tables XII, XIII.

310.

Record of commission's test well. near Floral Park.

Wisconsin:

Feet.

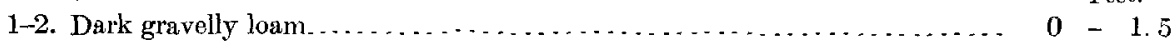

3-8. Grayish brown outwash sand and gravel...................... 4 - 31

Tisbury:

9-12. Medium to fine gray sand.............................. $35-53$

13. Medium gray sand, with small gravel. . . . . . . . . . . . . . . . . . . $55-56$

14-17. Fine grayish-brown gravel, with some sand; contains some erratic material. $60-77$

Cretaceous:

18. Very fine yellow sand............................. $80.5-81.5$

19-23. Light zellow, medium, quartz sand lacking the erratic particles in the

upper samples ......................................... $84-106$

24-26. Very fine, yellow, silty sand . . . . . . . . . . . . . . . . . . . . . $109 \quad-117$

27. Dark sandy clay .................................... $120 \quad-121$

28. Very fine dark-gray sand ................................. 130 . 130

29. Very fine, dirty yellow sand............................. $148 \quad-149$

Sample No. 25 was obtained when the small hand pump was changed to a larger force pump. It consists of coarse, varigated gravel with many glacial pebbles, and represents the accumuluted coarser material from the upper part of the well. It does not represent material from the depth, 113 feet, from which it was obtained. See Tables XII, XIIT.

312.

Record of commission's test well near Creedmoor.

Feet.

1-18. Outwash sands and gravel See Table XII.

315. See fig. 35 and Pl. XIV.

316. J. H. Herbert reports the following section for this well:

Record of Jagnow Brothers' well near Douglaston.

Wisconsin and Tisbury:

1. Yellow clay and sand

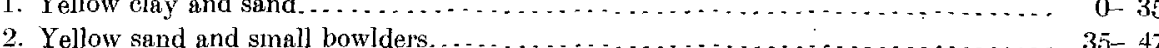

3. Coarse yellow sand and gravel............................. $47-5 . \ldots$

4. Coarse brown sand and iron gravel. . . . . . . . . . . . . . . . . . . . . . $55-6 r$

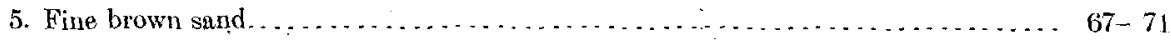

6. Fine yellow sand.......................................... $71-77$

7. White beach sand......................................... $77-89$

8. White and yellow sandy clay . . . . . . . . . . . . . . . . . . . . . . . . . . $89-10 t$

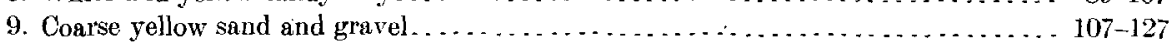

317. 'The well at this place was started by Stotthoff Brothers, who furnished the following samples: Record of well of W.K. Vanderbilt, jr., near Lake Success.

1. No record..... Wisconsin, Tisbury, and Mannetto:

2. Fine sand to large gravel, with a large percentage of erratic material ......... 40-125

3. Reddish-yellow medium sand, with small gravel (contains glacial material)........ 125-145 Cretaceous:

4. Yellow medium sand (not glacial). 
The water in this well stood 116 feet from the surface and tested 21 gallons per minute for twentyfour hours, when the test was pushed up to 40 gallons per minute. The elevation of the ground is 171 feet as determined by the engineers of the commission on additional water supply. The screen was placed from 166 to 186 feet. Later this well was deepened by Thomas B. Harper, of Jenkinstown, Pa. The following record has been transmitted to the Survey by Mr. Alexander S. Farmer:

Pleistocene:

$$
\text { Record of well of W. K. Vanderbilt, jr., near Lake Success. }
$$

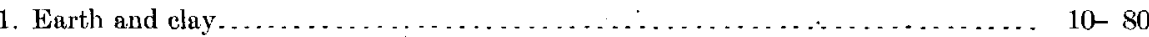

2. Yellow sand............................................. $80-100$

Transition:

Cretaceous:

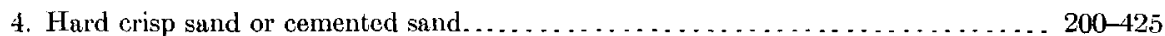

5. Sand and clay in layers; light-colored clay and yellowish-white sand.......... 425-460

6. Organic matter-wood; becomes black after exposure . . . . . . . . . . . . . . . . 460-538

7. Red clay ............................................. $560-660$

8. Fine yellowish-white sand; Lloyd sand. ......................... . 660-700

9. White and coarse gravel; free water-bearing strata; Lloyd sand. . . . . . . . . . . . 700-750

10. Blue clay; becomes light colored upon exposure.................. $750-755$

\section{8.}

Record of commission's test well near Lake Success.

Wisconsin:

Feet.

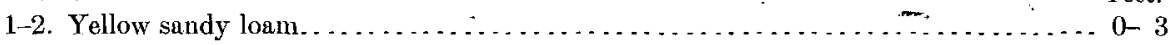

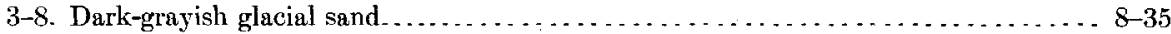

See Table XII

319. According to Mr. E. Lewis, oyster and clam shells were taken from the sands beneath the bowlder drift at Lakeville at a depth of 140 feet. $a$

320. Record of commission's test well between New Hyde Park and Lake Success.

Wisconsin and Tisbury?

Feet.

1. Dark sandy loam.

$0-0.4$

2. Yellow loamy sand.

$0.4-1.5$

3. Black sandy loam.

1. $5-4.4$

4-6. Yellowish-brown outwash sand and gravel.

4. 4-24

$7-9$. Very fine, yellow to gray, silty sand $24-36$

10. Yellow sand to fine gravel containing many erratics. . . .

11. Medium gray sand................................... $41 \quad 42$

12. Fine gravel with many erratics........................... $43 \quad-45$

321.

Record of E. C. Willetts's well near Plattsdale.

Wisconsin:

Feet.

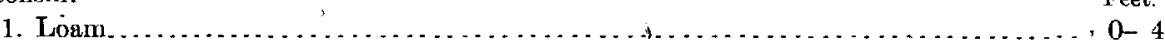

2. Red clay and stones.................................... $4-36$

3. Hardpan; very hard substance containing many angular stones cemented together

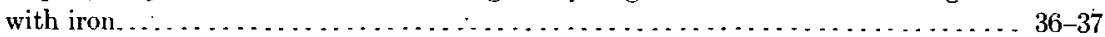

4. Very fine white sand containing dark-colored mica; water bearing $\ldots \ldots \ldots \ldots \ldots \ldots . . .67$ -

In spring the water comes up to within a few feet of the surface; in the dry season it is within 21 or 22 fect of the surface: evidently a perched water table.

322.

Record of A. Kiefer's well near Plattsdale.

Wisconsin:

1. Very hard marl with some cobbles

Feet. retaceous:

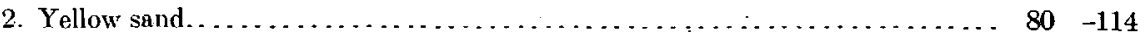

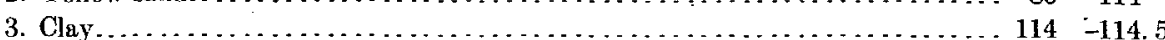

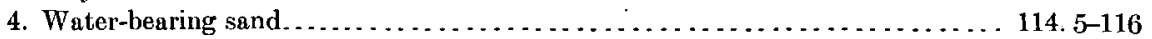

a Pop. Sci. Monthly, vol. 10, J877, p. 442. 
323.

Record of commission's test well 1 mile south of Manhasset.

Wisconsin and Tisbury?

1-2. Sandy loam.

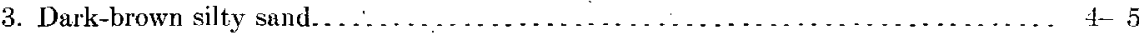

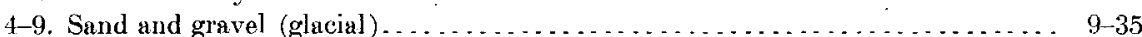

10-11. Brownish-yellow silty sand (apparently glacial) . . . . . . . . . . . . . . 39-47

See Tables XIT, XITI.

324.

Record of W. J. Hamilton's well neur Little Neck.

Wisconsin? and Tisbury:

1. Fine yellow sand.

Fcet

2. Coarse yellow sand

$0-45$

3. White beach sand. $45-55$

Cretaceous?

4. White coarse sand and small gravel......................... $63-71$

5. White fine sand and yellow fine gravel.......................... $71-83$

Cretaceous:

6. White, fine, beach sand . . . . . . . . . . . . . . . . . . . . . . . . 8 $83-99$

7. White, course beach sand . . . . . . . . . . . . . . . . . . . . . . . . . . . . . . . 99-102

8. Blue clay . . . . . . . . . . . . . . . . . . . . . . . . . . . . . . . . . . . . . 102-125

9. White coarse sand. . . . . . . . . . . . . . . . . . . . . . . . . . . . . . . . . 125-143

10. White small gravel. . . . . . . . . . . . . . . . . . . . . . . . . . . . . . . . . 143-147

325. Same section as 324 .

326.

Record of commission's test well near Thomaston.

Wisconsin:

1-3. Yellow sandy clay ........................................ 0

4-5. Dark, multicolored, fine sand to coarse gravel, pronouncedly glacial........ $8 \quad-12$

6-8. Yellowish-hrown clayey silt with gravel.................... $16 \quad-27$

Tisbury:

$9-12$. Fine to coarse glacial sand and gravel........................ $32 \quad-41.5$

Cretaceous:

13. Light-yellow, highly micaceous, clayey sand . . . . . . . . . . . . . . . . 42.7-43.5

14-17. White, clayey, highly micaceous sand . . . . . . . . . . . . . . . . . $44.5-60.5$

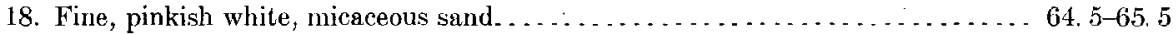

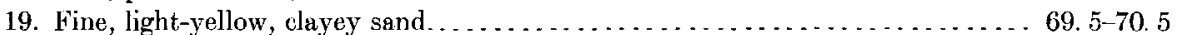

20. Fine to coarse yellow sand.............................. 75,76

21. Medium to coarse white sand; water bearing...................... $78 \quad-79$

327.

Record of J. B. Hixon's well near Thomastom.

Wisconsin:

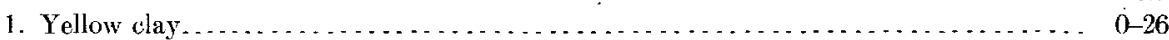

Transition:

2. Yellow fine sand . . . . . . . . . . . . . . . . . . . . . . . .

Tisbury:

3. Coarse, brown, iron-stained gravel . . . . . . . . . . . . . . . . . . . . . . . . . 44-52

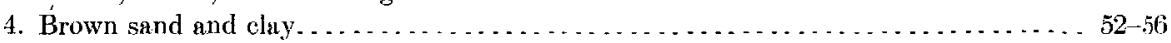

Cretaceous:

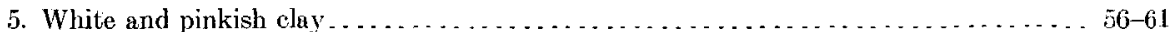

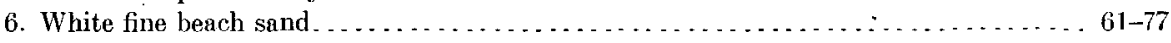

7. Yellow fine beach sand...................................... $77-83$

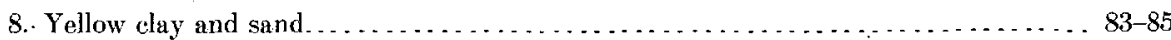

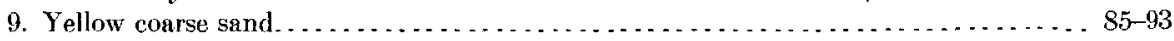


329. Phillips \& Worthington report the following section:

Record of railroad well at Great Neck station, Thomaston.

'Tisbury:

Feet.

1. Sand.............................................. 0

Cretaceous:

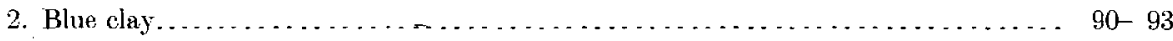

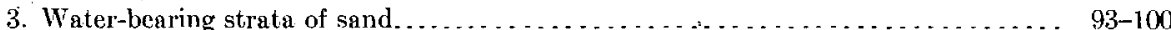

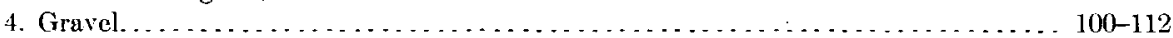

330.

Record of commission's test well near Manhasset.

Wisconsin: $\quad$ Feet.

1-3. Dark, brownish-yellow, clayey sand . . . . . . . . . . . . . . . . . . . .

Cretaceous?:

4-7. Fine, dark-gray or bluish-gray silty sand . . . . . . . . . . . . . . . . . . $9-25$

332. Water rises to a height of 13 feet above the surface, which is perhaps 5 feet above extreme high tide.

Mr. Hamilton reports that the only change ever noticed in this well was during the earthquake that occurred in September, 1898. Then the well commenced to flow very strongly and continued to do so for eight or ten hours, when it became normal and has remained so ever since.

Record of J.F. Hamilton's well at Manhasset.

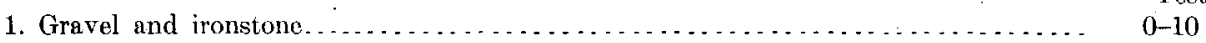

2. Quicksand.................................................... $10-70$

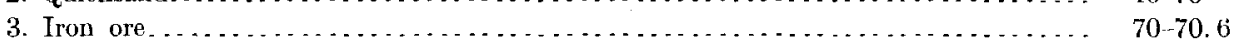

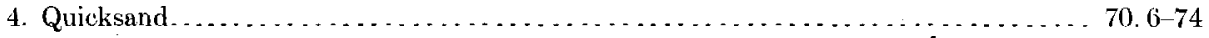

5. Iron ore . . . . . . . . . . . . .

6. Quicksand, with artesian water.................................... $75-78$

7. Iron ore . . . . . . . . . . . . .

335. Fig. 33 illustrates a typical case of a flowing well having many of the aspects of a spring. In this case the pipe was driven to a depth of 10 feet and flowing water obtained, as illustrated. It also shows the difference in flow at high and low tide, which is common in nearly all of the wells along the shore and the mud springs or mud cones on the bottom of the bay; these latter are evidently the same as the cones which were studied near Douglaston.

337. . Record of commission's test well near Manhasset Hill.

Wisconsin and Tisbury?:

1-3. Dark, bowldery, surface loan

4-10. Reddish brown glacial sand and gravel, with large percentage of erratic material.

338.

Record of H. Lustgarten's well near Manhasset Hill.

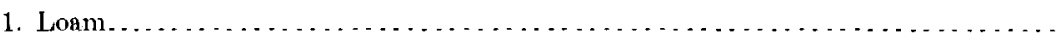

Transition:

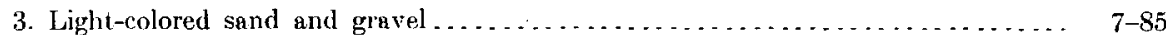
Tisbury:

4. Quicksand and gravel

340.

Record of commission's test well near Manhasset Hill.

Wisconsin:

$1-3$. Brownish yellow silty sand $\ldots \ldots \ldots \ldots \ldots \ldots \ldots \ldots \ldots \ldots \ldots \ldots \ldots \ldots \ldots \ldots . \ldots \ldots$

4-11. Dark fine sand and small gravel, containing much glacial débris......... 9-45

Cretaceous:

12-19. Fine white, micaceous, clayey sand 
311.

Record of Great Neck School well at Great Neck.

Tisbury:

1. Surface sand and gravel.

2. Clay.

Mr. Kasteard left this well one night without having encountered water; when he came back the next morning there was 9 feet of water in the well, and the water gradually rose until it came within 30 feet of the surface; it is probable that he had gotten down very near the bottom of the clay layer and that during the night the water worked its way through.

342. Record of Mrs. M. G. King's well near Great Neck.

Wisconsin:

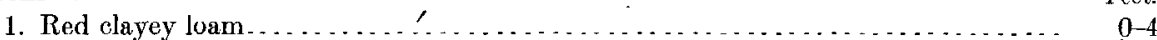

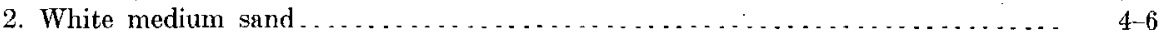

3. Hard pan . . . . . . . . . . . . . . . . . . . . . . . . . . . . . . . . . .

Tisbury:

4. White sand with occasional streaks of iron $\ldots \ldots \ldots \ldots \ldots \ldots \ldots \ldots \ldots \ldots \ldots \ldots \ldots$

5. White sand, described as good building sand . . . . . . . . . . . . . . . . . . . 22-45

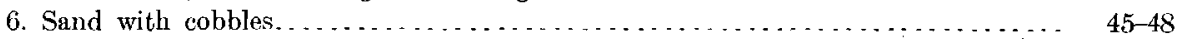

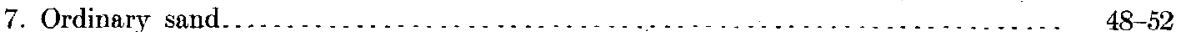

\section{4.}

Record of H. B. Booth's well, Great Neck.

Pleistocene:

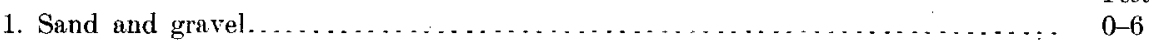

Cretaceous:

2. Clay of various colors; some dark, some light, some reddish.............. . 6-240

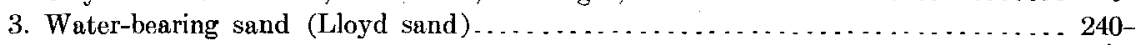

345. The following section should probably be regarded as only approximate:

Pleistocene:

Record of H. B. Anderson's well, Great Neck.

1. Hard clay and gravel; some bowlders. . . . . . . . . . . . . . . . . . . . . Cretaceous:

2. Quicksand and very fine white sand . . . . . . . . . . . . .

347.

Record of Wm. R. Grace's well, Great Neck:

1. Various sands . . . . . . . . . . . . . . . . . . . . . . . . . . . . . . . . 0.103

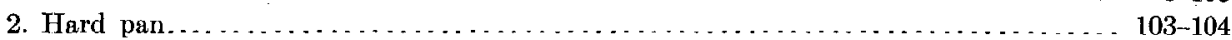

348. Record of V.P. Travis's well, Great Neck.

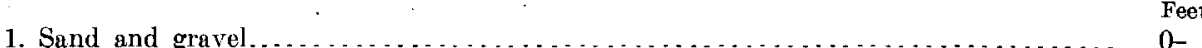

2. Hard yellow clay.

3. Yellow water-bearing gravel . . . . . . . . . . . . . . . . . . . . . . . . . .

Surface water was encountered at 24 feet; the water from the lower horizon stands 77 feet from the surface.

350. Mr. Herbert has kindly furnished the following samples from this well:

Tisbury:

Record of Robert Cox's well, Great Neck.

Clean glacial gravel.

Cretaceous:

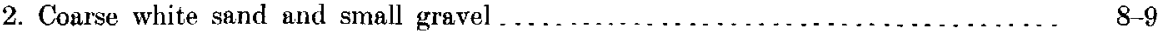

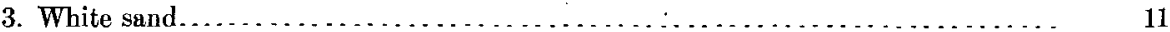

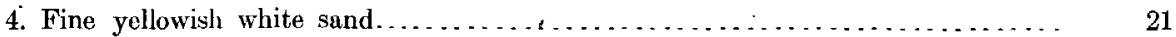

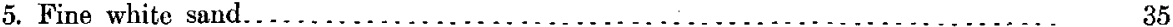

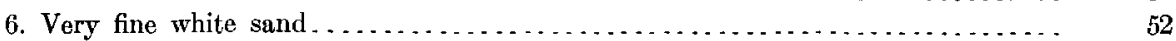


351. Mr. Isaac Kasteard, who dug the upper part of this well, reports the following section:

Wisconsin: 'Record of Robt. Seizer's well near Plandome Mills.

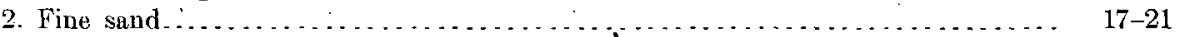

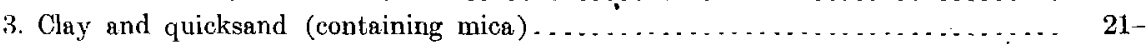

Mr. George Schmidt, who completed this well, gives the following data:

Wisconsin:

Record of Robt. Seizer's well near Plandome Mills.

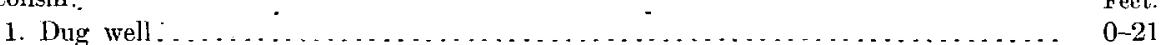

Tisbury?:

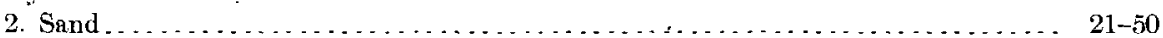

Sankaty?:

3. Bay mud and sand...................................... $-50-100$

Jameco?:

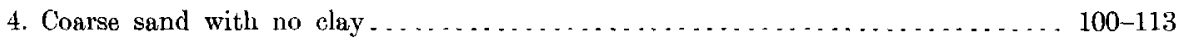

352.

Record of Charles Vanderbilt's well near Port Washington.

Wisconsin and Tisbury:

1. Surface loam.

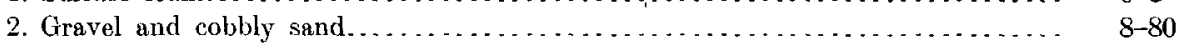

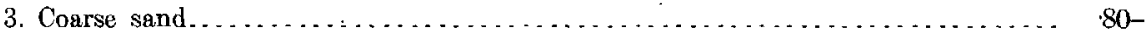

354.

Record of commission's test well near Port Washington.

Wisconsin:

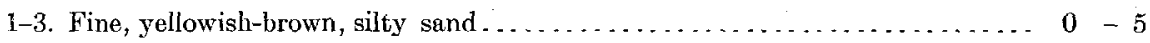

Transition:

3-6. Dark yellowish brown sand and gravel of glacial origin.........

Tisbury:

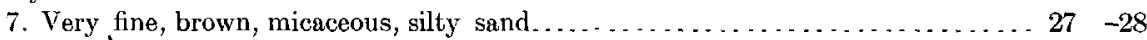

8 . Very fine, yellow-white, silty sand....................... $31.5-32.5$

9-10. Fine, dark-brown, micaceous sand . . . . . . . . . . . . . . . . . . . . $36.5-40$

11. Dark-gray micaceous sand to small gravel; looks like glacial rock débris . . . $45-46$

12. Very fine, brown, silty sand............................. 49.5 50.5

13. Yellowish brown sand with small gravel (glacial material) $\ldots \ldots \ldots \ldots \ldots \ldots 54 \quad-55$

Cretaceous?:

14-16. Very fine reddish-brown to steel-gray, silty, micaceous sand . . . . . . . . 59 59

17-18. Dark, steel-gray, very fine, clayey silt (blue clay) . . . . . . . . . . $74 \quad-79$

19-20. Dark, grayish-brown, micaceous, silty sand ................... . 82.5-87

$35 \%$.

Record of T. Valentine's well near Port Washington.

Wisconsin: . $\quad$ Feet.

1. Hardpan and dark iron soil, very hard ......................... 0 . 15 Transition:

2. Cobbles. ........................................ $15-33$

Tisbury:

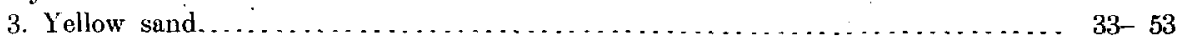

Manhasset bowlder bed:

4. A very compact layer of stones, which appeared to be put in almost artificially... $53-60$ Tisbury:

5. White sand, described as good building sand . . . . . . . . . . . . . . . . . $60-80$

Tisbury?:

6. Yellow sand.......................................... $8{ }^{80-123}$

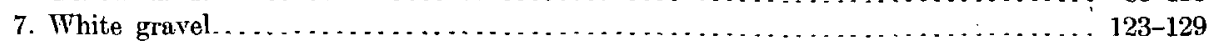




\section{UNDERGROUND WATER RESOURCES OF LONG ISLAND, NEW YORK.}

358.

Record of N. H. Jacobs's well near Port Washington.

1. Dug well.

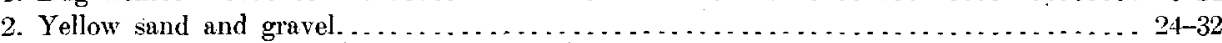

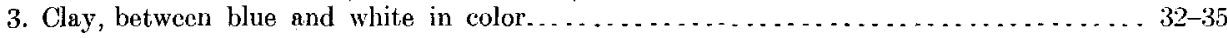

4. Sand and gravel; water-bearing. . . . . . . . . . . . . . . . .

360. Mr. George Schmidt reports the following section:

Record of T. E. Webb's well near Port Washington.

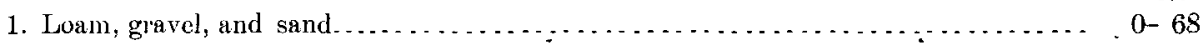

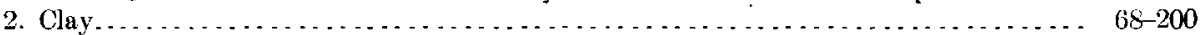

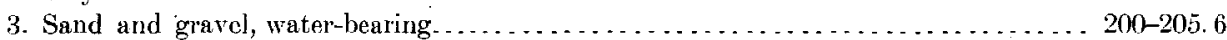

At 207 feet carbonized wood was found.' Top of well is 73 feet above high water.

Mr. John Fischer, who drilled the first 145 feet of this well, reports that water was found in soft, clayey sand, and that the greater part of the well was in soft clay or clayey sand; at 140 feet a lignitized log was struck.

361.

Record of Isaac Kasteard's well near Port Washington, N.Y.

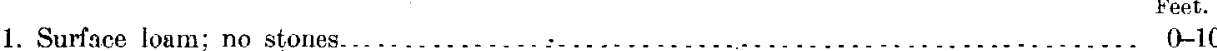

2. Cobbles and iron ore (size of cobbles, 4 to 8 inches in diameter) $\ldots \ldots \ldots \ldots \ldots \ldots \ldots$. $10-18$

3. Váricolored sands, each stratum about 4 feet thick (described as good building sand) _. 18-69

362. The Long Island Railiond Company have furnished the following partial analysis from their 60 to 70 foot driven well:

Analysis of railroad well near Port Washington.

Parts per million

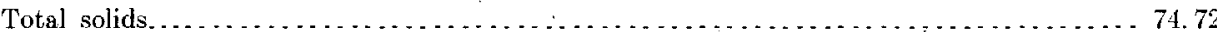

Chlorine..................

363. Record of F'. Vanoski's well near Port Washington. -

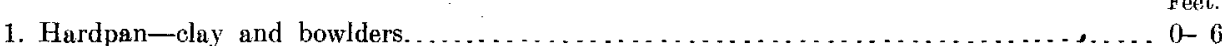

2. Varicolored coarse sand, coritaining occasional streaks of iron.................

364. Record of Charles H. Mason's well near Port Washington'.

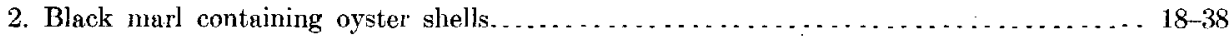

3. Clayey loam . . . . . . . . . . . . . . . . . . . . . . . . . . . . . . . . . . . . . . . $38-42$

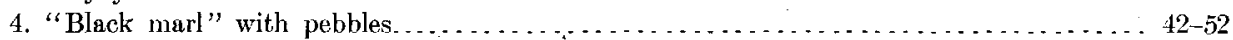

5. Very fine sand with iron; water-bearing . . . . . . . . . . . . . . . . . . . . . . $52-79$

6. White sand and gravel mixed . . . . . . . . . . . . . . . . . . . . . . . . . . . . $79-83$

A shell from stratum No. 2 has been identilied by Dr. W. H. Dall as Ostrea virginica and is regarded as probably Pleistocone.

365.

Record of Catholic Church well near Port Washington.

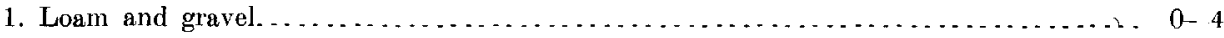

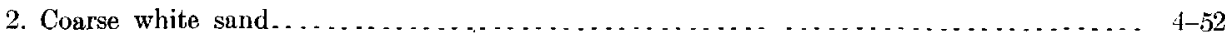

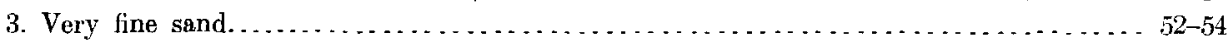

366. . Record of well of Dodge estate near Port Washington.

Wisconsin: - Feet.

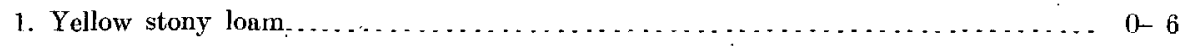
Tisbury:

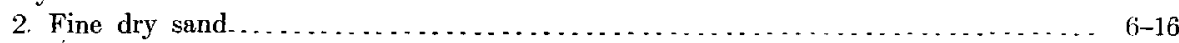


Manhasset bowlder bed?

Feet.

3. Rough stratum of cobbles with scarcely any sand between $\ldots \ldots \ldots \ldots \ldots \ldots \ldots$ 16-22 Tisbury:

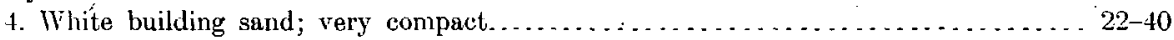

5. White loose dry sand . . . . . . . . . . . . . . . . . . . . . . . . . . . . . . $40-50$

Sankaty or Cretaceous:

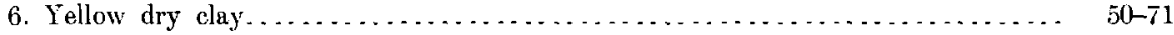

7. Blue clay, containing some water............................. $71-91$

The pipe broke of at the last depth given and the clay was not penetrated.

368.

Record of G. Zabriskie's well near Sands Point.

Feet.

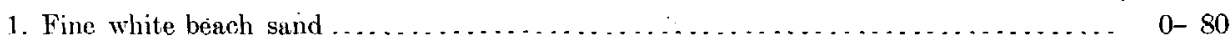

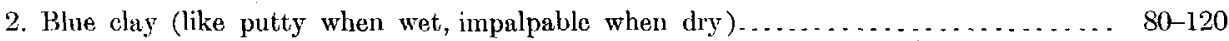

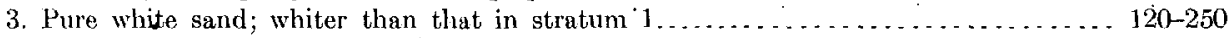

In the sand at 120 feet lignite, clam shells, and oyster shells were found. At 250 feet a hard substance was encountered upon which drilling made no inpression; Mr. Schmidt then abandoned the job, and Mr. A. J. Connolly attempted to drill farther; after working. three weeks, he also abandoned the well. Mr. Schmidt says that none of the hard material was brought to the surface. This probably represents hed rock.

369. Record of well at Castle Gould, near Port Washington.

Wisconsin:

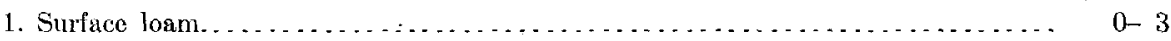

2. Black hardpan (rough, stony material, with no clay) $\ldots \ldots \ldots \ldots \ldots \ldots \ldots . .26$ Tisbury:

3. Coarse gravel............................................ $26-30$

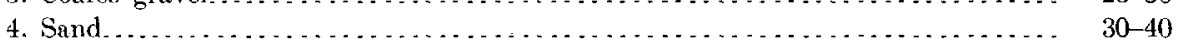

5. Fine sand containing mica....................................... $40-42$

6. Coarse sand (described as good building sand) ................... $42-88$

370. Mr. C. H. Danis reports that he put down a test well at this point to a depth of about 300 feet; the material passed through was successive small layers of sand and clay, none exceeding 4 to 6 inches in depth. At last a thick bed of gravel was reached, when the pipe broke; the water in the well rose to a point 12 feet above mean high tide; it would, therefore, have been a flowing well on the beach.

371 .

Record of well at Castle Gould, near Port Washington.

Wisconsin and Tisbury

1. Coarse gravel, with very little water........................ 6- 51

Tisbury and Cretaceous:

2. White sand

Cretaceous:
3. Gray clay...

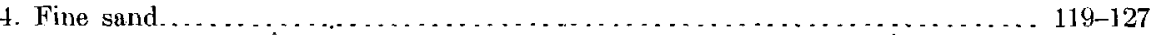

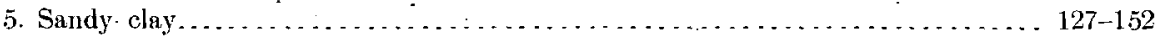

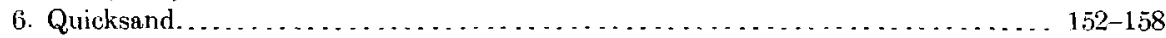

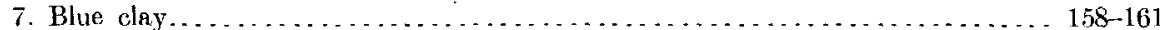
8. Water-bearing sand. . . . . . . . . . . . . . . . . . . . . . . . . . . . 161-166
9. Fine sand . . . . . . . . . . . . . . . . . . . . . . . . . . . . . . . . . . . $166-169$

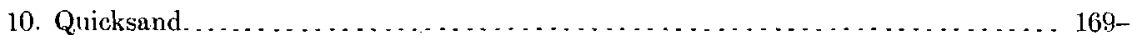

"We placed a Cook patent strainer between 161 and 166 feet, and although at the first test the well only showed $2 \frac{1}{2}$ gallons per ninute, we were able, after developing the well, to get over 30 gallons per minute. Lower down the hill, at a difference in elevation of about 40 feet, where we had 10 feet of this coarser waterbearing sand, we obtained 102 gallons per minute."-J. D. Kilpatrick.

372. Mr. Danis reports the material penetrated in this well as all white sand. This well flows at times, and would flow continually if the sand were coarser, the stoppage of the flow apparently being due to clogging with fine sand. The elevation is about 15 feet above mean high tide. 


\section{UNDERGROUND WATER RESOURCES OF LONG ISLAND, NEW YORK.}

373. The following section to a depth of 1.58 feet has been prepared from samples lurnished by Mr. Paul K. Ames, of the Long Beach Association; the remainder is from the record of the driller, Mr. W. C. Jaegle:

Recent:

Record of well of Long Beach Association at Long Beach.

1. White beach sand, with water-worn fragments of shells $\quad$ Feet.

2. Dirty gray sand, with small quartz pebbles and particles of vegetable matter.. $36-40$

Tisbury

3. Fine to coarse gray sand, with a few small quartz pebbles (salt water) $\ldots \ldots . .40-.50$

4. Medium gray sand; no gravel............................. $51-55$

5. Grayish yellow sand and small gravel, with a few greensand grains ........ $55-65$

6. Yellowish gray sand ....................................... $65-70^{\circ}$

7. Orange-yellow sand and gravel, similar to Rockaway material............ $70-73$ Sankaty:

8. Gray sand and gravel, similar to No. 7 in texture, but not iron-stained....... $73-76$

9. Large quartz gravel and pieces of blue clay containing sand and gravel........ $76-82$ Jameco:

10. Dark, multicolored coarse sand and gravel; considerable percentage of flattened shale pebbles; only 50 to 60 per cent of quartz; some biotite; looks as if it might be a sumple taken from the Wisconsin moraine in the center of island. . . 82-90 Cretaceous:

11. Black sand composed of fine, gray, quartz sand with a large percentage of lignite; some FeS and S; several large pieces of lignitized wood at 99 feet....... $90-99$

12. Grayish sand with some free sulphur and a few particles of lignite........ 99-107

13. White sand with occasional patches tinged lemon yellow, perhaps due to iron stains; a few particles of free sulphur........................ 107-111

14. Dark-gray silty sand.................................... 111-119

15. White sand with small pieces of lignite; note on bottle says "120, petrified wood". 119-121.

16. Very dark colored clay ("blue clay") ........................... 121-135

17. Coarse, gray, clayey sand, with particles of sulphur.................. 13j-143

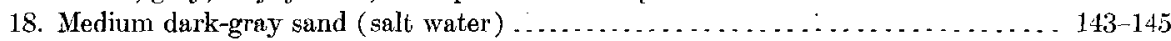

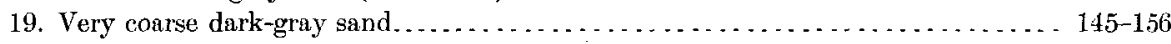

20. Olive-green sind and small quartz gravel; some sulphur salt water) $\ldots \ldots \ldots \ldots$. $156-158$

21. Very dark lead-colored clay .......... . . . . . . . . . . . . . . . . . . . 158-174

22. White sand, containing at 190 a $\log$ of lignitized wood $\ldots \ldots \ldots \ldots \ldots \ldots \ldots \ldots$ 174-192

23. White gravel and salt water.................................. $192-196$

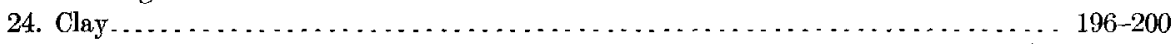

25. Fine sand............................................ 20 200-220

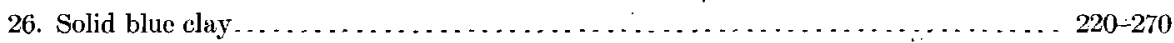
(At 270 fresh water, sweet and chalybeate.)

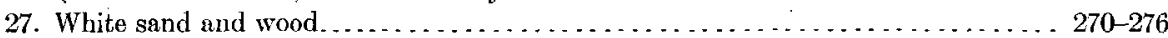

28. Clay ..... . . . . . . . . . . . . . . . . . . . . . . . . . . . . . . . . . . 276-282

29. White sand and wood . . . . . . . . . . . . . . . . . . . . . . . . . . . . . . 282-297

30: Blue clay . . . . . . . . . . . . . . . . . . . . . . . . . . . . . . . . . . . . . 297-305

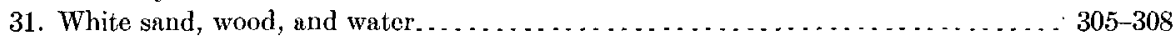

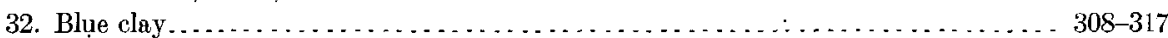

33. White sand containing wood and artesian water................... $317-325$

34. Blue clay ................................................. $325-340$

35. White sand and mineral water; has considerable $\mathrm{CO}_{2}$, sparkling and effervescent. $340-356$

36. Blue clay ............................................ $356-360$

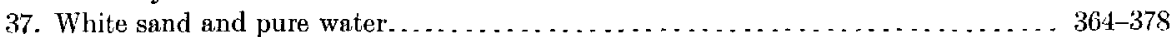

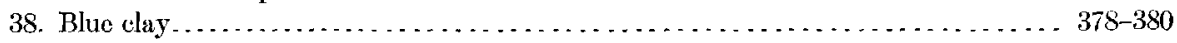

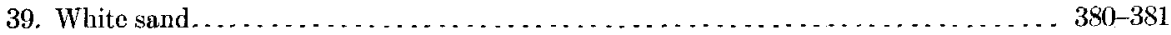

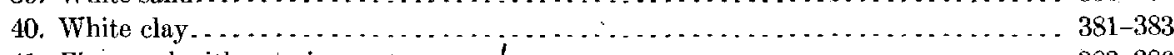

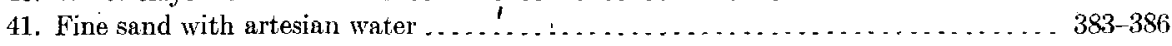


On May 6, 1903, well was flowing 5 gallons per minute, at a height of about 1 foot above the surface of the ground; it was from this well that the tide curve shown in fig. 34 , was obtained.

The water from a depth of 270 feet has been analyzed by Endermann and Saarbach, analytical chemists of New York, with the following results:

Analysis of water from depth of 270 feet in Long Beach Association's well at Long Beach.

Parts per million.

Alkali 125.000

Lime. 3.525

Magnesia.

4. 276

Oxide of iron

7.057

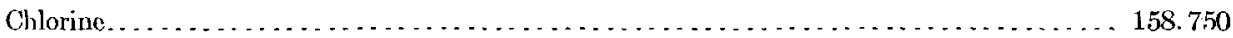

Sulphuric acid. . . . . . . . . . . . . . . . . . . . . . . . . . . . . . . . . . . . . . . . . . . . . . . . . . . . . . . . . . . . . .

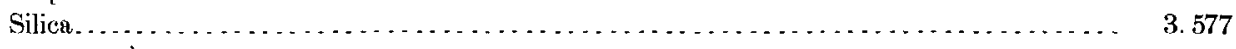

Total

317.545

Analysis of water from 383 to 386 feet by Doctors Endermann and Saarbach:

Analysis of water from depth of 383-386 feet in well of Long Beach Association at Long Beach.

Parts per million.

Total residue.

157. 32

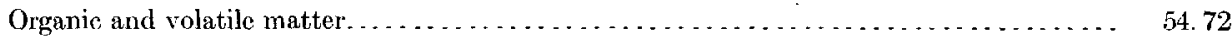

Mineral residue . . . . . . . . . . . . . . . . . . . . . . . . . . . . . . . . . . . . 102.6

Free ammonia. . . . . . . . . . . . . . . . . . . . . . . . . . . . . . . . . . 07

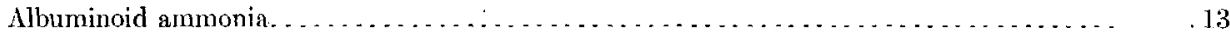

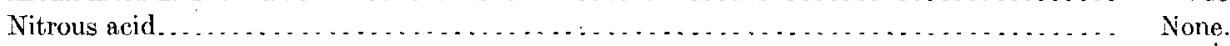

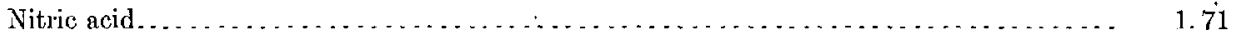

Oxygen required for organic matter. $\ldots \ldots \ldots \ldots \ldots \ldots \ldots \ldots \ldots \ldots \ldots \ldots \ldots \ldots \ldots \ldots \ldots \ldots \ldots \ldots$

Chlorine. . . . . . . . . . . . . . . . . . . . . . . . . . . . . . . . . . . . . . . 29. 07

374. The following section has been prepared from samples preserved in the museum of the Long Island Historical Society:

Tisbury:

Record of well at Hempstead poorhouse, Barnum Island, New York.

1. Orange sand and gravel

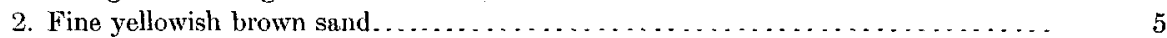

4. Orange sand and gravel. . . . . . . . . . . . . . . . . . . . . . . . . . . . . .

5. Very, dark-gray, clayey sand, with a few quartz pebbles............... 22

6. Small and medium quartz pebbles orange yellow . . . . . . . . . . . . . . . . . . 29

7. Fine to conse orange-yellow sand . . . . . . . . . . . . . . . . . . . . .

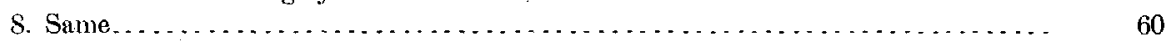

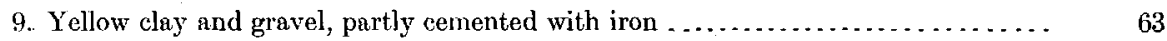

10. Fine yellow sand . . . . . . . . . . . . . . . . . . . . . . . . . . . . . .

Transition:

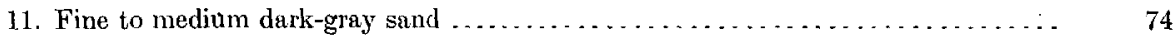
Sankaty:

12. Very fine dark-gray clay, with a little lignitized wood $\ldots \ldots \ldots \ldots \ldots \ldots \ldots \ldots \ldots \ldots$

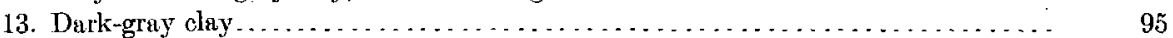

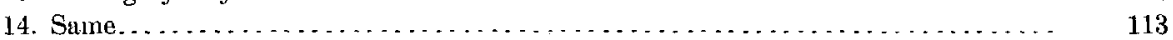

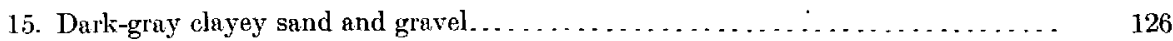
Jameco?:

16. Gravel of quartz and chert; has no recognizable erratics, but colors suggest glacial material; quite different from the orange sand at the top of the section........ 


\section{UNDERGROUND WATER RESOUROES OF LONG ISLAND, NEW YORK.}

Cretaceous:

Feet.

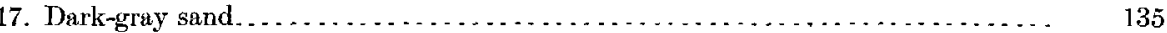

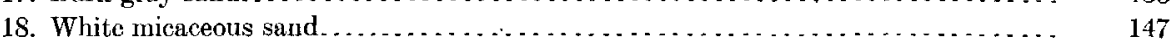

19. White sand and orange gravel; a few fragments of red quartzite $\ldots \ldots \ldots \ldots \ldots$

20. Coarse gray sand . . . . . . . . . . . . . . . . . . . . . . . . . . . . . . . . . . . . 170

21. Fine to coarse dirty yellowish gray sand $\ldots \ldots \ldots \ldots \ldots \ldots \ldots \ldots \ldots \ldots \ldots \ldots \ldots \ldots \ldots \ldots$

22. Coarse white sand with lignite . . . . . . . . . . . . . . . . . . . . . . . 180

23. Lignite. ... . . . . . . . . . . . . . . . . . . . . . . . . . . . . . . . . 200

24. Very fine gray clayey sand. . . . . . . . . . . . . . . . . . . . . . . . . . 225

25. Coarse grayish white sand . . . . . . . . . . . . . . . . . . . . . . . . . . . . . . . . . 243

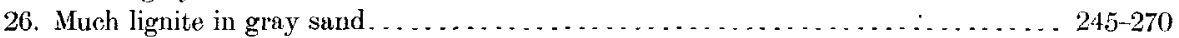

27. Gray sand and lignite. . . . . . . . . . . . . . . . . . . . . . . . . . . 2 270-370

28. Gray day . . . . . . . . . . . . . . . . . . . . . . . . . . . . . . . . . 380

29. Carbonaceous clay. ... . . . . . . . . . . . . . . . . . . . . . . . 393

These samples werc furnished by the driller, Mr. Theodore A. Carmen, who gave the following data regarding this well in a letter dated April 24, 1895:

"Some years ago I attempted to bore a well near the shore; at 123 feet reached fresh water; we continued boring to a depth of 380 feet; the soil was fine beach sand and clay, but the water was not good and did not rise to the surface."

A record of the well has been published by Lewis, "who adds the following remarks on the section:

"The deposit of clay between 70 and 126 feet seems closely analogous to many clays now found upon, and at various depths beneath, the surface of the island; it is evidently a local deposit, such as might occur in the depressions of the surface. Two tube wells have been driven at no great distance from Barnums Island, one 97 and the other 194 feet, in which no similar layer of clay was detected." Other records have been published by Merrill," Darton, " and Woolman."

375. As the artesian water obtained from the deep well at Long Beach, No. 373 , was so chalybeate that it was undesirable for domestic use, a pumping plant was established at East Rockaway which draws its water from shallow wells in the surface gravels.

The following analysis by Doctors Endermann and Saarbach has been lurnished by Mr. Paul K. Ames:

Analysis of water from pumping plant of Long Beach Association near East Rockaway.

Parts per million.

Total residue ...... . . . . . . . . . . . . . . . . . . . . . . . . . . . . . . . . 94.05

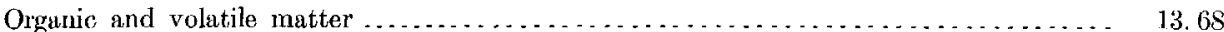

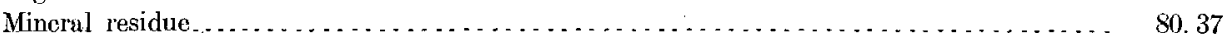

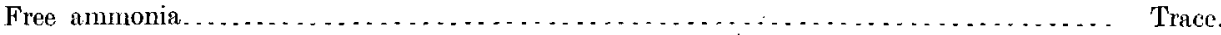

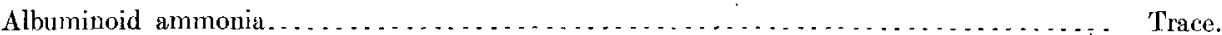

Nitrous acid. . . . . . . . . . . . . . . . . . . . . . . . . . . . . . . . . None.

Nitric acid. . . . . . . . . . . . . . . . . . . . . . . . . . . . . . . . . . . . .

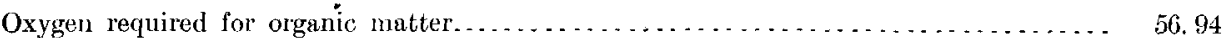

Chlorine. . . . . . . . . . . . . . . . . . . . . . . . . . . . . . . . . . . . . . . . . . . . . . . . . . 19.32

375A. Record of J. H. Clark's well at East Rockaway.

Tisbury:

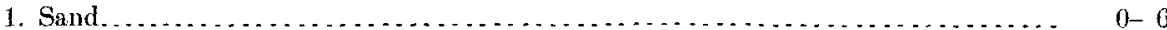

2. Coarse white gravel...................................... $6-8$

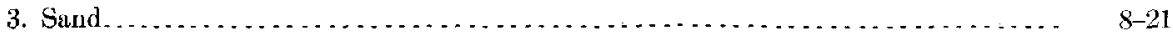

4. Coarse white gravel. . . . . . . . . . . . . . . . . . . . . . . . . . . . . . . . . . $21-24$

Tisbury? :

5. Bright-yellow elay which tasted like alum . . . . . . . . . . . . . . . . . . . . 24-27

Pop. Sci. Monthly, vol. 10, 18דi, p. 442

h Anmals N. Y. Acad. Sci, vol. 3, 1886, p. 350

c Bull. N. Y. Geol. Survey No. 138, 1896, pp, 32-33.

'Ann. Rept. Geol. Survey New Jersey for 1896,1897 , p. 160 
The driller, Mr. Fass, did not penetrate the clay in this well, but pulled up the pipe and obtained the water from the gravel above it.

376.

Record of J. M. Smith's well near Rockville Center.

Tisbury:

1. Stratified, yellow sand and quartz gravel, containing a small percentage of erratic

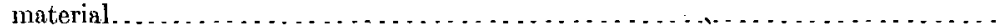

2. Cobble bed; large, yellow, iron-stained quartz bowlders

Feet.

$0-17$

$17-18$

Mr. McCarten says that eight or ten attempts have been made to drive wells on the property of Mr. Smith, all of which have been unsuccessful on account of the pipe bending in the attempt to pass through the layer of stones, which is water bearing and 26 inches in thickness. The sand and gravel below the layer of cobbles is said to be relatively dry.

377. The following record has been copied from a blueprint kindly furnished by Chief Engineer I. M. De Varona:

$$
\text { Record of Broollyn test well No. 26, near Smith Pond. }
$$

Recent to Tisbury: Fcet.

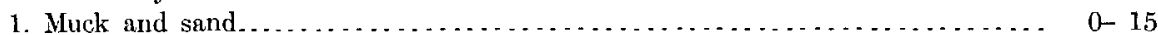

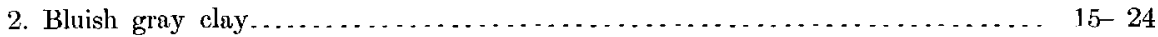

3. Yellow sand and gravel.................................... 24-56

Cretaccous?:

4. Bluish gray clay mixed with fine sand.......................... 56- 64

5. Bluish gray and yellow clay mixed with fine sand.................. 64- 71 Cretaceous:

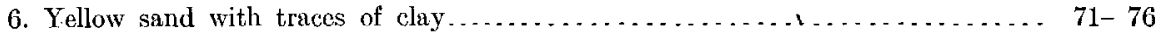

7. Gray sand, gravel, clay, and wood................................ $76-84$

8. Yellow sand, clay, and wood.............................. 84- 96

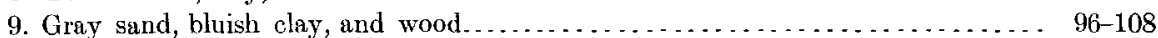

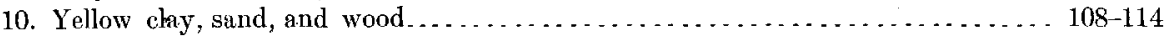

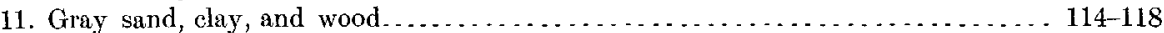

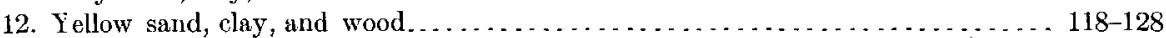

13. Bright-yellow sand, clay, and wood................................ 128-134

14. White sand, clay, and wood (slightly water-bearing from 170.7) . . . . . . 134-184

15. White sand, brown clay, and wood. . . . . . . . . . . . . . . . . . . . . 184-202

16. Solid gray clay; no water.................................... 202-214

17. Gray clay, sand, and wood; slightly water-bearing ................. 214-235

18. Gray clày, fine sand, and wood; slightly water-bearing $\ldots \ldots \ldots \ldots \ldots \ldots \ldots \ldots .235-279$

19. White sand, clay, and wood; slightly water-bearing . . . . . . . . . . . . . 279-510

20. Solid clay of dark bluish gray color .......................... $510-518$

21. Clay, sand, and wood; slightly water-bearing..................... . 518-522

22. Solid clay; no water................................... 522-527

23. Light-gray clay, sand, and wood; slightly water-bearing.............. 527-554

24. Sand, gravel, clay, and wood; water-bearing....................... $554-578$

25. Sharp white sand and white clay; no wood; flows 5 gallons per minute.... 578-579

26. Small gravel, white sand, and white clay; flows 5 gallons per minute...... 579-587

Elevation of surface, 8.3 feet. "First water at 25 feet; rises to 6 feet below at the surface; the best supply of water is from $42-45$ feet."

379.

Record of commission's test well near Rockville Center.

Wisconsin and Tisbury:

1-2. Dark-brown loamy sand.

$0.5-1.5$

3. Reddish yellow fine sand.

4. Very fine white sand to small gravel.

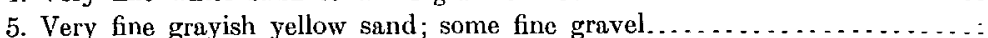

$13-14$

6. Yellow silty clay, mottled red. 


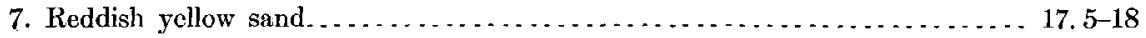

8. Red sand, fine to medium, with biotite . . . . . . . . . . . . . . . . . . 18.5 5

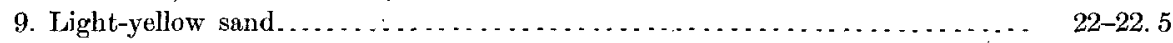

10. Same, with considerable yellow clay. ....................... 23-23.5

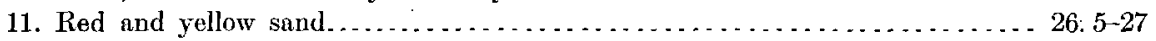

12. Fine, dark gray-hlue clay, with quartz sand and lignite.............. $27 \quad-27.5$

13. Grayish sand and lignite..................................... $29 \quad 39$

14. Light reddish yellow sand, no erratics........................ $33 \quad-34$

15-16. Medium white sand ................................... $36 \quad 42$

17. Medium yellow sand.................................. $43.5-44$

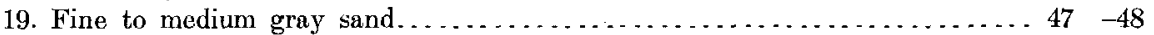

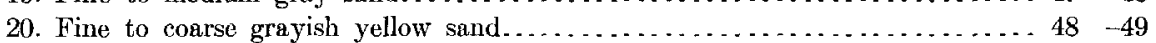

21. Fine to very coarse brownish yellow sand and quartz gravel (bowlder struck in this )

22. Bowlder ........................................... $51-52$

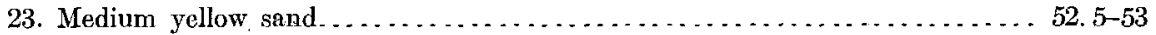

Cretaceous:

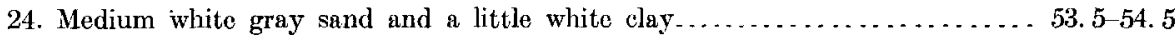

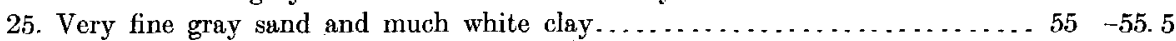

26. Very coarse white sand ...................................... 55.5-56

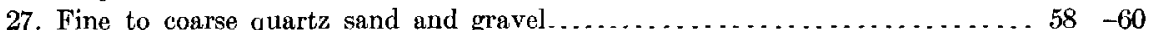

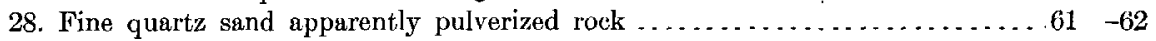

29. Medium grayish yellow sand ............................. $65-66$

30. Fine to medium yellowish gray sand and yellow clay $\ldots \ldots \ldots \ldots \ldots \ldots \ldots \ldots, 6,67.5$

31. Medium to coarse yellow sand and clay ...................... $71.6-72$

32. Dairk-gray clay . . . . . . . . . . . . . . . . . . . . . . .

379. Two of the wells used in this plant were completed in 1895 and the other two in 1892 . The village clerk gives the following data regarding the daily pumping during 1902:

Yield in 1902 of wells of Rockville Center waterworks, Rockville Center.

Maximum daily yield.

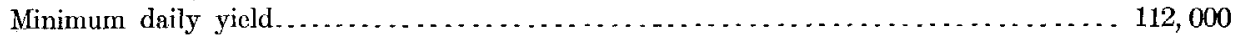

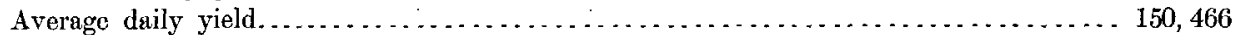

380. Record of commission's test well near Rockville Center.

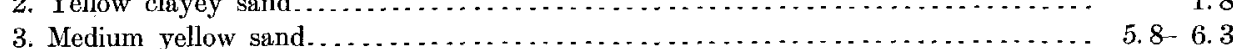

4. Medium to coarse gray sand................................... 9 . 10

5-7. Medium to coarse reddish brown sand; glacial . . . . . . . . . . . . . . . . . 10 . 19

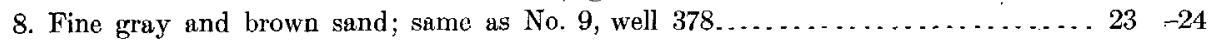

381-392. These were test wells put down by the commission on additional water supply around,the Millburn reservoir; the decpest was No. 382, of which the section is as follows:

Record of commission's "deep" test well near Millburn reservoir.

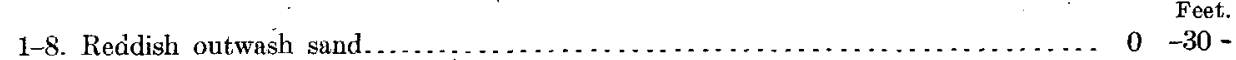

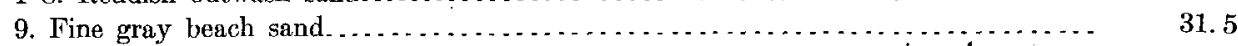

10. Yellow quartz gravel............................................. $34.5-35$

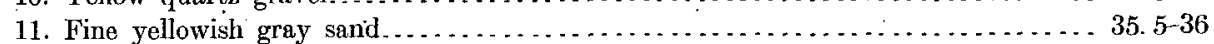

12. Reddish yellow sand....................................... $40-41$

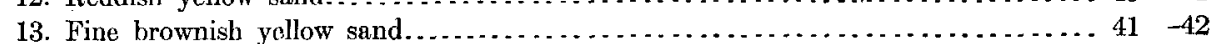




\begin{tabular}{|c|c|c|}
\hline & Fee & \\
\hline Coarser yellowish & & -51 \\
\hline Light gray highly micaceous sand... & 54 & -55 \\
\hline Brownish beach sand $\ldots \ldots \ldots \ldots \ldots \ldots \ldots$ & 56 & -57 \\
\hline -20. Fine, light-gray, highly micaceous sand......... & 58 & -69 \\
\hline 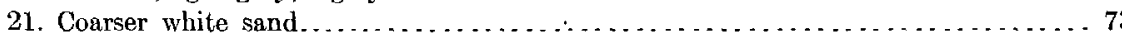 & 73 & -74 \\
\hline yellowish sand. & 78 & -79 \\
\hline Fine gray sand with a little white clay....... & 81 & -92 \\
\hline ark gray micaceous sand with a little lignite.... & 93 & -94 \\
\hline . Very dark-colored sandy clay.... & & -97 \\
\hline
\end{tabular}

The materiuls penetrated in the other wells are summarized in the following table:

Records of commission's wells near Millburn reservoir.

\begin{tabular}{|c|c|c|c|c|c|c|c|c|c|c|c|}
\hline Well number.. & 381. & 383. & 384. & 385. & 386. & 387. & 388. & 389. & 390. & 391. & 392. \\
\hline $\begin{array}{l}\text { 1. Reddish yellow outwash } \\
\text { sand and gravel............ } \\
\text { 2. Fine gray beach sand......... }\end{array}$ & $\begin{array}{r}0-29 \\
29-38\end{array}$ & $0-31$ & $\begin{array}{r}4-29 \\
29-32\end{array}$ & $\begin{array}{r}0-30 \\
30-31\end{array}$ & $\begin{array}{r}0-22.5 \\
22.5-22.5\end{array}$ & $\begin{array}{r}0-21 \\
21-25\end{array}$ & $\begin{array}{r}0-24 \\
24-25\end{array}$ & $\begin{array}{r}0-21 \\
21-22.5\end{array}$ & $\begin{array}{r}0-29 \\
29-30\end{array}$ & $\left\{\begin{array}{r}0-26.5 \\
26.5-32\end{array}\right.$ & $\begin{array}{r}0-26 \\
26-32\end{array}$ \\
\hline
\end{tabular}

See Tables XII, and XIII.

393. Mr. Hancock reports that all shallow wells in this neighborhood are sunk through about the same material and that one description will fit all. They vary in depth from 8 to 20 feet, according to the elevation of the surface.

Record of M. S. Thomas's well at Baldwin.

2. Loose sand, gray in color

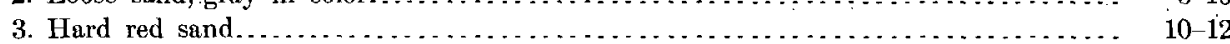

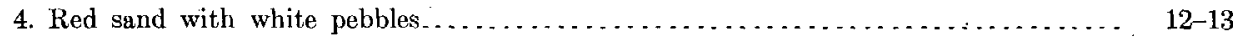

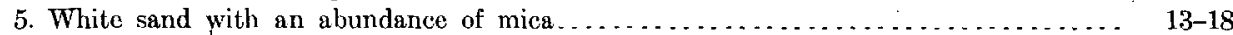

394.

Record of C. H. Southard's well at Baldwin.

1. Surface loam......................... Feet.

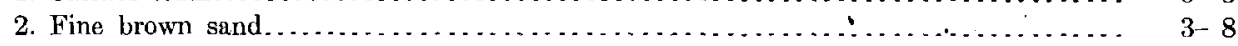

3. Very coarse light-colored gravel................................... $8-11$

4. Finer gravel, decreasing in size. . . . . . . . . . . . . . .

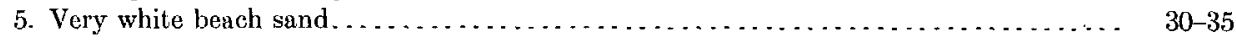

395. Mr. Wortman reports that at 50 feet he encountered a very black mud which choked the well point. He reports that the clay at Lynbrook is about $\mathbf{1 2}$ feet below the surface and is of great thickness. Above the clay is a very coarse stony material. He also reports that a black mud was encountered in driving a well at the railroad station at Baldwin.

396. This well was sounded July 10 by Francis Whitney, field assistant, and found to be 288.6 feet deep from the top of the old pipe: Lignite was reported from 300 to 370 feet.

399. Record of commission's test well near Norwood.

Wisconsin:

1-2. Brown loamy sand.

3-4. Medium reddish yellow sand

5. Fine to medium brownish yellow sand with much biotite $\ldots \ldots \ldots \ldots \ldots \ldots \ldots .10 .5-11.5$ Tisbury:

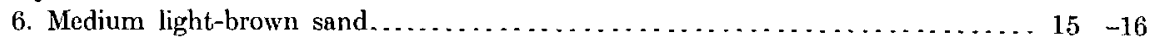

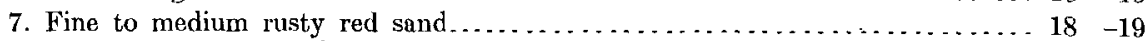

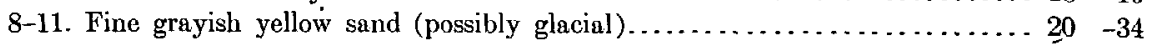


400. Record of commission's test well between Rockville and Hempstead.

Wisconsin:

1-2. Yellow gravelly loam.

9. Medium light-yellow sand.

See Tables XII, XIII

401.

Record of commission's test well south of Hempstead.

Wisconsin:

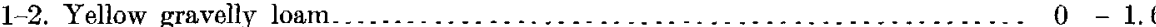

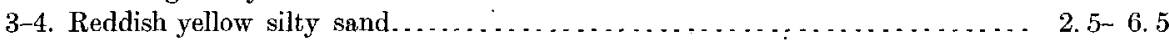

5. Fine reddish brown sand.................................. 9. 5-10.5

6-9. Dark reddish yellow sand and gravel (glacial) ................... 14. 5-22

Tisbury ? :

10-12. Fine yellowish silt to coarse gravel, becoming lighter and coarser below, not sharply glacial ................................. $20 \quad-32$

See Tables XII, XIII.

402. Record of commission's test well near Greenwich Point.

Wisconsin and Tisbury

1-2. Surface dark loamy clay ....................

3-4. Tough sandy clay with bowlders. . . . . . . . . . . . . . . . . . . . . . 2.5-3.4

5-8. Yellow sand to fine gravel. . . . . . . . . . . . . . . . . . . . . . . . . . . . . . . $66^{-17}$

9-10. Reddish yellow sand and small gravel, with a considerable percentage of

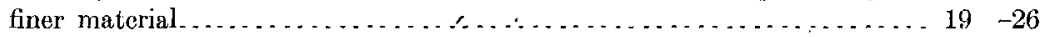

11. Coarse reddish yellow sand (same as No. 7 in well 411) . . . . . . . . . . . 29 $\quad 30$

All the section, with the exception of the upper 3.5 feet, appears to be regular outwash material.

403.

Record of commission's test well near Hempstead.

Wisconsin :

1. Dark loamy sand . . . . . . . Fect.

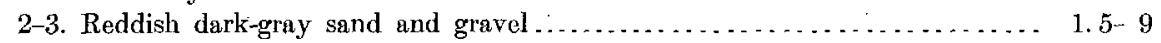

4. Medium gray sand with considerable glacial débris................... $10 \quad-11$

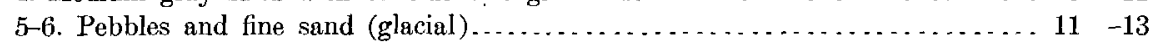

Tisbury? :

7. Dark reddish sand ... . . . . . . . . . . . . . . . . . . . . . . . . . . . . . . . 15 -16

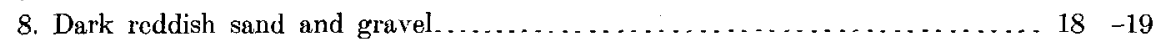

9 10. Medium reddish yellow sand . . . . . . . . . . . . . . . . . . . . . . . . . . . . 23.29

11. Light reddish yellow sand; no erratics (not clearly glacial) ............ . 33 . 33.5

See Tables XII, XIII.

404.

Record of commission's test well near Hempstead.

Wisconsin

1. Black sandy loam

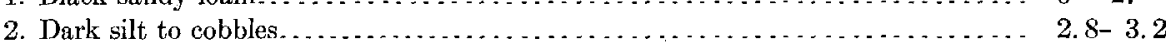

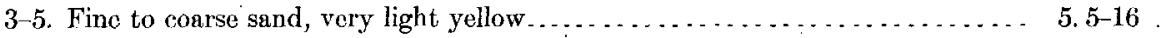

6. Medium reddish sand $\ldots \ldots \ldots \ldots \ldots \ldots \ldots \ldots \ldots \ldots \ldots \ldots \ldots \ldots \ldots \ldots \ldots \ldots \ldots \ldots$ Tisbury:

7. Yellow sand and gravel with some erratics....... . . . . . . . . . . . . . $18 \quad 19$

Tisbury and Cretaceous:

8-16. Red sand (doubtfully glacial)

$20.5-61$ 
Cretaceous:

17. Fine dark-colored sand with lignite

18-23. Fine, light-colored, silty, micaceous sand, suggesting material of Cretaceous

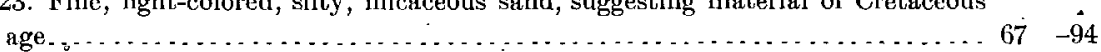

24. Very black, micaceous, sandy clay ... . . . . . . . . . . . . . . . . . . $95.5-97$

406.

Record of commission's test well near Hempstead.

Wisconsin and Tisbury?:

Feet.

1-2. Yellow surface loam . . . . . . . . . . . . . . . . . . . . . . . . . . 0 . 2

3-11. Glacial sand and gravel . . . . . . . . . . . . . . . . . . . . . . . . 2.52 .7

$40 \%$.

Record of commission's test well near East Meadow Brook.

Wisconsin:

Feet.

1-2. Light-yellow surface loam.............................. 0.1 .5

3-5. Coarse sand, with some erratic material $\ldots \ldots \ldots \ldots \ldots \ldots \ldots \ldots \ldots \ldots \ldots \ldots .5 \ldots$

6. Considerable gravel, with much erratic material . . . . . . . . . . . . . . . . . . . . .

Cretaceous:

7-14. Fine, white, highly micaceous, clayey sand . . . . . . . . . . . . . . $22 \quad-51$

15. Dark-brown, very fine, micaceous, clayey sand $\ldots \ldots \ldots \ldots \ldots \ldots \ldots \ldots \ldots \ldots 5 . \ldots \ldots$

16. Light-yellow clayey sand. . . . . . . . . . . . . . . . . . . . . . . . . . . $58.5-60$

17. Greenish yellow fine silt to medium sand, highly micaceous. . . . _...... 65 - 66

18. Gray silt to medium sand, highly micaceous..................... $70 \quad 71$

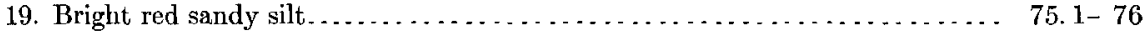

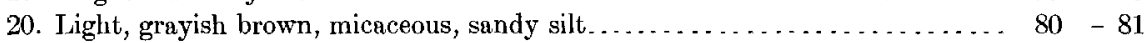

21-22. Greenish yellow, micaceous, sandy silt. . . . . . . . . . . . . . . $85 \quad 91$

23. Grayish brown, micaceous, silty sand, with some lignite . . . . . . . . . . 91.7-91.8

24. Fine light-yellow sund . . . . . . . . . . . . . . . . . . . . . . . . . 93.5-94

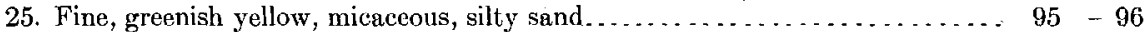

26. Dark-gray, micaceous, silty sand . . . . . . . . . . . . . . . . . . . . . . . . . . . . $100 \quad-101$

27-28. Medium light yellowish white sand . . . . . . . . . . . . . . . . . . . . . 105 111

29. Brownish white silty sand ................................. $115 \quad-116$

30. Dark yellowish gray silty sand. . . . . . . . . . . . . . . . . . . . . . . . . . . . $120 \quad-121$

31. Laminated black and white sandy clay . . . . . . . . . . . . . . . . . 123.5-125

408.

Record of commission's test well near East Meadow Brook.

Wisconsin and Tisbury?:

1-8. Light-yellow outwash sand and gravel.

Feet.

$0-35$

409.

Record of commission's test well near Garden City.

Wisconsin and Tisbury?:

1-9. Light-yellow outwash sand and gravel.

Sce Table XII.

410.

Record of commission's test well at Garden City.

Wisconsin:

1-2. Dark-colored gravelly loam

3-7. Brownish-yellow outwash sands and gravel, with much glacial material. ..... 2. 3-23

Tisbury?:

8-10. Fine to coarse reddish yellow sand, not clearly glacial $\ldots \ldots \ldots \ldots \ldots \ldots \ldots \ldots$

See Tables XII, XIII. 


\section{UNDERGROUND WATER RESOURCES OF LONG ISLAND, NEW YORK}

411.

Record of commission's test well at Garden City.

Wisconsin and Tisbury:

1. Black loamy sand

Feet.

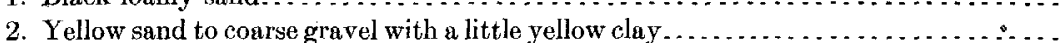

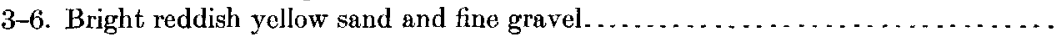

7. Fine yellow gravel with little, if any, glacial material (same as 11, well 402).

413. Record of commission's test well at Garden City.

Wisconsin:

1. Dark sandy loam with gravel. . . . . . . . . . . . . . . . . . . . . . . . .

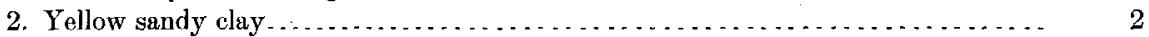

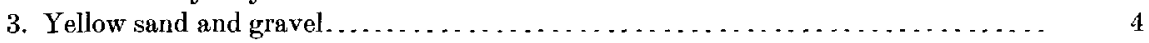

4-10. Grayish yellow sand to fine gravel. . . . . . . . . . . . . . . . . . . . . . . . 8.5-38.5

All samples apparcntly represent outwash material, and contain much biotite.

414. Mr. George L. Hubbell, general manager, states that the water level in the well owned by the Garden City Water Supply Company can be reduced 12 fect by excessive pumping, and that when the water level falls after several months' sterdy pumping the hydrants are opened and the pumps are run at their full capacity night and day for from twenty-four to thirty-six hours. When the normal rate of pumping is resumed the water level rises 5 feet. A layer of clay is encountered between 20 and 24 feet, which is overlain and underlain by sand and gravel.

416. Record of commission's test well near Mineola.

Wisconsin and Tisbury?:

Feet.

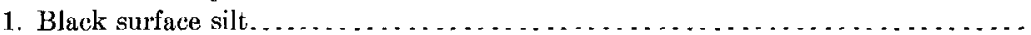

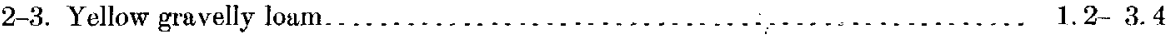

4-8. Fine sand to small gravel (outwash glacial material) $\ldots \ldots \ldots \ldots \ldots \ldots \ldots 6$. 6 .27

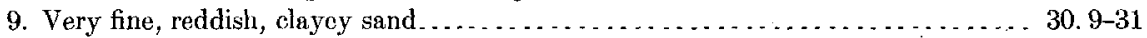

10-12. Fine silt to small gravel (outwash material) ..................... $31 \quad-42.5$

See Table XII.

418.

Record of commission's test well near Mineola.

Wisconsin:

1. Black surface loam

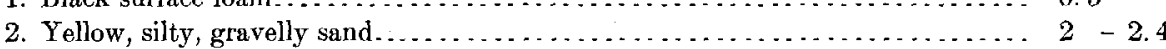

3-10. Fine silt to small gravel (outwash glacial material) ................. 6.5-42 Tisbury:

11.* Very fine, light-yellow, silty sand....................... $50 \quad-51$

12. Medium yellow sand (doubtfully glacial) ....................... $51 \quad-53.8$

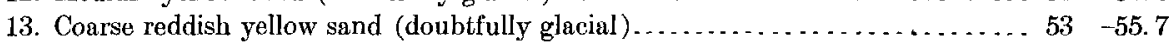

See Tables XII, XIII.

419.

Record of commission's test well near Mineola.

Wisconsin:

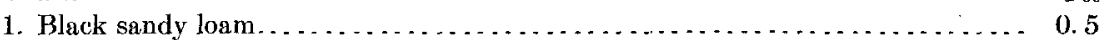

2. Dark loamy sand with gravel......................................

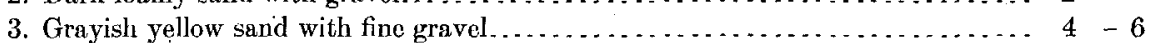

4. Same, but with more gravel . . . . . . . . . . . . . . . . . . . . . . . . . . 10 . 11.5

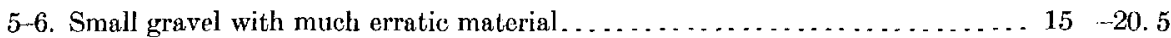

Tisbury:

7. Yellow sand with small gravel. . . . . . . . . . . . . . . . . . . . . . . 20. 5-21

8. Same, with a little clay . . . . . . . . . . . . . . . . . . . . . . . . . 25.26

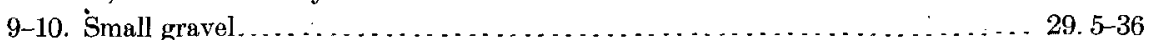

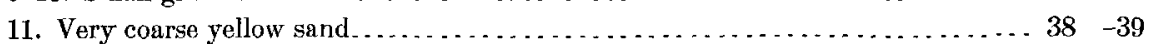

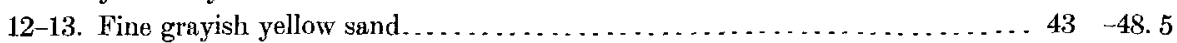

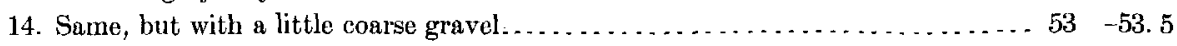


1. Coarse sand

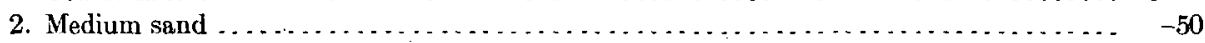

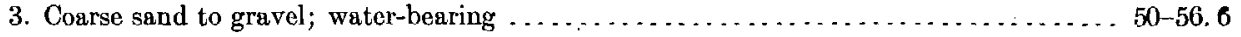

421.

Record of commission's test well near East Williston.

Wisconsin:

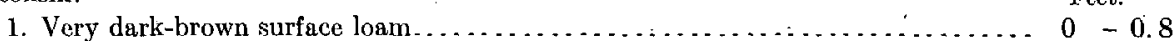

2. Reddish brown loamy sand............................ $2.7-2.9$

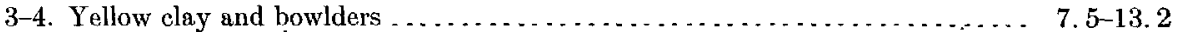

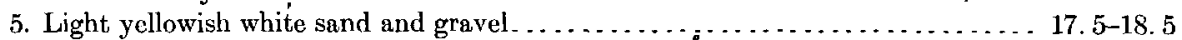

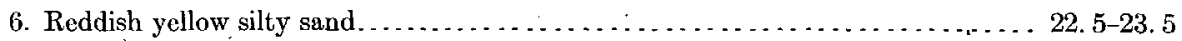

7. Very black sand, full of mica, looks like ground-up bowlder.............. $25 \quad-26.5$ Tisbury:

8-9. Fine to medium yellowish white sand........................ $30 \quad-36$

10. Fine yellowish white sand to medium gravel................... $40 \quad-41$

11. Small light-colored gravel (considerable percentage of glacial material)..... $41 \quad-42$

12-13. Fine to medium yellowish white sand ...................... $45 \quad-51$

14. Small light-colored gravel with glacial material .................... $54-55$ See Tables XII, XIII.

422.

Recorä of commission's test well near Albertson.

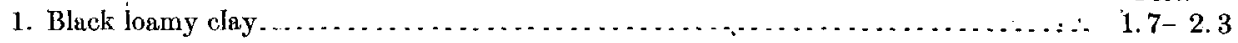

2. Brownish yellow clay with a few pebbles very similar to the clay at East Williston $\ldots=3-3.5$

3. Brown and yellow clay with reddish brown sand and gravel (glacial) .......... 8 - 9.5

4. Dark grayish sand with much fresh biotite; evidently débris from a glacial bowlder. 10.5-11.5

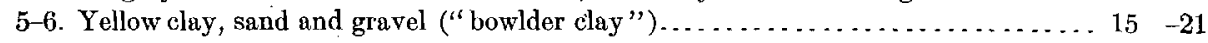

7. Sand and coarse gravel (glacial) ................................... $23 \quad 24$

8-10. Fine yellow sand with a noticeable percentage of glacial material . . . . . . . . $27 \quad-37$

See Tables XII, XIII.

423. The greater portion of this well is in light yellow sand and gravel. Near the bottom fine gray sandy clay was encountered.

424.

Record of W. P. Kelsey's well near old Westbury.

Wisconsin:

Feet.

$0-50$

1. Coarse

2. Alternate layers of sand and clay

425.

Record of J.E. Brady's well near old Westbury.

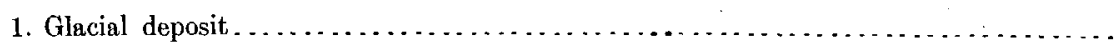

2. Clay and sand, mixed (white beach sand and greasy, slippery clay)

3. Coarse gravel, the pebbles of which were highly colored-black, red, and all gradations to yellow.

426.

Record of R. L. Cottnet's well near Old Westbury.

Wisconsin:

Feet.

1. Gravel and large stones. .................................. 0 . 50

2. Black hardpan containing a great many stones and a great deal of mica..... $50-62$ Mannetto:

3. Very coarse gravel, quite hard and with no water................ $62-75$

Cretaceous:

4. Sand with little water, quite black, and with a bad odor............ $75-80$

5 . Very fine muddy sand .................................. $80-85$ 
Cretaceous-Continued

6. Whitish blue clay, lighter than other clays.

7. White beach sand, water-bcaring. . . . . . . . . . . . . . . . . . . . . $88-170$

8. Quicksand............................................ 170 175

9. A very large stone was encountered at. . . . . . . . . . . . . . . . . . 175

10. Coarse white sand . . . . . . . . . . . . . . . . . . . . . . . . . . . . . . $175-180$

427. The following section has been prepared from samples preserved in the museum of the Long Island Historical Society. The location given is only approximate:

Mannetto:

Record of J.F. D. Lanier's well near Old Westbury.

1. Yellow surface sand and gravel, no glacial máterial.

2. Pinkish white clay interbedded with white sand, suggesting the upper part of the

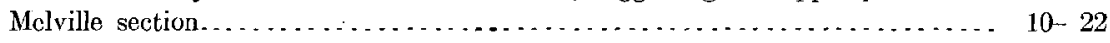

3. Fine yellow sand with supply of water. . . . . . . . . . . . . . . . . . . . . $22-37$

4. Pinkish white clay, marked "clay in thin layers" . . . . . . . . . . . . . . . $37-57$

5. Fine to medium yellow sand, marked "quicksand" . . . . . . . . . . . . . . 57-103

6. Ferruginous crusts in clayey sand . . . . . . . . . . . . . . . .

That stratum 3 should have contained an abundant supply of water, is rather surprising, considering the height of the well. The probable explanation is that the well was put down in the wet season and that this represents a perched water table.

428. The quicksand in the section below rose in the pipe three or four times. It was at last kept down by putting gravel in the bottom of the well. The water was obtained from quicksand. It was tested for twenty-four hours at a rate of 25 gallons per minute.

Wisconsin:

Record of J. F. D. Lanier's well near Old Westbury.

1. Fordpam Feet.

Transition:

2. Brown or gray clay, with plenty of flinty stones at the top . . . . . . . . . 20-100

Cretaceous:

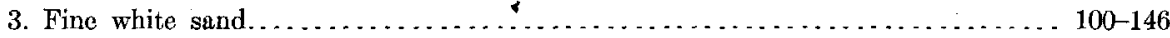

4. Quicksand, water-bearing . . . . . . . . . . . . . . . . . . . . . . . . . . 146-150

429. This well is about 40 feet higher than well 430 .

430. The following record has been prepared from the samples furnished by. Mr. John Tart c nd the record of Mr. F. Wankel, foremen for the Hudson Engineering and Contracting Company:

Record of H. B. Duryea's well near Old Westbury.

Wisconsin and Mannetto:

1. Loam . . . . . . . . . . . . . . . . . . . . . . . . . . . . . . .

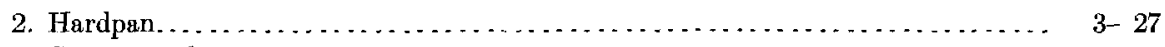

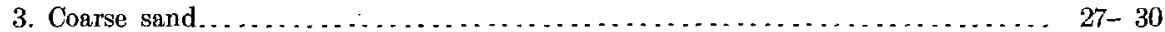

Cretaceous?:

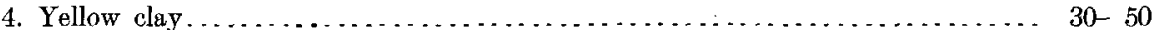

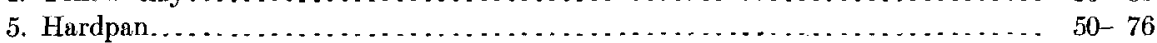

6. Quicksand . . . . . . . . . . . . . . . . .

Cretaceous:

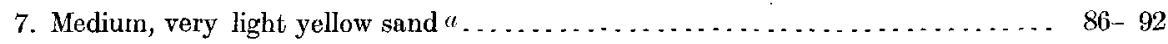

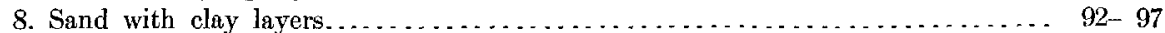

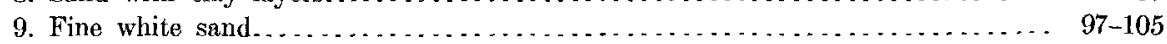

10. Medium yellow sand with some clay .......................... 105-121

11. Yellow sand with lumps of clay . .............................. 121-140 
Cretaceous-Continued.

Feet.

12. Bright reddish brown sand, with some ferruginous sandstone. . . . . . . . . . 140-152

13. Medium yellow sand, with lumps of white clay . . . . . . . . . . . . . . . . . . . 152-171

14. "Quicksand;" a very fine, light yellow, nicaceous, clayey sand............. 171-190

15. "Sandy white clay;" samples show only very fine, light yellow, micaccous, clayey sand ................................................. 190-225

16. "Quicksand," fine to medium, yellow, clayey sand.................. 225-258

17. "Dark clay.", samples show very dark, micaceous, sandy clay . . . . . . . . . . . . 258-280

18. Coarse soapstone sand . . . . . . . . . . . . . . . . . . . . . . . . . . . . . 280-286

19. Medium to coarse gray sand . . . . . . . . . . . . . . . . . . . . . . . . . . . 286-290

20. Medium to coarse sand ... . . . . . . . . . . . . . . . . . . . . . . . . . . . 290-308

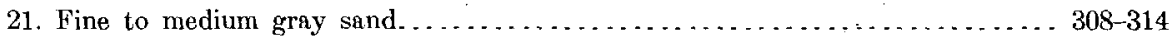

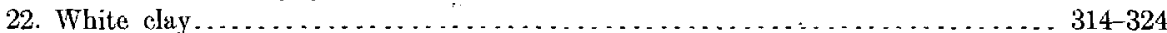

23. Coarse white sand . . . . . . . . . . . . . . . . . . . . . . . . . . . . . . . . . 324-329

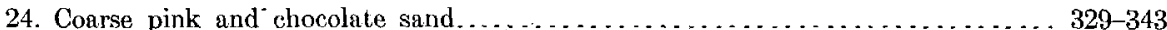

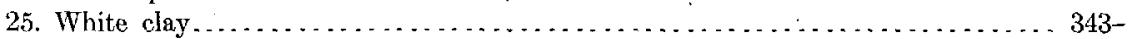

Strainers are placed from 300 to 308 feet and from 330 to 340 feet. Tlevation of ground, 197.5 feet.

431. The Cretaceous sand which underlies the Wheatley Hills, while water-bearing, is so fine that it is difficult to finish a well in it. Mr. E. D. Morgan has been particularly persistent in his search for a coarser layer that would yield an adequate supply of water. The records of several of the wells drilled at this place are given below.

The section of the well completed by Mr. John Fisher is reported as follows:

Wisconsin:

Record of E. D. Morgan's well in Wheatley Hills.

1. Hardpan

Cretaceous?:

2. Reddish clay.

Cretaceous:

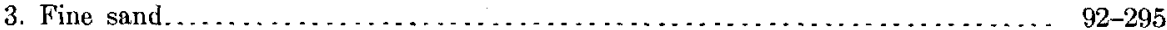

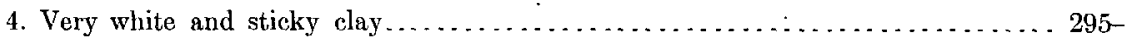

Water found at the top of the clay.

Five other parties (among whom was Gallienne) made failures in the same locality. Fisher ascribes their failure as due to their having passed through the clay. Below this there is a fine sand which sometimes rises in the pipe to a distance of 100 feet. In one case, where Fisher went through the clay for an experiment; the sand rose in his pipe to a distance of 60 feet. Down to 40 feet in this well the material passed through was so hard that no pipe was required.

A foreman in the employ of Mr. A. J. Connolly reports the following section:

Record of 'E. D. Morgan's well in Wheatley Hills.

Wisconsin:

1. Sand and clay with bowlders.

Fiet.

$0-90$

Mannetto?:

2. Coarse gravel, white and yellow

90

Cretaceous:

3. Yellow clay with fine sand.

4. Whitish clay (60 feet thick).

Mr. Alfred Wisson reports that in the well which he completed the section is almost the same as that which he reported from well 434. Of the wells drilled by Mr. A. W. Gallienne, Mr. Ed. Danis reports that the material penetrated was very similar to that encountered in the Harriman well (No. 512), on which Mr. Danis was working at the same time. 
In the summer of 1903 a new well was drilled at this place by Messrs. Wankel and Tart, of the Hudson Engineering and Contracting Company, who have furnished the Survey with the following record and samples:

Record of E. D. Morgan's well in Wheatley Hills.

Wisconsin: [Section by F. Wankel.]

1. Hardpan

Feet. Mannetto:

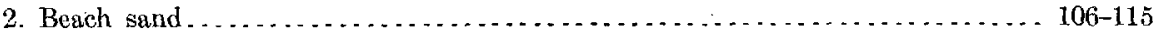

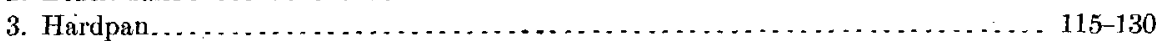

Mannetto ? and Cretaceous:

4. Beach sand . . . . . . . . . . . . . . . . . . . . . . . . . . . . . . . . . . . . . . . . 130-201 Cretaceous:

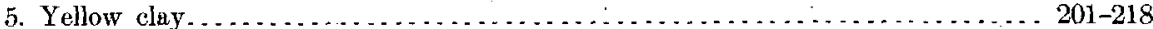

6. Beach sand (here I reached the surface of the water) $\ldots \ldots \ldots \ldots \ldots \ldots \ldots \ldots .218-236$

7. Gravel................................................... 236-243

8. Medium course sand . . . . . . . . . . . . . . . . . . . . . . . . . . . . . 243 252

9. Yellow clay . . . . . . . . . . . . . . . . . . . . . . . . . . . . . . . . . . 252-277

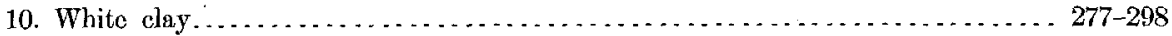

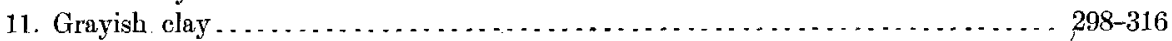

12. Yellow clay .............................................. $316-334$

13. Gravel, chunks of iron ore, hollow sandstones and big flow of water . . . . . 334-336

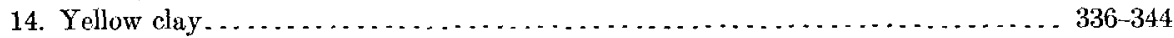

15. Yellow beach sand, very fine . . . . . . . . . . . . . . . . . . . . . . . . . . . . 344=350

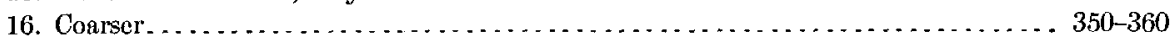

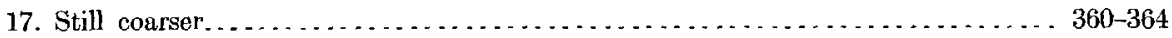

18. Quicksand . . . . . . . . . . . . . . . . . . . . . . . . . . . . . . . . . . $364-368$

19. Coarse, yellow sand . . . . . . . . . . . . . . . . . . . . . . . . . . . $368-393$

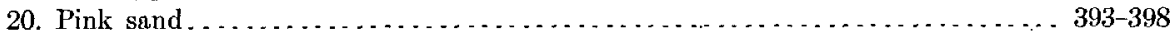

21. White sand, growing continually coarser to almost gravel ............. $398-427$

22. White medium coarse sand................................ $427-434$

23. White clay, not penetrated ................................. $434-$

From samples received from Wankel and Tart the following record has been compiled (elevation 328.5 feet, Geological Survey base):

\section{Record of E. D. Morgan's well in Wheatley Hills.}

$\dot{W}$ isconsin and Tisbury?: $\quad \cdot \quad$ Feet.

1. Quartz, sand, and gravel, with a considerable percentage of glacial material... 0-106 Mannetto:

2. Fine sand to small gravel; quartz, with fragments of red ferruginous sandstone and

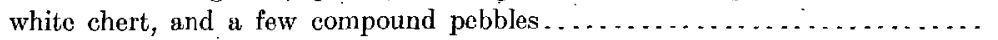

3. Quartz, sand, and gravel, many pebbles of ferruginous sandstone, and a few

fragments of compound pebbles (mica schist) ..................... . 115-130 Mannetto?:

4. Quartz, sand, and gravel, with some pebbles of ferruginous sundstone (no com-

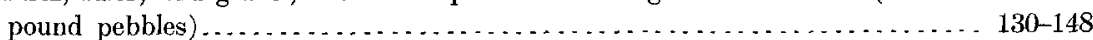

5. Orange-yellow quartz gravel, with a few pebbles of very much decomposed white chert and a few fragments of compound pebbles................... 148-149 Cretaceous:
6. Light-yellow quartz, sand, and gravel; white chert (no erratics).
7. Light-yellow quartz, sand, and gravel (no erratics)
8. Very light-yellow quartz, sand, and gravel ("water sand") .............. 201-218
9. Fine to medium yellowish-white sand, with a few small pebbles (no erratics)... 218-236
10. Small quartz gravel; many pebbles and fragments of white chert......... 236-243
11. Medium light-yellow sand . . . . . . . . . . . . . . . . . . . . . . . . . . . 243-251 
Cretaceous-Continued.

12. Yellow sandy clay (very fine yellow sandy silt) $\ldots \ldots \ldots \ldots \ldots \ldots \ldots \ldots \ldots .251-277$

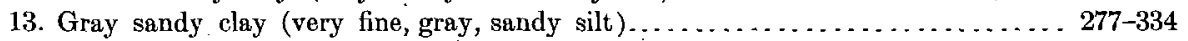

14. Quartz, sand, and gravel with a large percentage of rounded pebbles of ferruginous sandstone. A large fragment ( $3 \frac{1}{2}$ by 2 inches) of a very much decayed, rounded, granitic bowlder was obtained from this layer. This is the only piece of erratic material in the sample . . . . . . . . . . . . . . . . . . . . . . . 334-336

15. Yellow sandy silt or clay, with a few small quartz pebbles and fragments of ferruginous sandstone . . . . . . . . . . . . . . . . . . . . . . . . . . . $336-344$

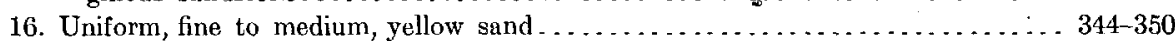

17. Same . . . . . . . . . . . . . . . . . . . . . . . . . . . . . . . . . . . . . . . . . 350-354

18. Same ... . . . . . . . . . . . . . . . . . . . . . . . . . . . . . . . . . . . . 354-368

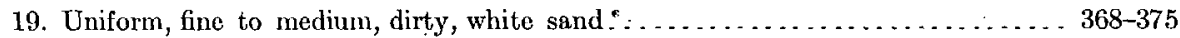

20. Medium yellow sand . . . . . . . . . . . . . . . . . . . . . . . . . . . . . 375-393

21. Uniform, coarse, reddish-brown sand. . . . . . . . . . . . . . . . . . . . . 393-398

22. Coarse light-yellow sand .................................. 398-418

23. Fine, white and yellow, quartz gravel, with fragments of ferruginous sandstone. . 418-435

A few specimens are preserved in the museum of the Long Island Historical Society, marked "Specimens obtained in boring a well at Wheatley Hills, Loug Island, summer of 1890." These are all normal Cretaceous material, but as no depths are given have no definite stratigraphic value. In one case a small tray marked "460 feet" contained a medium dirty yellow sand. This probably belongs to another section.

432. Mr. Thomas Griffin, foreman for W. C. Whitney, reports that the material penetrated was chiefly "hardpan and fine white sand."

433. Material penetrated very similar to that found in well 432 , but the sand is finer and contains a large amount of mica. The water is raised by steam pump and if a greater amount than 4 or 5 gallons per minute is pumped the water becomes cloudy.

434. Mr. Alfred Wisson reports the following section:

1. Hardpan (mixed clay and gravel with occasional streaks of clay) Cretaceous:

2. Soft mushy clay with much mica; the clay was black and had a very bad odor... 100-105

3. Soft reddish clay, loam-like in texture with some pure sand intermingled; some clear water was found at the bottom of the clay: yield, about 5 gallons a minute - 105-205

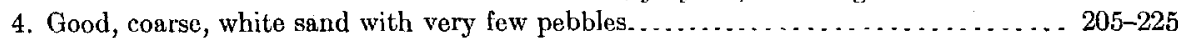

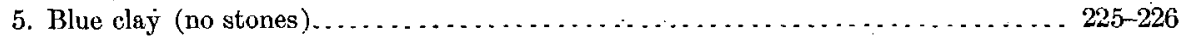

6. Good clear gravel............................................. 226-300

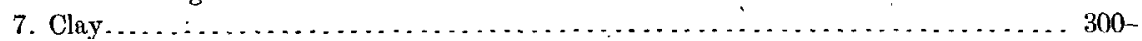

This well was tested for three weeks at a rate of 25 gallons a minute and occasionally 50 gallons a minute No effect was produced on the water level.

The following section is reported by Mr. John Fisher:

\section{Record of S. Mortimer's well in Wheatley Hills.}

Wisconsin and Mannetto:

1. Coarse gravel and loamy sand, like surface material.

2. Light baky clay; no water on top of the clay ....................... 150-160

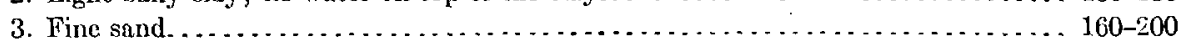

4. Coarse gravel, about the size of hickory nuts ....................... 200-205

Mr. Fisher states that this is the only well in this neighborhood in which water was found in gravel. He regards it as the best well in the vicinity of Wheatley Hills.

435. According to Mr. John Heerdegan the material penetrated in this well is as follows: 
 Record of W. Stowe's well in Wheatley Hills.}

Wisconsin:

1. Bowlders and clay (till)..................

Cretaceous?:

2. Alternating series of coarse and fine white sands... . . . . . . . . . . . . . . $40-190$

Cretaceous:

3. Fine sand and clay mixed . . . . . . . . . . . . . . . . . . . . . . . . . . $190-228$

4. Material gradually coarser until gravel is reached at $240 \ldots \ldots \ldots \ldots \ldots \ldots \ldots \ldots \ldots \ldots$. . . . . . . . . . . . . 245

5. Clay and sand mixed . . . . . . . . . . . . . . . . . . . . . . . . . . . . . . 245-295

Water was found in the sandy layers between 245 and 295 feet, but was cloudy, and the well was plugged at 240 feet and $\dot{i}$ strainer put in from 230 to 240 . No water was encountered until a depth of 228 feet was reached. 'The gravel in this well is reported as coarser than in any of the wells of the Nassau Electric Light and Power Company, No. 437.

Mr. I. H. Ford reports that in the well which he put down at this place he found nothing but sand. The first water was found at a depth of 130 feet.

436.

Record of Mrs. I. Vowman's well near Roslyn.

Wisconsin and Tisbury:

1. Coarse red gravel; very hard, and with no stones.

Feet

2. Coarse sand, quite red in streaks. ............................. $20-50$

Manhasset bowlder bed?:

3. Sand, with a thin layer of bowlders.............................. $50-53$

Tisbury and Mannetto:

4. Fine sand.

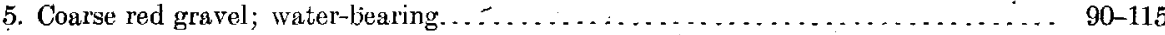

437. Record of well of Nassau Electric Light and Power Company, Rostyn.

Pleistocene:

1. Sand and gravel, similar to that at surface beneath loam (water-bearing between 80 and 82 feet)

Feet.

Cretaceous :

2. Ordinary, white, "beach" sand . . . . . . . . . . . . . . . . . . . . . . . . $100-180$

3. Lignite, with sand. . . . . . . . . . . . . . . . . . . . . . . . . . . . . . . . 180-182

4. Clay, containing a very small percentage of sand ("almost solid clay") . . . . . . . . 182-220

5. Water-bearing gravel. . . . . . . . . . . . . . . . . . . . . . . . . . . . . . . . . . . 220-238

6. Fine sand and white clay mixed. . . . . . . . . . . . . . . . . . . . . . . . 238-250

At 250 feet a shell was found in white clay which was identified by Dr. W. H. Dall as Terebratula filosa.

According to tests made by the Nassau Light and Power Company the whole series of 4 wells when pumped together yields 176,000 gallons in 24 hours. Individually the wells yield as follows:

Yield of wells of Nassau Electric Light and Power Company at Roslyn.

Well No. 1

120,000

Well No. 2... . . . . . . . . . . . . . . . . . . . . . . . . . . . . . . . . . .

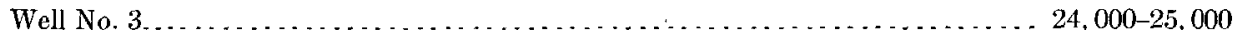

Well No. 4. . . . . . . . . . . . . . . . . . . . . . . . . . . . . . . . . . . . . . . . . 60 600

The strainers in these wells are 10 feet long and are between 228 and 238 feet below the surface. The wells were sunk to a depth of 250 feet, 12 feet below the strainers, in order that material passing the screens would fall below the screens and not clog up the wells.

138. Mr. Schmidt could not give a complete $\log$ of this well, but furnished the following data: Two bowlder beds were encountered, one at 82 feet, 4 feet thick, and another at 124 feet, 2 leet thick. Many of the stones were the size of a double fist and not a few were as large as one's head or even larger. Occasional streaks of clay were encountered, but these were not of any considerable thickness. There were some layers 
of coarse sand and gravel of the ordinary color, containing many quartz pebbles the size of hickory nuts One such bed was found at 126 feet, from which the supply of water comes.

Mr. Schmidt says that the above conditions are frequently met with north of the Jericho turnpike, and at Ieast as far east as Westbury. He has also encountered such bowlder beds at East Williston and always found water in them. He says that some of the stones are so large that it is all two men can do to pull one out with the windlass.

440. Record of commission's test well near Roslyn.

1-2. Dark sandy loam

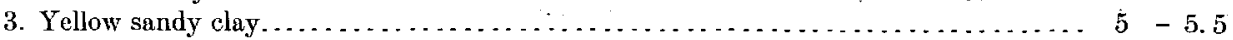

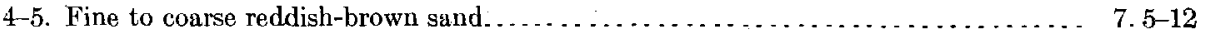

6. Fine, reddish-brown, clayey sand with bowlders and cobbles.............. $14 \quad-14.6$

7-9. Dark; yellowish gray, fine sand and small gravel, with a considerable percentage of erratics. . . . . . . . . . . . . . . . . . . . . . . . .

10-11. Very fine, light, yellowish-brown, micaceous clayey sand; resembles Cretaceous. $34 \quad-40$

12-14. Fine to coarse reddish-brown sand, mostly quartz (glacial)............... 44 -52

41.

Record of commission's test well near. Roslyn.

Wisconsin:

1. Black surface loam.

2-6. Dark, yellowish-brown, clayey sand and gravel containing a very large amount of glacial débris.

44. Mr. Corcoran reports that a clay bed was encountered between 90 and 190 feet, on penetrating which, water rushed up with considerable force, bringing sand with it.

Mr. Jesse Conklin writes (April 25, 1895), regarding a well at Roslyn, which is probably this well: "In Roslyn, near the Long Island Sound, I drove a 6-inch well 210 feet. At 74 feet I struck water, drove 10 feet in water and got a poor supply. I drove on 116 feet through fine sand and some clay; all through this 116 feet I found clam and oyster shells. At 200 feet I struck white gravel and drove 10 feet and got an unlimited supply of water. I pumped from this well 100 gallons per minute and could not lower the water a particle."

445.

Record of well at W. J. Post's brickyard, Glenwood Landing.

Tisbury?

1. Sand

$0-31$

Cretaceous:

2. Solid clay.

$31-.61$

3. Quicksand.

61- 69

4. Coarse gravel. $69-$

446.

Record of A. A. Knowles's well near Glentiead.

Wisconsin:

1. Brownish loam

Tisbury:

2. Yellow sand and gravel. . . . . . . . . . . . . . . . . . . . .

Manhasset bowlder bed:

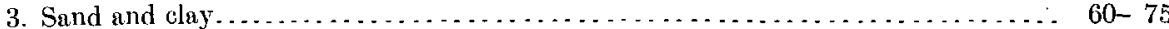

Tisbury:

4. Sand

Cretaceous:

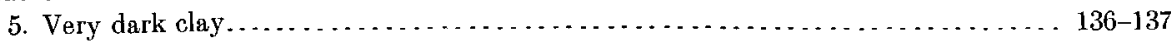

6. White sand, with water 


\section{UNDERGROUND WATER RESOURCES OF LONG ISIAND, NEW YORK.}

450.

Record of Kersona well near Sea Cliff.

Wisconsin and Tisbury:

1. Brown sand; slight flow of water at 45 feet

Feet. '

$0-45$

Cretaceous:

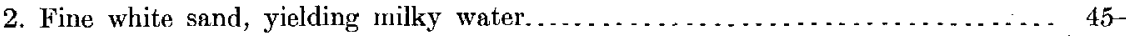

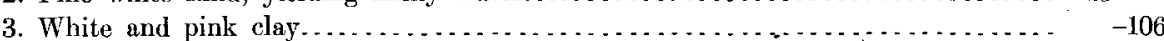

452. This plant draws its water from six 6 -inch wells sunk between 1897 and 1903, which yield 250,000 gallons in ten hours. The original supply was from springs.

453. Mr. Dubois states that at 80 feet he struck hardpan with some stone. This probably represents the Manhasset bowlder bed.

454. * Record of commission's test well 2 miles south of Locust Valley.

Wisconsin:

Feet 1. $5-10$

1-2. Grayish-ycllow fine sand to coarse gravel

3. Medium dark brownish gray sand ............................. 21-25

Tisbury:

4. Light yellow, very coarse sand and fine gravel; looks like Tisbury material... 37-40

5. Same, rather finer.......................................... $47-52$

See Table XIII.

455. Record of well of Nassau County Water Company, near Glen Cove.

Recent:

1. Marsh mud.

2. Brown sand

Tisbury:

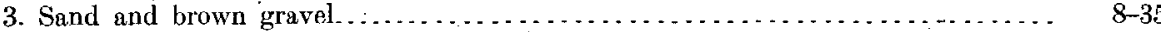

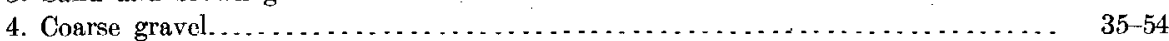

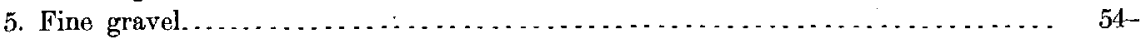

"The gravel [in this well] is as large as a man's fist; the sand is white and coarse from 8 to 54 . This well flows 6 feet in the air, and its pumping capacity is 250 gallons per minute."

Samples in the office of Mr. Oscar Darling, consulting engineer, show the following section for the first 10-inch well:

Tisbury:

Record of well of Nassau County Water Company, near Glen Cove.
1. Light-yellow sand and gravel with small percentage of glacial material

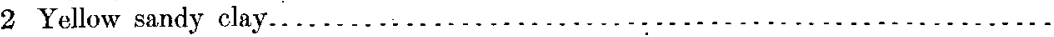
$0-23$
27

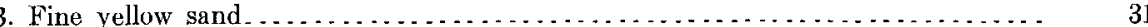

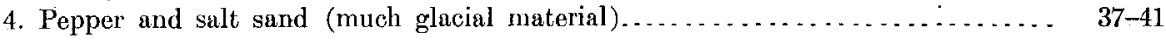
5. Coarse white sand . . . . . . . . . . . . . . . . . . . . . . . . .

Feet.

This well flowed first at 41 feet.

The 2-inch test well flowed first at a depth of 34 feet; it flowed, at 18 inches above the surface, 20 gallons per minute.

Mr. Darling gives the elevation of the ground at the pumping station as 50 feet above mean high tide.

456. Record of Friends' Academy well near Locust Valley.

Tisbury:

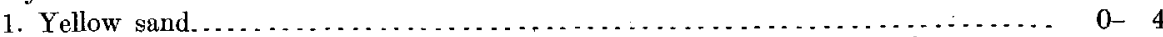

2. Light-colored sand and gravel................................. $4-80$

Manhasset bowlder bed?

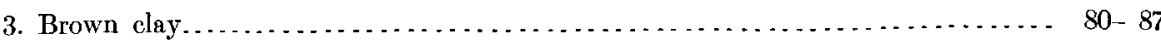

Tisbury:

4. Reddish brown sand, with some water....................... $87-90$ 
Cretaceous?:

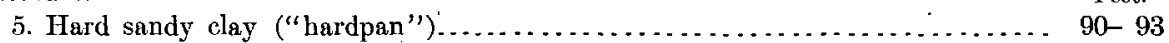

6. Gray sand and gravel. ...................................... 93-156

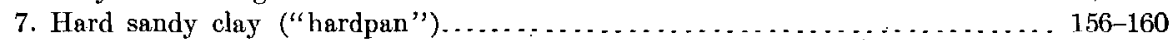

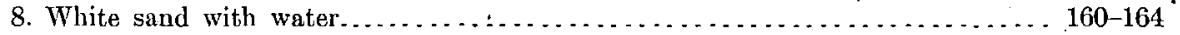

Cretaceous:

9. "Hardpan" . . . . . . . . . . . . . . . . . . . . . . . . . . . . . . . . . . . . . . 164-168

10. Brownish yellow to white sand . . . . . . . . . . . . . . . . . . . . . . . . . . . 168-197

11. White clay, becoming pink below. . . . . . . . . . . . . . . . . . . . . . . 197-209

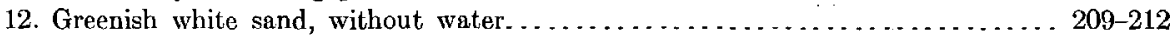

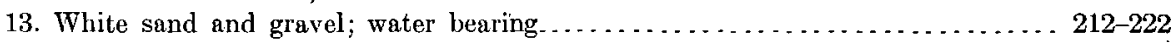

457.

Record of F. E. Willets's well near Glen Cove.

Wisconsin and Tisbury: Fect.

1. Brown loam............................................ 9

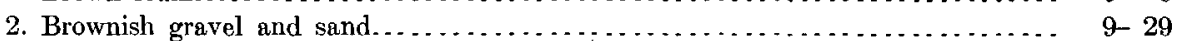

Manhasset bowlder bed:

3. "Hardpan;" clay with bowlders . . . . . . . . . . . . . . . . . . . . . $29-44$

Tisbury:

4. Light-colored sand. . . . . . . . . . . . . . . . . . . . . . . . . . . . $44-70$

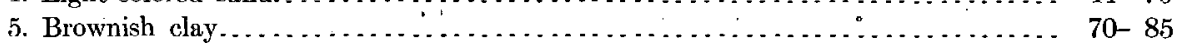

6. Sand, growing whiter as the depth increases.................... $85-158$

Cretaceous?

7. Clay, with enough grit to make it hard ("hardpan") .................. 158-164

8. White gravel (no water) .................................. 164-182

9. White coarse sand, with an abundant supply of water $\ldots \ldots \ldots \ldots \ldots \ldots \ldots \ldots$ 182-186

458.

Record of S. Seeman's well near Glen Cove.

Wisconsin:

1. Hard brown clay - Feet.

Tisbury:

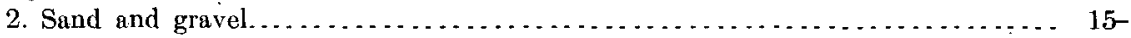

Transition:

3. White sand.

Cretaceous:

4. White clay, becoming pink below.

5. White sand; water bearing -

459.

Record of S. Burke's well near Glen Cove.

1. "Hardpan" " brown clay with grit; no bowlders.

Tisbury:

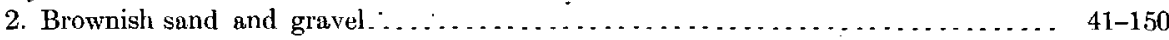
Cretaceous:

3. Whitish clay, becoming pink in its lower portion................ 150-165

4. White sand, containing water................................. 165-170

Mr. Dubois states that this bed of white or pink clay commonly overlies the watcr-bearing strata in this section.

460.

Tisbury: Record of North Country Club well near Glen Cove.

1. Surface gravel. .

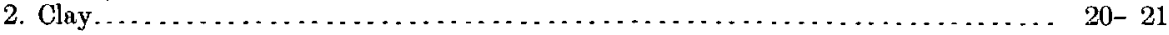

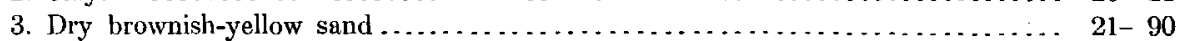

Cretaceous:

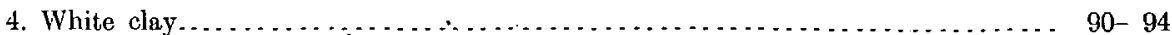

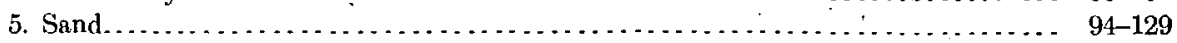


264 UNDERGROUND WATER RESOURCES OF LONG, ISLAND, NEW YORK.

462.

Record of John Minniken's well near Glen Cove.

Recent:

1. Filled ground.

Feet.

2. Peat

Tisbury:

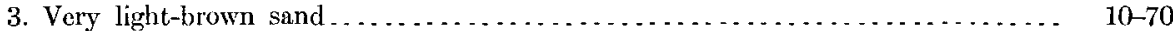

Cretaceous:

4. Pink clay

5. White sand and gravel, water bearing; similar to the gravel in Baldwin's well (No. 476)

This well flows at a height of about 40 feet above sea level.

463.

Record of Crystal Springs Ice Company's wells near Glen Cove.

Recent:

1. Marsh deposit. ............................................... $0-20$

Tisbury:

2. Sand, with water (small flow of fresh water at 28 feet) $\ldots \ldots \ldots \ldots \ldots \ldots \ldots .20-28$

Cretaceous:

3. Bluish clay, becoming white below . . . . . . . . . . . . . . . . . . . . . . $28-70$

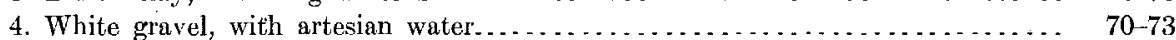

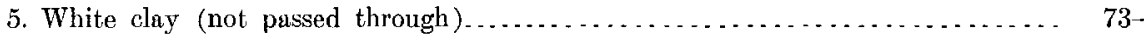

Two-inch well flowed 18 gallons per minute when first drilled, but the flow seems to have decreased slightly; 4-inch well flowed 30 gallons, with no decrease noticed. Water was piped up to $14 \frac{1}{2}$ feet above ground in 1899. Mr. Oscar Darling reports the surface at the 4-inch well to be 30 feet above mean high tide.

464. Mr. Ralph D. Carter gives the following section of this well:

Record of J. P. Tangeman's well near Glen Cove Landing.

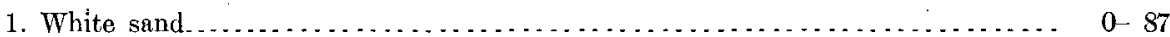

2. Hardpan. .............................................. $87-90$

3. Water-bearing stratum of gravel, sand, and clay, containing mica. ........ $90-100$

4. Gray-colored clay . . . . . . . . . . . . . . . . . . . . . . . . . . . . . . . . . . 100 105

465.

Record of W. M. Valentine's well near Dosoris.

Wisconsin and Tisbury:

1. Brown loam.............................................. 6

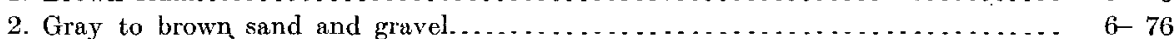

Manhasset bowlder bed:

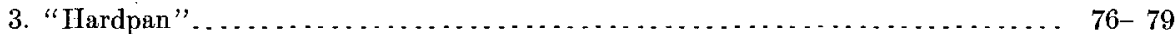

Tisbury:

4. Brownish sand with water...................................... 79

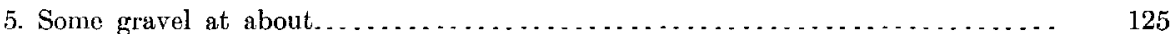

Cretaceous:

6. Very white clayey sånd.................................... 144-200

7. Blue elay, with a very hard layer at the base. . . . . . . . . . . . . . . . 200-215

166. This plant was originally constructed to supply the Pratt estate, but the mains were ultimately extended to Lattingtown and Glen Cove.

Mr. Frederick Miller put in the first two wells near the pumping station, after he had made the two tests mentioned under well 467 . These were both 6 -inch wells, one 38 and the other 48 feet deep. The 38-foot well has an elevation of about 48 feet, and flowed when first put down. Since the wells have been pumped, this well has ceased flowing.

In $1900 \mathrm{Mr}$. Munger put in two additional 6-inch wells, one 38 feet and the other 82 feet deep. The 82 -foot well is on the lowest ground of the series, being only about 30 feet above high tide; it is reported to flow 4 to 5 gallons per minute. Its section is approximately as follows: 
Record of Pratt estate well near Dosoris.

1. Soil.

Cretaceous:

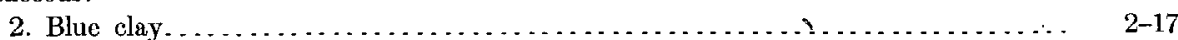

3. Reddish sand and gravel. . . . . . . . . . . . . . . . . . . . . . . . . .

4. Blue clay . . . . . . . . . . . . . . . . . . . . . . .

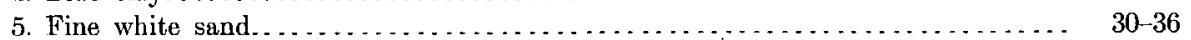

6. Alternate layers of gravel, sand, and clay ......................... $36-82$

Water was first struck at about 40 feet, but did not flow; at 70 feet water was again encountered, which filled the pipe almost to the top; at 75 fect the water flowed over the top of the pipe. The yield of these 4 wells is given as about 100,000 gallons per day.

The standpipe, which has a capacity of 158,000 gallons, is situated on the top of a hill, at an elevation of 160 fcet. About 250 feet southwest of one of the good wells at the pumping station, Mr. Munger put down four $1 \frac{1}{2}$-inch test wells to a depth of 125 feet without getting water. He reports the same character of soil, but no water.

467. Mr. Munger reports two wells sunk at this point to a depth of 125 feet, through sand and clay, without any results.

469.

Record of D.F. Bush's well near Dosoris Pond'.

Recent:

1. Yellowish brown sand ................................... 0 . 4

2. Marsh deposit ..................................... 4 - 7

Wisconsin?

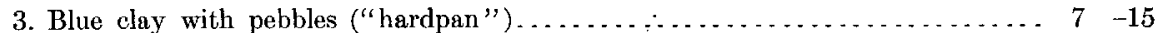

Tisbury:

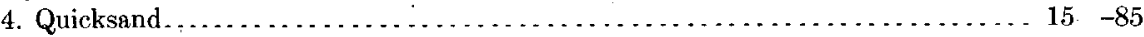

Sankaty:

5. Reddish gravel and clay................................ $85-88$

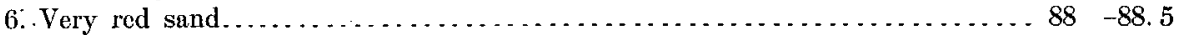

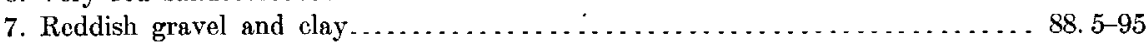

Jameco:

8. Light-colored gravel with a considerable percentage of glacial material; furnishes artesian water. $95 \quad-97$

Mr. Dubois has furnished a sample from stratum 8 .

470. The following section has been compiled from samples furnished through the kindness of Messrs. P. H. and J. Conlan:

Pleistocene:

Record of C. O. Gates's well near Peacock Point.

1. Sand and gravel................................... 0 . 40

2. Greenish gray sandy clay, with a few quartz pebbles................. 45

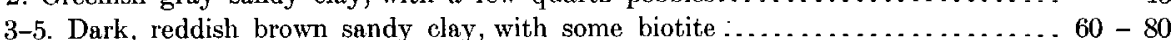

Transition:

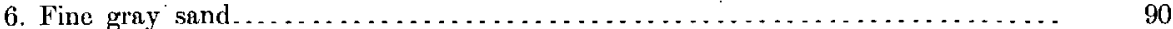

Cretaceous:

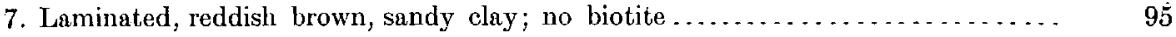

8. Very fine, pinkish white, micaceous sand............................ 100

9-14. Light-gray, medium, micaceous sand . . . . . . . . . . . . . . . . . . . . . 105-130

15. Dark, grayish brown, sandy clay . . . . . . . . . . . . . . . . . . . . . . . . . .

16. Pebbles of ferruginous sandstone . . . . . . . . . . . . . . . . . . . . 140

17-20. Laminated red and white clay. In the fragments furnished, the laminations show very great distortion; whether this is the natural condition of bed or is the result of method of taking samples is not known. Sample 17 contains a few fragments of a lamellibranch, but the sample shows $17116-$ No. 44-06-18 
evidence of having been laid out on the ground before it was packed in the bottle, and the shell may have been picked up there.......... . 145-160

21-26. Brick red, very plastic clay................................... 165-190

Lloyd sand:

27-30. Fine reddish sand; the red color seems to be due in a great measure to the red clay from the overlying bed........................ 195-210

31-33. Medium light-yellow sand................................ 215-225

34. Fine to coarse, light yellow, quartz gravel, with a few fragments of white, chalky looking chert........................................

Elevation 9.0 feet, Geological Survey base.

Mr. Bowman reports that the well flows 30 gallons per minute. For partial analysis by Prof. Charles S. Slichter, see page 68 .

471. Mr: E. K. Hutchinson reports the following section for this well:

Pleistocene:

$$
\text { Record of C. O. Gates's well near Peacock Point. }
$$

Cretaceous:

2. Alternate layers of red, black, gray, and milky-white clay $\ldots \ldots \ldots \ldots \ldots \ldots \ldots$. $80-200$ Lloyd sand:

3. Fine sand, gradually growing coarser.......................... 200-225

172. Water brings up a fine, micaceous, white sand, which settles with difficulty. It is claimed that storms from the north do not affect the water, but that storms from the east cause it to be very turbid.

Record of well near Peacock Point.

Pleistocene

1. Beach sand and gravel.................................... $0-138$

Cretaceous:

2. Red clay

Lloyd sand:

3. Sand with artesian water

473. Mr. Hutchinson reports the following section:

Record of W. D. Qutherie's well near Lattingtown.

Wisconsin and Tisbury:

Feet.

1. Sand and gravel

$0-80$

Cretaceous:

2. Clay, blue, white, and red, encountered in order given............... 80-260

Lloyd sand:

3. Varicolored sand and gravel, becoming coarse

$260-340$

This well began flowing at 260 and continued to 340 feet.

One of the workmen engaged on well gives the following record:

Record of W. D. Gutherie's well near Lattingtown.

Wisconsin and Tisbury:

1. Sand and grave

Feet.

Cretaceous:

2. White, blue, brown, and red clay, encountered in the order given $110-260$ Lloyd sand:

3 . White and yellow sand, in layers of 3 or 4 feet, alternating with layers of white clay. $.260-342$

Elevation 13.0 fect, Geological Survey base: 
474.

Record of W. D. Gutherie's well near Lattingtown.

Recent:

1. Swamp muck...
Wisconsin and Tisbury

Feet.

0- 5

2. Brownish sand.

Cretaceous?:

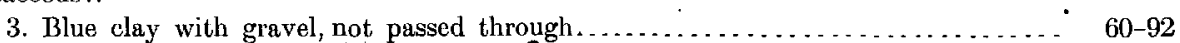

At 13 feet the water rose in a pipe 2 feet above the surface, and at 25 feet, 2.5 feet.

485.

Record of W. Price's well near Lattingtown.

Wisconsin?:

Feet.

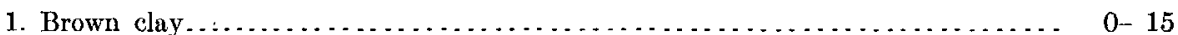

Transition:

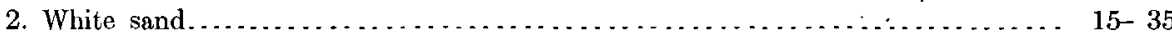

Cretaccous:

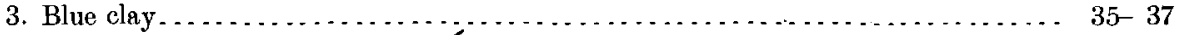

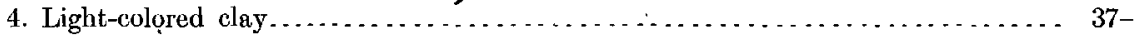

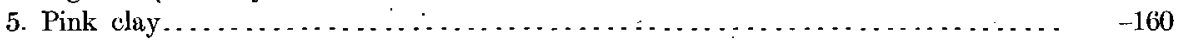

6. Light-yellow gravel . . . . . . . . . . . . . . . . . . . . . . . . . . . . . . . . . . . . 160-162

Elevation of surface 140 feet above mean sea level.

476. Mr. W. H. Baldwin, jr., has kindly furnished the following record of this well:

Wisconsin:

Record of well of W. H. Baldwin, jr., near Lattingtown.

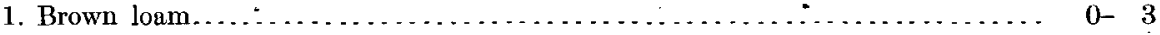

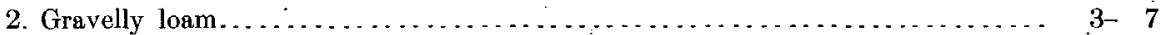

Transition:

3. Yellowish sand and gravel................................... $7-12$

Tisbury:

4. Sand and gravel, with occasional thin streaks of clay................ 12-107

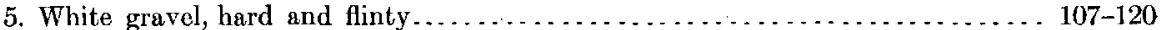

Cretaceous:

6. Clay ................................................... 120 130

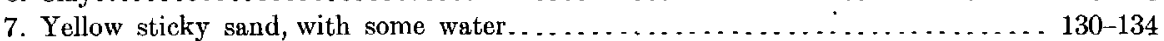

8. Gravel, with occasional streaks of very hard hardpan. . . . . . . . . . . . . . . . 134-199

9. Very hard clay bed........ . . . . . . . . . . . . . . . . . . . . . . . . . 199-255

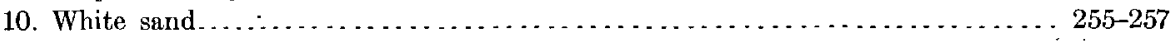

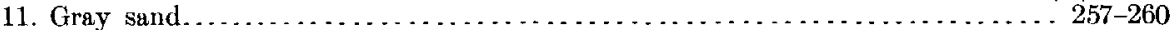

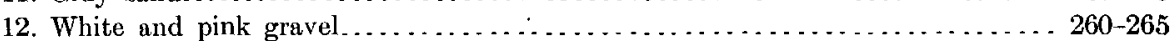

Elevation of surface 179.5 fect above sea level, Geological Survey base.

Analysis of water from well of $W . H$. Baldwin, jr., near Lattingtown.

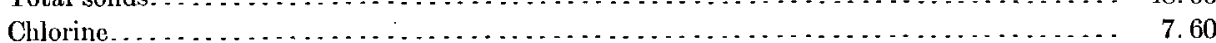

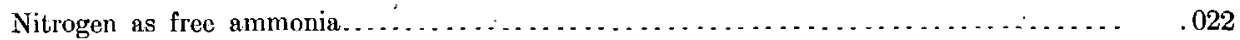

Nitrogen as albuminoid ammonia. . . . . . . . . . . . . . . . . . . . . . . . . . . 024

Nitrogen as nitrites. . . . . . . . . . . . . . . . . . . . . . . . . . . . . . . . 003

Nitrogen as nitrates. . . . . . . . . . . . . . . . . . . . . . . . . . . . . 3.300

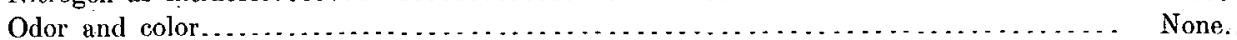

The solid matter is all in solution and is practically all sulphates. The amount (2.8 grains per gallon) is extremely small. This is an unusually soft water (almost as soft as rainwater) and bears no evidence of contamination.-C. N. Forrest, chemist and inspector, Long Island Railroad. 


\section{UNDERGROUND WATER RESOURCES OF LONG ISLAND, NEW YORK.}

479.

Record of L.C. Wier's well near Lattingtown.

Pleistocene:

1. Same as No. 481

retaceous:

2. White clay ............................................ $124-130$

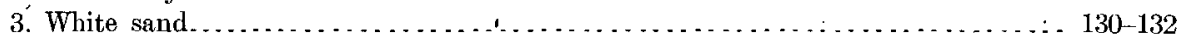

480.

Record of L. C. Wier's well near Lattingtown.

1. Sand with an occasional stratum of impervious clay .................

2. Beach sand and gravel...................................... 117.2-123.6

481.

Record of L. C. Wier's well near Lattingtown.

Tisbury:

1. Sand and gravel

Manhasset bowlder bed?

2. Red clay with gravel (hardpan).

Tisbury and Mannetto?

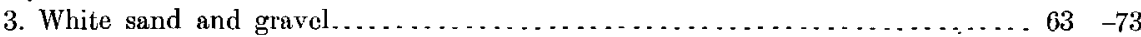

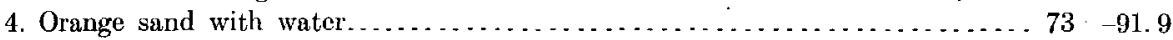

Cretaceous:

5. White clay

91.9

482. Record is reported as very similar to that of 481 .

483.

Record of E. Latting's well near Lattingtown.

Tisbury:

1. Sand and gravel.

1. Sand and gravel.

$0-60$

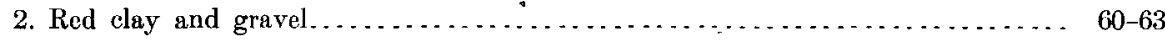

Tisbury:

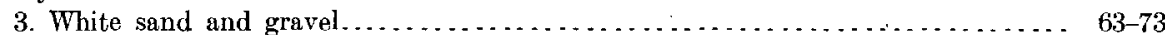

Mannetto?

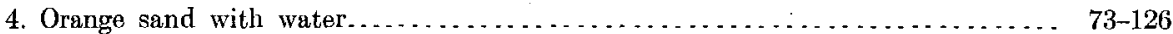

Cretaceous:

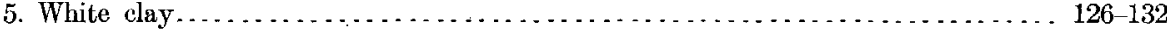

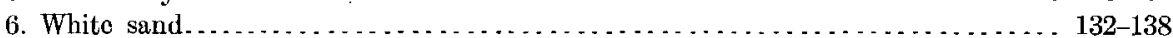

484.

Record of W. D. Gutherie's well near Lattingtown.

Tisbury:

Feet.

1. White gravel...

$0-60$.

Manhasset bowlder bed:

2. Red clay with gravel (hardpan)

$60-64$

Tisbury and Mannetto?:

3. White sand

64-83

Mannetto? and Cretaceous:

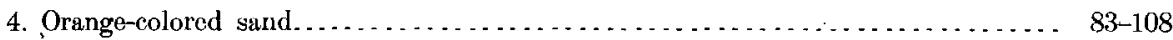

Cretaceous:

5. White clay

108

485.

Record of W. $\dot{D}$. Gutherie's well near Lattingtown.

Tisbury:

1. Stratified sand and gravel.

nhasset bowlder bed:

2. Large bowlder.

0- 38 isbury:

38

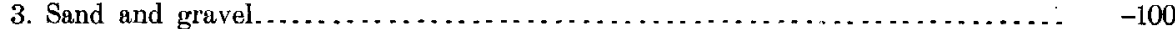


Cretaceous:

Feet.

4. White clay.

$100-110$

5. Orange-colored sand; water bearing.

110-144

487. Mr. Ward reports that the plant at this station consists of thirty-two 6-inch wells, 33 to 91 feet deep. 'Samples from a number of these are preserved in the archives of the Brooklyn department of water supply, and while the sections shown are quite irregular, the following selected records will indicate something of their general nature:

Record of well No. 2 E, Agawam pumping station.

1. No samples

2. Fine, gray, micaceous sand, and medium to coarse yellow sand

3. Fine, gray, micaceous sand.

4. Dark yellowish-gray silt to small gravel.

5. Very white, medium, micaceous sand.

7. Mixture of medium yellow and fine gray sand with some small pebbles

Nothing recognizably glacial.

Record of well No. 10 E, Agawam pumping station.

1. No samples

Feet.

$0-30$

2. Dark-gray clay

$30-35$.

3. Fine, gray, micaceous sand

$35-45$

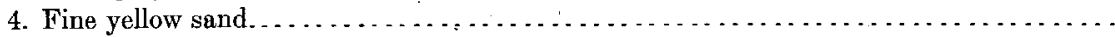

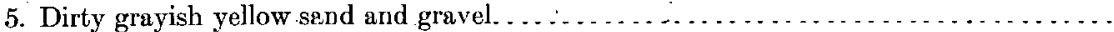

$45-50$

$50-65$

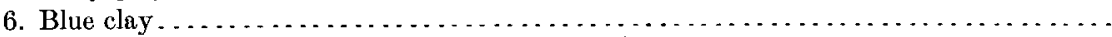

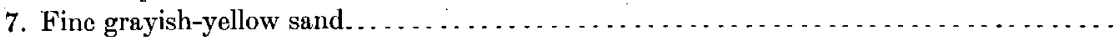
$65-70$

$70-75$

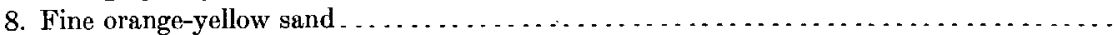

9. Medium grayish-yellow sand, with a considerable percentage of very small orangecolored pebbles.

$75-80$

$80-94$

Record of well No. 5 W, Agawam pumping station.

1. No samples.

Feet.

$0-54$.

$54-65$.

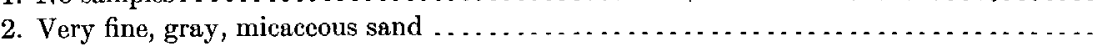

$65-69$.

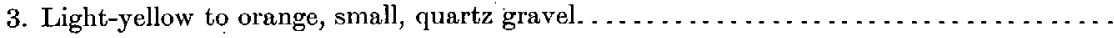

4. Fine gray sand, with lignite

$69-79$

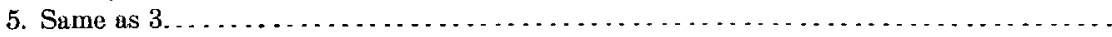

6. Gray and yellow fine to medium sand, with lignite

$79-84$.

$84-89$

Record of well No. $10 \mathrm{~W}$, Agawam pumping station.

1. No samples

2. Fine grayish yellow sand with a few pebbles. $\ldots \ldots \ldots \ldots \ldots \ldots \ldots \ldots \ldots \ldots \ldots \ldots \ldots$

3. Very fine dark-gray sand. . . . . . . . . . . . . . . . . . . . . . . . . . . .

4. Gray to light yellowish gray fine sand.

$35-45$

5. Fine, light, yellow or grayish yellow sand.

Record of well No. $11 \mathrm{~W}$, Agawam pumping station.

1. No samples . . . . . . . . . . . . . . . . . . . . . . . .

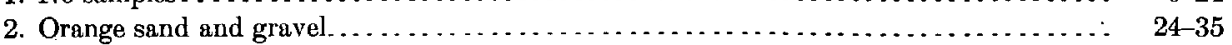

3. Fine, dark, gray and light gray sands in alternating beds.................. $35-8 a$ 
Record of well No. 12W Agawam pumping station.

1. No samiples.

\author{
i
}

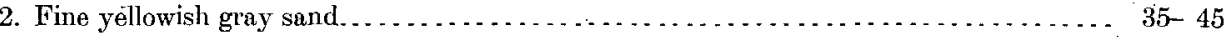

3. Fine gray sand ...... . . . . .

4. Fine dark-gray sand, with considerable lignite . . . . . . . . . . . . . . . . . . $60-110$

This section is much more uniform than the other rapidly alternating sections of the same series.

Record of well No. 6 B, E, Agawam pumping station.

1. No samples . . . . . . . . . . . Feet.

2. Fine gray micaceous sand, with a little yellow sand $\ldots \ldots \ldots \ldots \ldots \ldots \ldots \ldots \ldots \ldots \ldots 24,6 \ldots \ldots 2$

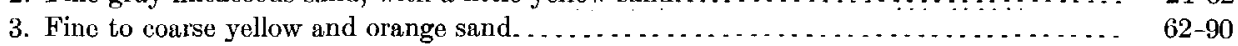

488. This was the site of the original Freeport or Agawam station, and was abandoned because of the large amount of chlorine which the water contained. The following summarized section has been prepared from the samples preserved by the Brooklyn waterworks:

Record of wells at old Freeport pumping station.

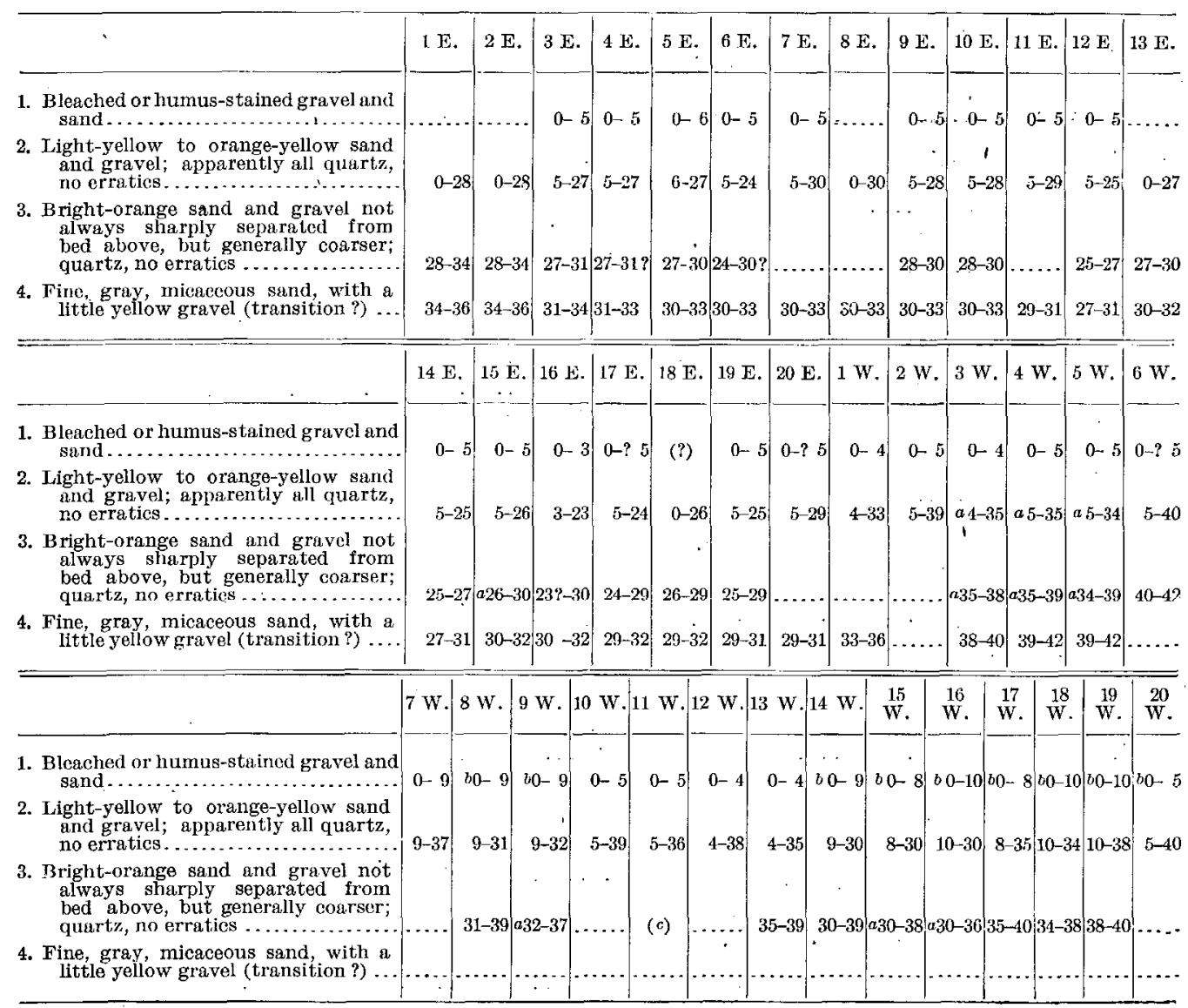

a Medium sand.

$b$ Weathering shows sharply to 5 feet and less markedly to depth indicated

c 37 to 40 feet white quartz gravel tinged with yellow. 
Analysis of water from wells of old Freeport pumping station.

[By Brooklyn health department.]

Parts per million.

Total solids. ...

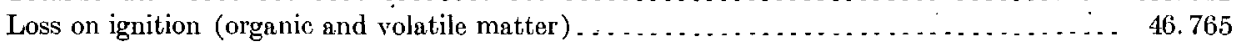

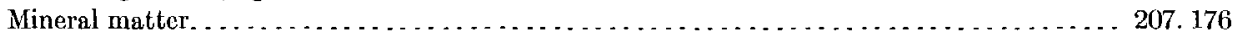

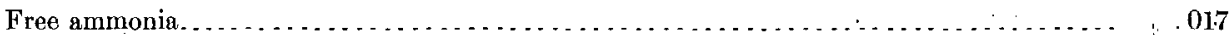

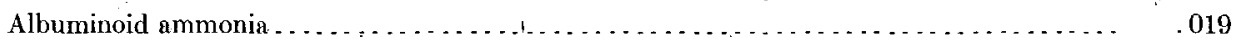

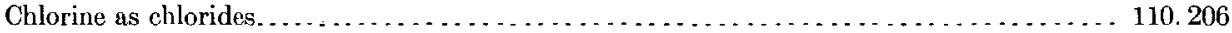

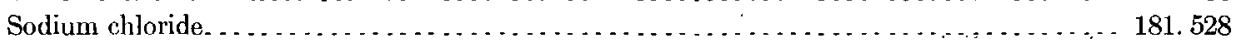

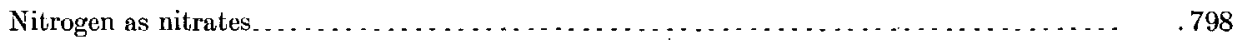

Nitrogen as nitrites . . . . . . . . . . . . . . . . . . . . . . . . . . . . . . . . . . . .

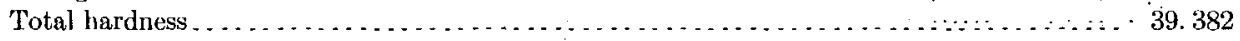

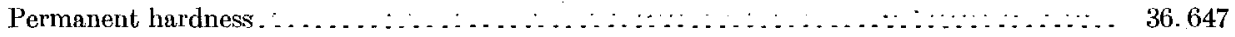

489. The plant at this station consists of sixty-two $4 \frac{1}{2}$-inch wells. The following summarized record has been prepared from the samples preserved by the Brooklyn waterworks:

Record of wells at Merrick pumping station.

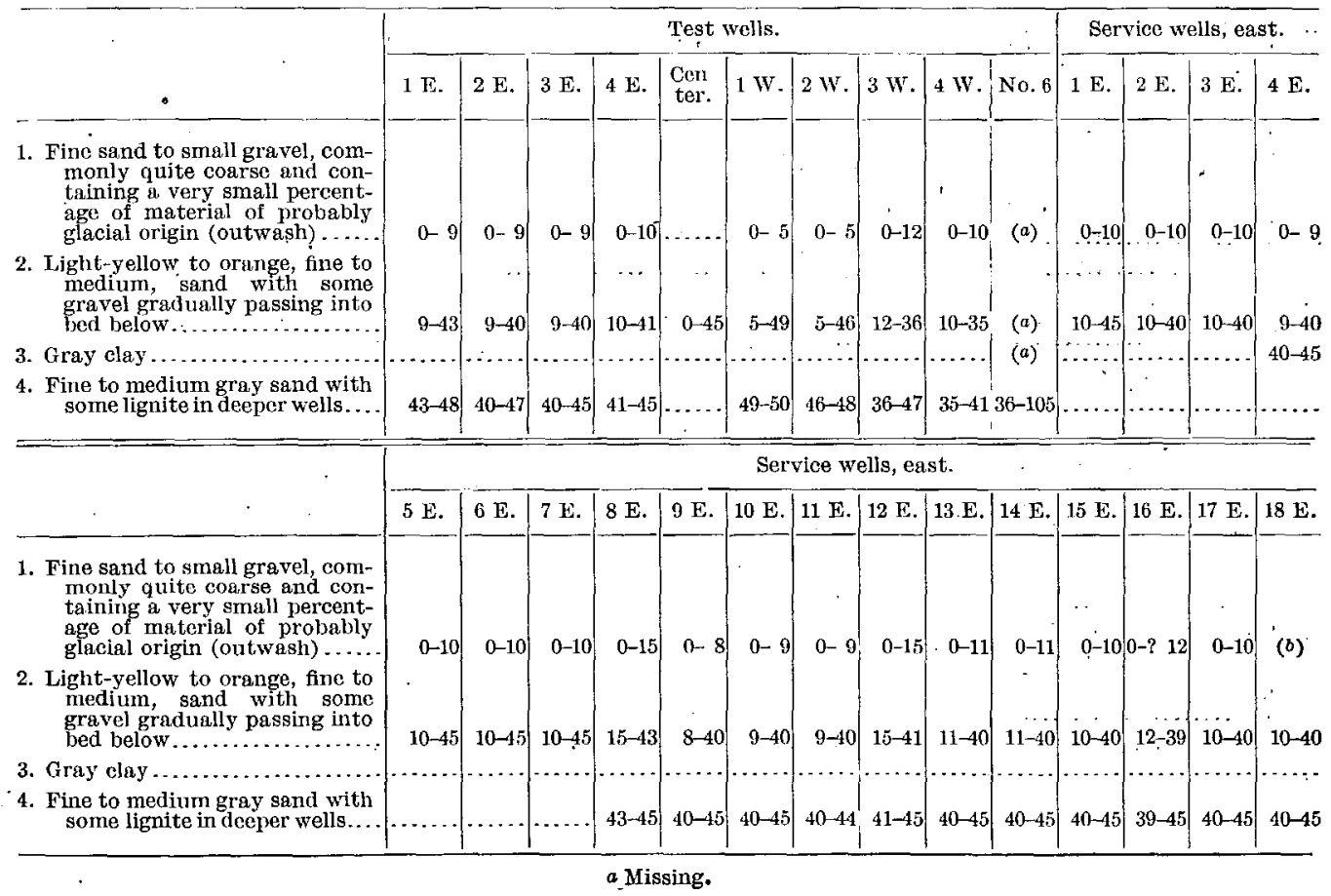


Record of wells at Merrick pumping station $<$ Continued.

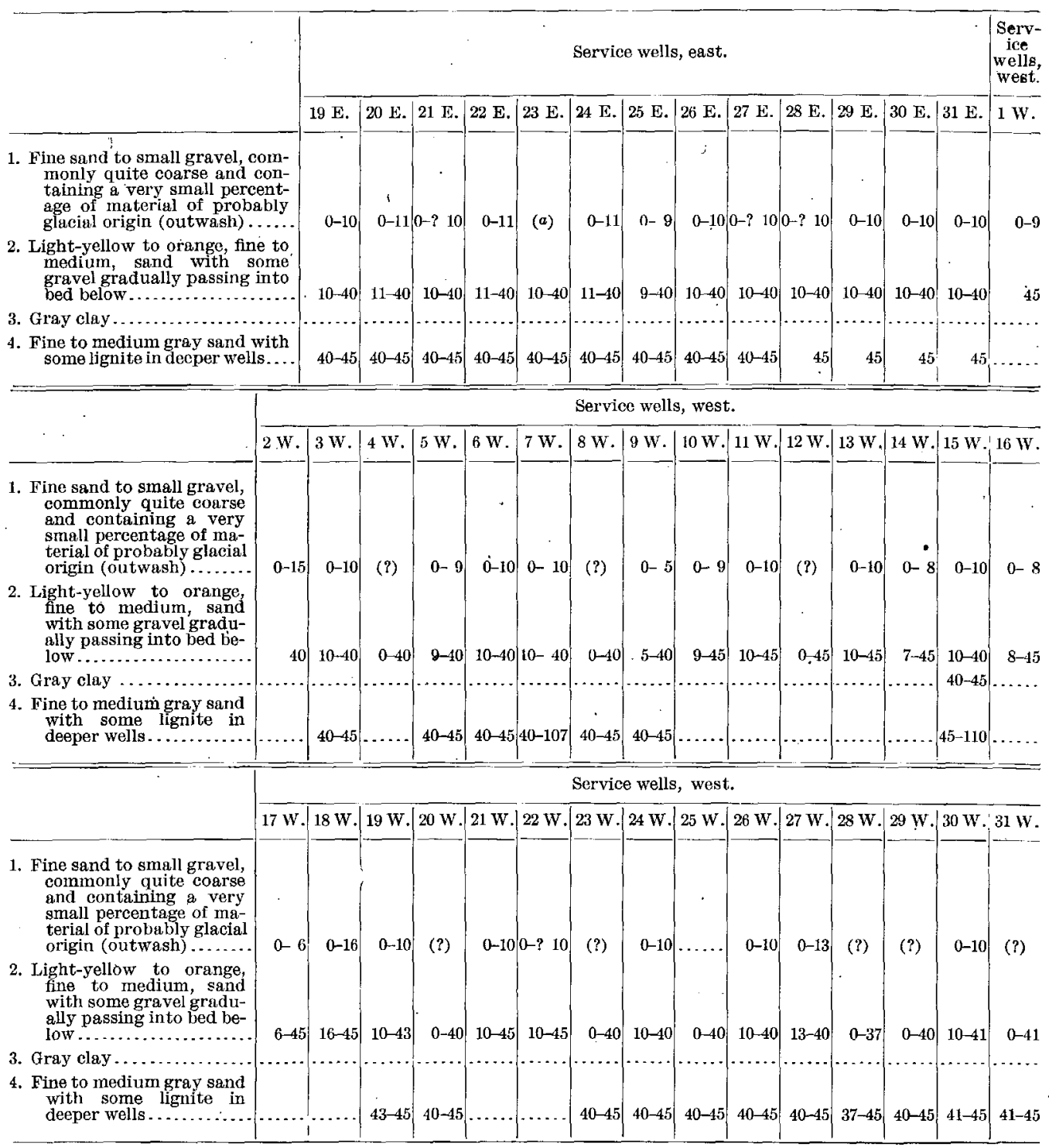

$a$ Very fine white silt with gravel of very doubtfui origin. 
The elevation of a number of the wells is given below:

Elevation of wells at Merrick pumping station.

7 E.

Feet.

$15 \mathrm{E}$

13. 5

$7 \mathrm{~W}$

$15 \mathrm{~W}$

During the month of June, 1900, when the station was not in use, the average height of the water in the decp wells was 9.01 feet above the Brooklyn base; in the shallow wells, 7.11 feet. In August, 1900, after pumping had begun, the average height of the water in the deep wells was 3.98 feet above, and in the shallow wells 2.32 feet below, the Brooklyn base.

490. The plant of the Merrick Water Company consists of 8 or 10 shallow wells pumped by a 16 -foot windmill; the water is discharged into a number of tanks and is distributed by a 3-inch pipe to the adjacent cottages.

491. According to Ward, the plant at this station consists of forty-six $4 \frac{1}{2}$-inch wells, 38 to 97 feet deep. When not pumped all of the deep wells will flow at a height of 11.11 feet above the Brooklyn base. The following summarized section has been prepared from the samples preserved by the Brooklyn water department:

Record of wells at Matowa pumping station.

\begin{tabular}{|c|c|c|c|c|c|c|c|c|c|c|c|c|c|}
\hline & $1 \mathrm{~s}$. & $2 \mathrm{~s}$. & $3 \mathbf{s}$ & $4 \mathrm{~S}$. & $5 \mathrm{~S}$. & $6 \mathrm{~S}$. & $7 \mathrm{~S}$. & $8 \mathrm{~S}$. & $9 \mathrm{~S}$. & $10 \mathrm{~S}$. & $11 \mathrm{~s}$. & $12 \mathrm{~S}$. & $1 \mathrm{~W}$. \\
\hline \multirow{5}{*}{ 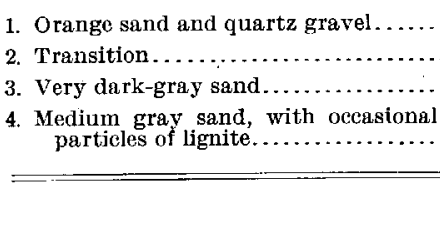 } & $0-35$ & 035 & $0-35$ & $0-35$ & $0-35$ & $0-35$ & $0-35$ & $0-35$ & $0-31$ & $0-35$ & $0-35$ & $0-30$ & $0-35$ \\
\hline & $35-40$ & $35-45$ & & $35-44$ & $35-44$ & & $35-44$ & & $31-38$ & & ...... & & $\ldots$. \\
\hline & $40-50$ & $45-50$ & & $44-55$ & & & $44-50$ & & & $35-38$ & $35-38$ & $30-38$ & $35-36$ \\
\hline & $50-100$ & 5098 & & $55-100$ & $44-99$ & & $50-97$ & $55-104$ & & & & & \\
\hline & $2-3 \mathrm{~W}$. & $\stackrel{5-6}{W}$ & $11 \mathrm{~W}$. & $14 \mathrm{~W}$. & $18 \mathrm{~W}$. & $1 \mathrm{E}$. & $2 \mathrm{E}$. & $3 \mathrm{E}$. & $4 \mathrm{E}$. & $\begin{array}{l}5-7 \\
\mathrm{E} .\end{array}$ & $8 \mathrm{E}$. & $9 \mathrm{E}$. & $\begin{array}{c}10-19 \\
\mathrm{E} .\end{array}$ \\
\hline 1. Orange sand and quartz gravel.. & $0-30$ & $0-30$ & & $0-30$ & $0-30$ & $0-35$ & $0-36$ & $0-35$ & $0-40$ & $0-40$ & $0-30$ & $0-35$ & $0-35$ \\
\hline 2. Transition . . . . . . . . . . . . . & $30-35$ & $30-35$ & & & & $35-38$ & $36-39$ & $35-40$ & & & $30-40$ & & $\ldots .$. \\
\hline 3. Very dark-gray sand ............... & & $\{\cdots \cdots$ & & $30-50$ & $\ldots$ & & $\ldots$ & & & & & & $\ldots$ \\
\hline $\begin{array}{l}\text { 4. Medium gray sand, with occasional } \\
\text { particles of lignite................... }\end{array}$ & $35-98$ & & & $50-73$ & & & & & & & & $35-40$ & $35-40$ \\
\hline
\end{tabular}

492. Record of commission's test well at Matowa pumping station.

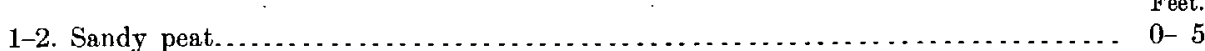

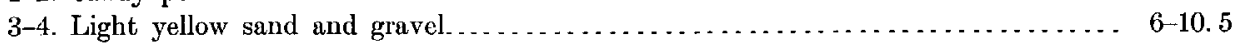

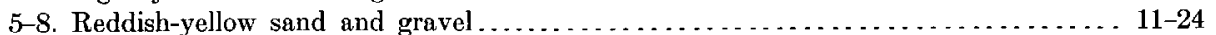

The whole section is probably of glacial origin, although the lower samples show a very small percentage of glacial material. 


\section{UNDERGROUND WATER RESOURCES OF LONG ISLAND, NEW YORK.}

493. According to Ward, the plant at this station consists of forty-three $4 \frac{1}{2}$-inch wells, 24 to 89 feet deep, and six 6 -inch wells 92 feet deep. The shallow wells do not flow, but the deeper wells do when the station is not in operation. The following section has been prepared from the samples preserved by the Brooklyn water department:

Record of wells at Wantagh pumping station, New York.

\begin{tabular}{|c|c|c|c|c|c|c|c|c|}
\hline & $12 \mathrm{E}$. & \multicolumn{2}{|c|}{$14 \mathrm{E}$. } & $16 \mathrm{E}$. & $18 \mathrm{E}$. & $20 \mathbf{E}$ & $14 \mathrm{~W}$. & $16 \mathrm{~W}$. \\
\hline 1. Grayish yellow sands and gravels, in part glacial. & $a 0-25$ & \multicolumn{2}{|c|}{$a 0-25$} & $a 0-30$ & $a 0-25$ & $6^{40-25}$ & $0-20$ & $0-25$ \\
\hline $\begin{array}{l}\text { 2. Fine dark-gray sands, occasionally showing yel- } \\
\text { low sands, possibly from the bed above....... }\end{array}$ & $25-49$ & \multicolumn{2}{|c|}{$25-40$} & $30-40$ & $25-40$ & $25-40$ & $20-40$ & $25-46$ \\
\hline $\begin{array}{l}\text { 3. Dark-gray clay or silty clay; containing a few } \\
\text { quartz pebbles in } \mathrm{S} \text { series of wells.................... }\end{array}$ & $b 49-50$ & \multicolumn{2}{|c|}{$40-50$} & $b 40-50$ & b $40-50$ & $40-50$ & $40-50$ & $46-50$ \\
\hline $\begin{array}{l}\text { 4. Fine gray sands, occasionally yellow or reddish } \\
\text { yeliow } \ldots \ldots \ldots \ldots \ldots \ldots \ldots \ldots \ldots \ldots \ldots\end{array}$ & $50-91$ & \multicolumn{2}{|c|}{$50-100$} & $50-70$ & $50-65$ & $50-65$ & $50-66$ & \multirow[t]{2}{*}{$50-75$} \\
\hline \multirow{3}{*}{ 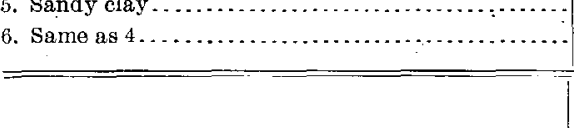 } & & & & & & & $66-70$ & \\
\hline & & & & & 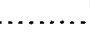 & & $70-90$ & \\
\hline & $18 \mathrm{~W}$. & $20 \mathrm{~W}$. & $22 \mathrm{~W}$. & $1 \mathrm{~s}$. & $2 \mathrm{~S}$. & $3 \mathrm{~s}$. & $4 \mathrm{~S}$ & $5 \mathrm{~S}$. \\
\hline I. Grayish yellow sands and gravels, in part glacial. & $0-20$ & $0-20$ & $0-20$ & $0-20$ & $0-20$ & $0-20$ & $0-25$ & $0-20$ \\
\hline $\begin{array}{l}\text { 2. Fine dark-gray sands, occasjonally showing yel- } \\
\text { low sands, possibly from the bed above ........ }\end{array}$ & $20-40$ & $20-41$ & $20-50$ & $20-40$ & $20-40$ & $20-40$ & $25-55$ & $20-45$ \\
\hline $\begin{array}{l}\text { 3. Dark-gray clay or silty clay; containing a few } \\
\text { quart } \% \text { pebbles in } \mathrm{S} \text { series of wells.............. }\end{array}$ & $40-44$ & $b 41-45$ & $c 50-55$ & $d 40-56$ & $d 40-56$ & $d 40-56$ & $55-60$ & $45-55$ \\
\hline $\begin{array}{l}\text { 4. Fine gray sands, occasionally yellow or reddish } \\
\text { yellow } \ldots \ldots \ldots \ldots \ldots \ldots \ldots \ldots \ldots \ldots \ldots \ldots \ldots \ldots\end{array}$ & $44-85$ & $45-85$ & $55-90$ & $56-90$ & $56-91$ & $55-90$ & c $60-70$ & $55-85$ \\
\hline 5. Sandy clay $\ldots \ldots \ldots \ldots \ldots \ldots \ldots \ldots \ldots \ldots \ldots \ldots \ldots$ & & & & & & & $70-75$ & \\
\hline 6. Same as $4 \ldots \ldots$....... & & & & & & & $75-90$ & \\
\hline
\end{tabular}

The deep test well No. 2 at this station has an elevation of 7.69 feet, and furnishes flowing water when the station is not in operation. In September, 1900, when the station was actively pumping, the arerage height of the water in the decp wells was 1.8 feet above the Brooklyn base, while in the shallow wells it was 2.8 feet below the Brooklyn base.

494.

Record of commission's test well at Wantagh pumping station.

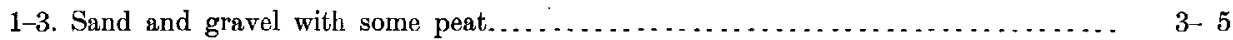

4-6. Reddish-brown fine to coarse sand ... . .

495. According to the report of the commission's inspector this well began to flow at 62 to 63 feet.

Record of commission's test well at Wantagh pumping station.

6-8. Very light-yellow quartz gravel, with very few, if any, erratics............ 16-30

9. Very fine, dark-gray, micaceous sand.................................. $30-31$

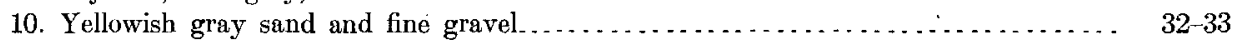

11-13. Very fine, dark-gray, micaceous sand......................... $34-46$

14. Blue clay, with quartz pebbles.................................. $48-60$

15-18. Very fine, dark-gray, micaceous sand, with lignite.................. $63-71$

Sec Table XIII.

496. From the upper part of this well no samples were reccived, but Prof. C. S. Slichter has furnished the following data: "Clay was encountered at a depth of 44 feet. At 62 feet an artesian head of about 32 inches was developed." 
Record of commission's test well near Wantagh pumping station.

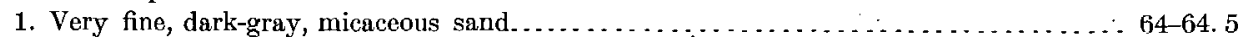

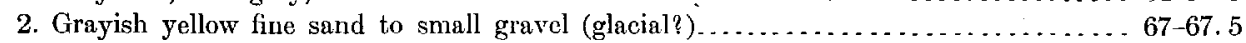

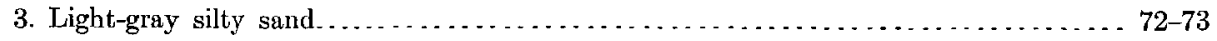

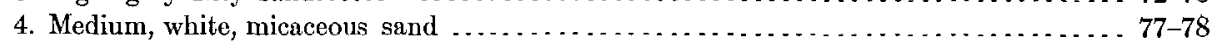

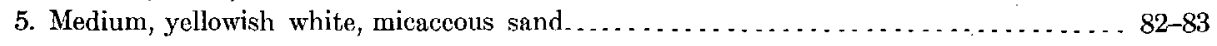

497. Record of commission's test well near Wantagh pumping station. Feet.

1. Humus-stained loamy sand. . . . . . . . . . . . . . . . . . . . .

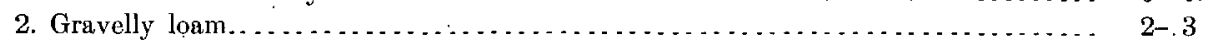

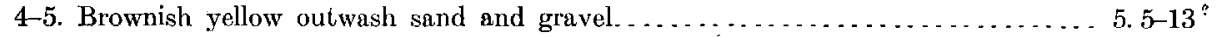

498: 'Recard of commission's test well near Camp $\dot{M}$ eeting grounds.

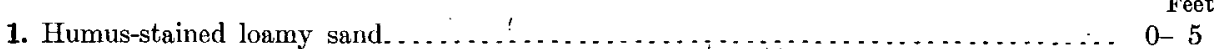

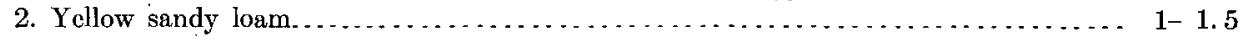

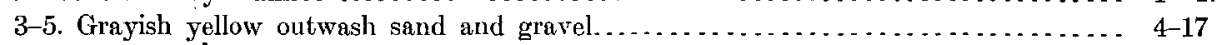

499 .

Record of commission's test well near Smithville south.

1. Humus-stained loam.

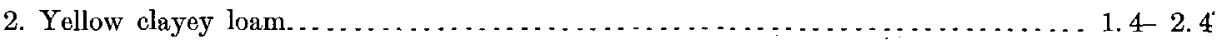

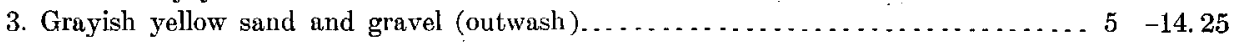

500. This was one of the wells put down at Camp Black during the Spanish-American war; its exact ocation was not learned.

Record of United States Army well on Hempstead Plains.

1. Top soil

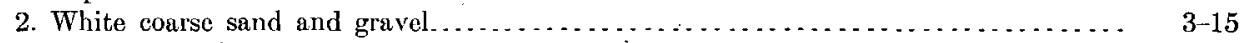

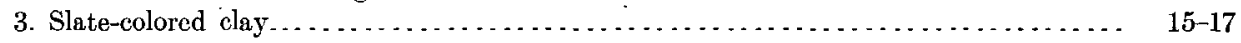

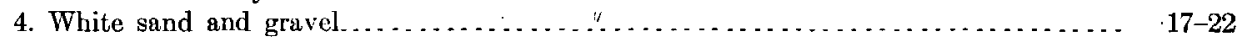

501.

Record of commission's test well near Hicksville.

Wisconsin and Tisbury:

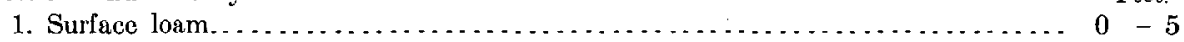

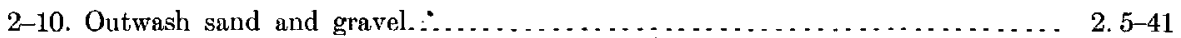

11. Fine to coarse yellowish sand with small particles of glacial material $\ldots \ldots \ldots 45-46$

Cretaceous?:

12-13. Fine light-yellow sand with considerable mica (probably not glacial); suggests the older sands exposed in the Melville section. . . . . . . . . . . . . . . $50 \quad-56$ See Table XII.

502.

Record of commission's test well near Hicksville.

Wisconsin:

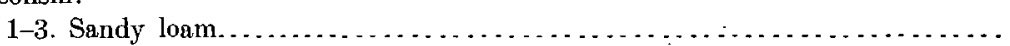

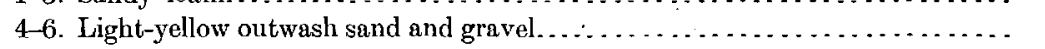

Tisbury:

7-8. Sand and gravel with a considerable percentage of black silt; looks very much like an old land surface (no glacial pebbles) ............... $15-22$

9-15. Very light, yellowish white, fine sand to small gravel, containing a very small percentage of glacial pebbles........................ $25-57$ 
Cretaceous?:

16. Very fine yellowish white sand, with a little lignite.

Feet.

17-25. Light, yellowish white, speckled, fine sand and small gravel; gravel is white quartz, with occasional particles of ferruginous sandstone; no pebbles of recognizable glacial material.

Cretaceous:

26-32. Uniform, light-yellow to white, micaceous sand..

33. Fine sand to small gravel, containing a considerable number of small, ferruginous, sandstone fragments, which give sample a speckled appearance. 132. 5-135.5

See Table XII. section:

503. Samples preserved in the office of Mr. Osear Darling, consulting engineer, show the following Record of Nassau County Water Company's well near Hicksville.

Wisconsin and Tisbury:

1. Glacial sand and gravel.

The well plant consists of two 8-inch wells placed in the bottom of a pit 50 fect deep, in which the direct suction pump is also placed. An Acme system is used having a storage capacity of 25,000 gallons.

505. Record of well of H.J. Heinz Company near Hicksville.

Wisconsin and Tisbury:

1. Sand and gravel

506.

Record of commission's test well near Hicksville.

Wisconsin and Tisbury:

1. Light-yellow surface loam........................

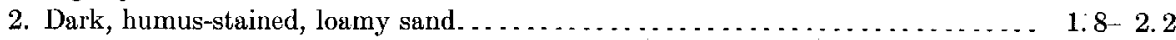

3-13. Light-colored outwash sand and gravel........................ $3 \quad-53$

Cretaceous:

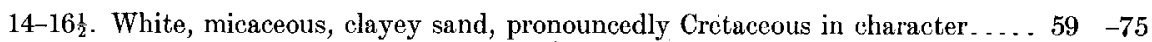

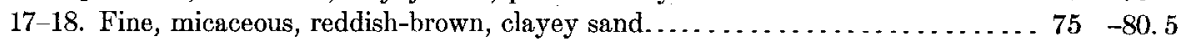
See Table XII.

\section{0\%.} Record of Jos. Steinart's well near Hicksville. '

Wisconsin and Tisbury:

1. Gravel.

Cretaceous:

2. Gravel with lignite and white clay, water bearing; water would not clear....... 75-120

3. Very black clay.

4. Gray sand with abundant supply of water...................... $130-150$

508. Mr. F. K. Walsh reports the following section:

Record of St. John's Protectory well near Hicksville.

Wisconsin and Tisbury:

1. A very compat sand with no gravel.

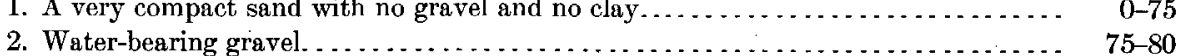

509. Record of well of Colored Children's Home near Westbury.

Wisconsin:

Feet.

1. Sharp dirty-white sand.

$0-20$

Cretaceous?:

2. Mixture of gray quicksand and clay. 
511. The following section has been prepared from the record and samples furnished by Mr. George $\mathrm{H}$. Pease, foreman:

Wisconsin: $\quad$ Record of W. P. Thompson's well near Old Westbury.

Mannetto:

2. Yellow quartz sand and gravel (no glacial material)................. $23-56$

Cretaccous? :

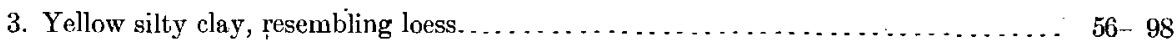
Cretaceous:

4. Fine to coarse yellow sand. . . . . . . . . . . . . . . . . . . . . . . . . . $98-108$

5. Very coarse light-yellow sand, with some gravel; slightly water bearing. . . . . . . $108-128$

6. Fine sand . . . . . . . . . . . . . . . . . . . . . . . . . . . . . . . . . 128 -131

7. Fine light-yellow sand; slightly water bearing. . . . . . . . . . . . . . . . . . . 131-144

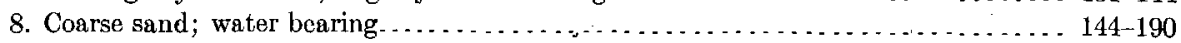

9. Coarse light-yellow sand and gravel, becoming finer below . . . . . . . . . . . . . . . 190-209

Strainer was placed between 195 and 205 feet. The well tested about 60 gallons per minute. Test was made on two consecutive days, and each test was continued ten hours.

512.

Record of $J$ H. Harriman's well in Wheatley Hills.

Wisconsin and Mannetto:

1. Loam and bowlders (some yellow gravel) ......................... 0 . 70

Cretaceous? :

2. Clay, with very little grit and no gravel (yellow, almost a loam, resembling loess

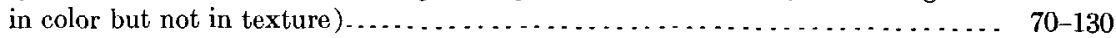
Cretaceous:

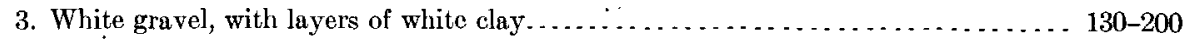

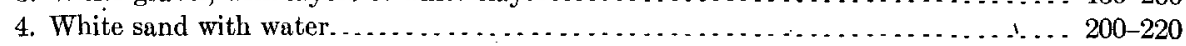

513.

Record of commission's test well near Jericho.

Pleistocene: Feet.

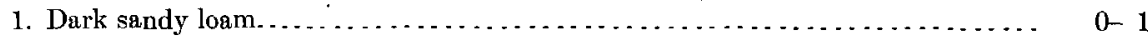

2. Yellow clayey sand.................................... 4 -5

3-6. Yellowish-brown fine to coarse glacial sand..................... 8 -18

7. Yellowish-white coarse to fine gravel (doubtfully glacial) .............. 18,5-19.5

8. Fine to medium yellowish-brown sand. . . . . . . . . . . . . . . . . . . . 23 . 23.5

9-10. Yellowish-white medium to coarse sand..................... 28.5-35. 5

11. Fine reddish-brown sand with considerable muscovite................. $39-40$

12-13. Medium to coarse yellowish-white sand with some biotite........... $44 \quad-50$

Cretaceous?:

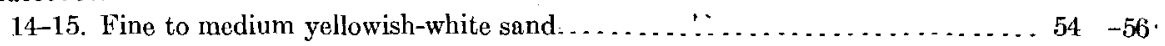

16. Very fine reddish-white sand. ........................... $59 \quad 60$

514.

Record of H.R. Winthrop's well near Jericho.

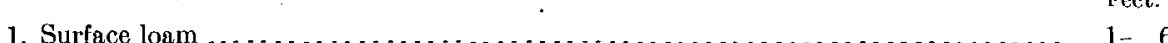

Pleistocene and Cretaceous:

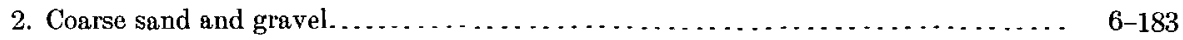

No change in the material from 6 to 183 feet. It was all of the same degree of fineness. Water was first encountered at 150 feet; down to that depth the material was almost perfectly dry. Four samples from the well, ranging from 171 feet 9 inches to 182 feet 10 inches, show very light-yellow sand and gravel, with no erratic material. It is therefore impossible to tell how much of this section is to be considered Pleistocene and how much pre-Pleistocene. 


\section{8}

UNDERGROUND WATER RESOURCES OF LONG ISLAND, NEW YORK.

515.

Record of T. Willis's well near Jericho.

Wisconsin:

1. Ordinary sand with an occasional bowlder (several blasts were necessary) ...... 0 - 50

Mannetto? and Cretaceous:

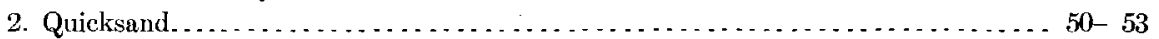

3. Red sand, with alternate layers of yellow and reddish-yellow gravels. . . . . . . . 53-175.5

See record and sample from well No. 514, which indicate that part of this gravel should be considered pre-Pleistocene.

516. The following record has been compiled from information furnished by Mr. John $\mathfrak{J}$. Hicks and Mr. William C. Jaegle:

Pleistocene: Record of Jacob Jackson's well near Jericho.

Cretaceous:

2. Black sticky clay, containing lignitized wood....................... $40-80$

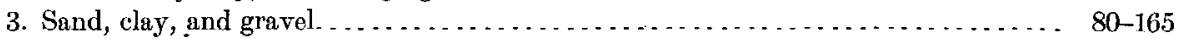

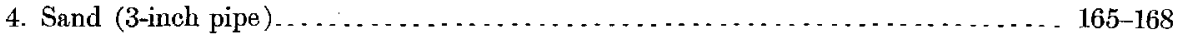

Mr. Hicks reports that this well was drilled from 165 to 210 feet by A. W. Gallienne. Mr. Jaegle, however, drove a new pipe in the same well to a depth of 3 feet and found good water, so the Gallienne well is to be discounted.

517. Samples from this well, together with a section drawn by W. Goold Levison December 28, 1881, are preserved in the museum of the Long Island Historical Society.

In the following section the record given on the left is from Mr. Levison's drawing, and that on the right is from the samples:

Records of Jules Kunz's well near Jericho.

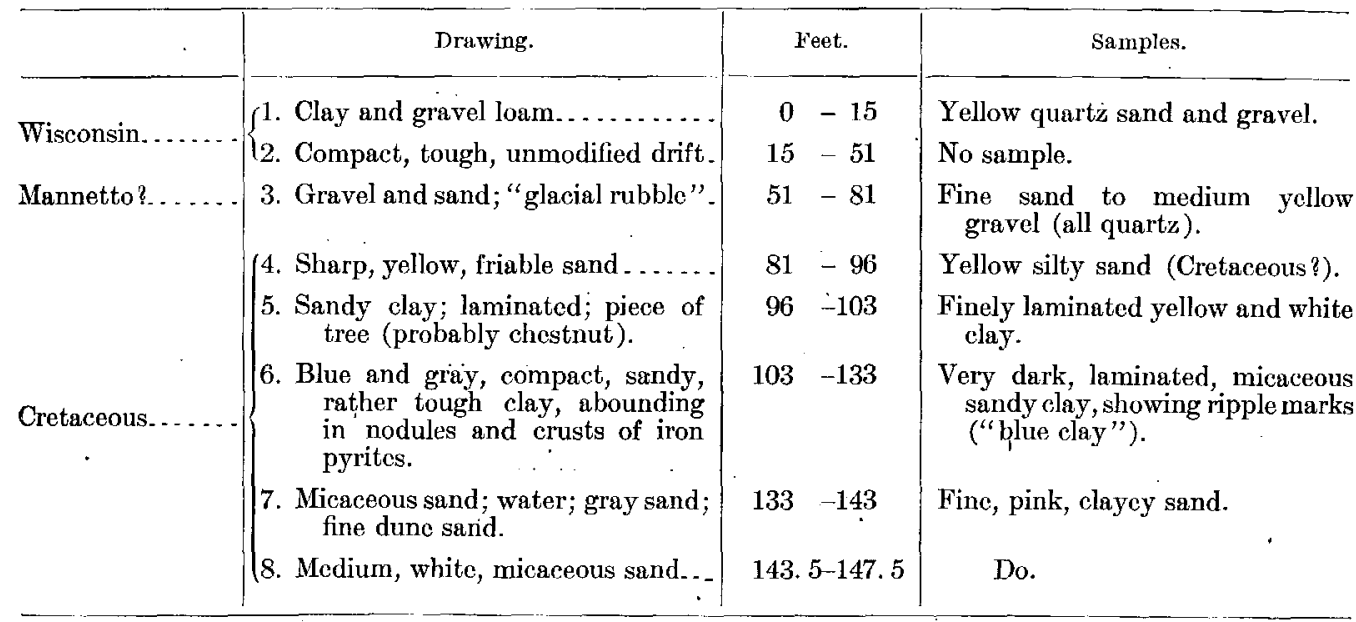

Merrill $a$ and Darton $b$ have both published records of this well in which an error has evidently been made in copying in the thickness of the yellow gravel, which extends from 51 to 81 feet.

518. This is a dug well from which the supply is now obtained from four 3 -inch strainers 12 feet long, placed horizontally in the water-bearing gravel just above the clay, and connected dircetly with the suction pipe from the pump. 
Pleistocene:

Record of Allard \& McGuire's well near Syosset.

1. Sand and gravel . Fect.

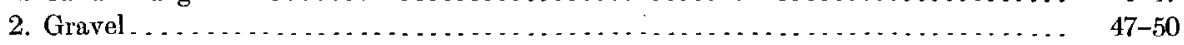

Cretaceous:

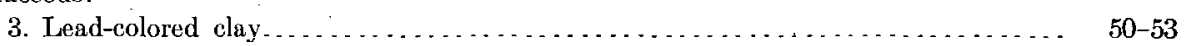

519. Mr. Jaegle states that in sinking this well he encountered, at a depth of 150 feet, a stratum of fine gravel, overlaid by gravelly elay, from which the air rushed with considerable force. This is probably a blowing well similar to those which have been described in many parts of the West (see p. 74).

520,

Record of county poor farm well near Brookville.

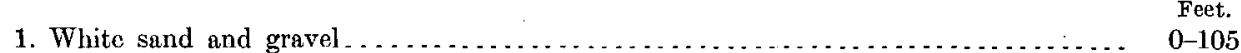

2. Quicksand; fine dark-colored sand with coarse material at bottom............ 105-278

521.

Recdrd of H. Rushmore's well near Brookville.

1. Surface loam and then ordinary sand...

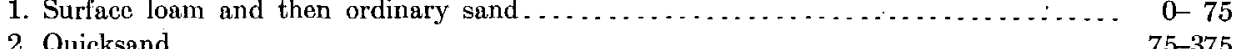

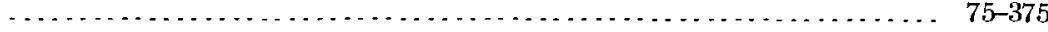

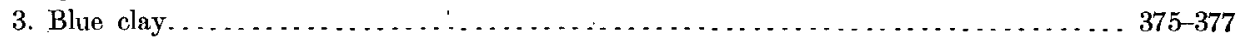

4. Hardpan (gravel and sand packed very hard) . . . . . . . . . . . . . . . . . 377-396

This record was reported by Mr. J. L. Bogart, who lives on the adjoining property and who was much interested in the well at the time it was sunk.

522.

Record of commission's well near East Nomich.

Wisconsin:

Record of commission's well near Last Nomich.

Dark surface loam and gravel ............... 1 - 5

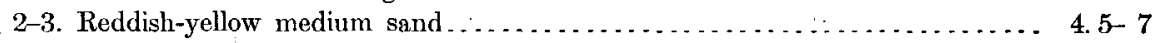

4. Ycllowish-gray clayey sand.............................. 12.5 5 .13.8

Wisconsin?:

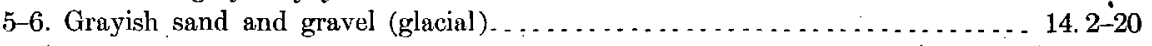

7. Light yellowish-white silt to coarse sand . . . . . . . . . . . . . . . . . 20 -23

523. Record of Quinan well near East Norwich.

Wisconsin and Tisbury: . Feet.

1. Very stony sand and gravel . . . . . . . . . . . .

Tisbury and Cretaceous:

2. Yellowish-red sand . . . . . . . . . . . . . . . . . . . . . . . . . . . . . . 100-120

Cretaceous:

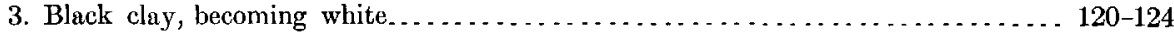

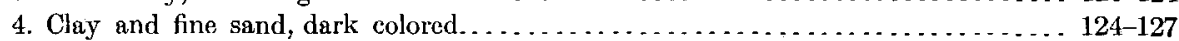

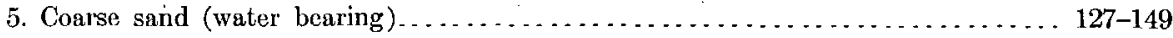

6. Clay . . . . . . . . . . . . . . . . . . . . . . . . . . . . . . . . . . . . 149

524.

Record of Ludlum well near East Nomwich.

1. Gravel ......

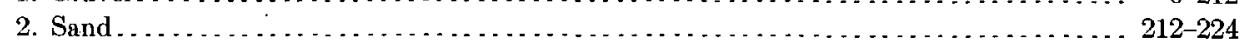

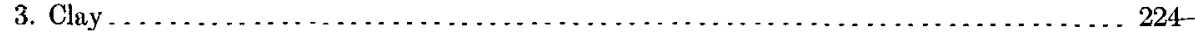

525. The first test well at this place, which was put down about 25 feet from the engine house, was unsuccessful. The samples preserved in the office of Mr. Oscar Darling, consulting engineer, show the following section:

Pleistocene:

$$
\text { Record of Nassau County Water Company's well at. Oyster Bay. }
$$

1. White sand and gravel . . . . . .

2. Coarse gravel. . . . . . . . . . . . . . .

3. Medium yellow' sand. . . . . . . . . . . . . . . 60

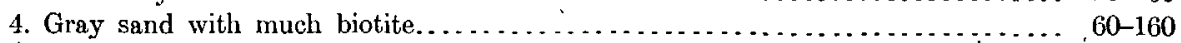




\section{UNDERGROUND WATER RESOUROES OF LONG ISLAND, NEW YORK.}

Water was found in abundance in the coarse gravel from 5 to 15 feet, but the sand below this point while water bearing, was regarded as too fine to furnish water for waterworks purposes. An attempt was then made to develop the stratum at 10 feet by a series of gang wells, but it was found to be only a small pocket. About 300 feet north of the pumping station, and down the valley, coarse water-bearing gravel was found at a depth of 10 feet which had a thickness of from 10 to 30 feet. It is expected that a gang of twelve 4-inch wells of an average depth of 35 feet will be put down at this point. The water from these wells stands just level with the surface, which is 23.5 feet above mean high tide.

526. This well was driven in 1900 . At a depth of 3 or 4 feet from the surface clay was encountered, below which there was gravel, and then clay to a depth of 50 feet, where water was encountered which flowed 8 to 9 gallons par minute. Below this was sand and gravel, which furnished a small flow of artesian water, to a depth of 160 feet, where a layer of clay 2 feet thick was encountered. At 62 feet a strong artesian head was encountered which forced the water 11 inches above the 3 -inch pipe, and furnished over 100 gallons per minute. As the water did not clear, it was driven through clay and sand to 165 feet, where it was stopped in sand and gravel. At this point it furnished about 80 gallons per minute of clear water. At 17 feet above the surface of the ground the well delivers 5 gallons per minute.

The following partial analysis was made by Prof. C. S. Slichter:

Analysis of water from Tounsend Underhill's well near Oyster Bay.

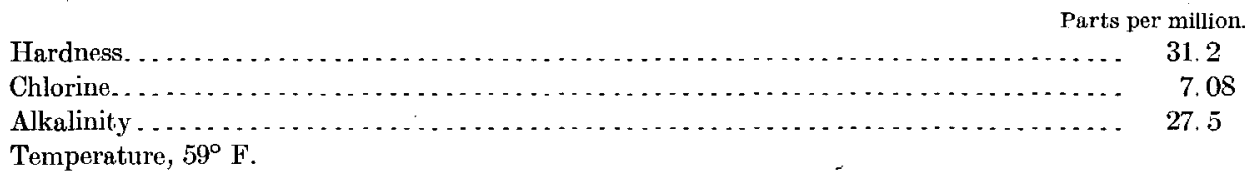

527. This well was driven in 1900 and now furnishes 15 gallons per minute at a height of 3 fect above the ground. The well is about 20 feet above mean sea level.

\section{Record of Charles Weeks's well near Oyster Bay.}

Wisconsin and Tisbury:

Feet.

$0-15$

1. Sand and gravel.

Sankaty?:

2. Clay

Jameco?:

3. Micaceous sand, gradually growing coarser.

528.

Record of J. M. Sammis's well near Oyster Bay.

Wisconsin and Tisbury:

Feet.

1. Sand and gravel with poor water.

$0-30$

Sankaty?:

2. Clay

$30-35$

Cretaccous?

3. Fine white sand with little water. . . . . . . . . . . . . . . . . . . . . . $35-140$

See fig. 16 .

529. Mr. E. K. Hutchinson, under date of April 29, 1896, gives the following data regarding this well:

Record of well of Van Sise \& Co. near Oyster Bay.

Wisconsin and Tisbury:

Feet.

1. Sand and gravel.

Sankaty?

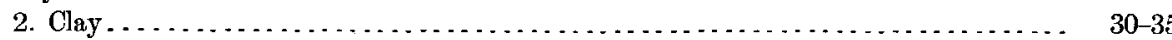

3. Clay and sand no water.......................................... 35-53

Jameco?:

4. Yollow sand and gravel. .................................. $53-57$

Flows 9 gallons per minute. 
The flow of this well was measured by W. H. C. Pynchon, April 11, 1903, and found to be 3 gallons per minute, at a height of 18 inches above the surface, or 10 to 12 feet above mean sea level (see fig. 16).

530. On April 27, 1903, Mr. Pynchon found the flow to be 5 gallons per minute from a reduced nozzle at 18 inches above the surface. He,reports that the water will rise $2 \frac{1}{2}$ feet above the surface.

Record of D. W. Smith's well at Oyster Bay.

Wisconsin and Tisbury:

Feet.

1. Sand and gravel.

$0-35$

Sankaty?:

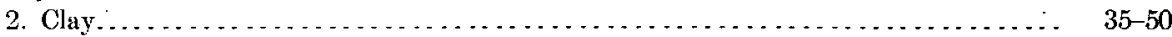

Jameco?:

3. Fine yellow sand, growing coarser............................... $50-65$

531. Mr. Hutchinson states that the original flow was 15 gallons per minute. On May 27, 1903, Mr. Pynchon found it to be 8.5 gallons. The water will rise about 6 feet above the surface of the ground.

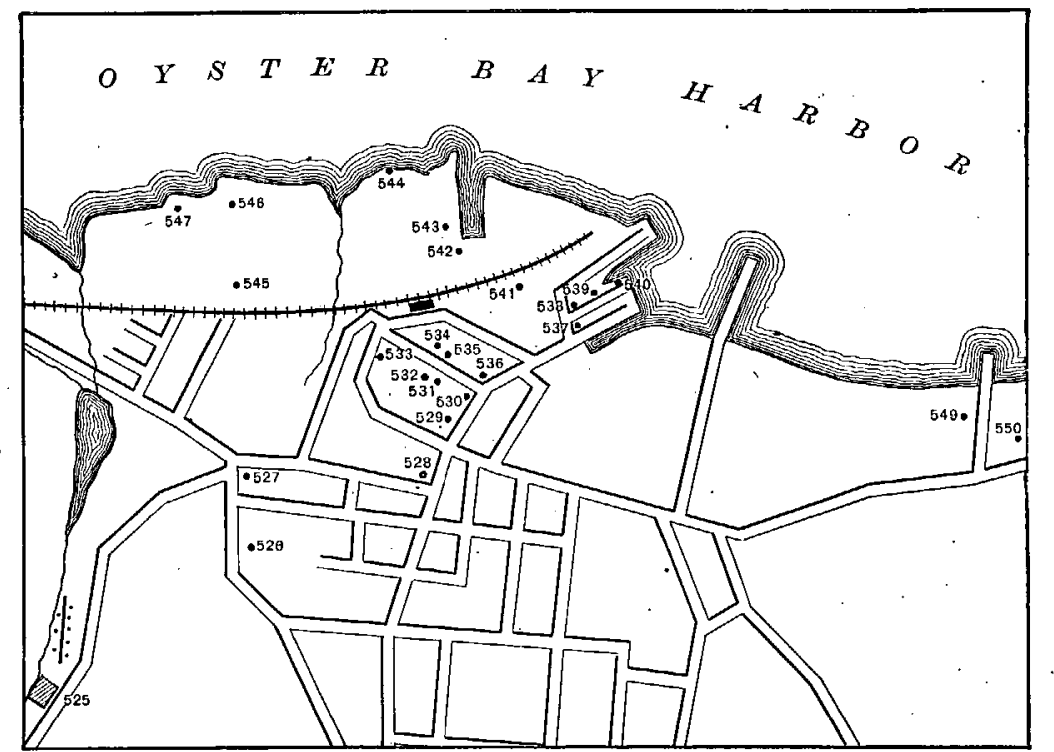

FIG. 67.-Sketch map showing locations of wells described at Oyster Bay.

532. The water-bearing gravel is reported to be unusually coarse in this well. When first completed, it flowed 21 gallons per minute.

Record of E.K. Hutchinson's well at Oyster Bay.

Wisconsin and Tisbury:

Feet.

1. Sand and gravel

$0-35$

Sankaty?:

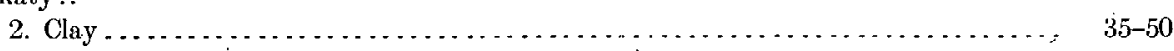

Jameco?:

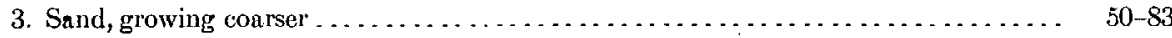

533. The clay layer usually encountered in this vicinity is reported as very thin in this well.

534. The original flow is reported as 10 gallons per minute. When masured by Mr. Pynchon April 27,1903 , it was 4 gallons per minute at a height of 2 feet above the surface.

535. The original flow was 9 to 10 gallons per minute. The flow April 27,1903 , was 2 gallons per minute at a height of 2 feet and 4 inches above the surface.

$17116-$ No. $44-06-19$ 


\section{UNDERGROUND WATER RESOURCES OF LONG ISLAND, NEW YORK.}

539. The following section of this well was furnished by Mr. E. K. Hutchinson in a letter dated $\Lambda$ pril 29, 1895:

Record of A.J. \& A. S. Hutchinson well at Oyster Bay.

Wisconsin and Tisbury: $\quad$ Feet.

1. Sand and gravel, with plenty of water of poor quality $\ldots \ldots \ldots \ldots \ldots \ldots \ldots+30$

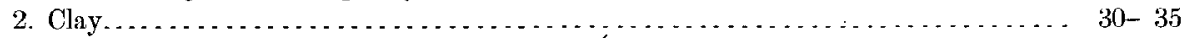

3. Sand and gravel; plenty of water raised 6 féet above level of first water. . . . . . 35-120 Sankàty?:

4. Clay; no water . . . . . . . . . . . . . . . . . . . . . . . . . . . . . . . . 120 185

Jameco?:

5. Yellow sand and gravel with artesian water....................... 185-190

The original flow from stratum 5 was about 70 gallons per minute at 3 feet above the ground. The water will rise to a point $\mathbf{1 7}$ feet above the surface at low tide and will overflow at high tide. The surface is 2 to 3 feet above mean high tide.

542. Mr. W. H. C. Pynchon reports the following history of this well:

"First position: Driven to a depth of 106 feet through sand and gravel with water all the way for 80 feet, then clay to 105 feet. It was left on Saturday night with water just dripping from the well pipe which stood 2 feet above ground. The flow kept on increasing until at the end of eight days it was flowing 50 gallons a minute from 2-inch pipe, about as much sand as water. It ran so for one week and then began to fall off, until at the end of one week more it was not running at all. 'Second position: It was then driven to 130 feet, but no flow Pipe was pulled up and its lower 6 feet perforated and covered with 40-mesh wire gauze. Third position: The pipe was then reinserted in the hole to a depth of about 125 feet, with the result that the water came up on the outside of the pipe instead of the inside so that carth had to be ranımed in all around the pipe. It then flowed 18 gallons per minute at 3 feet above the surface, though the water will rise to a level of about 9 feet. The wellhead is now 3.50 feet above high tide." (For general relations see fig. 16.)

543. The flow at low tide, June 30,1903 , was 26.5 gallons per minute.

Record of Dr. O. L. Jones's well at Oyster Bay.

\begin{tabular}{|c|c|}
\hline $\begin{array}{l}\text { Wisconsin and Tisbury: } \\
\text { 1. Gravel.......... }\end{array}$ & $\begin{array}{l}\text { Feet. } \\
0-60\end{array}$ \\
\hline Sankaty?: & \\
\hline 2. Clay... & $60-135$ \\
\hline Jameco?: & \\
\hline 3. Little flow at $\ldots$ & $135-140$ \\
\hline
\end{tabular}

Cretaceous:

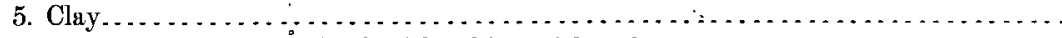

6. Very heavy gravel mixed with white sticky clay $\ldots \ldots \ldots \ldots \ldots \ldots \ldots \ldots \ldots \ldots$

Prof.' C. S. Slichter has made the following partial analysis of this water:

Analysis of water from Dr. O. L. Jones's well at Oyster Bay.

Iardness . . . . . . . . . . . . .

Chlorine ... . . . . . . . . . . 4.25

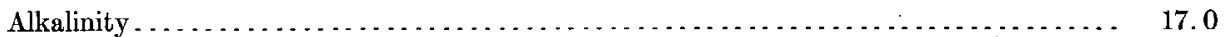

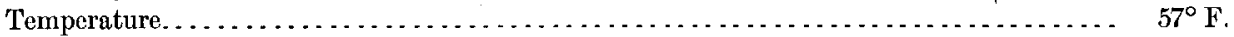

544. "Driven in July, 1896 . It is located on the beach at the edge of the salt marsh, and the tide rises ordinarily about 1 foot over the wellhead, i. e., well is about 6 feet above low-tide mark. At low tide the flow is not over 20 gallons per minute, but just before the tide goes over the wellhead it flows 100 gallons a minute. Water comes from gray and black sand, but is free from iron."

This well was sounded in connection with observations on the effect of the tide on the rate of flow and found to be 93.1 feet deep. (For general relations see fig. 16.) 
The sample of water, marked Mohannes Spring, Oyster Bay, Long Island, submitted to me for examination contains:

$$
\text { Analysis of water from Mohannes Casino well at Oyster Bay. }
$$

Sediment. . . . . . . N .

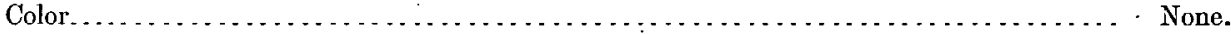

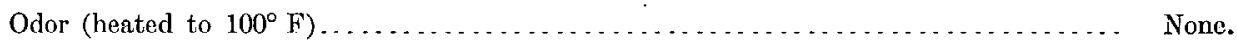

Chlorine in chlorides. . . . . . . . . . .

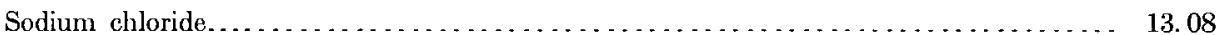

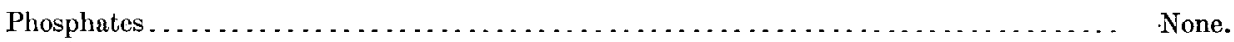

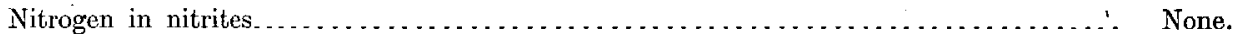

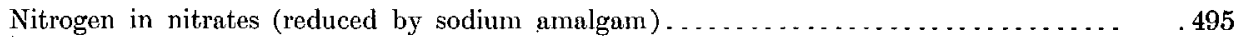

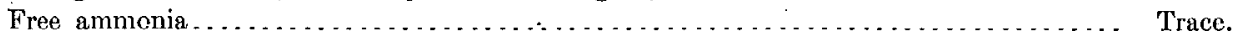

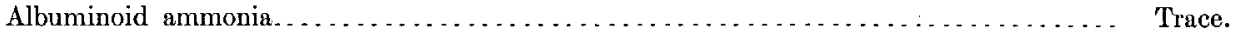

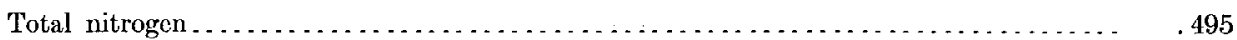

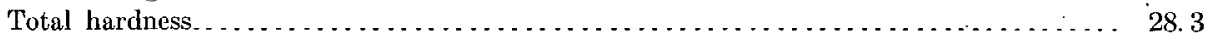

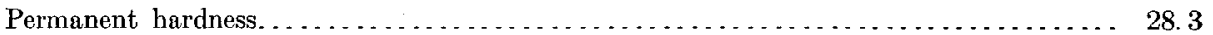

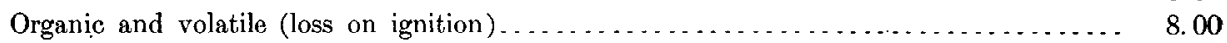

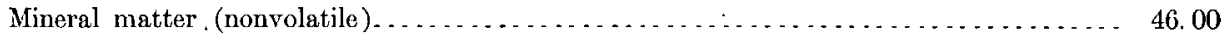

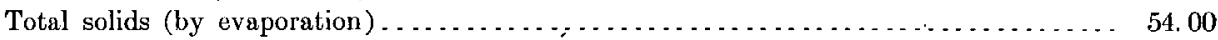

This sample of water is of great organic purity; it is very soft and is admirably adapted for use as a drinking water as well as for domestic purposes.--Ernst J. Lederle, Ph. D.

The "Mohannes Spring" is the 99-foot artesian well described in the table of wells.

545. The water is so strongly impregnated with iron that it is unfit to drink. Yicld 2 feet above the ground, 18 gallons per minute, at high tide.

Recent to Tisbury:

Record of T: Underhill's well at Oyster Bay.

Feet.

Sankaty:

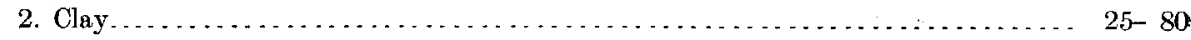

Jameco:

3. Fine gray and black sand, growing coarser..................... 80-107

Total depth according to sounding, 114 feet.

Analysis of water from T. Underhill's well at Oyster Bay.

[By Prof. C. S. Slichter.]

Parts per million.

Hardness

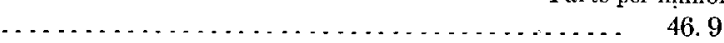

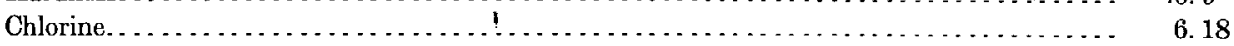

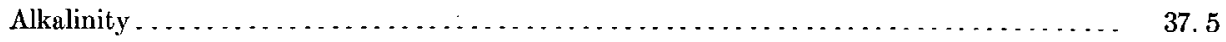

Temperature . . . . . . . .

546. Record of Lee well at Oyster Bay.

1. Heavy sand and gravel ........

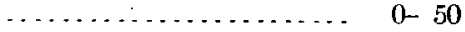

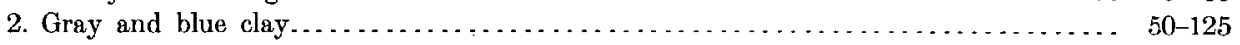

3. Beach sand, growing coarser, well commenced to flow at 160 feet.......... 130-200

Depth by sounding, 188.3 feet.

Analysis of water from Lee well at Oyster Bay.

[By Prof. C. S. Slichter.]

Parts per million.

Hardness. . . . . . . . . . . . . .

Chlorine. . . . . . . . .

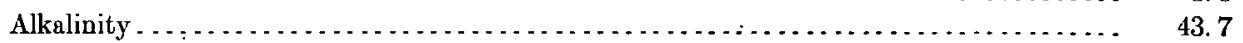

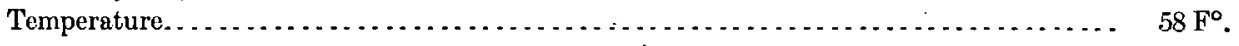




\section{UNDERGROUND WATER RESOURCES OF LONG ISLAND, NEW YORK.}

547.

Analysis of water from Burgess well at Oyster Bay.

[By Prof. C. S. Slichter.]

Purts per million.

Hardness.

Chlorine-..74

Temperature. . . . . . . . .

See Pl. XIII, $A$.

548.

Record of Hamilton well near Oyster Bay.

Wisconsin and Tisbury:

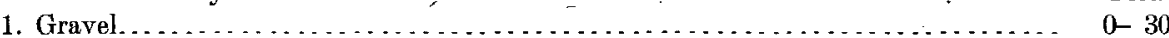

Sankaty?

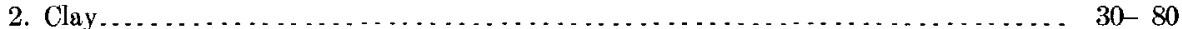

Jameco?:

3. Sand with water, not artesian................................. ${ }^{80-130}$

Cretaceous:

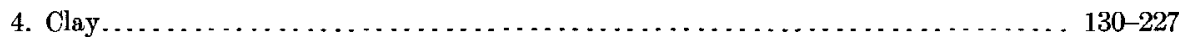

$\Lambda$ second well was drilled near this one and a good flow obtained at 105 feet.

549.

Record of William Trotter's well near Oyster Bay.

Wisconsin and Tisbury:

1. Gravel.

Sankaty?:

2. Cla

$10-70$

Jameco?:

3. Gravel, with artesian water.

$70-90$

Analysis of water from William Trotter's well near Oyster Bay.

[By Prof. C. S. Slichter.]

Parts per million.

Hardness.

21.9

Chlorine.

6.2

Alkalinity.

21.0

Temperature.

$56^{\circ} \mathrm{F}$.

551. Water is reported for the whole depth of the well, but did not flow until a depth of 259 feet was reached

Record of $H$. Dollard's well near Oyster Bay.

1. Surface sandc and gravel with some water.

2. Fine dark-colored brown sand, becoming coarser at the bottom and passing into a leadcolored gravel

Analysis of water from II. Dollard's well near Oyster Bay.

[By Prof. C. S. Slichter.]

Parts per million.

Hardness. 33. 7

Chlorine. . .

6.02

Alkalinity

19.95

Temperature.

552. The following section is from Mr. Ed. Schmidt:

Record of Edward Swan's well near Oyster Bay.

1. Coarse sand, slightly yellow in color, with occasional layer of gravel 
553. This well was very easily drilled. The material became coarser and coarser until at 465 feet an excellent flow was obtained. There was no red clay here and no hard red stratum. A little blue clay was found at 150 feet.

$$
\text { Record of E. Roosevelt's well near Oyster Bäy. }
$$

Wisconsin and Tisbury: Feet.

1. Sand and gravel, water bearing. . . . . . . . . . . . . . . . . . . . .

Cretáceous:

2. Brown sandy clay, grading into gray sandy clay.

$100-465$

554. For partial analysis see page 68 . (See fig. 16 for general relations.)

555.

Record of G. M. Fletcher's well on Center Island, New York.

Pleistocene and Cretaceous?:

1. Sand, with an occasional stratum of clay

Feet.

$0-160$

2. Alternate layers of yellow, black, red, blue (hard like flint), and milky-white clay............................................. 1600 -316

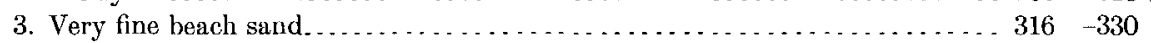

4. Thin stratum of brown shale............................... $330 \quad-330.4$

5. Coarse sand (with some water) . . . . . . . . . . . . . . . . . . . . . . 330.4-360

6. Thin stratum of brown shale . . . . . . . . . . . . . . . . . . . . . . . $360 \quad-360.2$

7 . Very fine sand, gradually growing coarser . . . . . . . . . . . . . . . . . . . . . . . 360. 2-370. 10

Elevation above tide 12 feet.

At the last depth given the particles ranged from onc-eighth to one-half inch in diameter. A concretion was encountered at 230 feet, and lignite at 330,350 , and 370 feet.

Mr. Frank Nichols, foreman in charge of the drilling of this well, reports that salt water was encountered at 18 feet and again at 100 feet. Fresh water was first encountered at 360 feet.

556. For partial analysis see page 68 .

557. Nichols states that the natural pressure is "lower" in this well than in the others, and this, together with the fact that salt water was used in drilling the well, necessitated long pumping before the water became fresh. The clay contains a great deal of sand and is very micaceous.

The low pressure is probably due to the fact that the main artesian gravel was not reached. It will be noticed on Pl. II that this well lacks 50 feet of reaching the coarse Lloyd gravel, in which the other wells are finished.

Pleistocene:

Record of S.T. Shaw's well on Center Island, New York.

Pleistoane and Cretaceous:

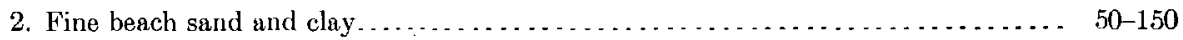

Cretaceous:

3. White, blue, and gray clay; red clay and sand and gray clay; encountered in the order named ........................................... 150-295

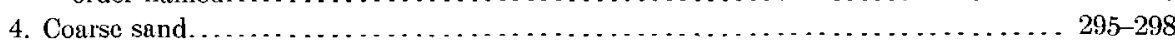

558. Mr. R. F. Nichols, foreman in charge of the drilling of this well, reports the following section: Record of C. Hoyt's well on Center Island, New York.

Pleistocene:

1. Very coarse gravel, coarse as black walnuts..................... 0 . 60

Cretaceous:

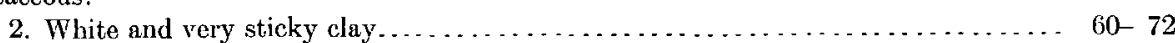

3. White beach sand . . . . . . . . . . . . . . . . . . . . . .

4. "Very pretty blue" clay................................... 90-130

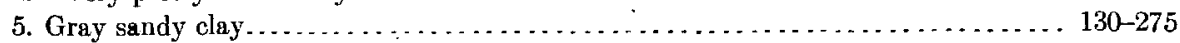


Cretaceous:

6. Very hard stratum, brownish red in color, were two days in drilling 8 inches; described as very similar to hard stratum reported in Fleteher well (No. 555) . . 275-

7. Gray sandy clay....

8. $\Lambda$ second hard stratum

9. Sand, becoming coarser and passing into white gravel-like peas. . . . . . . . . . . . . . 300-321

Well began to flow at 300 feet. Elevation above mean high tide, 4 feet.

559. Mr. R. F. Nichols, foreman, reports that this well began to flow at night. The screen was put in and the well was left at the depth to which it had been sunk.

Pleistocene:

$$
\text { Record of } C . W . \text { Wetmore's well on Center Island, New York. }
$$

Cretaceous:

2. Clay, no bowlders. . . . . . . . . . . . . . . . . . . . . . . . . . . . . 60 . 300

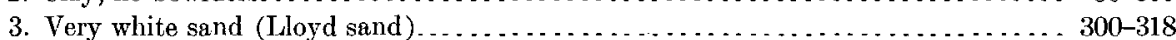

Elevation above mean high tide, 3 feet.

For partial analysis, see page 68 .

560. The material encountered in this well is very similar to that found in No. 558. Below 150 feet considerable lignite was found.

562. Mr. A. Neilson, superintendent of the Pierce estate, reports the following: "The writer was not managing the property when the well was put down, and so can not give record of strata. There was originally an old open well 30 fect deep, which was a good one, but to get more water a 6-inch pipe was put down 10 feet below the bottom of the open well. This well is about 600 feet back from the shore of the sound, and the top is about 30 feet above high water. 'The tides do not change the water in any way. About 150 fect from the one described there is another well about 80 feet deep, all 6 -inch pipe, which I believe is a better well, though it has never been tested to its full capacity."

Mr. Frank. Wankel, now foreman of the Hudson Engineering and Contracting Company, reports that a number of years ago he sunk a 6 -inch well for Colonel Kruger, and it may be that this is the woll referred to in the above lettcr. Mr. Wankel gives the following data regarding it:

Wisconsin and Tisbury:

Record of Colonel Kruger's well near Bayville.

2. Coarse gravel.

At this depth a fine material was encountered and the driving was discontinued. No clay was encountered. The well is 200 feet from the water's edge, and 50 or 60 feet above sea level, and tests 15 gallons a minute at full capacity.

564. Mr. Danis stated that early in July, 1903, the pipe, which originally extended 9 feet above the surface, was cut off even with the ground, and the flow increased very rapidly from 75 to 120 gallons per minute, weir measurement. Sand then followed and the water finally became very red. After a time it cleared and continued to flow at the increased rate.

Record of I. Cox's well near Mill Neck.

Tisbury:

Fect.

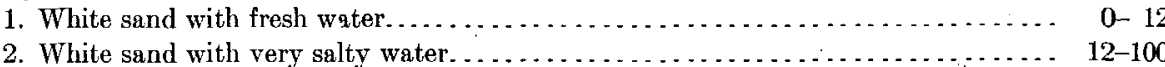

Sankaty?

3. "Black muck".

Cretaceous:

4. Thin layers of clay and quicksand. . . . . . . . . . . . . . . . . . . . . . . . .

5. Red clay, with occasional layers of gray clay containing lignitized wood . . . . . . 200-300

6. Sand, becoming coarser and filled with water (Lloyd sand) ............... $300-330$ 
566.

Record of commission's test well at Massapequa pumping station.

1-2. Peat with sand and gravel

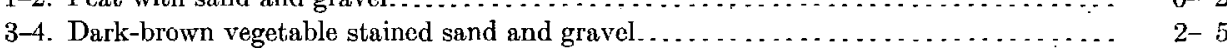

5-9. Yellow-brown sand and gravel (probably glacial outwash) ................... 5 . 5 .

567. According to Mr. Ward this plant consists of fifty-three $4 \frac{1}{2}$-inch wells, 37 to 106 fect deep. All the deeper wells furnish artesian water. Samples of the shallower wells, preserved in the municipal building, Brooklyn, show the following generalized section:

Generalized section of Brooklyn waterworks wells at Massapequa pumping station.

1. Light yellowish gray sand and gravel; nothing readily recognizable as of glacial origin. $\quad 0-25$

2. Fine reddish brown to yellowish gray sand............................ $25-40$

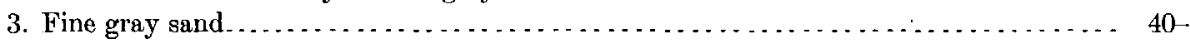

The elevation of deep test well No. 1, which is a flowing well, is 10.1 feet Brooklyn base.

568. Mr. Solomon Ketchem, secretary, reports that the supply of the Amityville Water Company is derived from 6-inch wells, 40 feet deep, sunk in 1893; the water level is 12 fect below the surface and is lowered 4 feet by pumping. The yield in 1900 was as follows:

Yield of Amityville Water Company's wells in 1900.

Gallons.

Maximum daily

53,000

Minimum daily 104,000

569. The whole section given below is glacial outwash.

Record of commission's well near Massapequa pumping station.

Feet.

1-2. Yellow sandy loam

3-9. Fine reddish-ycllow sand to small gravel See Table XII.

570.

Record of commission's well, near Massapequa pumping station.

Feet.

1. Humus-stained loamy sand and gravel.

$2-3$. Reddish-yellow loamy sand and gravel

4-6. Light yellowish-white outwash sand and gravel

6. $0-17.75$

571.

Record of commission's well near Massapequa pumping station.

1-3. Surface loan

3-9. Light-colored sand and quartz gravel only a very small percentage of erratic material.

572.

Record of commission's well near Massapequa pumping station.

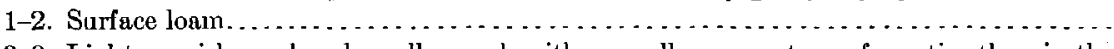

3-9. Light grayish sand and small gravel, with a smaller percentage of erratics than in the wells farther west.

573.

Record of commission's well near Massapequa pumping station.

1-2. Yellow sandy loam . . . . . . . . . . . . . . . . . . . . . . . . . . . . .

3-7. Reddish yellow fine to coarse sand (glacial outwash) $\ldots \ldots \ldots \ldots \ldots \ldots \ldots \ldots \ldots \ldots . .25$

574. Record of commission's well near Massapequa pumping station.

Wisconsin:

1-2. Surface loam.

3. Yellow loamy sand and gravel.

$2.7-3.3$

4-9. Coarse outwash sand and gravel 


\section{UNDERGROUND WATER RESOURCES OF LONG ISLAND, NEW YORK.}

Tisbury:

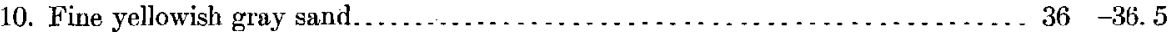

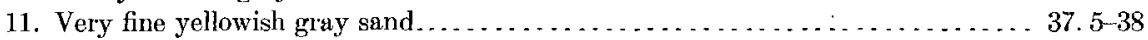

12. Medium sand ........................................ $40 \quad-41$

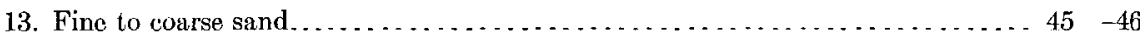

14-15. Coarse sand to fine gravel, with small layer of silt .............. . 50.5-53

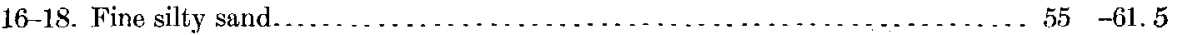

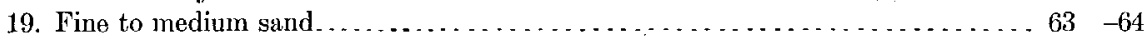

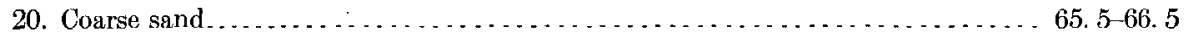

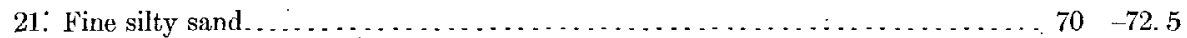

Cretaceous:

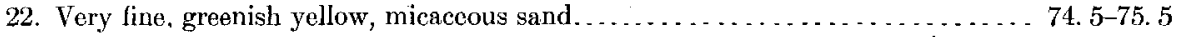

23-24. Medium, white, coarse sand. . . . . . . . . . . . . . . . . . . . . . . . 80 . 85

This series of samples shows apparently four stages of deposition above a depth of 70 feet. Sce Table XII.

575.

Record of commission's well near Massapequa pumping station.

Wisconsin and Tisbury?:

Feet.

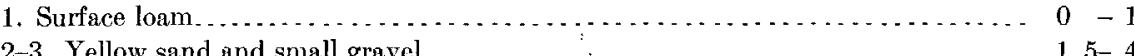

4-5. Yellowish white sand and small gravel. . . . . . . . . . . . . . . . . . .

6. Small gravel, with considerable percentage of erratics................. $15-16$

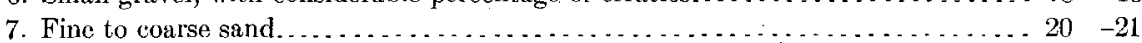

Cretaceous?:

8-12. Fine white sand with tendency toward a yellow color in the upper samples, possibly due to an old land surface. . . . . . . . . . . . . . . . . . . . $24 \quad-41.5$

See Table XII

576.

Record of Dryfuss \& Nibbe's well near Central Park.

Pleistocene:

1. Surface gravel.

Cretaccous:

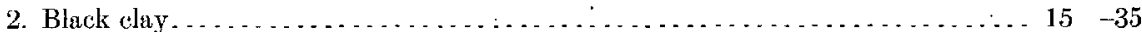

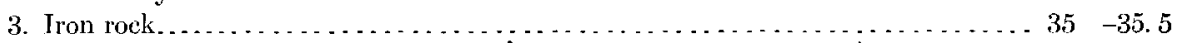

4. Fine dark sand, becoming coarser and containing water. .............. $35.5-55$

Mr. J. Elliott reports having dug a well at this place in which he struck clay very near the surface and passed through 3 feet of iron rock.

Analysis of water from Dryfuss \& Nibbe's well near Central Park.

[By Prof. C. S. Slichter.]

Parts per million

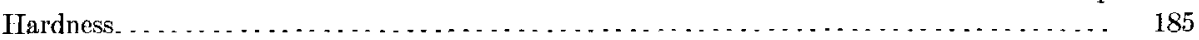

Chlorine ... . . . . . . . . . . .

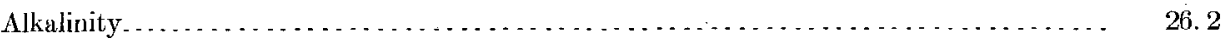

Temperature . . . . . . . . .

The high hardness and chlorine indicates that this well has become contaminated with the brines from the pickle factory.

$\mathbf{5 7 7}$. Mr. Elliott reports that in some of these wells thin layers of elay were found at 4 feet and 20 feet. He adds that similar layers of clay are often found in wells at a distance of half a mile from the foot of the hills, at which point the silty or clayey layers disappear.

578. The high chlorine in the analysis below is doubtless due to brine from the pickle factory. 
Analysis of uater from well of $J$ Keller \& Sons near Farmingdale

[By Prof C s slichter]

Parts per mullon

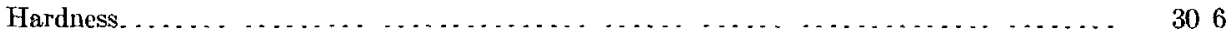

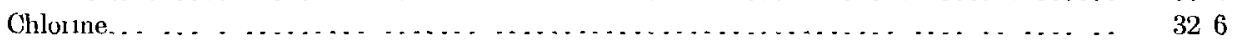

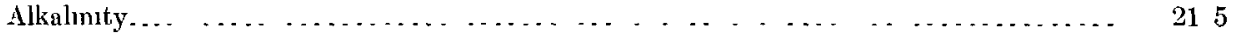

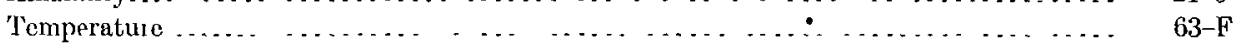

580. Record of commission's test well near Farmingdale . Feet

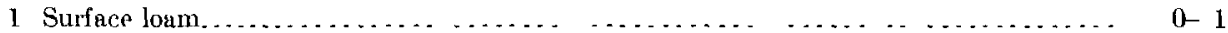

2-6 Ijght sands, passing into small giavel, with a very small peicentage of eriatics $\ldots . \ldots . .1-21$

582. Mr J H Guthel gives the following data "Diameter, 3 feet from 0 to 81 . $1 \frac{1}{4}$ inches from 81 to 111 feet The surface of the ground is black sol mixed with coarse gravel, yellow clay is underneath, then puse sand in depths of 10 to is feet, sepurated by iron ore and hardpan About the middle of the distance in depth I found a coarse yellow sand, very sticky, as if mixed with mud"

58 :3.

Record of Harms estate well near Plannvew

Pleistocene

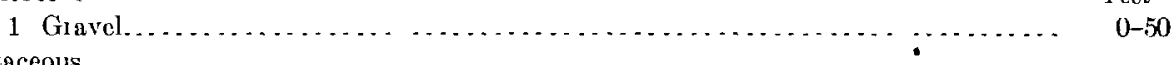

Cretaceous

2 Niternate layeis of hight giay and black clay . . . . . . . . . . . . . . . . $50-70$

3 Dark, rather coarse sand, with water .......................... 70-75

584. Mr Ellott furnished fou samples sepresenting maten ial between 58 and 70 feet, all are fine yellow Cretaceous ( ${ }^{2}$ ) sand

\section{Analyses of water from John T'tus's well near Plaınvew}

[By Proí C S Slichter ]

Parts pei million

Hai dness

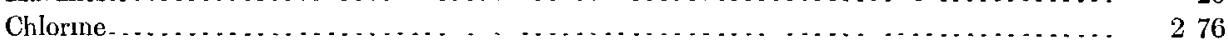

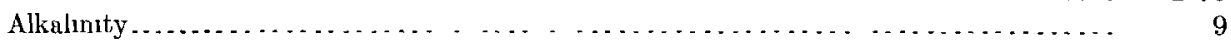

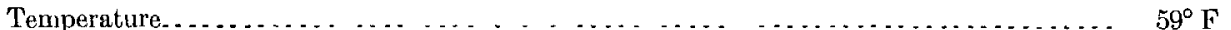

58.5.

Recond of Oscar Jackson's well in West Hulls

Pleistocene

1 Dark surface soll mixed with large field stones. Feet

Cretaceous

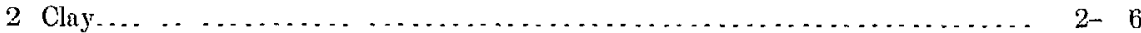

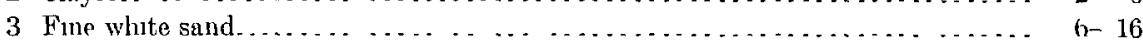

4 Coarse giavel mixed with elay, paited by veins of uron ore and hardpan...... 16-56

5 Sand with vems of black and blue clay 2 to 3 feet thick . . . . . . . . . . . . . . 56-119

6 Driven, mateiral not known. . . . . . . . . . . . . . . . . . . . . . . . $119-1415$

586. Mr Dubois has funnshed the following samples from this well

Record of $H L$ Stimpson's well in the West Hills

Clayey sand and gravel with many compound pebbles... Mannetito

4 Orange-yellow quartz pebbles, with a very few fragments of compound rocks, the Iatter probably derved from the oveslyng beds. . . . . . . . . . . . . .

5 Orange-yellow quartz pebbles, with considerable sand and yellow clay, and many tragments of decayed white chert. . . . . . . . . . . . . . . . . . . . .

6 White quarts sand, with much fine-gramed red ironstone and decayed chert .. 


\section{UNDERGROUND WATER RESOUROES OF LONG ISLAND, NEW YORK.}

Cretaceous ?:

7-17. White to light-yellow quartz sand and gravel containing fragments of decayed

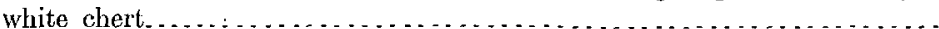

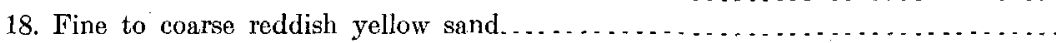

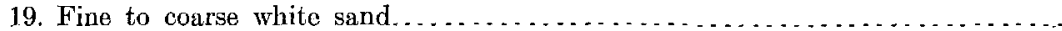

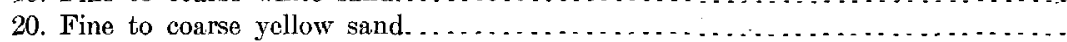

21-22. Medium, yellow, silty sand, with many small, brown, ferruginous nodules and a few pellets of clay ................................. 140-145

23-24. Medium to coarse light-yellow sand with many fragments of dark-brown ferruginous sandstone. . . . . . . . . . . . . . . . . . . . . . . . . . . . . 150-155

$58 \%$.

Record of Richard Collier's well near Woodbury.

Pleistocene:

1. Surface loam; no gravel.

Feet.

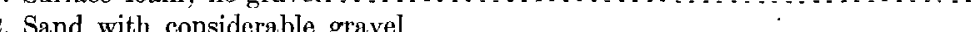

$0-15$

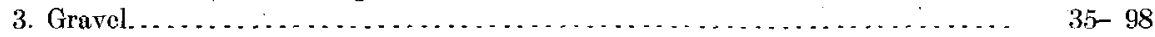

Cretaceous:

4. Black clay . . . . . . . . . . . . . . . . . . .

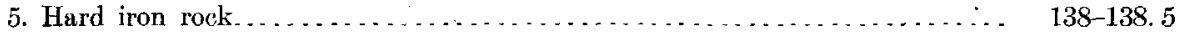

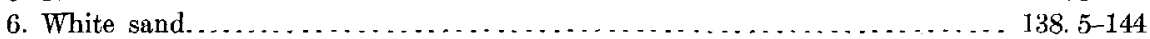

Analysis of water from Richard Collier's well near Woodbury.

[By Prof. C. s. Slichter.]

Parts per million.

Hardness

52.5

Chlorine.

16.6

Alkalinity....

12.5

Temperature

588. Mr. William Jaegle, who drilled this well, reports that between 120 and 150 feet he encountered a dry gravel from which the air rushed with considerable force, and that it blows intermittently between the 4-inch and 6-inch casing. The 6-inch casing extends to a depth of 120 feet and the 4-inch to a depth of 185 feet.

589. Mr. William Jaegle reports that a blowing well formerly existed at this place, but that it was destroyed in an attempt to find the hidden treasure which this blowing was thought to indicate.

590. It is stated that this well blows before a storm, and that it makes enough noise to be heard in the house.

591. These samples were taken from the dump by one of the men who had been with the well from the start. The surface about the woll is distinetly morainal in eharacter, but the samples indicate that tho Pleistocene material is of no very great thickness. The sands are apparently the same as the sands shown in the Melville section.

Cretaceous:

Record of Cold Spring Creamery well near Cold Spring station,

1. Dark clayey sands. Feet.

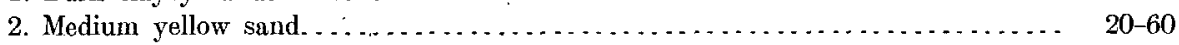

3. Medium reddish yellow sand, containing water ................... $60-96$

592.

Record of H. A. Monfort's well near Cold Spring station.

Wisconsin:

1. Loam and gravel. ........................................ $0-4$

Wisconsin and Cretaceous:

4- 90

2. White sand (dry)

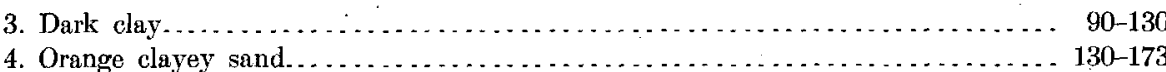

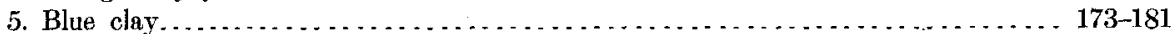

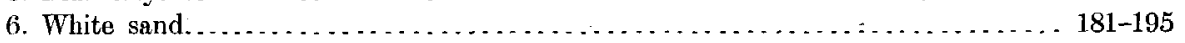


593.

[By G. J. Volckening, F. M., Fel. 21, 1898.]

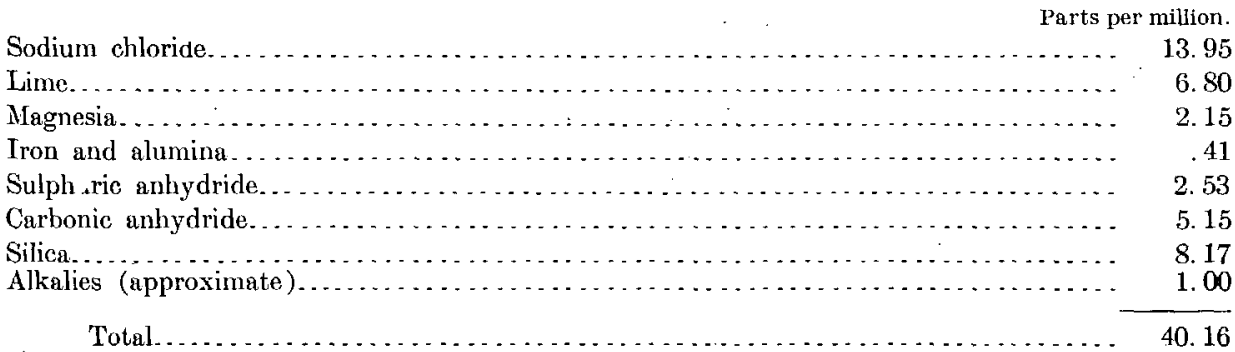

594. The section in this well is reported as very similar to that of woll No. 595 .

595. Record of Columbia farm well near Cold Spring Harbor.

Wisconsin and Tisbury:

1. Sands and gravel.

Cretaceous:

2. Water-bearing sand, yielding milky water at....................... 186

3. Alternate layers of fine white or lead-colored clay and sands, the sands containing water. . .

596. At 160 feet the well is reported to have furnished quite a littlo gas, which has very much the odor of marsh gas.

Tisbury:

Record of W, R. Jones's well near Cold Spring Harbor. Cretaceous:

2. Black clay, becoming whiter below............................... 190-200

3. White sand with water........................................ 200-228

598.

Record of Mrs. W. Wood's well near Cold Spring Harbor.

Tisbury: Fect.

1. Sand and gravel with an abundant supply of clear water, which turned dark on boiling.

2. Alternate layers of white or lead-colored sands and clays

$40-163$

599.

Record of well of Van Wyke heirs near Cold Spring Harbor.

Tisbury:

1. Surface sand and gravel.................................. 0 - 35

2. Sand and gravel, with a little water........................... $35-40$ Cretaceous:

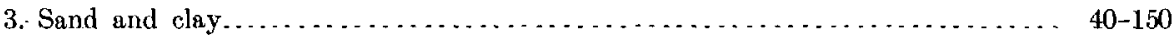

4. Water-bearing sand.....................................

601.

Record of W.E. Jones's well near Cold Spring Harbor.

Pleistocene:

3. Gravel with glacial pebbles. . . . . . . . . . . . . . . . . . . . . . . . .

602. This is stated to be the well which in Darton's report is given as "Cold Spring Harbor, 125 feet deep; flow 18 gallons per minute." 
603.

Record of G. E. Brightson's well near Cold Spring Harbor.

1. Gravelly clay, quite hard.

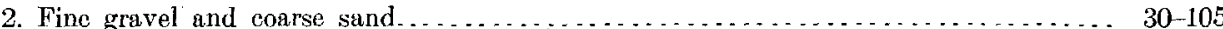

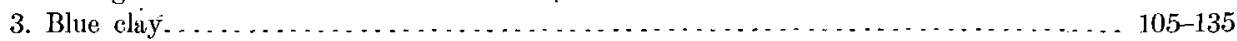

4. Bluish sand. ............................................... 135-170

5. Very coarse sand, water bearing...............

604. Record of L. C.Tiffany's well near Cold Spring Harbor.

Tisbury:

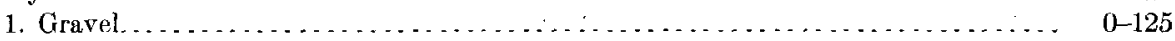

Cretaceous?:

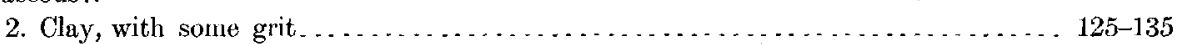

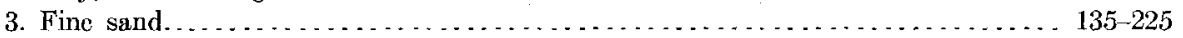

4. Blue clay. . . . . . . . . . . . . . . . . . . . . . . . . . . . . . . . . . . 225-235

5. Coarse sand, with abundant supply of water.................... $235-243$

607. Record of Wm: White's well near Cold Spring Harbor.

Tisbury: Feet.

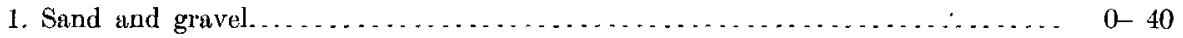
Cretaceous?:

2. Black clay ................................................. $40-41$

3. Sand and gravel................................................. $41-95$

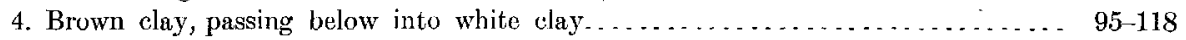

5. Red sand. . . . . . . . . . . . . . . . . . . . . . . . . . . . . . . . . . . . . 118 120

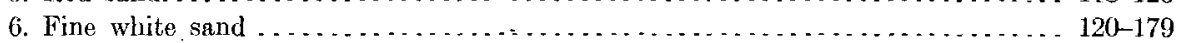

The well began to flow at 120 fect, but choked with sand, and a free flow was not obtained until a depth of 179 feet was reached. This well flowed 12 feet above high tide.

608.

Record of J. T. Jones's well near Cold Spring Harbor.

Tisbury:

1. Top soil and gravel, with highly mineral water

Feet. Tisbury?:

2. Black muck ..................

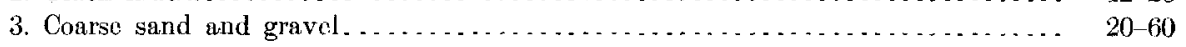

Cretaceous:
4. White clay.
$60-65$

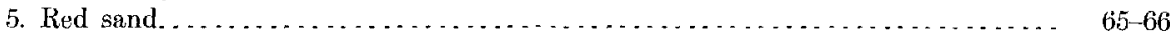

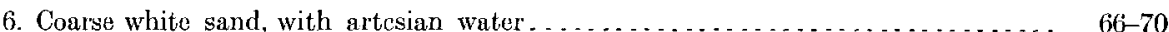

609.

Record of L. C. Tiffany's well near Cold Spring Harbor.

Tisbury:

Feet.

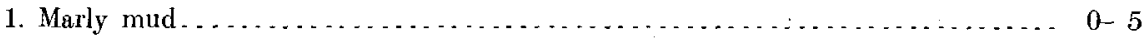

2. Beach gravel, with large stoncs............................. $5-50$

Cretaceous?:

3. Clay, black on top, becoming white below . . . . . . . . . . . . . . . . . . $50-58$

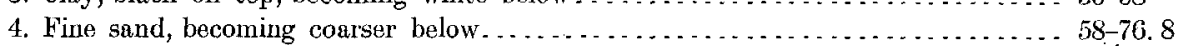

When the artesian sand was first struck, it is estimated that the well flowed 120 gallons per minute, but the water contained a large amount of fine, white, micaceous sand. To cut off this, the well was driven deeper and the flow reduced to 75 gallons per minute (measured). This is the maximum yield, the flow being less at low tide. 
610.

Record of H. De Forest's well near Cold Spring Harbor.

Tisbury:

Cretaceous:

2. White clay

3. Orange sand

$80-80.5$

4. Sand, bright

5. White clay

$80.5-95$

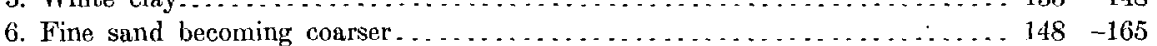

612. The following record has been prepared from samples preserved from this well:

Wisconsin:

Record of R. De Forest's well near Cold Spring Harbor:

1. Glacial sand and clay

2. Large quartz and granite pebbles. Cretaceous:

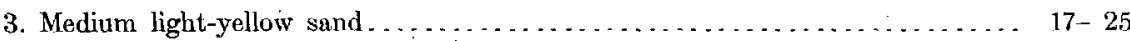

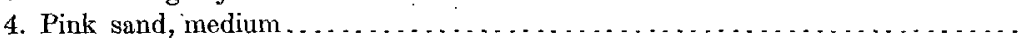

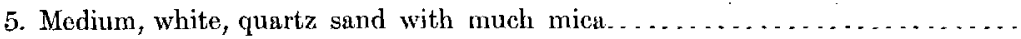

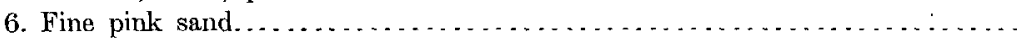

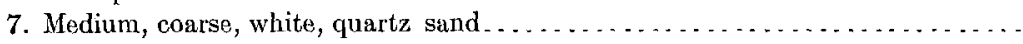

8. Coarse quartz with large pieces of FeS. . . . . . . . . . . . . . . . . . . .

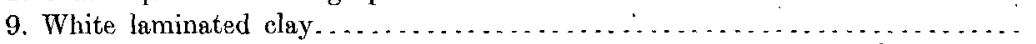

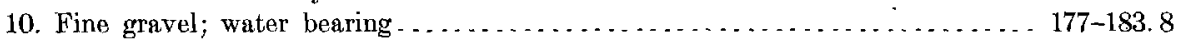

613.

Record of Eagle fdock well near Cold Spring Harbor.

Recent:

Feet.

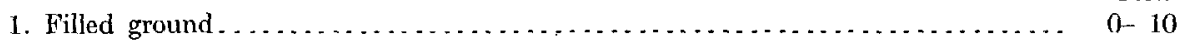

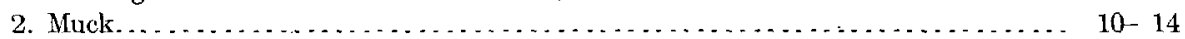

Tisbury:

3. Beach gravel with salty water.............................. 14-100

Sankaty:

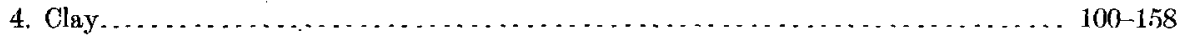

Jameco:

5. Fine sand, passing into coarse gravel containing artesian water. . . . . . . . . 158-176

The samples of the water-bearing gravel preserved by Capt. W. R. Bingham show a very large percentage of erratic material.

614. Mr. Webster reports that the measured flow of this well at 12.10 p. m., December 31,1902 , was 39 gallons per minute. Mr. J. G. Hannah, the former owner of the well, reports that on November 5, 1902, the flow at low tide was 16 gallons per minute and at high tide 50 gallons per minute. Mr. Webster has kindly furnished the following analysis, and notes by Prof. Herbert E. Smith, State chemist of Connecticut:

$$
\text { Analysis of water from James Bowen's well near Cold Spring Harbor. }
$$

\begin{tabular}{|c|c|}
\hline & \\
\hline & \\
\hline & \\
\hline & \\
\hline & \\
\hline & \\
\hline & \\
\hline & \\
\hline & \\
\hline & \\
\hline & \\
\hline esidue on evaporation: & i million. \\
\hline Total ............. & 39.0 \\
\hline Volatile. & 13.5 \\
\hline 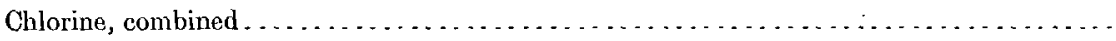 & 4.00 \\
\hline monin. . & 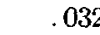 \\
\hline ammonia. . & 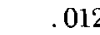 \\
\hline 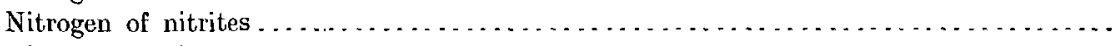 & .001 \\
\hline 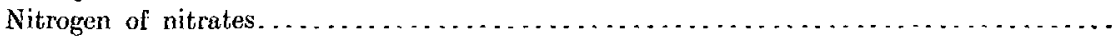 & .55 \\
\hline Oxygen consumed from permanganate in one-half hour at $100^{\circ} \mathrm{C} \ldots \ldots \ldots \ldots$ & .2 \\
\hline ardness as carbonate of calcium. & 10.00 \\
\hline olor....... & 0.0 \\
\hline
\end{tabular}




\section{UNDERGROUND WATER RESOURCES OF LONG ISLAND, NEW YORK.}

Professor Smith says: "The sample was clear, free from sediment, colorless, and odorless. These results show that the water contains a very small amount of mineral matter, that it is soft, and that it is of high organic purity. The figure for chlorine is subnormal for the locality of the well, and the nitrogen of nitrates is not much, if any, above the normal. 'These results indicate, in my opinion, that the water is free from sewage or drainage contamination and excellent for drinking and other domestic uses.

"The figures for organic matter are very satisfactory indeed. The figure for chlorine (4) is the chlorin? that is normal to a narrow strip in the central portion of Long Island. According to the chlorine map, this area is about 35 miles long, with an average width of not over 2 miles, and runs through Suffolk County, back of Cold Spring Harbor. As the nomal on the coast is 6 or above, the result in this sample would indicate that the water comes from the interior portion of the island.

"I of course do not wish to make too strict an interpretation of a single analysis, but where the diflerence between the local chlorine and that found is so distinct as in this case, I think it pretty safe to conclude that the water sent me comes from the interior."

In this connection see the analysis from the deep wells given on page 68 and analyses of wells Nos. 526 , $543,545,546,547,549,554,556$, and 559 .

\section{5. \\ Record of L. V. Bell's well near Cold Spring Harbor.}

1. Coarse sand and gravel, with one or two layers of cobbles; no clay .............

616. This well is in the basement and begins in pink Cretaceous sand.

617. Mr. Matthew King, foreman for P. H. \& J. Conlan, reports that this well is 325 feet deep, but Mr. J. Conlan states that it was finished at 140 feet.

620.

Record of T. S. Williams's well near Cold Spring Harbor.

Wisconsin:
1. Gravel.
$0-30$

Cretaceous:

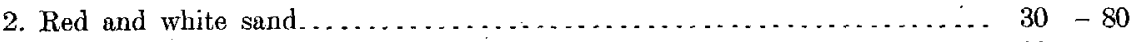

3. White clay. ....................................... 80 . 86

4. Fine white sand; fresh water.......................... $86 \quad-136$

5. White clay and sand.................................... $136 \quad-146$

6. Yellow sand and gravel (looks like brown sugar) ............... $146 \quad-160$

7. Red and white clay..................................... $160 \quad-162$

8. Fine white sand ...................................... $162 \quad-178$

9. Coarse quartz gravel; no sand; gravel about 1 inch diameter......... $178-200$

10. Fine white sand becoming pinkish.......................... $200 \quad-230$

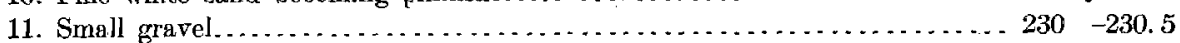

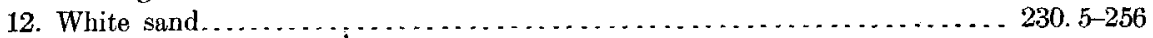

13. Yellow clay . . . . . . . . . . . . . . . . . . . . . . . . . . . . $256-257$

14. Reddish sand........................................ $257-262$

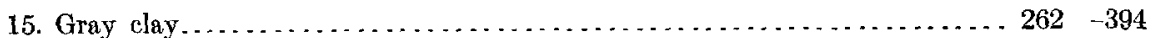

16. Coarse yellow gravel. . . . . . . . . . . . . . . . . . . . . . . . . . . $394 \quad-398$

This well flows 10 gallons per minute at a height of 8.5 feet above mean high tide.

621. Mr. Danis reports the following section for this well:

Wisconsin:

Record of Walter Jennings's well near Cold Spring Harbor.

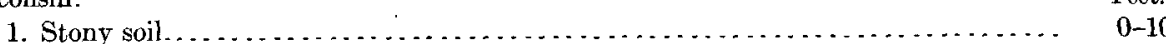

Cretaceous:

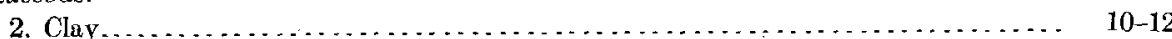

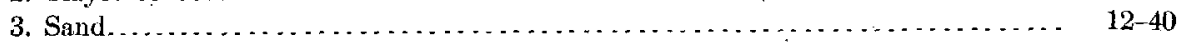

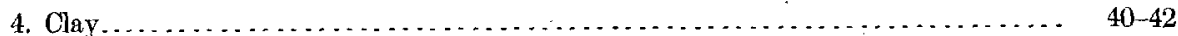

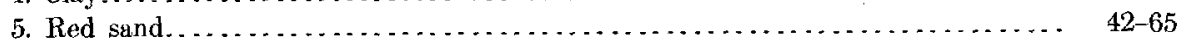

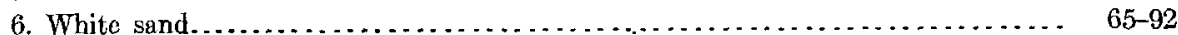


622. This well was first driven 42 feet and water obtained which was used for a time, but proved unsatisfactory. It was then deepened and two more water-hearing sands encountered. The present supply from the lower layer is reported to be very good.

\section{3.}

Record of R: De Forest's well, West Neck.

Cretaceous:
1. Brownish-red sandy clay .

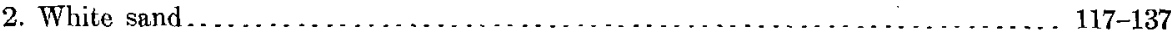
3. Clay . . . . . . . . . . . . . . . . . . . . . . . . . . . . . . . . . . . . . . 137-157

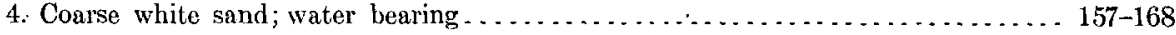

Feet.

624.

Record of Alex. Denton's well near Huntington.

Wisconsin

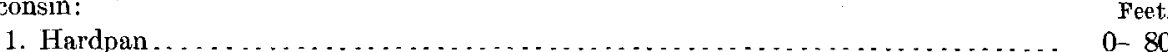

Tisbury?:

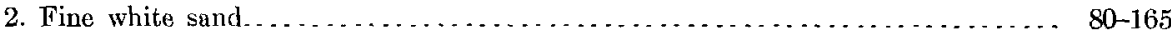

Cretaceous:

3. Light-colored clay . . . . . . . . . . . . . . . . . . . . . . . . . . . . . 165-175

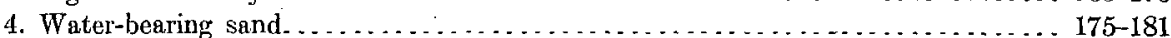

625.

Record of H.J. Dubois's well near Huntington.

Wisconsin and Tisbury:

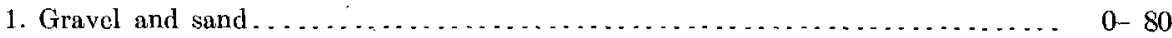

Fect.

Tisbury and Cretaccous:

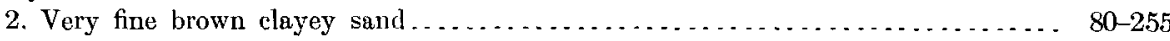

Cretaceous:

3. Fine to coarse light-yellow sand

$255-264$

626. On May 11, 1903, gage readings were begun on this well. Observations for six hours showed no fluctuations in the level of the water, which rose about 10 inches above the top of the pipe out of which it was flowing before being piped up. Mr. Sammis says that when first driven the water did not reach the top of the pipe but stood several inches below it. After one or two weeks' pumping the well began to flow.

627. This well was all in sandy gravel, with the exception of a thin layer of clay just above the white water-bearing gravel.

628. This well was abandoned in the summer of 1903, but Mr. Dubois states that they intend to sink it deeper. It will be seen from Pl. XVI that the Lloyd gravel should be encountered at this place at about 500 feet below sea level, or about 125 feet below the present bottom of the well. The chances of getting a good supply of water by deepening the well 150 feet are, therefore, regarded as extremely good.

Record of B. Ward's well, West Neek.

Wisconsin:

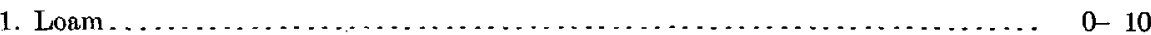

Tisbury:

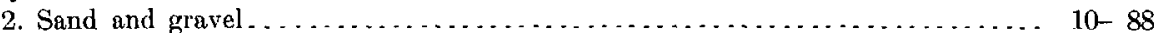

Sankaty? :

3. Blue clay $\ldots \ldots \ldots \ldots \ldots \ldots \ldots \ldots \ldots \ldots \ldots \ldots \ldots \ldots \ldots \ldots \ldots, 8,116$

Jameco?:

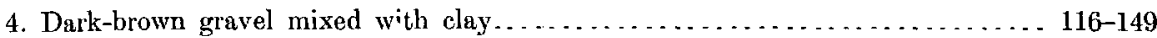

Transition:

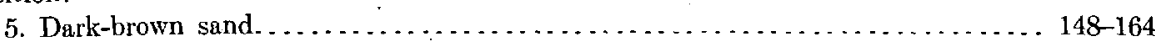

Cretaceous:

6. Blue hardpan .

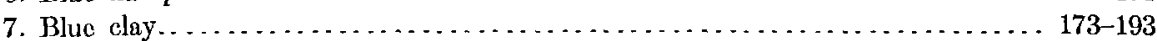

8. Pink clay ..................................................... 193-273

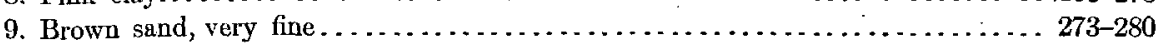


Cretaceous--Continued. Feet.

10. Pink clay................................................ 28 280 335

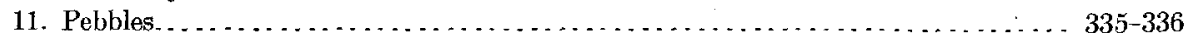

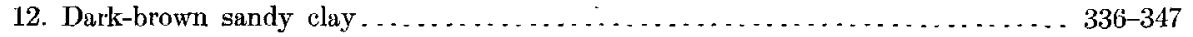

13. Sandstone. . . . . . . . . . . . . . . . . . . . . . . . . . . . . . . . . . . . 347-350

14. Pink clay . . . . . . . . . . . . . . . . . . . . . . . . . . . . . . . . . $350-386$

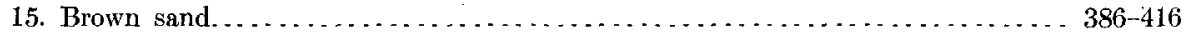

16. Fine sand, like quicksand . . . . . . . . . . . . . . . . . . . . . . . . . . 416-417

17. Brown clay . . . . . . . . . . . . . . . . . . . . . . . . . . . . $417-429$

18. Water sand . . . . . . . . . . . . . . . . . . . . . . . . . . . . . . . . . $429-430$

19. Light-blue clay . . . . . . . . . . . . . . . . . . . . . . . . . . . . . . . $430-432$

20. Very black clay ............................................. $432-435$

21. Light-blue clay, turning to reddish color near bottom of stratum. . . . . . . . 435-439

22. Brick-red clay ........................................... $439-444$

23. Slate-colored clay.......................................... 444447

24. White clay, like kaolin. . . . . . . . . . . . . . . . . . . . . . . . . . . . . 447-449

25. Very dark-blue clay ..................................... 449-453

26. Blue clay with charcoal . . . . . . . . . . . . . . . . . . . . . . . . . . $453-455$

27. Light-blue clay. . . . . . . . . . . . . . . . . . . . . . . . . . . . . . . . . $455-461$

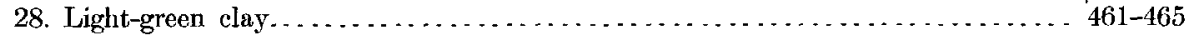

29. Red clay . . . . . . . . . . . . . . . . . . . . . . . . . . . . . . . . . . . $465-471$

30. Dark-gray clay . . . . . . . . . . . . . . . . . . . . . . . . . . . $471-476$

31. Light-blue clay . . . . . . . . . . . . . . . . . . . . . . . . . . . . . . . . 476-479

32. Dark-brown sand. . . . . . . . . . . . . . . . . . . . . . . . . . . 4794480

33. Green clay. .............................................. 4 480-482

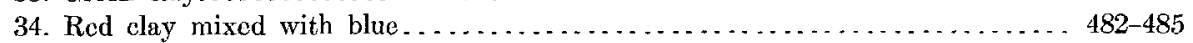

35. Very fine brown sand mixed with clay. ...................... 485-487

36. Very white clay. . . . . . . . . . . . . . . . . . . . . . . . . . . . . . . . . 487-488

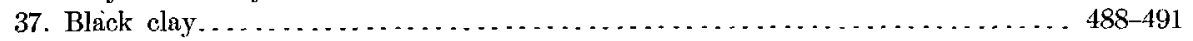

38. Dark-brown clay . . . . . . . . . . . . . . . . . . . . . . . . . . . . . . . . . 491-495

39. Drab-colored clay. . . . . . . . . . . . . . . . . . . . . . . . . . . . . . . . . . $495-497$

40. Hardpan, or sand rock; looks like an oolitic limestone............... 497-498

629. Record of Mrs. M. H. Clots's well, West Neck.

Wisconsin and Tisbury: Feet.

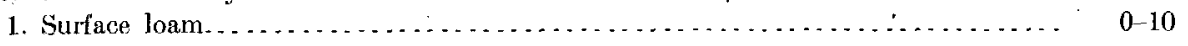

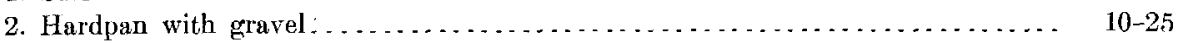

Tisbury and Sankaty: Sankaty

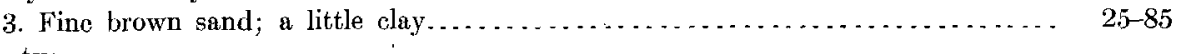

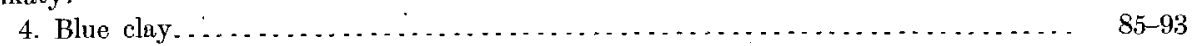

Jameco?:

5. Brown gravelly sand; water hearing. . . . . . . . . . . .

630. The 4-inch pipe is cut off 17 feet from surface, and the well flows 10 gallons per minute into an! underground cistern. Water would rise to within. 4 fect of the surface.

A 5 -inch well was sunk about 10 feet from the 4-inch well, to a depth of 147 feet, and flows into the underground cistern 18 gallons per minute.

Wisconsin:

Record of A. Heckscher's well near Halesite.

1. Surface . . . . . . . . . . . . . . . . . . .

Cretaceous:

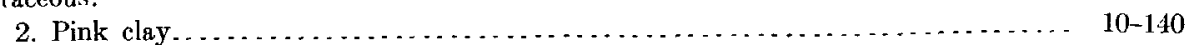

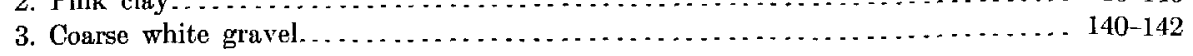


631.

Record of Mrs. A. W. Marsh's well, West Neck.

1. Dug well. ....... Feet.

Cretaceous:

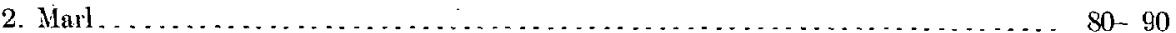

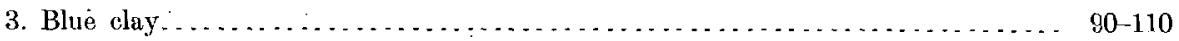

4. Fine sand, white ......................................... 110-111

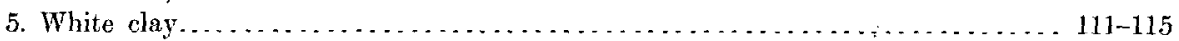

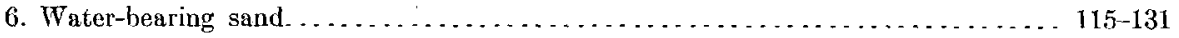

632. Record of R. B. Conklin's well, West Neck.

Wisconsin: $\quad$ Feet.

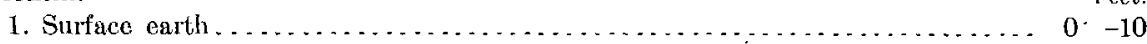

2. Bowlders ............................................. $10 \quad-12.5$

3. Gravel and clay mixed with surface material ................... 12.5 5-24

Tisbury

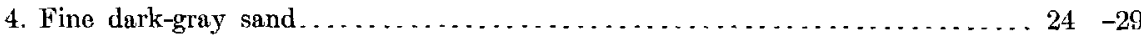

5. Dark-gray gravel ..................................... $29 \quad-30$

Cretaceous?

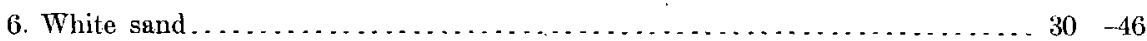

7. White quartz gravel; water bearing. . . . . . . . . . . . . . . . . $46 \quad-51$

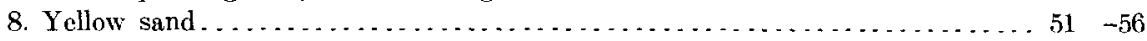

633. This well was visited in company with Dr. O. L. Jones on April 24, 1903, and the following samples and records obtained. At this time the pipe had been cut off about a foot below the mean level of the ground ( 5 to 6 feet above high tide), and the well was flowing about 5 gallons per minute. The foreman stated that at low tide the water ceased to flow, but when the tide had risen 1 foot the well commenced to How and the flow increased until high tide.

\begin{tabular}{|c|c|}
\hline 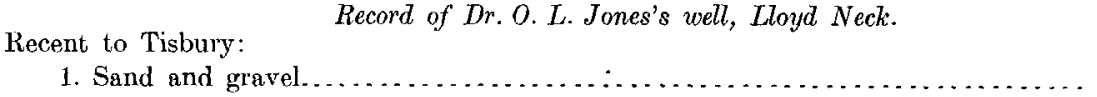 & $\begin{array}{l}\text { - Feet. } \\
0-95\end{array}$ \\
\hline Sankaty?: & \\
\hline $\begin{array}{l}\text { 2. Dark-gray laminated clay, with pieces of partly lignitized wood............. } \\
\text { Jameco?: }\end{array}$ & $95-105$ \\
\hline 3. Fine to coarse yellow sand (glacial?) $\ldots \ldots \ldots \ldots \ldots \ldots \ldots \ldots$ & $105-122$ \\
\hline Cretaceous: & \\
\hline 4. Dark-yray laminated clay $\ldots \ldots$ & $122-222$ \\
\hline Lloyd sand: & \\
\hline $\begin{array}{l}5-6 \text {. Very light-yellow sand, with fragments of white, very much decayed chert... } \\
\text { 7. White quartz gravel, with a few pebbles of ferruginous sandstone and white chert. }\end{array}$ & $\begin{array}{l}222-243 \\
243-246\end{array}$ \\
\hline 8. Coarse, light-yellow sand, with a few fossil fragments $\ldots \ldots \ldots \ldots \ldots \ldots \ldots$ & \\
\hline
\end{tabular}

The fossils from No. 8 were submitted to Dr. T. W. Stanton and he regarded them as Upper Cretaceous. They show: (1) Crinoid stem; (2) fragment of shell; (3) Clausa americana, a bryozoan very common in the Rancocas formation in New Jersey. (Identified by Ray S. Bassler.)

635. Record of commission's test well 1 mile northeast of Amityville.

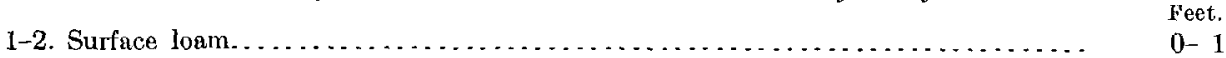

3. Gravel and yellow loam......................................... $1-2$

4-8. White quartz, sand, and gravel; very few if any erratics.............. See Table XII.

636. Record of commission's test well 1 mile north of Lindenhurst.

1. Very dark sandy clay.... Feet.

2-4 Sand and gravel with a small percentage of erratios...

5-6. Medium gray sand (possibly glacial)

17116-No. 44-06-20 


\section{UNDERGROUND WATER RESOURCES OF LONG ISLAND, NEW YORK.}

637.

Record of commission's test well $\mathscr{2}$ miles northwest of Lindenhurst.

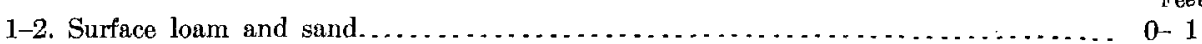

3-9. Sand and small gravel; very small percentage of erratics................ 1-29.5

See Table XII.

638. Record of commission's test well near Maywood.

1-2. Surface loam Feet.

3-9. Light grayish-white sand and quartz gravel; very small percentage of erratics.. $5-30$

639 . Record of commission's test well 1.5 miles south of Pinelawn.

Wisconsin and Tisbury:

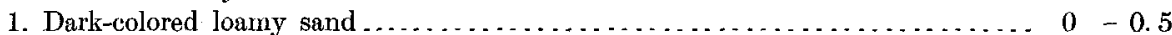

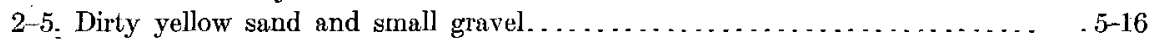

6-7. White sand and gravel; some erratics......................... $20 \quad-26$

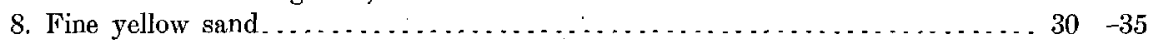

9-14. Medium grayish-yellow sand; some erratics ................... $40 \quad-60$

Cretaceous:

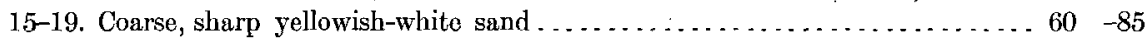

20-21. Very dark, fine lignitic sand. . . . . . . . . . . . . . . . . . . . . . . . $87 \quad-92$ See Table.XII.

640.

Record of commission's test well 2 miles north of Lindenhurst.

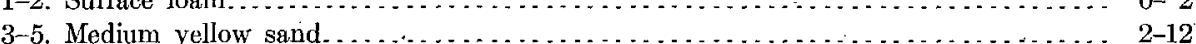

6. Very light-colored sand and gravel; small percentage of glacial material........ 14-30.5

641.

Record of commission's test well 2 mites south of Wyandanch.

1-2. Surface gravelly loam

3-8. Very light-colored sand and gravel with a very small percentage of glacial material. $\quad 5-31$

642.

Record of commission's test well near Pinelawn.

2. White sand and gravel with some erratics...........

643. The following analysis was made February 5, 1894, by C. F. Chandler, Ph. D. and Charles E. Pellew, E. M.:

Analysis of water from Colonial spring near Wyandanch.

Potassic sulphate. .......

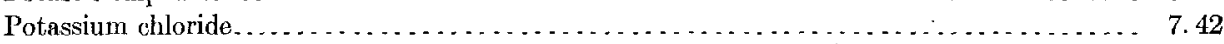

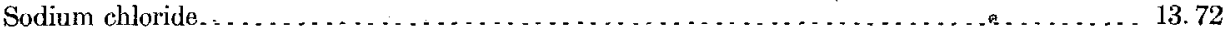

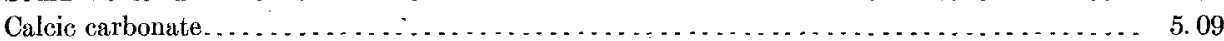

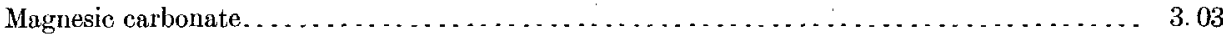

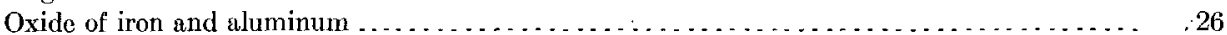

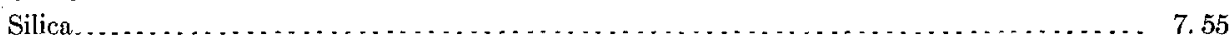

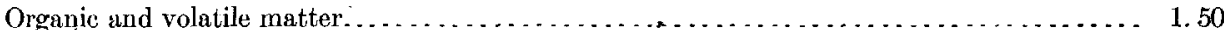

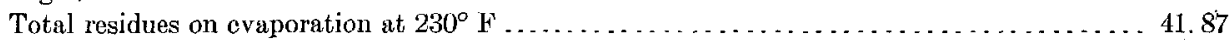

"The 'Colonial' is a pure alkaline water, showing unusual freedom from organic matter."

The analysis of the Mo-Mo-Ne spring was made by the same chemists, who pronounced it the purest water they had ever examined. 
Analysis of the Mo-Mo-Ne spring near Wyandanch.

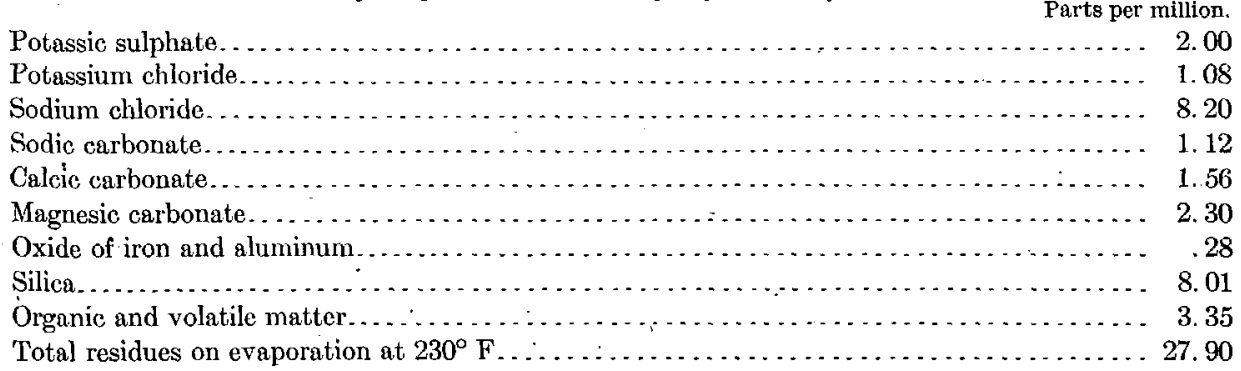

644. Mr. George Carll reports regarding this region: "My well was first dug 130 feet and gave a fair supply of good water. When the well was finished the bottom was a kind of a quicksand and clay, that at times would make the water of a whitish color. I afterwards sunk two terra-cotta tubes, making it 136 feet deep, and the water was from 12 to 15 feet in depth. About 500 or 600 yards to the north are never-failing springs. The wells north and south range from 20 to 50 feet in depth. I struck the same bed of clay at 47 feet, but there was nearly 3 feet difference in striking it in just the width of the well, and consequently I could get but.little water.'

645. Mr. Elliott states that the thin clay layer which occurs very near the surface in this well extends for about 1 mile south of his house, and north as far as Huntington.

Wisconsin and Tisbury:

Record of $J$. Elliott's well near Melville.

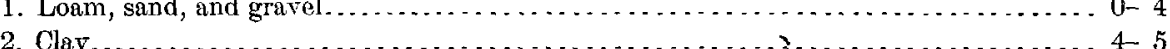

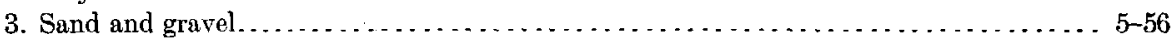

648. All deep wells in this neighborhood lost more or less water in 1900-1901.

Record of A. C. Soper \& Company's well near Fairground.

Wisconsin and Tisbury:

Feet.

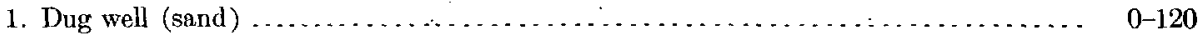

Cretaceous:

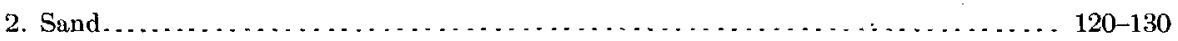

3. Clay .................................................. 130-200

4. Very fine sand, mixed with a little clay; plenty of water but could not pump on account of stopping up..................................... 200-260

5. Coarse sand; very good water; pumps without trouble.................. 260-267

649. Record of $F$. Gallienne's well near Huntington.

1. Glacial sand and gravel................................... 0 . 65

2. Dark-gray sand and clay .......................................... $65-70$

3. Dark dirty-gray sand and gravel (probably glacial) $\ldots \ldots \ldots \ldots \ldots \ldots \ldots \ldots \ldots \ldots$ Cretaceous:

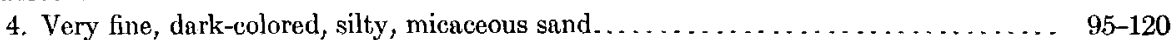

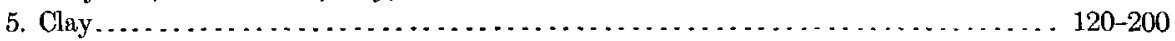

650. Mr. Darling states that this is a gravity system depending on a ground reservoir situated 170 feet above mean high tide. The material penetrated in the group of driven wells is as follows: 
Wisconsin:

Record of wells of Huntington waterworks, Huntington.

Tisbury:

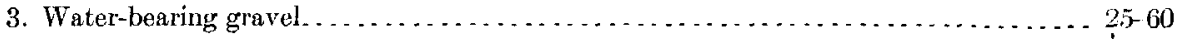

Each well will yield 150 gallons per minute without lowering the water below the suction limit.

652. Record of well of Huntington Light and Power Company near Halesite.

Recent:

1. Filled ground

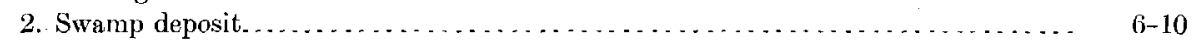

Tisbury:

3. Dark sand and gravel........................................ 10-70

Sankaty?:

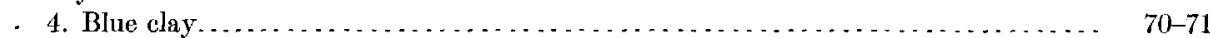

Jameco?:

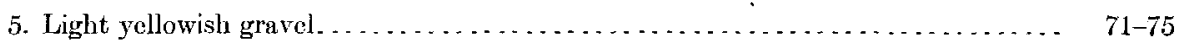

653.

Record of R. F. Carmen's well near Centerport.

Tisbury:

Feet.

1. Sand and gravel............................................ $0-154$

Cretaceous:

2. Blue clay....

3. White gravel

654.

Record of R. S. MeCrary's well near Centerport.

Wisconsin and 'Tisbury:

$0-16$

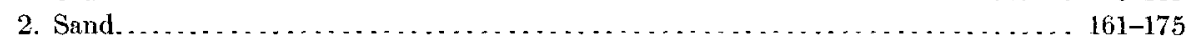

Jameco?:

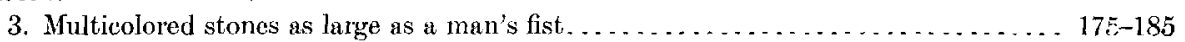

655.

Wisconsin:

Record of C. A. Hallock's well near Centerport.

1. Surface gravel . . . . . . . . . . . .

Cretaceous:

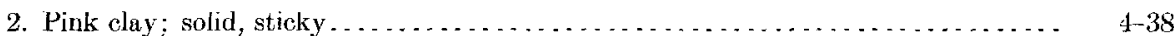

3. Dark-colored gravel; water bearing............................ $38-42$

657.

Record of J. J. Robinson's well near Centerport.

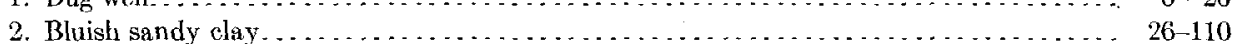

3. Yellow gravel. . . . . . . . . . . . . . . . . . . . . . . . . . . . . . . . . . $110-117$

658. This plant was originally supplied from śprings which yielded about 200 gallons per minute. The water from these was collected in a basin at the pumping station and then lifted to a ground reservoir having a capacity of about 250,000 gallons. Early in 1903 two very successful artesian wells were completed, and the spring supply has now been abandoned. The wells, which are 8 inches in diameter and 47 feet deep, are sitnated about 32 feet above mean high tide, and it has been found that 250 gallons per minute must be pumped from them to cause them to cease flowing. The ground reservoir is still used to supply the lower parts of the town, and an Acme system, having storage capacity of 25,000 gallons, has been installed for high-level service.

The following samples from one of the wells have been furnished by Mr. Henry Cabre, driller: 
Wisconsin:

Record of well of Northport waterworks, Northport.

1. Clayey gravel.

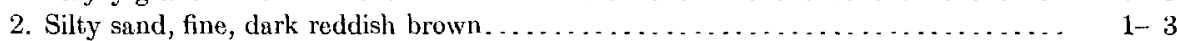

3. Very fine, reddish brown, clayey sand........................... $3-5$

4. Reddish brown silt to small gravel; contains a considerable percentage of erratics. . $5-10$

Tisbury:

5-6. Medium, dirty, yellow sand . . . . . . . . . . . . . . . . . . . . . . . . . . . $\quad 10-20$

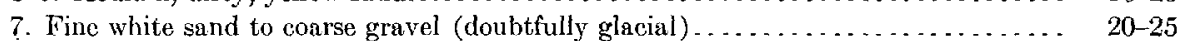

8. Fine, dirty, yellow sand . . . . . . . . . . . . . . . . . . . . . . . . . . . 25-30

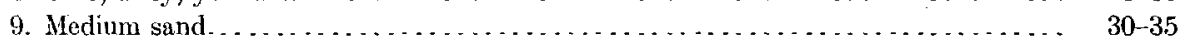

10-13. Medium sand to small gravel .............................. $35-48$

14. Medium, dirty, yellow sand..... . . . . . . . . . . . . . . . . . . . . . . . . . 48-51

This whole section, while not pronouncedly glacial, is probably to be regarded as composed of reworked material of Glacial age.

659.

Record of A. O. Gildersleeve's well near Larkfield.

Wisconsin and Tisbury: .

1. Coarse sand, mixed with gravel and small stones. . . . . . . . . . . . . 0 . 50

2. Coarser sand.............................................. 50-186

660. Record of Fred Nevins's well near Northport.

Wisconsin: $\quad$ reet.

Tisbury:

1. Loany clay.

2. Sand, becoming coarser with increasing depth

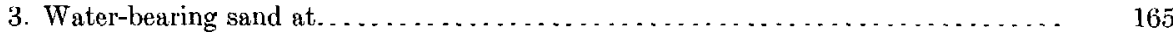

4. Sharp white sand, water bearing, at. . . . . . . . . . . . . . . . . . . . . 196

662. The following analysis was made by George A. Ferguson and Raymond J. Nestell, November 30, 1901:

\section{Analysis of water from $F . J$. Smith's well near Northport.}

Chlorine in chlorides.

.

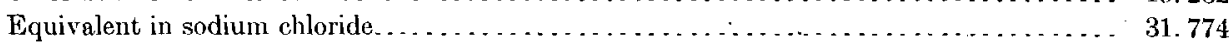

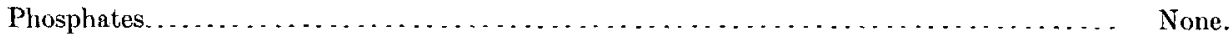

Nitrogen in nitrates........................................... 4.490

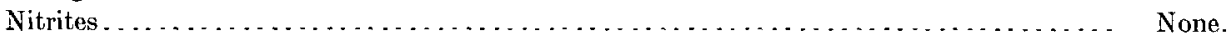

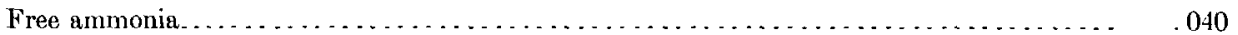

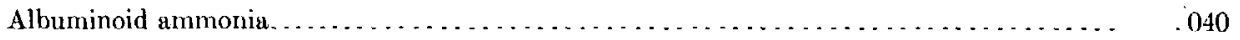

Hardness equivalent in calcium carbonate: .

Temporary............................................... 56.000

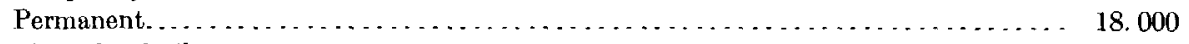

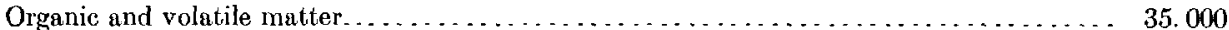

Mineral matter after ignition. . . . . . . . . . . . . . . . . . . . . . . . . . . . 125. 000

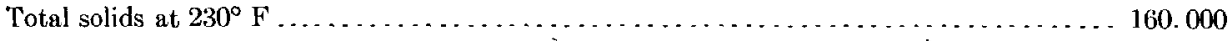

663. This flowing well consists of a short pipe driven into an old spring site. The water rises 5 feet in the pipe. It is a good example of a type of well common on the north shore which is on the border line between a spring and an artesian well. (See fig. 33.)

664. Record of D. B. Moss's well near. Little Neck.

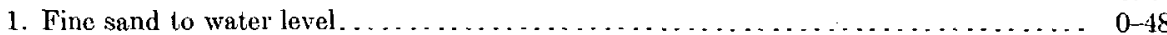

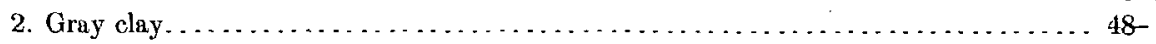

3. Very fine brown sand, some water................................ -67

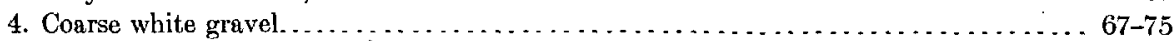


Tisbury:

1. Light-colored coarse sand and gravel

669. This is the Port Eaton well reported by Darton, "who gives the following record, furnished to him by Mr. Nimmo:

\section{Record of Dr. O. L. Jones's well on Eaton Neck.}

1. Quartz gravel and sand.

Feet.

3. Fine sand mixed with clay.

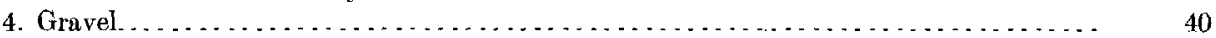

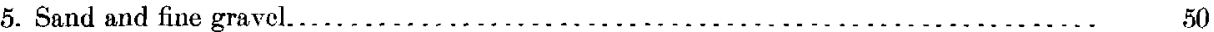

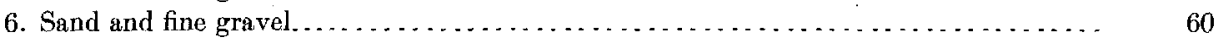

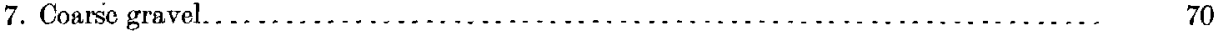

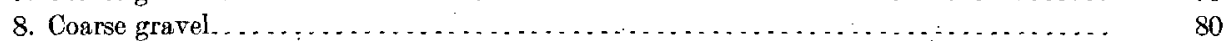

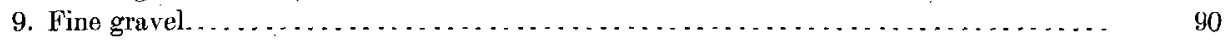

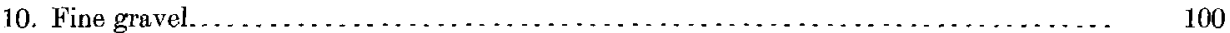

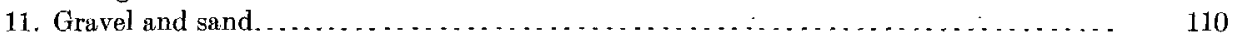

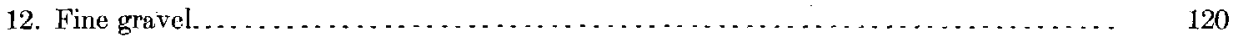

13. Coarse gravel . . . . . . . . .

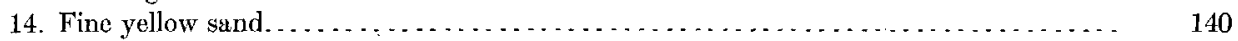

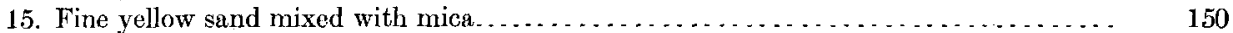

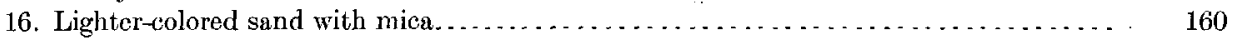

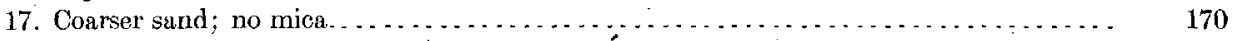

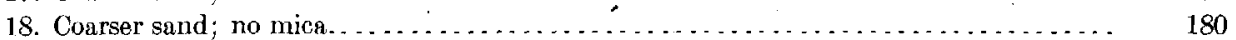

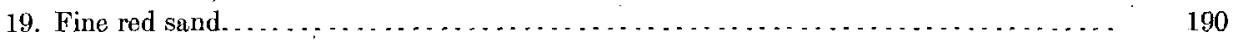

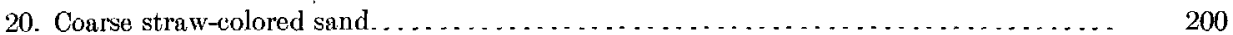

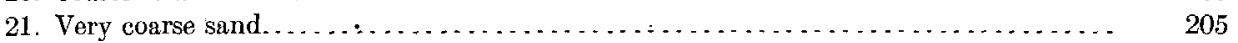

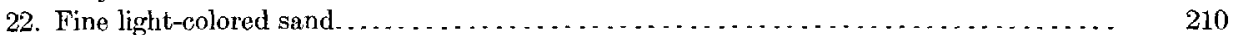

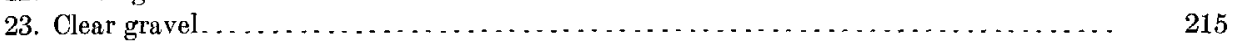

24. Light coarse sand . . . . . . . . . . . . . . . . . . . . . . . . . . . . . . . . . . 220

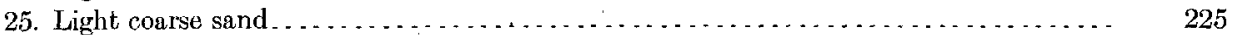

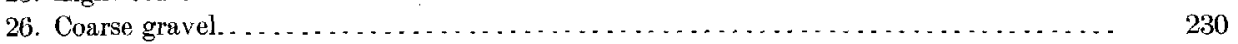

27. Coarse gravel. . . . . . . . . . . . . . . . . . .

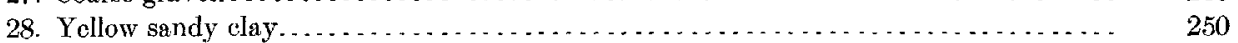

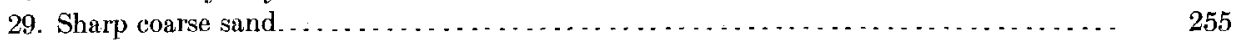

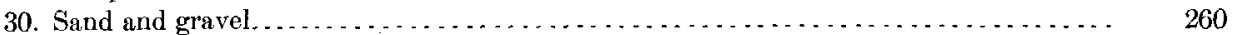

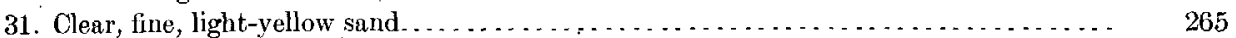

While salt water was reported in this well to a depth of 205 feet, at 263 feet an excellent supply of freshí water is said to have been obtained which flowed slightly above the surface. Attempts to develop this brought in salt water, and Mr. C. H. Danis, who afterwards worked on the well, reported that he could get no fresh water. The well was deepened to 340 feet through sand and gravel containing salt water.

670. Mr. Bevin has kindly furnished the Survey with the following samples from this well:

Record of L. A. Bevin's well on Eaton Neck.

Pleistocene:

1-2. White sand and gravel, with a percentage of erratics ................ $15-30$

Cretaccous:

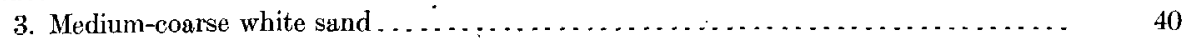

4-6. Sand and small pebbles with a rather pinkish cast ................... $50-75$

$a$ Darton, N. H., Artesian-well prospects in the Atlantic Coastal Plain region: Bull. U. S. Geol. Survey No. 138, 1896, p. 35 . 
Cretaceous - Continued.

Feet.

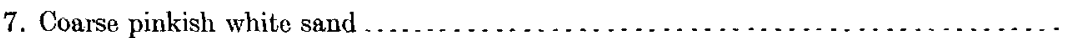

8-9. Medium, white, micaceous sand . . . . . . . . . . . . . . . . . . . . . . . . . . . 90-100

10. Very fine, gray, micaceous sand. . . . . . . . . . . . . . . . . . . . . . . . . 110

11. Medium to coarse white sand . . . . . . . . . . . . . . . . . . . . . . . . . . . . 120

12. Small angular quartz pebbles, evidently broken from larger ones. . . . . . . . . . . . 130

13. Medium to coarse white sand. . . . . . . . . . . . . . . . . . . . . . . . 130

14. Medium white sand. . . . . . . . . . . . . . . . . . . . . . . . . . . . . . . 150

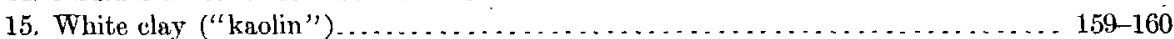

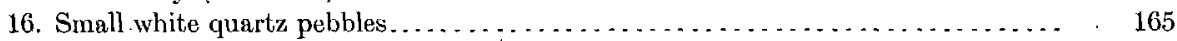

17-20. Fine, gray, micaccous sand. . . . . . . . . . . . . . . . . . . . . . . . . . 250-300

21-25. Medium-coarse white sand. . . . . . . . . . . . . . . . . . . . . . . . . . . . 215-240

26-31. Fine, white, micaceous sand. . . . . . . . . . . . . . . . . . . . . . . 250-300

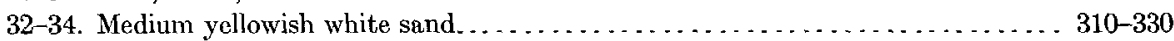

35-39. Fine sand and small quartz pebbles. . . . . . . . . . . . . . . . . . . . . $335-350$

Nearly all the samples contain fragments of milk-white chert, generally quite'soft; when first seen they may be mistaken for white, calcareous concretions.

Mr. Danis reports that fresh water was encountered at a depth of 12 feet, and that below that nothing but salty water was found. The well is about 5 feet above high tide, and flows a little salty water at high tide.

67 1. The following record has been furnished by Mr. E. K. Hutchinson to the New Jersey Geological Survey:

Record of Dr. E. H. Muncie's well on Muncie Island, New York.

Recent:

1. Muck and sand with shells.

Feet.

$0-10$

Wisconsin? and Tisbury:

2. Heavy, yellow, micaceous sand and gravel, with water salt as the ocean, standing nearly at the surface of the meadow; this stratum is very similar to that obtained from most of the shallow wells on Long Island.

Sankaty? and Jameco?

3. Clay; fine sand like beach sand; sand and clay mixed; color, blue and gray .... . 45-150 Jameco? and Cretaceous:

4. Clay, sand, etc, much like the last, only darker, with water which flowed 14 gallons a minute over the top of the casing, which was 2 feet above the ground. This water was fresh, but was colored black; about three wheelbarrow loads of wood (lignite) was pumped out; the pipe seemed to be in wood.

Cretaceous:

5. Lighter colored sand and clay mixed; amount of lignite gradually decreased. Sand a little heavier at the base where good water was obtained. Water flowed 8 gallons a minute from 2-inch pipe 2 feet above the surface . . . . . . . . . 200-270

Doctor Muncie reports: "The present well flows 15 gallons per minute; the first water obtained flowed for about 6 months and then stopped. At first the flow was about 30 gallons per mimute, but the water ontinued dirty.' 1901 :

673. The Long Island Railroad Company have furnished the following partial analysis made January,

$$
\text { Analysis of water from raitroad well near Babylon. }
$$

'otal solids.

Parts per million.

674. Mr. E. Camerdon, chief engineer, reports that two of the wells at this place were put down in 1893, and two in 1898; each well will yield 300 gallons per minute. The section is as follows:

a Ann. Rept. Geol. Survey New Jersey for 1899,1890 , p. 79 
Wisconsin and Tisbury:

Record of Sumpwams Water Company's well near Babylon.

1. Surface loam...

3. Coarse sharp sand. ... .

4. Very clcan white gravel. . . . . . . . . . . . . . . . . . . . . . . . . . . . . . 60.70

The water contains no iron, but shows slight traces of alum and salt. A detailed description of this plunt will be found in the Engineering Record, volume 43, 1901, pages 28-30.

675. When the original site of the Great South Bay Water Company plant, No. 691, was abandoned, the station was moved to this place. The present plant consists of twenty 5 -inch walls, 40 to 45 feet deep, with a capacity of 2,250,000 gallons per day.

680.

Record of C.S. Burr's well near Kings Park.

1. Dug well

Feet. retaceous:

2. Pink sand

3. White sand

Record of Captain Clarke's well near Elwood.

Cretaceous:

2. Dark, quite fine sand, sticky, no water.

$0-90$

683.

Record of Wm. Herod's well near Kings Park.

Fect.

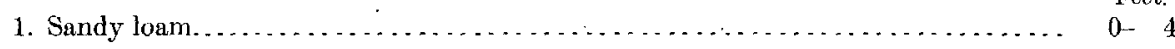

Tisbury and Cretaceous?

2. Medium white sand with occasional thin streaks of clay

$4-152$

685. Mr. Thompson reports: "I have put down five 6-inch flowing wells, the water from which is used for trout batching and growing. The first woll was sunk about ten years ago. I sunk a $1 \frac{1}{4}$-inch pipe, and got a good flow at 33 feet. A 6 -inch pipe gave 50 gallons per minute at 33 feet. I then drove a $1 \frac{1}{4}$-inch pipe inside of the 6-inch pipe, and at a depth of 45 feet got a nice flow."

Mr. H. J. Dubois, the driller, reports the following section for two wells on the south side of the ravine:

Record of Edw. Thompson's wells near Middleville.

Wisconsin and Tisbury:

1. Red loamy sand at the surface, becoming coarser and passing into gravel below.

A deeper well, put down on the northern side of the ravine, showed the following section:

Record of Edw. Thompson's well near Midalleville.

Wisconsin and Tisbury

1. Gravel with only a small amount of water

Feet.

$0-30$

Cretaceous?

2. Dark-brown clayey sand, becoming coarser below and yielding artesian water... 30 -160

686.

Record of J.F. McGiff's well near Fort Salonga.

Wisconsin:

1. Soil

Tisbury:

2. Ferruginous saud

3. Clean, light-colored, pebbly sand.

Feet.

$0-5$

$5-6$

$6-113$

Cretaceous:

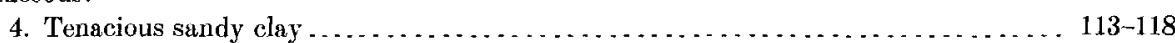

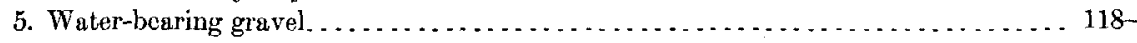


688. Mr. Velsor has furnished the following samples from this well:

Wisconsin:

$$
\text { Record of Doctor Gillette's well near Fort Salonga. }
$$

2. Glacial sand and gravel, for the most part quite clean, but containing a little silt between 35 and 38

Below 45 feet the samples show quite a little ferruginous cóncretionàry material.

691. This was the site of the original pumping station of the Great South Bay Water Company. 'The supply was from a gang of 5-inch wells, 60 fect deep, of which Mr. C. A. Lockwood gives the following data:

\section{Record of old wells of Great South Bay Water Company at Bayshore.}

1. White beach sand becoming finer near the bottom of the well ............... 0 . 60

For the first two years these wells, of which there were 12 or 15 in all, yielded a suffieient supply, but at the expiration of that time the demand increased, and a 350 -foot well was sunk to obtain a greater supply The material encountered in putting down this well was all white beach sand with some lignite at 300 feet. The water from the gang of 60-foot wells became more and more charged with iron, and had a smell similar to that of decayed vegetation; its taste was also bad. It was for this reason, together with the fact that a greater supply. was desired, that the deep tests were sunk and, when these failed, the station was moved to No: 675 .

Mr. Sands, the superintendent, has furnished the following notes regarding these deep test wells made by Mr. John C. Lockwood, the former president of the Great South Bay Water Company, who had charge of the drilling:

Record of deep test wells of Great South Bay Water Company at Bayshore.

Wisconsin and Tisbury: Feet.

1. Yellow sand and gravel ........................ 59

Sankaty?:

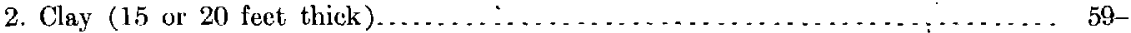

Cretaceous:

3. No record

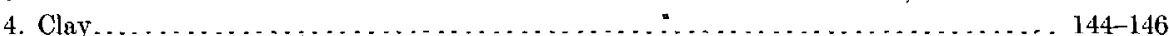

5. No record.

6. Clay (15 or 20 feet thick)

"At 262 feet got strong flow, water rising 9 feet 6 inches above surface when casing was rum unward."

The following analysis was published in the first rules and regulations of the company:

Analysis of water from old wells of Great South Bay Water Company at Bayshore.

[Analysis by C. F. Chandler, Ph. D., New York, December 5 , 1889.]

Parts per million

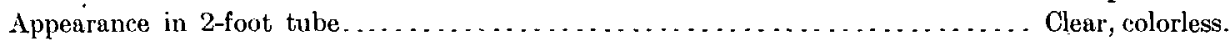

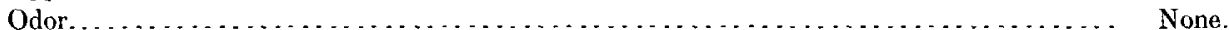

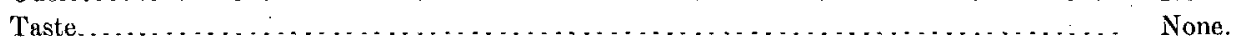

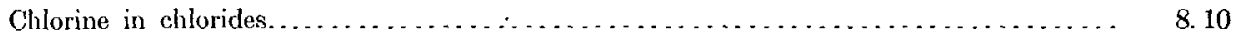

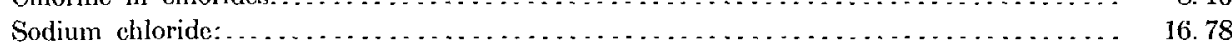

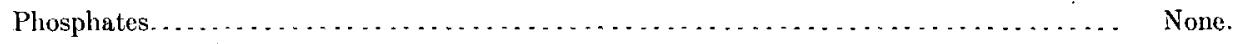

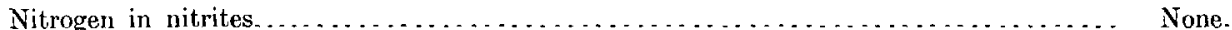

Nitrogen in nitrates. . . . . . . . . . . . . . . . . . . . . . . . . . . . . . 02

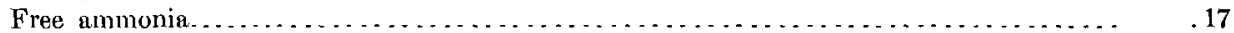

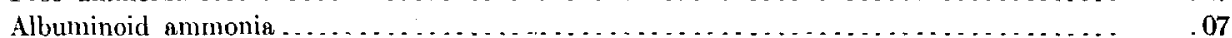

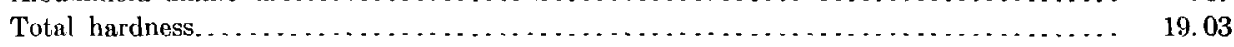

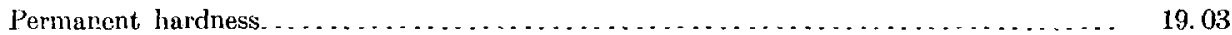


Organic and volatile matter.

Parts per million

3.99

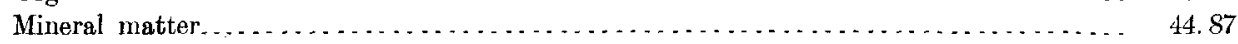

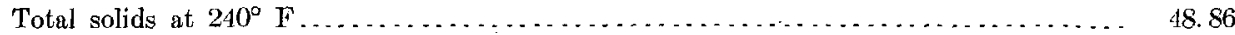

"The total amount of solid matter contained in the water is extremely small. There are no phosphates and no nitrites, both of which are regarded as evidences of contamination when present. The nitrogen in the form of nitrates is very small, and the free and albuminoid ammonia is moderate."

692.

Record of Strong well near Bayshore.

Feet.

1. Sand; no change in texture or color.

694.

Record of commission's test well near Bayshore.

Pleistocene:

1-2. Yellow surface loan...................................... 0 - 1.5

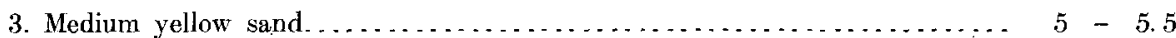

4-5. White sand and gravel................................... 10 - 16

6-21. Grayish white sand and gravel.............................. $20 \quad-100.5$

Cretaceous?:

22. Very dark brownish gray, micaceous, clayey sand ............... 101.5-102.5

Samples 1 to 21 apparently represent glacial outwash. See Table XII.

695.

Record of commission's test well near Bayshore.

1-2. Surface sandy loan. . . . . . . . . . . . . . . . . . . . . . . . . . . . . .

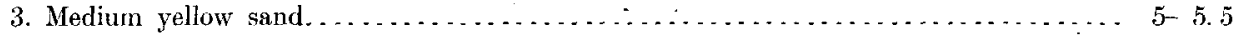

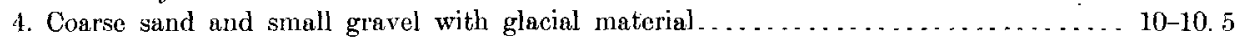

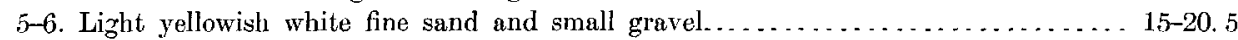

7. Small gravel with a little fine sand, containing some glacial material . . . . . . . . 25-26

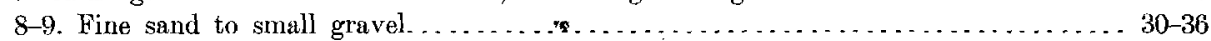

The whole section of this well is composed of glacial outwash. See Table XII.

696.

Record of commission's test well near East Islip.

Wisconsin and Tisbury:

Feet.

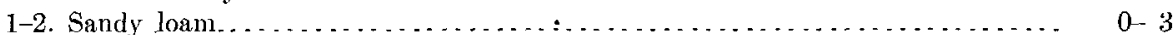

3. Light-ycllow fine sand to small gravel.......................... $3-5$

4. Grayish white sand and gravel, with considerable glacial material. . . . . . . . . 5 -10

$5-6$. Light reddish brown sand and gravel, with a small percentage of glacial material. . $\quad 10-20$

7-8. Light yellowish white fine to medium sand, not clearly glacial . . . . . . . . . . 20-30

697.

Record of commission's test well near Brentwood.

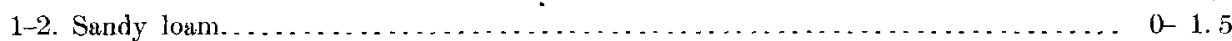

3-8. Griyish white sand and gravel; probably glacial outwash. . . . . . . . . . . $5-30$

See Table XIII.

698.

Record of commission's test well near Brentwood.

1-3. Surface loam; some gravel................................... $0-5.5$

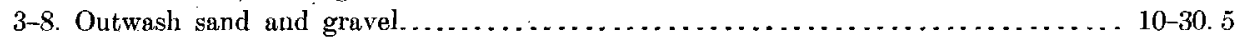

See Table XIII. 
699.

Record of commission's test well near Islip.

Feet.

1-2. Yellow surface loam.......................................... 0 . 2

3. Dark, humus-stained, medium sand. . . . . . . . . . . . . . . . $\ldots \ldots \ldots \ldots \ldots \ldots$

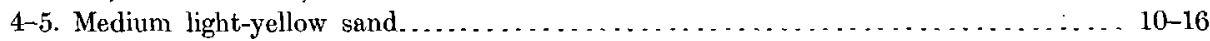

6. Small gravel, with a noticeable percentage of glacial material. . . . . . . . . . . 20 21

7-9. Dirty, yellow, fine sand to small gravel . . . . . .

This whole section appears to be of glacial origin.

700.

Record of commisçion's test well near Islip.

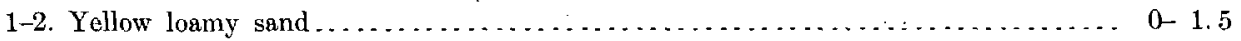

3-8. Light grayish sand and gravel, with a small percentage of glacial material. . . . . . . . $5-31$

9-10. Medium light-yellow sand; age very doubtful....................... $35-41$

701.

Record of commission's test well near Islip.

1-2. Yellow gravelly loam

3. Dark reddish brown sand and gravel with considerable glacial material.......... 2-5

4. Light-yellow medium sand to coarse gravel, with only a small percentage of glacial

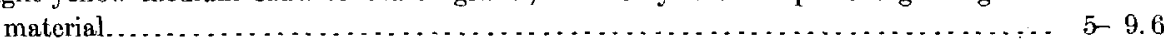

5. Very dark reddish-brown sand and gravel; very doubtfully glacial. $\ldots \ldots \ldots \ldots \ldots$. $15-35$

702.

Record of cammission's test well near Central Islip.

1. Black loamy sand...................................... 0

2-4. Fine to medium light yellow sand. . . . . . . . . . . . . .

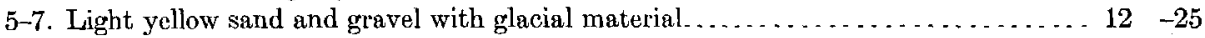

703.

Record of commission's test well near Central Istip.

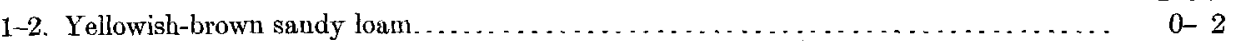

3. Medium light yellow sand:..................................... $2-5$

4-9. Light yellow sand and gravel with a little glacial material.................. 5 . 55

705. Mr. Darling states that the two wells at this point furnish 150 gallons of water per minute with deep-well pumps; with direct suction he believes they would yield 250 gallons each. He has installed an Acme system with a storige capacity of 10,000 gallons.

Record of wells at St. Joseph's in the Pines, near Brentwood.

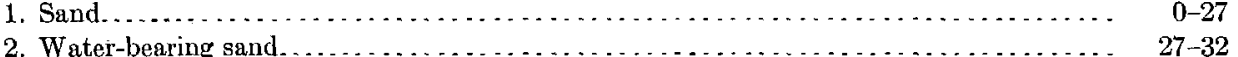

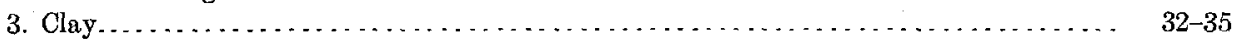

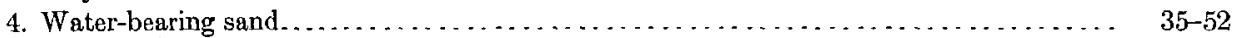

706. Mr. Codman writes regarding this locality: "In excavating for cellars or wells there is often found at a depth of 2 to 5 feet a layer of grayish-blue deposit, locally called 'blue clay.' This layer, which is 2 or 3 feet thick, on drying shows a clayey fracture, though it is wholly devoid of plastic qualities. I have washed it and find it a very fine sand. I consider it rock flour."

One mile south of the main line of the railroad the water stratum is found at a depth of 28 feet; 1 mile north of the track the depth gradually increases to 52 feet.

707. Record of commission's test well near Brentwood.

Wisconsin and Tisbury:

Feet.

$0-3$

3. Medium yellow sand with a little gravel. $\ldots \ldots \ldots \ldots \ldots \ldots \ldots \ldots \ldots \ldots \ldots \ldots \ldots \ldots \ldots \ldots \ldots$

4. Dark-drab silty sand, with a few pebbles.......................... 5 . 21 
Wisconsin and Tisbury-Continued.

5-8. Yellowish white sand and gravel.

9-10. Coarse sand and gravel, with a very noticeable percentage of erraties for this region.

11-12. Fine to coarse light yellow sand and gravel

13. Fine to coarse gravel with some sand and a small percentage of glacial material ..

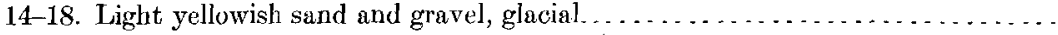

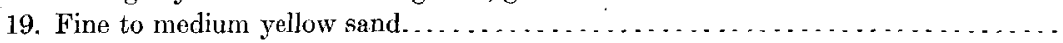
Cretaceous?:

21. Fine, yellowish brown sand and gravel. .......................... $97-100$

22. Fine to medium, grayish-yellow sand. . . . . . . . . . . . . . . . . . . 100-103 See Table XIII.

708. Record of commission's test well near Brentwood.

Wisconsin and Tisbury?

1. Black sandy loam

2. Light-yellow silt.

3-11. Light-yellow or grayish yellow sand, with considerable erratic material (glacial

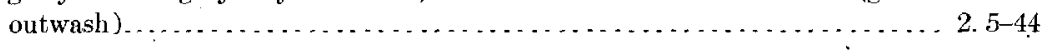

\section{See Table XIII}

\section{0.}

Record of Charles Blyndenburgh's well near Hauppauge.

Wisconsin:

1. Coarse gravel.

Wisconsin and Tisbury:

- 2. Fine sand, with thin layer of clay at $25 \mathrm{fcet}$

Tisbury:

3. Coarse gravel.

711. Mr. Price has kindly furnished the following samples from this well:

Pleistocene:

\section{Record of C. B. Pedrick's well near Smithtown.}

1. Very fine, brown, micaceous sand.

2. Grayish yellow silty sand and small, rather angular, quartz gravel; containg a few

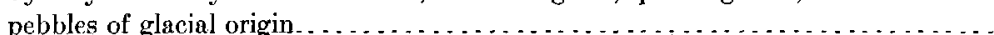

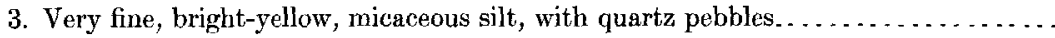

4. Medium yellow sand. $45-50$ $50-60$ $60-65$ $5-88$ $88-92$ $-100$ 03 
1. Sand, with surface water-

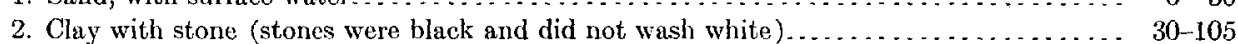

3. Black material. ................................................ . 105-110

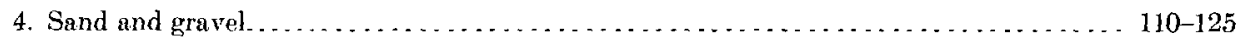

The sand from stratum 4 of this well rose in the pipe and was cleaned out and the water at once rose to within 50 fest of the surface.

714. Mr. Redwood has kindly furnished a sample from a depth of 95 fect; it is a glacial gravel, similar to that found in upper part of wells in this section.

715.

Record of E.M. Smith's well near Smithtown Branch.

Wisconsin:

Feet.

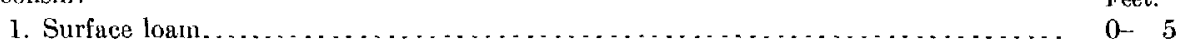

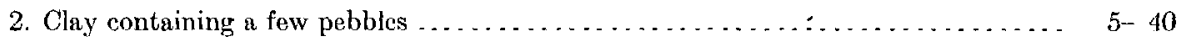

Tisbury:

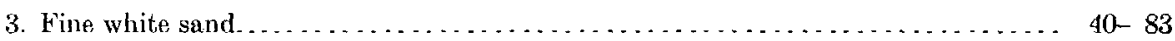

4. Good gravel........................................ $83-100$

716.

Record of C.F. Leeman's well near Smithtown Branch.

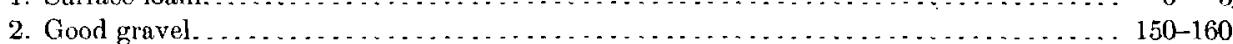

Mr. Rogers was unable to finish a complete log of this well. He thinks no clay was encountcred.

ร17. Record of Rassapeaque Club's well near Smithtown Branch.

Wisconsin or Tisbury: Feet.

1. From medium white sand at the top gradually becoming coarser until coarse gravel

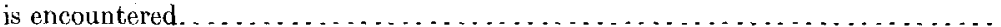

The coarse gravel in this well at 18 feet furnishes artesian water.

718. Mr. George Schmidt reports that one bed of clay was encountered in this well. He could give . no further information regarding it."

7 19. These wells were completed in 1899 and are pumped with an air lift. The supply is stated to have decriased and the water to be hard and salty.

Pleistocene? : Record of well of Society of St. Johnsland at Kings Park.

1. Sand and gravel, with surface water below 10 feet ........................ Feet.

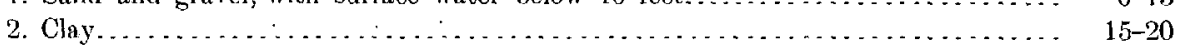

3. Sand, with main water-bearing horizon at about 40 feet................. $20-90$

720.

Record of W.W. Kenyon's well on Nissequogue River.

Tisbury:

Feet.

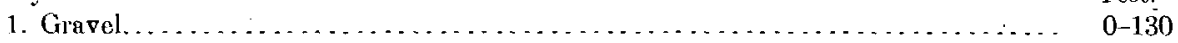

Cretaceous:

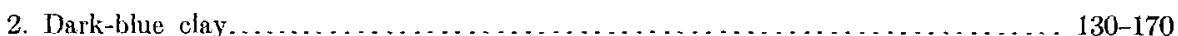

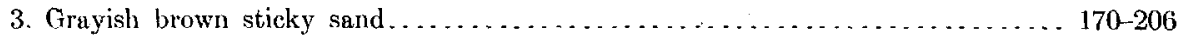

4. Coars 3 white gravel........................................ 206-212

72 $\mathrm{s}$. It is statcd that the water in this well at first stood 12 feet below the surface, but that after pumping it rose to 8 feet.

Record of W.J. Matherson's well on Nissequogue River.

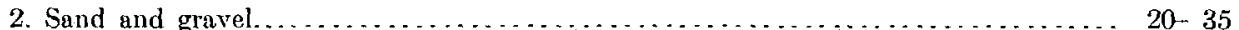

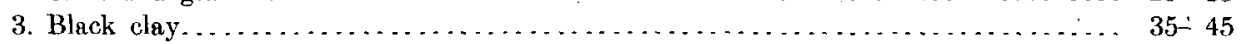

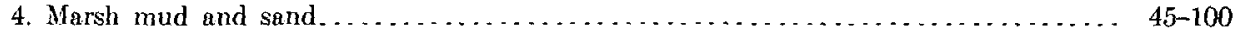

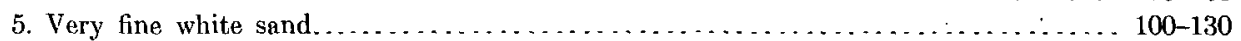

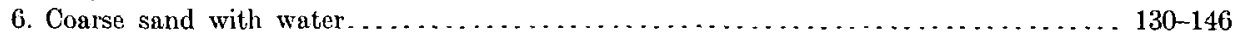




\section{UNDERGROUND WATER RESOURCES OF LONG ISLAND, NEW YORK.}

722. Mr. Rogers has kindly furnished a sample from this well from a depth of 80 fcet; it consists of brown glacial sand and gravel.

723.

Record of R. H. Smith's well near Stony Brook Harbor.

Wisconsin and Tisbury:

1. Sand................................................... 0 . 60

2. Mixture of clay and sund ........................................ $60-90$

3. Fine sand and gravel, growing coarser............................ 90-117

724. No clay or quicksand was encountered in this well, the material being entirely sand and gravel.

$72 \%$.

Record of $O$.R. Roberts's well near Oakdale.

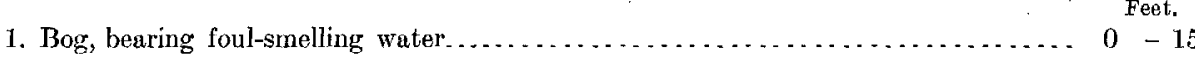

2. Fine black gravel.......................................... $15-17.5$

3. Muck, bearing foul-smelling water........................... 17. 5-170

At the depth of 170 feet, no better water having been encountered than that found at the top, the well, was abandoned. Mr. Kirk reports that in the vicinity of Swan Creck the same conditions are often encountered.

729.

Record of commission's test well near Sayville.

1. Black loamy sand.

Feet.

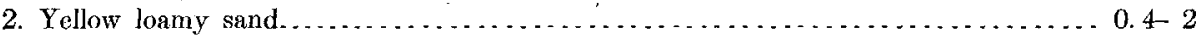

3-11. Light-yellow or grayish yellow fine sand to small gravel, with a few glacial pebbles $2-45$ See Table XIII.

730.

Record of commission's test well near Ronkonkoma.

Feet.

1. Black loamy sand.

Surface

2. Yellow loamy sand. Subsoil

7. Fine to medium white sand, with truces of lignite

8-14. Light-yellow fine sand to small gravel, with a few cratic fragments in the lower samples

$29-62$

731.

Record of commission's test well near Ronkonkoma.

2 Yellow loamy sand .

$3-5$. Grayish yellow sand and gravel with a few erratics................ $4-5$

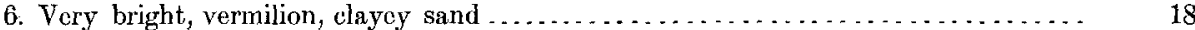

7-13. Very light grayish yellow sands and gravel, with a small percentage of glacial

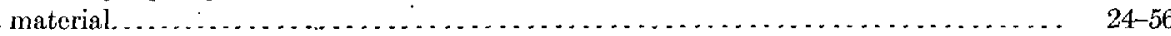

See Table XIII.

732. Record of commission's test well near Ronkonkoma.

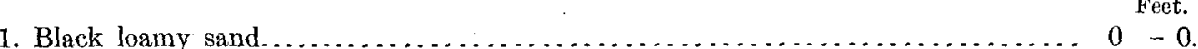

2. Yellowish loamy sand.

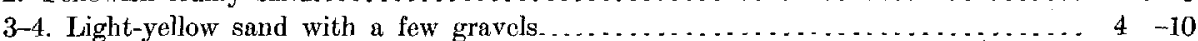

5. Very fine to medium grayish brown sand .......................... 14 .

6-9. Reddish ycllow sand and gravel, with pronounced glacial pebbles. . . . . . . . . 19 -35 See Table XIII.

734. Record of John Klaiber's well near Ronkonkoma.

Wisconsin and Tisbury:

1. Sandy loam

2. Coarse sharp sand; no stones nor clay. 
736. The location of this well as given on the map is probably slightly in error.

\section{Record of William Ralston's well near Lake Ronkonkoma.}

Wisconsin and Tisbury:

1. Surface Ioam.

- Feet.

(1)

2. Coarse gravel................................................. $5-12$

3. Medium white sand ....................................... 12-22

1. Black hardpan, with stones about the size of walnuts................. 22-25

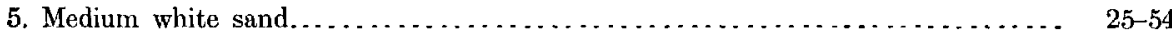

$73 \%$.

Record of J. Weber's well near Lake Ronkonkoma.

Pleistocene:

1. Sand ... :

2. Sand, with a little gravel and occasional streaks of clay . . . . . . . . . . . $13-25$

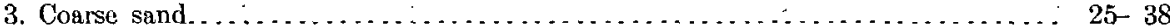

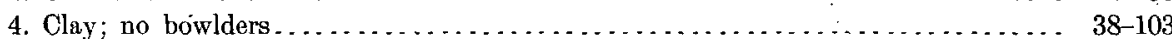

5. Water-bearing sand . . . . . . . . . . . . . . . . .

Mr. W. T. Arthur has kindly furnished the following samples from this well:

Record of J. Weber's well near Lake Ronkonkoma.

1. Medium to very coarse, dirty, quartz sand, with some small gravel; has the general aspect of glacial material, and contains a few rounded fragments of soft, fine-grained, mica schist, with biotite......................................... 103-117

2. Very fine, light-gray, silty sand, with much muscovite................. 117-

The water from the sand and gravel between 112 and 117 rises just to lake jevel.

738. There was 8 feet of water in.pipe when the well was completed; the lake level was then said to below. Later the lake level rose and a corresponding rise of the water in the well occurred.

Record of G. E. Plunkett's well near Lake Ronkonkoma.

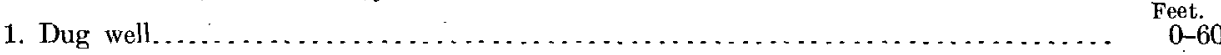

2. Coarse sand . . . . . . . . . . . . . . . . . . . . . . . . . . . . . . . . . $60-70$

739.

Record of R.W. Newton's well near Lake Ronkonkoma.

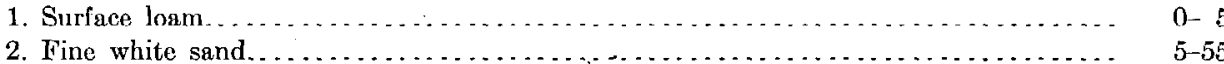

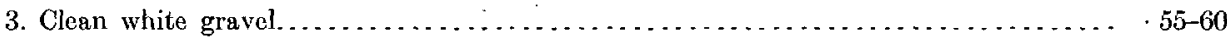

Mr. Rogers reports that where waterworn gravel is encountered at any depth exceeding about 50 feet the supply of water is always abundant and good. This statement of Mr. Rogers is equivalent to saying that wherever a coarse gravel is found below the main water table an abundant supply is obtained (p. 67).

A sample furnished by Mr. Rogers, marked "Newton well, 60 feet, 1896," is fine light-yellow glacial sand with some gravel.

740. Water stands 8 feet below surface; this is said to be at the same level as Lake Ronkonkoma.

Pleistocene: Record of well of W. Imhauser estate near Lake Ronkonkoma.

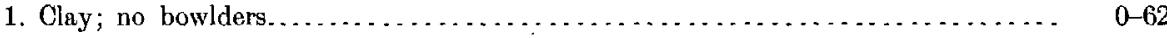

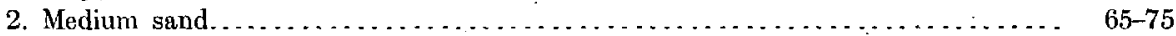

741. Mr. Ralston, who has lived near Lake Ronkonkoma all his life, reports that in digging or driving wells on the west side of the lake a considerable thickness of clay is encountered in nearly every instance, while on the east side the material is for the most part sandy, the sand being of the kind known as "beach sand." 


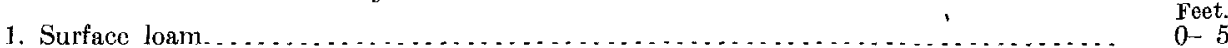

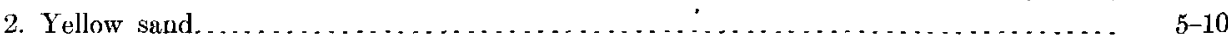

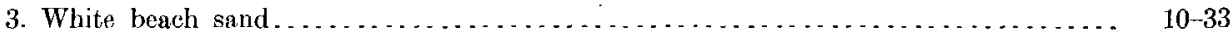

743. Record of W. H. Warner's well near Lake Ronkonkoma.

Wisconsin and Tisbury:

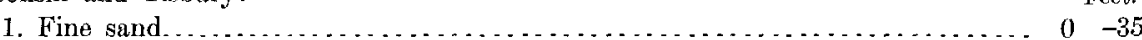

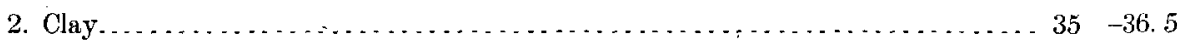

3. Sand and gravel........................................... $36.5-47$

744. In wet weather the water is milky, indicating, Mr. Terry thinks, that clay lies a short distance below 86 feet.

Wisconsin and Tisbury

Record of John Morrissey's well near Lake Grove.

1. Stony sand....

2. Coarse sand; no stones. ................................... 8

3. Yellow sand, described as being like the subsoil in sandy places. . . . . . . . . . $15-17$

4. Coarse sand, with stones varying in diameter from 4 to 6 inches......... $17-45$

5. Stones and gravel........................................ $45-58$

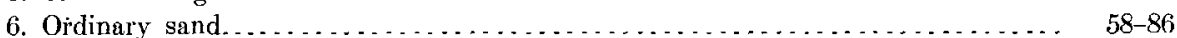

745. Record of Irving Overton's well near Lake Grove.

Wisconsin and Tisbury:

1. Stony top soil.

Finer material to fino sand....

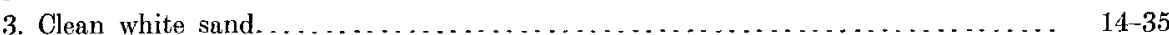

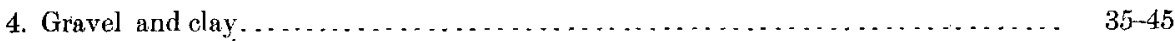

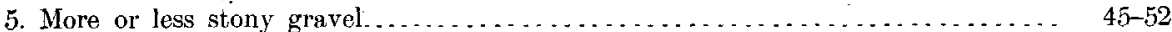

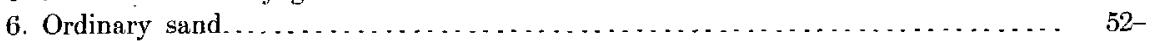

746.

Record of Doctor Monecke's well near Lake Grove.

1. Light sandy top soil

Feet.

2. Yellow sand subsoil, no stones.................................... 4- 8

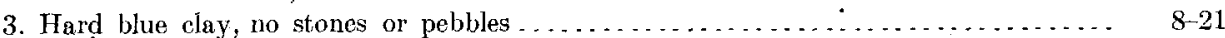

4. "Mica mud"................................................ 21-24

Water rose in the pipe, stopping further work. The water was muddy, but had no bad odor or bad taste.

In putting down another well on this same property for Doctor Monceke, leaves and muck were cncountered at 23 fect. There was a 14-inch stratum of this material, and the water coming from it had a very bad odor.

749.

Record of commission's test well near St. James.

Feet.

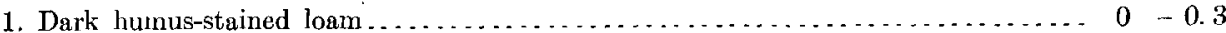

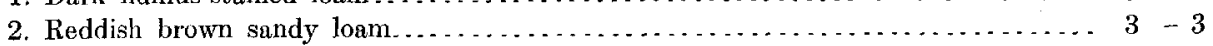

3. Brownish yellow sand and gravel.............................. $3-.6$

4-11. Dirty, gray, fine sund to coarse gravel; a small quantity of glacial material... $6-45$

12-14. Dark, yellowish gray, very fine to coarse sand, glacial . . . . . . . . . . . . . 45 -59

750.

Record of Father Ducey's well near st. James.

Wisconsin:

1. Hardpan, a compact mixture of sand and gravel, containing bowlders

Feet.

$0-60$ Tisbury:

2. Gravel and sand ........................................... 60-150

Mr. Rogers has sent the following sample from this well:

1-2 Clean, light-colored sand and gravel, clearly glacial, perhaps Tisbury $140-150$ 
$\% 51$.

Record of Jerome Saxe's well near St. James.

Pleistocene:

Feet.

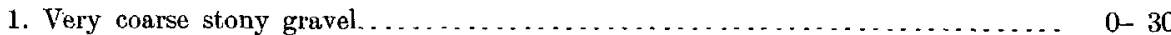

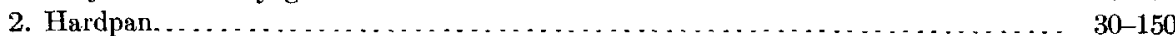

3. Clay containing a few stones.............................. 150-160

4. Gravel, etc.... . . . . . . . . . . . . . . . . . . . . . . . . . . . . . . . . . 160 . 208

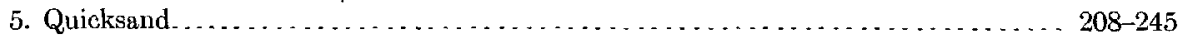

6. Clean white gravel...................................... 245-250

Mr. Rogers has furnished the following sample from this well:

1. Clean, light-colored glacial sand and gravel at . .

752. Record of D. Emmett's well near St. James.

1. Sand and gravel stones from the size of a fist to a robin's egg $\ldots \ldots \ldots \ldots \ldots \ldots . . . .60$

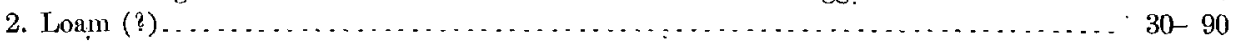

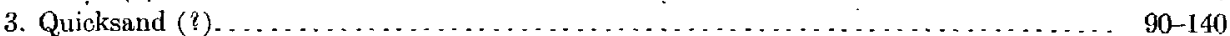

4. Clay, with thin layers of gravel. . . . . . . . . . . . . . . . . . . . . . . . . . .

5. Quicksand................................................... 190 .290

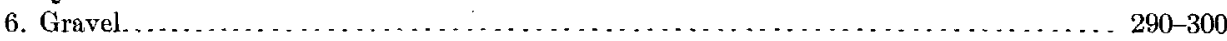

753. Mr. Rogers reports this well to be 82 or 83 feet above the level of the Sound, and that the water in the well rose to the level of the water in the Sound. He has furnished a sample of clean, light-colored glacial sand and gravel taken at a depth of 97 feet:

Wisconsin and Tisbury:

Record of D. Emmett's well near St. James.

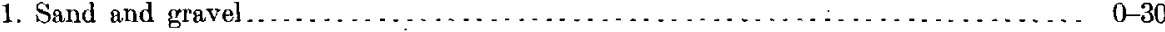

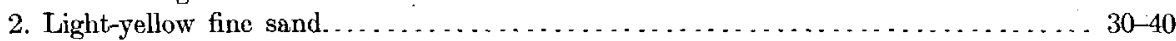

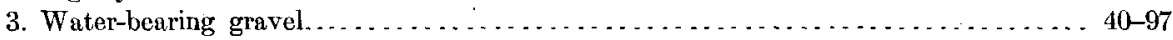

754. According to the location indicated on the map, the depth to water in this well is abnormally great, and it is quite possible that the location is an error.

Wisconsin and Tisbury: Feet.

1. "Till" (probably also including outwash gravel) $\ldots \ldots \ldots \ldots \ldots \ldots \ldots \ldots \ldots . . \ldots \ldots \ldots$

Cretaceous:

2. Fine pink sand.

$140-160$

755.

Record of commission's well near St. James.

Wisconsin:

Feet.

1-2. Brown surface loam $\ldots \ldots \ldots \ldots \ldots \ldots \ldots \ldots \ldots \ldots \ldots \ldots \ldots \ldots \ldots \ldots \ldots \ldots \ldots \ldots \ldots \ldots$
3. Very fine yellow loam and sand $\ldots \ldots \ldots \ldots \ldots \ldots \ldots \ldots$

Wisconsin and Tisbury:

4-16. Fine sand and small gravel, yellowish gray above and darker below (glacial) . . . 5-69

Tisbury:

17-20. Dark, yellowish gray, fine to medium sand, probably glacial. . . . . . . . . 69-90

The material shown in samples $17-20$ is the same as that shown in samples 12-14 in well No. 749. Sec Table XIII.

756.

Record of commission's test well near St. James.

Wisconsin and Tisbury:

1. Humus-stained surface loan

Feet.

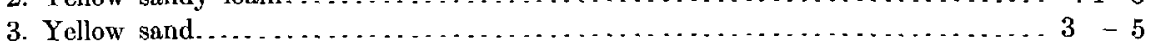

4-16. Gray sand and gravel (considerable glacial material) . . . . . . . . . . . . $5 \quad-70$

757. This well was first dug to 94 feet and then the 6 -inch pipe sunk 40 feet. The sinking of the pipe did not in any way aflect the water level in the well.

$17116-$ No. $44-06-21$ 


\section{UNDERGROUND WATER RESOURCES OF LONG ISIAND, NEW. YORK.}

759.

1. Surface loam.

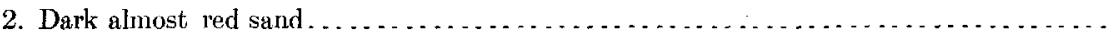

3. Quicksand

$10-60$

4. Coarse, dark, almost red sand ...................................... 106-107

760.

Record of W. Rowland's well near Setauket.

Wisconsin:

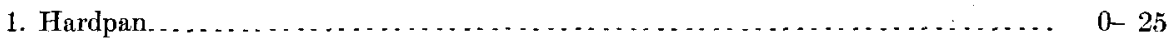

Tisbury:

2. Sand and gravel with one or two strata of hardpan $\ldots \ldots \ldots \ldots \ldots \ldots \ldots \ldots$ 25-60

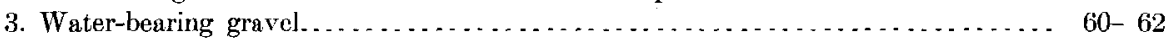

Cretaceous?:

4. Quicksand mixed with some clay ............................. $62-251$

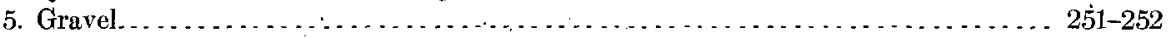

761.

Record of William Clarke's well near Setauket.

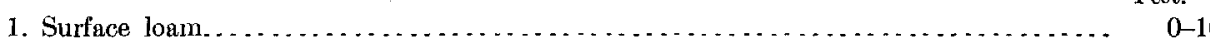

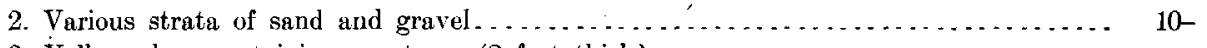

3. Yellow clay, containing no stones (2 feet thick).

4. Quicksand.

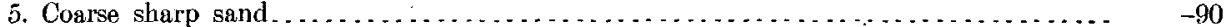

762.

Record of Howard Wallace's well near Setauket.

Wisconsin and Tisbury:

1. Surface loam.

2. Coarse sand and gravel. ...................................

3. Quicksand ............................................... $50-52$

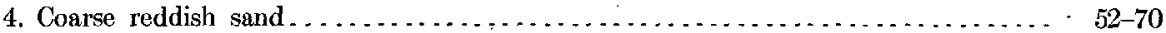

Mr. Rogers has furnished a sample marked " 70 feet, 1896," which is clearly glacial material.

763. The following samples have been received from this well:

Tisbury:

Record of W.T.Cox's well near Setauket.

1. Light, brownish yellow glacial sand and gravel, possibly Tisbury .....

Cretaceous:

2-3. Very fine, dark-gray, micaceous, sandy clay .................... $85-132$

4. Medium to coarse, white, quartz sand,.with some mica and white clay . . . . . . 132-145

5. Fine and coarse, gray, micaceous sand ....................... 145-155

6. Light-yellow medium sand . . . . . . . . . . . . . . . . . . . . . . . . . . . . . . 155-180

7. Light-yellow clayey sand. . . . . . . . . . . . . . . . . . . . . . . . . . . . . . . $180-188$

Water is reported to have stood only 4 feet below the surface when well was between 145 and 155 feet, but at 188 feet it stood 10 feet below the surface. Under date of October 5, 1903, Mr. W. T. Cox reports: "The water came from.fine gravel inixed with sand, which looked like brown sugar. The water was obtained, Mr. Hutchinson told me, at 320 feet. He measured the flow carefully and stated that it was 18 gallons a minute at low water and considerably more at high water. Water was abundant from 188 feet to the bottom of the well, but the fineness of the material prevented a flow, which commenced when a slightly coarser layer was encountered at 320 feet."

764.

Record of Nort House well at Setauket.

Wisconsin:

1. Hardpan

Tisbury:

2. Medium white sand. 
765.

Record of Charles Benner's well near Setauket.

Wisconsin:

Feet.

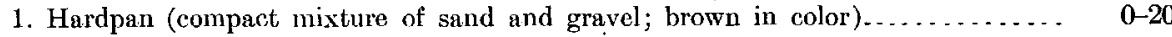
Tisbury:

2. Medium white sand.

$20-50$

7 66. The elevation of this well is said to be 6 feet above tide level. At a depth of 38 feet it was abandoned on account of the constantly increasing supply of salty water. Mr. Rogers reports that several other wells on the same property 20 to 30 feet deep gave fresh water. One of these wells is about 10 feet above high water and the other 5 feet.

768.

Record of John Thatcher's well, Crane Neck.

$$
\begin{aligned}
& \text { Feet. } \\
& 0-10 \\
& 10-50
\end{aligned}
$$

2. Gravel, with occasional streaks of hardpan $\ldots \ldots \ldots$
3. Clay and quicksand $\ldots \ldots \ldots \ldots$

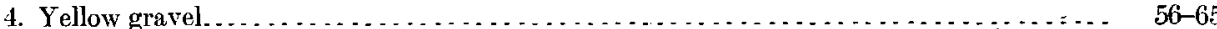

769.

Record of well near Old Field Point.

1. Hardpan

Feet.

$0-40$

2. Clean, fine gravel . . . . . . . . . . . . . . . . . . . . . . . . . . . . . . . . . . . . $40-50$

At the depth of 50 feet salt water was encountered and the well was abandoned.

7 70. A good water-bearing strata was encountered at 36 feet. Mr. Rogers reports that a number of wells in this immediate vicinity give a good yield of fresh water at about the same depth. Well No.769 is the only exception of which he knows.

771.

Record of well near Mount.Misery Point.

Wisconsin and Tisbury:

Feet.

1. Sand, with salt water

$0-110$ Cretaceous?

772.

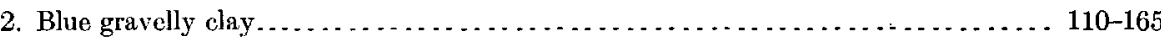

1. Fine sand of different colors, sometimes red and sometimes white, changing to good, clear, water-bearing gravel.

773. Wisconsin and Tisbury:

Record of Long Island Railroad well at Bayport station.
1. Surface loam
2. Yellow sand
3. Clay; no bowlders.
4. White sand

There was no change in the coarseness of the material in stratum 4. Mr. Arthur reports that in digging wells near Bayport occasional patches of clay may be found, but that such occurrences are rare.

774.

Record of Sea Cliff Hotel well at Patchogue.

Wisconsin and Tisbury:

1. Loam

2. Medium reddish gravel

$0-4$

3. Blue clay; no stones. .....

4. Very fine sand. 
316 UNDERGROUND WATER RESOUROES OH LONG ISLAND, NEW YORK.

775.

Record of Nassau Oyster Company's well at Patchogue.

1. Limbs, stumps, and trunks of trees.

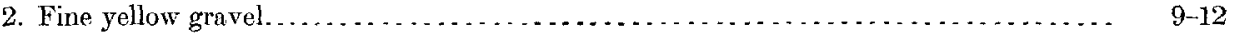

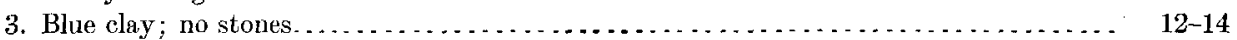

4. Muck and black loam.......................................... 14-49

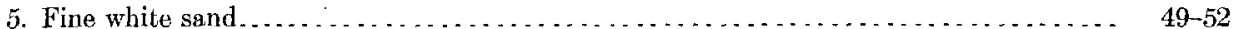

6. Black mud.................................................. $52-72$

At this depth very black water was encountered and the well was abandoned. The pipe was then pulled and the location changed 90 or 100 feet north, where the pipe was driven 19 feet through the following material:

Record of Nassau Oyster Company's well at Patchogue.

1. Sandy luam.

2. Medium yellow gravel.

3. Clean medium sand.

1. Loam.

2. Yellow gravel

3. Fine white sand

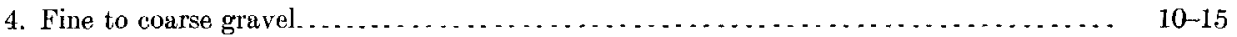

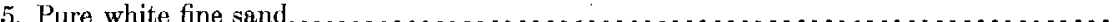

788.

Record of commission's test well near Patchogue.

Wisconsin and Tisbury:

1-2. Surface loam.

3-13. Light, yellowish gray, speckled sand (nothing clearly glacial) -...-....

14-20. Reddish brown fine to coarse sand (glacial) . . . . . . . . . . . . . . . $59-90$

Cretaceous:

22-28. Very fine, micaceous, gray to olive-green sand.................... 99-129.50

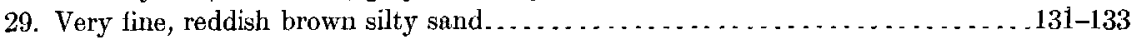

30. Dark brownish gray, very fine silty sand .......................... 134-135

31. Dark yellowish brown silt to coarse sand . . . . . . . . . . . . . . . . . . . 139-140

See Table XIII.

779.

Record of commission's test well near Patchogue.

Wisconsin and Tisbury:

1-2. Medium silty sand........................................... 0 1

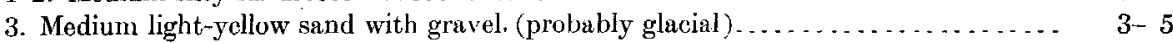

4-12. Fine to medium light-yellow sand (glacial) .................... 10-50

780.

Record of commission's test well near Patchogue.

Wisconsin and Tisbury?:

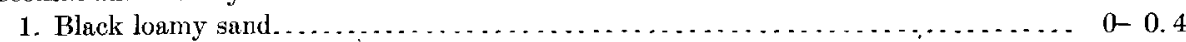

2. Medium yellow sand ...................................... 4 . 2

3-12. Yellowish white fine to medium sand, with a few pebbles (age very doubtful) _ _ 3-50

781. Record of commission's test well near Patchogue.

Wisconsin and Tisbury:

1-2. Yellow silt.

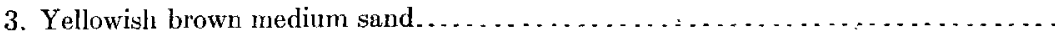

4-9. Light grayish white sand and gravel; the gravel is quite mottled and is probably

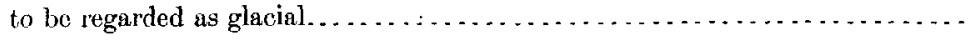

Cretaceous:

10. Grayish white medium sảnd, with much silvery white muscovite; suggests Cretaceous material . . . . . . . . . . . . . . . . . . . . . . . . . . . . .

11-12. Yellowish white fine to coarse sand.......................... 44-51

See Table XIIII. 


\section{DESCRIPTIVE NOTES ON WELLS.}

782.

Record of Reynolds well near Holbrook.

Pleistocene:

1. White sand and gravel.

Mr. Kirk reports that there was no change in the material at increasing depths. At 65 feet a bowlder the size of a man's head was encountered.

784.

Record of commission's well near Farmingville.

Wisconsin:

1. Dark humus-stained sand $\ldots \ldots \ldots-0.2$

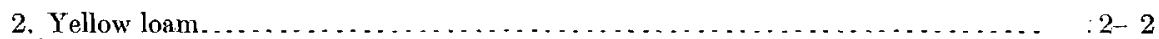

3. Bright-yellow medium sand ............................... $3-5$

4. Dark-gray sand and gravel, with much glacial material $\ldots \ldots \ldots \ldots \ldots \ldots \ldots$ Tisbury:

$5-6$. Fine to coarse yellow sand . . . . . . . . . . . . . . . . . . . . . . . . 14 -20

7-12. Light-gray sand and gravel, with some glacial pebbles............. $24 \quad-50$

785.

Record of A.P. Terry's well near Farmingville.

Wisconsin:

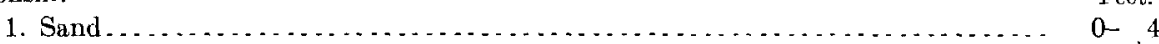

2. Gravel and stones 2 to 12 inches in diameter...................... 4- i6 Tisbury:

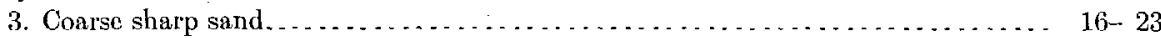

4. Gravel.................................................. 23- 37

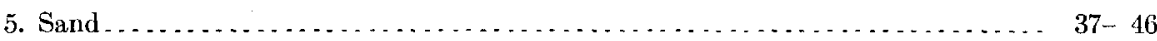

6. Gravel and stones....................................... $46-63$

7. Coarse sand ................................................... $63-71$

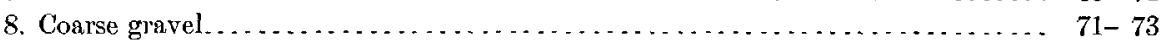

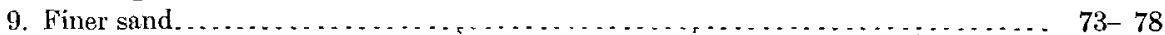

10. Sharp, white, coarse sand, with black specks...................... $78-86$

11. Coarse gravel. ........................................... $86-90$

12. Coărse sand . . . . . . . . . . . . . .

13. Sandy gravel . . . . . . . . . . . . . . . . . . . . . . . . . . . . . . . . $94-104$

14. Coarse gravel and small stoines.................................. 104-110

At 54 feet a stone 10 by 14 inches was taken out of this well.

786.

Record of August Fuch's well near Farmingville.

Wisconsin:

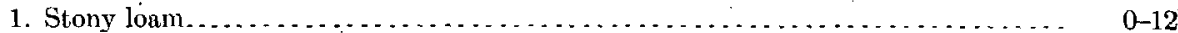
Tisbury:

2. Coarse white sand, with occasional stones 3 to 5 inches in diameter......... $12-40$

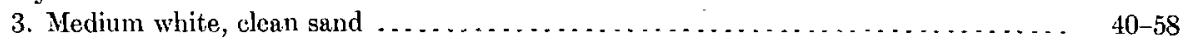

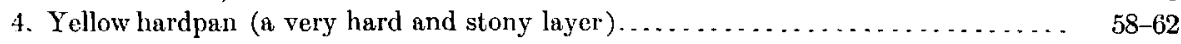

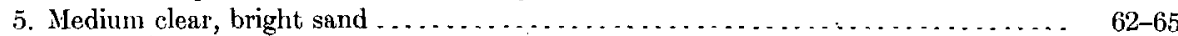

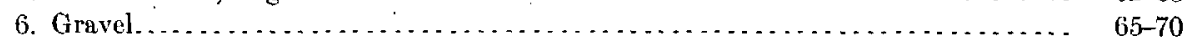

Mr. Terry reports that at 70 feet he struck "real" hardpan, on top of which water was found.

$78 \%$

Record of D. Schwarting's well near Farmingville.

Wisconsin:

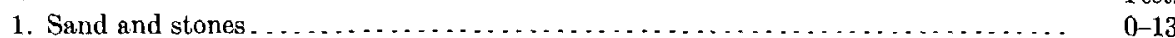

2. Coarser material; mixture of loam, gravel, and stones............... Tisbury:

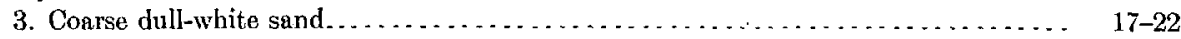

4. Sandy material, with some mica and an occasional stone............... $22-27$ 


\section{UNDERGROUND WATER RESOURCES OF LONG ISLAND, NEW YORK.}

788. Record of William Clark's well near Farmingville.

Wisconsin and Tisbury:

1. Heavy gravelly loam ... . . . . . . . . . . . . . . . . . . . . . . . . $6 \ldots$

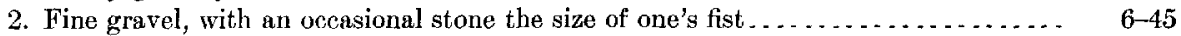

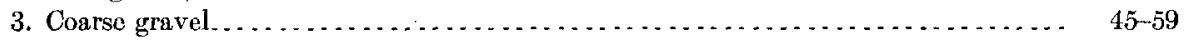

Material became coarser at increasing depths, and water was found in very coarse stony gravel.

789.

Record of Mrs. Max Richler's well near Farmingville.

Wisconsin and Tisbury:

1. Gravelly loan

Feet.

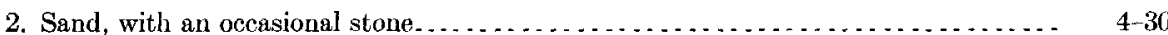

3. White clean sand. . . . . . . . . . . . . . . . . . . . . . . . . . . . . . . . . . . . . . 30 .

790. This well is described as at "Waverly, 3 miles northwest of Holbrook," and its exact location not known.

Record of Frank: Franz's well at Waverly.

1. Sund and gravel.

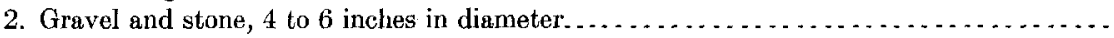

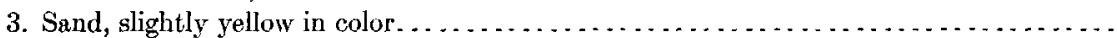

4. Ordinary sand.

791.

Record of J.F. Byrne's well near Selden.

1. Sand and gravel.

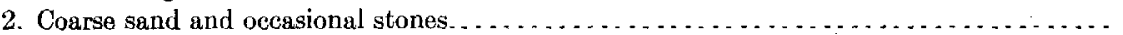

3. Coarse stony gravel.

$55-80$

4. Coarse, sharp, white sand, containing black specks which were thought to be iron pyrites.

ร92.

Record of Doctor Emerson's well near Selden.

1. Sandy top soil.

Feet.

$0-14$

14-30

$30-45$

$45-64$

2. Medium, white, fino sand; no mica

3. Very fine, hard, gray material, with a great deal of mica; soft and velvety to the touch.

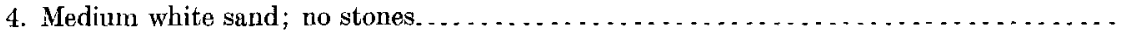

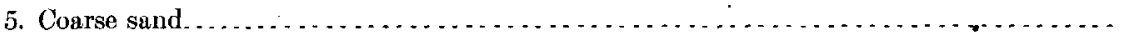

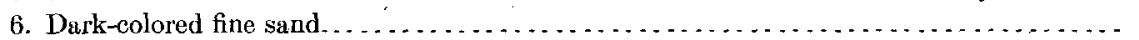

7. Coarse sand

1. Light sand.

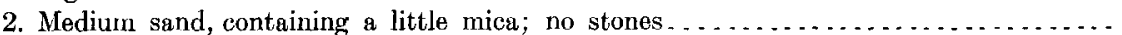

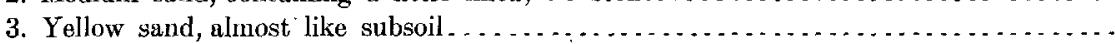

4. Mica sand, said to glisten in the sun like silver, this being probably due to the presence of muscovite; there were no stones in this stratum $\ldots \ldots \ldots \ldots \ldots \ldots \ldots \ldots \ldots \ldots$

On account of the increasing stickiness of the sand Mr. Terry believes that clay underlies it.

794.

Record of Adolph Sembler's well at New Village.

1. Medium dull sand; no top soil.

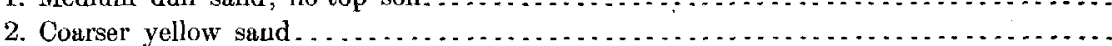

3. Very fine mica sand; from 8 to 32 feet the sides of the well stood-up like a wall; there

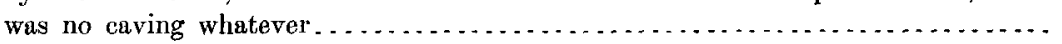

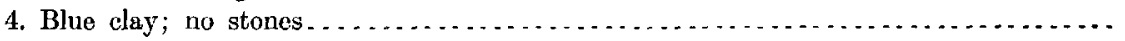

5. "Mica mud". 
795.

Record of commission's test 'well rear Terryville.

Wisconsin and Tisbury?:

1. Fine yellowish gray sand, evidently filling $\ldots \ldots \ldots \ldots \ldots \ldots \ldots \ldots \ldots \ldots, 0 \ldots \ldots$

2-3. Yellow sandy loam . . . . . . . . . . . . . . . 6

4-5. Yellowish gray sand with a few erratics..................... $66^{-}-15$

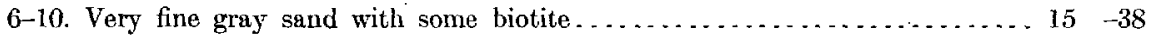

Cretaceous?:

11-16. Fine to coarse reddish yellow sand........................ $40 \quad-70$

796. $\quad$ Record of commission's well near Terryville.

Wisconsin and Tisbury: Feet

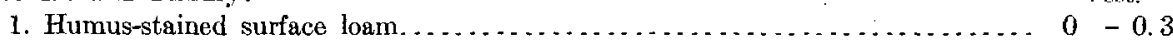

2. Dark reddish sandy loam........................................ 3

3-4. Light-yellow medium sand, passing gradually into bed below .......... $3-10$

$5-11$. Fine grayish white sand with muscovite and biotite.............. $10 \quad-45$

12-14. Medium to coarse light-yellow sand . . . . . . . . . . . . . . . . . . . $45 \quad-57$

See Table XIII.

797.

Record of commission's test well near Echo.

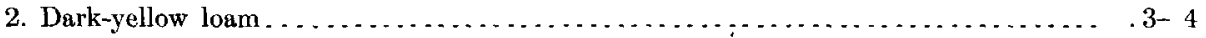

3. Medium yellow sand . . . . . . . . . . . . . . . . . . . . . . . . .

4-19. Dirty gray sand to small gravèl; small percentage of giacial material. . . . . 5 - 45

Samples 13 and 14,50 to 60 feet below the surface, show a very considerable amount of glacial material.

798.

Record of commission's test well near Echo.

2. Dark-yellow sandy loam....

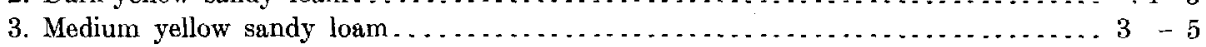

4-21. Dirty gray fine sand to small gravel; very small percentage of glacial material. $5-95$

See Table XIII.

800. This well is approximately 5 feet above mean high tide. Mr. Overton reports it to be 63 feet deep, which would more nearly agree with the depth of the Port Jefferson Water Company's wells, which obtain their water at about a depth of 50 feet. They are also flowing wells. As the well was attached to a ram, the depth could not be measured readily.

Record of J.J. Overton's well near Port Jefferson.

Feet. $0-4$

1. Loam

Tisbury:

2. Coarse white sand with occasional layers of white gravel.............. 4-20

801.

Record of J.L. Darling's well near Port Jefferson.

Tisbury:

1. Sandy loam...

2. Medium yellow to white sand $\ldots \ldots \ldots \ldots \ldots \ldots \ldots \ldots \ldots \ldots \ldots \ldots \ldots \ldots \ldots \ldots \ldots . . \ldots \ldots$

Cretaceous?

3. Sticky brown to drab colored clay . . . . . . . . . . . . . . . . . . . . . . . 20-40

4. Medium white sand ....................................... 40.96

The elevation of the surface at this well is approximately 50 feet above mean high tide. The clay described in stratum 3 is similar to that found in the brickyard 150 yards south of Mr. Darling's house.

803. The supply of the Port Jefferson Water Company is from two 6-inch wells, 54 feet deep, which will normally flow about 4 feet above the surface. One well tests 7,000 to 8,000 gallons per hour, while the two together give only 8,000 to 9,000 . Mr. T. B. Rogers gives the following section of these wells: 
Wisconsin and Tisbury?:

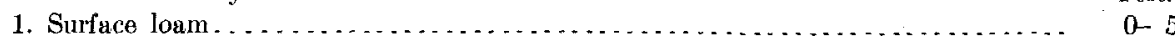

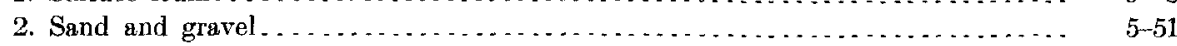

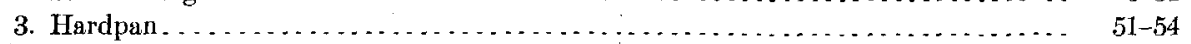

Mr. Rogers also furnishes a sample of the main water-bearing stratum, which is a clean, highly erratic, glacial sand.

The Long Island Railroad Company has furnished the following partial analysis of water from the mains of the Port Jefferson Water Company (March 30, 1903):

Analysis of water from Port Jefferson Water Company's wells, Port Jefferson.

Parts per million.

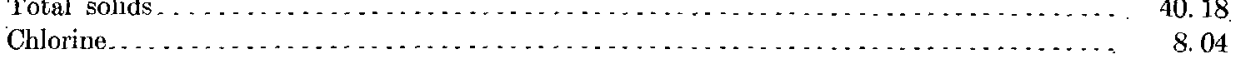

804. This well will flow about 5 feet above high tide. By means of a ram it supplies the bank and adjoining buildings.

Tisbury:

Record of N.W. Davis's, well, Port Jefferson.

1. Medium white sand.

Feet.

$0-7.5 .5$

806. Mr. Davis reports that this is a closed-point well and that he can give no record of the material passed through, but that it appeared to be very fine sand with probably some clay. He bases this opinion on the amount of fine sand which was pumped out during the water tests at different depths. At 140 feet very coarse material, probably coarse gravel, was encountered.

807. Record of J.W. Brown's well near Port Jefferson.

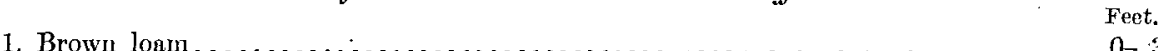

Tisbury:

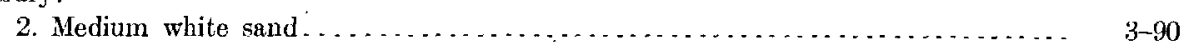

808. Record of J. Biddle's well near Port Jefferson.

Wisconsin: $\quad$ Feet.

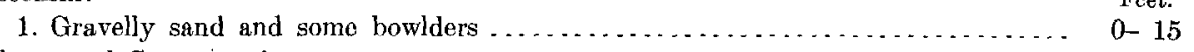

Tisbury and Cretaceous?:

2. Medium white sand with a little brown, sticky clay at about 100 feet . . . . 15-120

Attempts were made to dig a well on ground 20 feet higher, but the effort was abandoned on account of bowlders.

811. The following samples have been received from Mr. Rogers:

Tisbury:

$$
\text { Record of well of Port Jefferson Company, Port Jefferson. }
$$

1. Medium light-colored sand (glacial) . . . . . . . . . . . . . . . . . . . . . . . Feet.

2. Light-colored sand and gravel; fragments of ferruginous concretions and considerable erratic material . . . . . . . . . . . . . . . . . . . . . . . . . . . .

3. Light-colored glacial sand and gravel . . . . . . . . . . . . . . .

4. Same as 3 .

Cretaceous:

5. Dark-drab clay, containing some coarse quartz sand; leaves the fingers white as does Cretaceous material . . . . . . . . . . . . . . . . . . . . . . . . . . . .

6. Light-drab clay, containing sorne coarse quartz sand, evidently from laminated

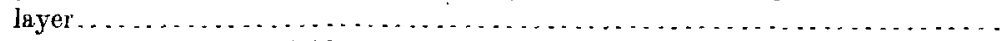

7. Fine to medium, white, highly micaceous, quartz sand ("not much water") ..... 
812. Mr. Davis reports that the surface in the vicinity of this well is rather thickly covered with bowlders and that he expected to encounter them in putting down this well, but that not a single bowlder was encountered, nor even coarse gravel.

Record of J.H.Hopkins's well near Mount Sinai.

Feet.

$0-3$

1. Surface loam..

2. Medium white sand.

813.

Record of J.M. Shaw's well near Bellport.

1. Surface loam and yellow sand

Fect.

$0-4$ 'Tisbury:

2. White sand with no change in coarseness. . . . . . . . .

814.

Record of W. McGee's well, 2 miles west of Yaphank station.

Wisconsin and Tisbury:
1. Loamy top soil, no stones.
2. Coarse sand.

Mr. Terry reports that the material of this well was the most even in character that he ever found.

818.

Record of Judge Bartlett's well near Middle Island, New York.

Wisconsin:

1. Hardpan.

Mr. Terry reports that the stones were embedded in a very heavy loam. Water was encountered at 8 feet, in a 6 -inch stratum of yellow mud. Another similar stratum was found at 32 feet. In the spring of the year the water stands 8 feet below the surface; in the dry season at 30 or 32 feet. The water at the 8 -foot level is impure.

819. Record of Hawman Brothers' well near Rocky Point.

1. Surface loam

Feet.

$0-3$ Tisbury:

2. Medium white sand with no gravel nor clay

822.

Record of Mrs. Groty's well near Manor.

Wisconsin?:

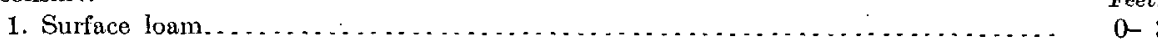

2. Clay, no stones. . . . . . . . . . . . . . . . . . . . . . .

3. Sand . . . . . . . . . . . . . . . . . . . . . . . .

824. Mr. Davis says that none of the water-bearing material in this well can be called gravel: In most of the wells in the vicinity of Port Jefferson he calculates on getting water a little above sea level, the elevation of the water level being greater at greater distances from the sea.

Record of G. E. Hageman's well near Wardenclyffe. Tisbury:

2. Medium white sand.

25. Mr. Nikola Tesla reports the following section:

Record of Nikola Tesla's well near Wardenclyffe.

Tisbury and Cretaceous?:

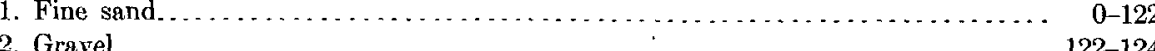

3. Alternating layers of sand and gravel........................ 124-166 
"At a greater depth than that shown in this well layers of fine sand and gravel, ench about 2 feet thick, alternate seemingly to an infinite depth. This was observed in digging a large shaft near the well, and it is assumed that the soil in the well is of the same character."

A sample furnished by Mr. T. B. Rogers, the driller, from a depth of 167 feet, is a clean glacial sand and gravel. The shaft referred to in Mr. Tesla's letter was 135 feet deep. In the bottom of this two pipes were driven at angles of 45 degrees. According to $\mathrm{Mr}$. W. H. Beers, the driller, the section is as follows, depths along the pipe having been reduced to vertical depths:

Tisbury:

$$
\text { Record of shaft sunk near Nikola Teṣla's well, Wardenclyffe. }
$$

1. Sand and gravel in dug well

Cretaceous?

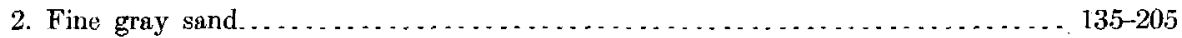

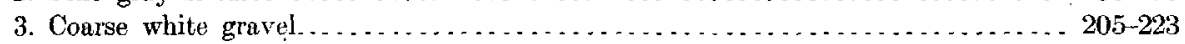

Cretaceous:

4. Fine white sand. . . . . . . . . . . . . . . . . . . . . . . . . . . . . . $223-347$

826. According to Mr. Saxe, this well shows medium-white fine sand all the way to its bottom at 90 feet. A sample from a depth of 80 feet shows light-yellow sand not clearly glacial.

Mr. Warden reports the following section:

Record of well of the North Shore Industrial Company near Woodville Landing.

Wisconsin :

1. Sand and clay.

Feet.

isbury and Cretaceous?

2. White sand, very fine.

3. Gravel, growing coarser.

827. Record of Wardenclyffe Brick and Tile Company's well, Woodville Landing.

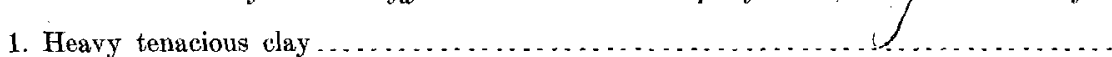

2. Coarse gravel.

Feet.

$0-4 \overline{7}$

$47-$

828.

Record of well of Long Island Railroad at Wading River.

Feet.

$0-5$

$5-110$

2. Coarse white sand, passing below into course gravel

Feet.

1. Creck mud, bearing very black water..............................

Wisconsin:

2. Hardpan (an iron cemented mixture of clay and stones).

$20-38$

The pipe broke at a depth of 38 feet and the well was abandoned.

830.

Record of $\dot{S} . W$. Wheeler's well near Wading River.

1. Surface loam

Feet. Tisbury:

2. Medium white sand.

Mr. Davis reports that in all his well experience he has not encountered bowlders below the surface between Wading River and Port Jefferson.

831 . Record of Dr. William Carr's well near Center Moriches.

Wisconsin?: Feet.

1. White sand and gravel, with many stones............................ $0-18$

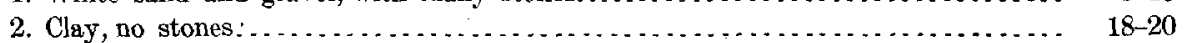

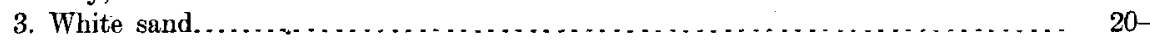


832. Record of Otto Lauraman's well near Center Moriches.

Wisconsin?:

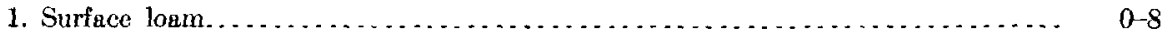

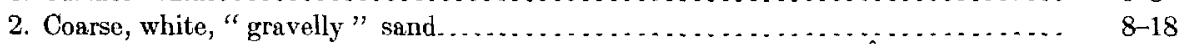

3. Hard, dry, yellow clay ...................................... 18-24

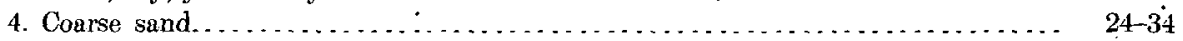

833.

Record of William Hallock's well near Center Moriches.

Wisconsin?:

1. Surface loam..

2. White "gravelly" sand -

3. Hard, dry, yellow clay with an occasional stone $15-20$

836.

Record of W. Frank Smith's well near East Moriches.

Wisconsin and Tisbury:
1. Loam
2. Sand.
3. Gravel.
4. White sand
5. Quicksand.

838.

Record of Wesley Young's well near South Manor.

Wisconsin:

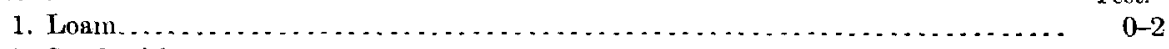

2. Sand with stones. . . . . . . . . . . . . . . . . . . . . . . . . . . . . .

839. Record of Alfred Steele's well near South Manor.

Wisconsin:

1. Surface loam.

2. Sand.

840.

Record of Benj. Raynor's well near South Manor.

Wisconsin?:

1. Surface loam . . . . . . . . . . . . . . . . .

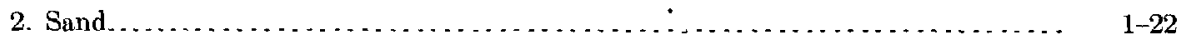

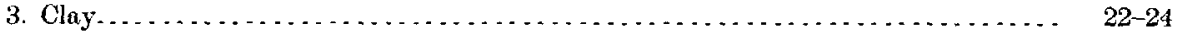

Mr. Nichol reports that he drove the pipe several feet into the clay bed and then pulled it up again, and obtained the water from above the clay.

841.

Record of Wallace Raynor's well near South Manor.

Wisconsin? :

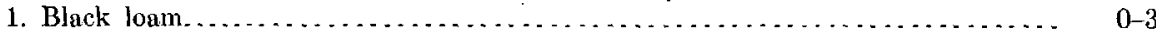

2. Clay, with occasional layers of sand; no stones.................... $3-15$

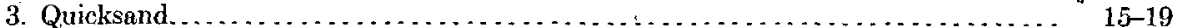

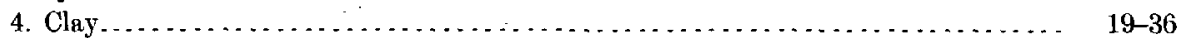

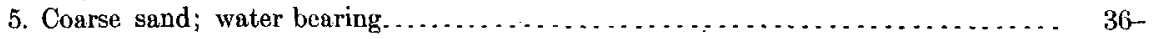

842.

Record of Porter Howell's well near South Manor.

Wisconsin?

Feet.

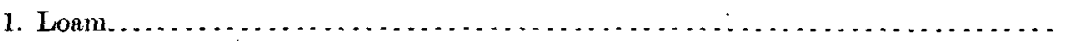

2. Sand, with a little clay. 


\section{UNDERGROUND WATER RESOURCES OF IONG ISLAND, NEW YORK.}

843.

Record of J. W. Nichol's well near Manorville.

Wisconsin ?:

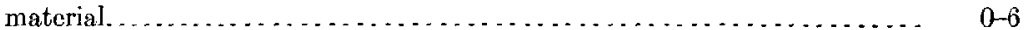

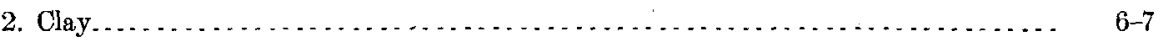

3. Coarse white sand............................................ $7-12$

844. Record of M. E. Raynor's well near Manorville.

Wisconsin?: $\quad$ Fect.

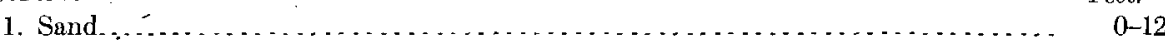

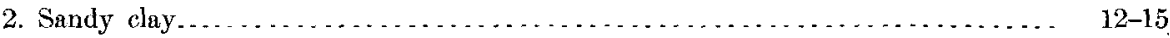

The clay in this well is described as being heavier (purer) at increasing depths. Water was found in a thin stratum of sand overlain and underlain by clay.

845. The Long Island Railroad Company report the following partial analysis:

Analysis of water from railroad well at Manorvitle.

Total solids Parts per million.

846. Record of Mrs. Jones's well near. Manorville.

Wisconsin?

1. Surface loam

3. Water-bearing sand $42-$

Mr. Nichol reports that the clay in stratum 2 was the color of putty, and that he has often encountered it in digging near the surface. He has never found stones in it.

847. Mr. Preston Raynor reports the following sections from two wells on his place:

Wisconsin?:

Record of Preston Raynor's well No. I, Manorville.

1. Iorm and yellow sand - Feet.

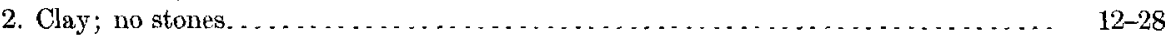

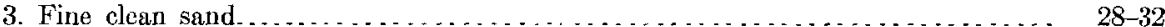

Wisconsin?

Record of Preston Raymor's well No. 2, Manorville.

Black Joam. ................................................ $0-3$

2. Hard clay; no stones....................................... $3-40$

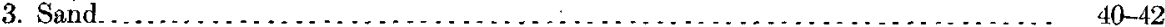

Mr. Raynor reports that he has never found a single stone in his vicinity. Clay is exposed in many of the ponds at low water, and several firms have made brick in this vicinity.

848. Mr. W. H. Beers has reported the following partial record:

Record of Dr. J. H. Darlington's well near Hulse Landing.

Wisconsin:

1. Black surface loam

Feet.

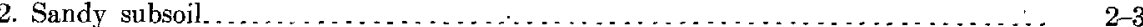

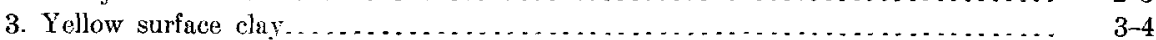

Transition:

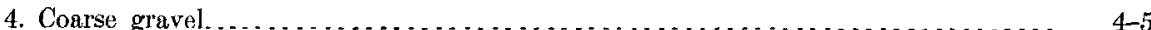

Tisbury:

5. Fine white beach sand

Sankaty?

6. Dark-red clay, like brick in color . . . . . . . . . . . . . . . . . . . . . . . $35-43$

7. Black sand, like that in sluggish creek ponds....................... $43-60$ 
849.

Record of R. B. Dayton's well near Remsenburg.

Wisconsin?:

Feet.

1. Joam

0

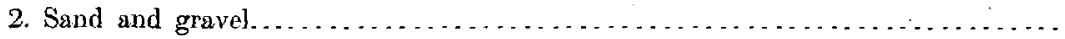

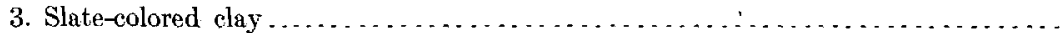

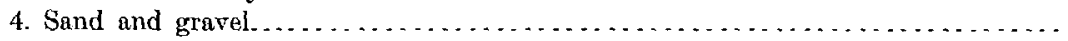

850.

Record of Jacob Raynor's well near Speonk.

Wisconsin?:

1. Surface loam and yellow sand.

2. Medium sand. .

3. Brown clay...

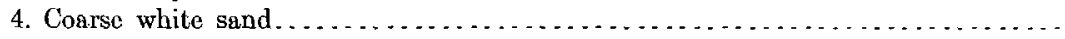

8.51.

Record of Ellsworth Raynor's well near Speonk.

Wisconsin?:

1. Surface loam and yellow sand

2. White send and yellow sand................................ $0-4$

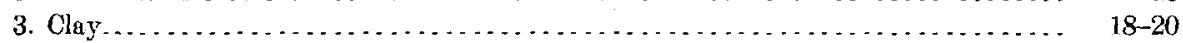

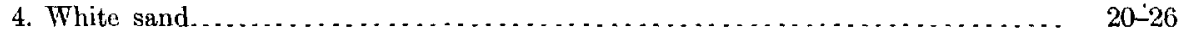

852. Record same as 850 .

854.

Record of C. H. Wells's well near Baiting Hollow.

Wisconsin and Tisbury:

1. Sandy loam...

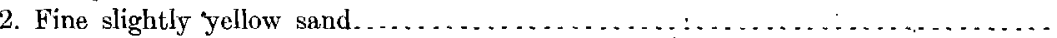

3. Hardpan (a hard stratum which carries no water and is composed of a mixture of clay and quite coarse gravel, seemingly cemented together with iron..

4. White sand.

Mr. Young says that the record above is duplicated in every well which he has put down in the vicinity of Baiting Hollow. The surface loam is of about. the same thickness in cach place, while the depth to stratum No. 2 varies from 45 to 60 feet, and the total depth of the wells from 90 to 110 feet. The depth to water varies with the elevation.

Mr. Young gives the following owners of wells in this vicinity, which have similar sections: Howell Benjamin, John B. Warner, Charles Warner, George F. Terry, John W. Fanning, J. C. Young, J. L. Young, Sydney Shaw, F. Hallock, Frank O. Reeves.

855. Record of Charles Warner's well near Baiting Hollow.

1. Heary surface loam.

2. White sand with occasional streaks of clay; no change in coarseness of sand at increasing depths.

856.

Record of Howell Sandford's weill near Baiting Hollow.

Tisbury:

1. Heavy surface loam............................... 5

2. White sand with occasional streaks of clay.

The material in this well is almost exactly similar to that in No. 855. There is no change in coarseness of sand at increasing depths. 
856A. Mr. Terry has furnished the following samples from this well:

Tisbury:

$$
\text { Record of Sydney Shaw's well at Centerville. }
$$

1-10. Light yellowish outwash sands with a little gravel

Below 65 feet the well was driven with a elosed point and no samples were obtained.

857.

Record of A. Zabriskie's well near West Hampton Beach.

Wisconsin?:

1. Surface loam and yellow sand.

2. Hardpan (described as a clay mixture of a slaty color whose particles seem to be cemented together).

Record of' Hallock d Small's well near Quogue Beach.

Recent:

2. Medium white sand bearing salt water.......................... 10-150

Cretaceous?:

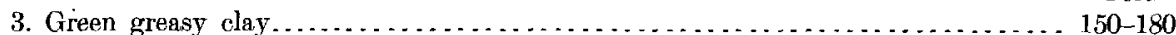

4. Medium white sand, containing a great deal of lignite............... 180-225

The sand gradually grew coarser until at 225 feet it was quite coarse. The top of the well is at tide level, and at the time it was drilled the water would rise in a pipe 12 feet above the surface.

$$
\begin{aligned}
& \text { Analysis of water from Hallock \& Small's well, Quogue Beach. } \\
& \text { [By F. E. Chandler, New York, April 2s, 1899.]. }
\end{aligned}
$$

App

Parts per million.

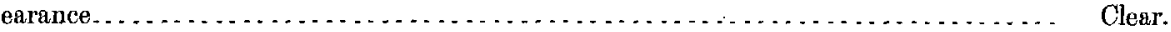

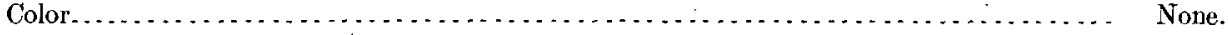

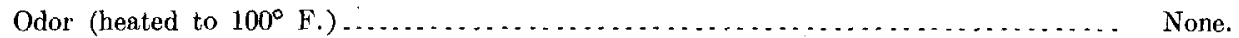

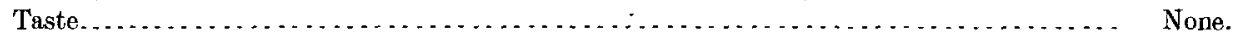

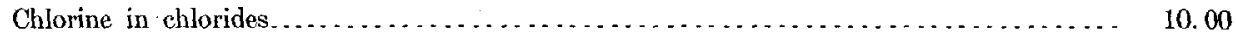

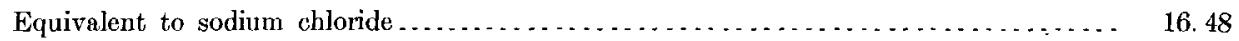

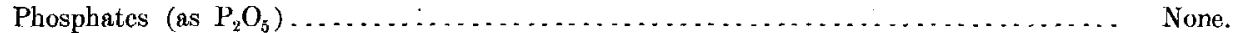

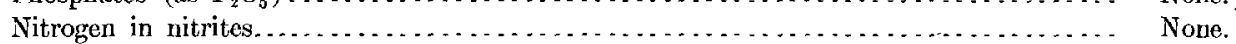

Nitrogen in nitrates.............................................. 1.20

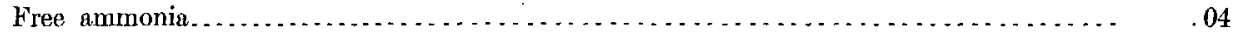

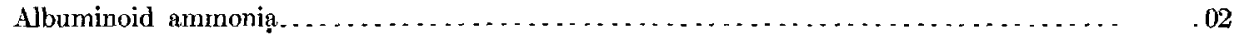

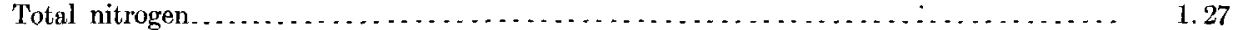

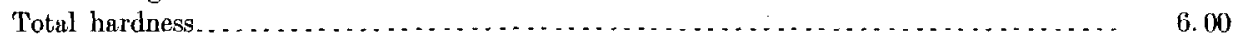

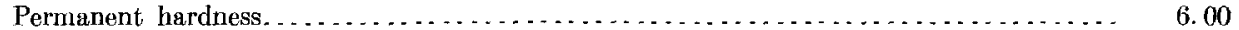

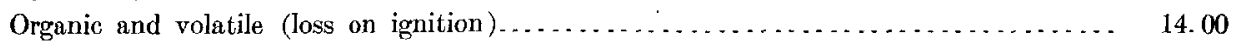

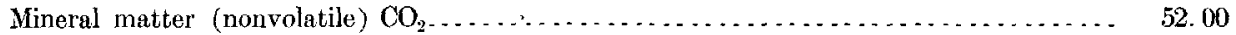

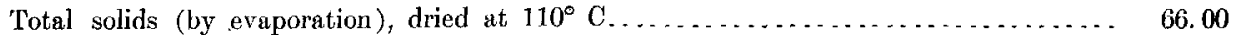

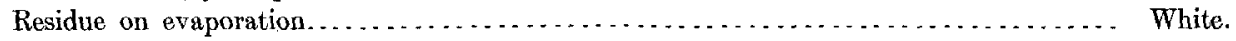

"This is a remarkably pure water, and is entirely free from contamination of every kind."

859. Mr. Asha B. Hallock has furnished the following samples from this well:

Sankaty:

Record of A. B. Hallock's well near Quogue.

1. Fragments of shells. .

\section{Cretaceous:}

2. Green sand marl...................................... 156-192

3. Coarse white quartz sand with pieces of gray clay and mica............. 192

4. Very fine dark-gray sand..................................... 200-224 
Cretaceous-Continued

5. Gray clay.

6. White micaceous sand with fragments of lignitized wood .............. 230-235

7. Very coarse quartz sand with mica and lignitized wood ................ 235-247

The fragmentary material from 135 feet was referred to Dr. W. H. Dall, who' reports as follows: "Contains fragments of Mulina, Astarte, an unidentifiable bivalve, a specimen of Nassa trivittata Say and fragments of an echinoderm. This is probably Pleistocene."

860. The well is on high ground and will flow from 1 to 2 gallons a minute.

Record of J. Wendell's well near Quogue.

Pleistocene:

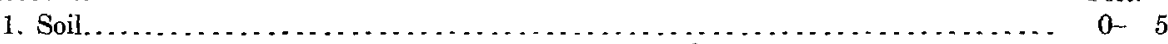

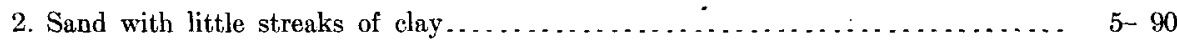

Pleistocene and Cretaceous:

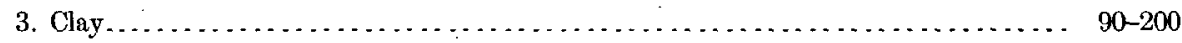

Cretaceous:

4. Clay with lignite. ...................................... 200-265

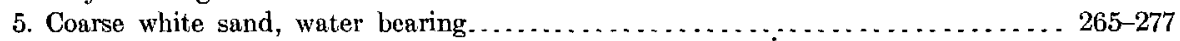

861. The driller, Mr. F. K. Walsh, gives the following record:

Recent:

Record of Quantuck Water Company's well near Quogue.

1. Bog material ...

Wisconsin and Tisbury:

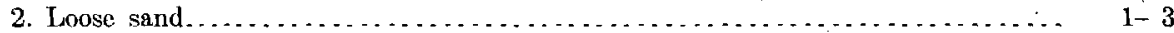

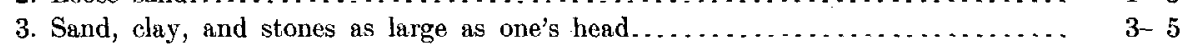

4. Very coarse sand with a little gravel.......................... 5 5-20

5. Very coarse sand and fine gravel with coarse stones.................. $20-40$

Analysis of water from Quantuck Water Company's well, Quogue.

[By C. F. Chandler, New York, December 1̄, 1902.]

Parts per million.

Appearance $\ldots \ldots \ldots \ldots \ldots \ldots \ldots$ Clear, with very slight sediment on bottom.

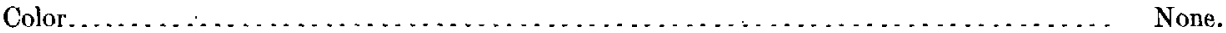

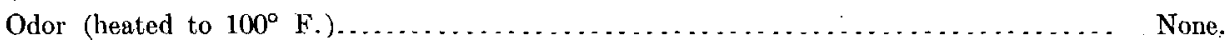

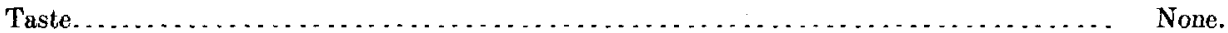

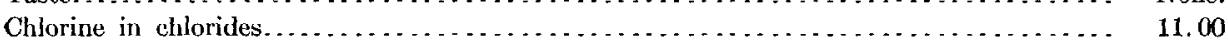

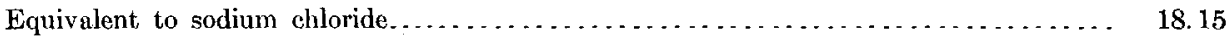

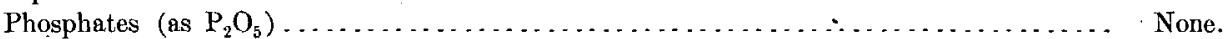

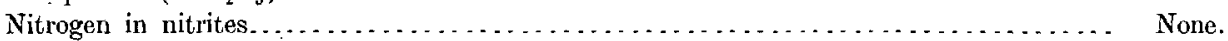

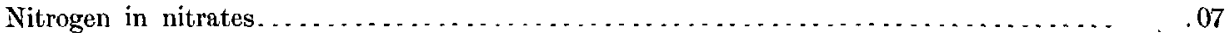

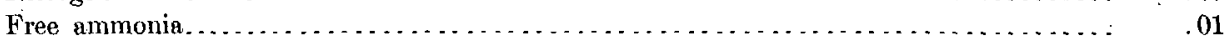

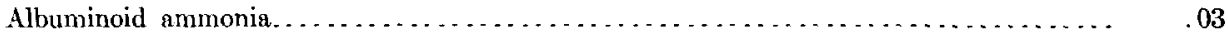

Total nitrogen............... 10

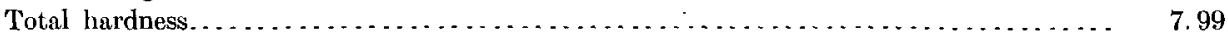

Permanent hardness. . . . . . . . . . . . . . . . . . . . . . . . . . . . . . . . . . $\quad 53$

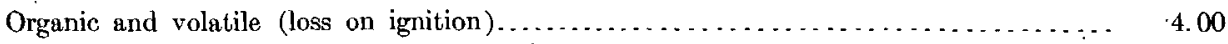

Minernl matter (nonvolatile) $\mathrm{CO}_{2}$ restored with ammonium carbonate............. 28.50

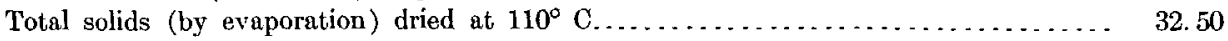

"This water is veiy pure indeed. It shows no signs whatever of contamination of any kind. The water does not contain any appreciable quantity of iron."

863. The pumping station of the Riverhead waterworks is in the Tower rolling mill and the pumps are 1 Knowles vertical triplex water-power pump, capacity 135 gallons per minute; and 1 single-stroke waterpower pump in reserve. The water is delivered into a tank baving a capacity of 40,000 gallons, which is situated in the tower of the mill. 


\section{UNDERGROUND WATER RESOURCES OF LONG ISTAND, NEW YORK.}

Mr. John R. Perkins, the former president of the company, reports the following data: "At the depth of 83 feet the first well flowed at the rate of 3 barrels a minute, but the water contained so much iron that the well was sunk deeper, to an approximate depth of 300 feet. The second well was sunk to a depth of 320 feet. Both contained a great deal of iron. The minimum amount pumped is 4,000 gallons an hour; the maximum amount is 8,000 ."

The driller, Mr. N. W. Davis, gives the following information: "Two wells, one 8 inches (225 feet deep), one 6 inches ( 305 feet deep). Lignitized wood at 180-200 feet. No clay beds found, but there were occasional beds of clay mixed with sand. Water first flowed over the pipe at 60 feet. The first well for this company was 85 feet deep and flowed a large amount of very chalybeate water. The well was made deeper to obtain a purer water. The flow becomes less at greater depths."

In a letter dated April 25, 1903, he states, regarding the first well: "Sunk an 8-inch well for the Riverhead waterworks 82 feet deep. Formation was sand, dark gravel, thick bed of elay, then sand mixed with jittle gravel. This yiclded about 120 to 130 gallons per minute."

864. Mr. Young reports that the points in all the wells in the vicinity of Riverhead corrode very quickly. Surface wells in the vicinity of Riverhead average a depth of 18 feet.

$$
\text { Record of Yetter \& Moore's well near Riverhead. }
$$

Wisconsin?:

1. Dark-brown sand and gravel containing dark-colored stones; the whole discolored by iron

Fect.

869.

Record of Capt. Jas. Downs's well near Jamesport.

Feet.

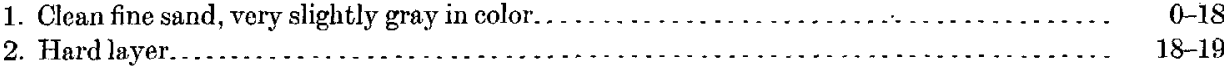

1. Clean fine sand, very slightly gray in color $\ldots \ldots \ldots \ldots \ldots \ldots$
2. Hard layer........

3. Coarse light-colored sand containing less mica than usual . . . . . . . . . . . . . . . $19-45$

870.

Record of J. J. McLaughlin's well near Jamesport.

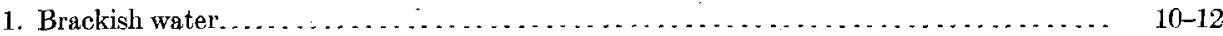

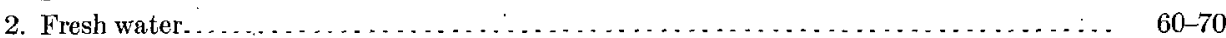

872. The Long Island Railroad Company has furnished the following partial analysis, dated February, 1899 , of the water from their 20-foot driven well:

Analysis of water of railroad well at Mattituck.

Total solids. Parts per million.

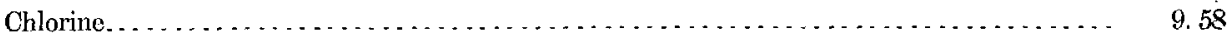

874.

Record of the Thane well near Shinnecock Hills.

Feet.

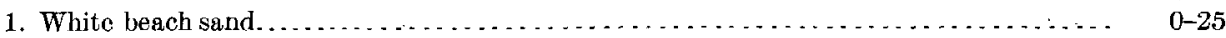

2. Coarse sand to gravel............................................... $25-35$

Several attempts were made to drive a well on the hilltop near the above well, but too many cobbles were encountered and the holes were abandoned.

875.

Record of C. W. Payne's well near North Sea.

1. Surface loam and yellow sand with some gravel. . . . . . . . . . . . . . . . . . .

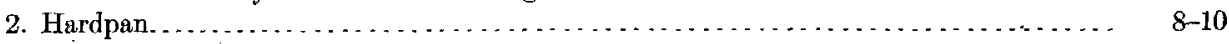

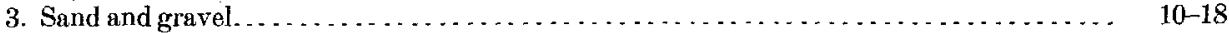

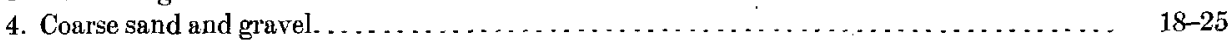

$87 \%$.

Record of Reid well near New Suffolk.

Wisconsin:

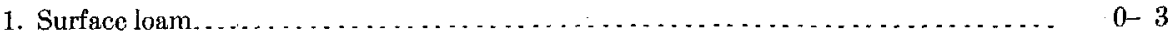

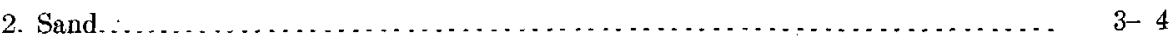

Sankaty? :

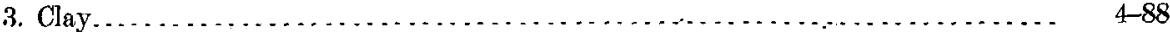

The clay is said to be distinctly stratified, the strata dipping $30^{\circ} \mathrm{W}$; it is also stained with iron. 
879. Mr. George Elliston, engineer, gives the following record for 1902:

"Maximum daily yield (August 29), 733,000 gallons; minimum daily yield (January 10), 114,000 gallons; average daily yield for year, 340,500 gallons; greatest amount pumped from the three wells, 41,000 gallons per hour; this was accomplished without difficulty, indicating a capacity of about a million gallons per day."

The original water level, according to Mr. Darling, constructing engineer, was 27 feet from the surface, while the present level reported by Mr. Elliston is 35 feet.

Analysis of water from well of Southampton Water Company, Southampton. .

[By Frazer \& Co., New York, June 30, 1903.]

Parts per million.

Color

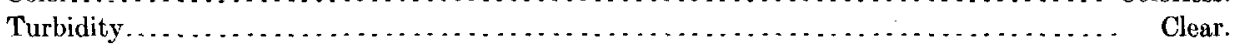

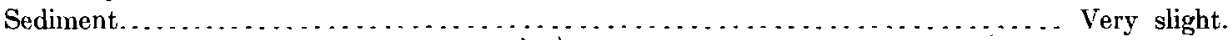

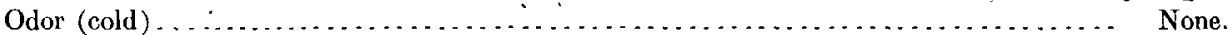

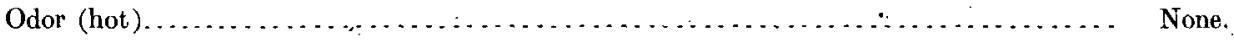

Total solids . . . . . . .

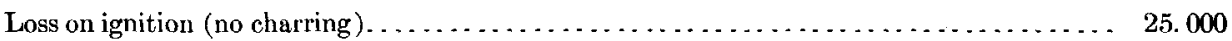

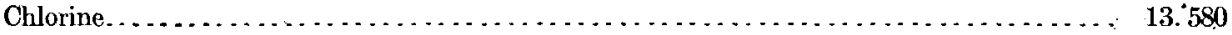

Nitrogen as free ammonia. . . . . . . . . . . . . . . . . . . . . . . . . . . . . . . 0.008

Nitrogen as albuminoid ammonia..................................... 0.018

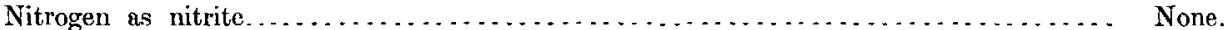

Nitrogen as nitrate . . . . . . . . . . . . . . . . . . . . . . . . . . . . . . . . . . . 800

Temporary härdness............................................. 5.000

Permanent hardness............................................... 20.000

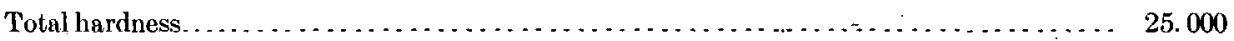

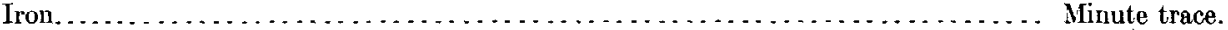

"The bacteriological examination shows the absence of any bacteria that indicate contamination by human or animal waste. The analysis of this water shows that it is pure and suitable for drinking purposes and general domestic use. 'The water is soft and does not show evidence of sewage contamination.'

When this system was first projected Fresh Pond was very seriously considered as a source of supply. Gagings showed sufficient water and analyses showed no contamination.

880. Mr. Arthur states that the water in this vicinity is found in pockets of clay, which, through surface wash, have become filled with gravel.

Wisconsin:

Record of Mrs. S. F. McDonald's well near Hampton Park.

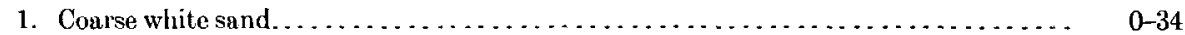

Sankaty?:

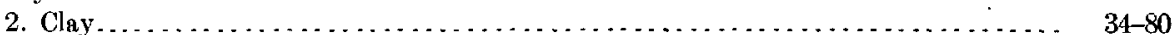

881.

Record of E. G. Whittaker's well near Hampton Park.

Sankaty? :

2. Very hard clay $\ldots \ldots \ldots \ldots \ldots \ldots \ldots \ldots \ldots \ldots \ldots \ldots \ldots \ldots \ldots \ldots \ldots \ldots \ldots \ldots \ldots, 2-82$

Jameco?

3. Sand.

$82-111$

At 18 feet clay was taken out which contained the imprint of shells, which, from the description given, were probably pectens.

881 A. Mr. Frederick $H$. Rose reports the following: "Our main spring-water supply seems to come from near sea level, and as we go in and up from the sea the wells deepen from a few feet to perhaps 60 , with a few hilltop clay or upper springs. My well here at Water Mill is about 18 feet deep, springs bubbling up through sand and gravelstones."

$17116-$ No. $44-06-22$ 
330、 UNDERGROUND WATER RESOURCES OF LONG ISLAND, NEW YORK.

882.

Record of J. F. Becker's well on Sihelter Island, New York.

1. Toamy clay.

Feet.

2. "Silver" sand

883.

Record of the Ulmer well on Shelter Island, New York.

Wisconsin:

Feet.

$0-10$

$10-14$

2. Gravel mixed with loam

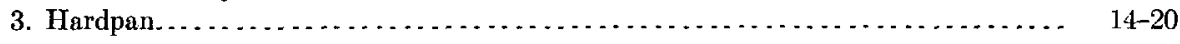

isconsin and Tisbury:

Tisbury:

4. Sand.

$20-43$

884.

Wisconsin:

1. Stony, dark-colored, almost red clay.

Record of John Weber's well on Shelter Island, New York.

Feet.

Tisbury:

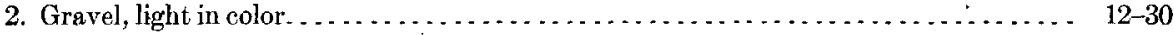

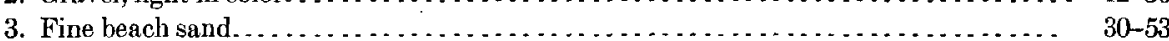

At 53 feet a big bowlder was encountered and the well was sunk no farther.

885. , Record of J. N. Stearns's well on Shelter Island, New York.

Wisconsin:

1. Red sandy loam

Feet.

2. Hard mixture of clay, sand, and gravel Sankaty:

3. Red and blue clay in strata 5 or 6 inches thick, alternating with strata of fine white and red sands.

888.

Record of A. O. Ryder's well on Shelter Island, New York.

Tisbury and Sankaty:

1. Ordinary sund in alternate layers of fine and coarse, containing a variety of shells at a depth of 60 feet.

889. The main source of supply at this pumping station consists of a dug well about 70 yards south of the Shelter Island Heights landing. In the bottom of the dug well there is a 6 -inch pipe 12 feet long.

Record of well of Shelter Island Heights Association, Shelter Island, New York.

1. Sand.

2. Gravel.

During the summer of 1903 the maximum amount pumped was 5,000 gallons per hour and the average about 4,000 gallons. If the well is pumped at the rate of 10,000 gallons per hour the water becomes brackish from the influx of the sea water.

Five hundred feet from this well a test boring was made in which the following material was encountered:

Record of test boring of. Shelter Island Heights Association on Shelter Island, New York.

Wisconsin and Tisbury:

Feet.

1. Sand and grave

$0-20$

2. Quicksand

20-60

Sankaty:

3. Red clay

$60-$ 


\section{DESORIPTIVE NOTES ON WELLS.}

Seven hundred feet west of the first well at the same elevation above the mean high tide and at the same distance from the shore the following section was obtained:

Record of test boring of Shelter Island Heights Association on Shelter Island, New York.

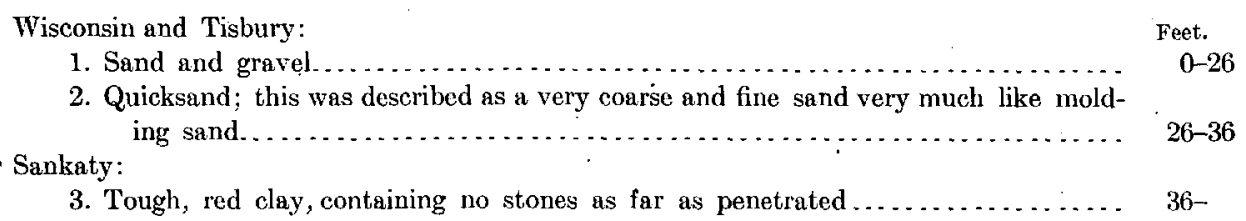

890. Mr. Havens reports that a group of 18 wells supplies the Manhanset House and the cottages adjacent to it. The amount pumped varies so greatly from day to day, according to the needs of the people in the cottages, and from summer to winter, according to the needs of the hotel, that no average could be given by Mr. Havens, nor could he estimate the maximum or minimum amount pumped.

Record of wells of Manhanset House, Shelter Island, New York.

Wisconsin and Tisbury:

Feet.

1. Stony and sandy loam. . . . . .

2. Hardpan................................................. $8-12$

3. White beach sand (coarse and fine mixed, running in places into "sandy gravel"). 12-17"

4. Hardpan ...... . . . . . . . . . . . . . . . . . . . . . . . . . . . . . . . . . . . . $\quad 17-20$

5. Quicksand, described by Mr. Havens as a good beach sand containing both black and white mica

841.

Record of J.M.Wells's well near Greenport.

1. Dry, yellow clay, containing a few small stones (the auger was twice broken and the fourth hole was begun before the attempt to complete the well was successful)....

. Medium red sand

892. Mr. Camerdon, of the Sumpwams Water Company, who was formerly engineer at this place, reports the following section for the first four wells:

Record of Greenport waterworks well, Greenport.

1. Hard, yellow sand and some yellow clay -at.

2. Fine, white sand, gradually growing coarser $\ldots-20$

3. Coarse gravel with pebbles 2 to 3 inches in diameter......................

In April, 1903, 5 additional wells were sunk, ranging in depth from 28 to 38 feet. As the water from these shallow wells showed a considerable percentage of chlorine a deep test well was sunk. Mr. N. W. Davis, who began this well, reports the following section for the upper 225 feet:

Wisconsin:

Record of test well of Greenport waterworks, Greenport.

Tisbury:

2. Alternate series of sands and gravel............................ 20-100 Sankaty:

3. Brown clay similar to that in Sanford's brickyard. ................ 100-150 Jameco:

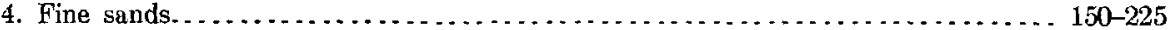




\section{UNDERGROUND WATER RESOURCES OF LONG ISLAND, NEW YORK.}

The well was then completed by Mr. E. K. Hutchinson, the only record kept being a few samples in a test tube preserved by Mr. Fred Klip. These show the following materials:

Record of test well of Greenport waterworks, Greenport.

2. Coarse quartz sand . . . . . . . . . . . . . . . . . . . . . . . . . . . . . .

3. Coarse quartz pebbles (one granite pebble).

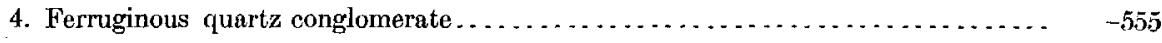
Cretaceous:

5. White, highly micaceous sand. . . . . . . . . . . . . . . . . . . . . . 555-605

6. Fine, white sand . . . . . . . . . . . . . . . . . . . . . . . . . . . . . . $605-612$

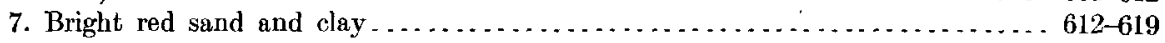

8. Brick red clay . . . . . . . . . . . . . . . . . . . . . . . . . . . . 619-635

9. Yellow sand and clay..................................... $635-640$

10. Yellowish-white clay ...................................... 640-645

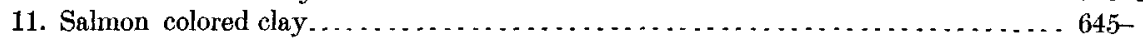

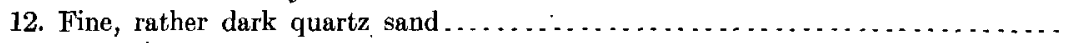

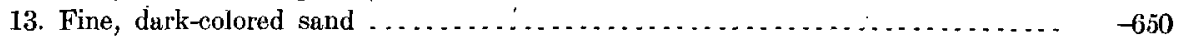

14. Coarse quartz sand containing fresh water (Lloyd sand?) .............. $650-670$

Pre-Cretaceous:

15. Schist . . . . . . . . . . . . . . . . . . . . . . . . . . . . . . . . $670-690$

One of the drillers reports that at 665 feet fresh water flowed over the top of the casing in a stream about the size of a pencil; the supply, however, was not deemed sufficient for pumping. Salt water was encountered between 225 and 555 feet.

The Long Island Railroad Company furnished the following analysis of water taken from the mains of the Greenport waterworks, November, 1901:

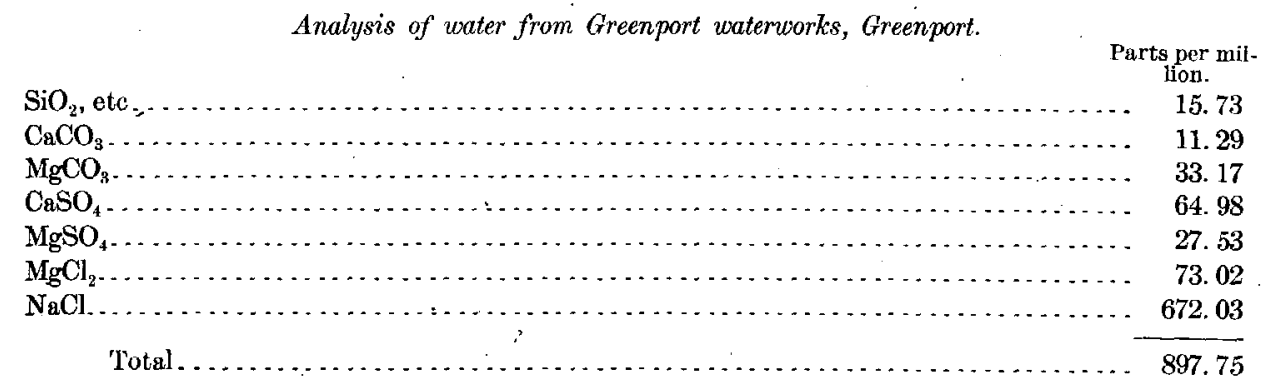

893. The Long Island Railroad Company report the following analysis from a 12 -foot driven well about 600 or 700 feet from tide water:

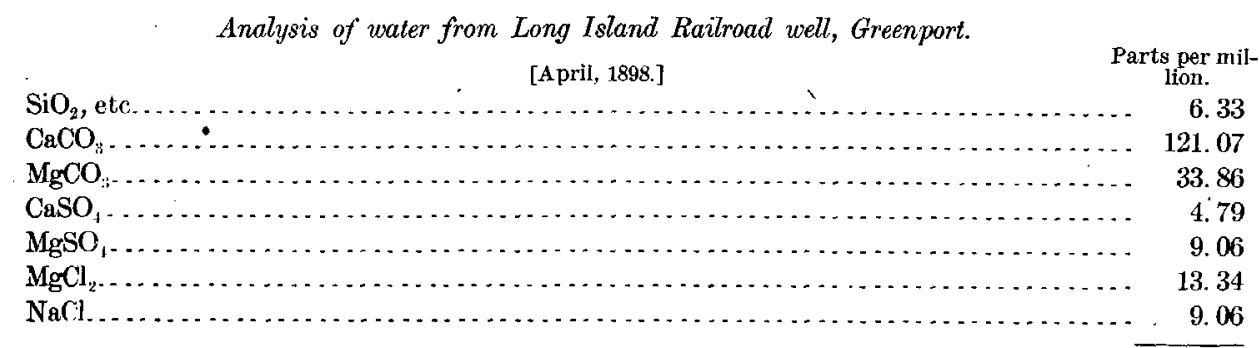

Total. 
They also report the following analysis from a 15 to 20 foot dug well 200 to 300 feet from tide water:

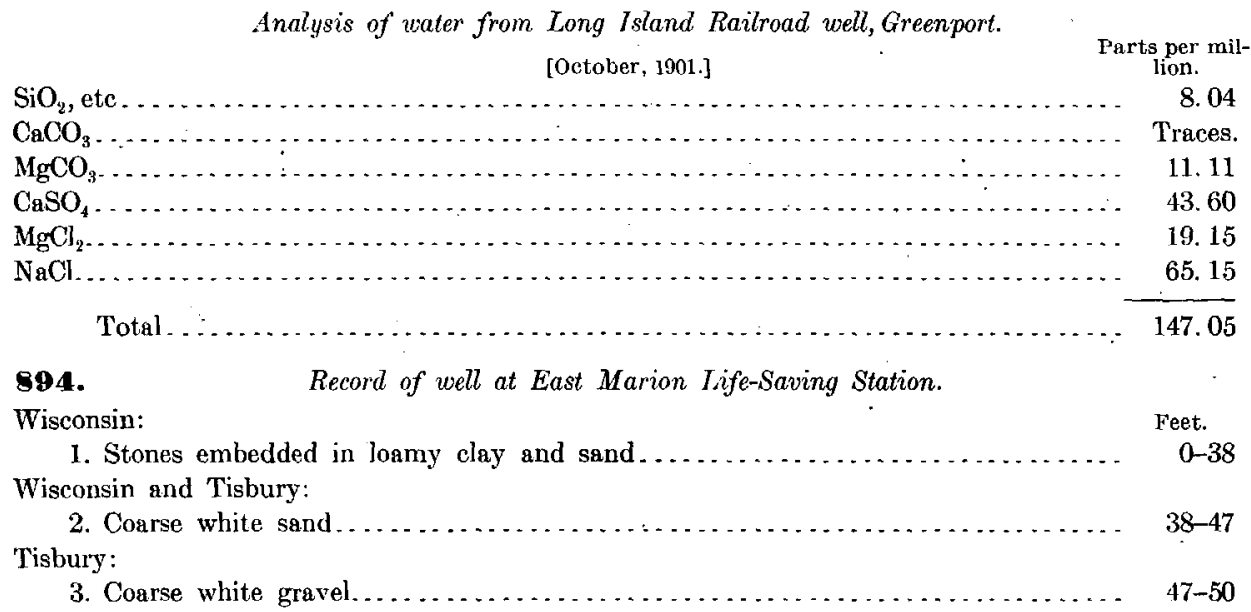

Some of the stones taken out of this well weighed at least 1,000 pounds. Many of them had to be blasted in order to be removed. The sand and gravel is reported as dipping about $45^{\circ} \mathrm{N}$.

895. Record of W.F.Furst's well, East Marion.

Wisconsin:

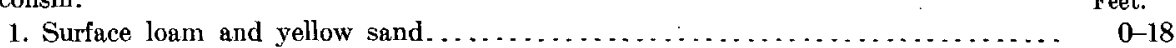

2. Hardpan (sand and gravel packed hard) .................... 18-22

Tisbury?:

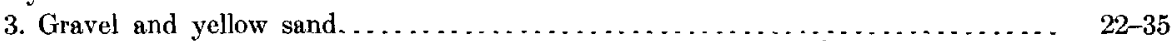

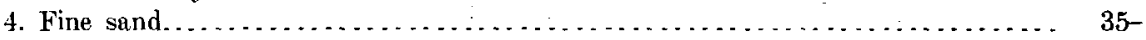

897. Mr. Sanford has furnished the following samples from this well:

Tisbury?:

Record of Sanford \& Son's well at Bridgehampton. Sankaty:

1. Gray micaceous clay, with a few small quartz pebbles................. $\quad 70$

2. Medium grayish white sand and gravel, with pieces of greenish clay containing Jameco:

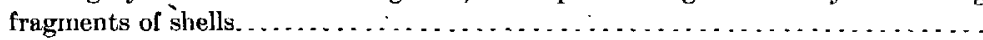

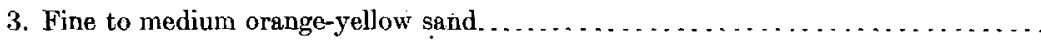

4. Orange-yellow gravel, apparently identical with that of the old glacial bed on Gardiners Island . . . . . . . . . . . . . . . . . . . . . . . . . . . . .

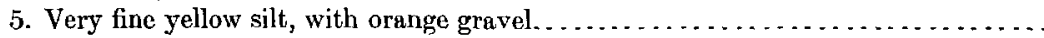
Cretaceous:

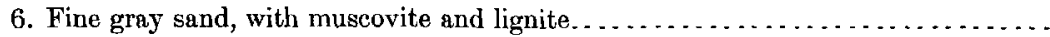

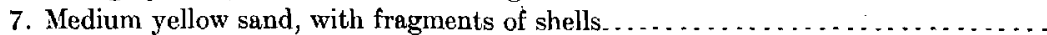

8. Gray clayey sand, with fragments of shells. . . . . . . . . . . . . . . . . . .

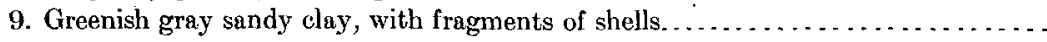

10. Very fine dark-gray sand, with some coarse white quartz sand................

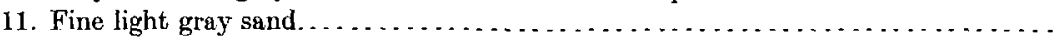

12. Fine to coarse light gray sand with partially lignitized wood $\ldots \ldots \ldots \ldots \ldots \ldots$

13. Medium white micaceous sand . . . . . . . . . . . . . . . . . . . . . . .

14. Fine light gray sand with lignite $\ldots \ldots \ldots \ldots \ldots$

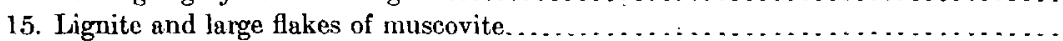

16. Medium white micaceous sand................................ 235

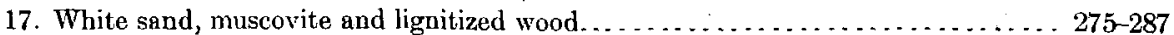

18. Fragments of iron pyrite . . . . . . . . . . . . . . . . . . . . . . . . . . 287-288

19. Fine to medium grayish-yellow sand........................... 288 -300 


\section{UNDERGROUND WATER RESOUROES OF LONG ISLAND, NEW YORK.}

Mr. Sanford reports that no record was kept, but that the samples were taken whenever he noticed a change in the material. In the above record the beds, therefore, probably extend from one sample to the next.

900. Mr. J. Wilkes Hedges reports: "From within one-eighth of a mile of the Atlantic Ocean to one and one-half miles north, the depth to water varies from 15 feet to 40 feet. As regards the strata, the first 15 inches is vegetable mold; the next 3 feet subsoil; then a layer of blue clay from 18 inches to 30 inches; then sand to water."

901. Mr. S. Shipperley, foreman for I. H. Ford, has furnished the following sarnples from this well:

Record of J. K. Morris's well near Sag Harbor.

Wisconsin and Tisbury: $\quad$ Feet.

1. Light-yellow sand and gravel, with a noticeable percentage of erratics, the material coarser in the lower portions.............................. $11-90$

2. Orange-yellow quartz sand and gravel. . . . . . . . . . . . . . . .

Sankaty?:

3. Grayish yellow, micaceous, silty clay, with a few pebbles................ 110-113

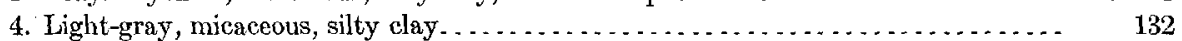
Jameco?:

5. Fine to very coarse, sharp, white sand, with a few scales of biotite $\ldots \ldots \ldots \ldots \ldots \ldots$ 143-145

903. Mr. Henry F. Cook, president of the Sag Harbor Waterworks Company, reports the following: "In 1888 four wells were sunk to a depth of 40 feet noar the pumping plant. These were pumped for a little less than a year, when the water became so red that it did not seem suitable for waterworks use; the wells were then driven to about 100 feet, and after being pumped for a time the water again became red. A large well, 15 by 15 , was then sunk about 900 feet south of the pumping station in the edge of a pond, and four 49 -foot wells were sunk, with the same result. The driven well system was then abandoned, and the water piped from Ligonee Brook into the large well. Ligonee Brook drains Long Pond, which may be regarded as the real source of the water."

Mr. E. Camerdon, of Sumpwams Water Company, at one time engineer at this point, states that 3 wells were put down in 1894 or 1895 , to a depth of 60 feet. No gravel was encountered, the section being entirely white sand. The wells were sunk to 60 feet, not in search of a different water-bearing horizon but to reach sand so coarse that it would not pass the screens.

904.

Record of Fahy Watch Case Company's well, Sag Harbor.

Tisbury:

1. Sand and gravel, varying a trifle from fine to coarse $\ldots \ldots \ldots \ldots \ldots \ldots \ldots \ldots \ldots \ldots \ldots$
2. Quicksand $\ldots \ldots \ldots \ldots \ldots \ldots \ldots \ldots \ldots \ldots \ldots$

The moment quicksand was encountered in this well driving was discontinued. The water from this well was obtained from four strata at the following depths:

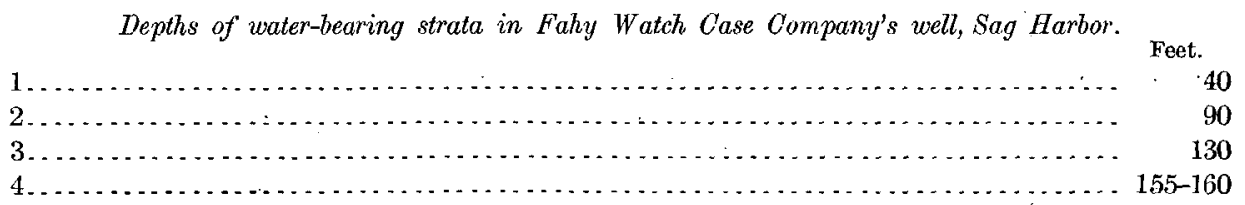

The well was tested to its full capacity and yielded 500 gallons per minute. 'The elovation above high tide is approximately 20 feet, and the water in the weIl rose to within 14 feet of the surface. The homogeneity of material is indicated by the fact that the same number of feet of pipe was driven daily.

905. Brackish water was found from a few feet below the surface down to 15 and 20 ; no water was encountered between 20 and 80 , when an abundant supply was obtained.

907.

Record of Doctor Benjamin's well at Shelter Island, New York.

Tisbury and Sankaty:

1. Very soft, white, medium, coarse sand 
Shells were encountered at 45 feet and continued to the bottom of the well. A fragment of Venus, apparently Venus mercenaria, has been forwarded by Doctor Benjamin, this being the only specimen saved from this shell-bearing layer.

\section{8.}

Record of J. E. Parker's well at Shelter Island, New York.

Wisconsin:

Seet.

$0-30$

isbury:

2. Sand and gravel in alternate layes, each layer about 8 or 10 feet thick.

$30-76$

909. Mrs. Hattic Conover, daughter of Mr. Uriah White, artesian-well driller, reports: "My father drilled the well at Orient in 1891 for the Orient Manufacturing Company. I am unable to give you any information regarding the well, except that I find one letter referring to it, giving its depth at that time as 406 feet, but the work was continued about three months longer. The water obtained was very salt, and they encountered a hard rock, and had to abandon the well, at a heavy loss."

910. According to Mr. Van Scoy, president of the Easthampton Home Water Company, the supply is derived from three 4 -inch wells 70 to 75 feet dcep, driven in the bottom of a pit 20 feet in diameter and 25 feet deep. On testing the wells a single well yielded 10,000 gallons per hour and two wells 15,000 and 16,000 gallons. Mr. Joe Seaman, foreman for Mr. W. C. Jaegle, gives the following section:

Record of Easthampton Home Water Company's well near Easthampton.

Wisconsin to Tisbury:

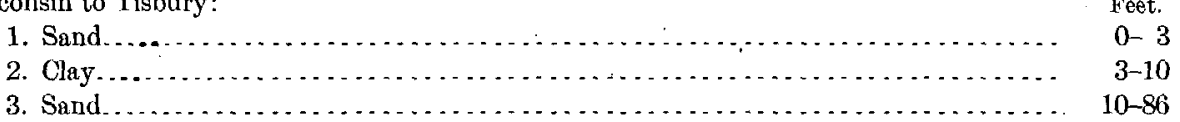

The sample, from a depth of 86 feet, which Mr. Seaman has furnished is a light-colored glacial gravel. Analysis of water from Home Water Company's wells, Easthampton.

[By Fraser \& Co., April 15, 1899.]

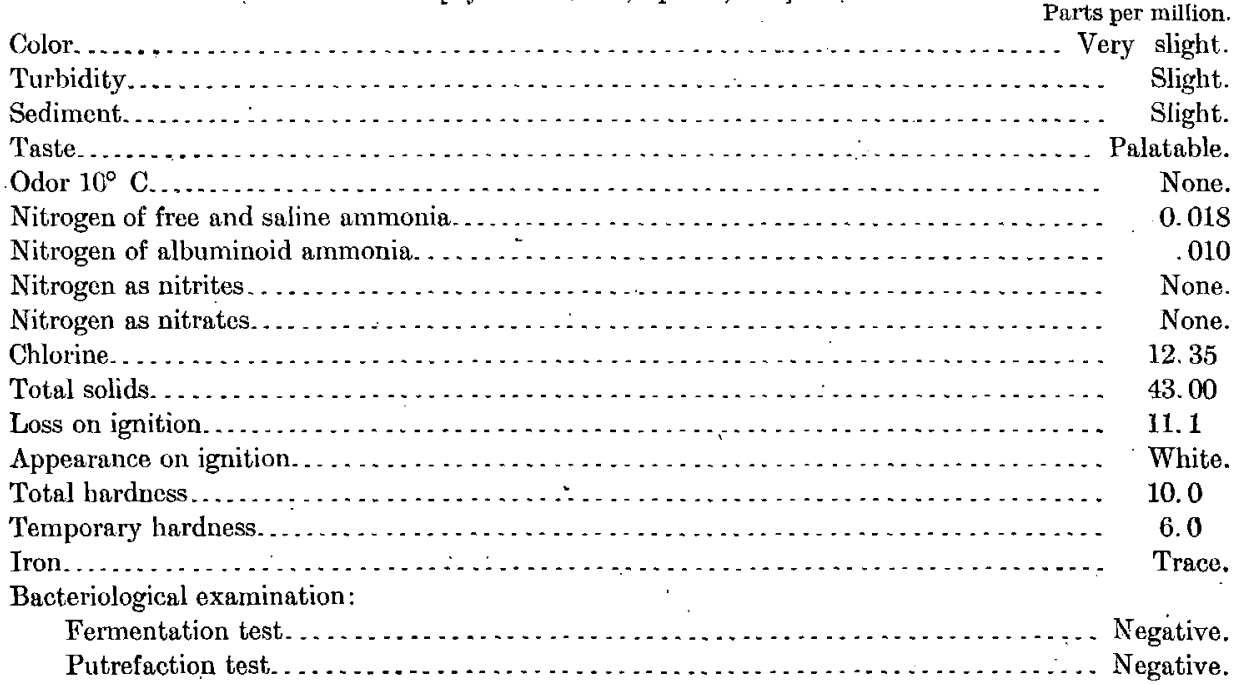

"The examination indicates that these specimens of water are soft and free from pollution and any excess of organic matter. The water is, therefore, recommended for drinking and general domestic purposes." 
911. Reçord of United States Army well on Plum Island, New York.

Wisconsin:

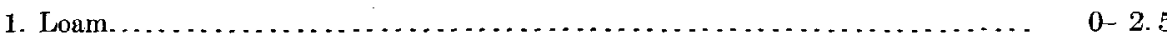

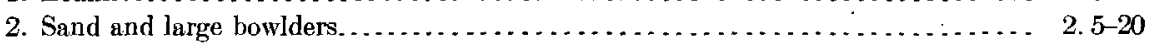

Tisbury?:

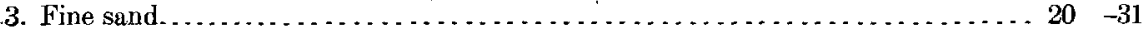

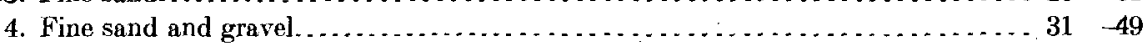

5. Coarse sand and fine gravel. ................................ $49 \quad-89$

"We crected a pumping plant here, which has been in operation since 1899."

$913 . \quad$ Record of Long Island Railroad well at Amagansett.

Wisconsin and Tisbury: Feet.

1. Coarse reddish brown sand, turning to white gravel. ................ $0-107$

914. Record of United States Army well at Gull Island, New York. Feet.

1. Loam and sand . . . . . . . . . . . . . . . . . . . . . . . . . . . . . . . . . . . . . 0

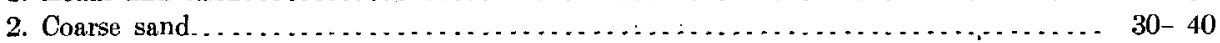

3. Very coarse sand. ........................................ $40-46$

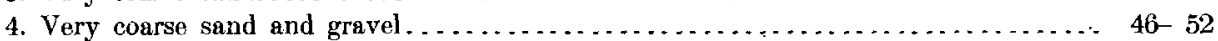

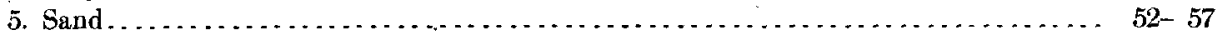

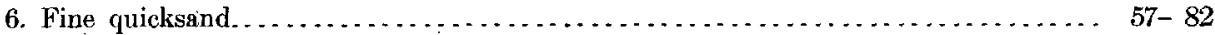

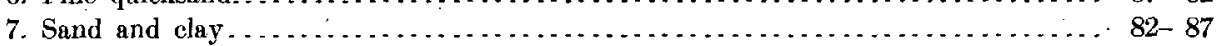

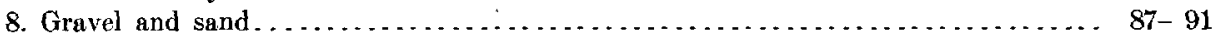

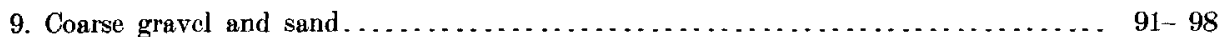

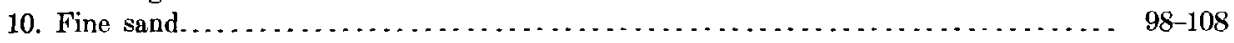

11. Light-colored clay . . . . . . . . . . . . . . . . . . . . . . . . . . . . . . . . . 108-112

12. Dark-blue clay, rather oily; when exposed to the air became very hard . . . . . . 112-291

Water was struck at 15 feet, but was very salty; the well flowed at 91 fect, also very salty; no water below 110 .

915. Record of United States Army well at Montauk.

1. Hardpan (very compact mixture of clay, gravel, and sand) Feet.

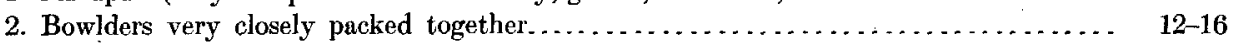

3. Coarse reddish brown sand . . . . . . . . . . . . . . . . . .

Surface water encountered at 9 feet.

Mr. Lockwood put down 3 wells at this place during the Spanish-American war. The second well was similar to the above, but a third well driven some distance from the two former ones had the following section:

Record of United States Army well at Montauk.

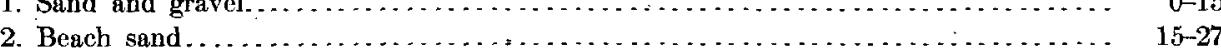

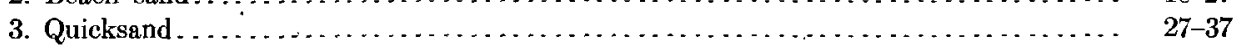

Mr. Lockwood reports that this well would be exhausted in a minute, and that it took an hour to fill up, so the pipe was pulled up 10 feet, when the well yielded 100,000 to 103,000 gallons a day.

916. The Long Island Railroad Company have furnished the following partial analysis of water from their driven well:

$$
\text { Analysis of railroad well at Montauk. }
$$

[July, 1898.]

Parts per million.

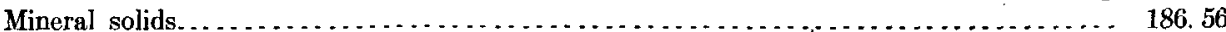

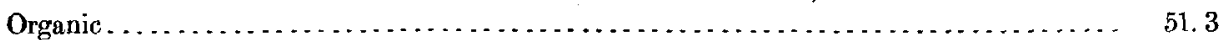

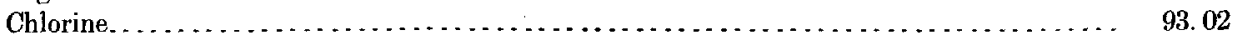


917. The following analysis of Fort Pond water was made by the Long Island Railroad Company, September, 1897 :

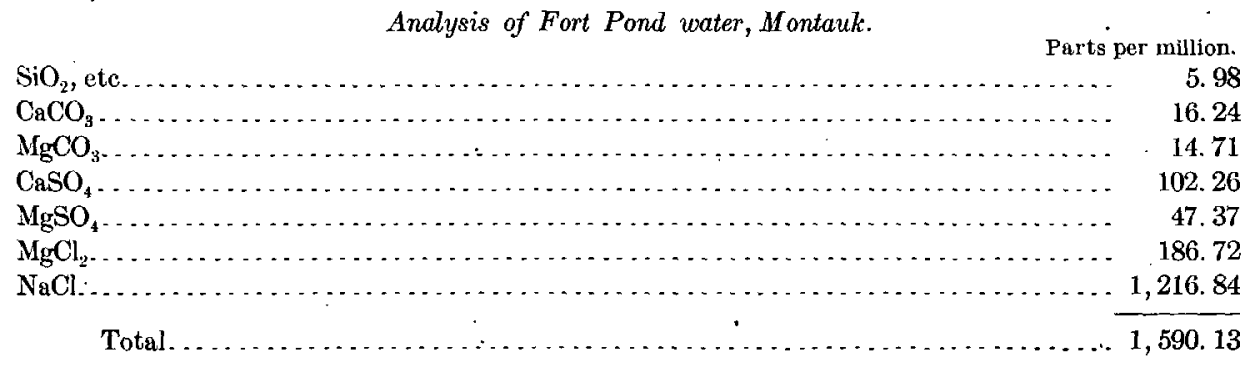

Not used for boilers.

918. The following analysis of Great Pond water was made by the Long Island Railroad Company, September, 1897 :

Analysis of water of Great Pond Lake, Montauk.

Parts per million.

$\mathrm{SiO}_{2}$ 14. 71

$\mathrm{CaCO}_{3}$

25. 14

$\mathrm{MgCO}_{3}$

33. 17

$\mathrm{CaSO}_{4}$

249. 15

$\mathrm{MgSO}_{4}$

353.29

$\mathrm{MgCl}_{2}$.

614.74

$\mathrm{NaCl}$.

Total

$6,145.74$

Not used for boilers.

919.

Record of Ferguson well on Fishers Island, New Yorl.

Pleistocene in part:

1. Gravel, bowlders, and samd.

Cretaceous?:

2. Blue clay.

Pre-cretaceous:

3. Rock, light-gray granite

$281-485$

Salt water was encountered at 201 feet, fresh water at 328 , and salt water at 485 feet. 


\section{CHAPTER V. \\ RESULTS OF SIZING AND FIUTRATION TESTS.}

By W. O. Crosbr.

SIZING TESTS.

In the detailed study of the underground water resources of any area it is important to know the extent to which the soil or underlying rock will absorb and transmit water. As both absorption and transmission depend more or less directly on the porosity of the strata, which in turn depends upon the relative size and arrangement of the particles composing them, one method of approaching the problem is to mechanically separate representative samples by means of sieves of known sizes and to construct from the data thus obtained a curve showing at a glance the relative proportions of coarse and fine materials and the degree of uniformity in the composition. From this curve may readily be deduced the effective size and the uniformity coefficient.

The effective size is the size of grain that would allow a sand to have its actual transmission capacity if all the grains were of the same diameter. It may be determined from the dimensions of the mesh of a sieve that will permit 10 per cent of the sample to pass through it, but will retain the other 90 per cent. Thus in any soil 10 per cent of the grains are smaller than effective size and 90 per cent are larger.

The uniformity coefficient is the ratio of the effective size to the size of grain which is larger than 60 per cent of the particles and smaller than 40 per cent.

The actual degree of uniformity "of the grains in any sample varies inversely. as the coefficient; and hence porosity and transmission must, in general, vary indirectly as the uniformity coefficient and directly as the effective size. Other things being equal, they are low, when the coefficient is high, that is, when the grains are diversified in size and the constitution of the sand highly composite, and also when the effective size is small. Otherwise stated, uniformity of grain . tends to the maximum values for both porosity and transmission and a high effective size favors transmission, especially by minimizing friction. It will thus be seen that these elements afford a check upon the porosity and transmission values as determined by actual trial in the filtration tests, and that they also afford a means of rating or grading, at least approximately, materials for which filtration tests have not been made.. 
- The determinations of the effective size and uniformity coefficient are, naturally, more accurate for relatively coarse than for fine materials because of the difficulty of separating and measuring minute particles; and hence it is especially desirable to supplement these determinations by filtration tests for fine-grained samples, or for those containing large proportions of quartz flour and clay. Theoretically, it should be possible to deduce a factor or formula for the conversion of sizing results into filtration results, and vice versa; but under the existing limitations of the sizing tests this is manifestly impossible, at least for relatively impalpable materials.

TABLE XII.-Results of sizing tests.

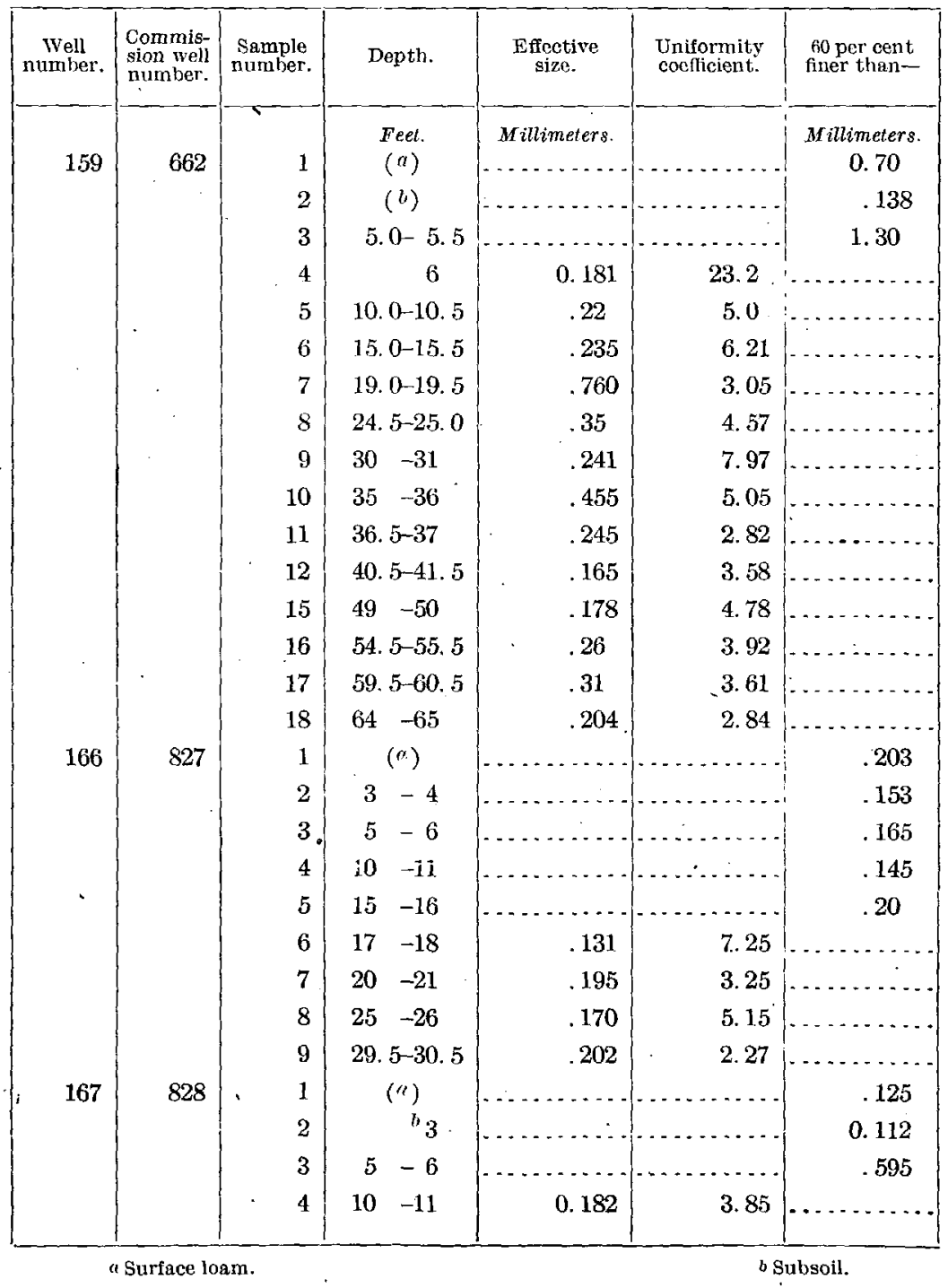


TABLE XII.-Results of sizing tests-Continued.

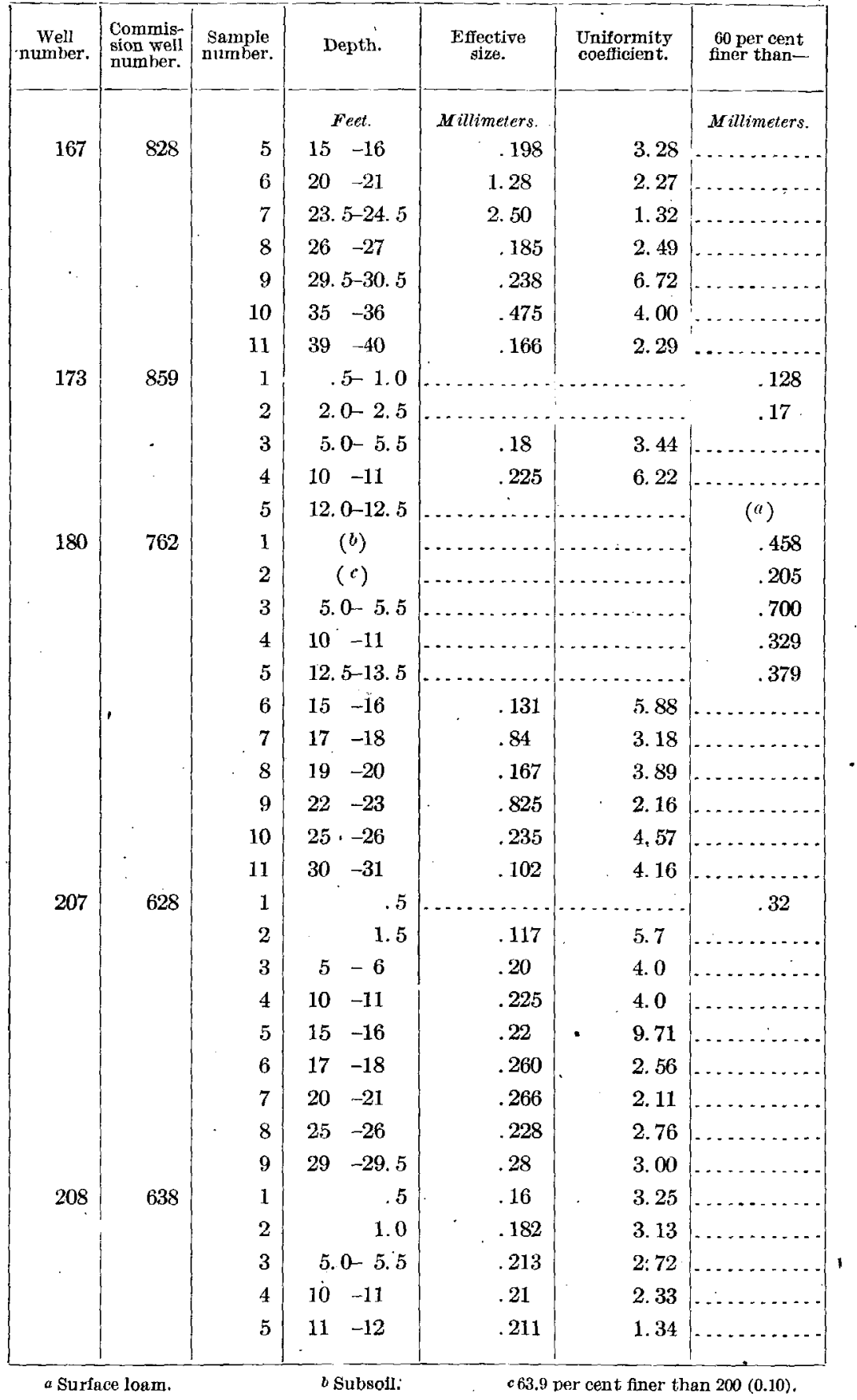


SIZING TESTS.

TABLE XII.-Results of sizing tests-Continued.

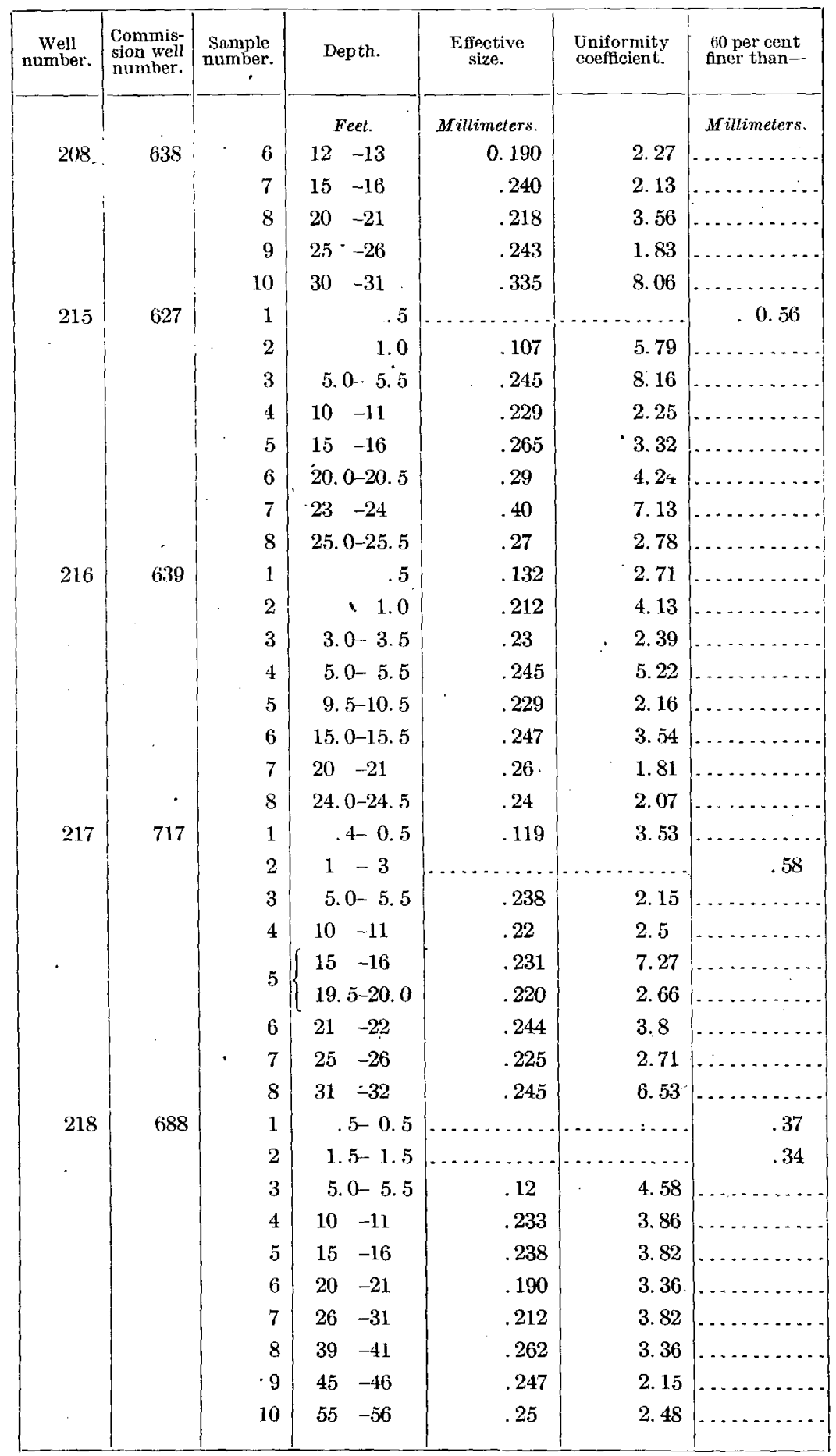


UNDERGROUND WATER RESOUROES OF LONG ISLAND, NEW YORK.

TABLE XII.-Results of sizing tests--Continued.

\begin{tabular}{|c|c|c|c|c|c|c|}
\hline $\begin{array}{c}\text { Well } \\
\text { number. }\end{array}$ & $\begin{array}{l}\text { Commis- } \\
\text { sion wel } \\
\text { number. }\end{array}$ & $\begin{array}{c}\text { Sample } \\
\text { number. }\end{array}$ & Depth. & $\begin{array}{l}\text { Effective } \\
\text { size. }\end{array}$ & $\begin{array}{l}\text { Uniformity } \\
\text { coefficient. }\end{array}$ & $\begin{array}{l}60 \text { per cent } \\
\text { finer than- }\end{array}$ \\
\hline & & & Feet. & Millimeters. & & Millimeters. \\
\hline 218 & 688 & 11 & $59-60$ & 0.283 & 2.86 & \\
\hline 221 & 687 & 1 & .4 & $\cdots$ & . & 0.22 \\
\hline \multirow[t]{7}{*}{ - } & & 2 & 1.5 & $\ldots .$. & 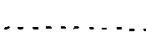 & .26 \\
\hline & & 3 & 5. 0 & . & $\cdots \cdot$ & .435 \\
\hline & & 4 & $\begin{array}{ll}10 & -11\end{array}$ & .172 & 4. 65 & \\
\hline & & 5 & $15-16$ & .310 & 2.99 & \\
\hline & & 6 & $\begin{array}{ll}20 & -21\end{array}$ & .518 & 5.79 & \\
\hline & & 7 & $25 \quad-26$ & $\therefore 282$ & 3. 72 & \\
\hline & & 8 & $\begin{array}{ll}28 & -29\end{array}$ & .295 & 1.88 & $\cdots$ \\
\hline \multirow[t]{10}{*}{229} & 695 & 1 & $.3-0.4$ & $\ldots .$. & .... & .255 \\
\hline & & 2 & $1.5-1.6$ & $\ldots$ & $\cdots$ & .28 \\
\hline & & 3 & $5.0-5.5$ & .221 & 4. 12 & \\
\hline & & 4 & $\begin{array}{ll}10 & -11\end{array}$ & .208 & 3.66 & \\
\hline & & 5 & $15-16$ & .205 & 2.24 & \\
\hline & & 6 & $\begin{array}{ll}20 & -21\end{array}$ & .240 & 2.63 & \\
\hline & & 7 & $25-26$ & .22 & 2.14 & \\
\hline & & 8 & $\begin{array}{ll}30 & -31\end{array}$ & . . .229 & 1.49 & $\cdots$ \\
\hline & & 9 & $\begin{array}{ll}34 & -35\end{array}$ & 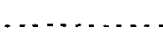 & & .112 \\
\hline & . & 10 & $\begin{array}{ll}37 & -38\end{array}$ & .. & . & .188 \\
\hline \multirow[t]{9}{*}{235} & 1090 & 1 & . $.5-1.0$ & $\ldots$. & $\ldots$ & .11 \\
\hline & & 2 & $2.0-2.5$ & $\ldots$. & $\ldots \ldots$ & .10 \\
\hline & & 3 & $5-6$ & .134 & 4.04 & \\
\hline & & 4 & $10-11$ & .195 & 2.8 & \\
\hline & & 5 & $\begin{array}{ll}15 & -16\end{array}$ & .209 & 3.21 & \\
\hline & & 6 & $\begin{array}{ll}20 & -21\end{array}$ & .245 & 7.00 & \\
\hline & & 7 & $\begin{array}{ll}22 & -23\end{array}$ & .22 & 7.05 & \\
\hline & & 8 & $\begin{array}{ll}26 & -27\end{array}$ & .206 & 3.4 & \\
\hline & & 9 & $28.0-28.5$ & $\ldots$ & . & .223 \\
\hline \multirow[t]{9}{*}{294} & 659 & 1 & & .... & $\cdots$ & .38 \\
\hline & & 2 & 1.0 & .215 & 1.47 & \\
\hline & & 3 & $5.0-5.5$ & .192 & 2.24 & \\
\hline & . & 4 & $10-11$ & .224 & 1. 83 & \\
\hline & & 5 & $15.0-15.5$ & .233 & 2. 11 & \\
\hline & & 6 & $20-21$ & .212 & 1.25 & \\
\hline & & 7 & $23.0-23.5$ & .245 & 1.9 & \\
\hline & & 8 & $25 \quad-26$ & .243 & 1.87 & \\
\hline & & 9 & $30.0-30.5$ & .250 & 1.79 & \\
\hline \multirow[t]{3}{*}{296} & 660 & 1 & .4 & .128 & 2.91 & \\
\hline & & 2 & 1.0 & .20 & 2.2 & \\
\hline & & 3 & $5.0-5.5$ & .22 & 2. 77 & \\
\hline
\end{tabular}


SIZING TESTS.

TABLE XII.-Results of sizing tests-Continued.

\begin{tabular}{|c|c|c|c|c|c|c|}
\hline $\begin{array}{c}\text { Well } \\
\text { number. }\end{array}$ & $\begin{array}{l}\text { Cornmis- } \\
\text { sion well } \\
\text { number. }\end{array}$ & $\begin{array}{l}\text { Sample } \\
\text { number. }\end{array}$ & Depth. & $\begin{array}{l}\text { Effective } \\
\text { size. }\end{array}$ & $\begin{array}{l}\text { Uniformity } \\
\text { coefficient. }\end{array}$ & $\begin{array}{l}60 \text { per cent } \\
\text { finer than- }\end{array}$ \\
\hline \multirow{8}{*}{296} & \multirow{8}{*}{660} & & Feet. & Millimeters. & & Millimeters. \\
\hline & & 4 & $10-11$ & 0.22 & 2.09 & \\
\hline & & 5 & $15-16$ & .258 & 2. 33 & . \\
\hline & & 6 & $\begin{array}{ll}20 & -21\end{array}$ & .207 & 1.75 & $\cdots$ \\
\hline & & 7 & $23-24$ & .212 & 1.8 & $\cdots$ \\
\hline & & 8 & $25-26$ & .226 & 2.57 & \\
\hline & & 9 & $29-30$ & .215 & 2.09 & $\ldots$. \\
\hline & & 10 & $35.0-35.5$ & .216 & 2.64 & $\ldots . \ldots$ \\
\hline \multirow[t]{10}{*}{303} & \multirow[t]{2}{*}{607} & 1 & $.5-0.5$ & $\ldots . . .$. & $\ldots \ldots \ldots$ & 0.442 \\
\hline & & 2 & $1-1$ & .215 & 2.68 & $\ldots$ \\
\hline & \multirow[b]{8}{*}{. } & 3 & $5.0-5.5$ & .229 & 5.07 & \\
\hline & & 4 & $10.0-10.5$ & .221 & 2.35 & $\cdots$ \\
\hline & & 5 & $15.0-15.5$ & .23 & 4.87 & \\
\hline & & 6 & $20.0-20.5$ & .206 & 3. 16 & \\
\hline & & 7 & $25-26$ & .22 & 2.05 & $\ldots$. \\
\hline & & 8 & $30.0-30.5$ & .25 & 8.00 & - \\
\hline & & 9 & $35-36$ & .218 & 3.03 & \\
\hline & & 10 & $\begin{array}{ll}40 & -41\end{array}$ & .23 & 7.43 & 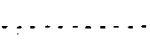 \\
\hline \multirow[t]{15}{*}{308} & \multirow[t]{10}{*}{907} & 1 & $.3-0.4$ & $\cdots$ & $\ldots \ldots$ & .28 \\
\hline & & 2 & $.8-1.0$ & - & $\ldots \ldots \ldots$ & .282 \\
\hline & & 3 & $2.5-3.0$ & .275 & 8.73 & 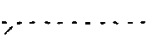 \\
\hline & & 4 & $6-7$ & .231 & 2.56 & - . \\
\hline & & 5 & $10-11$ & .347 & 5.48 & : \\
\hline & & 6 & $.15 .0-15.5$ & .274 & 4.05 & - \\
\hline & & 7 & $20.0-20.5$ & .264 & 3.51 & $\ldots$ \\
\hline & & 8 & $25-26$ & $\because 268$ & 6.34 & $\cdots$ \\
\hline & & 9 & $30.5-31.5$ & .36 & 9.78 & \\
\hline & & 10 & $\begin{array}{ll}32 & -33\end{array}$ & .228 & 11.4 & \\
\hline & \multirow[t]{5}{*}{ • } & 11 & $\begin{array}{ll}35 & -36\end{array}$ & .257 & 11.28 & \\
\hline & & 12 & $40-41$ & .26 & 8.12 & \\
\hline & & 13 & $44-45$ & .22 & 2.50 & \\
\hline & & 14 & $50-51$ & .216 & 4. 31 & \\
\hline & & 15 & $55.5-56.5$ & .226 & 5.66 & $\Leftrightarrow$ \\
\hline \multirow[t]{8}{*}{310} & \multirow[t]{8}{*}{$829^{\circ}$} & 1 & $.3-\quad .5$ & $\ldots$ & $\cdots$ & .23 \\
\hline & & 2 & $1.0-1.5$ & & & 38 \\
\hline & & 3 & $4.0-4.5$ & .23 & 3.83 & $\ldots$. \\
\hline & & 4 & $\begin{array}{ll}10 & -11\end{array}$ & .358 & 4. 47 & \\
\hline & & 5 & $15.0-15.5$ & .225 & 3.78 & \\
\hline & & 6 & $\begin{array}{ll}20 & -21\end{array}$ & .23 & 3.00 & \\
\hline & & 7 & $25-26$ & $.2 i$ & 3.85 & \\
\hline & & 8 & $\begin{array}{ll}30 & -30\end{array}$ & .239 & 686 & $\cdots$ \\
\hline
\end{tabular}


UNDERGROUND WATER RESOURCES OF LONG ISLAND, NEW YORK.

TABLE XII.--Results of sizing tests-Continued.

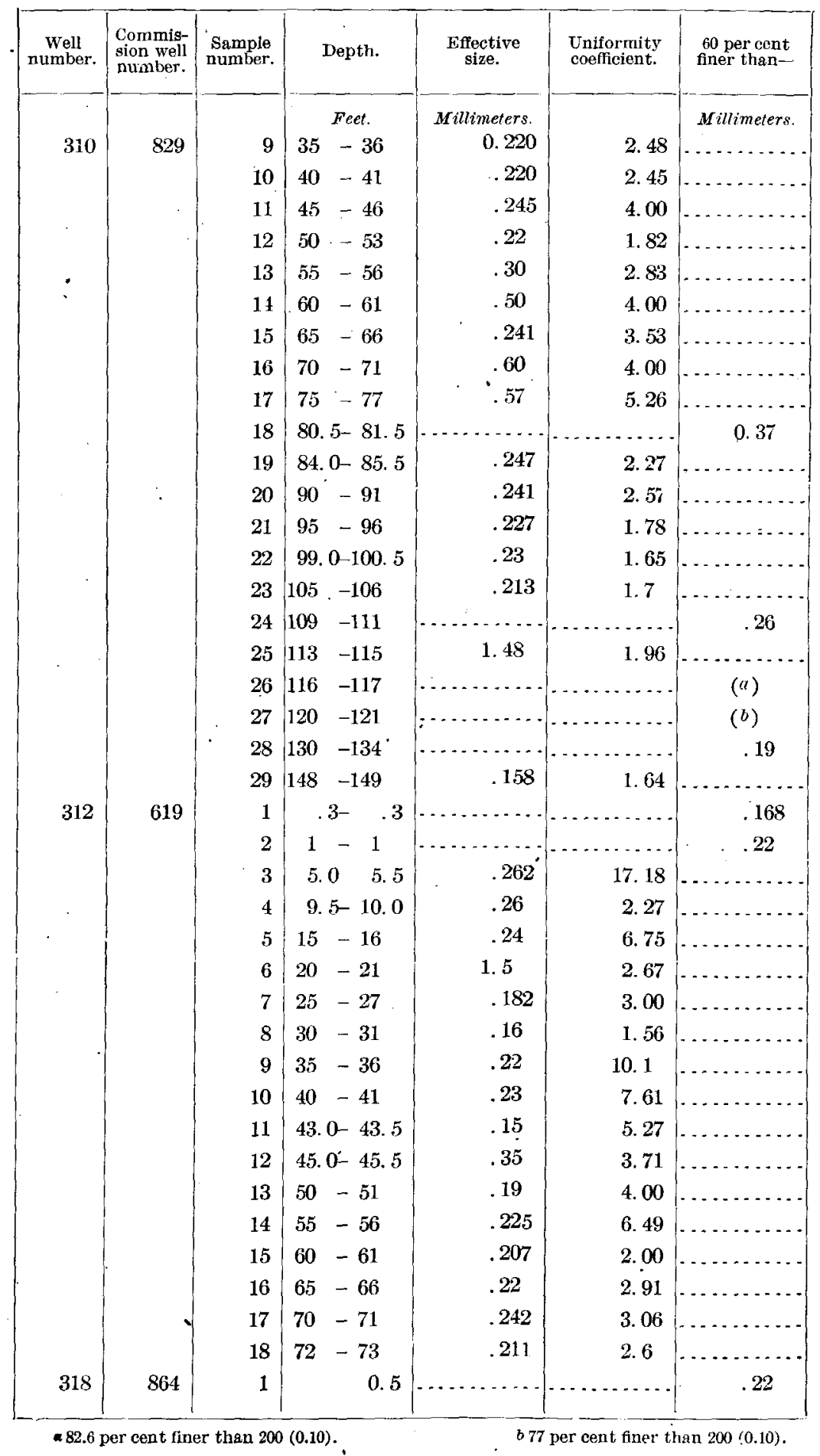


SIZING TESTS.

TARte XII.-Results of sizing tests-Continued.

\begin{tabular}{|c|c|c|c|c|c|c|}
\hline $\begin{array}{c}\text { Well } \\
\text { number: }\end{array}$ & $\begin{array}{l}\text { Commis- } \\
\text { sion well } \\
\text { number. }\end{array}$ & $\begin{array}{l}\text { Sample } \\
\text { number. }\end{array}$ & Depth. & $\begin{array}{l}\text { Effective } \\
\text { size. }\end{array}$ & $\begin{array}{l}\text { Uniformity } \\
\text { coefficient. }\end{array}$ & $\begin{array}{l}60 \text { per cent } \\
\text { finer than- }\end{array}$ \\
\hline \multirow{7}{*}{318} & \multirow{7}{*}{864} & 2 & $\begin{array}{l}\text { Feet. } \\
\qquad 3.0\end{array}$ & Millimeters. & & $\begin{array}{r}\text { Millimeters. } \\
0.359\end{array}$ \\
\hline & & 3 & $8-9$ & 0.137 & 3.0 & $\ldots \ldots$ \\
\hline & & 4 & $14--15$ & .13 & 2.7 & \\
\hline & & 5 & $\begin{array}{ll}19 & -20\end{array}$ & .136 & 2.71 & $\ldots$ \\
\hline & & 6 & $24 \quad-25$ & .141 & 2.73 & $\ldots$ \\
\hline & & 7 & $29-30$ & .11 & 2.82 & $\ldots \ldots \ldots$ \\
\hline & & 8 & $34^{\circ}-35$ & .15 & 2.53 & . \\
\hline \multirow[t]{11}{*}{323} & \multirow[t]{11}{*}{956} & 1 & $(a)$ & $\ldots$ & $\ldots \ldots$. & .11 \\
\hline & & 2 & $1.0-1.5$ & $\ldots$ & $\ldots$. & .345 \\
\hline & & 3 & $4-5$ & .130 & 3.35 & $\therefore . . . . .$. \\
\hline & & 4 & $\begin{array}{ll}9 & -10\end{array}$ & .15 & 300 & - \\
\hline & & 5 & $14-15$ & .225 & 3.82 & - \\
\hline & & 6 & $19-20$ & .22 & 4.05 & $\ldots \ldots$ \\
\hline & & 7 & $24 \quad-25$ & .209 & 2. 11 & \\
\hline & & 8 & $29-30$ & .181 & 3.15 & \\
\hline & & 9 & $34-35$ & .290 & 2.24 & \\
\hline & & 10 & $\begin{array}{ll}39 & -40\end{array}$ & .198 & 1.77 & $\ldots \ldots$ \\
\hline & & 11 & $\begin{array}{ll}45 & -47\end{array}$ & .172 & 3.26 & $\ldots$ \\
\hline \multirow[t]{10}{*}{381} & \multirow[t]{10}{*}{697} & 3 & $11.5-13.0$ & .18 & 2.02 & \\
\hline & & 4 & $16.5-17.0$ & .224 & 1.72 & \\
\hline & & 5 & $19.0-19.5$ & .29 & 3.17 & \\
\hline & & 6 & $24 \quad-25$ & .233 & 2.15 & \\
\hline & & 7 & $25.5-26.0$ & .27 & 11. 4 & $\ldots$ \\
\hline & & 8 & $27.0-27.8$ & .240 & 2.125 & \\
\hline & & 9 & $29-30$ & .187 & 4. 81 & $\ldots$. \\
\hline & & 10 & $34-34.5$ & .148 & 1.34 & \\
\hline & & 11 & $34.5-35$ & .169 & 1.65 & \\
\hline & & 12 & $\begin{array}{ll}37 & -38\end{array}$ & .169 & 1.95 & \\
\hline \multirow[t]{13}{*}{382} & \multirow[t]{13}{*}{658} & 3 & $9.5-10.5$ & .212 & 3.30 & \\
\hline & & 4 & $\begin{array}{ll}10 & -11\end{array}$ & .237 & 3.54 & $\therefore$ \\
\hline & & 5 & $15-16$ & .228 & 2.06 & \\
\hline & & 6 & $\begin{array}{ll}20 & -21\end{array}$ & .216 & 2. 69 & \\
\hline & & 7 & $25-26$ & .28 & 2. 18 & \\
\hline & & 8 & $29-30$ & .238 & 1. 97 & \\
\hline & & 9 & $31.5-32.3$ & .165 & 1. 4 & \\
\hline & & 10 & $34.5-35.0$ & .189 & 1. 42 & \\
\hline & & 11 & $35.5-36.0$ & .124 & . 2.00 & \\
\hline & & 12 & $40-41$ & .196 & 2. 32 & \\
\hline & & 13 & $41-42$ & .138 & 1. 42 & $\ldots$ \\
\hline & & 14 & $46-47$ & .198 & 2.65 & $\ldots$ \\
\hline & & 15 & $50-51$ & .282 & 1.88 & $\ldots$ \\
\hline
\end{tabular}

$17116-$ No. 44-06-23

a Surface. 
UNDDERGROUND WATER RESOURCES OF LONG ISLAND, NEW YORK.

TABLE XII-Results of sizing tests-Continued.

\begin{tabular}{|c|c|c|c|c|c|c|}
\hline $\begin{array}{c}\text { Well } \\
\text { number. }\end{array}$ & $\begin{array}{l}\text { Commis- } \\
\text { sion well } \\
\text { number. }\end{array}$ & $\begin{array}{l}\text { Sample } \\
\text { number. }\end{array}$ & Depth. & $\begin{array}{l}\text { Eiffective } \\
\text { size. }\end{array}$ & $\begin{array}{l}\text { Uniformity } \\
\text { coefficient. }\end{array}$ & $\begin{array}{l}60 \text { per cent } \\
\text { finer, than- }\end{array}$ \\
\hline \multirow{14}{*}{382} & \multirow{14}{*}{658} & & Feet. & Millimeters. & & Millimeters. \\
\hline & & 16 & $54 \quad-55$ & 0.245 & 1.51 & \\
\hline & & 17 & $\begin{array}{ll}56 & -57\end{array}$ & .187 & 1. 26 & \\
\hline & & 18 & $\begin{array}{ll}58 & -59\end{array}$ & $.2: 3$ & 1.46 & \\
\hline & & 19 & $63-64$ & .213 & 1. 62 & \\
\hline & & 20 & $\begin{array}{ll}68 & -69\end{array}$ & .170 & 2.03 & \\
\hline & & 21 & $\begin{array}{ll}73 & -74\end{array}$ & .378 & 2.07 & \\
\hline & & 22 & $\begin{array}{ll}78 & -79\end{array}$ & .280 & 1. 48 & \\
\hline & & 23 & $\begin{array}{ll}81 & -82\end{array}$ & .239 & 1. 76 & \\
\hline & & 24 & $\begin{array}{ll}86 & -87\end{array}$ & .209 & 1.45 & \\
\hline & & 25 & $\begin{array}{ll}91 & -92\end{array}$ & .177 & 2.06 & \\
\hline & & 26 & $93 \quad-94$ & .1 & 2.04 & \\
\hline & & 27 & $95-96.5$ & .169 & 1.38 & \\
\hline & & 28 & $96.5-97.0$ & .172 & 1. 6 & \\
\hline \multirow[t]{11}{*}{390} & \multirow[t]{11}{*}{617} & 1 & .5 & $\ldots \ldots$ & $\ldots$. & 0. 305 \\
\hline & & 2 & $1.8-2.0$ & $\ldots$. & $\ldots \ldots$ & .25 \\
\hline & & 3 & $2.3-2.5$ & .35 & 3.57 & \\
\hline & & 4 & $5-6$ & .346 & 4.05 & \\
\hline & & 5 & $8.5-9.0$ & .26 & 2.77 & \\
\hline & & 6 & $10-11$ & .22 & 2. 41 & \\
\hline & & 7 & $\begin{array}{ll}15 & -16\end{array}$ & .22 & 1.9 & \\
\hline & & 8 & $\begin{array}{ll}.16 & -17\end{array}$ & .24 & 4.00 & \\
\hline & & 9 & $\begin{array}{ll}20 & -21\end{array}$ & .236 & 3. 18 & \\
\hline & & 10 & $25 \quad-26$ & .22 & 1.73 & \\
\hline & & 11 & $29.0-29.8$ & .141 & 1.38 & \\
\hline \multirow[t]{12}{*}{391} & \multirow[t]{12}{*}{618} & 1 & 0.4 & $\ldots$. & ...... & .42 \\
\hline & & 2 & 1.5 & $\ldots$. & $\ldots$ & .35 \\
\hline & & 3 & 2. 5 & .345 & 6.67 & \\
\hline & & 4 & $5-6$ & .24 & 10.83 & \\
\hline & & 5 & $8-9$ & $\therefore 4$ & 2.35 & \\
\hline & & 6 & $10.5-11.5$ & .247 & 2.00 & \\
\hline & & 7 & $14-15$ & .251 & 2. 11 & \\
\hline & & 8 & $\begin{array}{ll}16 & -17\end{array}$ & .211 & $1 . \Omega 6$ & \\
\hline & & 9 & $18.5-19.0$ & .31 & 3. 32 & \\
\hline & & 10 & $23 \quad-24$ & .39 & 7. 12 & \\
\hline & & 11 & $26.5-27.5$ & .159 & 2. 52 & \\
\hline & & 12 & $31.0-32.3$ & .17 & 1. 4 & . \\
\hline \multirow[t]{4}{*}{400} & \multirow[t]{4}{*}{845} & 1 & $0-0.8$ & $\ldots .$. & ..... & .225 \\
\hline & & 2 & $1.3-1.5$ & .41 & 15. 24 & \\
\hline & & 3 & $2.0-2.2$ & .415 & 6. 14 & \\
\hline & & 4 & $6-7$ & .289 & 2. 01 & \\
\hline
\end{tabular}


SIZING TESTS.

TABLE XII.-Results of sizing tests-Continued.

\begin{tabular}{|c|c|c|c|c|c|c|}
\hline $\begin{array}{c}\text { Well } \\
\text { number. }\end{array}$ & $\begin{array}{l}\text { Commis- } \\
\text { sion well } \\
\text { number. }\end{array}$ & $\begin{array}{l}\text { Sample } \\
\text { number. }\end{array}$ & Depth. & $\begin{array}{l}\text { Effective } \\
\text { size. }\end{array}$ & $\begin{array}{l}\text { Uniformity } \\
\text { coefficient. }\end{array}$ & $\begin{array}{l}60 \text { per cent } \\
\text { finer than- }\end{array}$ \\
\hline \multirow{6}{*}{400} & \multirow{6}{*}{845} & & Feet. & Millimeters. & & Millimeters. \\
\hline & & 5 & $11.0-11.8$ & 0.225 & 3.02 & $\ldots \ldots . . . .$. \\
\hline & & 6 & $\begin{array}{ll}16 & -17\end{array}$ & .313 & 3.04 & \\
\hline & & 7 & $21-22$ & .240 & 2.06 & \\
\hline & & 8 & $\begin{array}{ll}26 & -27\end{array}$ & .29 & 2.76 & \\
\hline & & 9 & $31-32$ & .211 & 1. 96 & \\
\hline \multirow[t]{11}{*}{401} & \multirow[t]{11}{*}{846} & 1 & $0 .-0.7$ & $\ldots \ldots$ & $\ldots \ldots \ldots$ & 0.63 \\
\hline & & 2 & $1.2-1.6$ & $\ldots$ & $\ldots$. & .3 \\
\hline & & 3 & $2.5-2.7$ & .38 & 3.74 & $\ldots$ \\
\hline & & 4 & $5.5-6.5$ & .22 & 4. 9 & \\
\hline & & 5 & $9.5-10.5$ & .256 & 3.28 & \\
\hline & & 6 & $14.5-15.5$ & .205 & 2.63 & \\
\hline & & 7 & $19-20$ & .300 & 7.83 & - \\
\hline & & 9 & $21.5-22.5$ & .12 & 3.5 & \\
\hline & & 10 & $23 \quad-24$ & 18 & 3.00 & \\
\hline & & 11 & $28-29$ & .23 & 2.39 & \\
\hline & & 12 & $\begin{array}{ll}31 & -32\end{array}$ & .30 & 6.83 & \\
\hline \multirow[t]{11}{*}{403} & \multirow[t]{11}{*}{847} & 1 . & $0.2-0.4$ & $\ldots$ & $\ldots \ldots$ & 1.38 \\
\hline & & 2 & $1.5-1.9$ & .29 & 12.76 & $\ldots \ldots$ \\
\hline & & 3 & $3.0-3.4$ & .45 & 11.1 & \\
\hline & & 4 & $8-9$ & .223 & 2.24 & \\
\hline & & 5 & $\begin{array}{ll}10 & -11\end{array}$ & .228 & 2.63 & \\
\hline & & 6 & $\begin{array}{ll}12 & -13\end{array}$ & .41 & 12.7 & \\
\hline & & 7 & $\begin{array}{ll}15 & -16\end{array}$ & .315 & 3. 1 & \\
\hline & & 8 & $\begin{array}{ll}18 & -19\end{array}$ & $\therefore 4$ & 8. 15 & \\
\hline & & 9 & $23 \quad-24$ & .216 & 2.5 & \\
\hline & & 10 & $\begin{array}{ll}28 & -29\end{array}$ & .23 & 2.7 & \\
\hline & & 11 & $33.0-33.5$ & .24 & 2.04 & \\
\hline \multirow[t]{9}{*}{409} & \multirow[t]{9}{*}{422} & 1 & $(a)$ & $\ldots \ldots$ & $\ldots$. & 3. 30 \\
\hline & & 2 & $\quad b 1.5$ & .51 & 12.16 & \\
\hline & & 3 & 5 & .23 & 6.52 & \\
\hline & & 4 & 10 & .30 & 4. 4 & \\
\hline & & 5 & 15 & .252 & 4. 48 & \\
\hline & & 6 & 22 & .207 & 2.32 & \\
\hline & & 7 & 28 & .10 & 6.8 & \\
\hline & & 8 & 35 & .20 & 2.05 & \\
\hline & & 9 & 37 & .231 & 2.1 & \\
\hline \multirow[t]{4}{*}{410} & \multirow[t]{4}{*}{862} & 1 & $.2-\quad .6$ & .161 & 1.32 & \\
\hline & & 2 & $1.2-1.6$ & .753 & 8.13 & \\
\hline & & 3 & $2.3-2.5$ & .318 & 1. 8 & \\
\hline & & 4 & $7-8$ & .23 & 2.39 & \\
\hline
\end{tabular}


TABle XII.-Results of sizing tests-Continued.

\begin{tabular}{|c|c|c|c|c|c|c|}
\hline $\begin{array}{c}\text { Well } \\
\text { number. }\end{array}$ & $\begin{array}{l}\text { Commis- } \\
\text { sion well } \\
\text { number. }\end{array}$ & $\begin{array}{l}\text { Sample } \\
\text { number. }\end{array}$ & Depth. & $\begin{array}{l}\text { Effective } \\
\text { size. }\end{array}$ & $\begin{array}{l}\text { Uniformity } \\
\text { coefficient. }\end{array}$ & $\begin{array}{l}60 \text { per cent } \\
\text { finer than- }\end{array}$ \\
\hline \multirow{7}{*}{410} & \multirow{7}{*}{862} & & Feet. & Millimeters. & & Millimeters. \\
\hline & & 5 & $\begin{array}{ll}12 & -13\end{array}$ & 0.27 & 4.26 & \\
\hline & & 6 & $17-18$ & .41 & 11.00 & \\
\hline & & 7 & $\begin{array}{ll}22 & -23\end{array}$ & .459 & 7.01 & \\
\hline & & 8 & $27 \quad-28$ & .215 & 2.8 & \\
\hline & & 9 & $32^{\circ}-33$ & .241 & 5. 18 & \\
\hline & & 10 & $\begin{array}{ll}36 & -36.8\end{array}$ & .232 & 3.44 & \\
\hline \multirow[t]{12}{*}{416} & \multirow[t]{4}{*}{863} & 1 & $0-0.6$ & $\ldots$. & $\ldots$. & 0.236 \\
\hline & & 2 & $1.2-1.4$ & $\ldots$. & - . . . & 2.08 \\
\hline & & 3 & $32-3.4$ & .28 & 16.07 & \\
\hline & & 4 & $6-7$ & .28 & 4. 43 & \\
\hline & \multirow[t]{8}{*}{$\because$} & 5 & $11-12$ & .38 & 6.58 & \\
\hline & & 6 & $16-17$ & .445 & 6. 14 & \\
\hline & & 7 & $21-22$ & .41 & 8. 78 & \\
\hline & & 8 & $26-27$ & .38 & 6.32 & \\
\hline & & 9 & $30.9-31.0$ & & & .229 \\
\hline & & 10 & $31-32$ & .365 & 9.32 & \\
\hline & & 11 & $\begin{array}{ll}36 & -37\end{array}$ & .33 & 9.09 & \\
\hline & & 12 & $41.5-42.5$ & .365 & 8.22 & $\cdots$ \\
\hline \multirow[t]{13}{*}{418} & \multirow[t]{13}{*}{901} & 1 & $.3-.5$ & $\ldots$ & ..... & .44 \\
\hline & & 2 & $2.0-2.4$ & .48 & 13. 33 & \\
\hline & & 3 & $6.5-8.0$ & .368 & 6.71 & \\
\hline & & 4 & $11 \quad-12$ & .35 & 11. 14 & \\
\hline & & 5 & $\begin{array}{ll}16 & -17\end{array}$ & .362 & 9. 67 & \\
\hline & & 6 & $\begin{array}{ll}21 & -22\end{array}$ & .362 & 8.01 & \\
\hline & & 7 & $\begin{array}{ll}26 & -27\end{array}$ & .325 & 7.88 & \\
\hline & & 8 & $\begin{array}{ll}31 & -32\end{array}$ & .33 & 9.09 & \\
\hline & & 9 & $36.4-36.9$ & .198 & 3.8 & \\
\hline & & 10 & $41-42$ & .350 & 6.91 & \\
\hline & & 11 & $45-51$ & $\cdots$ & $\ldots$ & .242 \\
\hline & & 12 & 51. $0-53.8$ & .221 & 1.81 & \\
\hline & & 13 & $53.8-55.7$ & .35 & 2.29 & \\
\hline \multirow[t]{9}{*}{421} & \multirow[t]{9}{*}{906} & 1 & $0-0.8$ & $\ldots$. & $\ldots$. & .34 \\
\hline & & 2 & $2.7-2.9$ & .206 & 5. 34 & \\
\hline & & 3 & $7.5-8.5$ & .269 & 14.5 & \\
\hline & & 4 & $12.2-13.2$ & .22 & 6.27 & \\
\hline & & 5 & $17.5-18.5$ & .243 & 5.1 & \\
\hline & & 6 & $22.5-23.5$ & .23 & 3.91 & \\
\hline & & 7 & $25.0-25.5$ & .22 & 7. 73 & \\
\hline & & 8 & $30-31$ & .215 & . 3.02 & \\
\hline & & 9 & $\begin{array}{ll}35 & -36\end{array}$ & .228 & 2. 76 & \\
\hline
\end{tabular}


SIZING TESTS.

TABLE XII.--Results of sizing tests-Continued.

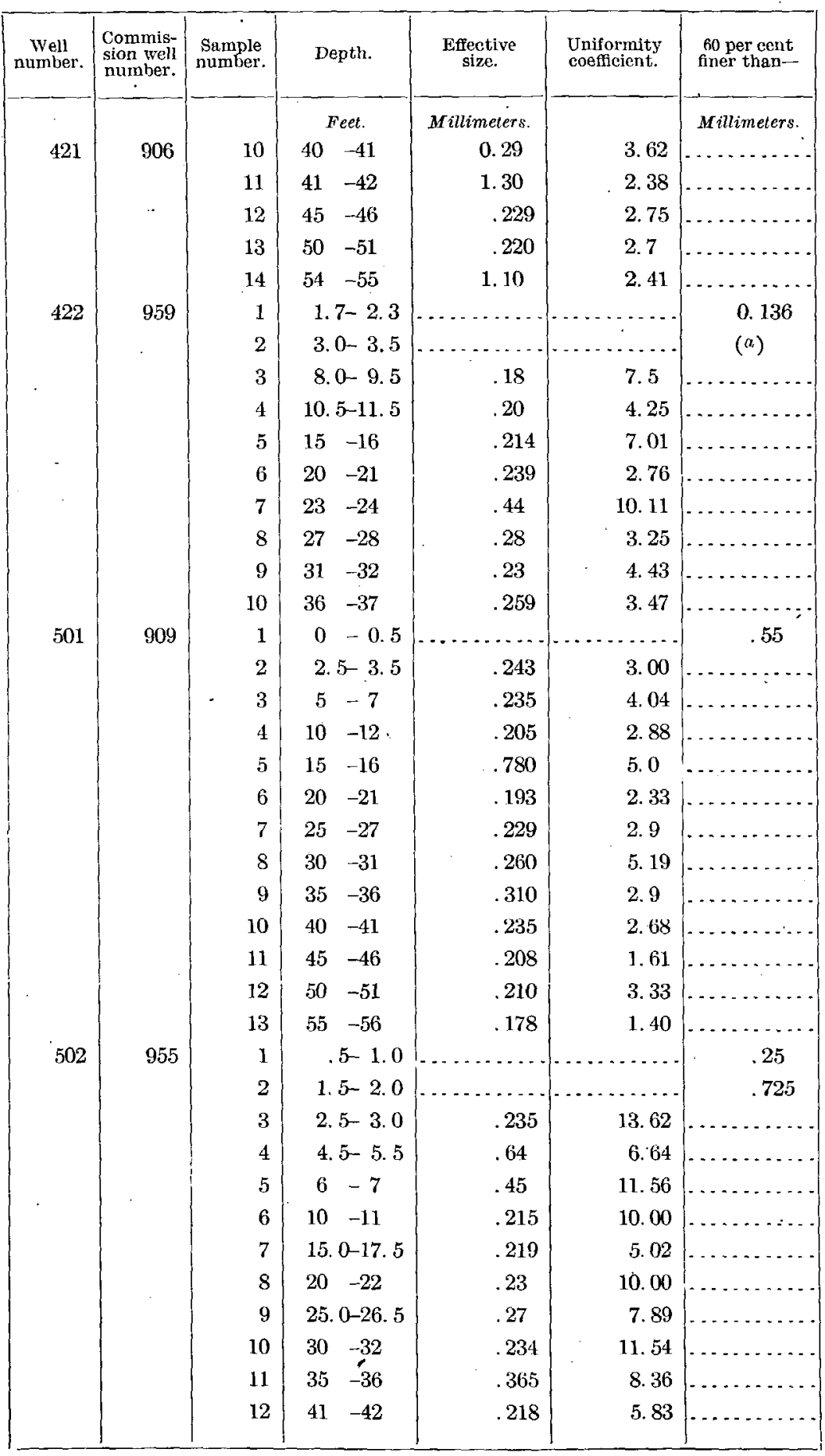

a 61 per cent finer than 0.10 
TABLE XII.-Results of sizing tests-Continued.

\begin{tabular}{|c|c|c|c|c|c|c|}
\hline $\begin{array}{c}\text { Well } \\
\text { number. }\end{array}$ & $\begin{array}{l}\text { Commis- } \\
\text { sion well } \\
\text { number. }\end{array}$ & $\begin{array}{l}\text { Sample } \\
\text { number. }\end{array}$ & Depth. & $\begin{array}{l}\text { Effective } \\
\text { size. }\end{array}$ & $\begin{array}{l}\text { Uniformity } \\
\text { coefficient. }\end{array}$ & $\begin{array}{l}60 \text { per cent } \\
\text { finer than- }\end{array}$ \\
\hline \multirow{22}{*}{502} & \multirow{22}{*}{955} & & Feet. & Millimeters. & & Millimeters. \\
\hline & & 13 & $45.0-46.5$ & 0.54 & 4.91 & ........... \\
\hline & & $a 14$ & $50-52$ & & $\cdots$ & \\
\hline & & . 15 & $55-57$ & 2.00 & 1.26 & \\
\hline & & 16 & $59-61$ & $\ldots$ & $\ldots$. & 0.535 \\
\hline & & 17 & $64.5-65.5$ & .42 & 2.26 & $\ldots$. \\
\hline & & 18 & $70.0-71.5$ & .365 & 2.66 &. \\
\hline & & 19 & $75-76$ & .42 & 3. 52 & \\
\hline & & 20 & $80-81$ & .225 & 4.00 & \\
\hline & & 21 & 83 & 2.21 & 1.35 & \\
\hline & & 22 & $85-86$ & .63 & 4. 41 & \\
\hline & & 23 & $90.0-90.5$ & .38 & 9.37 & \\
\hline & & 24 & $90.0-95.5$ & .22 & 5. 45 & \\
\hline & & 25 & $100.0-105.5$ & .37 & 5. 14 & \\
\hline & & 26 & $101-102$ & .23 & 2. 26 & \\
\hline & & 27 & $104.5-105.0$ & .219 & 1.89 & \\
\hline & & 28 & $109-110$ & .20 & 1.65 & \\
\hline & & 29 & $114.5-115.5$ & .185 & 1.52 & \\
\hline & & 30 & $120-121$ & .218 & 1.66 & \\
\hline & & 31 & $124.5-125.5$ & .218 & 1. 66 & $\ldots$ \\
\hline & & 32 & $129.5-131.0$ & .226 & 1.61 &. \\
\hline & & 33 & $132.5-135.5$ & .233 & 4. 68 & - \\
\hline \multirow[t]{18}{*}{506} & \multirow[t]{18}{*}{1142} & 1 & $0-1.5$ & $\ldots$ & $\ldots \ldots$ & .23 \\
\hline & & 2 & $1.8-2.2$ & .275 & 7.27 & $\ldots$ \\
\hline & & 3 & $3-6$ & .226 & 4. 56 & \\
\hline & & 4 & $10-11$ & .51 & 7.25 & . \\
\hline & & 5 & $15-16$ & .29 & 3.72 & - \\
\hline & & 6 & $20-21$ & .48 & 6.04 &. \\
\hline & & 7 & $25-26$ & .50 & 4.6 & \\
\hline & & 8 & $30-32$ & .23 & 3.91 & \\
\hline & & 9 & $35-37$ & .40 & 7.75 & \\
\hline & & 10 & $40-41$ & .35 & 5.71 & \\
\hline & & 11 & $45-46$ & .335 & 5.08 & \\
\hline & & 12 & $50-51$ & .275 & 3. 42 & \\
\hline & & 13 & $55-56$ & .23 & 9.13 & \\
\hline & & 14 & $60-61$ & .22 & 2.09 & \\
\hline & & 15 & $65-66$ & .218 & 1.51 & \\
\hline & & 16 & $70-71$ & .218 & 1.82 & $\ldots$ \\
\hline & & 17 & $75-76$ & .20 & 2.25 & $\cdots$ \\
\hline & & 18 & $79.5-80.5$ & .19 & 1.21 & $\ldots$ \\
\hline 569 & 849 & . 1 & $.2-\quad .3$ & $\ldots$ & - & .41 \\
\hline
\end{tabular}


SIZING TESTS.

TABLE XII.-Results of sizing tests--Continued.

\begin{tabular}{|c|c|c|c|c|c|c|}
\hline $\begin{array}{c}\text { Well } \\
\text { number. }\end{array}$ & $\begin{array}{l}\text { Commis- } \\
\text { sion well } \\
\text { number. }\end{array}$ & $\begin{array}{l}\text { Sample } \\
\text { number. }\end{array}$ & Depth. & $\begin{array}{l}\text { Effective } \\
\text { size. }\end{array}$ & $\begin{array}{l}\text { Uniformity } \\
\text { coefficient. }\end{array}$ & $\begin{array}{l}60 \text { per cent } \\
\text { finer than-- }\end{array}$ \\
\hline \multirow[t]{8}{*}{569} & \multirow[t]{8}{*}{849} & 2 & $\begin{array}{c}\text { Feet. } \\
2.0-2.4\end{array}$ & Millimeters. & & $\begin{array}{c}\text { Mrilimeters } \\
0.605\end{array}$ \\
\hline & & 3 & $4.0-4.5$ & 0.239 & 7. 66 & \\
\hline & & 4 & $\begin{array}{ll}10 & -11\end{array}$ & .27 & 3.89 & \\
\hline & & 5 & $\begin{array}{ll}11 & -12\end{array}$ & .27 & 3.89 & 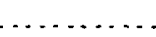 \\
\hline & & 6 & $\begin{array}{ll}15 & -16\end{array}$ & .34 & 2.91 & \\
\hline & & 7 & $20.5-21.5$ & .26 & 4. 12 & . \\
\hline & & 8 & 25. -26 & .238 & 2.48 & . \\
\hline & & 9 & $30-31$ & .49 & 3.67 & $\ldots$ \\
\hline \multirow[t]{24}{*}{574} & \multirow[t]{15}{*}{865} & 1 & $0.5-1.0$ & $\ldots \ldots$ & $\ldots$ & .216 \\
\hline & & 2 & $1.7-2.3$ & & & .205 \\
\hline & & 3 & $2.7-3.3$ & $\ldots$ & $\ldots \ldots$ & .23 \\
\hline & & 4 & $5-6$ & .31 & 2. 97 & \\
\hline & & 5 & $9-10$ & .30 & 10.67 & \\
\hline & & 6 & $15-16$ & .54 & 6.3 & \\
\hline & & 7 & $20-21$. & .375 & 2.47 & \\
\hline & & 8 & $25 \quad-27$ & .26 & 3.9 & \\
\hline & & 9 & $\begin{array}{ll}30 & -32\end{array}$ & .65 & 4.06 & \\
\hline & & 10 & $35.0-36.5$ & .213 & 2.07 & \\
\hline & & 11 & $37.5-38.0$ & .144 & 2. 19 & \\
\hline & & 12 & $40-41$ & .242 & 2.4 & \\
\hline & & 13 & $45 \quad-46$ & .228 & 4. 17 & \\
\hline & & 14 & $50.5-53.0$ & .52 & 4. 33 & \\
\hline & & 15 & $52.4-52.6$ & .11 & 7.82 & \\
\hline & $\sim$ & 16 & $\begin{array}{ll}55 & -58\end{array}$ & .105 & 8. 39 & \\
\hline & \multirow[t]{8}{*}{. } & 17 & $58.5-59.5$ & .118 & 3. 31 & \\
\hline & & 18 & $60.5-61.5$ & .220 & 1.85 & \\
\hline & & 19 & $63-64$ & .19 & 2.8 & \\
\hline & & 20 & $65.5-66.5$ & .65 & 1.77 & \\
\hline & & 21 & $70.0-72.5$ & .20 & 2.9 & \\
\hline & & 22 & $7.4 .5-75.5$ & .22 & 1,66 & \\
\hline & & 23 & $80-81$ & .232 & 2.41 & \\
\hline & & 24 & $84 \quad-85$ & .332 & 1.78 & - \\
\hline \multirow[t]{8}{*}{575} & 908 & 1 & $.5-1.0$ & .145 & 7.93 & \\
\hline & . & 2 & $1.5-2.0$ & .62 & 6.05 & \\
\hline & & 3 & $3-4$ & .33 & 6.06 & \\
\hline & & 4 & $6-7$ & .37 & 7.03 & \\
\hline & & 5 & $10-11$ & .363 & 7.71 & \\
\hline & & 6 & $15-16$ & .529 & 7.37 & •. \\
\hline & & 7 & $20-21$ & .213 & 2.63 & \\
\hline & & 8 & $24 \quad-25$ & .195 & 1. 13 & $\cdots$ \\
\hline
\end{tabular}


TABLE XII.-Results of sizing tests-Continued.

\begin{tabular}{|c|c|c|c|c|c|c|}
\hline $\begin{array}{c}\text { Well } \\
\text { number. }\end{array}$ & $\begin{array}{l}\text { Commis- } \\
\text { sion well } \\
\text { number. }\end{array}$ & $\begin{array}{l}\text { Sample } \\
\text { number. }\end{array}$ & Depth. & $\begin{array}{l}\text { Effective } \\
\text { size. }\end{array}$ & $\begin{array}{l}\text { Uniformity } \\
\text { coefficient. }\end{array}$ & $\begin{array}{l}60 \text { per cent } \\
\text { finer than- }\end{array}$ \\
\hline$\circ$ & \multirow{5}{*}{908} & & Feet. & Millimeters. & & Millimeters. \\
\hline \multirow[t]{4}{*}{575} & & 9 & $27.0-28.5$ & 0.215 & 1.08 & $\ldots . .$. \\
\hline & & 10 & $31-32$ & .218 & 1.09 & $\cdots$ \\
\hline & & 11 & $35-37$ & .218 & 1.3 & \\
\hline & & 12 & $40.5-41.5$ & .228 & 1.5 & $\ldots$ \\
\hline \multirow[t]{8}{*}{635} & \multirow[t]{8}{*}{743} & 1 & $0-0.5$ & $\ldots \ldots$ & $\ldots \ldots \ldots$ & 0.605 \\
\hline & & 2 & $.5-1.0$ & & $\ldots \ldots \ldots$ & 2.00 \\
\hline & & 3 & $1-3$ & .53 & 6.51 & $\ldots \ldots$ \\
\hline & & 4 & $5-6$ & .38 & 7.37 & - \\
\hline & & 5 & $10-11$ & .341 & 7.68 & \\
\hline & & 6 & $15--16$ & .398 & 4. 22 & $\cdots$ \\
\hline & & 7 & $19-20$ & .28 & 4.57 & \\
\hline & & 8 & $24 \quad-25$ & .38 & 2.6 & $\ldots \ldots$ \\
\hline \multirow[t]{9}{*}{637} & \multirow[t]{9}{*}{729} & 1 & $(\alpha)$ & $\cdots$. & $\ldots$. & 1.13 \\
\hline & & 2 & $.5-1.0$ & & . & .80 \\
\hline & & 3 & $1-3$ & .308 & 8.08 & $\ldots . .$. \\
\hline & & 4 & $5-6$ & .358 & 8.1 & $-2 .-2$ \\
\hline & & 5 & $10-11$ & .435 & 7.13 & \\
\hline & & 6 & $15-16$ & .25 & 4.8 & \\
\hline & & 7 & $20-21$ & .23 & 2.7 & \\
\hline & & 8 & $\begin{array}{ll}24 & -26\end{array}$ & .345 & 2. 43 & $\cdots \ldots$ \\
\hline & & 9 & 29 & .338 & 5.47 & $\ldots \ldots$ \\
\hline \multirow[t]{19}{*}{639} & \multirow[t]{19}{*}{826} & 1 & $(a)$ & & $\ldots$. & .74 \\
\hline & & 2 & $.5-2.0$ & .156 & 6.73 & $\ldots \ldots \ldots$ \\
\hline & & 3 & $5-6$ & $\cdot .259$ & 4.02 & - \\
\hline & & 4 & $10-12$ & .434 & 4.61 & \\
\hline & & 5 & $15-16$ & .219 & 4. 93 & $\ldots \ldots$ \\
\hline & & 6 & $20-21$ & .365 & 2.8 & \\
\hline & & 7 & $25-26$ & .42 & 6.21 & \\
\hline & & 8 & $30-31$ & .22 & 1. 59 & \\
\hline & & 9 & $35-36$ & .209 & 1.55 & \\
\hline & & 10 & $40-41$ & .314 & 3. 12 & \\
\hline & & 11 & $45-46$ & $.265^{\circ}$ & 3.21 & \\
\hline & & 12 & $50-51$ & .34 & 1.76 & \\
\hline & & 13 & $\begin{array}{lll}55 & -56\end{array}$ & 266 & 2.03 & $\ldots \ldots \ldots$ \\
\hline & & 14 & $60-61$ & .22 & 1.93 & \\
\hline & & 15 & $65-66$ & .37 & 2.51 & \\
\hline & & 16 & $\begin{array}{ll}70 & -71\end{array}$ & .382 & 2. 49 & $\ldots \ldots$ \\
\hline & & 17 & $75 \quad-76$ & .281 & 3.47 & \\
\hline & & 18 & $\begin{array}{ll}80 & -81\end{array}$ & .231 & 2.86 & . . . . . . . \\
\hline & & 19 & $\begin{array}{ll}85 & -86\end{array}$ & .46 & 2.72 & $\ldots \ldots$ \\
\hline
\end{tabular}


TABLE XII-Results of sizing tests-Continued.

\begin{tabular}{|c|c|c|c|c|c|c|}
\hline $\begin{array}{l}\text { Well } \\
\text { number. }\end{array}$ & $\begin{array}{l}\text { Commis- } \\
\text { sion well } \\
\text { number. }\end{array}$ & $\begin{array}{l}\text { Sample } \\
\text { number. }\end{array}$ & Depth. & $\begin{array}{l}\text { Effective } \\
\text { size. }\end{array}$ & $\begin{array}{l}\text { Uniformity } \\
\text { coefficient. }\end{array}$ & $\begin{array}{l}60 \text { per cent } \\
\text { finer than- }\end{array}$ \\
\hline \multirow{3}{*}{639} & \multirow{3}{*}{826} & & Feel. & Millimeiers. & & Millimeters. \\
\hline & & 20 & $87.0-87.5$ & 0.125 & 1.72 & ........ \\
\hline & & 21 & $91-92$ & $\ldots \ldots \ldots$ & $\ldots \ldots \ldots$ & 0.182 \\
\hline \multirow[t]{22}{*}{694} & \multirow[t]{22}{*}{861} & 1 & 0.3 & . & $\ldots$. & .5 \\
\hline & & 2 & $1.0-1.5$ & & $\ldots \ldots$ & 39 \\
\hline & & 3 & $5.0-5.5$ & .224 & 2.63 & $\ldots \ldots$ \\
\hline & & 4 & $10.0-10.5$ & .22 & 15. 23 & \\
\hline & & 5 & $15-16$ & .195 & 3. 33 & \\
\hline & & 6 & $20-21$ & 1. 10 & 3.19 & \\
\hline & & 7 & $30.0-30.5$ & .3 & 5.67 & \\
\hline & & 8 & $35.5-35.5$ & .33 & 2. 73 & \\
\hline & & 9 & $40.0-40.5$ & .23 & 3. 00 & \\
\hline & & 10 & $45.0-45.5$ & .214 & 1. 54 & \\
\hline & & 11 & $50.0-50.5$ & .238 & 5.25 & \\
\hline & & 12 & $55 \div 56$ & .222 & 3.47 & \\
\hline & & 13 & $60.0-60.5$ & .19 & 1.37 & \\
\hline & & 14 & $65.0-65.5$ & .224 & 2.28 & \\
\hline & & 15 & $69.0-69.5$ & .229 & 2.1 & \\
\hline & & 16 & $75.0-75.5$ & .242 & 1.9 & \\
\hline & & 17 & $80.0-80.5$ & .208 & 1. 25 & \\
\hline & & 18 & $85.0-85.5$ & .209 & 1.82 & \\
\hline & & 19 & $90.0-90.5$ & .200 & 1.17 & \\
\hline & & 20 & $95.0-95.5$ & .22 & 1.59 & \\
\hline & & 21 & $100.0-100.5$ & .18 & 1.23 & \\
\hline & & 22 & $101.5-102.0$ & $\ldots$ & $\ldots$ & .22 \\
\hline \multirow[t]{9}{*}{695} & \multirow[t]{9}{*}{843} & 1 & .5 & $\ldots$ & $\ldots$ & .4 \\
\hline & & 2 & $1.5-2.0$ & . & $\cdots$ & .6 \\
\hline & & 3 & $5.0-\quad 5.5$ & .22 & 4. 64 & \\
\hline & & 4 & $10.0-10.5$ & .27 & 1.73 & \\
\hline & & 5 & $15.0-15.5$ & .22 & 4.5 & \\
\hline & & 6 & $20.0-20.5$ & .22 & 2.73 & \\
\hline & & 7 & $25-26$ & .42 & 7.33 & \\
\hline & & 8 & $30.0-30.5$ & .23 & 2.52 & \\
\hline & & 9 & $35-36$ & .235 & 3.83 & \\
\hline
\end{tabular}


FILTRATION TESTS.

Filtration tests were made with columns of carefully packed material, 6 inches long and one ten-millionth of an acre in section, under a 5 -foot head of water. Recently boiled water of normal room temperature was used. Before beginning the filtration test with a sample the air was expelled by admitting water from a burette slowly at the bottom of the column, and the volume of water thus required to fill the sand was carefully measured. This volume expressed in cubic centimeters and also in percentages constitutes the porosity determination.

The upper end of the tube was then connected with the 5-foot head of water for the filtration test. Water was allowed to flow unmeasured for several minutes until the finer sand particles should have time to adjust themselves and until any residuum of air left in the sand should have been dissolved out; the flow was then carefully measured for five minutes and multiplied by 12 to get the rate per hour.

Under the conditions of the tests it is, obviously, impossible to reproduce the structure of the material as it existed in the ground; and yet this structure-the mode of association and arrangement of the grains of varying sizes-must profoundly influence the filtration rate. This is, probably, the most serious limitation of the filtration tests; for while we may fairly assume that the material in the ground is closely packed (hard-packed), we have, in general, or with ordinary boring samples, no means of knowing whether it is a homogeneous mixture or, as must commonly be the case, distinctly laminated, coarse, pervious layers alternating with fine, impervious layers, in a way to insure the maximum flow in a horizontal direction. If a general assumption must be made, it were, doubtless, most conservative to assume the horizontal flow as greater and the vertical flow as less than the filtration rate, which may be, in many cases, an approximate mean.

TABLE XIII:-Results of filtration tests.

\begin{tabular}{|c|c|c|c|c|c|c|c|}
\hline \multirow{2}{*}{$\begin{array}{c}\text { Well } \\
\text { number. }\end{array}$} & \multirow{2}{*}{$\begin{array}{l}\text { Commis- } \\
\text { sion well } \\
\text { number. }\end{array}$} & \multirow{2}{*}{$\begin{array}{l}\text { Sample } \\
\text { number. }\end{array}$} & \multirow{2}{*}{\multicolumn{2}{|c|}{ Depth (feet). }} & \multicolumn{2}{|c|}{ Porosity. } & \multirow{2}{*}{$\begin{array}{l}\text { Filtration: } \\
\text { Cm.3 per } \\
\text { hour. }\end{array}$} \\
\hline & & & & & $\mathrm{Cm} .{ }^{3}$. & Per cent. & \\
\hline \multirow[t]{3}{*}{148} & \multirow[t]{12}{*}{1204} & 9 & & $30-31$ & 17 & 32 & 960 \\
\hline & & 10 & & $35-36$ & 17 & 32 & 672 \\
\hline & & 11 & & $36-37$ & 14.5 & 27.3 & 2,880 \\
\hline \multirow[t]{9}{*}{. } & & 12 & & $44-45$ & 17 & 32 & 5,520 \\
\hline & & 13 & & $50-51$ & 16.5 & 31.1 & 540 \\
\hline & & 14 & & $55-56$ & 18 & 33.9 & 960 \\
\hline & & 15 & & $62-63$ & 16 & 30.1 & 4,320 \\
\hline & & 16 & & $67-68$ & 14.5 & 27.3 & 3,144 \\
\hline & & 17 & & $74-75$ & 17.5 & 33 & 3,780 \\
\hline & & 18 & & $82-83$ & 19 & 35.8 & 5,100 \\
\hline & & 19 & & $88-89$ & 14.5 & 27.3 & 2,204 \\
\hline & & 20 & & $95-96$ & 17 & 32 & 828 \\
\hline \multirow[t]{2}{*}{303} & \multirow[t]{2}{*}{607} & 5 & 15 & -15.5 & 18 & 33.9 & 6,180 \\
\hline & & 6 & & -20.5 & 18 & 33.9 & 2,040 \\
\hline
\end{tabular}


FILTRATION TESTS.

TABLE XIII.-Results of filtration tests-Continued.

\begin{tabular}{|c|c|c|c|c|c|c|}
\hline \multirow{2}{*}{$\begin{array}{c}\text { Well } \\
\text { number. }\end{array}$} & \multirow{2}{*}{$\begin{array}{l}\text { Commis- } \\
\text { sion well } \\
\text { number. }\end{array}$} & \multirow{2}{*}{$\begin{array}{l}\text { Sample } \\
\text { number. }\end{array}$} & \multirow{2}{*}{ Depth (feet). } & \multicolumn{2}{|c|}{ Porosity. } & \multirow{2}{*}{$\begin{array}{l}\text { Filtration: } \\
\text { Cm. per } \\
\text { hour. }\end{array}$} \\
\hline & & & & $\mathrm{Cm} .^{3}$. & Per cent. & \\
\hline \multirow[t]{4}{*}{303} & \multirow[t]{4}{*}{607} & 7 & $25-26$ & 19 & 35.8 & 4,200 \\
\hline & & 8 & $30-30.5$ & 17 & 32 & 7,380 \\
\hline & & 9 & $35-36$ & 18 & 33.9 & 2,616 \\
\hline & & 10 & $40 \quad-41$ & 15. 5 & 29.2 & 3,660 \\
\hline \multirow[t]{5}{*}{; 308} & \multirow[t]{5}{*}{907} & 11 & $35-36$ & 15 & 28.3 & 4,800 \\
\hline & & 12 & $40-41$ & 16.5 & 31.1 & 6,240 \\
\hline & & 13 & $44-45$ & 20 & 37.7 & 7,440 \\
\hline & & 14 & $\begin{array}{ll}50 & -51\end{array}$ & 15.5 & 29.2 & 3,360 \\
\hline & & 15 & $55.5-56.5$ & 17 & 32 & 7,560 \\
\hline \multirow[t]{11}{*}{310} & \multirow[t]{11}{*}{829} & 8 & $\begin{array}{ll}30 & -30\end{array}$ & 16.5 & 31.1 & 5,640 \\
\hline & & 9 & -36 & 16 & 30.1 & 3,540 \\
\hline & & 10 & $40-41$ & 18 & 33.9 & 4,620 \\
\hline & & 11 & $45 \quad-46$ & 17 & 32 & 8,760 \\
\hline & & 12 & $\begin{array}{ll}50 & -53\end{array}$ & 24 & 45.2 & 4,920 \\
\hline & & 13 & $55-56$ & 19.5 & 36.7 & 14,400 \\
\hline & & 14 & -61 & 19.5 & 36.7 & 17,940 \\
\hline & & 15 & -66 & 18.5 & 34.9 & 10,200 \\
\hline & & 16 & $70-71$ & 21 & 39.6 & 20,880 \\
\hline & & 17 & $\begin{array}{ll}75 & -77\end{array}$ & 15 & 28.3 & 21,840 \\
\hline & & 18 & $80.5-81.5$ & 26 & 49 & 84 \\
\hline \multirow[t]{6}{*}{323} & \multirow[t]{6}{*}{956} & 6 & $\begin{array}{ll}19 & -20\end{array}$ & 16 & 30.1 & 4,080 \\
\hline & & 7 & -25 & 16.5 & 31.1 & 2,136 \\
\hline & & 8 & -30 & 15 & 28.3 & 936 \\
\hline & & 9 & -35 & 21 & 39.6 & 14,880 \\
\hline & & 10 & -40 & 12.5 & 23.5 & 2,640 \\
\hline & & 11 & $\begin{array}{ll}45 & -47\end{array}$ & 14 & 26.4 & 636 \\
\hline \multirow[t]{8}{*}{382} & \multirow[t]{8}{*}{ a 658} & 3 & $9.5-10.5$ & 24 & 47 & 624 \\
\hline & & 4 & $10-11$ & 15 & 29.4 & 3,960 \\
\hline & & 5 & $15-16$ & 22 & 43. 1 & 9,840 \\
\hline & & 6 & $20-21$ & $11: 5$ & 22.5 & 4,740 \\
\hline & & 7 & $25 \quad-26$ & 21 & 41.1 & 9,120 \\
\hline & & 8 & $29-30$ & 12 & 23.5 & 2,940 \\
\hline & & 9 & $31.5-32.3$ & 22 & 43.1 & 852 \\
\hline & & 10 & $34.5-35.0$ & 17.5 & 34.3 & 80,000 \\
\hline \multirow[t]{5}{*}{400} & \multirow[t]{5}{*}{845} & 3 & $2-2.2$ & 18 & 33.9 & 7,200 \\
\hline & & 4 & -7 & 35 & 66 & 11,880 \\
\hline & & 5 & $\begin{array}{ll}11-11.8\end{array}$ & 18.5 & 34.9 & 4,680 \\
\hline & & 6 & $\begin{array}{ll}16 & -17\end{array}$ & 18 & 33.9 & 8,280 \\
\hline & & 7 & $21 \quad-22$ & 17.5 & 33 & $4,320^{\circ}$ \\
\hline
\end{tabular}

a Wells 658 and 846 have porosity percentages reckoned on basis of $51 \mathrm{~cm} .^{3}$ capacity for filtration tube; all others $53 \mathrm{~cm} .^{2}$ capacity. 
356 UNDERGROUND WATER RESOURCES OF LONG ISLAND, NEW YORK.

TABLE XIII.--Results of filtration tests - Continued.

\begin{tabular}{|c|c|c|c|c|c|c|}
\hline \multirow{2}{*}{$\begin{array}{c}\text { Well } \\
\text { number. }\end{array}$} & \multirow{2}{*}{$\begin{array}{l}\text { Commis- } \\
\text { sion well } \\
\text { number. }\end{array}$} & \multirow{2}{*}{$\begin{array}{l}\text { Sample } \\
\text { number. }\end{array}$} & \multirow{2}{*}{ Depth (feet). } & \multicolumn{2}{|c|}{ Porosity- } & \multirow{2}{*}{$\begin{array}{l}\text { Filtration: } \\
\text { Cm. }{ }^{3} \text { per } \\
\text { hour. }\end{array}$} \\
\hline & & & & $\mathrm{Cm} .{ }^{3}$ & Per cent. & \\
\hline \multirow[t]{2}{*}{400} & \multirow[t]{2}{*}{845} & 8 . & $26-27$ & 17.5 & 33 & 8,340 \\
\hline & & 9 & $\begin{array}{ll}31 & -32\end{array}$ & 19.5 & 36.7 & 3,540 \\
\hline \multirow[t]{9}{*}{401} & \multirow[t]{9}{*}{ a 846} & 3 & $2.5-2.7$ & 12.5 & 24.5 & 1,200 \\
\hline & & 4 & $5 . j-6.5$ & 13.5 & 26.4 & 3,300 \\
\hline & & 5 & $9.5-10.5$ & 17 & 33.3 & 4,740 \\
\hline & & 6 & $14.5-15.5$ & 17 & 33.3 & 1,740 \\
\hline & & 7 & $19-20$ & 16 & 31.3 & 9,420 \\
\hline & & 9 & $21.5-22.5$ & 14.5 & 28.4 & 444 \\
\hline & & 10 & $23-24$ & 13.5 & 26.4 & 288 \\
\hline & & 11 & $\begin{array}{ll}28 & -29\end{array}$ & 19 & 37.2 & 2,880 \\
\hline & & 12 & $\begin{array}{ll}31 & -32\end{array}$ & 17 & 33.3 & 8,280 \\
\hline \multirow[t]{6}{*}{403} & \multirow[t]{6}{*}{847} & 5 & $\begin{array}{ll}10 & -11\end{array}$ & 16 & 30.1 & 4,680 \\
\hline & & 6 & $12-13$ & 16 & 30.1 & 16,680 \\
\hline & & 7 & $15-16$ & 17.75 & 33.4 & 9,420 \\
\hline & & 8 & $18 \quad-19$ & 14 & 26.4 & 12,960 \\
\hline & & 9 & $23 \quad-24$ & 18.5 & 34.9 & 4,380 \\
\hline & & 10 & $\begin{array}{ll}28 & -29\end{array}$ & 16.5 & 31.1 & 5,340 \\
\hline \multirow[t]{8}{*}{410} & \multirow[t]{8}{*}{862} & 3 & $2.3-22.5$ & 20 & 37.7 & 3,060 \\
\hline & & 4 & $7-6$ & 16 & 30.1 & 2,700 \\
\hline & & 5 & $\begin{array}{ll}12 & -13\end{array}$ & 12.5 & 23.5 & 6,060 \\
\hline & & 6 & $\begin{array}{ll}17 & -18\end{array}$ & 13.5 & 25.4 & 8,760 \\
\hline & & 7 & $\begin{array}{ll}22 & -23\end{array}$ & 13 & 24.5 & 7,740 \\
\hline & & 8 & $\begin{array}{ll}27 & -28\end{array}$ & 15 & 28.3 & 2,880 \\
\hline & & 9 & $32-33$ & 23 & 43.3 & 3,180 \\
\hline & & 10 & $36.0-36.8$ & 16.5 & 31.1 & 6,000 \\
\hline \multirow[t]{6}{*}{418} & \multirow[t]{6}{*}{901} & 8 & $\begin{array}{ll}31 & -32\end{array}$ & 16.5 & 31.1 & 13,260 \\
\hline & & 9 & $36.4-36.9$ & 14.5 & 27.3 & 1,944 \\
\hline & & 10 & $\begin{array}{ll}41 & -42\end{array}$ & 17.5 & 33 & 13,704 \\
\hline & & 11 & $45-51$ & 23 & 43. 3 & 2,880 \\
\hline & & 12 & $51.0-53.8$ & 20 . & 37.7 & 7,860 \\
\hline & & 13 & 53. $8-55.7$ & 36 & 67.9 & 21,840 \\
\hline \multirow[t]{6}{*}{421} & \multirow[t]{6}{*}{906} & 9 & $35-36$ & 18.5 & 34.9 & 9,480 \\
\hline & & 10 & $40 \quad-41$ & 18 & 33.9 & 15,420 \\
\hline & & 11 & $\begin{array}{ll}41 & -42\end{array}$ & 24 & 45.2 & 60,000 \\
\hline & & 12 & $45-46$ & 21 & 39.6 & 12,504 \\
\hline & & 13 & $50-51$ & 20.5 & 38.6 & 11,340 \\
\hline & & 14 & $54-55$ & 19 & 35.8 & 52,800 \\
\hline \multirow[t]{2}{*}{422} & \multirow[t]{2}{*}{959} & 8 & $\begin{array}{ll}27 & -28\end{array}$ & 16.5 & 31.1 & 11,340 \\
\hline & & 9 & $31 \quad-32$ & 16 & 30.1 & 8,400 \\
\hline
\end{tabular}

a Wells 658 and 846 have porosity percentages reckoned on basis of $51 \mathrm{~cm}^{3}$ capacity for filtration tube; all others $53 \mathrm{~cm} .{ }^{3}$ capacity. 
FILTRATION TESTS.

TABLE XIII.-Results of filtration tests-Continued.

\begin{tabular}{|c|c|c|c|c|c|c|}
\hline \multirow{2}{*}{$\begin{array}{l}\text { Well } \\
\text { number. }\end{array}$} & \multirow{2}{*}{$\begin{array}{l}\text { Commis- } \\
\text { sion well } \\
\text { number. }\end{array}$} & \multirow{2}{*}{$\begin{array}{c}\text { Sample } \\
\text { number. }\end{array}$} & \multirow{2}{*}{ Depth (fcet). } & \multicolumn{2}{|c|}{ Porosity- } & \multirow{2}{*}{$\begin{array}{l}\text { Filtration: } \\
\text { Cm. } \\
\text { hour. }\end{array}$} \\
\hline & & & & $\mathrm{Cm}^{\mathrm{a}}$. & Per cent. & \\
\hline 422 & 959 & 10 & $\begin{array}{ll}36 & -37\end{array}$ & 17 & 32 & 12,060 \\
\hline \multirow[t]{5}{*}{454} & \multirow[t]{5}{*}{960} & 1 & $5-6$ & 16.5 & 31.1 & 6,000 \\
\hline & & 2 & $\begin{array}{ll}8 & -10\end{array}$ & 19.5 & 36.7 & 3,480 \\
\hline & & 3 & $21.1-25.0$ & 18 & 38.9 & 3,480 \\
\hline & & 4 & $\begin{array}{ll}37 & -40\end{array}$ & 18 & 33.9 & 18,240 \\
\hline & & 5 & $41 \quad-52$ & 17 & 32 & 1,980 \\
\hline \multirow[t]{18}{*}{495} & \multirow[t]{18}{*}{1272} & 1 & $0.5-1.0$ & 16.5 & $31: 1$ & 3,360 \\
\hline & & 2 & $1.5-2.0$ & 15 & 28.3 & 9,720 \\
\hline & & 3 & $5-6$ & 16 & 30.1 & 17,400 \\
\hline & & 4 & $10-11$ & 11 & 20.7 & 2,940 \\
\hline & & 5 & $15-16$ & 17.5 & 33 & 7,260 \\
\hline & & 6 & $20 \quad-21$ & 17 & 32 & 8,940 \\
\hline & & 7 & $24 \quad-25$ & 18 & 33.9 & 7,260 \\
\hline & & 8 & $29-30$ & 19.5 & 36.7 & 8,760 \\
\hline & & 9 & $\begin{array}{ll}30 & -31\end{array}$ & 20 & 37.7 & 1,080 \\
\hline & & 10 & $32-33$ & 16 & 30.1 & 2,160 \\
\hline & & 11 & $34-35$ & 24 & 45.2 & 1,500 \\
\hline & & 12 & $\begin{array}{ll}40 & -41\end{array}$ & 20.5 & 38.6 & 852 \\
\hline & & 13 & $45-46$ & 20 & 37.7 & 1,560 \\
\hline & & 14 & $48 \quad-49$ & - & 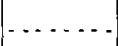 & $(a)$ \\
\hline & & 15 & $60-61$ & 26 & 49 & 408 \\
\hline & & 16 & $63-65$ & 20.5 & 38.6 & 756 \\
\hline & & 17 & $65.5-67.5$ & 23 & 43.3 & 372 \\
\hline & & 18 & $70-71$ & 21.5 & 40.5 & 251 \\
\hline \multirow[t]{6}{*}{697} & \multirow[t]{6}{*}{1087} & 3 & $5.0-5.5$ & 22 & 41.5 & 7,320 \\
\hline & & 4 & $10-12$ & 17 & 32 & 7,860 \\
\hline & & 5 & $15-16$ & 16.5 & 31.1 & 7,560 \\
\hline & & 6 & $20 \quad-21$ & 21.5 & 40.5 & 6,000 \\
\hline & & 7 & $25-26$ & 16.5 & 31.1 & 8,940 \\
\hline & & 8 & $29-30$ & 17 & 32 & 5,580 \\
\hline \multirow[t]{5}{*}{698} & \multirow[t]{5}{*}{1088} & 4 & $10.0-10.5$ & 16 & 30.1 & 60,600 \\
\hline & & 5 & $15.0-15.5$ & 15. $5^{2}$ & 29.2 & 10,200 \\
\hline & & 6 & $20.0-20.5$ & 16 & 30.1 & 10,620 \\
\hline & & 7 & $25.0-25.5$ & 17 & 32 & 6,600 \\
\hline & & 8 & $30.0-30.5$ & 16 & .30 .1 & 9,360 \\
\hline \multirow[t]{4}{*}{707} & \multirow[t]{4}{*}{1141} & 3 & $3-5$ & 13.5 & 26.4 & 4,140 \\
\hline & & 4 & $5 \quad-21$ & 16 & 30.1 & 1,320 \\
\hline & & 5 & $21 \quad-25$ & 16 & 30.1 & 8,760 \\
\hline & & 6 & $25 \cdot-30$ & 15.5 & 29.2 & 15,360 \\
\hline
\end{tabular}

a Does not filter. 
358 UNDERGROUND WATER RESOURCES OF LONG ISLAND, NEW YORK.

TABLE XIII.-Results of filtration tesls-Continued.

\begin{tabular}{|c|c|c|c|c|c|c|}
\hline \multirow{2}{*}{$\begin{array}{c}\text { Well } \\
\text { number. }\end{array}$} & \multirow{2}{*}{$\begin{array}{l}\text { Commis- } \\
\text { sion well } \\
\text { number. }\end{array}$} & \multirow{2}{*}{$\begin{array}{l}\text { Sample } \\
\text { number. }\end{array}$} & \multirow{2}{*}{ Depth (feet). } & \multicolumn{2}{|c|}{ Porosity- } & \multirow{2}{*}{$\begin{array}{c}\text { Filtration: } \\
\text { Cm. } \\
\text { hour. }\end{array}$} \\
\hline & & & & $\mathrm{Cm}^{3}$. & Per cent. & \\
\hline \multirow[t]{16}{*}{707} & \multirow[t]{16}{*}{1141} & 7 & $30-35$ & 16 & 30.1 & 42,960 \\
\hline & & 8 & $35-40$ & 16 & 30.1 & 10,560 \\
\hline & & 9 & $40-45$ & 18 & 33.9 & 58,200 \\
\hline & & 10 & $45-50$ & 18.5 & 34.9 & 12,660 \\
\hline & & 11 & $50-55$ & 15.5 & 29.2 & 12,960 \\
\hline & & 12 & $55-60$ & 18 & 33.9 & 9,840 \\
\hline & & 13 & $60-65$ & 14 & 26.4 & 15,180 \\
\hline & & 14 & $65-70$ & 15.5 & 29.2 & 8,700 \\
\hline & & 15 & . $\quad 70-78$ & 16 & 30.1 & 9,360 \\
\hline & & 16 & $78-81$ & 19 & 35.8 & 6,960 \\
\hline & & 17 & $81-85$ & 17 & 32 & 8,280 \\
\hline & & 18 & $85-88$ & 18 & 33.9 & 5,760 \\
\hline & & 19 & $88-92$ & 20.5 & 38.6 & 10,800 \\
\hline & & 20 & $92-97$ & 17 & 32 & 21,420 \\
\hline & & 21 & $97-100$ & 17 & 32 & 9,240 \\
\hline & & 22 & $100-103$ & 20 & 37.7 & 5,640 \\
\hline \multirow[t]{8}{*}{.708} & \multirow[t]{8}{*}{1195} & 4 & $5-10$ & 14.5 & 27.3 & 2,820 \\
\hline & & 5 & $10-15$ & 13.5 & 26.4 & 6,828 \\
\hline & & 6 & $15-20$ & 17 & 32 & 8,040 \\
\hline & & 7 & $25-28$ & 16.5 & 31.1 & 7,940 \\
\hline & & 8 & $29-30$ & 16 & 30.1 & 3,744 \\
\hline & & 9 & $30-35$ & 14.5 & 27.3 & 8,280 \\
\hline & & 10 & $35-40$ & 17 & 32 & 11,040 \\
\hline & & 11 & $40-44$ & 14.5 & 27.3 & 5,796 \\
\hline \multirow[t]{8}{*}{729} & \multirow[t]{8}{*}{1198} & 4 & $9-10$ & 15.5 & 29.2 & 6,420 \\
\hline & & 5 & $14-15$ & 14.5 & 27.3 & 4,020 \\
\hline & & 6 & $19-20$ & 13 & 24.5 & 3,984 \\
\hline & & 7 & $24-25$ & 15 & 28.3 & 8,520 \\
\hline & & 8 & $29-30$ & 16.5 & 31.1 & 9,360 \\
\hline & & 9 & $34-35$ & 20 & 37.7 & 9,960 \\
\hline & & 10 & $39-40$ & 17 & 32 & 10,920 \\
\hline & & 11 & $44-45$ & 18 & 33.9 & 8,760 \\
\hline \multirow[t]{8}{*}{731} & \multirow[t]{8}{*}{1200} & 3 & $4-5$ & 13.5 & 25.4 & 9,240 \\
\hline & & 4 & $9-10$ & 16 & 30.1 & 9,780 \\
\hline & & 5 & $14-15$ & 14.5 & 27.3 & 7,260 \\
\hline & & 7 & $24-25$ & 16.5 & 31.1 & 10,620 \\
\hline & & 8 & $29-30$ & 17 & 32 & 7,560 \\
\hline & & 9 & $34-35$ & 16.5 & 31.1 & 7,260 \\
\hline & & 10 & $39-40$ & 17 & 32 & 3,060 \\
\hline & & 11 & $44-45$ & 17 & 32 & 10,740 \\
\hline
\end{tabular}


FLLTRATION 'TESTS.

TABLE XIII.-Results of filtration tests-Continued.

\begin{tabular}{|c|c|c|c|c|c|c|}
\hline \multirow{2}{*}{$\begin{array}{c}\text { Well } \\
\text { number. }\end{array}$} & \multirow{2}{*}{$\begin{array}{l}\text { Commis- } \\
\text { sion well } \\
\text { number. }\end{array}$} & \multirow{2}{*}{$\begin{array}{l}\text { Sample } \\
\text { number. }\end{array}$} & \multirow{2}{*}{ Depth (feet). } & \multicolumn{2}{|c|}{ Porosity- } & \multirow{2}{*}{$\begin{array}{l}\text { Filtration: } \\
\text { Cm. } \text {. }^{3} \text { per } \\
\text { hour. }\end{array}$} \\
\hline & & & & $\mathrm{Cm} \cdot{ }^{3}$ & Per cent. & \\
\hline \multirow[t]{2}{*}{731} & \multirow[t]{2}{*}{1200} & 12 & $49-50$ & 16.5 & 31.1 & 10,980 \\
\hline & & 13 & $54-55$ & 15.5 & 29.2 & 6,480 \\
\hline \multirow[t]{6}{*}{732} & \multirow[t]{6}{*}{1202} & 4 & $9-10$ & 14.5 & 27.3 & 3,840 \\
\hline & & 5 & $14-15$ & 17.5 & 33 & 1,800 \\
\hline & & 6 & $19-20$ & 17.5 & 33 & 27,000 \\
\hline & & 7 & $24-25$ & 17 & 32 & 8,820 \\
\hline & & 8 & $29-30$ & 17.5 & 33 & 1,860 \\
\hline & & 9 & $34-35$ & 17 & 32 & 19,800 \\
\hline \multirow[t]{9}{*}{755} & \multirow[t]{9}{*}{1206} & 4 & $9-10$ & 16.5 & 31.1 & 840 \\
\hline & & 6 & $19-20$ & 14 & 26.4 & 14,520 \\
\hline & & 8 & $29-30$ & 17 & 32 & 3,000 \\
\hline & & 10 & $39-40$ & 17 & 32 & 3,240 \\
\hline & & 12 & $49-50$ & 15.5 & 29.2 & 4,140 \\
\hline & & 14 & $59-60$ & 16 & 30.1 & 7,980 \\
\hline & & 16 & $69-70$ & 18 & 33.9 & 5,580 \\
\hline & & 18 & $79-80$ & 19.5 & 36.7 & 2,700 \\
\hline & & 20 & $89-90$ & 21 & 39.6 & 4,860 \\
\hline \multirow[t]{11}{*}{778} & \multirow[t]{11}{*}{1145} & 3 & 45 & 19 & 35.8 & 9,360 \\
\hline & & 6 & $19-20$ & 19.5 & 36.7 & 7,200 \\
\hline & & 9 & $34-35$ & 18.5 & 34.9 & 7,320 \\
\hline & & 12 & $49-50$ & 20.5 & 38.6 & 5,340 \\
\hline & & 15 & $64-65$ & 19.5 & 36.7 & 6,540 \\
\hline & & 18 & $79-80$ & 19 & 35.8 & 3,480 \\
\hline & & 21 & $94-95$ & 19.5 & 36.7 & 3,960 \\
\hline & & 24 & $109-110$ & 19.5 & 36.7 & 6,360 \\
\hline & & 27 & $124-125$ & 21 & 39.6 & 2,700 \\
\hline & & 30 & $134-135$ & 22 & 41.5 & 54 \\
\hline & & 33 & $149-150$ & 20.5 & 38.6 & 4,344 \\
\hline \multirow[t]{9}{*}{781} & \multirow[t]{9}{*}{1169} & 4 & $9-10$ & 18 & 33.9 & 7,620 \\
\hline & & 5 & $14-15$ & 21 & 39.6 & 8,580 \\
\hline & & 6 & $19-20$ & 16 & 30.1 & 9,540 \\
\hline & & 7 & $24-25$ & 19 & 35.8 & 8,940 \\
\hline & & 8 & $29-30$ & 18.5 & 34.9 & 10,080 \\
\hline & & 9 & $34-35$ & 20.5 & 38.6 & 14,640 \\
\hline & & 10 & $39-40$ & 22 & 41,5 & 5,280 \\
\hline & & 11 & $44-45$ & 22 & 41.5 & 14,700 \\
\hline & & 12 & $49-51$ & 20.5 & 38.6 & 13,380 \\
\hline \multirow[t]{2}{*}{796} & \multirow[t]{2}{*}{1214} & 4 & $5-10$ & 20 & 37.7 & 3,300 \\
\hline & & 6 & $15-20$ & 19.5 & 36.7 & 6,360 \\
\hline
\end{tabular}


TABLE XIII.--Results of filtration tests-Continued.

\begin{tabular}{|c|c|c|c|c|c|c|}
\hline \multirow{2}{*}{$\begin{array}{l}\text { Well } \\
\text { number. }\end{array}$} & \multirow{2}{*}{$\begin{array}{l}\text { Commis- } \\
\text { sion well } \\
\text { number. }\end{array}$} & \multirow{2}{*}{$\begin{array}{l}\text { Sample } \\
\text { number. }\end{array}$} & \multirow{2}{*}{ Depth (feet). } & \multicolumn{2}{|c|}{ Porosity-- } & \multirow{2}{*}{$\begin{array}{l}\text { Filtration: } \\
\text { Cm." per } \\
\text { hour. }\end{array}$} \\
\hline & & & & $\mathrm{Cm}^{3}$. & Per cent. & \\
\hline \multirow[t]{4}{*}{796} & 1214 & 8 & $25-30$ & 20 & 37.7 & 5,640 \\
\hline & & 10 & $35-40$ & 19.5 & 36.7 & 3,060 \\
\hline & & 12 & $45-50$ & 20.5 & 38.6 & 3,480 \\
\hline & & 14 & $55-57$ & 19 & 35.8 & 3,600 \\
\hline \multirow[t]{9}{*}{798} & 1215 & 4 & $5-10$ & 17 & 32 & 9,180 \\
\hline & & 6 & $15-20$ & 17 & 32 & 7,200 \\
\hline & & 8 & $25-30$ & 16.5 & 31.1 & 6,780 \\
\hline & & 10 & $35-40$ & 17.5 & 33 & 5,940 \\
\hline & & 12 & $45-50$ & 18.5 & 34.9 & 5,280 \\
\hline & & 14 & $55-60$ & 17.5 & 33 & 10,320 \\
\hline & & 16 & $65-70$ & .15 & 28.3 & 4,440 \\
\hline & & 18 & $75-80$ & 17 & 32 & 3,240 \\
\hline & & 20 & $85-90$ & 16 & 30.1 & 2,736 \\
\hline
\end{tabular}




\section{CHAPTER VI. \\ THE SURFACE STREAMS OF LONG ISLAND.}

By Robert E. Honton.

\section{CHARACTER OF LONG ISLAND STREAMS.}

In a region of moderate rainfall a sloping valley which is continuously depressed below the ground-water horizon will contain a perennial stream. If the bed of the valley is in some degree impervious, the stream may continue over regions where the ground-water horizon lies at greater depth, or a perennial stream may be fed from natural or artificial surface storage in lakes in impervious basins lying above the general ground-water bed.

In general, however, a stream whose channel lies above the ground-water horizon will be intermittent, and such an intermittent stream may flow, under the following conditions: (a) Whenever the ground-water plane, in its periodic fluctuation, rises above the topographical elevation of the stream bed; $(b)$. whenever the surface supply from rainfall or melting snow is in excess of the amount absorbed by the soil, so that surface run-off takes place.

The great sand and gravel deposits of Long Island afford streams differing in character from those generally found elsewhere in New York and in the New England States, where rock is generally found near the surface.

Many of the Long Island catchment areas may be described as narrow strips extending inland from the south shore of the island, having in many cases a nearly uniform slope of about 20 feet per mile. The soil is coarse grained and permeable, and the ground-water table slopes toward the south shore at a rate of 10 or 12 feet per mile. In other words, the ground-water table approaches the surface at a rate of 8 to 10 feet per mile, and in the first few miles back from the coast the ground water lies very near the general ground surface. The general ground surface and ground-water planes intersect at tide water. The stream valleys are flat bottomed and generally marshy', and are depressed a few feet below the general surface.

The bed of the stream valley, running parallel to the general slope of the surface, intersects the ground-water horizon a short distance inland, commonly 1 to 5 miles, and it is at this point of intersection that the surface streams usually have their visible sources.

The level of the ground water is subject to periodic fluctuations of a few feet; hence the point of its intersection of the stream valley is not invariable, but may recede and advance with the season or with the rise and fall of ground water, as was observed by the writer in 1903. These conditions are illustrated for an ideal stream in figs. 68 and 69 . From tide water to the point $A$ of intersection of the stream valley with the minimum ground-water level the stream is perennial.

$$
\text { 17116-No. 44-06-24 }
$$


From $A$ to $B$, covering the range of fluctuation of ground water, the stream is intermittent, having its source at $A$ in times of low ground water and at $B$ in times of high ground water. The distance A-B is usually slight. Above the point B

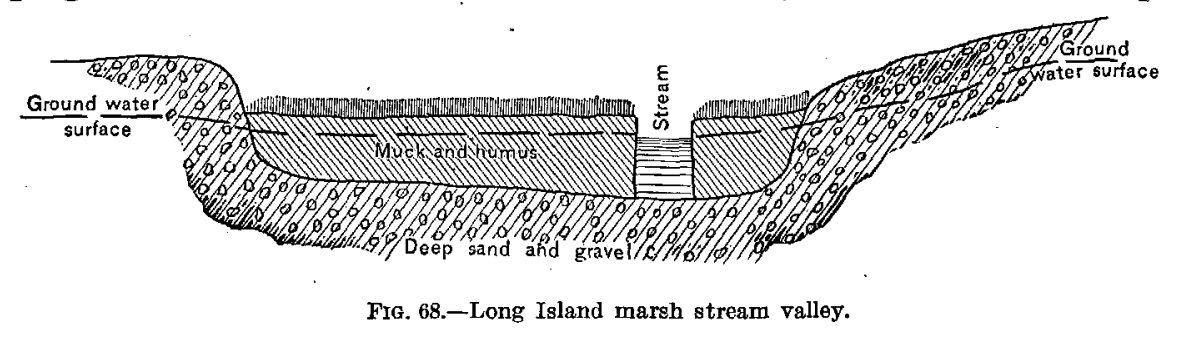

the stream flows only in times of freshets, when supplied by surface run-off. There are also flats and glacial depressions, as at $\mathrm{C}$, from which no surface run-off ever takes place.

If for any reason, as, for example, the existence of an outcrop of impervious material in the gravel slope, a permanent stream supply is brought to the surface at some point in the catchment basin above the point $B$, a disappearing stream may result, similar in character to streams from the Rocky Mountains which are lost in the porous soils of the Great Plains, but of course very much smaller. A number of such streams arising in springs have been observed.

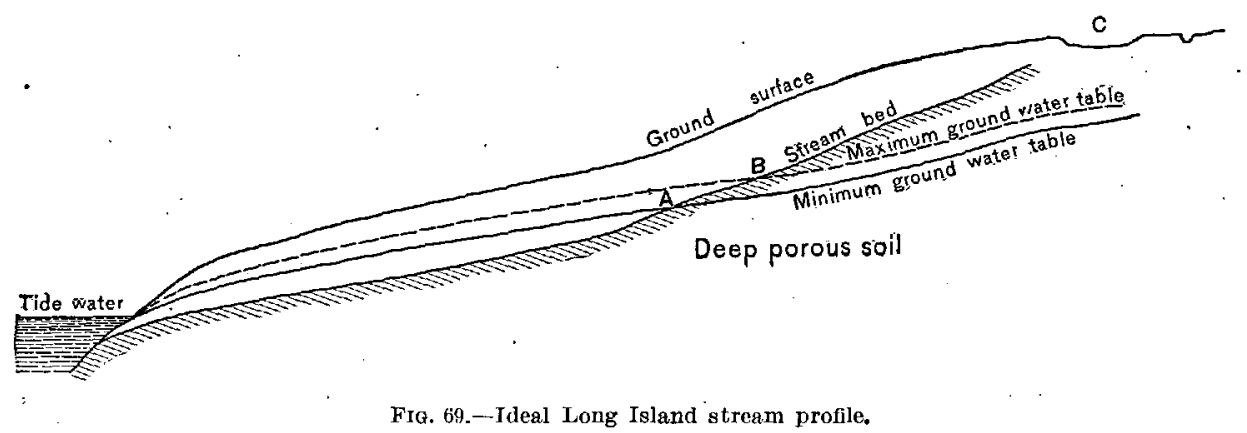

UTILIZATION OF LONG ISLAND SURFACE STREAMS.

The streams are too small in volume and declivity to afford extensive water powers.

In constructing the South Shore highway it was necessary to build earth dikes across the flat stream valleys, and these dikes have been utilized in many instances as mill dams, the absence of severe freshets on these streams making large overflow or wasteway channels unnecessary.

The second important use of the surface streams has been in the formation of numerous private ponds for landscape effect in private parks and for water supply for estates and summer residences.

Certain streams are utilized in cranberry culture and to a limited extent for irrigation of truck lands.

The streams and ground waters are utilized as sources of water supply for Brooklyn and for the extensive summer-resort population at towns along the south shore of the island. 
THE WATER SUPPLY OF BROOKLYN, N.Y. $a$

Brooklyn was incorporated as a city in 1834 ; it then contained a population of 23,000 . The question of constructing a system of public water supply was almost continually agitated from this date until 1856 , when the construction of a waterworks sysiem was undertaken, including supply ponds on a number of streams near Brooklyn, on the south shore of Long Island.

The original works were completed in 1862 , and comprised six supply ponds receiving the drainage from an aggregate catchment area of 65.6 square miles, including additions made to the waterworks previous to their later extension eastward. The cost of the original works was $\$ 4,200,000$.

Previous to the construction of the municipal waterworks, franchises had been secured and small supplies had been developed by a number of private water companies.

In 1889 the extension of the waterworks eastward from Rockville Center to Massapequa was undertaken. The extension of the system added a drainage area of 88.5 square miles, making the total area tributary to the complete system 154.1 square miles.

The names and capacities of the supply ponds in the old and new systems are given in the following tables, together with the population and annual consumption of water from the municipal system of Brooklyn: ${ }^{b}$

Area, elevation, and capacity of supply ponds for Broollyn waterworks.

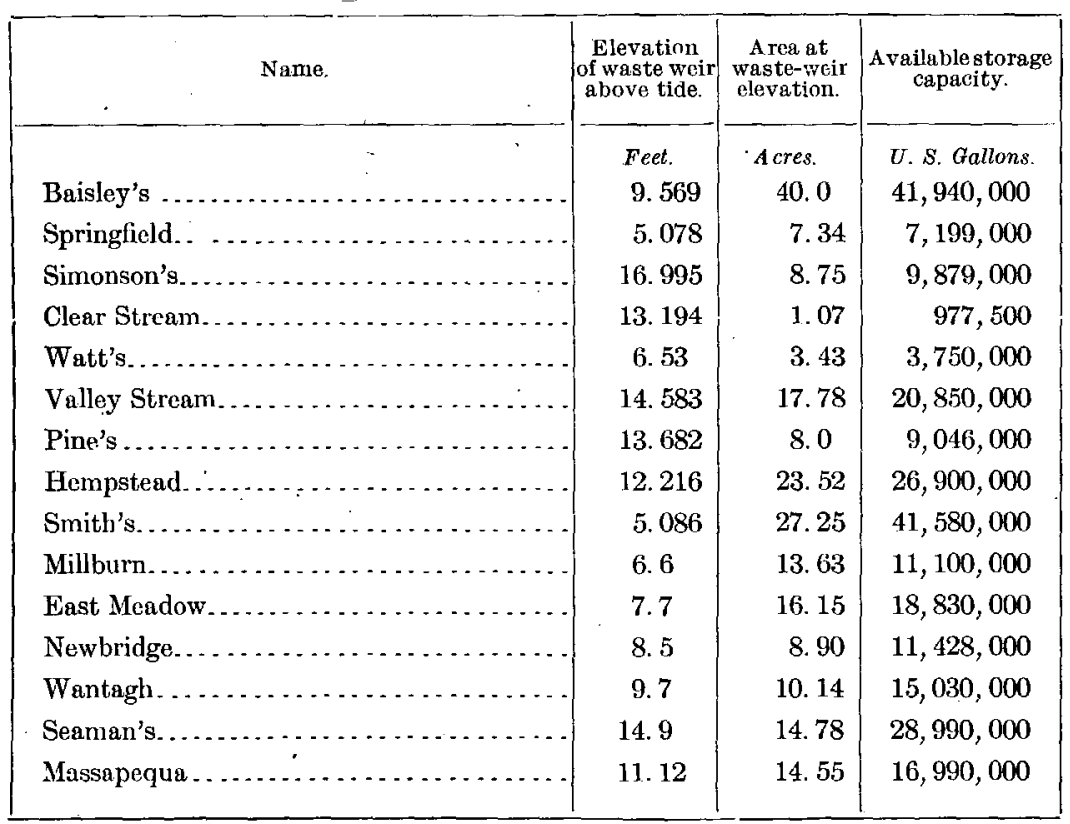

a See The Brooklyn Water Works and Sewers, memoir by James P. Kirkwood, 1857; also History and Description of the Water Supply of the City of Brooklyn, by I. M. De Varona, 1806.

$b$ From report of I. M. De Varona, 1896. 
ropulation of Brooklyn and daily water consumption per capita.

\begin{tabular}{|c|c|c|c|c|c|}
\hline \multirow[t]{2}{*}{ Year. } & \multirow[t]{2}{*}{ Population. } & \multirow[t]{2}{*}{$\begin{array}{l}\text { Population de- } \\
\text { pendent on } \\
\text { Brooklyn water } \\
\text { supply. }\end{array}$} & \multicolumn{2}{|c|}{$\begin{array}{l}\text { Average daily consumption, } \\
\text { Brooklyn water supply. }\end{array}$} & \multirow{2}{*}{$\begin{array}{c}\text { Consump- } \\
\text { tion per } \\
\text { capita per } \\
\text { day, Brook- } \\
\text { lyn water } \\
\text { supply. } \\
\text { U.S.gallons. }\end{array}$} \\
\hline & & & U. S. gallons. & Second-jeet. & \\
\hline 1860. & 266,714 & 266,714 & $3,293,000$ & 5.004 & 12.3 \\
\hline 1861. & 272,350 & 272,350 & $4,064,000$ & 6.299 & 14.9 \\
\hline 1862 & 278,106 & 278,106 & $5,026,000$ & 7.790 & 18.1 \\
\hline 1863. & 283,983 & 283,983 & $6,494,000$ & 10.065 & 22.9 \\
\hline 1864 & 289,985 & 289,985 & $8,105,000$ & 12.562 & 27.9 \\
\hline 1865. & 296,112 & 296,112 & $9,232,000$ & 14. 310 & 31.2 \\
\hline 1866. & 313,852 & 313,852 & $10,908,000$ & 16.907 & 34.8 \\
\hline 1867. & 332,656 & 332,656 & $12,351,000$ & 19.144 & 37.1 \\
\hline 1868. & 352,585 & 352,585 & $15,709,000$ & 24.349 & 44.6 \\
\hline 1869 & 373,710 & 373,710 & $17,629,000$ & 27.325 & 47.2 \\
\hline 1870. & 396,099 & 396,099 & $18,654,000$ & 28.914 & 47.1 \\
\hline $1871 \ldots$ & 412,403 & 412,403 & $19,351,000$ & 29.994 & 46.9 \\
\hline 1872 & 429,380 & 429,380 & $22,714,000$ & 35.207 & 52.9 \\
\hline 1873. & 447,054 & $\sim 447,054$ & $24,875,000$ & 38.556 & 55.6 \\
\hline 1874. & 465,455 & 465,455 & $24,755,000$ & 38.370 & 53.2 \\
\hline 1875 & 484,616 & 484,616 & $27,150,000$ & 42.082 & 56.0 \\
\hline 1876 & 500,014 & 500,014 & $28,109,000$ & 43.569 & 56.2 \\
\hline 1877. & 515,903 & 515,903 & $30,345,000$ & 47.035 & 58.8 \\
\hline 1878 & 532,296 & 532,296 & $30,507,000$ & 47.286 & 57.3 \\
\hline 1879. & 549,211 & 549,211 & $32,912,000$ & 51.014 & 59.9 \\
\hline 1880 & 566,663 & 566,663 & $30,745,000$ & 47.655 & 54.3 \\
\hline 1881. & 584,659 & 584,659 & $32,722,000$ & 50.719 & 56.0 \\
\hline 1882. & 601,103 & 601,103 & $34,623,000$ & 53.665 & 57.6 \\
\hline 1883. & 617,517 & 617,517 & $36,149,000$ & 56.031 & 58.5 \\
\hline 1884. & 634,887 & 634,887 & $38,880,000$ & 60.264 & 61.2 \\
\hline 1885. & 673,050 & 673,050 & $43,379,000$ & 67.237 & 64.5 \\
\hline 1886. & 750,000 & 728,929 & $45,304,000$ & 70.221 & 62.2 \\
\hline 1887. & 765,000 & 741,104 & $46,278,000$ & 71.731 & 62.4 \\
\hline 1888. & 782,221 & 756,195 & $49,794,000$ & 77.181 & 65.8 \\
\hline 1889. & 852,467 & 823,367 & $52,197,000$ & 80.905 & 63.4 \\
\hline 1890. & 853,945 & 853,587 & $55,201,000$ & 85.562 & 67.0 \\
\hline $1891 \ldots$ & 880,780 & 846,330 & $58,083,000$ & 90.029 & 68.6 \\
\hline 1892 & 957,958 & 919,417 & $67,566,000$ & 104.727 & 73.5 \\
\hline 1893. & $1,003,781$ & 961,039 & $75,823,000$ & 117.526 & 78.9 \\
\hline 1894 & $1,080,000$ & 996,500 & $71,360,000$ & 110.608 & 71.6 \\
\hline 1895. & $1,105,000$ & $1,013,500$ & $75,735,000$ & 117.389 & 74.7 \\
\hline
\end{tabular}


The principal results of gagings made prior to the year 1903 are shown in the accompanying tables. ${ }^{a}$

The following gagings, by William McAlpine, were made by inserting in the streams wooden sluiceways, through which all the surface flow was passed. The drainage basin of Parsonage Creek is given as 21.74 square miles, and the combined areas tributary to all the streams which extended along the south shore from Jamaica Creek to East Meadow Brook is stated to be somewhat in excess of 100 square miles.

The precipitation at Erasmus Hall during the period of gaging was as follows:

Precipitation at Erasmus Hall, Long Island.

\begin{tabular}{|c|c|c|}
\hline \multirow{2}{*}{ Month. } & \multicolumn{2}{|c|}{ Precipitation. } \\
\hline & 1851. & Normal. \\
\hline July $\ldots \ldots \ldots \ldots \ldots \ldots \ldots$ & $\begin{array}{l}\text { Inches, } \\
3.85\end{array}$ & $\begin{array}{r}\text { Inches. } \\
3.21\end{array}$ \\
\hline August. . . . . . . . . . $\ldots \ldots \ldots$ & 3.23 & 4. 44 \\
\hline September. . . . $\ldots \ldots \ldots$ & 1.06 & 3. 09 \\
\hline October. ........ & 4. 47 & 3.39 \\
\hline November. . . . . . . . . . . & 3.99 & 3.24 \\
\hline December...................... &.- .2 .01 & 3.74 \\
\hline Period . . . . & 18.61 & 21. 11 \\
\hline
\end{tabular}

Gagings of Long Island streams by Wm. J. McAlpine and L. S. Nash in 1851.

\begin{tabular}{|c|c|c|c|c|c|c|c|c|c|}
\hline \multirow[t]{2}{*}{ Body of water. } & \multirow{2}{*}{$\begin{array}{l}\begin{array}{c}\text { Dura- } \\
\text { tion of } \\
\text { gaging. }\end{array} \\
\text { Hours }\end{array}$} & \multicolumn{2}{|c|}{$\begin{array}{l}\text { McAlpine and Stod- } \\
\text { dard, Oct. } 11,1851 .\end{array}$} & $\begin{array}{l}\text { Dura- } \\
\text { tion of } \\
\text { gaging. }\end{array}$ & \multicolumn{2}{|c|}{$\begin{array}{l}\text { Mean of I, S. } \\
\text { Nash's gagings } \\
\text { Nov. } 6,7,8,1851 \text {. }\end{array}$} & \multirow{2}{*}{$\begin{array}{l}\begin{array}{c}\text { Dura- } \\
\text { tion of } \\
\text { gaging. }\end{array} \\
\text { Hours. }\end{array}$} & \multicolumn{2}{|c|}{$\begin{array}{c}\text { Mean of L. B. } \\
\text { Nash's gagings, } \\
\text { Nov.17,18,19,1851. }\end{array}$} \\
\hline & & $\begin{array}{c}\text { Gallons } \\
\text { per day. }\end{array}$ & $\left|\begin{array}{c}\text { Second } \\
\text { feet. }\end{array}\right|$ & Hours. & $\begin{array}{l}\text { Gallons } \\
\text { per day. }\end{array}$ & $\begin{array}{c}\text { Second- } \\
\text { feet. }\end{array}$ & & $\begin{array}{c}\text { Gallons } \\
\text { per day. }\end{array}$ & $\begin{array}{l}\text { Second- } \\
\text { feet. }\end{array}$ \\
\hline Baisley's Pond (Jamaica Creek) ...... & 6 & $5,280,000$ & 8.18 & 12 & $6,233,172$ & 9.67 & $7 \frac{1}{2}$ & $8,440,312$ & 13.08 \\
\hline $\begin{array}{l}\text { Springfield Stream (Nostrand's } \\
\text { Pond), West B ranch................ }\end{array}$ & 8 & $1,600,000$ & 2.48 & 11 & $1,689,160$ & 2.62 & 8 & $1,890,864$ & 2.93 \\
\hline Springfield Stream, East Branch..... & 24 & 264,000 & .41 & 24 & 300,072 & .46 & 24 & 354,384 & .55 \\
\hline $\begin{array}{l}\text { Hook Creek, West Braneh (or Brook- } \\
\text { field Streqm) ............................ }\end{array}$ & 13 & $4,095,000$ & 6.34 & 12 & $4,339,720$ & 6.73 & 14 & $4,989,782$ & 7.73 \\
\hline $\begin{array}{l}\text { Hook Creek, Middle B ranch (or Clear } \\
\text { Stream) } \ldots \ldots \ldots \ldots \ldots \ldots \ldots \ldots \ldots \ldots \ldots \ldots\end{array}$ & 24 & 540,000 & .84 & 24 & 771,816 & 1.20 & 24 & 969,600 & 1.50 \\
\hline 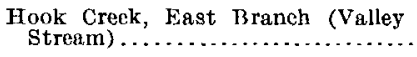 & 6 & $2,430,000$ & 3.77 & 6 & $2,510,643$ & 3.88 & 6 & $3,374,742$ & 5.22 \\
\hline Pine Creek (Pine's Brook) ........... & 6 & $2,400,000$ & 3.72 & 6 & $2,832,240$ & 4.39 & 63 & $3,249,423$ & 5.04 \\
\hline Parsonage Creek.......... & 7 & $8,330,000$ & 12.91 & 12 & $10,543,464$ & 16.28 & 12 & $12,594,348$ & 19.53 \\
\hline Millburn, West Branch... & $\cdots$ & $\because \ldots \ldots \ldots \ldots$ & ...... & 24 & 473,328 & .73 & 24 & 518,400 & .80 \\
\hline Millburn, Middle Branch . & 6 & 504,000 & .78 & 24 & 299,616 & .46 & 24 & 375,840 & .58 \\
\hline Millburn, East Branch ...... & ........ & ........... & ….. & 24 & $2,836,152$ & 4.40 & 24 & $4,276,800$ & 6.63 \\
\hline East Meadow Stream .......... & 10 & $5,340,000$ & 8.28 & 12 & $5,601,756$ & 8.68 & 12 & $6,280,800$ & 9.73 \\
\hline
\end{tabular}


Mean monthly discharge of Long Island streams from gagings made by Artemus Whitlock in 1852.

\begin{tabular}{|c|c|c|c|c|c|c|c|c|c|c|}
\hline \multirow[t]{2}{*}{ Body of water. } & \multicolumn{2}{|c|}{ August. } & \multicolumn{2}{|c|}{ September. } & \multicolumn{2}{|c|}{ October. } & \multicolumn{2}{|c|}{ Novernber. } & \multicolumn{2}{|c|}{ December. } \\
\hline & $\begin{array}{l}\text { Gallons } \\
\text { per day. }\end{array}$ & $\mid \begin{array}{c}\text { Second }- \\
\text { feet. }\end{array}$ & $\begin{array}{l}\text { Gallons } \\
\text { per day. }\end{array}$ & $\begin{array}{l}\text { Second- } \\
\text { feet. }\end{array}$ & $\begin{array}{l}\text { Gallons } \\
\text { per day. }\end{array}$ & $\begin{array}{l}\text { Second- } \\
\text { feet. }\end{array}$ & $\begin{array}{l}\text { Gallons } \\
\text { per day. }\end{array}$ & $\left|\begin{array}{c}\text { Second }- \\
\text { feet: }\end{array}\right|$ & $\begin{array}{l}\text { Gallons } \\
\text { per day. }\end{array}$ & $\begin{array}{l}\text { Second- } \\
\text { feet. }\end{array}$ \\
\hline $\begin{array}{l}\text { Baisley's Pond (Ja- } \\
\text { maica Creek)....... }\end{array}$ & $6,387,000$ & 9.90 & $6,863,000$ & 10.65 & $6,154,000$ & 9.53 & $7,804,000$ & 12.09 & $8,137,000$ & 12.62 \\
\hline $\begin{array}{l}\text { On West Branch } \\
\text { Hook Creek, or } \\
\text { Brookficld Stream: }\end{array}$ & & & & & & & & & & \\
\hline Simonson's Pond. & & & & & & , & $2,701,000$ & 4.18 & & ..... \\
\hline Conselyca's Pond. & $2,847,000$ & 4.42 & $4,793,000$ & 7.42 & $2,493,000$ & 3.85 & $3,121,000$ & 4.84 & $4,769,000$ & 7.39 \\
\hline $\begin{array}{c}\text { Valley stream (P. } \\
\text { Cornell's) } \ldots \ldots \ldots\end{array}$ & & - & & & & & $4,616,000$ & 7.16 & & \\
\hline $\begin{array}{l}\text { On Fast Braneh } \\
\text { Hook Creek......... }\end{array}$ & $3,078,000$ & 4.74 & $5,445,000$ & 8.43 & $3,319,000$ & 5.15 & $3,601,000$ & 5.58 & $5,257,000$ & 8.15 \\
\hline Pine's Brook......... & $2,714,000$ & 4. 20 & $3,447,000$ & 5.35 & $2,464,000$ & 3.81 & $3,121,000$ & 4.84 & & ..... \\
\hline On Parsonage Creck: & & & & & & & & & & \\
\hline Hempstead Pond. & & & $a 16,682,000$ & 26.20 & & & & & & \\
\hline $\begin{array}{l}\text { Hempstead stor- } \\
\text { age reservoir... }\end{array}$ & & & $10,228,000$ & 15.81 & $8,993,000$ & 13.93 & $12,003,000$ & 18.60 & & \\
\hline
\end{tabular}

a The increase of flow was caused by heavy rains just before taking the observations.

Miscellaneous gagings of Long Istand streams. .

\begin{tabular}{|c|c|c|c|c|c|c|c|c|}
\hline \multirow{2}{*}{ Body of water. } & \multicolumn{2}{|c|}{$\begin{array}{l}\text { Gagings made by } \\
\text { Leigh, st od- } \\
\text { dard, and Bre- } \\
\text { voort, com- } \\
\text { pleted Sept. 9, } \\
1854 \text {. }\end{array}$} & \multicolumn{2}{|c|}{$\begin{array}{l}\text { Gagings made un- } \\
\text { der direction of } \\
\text { Jas. P. Kirk- } \\
\text { wood, 1856-57. }\end{array}$} & \multicolumn{2}{|c|}{$\begin{array}{l}\text { Grgings in a d e } \\
\text { Sept. } 19 \text { to Oct. } \\
12,1885 \text {. }\end{array}$} & \multicolumn{2}{|c|}{$\begin{array}{l}\text { Eatimate of the } \\
\text { minimum flow } \\
\text { based on gag } \\
\text { ings made Aug. } \\
30 \text { to Oet. } 5,1894 .\end{array}$} \\
\hline & $\begin{array}{l}\text { Gallons } \\
\text { per day. }\end{array}$ & $\begin{array}{c}\text { Second- } \\
\text { feet. }\end{array}$ & $\begin{array}{c}\text { Gallons } \\
\text { per day. }\end{array}$ & $\left|\begin{array}{c}\text { Second- } \\
\text { feet }\end{array}\right|$ & $\begin{array}{c}\text { Gallons } \\
\text { per doy. }\end{array}$ & $\begin{array}{c}\text { Second }- \\
\text { feet. }\end{array}$ & $\begin{array}{l}\text { Gallons } \\
\text { per day. }\end{array}$ & $\begin{array}{l}\text { Second- } \\
\text { feet }\end{array}$ \\
\hline Baiseley's Pond (Jamaica Creek) ... & $6,732,000$ & 10.43 & $2,924,000$ & 4.53 & & & & \\
\hline Springfield Stream, West Branch........ & $3,487,000$ & 5.41 & 607,000 & .94 & & & & \\
\hline \multicolumn{9}{|l|}{ Springfield Stream, East Branch........ } \\
\hline $\begin{array}{l}\text { Hook Creek, West Branch (Simonson's } \\
\text { Pond), or Brookfield Stream.............. }\end{array}$ & $2,501,000$ & 3.88 & $1,798,000$ & 2.79 & $1,266,000$ & 1.97 & 2,$000 ; 000$ & 3.10 \\
\hline 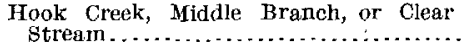 & & & 708,000 & 1.10 & & & 200,000 & .31 \\
\hline $\begin{array}{r}\text { Hook Creek, East Braneh, or Valley } \\
\text { Stream } . . . . . . . \ldots \ldots \ldots \ldots\end{array}$ & $4,212,000$ & 6.53 & $2,287,000$ & 3.55 & $1,879,000$ & 2.91 & $1,300,000$ & 2.01 \\
\hline \multicolumn{9}{|l|}{$\begin{array}{l}\text { Watt's Pond, on East Branch Hook Creek, } \\
\text { below Valley Stream Pond .............. }\end{array}$} \\
\hline 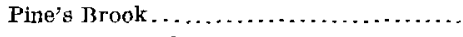 & & & $2,460,000$ & $3.8 \mathrm{I}$ & $1,050,000$ & 1.63 & 600,000 & .93 \\
\hline Sehodack Brook................ & & & $\cdots$ & -......... & 695,000 & 1.08 & $1,000,000$ & 1.55 \\
\hline Hempsteud Pond (I. Cornell's) .... - & $11,266,000$ & 17.51 & $7,326,000$ & 11. 36 & & & & $\cdots$ \\
\hline Hempstead storage reservoir..... . & & & & & $7,149,000$ & 11.08 & $8,000,000$ & 12.40 \\
\hline
\end{tabular}

In thirty days preceding the gagings of October 11, 1851, 1.62 inches of rain fell, an additional precipitation of 3.85 inches preceded the second series of gagings, and a further increase of 0.92 inch of rainfall occurred before the third series of gagings were made. ${ }^{a}$

Details as to the methods of gaging, precise location, and drainage areas above the points of gaging, or daily discharge results, are unavailable.

It is known, however, that, beginning with McAlpine's gagings in 1851, most of the measurements have been made in flumes or sluiceways constructed for the purpose, the velocity and area of cross section being determined without 
disturbing the ground-water conditions or affecting the relative ground-water level above and below.

Most of the streams flow in flat swamp valleys underlain by gravel so porous that the flow from a large spring that was observed was absorbed or lost in the soil within a short distance from its origin. The velocity of flow of ground water adjacent to an earthen dam has been observed by Professor Slichter ${ }^{a}$ to be many times greater than the normal velocity in places where the ground-water level is undisturbed.
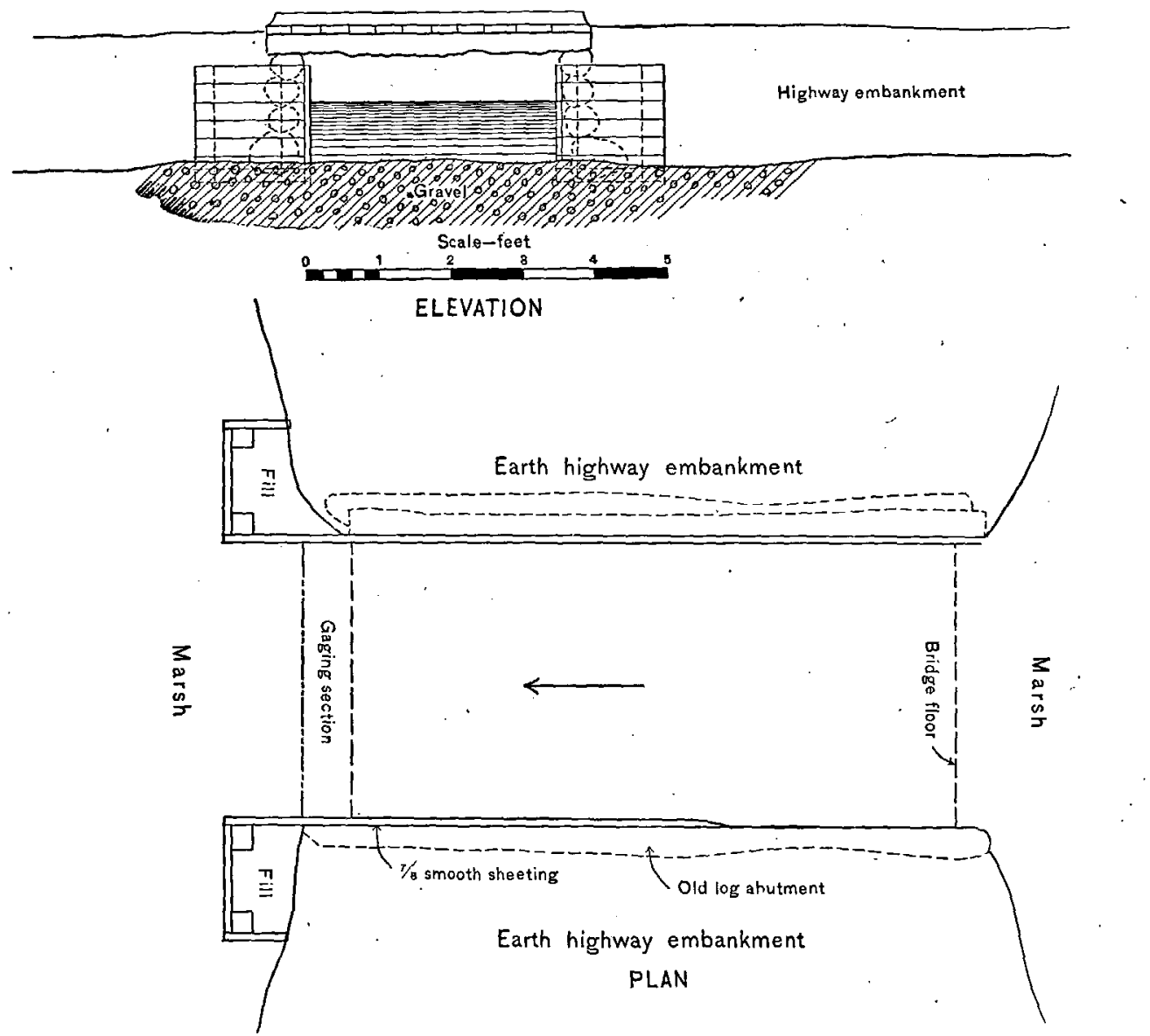

FIG. 70.-Temporary gaging station of the United States Geological Survey, Orowoc Creek, Islip, Long Island, New York, June 7, 1903. Plan shows bridge floor removed.

Care must be exercised in gaging such streams to properly differentiate between the surface discharge of the streams and the underflow in the porous valleys. The method of gaging in open sluiceways, generally used by the earlier engineers, was followed in the investigations of the United States Geological Survey in 1903, except on Orowoc Creek, Doxsee Creek, and Massapequa Creek, streams on which suitable sites for gaging at moderate expense could be found only in conjunction with existing weirs at private ponds. 
The gagings of the United States'Geological Survey in 1903 included most of the remarkable period of light rainfall in May and June. They were discontinued early in July, after heavy rains had fallen, disturbing the ground-water conditions.

In the following pages are also given results obtained at a number of elaborate. small weirs erected by the New York water supply commission, chiefly in the swamp stream valleys on the property of the city of New York, in Nassau County.

Most of the current-meter measurements of the Geological Survey were made with a specially rated Fteley meter. Vertical velocity curves were taken to determine the distribution of currents in the stream channel. The steady regimen and smooth currents of many of the streams favor accurate results by this method. Some of the stations were not fully completed and none of the rating curves were finished at the time of discontinuance. Points were obtained on the curves in most cases to cover nearly the full range of observed gage heights and to afford a reliable basis of estimating the discharge. The streams gaged were distributed along the south shore of the island, and the individual cost of gagings was a small fraction of that required to build individual weirs.

In conjunction with the surface-stream and driven-well supply stations of the Brooklyn waterworks, records have been kept of the ground-water level, showing its reduction by pumping in a most interesting manner. ${ }^{a}$ Records of the pumpage and diversion and of the supply pond levels have also been kept. The waste over the spillways when observed in 1903 was largely in the nature of wave wash and not susceptible of accurate estimation. Unfortunately, these records do not furnish a reliable basis for estimating the yield of the streams flowing into the water-supply ponds. The regimen of these streams is further subject to the influence of pumping from the adjacent ground water.

Prior to the gagings of 1903 very few definite data were obtained concerning the regimen of the surface streams of Long Island. The results for 1903 do not of themselves form a sufficient basis for estimating either the average or the minimum yield of the Long Island catchment areas.

EAST MEADOW BROOK, NEAR FREEPORT, LONG ISLAND.

East Meadow Brook has its visible source 5 miles from the south shore of Long Island. Well-defined stream channels, somewhat branching, extend nearly to the northern limit of the catchment basin; receiving the surface drainage from its east and west portions.

The drainage basin extends inland 14 miles and has a nearly uniform width, varying from 2 to 3 miles. The topography is moderately rolling and the surface slope quite uniform, the northerly divide being about 300 feet elevation above tide.

Five ponds and dams are on the main stream. Small water powers for grist mills and a paper mill were formerly in use.

The stream is tributary to the Brooklyn water supply through an intercepting conduit, which follows the south shore of the island.

a See De Varona, Brooklyn Water Supply. 
A portion of the drainage basin lies north of the ground-water divide of Long Island. The catchment basin contains 28 per cent of forest cover, chiefly scrub oaks and conifers, 44 per cent pasture and other grass land, and about 28 per cent of cultivated land.

Earlier gagings of East Meadow Brook at Freeport, Long Island.

\begin{tabular}{|c|c|c|c|c|}
\hline Date. & Observer. & Gallons per day. & Second-feet. & $\begin{array}{l}\text { Second-feet } \\
\text { per square } \\
\text { mile ( } 31 \\
\text { square } \\
\text { miles). }\end{array}$ \\
\hline October $11,1851 \ldots \ldots \ldots$ & McAlpine and Stoddard . . . . . . . & $6,410,000$ & 9.93 & 0.32 \\
\hline November $6,7,8,1851 \ldots$ & L. S. Nash. . . . . . . . . . . . . . . . . . & $6,724,000$ & 10.42 & .33 \\
\hline November $17,18,19,1851$. & $\ldots$ do....... & $7,539,000$ & 11. 69 & .38 \\
\hline August, $1852 \ldots \ldots \ldots$ & Artemus Whitlock. . . . . . . . . . & $\ldots \ldots \ldots$ & . & $\cdots$ \\
\hline September, 1852 . & ... do. & $\left\{\begin{array}{r}a 16,270,000 \\
9,583,000\end{array}\right.$ & $\begin{array}{l}25.26 \\
14.85\end{array}$ & $\begin{array}{l}.81 \\
.48\end{array}$ \\
\hline October, 1852. . . & ... do. . . . . . . . . & $7,324,000$ & 11.35 & .37 \\
\hline November, $1852 \ldots . .$. & .... do. . . . . . . . & $7,324,000$ & 11.35 & .37 \\
\hline December, $1852 \ldots . . .$. & .....do.... & $\ldots \ldots \ldots$ & $\mid \ldots \ldots \ldots$. & $\ldots \ldots . . . .$. \\
\hline June 1 to October $15,1883^{h}$. & & $5,200,000$ & 8.06 & .26 \\
\hline $\begin{array}{l}\text { September } 19 \text { to October } \\
2,1885 \text {. }\end{array}$ & & $4,217,000$ & 6.54 & .21 \\
\hline
\end{tabular}

a This increase in flow caused by heavy rains just before observations were taken. $b$ For conduit east of Rockville Center.

Mean daily discharge, in second feet, of East Meadow Brook near Freeport, Long Island, for 1903.“

\begin{tabular}{|c|c|c|c|c|c|c|c|c|c|c|c|c|c|c|c|}
\hline Day. & Apr. & May. & June. & July. & Altg. & Sept. & Oet. & Day. & Apr. & May. & June. & July. & Aug. & Sept. & Oct. \\
\hline 1.. & & $b 28.42$ & 18.04 & 24.38 & 14.41 & 15.49 & 13.56 & 18. & & & 20.22 & 24.05 & 15.36 & 16.81 & 32.22 \\
\hline 2. & & & 18.13 & 21.82 & 13.38 & 16.07 & 12.71 & $19^{\circ}$. & & & 22.97 & 29.07 & $14.12^{\circ}$ & 16.07 & 22.45 \\
\hline 3. & & & 17.89 & 24.30 & 15.43 & 16.02 & 13.72 & 20. & & & 23.84 & 20.36 & 15.28 & 14.72 & 16.00 \\
\hline 4. & & & 17.48 & 23.14 & 14.94 & 15. 20 & 16.30 & 21. & & & 25.98 & 20.29 & 16.09 & 13.35 & 16.49 \\
\hline 5. & & & 17,48 & 22.00 & 63.95 & 16.88 & 14.30 & 22. & $b 10.73$ & & 24.28 & 18.79 & 35.41 & 13.44 & 16.23 \\
\hline 6.. & & & 18.15 & 21.41 & 23.87 & 17.27 & 13.07 & $23 .$. & & & 24.31 & 23.68 & 14.89 & 13.02 & 16. 12 \\
\hline $7 .$. & & & 24.95 & 20.80 & 29.32 & 15.56 & 12.77 & $24 \ldots$ & & & 28.37 & 17.26 & 18. 36 & 13.21 & 15.80 \\
\hline 8. & & & 31.59 & 19.16 & 18.50 & 13.32 & 13.68 & 25. & & & 25.21 & 17,66 & 14.18 & 13.09 & 15.90 \\
\hline $9 .$. & & & 26.92 & 17.50 & 18.79 & 14.02 & 35.93 & $26 .$. & & & 21.96 & 16.08 & 15.65 & 13.25 & 16.09 \\
\hline $10 .$. & & & 19.35 & 19.20 & 16.09 & 13.72 & 29.70 & $27 \ldots$ & & & 21.37 & 16.20 & 17.21 & 13.76 & 15.71 \\
\hline $11 \ldots$ & & & $19: 87$ & 18.86 & 18.70 & 13.49 & 14.91 & $28 \ldots$ & & & 20.56 & 15.58 & 22.69 & 16.17 & 15. 59 \\
\hline 12. & & & 41.89 & 19.97 & 19.73 & 13.32 & 19.93 & $29 \ldots$ & & & 28.85 & 15.62 & 32.55 & 13.41 & 15.80 \\
\hline 13. & & & 40.37 & 24.20 & 17.19 & 13.45 & 15.30 & & & & 36.92 & 16.38 & 25.99 & 13.84 & 15.68 \\
\hline $14 .$. & & & 22.59 & 21.20 & 16.72 & 14.38 & 14.49 & \multirow{4}{*}{ Mean. } & & & & 16.08 & 17.65 & & 15.88 \\
\hline $15 .$. & & & 38.42 & 18.31 & 15.58 & 15.06 & 13.88 & & & & 25.17 & 20.02 & 20.36 & 14.78 & 17.11 \\
\hline 16. & & & 32.24 & 17.95 & 15.55 & 19.85 & 12.90 & & & & & & & & \\
\hline & & & 24.82 & 19.32 & 16.79 & 15.12 & 21.30 & & & & & & & & \\
\hline
\end{tabular}

a Weir of New York water supply commission in swamp at head of Brooklyn waterworks supply pond. $b$ Current meter measurement by U. S. Geological Survey. 


\section{NEWBRIDGE STREAMS NEAR MERRICK, LONG ISLAND.}

These streams drain the first catchment area lying east of East Meadow Brook. The surface drainage is 3.3 square miles. The larger tributary has its visible source 2 miles from tide water.

The stream valley extends inland nearly to the head of the catchment basin. This catchment basin has a maximum width of 1 mile near the foot, decreasing in width toward the northern end. The topography is flat and the surface slope is quite uniform, the head of the basin being at altitude 100 feet above tide. About 58 per cent of the catchment area of these streams has forest cover, 19 per cent is sodded, and about 22 per cent is under cultivation. The Newbridge streams are tributary to the Brooklyn waterworks.

Mean daily discharge, in second feet, of Newbridge streams near Freeport, Long Island, for 1903.a

\begin{tabular}{|c|c|c|c|c|c|c|c|c|c|c|c|}
\hline Day. & Aug. & Sept. & Oet. & Day. & Aug. & Sept. & Oet. & Day. & Alig. & Sept. & Oct. \\
\hline $1 \ldots$ & 4.07 & 3.76 & 2.65 & $12 \ldots$ & 4.91 & 3.10 & 3.00 & $23 .$. & 3.33 & 2.83 & 3.42 \\
\hline $2 \ldots$ & 4.02 & 3.59 & 2.55 & $13 \ldots$ & 4.37 & 3.16 & 3.00 & $24 \ldots \ldots$ & 3.44 & 2. 78 & 3. 36 \\
\hline $3 \ldots$ & 3.92 & 3.54 & 2.79 & $14 \ldots$ & 4.22 & 3.03 & 2.99 & $25 \ldots \ldots \ldots$ & 3.34 & 2.73 & $3.3 i$ \\
\hline $4 \ldots$ & 4.66 & 3.47 & 2.47 & $15 \ldots$ & 4.30 & 3.00 & 2.94 & $26 \ldots \ldots$ & 3.57 & 2.74 & 3.43 \\
\hline $5 \ldots$ & 12.40 & 3.69 & 2.57 & $16 \ldots$ & 4. 23 & 4. 42 & 2.90 & $27 \ldots \ldots$ & 3.26 & 2.69 & 3.36 \\
\hline $6 \ldots$ & 6.34 & 3.18 & 2.66 & 17. & 4. 11 & 3.45 & 4.01 & $28 \ldots$. & 4.41 & 2.99 & 3.43 \\
\hline $7 . \therefore$ & 5.65 & 3.26 & 2.55 & $18 .$. & 3.78 & 3.39 & 4.63 & 29. & 4.05 & 2.72 & 3.43 \\
\hline $8 \ldots$ & 4.94 & 3.27 & 2.55 & 19. & 3.61 & 3.01 & 3.75 & $30 \ldots \ldots$ & 4.63 & 2.84 & 3.35 \\
\hline $9 \ldots$ & 4.81 & 3.27 & 5.25 & 20. & 3.71 & 2.94 & 3.61 & $31 \ldots \ldots \ldots$ & 4.02 & & 3.37 \\
\hline $10 \ldots$ & 4.73 & 3.28 & 3.66 & $21 .$. & 3.42 & 2.93 & 3.51 & \multirow[t]{2}{*}{ Mean ... } & \multirow{2}{*}{.4 .47} & \multirow[t]{2}{*}{3.15} & \multirow[t]{2}{*}{3.27} \\
\hline $11 \ldots \ldots$ & 5.29 & 3.23 & 3.33 & & 3.38 & 2.87 & 3.47 & & & & \\
\hline
\end{tabular}

a At weirs of New York water supply commission.

\section{WANTAGH STREAMS AT WANTAGH, LONG ISLAND.}

A group of short branching streams, having their visible sources 3 miles inland, drain a relatively flat area, the topographic boundary of which is difficult of precise determination, but has been estimated at 17.6 square miles. This area lies entirely south of the ground-water divide of Long Island. The drainage area is lenticular, the northern end lying at elevation 250 feet. A dry stream valley, not very clearly demarcated, extends nearly to the northern limit of the basin.

The drainage basin contains about 40 per cent cultivated land and an equal percentage of pasture and grass areas; the remainder being chiefly. wooded. There are several private ponds near the mouth of the stream. Water has also been diverted to the conduit of the Brooklyn waterworks since 1891."

The stream above Seamans Pond is divided between three channels. Two low weirs were erected just above Seamans Pond, and gaging records were maintained there from July 21 to November 8, 1903, by the New York water supply commission.

The discharge determined from gagings June 1 to October 15, 1883, is stated at $3,400,000$ gallons ( 5.25 second-feet). The year 1883 was preceded by several years of somewhat deficient rainfall. Details of these gagings are not available. 
Mean daily discharge, in second-feet, of Wantagh streams at Wantagh, Long Istand, for 1903.a

\begin{tabular}{|c|c|c|c|c|c|c|c|c|c|c|c|c|c|c|}
\hline Day. & July. & Aug. & Sept. & Oct. & Day. & July. & Aug. & Sept. & Oct. & Day. & July. & Aug. & Sept. & Oct. \\
\hline $1 .$. & & 11.63 & $14.0 \overline{5}$ & 11.20 & $12 \ldots$ & & 16.73 & 12.52 & 14.02 & 23. & 16.62 & 12.24 & 12.19 & 12.80 \\
\hline 2. & & 12.21 & 11.84 & 10.52 & 13. & & 13. 18 & 11.33 & 12.51 & 24. & 13.12 & 11.93 & 10.18 & 12.12 \\
\hline 3. & & 12.07 & 12.92 & 11.51 & $14 .$. & & 14.89 & 9.93 & 10.11 & 25. & 12.75 & 10.73 & 11.03 & 12.13 \\
\hline $4 .$. & & 13.10 & 14.91 & 9.85 & $15 .$. & & 13.62 & 11.90 & 12.60 & 26. & 8.53 & 13.86 & 10.52 & 11.40 \\
\hline 5. & & 48.30 & 14.67 & 13.07 & 16. & & 13.66 & 14.22 & 11.30 & 27. & 9.82 & 12.56 & 11.27 & 12.30 \\
\hline 6. & & 21.19 & 14.11 & 9.81 & 17. & & 14. 19 & 13.76 & 19.28 & 28. & 11.36 & 18.70 & 12.68 & 12.67 \\
\hline 7. & & 17.31 & 12.61 & 10.31 & 18. & & 10.88 & 13.09 & 19.90 & 29. & 12.52 & 25.65 & 10.06 & 12.67 \\
\hline 8. & & 16.00 & 10.85 & 10.23 & 19. & & 13.35 & 10.71 & 14.06 & 30. & 12.19 & 19.92 & 13.75 & 12.56 \\
\hline 9. & & 15.67 & 13.21 & 29.81 & 20. & & 13. 72 & 12.75 & 12.86 & \multirow{3}{*}{ Mcan. } & 12.30 & 10.07 & $\because$ & 12.06 \\
\hline 10. & & 16.23 & 12.79 & 14.99 & 21. & 13.55 & 12.26 & 9.68 & 11.17 & & & \multirow[t]{2}{*}{15.68} & \multirow{2}{*}{12.17} & \multirow{2}{*}{13.13} \\
\hline 11. & & 18. 46 & 10.78 & 13.59 & 22. & 14.35 & 12,36 & 11.10 & 13.12 & & & & & \\
\hline
\end{tabular}

a At weirs of New York water supply commission.

MASSAPEQUA CREEK AT FARMINGDALE AND FREEPORT, LONG ISLAND.

Massapequa Creek drains an area extending inland a distance of 14 miles from the mouth of the stream. In shape, the drainage basin is irregular, but gradually increases from a width of 1 mile at the mouth to a maximum width of 5 miles near the northern divide. The topography is diversified, including a flat valley on the east; a table-land on the north, comprising about 5 square miles, the drainage from which is chiefly into depressions; a group of hills rising to altitude 300 feet near the center of the basin; and a generally southerly slope, somewhat rolling, in the southwest portion of the surface catchment area.

The stream comprises three short branches which unite 2 miles above the outlet of the stream into tide water. The longest branch has its visible source at a distance of 5 miles inland. A gaging station was established on this branch of the stream May 6, 1903, at a small weir forming the outlet of a private pond. The area of the pond is so small as to exert but little regulating influence, and the stream entering the pond is entirely unregulated.

In order to procure a record at as early a date as possible readings were taken on the weir without modification, until such changes could be made as were desirable to secure the best results during low water. The weir was located just above the head of the property of the Brooklyn water department.

The stream below the weir flows through a marsh valley, bordered by sandy slopes. The bed of the marsh is porous gravel overlain by 2 or 3 feet of muck and vegetation, through which the surface waters percolate.

Springs enter the margin of the small pond at the Farmingdale weir, and the stream has its visible sources a short distance above this pond. The precise point at which the stream rises apparently varies with the season and stage of ground water, which also determine the position of the seepage or wet sand areas observed on the slopes in certain places in this catchment basin. Throughout the lower portion of the drainage basin the ground-water horizon lies within a few feet of the surface. The entire drainage basin lies south of the summit of the ground-water table of Long Island. 
Massapequa Creek is utilized in conjunction with the water-supply system of Brooklyn. The drainage basin and the position of the conduit line are shown on the Northport and Babylon sheets of the United States Geological Survey's topographic map. The drainage areas are as follows:

\section{Drainage areas, Massapequa Creek.}

Square miles.

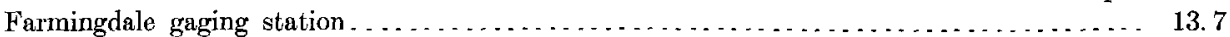

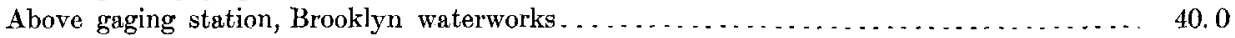

Above outlet Brooklyn waterworks supply pond ......................... 40.9

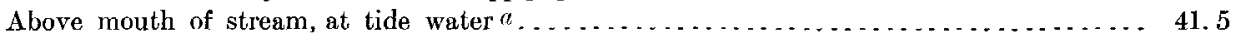

The catchment area comprises about 60 per cent woodland, 20 per cent sodded areas, and 20 per cent of land under cultivation.

A series of gagings of this stream, June 1 to October 15, 1883, at a point near the mouth showed a mean discharge of $3,097,000$ gallons, or 4.8 second-feet. Details are not available.

Mean daily discharge, in second-feet, of Massapiequa Creek at Farmingdale, Long Island, for 1903.

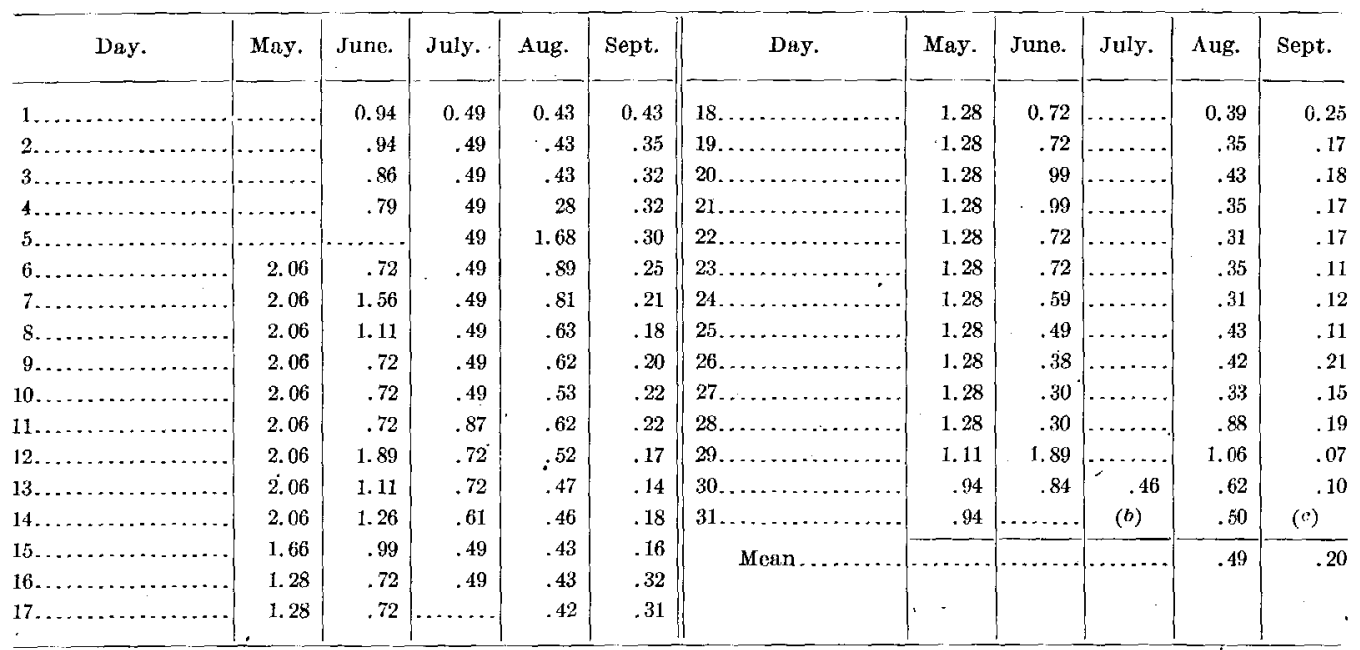

a The Brooklyn waterworks supply from this stream is deseribed in. The Water Supply of Brooklyn, by I. M. De Varona, 1896, pp. $75-76$

$\checkmark$ Gage readings by New York water-supply commission beginning July 31, 1903.

c Stream reported to go dry at times. 
CARLLS RIVER.

Mean daily discharge, in second-feet, of Massapequa Creek at Massapequa, Long Island, for 1903.a

\begin{tabular}{|c|c|c|c|c|c|c|c|c|c|c|c|}
\hline Day. & June. & July. & Aug. & Sept. & Oct. & Day. & June. & July. & Aug. & Sept. & Oct. \\
\hline 1.. & & 20.51 & 10.77 & 13.59 & 8.92 & 18. & & 12.18 & 10.62 & 12.31 & 16.12 \\
\hline $2 \ldots$ & & 18.07 & 10.27 & 12.08 & $8.7 \overline{5}$ & 19. & & 15. 53 & 10.32 & 10.32 & 13.50 \\
\hline $3 \ldots$ & & 17.55 & 9.61 & 11.25 & 9.21 & 20. & 17.33 & 14.57 & 10. 85 & 9.71 & 11.47 \\
\hline $4 \ldots$ & & 16.88 & 10.47 & 11.10 & 8.90 & $21, \ldots \ldots$ & 20,13 & 16.07 & 10.42 & 9.24 & 11.09 \\
\hline 5. & & 16.16 & 29.50 & 11.65 & 8.71 & $22 \ldots$ & 15.65 & 14.40 & 9.87 & 9.18 & 10.62 \\
\hline $6 .$. & & 16.37 & 24.54 & 11.47. & 8.70 & $23 \ldots$ & 18.70 & 13.99 & 9.82 & 9.14 & 10.50 \\
\hline $7 \ldots$ & & 16.06 & 20.76 & 10.69 & 8.64 & $24 \ldots$ & 23.51 & 13.03 & 9.91 & 9.06 & 10.35 \\
\hline $8 \ldots$ & & 15.16 & 15.69 & 10.37 & 8.50 & 25 .. & 18.54 & 12.97 & 10.69 & 8.86 & 10.25 \\
\hline $9 .$. & & 14.83 & 14.39 & 10.34 & 19.72 & $26 \ldots$ & 17.16 & 12.42 & 12.02 & $8.6 \overline{5}$ & 10.17 \\
\hline 10.. & & 14.28 & 13.70 & 10.33 & 18.98 & $27 \ldots$ & 16.39 & 11,23 & 10.82 & 9,12 & 9.98 \\
\hline 11.. & & 15.39 & 14.63 & 10.13 & 13.86 & 28. & 15.75 & 11.05 & 16.28 & 11.30 & 9.98 \\
\hline $12 .$. & & 14.86 & 14.91 & 9.81 & 12.23 & $29 \ldots \ldots \ldots$ & 21.18 & 10.81 & 26.54 & 9.36 & 9.82 \\
\hline $13 \ldots$ & & 16.01 & 11.59 & 9.60 & 10.99 & $30 \ldots$ & 28.58 & 10.99 & 21.62 & 8.89 & 9.76 \\
\hline 14. & & 15.29 & 12.21 & 9.51 & 10.24 & $31 \ldots$ & & 10.99 & 15.74 & & 9.76 \\
\hline $15 \ldots$ & & 13.98 & 11.70 & 9.42 & 9.71 & Mean. & & 14.47 & 13.96 & 10.29 & 11.02 \\
\hline $16 \ldots$ & & 13.62 & 11.29 & 10.70 & 9.53 & & & & & & \\
\hline 17. & & 13.11 & 11.10 & 11.26 & 12.72 & & & & & & \\
\hline
\end{tabular}

a At weir of New York water-supply commission.

CARLLS RIVER AT BABYLON, LONG ISLAND.

The drainage basin of this group of streams extends northward to within 2 miles of tide water, where it drains a small flat area at elevation 200 feet. A surface drainage valley can be traced from the head of the area following closely the right-hand watershed line to the head of the surface stream near Wyandanch.

The central portion of the drainage basin is 5 miles wide and includes the Half Hollow and Dix group of hills. The Colonial Springs issue from the south slope of Half Hollow Hills. The outflowing streams are absorbed by the soil, after running for a short distance.

The surface stream is branching and its valley is flat and marshy. Its longest branch is visible about 5 miles above the mouth. Five large private ponds have been constructed on the main branches, and the regimen of flow is largely arbitrary.

The drainage basin is shown on the Northport and Babylon sheets of the United States Geological Survey's topographic map, and covers 365 square miles above the gaging stations.

Highway embankments across the swamp valley serve to concentrate the flow of surface waters into narrow bridge openings, but some water may be lost by diversion into the surrounding gravels, due to the ponding. Temporary gages were erected at bridges crossing the two main branches of the stream above Kennel Club Pond, May 6, 1903. The gaging stations were completed early in June by planking the side walls of the bridges, affording smooth rectangular channels in which current-meter measurements were made. The combined discharge at the two gaging stations represents the total surface flow of the stream, and is shown in the following table. 
List of discharge measurements of Carlls River (East and West branches) at Babylon, Long Island, for 1903. WEST BRANCH.

\begin{tabular}{|c|c|c|c|}
\hline Date. & Hydrographer. & Gage height. & Discharge. \\
\hline May 6... & 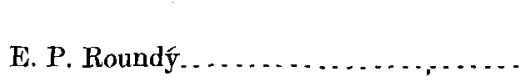 & $\begin{array}{l}\text { Feet. } \\
1.50\end{array}$ & $\begin{array}{c}\text { Second-feet. } \\
9.60\end{array}$ \\
\hline June $13 \ldots$ & E. P. Roundy and A. P. Porter......... & 1.735 & 15.96 \\
\hline July $6 \ldots$ & A. P. Porter . . . . . . . . . . . . & 1.66 & 12.73 \\
\hline
\end{tabular}

EAST BRANCH.

\begin{tabular}{|c|c|c|c|}
\hline May 6. . & E. P. Roundy $\ldots \ldots \ldots \ldots \ldots \ldots \ldots \ldots$ & 1. 10 & 26.58 \\
\hline June 13. & E. P. Roundy and A. P. Porter......... & 1.395 & 37.13 \\
\hline July $6 .$. & A. P. Porter . . . . . . . . . . & 1. 185 & 28.37 \\
\hline
\end{tabular}

BOTH BRANCHES.

\begin{tabular}{|c|c|c|}
\hline May 6 . & $\ldots \ldots$ & 36.18 \\
\hline June 13. & $\ldots \ldots \ldots \ldots \ldots \ldots \ldots \ldots \ldots$ & 53.09 \\
\hline July $6 .$. & $\ldots$ & 41.10 \\
\hline
\end{tabular}

Combined mean daily discharge, in second-feet, of East and West branches of Carlls River, at Babylon, Long 1sland, for 1903 .

\begin{tabular}{|c|c|c|c|c|c|c|c|c|c|c|c|}
\hline Day. & May. & June. & July. & Day. & May. & June. & July. & Day. & May. & June. & July. \\
\hline $1 \ldots$ & & 34.9 & 42.5 & $12 \ldots$ & (a) & 60.2 & 35.5 & $22, \ldots \ldots \ldots$ & 41.4 & 36.5 & \\
\hline $2 \ldots$ & 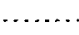 & 34.4 & (a) & $13 \ldots$ & (a) & 54.4 & .42 .2 & $23 \ldots \ldots \ldots$ & 37.1 & 41.4 & \\
\hline $3 \ldots t$. & $\ldots .$. & 34.4 & 59.6 & $14 \ldots \ldots$ & (a) & 43.3 & 47.9 & $24 \ldots \ldots \ldots$ & 35.6 & 47.5 & \\
\hline $4 \ldots$. & $\cdots \cdot$ & 33.7 & 36.6 & $15 \ldots \ldots$ & 32.1 & 37.8 & 45.8 & $25 \ldots \ldots$ & 35.6 & 46.0 & \\
\hline $5 \ldots$ & ........ & 34.0 & 37.2 & $16 . .$. & 32.1 & 33.7 & 42.5 & $26 \ldots \ldots$ & 36.6 & 43.2 & \\
\hline $6 \ldots$ & 35.6 & 34.5 & 40.5 & $17 \ldots$ & 32,1 & 40.5 & 42.4 & $27 \ldots \ldots$ & 37.7 & 38.0 & \\
\hline $7 .$. & 35.6 & 44.8 & 43.2 & $18 .$. & 32.1 & 48.2 & & $28 .$. & 42.4 & 35.9 & \\
\hline $8 .$. & 35.6 & 60.5 & 40.9 & 19. & 32.1 & 58.2 & & $29 \ldots \ldots$ & 39.4 & 44.9 & \\
\hline $9 \ldots \ldots \ldots$ & 35.6 & 46.3 & 37.6 & $20 \ldots$ & 34.5 & 55.6 & & $30 \ldots \ldots$ & 37.7 & & \\
\hline $10 \ldots \ldots \ldots$ & 35.6 & 46.9 & 39.1 & $21 .$. & 53.2 & 40.8 & & 31. & 35.6 & & \\
\hline $11 \ldots \ldots$ & 32.1 & 42.5 & 56.9 & & & & & & & & \\
\hline
\end{tabular}

The current-meter rating curves were never completed, but the measurements were made with great care and covered nearly the full range of fluctuation of the streams during the period of gaging. They are sufficient to afford a reliable estimate of the flow during the remarkable drought of May-June, 1903.

A small portion of the surface area of this stream lies north of the groundwater divide. 


\section{SAMPAWAMS CREEK.}

The surface drainage area of this stream extends inland 14 miles. The basin is narrow and elongated, the average width for 9 miles from the coast being 1 mile. The northern end of the basin broadens out, and includes the Commack plateau, which lies at elevation 180 to 200 feet above tide. The visible stream rises within 5 miles of the coast, but a well-defined stream valley extends much farther inland and may be traced on the topography quite to the northern divide.

The stream flows through a broad, flat valley, having a firm gravel soil overlain with muck, bog, and swamp vegetation. The Long Island Railroad embankment at Babylon forms an effectual cut-off for the flow of surface water through the swamp, and at the same time does not materially change the surface level or modify the ground-water conditions. The gaging station was selected at the point where the surface waters of the swamp valley are concentrated at the opening under this embankment.

In order to secure results as early in the season as possible, a gage was erected and measurements were begun early in May, 1903. In June a temporary measuring flume was constructed just above the railroad embankment. Meter measurements were made with a specially rated Fteley meter in every square foot of the cross section or oftener where necessary, in order to determine local current irregularities, and vertical velocity curves were determined at 0.1 -foot intervals from surface to bottom. The data of two of these velocity curves, showing the current in a shallow stream flowing over a smooth hard gravel bed, are given below:

Data from vertical velocity curves, Sampawams Creek, Babylon, Long Island.

[Measurements by A. P. Porter. Meter, F teley No. 107.]

\begin{tabular}{|c|c|c|c|}
\hline Data. & June 26, 1903. & June 30, 1903. & Mean of two. \\
\hline Station ... . . . $\ldots \ldots \ldots \ldots$ & 15 & 8 & \\
\hline Gage $\ldots \ldots \ldots \ldots \ldots \ldots \ldots$ fect. & 0.58 & 0.74 & 0.61 \\
\hline Depth......................do... & .92 & 1.02 & .97 \\
\hline Mean velocity $\ldots \ldots \ldots \ldots \ldots \ldots \ldots$ & .923 & 1.125 & 1.024 \\
\hline Surface velocity....... & .89 & 1.189 & 1.04 \\
\hline Per cent of mean.... & .964 & 1.048 & 1.006 \\
\hline Bottom velocity...... & .80 & .88 & .84 \\
\hline Per cent of mean...... & .866 & .782 & .824 \\
\hline Maximum velocity.... & .965 & 1. 191 & 1.078 \\
\hline Per cent of mean.............. & 1. 045 & 1.058 & 1.051 \\
\hline Depth of maximum........... feet. & .30 & .15 & $.2 \dot{25}$ \\
\hline Per cent of depth.................. & .326 & .147 & .236 \\
\hline Depth of point of mean velocity...feet. . & .62 & .61 & .615 \\
\hline Per cent of total depth. . . . . . . . . & .674 & .60 & .637 \\
\hline
\end{tabular}


UNDERGROUND WATER RESOURCES OF LONG ISLAND, NEW YORK.

Mean daily discharge, in second-feet, of Sampawams Creek at Babylon, Long Island, for 1903.

\begin{tabular}{|c|c|c|c|c|c|c|c|c|c|c|c|}
\hline Day. & May. & June. & July. & Day. & May. & June. & July. & Day. & May. & June. & July. \\
\hline $1 \ldots$ & & 12.3 & 23.5 & $12 \ldots$ & 13.4 & (d) & 15.0 & $22 .$. & 12.9 & 13.4 & \\
\hline $2 \ldots$ & $\cdots$ & 12.0 & 15.4 & $13 \ldots$ & 15.4 & 15.0 & 15.4 & 23. & 13.2 & 15.0 & \\
\hline 3.... & ........ & 12.2 & 13.4 & $14 \ldots \ldots \ldots$ & 15.4 & 17.1 & 14.6 & $24 .$. & 13.4 & I4. 4 & \\
\hline $4 \ldots$ & ........ & 12.2 & 12.6 & $15 \ldots \ldots \ldots$ & 15.4 & 15.0 & 13.8 & 25. & 12.9 & 13.2 & \\
\hline $5 \ldots$ & ........ & 12.3 & 12.4 & $16 \ldots$ & 15.4 & 13.6 & & $26 .$. & 12.3 & 13.6 & \\
\hline $6 \ldots$ & 21.4 & 12.4 & 12.4 & $17 \ldots$ & 13.2 & 13.2 & & 27. & 12.6 & 13.6 & \\
\hline $7 \ldots$ & 16.8 & $(a)$ & 12.4 & 18. & 13.2 & 13.2 & & $28 \ldots \ldots \ldots$ & 13.4 & 13.0 & \\
\hline $8 \ldots$. & 15.4 & 20.9 & 12.5 & $19 .$. & 12.6 & 14.4 & & $29 \ldots$ & 13.4 & 20.1 & \\
\hline $9 \ldots \ldots$ & 15.4 & 18.8 & 13.1 & $20 \ldots$ & 14.4 & 17.8 & & $30 .$. & 12.2 & (1) & \\
\hline $10 \ldots$ & 14.2 & 13.8 & 13.2 & $21 \ldots \ldots \ldots$ & 13.2 & 13.1 & & $31 \ldots \ldots \ldots$ & 15.0 & & \\
\hline $.11 \ldots$. & 12.8 & 13.8 & 13.4 & & & & . & & & & \\
\hline
\end{tabular}

The drainage basin of Sampawams Creek is shown. on the Northport and Babylon sheets of the United States Geological Survey's topographic map. The surface drainage area above the gaging station is 23 square miles, and above the mouth of the stream 24 square miles.

The ground-water divide crosses the basin near the foot of the upper broad area. The surface drainage area below the ground-water divide is about 10 square miles. Water power is developed at tide water; an earth dam affords 6 feet head to a whip factory and sawmill. There are also several small private ponds on the stream. At one of these, at the Unkeway Nurseries, a small turbine is in use under 5 feet head.

\section{OROWOC AND DOXSEE CREEKS, ISLIP.}

These streams drain a long, narrow area extending 6 miles inland and comprising topographic areas as follows:

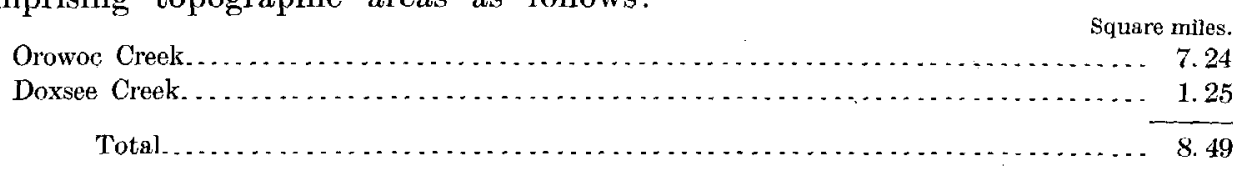

The ground-water divide at the north of the drainage areas of these streams probably lies some distance north of the surface divide. Both streams flow through swampy valleys crossed by fairly impermeable dikes constructed for highways. A temporary gaging station was maintained on Orowoc Creek, May 9 to July 16, 1903, inclusive. The gage, a finely divided scale, was erected at the first highway bridge above the Long Island Railroad in Islip.

The stream passes through a single rectangular span 4 feet in width, having timber sides and smooth gravel bottom. The section was rendered suitable for accurate gaging by moderate repair, and the following measurements were made with a specially rated current. meter, velocities being taken in every square foot of cross-sectional area or less: 
Discharge measurements of Orowoc Creek for 1903.

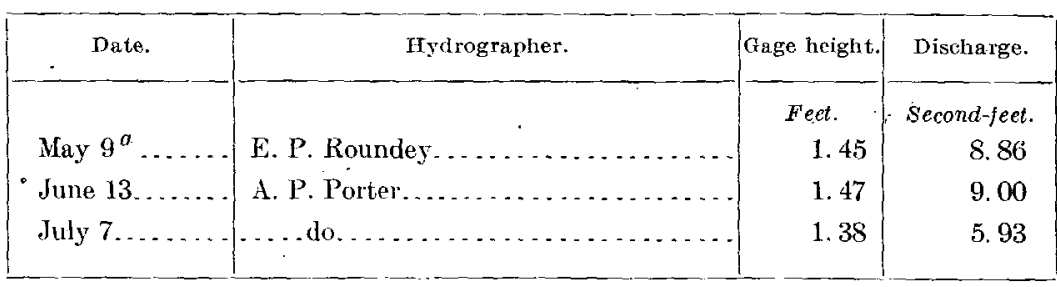

a Gaging station not completed.

Orowoc Creek is utilized as a supply for a small private pond at the South Shore Highway. A second small pond lies above the gaging station. The stream above the point of gaging is practically uncontrolled.

Doxsee Creek was gaged at a weir forming the outlet of a private pond. The regimen of the stream was arbitrarily controlled at times as required for flooding of cranberry' flats.

In order to secure a record as early as possible in the season, a gage was erected at the existing weir May 6, 1903. A metal crest weir conforming essentially to fixed standards was installed June 6,1903 , and the record continued until July 15, 1903.

Combined mean daily discharge, in second-feet, of Orowoc and Doxsee creeks at Islip, Long Island, for 1903.

\begin{tabular}{|c|c|c|c|c|c|c|c|c|c|c|c|}
\hline Day. & May. & June. & July'. & Day. & May. & June. & July. & - Day. & May. & June. & July. \\
\hline $1 .$. & & & 11.82 & $12 \ldots$ & 27.06 & 17.30 & 7.14 & 22. & b6.77 & & \\
\hline 2. & & & 11.80 & $13 .$. & 26.67 & 10.52 & 8.67 & 23. & & & \\
\hline $3 .$. & & & 11.15 & $14 \ldots$ & 26.67 & 14.74 & 7.57 & $24 \ldots$ & & & \\
\hline $4 \ldots$ & & & 11.15 & $15 .$. & 26.67 & 10.84 & 6.99 & $25 .$. & & 11.42 & \\
\hline $5 .$. & & $\ldots \ldots$ & 11.06 & $16 .$. & 26.60 & 10.11 & 7.14 & $26 \ldots$ & & 10.72 & \\
\hline b... & & $\ldots \ldots$ & 10.97 & $17 .$. & 25.35 & & & 27. & & 10.20 & \\
\hline 7. & & 13.91 & 10.57 & & 24.61 & & & 28. & & 10.05 & \\
\hline $8 .$. & & 14.36 & 9.07 & $19 .$. & 21.87 & & & 29. & & 12.96 & \\
\hline $9 \ldots \ldots$ & 29.70 & 10.11 & 7.98 & $20 \ldots \ldots \ldots$ & 18.17 & & & $30 \ldots \ldots$ & & 12.42 & \\
\hline $10 \ldots \ldots \ldots$ & 30.99 & 3.31 & 7.57 & $21 \ldots \ldots$ & 12.83 & 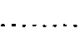 & .... & $31 \ldots \ldots$ & & & \\
\hline $11 \ldots \ldots \ldots$ & 27.19 & 3.13 & 7.64 & & & & & & & & \\
\hline
\end{tabular}

a May 7 to 21, inclusive, drawing water from pond above gage on Doxsee Creek.

b May 22 to June 6, inclusive, refilling pond above gage on Doxsee Creek.

The drainage areas of these streams are shown on the Setauket and Fire Island sheets of the United States Geological Survey's map.

Champlin Creek, lying immediately east of Islip, drains a surface area of 6.9 square miles above the South Shore highway. The drainage basin is long and narrow and the surface stream extends well inland, the lower course being turned into private ponds.

17116--No. $44-06-25$ 
CONNETQUOT BROOK, NEAR GREAT RIVER, LONG ISLAND.

The catchment area of several small streams entering Connetquot Bay comprises an irregular rectangle 2 miles wide at tide water, extending inland 7 miles, and 6 miles wide at the northern divide. The topographic area is 19.6 square miles above tide limit at the South Shore highway. Of this area, 5.6 square miles is tributary to Cutting Creek, on which a separate gaging station was temporarily maintained in 1903. The remainder is tributary to the Connetquot streams.

Ponds have been constructed for pleasure parks above the South Shore highway, and these ponds control the outflow of the several branches. The central branch heads near the northern watershed divide. The discharge of this branch was determined as follows, at the South Shore highway crossing:
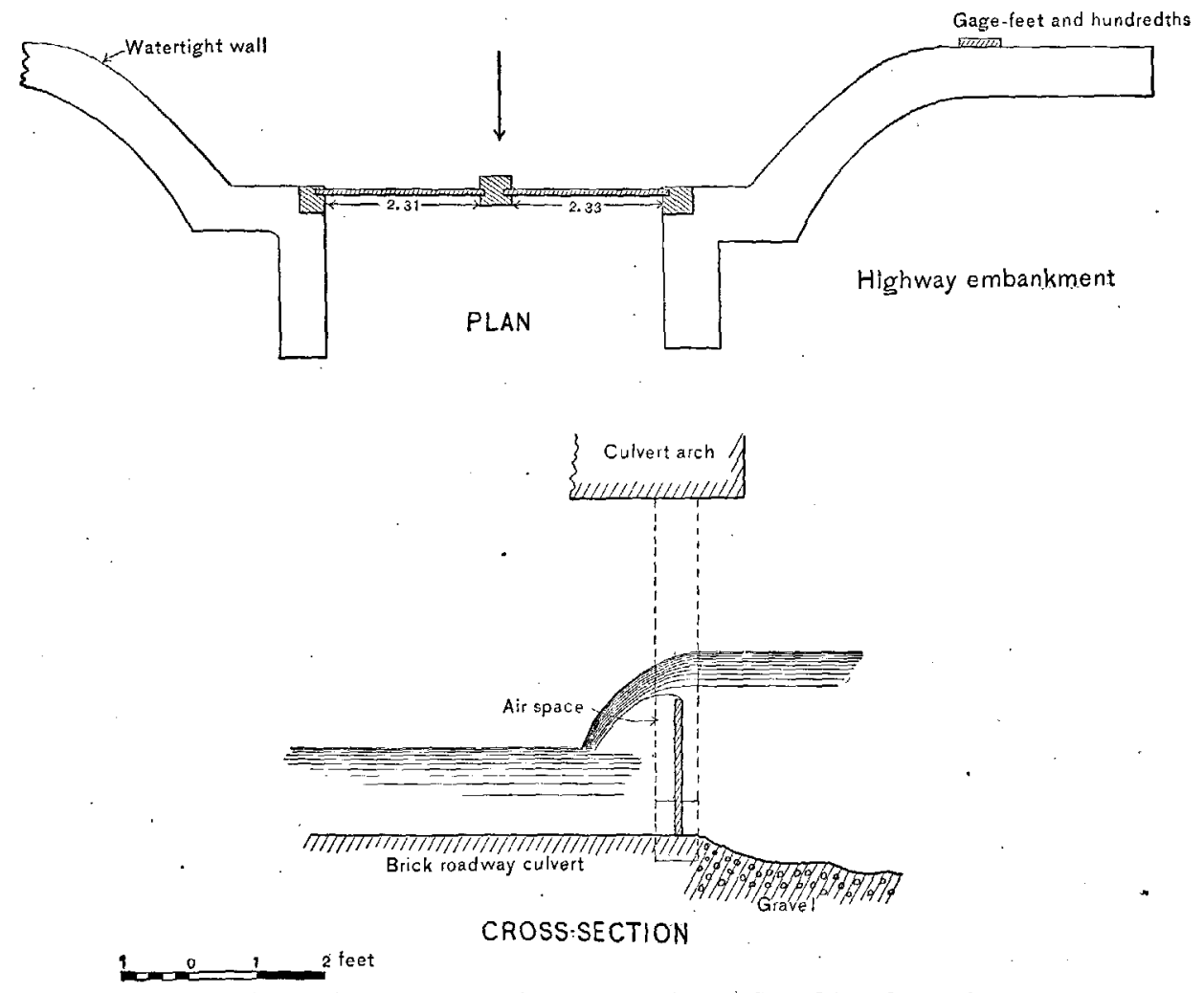

FIG. 71.-Weir on private pond, Cutting Creek, near Great River, I.ong Island.

- Discharge measurements of central branch of Connetquot Brook, for 1903.

\begin{tabular}{|c|c|c|c|}
\hline Date. & Hydrographer. & $\begin{array}{c}\text { Gage } \\
\text { height. }\end{array}$ & Discharge. \\
\hline & & Feet. & Second-feet. \\
\hline Apr. 22 & R. E. Horton.... & $\ldots \ldots$ & 69.5 \\
\hline June 20 & E. P. Roundey. & 2.57 & 58.3 \\
\hline July $1 .$. & A. P. Porter ..... & 2.72 & 82.3 \\
\hline
\end{tabular}


The results of gagings of Cutting Creek are given in the following table. The regimen is rendered artifical by pondage.

The discharge was obtained at an existing pond weir having a level crest, free discharge, four complete contractions, and no velocity of approach. The discharge usually varied but little during a single day, and the mean of two readings on a finely divided scale has been used in conjunction with the Francis formula in calculating the discharge.

Mean daily discharge in second-feet of Cutting Creek at East Islip, Long Island, for 1903.

\begin{tabular}{|c|c|c|c|c|c|c|c|c|c|c|c|}
\hline Day. & May. & Jume. & July, & Day, & May. & June. & July. & Day. & May. & June. & July. \\
\hline $1 \ldots$ & $\therefore$ & (a) & 6.19 & 12. & 4.12 & 13.60 & & 23. & 0.64 & 6.22 & \\
\hline $2 \ldots \ldots \ldots$ & & (a) & 6.19 & $13 \ldots \ldots$ & 4.12 & 6. 78 & & $24 \ldots$ & .32 & 6.40 & \\
\hline $3 \ldots \ldots$ & & (a) & 6.01 & $14 \ldots$ & 3.41 & 13.60 & & $25 \ldots$ & .64 & 6. 19 & \\
\hline $1, \ldots \ldots \ldots$ & & (a) & 6.22 & $15 \ldots \ldots$ & 2.17 & 14. 17 & & $26 \ldots$ & .32 & 6.40 & \\
\hline $5 \ldots \ldots$. & & (a) & 6.01 & $16 \ldots \ldots$ & 2.17 & 12.64 & & $27 \ldots$ & .64 & 6.01 & \\
\hline $6 . \ldots \ldots$ & & (a) & & $17 \ldots \ldots$ & .32 & 9.52 & & $28 \ldots \ldots$ & .64 & 6.01 & \\
\hline$T \ldots \ldots$. & & (a) & & $18 \ldots \ldots$ & .32 & 5. 19 & & $29 \ldots \ldots$ & .46 & 6.19 & $\ldots \ldots$ \\
\hline s....... & & 5.58 & & 19. & $\cdots \cdots$ & 5.33 & & $30 \ldots \ldots$ & .64 & 6.22 & \\
\hline $9 \ldots \ldots \ldots$ & ...... & 7.20 & & $20 .$. & $\cdots \cdots$ & 5.58 & & $31 \ldots \ldots$ & $\ldots$ & & \\
\hline $10 \ldots \ldots \ldots$ & 5.58 & 8.04 & & $21 .$. & .37 & 5.54 & & & & & \\
\hline $11 \ldots \ldots$. & 5.58 & 9.83 & & $22 .$. & $\ldots$. & 6.01 & & & & & \\
\hline
\end{tabular}

a Water reserved as pondage, June 1 to 7 , inclusive.

\section{LAKE RONKONKOMA AND ADJACENT STREAMS.}

The inland basin of Take Ronkonkoma lies immediately north of the topographical catchment area of the Connetquot streams. It appears probable that the ground-water divide lies considerably north of the surface divide between the catchment basins, so that the effective catchment tributary to the Connetquot streams is thereby increased.

Lake Ronkonkoma has a surface area of about 0.5 square mile, and receives the surface run-off from a total area of 8 square miles. This remarkable depression extends somewhat below sea level and represents virtually a natural well. The range of surface fluctuations of this lake has not been reliably determined.

Lake Ronkonkoma lies in a catchment basin topographically tributary to Nissequogue River, a northward-flowing stream having a surface drainage basin. of 44 square miles. This is the largest basin on the north slope of Long Island. The surface stream extends inland nearly to the watershed line.

A brief reconnaissance of streams east of the Connetquot area was made April 23, 1903. Edwards and Tuttle creeks have surface drainage basins of 6.7 and 9 square miles, respectively, and are utilized at private ponds. Patchogue Creek, in addition to supplying private ponds, is utilized to furnish water power at the lace factory of the Patchogue Manufacturing Company, under a head of 14 feet. This stream drains a surface area of 14 square miles.

Swan River at East Patchogue affords a fall of about 12 feet at tide water, with good pondage. This stream drains a surface area of 7.8 square miles. The visible stream extends inland 4.5 miles. Its catchment area is narrow and elongated, extending 7 miles inländ. 
The South Shore highway dike is utilized as a dam on Mud Creek, affording. a pond of perhaps 10 acres area, and about 6 feet fall, where a drag sawmill, with a breast water wheel, was formerly operated.

The estimated discharge of Mud Creek, April 23, 1903, was 6.7 second-feet, from a surface drainage area of 5 square miles.

\section{CARMANS RIVER, OR CONNECTICUT RIVER OF LONG ISLAND.}

The drainage area of this stream is an irregular triangle, having its apex at the mouth of the stream, and its base, which is about 10 miles in length, extending parallel to the north shore of Long Island, at an average distance of 1.5 miles therefrom, and at elevation 150 to 200 feet. The surface of the drainage basin slopes from elevation 150 near the northern divide to tide-water elevation at the mouth, in a distance of 12 to 13 miles. The surface stream extends inland only 10 miles. There are no perennial streams in the upper catchment area, although the topographic stream valley is defined nearly to the northern divide of the basin. The northwestern portion of the drainage basin lies in the Saint James plateau, north of the group of sand hills which lie near the center of the island at Selden, and reaches westward nearly to Lake Ronkonkoma.

The general topography is moderately rolling. A few undrained depressions of one-fourth square mile area or less are found near the northern divide, and the upper 2.5 miles of the course of a dry tributary valley on the left of the main stream have been cut off and form a depression.

The catchment area is overlain by sand and gravel, and is largely covered with scrub oaks and conifers.

From South Haven to the mouth of the stream, a distance of 3 miles, the channel is bordered by marsh and the current is sluggish. Tidal influence extends to South Haven.

A temporary gaging station was established at South Haven May 8, 1903, but was discontinued July 16, 1903, before an opportunity had been found to determine the range and cycle of daily tides at this point. The gage readings are not at present available. Discharge measurements were made as follows:

Discharge of Carmans River, Long Island, for 1909.

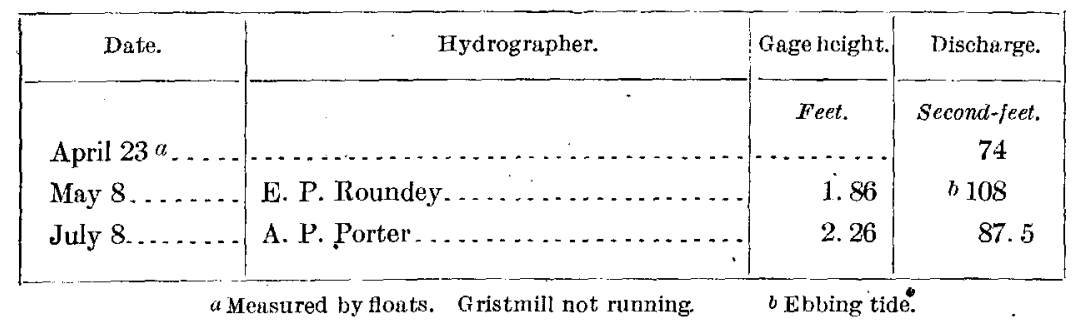

An earthen dike at South Haven affords a fall of 6 feet and a storage pond that is utilized by a gristmill and a sawmill.

An earthen dam at Yaphank affords extensive pondage. A custom saw and grist mill operate under a head of 10 feet. 
One mile above Yaphank is a third dam, about 6 feet high, which has been abandoned.

The course of the stream intermediate to the dams is through a swamp channel. The major portion of the fall is utilized at existing dams.

The drainage basin is shown on the Moriches and Setauket sheets of the United States Geological Survey's topographic map:

Drainage areas of Carmans River, Long Island.

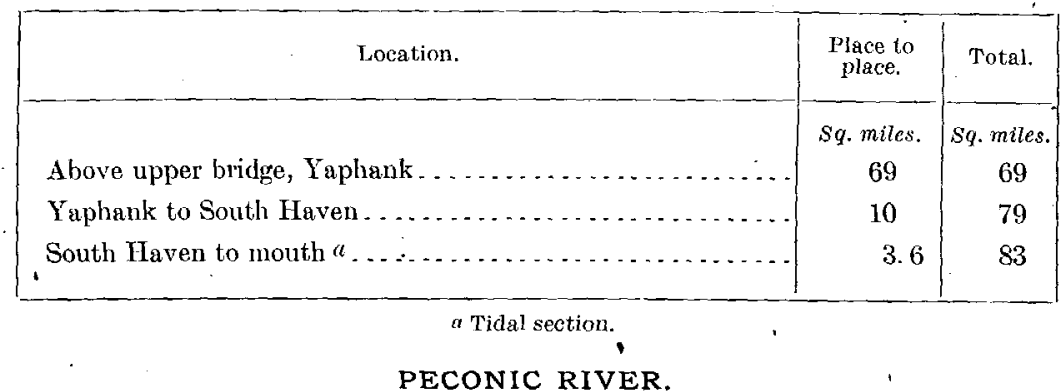

This stream has the most extended surface-drainage basin on Long Island. Its drainage comprises an irregular rectangular area, of which the northern divide is within one-half mile to $1 \frac{1}{2}$ miles of Long Island Sound and at an altitude of 150 to 200 feet above tide. The general slope of the basin is toward the southceast. The surface divide on the west is not sharply defined, but is at an average elevation of 120 feet above tide.

The southern watershed is about 5 miles from the seacoast.

The drainage area above Calverton is in general flat and contains about 25 undrained depressions, chiefly of but a few acres area. Deep Pond, at an elevation of 23 feet, lies in the largest depression and receives the drainage from an area of 0.35 square mile.

Below Calverton the drainage on the north is comparatively flat and is besprinkled with undrained hollows.

Farther south the topography of the drainage basin is intricate and precipitous, the river valley being at an average elevation of 20 feet, and the southern divide, 2.5 miles distant, at elevations of 200 to 300 feet. Small undrained depressions are very numerous on this slope and receive a large proportion of the precipitation.

- The drainage basin is shown on the Moriches and Riverhead sheets of the United States Geological Survey's topographic map, from which the following areas have been deduced:

Drainage areas of Peconic River, Long Island.

\begin{tabular}{|c|c|c|}
\hline Location. & $\begin{array}{l}\text { Place to } \\
\text { place. }\end{array}$ & Total. \\
\hline , & sq. miles. & Sq. miles. \\
\hline Above Long Island R. R. bridge, near Calverton & 59 & 59 \\
\hline Above Riverhead dam $\ldots \ldots \ldots \ldots \ldots$ & 25 & 84 \\
\hline
\end{tabular}


Peconic River is tributary to Peconic Bay, which bifurcates the eastern end of Long Island. The surface stream is formed by the junction of two short branches 9 miles from Riverhead. At this point the stream is 40 feet above tide. Almost the entire fall from its source is taken up at flood dams for cranberry marshes and at water-power dams.

Water power on Peconic River.

Riverhead: Earth dike of usual Long Island type. Five to 7 feet head, varying with tide. Good pondage. Water privilege divided between the Tower mill and the Peconic gristmill.

Electric-light plant: One and one-half miles above Riverhead. Earth dike. Extensive pondage. Formerly a woolen mill, stated to have 95 horsepower, 6.5 feet head.

Dam No. 3: Forge and gristmill. Unused for many years. Earth dike; 5.5 feet fall.

Calverton: E. L. Brown gristmill. Five feet fall. Also unused drag sawmill. Antiquated "tub and flutter" water wheels.

A brief reconnaissance of this stream was made April 24, 1903. Slack water exists throughout almost the entire course of the stream, affording no opportunity for the erection of a weir. Sufficient fall exists at the Long Island Railroad bridge near Calverton to afford an opportunity for gaging. The discharge at this point was roughly estimated at 45 second-feet April 24, 1903, and as measured by E. P. Roundey May 11,1903, was 48 second-feet; gage height, 1.3 feet. April 24, 1903, the discharge at Manorville highway bridge, estimated from surface floats, was 33 second-feet. April 24, 1903, the discharge estimated from float measurements at the junction of the north and south branches 1.5 miles above Manorville was 22.5 second-feet.

The stream flows in a flat valley of the usual Long Island type. The channel is bordered by marshes, forming a fat having an average width of perhaps 1,000 feet. This marshy flat is subdivided transversely by low dikes having gates utilized in flooding the inclosed cranberry marshes. The soil is gravel, with sand at the surface and forming the hills. Much of the area is covered with scrub oak.

The drainage basin extends 4 miles westward from the visible heads of the two main branches, including an area of 25 square miles in which there are no visible streams. A well-marked dry stream valley reaches, however, from the junction of the branches to the westerly watershed line.

The drainage south of Peconic River basin comprises numerous small water courses in marshy valleys, the surface streams heading at but little distance above the limit of tide water.

The two eastern prongs of Long Island are comprised chiefly of absorbent . sandy soils, affording very little surface run-off, and consequently having scarcely any surface streams. 
Mean daily gage height, in feet, of Peconic River, at Calverton, Long Island, for 1903.

\begin{tabular}{|c|c|c|c|c|c|c|c|c|c|c|c|}
\hline Day. & May. & June. & July. & Day. & May. & June. & July. & Day. & May. & June. & July. \\
\hline $1 \ldots$ & & 1.10 & $1 . \dot{20}$ & $12 \ldots \ldots \ldots$ & 1. 22 & 1.42 & 1.15 & 22. & 1.20 & 1.30 & \\
\hline $2 \ldots$ & & 1.10 & 1. 15 & $13 \ldots \ldots$ & 1.25 & 1.50 & 1.15 & $23 \ldots \ldots$ & 1.20 & 1.30 & \\
\hline 3. & & 1.10 & 1.15 & $14 \ldots \ldots$ & 1.30 & 1.40 & 1.15 & $24 \ldots \ldots$ & 1.20 & 1. 28 & \\
\hline 4. & & 1.20 & 1.15 & $15 \ldots \ldots$ & 1.27 & 1. 35 & 1.15 & $25 \ldots$ & 1. 20 & 1.225 & \\
\hline 5. & & 1.20 & 1.15 & $16 .$. & 1.25 & 1. 30 & 1. 12 & 26. & 1.20 & 1.20 & \\
\hline $6 \ldots$ & & 1.15 & 1.15 & $17 \ldots$ & 1.20 & 1.30 & & 27. & 1.20 & 1.15 & \\
\hline $7 \ldots$ & & 1. 15 & 1.15 & $18 \ldots \ldots$ & 1.20 & 1.30 & & 28 & 1.20 & 1.15 & $\ldots \ldots$ \\
\hline 8. . & & 1.37 & 1.10 & $19 \ldots \ldots$ & 1.20 & 1.225 & & 29 & 1.15 & 1.15 & $\ldots \ldots$ \\
\hline 9. & & 1.40 & 1.10 & $20 \ldots \ldots$ & 1.20 & 1.20 & & 30. & 1.15 & 1.22 & $\ldots$ \\
\hline $10 .$. & & 1.40 & 1.10 & $21 \ldots \ldots$ & 1.20 & 1.30 & & 31. & 1.15 & & \\
\hline $11 \ldots$ & & $\cdot 1.32$ & 1.10 & & & & & & & & \\
\hline
\end{tabular}

HYDROLOGIC CONDITIONS ON LONG ISLAND DURING 1903.

At the time the stream gaging was undertaken by the United States Geological Survey in April, 1903, the ground-water level was very high, as a result of heavy precipitation during the first sixteen days of April.

Beginning on April 17 a period of fifty-one days ensued with no precipitation of consequence. During this period the ground-water level in wells on Long Island fell steadily. The average lowering of ground water in a number of wells was as follows:

\begin{tabular}{|c|c|}
\hline Minimum depth to ground water. & $\begin{array}{l}\text { Depletion of ground } \\
\text { water during } \\
\text { drought of April } \\
\text { to June, } 1903 \text {. }\end{array}$ \\
\hline & Feet. \\
\hline 0 to 5 feet. . . & 2.17 \\
\hline 5 to 10 feet..... & 1.62 \\
\hline 10 to 15 feet. . . . . . . . . . . & 1.42 \\
\hline 15 to 20 feet......... & 1.35 \\
\hline 20 to 25 feet. . . . . . . . . . . . & .92 \\
\hline Over 25 feet...... & .2 to .3 \\
\hline
\end{tabular}

Data concerning the ground-water stages during this drought, in conjunction with the contemporary gagings, afford an opportunity to study the regimen of the streams in relation to ground water. The rainfall was so slight that little or no accretion to the ground-water supply occurred from this source. The condition of the soil above the ground-water table appears to have been that of continually decreasing saturation.

June 7 to 15, 1903, abnormally heavy. rainfall occurred and was followed by considerable amounts of precipitation at frequent but irregular intervals to the end of the season, a condition tending to produce strata of differing saturation in the soil above the ground-water table, a disturbing factor in any attempted solution of the relations of rainfall, ground water, and run-off. 


\section{UNDERGROUND WATER RESOURCES OF LONG ISLAND, NEW YORK.}

In order to facilitate comparison of the earlier gagings with the later data, the mean annual precipitation on Long Island and its departure from the normal has been presented in the following table, as given in the records compiled by the New York Water Supply Commission in 1903:

Precipitation on Long Island, 1903, at United States Weather Bureau stations.

\begin{tabular}{|c|c|c|c|}
\hline$\cdot$ & Cutehogue. & Setnuket. & $\begin{array}{l}\text { Southimp- } \\
\text { ton. }\end{array}$ \\
\hline March precipitation..... . & $\begin{array}{l}\text { Inches. } \\
1.26\end{array}$ & $\begin{array}{l}\text { Inehes. } \\
1.05\end{array}$ & $\begin{array}{r}\text { Inches. } \\
\text { 1. } 20\end{array}$ \\
\hline April 1-16, inclusive... & 4.26 & 3.61 & 4. 19 \\
\hline April $24 \ldots$ & Tr. & $\mathrm{Tr}$. & \\
\hline May $3 \ldots$ & $\ldots$. & $\operatorname{Tr}$. & $\ldots$ \\
\hline May $4 \ldots \ldots \ldots \ldots$ & Tr. & .08 & .14 \\
\hline May $5 \ldots \ldots \ldots \ldots$ & Tr. & .07 & 01 \\
\hline May $6 \ldots \ldots \ldots$ & $\operatorname{Tr}$. & $\ldots$ & 09 \\
\hline May $7 \ldots .$. & $\ldots$ & .02 & .06 \\
\hline May $8 \ldots \ldots$ & .05 & & \\
\hline May $20 \ldots \ldots . .$. & & & $\cdots$ \\
\hline May $21 \ldots . . . . .$. & .25 & $\ldots$ & $\operatorname{Tr}$. \\
\hline May $22 \ldots . . . . . .$. & .15 & .14 & .15 \\
\hline May $23 \ldots . . . . . . .$. & $\ldots \ldots$ & .... & .03 \\
\hline May $24 \ldots \ldots \ldots \ldots \ldots \ldots \ldots$ & .01 & Tr. & $\mathrm{T}_{\mathrm{l}}$ \\
\hline May $28 \ldots \ldots \ldots$ & $\ldots$. & .19 & $\ldots$ \\
\hline May $30 \ldots \ldots . .$. & .48 & .... & .78 \\
\hline 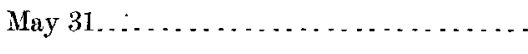 & $\therefore$. & $\cdots$ & $\operatorname{Tr}$. \\
\hline Total, April 17 to June 6, inclusive. & 94 & 50 & 1.26 \\
\hline June $7-25$, inclusive. . . . . . . . . . . . & 5.17 & 6.49 & 4.47 \\
\hline June $29 \ldots \ldots \ldots \ldots \ldots \ldots \ldots \ldots$ & .91 & 1.35 & .20 \\
\hline July $\ldots \ldots \ldots \ldots \ldots \ldots \ldots$ & 1.89 & 2.26 & 1.66 \\
\hline August................... & 7.77 & 6.28 & 7.37 \\
\hline September . . . . . . . . . . . & 1.25 & 2.61 & 1.24 \\
\hline October............................. & 4. 53 & 3. 66 & 3. 42 \\
\hline
\end{tabular}


HYDROLOGIO OONDITIONS IN 1903.

Mean annual precipitation on Long Island. ${ }^{t}$

\begin{tabular}{|c|c|c|c|c|c|c|c|c|c|c|c|}
\hline Year. & $\begin{array}{l}\text { Precipi- } \\
\text { tation. }\end{array}$ & $\begin{array}{c}\text { De- } \\
\text { par- } \\
\text { ture } \\
\text { from } \\
\text { nor- } \\
\text { mal. }\end{array}$ & $\begin{array}{l}\text { Aggre- } \\
\text { gate } \\
\text { de- } \\
\text { par- } \\
\text { ture. }\end{array}$ & Year. & $\begin{array}{l}\text { Precipi- } \\
\text { tation. }\end{array}$ & $\begin{array}{l}\text { De- } \\
\text { par- } \\
\text { ture } \\
\text { from } \\
\text { nor- } \\
\text { mal. }\end{array}$ & $\begin{array}{c}\text { Aggre- } \\
\text { gate- } \\
\text { de- } \\
\text { par- } \\
\text { ture. }\end{array}$ & Year. & $\begin{array}{l}\text { Precipi- } \\
\text { tation. }\end{array}$ & $\begin{array}{l}\text { De- } \\
\text { par- } \\
\text { ture } \\
\text { from } \\
\text { nor- } \\
\text { mal. }\end{array}$ & $\begin{array}{c}\text { Aggre- } \\
\text { gate } \\
\text { de- } \\
\text { par- } \\
\text { ture. }\end{array}$ \\
\hline & Inches. & Inches. & Inches. & & Inches. & Inches. & Inches. & & Inches. & Inches. & Inches. \\
\hline 1826. & 54.31 & +11.75 & +11.75 & 1852. & 36.91 & -5.65 & -75.09 & 1878. & 46.91 & +3.35 & -69.19 \\
\hline $1827 \ldots$ & $50 \div 9$ & +8.23 & +19.48 & 18.53. & 47.88 & +5.32 & -69.77 & 1879. & 40.07 & -2.49 & -71.68 \\
\hline 1828. & 43.95 & +1.39 & +21.37 & 1854. & 47.23 & +4.67 & -65.10 & 1880. & 39.19 & -3.37 & -75.04 \\
\hline 1829. & $45 \overline{3} .07$ & +2.51 & +23.88 & 1855 & 43.03 & +.47 & -64.63 & 1881. & 35.53 & -7.03 & -82.08 \\
\hline 1830. & 45.41 & +2.85 & +26.73 & 1856. & 38.26 & -4.30 & -68.93 & $1882 .:$ & 39.40 & -3.16 & -85.24 \\
\hline $1831 \ldots$ & 39.16 & -3.40 & +23.33 & 1857. & 41.39 & -1.17 & -70.10 & $1883 \ldots$ & 36.95 & -5.61 & -90.85 \\
\hline 1832. & 40,37 & -2.19 & +21.14 & 1858. & 39.37 & -3.19 & -73.29 & $1884 \ldots$ & 46.81 & +4.25 & -86.60 \\
\hline 1833. & 38,45 & -4.11 & +17.03 & 1859. & 58.29 & +15.73 & -57.56 & 1885. & 39.09 & -3.47 & -90.07 \\
\hline 1834. & 39.24 & -3.32 & +13.71 & 1860. & 30.43 & +12.13 & -69.69 & $1886 \ldots$ & 48.64 & +6.08 & -83.99 \\
\hline 1835. & 30.37 & -12.19 & +1.52 & 1861. & 39.27 & -3.29 & -72.98 & 1887. & 47.07 & +4.51 & -79.48 \\
\hline 1836. & 37.23 & -5.33 & -3.81 & $1862 \ldots$ & 43.35 & +.79 & -72.19 & $1888 \ldots$ & 50.48 & +7.92 & -71.56 \\
\hline $1837 \ldots$ & 35.29 & -7.27 & -11.08 & 1863. & 41. 18 & -1.38 & -73.57 & '1889.. & 55.66 & +13.10 & -58.46 \\
\hline $1838 \ldots$ & 34.19 & -8.37 & -19.45 & $1864 \ldots$ & 38.10 & -4.46 & -78.03 & $1890 \ldots$ & 52.19 & +9.63 & -48.83 \\
\hline $1839 \ldots$ & 38.90 & -3.66 & -23.11 & $1865 \ldots$ & 43.49 & +.93 & -77.10 & $1891 \ldots$ & 45.61 & +3.05 & -45.78 \\
\hline $1840 \ldots$ & 37.34 & -5.22 & -28.33 & 1866. & 45.79 & +3.23 & -73.87 & $1892 \ldots$ & 40.32 & -2.24 & -48.02 \\
\hline 1841. & 44.94 & +2.38 & -25.95 & $1867 \ldots$ & .45 .80 & +3.24 & -70.63 & $1893 \ldots$ & 46.81 & +4.25 & -43.77 \\
\hline 1842. & 39.47 & -3.09 & -29.04 & 1868. & 45.01 & +2.45 & -68.18 & 1844. & 44.30 & +1.74 & -42.03 \\
\hline $1843 \ldots$ & 36.69 & -5.87 & -34.91 & 1860. & 45.67 & +3.11 & $-65.07^{-}$ & 1895. & 38.33 & -4.23 & -46.26 \\
\hline 1844. & 39.02 & -3.54 & -38.45 & 1870. & 35.02 & -7.54 & -72.61 & $1806 \ldots$ & 39.79 & -2.77 & -49.03 \\
\hline $1845 .$. & 33.68 & -8.88 & -47.33 & $1871 \ldots$ & 43.72 & +1.16 & -71.45 & $1897 \ldots$ & 50.95 & +7.39 & -41.64 \\
\hline 1846. & 38.50 & -4.06 & -51.39 & 1872. & 42.31 & -.25 & -71.70 & 1898. & 57.89 & $+15,33$ & -26.31 \\
\hline $1847 \ldots$ & 46.77 & +4.21 & -47.18 & $1873 \ldots$ & 39.27 & -3.29 & -74.99 & $1899 \ldots \ldots$ & 43.10 & +.54 & -25.77 \\
\hline 1848. & 33.14 & -9.42 & -56.60 & $1874 \ldots$ & 41.47 & $-1,09$ & -76.08 & $1900 \ldots$ & 45.19 & +2.63 & -23.14 \\
\hline 1849 & 30.40 & -12.16 & -68.76 & $1875 \ldots$. & 44.43 & +1.87 & -74.21 & $1901 \ldots$ & 50.62 & +8.06 & $-15,08$ \\
\hline 1850 & 45.39 & -2.83 & -65.93 & $1876 \ldots$. & 45.67 & +3.11 & -71.10 & $1902 \ldots$ & 50.49 & +7.93 & -7.15 \\
\hline 1851. & 39.05 & -3.51 & -69.44 & 1877. & 41.12 & -1.44 & -72.54 & 1903. & 73.23 & & \\
\hline
\end{tabular}

a from records of U. S. Signal Service, U. S. Weather Bureau; U. S. Army posts, New York State, etc., as compiled by New York Water Supply Commission. 



\section{GENERAL INDEX.}

[An index of wells by names of owners is given on pp. 391-394.]

Agawam pumping station, underflow at, diagrams Page. illustrating .......... 99-100 Amityville Waterworks Co., system of, data concerning ...................... 82-83,150-151, 287 water system of, map showing...... Pl. xix, in pocket. Ammeter. See Meter, recording. .

Analyses of well waters . . ................ 68-69, 169-170, $183,185,190-193,199,202-203,206-207,211,213-$ $214,226,228,231-233,244,247-248,267,271,282-$ 284, 288-291, 293, 298, 301, 303, 305, 333, 335̃, 337 Assistance, acknowledgmen ls to those rendering.... 116-117 Babylon Sumpwams Water Co., water system of, data concerning.............. 82-83, 154-155, 303-304 water system of, map showing ...... Pl. xix, in pocket.

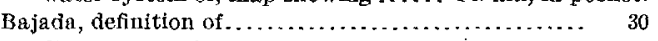
diagram showing. ........................... 29

Ba rometic changes, effect of, on ground water..... 72,74 effect of, on ground water, diagram showing ..... 72 Basement rocks, occurrence and eharacter of........ 16-17

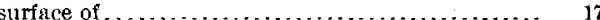

Bayshore, water system of, map showing. Pl. xix, in pocket. Bayside, water system of, data concerning ......... 80-81 water system of, map showing ....... Pl. xix, in pocket. Bayside pumping station, borings at, location of, map showing ........................... 217

Bed rock, character and occurrence of ............. 16-17 position of, map showing ................... 16 sections showing............................ 17,36 Belleview road stations, underflow at, figures showing ........................ 106, 107,108

Berry, E. W., on Matawan formation............. 24,25

Bibliography of Long Island.

Blythbourne Water Co., water system of, data concerning .................. 80-81,118-119, 168 water system of, map showing...... Pl. xix, in pocket.

Bowery Bay Building and Improvement Co., system of, data concerning .......... 82-83,130-131, 197 water system of, map showing....... Pl, xix, in pocket. Bowman, Isaiah, Veateh, A. C., and, well records by. 126-337 Broken Grounds, view of ..................... 38 Brooklyn, sewer tunnel in south, plan and sections of.. 168 Brooklyn Borough, water supply of ......... $71-81 \quad 363-364$ water supply of, map showing systems of........

Pl. $x i x$, in pocket.

Brooklyn Department of Water Supply, data concerning

pumping stations of ............ $74-79$ map showing systems of........ Pl. xix, in pocket. storage reservoirs of $\ldots \ldots \ldots \ldots \ldots \ldots \ldots \ldots \ldots .74-79$ map showing................ Pl. xix, in pocket.

water level in watershed of effect of pumping on diagram showing

wells of, in lex to

at, diagram showing.......... 49

Burgess well, Oyster Bay, view of ................ 64

Carns River, data concerning .................... $373-374$
Carmans River, data concerning............... $380-381$

$380-381$

Cedar Brook station, underflow at, figure showing.... 103

Center Island, section at, diagram showing......... 38

Cherry Hill Point, section near, diagram showing.....
Citizens Water Supply Co., water system of, data concerning .. 80-81, 82-83, 128-129, 132-133, 194, 195, 214 water system of, map showing....... Pl. xix, in pocket. well of, effect of tides on, figure showing........ 71

Clark, W, B., on New Jersey geology ............. 21,23 Coastal plain, drainage of development of, diagram showing................................ Cold Spring Harbor, well at, view of ............. 64 College Point, water system of, data concerning ..... 78-79 water system of, map showing...... Pl. xix, in pocket. Commutator clock, views of .................... 92,98 Connecticut, well water from, analyses of . . . . . . . . 68-69 Connecticut River of Long Island. See Carmans River.

Connetquot Brook, data concerning............ 378-379 Cook, G. H., on New Jersey geology............... 21 Creedmoor, moraine near, view of ................. 44 Cretaceous rocks of Long Island, artesian area of, map

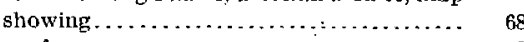
character of ............................ 18 comparison of New Jersey Cretaceous and....... 21-25 distribution of ......................... 19-20 maps showing $: \ldots \ldots \ldots \ldots \ldots \ldots \ldots \ldots \ldots \ldots \ldots \ldots \ldots \ldots \ldots, 20,68$ position of, diagrams showing ............... $34-39$ relations of . . . . . . . . . . . . . . . . . . . . . . . . 21-25 map showing .............................. 18

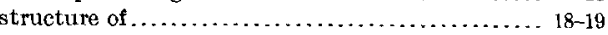
maps showing ...................... 18, 19,68 summary of ................................ ${ }_{26}$ view of, at Melville ........................ 22 water supply in....................... $55-56,65-67$ wells to .

Cretaceous rocks of. New Jersey, comparison of Long Island cretaceous and $\ldots \ldots \ldots \ldots \ldots \ldots \ldots 21-23$ map showing ................................. 18 section of $\ldots \ldots \ldots \ldots \ldots \ldots \ldots \ldots \ldots \ldots \ldots \ldots, 22$ Cretaceous sand, view of .......................... 22 Crosby, W. O., sizing and filtration tests by . . . . . . . 338-360

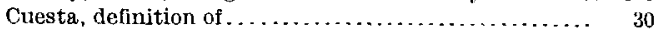
diagram showing $\ldots \ldots \ldots \ldots \ldots \ldots \ldots \ldots \ldots \ldots, \quad 29$ Cutting Creek, weir on, view of . . .............. 378 Dams, effect of, on ground water................ $62,73-74$ seepage from............................ 106-1 10

Darton, N. H., on deflection of rivers in Hightstown Vale ............................ 31,32

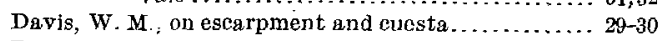
Douglaston, well at, effect of tide on............. 7: well at, effect of tide on, diagram showing........ 71

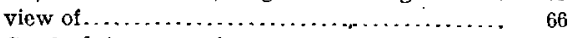
Doxsee Creek, data concerning . . . . . . . . . . . . . Drainage of North Atlantic coastal plain, develop-

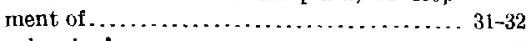
diagram showing $\ldots \ldots \ldots \ldots \ldots \ldots \ldots \ldots \ldots \ldots \ldots \ldots, 3_{2}$ Drillers of weils, list of ....................... 116-117 Dunton, well at, diagram of . ................... . 213 East Meadow Brook, data concerning . ........... 368-369 underflow at, figure showing ................. 101 East Meadow Pond, seepage from ................ 107-108 Easthampton Water Co., system of, data concerning. . 84-85, $166-167,33 \overline{5}$ water system of, map showing...... Pl. $\mathrm{xdx}$, in pocket. Electrolyte, spreading of, figures showing .......... 93,94 


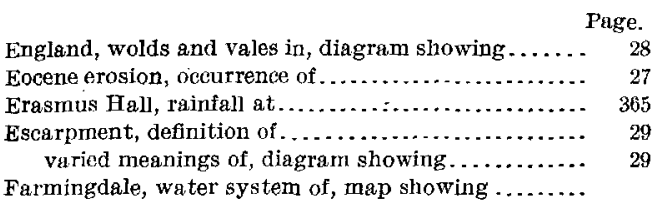

Pl. xix, in pocket.

Filtration tests of difierent sands, results of ....... 354-360 Flatbush Waterworks Co., system of, data concerning...................... 80-81, 118-119

water system of, map showing...... Pl. xix, in pocket.

Fleischman Manufacturing Co., wells of, location of, map showing.

Flushing, water system of, data concerning .......... $80-81$ water system of map showing . . ... Pl. xix, in pocket.

Folding, occurrence and cause of................. 37-40

Fordham gneiss, occurrence and character of ........ 16

F reeport, underflow measurements near............ 86 water system of, map showing ...... Pl. xix, in pocket. wa terworks of, data concerning......... 82-83,142-143 Garden City Water Supply Co., system of, data concerning ........... 82-83, 142-143, 254

Gardiner crosion, occurrence and character of ........ 40

Gardiners Is]and, erosion on .................. 40 sections of, diagrams showing. ................ 37,39

Gay Head, folding at, character and cause of . ....... 37-40 section at, diagram showing................. 39

Geologic history of Long Island.................. 48-50 Geology, outlines of ....................... $15-52$

German-American Improvement Co., waterworks of data concerning . .......... 80-81, 126-127, 189

Glaciation on Long Island, conditions of ....... 33-48, 50-52 Glen Cove, water systems of, map showing ..........

Pl. xix, in pocket.

Grand avenue and Newbridge Brook station, underflow at, diagram showing................

Great South Bay Water Co. water syster concerning.......... 82-85, 154-155, 160-161,304 water system of, map showing....... Pl. $x i x$, in pocket. Greenport, water system of, data concerning....... 84-85, $166-167,331-332$

water system of, map showing. P1. $x i x$, in pocket. Ground-water table. See Water table.

Iarbor Hill glacier, moraine and outwash plain of, views of.

Harbor Hill stage, position of ice during, map showing.

Hempstead, watersystem of, data concerning. . 82-83, 142-143 water system of, map showing....... Pl. xix, in pocket.

Hempstead Brook, flow of ...................... 58-58 now of, increase of, map showing .............. 59 Hempstead reservoir, discharge of................. 62 Hicksville, water system of, map showing. P1. xix, in pocket.

Hightstown Vale, deflection of rivers in. ........... 31-32 deflection of rivers in, figure showing ........... 32 map showing.....

location and character of . section showing.

Hollick, A., on Iong Island geology ............. 16,48 Holliswood, water system of, data concerning ....... 80-81 water system of, map showing....... Pl xix, in pocket.

Horton, R. F., on surface streams of Long Island... 361-385 Huntington, outwash plain near, view of...

Huntington Water Works Co:, water system of, dat concerning............. 82-83,154-155, 299-300 water system of, map showing....... Pl. $x i x$, in pocket. Ice sheet, folding due to . . . . . . . . . . . . . . . . . . $39-40$

Islip, gaging station at, view of ................ 367 gagings near . . . . . . . . . . . . . . . . . l’ape.

Islip, water system of, map showing..... Pl. xix, in pocket. Jamaica Water Supply Co, water system of, data concerning ................. 80-81,132-133,210-211 wate $r$ system of, map showing........ Pl. xix, in pocket. Jameco gravel, artesian area of, map showing.......... 66 deposition and occurrence of................. $34,35,55$ position of, diagrams showing ............... 34-39,56 water supply in ........................ $55-56,64-65$ wells to . . . . . . . . . . . . Jones well, Cold Spring Irarbor, view of .............. 64 King's sand pit, view in ......................... 40 Knapp, G. W., on Long Island Miocene. . . . . . . . . . 25 Lafayette submergence, occurrence of ................. 28 Lakes, effect of, on ground water................ 106-110 occurrence and causes of . . . . . . . . . . . . . . . 61-63

Landslip phenomena, view of. .................. 38 Lindenhurst, fire department wells of, data concern-

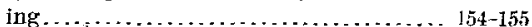
fire department wells of, map showing. $\mathrm{Pl}$. $\mathrm{xix}$, in pocket. Lloyd sand, depth to, maps showing ............. 18,68 position and importance of ............. 19,23,2ti,6; 67 diagram showing........................ 56 water of, analyses of . . . . . . . . . . . . . . . . . . $68-69$ water supply in $\ldots \ldots \ldots \ldots \ldots \ldots \ldots \ldots \ldots \ldots \ldots \ldots \ldots+6,67$ wells to ..................................... $65-66$ Long Beach, well at, relation of tide and, figure showing ............................. 70

Long Beach Association, waterworks of, dataconcerning . . . . . . . . . . . . . . . . . Long Island City, water system of, data regarding . . 78-79 map showing.................. Pl. xix, in pocket. Long Island City pumping station No. B, borings at, diagram showing ..................... 188

Mackay, C. H., waterworks of, data concerning. ....... $82-83$ water system of, map showing ....... IJ. xix, in pocket. McAlpine, W. T., stream measurements by .......... 365 McGee, W. J., on deflection of rivers in Hightstown Vale. 31-32 Manhasset, well at, figure showing............... 64 Manhasset bowlder bed, view of .................. 40 Manhasset gravel. See Tishury gravel.

Manhanset Fouse, water system of, data concerning. 84-85, $164-165,331$ water system of, map showing...... Pl. xix, in pocket. Manhattan Island, sections of, figure showing....... 17 Mannetto gravel, deposition and distribution of . . . . . 33-34

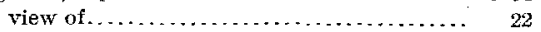

Map showing Cretaceous artesian well area........... 86 showing depth of Lloyd sand .................. $\quad 68$ showing distribution of Cretaceous......... 18,20,68 showing distribution of Miocene ................ 27 showing distribution of water-power development. $\quad 60$ showing defection of streams................. 32 showing development of drinage on North Atlantic coastal plain.................. showing increase of flow of Hempstend Brook.... showing Jameco artesian-well area : ............... showing location of borings for Pennsylvania, New York and Long Island Railroad..... 182, 184, I86 showing location of underlow stations..........' 87 $91,92,99-103,105-114$ showing loeation of wells............ 180,217,223,281 $\mathrm{Pl}$, xix, in pocket. showing north shore artesian-well area........... 66 showing position of bed rock.................. 16 showing position of ground-water table..............

Pl. xix, in pocket. showing rela tive position of ice at different stages.. 44 showing structure of Cretaceous beds........... 18, 19 
Māp showing waterworks srstems of Lōng Island....

Pl. xix, in pocket.

Marl series of New Jersey, occurrence and character of .

Marthas Vineyard, section of, diagram showing ...... 39

Massapequa, underflow measurements near . . . . . . 86,95-96

Massapequa Creek, data concerning . ............... 371-373

Matawan formation, oceurrence and character of ..... 22

Mather, W. W., figure cited from................. 39

Helville, N. $Y_{.}$, section near .................... 20

views of Cretaceous and Manetto deposits near...

Merrick pumping station. underflow at, figure showing-

Merrick Water Co., water system of, data concern-

$$
\text { ing.................... } 82-83,146-147,273
$$

water system of, map showing....... Pl. xix, in pocket.

Merrill, F. J. II, on geology of Long Island.

Meter, direct-reading, description and use of . . . . . . . . . 90-97

use of figure showing ....................... 92

view of.

Meter, self-recording, charts of, view of ...............

clock for, views of $\ldots \ldots \ldots \ldots \ldots \ldots \ldots \ldots \ldots \ldots, \ldots 2,98$

description and use of . . . . . . . . . . . . . . . . . 97-99

view of..

Meters, types of for underflow measurements....... 90

Miocene rocks, distribution of . . . . . . 27-28

distribution of, map showing ................. 27

Miocene sulmergence, occurrence of .

Montauk Water Co., water system of data concerning. 80-81, $132-133,213-214$

well of, figure of $\ldots \ldots \ldots \ldots \ldots \ldots \ldots \ldots \ldots \ldots . .213$ water system of, map showing ....... Pl. xix, in pocket.

Mountain Mist Springs, character of...............

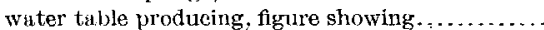

Nash, L.S., stream measurements by...

Nassau County Water Co., water system of, data concerning.... 82-83, $144-145,148-151,262-276,279-280$

New Jorsey, Cretaceous rocks of, position of, map

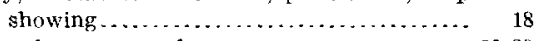

sections of $\ldots \ldots \ldots \ldots \ldots \ldots \ldots \ldots \ldots \ldots \ldots \ldots \ldots, 22,30$

location of map showing...

Newbridge Brook, underflow at, figure illustrating..... 105

Newbridge streams, data concerning............. $\quad 370$

Newtown, water system of, map showing. .Pl. xix, in pocket.

New York City department of water supply, water systens of ........................ $70-81$ water system of, map showing ........... xix, in pocket. wells of, index to ................................ 393

New York City commission on additional water supply, acknowledgements to............ 87,116 maps and diagrams from........ $70, \mathrm{Pl}$. xix, in pocket. test wells of, index to . . . . . . . . . . . . . . . . . 391

North shore, artesian area of, map of.............. 66 valleys of, origin of $\ldots \ldots \ldots \ldots \ldots \ldots \ldots \ldots \ldots, 43-44$ wells on, views of.

Northport Water. Works Co., water system of, data concerning............... 82-83,154-155, 300-301 water system of, map showing ....... Pl. xix, in pocket.

Oak Park, water system of, map showing . Pl. xix, in poeket

Orowoc creek, data concerning ................ 376-377 gaging station on, figure of . . . . . . . . . . . . . . . 367

Oyster Bay, water system of, map showing. Pl, xix,in pocket. well at, view of.

wells at, location of, map showing.

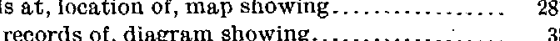

Patchogue, water system of, map showing. Pl. xix, in pocket. Pennsylvania, New York and Long Island Railroad, borings of, maps and diagrams showing. $182,184,186$

Perrineville wold, location and character of ......... 31 sections showing......................... 30
Co of, data concerning ..................... 80-81, 118-119, 169 Pleistocene time, glaciation in . . . . . . . . . . . . $33-48$ Pliocene erósion, occurrence of.................... 28 Ponds, effect of, on ground water..........62-63, 106-110 effect of, on ground water, figure showing......... 62 leakage from, figure showing . . . . . . . . . . . . . . 62

occurrence and cause of , ....................... $61-03$ Porosity. See Filtration tests.

Port Jefferson Water Co., water system of, data concerning............... 84-85,160 161,319-320

Pratt estate, water system of, data concerning _._. 82-83, $144-145,264-265$ water system of, map showing...... Pl. xix, in pocket. Pumping, effect of, on ground water......... 73-74,111-114 effect of, on ground water, figure showing ........ 72 Pumping stations, underflow at, diagrams showing. . 98-103,

Quantuck Water Co., water system of, data concerning ...................... 84-85, 164-165,327 Quaternary time glaciation in .............. 33-48,49-52 Queens Borough, waterworks of, data concerning... $78-81$ water system of, map showing...... Pl. xix, in pocket. Queens County Water Co., water system of, data concerning............... 82-83,136-13i, 224-226 water system of, map showing...... Pl, xix, in pocket. wells of, location of, map showing ............. 223 sections of, figure, showing ................. 225 Quogue, water system of, map showing.. Pl. xix, in pocket. Ruinfall, effect of, on ground water ........ 69-71,104-106 Rit nfall in 1903, data of . . . . . . . . . . . . . . . . . . $415-417$ Rapid transit commission, borings of, diagrams showing $\ldots \ldots \ldots \ldots \ldots \ldots \ldots \ldots \ldots \ldots \ldots, 170,172$ Raritan formation, age of .................... $25-26$ occurrence and character of ............ 22 Rathbun, $F, D$., record of well fuctuations furnished by ............................... 70 Recent time, submergence in................... 48 Reservoirs, data concerning . . ................. $76-85$ Riverhead waterworks, water system of, data concerning................... 84-85, 164-165,327-328 water system of, map showing ...... Pl. xix, in pocket. Rockaway Ridge, folding at, section showing........ 36 Rockville Center, water system of, data concerning . . 82-83, $140-41,250$

water system of, map showing...... . Pl. xix, in pocket. Ronkonkoma, Lake, character of ............63,379-380 character of, figure showing .................... 63 Ronkonkoma stage, position of ice in, map showing... 44 Sag Harbor Water Co., water system of, data concerning ..................... 84-85, 166-167, 334 water system of, map showing...... Pl. xix, in pocket. Sal ammoniac, use of . . . . . . . . . . . . . . . . . . . . 96-97 Salisbury, R. D., on New Jersey geology ........... 16,21 Sampawams Creek, data concerning............. 375-376 San Gabriel River, Cal., underflow measurements on, figure illustrating.................... . 91

Sand grains, effective size of definition of filtration tests with different sizes of . . . . . . . . . $354-360$ sizíng tests of ............................ $338-353$ Sand spit, view of .............................. Sankaty formation, deposition, character, and distribution of .......................... $36-37$ position of, diagrams showing .................... $34-39$ Sayville, water system of, map showing. Pl. xix, in pocket. Seacliff Water Co., water system of, data concerning. 82-83, $144-145,262$

water system of, map showing ..... Pl, xix, in pocket. 


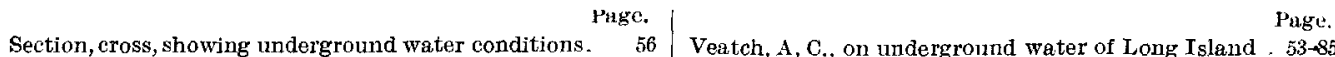
Section, generalized, of pre-pleistocene rocks . . Sections, geologic, of Long Island............. 20,33,35, diagrams showing ........... 17,34,35,36,37,38,39,56 Sections, geologic, of Long Island and New Jersey, location of, diagram showing...............

Setauket, structure near, diagram showing......... Shelter Island Heights Association, water system of, data concerning ........ 84-85, 166-167 water system of, map showing...... Pl. xix, in pocket. Slichter, C. S., on velocity of underflow on Long Island. 86-115 Smithtown Harbor, sand spit at, view of ........... 52 Southampton Watcr Co., water system of, data concerning ................. 84-85, 164-165,329 water system of, map showing..... Pl. xix, in pocket.

Springs, discharge of . . . . . . . . . . . origin of . . . . . . . . . . . . . . . . . . . . . . . . . $58-59$ Springs, mineral, occurrence of ................. 59 Steinway and Son, water system of, data concerning. 82-83, water system of, map showing..... . Pl. xix, in pocket. Stockbridge dolomite, occurrence and character of... 16 cross section showing............ 17

Streams, channels of, analogy of wclls and.......... 58 character of .................... $60,361-362,368-383$ measurements of. .................... 58-59,365-383 occurrence of. . . . . . . . . . . . . . . . . . . . .60,361-383 origin of . . . . . . . profile of, ideal........................... 362 utilization of ...................60-61,74,76-78,84,362

Success, Lake, character of ................... character of, figure showing ...................

Temperature, effect of, on ground water........... 72 effect of, on ground water, diagram showing..... 72

Tertiary rocks, section of...................... 20-21

Tertiary rocks of New Jersey, comparison of Long Island Tertiary and .................. 21-25 section of .............................. 21

Tertiary time, history of, on Long Island.......... 26-32

Test wells for underflow measurements, description of . ............................... $88-90$ plan of, figure illustrating....

Texas bars, theoretical deflection of rivers by ........... theoretical deflection by, figure showing........ 32

Tides, effect of, on ground water............... $71-72$ effect of, on ground water, diagrams showing . . $64,70,71$

Tisbury gravel, deposition and occurrence of . . . . . . 41-43 position of, diagrams showing............... 34,38

Tobacco Point, section at, diagram showing....... $\quad 37$

Topography, development of . . . . . . . . . 28 28 $32,46-48,50-52$ sketch of

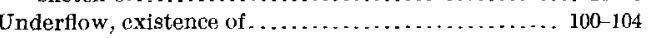
measurements of........................... $86-104$ figure illustrating.

velocity of . ................... 92-94,86-88, 104-113 figures jllustrating............ 91-92,98-103,105-113

Underflow apparatus, character of . . . . . . . . . . 88-98 principles involved.in........................ 99 views of ............................ $90,92,98,100$ Underflow stations, location of, maps showing ...... 87,91 ,

Underground water. See Water, underground.

Vale, definition of ............................ 29 diggrams of . . . . . . . . . . . . . . . . . . . . . . . . . 28-30

occurrence of. . . . . . . . . . . . . . . . . . . . . 28 32

Veatch, A. C., on geology of Long Island............ 15-52

Veatch, $c$ and Bowman, Isaiah, well records by . 116-33?

Vincyard interval, character of .................. 43-44 Wantagh Fond, seepage from.................. 108-110 underflow near figure illustrating . _ 109-112 Wantagh pumping stations, location of, figures showing............... $87,114, \mathrm{Pl}$. xix, in pocket. underflow at, diagran illustrating . ............ 98 Wantagh streams, data concerning . . . . . . . . . . . 370-371 Ward, L. F., on Island series....................

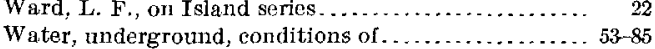
underground, general principles of . ............ 53-55 geologic conditions of ................... $55-50$ source of . . . . . . . . . . transmission of . . . . . . . . . . . . . . . . . $53-54$

Water, well, analyses of. See Analyses of well waters.

Water powers, development of.............. $60-61,362$ distribution of, map showing. . . . . . . . . . . . . . . 60

Water table, definition of ......................... 54

fluctuations of . figures showing ....................... 70,72 perched type of . . . . . . . . . . . . . . . . . . $57-\tilde{n} 8,61-62$ figures showing . ....................... $56-58,61$ position of . . . . . . . . . . . . . . . . . . . . $57-59$ figures showing . . . . . . . . . . $54,56,57,58,61-63,70$ map showing................ Pl. xix, in pocket. springs dependent on.................... 58-59

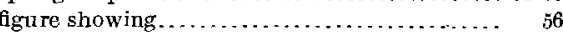
Waterworks, data concerning . . . . . . . . . . . . . . . 74-85 map showing................... Pl. xix, in pocket. Woll drillers, list of those assisting. . . . . . . . . . . 116-117 Well owners, list of ...................... 391-394 Wells, analogy of dcep-cut channels and, diagram showing ............................. 58

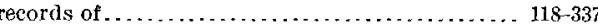

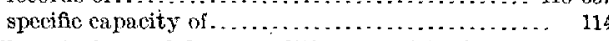
Wells, artesian and deep, conditions requisite for .. 54-55,67 artesian and deep, distribution of ............. $63-67$ location of, maps showing. - 70-72, 180, Pl. XIx, in pocket.

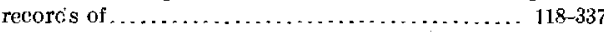
views of waters of anulyses of. See Analyses.

Wells, blowing, occurrence of .................. 74

Wells in Connecticut, water of, analyses of ............. $68-69$

West Hills, section in strata iл, views showing...................... 22 water conditions in, diagram showing............ 57

White, David, on Raritan formation ................

Whitestone, water system of data regarding........ 80-81 water system of, map showing....... Pl. XIX, in pocket.

Whitlock, Artemus, stream measurements by ....... 360

Whitney, F. L., record of well fluctuations furnished by ............................, 71

Wisconsin deposits, deposition and occurrence of . . . . 44 48

Wolds, definition of . . . . . . . . . . . . . . . . . . . .

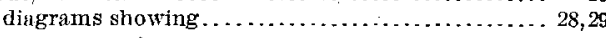
occurrence of. ........................... 30,31

Woodhaven Water Supply Co., water system of, data concerning ................ 80-81,126-127, 192 water system of, map showing....... PI. xix, in pocket.

Woodside Water Co., water system of, data concorning ...................... 80-81,128-131,194 water system of, map showing...... Pl. xIx, in pocket. Woodworth, J. B., on Tisbury gravel............ 37,41 figure cited from . .

Woolman, Lewis, on wells and geology of Long Island and New Jersey..................... 23-24 


\section{INDEX OF WELL DATA.}

(By names of owners.)

[For wells by locality, see map (PI. XXIV, in pocket), from which numbers used in tables and notes, pp. 123-368, can be obtained.]

\begin{tabular}{|c|c|c|}
\hline & & Page. \\
\hline Abrames, Jirde. ............ 13 & Breslau fire department ....... 154 & College Point. See New \\
\hline diagram showing ........... & B ridgehampton ............... & department of \\
\hline Ackerly, Hiram .............. 154-155 & Brightson, G. E ..... & snpply (Queens Borough). \\
\hline Adams, Maude. . . . . . . . . . 154-155 & Brookhaven ................ 162-163 & Collier, Richard... \\
\hline Albertson, J. A......... 142-143, 256 & Brooklyn Borough Gas Co..... 118-119 & Colonial springs. ... . \\
\hline Allard \& MeGuirc. . . . 148-149, 278-279 & Brooklyn department of water & Columbia farm.......... \\
\hline Amagansett $\ldots \ldots \ldots \ldots \ldots \ldots \ldots, 160-167$ & supply, See New York City & Commack ................ 156-157 \\
\hline $\begin{array}{l}\text { American Cordage and Manufac- } \\
\text { turing Co } \ldots \ldots \ldots \ldots \ldots \ldots, 122-123\end{array}$ & $\begin{array}{l}\text { water supply } \\
\text { augh). }\end{array}$ & $\begin{array}{l}\text { Commission on additional water } \\
\text { supply. See New York City. }\end{array}$ \\
\hline American Hard Rubber Co. $130-131,198$ & lyn Rapid Transit Co. 118-119, 168 & Congress Brewing Co . . . . . . 120-121 \\
\hline $83,150,151,287$ & Brooklyn sewer department.... 118- & Conklin, Fred.......... \\
\hline .. $138-139,242$ & 119,168 & Conklin, R. B...... \\
\hline & Brooklyn Union Gas Co....... 118- & Consolidated Gas Co...... 1 \\
\hline kle Brothers....... 120-121,175 & $123,172,177,178$ & Consolidated Ice Co.... 64, 15 \\
\hline $\begin{array}{r}\text { Army, U. S........... 118-119,134- } \\
135,146-147,166-169,220,275,336\end{array}$ & $\begin{array}{l}\text { Brooklyn waterworks. See New } \\
\text { York City department of water }\end{array}$ & $\begin{array}{l}\text { Consumers Hygeia Ice Co.. } 12 \\
\text { Cottnet, R. L......... 142-14 }\end{array}$ \\
\hline$\ldots \ldots \ldots \ldots 134-135,222$ & & Cox, Irving . . . . . . 21,66, ] \\
\hline 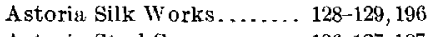 & el........ 134-135, 224 & obert . . . . . . 140-1 \\
\hline$\ldots \ldots \ldots 126-127,187$ & $n \ldots \ldots \ldots \ldots \ldots .134-135$ & T. $\ldots \ldots \ldots \ldots 19,1$ \\
\hline $\begin{array}{r}\text { Babylon Sumpwams Water Co . 82-83, } \\
154-155,303-304\end{array}$ & $\begin{array}{l}\ldots \ldots \ldots \ldots \ldots \ldots 156-157 \\
\ldots \ldots \ldots \ldots \ldots 160-161,320\end{array}$ & $\begin{array}{l}\text { Coyson, A. \& s. B...... } 1 \\
\text { Cravath, P. D......... }\end{array}$ \\
\hline$A, \ldots \ldots \ldots \ldots \ldots \ldots, 162-163$ & as............ 16 & Crescent Chemical Co........ 118-119 \\
\hline$\ldots 134-13$ & $\ldots \ldots \ldots 12$ & Crystal Springs Ice Co.......... \\
\hline rt........... 134 & $\ldots \ldots \ldots 150-15$ & $144-145,264,285$ \\
\hline$j x \ldots \ldots .146-147$ & $144-14$ & $-159,313$ \\
\hline & & $1 \ldots \ldots \ldots \ldots, 160$ \\
\hline Co. $118-119,169$ & $146-14$ & ton; J. H......... 162-163,324 \\
\hline$\ldots \ldots \ldots 162-163$ & $\operatorname{stin} \ldots \ldots \ldots \ldots .15$ & 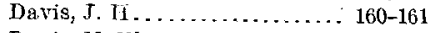 \\
\hline $\begin{array}{l}\text { ee New York City de- } \\
\text { of water supply } \\
\text { orough). }\end{array}$ & $\begin{array}{l}\ldots \ldots \ldots \ldots 160-16 \\
\ldots \ldots \ldots 130-131,19 \\
\text { tery } \ldots \ldots \ldots 122-12\end{array}$ & 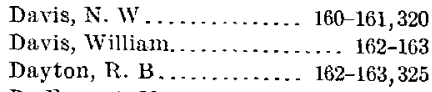 \\
\hline$\ldots \ldots \ldots \ldots \ldots, 164-165,330$ & $\ldots \ldots \ldots \ldots \ldots \ldots 162-163$ & Henry ........ \\
\hline $\mathrm{C} \ldots \ldots \ldots \ldots \ldots 146$ & $\ldots \ldots \ldots 154-15$ & ... $152-153,293,29$ \\
\hline$\ldots \ldots \ldots \ldots \ldots \ldots 1^{152-153,}$ & $\ldots \ldots \ldots 154-1$ & rs. $-\ldots \ldots \ldots$. 10 \\
\hline Dr. $\ldots \ldots \ldots 166-167,334$ & $\ldots \ldots \ldots \ldots \ldots, 162-1$ & M..... \\
\hline taries.......... $158-159,315$ & $\ldots \ldots \ldots \ldots \ldots 130-1$ & 3,290 \\
\hline$\ldots \ldots \ldots \ldots \ldots \ldots, 146$ & $0 . \ldots \ldots .132-1$ & Dedr \\
\hline Frank........... 144-1 & & 3,295 \\
\hline$\ldots \ldots \ldots 21,154-155,302-303$ & .... 160 & 29,196 \\
\hline Charles.... 126-127, 187,198 & n.e. & $23-224$ \\
\hline$\ldots \ldots \ldots \ldots 160-161$ & nhasset Hil & 44-245 \\
\hline$\ldots \ldots \ldots \ldots 152$ & $\ldots \ldots \ldots \ldots 120-121$ & $\ldots 150-151,284$ \\
\hline gh, Charles ... 156-157 & Citizens Water Supply Co ...... 71. & $\ldots \ldots 164-166,328$ \\
\hline Blythbourne Water Co...$\ldots \ldots 80$ & $\begin{array}{r}80-81,128-129,132-133 \\
138-139,194,195,214,238\end{array}$ & $\begin{array}{l}\text { Dryer, }-\ldots \ldots \ldots \ldots \ldots \ldots \ldots \text { 160-161 } \\
\text { Dryfuss \& Nibbe......... 150-151,288 }\end{array}$ \\
\hline & & . $152-153,295,320$ \\
\hline $\mathrm{Co}_{0}$ & & $\ldots 158-159,312$ \\
\hline$\ldots \ldots \ldots 72,152-1$ & $\ldots \ldots \ldots \ldots 160-161,318$ & $, F, W \ldots \ldots \ldots \ldots \ldots, 132-133$ \\
\hline$\ldots \ldots \ldots \ldots, 128-129,194$ & $1-\ldots \ldots \ldots 156-157,304$ & Duryea, . .T. T3..... 67, 142-143,256-257 \\
\hline & $\ldots \ldots \ldots 158-159,314,324$ & diagram skowing......... 58 \\
\hline owen, James........ 152-153, 293-294 & M. II . . . . . 152-153, 296 & Eagle Dock........... 152-153,293,318 \\
\hline $\begin{array}{l}\text { owery Bay Building and } \mathrm{Im}-\text {. } \\
\text { provement Co..... } 82-83,130-131,197\end{array}$ & $\begin{array}{l}\text { Cockran, Bourke.......... } 140-141,245 \\
\text { Cold Spring Creamery } \ldots \ldots .152-153,290\end{array}$ & $\begin{array}{r}\text { East Marion life-saving station.., } 166- \\
167,333\end{array}$ \\
\hline rady, J. F . . . . . . . . 142-143, 255 & Cold spring Hatchery........ 152-153 & $-166-167$ \\
\hline ragnaw estate........... 124-125, 183 & xter $\ldots \ldots \ldots \ldots \ldots \ldots$ 154-155, 301 & East River Gas Co........ \\
\hline rentwood . . . . . . . . . . 156-158, 307 & $\ldots \ldots .134-135,220$ & Eastern Brewing Co..... \\
\hline
\end{tabular}




\begin{tabular}{|c|c|c|}
\hline & & Kasteard $T$ \\
\hline $\begin{array}{l}\text { Easthampton Home Water Co.. 84-85, } \\
\qquad 166-167,335\end{array}$ & $\begin{array}{l}\text { Hallock, F. G............. 158-159 } \\
\text { Hallock, Willigin......... } 162-163,323\end{array}$ & eene, Foxhall...... \\
\hline Edison, Charles . ...... 142-143,254,274 & Hallock \& Small ........ 20,164-165, 326 & Eeene, James . . . . . . . . \\
\hline Slliott, $J \ldots \ldots \ldots \ldots \ldots \ldots$ 154-155,299 & Hamilton, $-\ldots \ldots \ldots \ldots, 150-151,284$ & Keil, Charles........ \\
\hline Emerson, Dr. — . . . . 160-161,318 & Hamilton, J. F : . . . . . . 138 139,241 & $150-151,288-289$ \\
\hline Emken Chemical Co ......... 124-125 & Iamilton, W. J.......... 138-139,240 & ..... 142-143, 255 \\
\hline inmett, D . . . . . . . 19, 158-159,313 & Iarek, Rudolph... . . . 124-125, 186-187 & $74,148-149,279$ \\
\hline Impire Oil Refinery . . . . . . . . 122-123 & $\therefore \quad 150-151,289$ & $\ldots 158-159,309$ \\
\hline pping, Joseph . . . . . . . . . . . 120 121 & Dr. & Ty \\
\hline rland, George, sr........ 158-159,314 & an, J.H. . ........ 148-149,277 & 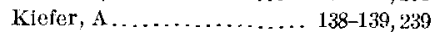 \\
\hline xcelsior Brewing Co ..... 120-121,173 & rge $\ldots \ldots \ldots \ldots \ldots \ldots 162-163$ & 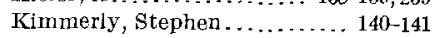 \\
\hline Vatch Case Co..... $1666-167,334$ & $\ldots \ldots \ldots \ldots \ldots \ldots, 158-159,310$ & \\
\hline$\ldots \ldots \ldots \ldots \ldots 150-151,2$ & ( & Tany $\mathrm{F}$ \\
\hline nde........ 148 & 21 & \\
\hline$\& W \ldots 146-147,337,368$ & IIst ....... 15 & $\mathrm{~h}, \mathrm{~F} . \mathrm{H}, \mathrm{Co} . . . . .$. \\
\hline Fatbush Waterworks Co . . ... 80-81, & (1) & \\
\hline & (1) & an \\
\hline Fleishman Manufacturing Co. 122- & & i \\
\hline & & $1414=0$ \\
\hline$-\ldots \ldots \ldots .126-127,187$ & ring .......... $\quad 36$ & $-119,172$ \\
\hline G. M...... $66,150-151,285$ & Co... 82-83, 142-143 & $162-163$ \\
\hline [rs. Julia . . . . . . 136-137,227 & 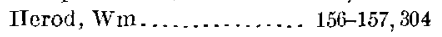 & Kruger, \\
\hline tate. . . . . . . 122-123, 181 & tter............ & T 1 \\
\hline See New York City & & \\
\hline & & \\
\hline & & \\
\hline$\dot{y} \ldots \ldots \ldots \ldots \ldots .128-129$ & म) & ............. \\
\hline . $\ldots \ldots \ldots \ldots 160-16$ & & $0 \ldots \ldots \ldots$ \\
\hline works. .. 82-83, 142-143 & rewing $\mathrm{Co}$. & hn........... \\
\hline$-\ldots \ldots \ldots \ldots \ldots 138-139$ & 0.10010 & e Beach Bathing $A$ \\
\hline $\operatorname{demy} \ldots \ldots .144-145,262-263$ & te..... $66,150-1$ & 35,222 \\
\hline & showing ......... $\quad 38$ & \\
\hline $1 k \ldots \ldots \ldots 124-125,185$ & $y \ldots \ldots \ldots \ldots \ldots 138-139$ & 283 \\
\hline ust . . . . . . . $160-161,317$ & s..... 118-110 & C. F $\ldots \ldots \ldots$ \\
\hline F. . . & ......... 124 & dieu's, J.IT., Sons $138-139,241$ \\
\hline$\ldots \ldots \ldots \ldots \ldots 154-155,299$ & $0 \ldots \ldots \ldots 154-155$ & m showing ........... 64 \\
\hline & ght and Power Co_ 154 & Liebmann, S., Sons Brewing Co. \\
\hline & & \\
\hline & Huntington Water Works & 年 \\
\hline $66,146-147,26$ & 15 & Long Beach Associution......... \\
\hline$\ldots \ldots \ldots 14$ & 04 & \\
\hline$\ldots \ldots \ldots .12$ & $\ldots \ldots \ldots, 1$ & 119 , \\
\hline vement & 1.s... 1 & 148 \\
\hline 189 & & 183 , \\
\hline 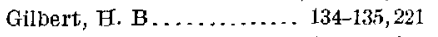 & 1 & 303 \\
\hline$\ldots \ldots \ldots 154-15$ & $\mathrm{Co} \ldots \mathrm{I}$ & \\
\hline a & ........ 1 & -153 \\
\hline$-\ldots \ldots \ldots \ldots 156-15$ & (n) & $158-159,336$ \\
\hline Sons........ 122-12 & & $D \ldots \ldots \ldots$ \\
\hline$\ldots \ldots \ldots \ldots \ldots 16$ & &,$-\ldots . .$. \\
\hline$\ldots \ldots \ldots \ldots$ & $\ldots \ldots 1$ & 281 \\
\hline ald $\ldots \ldots \ldots \ldots, 16$ & $\ldots \ldots \ldots 13$ & -165 \\
\hline n........... & aica Water Supply Co...... 80 & , Henry .... \\
\hline & & 300 \\
\hline$\ldots \ldots \ldots \ldots .138-1$ & diagram showing ..... & rs. S. F. . \\
\hline . & $\ldots \ldots$ & Iter.......... \\
\hline $01 \ldots \ldots \ldots .138-139,242$ & and Machine & (................. \\
\hline ay Water Co.... 82-85, & & $\mathrm{H} \ldots \ldots$ \\
\hline $154-155,160-161,304-306$ & & .. $66,150-151,285$ \\
\hline aterworks....... 84 & $\ldots \ldots \ldots 152-153,291$ & ram showing. \\
\hline $85,166-167 ; 331-332$ & $-153,292$ & $\ldots 126-127,187$ \\
\hline$\ldots$... 134-135, 221-222 & . & 5,328 \\
\hline _. . . . . . 162-163, 321 & Jones, $0, \mathrm{~L} \ldots \ldots \ldots \ldots \ldots \ldots$ 65, & Is Coal Co ...... 134-15 \\
\hline D. . $66,146-147,266,267,268$ & $66,148,155,282,286,297,302$ & srewing $00 . \ldots \ldots \ldots$ \\
\hline $\mathrm{E} \ldots \ldots \ldots \ldots 162-163,321$ & diagram showing ........... 38 & ............ $118-119,10$ \\
\hline$\ldots \ldots 20,164-165,326-327$ & ........... 152-153,291 & ard ............ 134-135, 223 \\
\hline & & House ... 84-85, 164-165, 331 \\
\hline A........ 154-15 & $\ldots \ldots 120-121.175$ & n Beach Hotel. ...... 118-119 \\
\hline
\end{tabular}


Page.

Manhattan State Hospital. ... 156-157 Marsh, Mrs. A. W........ 152-153, 297 Marsh, Theodore............ 160-161 Martin, J. E.............. 134-135, 221 Mason, C. H.............. 140-141, 244 Massapequa IIotel ............. 150-151 Masury, J. W., \& Son..... 120-121, 175 Matherson, W. T. ........ 158-159, 309 Matherson, W. T. \& Co . 124-125, 185-186 Mattiluck.................. 164-165 Melville, Frank, jr........... 160-161 Morger \& Thrall . . . . . . . . 120-121, 174 Merrick Water Co.... 82-83, 146-147, 273 Metzner, M. A ................ 158-159 Miller, Mary ................ 162-163 Milliken Bros................. 118-119 Mineola Court-house............ 142-143 Minniken, John . . ......... 144-145, 264 Mohannes Casino ........ 150-151, 283 Mo-Mo-Ne Spring............ 298-299 Monecke, Dr. _ ......... 158-159, 312 Monfort, H. A . . . . . . . . . 152-15̃3, 290 Montauk Brewing Co ..... 128-129, 193 Montauk Water Co............ 80-81, 132-133, 213-214

Morgan, Charles _ f......138-130 Morgan, E. D . ....667, 142-143, 257-250 diagram showing ........... 58

Morrell, - . . . . . . . . . . . . 154-155

Morris, J. K............. 166-167, 334

Morrison, D. G............ 124-125

Morrissey, John......... 158-159, 312

Mortimer, Stanley . . . . . . . . 144-145, 250

Moss, D. B........... 154-155, 301,327

Mountain Mist Springs . ... 152-153, 291

Muncie, E. H. ........... 154-155, 303

Nassau County poor farm : 148-149, 279 Nassau County Water Co ...... 82-83, $144-145,148-151,262,276,279-280$

Nassau Electric Light and Power

Co ................... 144-145, 260 Nassau Oyster Co.......... 160-j61,316 Navy, U. S. . . . . . . . . 120-121, 176-177 Neptune Consumers Ice Co...... 122 $123,178-179$ Nevins, Fred ............ 154-155, 301 New Calvary Cemetery . .... 128-129, 195 New York and Queens County Railroad . ............. 128-129, 195 New York Architectural Terra Cotta Co .............. 124-125, 18 New York Asbestos Co.... 124-125, 186 New York-Brooklyn Rapid Transit Co ............... 118-119, 169

New York City commission on additional water supply . ... 126-151, 150-161, 187, 193-198, 209, 211-220, $235-241,243,249-255,261-262,273-$ $277,279,28 \bar{\gamma}-289,24 \overline{7}-298,306-308$, $310,312-313,316-317,319,339-360$ New York City department of watersupply (Brooklyn Borough)

Agewatn.... i8 - $79,146-147,269-270$ Baisleys......... $76-7 \overline{7}, 130-\mathrm{j} 31,203$ diagram showing........ 34

Clear Stream..... $76-\overline{7} 7,136-13 \overline{7}, 288$
New York City, department of water supply (Brooklyn Borough): Forest Stream....... 76-77, $136-137,233-234$ diagram showing ........ 34 Freeport ......... 146-147, 270-271 Gravesend ....... 76-77, 118-119,169 Jameco...... $76-77,130-131,204-206$ diagram showing ........ 34 Massapequa..... 78-79.150-151,287 Matowa........ 78-79, 146-147,273 Merrick ..... 78-79, 146-147, 271-273 New Lots ....... 76-77, 126-127, 18? diagram showing.

New Utrecht - $76-77,118-110$ Oconce............. 76-77,130-131 Shetucket .......... 76-77,130-131 Spring Creek . 76-77, 126-127, 190-191 Springfield 76-77, 130-131, 201-202 'Test wells ............... 126-127 130-133,136-137, 140-141, 189-190, $200,202-210,212-213,228-235,249$ diagrams showing.. $34,36,5$ Watts Pond.. 76-77, 136-137, 231-232 Wantagh....... 78-79, 116-147,274

New York City department of watersupply (Queens Borough),

Bayside....... 80-81, 134-135, 218-219 College Point. See Fresh

Meadow.

Flushing See Bayside.

Fresh Meadow........... 78-79 $132-133-215-216$ Long Island City No. 1..... 78-79, Long Island City No. $2 \ldots . \begin{array}{r}122 \cdot 123 \\ 78-79\end{array}$

130-131
Long Island City No. 3... . . 78-79 $124-125,184-185$ Whitestone No. 1........ 80-81, Whitestone No. 2......... $80-81$, $134-135,220$

New York Quarantine Station... 118 New York Quinine and Chemical

Co ............. 122-123, 179 New York Sanitary Utilization Co .............. 66, 126-127, 188 Newton, E. H. . . . . . . . . . . 158-159 Newton, Nelson. . . . . . . . 158-159, 312 Newton, R. W........... 158-159,311 Newwitter \& Migel . . . . . . . . . . 126-127 Nichol, J. W ........... 162-163, 324,353 Nichols Chemical Co . . . . . . . . 122-123 Noback, Frederick........ 156-157, 309 Nort IIouse . . . . . ........ 158-1.59, 314 North Country Club....... 144-145, 263 North Shore Industrial Co. 322, 362-363 Northport Waterworks Co.... 82-83, 154-155,300-301 ........... 160-161, 349 Norton, Jas............. 148-149,281 Nostrand, Frant............... 144-145 Obermeyer \& Liebmann....... 120-121 O'Kiefe, Ed................ 124-125
Page.

eld Point........... 160-161,328 O'Leary, D............. 138-139,240 Orient Manufacturing Co.. 166-167,335 Overton, Irving. . ...... 158-159,312,319 Overton, J. J . . . . . . . . . . . 160-161,319 Oysteman's Dock Co........... 148-149 Parker, J. E . . . . . . . . . . . . 166-167, 335 Parks, W. G............ 142-143,255 Parsons, Fred................ 156-157 Payne, C. W ... . . . . . . 164-167, 328, 334 Payne, J. B............. 156-157,308 Pedrick, C. B . . . . . . . . . . 156-157,308 Pennsylvania, New York and Long Island Railroad.... 122-123, 182 Peter Cooper Glue Co...... 122-123,178 Pfalzgraf, H. C., estate. ....... . 80-81 $118-119,169$

Pfeizer Chemical Co .....120-121, 173-174 Pierce, Winslow .......... 150-151, 280 Place, Howard. . . . . . . . . . . . 140-141 Plunkett, G. E.......... 158-159,311 Port Jefferson Co. . . .... 19, 160-16i, 320 Port Jefferson Fire Co..... 160-161,320 Port Jefferson Water Co........ 84-85, 160-161,319-320 Port Washington Catholic Church ............. 140-141, 244, 262 Post, W. J . . . . . . . . . . 144-145, 261, 282 Post, Mrs. - .................. 164-165 Powcll, L. F . . . . . . . . . . . . 144-145 Pratt estate .. 64, 82-83, 144-145, 264-265 Prico, William........... 146-147,267 Provost, D. C............... 134-135 Quantuck Water Co. . 81-85, 164-166,327 Qutens Borough. See New York City department of water supply (Queens Borough).

Queens County Wuter Co ...... 24,66, $77,82-83,130-131,136-137,200,224-228$ diagram showing ........... 36 Quinan, - . . . . ......... 148-149, 279 Ralston, William.......... 158-159,311 Rassapeaque Club. ....... 156-157, 309 Raynor, Benjamin........ 162-163,323 Raynor, Ellsworth ........ 162-163,325 Raynor, Jacob. . .......... 162-163, 325 Raynor, M. E........... 162-163, 324 Raynor, Preston. . ........ 162-163, 324 Raynor, Wallace .......... 162-163,323 Reboul, H. W . . . . . . . . . . 158-159, 310 Recknagle, C. F ............... 138-139 Reed, J................... 140-141 Reid, $-\ldots \ldots \ldots \ldots \ldots \ldots \ldots$ 164-165, 328 Reynolds, - ........... 160-161, 317 Rice, J. H ................ 138-139 Richter, Mrs. Max . . . . . . . 160-161,318 Rivererest sanitarium..... 128-129, 196 diagram showing .......... 58 Riverhead waterworks . . . . . . 84-85, Roberts, C. R........... 158-159, 310 Robinson, J. J............ 154-155, 300 Robinson, Mrs. -............ 162-163 Robinson Bros................ 122-123 Rockville Center.... 82-83, 140-141, 250 Rogers, W. C........ 162-163, 325, 355 
Page

Ronkonkoma............... 158-159 Roosevelt, E..........66, 150-151, 285 Roosevelt, Theodore...... 152-153, 294 Rowland, Woodhull.... 19, 158-159,314 Rowley, Edward............. 156-157 Rushmore, Henry......... 148-149, 279 Ryan, Mrs. Mary . . . . . . . . . . . . 124-125 Ryder, A. O........... 164-165, 330 Sag Harbor Waterworks Co . . . . 81-85, $166-167,334$ Sagaponak................. 166-167 St. John's Protectory...... 148-149, 276 St. Joseph's in the Pines. . . 156-157, 307 St. Paul School.............. 142-143 Sammis, J. M............ 148-149, 280 diagram showing............ 38 Sandford, Howell. ......... 164-165, 325 Sanford, J. A., \& Sons ......... 120 $166-167,333-334$ Saxe, Jerome . . ...... 158-159,313,340 Sayville............... 160-161,315 Scharman, H. B., \& Sons...... 120-121 Schrciber, A. . . . . ........ 142-143, 251 Schroiber, C............. 136-137, 231 Schwarting, D.......... 160-161,317 Scott, Mrs. M. E . . . . . . 134-135, 222, 237 Sca Cliff Hotel. . . . . . . . . . 160-161,315 Sea Cliff Water Co . . 82-83, 144-145, 262 Seaman, L. A., estate.......... 138-139 Seeman, S . .............. 144-145, 263 Seitz, N., Sons........... 122-123, 178 Seizer, Robert. ............ 140-141,243 Sembler, Adolf ............ 160-161,318 Shaw, J. M. . . . . . . . . . . 160-161,321 Shaw, S. T. . ..... 66, 150-151,285, 309 Shaw, Sydney ............. 164-165,326 Shelter Island Heights $A$ ssocia-

tion ......... 84-85, 164-165,330-331 Sherman, C. S .......66, 150-151,285 diagram showing

Shipman, William, estate...... 158-159 Shultz, J.H., Co.............. 120-12 Siebrecht, Wm........... 124-125, 18 Simpson, T. J., Co............ 136-137 Small, Lorenzo \&.............. 140-141

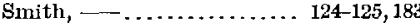
Smith, Brewster............ 156-157 Smith, C. D . . . . . . . . . . . 156-157, 309 Smith, D. W.............. 148-149,281 Smith, E. M ............ 156-157,309

Smith, F. J.......... 154-155,301,327 Smith, F. W ................ 166-167 Smith, J. M............ 140-141,267 Smith, J, Otis . .....156-157 Smith, R. II............. 158-159,310 Smith, Victor F . . . . . . . . . . . 156-157 Smith, W............. 150-151 Smith, W. Frank......... 162-163,323 Society of St. Johnsland ... 158-159, 309 Soper, A. C., \& Co...... 154-155, 299, 325
Page. Southard, C. H. . . . . . . . . . $\begin{array}{r}164-165,329 \\ 142-143,251\end{array}$ Standard Oil Co.... 122-123, 180, 181, 191 Stearns, J. N. . . . . . . . . . . . . 164-165,330 Steele, Alfred . . . . . . ........ 162-163,323 Steinart, Joseph........... 148-149,276 Steinhert, Auglustus. ... 124-125, 183, 276 Steinway \& Soń........ 82-83, 128-129 Still, E. S . . ... . . . . . . . Stimpson, H. L. . . . . . 150-151, 289-290 diagram showing.......... 57 Stonebanks, - $\ldots . . . . . . . .130-131$ Stowe, W.......... 144-145, 259-260 Streeter \& Dennison. . . . . . . . . 122-123 Strong, - . . . ............15.3-157,306 Sumpwams Water Co ......... . 82-83, $154-155,303-304$ Swan, Edward........... 150-151,284 Sweeney Manufacturing Co... 120-121 Talmon, Sarah.............. 152-153 Tangeman, J. P. . . . . . . . . . 144-145, 264 Tartar Chemical Co.... 118-119, 170-171 Terry, A. P.............. 160-161,317 Tesla, Nikola.......... 162 163,321-322 Thane, - . .............. 164-165,328 Thatcher, John........... 160-161,315 Thomas, M. S........... 142-143, 251 Thompson, Edward....... 154-155, 304 Thompson, w. P.......... 148-149, 277 Tiffany, L. C. . . ...... 152-153,292,294 Titus, John . . . . . . . . . . . . 150-151, 289 Totten, H. G............... 156-157 Touscher, L . . . . . . . . . . 134-135, 224 Townsend, E. M............. 150-151 Townsend heirs. . . . . . 72, 148-149, 281 diagram showing ........... 38 Transit Development Co....... 118Travis, V. P $\ldots \ldots \ldots \ldots$ 138-139, 242 Trotter, William......... 150-151,284 Ulmer, $-\ldots \ldots \ldots \ldots \ldots$ 164-165, 330, 360 Underhill, Townsend........... 72 , $148-149,280,283$ Valentine, W. M.......... 144-145, 264 Valentine, Theodore... . . . . . 140-141, $144-145,243,260-261$

Van Iderstine, P., Sons. . . . 154-155, 302 Van Sise \& Co........ 148-149,280-281 Van Wyke heirs ........... 152-153,291

Vanderbilt, Charles........ 140-141,243 - Vanderbilt, W. K. . . . . . . . . . 158-159 Vanderbilt, W. K., jr........... 21, $66,67,138-139,238-239$
diagrams showing ......... 58,61 Vanoski, Frank . . .......... 140-141,244 Vowman, Mrs. I . . . . . . . . . . 144-145, 260 Wakeman, E. L........... 132-133,214 Wallace, Howard ......... 158-159, 314 Walsh, F. K............... 134-135
Page. Walthers, Max. ............. 164-165 Ward, Barclay .. 21, 24, 152-153, 295-296 Ward's shipyards . . . . . . . . . 126-127 Warden, J. S............ 162-163,351 Wardenclyffe Brick and Tile Co.. 162Warner, Charles . . ....... 162-163, 325 Warner, W. H............ 158-159,312 Water Mill ....... . . . . . . . . 164-165, 329 Watt, T. C............. 144-155, 282 Webb, T. E. . . . . . . . . . . . 140-141,244 Weber, J................. 158-159, 311 Weber, John . . . . . . . . . . . . 164-165, 330 Weeks, Charles........... 148-149, 280 Wells, C. H............... 162-163, 325 Wells, J. M. . . . . . . . . . . 164-165, 331 Wendell, J ............ 164-165, 327 West Brooklyn Water Co.. 118-119, 178 West Sayville.............. 158-159 Westbury Colored Childrens'

Home................ 148-149, 276 Westcott Express Co...... 124-125, 183 Westinghouse Electric Co. 122-123, 18 Wetmore, C. W....... 65, 150-151, 286 Wheeler, S. W . . . . . . . . . 162-163, 322 Whitaker, E. G.......... 164-165, 329 White, Mrs. Coles . ........148-149 White, Thomas F., Co. . 66, 126-127, 188 White, Wm.............. 152-153, 292 White Lead Co ..... 126-127, 18 Whitestone. See New York City department of water supply (Queens Borough).

Whitney, W. C............ 144-145, 259 Wier, L. C................ 146-147,268 Willets, E. C............. 138-139, 239 Willetts, F. E........... 144-145, 263 Willetts, Walter.......... 144-145, 261 Willey, C. A., \& Co ....... 124-125, 183 Williams, T. S . . . . . . . 65, 152-153, 294 Willis, T.............. 148-149, 278 Wilson, G. B............. 134-135, 222 Winthrop, F. R ............. 148-149 Winthrop, Robert............ 148-149 Witherspoon \& Co. ............ 124-125 Wonder, Mrs. $\_\ldots \ldots \ldots . .124-125,185$ Wood, Mrs. Welton........ 152-153, 291 Wood, Wilton........... 152-153,295 Woodhaven Water Supply Co... 80-81, Woodruff, A. $J \ldots \ldots \ldots . .126-127,192$ Woodside Water Co ......... 162-163 $128-129,194,195,197$
19. Wortman, H............. 142-144, 251 Wright, W. De F............. 140-141 Yetter \& Moore........... 164-165, 328 Young, Wesley........... 162-163, 323 Young \& Metzner............ 124-125 Young Bag Co............... 124-125 Zabriskie, A ugustus ......... 164-165, 326 Zabriskie, George.......... 140-141,245 


\title{
CLASSIFICATION OF THE PUBLICATIONS OF THE UNITED STATES GEOLOGICAL SURVEY.
}

\author{
[Professional Paper No. 44.]
}

The serial publications of the United States Geological Survey consist of (1) Annual Reports, (2) Monographs, (3) Professional Papers, (4) Bulletins, (5) Mineral Resources, (6) Water-Supply and Irrigation Papers, (7) Topographic Atlas of the United States-folios and separate sheets thereof, (8) Geologic Atlas of the. United States-folios thereof. The classes numbered 2, 7, and 8 are sold at cost of publication; the others'are distributed free, A circular giving complete lists may be had on application.

Most of the above publications may be obtained or consulted in the following ways:

1. A limited number are delivered to the Director of the Survey, from whom they may be obtained, free of charge (except classes 2,7 , and 8 ), on application.

2. A certain number are delivered to Senators and Representatives in Congress, for distribution.

3. Other copies are deposited with the Superintendent of Documents, Washington, D. C., from whom they may be had at practically cost.

4. Copies of all Government publications are furnished to the principal public libraries in the large cities throughout the United States, where they may be consulted by those interested.

The Professional Papers, Bulletins, and Water-Supply Papers treat of a variety of subjects, and the total number issued is large. They have therefore been classified into the following series: $A$, Economic geology; B, Descriptive geology; C, Systematic geology and paleontology; D, Petrography and mineralogy; E, Chemistry and physics; F, Geography; G, Miscellaneous; H, Forestry; I, Irrigation; J, Water storage; K, Pumping water; L, Quality of water; M, General hydrographic investigations; N, Water power; O, Underground waters; P, Hydrographic progress reports. This paper is the seventy-first in Series $B$ and the thirty-ninth in Series $O$, the complete lists of which follow. ( $\mathrm{PP}=$ Professional Paper; $\mathrm{B}=$ Bulletin; WS =Water-Supply Paper. ).

\section{SERIES B, DESCRTPTIVE GEOLOGY}

B 23. Observations on the junction between the Eastern sandstone and the Keweenaw series on Keweenaw Point, Lake Superior, by R. D. Irving and T. C. Chamberlin. 1885. 124 pp., 17 pls. (Out of stock.)

B 33. Notes on geology of northern California, by J. S. Diller. 1886. 23 pp. (Out of stock.)

B 39. The upper beaches and deltas of Glacial Lake Agassiz, by Warren Upham. 1887. $84 \mathrm{pp} ., 1 \mathrm{pl}$. (Ont of stoek.)

B 40. Changes in river courses in Washington Territory due to glaciation, by Bailey Willis. 1887. $10 \mathrm{pp} ., 4 \mathrm{pls}$. (Out of stock.)

B 45. The present condition of knowledge of the geology of Texas, by R. T. Hill. 1887. 94 pp. (Out of stock.)

B 53. The geology of Nantucket, by N. S. Shaler. 1889, 55 pp., 10 pls. . (Out of stock.)

B 57. A geological reconnaissance in southwestern Kansas, by Robert Hay. 1890.49 pp., 2 pls.

B 58. The glacial boundary in western Pennsylyania, Ohio, Kentucky, Indiana, and Illinois, by G. F. Wright, with introduction by T. C. Chamberlin. 1890.112 pp., 8 pls. (Out of stock.)

B 67. The relations of the traps of the Newark system in the Now Jersey region, by N. H. Darton. 1890. 82 pp. (Out of stock.)

B 104. Glaciation of the Yellowstone Valley north of the Park, by W. H. Weed. 1893. 41 pp., 4 pls.

B 108. A.geological reconnaissance in central Washington, by I. C. Russell. 1893. 108 pp., 12 pis. (Out of stock.)

B 119. A geological reconnaissance in northwest Wyoming, by G. H. Eldridge. $1894.72 \mathrm{pp} .4 \mathrm{pls}$.

B 137. The geology of the Fort Riley Military Reservation and vicinity, Kansas, by Robert Hay. 1896.35 pp.. 8 pls.

B 144. The moraines of the Missouri Coteau and their attendant deposits, by J. E. Todd. 1896.71 pp., 21 pls.

B 158. The moraines of southenstem Sonth Dakota and their attendant deposits, by J. E. Todd. $1899.171 \mathrm{pp}$., $27 \mathrm{pls.}$

B 159. The geology of eastern Berkshire County, Massachusetts, by B. K. Emerson. 1899. 139 pp., 9 pls.

B 165. Contributions to the geology of Maine, by H. S. Willams and H. E. Gregory. 1900. 212 pp., 14 pls.

Ws 70. Geology and water resources of the Patrick and Goshen Hole quadrangles in eastern Wyoming and westem Nebraska, by G. I. Adams, 1902.50 pp., 11 pls.

B 199. Geology and water resources of the Snake River Plains of Idaho, by I. C. Russell. 1902. $192 \mathrm{pp} ., 25 \mathrm{plg}$.

PP 1. Preliminary report on the Ketchikan mining district, Alaska, with an introductory sketch of the geology of southeastern Alaska, by A. H. Brooks. 1902.120 pp., 2 pls.

PP 2. Reconnaissance of the northwestern portion of Seward Peninsula, Alaska, by A. J. Collier. $1902.70 \mathrm{pp} ., 11 \mathrm{pls}$.

PP 3. Geology and petrography of Crater Lake National Park, by J. S. Diller and H. B. Patton. 1902.167 pp., 19 pla. 
PP 10. Reconnaissance from Fort Ifamlin to Kotzebue Sound, Alaska, by way of Dall, Kanuti, Allen, and Kowak rivers, by W. C. Mendenhall. 1902.68 pp., 10 pls.

PP 11. Clays of the United Stutes east of the Mississippi River, by Heinrich Ries. 1903.298 pp., 9 pls.

PP 12. Geclogy of the Globe copper district, Arizona, by F. L. Runsome. 1903. 168 pp., 27 pls.

PP 13. Drainage modifications in southeastern Ohio and adjacent parts of West Virginia and Kentucky, by W. G. Tight. 1903. 111 pp., 17 pls.

B 208. Descriptive geology of Nevada south of the fortieth parallel and adjacent portions of California, by J. E. Spurr. 1903. 229 pp., 8 pls.

B 209. Geology of Ascutney Mountain, Vermont, by R. A. Daly. 1903. 122 pp., 7 pls.

WS 78. Preliminary report on artegian basins in southwestern Idaho and southeastern Oregon, by I. C. Russell. 1903.51 pp., 2 pls.

PP 15. Mineral resources of the Mount Wrangell district, Aluska, by W. C. Mendenhall and F. C. Schrader. 1903.71 pp., 10 pls.

PP 17. Preliminary report on the geology and water resources of Nebraska west of the one hundred and third meridian, by N. H. Darton. 1903.69 pp., 43 pls.

B 217. Notes on the geology of southwestern Idaho and southeastern Oregon, oy I. C. Russell. $1903.83 \mathrm{pp} ., 18 \mathrm{pls}$.

B 219. The ore deposits of Tonopah, Nevada (preliminary report), by J. E. Spurr. 1903. 31 pp., 1 pl.

PP 20. A reconnaissance in northern Alaska in 1901, by F. C. Sehrader. 1904.139 pp., 16 pls.

PP 21. The geology and ore deposits of the Bisbee quadrangle, Arizona, by F. L. Ransome. $1904.168 \mathrm{pp}$., $29 \mathrm{pls}$.

WS 90. Geology and water resourees of part of the lower James River Valley, South Dakota, by J. E. Todd and C. M. Hall. 1904. $47 \mathrm{pp}, 23 \mathrm{pls}$

PP 25. The copper deposits of the Encampment district, Wyoming, by A. C. Spencer. 1904. 107 pp., 2 pls.

PI 26. Economic resources of the northern Black Hills, by J. D. Irving, with contributions by S. F. Emmons and T. A. .Taggar, jr. 1904.222 pp., 20 pls.

PP 27. Geological reconnaissunce across the Bitterroot Range and Clearwater Mountains in Montana and Idaho, by Waldemar Lindgren. 1904. $122 \mathrm{pp},, 15 \mathrm{pls}$.

PP 31. Preliminary report on the geology of the Arbuckle and Wichita mountains in Indian Territory and oklahoma, by J. A. Taff, with an appendix on reported ore deposits in the Wichita Mountains, by H. F. Bain. $1904.97 \mathrm{pp}, 8$ pls.

B 235. A geological reconnaissance across the Cascade Range near the forty-ninth parallel, by G. O. Smith and F. C. Calkins. 1904. 103 pp., 4 pls.

B 236. The Porcupine placer district, Alaska, by C. W. Wright. $1904.35 \mathrm{pp} ., 10 \mathrm{pis}$.

B 237. Igneous rocks of the Highwood Mountains, Montana, by L. V. Pirsson. 1904. 208 pp., 7 pls.

B 238. Econornic geology of the lola quadrangle, Kansas, by G. I. Adams, Erasmus Haworth, and W. R. Crane. 1904.83 pp., 1 pl.

PP 32. Geology and underground water resources of the central Great Plains, by N. H. Darton. 1905.433 pp., 72 pls.

WS 110. Contributions to hyd rology of eastern United States, 1904; M. G. Fuller, geologist in charge. $1905.211 \mathrm{pp} ., 5 \mathrm{pls}$.

B 242. Geology of the Hudson Valley between the Hoosic and the Kinderhook, by T. Nelson Dale. $1904.63 \mathrm{pp} ., 3 \mathrm{pls}$.

PP 34. The Delavan lobe of the Lake Michigan Glacier of the Wisconsin stage of glaciation and associated phenomena, by W. C. Alden. 1904, 106 pp., 15 pls.

PP 35, Geology of the Perry Basin in southeastern Maine, by G. O. Smith and David White. 1905. 107 pp., 6 pls.

B 243. Cement muterials and industry of the United States, by E. C. Eckel. 1905. 395 pp., 15 pls.

B 246. Zine and lead deposits of northeastern Illinois, by H. F. Bain. 1904. 56 pp., 5 pis.

B 247. The Fuirhaven gold placers of Seward Peninsula, Alaska, by F. H. Moffit. 1905.85 pp., 14 pls.

B 249. Limestones of southwestern Pennsylvania, by F. G. Clapp. $1905.52 \mathrm{pp}$, 7 pls.

B 250. The petroleum fields of the Pacific coast of Alaska, with an account of the Bering River coal deposit, by G. C. Martin. 1905. $65 \mathrm{pp}, 7 \mathrm{pls}$.

B 251. The gold placers of the Fortymile, Birch Creek, and Fairbanks regions, Alaska, by L. M. Prindle. 1905.89 pp.,16 pls. WS. 1.18. Geology and water resources of a portion of east-central Washington, by F. C. Calkins. $1905.96 \mathrm{pp.,} 4$ pls.

B 252. Preliminary report on the geology and water resources of central Oregon, by I. C. Russell. $1905.138 \mathrm{pp} ., 24 \mathrm{pls}$.

PP 36. The lead, zinc, and fuorspar deposits of western Kentucky, by E. O. Ulrieh and W.S. Tangier Smith. 1905.218 pp., 15 pls.

PP 38. Economic geology of the Bingham mining district of Utah, by J. M. Boutwell, with a chapter on areal geology, by Arthur Keith, and an introduetion on general geology, by S. F. Emmons. 1905. 413 pp., 49 pls.

PP 41. The geology of the central Copper River region, Alaska, by W. C. Mendenhall. 1905.

B 254. Report of progress in the geological resurvey of the Cripple Creek district, Colorado, by Waldemar Lindgren and F. L. Ransome. $1904.36 \mathrm{pp}$.

B 255. The fluorspar 1eposits of southern Illinois, by H. Foster Bain. $1905.75 \mathrm{pp}, 6 \mathrm{pls}$.

B 256. Nineral resources of the Elders Ridge quadrangle, Pennsylvania, by R. W. Stone. 1905.85 pp., 12 pls.

B 257 . Geology and paleontology of the Judith River beds, by T. W. Stanton and J. B. Hatcher, with a chapter on the fossil plants, by F. H. Knowlton. 1905. 174 pp., 19 pls.

PP 42. Geology of the Tonopah mining district, Nevada, by J. E. Spur. 1905.295 pp., 24 pls.

WS 123. Geology and underground water conditions of the Jornada del Muerto, New Mexico, by C. R. Keyes. 1905. 42 pp., 9 pls.

Ws 136. Underground waters of Salt River Valley, Arizona, by W. T. Lee. 1905.196 pp., 24 pls.

PP 43. The copper deposits of the Clifton-Morenci district, Arizona, by Waldemar Lindgren. 1905.372 pp., 25 pls.

B 265. Geology of the Boulder district, Colorado, by N. M. Fenneman. 1905. 101 pp., 5 pls.

B 267. The copper deposits of Missouri, by H. Foster Bain and E. O. Ulrich. 1905. $52 \mathrm{pp}, 1 \mathrm{pl}$.

PP 44. Underground water resources of Long Island, New York, by-A. C. Veatch, C. S. Slichter, Isaiah Bowman, W. O. Crosby, and R. F. Horton. 1906. 394 pp., 34 pls. 


\section{SERIES O, UNDERGROUND WATERS.}

WS 4. A reconnaissance in southeastern Washington, by I. C. Russell. 1897, 96 pp., 7 pls. (Out of stock.)

WS 6. Underground waters of southwestern Kansas, by Erasmus Haworth. 1897. 6a pp., 12 pls. (Out of stock.)

WS 7. Seepage waters of northern Utah, by Samuel Fortier. 1897. 50 pp., 3 pls. (Out of stock.)

WS 12. Underground waters of southeastern Nebraska, by N. H. Darton. 1898. 56 pp., 21 pls. (Out of stock:)

WS 21. Wells of northern Indiana, by Frank Leverett. $1899.82 \mathrm{pp}, 2 \mathrm{pls}$.

Ws 26. Wells of southern Indiana (Continuation of No. 21), by Frank Leverett. 1899. $64 \mathrm{pp}$

WS 30. Water resources of the I.ower Peninsula of Michigan, by A. C. Lane. 1899. 97 pp., 7 pls. (Out of stock.)

WS 31. Lower Michigan mineral waters, by A. C. Lane. $1899.97 \mathrm{pp}, 4 \mathrm{pls}$

WS 34. Geology und water resources of a portion of sontheastern South Dakota, by J. E. Todd. $1900.34 \mathrm{pp} ., 19 \mathrm{pls}$

WS 53. Geology and water resources of Nez Perces County, Idaho, Pt. I, by I. C. Russell. 1901. 86 pp., 10 pls.

WS 54. Geology and water resources of Nez Perces Count,y, Idaho, Pt. II, by I. C. Rissell. 1901. $87-141$ pp.

WS 55. Geology und water resources of a portion of Yakima County, Wash., by G. O. Smith. $1901.68 \mathrm{pp} ., 7 \mathrm{pls}$ WS 57. Freiiminary list of deep borings in the United States, Pt. 1, by N. H. Darton, 1902. 60 pp. (Out of stock.) WS 59. Development and application of water in southern California, Pt. I, by J. B. Lippincott. 1902, 95 pp., 11 pls. (Ou1 of stock.)

Ws 60. Development and application of water in southern California, Pt. II, by J. B. Lippineott. 96-140 pp. (Out of stock.)

WS 61. Preliminary list of deep borings in the United States, Pt. II, by N. H. Darton. 1902. 67 pp. (Out of stock.)

WS 67. The motions of underground waters, by C. S. Slichter. $1902.106 \mathrm{pp}, 8 \mathrm{pls}$.

B 199. Geology and water resources of the Snake River Plains of Idaho, by I. C. Russell. $1902.192 \mathrm{pp} ., 25 \mathrm{pls}$.

WS 7 . Water resources of Molokai, Hawaian Islands, by Waldemar Lindgren, 1903. 62 pp., 4 pls.

WS 78. Preliminary report on artesian basins in southwestern Idaho and southenstern Oregon, by I. C. Russell. 1903. 51 pp., 2 pls.

WS 90. Geology and water resources of part of the lower James River Valley, South Dakota, by J. E. Todd and C. M. Hall. 1904. 45 pp., 23 pls.

WS 101. Underground waters of southern Louisiana, by G. D. Harris; with discussions of their uses for water supplies and for rice irrigation, by M. L. Fuller. 1904. $98 \mathrm{pp.,} 11 \mathrm{pls}$

WS 102. Contributions to the hydrology of eastern United States, 1903, by M. L, Fuller. 1904. $522 \mathrm{pp}$

Ws 104. The underground waters of Gila Valley, Arizona, by Willis T. Lee. 1904. 71 pp., 5 pls.

WS 106. Water resourees of the Philadelphia district, by Florence Bascom. 1904. 75 pp., 4 pls.

Ws 110. Contributions to the hydrology of eastern United States, 1904; M. L. Fuller, geologist in charge. 1904.211 pp., 5 pls.

PP 17. Preliminary report on the geology and water resources of Nebraska west of the one hundred and third meridian, by N. H. Darton. 1903 . 69 pp., 43 pls.

PP 32. Prelimintry report on the geology and underground water resources of the central Great Plains, by N. II, Darton. 1905. 433 pp., 72 pls.

WS 111. Preliminary report on underground waters of Washington, by Henry Landes. $1905.85 \mathrm{pp} ., 1 \mathrm{pl}$.

WS 112. Underflow tests in the drainage basin of Los Angeles River, by Homer Hamlin. $1905.55 \mathrm{pp} .7 \mathrm{pls}$.

WS 114. Underground waters of eastern United States, by M. L. Fuller und others. 1905.285 pp., 18 pls.

WS 118. Geology and water resources of east-central Washington, by F. C. Calkins. 1905. 96 pp., 4 pls.

B 252. Preliminary report on the geology and water resources of central Oregon, by I. C. Russell, $1905.138 \mathrm{pp}, 24 \mathrm{pls}$.

WS 120. Bibliographic review and inclex of papers relating to underground waters published by the United States Geologícal Survey, 1879-1904, by M. L. Fuller. 1905. 128 pp.

WS 122. Relation of the law to underground waters, by D. W. Johnson. $1905.55 \mathrm{pp}$.

WS 123. Geology and underground water conditions of the Jornada del Muerto, New Mexico, by C. R. Keyes. 1905.42 pp., $9 \mathrm{pls}$

WS 136. Underground waters of Salt River Valley, Arizona, by W. T. Lee. $1905.196 \mathrm{pp} ., 23 \mathrm{pls.}$

B 264. Record of deep-well drilling for 1904, by M. L. Fuller, E. F. Lines, and A. C. Veateh. $1905.106 \mathrm{pp}$

PP 44. Underground water resources of Long Island, New York, by A. C. Veatch, C. S. Slichter, Isaiah Bowman, W. O. Crosby, and R. E. Horton. 1906. 394 pp., 34 pls.

The following papers also relate to this subject: Underground waters of Arkansas Valley in eastern Colorado, by G. $\mathrm{K}$. Gilbert, in Seventeenth Annual, Pt. II; Preliminary report on artesian waters of a portion of the Dakotas, by N. H. Darton, in Seventeenth Annual, Pt. II; Water resources of Illinois, by Frank Leverett, in Seventeenth Annual, Pt. II; Water resources of Indiana and Ohio, by Frank Leverett, in Eighteenth Amnua!. Pt. IV; New developments in well boring and irrigation in eastern South Dakota, by N. H. Darton, in Eighteenth Annual, Pt. IV; Rock waters of ohio, by Edward Orton, in Nineteenth Annual, Pt. IV; Artesian well prospects in the Atlantic coastal plain region, by N. H. Darton, Bulletin No: 138

Correspondence should be addressed to

\section{The Director,}

United States Geological Sukvex;

Februaky, i.906.

Washington, D. C. 




\section{LIBRARY}

$$
\begin{aligned}
& \text { BHofoll 115 Mined } \\
& \begin{array}{l}
\text { BRARY } \\
\text { SPIJANE AASH. }
\end{array} \\
& \text { JUN - } 11971 \\
& \text { - Jasto } \\
& \text { DIEASE RETORE }
\end{aligned}
$$

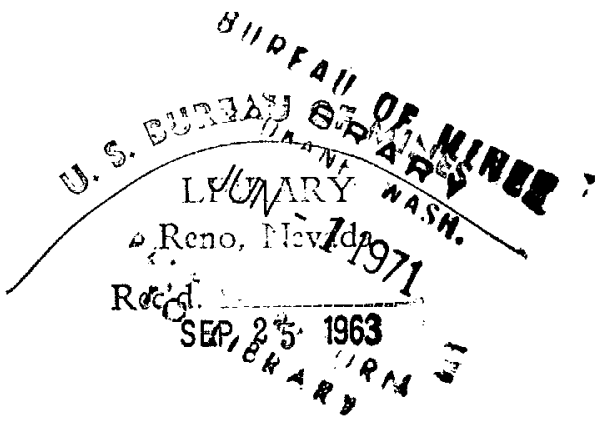

